\title{
Trends in Opioid Prescribing for Non-Cancer Pain and Associated Resource Utilisation in Wales
}

Submitted to Swansea University in fulfilment of the requirements for the Degree of Doctor of Philosophy

Emma Davies

BSc MPharm

Copyright: The author, Emma Davies, 2021. 

Yn olaf, mae'n cael ei wneud 



\section{Summary}

\section{Background}

Opioid prescribing in the UK has increased significantly since the start of the millennium and has been associated with a rise in chronic pain reporting. In Wales, despite concern about rising rates of opioid analgesic prescribing, no detailed examination of the data had been undertaken to assess the changes in prescribing and its consequent impact on the population.

\section{Methods}

In this study, anonymised, individual level data of people diagnosed with noncancer pain in Wales was extracted from the Secure Anonymised Information Linkage (SAIL) Databank and used to scrutinise opioid analgesic prescribing trends in people aged 18 years and over, establish whether legislation or clinical guidance impacted on those trends and examine associations with increased healthcare use. The study was conducted in two phases. Phase 1 included a retrospective, repeated cross-sectional analysis of opioid analgesics issued from Primary Care, stratified by gender, age and socioeconomic status. Phase 2 of the study evaluated differences in healthcare service use and costs between individuals receiving opioids for defined non-cancer pain-related diagnoses and matched patients not receiving opioids.

Results

Total opioid prescribing increased by $43.6 \%$ and strong opioids by $306.2 \%$ between 2005 and 2015. Women received 1.5 times more prescriptions than men. Increasing age was associated with higher prescribing rates. People in the most deprived areas received 2.4 times more prescriptions than in least deprived. People receiving opioid prescriptions accessed primary care four times more frequently than controls and had twice the number of hospital admissions. Opioid prescription was associated with $41 \%$ higher healthcare costs than noted in controls.

\section{Conclusion}

This research highlights the need to develop a national strategy to address pain management and opioid stewardship in Wales. We must consider how to address the wide variability observed, particularly between areas of differing socioeconomic status. Further research should investigate what underlies continued opioid prescribing and how alternative strategies can be implemented in practice to reduce population harm and optimise the use of limited healthcare resources. 


\section{Declaration}

This work has not previously been accepted in substance for any degree and is not being concurrently submitted in candidature for any degree.

Signed

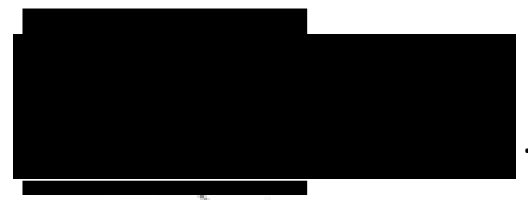

(candidate)

Date ...24 September 2021

\section{Statement 1}

This thesis is the result of my own work, except where otherwise stated. The work for this thesis was carried out at the College of Health and Human Sciences, Swansea University, Wales with data extracted from the Secure Anonymised Information Linkage (SAIL) Databank, College of Medicine, Swansea University. Other sources are acknowledged giving explicit references. A bibliography is appended.

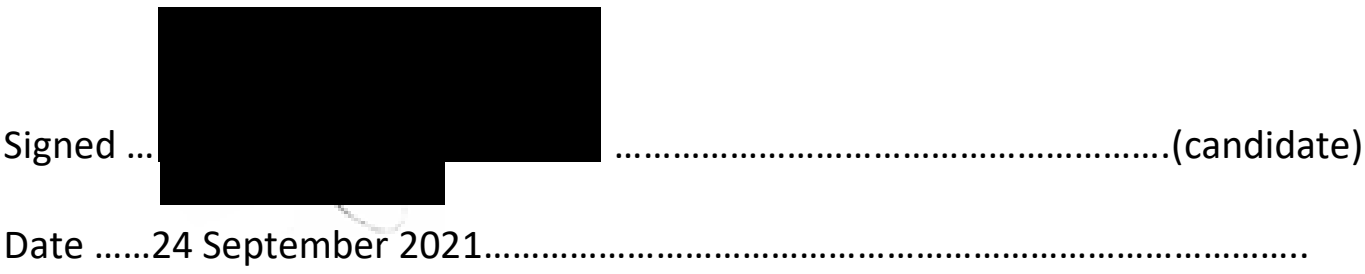

\section{Statement 2}

I hereby give consent for my thesis, if accepted, to be available for photocopying and for inter-library loan, and for the title and summary to be made available to outside organisations.

Signed ...

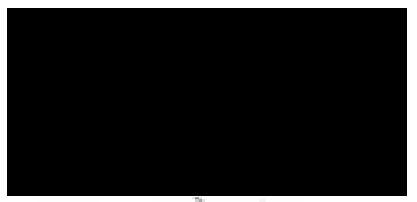

(candidate)

Date

24 September 2021 


\section{Table of contents}

Acknowledgements $\quad 1$

List of Tables $\quad 2$

List of Figures $\quad 4$

List of abbreviations $\quad 6$

$\begin{array}{lr}\text { Personal Statement } & 10\end{array}$

$\begin{array}{ll}\text { Chapter } 1 \text { - Introduction } & 12\end{array}$

1.1 Chapter overview $\quad 12$

1.2 Defining pain 13

1.3 Defining chronic pain 14

1.4 Global burden of pain $\quad 16$

1.4.1 Prevalence of Chronic Pain in the United Kingdom 19

$\begin{array}{ll}\text { 1.4.2 The situation in Wales } & 20\end{array}$

$\begin{array}{ll}1.4 .3 \text { Economic burden of pain } & 21\end{array}$

1.5 Pain management in general $\quad 22$

1.6 Contemporary use of opioids $\quad 24$

1.6.1 Acceptability of opioids in non-cancer pain $\quad 25$

$\begin{array}{ll}\text { 1.6.2 Arguments in support of opioids } & 27\end{array}$

1.6.3 Are opioids effective in all types of pain? 30

1.6.4 Why are opioids prescribed? $\quad 31$

1.7 Adverse effects of opioids

1.7.1 Effects associated with duration of opioid use $\quad 33$

1.7.2 Quality of life $\quad 34$

1.8 Gender differences in pain $\quad 35$

1.9 Does age impact on pain?

1.10 Inequality and deprivation $\quad 40$

1.10.1 Determinants of health in the Welsh population? 42

1.10.2 Prudent healthcare in Wales $\quad 43$

1.10.3 Links between pain and deprivation $\quad 44$

1.11 Role of legislation and guidelines in controlling opioid analgesic prescribing 46

1.11.1 Does anyone know how much opioid is too much? 48

1.12 Why is research needed in Wales?

1.13 Route into research

Chapter 2 - Methods $\quad 55$

2.1 Chapter overview $\quad 55$

2.1.1 What are the aims of this research?

2.2 Research objectives $\quad 56$

2.3 Research strategy $\quad 56$

2.4 Study Design

2.4.1 The SAIL Databank $\quad 58$

2.4.2 Anonymisation process and security $\quad 59$

2.4.3 Why the SAIL databank chosen for this study $\quad 60$

$\begin{array}{ll}2.5 \text { Ethical considerations } & 61\end{array}$

$\begin{array}{ll}\text { 2.5.1 Project Application, process and approvals } & 62\end{array}$ 
2.6 Data compilation $\quad 65$

2.6.1 Identifying Phase 1 study subjects $\quad 65$

2.6.2 Exclusion of individuals with cancer diagnoses $\quad 66$

2.6.3 Drug classification $\quad 66$

$\begin{array}{ll}\text { 2.6.4 Age and age-group formation } & 67\end{array}$

2.6.4.1 Age-group population calculations $\quad 68$

2.6.5 Standardising population estimates 68

2.6.5.1 Gender population standardisation $\quad 69$

2.6.5.2 Population standardisation for deprivation quintiles $\quad 70$

2.6.6 Condition-linked data $\quad 70$

$\begin{array}{ll}\text { 2.6.7 Data extraction and analysis } & 71\end{array}$

$\begin{array}{ll}\text { 2.6.8 Data security and quality assurance } & 73\end{array}$

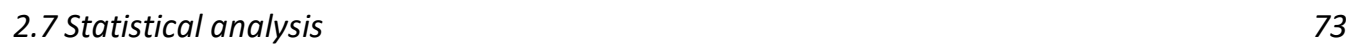

$\begin{array}{ll}\text { 2.7.1 Spearman's rank correlation } & 74\end{array}$

$\begin{array}{ll}\text { 2.7.2 Mann-Whitney U tests } & 75\end{array}$

$\begin{array}{ll}2.7 .3 \text { Kruskal-Wallis tests } & 75\end{array}$

$\begin{array}{ll}\text { 2.7.4 Time series analysis } & 76\end{array}$

Chapter 3 - General trends in opioid prescribing for non-cancer pain 78

$\begin{array}{ll}3.1 \text { Chapter overview } & 78\end{array}$

3.2 Study objective to be addressed in this chapter 78

$\begin{array}{ll}3.3 \text { Background } & 78\end{array}$

$\begin{array}{ll}3.4 \text { Method } & 79\end{array}$

$\begin{array}{ll}3.4 .1 \text { Trend analysis } & 79\end{array}$

3.4.2 Determining prescription persistence $\quad 80$

$\begin{array}{ll}3.4 .2 .1 \text { Trends analysis } & 80\end{array}$

3.4.2.2 Stratification of prescribing persistence data $\quad 80$

3.5 Results

3.5.1 Overview of prescribing trends $\quad 81$

3.5.2 Trends in prescribing by type of opioid analgesic $\quad 82$

3.5.3 Trends in prescribing by gender $\quad 83$

3.5.4 Prescribing by age $\quad 85$

3.5.5 Prescribing persistence $\quad 89$

$\begin{array}{ll}3.6 \text { Discussion } & 92\end{array}$

3.6.1 Summary and reflection on findings $\quad 92$

3.6.2 Comparison of general opioid prescribing trends in the UK 93

3.6.3 Weak versus strong opioids 96

$\begin{array}{ll}\text { 3.6.4 People versus prescriptions } & 97\end{array}$

3.6.5 Gender differences in opioid prescribing 98

3.6.6 Age differences in opioid prescribing 99

$\begin{array}{ll}\text { 3.6.7 Prescribing persistence or duration } & 100\end{array}$

$\begin{array}{ll}3.7 \text { Conclusion } & 102\end{array}$

Chapter 4 - Trends in prescribing by socio-economic deprivation 105

$\begin{array}{ll}4.1 \text { Chapter overview } & 105\end{array}$

4.2 Study objective to be addressed in this chapter 105

$\begin{array}{ll}4.3 \text { Background } & 105\end{array}$

4.4 Methods 106

$\begin{array}{ll}\text { 4.4.1 Welsh Index of Multiple Deprivation } & 106\end{array}$ 
4.4.2 Identification of socio-economic deprivation areas $\quad 108$

4.4.3 Calculation of WIMD populations 108

$\begin{array}{ll}\text { 4.4.4 Missing data } & 109\end{array}$

4.4.5 Data extraction and analysis 109

4.4.6 Log-linear regression 110

$\begin{array}{ll}4.5 \text { Results } & 110\end{array}$

4.5.1.1 All opioids $\quad 110$

4.5.1.2 Weak opioids $\quad 112$

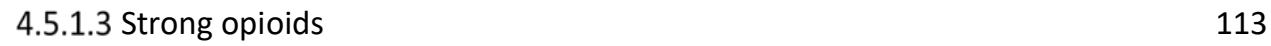

4.5.2 Gender differences $\quad 115$

$\begin{array}{ll}\text { 4.5.2.1 All opioids } & 115\end{array}$

$\begin{array}{ll}\text { 4.5.2.2 Weak opioids } & 117\end{array}$

$\begin{array}{ll}\text { 4.5.2.3 Strong opioids } & 120\end{array}$

$\begin{array}{ll}\text { 4.5.3 Log-linear regression outcomes } & 123\end{array}$

$\begin{array}{ll}\text { 4.6 Discussion } & 124\end{array}$

4.6.1 Summary and reflection on findings $\quad 124$

4.6.2 Contextualising results with existing literature $\quad 125$

$\begin{array}{ll}\text { 4.6.3 Gender differences and deprivation } & 131\end{array}$

$\begin{array}{ll}4.7 \text { Conclusion } & 134\end{array}$

Chapter 5 - Prescribing trends in oral morphine equivalent dose 136

$\begin{array}{ll}5.1 \text { Chapter overview } & 136\end{array}$

5.2 Study objective to be addressed in this chapter 136

$\begin{array}{ll}5.3 \text { Background } & 136\end{array}$

$\begin{array}{ll}5.4 \text { Method } & 140\end{array}$

5.4.1 Data extraction analysis $\quad 140$

$\begin{array}{ll}5.4 .2 \text { Opioid prescriptions } & 140\end{array}$

$\begin{array}{ll}\text { 5.4.3 Choice of opioid } & 141\end{array}$

5.4.4 Estimated oral morphine equivalent dose (OMEQ $) \quad 141$

5.4.5 Development of an estimated OMEQ 143

$\begin{array}{ll}5.5 \text { Results } & 144\end{array}$

$\begin{array}{ll}\text { 5.5.1 Number of prescriptions } & 144\end{array}$

5.5.2 Prescribing trends by oral morphine equivalence dose (OMEQ $)$

5.5.3 Gender differences in oral morphine equivalent dose (OMEQ $) \quad 149$

5.5.3.1 Gender differences in $\mathrm{OMEQ}_{\mathrm{e}}$ by drug $\quad 149$

5.5.3.2 Gender differences in $\mathrm{OMEQ}_{\mathrm{e}}$ per prescription $\quad 153$

5.5.4 Differences in oral morphine equivalent dose by deprivation 156

5.5.4.1 Differences in high dose oral morphine equivalent dose by deprivation $\quad 159$

5.5.5 Comparison of prescription numbers and $\mathrm{OMEQ}_{\mathrm{e}} \quad 161$

5.6 Discussion 163

5.6.1 Summary and reflection on findings 163

$\begin{array}{ll}\text { 5.6.2 Comparison with the literature } & 165\end{array}$

$\begin{array}{ll}\text { 5.6.2.1 Choice of opioid } & 165\end{array}$

5.6.2.2 Comparison of trends in opioid burden 167

$\begin{array}{ll}5.7 \text { Conclusion } & 172\end{array}$

Chapter 6-Time Series Analysis examining the effect of legislation and prescribing guidance on $\begin{array}{ll}\text { opioid prescribing in Wales } & 175\end{array}$

$\begin{array}{ll}6.1 \text { Chapter overview } & 175\end{array}$

6.2 Study objective to be addressed in this chapter $\quad 175$ 
$\begin{array}{ll}6.3 \text { Background } & 175\end{array}$

6.4 Method 176

6.4.1 Forms of time series analysis $\quad 176$

6.4.2 Calculating the moving mean and centred-moving mean for TSA 178

6.4.3 Seasonal adjustment $\quad 179$

6.4.4 Predicting future issue of prescriptions $\quad 180$

6.4.5 Legislation changes in the UK between 2005 and $2015 \quad 181$

6.4.6 Prescribing and condition guidance 183

$\begin{array}{ll}\text { 6.4.7 Prescription charges } & 184\end{array}$

6.5 Results $\quad 185$

6.5.1 Legislation and guidance changes $\quad 185$

6.5.2 Interrupted Time Series Analysis $\quad 187$

6.5.2.1 Weak opioids 188

6.5.2.2 Strong opioids 190

6.5.2.3 Changes examined by gender 191

6.5.2.4 Changes by socioeconomic deprivation $\quad 192$

6.5.2.5 Seasonal variation 193

$\begin{array}{ll}6.5 .3 \text { Predictions } & 194\end{array}$

6.6 Discussion 195

6.6.1 Summary and reflection on findings $\quad 195$

6.6.2 Is TSA a useful means of predicting prescribing trends or changes? 197

6.6.3 Effects of legislation on prescribing trends 198

6.6.4 Abolition of prescription charges $\quad 200$

6.6.5 Effects of national prescribing indicators and guidelines on prescribing trends 200

$\begin{array}{ll}6.7 \text { Conclusion } & 203\end{array}$

Chapter 7 - Healthcare resource utilisation and cost-analysis associated with opioid prescribing

206

$\begin{array}{ll}7.1 \text { Chapter overview } & 206\end{array}$

7.2 Study objectives to be addressed in this chapter 206

$\begin{array}{ll}7.3 \text { Background } & 207\end{array}$

$\begin{array}{ll}\text { 7.3.1 Efficiency } & 207\end{array}$

$\begin{array}{ll}\text { 7.3.2 Welfarism } & 208\end{array}$

$\begin{array}{ll}\text { 7.3.3 Extra-welfarism } & 208\end{array}$

7.3.4 What approach do we take to Heath Economic Analysis in Wales and the UK? 209

$\begin{array}{ll}\text { 7.3.5 Equity and equality } & 209\end{array}$

$\begin{array}{ll}\text { 7.3.6 Use of healthcare services } & 209\end{array}$

$\begin{array}{ll}7.4 \text { Method } & 210\end{array}$

$\begin{array}{ll}\text { 7.4.1 Study design } & 210\end{array}$

$\begin{array}{ll}\text { 7.4.2 Cohort identification } & 210\end{array}$

$\begin{array}{ll}\text { 7.4.2.1 Case Subject group } & 210\end{array}$

7.4.2.2 Control subject group $\quad 211$

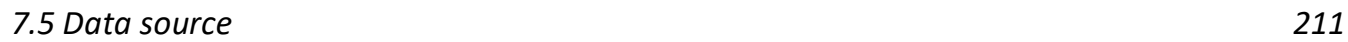

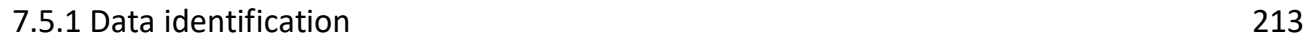

$\begin{array}{ll}7.6 \text { Cost Analysis } & 214\end{array}$

7.6.1 Costs included in the analysis $\quad 214$

$\begin{array}{ll}\text { 7.6.2 Cost of healthcare resource use } & 214\end{array}$

$\begin{array}{ll}7.7 \text { Statistical analysis } & 217\end{array}$

$\begin{array}{ll}\text { 7.7.1 Student's t-test } & 217\end{array}$

$\begin{array}{ll}\text { 7.7.2 One-way analysis of variance (ANOVA) } & 217\end{array}$

$\begin{array}{ll}\text { 7.7.3 Two-way ANOVA } & 218\end{array}$ 
7.7.4 Multiple linear regression

$\begin{array}{ll}\text { 7.7.5 Bonferroni-Holm correction } & 220\end{array}$

$\begin{array}{ll}\text { 7.7.6 Sensitivity analysis } & 221\end{array}$

$\begin{array}{ll}\text { 7.7.7 Threshold analysis } & 221\end{array}$

$\begin{array}{ll}7.8 \text { Results } & 222\end{array}$

$\begin{array}{ll}\text { 7.8.1 Primary Care attendance } & 223\end{array}$

$\begin{array}{ll}7.8 .2 \text { Out-Patient attendance } & 225\end{array}$

$\begin{array}{ll}\text { 7.8.3 Emergency Department Utilisation } & 227\end{array}$

7.8.4 In-Patient attendance and Length of Stay $\quad 229$

7.9 Regression Analysis $\quad 233$

7.9.1 Primary Care General Practice attendance $\quad 234$

7.9.2 Out-patient attendance $\quad 236$

$\begin{array}{ll}\text { 7.9.3 Emergency Department attendance } & 237\end{array}$

$\begin{array}{ll}\text { 7.9.4 Number of In-patient admissions } & 237\end{array}$

$\begin{array}{ll}7.10 \text { Cost Analysis } & 238\end{array}$

$\begin{array}{ll}\text { 7.10.1 Total Healthcare costs } & 238\end{array}$

$\begin{array}{ll}\text { 7.10.2 Costs for specified services } & 238\end{array}$

7.10.3 Examining costs by demographic factors $\quad 241$

$\begin{array}{ll}\text { 7.10.3.1 Gender } & 241\end{array}$

$\begin{array}{ll}\text { 7.10.3.2 Deprivation } & 241\end{array}$

7.10.3.3 Prescribing Persistence $\quad 243$

$\begin{array}{ll}\text { 7.10.3.4 One-way sensitivity analysis } & 243\end{array}$

7.10.3.5 Two-way sensitivity analysis $\quad 245$

$\begin{array}{ll}7.11 \text { Discussion } & 247\end{array}$

7.11.1 Summary and reflections on findings $\quad 247$

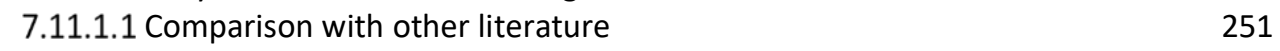

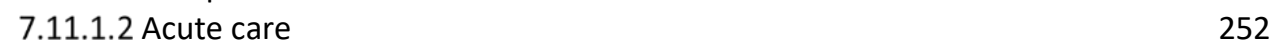

7.11.1.3 Emergency department utilisation $\quad 253$

$\begin{array}{ll}\text { 7.11.1.4 Prescription persistence and dose } & 254\end{array}$

7.11.1.5 Comparisons of costs 254

7.11.1.6 Healthcare use associated with pain, opioid analgesics, and demographic $\begin{array}{ll}\text { factors } & 257\end{array}$

$\begin{array}{ll}7.12 \text { Conclusion } & 261\end{array}$

Chapter 8- Discussion $\quad 264$

8.1 Chapter overview 264

8.2 Summary of study results $\quad 265$

8.3 Why are opioids prescribed? $\quad 267$

$\begin{array}{ll}\text { 8.3.1 Waiting for help } & 267\end{array}$

8.3.2 Does 'ability to pay' affect prescribing? $\quad 270$

8.3.3 Why might the legislation not have 'worked' in the UK? 270

8.3.4 Are current methods of monitoring prescribing fit for purpose? 272

8.3.4.1 Is there benefit to using OMEQ to monitor opioid prescribing burden? $\quad 274$

8.3.5 Are the trends in resource utilisation and costs a sign of equality in healthcare? 275

8.3.6 Will guidelines change prescribing in the future? 276

8.4 Policy direction for pain management and analgesic stewardship in Wales 279

8.4.1 Drug monitoring and prescribing databases $\quad 281$

8.4.2 Learning from our Celtic cousins 286

8.5 Strengths of this research $\quad 292$

8.5.1 Impact and influence of this research 293

8.6 Limitations of the research presented 294 
8.6.1 Limits of data extraction

8.6.2 Absence of dispensing data 296

8.6.3 Defining variables $\quad 297$

8.6.4 Absence of qualitative data 298

8.7 Ideas for future research 299

8.7.1 Integrating prescribing and dispensing data 299

8.7.2 Professional differences in prescribing 299

$\begin{array}{ll}\text { 8.7.3 Socioeconomic deprivation } & 300\end{array}$

8.7.4 Gender differences in opioid prescribing 301

8.7.5 Race and intersectional differences in prescribing 301

8.7.6 Determining effects of long-term opioid prescribing 302

$\begin{array}{ll}\text { 8.7.7 Cost-effectiveness studies } & 303\end{array}$

8.7.8 Examining outcomes 303

8.8 Study Conclusion 303

$\begin{array}{ll}\text { References } & 309\end{array}$

Appendices in Volume 2 360 


\section{Acknowledgements}

I would like to thank above all, my supervising team, Professors Ceri Phillips and Jaynie Rance who supported and guided this research and encouraged me to continue through some difficult times. Drs Berni Sewell and Mari Jones, who took me on and have been incredibly patient, helped shape my thinking and made me believe I could be a researcher after all.

In the College of Health and Human Sciences, Maria Davis and Katie Retallick for their tireless support of all of us post-grads. Maddie, Sara, Caitlin, Carol, Alyson and Amy for their friendship, laughter and coffee.

Thanks also to my 'housekeeper', Dr Cathy Price, who is always there when I need her and was again. Dr Karen Hodson, who has guided me throughout my career and continues to do so. Professor Ann Taylor and Dr Sue Jeffs for reminding me to pull myself together and get on with it! Similarly, Dr Roger Knaggs, who has quietly cleared the path for me and many others to follow.

I gratefully acknowledge the funding from Pharmacy Research UK. This report is independent research, commissioned by Pharmacy Research UK. The views expressed in this publication are those the author and not necessarily those of Pharmacy Research UK. OPharmacy Research UK, 2nd November 2015. I would particularly like to thank Desh Mofidi for providing me with support and advice, well beyond his job description.

Thank you, Research Capacity Building Collaboration Wales (RCBC), for providing financial support for my PhD fellowship and personal support through the Community of Scholars. Special mention to Nic, Liba, Helen, Nicole, Jess and Ed.

This study makes use of anonymized data generated by the Secure Anonymized Information Linkage (SAIL) system, which is part of the national e-health records research infrastructure for Wales. I would like to acknowledge all the data providers who make anonymised data available for research and also the staff who undertook the data extraction on my behalf.

Finally, Chris, Gethin and Awen xxx 


\section{List of Tables}

Table 1.1: Comparison of domains used to develop indices of deprivation for each country in the United Kingdom

Table 2.1: Study categorisation of weak and strong opioids

Table 2.2: Representation of General Practice within SAIL Databank by Health Board 68

Table 2.3: Principal metadata for prescription trend analysis (TOPAS_20170419) 72

Table 3.1: Changes in number of prescriptions issued between 2005 and 2015

Table 3.2: Gender trends in opioid prescription rates during the study period, 2005 - $2015 \quad 84$

Table 3.3: Change in rates of prescribing (adjusted to population) issued 2005 to 2015, stratified

by age-group

Table 3.4: Demographics for prescribing persistence adjusted to population

Table 4.1: Components of the Welsh Index of Multiple Deprivation (WIMD) (Welsh Government 2011)

Table 4.2: Comparison of quintile-population adjusted numbers of prescriptions for all opioid medicines by Welsh Index of Multiple Deprivation quintiles across Wales between 2005 and 2015.

Table 4.3: Comparison of quintile-population adjusted numbers of prescriptions for weak opioid medicines by Welsh Index of Multiple Deprivation quintiles across Wales between 2005 and 2015.

Table 4.4: Comparison of quintile-population adjusted numbers of prescriptions for strong opioid medicines by Welsh Index of Multiple Deprivation quintiles across Wales between 2005 and 2015.

Table 4.5: Comparison of quintile-population adjusted numbers of prescriptions for all opioid medicines by Welsh Index of Multiple Deprivation quintiles and gender across Wales between 2005 and 2015

Table 4.6: Comparison of quintile-population adjusted numbers of prescriptions for weak opioid medicines by Welsh Index of Multiple Deprivation quintiles and gender across Wales between 2005 and 2015

Table 4.7: Comparison of quintile-population adjusted numbers of prescriptions for strong opioid medicines by Welsh Index of Multiple Deprivation quintiles and gender across Wales between 2005 and 2015.

Table 4.8: Output from log-linear regression to determine variables which may increase the likelihood of receiving a strong opioid analgesic prescription

Table 5.1: Example of calculations for OMEQe (milligrams) using 2005 data for female subjects. Annualised total $=$ oral morphine equivalent of daily dose $\mathrm{x}$ annual number of prescriptions. Process repeated for each drug product e.g., buprenorphine $10 \mathrm{mcg} /$ hour patch, BuTrans $10 \mathrm{mcg} /$ hour patch and totalled for each year

Table 5.2: Examples of oral morphine equivalent (OME) dosing tables, used to develop OMEproxy measures for comparing opioid burden for the TOPAS study. The tables below are reproduced from Opioids Aware (April 2020) (Faculty of Pain Medicine 2021)

Table 5.3: Trends in the number of opioid prescriptions per 1,000 population $2005-2015$, stratified by drug

Table 5.4: Daily oral morphine equivalent dose (milligrams) issued on prescription, adjusted to 1000 population, stratified by drug

Table 5.5: Trends in Total Annualised Oral Morphine Equivalent Doses (milligrams) per 1,000 population issued by gender. P-values calculated using Mann-Whitney tests for each drug

Table 5.6: Trends in oral morphine equivalent dose (milligrams) per prescription issued and by drug, stratified by gender. P-values calculated using Mann-Whitney tests 
Table 5.7: Trends in total oral morphine equivalent dose prescribed stratified by deprivation (Welsh Index of Multiple Deprivation) and adjusted to population

Table 5.8: Trends in oral morphine equivalence stratified by deprivation (Welsh Index of Multiple Deprivation WIMD2011) and examined by dose per prescription and dose per person 158

Table 5.9: Trends in total oral morphine equivalent dose of 120 milligrams or higher prescribed stratified by deprivation (Welsh Index of Multiple Deprivation) and adjusted to deprivation quintile population.

Table 6.1: Output from testing for best-fit model of interrupted time series analysis $\quad 187$

Table 6.2: Results of interrupted time series analysis of opioid prescribing data $\quad 188$

Table 6.3: Results of interrupted time series analysis of weak opioid prescribing data $\quad 189$

Table 6.4: Results of interrupted time series analysis of strong opioid prescribing data $\quad 190$

Table 6.5: Output from Intermittent Time Series Analysis by gender (full data in 0) 191

Table 6.6: Output from Intermittent Time Series Analysis by socioeconomic deprivation quintile (full data in Appendix E)

Table 6.7: Average seasonal effects of opioid prescribing based on time series analysis and displayed by type of opioid prescribed

Table 6.8: Comparison of predicted trends in opioid prescribing using trendline analysis

Table 7.1: Unit costs for calculating Healthcare utilisation

Table 7.2: Demographic data for healthcare utilisation between cases and controls.

Table 7.3: Comparison of Primary Care General Practice utilisation depending on receipt of opioid prescriptions (case versus control)

Table 7.4: Comparison of out-patient resource utilisation depending on receipt of opioid prescriptions (case versus control)

Table 7.5: Comparison of Emergency department resource utilisation depending on receipt of opioid prescriptions (case versus control)

Table 7.6: Comparison of In-patient admission and length of stay depending on receipt of opioid prescriptions (case versus control)

Table 7.7: Output from multiple linear regression to predict Healthcare utilisation by visits or attendances

Table 7.8: Average costings per person for healthcare utilisation comparing all subjects within the study, cases and controls.

Table 7.9: Average costings by gender for healthcare utilisation, comparing cases and controls 241

Table 7.10: Average costings per person by deprivation for healthcare utilisation comparing cases and controls

Table 7.11: Average costings per person for healthcare utilisation comparing all opioid prescribing persistence of less or more than 6 months

Table 7.12: Comparison of costs per person for sensitivity analysis: study estimates of case subject costs compared to re-calculated control subject costs

Table 7.13: Comparison of costs per person for sensitivity analysis: study estimates of recalculated case and control subject costs 


\section{List of Figures}

Figure 1.1: Example literature database search strategy using MESH headings

Figure 1.2 Comparison of Welsh Index of Multiple Deprivation domains, contrasting health (left) with geographical access to services (right) using the 2011 WIMD (darker colours = more deprived) (Welsh Government 2011)

Figure 1.3: The four principles of prudent healthcare in Wales (Welsh Government and NHS Wales 2016)

Figure 1.5: Overview of study including outputs generated up until July 2021

Figure 2.1: SAIL data anonymisation process (Secure Anonymised Information Linkage Databank 2021)

Figure 2.2: Diagrammatic view of data extraction and organisation for phase 1 trend analysis of opioid prescribing $2005-2015$

Figure 2.3: Method for calculating SAIL databank population for the TOPAS study, using Abertawe Tawe Bro Morgannwg University Health Board (ABMUHB) population 2005 as an example factor

Figure 2.4: Calculating gender-adjusted population using male population in 2015 as an example factor

Figure 2.5: Example and explanation of SQL code for data extraction

Figure 3.1: Trends in number of prescriptions per 1,000 issued between 2005 and 2015, stratified by opioid-type

Figure 3.2: Trends in opioid analgesic prescribing rate 2005 to 2015, stratified by gender 84

Figure 3.3: Number of people receiving opioid analgesic prescriptions between 2005 and 2015, stratified by age-group

Figure 3.4: Trends in the number of weak opioid prescriptions issued between 2005 and 2015 and stratified by age-group. Data displayed by number of prescriptions per 1,000 age-group adjusted population per year

Figure 3.5: Trends in the number of strong opioid prescriptions between 2005 and 2015 and stratified by age-group. Data displayed by number of prescriptions per 1,000 age-group adjusted population per year

Figure 4.1: Map showing boundaries of Welsh Health Boards in 2014. These boundaries remained in place until March 2019

Figure 4.2: Trends in the number of all opioid prescriptions issued by deprivation area and adjusted to the population of each quintile of deprivation in Wales

Figure 4.3: Trends in the number of strong opioid prescriptions issued by deprivation area and adjusted to the population of each quintile of deprivation in Wales

Figure 4.4: Trends in the number of all opioid prescriptions issued to men and women by deprivation quintile and adjusted to quintile-population in Wales

Figure 4.5: Annual percentage change in the number of prescriptions for all opioids by each quintile of deprivation in Wales and gender

Figure 4.6: Trends in the number of weak opioid prescriptions issued to men and women by deprivation quintile and adjusted to quintile-population in Wales

Figure 4.7: Annual percentage change in the number of prescriptions for weak opioids by each quintile of deprivation in Wales and gender

Figure 4.8: Trends in the number of strong opioid prescriptions issued to men and women by deprivation quintile and adjusted to quintile-population in Wales

Figure 4.9: Annual percentage change in the number of prescriptions for strong opioids by each quintile of deprivation in Wales and gender

Figure 4.10: Welsh Index of Multiple Deprivation 2019 
Figure 5.1: Comparing Defined Daily Dose (DDD) to Oral Morphine Equivalent dose (OMEQ). Doses of codeine, morphine and oxycodone are equianalgesic

Figure 5.2: Trends in the number of prescriptions of named opioids by percentage of the total number of issued opioid prescriptions

Figure 5.3: Trend in annual total daily oral morphine equivalent dose (milligrams) per 1,000 population, stratified by drug

Figure 5.4: Trends in annualised $\mathrm{OMEQ}_{\mathrm{e}}$ (milligrams) per 1,000 population stratified by gender149

Figure 5.4: Trends in total Oral Morphine Equivalent Dose (milligrams) per 1,000 population, stratified by drug prescribed and examined by gender (Top - men, bottom - women) 152

Figure 5.5: Trends in Oral Morphine Equivalent Dose (milligrams) per prescription issued to men and stratified by drug

Figure 5.6: Trends in Oral Morphine Equivalent Dose (milligrams) per prescription issued to women and stratified by drug

Figure 5.7: Trends in $\mathrm{OMEQ}_{\mathrm{e}}$ (milligrams) per 1,000 population, stratified by deprivation using the Welsh Index of Multiple Deprivation 2011 (WIMD2011)

Figure 5.8: Trends in $\mathrm{OMEQ}_{\mathrm{e}}$ of 120 milligrams or higher per 1,000 population, stratified by deprivation using the Welsh Index of Multiple Deprivation 2011 (WIMD2011)

Figure 5.9: Comparison of the percentage contribution of each opioid prescribed by total prescriptions issued and total daily OMEQ dose $(\mathrm{mg})$ in Wales between 2005 and 2015161

Figure 5.10: Trends in oral morphine equivalence compared to number of prescriptions issued per 1,000 population between $2005-2015$

Figure 6.1: Screenshot of Excel spreadsheet showing calculation of moving means (1) and centred moving means (2) for opioid prescriptions as part of time series analysis

Figure 6.2: Screen shot of Excel spreadsheet showing calculation of average seasonal effect on the number of opioid prescriptions issued from Primary Care Practices registered with the SAIL databank

Figure 6.3: Monthly trend in the number of opioid prescriptions issued from Primary Care Practices who provide data to SAIL databank. Shown with a polynomial $\left(x^{2}\right)$ 'best fit' trendline, $\mathrm{R} 2=0.9882$. Shown with time markers for legislation changes and National Prescribing indicators which included targets for reducing prescribing

Figure 6.4: Plot of the number of opioid prescriptions between January 2005 (month 1) and December 2015 (month 132).

Figure 6.5: Plot of the number of weak opioid prescriptions between January 2005 (month 1) and December 2015 (month 132).

Figure 6.6: Plot of the number of strong opioid prescriptions between January 2005 (month 1) and December 2015 (month 132).

Figure 6.7: Plot of the number of opioid prescriptions issued by gender, between January 2005 (month 1) and December 2015 (month 132).

Figure 6.8: Graphical depiction of average seasonal variation in the number of opioid prescriptions, by type of opioid issued from time series analysis

Figure 6.9: Predicted trends in the number of opioid prescriptions issued using different timeseries trendline analysis

Figure 8.1: Trend of opioid prescribing in Wales from January 2016 - July 2020 using prescription dispensing data taken from CASPA. Linear trend lines added. A - Monthly number of prescriptions with linear trend line. B - Close up of monthly trends that shows similarity with seasonally adjusted data noted from TOPAS study results. 


\title{
List of abbreviations
}

\author{
$95 \% \mathrm{Cl} \quad 95 \%$ Confidence Interval \\ ABMUHB Abertawe Bro Morgannwg University Health Board \\ ABUHB Aneurin Bevan University Health Board \\ ADDE Annual District Death Extract \\ ADE Adverse Drug Event \\ AICc corrected Akaike Information Correction \\ ALF Anonymised Linkage Field \\ ALF-E Anonymised Linkage Field with additional encryption \\ ANOVA Analysis of Variance statistical test \\ BCUHB Betsi Cadwaladr University Health Board \\ BNF British National Formulary \\ CASPA Comparative Analysis System for Prescribing Audit \\ $\mathrm{CCl} \quad$ Charlson Comorbidity Index \\ $\mathrm{CHC} \quad$ Community of Health Councils for Wales \\ CIPHER Centre for Improvement in Population Health through e-Records \\ Research \\ CMM Centred moving mean \\ CNCP Chronic non-cancer pain \\ CNMP Chronic non-malignant pain \\ CP Chronic pain \\ CPRD Clinical Practice Research Datalink \\ CTUHB Cwm Taf University Health Board \\ CVUHB Cardiff and Vale University Health Board \\ DALYs Disability Adjusted Life Years \\ DDD Defined Daily Dose \\ DHCW Digital Health and Care Wales \\ DUP Drug usage period \\ EU European Union \\ FPM Faculty of Pain Management \\ GBD Global Burden of Disease study
}




\begin{tabular}{|c|c|}
\hline GBTM & Group based trajectory model \\
\hline GP & General Practitioner \\
\hline GPPC & General Practice Primary Care \\
\hline HB & Health Board \\
\hline HDUHB & Hywel Dda University Health Board \\
\hline HES & Hospital Episode Statistics \\
\hline HR & Hazard ratio \\
\hline HRG 3.5 & Healthcare Resource Groups version 3.5 (NHS England) \\
\hline HRG 4 & Healthcare Resource Groups version 4 (NHS England) \\
\hline HSE & Health Survey for England \\
\hline ICD-10 & $\begin{array}{l}\text { International Statistical Classification of Diseases and Health- } \\
\text { related problems, } 10^{\text {th }} \text { revision }\end{array}$ \\
\hline IGRP & Information Governance Review Panel (SAIL) \\
\hline ISE & Individual seasonal effect \\
\hline LSOA & Lower Super Output Area \\
\hline MESH & Medical Subject Headings \\
\hline MLE & Maximum likelihood method (for time series analysis) \\
\hline mg & milligrams \\
\hline $\mathrm{MM}$ & Moving mean \\
\hline NICE & National Institute for Health and Care Excellence \\
\hline NPI & National Prescribing Indicator \\
\hline NPIs & National Prescribing Indicators \\
\hline OLS & Ordinary Least Squares \\
\hline OMEQ & Oral morphine equivalent dose \\
\hline $\mathrm{OMEQ}_{\mathrm{e}}$ & Estimated oral morphine equivalent dose \\
\hline ONS & Office for National Statistics \\
\hline OPCS-4 & OPCS Classification of Interventions and Procedures version 4 \\
\hline OR & Odds Ratio \\
\hline $\mathrm{PHIH}$ & Prudent Healthcare Intelligence Hub \\
\hline PP & Prescription period \\
\hline PRUK & Pharmacy Research UK \\
\hline PTHB & Powys Teaching Health Board \\
\hline
\end{tabular}


QALY Quality-adjusted Life Year

RALF Residential Anonymised Linking Field

RMSE Root mean squared error

SAIL Secure Anonymised Information Linkage databank

SIGN Scottish Intercollegiate Guideline Network

SPSS Statistical Package for Social Sciences (historical name, now

commonly just referred to as SPSS)

SQL $\quad$ Structured Query Language

TSA Time Series Analysis

TOPAS Trends in Opioid Prescribing and Associated Resource Utilisation

in Wales

UK United Kingdom

USA United States

WDSD Welsh Demographic Service Dataset

WG Welsh Government

WHO World Health Organisation

WLGP Welsh Longitudinal General Practice

WMID Welsh Index of Multiple Deprivation

YLD Years Lived with Disability 


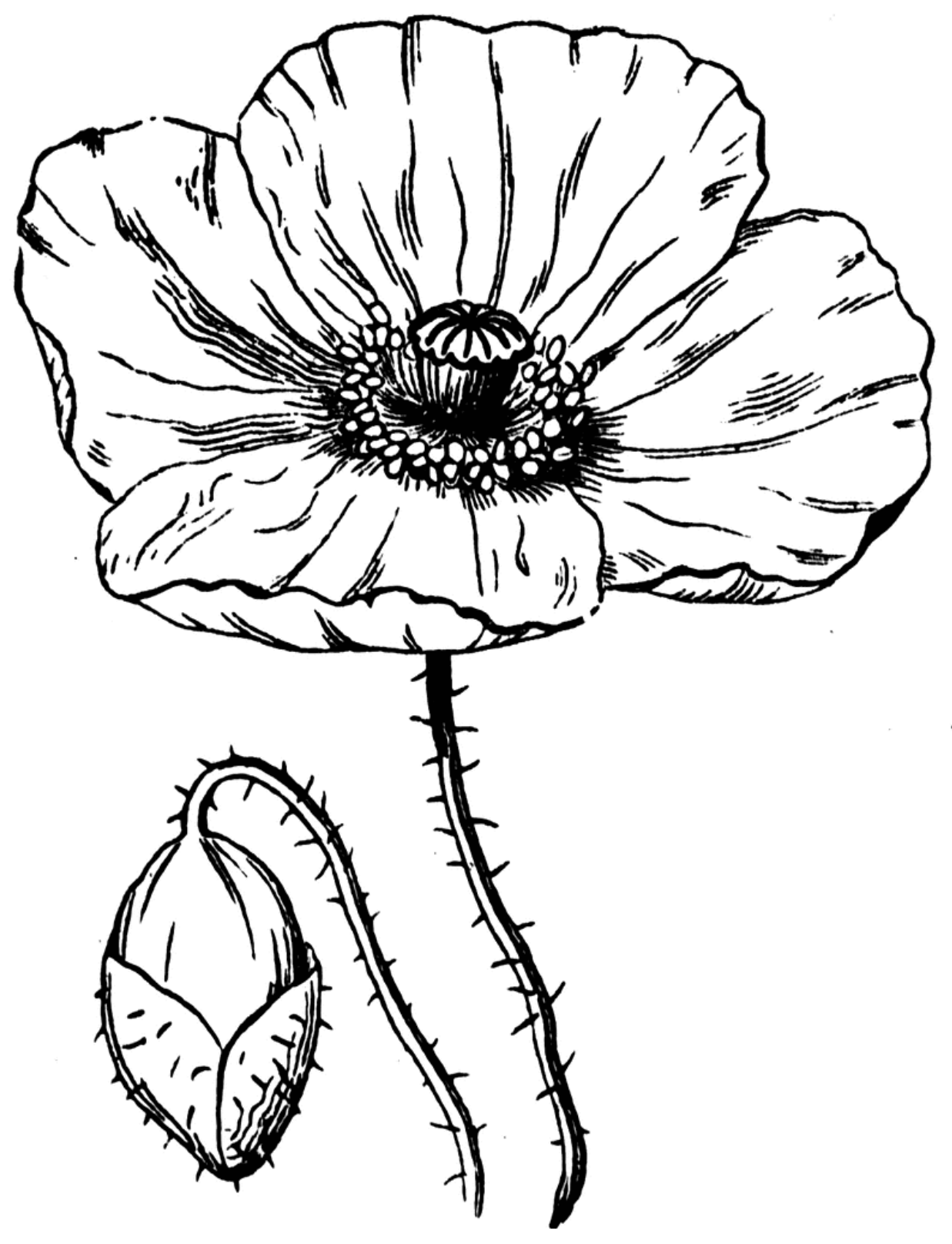

"When pain was unavoidable, we tolerated it. When pain became avoidable, it became intolerable. What we have created, with all of our painkillers and pain management strategies, is an intolerance and increased sensitivity to pain." Joanne Dahl, speaking in Southampton, 2007 


\section{Personal Statement}

I have worked as a pain management specialist pharmacist since early 2006 , working initially under the generous supervision of Dr Cathy Price in Southampton. Shortly after I started working in pain management, concerns from North America about the misuse of prescribed opioid analgesics began to gather pace. This set about a change in chronic pain management around the world. Mindful practitioners began to reassess their own practice and realise that whilst opioids, and analgesics in general, may be helpful for some people, it was perhaps a smaller percentage than had first been thought. Chronic pain management is a long-term pursuit. Enduring harms associated with opioids, including immune dysfunction, endocrine dysfunction, depression, and anxiety all began to feel a little more real. The question of whether opioids do more harm than good has become a topic of conversation at most chronic pain meetings and conferences.

It appears there is consensus in the UK that opioids are unlikely to benefit most people living with persistent pain in the long-term. We must be wary, however, not to remove them from those who do gain advantage. Increasingly, I spend my time in practise supporting people to manage long-term health conditions that opioids have contributed to. People who have been convinced that opioids are their only option, despite little evidence their lives have improved since taking them. Despite these concerns, little information exists about the potential numbers of people developing health problems other than misuse, from prescribed opioids in the United Kingdom. There is nothing at all available from Wales on this issue. Consequently, I had to start at the beginning and establish some baseline data on trends in opioid prescribing in Wales. I then, looked at drug selection and duration of prescribing, as this may help develop better targeted prescribing interventions nationally. Finally, I wanted to examine whether opioid use was associated with greater healthcare use in Wales and how much that was likely to be costing the National Health Service. Having an indication of the likely costs may assist in rethinking how limited funds are best used, supporting the large numbers of people we know live with pain in Wales. 
Chapter 1

Introduction

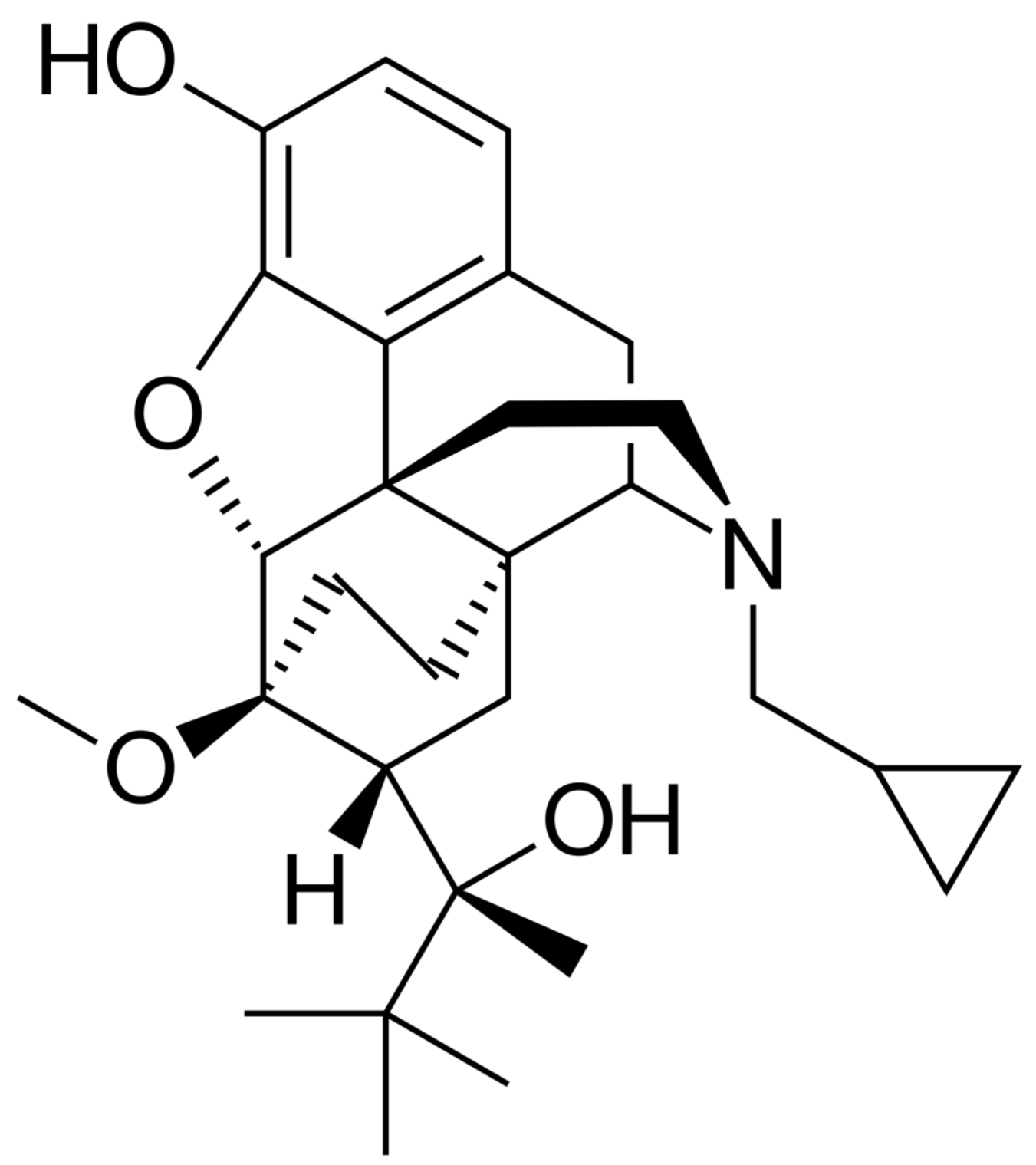

"You can't go back and change the beginning, but you can start where you are and change the ending."

C S Lewis 


\section{Chapter 1 - Introduction}

\subsection{Chapter overview}

This chapter provides a narrative of published literature relating to pain and the use of opioid analgesics. A pragmatic review of the literature was conducted using medical subject headings (MESH) to systematically search literature databases (Figure 1.1). Quality was appraised using Critical Appraisal Skills Programme (CASP) checklists. Snowballing was also used, especially in regard of determining seminal references, and grey literature was searched using Googlescholar and directly on Government and other organisations websites.

Included in this chapter is a discussion of pain, how it is defined and why it is important in societal and economic terms. Then, the management of pain and the place of opioid analgesics in it. In particular, the current acceptability of opioid use for chronic pain is considered, alongside concerns about potential adverse effects and long-term harms. Factors which are considered to impact on the prevalence of pain and consequently, possibly linked to opioid prescribing will also be covered. These include gender, age, socioeconomic deprivation, legislation and clinical guidance. Finally, the chapter ends with an explanation of why the research presented in the thesis was needed and how the research questions were generated. This introduction is intended to set the scene for the rest of the study and will be referred back to as the thesis progresses. 
Database search strategy for Burden of Chronic Pain

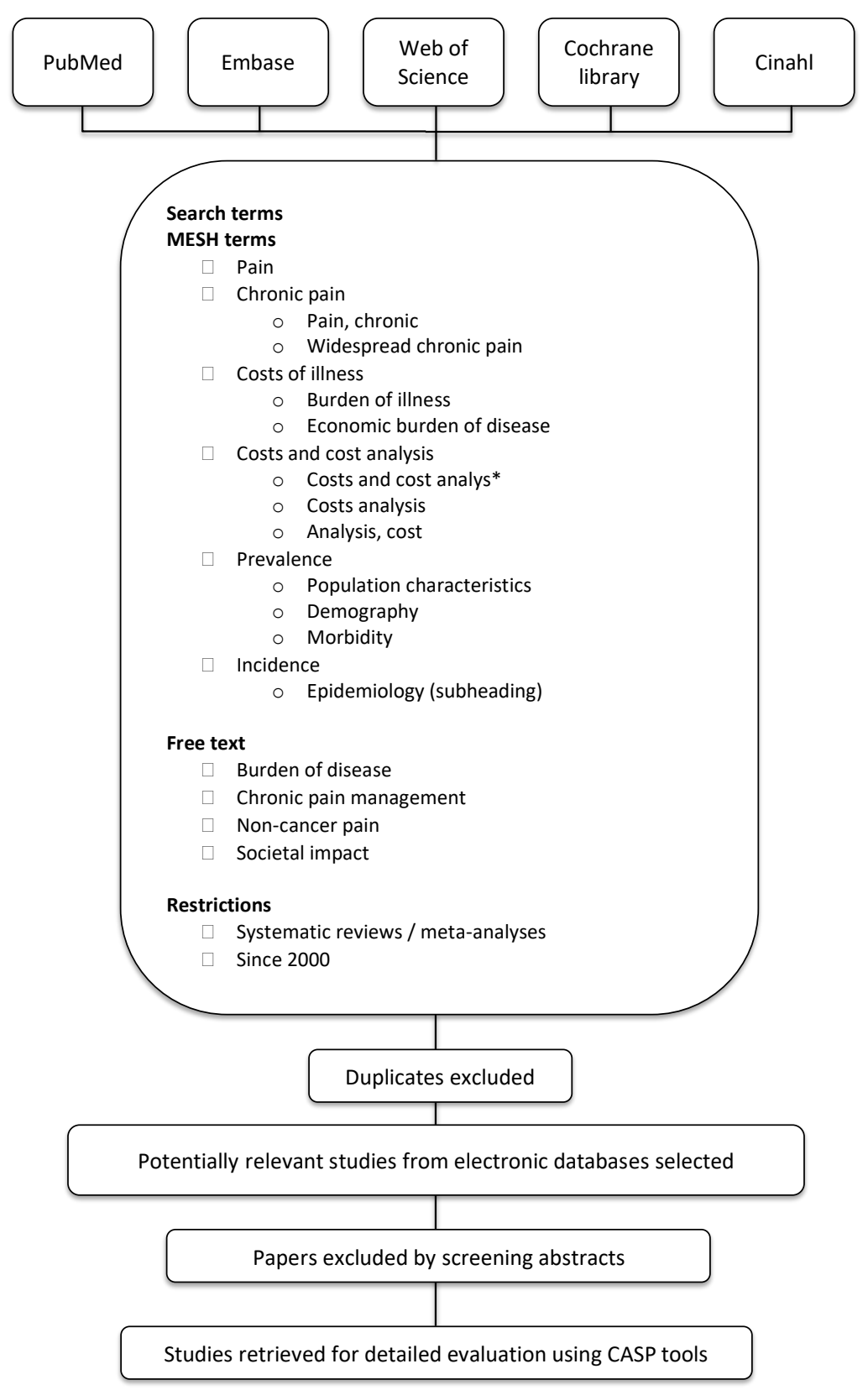

Figure 1.1: Example literature database search strategy using MESH headings

\subsection{Defining pain}

Pain is defined by the International Association for the Study of Pain (IASP) as, 'an unpleasant sensory and emotional experience associated with, or resembling that associated with actual or potential tissue damage' (Nicholas et al. 2019). 
Acknowledging an emotional component to the episode of pain leads to it being subjective and individual to the person having the experience (Eccleston 2011). Consequently, understanding, treating and managing pain from any source becomes reliant on self-report and this places it apart from many other conditions in medicine and undoubtedly is one reason it can be difficult to manage in clinical practice (Raffaeli and Arnaudo 2017).

Pain can be sub-divided into acute, malignant and chronic-non-malignant, which may then be further described by virtue of a known or postulated underlying source (Treede et al. 2015; Nicholas et al. 2019; Smith et al. 2019). Simply put, acute pain is of short duration and commonly caused by trauma of some sort (e.g., standing on a drawing pin, following a sports injury, broken bones or after surgery). The duration of pain expected from acute incidents are generally acknowledged to be related to the time needed to heal (Carr and Goudas 1999; Johnson et al. 2013). Malignant pain arises from cancer and is often referred to as chronic malignant pain, as its duration is generally linked to the period of disease, which is of a long-term nature (Cooney et al. 2013; Lozano-Ondoua et al. 2013; Bennett et al. 2019). Lastly, chronic non-malignant pain (CNMP) describes pain experienced for longer than three months, or beyond the time that healing would have been expected (Nicholas et al. 2019). Chronic non-malignant pain may develop following an episode of acute pain, could be a symptom of an underlying condition such as osteoarthritis or diabetes, but may also be thought of as a condition in its own right (Department of Health 2012) given it often persists after an original insult has resolved or may even occur for seemingly no known reason.

\subsection{Defining chronic pain}

Whilst acute pain is experienced by the majority of people at some point in life, pain that persists is a source of suffering estimated to affect up to $20 \%$ of people worldwide (IASP 2019). Chronic pain is an overarching term, which clinically, can encompass a range of presentations of pain and other symptoms. The International Association for the Study of Pain (IASP) agreed diagnostic criteria 
for a range of chronic pain conditions to be included in the International Classification of Diseases version 11 (ICD-11) and which will come into practice in 2022 (Nugraha et al. 2019a). Chronic primary pain, is newly designated a disease. It is characterised by pain persisting for more than 3 months which, cannot be attributed to another condition (Nicholas et al. 2019). Other symptoms emotional distress and / or functional disability (Nicholas et al. 2019) which has been described by the National Institute of Health and Care Excellence (NICE) as being disproportionate to any underlying pathology, (National Institute for Health and Care Excellence 2021). Conditions included under the diagnostic banner of chronic primary pain include fibromyalgia and complex regional pain syndrome (Nicholas et al. 2019; National Institute for Health and Care Excellence 2021).

Chronic primary pain can co-exist with other chronic pain conditions and IASP have classified six secondary chronic pain conditions. Other new additions to ICD11 include chronic cancer-related pain, caused by cancer or its treatment (Bennett et al. 2019) and chronic post-surgical or post-traumatic pain, which may result from or worsen as a consequence of tissue trauma (Schug et al. 2019). Chronic secondary musculoskeletal pain in bones, joints and tendons (Perrot et al. 2019), visceral pain which originates from internal organs (Aziz et al. 2019) and headache or orofacial pain (Benoliel et al. 2019) are not new classifications but have had characteristics updated for ICD-11. Chronic neuropathic pain has been in previous ICDs, but ICD-11 will now also include peripheral and central neuropathic pain (Scholz et al. 2019).

The new classifications do not guarantee, consensus in relation to defining chronic, non-malignant pain (Treede et al. 2015; Raffaeli and Arnaudo 2017). Indeed, over recent times, even the use of 'chronic' has been called into question and oftentimes, 'persistent pain' will be used as an alternative term (Gureje et al. 1998). Discussion over many years has also questioned whether acute and chronic pain can be differentiated by degrees of 'interference' with functioning (Grichnik and Ferrante 1991; Schofferman 2006; Nugraha et al. 2019). However, 
insufficient agreement on the assessment of interference has been reached and it is accepted that pain of any duration can have a marked impact on a person's ability to continue activities of daily life for the time it is experienced and is not singular to chronic pain (Jennings et al. 2009; Turk et al. 2016; Hayhurst et al. 2018).

From a research perspective, defining chronic non-malignant pain can be inconsistent and can lead to discrepancies in literature, when different classifications are used (Fayaz et al. 2016). Chronic pain as an all-encompassing definition is less commonly used than specific complaints, which may have ongoing pain as a major symptom. Lower back pain for example, would appear more straightforward to recruit for trials and research purposes (Reddy et al. 2012). However, even the area considered to be the lower back differs between studies and whether pain is associated with reduction in activity also (Buchbinder et al. 2013). This has led to authors developing their own definitions for pain and severity and propose them to be used to guide future research (Buchbinder et al. 2013; Hoy et al. 2014).

Pain can be a symptom of disease or injury or considered a condition in its own right (Nicholas et al. 2019; Perrot et al. 2019). Pain is a complex condition, which, despite efforts to better define it, remains contentious diagnostically, particularly when it becomes chronic or persistent. Importantly, pain is a ubiquitous experience and so, has potentially far-reaching consequences for global health.

\subsection{Global burden of pain}

The Global Burden of Disease study (GBD) is an international project, collecting data from more than 200 countries worldwide. It tracks and examines data from 1990 to the present to provide insight into risk factors and disease prevalence, then used to develop national and international policy and initiatives to improve health and wellbeing of the world's population (Institute for Health Metrics and Evaluation 2020). Over the duration the GBD has been running, there has been a noticeable shift in the number of chronic, long-term, non-communicable 
conditions contributing to the overall burden, one measure of which is disability adjusted life years (DALYs). It was reported from the GBD 2019, the burden of HIV/AIDS had substantially dropped since 2004 due to global availability of antiretroviral drugs (Vos et al. 2020). Headache disorders in people aged 25-49 years increased from rank 7 based on DALYs (3.1\% of DALYs) in 2009 to rank 5 (3.7\% of DALYs) in 2019 (Vos et al. 2020). Initially, the distribution was weighted more toward communicable diseases (Vos et al. 2020). Rice, Smith and Blyth (2016) explained the change is partly due to changing demographics in developing and developed countries. Populations are becoming older, more obese and less active. All of which contributes to long-term conditions such as type-2 diabetes, which, may also pertain to pain (Rice et al. 2016). In developing countries, there are increasing numbers of road traffic accidents, and road injuries have remained the number 1 cause of DALYs since 2009 for people aged between 10 and 49 years old worldwide (Vos et al. 2020). Road injuries are also associated with pain and possible persistence of it over time (Rice et al. 2016) although this is not necessarily captured in the GBD. Also, rurality and manual working, being more common in low- and middle-income countries (LMIC), is likely to lead to higher incidence of injury and consequently pain. Aging populations and the continued presences communicable diseases such as HIV/AIDs and herpes zoster, also contribute to a neuropathic pain burden (Blyth 2018; Sharma et al. 2019). However, low personal income and poor accessibility to healthcare affects the actions people in LMICs can take, even when they have pain. Access to opioids is limited for example, with up to $80 \%$ of world consumption in high income countries, predominantly North America (Sharma et al. 2019; Richards et al. 2020).

Using years lived with disability (YLDs) as the measure, taking account of prevalence and severity of disease, usefully demonstrates the rising burden of long-term conditions in a generally aging global population. Simply put, whilst life expectancy has increased worldwide, it has not been mirrored by longer time spent in good health (Rice et al. 2016). As improvements continue to be made in the treatment of infectious conditions, the rise in long-term, pain-associated 
conditions will likely continue unless global policy is developed to address it (Rice et al. 2016; Blyth et al. 2019).

The GBD 2010, reported in 2014 (Hoy et al. 2014) revealed low back pain to be the number one cause of disability worldwide and ranked $12^{\text {th }}$ of the common chronic conditions examined. Whilst ranked first for YLDs it was also ranked $6^{\text {th }}$ for DALYs. Low back pain is established as a major health and economic issue in the UK and other developed countries (Lambeek et al. 2011; Hong et al. 2013) with lifetime prevalence estimated between 51\% to 84\% (Henschke et al. 2015). The GBD 2010 was perhaps the first acknowledgment that low back pain had become a global health issue with large, reported increases in prevalence in developing nations (Hoy et al. 2014a). In part, this was as likely due to advances in data collection globally, as to a major change in pathology. The status of low back pain as the leading cause of YLDs worldwide was confirmed in the 2017 GBD. An estimated increase of 53\% (from 377.5million in 1990 to 577.0 million in 2017) in point prevalence was made for the 27 years, the study examines (Wu et al. 2020). Low back pain was specified the cause of age standardised YLDs for men in 133 of 195 countries and for women in 109 countries in the 2016 study (Blyth and Schneider 2018). In the 2019 GBD, low back pain was ranked $7^{\text {th }}$ and accounted for $3.2 \%$ of DALYs worldwide for people aged $10-24$ years (Vos et al. 2020), further illustrating it to be a condition with a significant and potentially lasting impact.

It is not just low back pain contributing to global pain burden, however. In the 2010 study, the most commonly occurring symptomatic chronic condition was recurrent tension-type headache (Rice et al. 2016). Musculoskeletal pain, which includes at least 150 discrete diagnoses including gout, rheumatoid arthritis, hip and knee osteoarthritis and back and neck pain is also examined as part of the GBD (Blyth et al. 2019). 'Other' musculoskeletal and osteoarthritis were listed in the top 25 conditions for people aged over 50 years in 2019 (Vos et al. 2020), which could be assumed to have pain as a symptom. Falls were the number 8 cause of global DALYs in 2019 for people aged 75 years and over and ranked $16^{\text {th }}$ 
for the $50-74$ years age-group (Vos et al. 2020). The contribution of falls to the experience of pain, is not included however, and herein lies a point of debate, in terms of estimating the true burden of pain, locally or globally (Rice et al. 2016; Blyth and Schneider 2018; Blyth et al. 2019). Given the methodology used to collect and analyse data on a global scale, including navigating various standards of healthcare services and efficiencies of data collection, it is inevitable prevalence figures are in fact an underestimate (Blyth and Schneider 2018). Further, there is a range of conditions for which pain might be experienced and yet, not recorded as part of the study (Rice et al. 2016).

\subsubsection{Prevalence of Chronic Pain in the United Kingdom}

The Chief Medical Officer's (England) report in 2009 estimated 5 million people each year develop chronic pain in the United Kingdom (UK) with an anticipated recovery rate of around 66\% (Chief Medical Officer of England 2009). The National Pain Audit published in 2012 quoted a figure of 7.8 million people living with chronic pain in the UK (Price et al. 2012). This figure, assumed from previous estimates, including a 2006 European survey of chronic pain which gave a prevalence approximation of $13 \%$ (Breivik et al. 2006), relates to people living with moderate to severe levels of pain.

Fayaz et al. (2016) produced an extrapolated estimate of 28 million UK residents living with chronic pain, a figure considerably higher than any previously published and equitable to approximately $43 \%$ of the population (Fayaz et al. 2016). In their meta-analysis, only studies pertaining to the UK were included and this limited included studies to nineteen. Less than half of those examined had been designed as prevalence studies. The resulting figure is not, however, markedly different from prevalence estimations (Tsang et al. 2008) of widespread chronic pain. The Fayaz paper differs from previous estimates such as Breivik's of 13\% (Breivik et al. 2006), gained through direct questioning of people living with pain, by moving the focus away from moderate to severe pain levels, to data relating to chronic, non-cancer pain of any severity. 
The Health Survey for England 2017 examined chronic pain. Results revealed a $34 \%$ prevalence of pain lasting for more than 3 months, in just under 8,000 people aged 16 years and over (England 2017). A similar prevalence was reported in Ireland (35.5\% community prevalence of chronic pain) (Raftery et al. 2011). There was an increase in pain reporting with advancing age and women were disproportionality represented in all age-groups. Pain prevalence exceeded the national average for all adults, at age $45-54$ years, when pain was reported by $39 \%$ of respondents (England 2017).

Racial disparities have been noted in pain reporting in the UK and elsewhere (Jimenez et al. 2011; Janevic et al. 2017; Public Health England 2017). In England, black people had an estimated chronic pain prevalence of $44 \%$, when the average for all ethnicities was 34\% (Public Health England 2017). As highlighted by Rice and colleagues (2016), chronic pain reporting becomes more prevalent with increasing levels of obesity and low physical activity (Rice et al. 2016). Healthy weight was associated with $29 \%$ chronic pain prevalence compared to 54\% in people identified as very obese (Public Health England 2017). Less than 30 minutes per week of moderate or vigorous activity resulted in a $14 \%$ difference in pain prevalence reporting, compared to those who exceeded 30 minutes (45\% compared to 31\% respectively) (Public Health England 2017).

\subsubsection{The situation in Wales}

No studies have to date examined chronic pain prevalence in Wales alone. Fayaz (2016) estimated UK prevalence to be around 44\%, based on a systematic review of 19 UK-based studied (Fayaz et al. 2016). Based on a population of 3.2 million (StatsWales 2021) this could mean up to 1.3 million people may live with chronic pain in Wales.

Wales is known to have poor levels of general health (Public Health Wales Observatory 2020). Data from StatsWales (StatsWales 2020) on general health and illness in the Welsh population 2017-18 stated that 70\% of people aged 16 years or over had good or very good general health. Ten percent of the adult 
population were listed as having bad or very bad health (StatsWales 2020). Data for England are presented differently. The last comparative data for England and Wales were released in 2013 (Office for National Statistics 2013) and presented findings from 2011. Then, just over $81 \%$ of people in Wales and England reported 'very good' or 'good' general health. Wales and the North East of England had the lowest levels of people rating their health in the higher categories. Blaenau Gwent local authority in Wales had the lowest percentage $(72.6 \%)$ of people who reported their general health and wellbeing in the top two classifications, which was over $15 \%$ lower than the authority with the highest ranking (Hart in Hampshire, 88.1\%) (Office for National Statistics 2013). It would appear that, since 2015, general health and wellbeing in Wales has deteriorated.

Musculoskeletal (MSK) complaints account for the highest reported percentage of illness type in Wales. Between 2016 and 2018, the proportion of people affected with MSK conditions remained at 17\% (StatsWales 2020). Mental Health disorders affected $8-9 \%$ of people aged 16 years and over in the same period of time (StatsWales 2020). Whilst these data do not, on their own, explain the $44 \%$ estimate of the population living with pain, it begins to build a picture of the Welsh population and the complexity of collecting and interpreting data about population health.

\subsubsection{Economic burden of pain}

The impact of pain is far-reaching, going well beyond healthcare systems. The socioeconomic burden is probably higher than for many other health conditions. Pain is often accompanied by poor mental health, high rates of absenteeism and reduced productivity (Phillips and Harper 2011; Henschke et al. 2015). Pain also impacts on more than the individual, posing a significant challenge for families, carers, employers and therefore society as a whole (Latham and Davis 1994; Phillips and Harper 2011; Henschke et al. 2015). High levels of disability are associated with pain (Section 1.4) which is a major influence on the economics of the condition (Phillips 2008). 
The economic burden of chronic pain has been estimated at 3 to $10 \%$ of gross domestic product in Europe (Breivik et al. 2013), which in 2015 would have been approximately $€ 441$ to $€ 1,471$ billion per year ( $£ 381$ to $£ 1,273$ billion) (Bourgeais and Krueger 2017). Estimates from the USA, based on 100 million people experiencing pain of any level, were calculated between $\$ 560$ and $\$ 635$ billion annually (Richard and Richard 2012). In Australia, with a population of 22.7 million people, total costs of pain in 2007 were $\$ 34$.3billion ( $\sim 19$ billion). As illustrated by the GBD study (Section 1.4), pain is a major burden globally, although estimates of subsequent costs from developing countries are more difficult to determine. More specifically, a UK estimate for back pain was made for over f10billion, based on a prevalence of between 36\% and 37\% (age range 16+ years, 47 million population) (Maniadakis and Gray 2000). A similar study in Germany estimated total costs of back pain to be $€ 48.96$ billion ( $₹ 42$ billion) (age 18 years and over, 61.8 million population) (Wenig et al. 2009). The UK total included an estimate of $£ 140.6$ million spent in primary care services such as general practice and home care, which, accounted for $13 \%$ of NHS costs although only $1-2 \%$ of the full economic cost estimate. Employment costs lost due to low back pain, were 5 to 10 times that of NHS healthcare (Maniadakis and Gray 2000).

As with disease burden, the full economic consequences of pain are likely to remain underestimated. This is in part due to its ubiquitous presentation as a symptom of other conditions, but also as the consequences of living with chronic pain are far-reaching and can be difficult to quantify.

\subsection{Pain management in general}

The multiple definitions of pain (Section 1.2) result in a variety of approaches to treating or managing it. It has been acknowledged for years, that pain, whilst felt physically, is a complex perception, influenced by more than physical change or injury (Eccleston 2001; Nicholas et al. 2019). In 1997, Gifford and Butler acknowledged that acute and chronic pain were not simple mechanisms but influenced by components including nociception, central processing (including 
how the brain modifies the response to incoming stimulation) and motor and autonomic responses. They highlighted that pain is an 'experience' and as such an individual's emotions and previous encounters with pain influence their presentation at any time (Gifford and Butler 1997). Moseley (2007) proposed that pain intensity is not related to tissue damage. The longer pain persists, the less predictable the relationship between tissues and pain becomes (Moseley 2007) implying a crossover between acute and chronic pain experiences, with the balance of biological and social or psychological influence varying with the individual and time.

Brena and Sanders (1992) were amongst the first to widen the desirable outcomes of chronic pain management beyond only a reduction in painful sensation. In addition to reduced discomfort, they listed the needs of people suffering chronic, non-cancer pain as increasing function, lifestyle improvements, reduced environmental stress and returning to work (Brena and Sanders 1992). Clearly, many of these factors will not be resolved by medicines. Recent painrelated guidelines from the National Institute for Health and Care Excellence (NICE) have been careful to stress the importance of taking into consideration the individual's experience, level of distress in addition to their physical debilitation (National Institute for Health and Care Excellence 2016; National Institute for Health and Care Excellence 2021). The Scottish Intercollegiate Guideline Network (SIGN) also promotes a holistic approach to management. As with NICE, SIGN promotes the offer of non-pharmacological strategies such as psychological support, increasing activity and addressing social concerns such as relationships and employment before medicines and especially opioids, are initiated (Scottish Intercollegiate Guideline Network 2019).

NICE recently faced criticism for reducing the number of medicines recommended for use in chronic primary pain (Eccleston et al. 2021; Smith et al. 2021), which includes conditions such as fibromyalgia and complex regional pain syndrome (CRPS). Although medicines are less commonly promoted as first line options for managing chronic, non-cancer pain conditions, they remain an 
important component of pain treatment regimens for some people. For example, low back pain may be helped by the use of non-steroidal anti-inflammatory medicines such as ibuprofen and naproxen (National Institute for Health and Care Excellence 2016; Scottish Intercollegiate Guideline Network 2019) and in osteoarthritis, paracetamol and non-steroidal anti-inflammatories are suggested as first line medicine options (National Institute for Health and Care Excellence 2014; Scottish Intercollegiate Guideline Network 2019). Other guidelines such as those from Royal College of Physicians for CRPS (2018) suggest more specific approaches to managing the named condition including the particular specialist services which may benefit the patient at different stages of their treatment (Goebel et al. 2018). There may not always be agreement on the overall approach of guidelines, especially those produced by NICE, which have potential to widely influence practice in the UK and further afield (Eccleston et al. 2021; Smith et al. 2021). However, what an increasing number of guidelines have in common is the suggestion that functional outcomes are more important than pain reduction per se and the promotion of analgesic medicines other than opioids, for the majority of commonly occurring non-cancer pain-related conditions (National Institute for Health and Care Excellence 2016; Scottish Intercollegiate Guideline Network 2019; Stewart et al. 2019; National Institute for Health and Care Excellence 2021).

\subsection{Contemporary use of opioids}

Opioid analgesics are widely prescribed in Wales and the UK as a whole (Ruscitto et al. 2015; Brinksman 2018; Davies et al. 2018; Curtis et al. 2019). They are acknowledged as effective analgesics (Faculty of Pain Medicine 2021). Opioids have been the mainstay of pain relief for a multitude of conditions for hundreds of years, in one form or another (Meldrum 2003; Sabatowski et al. 2004). Opioid analgesics including morphine are well established to treat pain caused by trauma or surgery and also in the management of pain due to cancer and at the end of life (Faculty of Pain Medicines 2021). It is likely from the history of opioid use, that differentiation of pain-type has not always been a consideration in the use of these drugs (El-Ansary and Galiongy 1984). 


\subsubsection{Acceptability of opioids in non-cancer pain}

Opioid analgesics were not considered a common part of chronic, non-cancer pain management for the majority of the 1900's (Meldrum 2003; Sabatowski et al. 2004). This changed following the publication of a seminal series of case reports by Portenoy and Foley in 1986 (Portenoy and Foley 1986). The authors described 38 cases of chronic non-cancer pain, where opioids were used for extended periods and examined retrospectively. Although morphine equivalence was used to describe the doses used, the patients did not use morphine - twelve receiving oxycodone with others having methadone, levorphanol (not used in the UK), codeine and others regarded as 'weak' opioids. The authors concluded the use of opioids for managing chronic, non-cancer pain could be "safe, salutary and more humane" alternative to current methods employed at the time of surgery or no treatment (Portenoy and Foley 1986).

McQuay (1989) focused on practical considerations such as efficacy, ceiling effect and toxicity but ultimately reinforced support for using opioid analgesics for chronic pain (McQuay 1989). In the USA, prescribing laws were relaxed, and liberalization of opioid prescribing led to a rapid escalation in their use (Franklin et al. 2012). The trend was quickly mirrored in other developed nations where rises in prescribing of opioids of all types were observed from the mid 1990's onwards (Ruscitto et al. 2015; Karanges et al. 2016). Opioid prescribing in noncancer pain was rationalised by statements such as 'there is consensus among pain specialists that opioid therapy is appropriate for selected patients with chronic pain and can provide sustained benefit to such patients' (Franklin et al. 2012). However, even though more people were being prescribed opioid analgesics, concern was also expressed. Less than 10 years after the Portenoy (1986) paper, Large and Schug questioned whether the use of opioid analgesics for non-cancer pain raised questions about 'the purpose of pain management' (Large and Schug 1995). The authors asked if opioids create self-sufficiency in people living with pain or made difficult lives more limited. Should the aim of 
pain management be total pain relief or improved function and quality of life? (Large and Schug 1995).

In the subsequent 25 years, the debate on using opioids for long-term pain management in particular has continued to rage. The idea that pain intensity and therefore, reducing it as the main goal of treatment, was promoted especially in the USA. In 1996, the then President of the American Pain Society proposed pain should be considered the ' $5^{\text {th }}$ vital sign' (Sullivan and Ballantyne 2016). The result of the initiative was not simply to raise the profile of pain as a condition in its own right. It also gave credence for both patients and practitioners to use analgesic medicines to reduce pain and opioids, so often reported as 'gold standard analgesics' (Bekkering et al. 2011; Wiffen et al. 2017) were the obvious choice to use. Further, the inclusion of pain intensity as an outcome measure is postulated by many, as the reason for the rapid rise in opioid prescribing in America and elsewhere (Franklin et al. 2012; Sullivan and Ballantyne 2016; Todd et al. 2018). Whilst rates of prescribing were increasing across the world, evidence was emerging of rising prescription opioid-related overdoses and deaths (Gomes and Juurlink 2016; Spooner 2016) in North America, leading to what is now referred to as the 'Opioid crisis' (McGreal 2018; Centers for Disease Control and Prevention 2021). Although opioid-related deaths and hospital admissions have increased in UK (Liddell 2019; Turner et al. 2019) only around $8.2 \%$ are related to prescription opioids, compared to $40 \%$ in the USA (Stannard 2018b). As importantly perhaps, opioid analgesics do not have good evidence of effect for the majority of people who use them (Rivat and Ballantyne 2016; Stannard 2018a; Faculty of Pain Medicine 2021) (Section 1.6.3). The use of opioid analgesics is also associated with significant adverse effects, which, have become better recognised in the last 20 years (Section 1.7).

High pain scores in chronic pain states are often a measure of distress, desire for support, depression and anxiety rather than physical pain per se (Blozik et al. 2009; Sullivan and Ballantyne 2016; Stannard 2018b). Continuing poorly effective medicines, aiming to reduce pain intensity and which do not seem to make life 
more bearable for all people living with pain is becoming questionable clinical practice in the UK (Large and Schug 1995; Ballantyne et al. 2016; Stannard 2018b). Despite that, opioid analgesics continue to be frequently prescribed (Curtis et al. 2019; Jani et al. 2020). In the USA, opioid prescribing remains highly politicised and a source of conflict between different clinicians and patientgroups, with starkly drawn views on the place of the medicines in pain management (Matthias et al. 2014; Mackey 2019; Schatman and Shapiro 2019; Matthias 2020; Nichols et al. 2020).

In the UK, discussion tends to be more nuanced. For instance, recently published National Institute for Health and Care Excellence (NICE) guidance for managing chronic primary pain in over 16's, recommended against offering all standard analgesics including opioids (National Institute for Health and Care Excellence (NICE) 2021). Authors have argued that NICE ignored swathes of literature which support commonly used interventions and treatments, leading to only a small number of recommendations for treatment being made (Eccleston et al. 2021; Smith et al. 2021). Whilst pro-analgesic American literature tends to focus on patients' 'right' to opioid analgesics and the expectation pain will be relieved, UK clinicians' focus is on ensuring individualised care, including non-pharmacological support, remains available for those who may benefit from it. In the UK, there is little disagreement with the notion that opioids should not be routinely offered for long-term pain conditions (Centre for Clinical Practice at NICE 2013; National Institute for Health and Care Excellence 2014; National Institute for Health and Care Excellence 2016; National Institute for Health and Care Excellence 2021), signalling perhaps they are becoming less acceptable for long-term pain management.

\subsubsection{Arguments in support of opioids}

At the point that opioid analgesic use for chronic non-cancer pain became more widespread, little trial data supported their use (Franklin and Neurology 2014; Faculty of Pain Medicine 2021). In the late 1990s, a lack of reasonably conducted clinical studies in chronic, non-cancer pain has been suggested to have allowed 
some treatment methods to "gain a foothold in the mythology of pain management before controlled trials are conducted" (Justins 1996). It was acknowledged even then that using opioids for chronic non-cancer pain was controversial (Justins 1996). However, patient surveys and case reports were published which supported the use of long-term opioids for nociceptive and neuropathic pain in carefully selected patient groups. It was recognised that people with chronic non-cancer pain would often state a preference for opioids over other medication choices (Collett 2001).

What studies were available, predominantly compared opioids to placebo and used a variety of administration routes including parenteral, where application in clinical practice is limited. The overall conclusions from these early trials demonstrated a reasonable effect of opioids in nociceptive pain although neuropathic pain responded less well and idiopathic pain appeared not to respond to opioids at all; albeit in mostly uncontrolled circumstances and with small patient numbers. (Collett 2001). Similarly, tentative support for using opioids was issued from a Cochrane systematic review and meta-analysis which suggested that "proper management of a type of strong painkiller (opioids) in well-selected patients with no history of substance addiction or abuse can lead to long-term pain relieffor some patients..." (Noble et al. 2010). The paucity of comparative data with other drug classes such as anti-inflammatories and antidepressants in studies examining the use of opioids in the management of chronic low back pain was also noted by Chaparro (2013). Where data are available, little difference between opioids and other drugs exists in either efficacy or their effect on functional improvement (Chaparro et al. 2013).

Attempts continue to determine the efficacy of opioids for chronic non-cancer pain compared to other medications as well as placebo. Previously, Furlan et al. (2006) tried to establish whether certain types of chronic non-cancer pain responded better to opioids than others using a systematic review of literature up until 2005 (Furlan et al. 2006). The group examined data on opioids in chronic non-cancer pain using pain for longer than six months as the working definition 
of the condition. However, in terms of opioid use, durations greater than 7 days were included which, in chronic pain practice would be an unusually short period of time of use. Data from 41 studies including over six-thousand people, published prior to 2005 were examined for the study. Eighty percent of people included in these studies had diagnoses of chronic nociceptive pain and $12 \%$ had neuropathic pain (Furlan et al. 2006). The longest mean length of opioid therapy was just under 9 weeks, which would not be regarded as 'long-term' use in practice. Following meta-analysis of 28 placebo controlled trials the authors conclude the findings were in favour of using opioids in respect of pain relief (Furlan et al. 2006). There was little difference between types of opioids in terms of efficacy.

Further, when compared to other analgesic medicines, including those used for neuropathic pain such as tricyclic anti-depressants, there was little variance in effectiveness for pain relief. The authors highlight inadequacies in the various study designs, which would affect the accuracy of the outcomes reported (Furlan et al. 2006). The majority of studies conducted in this area have included only small patient numbers and were often uncontrolled, which throws doubt on their subsequent findings (Collett 2001). In a later paper, Furlan's group undertook an analysis of a further 4 years of data which added twenty-one trials to the previous study. Head-to-head comparisons of opioids were excluded but they did look at a small number of studies comparing opioids to non-opioid analgesics such as non-steroidal anti-inflammatory drugs. Trials were again short-term, the longest being 24 weeks but the majority less than 6 weeks. Eighty-seven percent of the trials had significant input from pharmaceutical companies, which could further bias the outcomes (Furlan et al. 2011).

Overall, it appears opioids can provide reduction in pain levels for a small number of people for a relatively short period of time. The current evidence does not however, demonstrate that opioids can provide long-term pain relief or functional improvement (FAHMS et al. 2016, Häuser et al. 2021). High quality, long-term studies which are better able to identify which patient groups and 
conditions are most likely to benefit, are needed (Noble et al. 2010; Franklin and American Association of Neurology 2014).

\subsubsection{Are opioids effective in all types of pain?}

Commonly, discussions about the place of opioids state, "Opioids are very good analgesics for acute pain and for pain at the end of life but there is little evidence that they are helpful for long term pain" (Faculty of Pain Medicine 2021). However, this statement is not unequivocally borne out in the literature (Wiffen et al. 2017). There is a suggestion morphine and fentanyl may be more effective than other opioids in cancer pain although the evidence-base is perhaps, not as strong as perceived by most clinicians who commonly prescribe them in practice (Wiffen et al. 2017). A similar issue pervades acute pain management, where evidence of widespread opioid use has possibly been perceived as evidence of effectiveness (Schug et al. 2016). Currently therefore, opioids maintain an important and for many, useful part of their pain management regimens for acute pain and pain associated with cancer and end of life. Importantly, clinical practitioners and prescribers are familiar with using them.

Mounting interest in short-term prescribing becoming long-term use and the subsequent implications of it, have led to different approaches being advocated. Avoiding modified-release opioids and using multi-modal treatment for postoperative pain has been suggested as a means of reducing chronic use (Levy et al. 2021). Consideration of long-term opioid harms in cancer pain is increasingly important, particularly as more people are surviving or living for longer with the disease (Ballantyne 2003; Jones et al. 2020). Perhaps more so than with other pain types, it is generally acknowledged, in the UK, Europe and Australia at least, evidence to support the use of opioids in chronic, non-cancer pain is limited (Hansen et al. 2015; FAHMS et al. 2016). In North America, it seems lines are drawn between professional and patient groups with differing views of the place of opioids in chronic pain management. Advocates of opioid use are noted to refer to their 'rivals' as 'opioid zealots' and claim they are working to deny people with pain effective treatment (Schatman et al. 2016; Oliver and Carlson 
2020). Those labelled 'zealots' would claim to advocate a harm reduction approach to opioid use (Kolodny and Frieden 2017).

Theories abound as to the reasons behind the apparent lack of efficacy of opioids in chronic non-cancer pain (Chou et al. 2009; Chapman et al. 2010; Faculty of Pain Medicine 2021). Possible explanations include tolerance, whereby increasing doses of the drug are required to illicit the same analgesic effect, opioid-induced hyperalgesia and even loss of placebo effect with prolonged use (Ballantyne and Shin 2008). Concerns about the association of long-term opioid use and higher rates of impairment including misuse and dependence are often quoted in guidelines as a reason to avoid using them for extended periods of time (Franklin and American Academy of Neurology 2014; Dowell et al. 2016). There is limited data on the prevalence of chronic opioid use in individuals in the UK, however, Bedson (2016) demonstrated of people receiving opioids for periods greater than 2 years, the percentage prescribed long-acting controlled opioids increased from 3.5\% to 22.6\% between 2004 and 2013 (Bedson et al. 2016). Jani et al. (2020) observed $14.6 \%(n=1,968,742)$ of people newly started on opioid analgesics, became long-term users within the first year, albeit longterm in this instance was receipt of 3 prescriptions in 90 days (Jani et al. 2020). Increases in long-term opioid analgesic prescribing has been observed outside of the UK as well. In an Australian cohort ( $n=1,936,573)$ long-term opioid prescribing prevalence increased from 5.5\% in 2012 to 9.1\% in 2018 (Black-Tiong et al. 2021).

\subsubsection{Why are opioids prescribed?}

Prescribing opioids has been suggested to be a surrogate for access to alternative pain management support (Finestone et al. 2016). Pain service provision in Wales was not mapped as part of this study but, the National Pain Audit (2012) collected data demonstrating every health board in Wales had chronic pain service provision, with $80 \%$ claiming to have multi-disciplinary teams which, as a minimum would compose a medical doctor, physiotherapist and psychologist (Price et al. 2012). Average waiting time for those services at the time of the 
audit was 33 weeks (Price et al. 2012). Official figures on waiting times for pain services were not available, during the composition of this thesis, although it is acknowledged post-Covid 19-pandemic, waiting times will have increased substantially.

The Royal College of General Practice's (RCGP) guidance to its members is to continue to offer support and treatment to people, even if they are waiting for or receiving input from specialist providers. This may lead to GPs and other practitioners in primary care, resorting to prescribing opioids or maintaining them in the face of poor outcomes, simply because they feel they have nothing else to offer (Finestone et al. 2016).

McCrorie's (2015) study supported this notion, with GPs explaining during interviews, that continuing to prescribe opioids was often due to long waits for other treatments or support or patient's not being ready to consider alternatives to medicines (McCrorie et al. 2015). An examination of routinely collected, longitudinal data from primary care practices in England, noted increasing numbers of primary care appointments were more likely to result in the prescription of a strong opioid. An odds ratio (OR) of 3.04 (range $2.48-3.73$ ) where there were more than 12 visits per year was observed (Foy et al. 2016).

The same study demonstrated referral to chronic pain services also resulted in an increased likelihood of strong opioid prescribing that was also more likely to persist (OR 5.74, range 5.09-6.47) (Foy et al. 2016). Something commonly heard in practise, was also reported in McCrorie's study, "...every time I send somebody to chronic pain [clinic] they come out with more medication, or injections.' [GP, female; Leeds; 319]" (McCrorie et al. 2015). If this is the case, then whilst prescribing sits predominantly in primary care (Curtis and Goldacre 2018) it would seem influences on prescribing could be greater than simply 'prescriber preference' or lack of alternative. 


\subsection{Adverse effects of opioids}

Opioids are associated with an array of adverse effects (Breivik and Stubhaug 2014), a number of which have historically been considered less clinically relevant when the drugs are only being used for a short period of time, when a potentially life-threatening condition is causing pain or at the end of life. Commonly cited adverse effects of opioids in clinical trials are constipation, nausea and vomiting, dizziness and drowsiness (Furlan et al. 2006).

Studies have demonstrated correlation with endocrine dysfunction (Katz and Mazer 2009) increased risk of falls and fractures on older people (Baldini et al. 2012), sleep-disordered breathing (Baldini et al. 2012; Els et al. 2017), neonatal abstinence syndrome (Desai et al. 2015), cardiac issues e.g. QT prolongation with methadone (Everdingen et al. 2013), opioid-induced hyperalgesia (Higgins et al. 2019; Faculty of Pain Medicine 2020), non-fatal overdose (Brady et al. 2017; Holloway et al. 2018), increased number of emergency department visits (Braden et al. 2010; Nelson et al. 2015), death from unintentional poisoning (Coyle et al. 2018; Alho et al. 2020).

The wide-ranging effects and generic nature of adverse effects, many of which can be experienced by people with long-term pain or linked to co-morbid conditions, is likely to result in opioids being initially at least, overlooked as causative in their presentation.

\subsubsection{Effects associated with duration of opioid use}

Taking opioid analgesics for longer periods of time, have been associated with a number of negative health outcomes (Jain et al. 2018). There is limited evidence a minority of people remaining on opioids for more than 6 months, experience a persistent reduction in pain intensity (Noble et al. 2008; Faculty of Pain Medicine 2021 In one study, 50\% of people remaining on opioids for up to 10 years continued to report severe to unbearable pain (Jensen et al. 2006). This was followed by another study showing doses above $30 \mathrm{mg}$ oral morphine equivalent (OMEQ) dose for more than 1 year were associated with higher stimulated pain 
intensity than observed in non-opioid users or those receiving lower doses. Baseline pain levels were higher in people on long-term opioids as well (Cohen et al. 2008).

Use of opioid analgesics have been associated with lower quality of life (Jensen et al. 2006). A study of chronic pain in Denmark, observed $60 \%$ of people discharged from pain clinic on modified release opioids, remained on them 10 years later. Whilst dose reduction was as common as dose increase over that time, depression recurred or emerged in nearly $30 \%$ of opioid users and quality of life measures were statistically lower compared to non-users (Jain et al. 2018). Corroborating earlier findings of the risk of new-onset depression being $18 \%$ in people using opioids for 30 - 90 days, rising to $35 \%$ increased risk with more than 90 days use (Scherrer et al. 2016). The dose of opioid was not significantly associated with depression onset, which, as with Jain's (2018) findings, is suggestive that duration of use is a more important factor.

People using opioid analgesics for more than 6 months prior to undergoing spinal surgery were nearly $40 \%$ more likely to develop wound infections and just over $20 \%$ more likely to have the procedure repeated within a year(Jain et al. 2018).

\subsubsection{Quality of life}

Living with pain takes a huge toll on individual's quality of life (QoL) (Smith and Torrance 2012; Husky et al. 2018). Using opioid analgesics was assumed would lead to reduced levels of pain and consequently, improve all other aspects of a person's experience (Portenoy and Foley 1986; Trescot et al. 2008). A 2005 systematic review, funded by the Janssen-Cilag pharmaceutical company, reported improvements in pain and QoL for people using a range of opioid analgesics with doses of up to 1.2g OMEQ per day, 10 times the now recommended maximum $120 \mathrm{mg}$ OMEQ dose for chronic pain (Faculty of Pain Medicine 2021). On closer examination however, only 4 of 11 studies included could provide evidence of an improvement in QoL scores, following up to 2 years treatment with opioid analgesics. One of those 4, assumed improvement in 
physical function correlated to improved QoL (Devulder et al. 2005). An optimum dose of around 40mg OMEQ per day has been suggested, in terms providing physical or functional improvement, reduce pain intensity and increase overall well-being. High doses of opioids are associated with initial increases in feelings of well-being and quality of life, but this does not appear sustained (Dillie et al. 2008; Els et al. 2017)

Since 2005, there has been substantial change in the focus of pain management, from being aimed predominantly at reducing pain intensity to focusing on function and quality of life (Hanna 2012; Rice et al. 2016; Merriwether et al. 2018; Stilwell and Harman 2019; NICE 2020). Further, as time goes on, evidence continues to emerge of the detriment opioid analgesics can inflict on quality of life (Dillie et al. 2008; Baldini et al. 2012; Els et al. 2017; Solà et al. 2019). A sample of people in primary care living with chronic pain, demonstrated significantly lower health related QoL scores with doses of opioids greater than 105mg OMEQ (Dillie et al. 2008).

It is important to note, for a minority of people, who are able to tolerate any adverse effects that occur, there is a small amount of evidence opioids can be helpful to maintain pain levels at a manageable level, thus facilitating improved function and quality of life (Noble et al. 2008; Faculty of Pain Medicine 2021).

\subsection{Gender differences in pain}

Epidemiological and clinical studies have consistently, but not exclusively, reported women to have a higher prevalence of painful conditions and to experience higher levels of pain than men (Fullerton et al. 2018; Dance 2019).

Differences between male and females have been acknowledged for many years, yet a review in 2005 highlighted $79 \%$ of animal studies published in the preceding 10 years in a leading research journal, had only included male subjects. Only $4 \%$ of published work had examined gender differences (Mogil and Chanda 2005). 
Why do differences exist? It may not be possible to fully elucidate the answer due to the complexity of pain, particularly chronic pain and the numerous facets that impact upon pain perception. However, potential theories for gender differences in pain and analgesic response have been put forward (Fullerton et al. 2018). Most obvious is steroid hormone differences between male and female subjects, including changes depending on the stage of menstrual cycle or whether female subjects are pre- or post-menopause (Greenspan et al. 2007). Observed differences in pain symptoms between sexes, in the period after puberty and before menopause are highly suggestive that sex steroid hormones have a role in the experience of pain (Sullivan et al. 2000; LeResche et al. 2005; Vincent and Tracey 2008). Testosterone, which after puberty is significantly higher in males than females, has been postulated to be protective, and may have an analgesic role based on findings in particular painful conditions such as temporomandibular joint pain (Fischer et al. 2007; Vincent and Tracey 2008). These findings are supported by studies demonstrating reduced androgens in men and women with rheumatoid arthritis, where exogenous androgen improve pain symptoms in both sexes (Vincent and Tracey 2008). Differences are not seen in all pain modalities however (Ruau et al. 2012; Sorge and Strath 2018), highlighting it is too simplistic to assume sex hormones are the only explanation.

Emotional differences have also been suggested as a possible explanation for some observed differences (Rhudy and Williams 2005; Coll et al. 2012). Women tend to be more reactive to negative stimuli and perceived threat (Rhudy and Williams 2005). This might even include the extent to which pain in perceived in others, based on the perceived threat to the person being observed (Coll et al. 2012). Emotional responses such as frustration, depression and anxiety have been observed to have different effects on men and women's pain experience. For example, pain intensity was heightened by feelings of frustration in women, but by depression and anxiety in men, in one study (Riley et al. 2001). Catastrophic thinking, where experiencing pain is associated with rumination on worst case outcomes, appears to occur more frequently in women than men and is associated with greater pain reporting (Keefe et al. 2000; Sullivan et al. 2000), 
adding weight to the emotional component of pain being an important consideration when assessing and supporting men and women in pain.

Sex differences have also been shown in the response to opioid analgesics (Fillingim and Gear 2004; Niesters et al. 2010; Pieretti et al. 2016; Fullerton et al. 2018). Animal experimental models have shown sex differences in opioid responses, which are seemingly reversed in humans i.e., male rats have a more robust response than females, yet female humans appear to respond better to opioids (Fillingim and Gear 2004). A systematic review found women to be more responsive to parenteral morphine, but no sex differences in response to other forms of opioids (Niesters et al. 2010). Other studies have not shown analgesic differences but have demonstrated women to be more susceptible to adverse effects (Fillingim et al. 2005; Fullerton et al. 2018). Sex differences in metabolic pathways and inter- and intracellular mechanisms by which opioids take their effects have been put forward as contributing to the variation in response that is noted clinically (Fullerton et al. 2018).

\subsection{Does age impact on pain?}

It is no secret the population of the UK is getting older. Estimates suggest people age over 65 years will comprise to $36 \%$ of the UK population by 2050 (Schofield 2017a). Frequency of pain has been shown to increase with age up to around the sixth or seventh decade, at which point it appears to plateau or decrease (Molton and Terrill 2014).

Age in itself is not painful. There should be no assumption that pain is 'just part of getting older'. However, getting older brings with it a greater tendency to develop problems which can impact on quality of life and so increase the likelihood of experiencing pain (Gibson and Helme 2001; Gibson and Farrell 2004; Docking et al. 2011; Gibson and Lussier 2012). Functional disability reduced social and support networks and higher disease burden have all been associated with higher pain prevalence at any age but are more common as people get older (Gibson and Helme 2001; Schofield 2012). Pain prevalence has 
been reported as $73 \%$ for older people living independently and rising to $80 \%$ if living in a care home (Schofield 2012).

As with gender differences in pain, the reasons for changing perceptions of pain with aging are multifarious. Changes in brain connectivity are shown to increase pain threshold and increase pain intensity in older people (age 60-79 years) compared to younger people (age 18-26 years). In the experiments described pain in older folk appeared to be increased in due to a reduction in descending inhibition, a process which normally tempers intensity (González-Rold́an et al. 2020). The findings corroborated previous studies where pain perception was demonstrably altered in older people, by later activation and reduced inhibition resulting in a more intense perception (Marouf et al. 2014; Lautenbacher et al. 2017).

Other factors impacting pain experience in older people includes limited physical function, possibly due to pain but which can also further exacerbate it (Schofield 2012; Molton and Terrill 2014). Sleep disturbance is common in people living with pain (Lusa et al. 2015) but has been reported twice as often in older people with chronic pain (Molton and Terrill 2014). High impact pain, where pain intensity is reportedly low but functional impact and low mood is significant is found in around $25 \%$ of older people with pain (Corran et al. 1997). This is considered due to the greater impact of co-morbidities on mood and function in older people, which has a consequent effect on the individual's perception of pain (Gibson and Lussier 2012; Aguera-Ortiz et al. 2013).

Depression also links to dementia, which itself is a growing concern within an aging population. Pain assessment is difficult to undertake with people who are living with cognitive impairment or communication problems which may result in pain going untreated if it is misdiagnosed as agitation for example (Schofield and Abdulla 2018). Depression and early symptoms of cognitive impairment can be tricky to distinguish in clinical practise but if left unsupported, both can worsen the experience of pain in the individual (Zis et al. 2017). 
Another consideration for older people, is how analgesics fit into this picture. Polypharmacy appears to increase with age. In Wales, it was demonstrated that nearly a third of registered patients aged over 74 were prescribed at least 10 medicines in primary care (All Wales Medicines Strategy Group 2014). Opioids have a range of interactions with other medicines commonly prescribed in older people, such as sedative hypnotics (All Wales Medicines Strategy Group 2014). Side-effects from opioids are exceedingly common and increase in likelihood with age due to changes in drug handling e.g., reduced elimination due to renal insufficiency (Pergolizzi et al. 2017). Adverse effects including nausea, vomiting and constipation (BNF: British National Formulary - NICE. 2021) but also sedation (Tan et al. 2015), depression and anxiety (Mazereeuw et al. 2018), osteoporosis, falls and fractures (Katz and Mazer 2009) which are already more prevalent in older people (Stubbs et al. 2014a; Stubbs et al. 2014b), thus potentially worsening overall health and wellbeing. Concerns about harms of opioids have to be balanced against the risks of undertreating pain which has also been reported as more common in older people (Schofield 2017b). Prescribers report concern when issuing opioids for older people, not least due to fear of causing harm due to dose or interactions (O'Brien and Wand 2020). No evidence has been found to suggest opioids are more or less effective in older than younger people. However, considerations about the risks and benefits of prescribing opioid analgesics to older people need to be made in an individual context, taking account of a wide range of factors as described.

There is no previous research available from Wales, that has examined opioid prescribing trends, or has analysed whether gender or age differences exist in opioid use, outside of illicit drugs (Holloway et al. 2018; Walsh and NWIS 2019). Considering the evidence for using opioid analgesics and effects of gender and age on the experience of pain, does not on its own, explain why opioids are prescribed on an individual or population basis. Rather it is part of setting the context in which prescribing trends were formed. 


\subsection{Inequality and deprivation}

"Inequality kills slowly, gently, just a small additional effect every day." (p.1, Bambra 2016). This, written by Danny Dorling in Clare Bambra's book 'Health Divides: Where you live can kill you' (Bambra 2016), echoes the sentiment expressed in reports examining social determinants of health in the UK (Marmot \& Bell 2012; Marmot 2017). Connections between poor health, including drug misuse and deprivation are not disputed (Marmot 2005; Liddell 2019; Taylor et al. 2019). It is also acknowledged that pain is more prevalent in areas of poverty (Todd et al. 2018; Moore et al. 2020). Socio-economic deprivation is significant in the UK. It was estimated up to 1.5 million people were destitute at some time in 2017 (Unit 2013). In-work poverty has risen faster than employment, resulting in around a fifth of the UK population being classified as 'poor' (Barnard 2018). The coronavirus pandemic has resulted in heightened recognition of poverty within the UK population and the subsequent health divides that result (Whitehead et al. 2021)

The Joseph Rowntree Foundation highlight four areas of industry which have high rates of in-work poverty: accommodation and food services; agriculture, forestry and fishing; administrative and support services; and wholesale and retail (Joseph Rowntree Foundation 2017). These areas now form the basis of the Welsh economy since the decline of heavy industry, including coal-mining (Welsh Government 2016).

Differences exist between the four nations of the UK but also within each country (Bambra 2016; Joseph Rowntree Foundation 2017). Wales has maintained higher prevalence of poverty than England, Scotland or Northern Ireland since the late 1990's despite a small reduction in the percentage of population affected (Barnard 2018). Comparing rates of deprivation between the four nations is not as straightforward as it might appear, given each publishes indices of deprivation (Welsh Government 2011) undefined. Deprivation domains vary between countries (Table 1.1) and are subject to change. Welsh indices were updated in 2019, with component domains changing percentage 
contribution to the overall score (Welsh Government 2011). It has been suggested these differences, albeit small, may result in misleading outcomes (Abel et al. 2016). Differences in mortality rates appeared worse in Wales compared to England when using each country's own indices of deprivation, with the gap closing when a common schema was used (Abel et al. 2016). International comparisons can be further complicated by differences in welfare and regulatory systems (McAreavey \& Brown 2019), as well as cultural and social variances (Guillaume et al. 2016).

Table 1.1: Comparison of domains used to develop indices of deprivation for each country in the United Kingdom

\begin{tabular}{|c|c|c|c|c|}
\hline \multirow[t]{2}{*}{ Domain } & \multicolumn{4}{|c|}{ Percentage of each domain for final deprivation score (\%) } \\
\hline & Wales & Scotland & Northern Ireland & England \\
\hline Income & 23.5 & 28 & 25 & 22.5 \\
\hline Employment & 23.5 & 28 & 25 & 22.5 \\
\hline Education & 14 & 14 & 15 & 13.5 \\
\hline Health & 14 & 14 & 15 & 13.5 \\
\hline Crime / community safety & 5 & 5 & 5 & 9.3 \\
\hline Barriers to housing/services & - & - & - & 9.3 \\
\hline Housing & 5 & 2 & - & - \\
\hline Access to services & 10 & 9 & 10 & - \\
\hline Living environment & 5 & - & 5 & 9.3 \\
\hline
\end{tabular}

(Welsh Government 2011; Northern Ireland Statistics Research Agency 2017; Ministry of Housing 2019; National Records of Scotland 2020)

Whilst levels of poverty are higher in Wales than elsewhere in the UK, Scotland is said to have worse health outcomes and lower life-expectancy than the other home nations (Whynes 2008; Bevan et al. 2014; Bambra 2016). Other measures however, such as waiting times for surgery are generally lower in Scotland and England, with Wales and Northern Ireland consistently reporting much longer waits (Sutherland \& Coyle 2009; Bevan et al. 2014) which may then be associated with greater debilitation and potentially poorer outcomes.

Julian Tudor-Hart, a general practitioner working in the South Wales Valleys, published his seminal work, 'The Inverse Care Law' in 1971 (Tudor Hart 1971). It is as valid now, as it was when written, 23 years after the establishment of the National Health Service (NHS). An ideal of the NHS, declared by Nye Bevan “....to generalise the best health advice and treatment" (Delamothe 2008) was based on the intent for all people, regardless of circumstance, to receive the same 
service dependent on need, rather than the ability to pay. As Tudor-Hart

highlighted, despite the best efforts of those working in the NHS, "The

availability of good medical care tends to vary inversely with the need for it, in the population served" (Tudor Hart 1971).

Whilst NHS services are free at the point of delivery and available throughout Wales, there remains inequitable access to services, with some areas of overall low deprivation, being poorly served by medical and other public services (Figure 1.2 Comparison of Welsh Index of Multiple Deprivation domains, contrasting health (left) ).
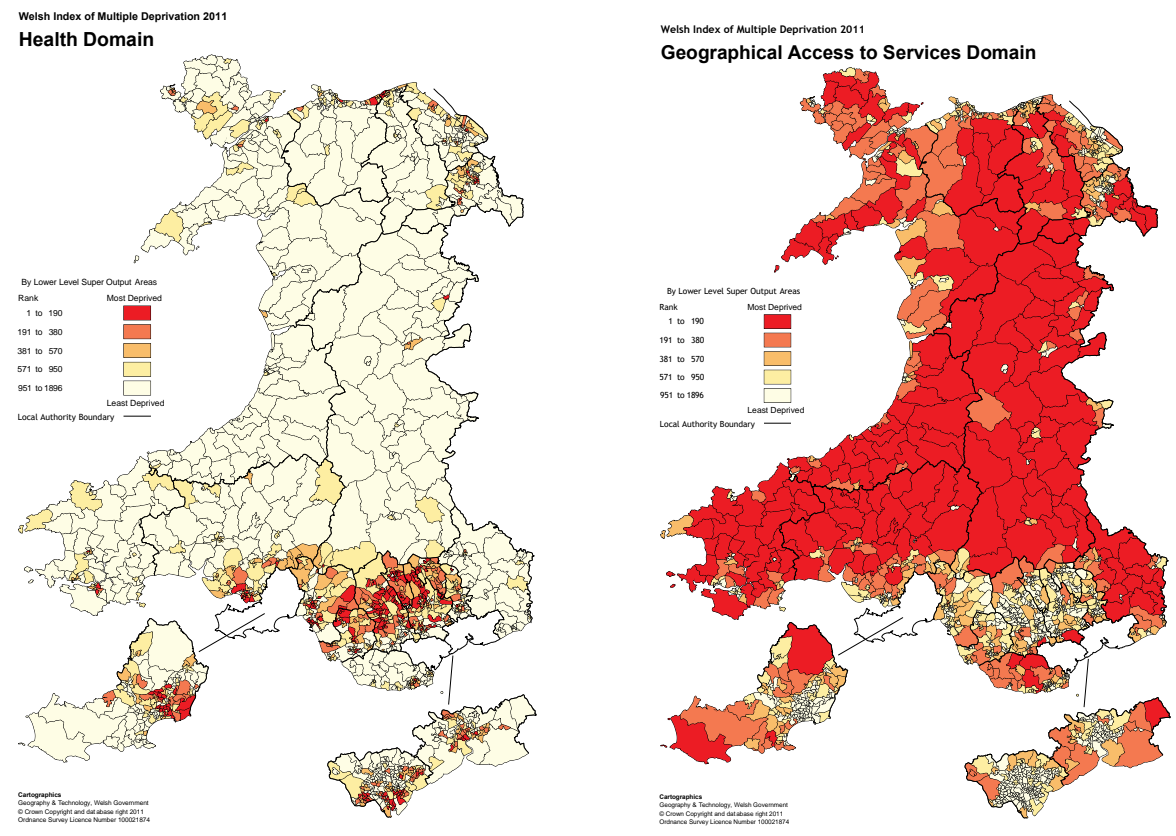

Figure 1.2 Comparison of Welsh Index of Multiple Deprivation domains, contrasting health (left) with geographical access to services (right) using the 2011 WIMD (darker colours = more deprived) (Welsh Government 2011)

\subsubsection{Determinants of health in the Welsh population?}

The areas of Wales with the greatest levels of deprivation now, are concentrated in the old coalfields such as the Rhondda and Merthyr valleys, Caerphilly, and Blaenau Gwent in the South, Flintshire in the North and Pembrokeshire in the West. The legacy of unemployment and lack of investment can be seen when travelling through those areas. "Worklessness' has been associated with 3 times 
higher reporting of ill health than areas with high employment (Del Roy Fletcher 2007). Direct links between social inequalities and health inequalities have been made and demonstrated time and again (Whynes 2008; Marmot \& Bell 2012; Galama \& van Kippersluis 2019). However, it is a likely over-simplification that economic deprivation alone is the cause of poor health. Levels of health are often worse than would be predicted by the level of deprivation alone (Whynes 2008). Referred to as the 'Scottish effect', as it was first described in that population, but has subsequently been demonstrated in English and Welsh populations (Whynes 2008).

Since the late 1990's social determinants of health have been considered more in policy formation than deprivation alone (Marmot 2005; Public Health England 2017; Welsh Government 2017). Social determinants of health are broadly defined as, 'the conditions in which people are born, grow, work, live and age and the wide set of forces and systems shaping the conditions of daily life' (World Health Organization 2021). They include education and employment opportunities, housing, social networks, location and how it facilitates other aspects of life such as diet, exercise and social connections (The Health Foundation 2019). It acknowledges that much more than 'medical' input is required for a healthy life. The Welsh Government (WG) set out a strategy in 2017, with one stated aim being to 'break the cycle' of '...the stubborn legacy of ill-health' (Welsh Government 2017). It supports the approach of viewing healthcare as separate to medical care (Braveman \& Gottlieb 2014), acknowledging the links between social prosperity and a healthier and happier population.

\subsubsection{Prudent healthcare in Wales}

In 2013, the Bevan Commission started the discussion on adopting a prudent healthcare approach to service delivery in NHS Wales (Aylward et al. 2013). Prudent healthcare aims to improve the health of the nation by following 4 principles (Figure 1.3) undefined 


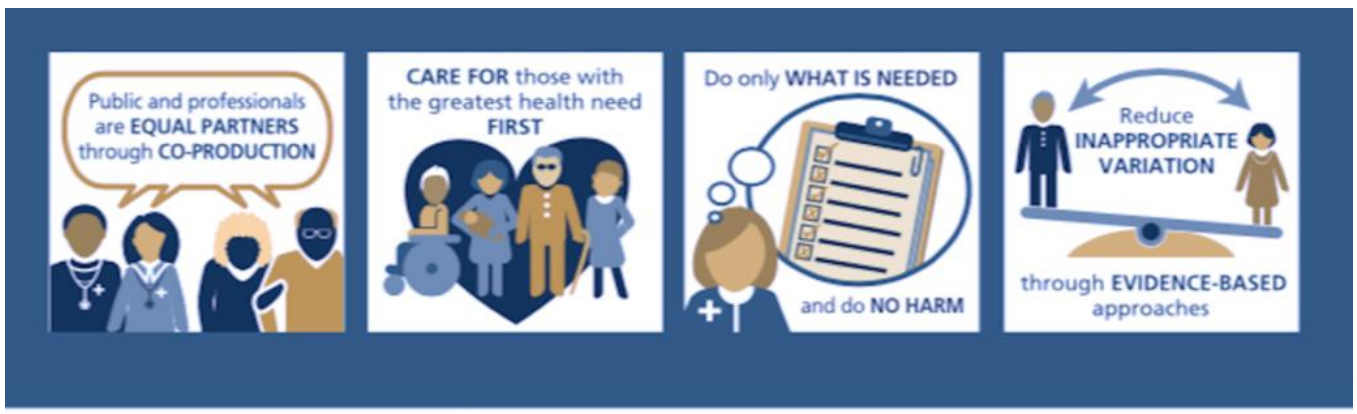

\section{www.prudenthealthcare.wales}

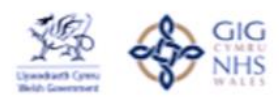

Figure 1.3: The four principles of prudent healthcare in Wales (Welsh Government and NHS Wales 2016)

Then Health Minister, Mark Drakeford set out the policy in January 2014 in a speech that included the line: "I think that we have to move beyond the 'do no harm' principle to one which is focused on what is normally called minimum appropriate intervention. The principle that treatment should being with the basic proven tests and interventions..." (Bradley et al. 2014). Chronic pain was one of the first four clinical services to be included in workshops used to test the principles. A 'Realistic Medicine' strategy was also launched in Scotland in 2016 (Calderwood 2017). Initiatives such as Quality Prescribing are based on the philosophy, as is most recently seen in guidelines for Chronic Pain (Harrison and Cormack 2018). Whilst there seems to be general agreement amongst healthcare professionals and organisations in Wales, that the prudent healthcare strategy is sensible, there remain barriers to its adoption (Addis et al. 2019). Outcome measures were reported by clinicians as a particular barrier to practising in an evidence-based way. Without understanding if what is currently being done is effective or not, it could be difficult to persuade people of the need for change (Addis et al. 2019). This is a pertinent consideration in relation to opioid medicines use, where there is a paucity of relevant data on clinically beneficial outcomes despite much evidence of their harms (Els et al. 2017).

\subsubsection{Links between pain and deprivation}

Pain is widespread in the UK population (Elliott et al. 2002; Fayaz et al. 2016). Prevalence estimates have ranged between 8 to $45 \%$ of the UK population living with some level of pain, since the early 1990s (Bowsher et al. 1991; Breivik et al. 
2006; Fayaz et al. 2016). Whilst the condition is acknowledged to occur more frequently in areas of greater deprivation (Morgan et al. 2011; Todd et al. 2018; Gulliford 2020), the reasons for this are less well-defined. Pain is acknowledged to be a bio-psychosocial phenomenon (Eccleston 2011; Meints and Edwards 2018; Nicholas et al. 2019). How much of a role each constituent part plays in each individual's experience is very difficult to gauge. Engel (1959) suggested pain, whilst evolving from impulses received from the periphery for example, becomes separated from purely biological mechanisms and become phylogenetic and ontogenetically-led experiences (Engel 1959). Whilst perhaps some of the terminology used by Engel has left medical parlance, his theory has not. By 1977, Engel's theory had formed into the biopsychosocial model of pain that many pain management practitioners and researchers currently use as the basis for treatment (Engel 1977; Gatchel et al. 2007). That pain is a perception which can be affected by emotional inputs, good and bad, is not in doubt (Moseley \& Vlaeyen 2015; Meints and Edwards 2018).

Of importance, when considering why some groups or individuals are more likely to experience long-lasting pain, is the impact of 'trauma' as being causative for some pain conditions. Conditions such as fibromyalgia and chronic fatigue, where there is not often a known cause of onset have been used as examples of trauma-linked pain (Scioli-Salter et al. 2015; Nicholas et al. 2019, ). However, there appears to be an association between persistent stressors such as posttraumatic stress disorder and worsening pain experience (Abdallah \& Geha 2017) for other conditions including back pain and knee pain (Asmundson and Katz 2009; Scioli-Salter et al. 2015; Meints and Edwards 2018).

There is similarity in neurobiology of stress or trauma responses and pain. Functional magnetic resonance imaging (MRI) has demonstrated specific areas of the brain respond similarly to stressful and painful stimuli (Tracey and Mantyh 2007; Tracey 2016). Pain and stress are considered to be adaptive responses. If unchecked, the response to either can become maladaptive, in other words, 
more permanent physiological changes occur which place the individual into a more permanent state of suffering (Saariaho et al. 2011).

The link with socioeconomic deprivation is not entirely elucidated, albeit acknowledged that living a more impoverished life is a keen source of ongoing stress (Engel 1959; Loyland 2016). Children growing up in difficult circumstances are more likely to demonstrate adverse behaviours, linked to neurodevelopmental changes (McLaughlin et al. 2014; Mackes et al. 2020). Adverse life events as children, led to greater pain reporting in adulthood in the British Birth Cohort Study (1958) (Macfarlane et al. 2009). Simplistically, the association could be deemed a form of Pavlovian response (Moseley \& Vlaeyen 2015, Abdallah \& Geha 2017).

However, it is almost a certainly more complex picture as other life factors have also shown to be impactful on pain experience (Elliott et al. 2002; Macfarlane 2005; Moseley \& Vlaeyen 2015). Indebtedness for example, has also been linked to chronic pain experience, with one study reporting a $30 \%$ increased likelihood of experiencing pain when indebted (Warth et al. 2019). Being in debt and experiencing deprivation were not directly linked, although pain experience was reportedly greater in the unemployed (Warth et al. 2019). Another theory which has been mooted is more mechanical in nature. Suggestions those living in more socioeconomically deprived areas are more likely to be employed in manual jobs (Crisp et al. 2009; Barnard 2018). Increased pain reporting has been observed in people, especially women who have physically active jobs (Rocha et al. 2017; Sorge and Strath 2018; Wu et al. 2020). This contrasts though, with evidence that being physically active is associated with reduced prevalence or longevity of painful conditions such as back pain (Smith et al. 2019).

\subsection{Role of legislation and guidelines in controlling opioid analgesic prescribing}

A major driver for changes to opioid management in the UK was the discovery and conviction of serial-killer Harold Shipman, who worked as a GP in Hyde, Manchester. In January 2000, Shipman was found guilty of killing 15 patients, 
although he is estimated to have murdered at least 215 using high doses of opioid medicines (Baker 2004; Gallagher 2006). Following the Public Inquiries (Dyer 2004) into the circumstances surrounding his activities, several changes were made to legislation around controlled drugs such as morphine and fentanyl. The purpose of legislation changes was, in part, to make such behaviours harder to carry out but also easier to detect (Dyer 2004; Baker 2004; Gallagher 2006). The legislation changes came into effect during the 2005 - 2015 study period. Recently, the Gosport inquiry (2018) revealed the extent of deaths related to the over-prescribing and failure to act on concerns of such (Gosport Independent Panel 2018). Similarities with the Shipman murders are stark. Although not investigated and reported for 20 years, the first reports about the use of opioids in Gosport were made in 1991 and continued throughout the same time period that Shipman was active. A population of older, vulnerable patients were given opioid medicines under the guise of pain relief, predominantly by a single prescriber (Gosport Independent Panel 2018). Concerns were raised by other staff, on the basis that often, there was no indication for the analgesics and the patients targeted were not considered to be imminently dying or receiving palliative care (Gosport Independent Panel 2018; Knights et al. 2018; Pocock et al. 2018). What is not clear in this awful case, is whether the laws introduced following Shipman, would have led to a different outcome in Gosport. The concerns raised by staff were mostly clinical and about the appropriateness of the prescriptions. The post-Shipman legislation made no mention of clinical suitability, suggesting as long as prescriptions are written correctly, not issued in large quantities or at an unusual frequency, they might still slip under the radar (Dyer 2004; Department of Health 2013).

The introduction of National Prescribing Indicators was agreed in Wales in 2003, as a way of promoting rational prescribing (All Wales Medicines Strategy Group 2021). The first opioid analgesic prescribing indicator was created for the 2012/13 financial year and since then, been included every year (All Wales Medicines Strategy Group 2013). NICE clinical guidelines are intended for use in England and Wales and focus on condition management. Over the years, NICE 
has progressed towards providing non-pharmacological as well as, pharmacological recommendations for treating and managing conditions. In relation to pain, NICE published guidelines for osteoarthritis (National Collaborating Centre for Chronic Conditions 2008), low back pain (National Institute for Health and Clinical Excellence 2008) and neuropathic pain (Centre for Clinical Practice at NICE 2013) between 2005 and 2015.

Since the early 2000's, significant increases in opioid prescribing have been observed in Wales (Davies et al. 2018) and across the UK (Zin et al. 2014; Jani et al. 2020). The trends noted in the UK, mirror what has been seen in other developed nations (Boudreau et al. 2009; Hamunen et al. 2012; Fischer et al. 2014; Levy et al. 2015; Wagemaakers et al. 2017). However, if pain prevalence is increasing or being maintained at a high level (Fayaz et al. 2016; Vos et al. 2017; Blyth and Schneider 2018), should it be a surprise to see increases in opioid analgesic prescribing?

Opioid analgesic prescribing is often discussed in terms of 'burden' and indeed, in Wales, prescribing indicators, developed to influence practise, refer to opioid burden (All Wales Medicines Strategy Group 2019). The implication of the term is of a 'heavy load' or at best, something that must be carried. In the case of opioids, who carries the burden? Is it the health service or the person taking the medicine?

\subsubsection{Does anyone know how much opioid is too much?}

More than 30 years ago, Portenoy (1986) discussed opioid safety in the context average doses of 40mg OMEQ per day (Portenoy and Foley 1986). Twenty years later, an average of 55mg OMEQ per day was reported (Korff et al. 2008). More recent studies however have shown a significant increase in the average dose of people reviewed, up to 140mg OMEQ in America (Dowell et al. 2016) and 86mg OMEQ in the UK (Zin et al. 2014). This, in spite of evidence that even when doses are significantly increased over a period of time, only a minority of people report meaningful improvement in pain and function (Trescot et al. 2008). 
The opioid crisis in the USA gained first prominence in Washington State, where doses of opioids increased by 50\% between 1996 and 2002 (Franklin et al. 2012). This led in 2007, to the development of state-wide, inter-agency guidelines on opioid dosing, including an opioid dose maximum of 120mg daily OMEQ. Within 5 years of the guideline's introduction, a 27\% reduction in daily OMEQ of modified release opioids and 35\% fewer people receiving more than $120 \mathrm{mg}$ OMEQ each day was reported (Franklin et al. 2012). Since then, recommended maximum OMEQ of $120 \mathrm{mg}$ per day have been incorporated into guidelines internationally, including here in the UK (All Wales Medicines Strategy Group and All Wales Therapeutics and Toxicology Centre 2020; Faculty of Pain Medicine 2021).

\subsection{Why is research needed in Wales?}

Pain is a major health and wellbeing concern worldwide (Blyth and Schneider 2018; Wu et al. 2020). It appears, from the last two Global Burden of Disease studies, the prevalence of long-term, disability related painful conditions are rising.(Hoy et al. 2014; Wu et al. 2020). An exploration of UK data from the 2016 Global Burden of Disease study revealed Wales to have higher rates of disabilityadjusted life years for low back and neck pain (1,692 per 100,000 population) than either Scotland (1,654 per 100,000 population) or Northern Ireland (1,645 per 100,000 population) although rates in England were higher (1,820 per 100,000 population)(Steel et al. 2018). This is important, not least due to the significant burden pain places on healthcare systems and society more widely (Elliott et al. 2002; Hanna 2012, Takura et al. 2015). Furthermore, the economic implications of having a noteworthy proportion of any population living with pain and possibly unable to work as a consequence, are substantial (Phillips and Harper 2011; Gustavsson et al. 2012).

There are clear associations between pain and socioeconomic deprivation (Morgan et al. 2011), as there are with other long-term health conditions (Whynes 2008; Newton et al. 2015). Added to this, concerns about rising rates of opioid prescribing in the UK have escalated since the start of the 2000's (Zin et al. 
2014; Ruscitto et al. 2015). This is in part, a result of the highly publicised problems experienced in North America (Weisberg and Stannard 2013; Vokinger 2018; Verhamme and Bohnen 2019) again, concentrated in areas of greatest socioeconomic deprivation (Kurani et al. 2020; Nowakowska et al. 2020). Wales is a country where a sizeable part of the population have poor health (Public Health Wales Observatory 2018; StatsWales 2020; NHS Wales 2021) and live in relative poverty (Barnard 2018). The number of people living with pain in Wales is unknown. An approximation of around 1.3million people affected, can be made based on prevalence estimations that up to $44 \%$ of the UK population live with pain (Fayaz et al. 2016).

It is known however, that analgesic prescribing has increased in Wales over the last 20 years (Welsh Analytical Prescribing Support Unit 2013; Statistics for Wales 2020). It is of particular concern not simply due to the risk of misuse (Amsterdam and Brink 2015; Morley et al. 2017) or disproportionate numbers of opioidrelated deaths (Turner et al. 2019; Office for National Statistics 2021) but also long-term harms to general health and wellbeing (Els et al. 2017) in an already 'sick' population. Whilst research has been conducted on opioid prescribing in the UK (Zin et al. 2014; Ruscitto et al. 2015; Mordecai et al. 2018; Todd et al. 2018; Torrance et al. 2018), none has thus far been conducted in Wales.

Basic prescribing data, whilst useful for monitoring trends in the population as a whole (Shared Services Partnership 2021) do not allow detailed analysis (Curtis and Goldacre 2018) and this possibly limits the effectiveness of initiatives aimed at influencing it (All Wales Medicines Strategy Group 2019). Since 2012, there have been arbitrary targets to reduce opioid analgesic prescribing across Wales (All Wales Medicines Strategy Group 2013). What is difficult to determine is an acceptable level of prescribing, given the rising prevalence of conditions that may be deemed to be painful. If Wales has a higher-than-average burden of painful conditions in the population, would it necessarily be 'wrong' to have higher than average opioid prescribing? 


\subsection{Route into research}

In 2013, Welsh Government's Advisory Panel on Substance Misuse asked for stakeholder evidence regarding misuse of prescribed analgesics. Whilst a final report was not published, a research briefing was released outlining the concerns about rising numbers of prescriptions, associated harms, misuse and deaths (Roberts 2016). The evidence gathered and research briefing, highlighted a general agreement amongst patients, practitioners and other stakeholders, that opioid prescribing was increasing and was creating problems for services and individuals. However, there were few detailed data on opioid prescribing, in particular examining who was receiving prescriptions or how prescribing differed across Wales. The challenge was laid down by the Chair of the Panel (Professor Phil Routledge) to find and analyse the data needed to provide some greater insight (Routledge 2013).

From those discussions and others with Welsh Government and organisations such as the All Wales Therapeutics and Toxicology Centre (AWTTC), issues of importance in relation to opioid prescribing were debated and developed into the following research questions:

- How has opioid analgesic prescribing changed over time?

- How do factors such as gender, age, or socioeconomic status effect the prescription of opioid analgesics?

- Have guidelines or legislation influenced opioid prescribing?

- Are there associations between receiving opioid analgesic prescriptions and use of healthcare services?

- Finally, and importantly for policy makers, how much does this cost the Welsh NHS?

Having this information might allow better targeted services and more prudent use of limited budgets. This thesis aims to make a start on answering those questions by using a large repository of Wales-specific healthcare data. The study will look back at the patterns of prescribing and healthcare use, and what might 
have influenced them. It is hoped that by developing a better understanding of what has happened in the past, the future can be more effectively shaped. 


\section{Trends in Opioid Prescribing for non-cancer pain and Associated resource utilisation in Wales}

Study aim: To provide insights into the trends in opioid analgesic prescribing in Wales, whether legislation or guidance has influenced it and assess how opioid prescribing is associated with healthcare utilisation and associated healthcare service costs

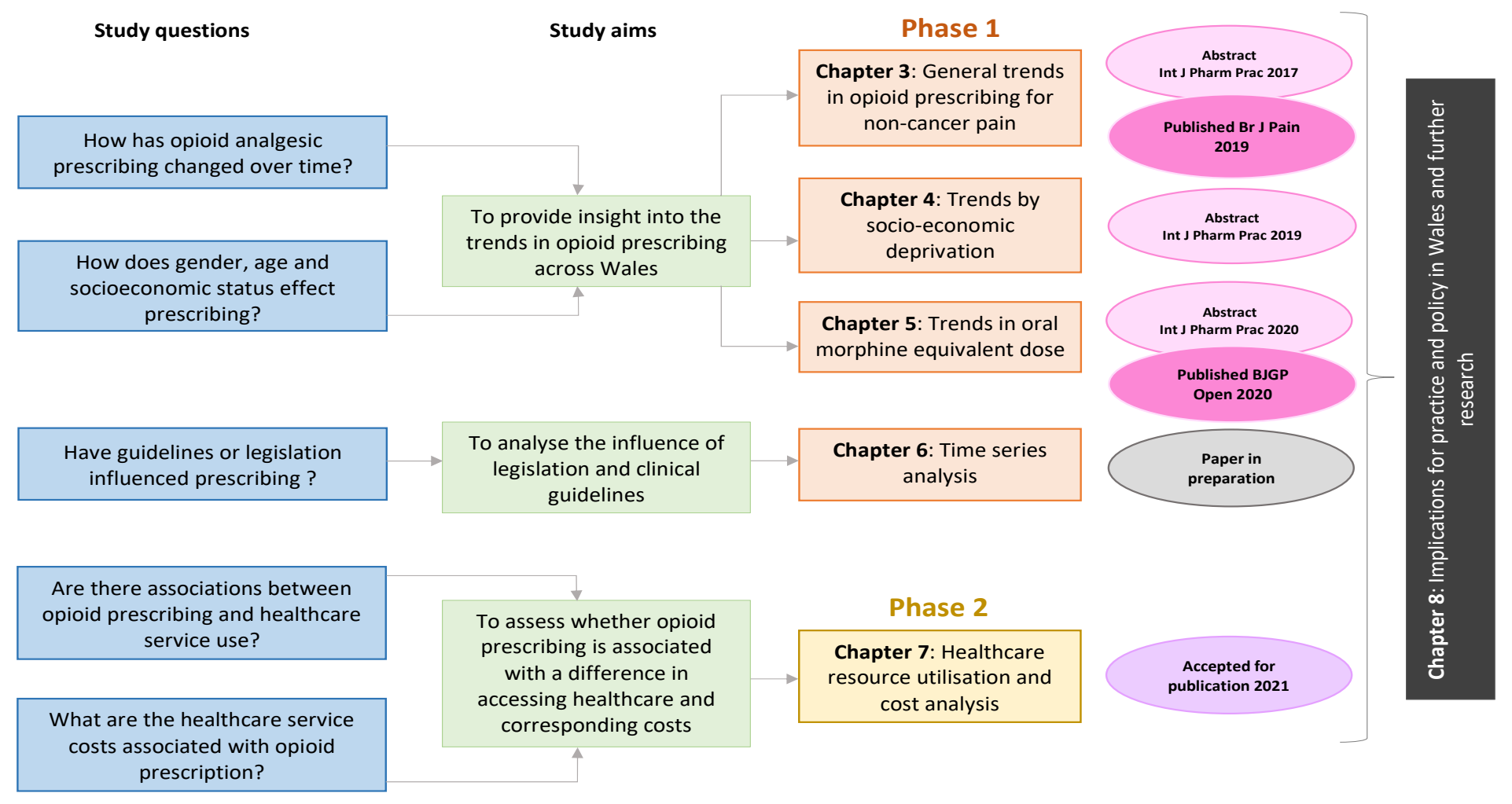

Figure 1.4: Overview of study including outputs generated up until July 2021 
Chapter 2

Methods

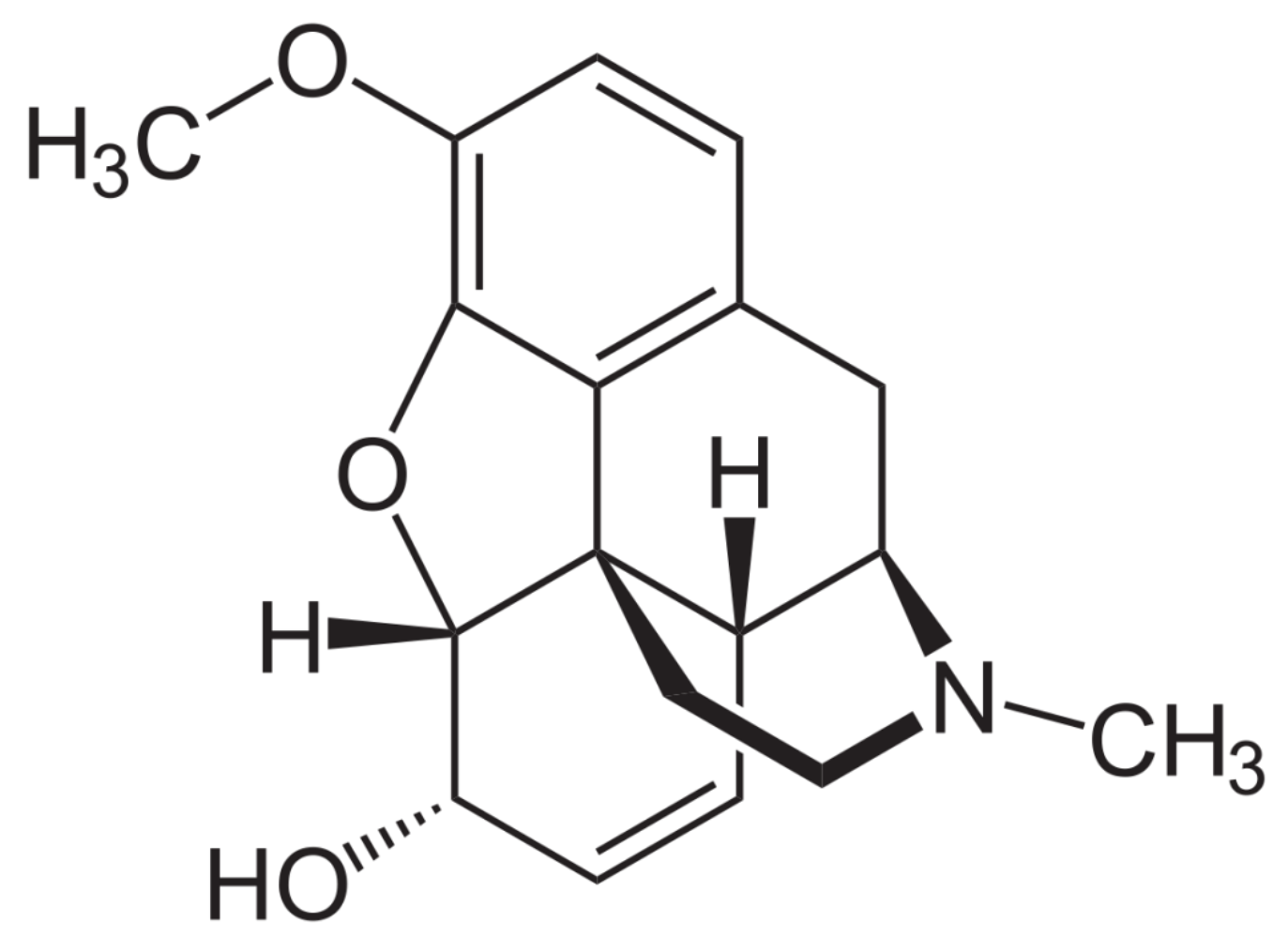

"Though this be madness, yet there is a method in't."

William Shakespeare (Hamlet) 


\section{Chapter 2 - Methods}

\subsection{Chapter overview}

In Chapter 1, how pain can be defined, and the social and economic burden of non-cancer pain were reviewed. How pain can be managed and where opioid analgesics fit into that was introduced, as were concerns about their potential harms. Aspects of socio-economic deprivation and how it affects healthcare outcomes across the UK and Wales in particular were presented. In this chapter, the research aims, and objectives are set out. The research strategy and overarching methodology is described, alongside statistical methods which are similar throughout the thesis. This chapter focusses on Phase 1 of the study, where prescribing trends were examined. Methods used in Phase 2, which analyses healthcare service use and associated costs, are found in Chapter 7.

\subsubsection{What are the aims of this research?}

There are three main aims to this research (Figure 1.4).

1. To provide greater insight of the trends in opioid analgesic prescribing across Wales, by examining individual demographic data for those people receiving prescriptions. This moves on from basic prescribing trends commonly used in practice. Gender, age and socioeconomic status are not routinely discussed or targeted by guidelines or prescribing initiatives (All Wales Medicines Strategy Group 2013; Welsh Analytical Prescribing Support Unit 2013; All Wales Therapeutics and Toxicology Centre 2021) but are likely to be important.

2. To analyse the influence of legislation and prescribing guidance on opioid prescribing. At the time of writing, Wales had not produced national guidelines for prescribing in pain management. Instead, guidelines from the National Institute for Health and Care Excellence (NICE) and National Prescribing Indicators (NPIs) are promoted to encourage rational, evidence-based prescribing. NPIs have included analgesic measures since 2009, (All Wales Medicines Strategy Group 2021) but, as with NICE, the 
impact on practice is difficult to measure. Included, is a question of whether current methods of examining prescribing trends are accurate, in terms of opioid burden.

3. To assess whether opioid prescribing is associated with any difference in accessing healthcare. A cost-burden study for healthcare attendance will be undertaken to estimate the impact on NHS Wales. The intention is to share this information with Welsh Government, to inform debate on future goals of prescribing measures and how this all fits with the wider aims for a Healthier Wales (Welsh Government 2019).

\subsection{Research objectives}

To address the study aims, the objectives of this research were set out as follows

1. To examine prescribing trends of opioid analgesics between 2005 and 20015 and scrutinise whether trends differ between gender or different age-groups (Phase 1 - Chapter 3)

2. To determine if opioid analgesic prescribing trends are affected by socioeconomic deprivation status (Phase 1 - Chapter 4)

3. To analyse trends in oral morphine equivalent doses and prescribing duration using estimated measures for each (Phase 1 - Chapter 5)

4. To determine if general opioid prescribing trends appear affected by legislative or clinical guidance changes during the study period using time series analysis (Phase 1 - Chapter 6)

5. To assess the frequency of primary and secondary healthcare attendance by patients with defined non-cancer pain conditions receiving opioid analgesic therapy (Phase 2 - Chapter 7)

6. To estimate healthcare service costs associated with the use of opioid analgesics (Phase 2 - Chapter 7)

\subsection{Research strategy}

Opioid prescribing in Wales has not been previously examined. This study aimed to provide the starting point from which more research may build in future. 
Qualitative enquiry around the reasons for prescribing, or attitudes of prescribers and people receiving prescriptions was not included in this research. A single hypothesis is not being tested here. Instead, there is an overarching study question, based on existing knowledge of opioid prescribing in the UK and associations with healthcare utilisation. Three research aims were developed and broken down into more specific analyses such as trends in opioid prescribing by age-group or gender.

Examining trends in prescribing, healthcare utilisation and associated costs is fundamentally a deductive process. A positivism paradigm would suggest that using quantitative methods to address the research questions posed would provide objective results (Kawulich 2012). Even with a study the size of the one described here, investigator bias could be introduced. For example, a clinical interest in opioid prescribing or professional experience of people coming to harm as a consequence of using them, might skew interpretation of the results. Consequently, it might be more accurate that the study epistemology is within a post-positivist framework, whereby perfect objectivity is aimed for but pragmatically, not achieved (Kawulich 2012; Khaldi 2017).

Both parts of this study required a form of census, with a range of population characteristics being measured (Lavrakas 2008). Use of questionnaires or data from individual primary care practices or Health Boards was ruled out as viable methods for data collection due to the quantity of data needed to enable a Wales-wide picture. Surveying the population directly would also have required a significant amount of time and resource, with potentially limited return for the effort.

\subsection{Study Design}

The Trends in Opioid Prescribing for non-cancer pain and ASsociated healthcare utilisation in Wales (TOPAS) study was designed in two phases. The first set out to examine trends in opioid prescribing for non-cancer pain, across Wales between 1 January 2005 and 31 December 2015. Using a retrospective, repeated 
cross-sectional design, similar to that used by other researchers in this area (Zin et al. 2014), trends in opioid prescribing and demographic influences were examined. Additionally, time series analysis was used to examine the effect of legislation and guidance on opioid prescribing. Phase 2 of the study was a retrospective, longitudinal case-controlled study. Frequency of healthcare service attendance by people with recorded diagnoses of conditions associated with pain, was assessed using linked data from primary and secondary care. Finally, each recorded interaction was attributed costs using nationally available unit cost data, in order to estimate healthcare service charges and determine associations with opioid analgesic prescribing.

\subsubsection{The SAIL Databank}

The SAIL databank was established in 2007 , to ensure the copious amounts of routinely collected data from services in Wales was used to improve the quality of healthcare and other public services. Data are used to review service use, treatment outcomes and impact on the population of other public health initiatives such as respiratory health changes following housing improvements (Ford et al. 2009, Jones et al. 2019) . It is now housed in its own building, which enables physical security and access restrictions, in addition to the multi-layer technological safeguarding measures (Jones et al. 2019). As with the Clinical Practice Research Datalink (CPRD) (Wolf et al. 2019) and other data sources data are anonymised at source or by other third-party providers such as the Digital Health and Care Wales (DHCW) before it is accepted into the databank. By doing so, it is not possible for dataset reconstruction in an identifiable way (Ford et al. 2009; Lyons et al. 2009).

Data is gathered from primary care when practices opt to share the data they hold. Consequently, individual patients need to request their general practitioner does not share their data if they do not want them used (Secure Anonymised Information Linkage Databank 2021). Secondary care data such as inpatient admission and emergency department attendances are recorded automatically by data coders working in Health Boards across the country, and the data shared 
nationally as part of the work of DHCW and Welsh Government. Whilst Wales has a relatively small population (3.15million in 2019), the SAIL databank provides data on $78 \%$ of it (2.5million) (Secure Anonymised Information Linkage Databank 2021). This makes the SAIL databank the most complete collection of health and social care data in the UK and reflective of the population it serves.

\subsubsection{Anonymisation process and security}

Ensuring the anonymisation and security of data for research purposes is crucial. Researchers should ensure their choice of data provider abides to high standards of security for data transfer and storage but also linkage, which are all measures of quality (Clinical Practice Research Datalink 2021; Doidge et al. 2020). The process of anonymisation used by the SAIL databank is similar to that used by other systems (Clinical Practice Research Datalink 2021). Probabilistic and deterministic linkage methods are used for linking datasets within the SAIL databank (Figure 2.1). When tested for accuracy, the SAIL databank was found to have rates of linkage accuracy greater than $90 \%$ and most, were greater than 99\% (Lyons et al. 2009).

- Datasets are split into
- demographic component e.g. commonly recognsied identifiers
- clinic or event component e.g. prcedures or prescribing event
- Demographic component transported to Trusted Third Party (TTP), NWIS
- Clinical component goes to SAIL databank via secure file upload
- TTP anonymise and encrypt demongraphic data and then subject it to quality assurance to ensure content anonymity
- Each individual's record assigned an Anonymous Linking Field (ALF) or Residential Anonymous Linking Field (RALF) for place of
residence
datasets
Anonymisation
and encryption
- Anonymised demographc elements sent to SAll databnk for loading
- Contain ALF, week of birth, gender code and area of residence (LSOA)
- Data recombined with clinical/event component
the datasets can now be linked to other datasets
- Data
- SAlL databank carried out further encryption of the ALF to form an ALF-E
- ALF-E used for linking datasets
- Where small numbers of individuals are included e.g. rare disease, data is aggregated for statistical analysis to avoid possible
indenitifcation
- At project completion, datasets are archived
safeguards

Figure 2.1: SAIL data anonymisation process (Secure Anonymised Information Linkage Databank 2021)

The security of data within the SAIL databank is closely monitored. All data extraction and analyses are undertaken via a secure server and accessed through an equally secure gateway (Jones et al. 2014). No data can be transferred in or 
out of the gateway without being reviewed by data analysts from SAIL, who verify data falls within the agreed parameters and does not allow the possibility of unmasking individuals. Of keen interest are data with low frequency, which, SAIL is very cautious about allowing removal from the secure gateway, as in theory, they might be more easily identified (Ford et al. 2009; Jones et al. 2014).

\subsubsection{Why the SAIL databank chosen for this study}

Prescription numbers for opioids are routinely collected and used to track trends and mark against National Prescribing Indicators (National Statistics, Knowledge \& Analytical Services 2015; All Wales Medicines Strategy Group 2019) (Chapter 6). Whilst data was and is available on prescription numbers and defined daily dose per 1,000 patients from Welsh Government sources, it is not possible to examine trends by anything more detailed than Practice level i.e., by the GP practice who supplies the prescription. Demographic exploration is also not possible with NHS prescribing data used by Health Boards and Government organisations in Wales (All Wales Therapeutics and Toxicology Centre 2021; Shared Services Partnership 2021). Whilst the socio-economic rank can be elucidated for the practice where a prescription originates, it will not necessarily correlate with the individual patient (Stats for Wales 2021). Prescribing data also does not permit examination of differences in gender or age. Without this knowledge, policy and guidance around prescribing may not reflect variance in practise and consequently, may be limited in effectiveness.

In order to understand the trends noted across Wales, being able to examine data to individual patient level is essential. The SAIL databank provides opportunity to trace individuals' interactions with the healthcare system as a whole, including conditions being managed and how they might fit with attendance and prescribing (Jones et al. 2014; Jones et al. 2019). The costanalysis part of this research would not be possible without access to data, only available from the SAIL databank (Jones et al. 2019; Secure Anonymised Information Linkage Databank 2021). There are currently no other means of accessing individual's linked data from a single source, which, spans primary and 
secondary care health services in Wales in addition to the demographic information necessary to make sense of it. Whilst other databanks such as Clinical Practice Research Datalink (CPRD) (Clinical Practice Research Datalink 2021) contain data from Wales, it is only a small part of the total data held and is focussed on primary care. Administrative Data Research UK (ADR UK) is another option for accessing Welsh data but uses the SAIL databank as its source for Wales in any case (Administrative Data Research UK 2021). A comparison of data sources within the UK, which were considered and rejected for use in this research is appended (Appendix A).

The research in this thesis required linked data from across Wales and coverage of all sectors of healthcare. The SAIL databank is the only available source of such complete data and is quality assured in terms of data collection, storage, linkage, analysis and release. Consequently, using the SAIL databank as the data source for this research, was the best option to determine the answers to the questions posed.

\subsection{Ethical considerations}

The SAIL databank provides anonymised person level routine data for research and evaluation (Ford et al. 2009; Jones et al. 2014). Data has commonly recognised means of identification removed before it is submitted to the SAIL Databank. It is considered impossible to reconstruct identities from within SAIL (Lyons et al. 2009). The Information Governance Review Panel (IGRP) of SAIL is an independent group of professionals from a range of organisations including Welsh Government, Public Health Wales and the local Health Board based in Swansea, as well as members of the public. Their role is to quality assure all applications for access to the databank (Ford et al. 2009) and they approved the TOPAS study in 2016 (Section 2.5.1). Consideration of the ethical implications of the study form part of the deliberations by the IGRP, prior to approval. Whilst people have the option of withdrawing their data from the SAIL databank, they must opt-out at the source of data entry e.g., their GP practice. It is therefore 
incumbent on those inputting data at source, to ensure people are aware of the potential for it to be included in research studies.

\subsubsection{Project Application, process and approvals}

The project was discussed with a Senior data analyst from SAIL, as per the internal processes of SAIL (Secure Anonymised Information Linkage Databank 2021). The SAIL analyst wrote a scoping document, based on the discussions and study protocol, to detail the datasets the project would need to access (Appendix A). Following the scoping exercise, an estimate of the costs for the data analyst to undertake the extraction process, was provided by SAIL. Research funding applications were made and secured from Pharmacy Research UK (Ref. PRUK2016-PA1-A), which allowed progression of the project. Approval for the data extraction was subsequently sought and obtained from the IGRP by submission of the project protocol (Appendix A) and application (scope) form (Appendix A). After consent for the research was gained, Safe Researcher Training was undertaken, and evidence of qualification submitted to SAIL, as part of the quality assurance processes for the organisation. On completion of these processes, access to the secure online gateway where the extracted datasets could be gained, was permitted and the project was able to start. Following study approval; a detailed specification for data extraction was developed with the Prudent Healthcare Intelligence Hub (PHIH) team from the Farr Institute, Swansea University (Appendix A). To enable effective dataset identification, a range of inclusions and exclusions were developed based upon the study protocol (Appendix A).

\subsubsection{Data sources}

A split file approach is used to import person level datasets into the secure environment of the SAIL databank. The method facilitates linkage whilst maintaining the anonymisation and confidentiality of the records (Ford et al. 2009; Lyons et al. 2009). Several datasets were used to build the TOPAS data tables: 
- Welsh Demographic Service Dataset (WDSD) includes demographic information such as week of birth, Low Super Output Area (LSOA), Residential Anonymised Linking Field (RALF) (in lieu of addresses), Welsh Index of Multiple Deprivation (WMID) scores, Health Board of residence for each residency period using Local super Output Area (LSOA) levels and registration to General Practitioners in Wales.

- Welsh Longitudinal General Practice (WLGP) data (GP event data) is a record of all interactions in primary care. It is formed by extracting data from $78 \%$ of Welsh General Practice. Data are recorded using specific event codes in Read-code format and an encrypted Anonymised Linkage Field (ALF) for each patient. The ALF can be used to link that individual's records to all other datasets available within the SAIL databank, where they have data recorded.

- Annual District Death Extract (ADDE), which the Office for National Statistics (ONS) provides SAIL with date of death, primary and secondary causes of death via the national repository of mortality data. 


\section{Phase 1}

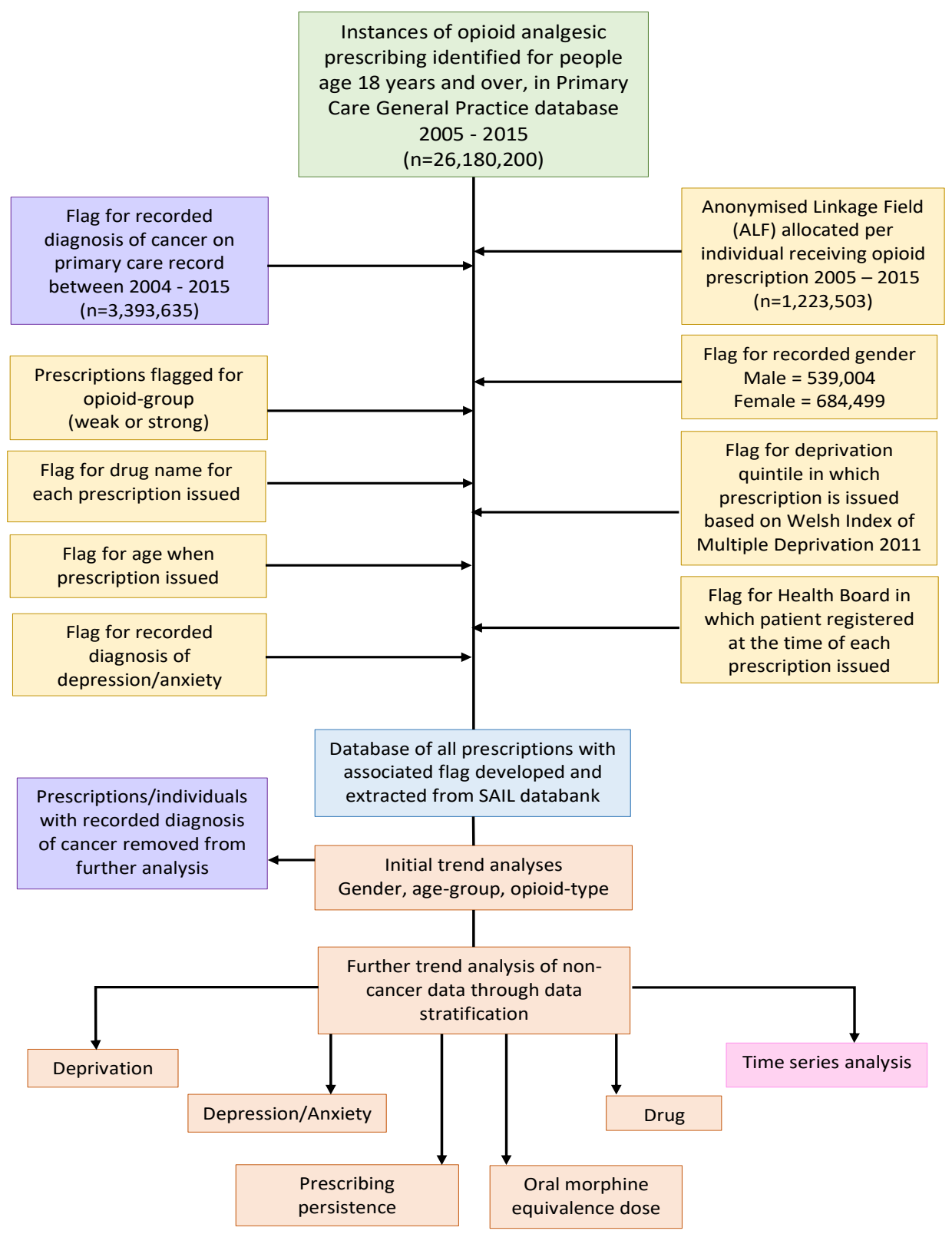

Figure 2.2: Diagrammatic view of data extraction and organisation for phase 1 trend analysis of opioid prescribing $2005-2015$

The methods used throughout the research are described below where they are common to more than one chapter. Methods used to undertake specific data preparation, extraction or analyses are set out in more detail in the corresponding chapters. 


\subsection{Data compilation}

Due to issues of data security, a Research Data Analyst from the Prudent Healthcare Intelligence Hub (PHIH), a section of the Centre for Improvement in Population Health through E-records Research (CIPHER) within the Farr Institute and based at Swansea University, undertook the compilation of data tables from the SAIL Databank. The compiled read-code lists, and other data specifications were used to assemble a series of data tables, which were then linked (Section Error! Reference source not found.). A master table was devised, which included separate columns, known as 'flags' for certain criteria such as 'weak' or 'strong' opioid in order to scrutinise the datasets.

\subsubsection{Identifying Phase 1 study subjects}

A list of opioid medicines was based upon a file shared by colleagues at University of Nottingham (Appendix A), who examined trends in strong opioid prescribing using CPRD, which provides predominantly English data (Zin et al. 2014). The Nottingham list was expanded on the basis of Welsh prescribing data taken from NHS Wales using the Comparative Analysis System for Prescribing Audit (CASPA) (Shared Services Partnership 2021), which demonstrated the range of opioid medications in use over the study period (2005 to 2015)

(Appendix A). Sixteen drug categories (buprenorphine, codeine, dextropropoxyphene, diamorphine, dihydrocodeine, dipipanone, fentanyl, hydromorphone, meptazinol, methadone, morphine, oxycodone, pentazocine, pethidine, tapentadol and tramadol) were included. Read-codes, a thesaurus of clinical terms, used to record all interactions, diagnoses and interventions throughout Primary Care in the NHS, until 2018, were used to develop a list of all products to be included. Lists of read-codes were compiled from the Clinical Terminology Browser available from the NHS Information Authority and accessed via the SAIL gateway. All analgesic products containing the 16 drugs, including where an opioid was combined with another medicine e.g., co-codamol (codeine and paracetamol), were included and identified by their individual read-code. 
The list was checked against both versions of read-codes available ( 22 and v3) to ensure maximum data extraction.

The WLGP database was searched, and every opioid prescription noted within the study period, 1 January 2005 to 31 December 2015 was identified. Corresponding event dates, ALF, demographic information (gender, LSOA, WIMD) were collected using the anonymised linkage systems in place within SAIL (Section 2.4.2). The data was subjected to repeated cross-sectional sampling to determine monthly and annual time-series, prescribing trends over the study period.

\subsubsection{Exclusion of individuals with cancer diagnoses}

This research was concerned with non-cancer related opioid analgesic prescribing. Therefore, following the identification of all relevant opioid prescriptions, data were stratified into cancer and non-cancer groups. A validated list of cancer read-codes was shared from University of Nottingham (Appendix A), checked and verified by the author to ensure both available versions of read-codes ( $\mathrm{v} 2$ and $\mathrm{v} 3$ ) were included. The cancer group included any individual with a cancer diagnosis in their Primary Care General Practice medical record at any time during the study period $(2005-2015)$ or with a qualifying cancer event recorded in the 12 months prior to the study period i.e., between 1 January and 31 December 2004. Data pertaining to individuals with cancer diagnoses were removed from the main dataset and so excluded from the study analyses presented in this thesis.

\subsubsection{Drug classification}

Within the data tables extracted from the main SAIL datasets, the exact product details and a grouping opioid name e.g., codeine were included to assist with categorisation. All opioid analgesics included were categorised into weak or strong (Table 2.1) based on recognized listing by the World Health Organisation (World Health Organisation 2019) and the British National Formulary (BNF: British National Formulary - NICE. 2021) and clinical guidelines, where drugs 
might be categorised on the basis of the order they get used in practice (Scottish Intercollegiate Guideline Network SIGN 2019).

\begin{tabular}{ll} 
Table 2.1: Study categorisation of weak and strong opioids \\
\hline Weak opioid & Strong opioid \\
\hline Buprenorphine $5,10,15,20$ & Buprenorphine - S/L preparations <2mg, \\
micrograms/hour patch & $\geq 35$ micrograms/hour patches \\
Codeine & Diamorphine \\
Dihydrocodeine & Fentanyl \\
Dextropropoxyphene & Hydromorphone \\
Dipipanone & Methadone - tablets only \\
Meptazinol & Morphine \\
Pentazocine (with paracetamol) & Oxycodone \\
Tramadol & Pentazocine \\
& Pethidine \\
& Tapentadol
\end{tabular}

\subsubsection{Age and age-group formation}

Age was calculated based on the week of birth (Monday preceding birth as per SAIL protocol) (SAILdatabank.com). Initially, the range of ages of people included in the study was calculated by assigning their age at an index date of $1^{\text {st }}$ January 2016. For those people who died during the study period; age at the date of death was reported. However, use of the index-date led to data being skewed, particularly in terms of people in the younger age-groups. Consequently, age at the time the prescription was issued, was used. Welsh Government use a 16 to 24 years age-group (StatsWales 2021 and this was adjusted to 18-24 years to bring the study in line with others from the UK where 18 years old was the youngest age included (Torrance et al. 2014; Zin et al. 2014). Data relating to patients aged less than 18 years were removed and only patients aged 18 years or over at any time during the study period $(2005-2015)$ were selected for further analysis. Extracted data were sorted into the designated age-groups chosen for this study, 18-24 years, 25-44 years, 45-64 years, 65-74 years, 75-84 years and $85+$ years groups, mirroring the groupings used by Welsh Government. Data were stratified by age-group for comparison of prescribing trends. 


\subsubsection{Age-group population calculations}

Annual population estimates by age, are available from StatsWales (StatsWales 2021). Data for each year, 2005 to 2015 , for each age from 18 years to the maximum recorded, were extracted in an Excel file. The percentage of the total Welsh population each age-group comprised, was calculated for each year. The percentages were then used to determine the SAIL population for each agegroup, using the previously calculated SAIL annual populations. The annual SAIL populations per age-group were used to adjust the number of people and prescriptions issued to each people within each age-group to per 1,000 agegroup adjusted figures (Appendix A), providing a more balanced comparison.

\subsubsection{Standardising population estimates}

At the time of this study, the SAIL databank contained data for $78 \%$ of the Welsh population Databank 2021). As data become available to SAIL, it is backdated in the databank, so there was consistent representation throughout the study period. However, representation of each Health Board within the SAIL databank is proportionally different (Table 2.2). To determine population adjusted data, a method for populace estimation was devised.

Table 2.2: Representation of General Practice within SAIL Databank by Health Board

\begin{tabular}{lccc}
\hline 2015 Population & (\% total) & $\begin{array}{c}\text { Number of GP } \\
\text { Practices } \\
\text { (\% Wales total) }\end{array}$ & $\begin{array}{c}\text { GP Practices } \\
\text { registered with } \\
\text { SAIL } \\
\text { (\% SAIL total) }\end{array}$ \\
\hline Wales total & $3,099,086(100)$ & $443(100)$ & $345(78)$ \\
\hline Health Board (HB) & & & \\
Abertawe Bro Morgannwg & $525,466(17)$ & $73(16)$ & $70(20)$ \\
Aneurin Bevan & $581,789(19)$ & $82(19)$ & $57(17)$ \\
Betsi Cadwaladr & $694,473(22)$ & $109(25)$ & $82(24)$ \\
Cardiff and Vale & $484,752(16)$ & $66(15)$ & $53(15)$ \\
Cwm Taf & $296,735(10)$ & $43(10)$ & $33(10)$ \\
Hywel Dda & $383,229(12)$ & $53(12)$ & $43(12)$ \\
Powys & $132,642(4)$ & $17(4)$ & $7(2)$ \\
\hline
\end{tabular}

The Health Board areas used for the TOPAS study came into force in 2009 when new organisations were formed (NHS Wales 2009). Mid-year populations estimates for each Health Board (Table 2.2) were taken from StatsWales (StatsWales 2021) for the years 2009 - 2015. The proportion of the total Welsh 
population living within each Health Board area was calculated. The percentages were used to calculate equivalent Health Board populations between 2005 and 2008 prior to the new organisations' emergence. The calculated or extracted population of each Health Board was adjusted to reflect their percentage representation in the SAIL databank. Population totals for each health board were added together to give an annual total for the SAIL databank. Calculated population numbers were divided by 1000 in order to calculate results per 1,000 population (Figure 2.3).

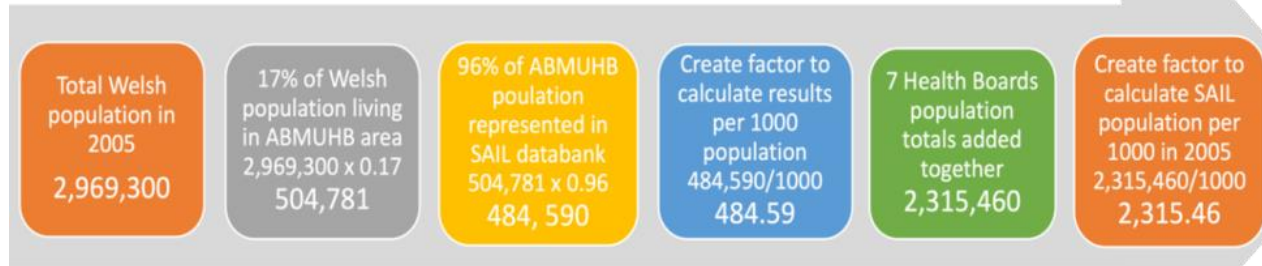

Figure 2.3: Method for calculating SAIL databank population for the TOPAS study, using Abertawe Tawe Bro Morgannwg University Health Board (ABMUHB) population 2005 as an example factor

\subsubsection{Gender population standardisation}

Yearly estimates of Welsh population by gender are available from StatsWales (StatsWales 2021). The population estimates for 2015 given by gender and by Health Board, for people aged 18 years and over, were extracted into an Excel spreadsheet (Appendix A). Percentage representation of women and men were calculated for each Health Board (Figure 2.4). The percentages were used to compute the population of men and women each year of the study and further adjusted to provide population per 1,000 that were subsequently used to amend the numbers of people and prescriptions throughout the study. 


\subsubsection{Population standardisation for deprivation quintiles}

To enable population-standardised data for the deprivation studies, local super output area data (LSOA) was used to calculate the annual population assigned to each deprivation quintile per Health Board area. LSOAs are small areas accounting for around 1500 people, which are assigned a deprivation score. Those scores are then ranked and further assigned into quintiles where 1 is the most deprived and 5 the least. These are then used to develop the Welsh Index of Multiple Deprivation (WIMD).

The percentage of each Health Board population living in each WIMD2011 (the WIMD for 2011 chosen for use in this study) quintile was calculated and used to adjust to the general practice primary care population represented in SAIL (Appendix A). Estimated populations for each quintile were totalled to provide national numbers and used to adjust results and provide 'per 1,000 quintile population' results. A more detailed description of LSOAs, WIMD and the population calculations is included in Chapter 4.

\subsubsection{Condition-linked data}

Initially, it was hoped data extraction would allow an estimation of prevalence of pain-associated conditions and/or depression and anxiety within the whole population and compared to those receiving opioid analgesic prescriptions (Section 1.7). Consequently, read-code lists were developed (Appendix F and Appendix $\mathrm{H}$ ) to identify individuals who had at least one of six pain-related 
conditions: rheumatoid and osteo-arthritis, back and neck pain, neuropathic pain and fibromyalgia, or codes related to depression and/or anxiety on their primary care records. For Phase 1 of the study, the pain-associated condition data was not used to examine the whole SAIL dataset. Instead, data was linked only to those also receiving opioid analgesic prescriptions, which did not therefore provide an accurate reflection of prevalence. Consequently, the condition data was used only to identify subjects for Phase 2 of the study (Section 7.4.2).

A request was made to SAIL for a table to contain data for all individuals aged 18 and over, with data in the SAIL databank who had a record of depression and/or anxiety on their Primary Care record. The table was cross-referenced with the main TOPAS data table (individuals receiving opioid analgesic prescriptions) and details of any individual whose data appeared in both tables was to be extracted into a third dataset. The new dataset was to contain data for any person, aged 18 or over, with a recorded diagnosis of depression and/or anxiety and who received a prescription for at least one opioid medicine between 2005 and 2015. Within the main TOPAS dataset, a flag was added to indicate individuals with a recorded diagnosis of depression and/or anxiety. Following analysis of the data, clarification of the method of subject identification was requested from SAIL, but not provided. The data could not, therefore, be validated for the main thesis but does show some interesting signals and so is included in Appendix $\mathrm{H}$ and should be subject to further research.

\subsubsection{Data extraction and analysis}

In order to extract data for descriptive analysis, Structured Query Language (SQL) coding based on the study meta-data (Table X) was used, to interrogate the main data tables.

SQL is standardised programming language used for managing relational databases and performing operations on the data in them. Developed in the 1970 's, SQL is used by data analysts for setting up and running analytical queries (Techtarget 2021). 
Table 2.3: Principal metadata for prescription trend analysis (TOPAS_20170419)

\begin{tabular}{|c|c|c|}
\hline Column name & Description & Project specific detail \\
\hline EVENT_DT & Date of event & Date of prescription \\
\hline ALF_PE & Unique patient identifier & \\
\hline GNDR_CD & Gender code & 1=Male 2=Female \\
\hline DRUG_CAT & Drug name & Tramadol, Morphine etc \\
\hline W_S & $\begin{array}{l}\text { Weak / Strong } \\
\text { categorization }\end{array}$ & \\
\hline CANCER & Cancer patient flag & $\begin{array}{l}\text { Cancer = } 1 \text { (if patient had } \\
\text { cancer diagnosis in study } \\
\text { period } 2005-2015 \text { ) }\end{array}$ \\
\hline LHB_DESC & $\begin{array}{l}\text { Local Health Board } \\
\text { Description }\end{array}$ & $\begin{array}{l}\text { 7A1 - Betsi Cadwaladr } \\
\text { 7A2 - Hywel Dda } \\
\text { 7A3 - Abertawe Bro } \\
\text { Morgannwg } \\
\text { 7A4 - Cardiff and Vale } \\
\text { 7A5 - Cwm Taff } \\
\text { 7A6 - Aneurin Bevan } \\
\text { 7A7 - Powys }\end{array}$ \\
\hline WIMD2011_5 ${ }^{\text {TH }}$ & 5 Decile & $\begin{array}{l}\text { Welsh Index of multiple } \\
\text { deprivation }\end{array}$ \\
\hline DRUG_USAGE_PERIOD & $\begin{array}{l}\text { Flag for short term / long } \\
\text { term use }\end{array}$ & $\begin{array}{l}1=\text { short term user }(<182.5 \\
\text { days) } \\
2=\text { long term user ( }>182.5 \\
\text { days) } \\
3=\text { other (includes non- } \\
\text { actives) }\end{array}$ \\
\hline
\end{tabular}

SQL coding was written and entered into the SQL Editor of Eclipse SDK software (Figure 2.5). Each search was run, and the results extracted from Eclipse SDK into Excel 2016 spreadsheets and subsequently to IBM SPSS Statistics v26 software for further analysis. Extracted data was presented as frequency tables and then rearranged for analysis by SPSS (IBM Corporation 2021).

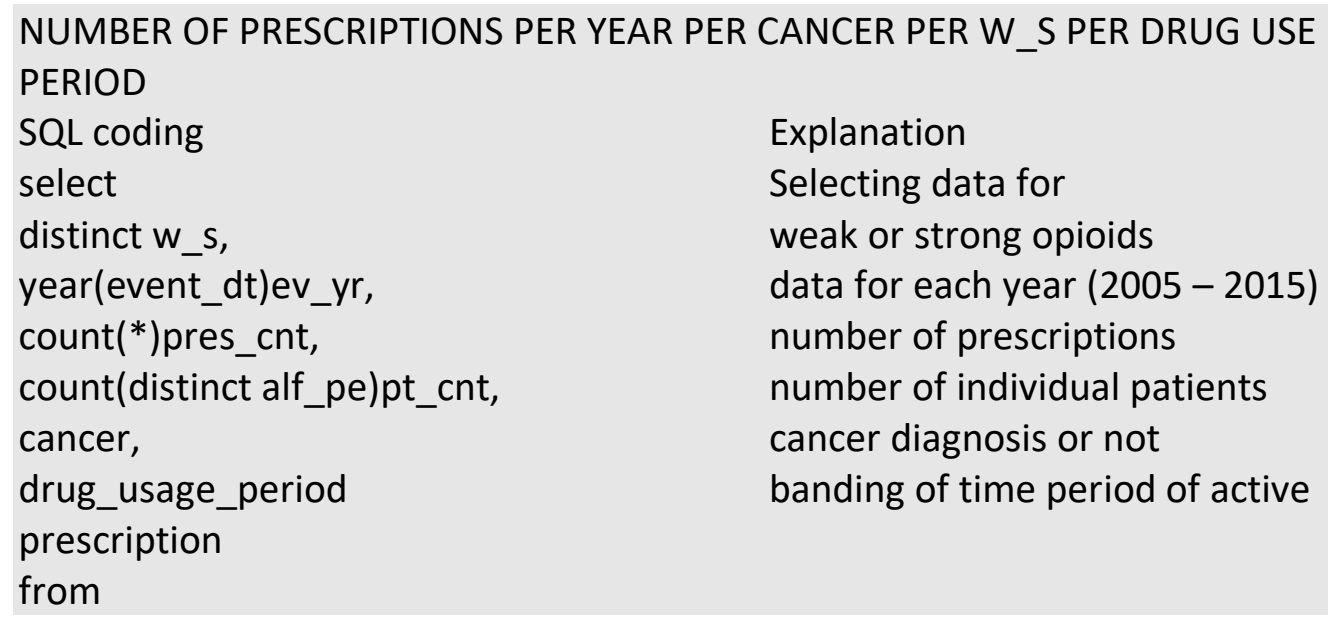

\section{Explanation}

Selecting data for weak or strong opioids data for each year (2005 - 2015) number of prescriptions number of individual patients cancer diagnosis or not banding of time period of active 
SAILW0507V.TOPAS_20170419

project

group by

columns

w_s,

year(event_dt),

cancer,

drug_usage_period

prescription

order by

ev_yr

Figure 2.5: Example and explanation of SQL code for data extraction main data table for the TOPAS

results table to have the following

weak or strong opioid

year

cancer diagnosis or not

banding of time period of active

data to be presented by

year $(2005-2015)$

In order to perform statistical analysis, the main data table from SAIL was downloaded into SPSS. The table was cleaned to remove unnecessary columnar data. For example, there were multiple gender codes taken from the Primary Care General Practice database; there were 8 possible causes of death available although it was rare that more than 2 were completed. Replicated or unneeded data were removed and then split into cancer and non-cancer tables. The noncancer table was further split into weak and strong opioid tables to improve the processing speed.

\subsubsection{Data security and quality assurance}

All data processing was performed within the secure SAIL gateway and stored on SAIL servers. The in-house quality assurance processes were applied to each data extraction undertaken by the SAIL analyst as the study tables were compiled (Jones et al. 2019). When data processing was complete, a request to extract results tables and graphs was made to SAIL. The SAIL Data Guardian who ensures any risk of disclosure has been assuaged, assesses all requests for data extraction. If the requests met the criteria for release, it was then downloaded from the secure gateway and could be used to develop tables and figures for inclusion in the thesis (Secure Anonymised Information Linkage Databank 2021).

\subsection{Statistical analysis}

The study produced a large amount of data and a range of variables which lent themselves to a number of different analyses. The study population for the 
Phase 1 study, were selected from the whole of the SAIL databank based on the presence of opioid prescriptions on the primary care medical record, therefore, normal distribution was not assumed. Shapiro-Wilks confirmed Phase 1 data were not normally distributed and so non-parametric tests were selected for the analysis (Chapter 3, Chapter 4 and Chapter 5). The trend analysis for phase 1 concentrated initially on examining the distribution of e.g., prescriptions over the 11-year study period and examined correlation between time and prescribing rate over that time. In addition to trend analysis, differences between groups e.g., ordinal variables such as socioeconomic quintiles and binary variables e.g., gender, weak or strong opioid groups, were used to stratify datasets before comparisons were made between the trends in each group. The rate of change was also used as the basis for investigating the effect of legislation or guidelines

Phase 2 of this study selected a cohort of individuals again from the whole SAIL databank population which represents $78 \%$ of the Welsh population. The study cohort was determined by diagnosis (Section Error! Reference source not found.) and then further stratified by opioid prescription. It might be assumed that due to the census-type data selection, normal distribution would not be likely. However, due to the large sample size in both arms of the study (case and control), we determined parametric tests could provide accurate analysis. As with Phase 1, descriptive statistics were used to examine patterns of healthcare use and associated costs in case and control groups and comparisons between and within groups, using categorical variables, to stratify groups. Regression analyses were also undertaken to examine the relationships between variables and the study outcome of healthcare utilisation. Whilst parametric testing was used for analysis, to ensure it was an accurate representation of the data, nonparametric tests were also performed. The output of those analyses is presented in Appendix F.

\subsubsection{Spearman's rank correlation}

Spearman's rank correlation (Spearman's Rho) is a non-parametric measure. It assesses how the relationship between two variables is described or the 
statistical dependence between them. For this study, Spearman's rank correlations were used to determine the relationship between time and the rate of prescribing (Chapter 3, Chapter 4 and Chapter 5). This method of examining change over time was described by Mordecai et al in 2018 (Mordecai et al. 2018). The closer to 1.000 or -1.000 the result of Spearman's rank correlation is, the stronger the relationship between the two variables e.g., Spearman's r>.999 indicates the rate of prescribing was strongly linked to time or, more simply, prescribing increased over time.

\subsubsection{Mann-Whitney U tests}

The Mann-Whitney $U$ test is a non-parametric test that examines differences between two groups or populations. It takes the null hypothesis to be that for randomly selected values ( $A$ and $B$ ) from two groups, the probability of $A$ being greater than $B$ is equal to the probability of $B$ being greater than $A$.

Mann-Whitney $U$ tests were used to examine differences between e.g. the number of strong opioid prescriptions per 1,000 population received by men or women (Chapter 3, Chapter 4 and Appendix B). For the purposes of this study, the tests examine all 11 years of data, rather than differences in the trends described.

\subsubsection{Kruskal-Wallis tests}

The Kruskal-Wallis test is an extension of the Mann-Whitney $U$ test. Again, it is a non-parametric test used to compare two or more groups. Whilst Kruskal-Wallis can determine if a difference exists e.g., if one group is dominant or greater for example, it does not pinpoint where exactly that difference lies. Consequently, pairs of data are also contrasted using Dunn's pairwise comparison and Bonferroni correction. These tests are used in this study to compare e.g., how the number of prescriptions issued between 2005 and 2015 differ between quintiles of deprivation (Chapter 4 and Appendix C). Kruskal-Wallis tests might suggest more prescriptions are issued in some quintiles than others but do not reveal where the differences lie. Dunn's pairwise comparisons provide that 
detail. For example, following a statistically significant Kruskal-Wallis test, Dunn's pairwise comparison might then illustrate more prescriptions are issued in quintile 1 compared to quintile 2 , but more prescriptions are issued in quintile 2 than in quintile 3.

\subsubsection{Time series analysis}

Interrupted and continuous times series analyses were conducted to examine whether changes in UK legislation or Welsh National Prescribing Indicators impacted prescribing trends between 2005 and 2015. An explanation of the methods used is provided in Chapter 6.

Continuous TSA was conducted using SPSS v26 software (IBM Corporation 2021) to sort the data and Microsoft Excel software (Microsoft Corporation 2018) to undertake calculations and plot graphs. Interrupted TSA analyses were completed using SAS 9.4 software (SAS Institute Inc 2021). 


\section{Phase 1}

Chapter 3

General trends in opioid prescribing for non-cancer pain

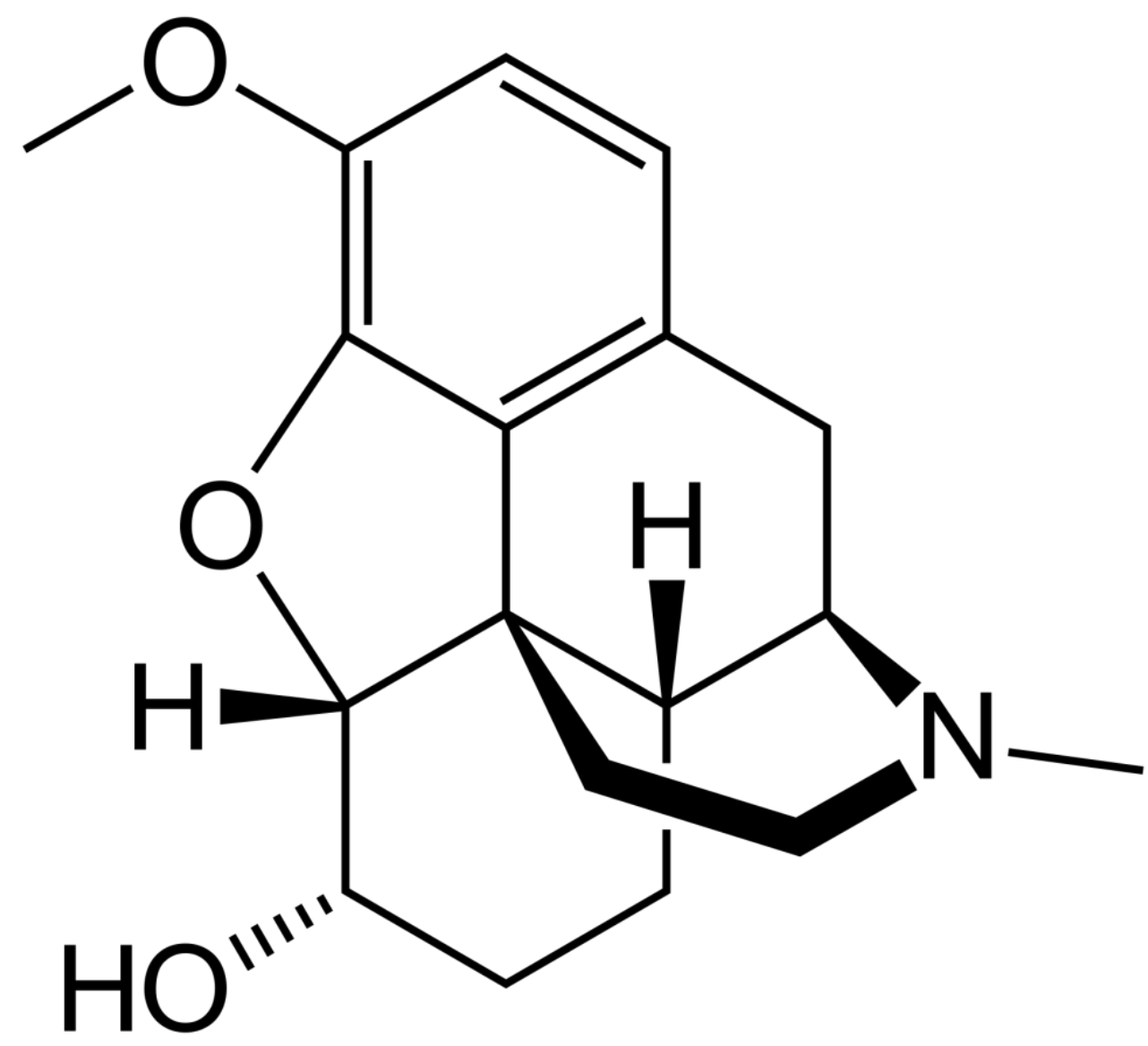

"If you do what you've always done, you'll get what you've always got." Henry Ford 


\section{Chapter 3 - General trends in opioid prescribing for non- cancer pain}

\subsection{Chapter overview}

In Chapter 1, the background information which formed the overarching aims for this research were introduced and discussed. In Chapter 2, the methods adopted for the project were set out. In this first chapter of Phase 1, the main trends in opioid analgesic prescribing in Wales, between 2005 and 2015 will be presented. In addition to analysing the overall trends in terms of number of prescriptions, the data will be stratified by opioid-type (weak or strong opioid), gender and age. An overview of prescribing persistence throughout the study period is also examined.

\subsection{Study objective to be addressed in this chapter}

- To examine prescribing trends of opioid analgesics between 2005 and 20015 and scrutinise whether trends differed between gender or different agegroups

\subsection{Background}

Before this study was conceived, the number of prescriptions for opioid analgesics being issued in Wales, was known to have increased (All Wales Medicines Strategy Group 2013). Studies from across the UK had been published, raising opioid prescribing as a concern. Zin et al. (2014) published one of the first UK studies, examining the changes to strong opioid prescribing and stratified by diagnosis (cancer or non-cancer), defined daily dose and oral morphine equivalent and days of supply (Zin et al. 2014). Ruscitto et al. (2015) examined prescribing trends of opioids and other analgesics over a 16-year period in Tayside, Scotland. Results were presented by numbers of prescriptions and stratified by drug, individuals age and gender (Ruscitto et al. 2015). Bedson et al. (2016) looked at changes in the incidence of long-term opioid prescribing in a UK primary care population, from 2002 until 2013. Trends were broken down by gender and age-group, as well as the release-profile of the opioid analgesic. 
Taking the example from previous authors, the results presented in this chapter will examine the numbers of opioid analgesic prescriptions issued each year between 2005 and 2015 and adjusted to population. Gender (Section 1.8) and age (Section 1.9) have been demonstrated to be factors which can impact pain prevalence and potentially therefore, prescribing. Consequently, data are examined in those stratifications. Opioid analgesics are divided into 'weak' or 'strong' classifications (Section 2.6.3) and so trends within those groups are analysed. Finally, as Bedson et al. (2016) demonstrated, long-term prescribing is known to have increased in the UK (Bedson et al. 2016) but has not been specifically examined in Wales previously. An estimated measure of opioid prescribing persistence was developed for this study and preliminary results presented here.

\subsection{Method}

\subsubsection{Trend analysis}

Individuals were identified and corresponding data relating to opioid prescriptions and demographics, extracted from the SAIL databank as described in Section 2.6. The number of prescriptions issued each year were totalled and also adjusted to population, with descriptive statistics used to describe the trends observed. Spearman's rank correlations (Section 2.7.1) were used to examine the change in prescribing rate over the study period, using all data between 2005 and 2015, in addition to calculating percentage changes over time.

Data were stratified into groups based on gender (Section 2.6.5.1), age-group (Section 2.6.4) and also the type of opioid prescribed on each individual prescription (Section 2.6.3). Comparisons of the number of prescriptions or prescribing rate, between genders and drug-type were made using MannWhitney $U$ tests (Section2.7.2). Comparisons between age-groups used KruskalWallis tests, with Dunn's pairwise comparison and Bonferroni corrections to confirm where any statistically significant differences were (Section 2.7.3). 


\subsubsection{Determining prescription persistence}

Controlled drug prescriptions (which include opioids), have a legally valid duration of 28 days, after which time the prescription cannot be dispensed. Practice guidelines advise prescribers not to prescribe greater than 30 days of controlled drugs on a single prescription (Royal Pharmaceutical Society of Great Britain 2021)

At the time of data extraction, the SAIL databank did not include dispensing data, which would allow analysis of dosing instructions provided with the prescription and quantity of medicine prescribed. From that data, it would be possible to determine the daily dose of each medicine prescribed and make an estimation of the duration of use for the prescription issued. Consequently, prescription persistence was estimated by tracking the days between prescriptions issued, for each individual in the dataset. The total number of days between the first prescription issued and the final one, where each prescription was issued within 31.5 days of the previous one, was listed as the total prescribing period. Where a second prescription was not issued within 31.5 days of the first, it was listed as a one-off prescription.

\subsubsection{Trends analysis}

Data was stratified by year, to develop trend analysis of prescribing persistence between 2005 and 2015. All prescriptions issued to any individual were examined. The year in which the prescribing period ended was used as the index date for each prescribing period. For example, an individual receiving 3640 days of prescription coverage, starting in 2005 and ending in 2014, would be counted in the 2014 total, despite having data in every year between.

\subsubsection{Stratification of prescribing persistence data}

For the purposes of this analysis, data were examined for an individual's opioid use over a period of time, rather than examining by each individual drug prescribed. Data were stratified by the type of opioid prescribed at the end of each prescribing period. For example, where a person had a period of prescribing 
which ended with morphine, a 'strong' opioid, they would be flagged as 'strong opioid' even if earlier in the prescribing period, they had been prescribed a 'weak' opioid. Data were also stratified by gender and socioeconomic deprivation quintile as previously described and adjusted to population (Appendix G).

\subsection{Results}

Between 2005 and 2015, 22,786,565 opioid analgesic prescriptions were issued to $1,099,026$ individuals with non-cancer diagnoses whose data was contained in the SAIL Databank. Trends in the number of people receiving prescriptions were analysed and followed similar patterns to those of the numbers of prescriptions. Consequently, those results are not presented in this chapter but are included in Appendices B, C and D.

\subsubsection{Overview of prescribing trends}

Total opioid prescribing rate increased by $49.7 \%$ between 2005 and 2015 (Table 3.1). Between 2005 and 2010, the annual number of opioid prescriptions issued increased $27.5 \%$ (from 696.8 to 888.2 prescriptions per 1,000 population). There was a slower increase of $12.7 \%$ (from 888.2 to 1000.7 prescriptions per 1,000 population), between 2010 and 2015 (Appendix B). However, while the increasing trend in the number of people being prescribed opioids had started to decline, the number of prescriptions issued each year was still increasing at the end of the study period (Error! Reference source not found.).

\begin{tabular}{|c|c|c|c|c|c|}
\hline Group & 2005 & 2015 & $\begin{array}{c}\text { Change in } \\
\text { variable }\end{array}$ & $\begin{array}{l}\text { Percentage } \\
\text { change (\%) }\end{array}$ & $\begin{array}{c}\text { Spearman's r, p- } \\
\text { value }\end{array}$ \\
\hline \multicolumn{6}{|l|}{ Total } \\
\hline Prescriptions & $1,613,417$ & $2,415,374$ & 801,957 & 49.7 & $>.999, p<.001^{*}$ \\
\hline Prescriptions per 1,000 & 696.8 & 1000.7 & 303.9 & 43.6 & $>.999, p<.001^{*}$ \\
\hline \multicolumn{6}{|l|}{ Weak opioids } \\
\hline Prescriptions & $1,520,441$ & $2,021,677$ & 801,957 & 33.0 & $0.945, p<0.05^{*}$ \\
\hline Prescriptions per 1,000 & 656.6 & 837.6 & 181.0 & 27.6 & $0.909, p<0.01 *$ \\
\hline \multicolumn{6}{|l|}{ Strong opioids } \\
\hline Prescriptions & 92,976 & 393,697 & 300,721 & 323.4 & $>.999, p<.001^{*}$ \\
\hline Prescriptions per 1,000 & 40.2 & 163.1 & 123.0 & 306.2 & $>.999, p<.001^{*}$ \\
\hline
\end{tabular}

$*$ p $<0.05$ = statistically significant. Annual data Appendix B 


\subsubsection{Trends in prescribing by type of opioid analgesic}

Between 2005 and 2015, weak opioid prescribing rates rose by $27.6 \%$, with a strong correlation confirmed by Spearman's Rho (Table 3.1). Weak opioids prescribing peaked in 2012 following a 30\% increase (from 656.6 to 853.8 prescriptions per 1,000 population) (Figure 3.1). Whilst overall annual numbers of prescriptions rose over the 11 years of the study, there was a $1.9 \%$ reduction (from 853.8 to 837.6 prescriptions per 1,000 population) in prescription rate between 2012 and 2015 (Figure 3.1 and Appendix B).

Strong opioid prescribing rates increased by $306.2 \%$ (from 40.2 to 163.1 prescriptions per 1,000 population) over the study period (Table 3.1). In the first 6 years of the study (2005 to 2010), prescribing increased 112.4\% (from 40.2 to 85.3 prescriptions per 1,000 population). From 2010 until the end of 2015 however, the overall change was $91.2 \%$ (from 85.3 to 163.1 prescriptions per 1,000 population) (Figure 3.1).

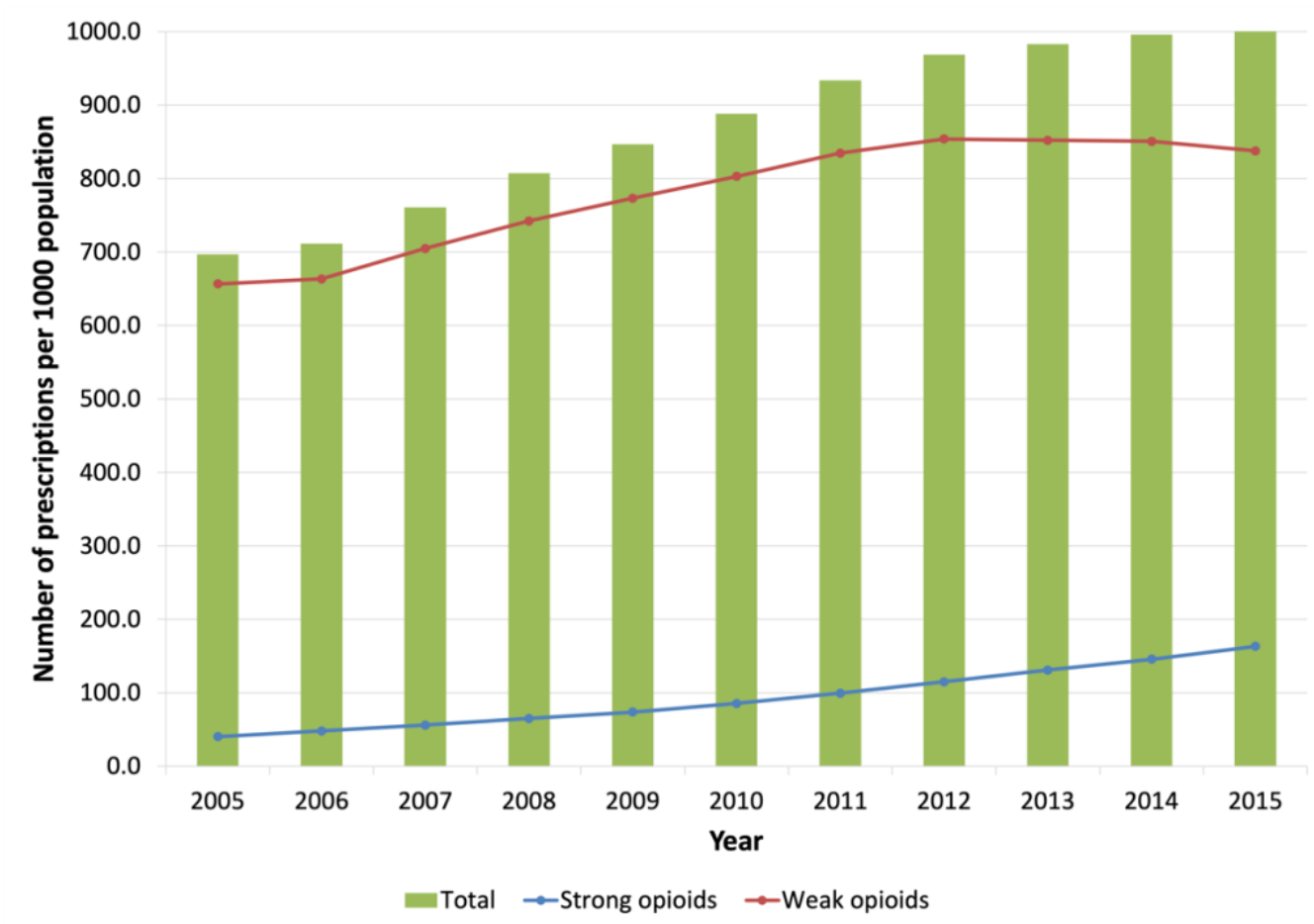

Figure 3.1: Trends in number of prescriptions per 1,000 issued between 2005 and 2015, stratified by opioidtype

Weak opioid prescribing covered $89.3 \%$ (20,350,394 of 22,786,565 prescriptions) of all opioid prescribing between 2005 and 2015. Whilst annual prescription 
numbers peaked in 2012, the percentage of weak opioid prescriptions as a percentage of all opioid prescriptions being issued, reduced annually. Strong opioids accounted for $10.7 \%$ of all opioid analgesic prescriptions issued between 2005-2015. However, the percentage of total prescribing accounted for by strong opioids rose from $5.8 \%$ (40.2 from 696.8 total prescriptions per 1,000 population) to $16.3 \%$ (163.1 from 1000.7 total prescriptions per 1,000 population) over that time.

\subsubsection{Trends in prescribing by gender}

Women comprised $56.4 \%$ of the total number of people receiving opioid prescriptions. Just under $30 \%$ percent more individual women $(n=620,288)$ than men $(n=478,738)$ received opioid prescriptions between 2005 and 2015. On an average basis however, $60 \%$ of people receiving prescriptions each year were women (Appendix B).

One and a half times more prescriptions for opioid analgesics were issued to women than men between 2005 and 2015 (Table 3.2 and Appendix B). Over the study period, women received 13,992,935 prescriptions, significantly more than the $8,793,630$ received by men (Mann-Whitney $U$ test, $U=121.00, S E=15.23$, $\left.p<.001, \eta^{2}=0.72, d_{\text {Cohen }}=3.19\right)$. 


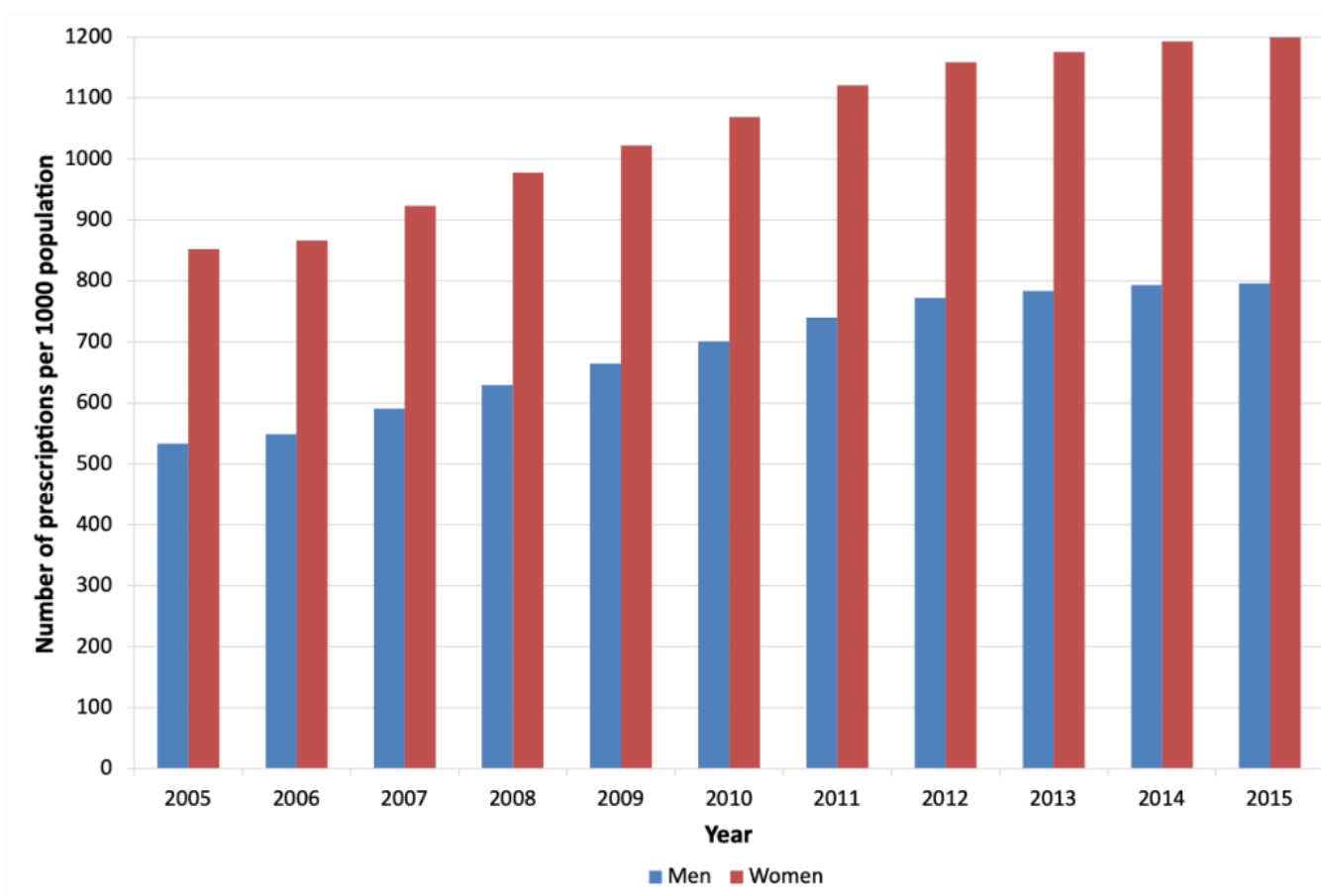

Figure 3.2: Trends in opioid analgesic prescribing rate 2005 to 2015, stratified by gender

Women steadily received the same proportion of all prescriptions throughout the study period, accounting for around $60 \%$ of all opioid prescriptions issued in total, consistent with results already set out (Figure 3.2).

Table 3.2: Gender trends in opioid prescription rates during the study period, 2005 - 2015

\begin{tabular}{|c|c|c|c|c|c|}
\hline & \multicolumn{2}{|c|}{$\begin{array}{l}\text { Per } 1,000 \text { gender } \\
\text { population }\end{array}$} & \multirow[t]{2}{*}{$\begin{array}{l}\text { Change in } \\
\text { variable }\end{array}$} & \multirow[t]{2}{*}{$\begin{array}{l}\text { Percentage } \\
\text { change (\%) }\end{array}$} & \multirow[t]{2}{*}{$\begin{array}{c}\text { Spearman's r } \\
\text { p-value }\end{array}$} \\
\hline & 2005 & 2015 & & & \\
\hline \multicolumn{6}{|l|}{ Total } \\
\hline Men & 533.1 & 795.6 & 262.5 & 49.3 & $>.999, p<.001^{*}$ \\
\hline Women & 852.6 & 1199.6 & 347.0 & 40.7 & $>.999, p<.001^{*}$ \\
\hline \multicolumn{6}{|l|}{ Weak opioid } \\
\hline Men & 496.4 & 665.6 & 169.2 & 34.1 & $0.909, p<.001^{*}$ \\
\hline Women & 809.1 & 1004.3 & 195.2 & 24.1 & $0.909, p<.001^{*}$ \\
\hline \multicolumn{6}{|l|}{ Strong opioid } \\
\hline Men & 36.6 & 130.0 & 93.4 & 255.2 & $>.999, p<.001^{*}$ \\
\hline Women & 43.5 & 195.2 & 151.7 & 348.7 & $>.999, p<.001^{*}$ \\
\hline
\end{tabular}

${ }^{*} \mathrm{p}<0.05=$ statistically significant. Annual data Appendix B.

Women and men saw year-on-year increases in the number of opioid prescriptions issued between 2005 and 2015, although the rates of increase were reducing towards the end of the study period (Error! Reference source not found.).

Weak opioid prescribing rates rose more for men than women in the time period examined. As previously illustrated (Figure 3.1), there were peaks in prescribing 
rates of weak opioids for men and women in 2012, followed by small reductions in annual numbers (Appendix B).

As was seen with overall prescribing rates, women received around $60 \%$ of all weak opioid prescriptions issued over the study period. Predictably, women received significantly more prescriptions than men for weak opioids (Table 3.2), as confirmed by a Mann-Whitney $U$ test $\left(U=121.00, S E=15.23, p<.001, \eta^{2}=0.72\right.$, $\left.d_{\text {Cohen }}=3.19\right)$.

Very large increases were observed in strong opioid prescribing rates for men and women between 2005 and 2015 (Table 3.2). Spearman's Rho correlations were very strong for both genders $(r>.999, p<.001)$, demonstrating a clearly positive upwards trend over time (Table 3.2).

Year on year increases in strong opioid prescribing rates were noted throughout the study period although for both genders, the rate of increase slightly slowed in the second half of the study period (Appendix B). Between 2005 and 2015, prescribing rose $83.7 \%$ (from 36.6 to 69.0 prescriptions per 1,000 population) for men and $91.8 \%$ (from 43.5 to 101.0 prescriptions per 1,000 population) for women. In the second half of the period, 2010 to 2015, men's strong opioid prescribing rates rose $81.7 \%$ (from 69.0 to 130.0 prescriptions per 1,000 population) and for women it was $87.3 \%$ (from 101.0 to 195.2 prescriptions per 1,000 population) (Appendix B).

\subsubsection{Prescribing by age}

Using the age on the date of prescription, the average age for people receiving an opioid prescription for any indication was 55.2 years (SD=18.0, range 18-110 years). In individual terms, people aged 45 to 64 years, were the most represented age group between 2005 and 2015 (Figure 3.3) with nearly half a million more people in that age-group receiving opioid prescriptions than in any other. 


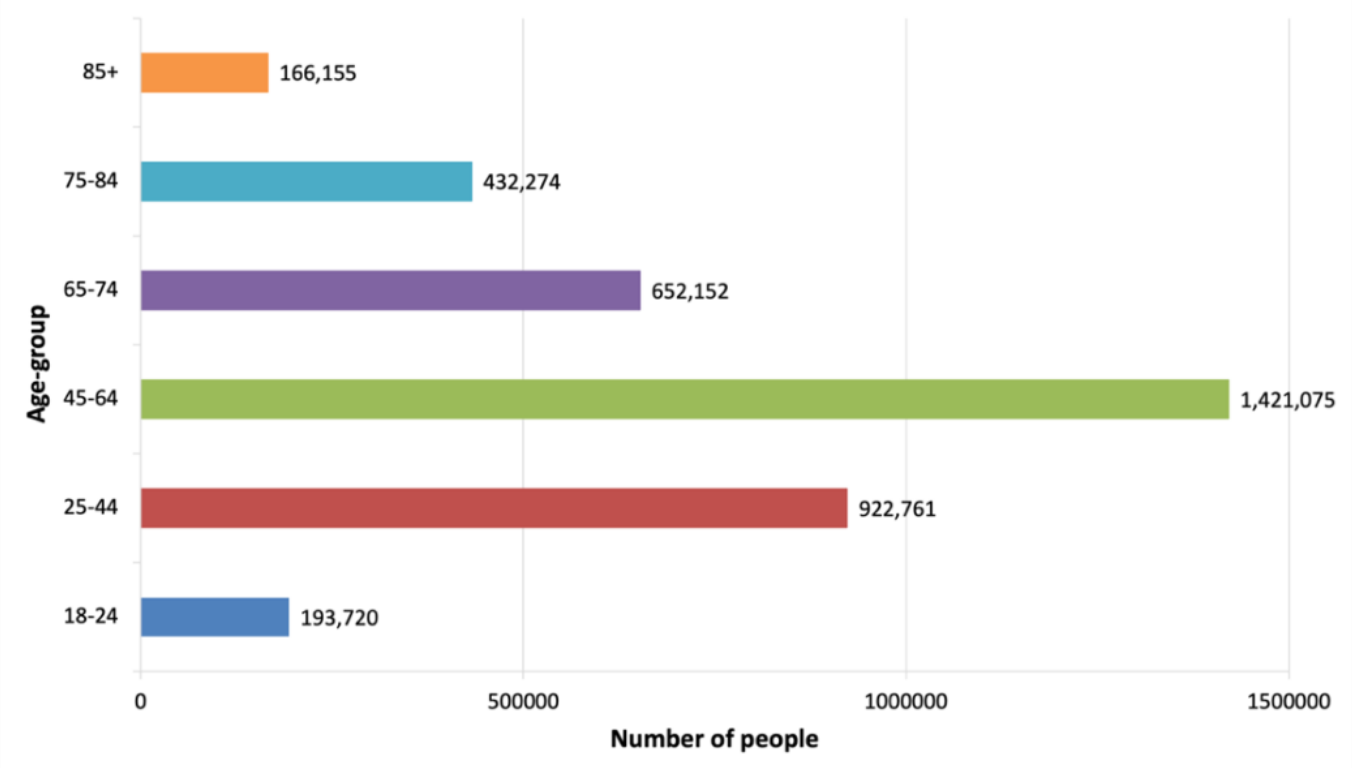

Figure 3.3: Number of people receiving opioid analgesic prescriptions between 2005 and 2015, stratified by age-group

People aged 65 years and above between 2005 and 2015, received more prescriptions than those in lower age-groups (Table 3.3). Eleven times more prescriptions (per 1,000 population) were issued to people aged 85 years and over, compared to those aged between 18 and 24 years old (Appendix B). In respect of number of prescriptions issued there was a 2-times difference between the oldest and youngest age-groups, which points to disproportionate prescribing by age (Error! Reference source not found.). Increases in the rates of prescribing (prescriptions per 1,000) were noted for all age groups over the study period, although the youngest age-group (18 to 24 years) demonstrated only moderate correlation compared to strong correlations noted in all other agegroups (Table 3.3).

Table 3.3: Change in rates of prescribing (adjusted to population) issued 2005 to 2015, stratified by agegroup

\begin{tabular}{|c|c|c|c|c|c|}
\hline \multirow[t]{2}{*}{$\begin{array}{l}\text { Age group } \\
\text { (years) }\end{array}$} & \multicolumn{2}{|c|}{$\begin{array}{l}\text { Per } 1,000 \text { age- } \\
\text { adjusted population }\end{array}$} & \multirow{2}{*}{$\begin{array}{c}\text { Change } \\
\text { in } \\
\text { variable }\end{array}$} & \multirow[t]{2}{*}{$\begin{array}{l}\text { Percentage } \\
\text { change (\%) }\end{array}$} & \multirow[t]{2}{*}{$\begin{array}{l}\text { Spearman's r } \\
\text { p-value* }\end{array}$} \\
\hline & 2005 & 2015 & & & \\
\hline \multicolumn{6}{|l|}{ Total } \\
\hline $18-24$ & 119.1 & 123.8 & 4.7 & 4.0 & $.418, p=0.201$ \\
\hline $25-44$ & 394.7 & 598.5 & 203.8 & 51.6 & $.991, p<.001^{*}$ \\
\hline $45-64$ & 822.1 & 1229.0 & 406.9 & 49.5 & $>.999, p<.001^{*}$ \\
\hline $65-74$ & 1211.8 & 1421.5 & 209.7 & 17.3 & $.909, p<.001^{*}$ \\
\hline $75-84$ & 1271.1 & 1596.2 & 325.1 & 25.6 & $.982, p<.001^{*}$ \\
\hline $85+$ & 1359.1 & 1573.0 & 214.0 & 15.7 & $.945, p<.001^{*}$ \\
\hline \multicolumn{6}{|l|}{ Weak opioid } \\
\hline $18-24$ & 110.9 & 114.2 & 3.2 & 2.9 & $.418, p=0.201$ \\
\hline
\end{tabular}




\begin{tabular}{rccccc}
\hline $\mathbf{2 5 - 4 4}$ & 362.2 & 489.8 & 127.6 & 35.2 & $.873, p<.001^{*}$ \\
$\mathbf{4 5 - 6 4}$ & 773.8 & 1010.1 & 236.3 & 30.2 & $.873, p<.001^{*}$ \\
$\mathbf{6 5 - 7 4}$ & 1162.1 & 1229.8 & 67.8 & 5.8 & $.582, p=0.060$ \\
$\mathbf{7 5 - 8 4}$ & 1213.7 & 1374.8 & 161.1 & 13.3 & $.873, p<.001^{*}$ \\
$\mathbf{8 5 +}$ & 1284.8 & 1301.3 & 16.5 & 1.3 & $.791, p<0.01^{*}$ \\
\hline Strong opioid & & & & & \\
$\mathbf{1 8 - 2 4}$ & 8.1 & 9.7 & 1.5 & 18.6 & $.041, p=0.905$ \\
$\mathbf{2 5 - 4 4}$ & 32.5 & 108.7 & 76.2 & 234.1 & $>.999, p<.001^{*}$ \\
$\mathbf{4 5 - 6 4}$ & 48.4 & 219.0 & 170.6 & 352.8 & $>.999, p<.001^{*}$ \\
$\mathbf{6 5 - 7 4}$ & 49.8 & 191.6 & 141.9 & 285.0 & $>.999, p<.001^{*}$ \\
$\mathbf{7 5 - 8 4}$ & 57.4 & 221.4 & 164.0 & 285.9 & $>.999, p<.001^{*}$ \\
$\mathbf{8 5 +}$ & 74.3 & 271.8 & 197.5 & 265.8 & $>.999, p<.001^{*}$ \\
\hline
\end{tabular}

in annual prescription numbers was noted in the $25-44$ years group (Table 3.3). The second largest increase was observed in the 45 to 64 years age group, although there were twice as many prescriptions issued compared to the $25-44$ years group over the period examined (Table 3.3).

Prescriptions for people aged 65 years and older accounted for $46.2 \%(8,707,403$ of $22,808,675$ ) of the total number of prescriptions issued between 2005 and 2015. However, when adjusted to each age-group population this changed to $71.3 \%$ of prescriptions per 1,000 population issued within to people aged 65 years and aboveError! Reference source not found.. Based on prescriptions per 1,000 age-adjusted population data, the group which had the highest rate of prescribing was aged 85 years and older.

The trend in the number of weak opioid prescriptions issued by age-group followed a similar trend to those seen in the number of people receiving prescriptions (Appendix B). The largest increase was in the 25 to 44 years age group, as with the overall trend. 


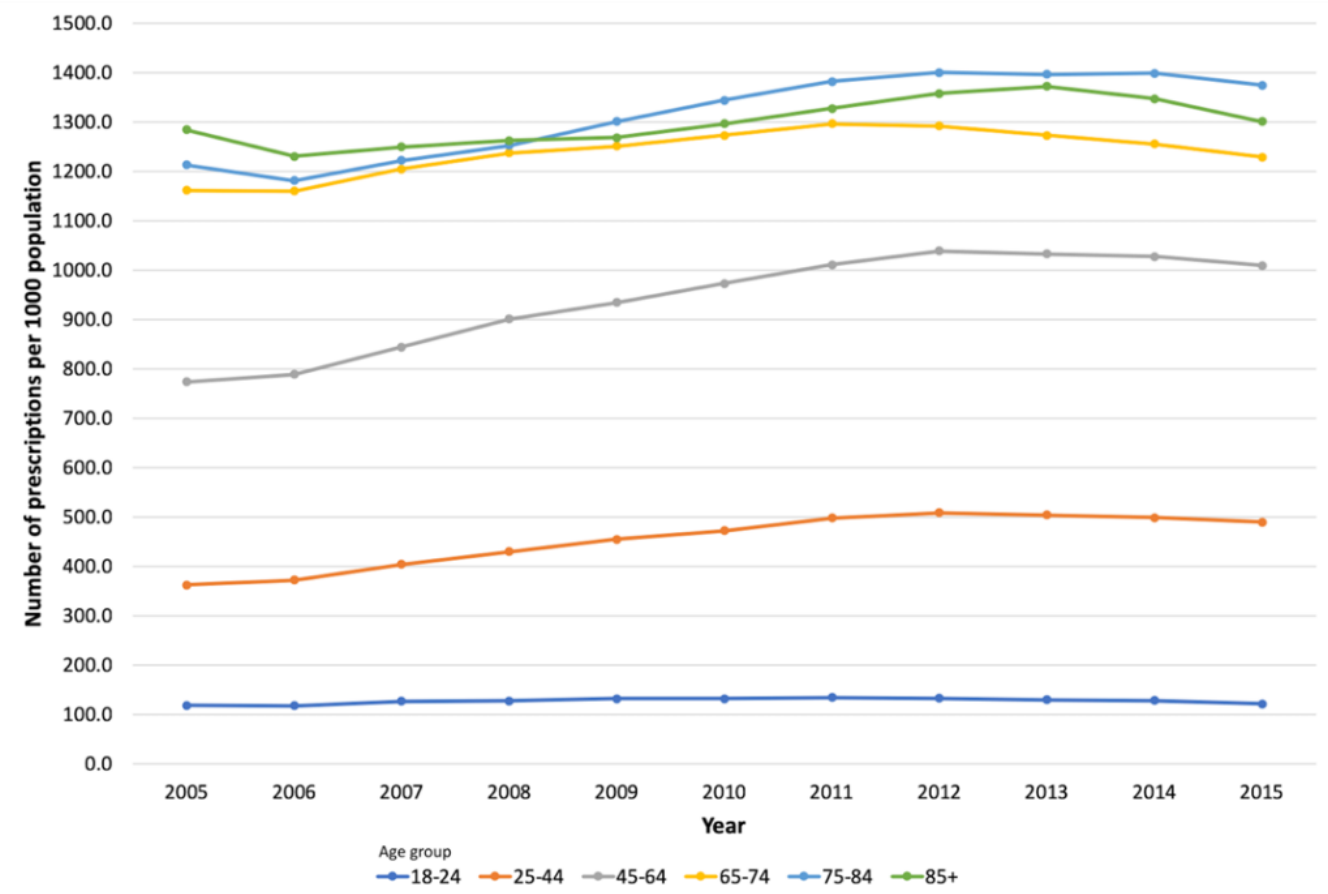

Figure 3.4: Trends in the number of weak opioid prescriptions issued between 2005 and 2015 and stratified by age-group. Data displayed by number of prescriptions per 1,000 age-group adjusted population per year

A Kruskal-Wallis test demonstrated a significant difference $(H=58.66, p<.001$, $\eta^{2}=0.89, \mathrm{~d}_{\text {Cohen }}=5.82$ ) between the number of prescriptions issued to the various age-groups over the study period. Dunn's pairwise tests, and Bonferroni corrections confirmed significantly more weak opioid prescriptions were issued to individuals aged 45 years and above, when compared to the 18 - to 24 - year group (Appendix B). Significantly fewer prescriptions were issued to 25- to 44year-olds compared to those aged 75 years and over. No other statistically significant differences in the rates of weak opioid analgesics were observed between age groups (Appendix B).

Strong opioid prescribing rates more than tripled in all age-groups other than the youngest group (18 - 24 years) (Figure 3.5 ) over the 11 years analysed. The largest percentage increase was noted in the 45 to 64 years age-group (Table 3.3). Differences were tested using a Kruskal-Wallis test and were statistically significant $\left(H=51.38, p<.001, \eta^{2}=0.77, d_{\text {cohen }}=3.69\right)$. The youngest age-group (1824 years) were confirmed to have significantly fewer prescriptions than all agegroups age 45 years and over. The older two age-groups were confirmed to have significantly more prescriptions than 24-44 year olds also (Appendix B). 


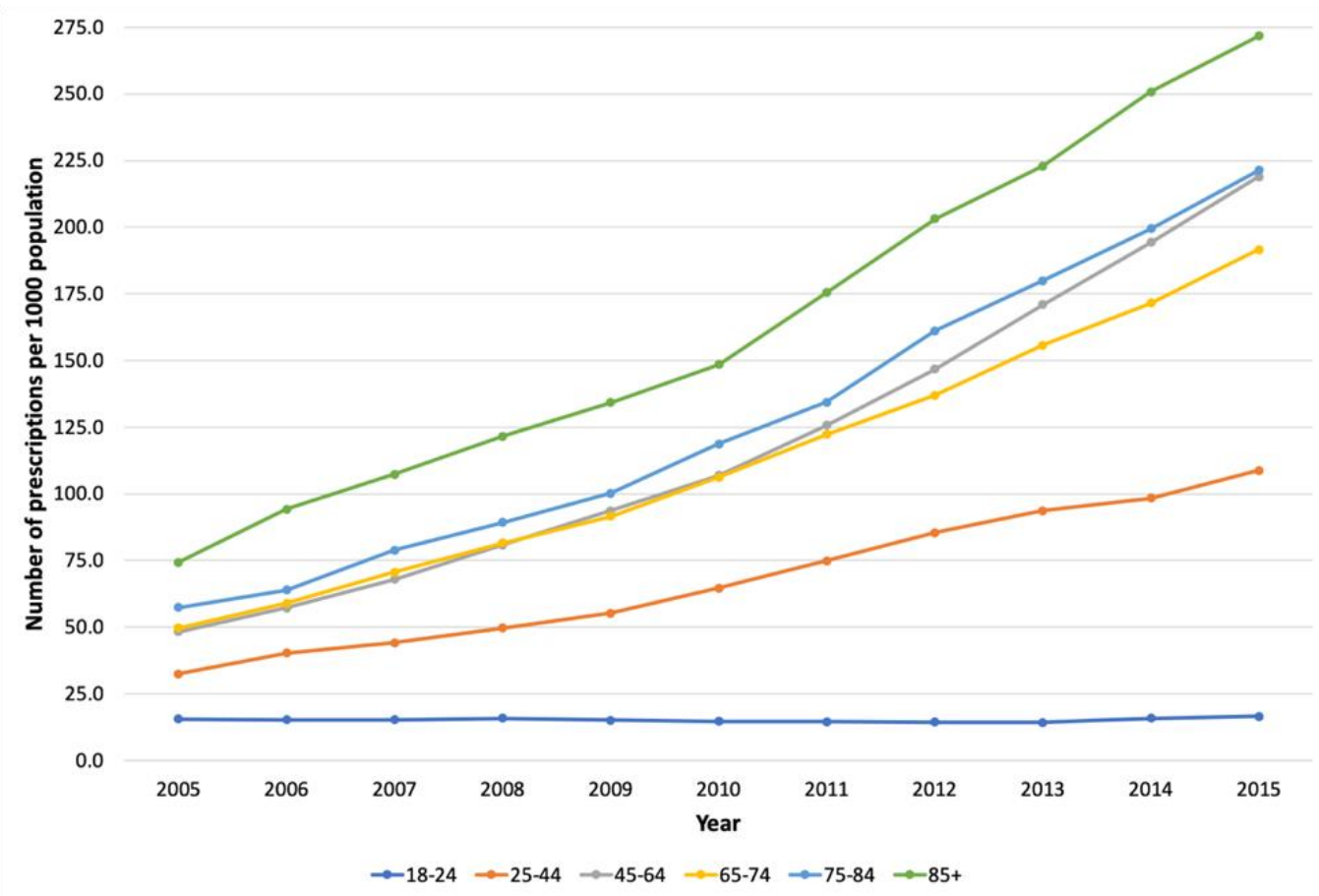

Figure 3.5: Trends in the number of strong opioid prescriptions between 2005 and 2015 and stratified by age-group. Data displayed by number of prescriptions per 1,000 age-group adjusted population per year

Two thirds of strong opioid prescriptions were issued to people aged 65 years and above (based on population-adjusted data) (Figure 3.5) between 2005 and 2015. Over the 11 years of the study, $28 \%$ more prescriptions were issued to people in the age-group aged 85 years and over than the group with the next highest number of prescriptions, aged $75-84$ years (Figure 3.5 ).

\subsubsection{Prescribing persistence}

Between 2005 and 2015, there were 4,308,035 opioid prescribing events in the 345 SAIL registered practices (Table 3.4). Of these, 4,095,952 (95\%) events had a prescribing persistence of 31 days or less, with 3,978,480 (92.4\%) events classified as single events, where a second prescription was not issued within 31.5 days of the first.

When all prescriptions were included in the analysis, average prescribing persistence was $8.6 \pm 63.3$ days (Table 3.4 ). Removing all single prescriptions (where another was not issued within 31 days), average persistence duration increased to $94.7 \pm 189.8$ days. 
For the 11 years analysed, 99,699 (3.8 events per 1,000 population) prescribing events occurred with a persistence of more than 3 months ( $\geq 91$ days) and an average prescribing period of $265.7 \pm 317.1$ days (Table 3.4). Of those, 46,378 (1.8 events per 1,000 population) had a prescribing persistence of over 6 months ( $\geq 181$ days) and an average period of $423.9 \pm 410.7$ (SD) days.

There were 4,837 ( 0.2 events per 1,000 population) prescribing periods of more than 2 years ( $>730$ days, mean average $1356.9 \pm 712.2$ days), 856 (0.03 events per 1,000 population) periods of more than 5 years ( $>1825$ days, mean average $2660.5 \pm 645.7$ days) and 110 periods that extended over 10 years (>3650 days, mean average $3794.1 \pm 84.8$ days). 
Table 3.4: Demographics for prescribing persistence adjusted to population

\begin{tabular}{|c|c|c|c|c|}
\hline \multirow[t]{2}{*}{ Mean data for period 2005-2015 } & \multicolumn{4}{|c|}{ Period of prescription persistence } \\
\hline & Total & $\leq 31$ days & $\geq 91$ days & $\geq 181$ days \\
\hline Number of events & 4308035 & 4095952 & 99699 & 46378 \\
\hline Mean prescription persistence (days) \pm SD & $8.60 \pm 63.309$ & $9.4 \pm 4.726$ & $265.73 \pm 317.085$ & $423.88 \pm 410.739$ \\
\hline Number of events per 1,000 population & 165.1 & 157.0 & 3.8 & 1.8 \\
\hline \multicolumn{5}{|l|}{ Gender } \\
\hline Male & $137.0(41.6)$ & $129.9(41.5)$ & $3.2(42.6)$ & $1.5(42.2)$ \\
\hline Female & $192.2(58.4)$ & $183.1(58.5)$ & $4.4(57.4)$ & $2.0(57.8)$ \\
\hline \multicolumn{5}{|l|}{ Last opioid in prescription period } \\
\hline Strong & $6.7(4.1)$ & $6.1(3.9)$ & $0.3(8.4)$ & $0.2(9.2)$ \\
\hline Weak & $158.4(95.9)$ & $150.9(96.1)$ & $3.5(91.6)$ & $1.6(90.8)$ \\
\hline \multicolumn{5}{|l|}{ Deprivation quintile WIMD2011 } \\
\hline WIMD1 & $209.2(26.4)$ & $197.3(26.2)$ & $5.7(31.3)$ & $2.7(31.7)$ \\
\hline WIMD2 & $173.6(21.9)$ & $164.7(21.8)$ & $4.3(23.2)$ & $2.0(23.9)$ \\
\hline WIMD3 & $165.8(20.9)$ & $157.7(20.9)$ & $3.8(20.7)$ & $1.8(20.9)$ \\
\hline WIMD4 & $128.7(16.2)$ & $123.4(16.4)$ & $2.4(13.1)$ & $1.1(12.7)$ \\
\hline \multicolumn{5}{|l|}{ Recorded diagnoses } \\
\hline Big six non cancer pain & $119.7(72.5)$ & $113.6(72.3)$ & $2.9(74.8)$ & $1.3(74.6)$ \\
\hline Depression/anxiety & $47.5(28.8)$ & $45.0(28.7)$ & $1.2(31.2)$ & $0.6(32.0)$ \\
\hline
\end{tabular}




\subsection{Discussion}

\subsubsection{Summary and reflection on findings}

In answer to the study question, 'how has opioid prescribing changed over time?' the results presented in this chapter demonstrate that between 2005 and 2015, the number of prescriptions for opioids in Wales increased considerably (Section 3.2). Large rises in the number of people being issued prescriptions were also observed (Appendix B). Even within the context of a substantial overall rise, the huge percentage increases in prescriptions for strong opioid analgesics such as morphine, oxycodone and fentanyl were striking. Many more women received prescriptions than men for the duration of the data collection period, with correspondingly higher numbers of prescriptions for all classes of opioids. Opioid prescribing rates also increased with age, again with trends consistent for both weak and strong opioid analgesics. Consequently, the study question of whether gender and age might affect prescribing can be answered in the affirmative.

It may be assumed that higher rates of prescribing in women compared to men is linked to their increased reporting of pain. There may be, as discussed earlier (Section 1.8), reasons women experience higher levels of pain and as a result, be offered opioid analgesics more frequently than their male counterparts (Section 3.6.5). Further analysis to examine differences in oral morphine equivalent dosing, between genders was conducted as part of this thesis and will be presented in Chapter 5. Pain prevalence has been shown to increase with age (Section 1.9), as does the occurrence of disease states such as musculoskeletal conditions, which, are also associated with pain (Ali et al. 2018). Consequently, it may not be entirely surprising to see more opioid prescriptions issued to the oldest people in the population. However, with increasing age, there is also a tendency to observe multiple morbidities and polypharmacy, which can make older people more susceptible to the adverse effects, interactions and harms associated with opioid analgesics, at any dose (Section 1.7). The much higher rates of prescribing of strong opioids, once again, are perhaps of greatest concern in an aging population. Of interest is the large rise in prescribing rates in people of working age. It raises the question of whether the rise of opioid 
analgesic prescribing is associated with changes in employment due to ill-health or if opioids allow people to remain in work (Hanna et al. 2020).

Prescription persistence was a measure devised for this study in the absence of information required to undertake an accurate calculation. Using an estimation there was a trend of increasing prescription persistence between 2005 and 2015. In other words, the data suggests more people are taking opioid analgesics for longer periods of time. Age did not appear to be a major factor in prescribing persistence, with little difference between all but the youngest age-group (1824 years). Whilst not included in the results shown in this chapter, analysis of prescribing persistence demonstrated people residing in areas of greatest deprivation were exposed to opioid analgesics for greater durations than those in areas with better socioeconomic conditions (Appendix G). Persistent durations of opioid use were also observed more frequently for people aged over 45 years. The implications of extended duration of use are significant clinically, given the wide range of harms opioids can cause. The prevalence of long-term prescribing in people of working age is something requiring further investigation, as repercussions in workforce availability have farther reaching consequences, economically as well as on healthcare services. Opioid analgesics have not been shown to be effective for most people. So, there are questions arising from this finding in terms of how people receiving opioid analgesics are being reviewed and advised in all healthcare settings across Wales.

\subsubsection{Comparison of general opioid prescribing trends in the UK}

A variety of different data collection methods have been used to examine opioid prescribing across the UK, making direct comparisons less straightforward (Zin et al. 2014; Mordecai et al. 2018; Torrance et al. 2018; Jani et al. 2020a). Zin et al. (2014) analysed changes in the number of prescriptions issued for four strong opioid medicines (buprenorphine, fentanyl, morphine, and oxycodone) between 2000 and 2010. Mordecai et al. (2018) looked at a larger range of opioid medicines but used trends in oral morphine equivalent quantity as the measure of change over a 43-month period (Mordecai et al. 2018). Recently, Jani et al. 
(2020) published prescribing trends in the UK between 2006 and 2017. The analysis included prescribing rates for individual opioid analgesics with for example, a 5-fold increase in codeine prescribing in that time (Jani et al. 2020). However, the trends in opioid prescription numbers noted in this study corroborate those observed in other UK-based analyses. Curtis and colleagues (2019) undertook a large review of opioid prescribing trends, using nationally available prescribing data from England (Curtis et al. 2019). Between 1998 and 2016, a 34\% (from 568 to 761 prescriptions per 1,000 population) increase in the number of oral and transdermal opioid prescriptions dispensed was found. Substantial prescribing variation across the country was described (Curtis et al. 2019), contributing to a percentage increase lower than that observed in Wales. A similar trend was observed in Tayside, Scotland, where a $29 \%$ increase (8.5\% RR to $11.0 \%$ RR standardised data) in opioid prescribing was reported between 1995 and 2010 (Ruscitto et al. 2015). These results are in line with the $43.9 \%$ rise in prescription numbers (per 1,000 population) reported, albeit the increases in Wales were observed over a shorter time period. Of interest is the peak in overall opioid prescribing rates noted in 2012, which was a similar finding observed by Jani (2020). In that study of UK-wide opioid analgesic prescribing rates between 1998 and 2016 (Curtis et al. 2019), tramadol, oxycodone and fentanyl prescribing were noted to begin to decline in 2012 although codeine and morphine continued to increase (Jani et al. 2020).

Primary care prescribers frequently express concern about prescribing strong opioids in particular (McCrorie et al. 2015; Kinnaird et al. 2019), which perhaps, contradicts the trends observed in the data. Furthermore, these rises have been noted over a period where evidence to support using opioids in long-term pain was rather rapidly falling away. Questions of how changes in practice or evidence for prescribing choices are communicated and practitioners' compliance with best practice is monitored are also relevant in this situation. The influence of guidance and legislation on opioid prescribing in Wales is examined in Chapter 6. 
Similar increases in opioid prescribing have been reported elsewhere in Europe (Gustavsson et al. 2012; Bosetti et al. 2019), North America (Silversides 2011; Kenan et al. 2012) and Australia (Karanges et al. 2016; Wagemaakers et al. 2017). It would be wrong however, to assume these trends are mirrored everywhere, even within recognised international boundaries. A Portuguese study, for example, suggested only a small percentage (4.37\%) of people living with pain were using opioid analgesics as part of their management, in spite of the majority of people (76\%) questioned using analgesic medicines (Azevedo et al. 2013). Whilst an increase in analgesic prescribing has been recorded in France, ranking it third for overall consumption, in comparison to other European countries, they have amongst the lowest strong opioid prescribing (HiderMlynarz et al. 2018).

At the end of the TOPAS study period (2015), the UK was observed to have the highest opioid prescribing in Europe for both weak and strong opioids. This was accounted for by high rates of codeine and morphine prescribing (Hider-Mlynarz et al. 2018), consistent with prescribing patterns noted in Wales at that time (see Chapter 5). European data also identified the UK as having the highest rates of high-risk (illicit) opioid use in the Europe (European Monitoring Centre 2020). However, the International Narcotics Control Board (INCB) released data which placed the UK $16^{\text {th }}$ from 22 European countries in terms of opioid consumption (DDD per million inhabitants per day) (International Narcotics Control Board 2016). Consumption of opioids (morphine equivalent in $\mathrm{mg} /$ capita) worldwide placed the UK around the middle of countries whose data was presented but still much lower than North America, Australia and Germany (International Narcotics Control Board 2016). These differences raise questions which warrant further investigation. Cultural differences in pain perception and the necessity of medicines in its management may play a part in decision making around prescribing (Meyer et al. 2020). This is likely to affect practitioners and individuals who might receive prescriptions, as well as determining whether other, non-pharmacological management options are available. Large differences in healthcare systems across the world also impact on service development and 
the role that different healthcare professionals are able to play in providing and reviewing pain management and prescribing. Meyer et al (2020) examined some of these issues in the context of opioid prescribing in Rhode Island (Meyer et al. 2020). The suggestion was that the USA should consider moving towards a more 'European' model of optimising health and wellness rather than simply addressing symptoms and conditions within a purely medicalised system. European controls on advertising pharmaceutical products was cited as one difference that was likely to influence patient-demand for medication and which has been demonstrated to have had direct impact on prescribing levels in the USA (McGreal 2018; Marks 2020; Meyer et al. 2020).

\subsubsection{Weak versus strong opioids}

An overall increase in all opioid types was observed across the 11 years of the study although rates of increase began to slow from around 2012 onwards. The reduced pace of growth could be traced back to reductions in prescribing of particular drugs, of note tramadol and fentanyl (Chapter 5). This finding was mirrored by a study of UK-wide opioid prescribing where a reduction in tramadol, oxycodone and fentanyl was observed from 2012 onwards (Jani et al. 2020). A reduction in the overall rate of opioid prescribing was reported in England between 2016 and 2017 (Curtis et al. 2019). Despite the deceleration of prescribing, the disproportionate increases in 'strong' opioid prescribing as a percentage of all opioids throughout this study mirrors observations of others who have monitored opioid prescribing from the early 2000's onwards (Torrance et al. 2018; Green et al. 2019). An increase of 554\% (from $0.13 \%$ to $0.85 \%$ of people receiving at least one opioid prescription) in the proportion of patients receiving strong opioid prescriptions was reported in a population of people accessing primary care services in Leeds and Bradford between 2008 and 2012 (Foy et al. 2018). Whilst this remains a small percentage of opioid users overall, the relative size of increase over 4 years reflects findings of other studies where strong opioid prescribing was examined. Zin et al (Zin et al. 2014), examined strong opioid prescribing in isolation and reported an $80.4 \%$ (from 1.8 to 9.2 patients per 1,000 ) increase in the number of people issued strong opioid 
prescriptions in a UK population (predominantly in England) between 2000 and 2010. This was a greater increase than the $66.9 \%$ seen in Wales between 2005 and 2015. A larger proportion of the Welsh population was included in this analysis relative to that evaluated by Zin and colleagues however, which may account for some differences.

\subsubsection{People versus prescriptions}

Zin et al. (2014) described a $411 \%$ increase in the number of people (from 1.8 to 9.2 people per 1,000 ) receiving prescriptions and a $60 \%$ increase in prescriptions (from 6.0 to 9.5 prescriptions per patient) for strong opioids between 2000 and 2010 in the UK. Using the same measures, this study observed a $202 \%$ increase (from 4.4 to 13.3 people per 1,000 population) and a 35\% rise (from 9.1 to 12.3 prescriptions per patient) in strong opioids over a similar period. Whilst the percentage increases in this study were lower, it points towards a higher overall level of strong opioid prescribing in Wales than noted in the predominantly English data (Zin et al. 2014). The implication of a large increase in the number of people compared to prescriptions per person suggests that the majority of prescriptions issued are short-term (e.g., for acute pain indications).

Comparisons can be drawn with similar findings elsewhere in Europe (Rosner et al. 2019). A systematic review of prescribing trend studies from Germany did not establish a percentage increase in annual prescription numbers but highlighted the biggest reported increase in prescription prevalence being $37 \%$ between 2000 and 2010 (Rosner et al. 2019). In Norway between 2004 and 2007, a 9\% increase in the number of people without cancer, receiving opioids was observed. Numbers of prescriptions were not measured in the study however (Fredheim et al. 2010). Regional variation in people and prescriptions were reported in Germany, with a north-south divide described (Rosner et al. 2019). Similar regional variances were observed in Wales, with the seven health boards showing slightly differing trends (Appendix I). 


\subsubsection{Gender differences in opioid prescribing}

The number of women compared to men across all age-groups increased in terms of receiving prescriptions and also the annual prescriptions issued. Similar findings have been noted by other UK-based studies (Zin et al. 2014; Ruscitto et al. 2015). In England and Scotland, greater differences between men and women were noted for the increases in strong opioid prescribing over the respective study periods (Zin et al. 2014; Ruscitto et al. 2015), which this study corroborated. Long-term prescribing rates for opioid analgesics in England were shown to be consistently higher in women and increased more between 2002 and 2013 than for men (Bedson et al. 2016), again corroborated by the TOPAS study. Other UK studies have documented women receiving opioids in greater numbers than men but without examining changes in those figures over time (Todd et al. 2018; Torrance et al. 2018). Data from the USA have shown large reductions in overall rates of opioid prescribing over the last 10 years (IQVIA 2020). Between 2008 and 2018, based on data from 92\% of retail pharmacies in the USA, reductions in the number of men and women receiving opioids were around $30 \%$ overall although statistically higher numbers of women consistently filled prescriptions (Schieber et al. 2020). The largest differences between genders was in the youngest age-group examined (20-24 years), where twice as many women as men filled prescriptions (Schieber et al. 2020). Whilst studies consistently report proportions of men and women included data trends by gender are much less frequently reported. Concerns about gender-disparities in accessing support for painful conditions have been highlighted recently in the UK (Barnett 2020; Connolly 2020; Marsh 2021; Warraich 2021), focussing on gynaecological-related pain in particular. However, gender differences in presentation and prevalence of other pain-related conditions have been observed including chronic back pain, where in women, associations with the menstrual cycle and age-associated changes in hormone levels may account for increased prevalence (Fehrmann et al. 2019; Rathbone et al. 2020). Variation between men and women, in response to pain rehabilitation have also been reported (Rovner et al. 2017) and could have implications for analgesic use as 
well. Further research into differences in opioid and analgesic prescribing between men and women is needed. The use of analgesics needs to be placed in the context of underlying causes of pain, genetic and hormonal variation and emotional or psychological differences between genders.

\subsubsection{Age differences in opioid prescribing}

The study data demonstrated that people in the oldest age-groups ( $85+$ years) had the highest rates of prescribing for all types of opioids between 2005 and 2015. Long-term prescribing in an English primary care population observed that people aged $65+$ years were more likely to receive an opioid prescription, and this was a consistent pattern noted over 11 years (Bedson et al. 2016). As prescribing persisted, the percentage of younger people maintained on opioids increased and was not dissimilar to that seen in the older group (Bedson et al. 2016). However, a number of UK studies have shown similar results, with higher rates of prescribing noted in older age groups (Zin et al. 2014; Ruscitto et al. 2015; Torrance et al. 2018). Jani et al. (2020) examined prescribing trends in England which, whilst not presenting age-group trend analysis, did show the factor with greatest odds ratio for long-term opioid use was being age 75 years and over (Jani et al. 2020), which findings presented here would corroborate. As in the TOPAS study, the likelihood of receiving strong opioid prescriptions also increased with age with $31 \%$ of strong opioids initiated in people aged 85 years and over (Jani et al. 2020).

An increase of $171 \%$ (from $4.2 \%$ to $11.4 \%$ of patients) in the percentage of people aged 65 years and older receiving opioid prescriptions was observed between 2005 and 2017 in the Netherlands. The percentage of strong opioid prescribing increased $438.5 \%$ (from 1.3 to $7.0 \%$ of prescriptions) The oldest patients (aged 85+ years) were more likely to be given a prescription for a strong opioid, with $40 \%$ having them prescribed for 3 months or more (Weesie et al. 2020). The increases reported appear much larger than those described in the TOPAS data. However, the overall trends are similar, not least with strong opioid prescribing increasing most in the oldest population. In the Weesie et al. (2020) 
study, cancer was the principal reason for opioid prescribing in the $85+$ years group (Weesie et al. 2020). This study specifically looked at non-cancer prescribing yet demonstrated similar patterns. High levels of opioid analgesics in older, more metabolically vulnerable people is a cause of concern due to the potential for inadvertent toxicity and overdose (Huang and Mallet 2013; Gazelka et al. 2020).

Despite overall reductions in percentages of age-grouped adults receiving opioid analgesics in the USA, the smallest reductions were observed in people aged 45 years and above. The number of prescriptions per person in those groups barely changed between 2008 and 2018. People aged 55-64 years actually had a small increase (Schieber et al. 2020) which corresponds to data presented here, which, revealed that some of the largest increases in prescribing were noted in the 4564 years age-group, whilst overall, the highest rates were maintained in the oldest age-groups.

\subsubsection{Prescribing persistence or duration}

By far the majority of opioid prescribing throughout this study (95\%), was shortterm with prescriptions not repeated within 31 days. This accords with Chevalier et al.'s (2014) observations of $50.5 \%$ of opioids being prescribed for less than $<31$ days, in a primary care population in the UK (Chevalier et al. 2014). In Chevalier et al.'s study, people were excluded from analysis once their indexed prescribing period finished, whereas this study allowed repeated presentations by individuals, with a break of more than 31 days between prescriptions considered a new prescribing period. This may have led to intermittent but persistent prescribing being observed as short-term when it actually covered longer periods. This is a potential limitation of any research in this area (Bedson et al. 2016; Jani et al. 2020). The only accurate method to determine longevity of using opioids would be to take a history from each individual patient. Consequently, all measures of prescription persistence or opioid use are essentially estimations (Zin et al. 2014; Lalic et al. 2018). Using a method to determine persistence used 
by other researchers, was decided to provide a reliable measure for this study (Chang et al. 2018).

People with prescription persistence of more than six months constituted $1 \%$ of this study's population with an average of 424 days exposure. The percentage of long-term users appears low in comparison to other study's findings. Chevalier et al. (2014) noted $21 \%$ of study participants had chronic opioid exposure with an average 170 days continuous use, using similar criteria as this study but where the population was on average nearly 10 years older (Chevalier et al. 2014). In the same study, chronic users made up $6.3 \%$ of the German cohort, with a similar average age to their UK counterparts with an average continuous prescribing duration of 567 days (Chevalier et al. 2014). In an Australian population, 2.6\% ( 430,000 people) met the criteria of persistent opioid use when a group-based trajectory model (GBTM) was used over a 12-month period (Lalic et al. 2018). The GBTM method avoids setting explicit criteria for persistence. Instead, it uses a probability calculation based on patterns of prescribing in the 12 months after the first prescription (Lalic et al. 2018). Kern et al. (2015) noted chronic use (>183 days) composed $6.8 \%$ of prescribing in an American population ( 256,000 people), substantially higher than in the analysis presented here, where the same persistence criteria was used (Kern et al. 2015). It was also higher than an earlier American study which had an average $2.7 \%$ of supply $>181$ days over 5 years (Sullivan et al. 2008), perhaps reflecting the rapid rise in opioid use across America since the early 2000s. Bedson et al. (2016) examined trends in long-term prescribing (more than 3 prescriptions in a 90-day period) in a population with musculoskeletal conditions, between 2002 and 2013, a similar time period to this study. Overall, a $38 \%$ increase in the incidence of long-term prescribing was reported, (Bedson et al. 2016). This was much lower than noted in the TOPAS data, where a 508\% (from 1736 to 10,560 events) increase in the number of prescribing events with a duration of more than 6 months was observed between 2005 and 2015 (Appendix G). 
The GBTM method adopted by Lalic's group aims to overcome the need for explicit criteria to define persistence (Lalic et al. 2018). Prescribing persistence has been differently defined by researchers. Durations of $<30$ days, 31-90 days, 91-179 days and $>180$ days have all been used in the literature to define periods of interest. Generally, durations over 90 days may be referred to as chronic or long-term prescribing (Portenoy and Foley 1986; Sullivan et al. 2008; Kern et al. 2015; Bedson et al. 2016; Jain et al. 2018).What all these studies demonstrate is that there is no clear definition of prescribing persistence. Further research would be beneficial to determine consensus on what measure could be used in Wales, to consistently measure prescribing persistence. Duration of use appears to be as important a factor in developing long-term harms as dose (Scherrer et al. 2016; Mundkur et al. 2017; Salas et al. 2020) and therefore, even if the overall number of prescription reduces, prescribers must still be vigilant for long-term use.

\subsection{Conclusion}

The data presented in this first results chapter, shows large increases in opioid prescribing in Wales between 2005 and 2015. Over the 11 years analysed, more people received a greater number of prescriptions for opioid analgesics each year. Whilst there was a signal that increases in overall prescribing rates were starting to slow and reduce towards the end of the study period, strong opioids, often associated with greater risks of harm and misuse, were continuing to rise.

Opioid prescribing appears to be directed disproportionately towards women, who receive substantially more prescriptions than their male counterparts. There is evidence women present more frequently with pain and also, that they respond more positively to opioids. It is probably insufficient to explain the difference in rates of prescribing between men and women, however. Subsequent chapters will return to gender discrepancies as other aspects of opioid prescribing are examined. 
People aged 45 years and above received most prescriptions. This might appear easily explained as pain reporting increases with age and this is well established. Consequently, perhaps it should not be surprising most of the prescribing is observed in older people. The risks of opioids in older populations are significant, however. They are likely to contribute to morbidity and even mortality in some cases, especially when combined with other high-risk medicines. This study is the first to attempt to examine prescribing persistence in Wales, given its growing importance as a risk factor for opioid-induced harm. The data signals the duration of use is rising in all ages and genders receiving opioid prescriptions. Rates of opioid prescribing in working aged people are a concern. If greater numbers are being initiated on opioid analgesics that persist for longer periods of time, this has potentially serious implications for the general health, wellbeing, and productivity of the population. 


\section{Chapter 4}

Trends in prescribing by socio-economic deprivation

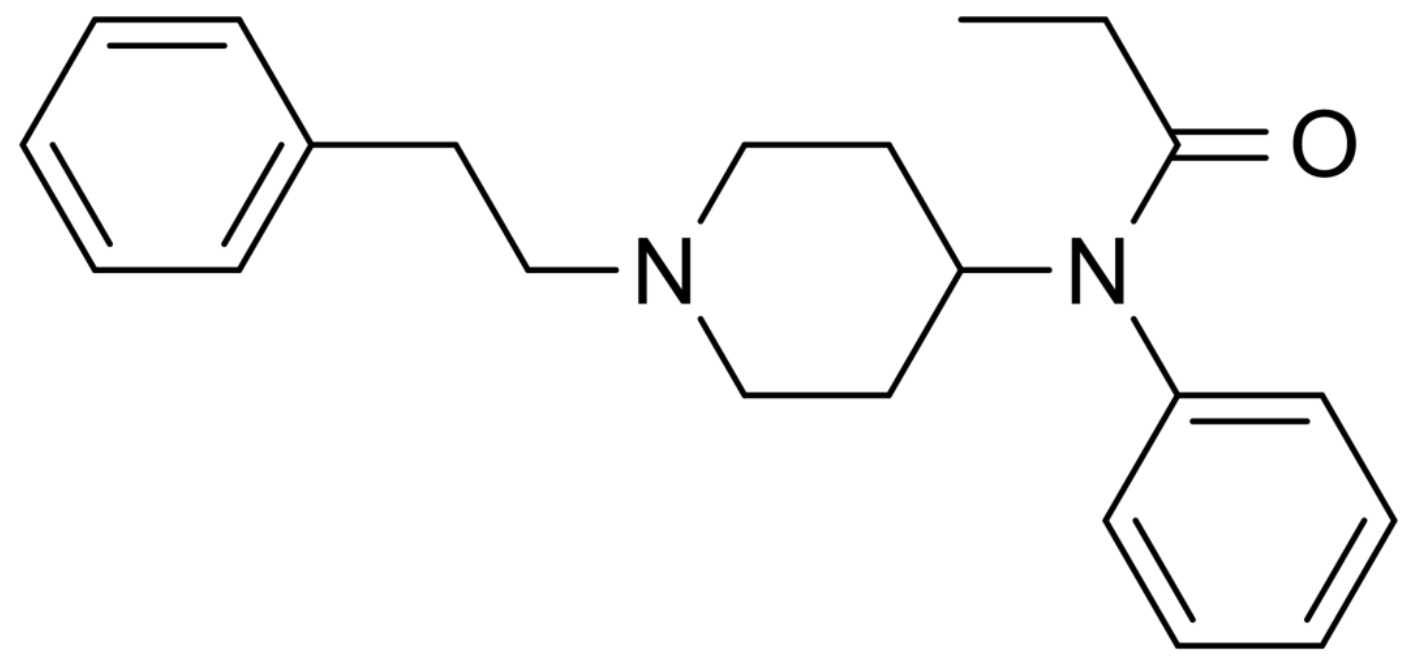

"...... any claim that the NHS has achieved its aim of providing equality in medical care is an illusion. In fact, absolute equality could never be achieved under any system of medical care, education, or other essential service to the community. The motives for suggesting otherwise are political and ignore human factors" British Medical Association, 1971 


\section{Chapter 4 - Trends in prescribing by socio-economic deprivation}

\subsection{Chapter overview}

The social and economic burden of pain and the role of opioids in managing it was considered in Chapter 1. Alongside that, population health in Wales and how socioeconomic deprivation impacts upon it was discussed. Chapter 2 set out the use of linked-data and the main methods used to extract and analyse data for the project. Chapter 3 set out the overarching trends in opioid prescribing across Wales, stratified by drug-group, gender, and age-group. Thus far, a significant increase in opioid prescribing across Wales, between 2005 and 2015 has been revealed. The rates of prescribing rose most sharply for strong opioids such as morphine and oxycodone. Opioid prescribing has been shown to be more prevalent in women than men and for people aged 45 and over in particular. Chapter 4 examines in more detail, trends in opioid prescribing in areas which have differing levels of socioeconomic deprivation. Initially, links between pain and deprivation will be considered, as a means of explaining the data explored in the results.

\subsection{Study objective to be addressed in this chapter}

- To determine if opioid analgesic prescribing trends are affected by socioeconomic deprivation status

\subsection{Background}

As described in Section 1.10, Wales has a history of poverty, exceeding that of other countries in the UK (Barnard 2018), even considering differences in how socioeconomic deprivation are calculated (Section 1.10). Whilst it is often acknowledged that higher levels of opioid use will be associated with deprivation (Taylor et al. 2019), no study has previously examined the data for Wales specifically. Other UK-focussed studies have described differences in opioid prescribing in areas with markers of socioeconomic deprivation. Notably, Todd et al. (2018) described a 'pain divide' between the north and south in England after 
scrutinising data from the Health Survey for England (HSE) (Todd et al. 2018). Similar percentages of people reported having high intensity pain but, more people in the north (12.3\%) stated being moderately or severely limited by it, compared to $9.2 \%$ in the south. Added to this, $2.5 \%$ of people in the north of England used opioids compared to $1.7 \%$ in the south (Todd et al. 2018). The north-south divide used by Todd et al. (2018) could be deemed arbitrary, in terms of determining socioeconomic deprivation. It perhaps draws on social stereotypes, when in fact different levels of deprivation are seen in all communities.

The results presented in this chapter will use a validated socioeconomic deprivation measure (Welsh Index of Multiple Deprivation, WIMD), as they are reliable and more easily comparable with data from other countries (Section 4.4.1). Jani et al. (2020) examined opioid prescribing data between 2006 and 2017 although deprivation data were not presented as trends (Jani et al. 2020). Higher opioid prescribing rates have been consistently demonstrated in the most socioeconomically deprived areas (Chen et al. 2019; Macfarlane et al. 2020; Torrance et al. 2020). The TOPAS study differs from other published studies by examining changes in prescribing rates, stratified by deprivation quintile, over time. To further build the picture of prescribing across Wales, trends in prescribing rates by opioid-type (weak or strong) and gender, which as previously discussed (Sections 3.5.2 and Section 3.5.3) are associated with different changes in prescribing (Chapter 3), are examined in the context of deprivation. This is a novel aspect of the TOPAS study that has not been found in other studies from the UK.

\subsection{Methods}

\subsubsection{Welsh Index of Multiple Deprivation}

The Welsh Index of Multiple Deprivation (WIMD) is the official measure used by the Welsh Government to determine relative deprivation of areas within Wales (Welsh Government 2011). For the TOPAS study, the 2011 Index was used (Welsh Government 2011) and presented in quintiles. It is notable that 
deprivation is multifactorial (Table 4.1) and the WIMD does not represent multilevel deprivation e.g. scores are not linear so areas in group 2 are not twice as deprived as those in group 4 (Welsh Government 2011).

Table 4.1: Components of the Welsh Index of Multiple Deprivation (WIMD) (Welsh Government 2011)

\begin{tabular}{rrc} 
Component & Percentage of WIMD Score \% \\
\hline Income & 23.5 \\
Employment & 23.5 \\
Health & 14 \\
Education & 14 \\
Geographical access to services & 10 \\
Community safety & 5 \\
Physical environment & 5 \\
Housing & 5
\end{tabular}

The most populated areas of Wales, concentrated in the South of the country, have been shown to have the highest overall levels of deprivation. These areas are principally within the areas of Aneurin Bevan Local Health Board (ABLHB), Cardiff and Vale University Health Board (CVUHB), Cwm Taff Local Health Board (CTLHB) and Abertawe Bro Morgannwg University Health Board (ABMUHB) although areas of substantial deprivation are found in every Health Board (Figure 4.1).

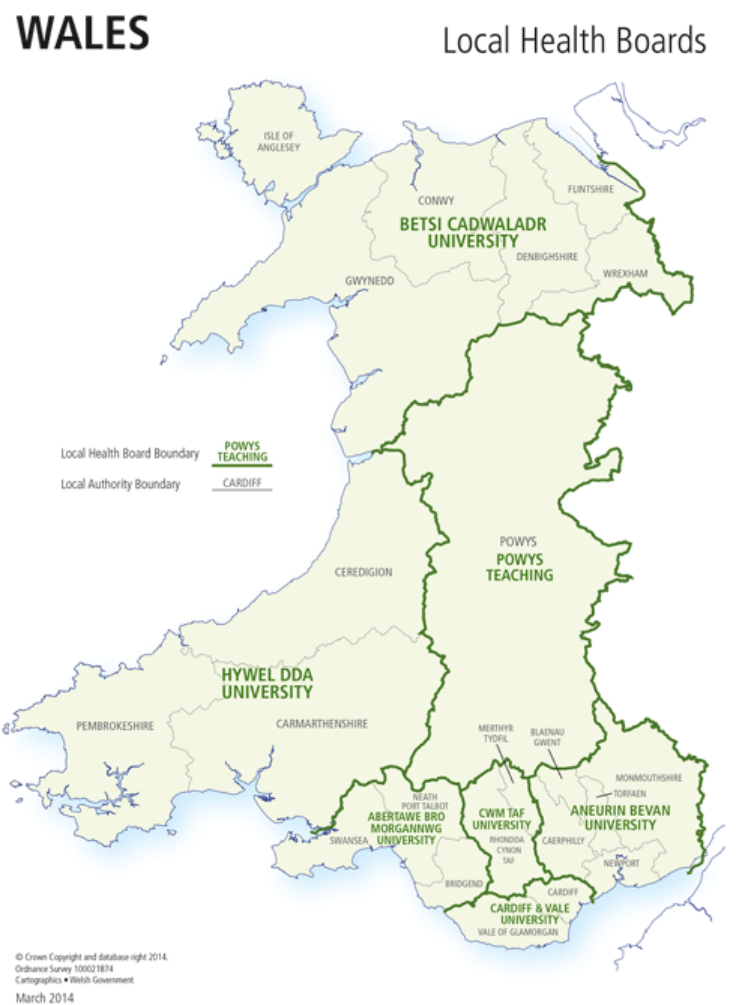

Figure 4.1: Map showing boundaries of Welsh Health Boards in 2014. These boundaries remained in place until March 2019

(Patient Knows Best 2014) 


\subsubsection{Identification of socio-economic deprivation areas}

Lower super output areas (LSOA) are geographic units used in the Welsh Index of Multiple Deprivation (WIMD) calculations. Each LSOA has a population of around 1500 people, although this does vary slightly as the areas have remained unchanged since their inception in 2004 (StatsWales 2019). There were 1896 LSOAs defined across Wales in 2011. The most deprived LSOA in Wales is ranked 1 and calculated using the 8 categories defined by the Welsh Index of Multiple Deprivation (Table 4.1).

Local super output area (LSOA) data was also accessed from StatsWales. The data was presented by LSOA and WIMD quintile and then manually stratified into Health Board areas based on the LSOA, using LSOA mapping from (StatsWales 2010). This data was used to determine the percentage population of each Health Board living in each WIMD quintile area. Those proportions were used to calculate SAIL populations for each quintile, per Health Board, per year from 2005 to 2015 . Those population estimates were used to calculate results by 1000 population per relevant area.

\subsubsection{Calculation of WIMD populations}

The LSOA and corresponding WIMD2011 quintile listed for each person was linked from the Welsh Demographic Service dataset (WDSD) (Appendix C) and added to the main study data set, subsequently extracted to analysis. This allowed data to be stratify by WIMD2011 quintile and had the potential to stratify by LSOA if required.

Welsh population data for people aged 18 years and older for each LSOA, within each Welsh county was available from StatsWales (StatsWales 2021) Population data for 2011 was downloaded and sorted by LSOA, numbers 1 to 1896 where LSOA1 is considered the most socio-economically deprived and LSOA1896 the least deprived. Each LSOA was further organised into WIMD2011 quintiles, where WIMD1, the most socioeconomically deprived included 380 LSOAs and the remaining 4 had 379. The percentage of total Welsh population (2011) located 
within each quintile was used to calculate SAIL databank quintile populations. The percentages were assumed to be the same for each year within the study and so used to adjust SAIL databank population 2005 to 2015, then used to adjust per 1,000 population (Appendix C).

Each LSOA population was also matched to the Health Board in which it was located, building up population numbers in WIMD quintiles. Once the WIMD quintile population for each of the seven Welsh health boards was calculated, the percentage of total Health Board population accounted for by each WIMD quintile was computed. The percentages were used to adjust the SAIL populations for each Health Board, thus providing estimates of WIMD2011 quintile population at national and Health Board level for the whole study period (Appendix C).

\subsubsection{Missing data}

The extracted data contained 785,056 (3.4\% of the 11-year total) prescriptions for 144,993 (3.8\% of the total) people between 2005 and 2015, where an LSOA or WIMD quintile were not allocated. These data were left out of analyses. SAIL was unable to explain why individuals were not allocated but it could include temporary residents receiving prescriptions whilst on holiday from outside of Wales for example. There are also people registered with Welsh GPs but who have an English address, due to proximity to the Wales-England border. Owing to the large number of data included in the study, it was not considered problematic for the overall analysis that they were not included.

\subsubsection{Data extraction and analysis}

Individual subjects were identified and their anonymised, linked data for opioid prescriptions and demographics extracted from the SAIL databank as described in Section 2.6. Annual number of prescriptions issued were totalled, stratified by WIMD2011 quintile and further adjusted to W quintile populations (Section 4.4.3). Descriptive statistics described the trends using the full data set from 2005 to 2015. Percentage change over time and Spearman's rank correlations 
(Section 2.7.1) were used to examine changes in prescribing rates over the 11year study period.

Data were stratified into groups based on gender (Section 2.6.5.1) and the opioid type prescribed per prescription (Section 2.6.3). Comparisons between WIMD2011 quintiles used Kruskal-Wallis tests, with Dunn's pairwise comparison and Bonferroni corrections used to confirm statistically significant differences (Section 2.7.3). Comparisons of the number of prescriptions or prescribing rate, between genders within the same quintiles were made using Mann-Whitney $U$ tests (Section 2.7.2).

\subsubsection{Log-linear regression}

In this study, log-linear regression models were developed to examine factors which may be associated with prescribing rates. Log-linear regression is used to examine the associations between more than two categorical variables. In this study, variables including gender, deprivation quintile, weak or strong opioid and Health Board were transformed into categorical variables to assist the analysis. For example, socioeconomic deprivation quintile 1 (WIMD1) was recoded as 1 and all others 0 , or strong opioids coded as 1 and weak opioids as 0.

\subsection{Results}

\subsubsection{All opioids}

In total, $6,493,712$ opioid prescriptions were issued in the most deprived areas compared to $2,752,198$ issued in the least deprived over the period examined (2.4 times more). Twenty-nine and a half percent of all opioid prescriptions $(n=22,001,509)$ were issued within the most deprived areas of Wales $(29.0 \%$ when adjusted to population). The least deprived areas (WIMD5) had $12.5 \%$ of all opioid prescribing across the study period (12.2\% when adjusted to 
population). The distribution of prescribing across the different areas of deprivation remained consistent for the 11-year period examined (Figure 4.2).

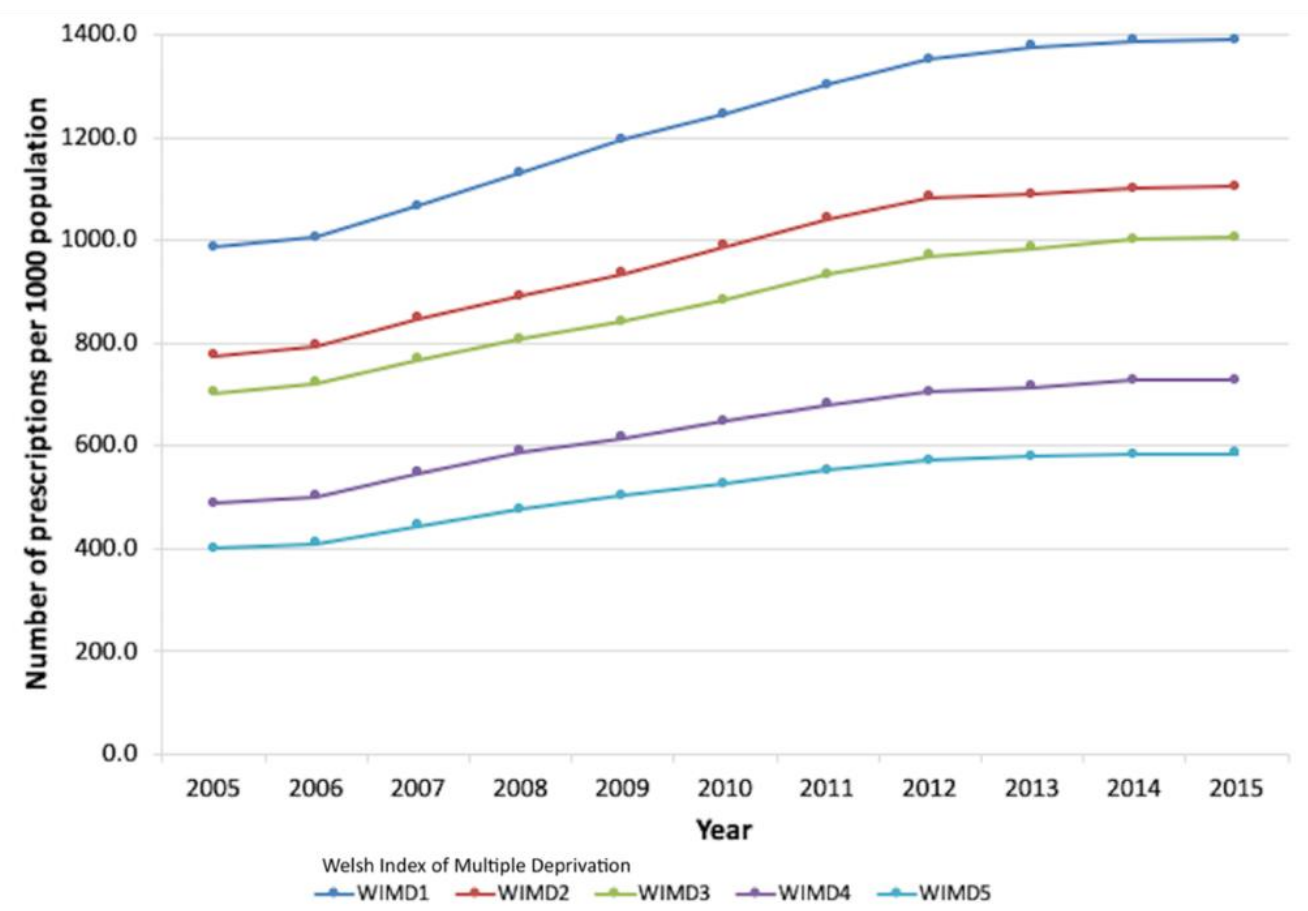

Figure 4.2: Trends in the number of all opioid prescriptions issued by deprivation area and adjusted to the population of each quintile of deprivation in Wales

$W I M D=$ Welsh Index of Multiple Deprivation where WIMD1 = most deprived and WIMD5 = least deprived

When examined as a whole, statistically significant differences between the numbers of opioid prescriptions being issued in the 5 areas of deprivation across Wales were detected (Kruskal-Wallis, $p<.001, \eta^{2}=0.839$, $\left.d_{\text {cohen }}=4.569\right)$. Post-hoc analysis confirmed that significantly more prescriptions were issued in the three most deprived quintiles when compared to the least deprived (WIMD1 $p<.001$, WIMD2 $p<.001$ and WIMD3 p=0.008) (Appendix C). Significantly more prescriptions were also issued in WIMD1 $(p<.001)$ and WIMD2 $(p<0.05)$ areas compared to in the WIMD4 quintile. No statistical differences in prescription numbers were detected between quintiles WIMD3 to 5 (Appendix C). Similar outcomes were noted for Kruskal-Wallis analysis of prescribing rate data (Appendix C).

Table 4.2: Comparison of quintile-population adjusted numbers of prescriptions for all opioid medicines by Welsh Index of Multiple Deprivation quintiles across Wales between 2005 and 2015.

Prescriptions per 1,000 population

$\begin{array}{lllll}\text { WIMD1 } & \text { WIMD2 } & \text { WIMD3 } & \text { WIMD4 } & \text { WIMD5 }\end{array}$




\begin{tabular}{rccccc}
\hline 2005 & 987.1 & 774.8 & 702.4 & 487.0 & 399.7 \\
2015 & 1389.8 & 1104.8 & 1005.2 & 727.0 & 585.1 \\
\hline $\begin{array}{r}\text { Change rate } \\
\text { (\%) } 2005-\end{array}$ & 40.8 & 42.6 & 43.1 & 49.3 & 46.4 \\
$\mathbf{2 0 1 5}$ & & & & \\
\hline $\begin{array}{r}\text { Spearman's r } \\
\text { *p-value }\end{array}$ & $>$ p $<$ <.099 & $>.999$ & $>.999$ & 0.991 & $>.999$ \\
\hline
\end{tabular}

WIMD1 = most socioeconomically deprived, WIMD5 = least socio-economically deprived $* p<0.05=$ statistically significant

The greatest increase in annual prescription rates were seen in the least deprived areas. WIMD4 areas had an increase of $49.3 \%$ and there was a $46.4 \%$ increase in WIMD5 (Table 4.2). There was strong correlation between prescribing rates and time for all quintiles over the study. There were fairly large annual increases in the number of prescriptions and corresponding prescribing rates issued in all quintiles in the first seven years of the study period. From 2012 onwards however, those increases became smaller and the trend appeared likely to start reversing beyond 2015 .

\subsubsection{Weak opioids}

Over the 11 years examined, 2.4 times more weak opioid prescriptions were issued in the most deprived areas (WIMD1) of Wales compared to the least deprived area (WIMD5) (Table 4.3). For the whole study period, prescribing rates were inversely related to level of deprivation.

Table 4.3: Comparison of quintile-population adjusted numbers of prescriptions for weak opioid medicines by Welsh Index of Multiple Deprivation quintiles across Wales between 2005 and 2015.

\begin{tabular}{|c|c|c|c|c|c|}
\hline & \multicolumn{5}{|c|}{ Number of prescriptions per 1,000 population } \\
\hline & WIMD1 & WIMD2 & WIMD3 & WIMD4 & WIMD5 \\
\hline 2005 & 937.1 & 730.5 & 659.1 & 455.4 & 377.2 \\
\hline 2015 & 1166.2 & 928.9 & 834.8 & 605.3 & 496.8 \\
\hline $\begin{array}{l}\text { Change rate } \\
\text { (\%) } 2005-2015\end{array}$ & 24.5 & 27.2 & 26.6 & 32.9 & 31.7 \\
\hline $\begin{array}{r}\text { Spearman's r } \\
*_{\text {p-value }}\end{array}$ & $\begin{array}{c}0.882 \\
p<.001^{*}\end{array}$ & $\begin{array}{c}0.873 \\
p<.001^{*}\end{array}$ & $\begin{array}{c}0.936 \\
p<.001^{*}\end{array}$ & $\begin{array}{c}0.945 \\
p<.001 *\end{array}$ & $\begin{array}{c}0.909 \\
p<.001^{*}\end{array}$ \\
\hline
\end{tabular}

WIMD1 = most socioeconomically deprived, WIMD5 = least socioeconomically deprived ${ }^{*} p$-value $<0.05=$ statistically significant. Full annual data Appendix $C$

Statistically significant differences in the number of weak opioid prescriptions issued between each deprivation quintile were identified (Kruskal-Wallis, $p<.001$, $\left.\eta^{2}=0.886, d_{\text {cohen }}=5.568\right)$. As with the overall trend analysis, significantly more prescriptions were issued in the three most deprived quintiles when compared 
to the least deprived (Appendix C). Significantly more prescriptions were issued in WIMD1 and WIMD2 quintiles, when compared to WIMD4. No statistically significant difference was detected between the three higher quintiles however, in terms of total number of prescriptions issued between 2005 -2015 (Appendix C).

As with overall opioid prescribing previously described, the larger increases in the numbers of prescriptions issued each year, were seen in the less deprived areas (WIMD4 and WIMD5) (Table 4.3). There were very strong Spearman's correlations between prescribing rate and time in all quintiles (Table 4.3). The annual number of weak opioid prescriptions peaked in two areas (WIMD2 and WIMD5) in 2012 and as with the number of people (Appendix C), prescribing in all areas started to slow and reduce in the subsequent three years (Error! Reference source not found.Error! Reference source not found.). The least deprived quintile demonstrated the smallest reductions in annual prescribing rate between 2013 and 2015, thus drawing prescribing rates within those areas closer to the quintile above.

\subsubsection{Strong opioids}

Over the study period, 2.3 times more strong opioid prescriptions were issued in the most deprived areas of Wales compared to the least deprived area (Figure 4.3). Rate of prescribing and the number of strong opioid prescriptions issued 
was inversely associated with deprivation quintile.

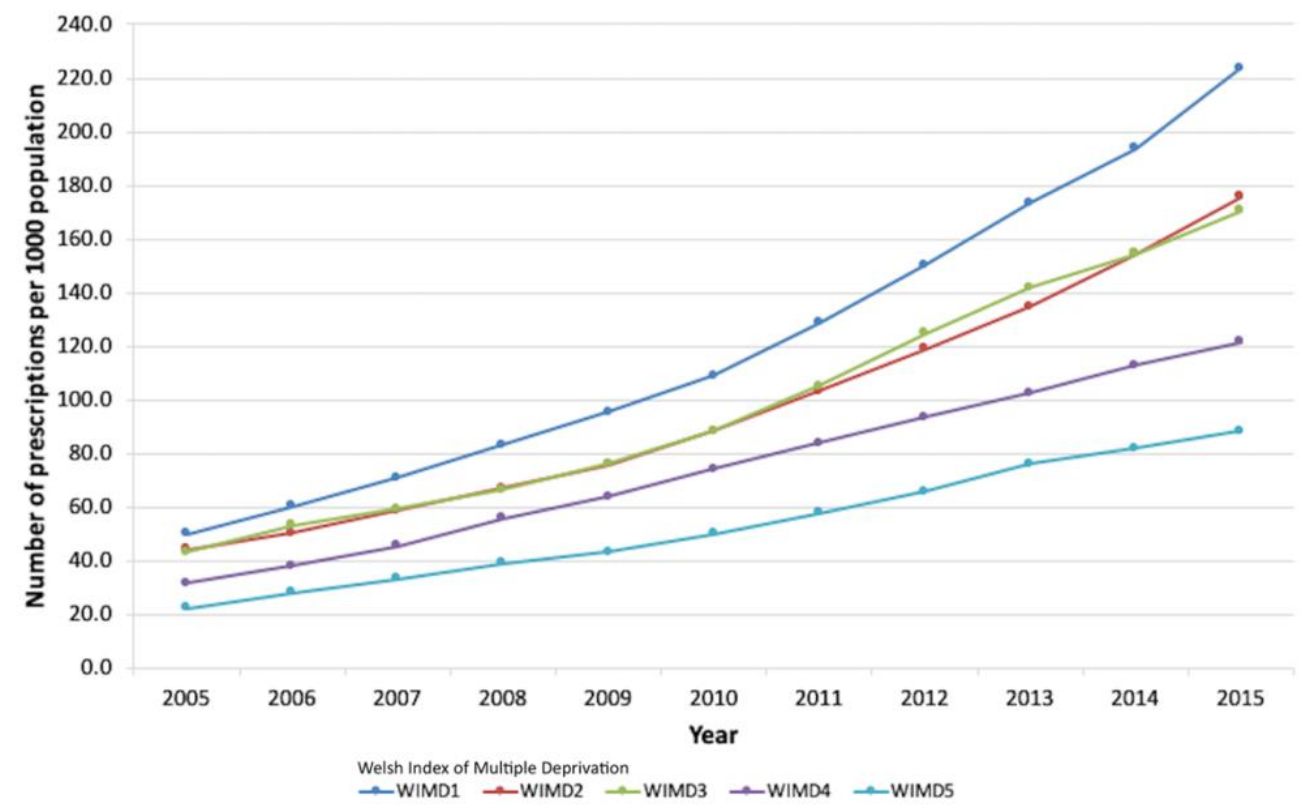

Figure 4.3: Trends in the number of strong opioid prescriptions issued by deprivation area and adjusted to the population of each quintile of deprivation in Wales

WIMD = Welsh Index of Multiple Deprivation where WIMD1 = most deprived and WIMD5 = least deprived

The most deprived areas also experienced the largest increase in the number of strong opioid prescriptions (Table 4.4). The smallest increase was seen in WIMD4 areas although those areas still had nearly 4 times increase in the annual number of prescriptions ( $285.2 \%$, from 31.6 to 121.6 prescriptions per 1,000 population).

Table 4.4: Comparison of quintile-population adjusted numbers of prescriptions for strong opioid medicines by Welsh Index of Multiple Deprivation quintiles across Wales between 2005 and 2015.

\begin{tabular}{rccccc}
\hline & \multicolumn{5}{c}{ Prescriptions per 1,000 population } \\
\cline { 2 - 6 } & WIMD1 & WIMD2 & WIMD3 & WIMD4 & WIMD5 \\
\hline $\mathbf{2 0 0 5}$ & 50.0 & 44.3 & 43.3 & 31.6 & 22.5 \\
$\mathbf{2 0 1 5}$ & 223.6 & 175.9 & 170.4 & 121.6 & 88.3 \\
\hline Change rate & 346.8 & 297.1 & 293.9 & 285.2 & 291.9 \\
(\%) 2005 - 2015 & & & & & \\
\hline Spearman's $r$ & $>.999$ & $>.999$ & $>.999$ & $>.999$ & $>.999$ \\
*p-value & p $<.001^{*}$ & $p<.001^{*}$ & $p<.001^{*}$ & $p<.001^{*}$ & $p<.001^{*}$ \\
\hline
\end{tabular}

WIMD1 = most socioeconomically deprived, WIMD5 = least socioeconomically deprived ${ }^{*} p$-value $<0.05=$ statistically significant. Full annual data Appendix C

The difference in the number of prescriptions issued within each deprivation quintile across Wales were noted as statistically significant $\left(p=.007, \eta^{2}=0.2\right.$, $\mathrm{d}_{\text {cohen }}=1.001$ ), as determined by a Kruskal-Wallis test. However, post-hoc analysis confirmed a statistically significant difference $(p<0.01)$ in the prescribing rate for strong opioids only between the most deprived (WIMD1) and least deprived (WIMD5 areas) (Appendix C). 
As the study period progressed, the overall rate of increase in annual prescribing remained consistently higher in the most deprived quintile when rates in the two least deprived quintiles began to level out (Figure 4.3). This resulted in divergence in the number of strong opioid prescriptions being issued in the most deprived areas of Wales, compared to those in the more socioeconomically advantaged areas (Figure 4.3).

Unlike overall opioid prescribing and weak opioid prescribing, there was no clear pattern in the rates of change for strong opioid analgesics. Unlike weak opioid prescribing, a peak in annual prescribing numbers had not been reached within the 11 years of data this study analysed.

\subsubsection{Gender differences}

\subsubsection{All opioids}

Women received more prescriptions for all types of opioids, regardless of the socio-economic deprivation quintile they lived in (Figure 4.4). The number of prescriptions issued to women in the two least deprived areas (WIMD4 and WIMD5) demonstrated a similar trend to that observed in men living in middle quintiles (WIMD2 and WIMD3) (Figure 4.4).

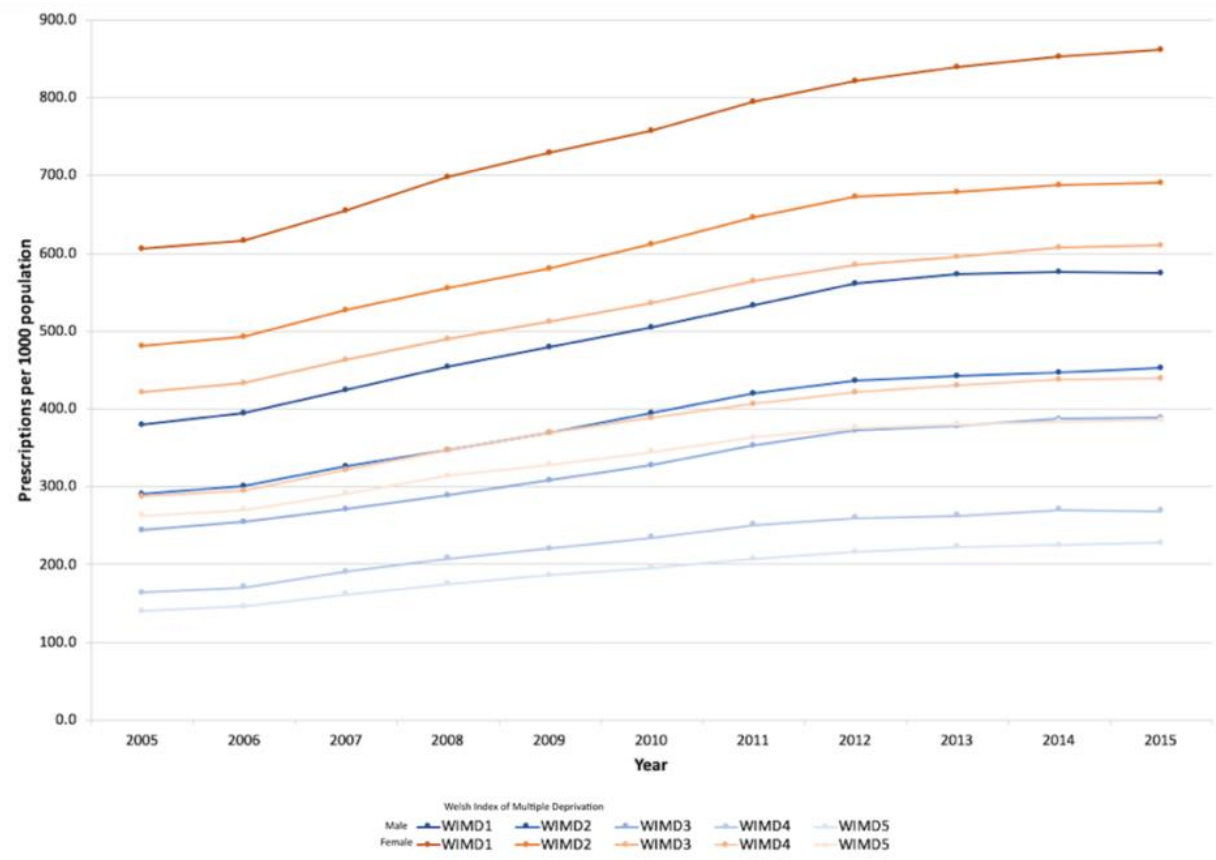

Figure 4.4: Trends in the number of all opioid prescriptions issued to men and women by deprivation quintile and adjusted to quintile-population in Wales

WIMD = Welsh Index of Multiple Deprivation where WIMD1 = most deprived and WIMD5 = least deprived 
Totaled over the 11 years examined, women received between 51\% (WIMD1) and $76 \%$ (WIMD5) more opioid prescriptions per 1,000 quintile-population, than men (Table 4.5) which was statistically significant $\left(p<.001, \eta^{2}=0.717\right.$, $\left.d_{\text {Cohen }}=3.817\right)$. The annual percentage increases in the number of prescriptions issued however, were higher for men than women in every quintile (Table 4.5).

Table 4.5: Comparison of quintile-population adjusted numbers of prescriptions for all opioid medicines by Welsh Index of Multiple Deprivation quintiles and gender across Wales between 2005 and 2015

\begin{tabular}{|c|c|c|c|c|c|c|c|c|c|c|}
\hline & \multicolumn{10}{|c|}{ Number of prescriptions per 1,000 population } \\
\hline & \multicolumn{2}{|c|}{ WIMD1 } & \multicolumn{2}{|c|}{ WIMD2 } & \multicolumn{2}{|c|}{ WIMD3 } & \multicolumn{2}{|c|}{ WIMD4 } & \multicolumn{2}{|c|}{ WIMD5 } \\
\hline & Male & Female & Male & Female & Male & Female & Male & Female & Male & Female \\
\hline 2005 & 380.5 & 606.6 & 290.4 & 480.5 & 244.0 & 421.2 & 163.7 & 287.0 & 140.3 & 262.7 \\
\hline 2015 & 575.1 & 861.5 & 452.4 & 690.0 & 388.4 & 610.1 & 268.6 & 439.9 & 227.4 & 385.3 \\
\hline $\begin{array}{l}\text { Change rate } \\
\text { (\%) } 2005-2015\end{array}$ & 51.1 & 42.0 & 55.8 & 43.6 & 59.2 & 44.9 & 64.1 & 53.3 & 62.1 & 46.7 \\
\hline $\begin{array}{r}\text { Spearman's } r, p- \\
\text { value }\end{array}$ & $\begin{array}{c}0.973 \\
p<.001 \\
*\end{array}$ & $\begin{array}{c}>.999 \\
p<.001 \\
*\end{array}$ & $\begin{array}{c}>.999 \\
p<.001 \\
*\end{array}$ & $\begin{array}{c}>.999 \\
p<.001 \\
*\end{array}$ & $\begin{array}{c}>.999 \\
p<.001 \\
*\end{array}$ & $\begin{array}{c}>.999 \\
p<.001 \\
*\end{array}$ & $\begin{array}{c}0.991 \\
p<.001 \\
*\end{array}$ & $\begin{array}{c}>.999 \\
p<.001 \\
*\end{array}$ & $\begin{array}{c}>.999 \\
p<.001 \\
*\end{array}$ & $\begin{array}{c}>.999 \\
p<.001 \\
*\end{array}$ \\
\hline $\begin{array}{r}\text { Mann- } \\
\text { Whitney } \\
\text { (between genders } \\
\text { in same quintile) }\end{array}$ & $\begin{array}{r}p<. c \\
\eta^{2}=0 \\
d_{\text {Cohen }}\end{array}$ & $\begin{array}{l}01 * \\
717 \\
3.187\end{array}$ & $\begin{array}{r}\mathrm{p}< \\
\eta^{2}= \\
\mathrm{d}_{\text {Cohen }}\end{array}$ & $\begin{array}{l}01^{*}, \\
.717 \\
=3.187\end{array}$ & $\begin{array}{r}p<. c \\
\eta^{2}=c \\
d_{\text {Cohen }}\end{array}$ & $\begin{array}{l}01 * \\
717 \\
3.187\end{array}$ & $\begin{array}{r}\mathrm{p}<.0 \\
\eta^{2}=0 \\
\mathrm{~d}_{\text {Cohen }}\end{array}$ & $\begin{array}{l}01 * \\
717 \\
3.187\end{array}$ & $\begin{array}{r}\mathrm{p}<. \mathrm{C} \\
\eta^{2}=0 \\
\mathrm{~d}_{\text {Cohen }}\end{array}$ & $\begin{array}{l}01^{*}, \\
.717 \\
=3.187\end{array}$ \\
\hline
\end{tabular}

WIMD1 = most socioeconomically deprived, WIMD5 = least socio-economically deprived $* p<0.05=$ statistically significant

Within gender groups, statistically significant differences were observed in the number of prescriptions issued in each quintile (Table 4.5). Between women, significantly more prescriptions were issued in quintiles with greatest deprivation compared to the least (Kruskal-Wallis, $p<.001, \eta^{2}=0.844$, $d_{\text {cohen }}=4.653$ ). Post-hoc analysis confirmed the number of prescriptions issued in adjacent quintiles (e.g., WIMD1 and WIMD2, WIMD4 and WIMD5) were not statistically different (Appendix C). Neither was there a statistically different number of prescriptions issued in WIMD1 and WIMD3 quintiles ( $p=0.128$ ) (Appendix C).

Comparison of the number of prescriptions issued to men in the five deprivation quintiles demonstrated the same differences as observed for women. Overall, the total number of prescriptions issued to men, per quintile between 2005 and 2015 were significantly different (Kruskal-Wallis, $p<.001, \eta^{2}=0.833, d_{\text {cohen }}=4.472$ ). Adjacent quintiles did not have confirmed statistical differences in number of prescriptions issued (Appendix C) and similarly, WIMD1 and WIMD3 areas were statistically similar in the number of prescriptions issued $(p=0.084)$. 
Trends in total opioid prescribing demonstrated a slowing in annual percentage increases as the study period drew to its end (Figure 4.5). Interestingly, increases in prescribing were inverse to deprivation, despite the number of prescriptions issued being so much higher in more deprived areas.

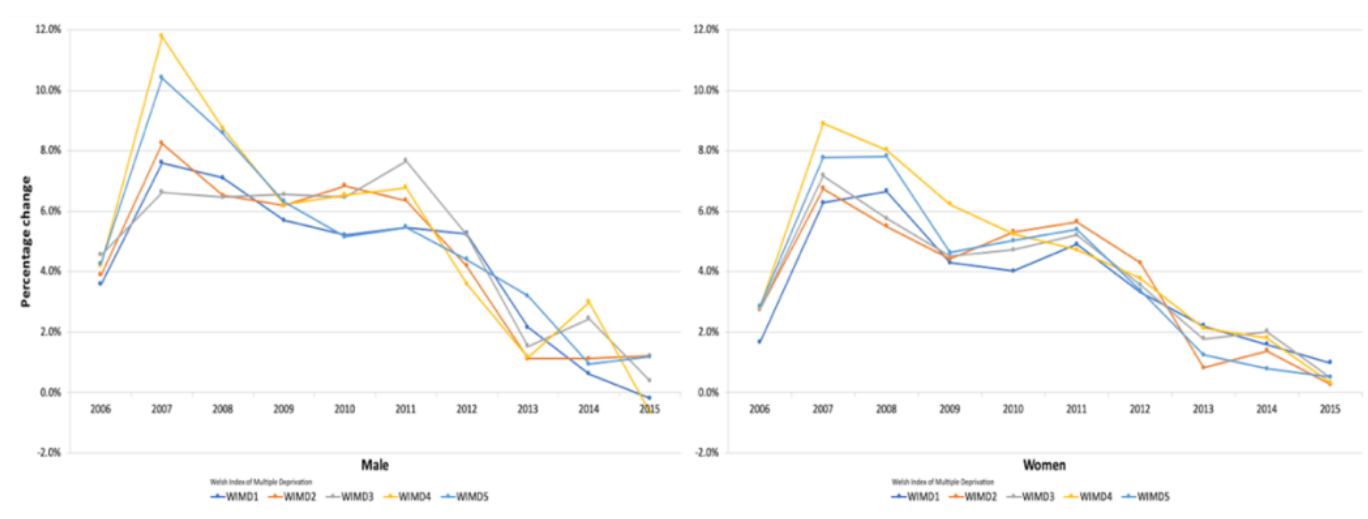

Figure 4.5: Annual percentage change in the number of prescriptions for all opioids by each quintile of deprivation in Wales and gender

WIMD = Welsh Index of Multiple Deprivation where WIMD1 = most deprived and WIMD5 = least deprived

\subsubsection{Weak opioids}

Similar trends in weak opioid prescribing were observed in men and women in each deprivation quintile over the study period (Figure 4.6). For both genders, large differences were noted between the most and least deprived quintiles especially. In total, 2.3 times the number of prescriptions per 1,000 population were issued to women living in the most deprived areas (WIMD1) compared to those in the least deprived (WIMD5) (Table 4.6).
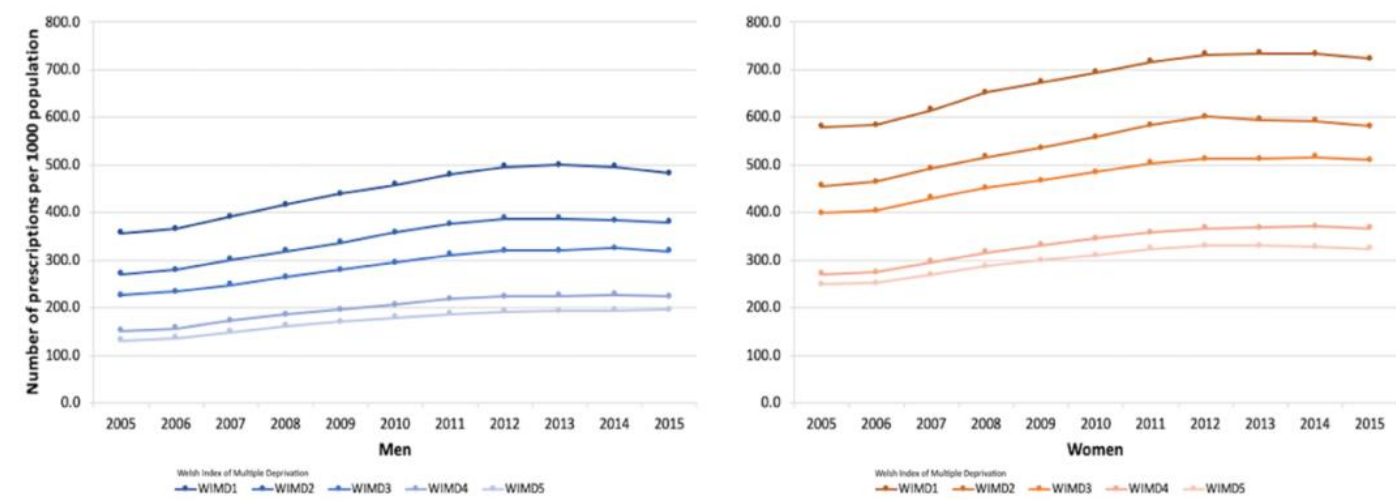

Figure 4.6: Trends in the number of weak opioid prescriptions issued to men and women by deprivation quintile and adjusted to quintile-population in Wales $W I M D=$ Welsh Index of Multiple Deprivation where WIMD1 = most deprived and WIMD5 = least deprived 
Table 4.6: Comparison of quintile-population adjusted numbers of prescriptions for weak opioid medicines by Welsh Index of Multiple Deprivation quintiles and gender across Wales between 2005 and 2015

\begin{tabular}{|c|c|c|c|c|c|c|c|c|c|c|}
\hline & \multicolumn{10}{|c|}{ Number of prescriptions per 1,000 population } \\
\hline & \multicolumn{2}{|c|}{ WIMD1 } & \multicolumn{2}{|c|}{ WIMD2 } & \multicolumn{2}{|c|}{ WIMD3 } & \multicolumn{2}{|c|}{ WIMD4 } & \multicolumn{2}{|c|}{ WIMD5 } \\
\hline & Male & Female & Male & Female & Male & Female & Male & Female & Male & Female \\
\hline 2005 & 357.0 & 580.1 & 270.6 & 456.2 & 225.9 & 398.2 & 151.2 & 270.3 & 131.3 & 249.0 \\
\hline 2015 & 482.9 & 722.5 & 379.7 & 580.9 & 319.2 & 510.0 & 223.3 & 366.6 & 196.1 & 324.1 \\
\hline $\begin{array}{r}\text { Change rate (\%) } \\
2005-2015\end{array}$ & 35.3 & 24.6 & 40.3 & 27.3 & 41.3 & 28.1 & 47.7 & 35.6 & 49.4 & 30.2 \\
\hline $\begin{array}{r}\text { Spearman's r, } \\
\text { p-value }\end{array}$ & $\begin{array}{c}0.882 \\
p<.001^{*}\end{array}$ & $\begin{array}{c}0.873 \\
p<.001 *\end{array}$ & $\begin{array}{c}0.873 \\
p<.001^{*}\end{array}$ & $\begin{array}{c}0.873 \\
p<.001^{*}\end{array}$ & $\begin{array}{c}0.936 \\
p<.001^{*}\end{array}$ & $\begin{array}{c}0.936 \\
p<.001 *\end{array}$ & $\begin{array}{c}0.945 \\
p<.001^{*}\end{array}$ & $\begin{array}{c}0.945 \\
p<.001^{*}\end{array}$ & $\begin{array}{c}>.999 \\
p<.001^{*}\end{array}$ & $\begin{array}{c}0.873 \\
p<.001^{*}\end{array}$ \\
\hline $\begin{array}{l}\text { Mann-Whitney } \\
\text { (between genders } \\
\text { in same quintile) }\end{array}$ & $\begin{array}{r}\mathrm{p}<.001^{*} \\
\mathrm{~d}_{\text {Cohe }}\end{array}$ & $\begin{array}{l}2=0.717 \\
3.187\end{array}$ & $\begin{array}{r}\mathrm{p}<.001^{*} \\
\mathrm{~d}_{\text {Cohe }}\end{array}$ & $\begin{array}{l}l^{2}=0.717 \\
3.187\end{array}$ & $\begin{array}{r}\mathrm{p}<.001^{*} \\
\mathrm{~d}_{\text {Cohe }}\end{array}$ & $\begin{array}{l}2=0.717 \\
.187\end{array}$ & $\begin{array}{r}\mathrm{p}<.001^{*} \\
\mathrm{~d}_{\text {Cohe }}\end{array}$ & $\begin{array}{l}1^{2}=0.717 \\
3.187\end{array}$ & $\begin{array}{r}\mathrm{p}<.001^{*} \\
\mathrm{~d}_{\text {Cohe }}\end{array}$ & $\begin{array}{l}2=0.717 \\
3.187\end{array}$ \\
\hline
\end{tabular}

WIMD1 = most socioeconomically deprived, WIMD5 = least socioeconomically deprived *p<0.05 = statistically significant. Annual data in Appendix C 
The overall rate of prescribing was less in men than women in every quintile although increases in prescribing were greater (Table 4.6). However, the percentage difference between each quintile was greater than observed between women. Men in the most deprived areas (WIMD1) received 2.6 times more weak opioid prescriptions than those in the least deprived (WIMD5) (Table 4.6).

Rates of weak opioid prescribing began to reduce from 2013 for both genders, resulting in the differences between quintiles beginning to narrow slightly at the end of the study period. Overall prescribing rates for women were significantly higher than for men in equivalent socio-economic quintiles (Figure 4.6).

Differences in the number of prescriptions issued to women in the five deprivation quintiles, were statistically significant (Kruskal-Wallis, $p<.001$, $\left.\eta^{2}=0.895, d_{\text {Cohen }}=5.824\right)$. Post-hoc analysis confirmed significantly more prescriptions were issued in quintiles WIMD1-3 compared to the least deprived quintile (WIMD5) (Appendix C). This was also true for the two most deprived quintiles compared to WIMD4 areas and it also confirmed significantly more prescriptions were issued in the most deprived quintile (WIMD1) compared to the areas of middle deprivation levels (WIMD3).

Fewer differences in the number of prescriptions issued in the different quintiles were observed in men, although the overall comparison was statistically significant (Kruskal-Wallis, $p<.001, \eta^{2}=0.878$, $d_{\text {Cohen }}=5.358$ ). Significantly more weak opioid prescriptions were confirmed to have been issued in the three most deprived quintiles (WIMD1 to WIMD3) when directly compared to the least deprived areas. Post-hoc analysis also confirmed statistically more prescriptions were issued in the two most deprived quintiles (WIMD1 and WIMD2) when directly compared to the $4^{\text {th }}$ quintile (WIMD4) (Appendix C). 


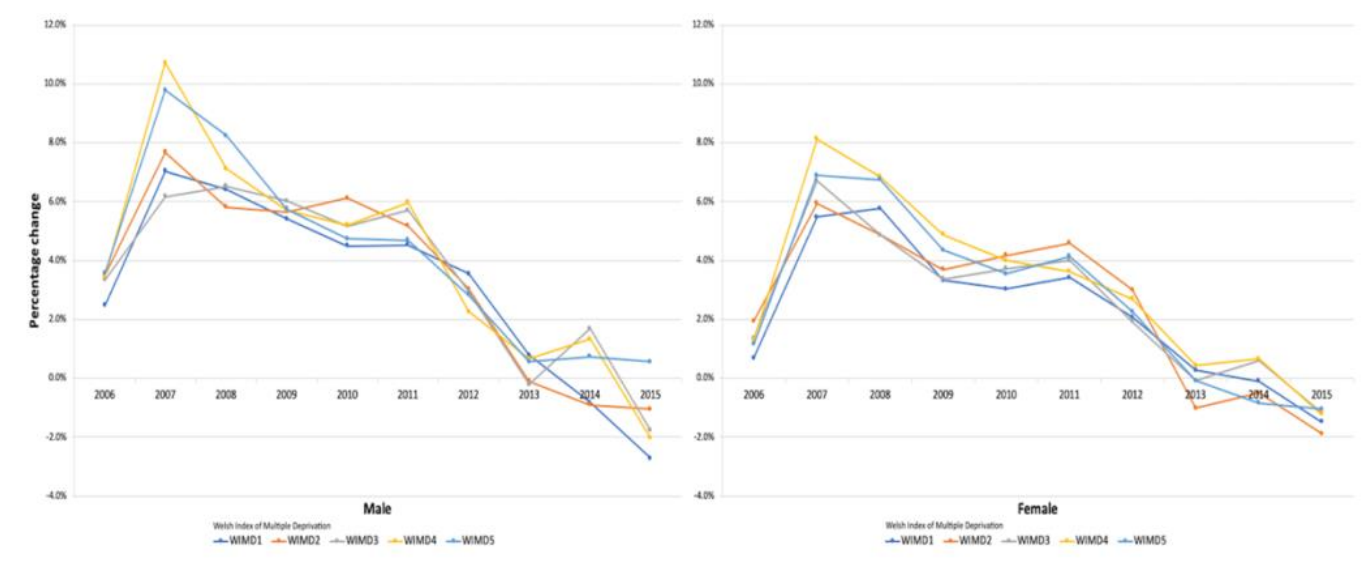

Figure 4.7: Annual percentage change in the number of prescriptions for weak opioids by each quintile of deprivation in Wales and gender

WIMD = Welsh Index of Multiple Deprivation where WIMD1 = most deprived and WIMD5 = least deprive)

The rate of increase in weak opioid prescribing slowed consistently in all quintiles and both genders over the 11 years examined (Figure 4.7). Reductions in prescriptions per 1,000 population were noted in all deprivation quintiles for women by 2015 and all but the least deprived areas for men (Figure 4.7).

\subsubsection{Strong opioids}

Women in each socio-economic deprivation quintile, received more prescriptions for strong opioid medicines, than men in the same areas. The greatest increase in prescribing rate was noted in the WIMD1 quintile (Table 4.7). Increases in prescribing rate were greater for women than men, in every quintile. Despite the empirical differences, strong opioid prescribing rates over the 11 years analysed were only statistically different in the least deprived quintile (WIMD5) (Table 4.7). Spearman's $r$ tests demonstrated strong correlation in all quintiles for both genders, between time and prescribing rate, confirming the upward trends observed between 2005 and 2015 (Table 4.7). 
Table 4.7: Comparison of quintile-population adjusted numbers of prescriptions for strong opioid medicines by Welsh Index of Multiple Deprivation quintiles and gender across Wales between 2005 and 2015.

\begin{tabular}{|c|c|c|c|c|c|c|c|c|c|c|}
\hline & \multicolumn{10}{|c|}{ Number of prescriptions per 1,000 population } \\
\hline & \multicolumn{2}{|c|}{ WIMD1 } & \multicolumn{2}{|c|}{ WIMD2 } & \multicolumn{2}{|c|}{ WIMD3 } & \multicolumn{2}{|c|}{ WIMD4 } & \multicolumn{2}{|c|}{ WIMD5 } \\
\hline & Male & Female & Male & Female & Male & Female & Male & Female & Male & Female \\
\hline 2005 & 23.5 & 26.5 & 19.8 & 24.3 & 18.0 & 22.9 & 12.5 & 16.7 & 9.0 & 13.7 \\
\hline 2015 & 92.2 & 138.9 & 72.7 & 109.2 & 69.1 & 100.2 & 45.3 & 73.3 & 31.3 & 61.2 \\
\hline Change rate (\%) & 292.0 & 423.7 & 267.5 & 349.5 & 283.3 & 336.7 & 262.0 & 338.3 & 246.6 & 346.9 \\
\hline \multicolumn{11}{|l|}{$2005-2015$} \\
\hline Spearman's r, p- & $>.999$ & $>.999$ & $>.999$ & $>.999$ & $>.999$ & $>.999$ & $>.999$ & $>.999$ & $>.999$ & $>.999$ \\
\hline value & $\mathrm{p}<.001^{*}$ & $\mathrm{p}<.001^{*}$ & $\mathrm{p}<.001^{*}$ & $\mathrm{p}<.001^{*}$ & $\mathrm{p}<.001^{*}$ & $\mathrm{p}<.001^{*}$ & $\mathrm{p}<.001^{*}$ & $\mathrm{p}<.001^{*}$ & $\mathrm{p}<.001^{*}$ & $\mathrm{p}<.001 *$ \\
\hline $\begin{array}{r}\text { Mann-Whitney } \\
\text { (between genders in } \\
\text { same quintile) }\end{array}$ & \multicolumn{2}{|c|}{$\begin{array}{c}p=.193, \eta^{2}=0.082 \\
d_{\text {Cohen }}=0.599\end{array}$} & \multicolumn{2}{|c|}{$\begin{array}{c}p=.116, \eta^{2}=0.118 \\
d_{\text {Cohen }}=0.73\end{array}$} & \multicolumn{2}{|c|}{$\begin{array}{c}\mathrm{p}=.101, \eta^{2}=0.127 \\
\mathrm{~d}_{\text {Cohen }}=0.764\end{array}$} & \multicolumn{2}{|c|}{$\begin{array}{c}p=.056, \eta^{2}=0.165 \\
d_{\text {Cohen }}=0.889\end{array}$} & \multicolumn{2}{|c|}{$\begin{array}{c}\mathrm{p}<.01^{*}, \mathrm{\eta}^{2}=0.298 \\
\mathrm{~d}_{\text {Cohen }}=1.303\end{array}$} \\
\hline
\end{tabular}

WIMD1 = most socioeconomically deprived, WIMD5 = least socio-economically deprived. ${ }^{*} p<0.05=$ statistically significant. Annual data in Appendix C 
Comparing prescribing rates between quintiles and by gender, differences were deemed statistically significant for women (Kruskal-Wallis, $p<0.05, \eta^{2}=0.113$, $\mathrm{d}_{\text {Cohen }}=0.715$ ) and men (Kruskal-Wallis, $\left.\mathrm{p}<0.01, \eta^{2}=0.323, \mathrm{~d}_{\text {Cohen }}=1.383\right)$. Pairwise comparisons of strong opioid prescribing rates for women in the different quintiles only confirmed a statically significant difference between the most (WIMD1) and least (WIMD5) quintiles however ( $p<0.05)$ (Appendix $C)$.
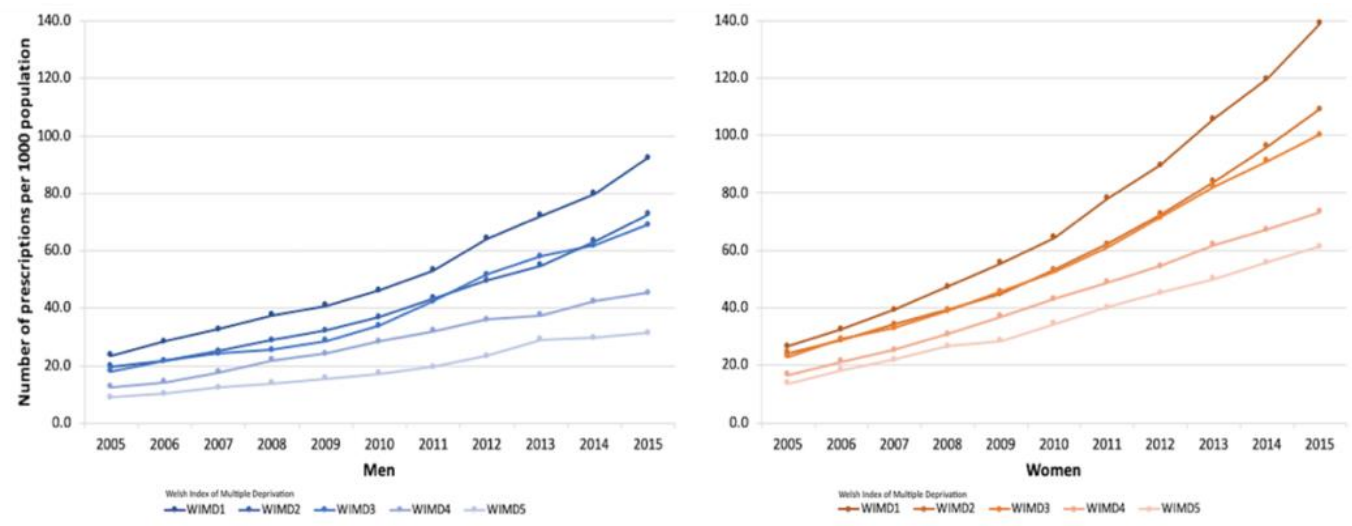

Figure 4.8: Trends in the number of strong opioid prescriptions issued to men and women by deprivation quintile and adjusted to quintile-population in Wales WIMD = Welsh Index of Multiple Deprivation where WIMD1 = most deprived and WIMD5 = least deprived

A slightly different observation was noted in men. Pairwise comparisons between number of prescriptions per 1,000 population issued $2005-2015$, confirmed statistically different rates between the 3 most deprived areas (WIMD1, WIMD2 and WIMD3) and the least deprived (WIMD5). No other differences were observed in prescribing rates between the other quintiles (Appendix C).

As with strong opioid prescribing in general, there was no pattern in terms of the annual percentage increases noted in any of the five quintiles or either gender. Both lower quintiles (WIMD1 and WIMD2) had consistent percentage increases each year (Figure 4.8). In quintiles WIMD3 to WIMD5, there appeared to be greater fluctuation in annual percentage change for men than women (Figure 4.9). Whilst slowing in the rate of increase in prescribing was observed in both genders towards the end of the study, strong opioid prescribing was still rising, with the highest rates in the most deprived areas (Figure 4.9). 


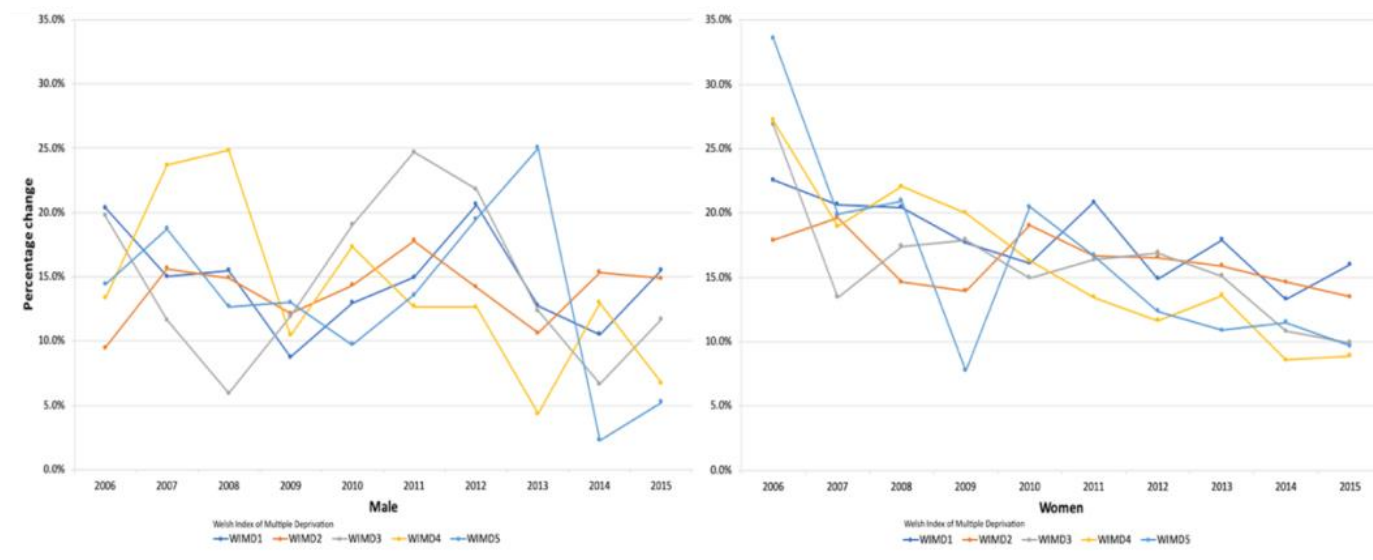

Figure 4.9: Annual percentage change in the number of prescriptions for strong opioids by each quintile of deprivation in Wales and gender

WIMD = Welsh Index of Multiple Deprivation where WIMD1 = most deprived and WIMD5 = least deprived

\subsubsection{Log-linear regression outcomes}

The log-linear regression results were used to examine which variables might predict the likelihood of receiving a prescription for a strong opioid analgesic. The variables used in the model were deprivation quintile, gender, age and Health Board in the Primary Care medical record. $X^{2}(8)=7947.33, p<0.001$. The model explained $2 \%$ (Nagelkerke $\mathrm{R}^{2}$ ) of the variance in strong opioid prescribing and correctly classified $89.4 \%$ of cases. The $ß_{0}$ (constant) for the equation was 4.272, SE=.006, $p<.001$.

Based on the regression output, people living in the Abertawe Bro Morgannwg, Cwm Taf and Aneurin Bevan University Heath Board areas were more likely and to receive a strong opioid prescription as individuals living in Powys Teaching Health Board (Table 4.8). Living in areas considered to be within the two most deprived quintiles slightly increased the chance of receiving a strong opioid prescription compared to those living in the least deprived. Living in quintiles WIMD3 and WIMD4 however, reduced the likelihood compared to being in WIMD5. Being male reduced the likelihood of receiving a strong opioid prescription, compared to being female. Every year of additional age over the age of 18 years led to an increased likelihood of receiving a strong opioid prescription. So, the receipt of a strong opioid prescription appeared to correlate with increasing age. 
Table 4.8: Output from log-linear regression to determine variables which may increase the likelihood of receiving a strong opioid analgesic prescription

\begin{tabular}{|c|c|}
\hline Variable & $\beta_{n}(S E), p$-value* \\
\hline \multicolumn{2}{|l|}{ Deprivation quintile } \\
\hline WIMD1 & $1.023(.002),<0.001$ \\
\hline WIMD2 & $1.045(.003), p<.001$ \\
\hline WIMD3 & $.980(.003), p<.001$ \\
\hline WIMD4 & $.931(.003), p<.001$ \\
\hline Gender (male) & $.952(.001), p<.001$ \\
\hline Age (per year over 18 years) & $1.012(.000), p<.001$ \\
\hline \multicolumn{2}{|l|}{ Health Board } \\
\hline Betsi Cadwaladr & $.751(.005), p<.001$ \\
\hline Hywel Dda & $.696(.005), p<.001$ \\
\hline Abertawe Bro Morgannwg & $1.378(.005), p<.001$ \\
\hline Cardiff and Vale & $.792(.005), p<.001$ \\
\hline Cwm Taf & $1.225(.006), p<.001$ \\
\hline Aneurin Bevan & $1.055(.005), p<.001$ \\
\hline
\end{tabular}

${ }^{*}$ p-value $<.05$ = statistically significant

This model was a poor predictor for strong opioid prescribing, which was likely to be due to all included data being initially selected by the presence of an opioid prescription (Chapter 2).

\subsection{Discussion}

\subsubsection{Summary and reflection on findings}

Nearly a third of all opioid prescriptions were issued to people living in the most deprived areas of Wales. Whilst this might have led to an assumption that prescribing would have increased more in the most deprived areas, compared to the least, it was not the case. In fact, overall opioid prescribing rates rose more in less deprived areas over the study period. With the possibility of a reduction in overall prescribing, it appears levels of prescribing might draw closer between quintiles. However, strong opioid prescribing rates were shown to be increasing more rapidly in the most deprived quintiles. Different to weak opioid prescribing, there were signs of increasing disparity between strong opioid exposure in areas of greatest and least deprivation across Wales. The log-linear regression reported here was not an effective means of predicting which of those variables might be most important. Future analysis should include data for people not receiving opioid analgesic prescriptions, to increase the model accuracy. 
It was already known from previous results that women received more prescriptions of weak and strong opioids than men (Chapter 3 ). Women in every quintile had increases in strong opioid prescribing that were much larger than those observed in their male counterparts. Overall, prescribing rates were highest for women living in the most socioeconomically deprived areas of Wales. Of interest was the largest gender difference in prescribing rate, noted in the least deprived quintile (WIMD5). However, as with overall prescribing, there was increasing separation of strong opioid prescribing rates between the quintiles of socio-economic deprivation for men and women as the study period drew to an end. In simple terms, there is evidence that the gap between the poorest and richest in Welsh society is widening.

\subsubsection{Contextualising results with existing literature}

The findings presented here are consistent with those from the rest of the UK which demonstrate opioid prescribing increases with deprivation (Todd et al. 2018; Macfarlane et al. 2020; Chen et al. 2019; Curtis et al. 2019; Torrance et al. 2018; Schifanella et al. 2020). The Welsh data revealed 2.4 times more prescriptions were issued to people living in the most socioeconomically deprived areas compared to those in more affluent areas. Most UK studies have highlighted correlates between deprivation quintile and opioid prescribing, but not reflected on trends in areas with differing socioeconomic scores. Schifanella et al. (2020) reported spatial association between rates of opioid prescribing and markers of deprivation such as unemployment, low educational attainment and poor housing. Their findings, whilst not reported as a trend analysis, were consistent between 2015 and 2018 and used LSOA level data rather than multiple deprivation quintiles (Schifanella et al. 2020). It revealed that particular geographical areas could be demonstrated to have significant changes in prescribing in that period. However, using LSOA spatial models showed that often, significant differences were only noted in relatively discrete units of area e.g., 40 out of 186 LSOAs in Sandwell, West Midlands, accounted for the average increase of 70mg OMEQ observed during the study period (Schifanella et al. 
2020). Practices in the most deprived areas of England were found to be more likely to prescribe all opioids and particularly high dose opioids (greater than $120 \mathrm{mg}$ oral morphine equivalent dose per day) (Curtis et al. 2019). Again, small reductions have been noted in overall opioid prescribing in England since the mid-2000s (Curtis et al. 2019; Taylor et al. 2019), as in Wales. High dose opioid prescribing was examined for this study and the results are presented in Appendix D.

TOPAS data confirms those observed in Scotland between 1995 and 2010, where increases in prescribing was noted and more so in areas with of greater socioeconomic deprivation (Ruscitto et al. 2015). Whilst strong opioid prescribing correlated with deprivation, percentage increases by quintile were not provided. However, as in this study, there was a small reduction in weak opioid prescribing and a significant increase in strong opioid prescribing by study end (Ruscitto et al. 2015). More recently, a larger study from Scotland calculated that people in the most deprived areas were 3 to 4 times more likely to receive weak and strong opioid prescriptions, respectively (Torrance et al. 2018). In this study, these figures were 1.7 and 1.8 times respectively, so almost half the difference noted in Scotland. The data in Scotland was complete for all prescriptions dispensed, whereas TOPAS data was more selective, based on prescriptions issued to $78 \%$ of the population (Chapter 2). However, the shape of the trends noted were similar between Wales and Scotland over a similar time period (Torrance et al. 2018). Deprivation is calculated differently in Scotland, compared to Wales (Table 1.1). Given Wales is classified as the most deprived country in the UK, it could be the differences between most and least deprived are not as great as elsewhere. What impact this has on prescribing is not clear, however.

This study observed a south-north divide, prescribing increased with deprivation, with the most deprived areas predominantly in the south of the country. A north-south divide has been used to explain patterns of English opioid prescribing, with northern England commonly defined as the North East, North West and Yorkshire and Humber regions (Todd et al. 2018). Regional variation 
has also been examined using geographical latitude as the measure of north or south (Mordecai et al. 2018). Others have reported on north-south variation in prescribing without clearly defining where the dividing line lies (Chen et al. 2019). Whilst geography cannot account for deprivation alone, it is known that there is more widespread socioeconomic deprivation in the north of England, compared to the south (Crisp et al. 2009; Joseph Rowntree Foundation 2017). Todd et al. (2018) observed demographic factors of people reporting chronic pain, such as age, gender and anxiety levels, appeared similar between those in the north and south. However, larger differences were noted in income levels (42.8\% of people in the north compared to $32.4 \%$ in the south in the lower 2 quintiles), self-reported health status ('bad' or 'very bad' reported by $7.6 \%$ of subjects in the north compared to $5.5 \%$ in the south) (Todd et al. 2018). Prescribers in the north of England have been observed to issue more opioid prescriptions than their southern counterparts (Todd et al. 2018; Mordecai et al. 2018; Curtis et al. 2019; Chen et al. 2019). 


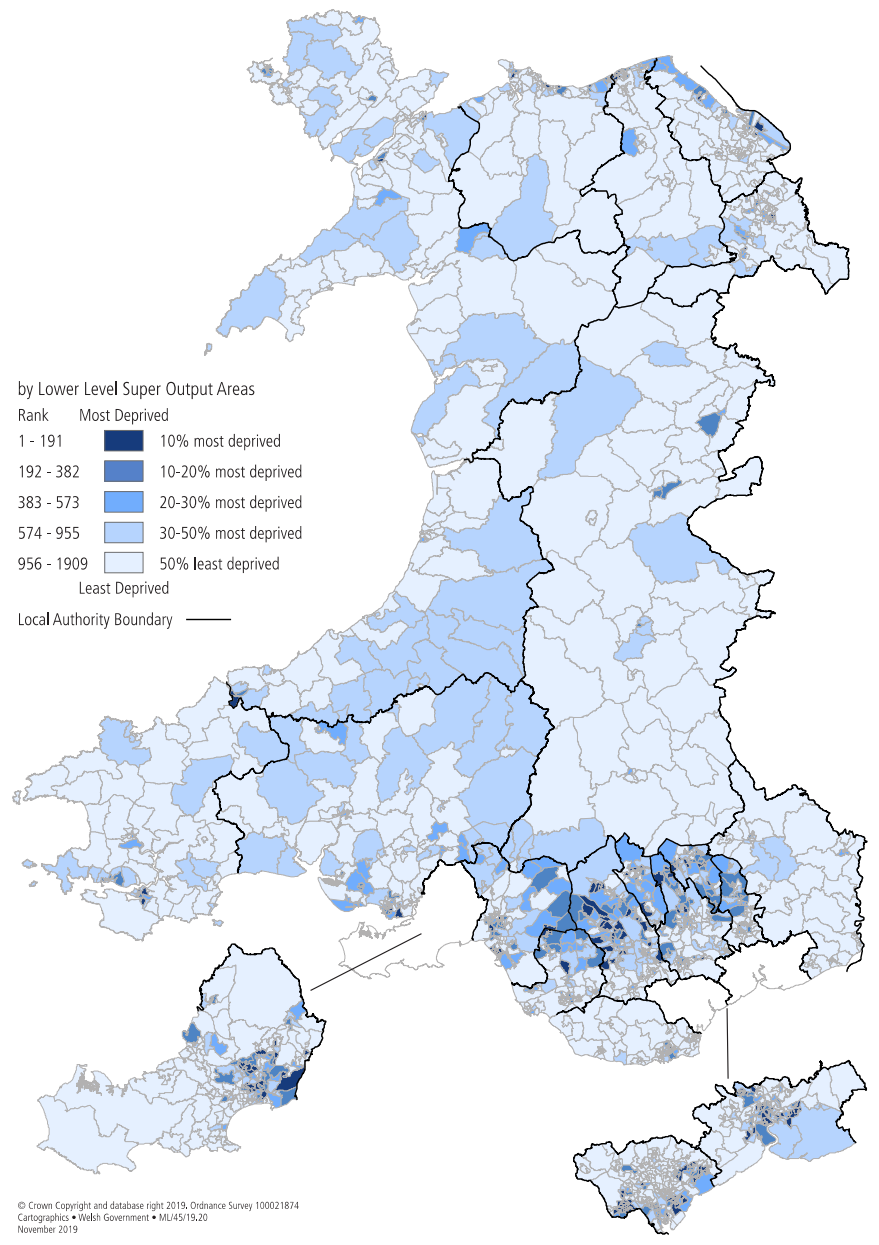

Figure 4.10: Welsh Index of Multiple Deprivation 2019

(StatsWales 2019)

Using oral morphine equivalence, a higher burden of weak and strong opioids was more commonly issued in the north (Mordecai et al. 2018; Curtis et al. 2019). A study examining data from a small geographic area of Leeds and Bradford, in the north of England, reported a greater likelihood of opioid prescribing with increased levels of deprivation. This was within an area that would be generally classed as 'deprived' relative to areas in the south of the country (Foy et al. 2016). Schifanella et al. (2020) confirmed that examining data at LSOA level, differences within areas that fall into the same index of multiple deprivation quintile, can be determined (Schifanella et al. 2020). This perhaps suggests that broad categories of deprivation or geographical areas, used in WIMD and others, are insufficient on their own to explain the patterns of 
prescribing noted. For example, Schifanella et al. (2020) describes an area of the West Midlands that as whole had a notable increase in opioid prescribing between 2016-2018. When analysed at LSOA level, the increase was accounted for by just over $20 \%$ of the total area's population. Whilst the LSOAs contributing to that area were grouped together in one part of the district, they were a small but exceptional part of a larger whole (Schifanella et al. 2020). Further analysis of opioid prescribing by LSOAs in Wales would be beneficial to allow more precise targeting of local communities for interventions to address it. In all likelihood a combination of broad and focused analysis would be helpful over time to examine changes in prescribing and monitor variation across the country.

Although not examined in this study, opioid prescribing has been correlated with greater perceived pain intensity or pain reporting in the UK (Toye et al. 2017; Todd et al. 2018; Torrance et al. 2018; Macfarlane et al. 2020; Jani et al. 2020). A prevalence of $36.7 \%$ in the north of England and 35\% in the south has been reported. Pain was moderately or severely limiting for $12.3 \%$ of respondents in the north and 9.2\% in the south (Todd et al. 2018). Pain prevalence in the general UK population have been estimated at up to $44 \%$ (Fayaz et al. 2016). No measure was available in Wales at the time of this study that could be used as a direct comparator. The Welsh Health Survey in 2015, however described $15 \%$ of people being limited by health problems or disability which may suggest a similar prevalence of moderate to severe pain. Self-reported pain in multiple sites has also shown correlation with opioid use. An adjusted risk ratio of 16.66 (Cl $15.42-$ 17.99) was noted for people from all over the UK, reporting pain in seven or more sites, compared to those without any sites of pain although receiving opioids (Macfarlane et al. 2020). High-dose opioid prescribing has also been shown to be more prevalent in primary care practices with greater numbers of patients on long-term condition registers (Curtis et al. 2019). Interestingly, pain intensity was not demonstrated to be significantly different between groups of people taking 99-199mg and more than 200mg oral morphine equivalent daily dose in a large Australian study. Socio-economic status did inversely correlate with dose however (Campbell, Nielsen, Bruno, et al. 2015). Pain intensity was 
also not found to be predictive for opioid prescribing in a predominantly rural, deprived cohort in the USA (Kapoor \& Thorn 2014).

Higher levels of opioid prescribing in areas of greater socioeconomic deprivation is a consistent finding around the world (McDonald et al. 2012; Smith et al. 2019; Bosetti et al. 2019; Islam \& Wollersheim 2019). The American 'opioid crisis' is probably the most documented pattern of prescribing worldwide (Weisberg and Stannard 2013; McGreal 2018; Stoicea et al. 2019; Wen and Sadeghi 2020). Between 2006 and 2012, the prescribing rate increased from 72.4 to 81.3 prescriptions per 1,000 people nationwide (Centers for Disease Control and Prevention 2021) but particularly in areas associated with higher levels of unemployment and deprivation (Weisberg et al. 2014; McGreal 2018; Stoicea et al. 2019). More recently, there is evidence that prescribing of opioids is reducing across north America (Kurani et al. 2020). Overall rates have dropped significantly from 81.3 prescriptions per 100 people in 2012 to 46.7 prescriptions per 100 people in 2019 (Centers for Disease Control and Prevention 2021). The finding of a closing gap between the most and least deprived areas has been observed in America. The percentage reduction in opioid prescriptions issued, was inversely related to deprivation quintile (Kurani et al. 2020). However, some of the most deprived counties continue to have much higher rates, for example Tift County in Georgia had an opioid prescribing rate of 178 prescriptions per 100 people in 2019 (Centers for Disease Control and Prevention 2021). Reports from Australia have illustrated higher levels of opioid prescribing in more rural and less affluent areas (Degenhardt et al. 2015; Islam \& Wollersheim 2019). Islam and Wollersheim (2019) demonstrated between 2013 and 2016, adjusted odd ratio $(A O R)$ for opioid prescribing was $1.59(95 \% \mathrm{Cl} 1.48-1.71)$ in the most deprived areas compared to an AOR of 1 for the least deprived areas of Australia (Islam and Wollersheim 2019). Rates of opioid prescribing also vary widely across European. Between 2014 and 2016, opioid consumption in Germany was observed to average 21,346 defined daily doses (DDDs) per 1,000,000 inhabitants, compared to 66 DDDs/1,000,000 inhabitants in Ukraine (Bosetti et al. 2019). 
Whilst opioid prescribing rates vary across developed countries, higher rates of prescribing in poorer areas are a consistent feature. Is deprivation a satisfactory reason for prescribing larger quantities of opioid analgesics, however? Whilst pain prevalence may be higher in more deprived areas, evidence would not support the widespread use opioids to address it. Reducing inequality would likely reduce the variation seen in the health of people with different socioeconomic status, given the links between deprivation and pain. Lessening inequity requires political solutions and a genuine commitment to improve social mobility and working conditions (Marmot 2017). Such aspirations are often cited and in Wales, they form the basis of Government policy (Welsh Government 2019). However, it takes time and whilst efforts to reduce inequality continue, measures still need to be put in place to address prescribing as a means of harm reduction.

\subsubsection{Gender differences and deprivation}

It is known women receive more prescriptions for opioid analgesics than men in the UK and worldwide, where opioids are commonly prescribed (Campbell et al. 2015; Bedson et al. 2016; Mazure and Fiellin 2018; Todd et al. 2018; Torrance et al. 2018; Taylor et al. 2019). Data presented here, confirmed this and that there were additional associations between gender and deprivation.

Bedson et al. (2016) examined trends in the incidence of opioid initiation, for musculoskeletal pain between $2002-2013$. Whilst it was shown women had a higher incidence than men, for opioid initiation every year, the rate of increase was higher overall for men (Bedson et al. 2016). Unlike results shared here, data was not stratified by deprivation and statistical significance of the differences was not provided. However, assuming rate of initiation and overall rates of prescribing are linked, the all-opioid trend findings in this study are consistent with those of Bedson et al. (2016), in that women had higher rates of prescribing, but lower rates of increase than men (Bedson et al. 2016). The reason for the observation cannot be elucidated from this study and warrants further research. Closing the gender gap in prescribing could be considered a sign of improving 
healthcare equity. However, if it resulted in high levels of opioid prescribing in men and women, would it necessarily be a success or, given what is known about the harms of opioids, actually a sign of failure?

Svendsen et al. (2014) observed Norwegian women in the lowest income quartile and who were either unemployed or in receipt of disability pensions, were more likely to receive persistent opioid prescriptions than women with higher incomes or who were employed. The study did not explore whether unemployment or receipt of financial support was the cause or result of living with pain. It is an issue worthy of further research. Women in all categories were more likely than men to receive long-term opioids, although the difference between odds ratio increasing as income also increased. Whilst this part of the study did not analyse prescribing persistence, that the largest gender differences in prescribing rates were in the least deprived quintiles, was supported by Svendsen's work (Svendsen et al. 2014).

Higher pain prevalence and reported intensity have been frequently cited to be higher in females than males (Todd et al. 2018; Mazure \& Fiellin 2018). Todd et al.'s. study of pain intensity and opioid utilisation, observed statistically significant increases in reporting of severe and moderately limiting pain in females, compared to males (Todd et al. 2018). A prescribing trend analysis did not form part of that study, however. The Scottish Family Health Study includes questions on pain experience. From that, $71.8 \%$ of people reporting severe pain were female, although women were over-represented in the study $(63.7 \%$ of respondents) (Torrance et al. 2018). Whilst trend analysis stratified by gender or deprivation, has not been reported in Scotland, a point prevalence study from 2012 confirmed significantly higher $(p<.001)$ rates of weak opioid prescribing for women, than men which TOPAS findings were consistent with (Torrance et al. 2018). As with data presented here, there was not a statistically significant difference in strong opioid prescribing rates between men and women (Torrance et al. 2018), although it was not stratified by deprivation quintile, so direct comparison was not possible. 
Of concern from this work was the trajectory of increase of strong opioid prescribing rate (Figure 4.8) for women in the most deprived quintile, which does not appear to have previously been reported. Overall, opioid prescribing rates in Wales since the end of the study period in 2015 have levelled, with some initial signs of reduction (Shared Services Partnership 2021). However, the rate of strong opioid prescribing was continuing to increase at the end of the study period. The difference in pain experience of men and women has recently been highlighted in mainstream media with particular focus on gynaecological conditions such as endometriosis and complications of vaginal mesh surgery (Barnett 2020; Connolly 2020; Marsh 2021; Warraich 2021). Women have described feeling disbelieved in respect of the pain they are reporting and consequently, often 'fobbed off' with painkillers, which are frequently opioids, as a consequence (Connolly 2020; Marsh 2021). It perhaps suggests the lack of understanding of female-specific conditions and ingrained gender constructs could be as influential on prescribing decisions as the presenting symptoms (Marsh 2021). Further research is clearly needed in terms of gender-differences in pain experience and its management.

Mazure and Fiellin (2018) commented on the impact gender difference in the context of the north American opioid crisis (Mazure \& Fiellin 2018). They highlighted women were more likely than men to access opioids on prescription than illicitly and for pain, which is supported by other studies including in Wales (Cicero et al. 2009; Holloway et al. 2018; Fischer et al. 2018). Although women are currently less likely to present with opioid use disorder (NWIS - Information Services 2019), there is evidence they develop problematic use more quickly than their male counterparts (Hernandez-Avila et al. 2004; McHugh et al. 2018). Substance misuse is also more common in areas of greater socioeconomic deprivation (Liddell 2019; Taylor et al. 2019). Adverse effects of opioids have been shown to differ between men and women (Fillingim et al. 2009; National Academies of Sciences, Engineering, and Medicine 2017), although it cannot be stated with certainty that women experience worse effects. The combination of rising strong opioid use in women living under the most challenging conditions is 
suggestive the prevalence of any resulting harm could be disproportionate and further widen health inequalities.

\subsection{Conclusion}

Opioid prescribing is significantly more common in areas with higher levels of socioeconomic deprivation. This is one of few studies which has examined opioid prescribing trends by deprivation. Whilst other studies have demonstrated large increases in opioid prescribing, this study goes further by showing how trends vary depending on deprivation and gender.

A high burden of opioid prescribing within deprived and unhealthy populations should be a cause of great concern for healthcare leaders and policymakers. The long-term harm of opioids is likely to be felt more severely in areas where levels of health and general well-being are already poor. An additional apprehension is the potential for prescribed opioids to be diverted or used in conjunction with illicit substances, as in Wales, areas of high prescribing are also those with high levels of substance misuse. Access to services is problematic, in no small part due to the geography of the country. This research has demonstrated however, prescribing is a nationwide concern and whilst certain areas have larger populations of concern, no area is devoid of problematic opioid use.

There is an urgent need to address the reasons that pain prevalence and opioid prescribing are so much higher in the populations specified in this study. Examining the motivation of prescribers and those receiving prescriptions is necessary to develop new strategies which target the most vulnerable people, in a manner that considers the complexities of their pain and the lives that they are leading. 


\section{Chapter 5}

Prescribing trends in oral morphine equivalent dose

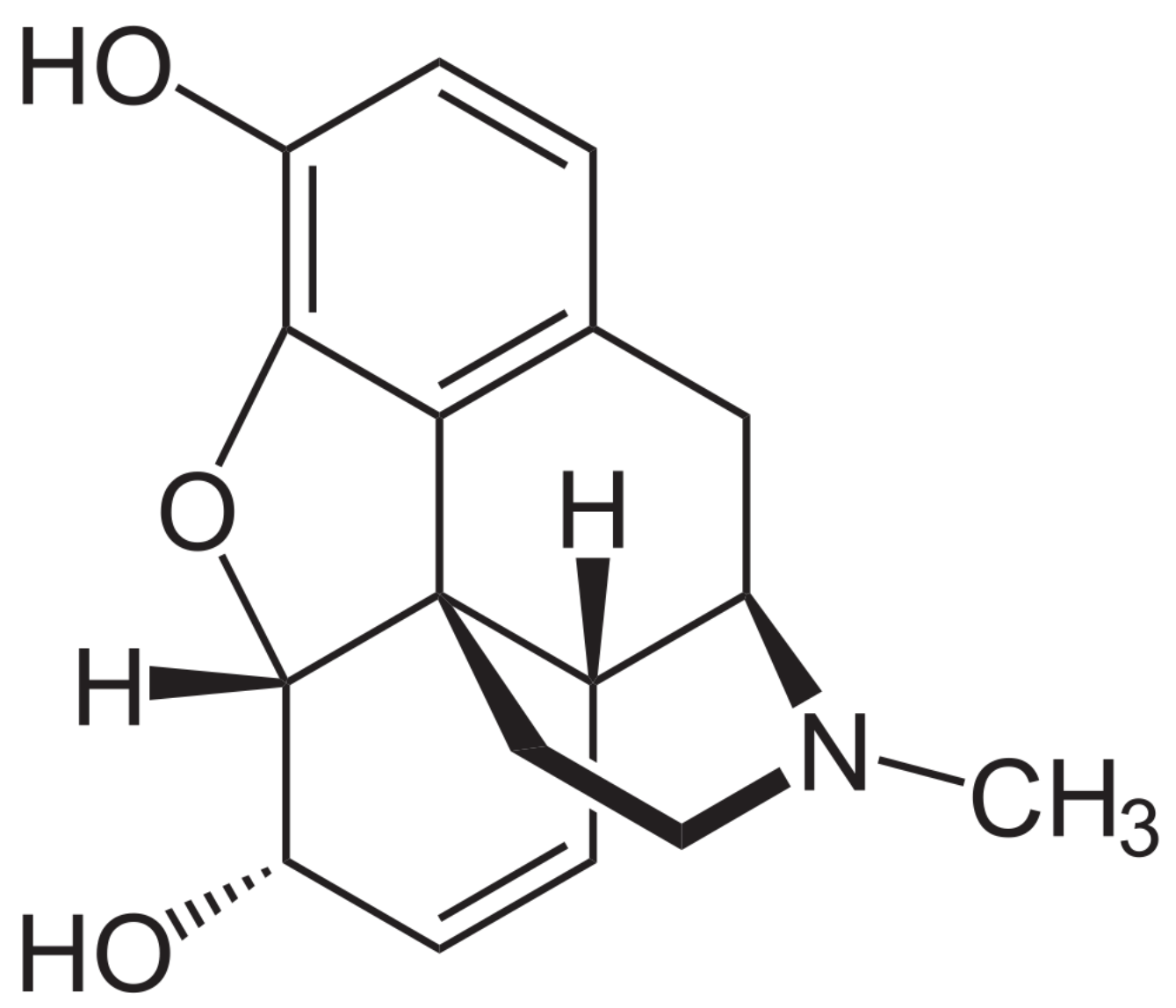

"No man ever sank under the burden of the day. It is when tomorrow's burden is added to the burden of today that the weight is more than a man can bear." George MacDonald 


\section{Chapter 5 - Prescribing trends in oral morphine equivalent dose}

\subsection{Chapter overview}

A review of non-cancer pain and the use of opioids in its management, as well as the use of large datasets was laid out in Chapter 1. The second chapter set out the methods used for the main analyses involved in Phase 1 of this research. The results so far have illustrated significant increases in all opioid prescribing and of strong opioids in particular. Women were more likely to receive opioid prescriptions than men. People aged 45 years and above have higher rates of prescribing than those who are younger. Opioid prescribing was much higher in areas of greatest socioeconomic deprivation and again, women appear disproportionately affected. This chapter scrutinises the data in a different way. Using a purpose-designed measure to determine oral morphine equivalent dosing changes, trends will be examined whilst also looking at which drugs were prescribed. Comparisons will also be made with the trends in prescription numbers to determine if it is a more effective method for determining 'opioid burden'.

\subsection{Study objective to be addressed in this chapter}

- To analyse trends in oral morphine equivalent doses and prescribing duration using estimated measures for each

\subsection{Background}

The internationally recognised daily defined dose (DDD) used by the World Health Organisation (WHO) is often used as the measure of burden in studies and data reviews in practice. The WHO define DDDs as 'the assumed average maintenance dose per day for a drug used for its main indication in adults' (WHO Collaborating Centre for Drug Statistics Methodology 2021). To decide DDDs for any drug, WHO classifies medicines according to the organ or body system they act upon, as well as their pharmacological properties. The number of DDDs is used as an expression of consumption (Figure 5.1Error! Reference source not 
found.). Whilst this system is defined and accepted internationally, it is not necessarily reflective of the indications or recommended doses in any particular country. This is pertinent for opioids, where there is considerable inequality of access worldwide (Richards et al. 2020).

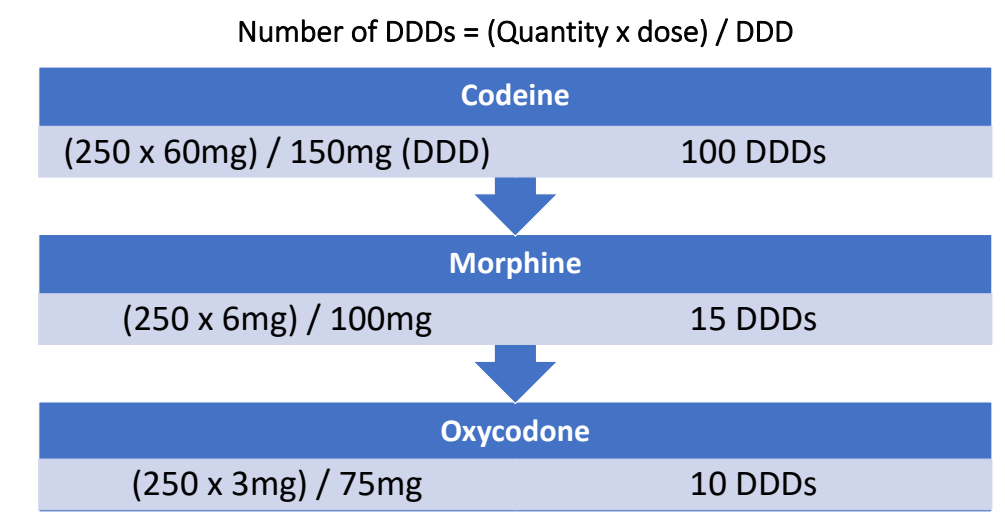

Figure 5.1: Comparing Defined Daily Dose (DDD) to Oral Morphine Equivalent dose (OMEQ). Doses of codeine, morphine and oxycodone are equianalgesic

(WHO Collaborating Centre for Drug Statistics Methodology 2021)

As DDDs are different for different drugs and for different formulations of the same drug, it does not allow easy comparison or perhaps, can foster the impression that the 'burden' of some drugs is less than it might be (Svendsen et al. 2011). Average daily quantity (ADQ) was developed in the UK by an expert group, using 'English' dosing as the basis, purportedly to reflect clinical practice more accurately (Walley and Roberts 2000). However, ADQs are not devised for all medicines, including newer opioids such as tapentadol, which does not have DDD measures listed. Consequently, neither DDDs nor ADQs can be used to consistently measure all opioid prescribing in the UK. Added to that ADQs cannot be used as a comparator to prescribing in other countries around the world.

In recent years, guidelines in countries with widespread use of opioids for chronic, non-cancer pain have included suggested maximum oral morphine equivalent doses of between $90 \mathrm{mg}$ and $120 \mathrm{mg}$ per day (Faculty of Pain Medicine 2021). To assess prescribing data compliance with guidance would necessitate the conversion of each opioid being used to an equivalent DDD or ADQ measures. In practice, DDDs and ADQs are used for reviewing prescribing data, 
rather than for guiding prescribing itself. Perhaps a question arises of how useful it is in guiding prescribers towards better practise.

Recent analyses of UK prescribing data have used OMEQ as a measure of opioid burden (Zin et al. 2014; Mordecai et al. 2018) alongside more conventional measures such as the number of prescriptions. Svendsen and colleagues (2011) have previously suggested OMEQ is a preferable measure to use as a comparator of prescribing between countries and more accurately reflects clinical practice (Svendsen et al. 2011). As with DDDs and ADQs however, OMEQ is not a faultless measure. Challenges exist insofar as gaining agreement on conversions which are unequivocal (Shaw and Fudin 2013; Schatman et al. 2016). However, the arguments around using OMEQ as a measure of prescribing burden, are perhaps conflated with disparate views on equianalgesic dosing in clinical practise (Natusch 2012; Shaw and Fudin 2013; Schatman et al. 2016).

Equianalgesic dosing is generally a clinical action, whereby a prescriber attempts to determine the dose of one opioid which provides the same level of analgesia as a specified dose of another (Natusch 2012). Disparities in how equianalgesic doses are calculated either by individuals (Rennick et al. 2016) or when using purposefully designed 'calculators' (Shaw and Fudin 2013) have been demonstrated however.

Some argue morphine equivalence is fundamentally flawed, due to pharmacogenomic differences and small pharmacokinetic differences between individual drugs. Using equianalgesic dosing to determine dose limits is suggested to be problematic as individuals handle medicines differently and dependent on multiple other factors including other illnesses, medicines, renal function and so on (Schatman et al. 2016). However, if an individual requires an opioid medicine but is unable to tolerate their current prescription, prescribers will require some idea of a roughly equivalent dose of an alternative to change them to (Natusch 2012). 
Using OMEQ as a measure of prescribing burden, potentially has another purpose. In terms of supporting prescribers to understand data and contextualise it within their practice, it might be helpful (Svendsen et al. 2011). Whilst understanding of equivalence or equianalgesic dosing is generally considered poor (Shaheen et al. 2009; Rennick et al. 2016), it is likely better than for DDDs or ADQs, which are not easily interpreted into common terms. OMEQ might enable prescribers to understand the implications of their prescribing, which would seem an important aspect of enabling change, over time.

Higher opioid burden in socioeconomically deprived areas has started to provoke great concern in health leaders and policy makers alike here in the UK (Taylor et al. 2019). Deprivation is inextricably linked with poorer health outcomes and a higher incidence of chronic pain. Further, higher levels of prescribing of dependency forming medicines, other than opioids, has been observed in areas of greater deprivation (Taylor et al. 2019; Torrance et al. 2020) and confer additional risk of harm to the user.

National and international concerns around opioids have tended to focus on strong analgesics such as morphine and oxycodone (Scherrer et al. 2016; Lin et al. 2017). This is likely due to the agenda being predominated by the USA opioid crisis, where strong opioids and oxycodone especially, are heavily implicated (McGreal 2018). There is evidence though, dose and duration are as likely indicators of harm or potential for dependence as the choice of drug (Gomes et al. 2011; Els et al. 2017). Estimates suggest as many as 78\% of people using opioids for extended periods, experience adverse effects (Els et al. 2017).

The results presented here, therefore, examine the trends in opioid prescribing between 2005 and 2015 using an estimated OMEQ (denoted as OMEQe) and compare them to prescription numbers issued over the same period. The data are stratified by individual drug as a means of assessing if one measure is a better reflection of prescribing burden than the other. As already discussed in this thesis, gender differences in pain perception and opioid prescribing have 
been observed (Section 1.8 and Section 3.6.5) and so gender differences in opioid burden were also analysed.

\subsection{Method}

\subsubsection{Data extraction analysis}

Identified subjects had their anonymised, linked data for opioid prescriptions and demographics extracted from the SAIL databank as previously described in Section 2.6Chapter 2. The number of prescriptions issued each year were totalled and stratified by drug and further adjusted to SAIL population (Section Error! Reference source not found.) for initial reporting. Estimated OMEQ was calculated for each drug product as set out in Sections 5.4.2 to 5.4.5 and then stratified into groups based on gender (Section 2.6.5.1) and also WIMD2011 quintile (Section 4.4.1 to Section 4.4.3). Descriptive statistics described the trends using the data sets created, from 2005 to 2015. Percentage change over time and Spearman's rank correlations (Section 2.7.1) analysed changes in prescribing rates and $\mathrm{OMEQ}_{\mathrm{e}}$ for each drug over the 11-year study period. Comparisons between OMEQ $_{e}$ for each drug and WIMD2011 quintiles used Kruskal-Wallis tests, with Dunn's pairwise comparison and Bonferroni corrections used to confirm statistically significant differences (Section 2.7.3). Comparisons of the prescribing rate and $\mathrm{OMEQ}_{e}$, between genders were made using MannWhitney $U$ tests (Section 2.7.2).

\subsubsection{Opioid prescriptions}

For each prescribing event listed in the main extraction data table, the prescribed opioid product was available from the Primary Care General Practice dataset. Each product was given a 'flag' for the main opioid within it e.g., codeine, morphine, oxycodone etc. Data could be sorted either by the drug group e.g., codeine or by the product prescribed e.g., co-codamol 30/500 (Table 5.1). For the purposes of annual trend analysis, the drug groupings were used. Products were used to determine estimated oral morphine equivalence (OMEQ). 
Table 5.1: Example of calculations for OMEQe (milligrams) using 2005 data for female subjects. Annualised total $=$ oral morphine equivalent of daily dose $x$ annual number of prescriptions. Process repeated for each drug product e.g., buprenorphine $10 \mathrm{mcg} /$ hour patch, BuTrans $10 \mathrm{mcg} /$ hour patch and totalled for each year

\begin{tabular}{|c|c|c|c|c|}
\hline \multirow[b]{2}{*}{ Drug product } & \multicolumn{3}{|c|}{ Units used for calculating annualised OMEQ } & \multirow[b]{2}{*}{$\begin{array}{c}\text { Annualised } \\
\text { total oral } \\
\text { morphine } \\
\text { equivalent } \\
\text { burden (mg) }\end{array}$} \\
\hline & $\begin{array}{l}\text { Recommended daily } \\
\text { dose*** }\end{array}$ & $\begin{array}{l}\text { Oral morphine } \\
\text { equivalent of } \\
\text { daily dose } \\
(\mathrm{mg})^{* * * * * * *}\end{array}$ & $\begin{array}{c}\text { Annual } \\
\text { number of } \\
\text { prescriptions }\end{array}$ & \\
\hline \multicolumn{5}{|l|}{ Buprenorphine } \\
\hline 10micrograms/hour & 1 patch per week & 24 & 28 & 672 \\
\hline 52.5 micrograms/hour & 1 patch twice a week & 126 & 354 & 44,604 \\
\hline \multicolumn{5}{|l|}{ Codeine } \\
\hline Co-codamol 8/500 & 2 tablets 4 times a day & 6.4 & 17,952 & 114,893 \\
\hline Codeine phosphate $30 \mathrm{mg}$ & 2 tablets 4 times a day & 24 & 16,293 & 391,032 \\
\hline Zapain capsules $(30 / 500)$ & 2 tablets 4 times a day & 24 & 112 & 2688 \\
\hline \multicolumn{5}{|l|}{ Dihydrocodeine } \\
\hline Co-dydramol 10/500 & 2 tablets 4 times a day & 8 & 153,047 & $1,224,376$ \\
\hline DHC Continus $90 \mathrm{mg}$ MR tablet & 1 tablet twice a day & 18 & 1,009 & 18,612 \\
\hline Remedeine tablet & 2 tablets 4 times a day & 16 & 1,295 & 20,720 \\
\hline \multicolumn{5}{|l|}{ Fentanyl } \\
\hline Durogesic $100 \mathrm{mcg} /$ hour patch & 1 patch every 3 days & 360 & 131 & 47,160 \\
\hline $\begin{array}{r}\text { Fentanyl 200micrograms SL } \\
\text { lozenge }\end{array}$ & 1 lozenge 4 times a day & 120 & 40 & 4,800 \\
\hline Fentanyl $25 \mathrm{mcg} /$ hour patch & 1 patch every 3 days & 90 & 3,429 & 308,610 \\
\hline \multicolumn{5}{|l|}{ Morphine } \\
\hline Morphgesic SR $10 \mathrm{mg} \mathrm{m} / \mathrm{r}$ tablet & 1 tablet twice a day & 20 & 73 & 730 \\
\hline MXL 60mg m/r capsule & I capsule once a day & 60 & 23 & 1,380 \\
\hline Oramorph $10 \mathrm{mg} / 5 \mathrm{~mL}$ liquid $100 \mathrm{~mL}$ & $5 \mathrm{~mL}$ every 2 hours & 120 & 573 & 68,760 \\
\hline Sevredol $20 \mathrm{mg}$ tablet & 1 tablet every 6 hours & 120 & 299 & 35,880 \\
\hline \multicolumn{5}{|l|}{ Oxycodone } \\
\hline Longtec $20 \mathrm{mg} \mathrm{m} / \mathrm{r}$ tablets & 1 tablet twice a day & 80 & 1 & 80 \\
\hline Oxycodone $\mathrm{HCl} 20 \mathrm{mg}$ capsule & 1 capsule every 4 hours & 240 & 250 & 60,000 \\
\hline OxyContin $80 \mathrm{mg} \mathrm{m} / \mathrm{r}$ tablet & I capsule twice a day & 320 & 262 & 83,840 \\
\hline \multicolumn{5}{|l|}{ Tramadol } \\
\hline Dromadol XL 200mg m/r tablet & 1 tablet once daily & 20 & 11 & 220 \\
\hline Tramadol 50mg capsule & 2 capsules 4 times a day & 40 & 93,918 & $3,756,720$ \\
\hline Tramacet $325 \mathrm{mg} / 37.5 \mathrm{mg}$ & 2 tablets 4 times a day & 30 & 4,450 & 133,500 \\
\hline \multicolumn{5}{|l|}{ Other } \\
\hline Co-proxamol $32.5 \mathrm{mg} / 325 \mathrm{mg}$ tablet & 2 tablets 4 times a day & 26 & 82,015 & $2,132,390$ \\
\hline Hydromorphone $\mathrm{HCl} 1.3 \mathrm{mg}$ capsule & 1 capsule every 4 hours & 58.5 & 6 & 351 \\
\hline Pethidine $\mathrm{HCl} 50 \mathrm{mg}$ tablet & 1 tablet every 4 hours & 30 & 2,381 & 71,430 \\
\hline
\end{tabular}

*(BNF: British National Formulary - NICE. 2021) **(Datapharm Ltd 2021) ***(Faculty of Pain Medicine 2021.) $* * * *$ (Pain Management Centre and Oxford University Hospitals NHS Foundation Trust 2020)

\subsubsection{Choice of opioid}

Opioid products commonly associated with the treatment of substance misuse e.g., buprenorphine $2 \mathrm{mg}$ and $8 \mathrm{mg}$, Subutex ${ }^{\circledR}$ and Suboxone ${ }^{\circledR}$ branded products and injectable opioids were removed from the main analysis to provide a picture of prescribing of opioids more likely to be for non-cancer related pain.

\subsubsection{Estimated oral morphine equivalent dose $\left(O M E Q_{e}\right)$}

At the time of this study, the SAIL datasets were not accessing prescription dispensing data. Whilst data for prescriptions filled in community pharmacies is 
available from Primary Care Services in Wales (Shared Services Partnership 2021) it was not available for the anonymisation process required by the data linkage used by SAIL (Ford et al. 2009). Without the anonymous linkage process (Section 2.4.2), individuals cannot be followed and consequently, the relationship between a prescription and an event in Primary Care cannot be determined.

SAIL have access to information about the product and strength prescribed e.g., 'Morphine sulphate MR tablets $10 \mathrm{mg}$ ' which is automatically coded when inputted into General Practice Primary Care (GPPC) notes systems when the prescription is generated during the appointment. However, the dosing instructions. e.g., 'Two tablets to be taken twice daily' and the quantity provided e.g., '56 (fifty-six) tablets' are classed as 'free text entry' and whilst coded within the prescription, are not coded within the GPPC system and consequently, not available to SAIL. Lack of dosing information meant it was not possible to calculate an accurate 'oral morphine equivalent' (OMEQ) dose. Using OMEQ allows improved comparison of opioid burden between different drugs and different products, by putting everything into the same form (Svendsen et al. 2011). Concerns around opioid use are linked to the doses being taken and the duration of use, more so than the individual drug itself.

Oral morphine equivalent dose (OMEQ) is normally calculated by multiplying the dose for each prescription (this can be a combination of doses e.g. $10 \mathrm{mg}+30 \mathrm{mg}$ tablets to give a total of $40 \mathrm{mg}$ per dose), by the equi-analgesic ratio of the opioid in question (Zin et al. 2014). The number of days' supply provided by each prescription is then divided by the numerical daily dose (NDD) which is a figure normally taken from the free text on the prescription (e.g., 'One tablet to be taken twice daily'). This method allows an OMEQ dose to be calculated per prescription or can be calculated per individual over longer periods to, for example, calculate annual OMEQ doses (Zin et al. 2014; Mordecai et al. 2018). 


\subsubsection{Development of an estimated OMEQ}

An estimated OMEQ (OMEQ $)$ was devised. Injections and buprenorphine products specified for the management of opioid misuse were cleansed from the data-tables. Oral and transdermal opioids in common use were retained for examination. Each product was allocated its OMEQ value based on conversion tables available for clinical practice (Appendix D) (Faculty of Pain Medicine 2021). The $\mathrm{OMEQ}_{\mathrm{e}}$ value was based on the opioid dose within the product and then multiplied by the recommended dose per day, as available from the British National Formulary (BNF: British National Formulary - NICE. 2021) or the summary of product characteristics (Datapharm Ltd 2021) (Appendix DError! Reference source not found.). The OMEQ for each product was multiplied by the number of prescriptions issued each year to determine annual totals.

Table 5.2: Examples of oral morphine equivalent (OME) dosing tables, used to develop OME-proxy measures for comparing opioid burden for the TOPAS study. The tables below are reproduced from Opioids Aware (April 2020) (Faculty of Pain Medicine 2021)

Oral opioids

\begin{tabular}{rcc}
\hline Codeine phosphate & $\begin{array}{c}\text { Potency ratio with oral } \\
\text { morphine }\end{array}$ & $\begin{array}{c}\text { Equivalent dose to 10mg oral } \\
\text { morphine }\end{array}$ \\
\hline Dihydrocodeine & 0.1 & $100 \mathrm{mg}$ \\
\hline Morphine & 0.1 & $100 \mathrm{mg}$ \\
\hline Oxycodone & 1 & $10 \mathrm{mg}$ \\
\hline Tapentadol & 2 & $5 \mathrm{mg}$ \\
\hline Tramadol & 0.4 & $25 \mathrm{mg}$ \\
\hline
\end{tabular}

Transdermal opioids

\begin{tabular}{rcccccc}
\hline $\begin{array}{r}\text { Buprenorphine } \\
\text { patch strength }\end{array}$ & $\begin{array}{c}5 \\
\text { microgram } / \mathrm{hr}\end{array}$ & $\begin{array}{c}10 \\
\text { microgram/hr }\end{array}$ & $\begin{array}{c}20 \\
\text { microgram } / \mathrm{hr}\end{array}$ & $\begin{array}{c}35 \\
\text { microgram } / \mathrm{hr}\end{array}$ & $\begin{array}{c}52 \\
\text { microgram } / \mathrm{hr}\end{array}$ & $\begin{array}{c}70 \\
\text { microgram } / \mathrm{hr}\end{array}$ \\
\hline $\begin{array}{r}\text { Oral } \\
\text { morphine }\end{array}$ & $12 \mathrm{mg}$ & $24 \mathrm{mg}$ & $48 \mathrm{mg}$ & $84 \mathrm{mg}$ & $126 \mathrm{mg}$ & $168 \mathrm{mg}$ \\
\hline $\begin{array}{r}\text { Fentanyl patch } \\
\text { strength }\end{array}$ & $\begin{array}{c}12 \\
\text { microgram } / \mathrm{hr}\end{array}$ & $\begin{array}{c}25 \\
\text { microgram } / \mathrm{hr}\end{array}$ & $\begin{array}{c}50 \\
\text { microgram } / \mathrm{hr}\end{array}$ & $\begin{array}{c}75 \\
\text { microgram } / \mathrm{hr}\end{array}$ & $\begin{array}{c}100 \\
\text { microgram } / \mathrm{hr}\end{array}$ \\
\hline Oral morphine & $45 \mathrm{mg}$ & $90 \mathrm{mg}$ & $180 \mathrm{mg}$ & $270 \mathrm{mg}$ & $360 \mathrm{mg}$ \\
\hline
\end{tabular}

Annual total $\mathrm{OMEQ}_{\mathrm{e}}$ (milligrams) for each drug grouping were calculated by adding together all product totals (Appendix DError! Reference source not found.). 


\subsection{Results}

\subsubsection{Number of prescriptions}

More than half of all opioid prescriptions (53.9\% mean average) issued in Wales between 2005 and 2015 were for codeine. In 2015, codeine prescriptions accounted for $54.3 \%$ of the total of all opioid prescriptions examined.

Prescriptions for codeine increased $54.8 \%$ over the study period (Table 5.3). 
Table 5.3: Trends in the number of opioid prescriptions per 1,000 population 2005 - 2015, stratified by drug

\begin{tabular}{|c|c|c|c|c|c|c|c|c|}
\hline & \multicolumn{8}{|c|}{ Prescriptions per 1,000 population } \\
\hline & Buprenorphine & Codeine & Dihydrocodeine & Fentanyl & Morphine & Oxycodone & Tramadol & Other \\
\hline 2005 & 8.2 & 350.9 & 157.4 & 6.2 & 16.9 & 5.5 & 95.1 & 56.6 \\
\hline 2015 & 34.9 & 543.3 & 111.1 & 18.3 & 104.2 & 25.7 & 156.3 & 6.9 \\
\hline $\begin{array}{l}\text { Rate change } \\
\text { (\%) 2005-2015 }\end{array}$ & 323.8 & 54.8 & -29.4 & 193.3 & 515.8 & 371.5 & 64.4 & -87.8 \\
\hline $\begin{array}{r}\text { Spearman's } r, \\
{ }^{*} p \text {-value }\end{array}$ & $\begin{array}{c}0.991 \\
p<.001^{*}\end{array}$ & $\begin{array}{c}>.999 \\
p<.001^{*}\end{array}$ & $\begin{array}{c}<-.999 \\
p<.001^{*}\end{array}$ & $\begin{array}{c}0.882 \\
p<.001^{*}\end{array}$ & $\begin{array}{c}>.999 \\
p<.001^{*}\end{array}$ & $\begin{array}{c}0.964 \\
p<.001^{*}\end{array}$ & $\begin{array}{c}0.882 \\
p<.001^{*}\end{array}$ & $\begin{array}{l}-0.724 \\
p<0.05^{*}\end{array}$ \\
\hline
\end{tabular}

$p$-value $<0.05=$ statistically significant. Annual data in Appendix $D$

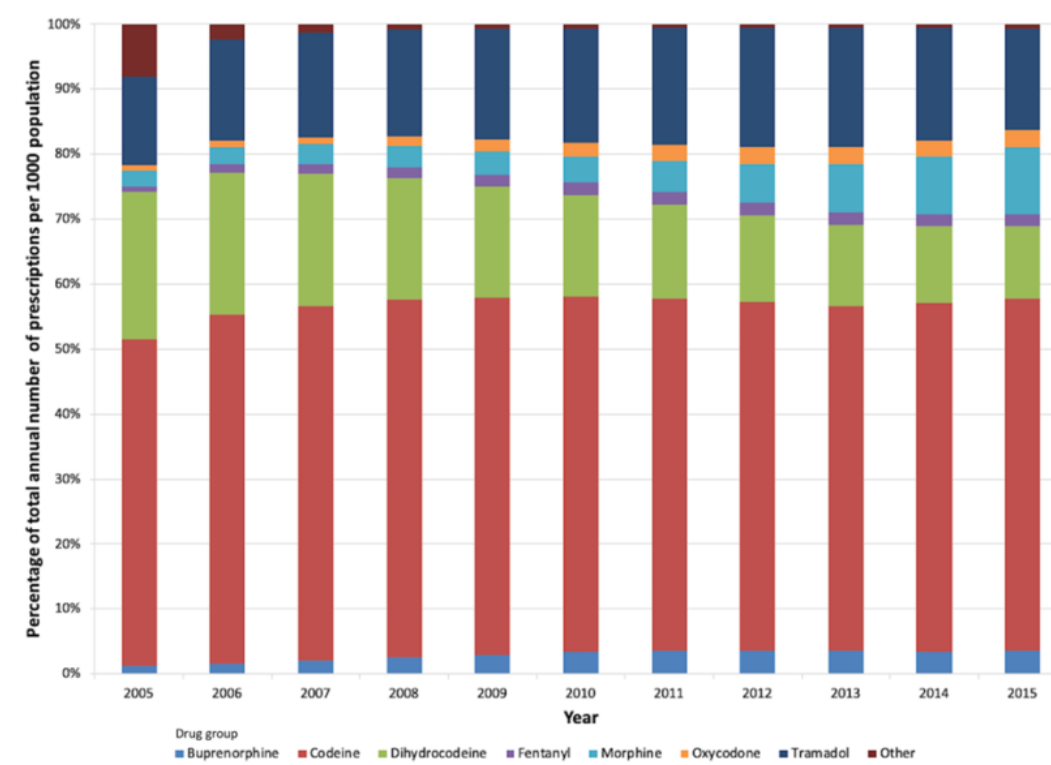

Figure 5.2: Trends in the number of prescriptions of named opioids by percentage of the total number of issued opioid prescriptions 
The annual number of morphine prescriptions had the greatest increase of any named drug across the eleven years examined. Prescriptions for morphine increased $515.8 \%$ (Table 5.3). Morphine prescriptions accounted for $2.4 \%$ of the total opioid prescriptions in 2005, rising to $10.4 \%$ by 2015 (Figure 5.2Error! Reference source not found.). Spearman's $r$ correlations between prescriptions per 1,000 population and time were strong for all drugs. Dihydrocodeine and 'other' opioids had negative correlations, confirming the reduction in prescribing rate over the study period (Table 5.3). In the 'other category, the reduction was mostly a result of dextropropoxyphene being withdrawn from the UK market in mid-2005 (Appendix D). Comparison of the annual prescribing rates revealed statistically significant differences between the total number of prescriptions per 1,000 population between 2005 and 2015, when compared by drug (KruskalWallis, $\left.p<.001, \eta^{2}=0.839 . d_{c o h e n}=4.56\right)$. Pairwise comparisons by drug, confirmed there were significantly more prescriptions for codeine issued than for all but dihydrocodeine and tramadol (Appendix D).

Post-hoc Dunn's pairwise tests, and Bonferroni corrections confirmed codeine prescribing rates were significantly higher for all drugs other than dihydrocodeine, tramadol. Those three drugs did not have statistically significantly different prescribing rates between 2005 and 2015. Dihydrocodeine and tramadol were also confirmed to have statistically higher rates of prescribing than all other drugs apart from morphine (Appendix D). Dihydrocodeine prescriptions decreased by $29.4 \%$ (Table 5.3) between 2005 and 2015. Whilst this was the only commonly prescribed opioid that reduced during the study period, it was still the third most frequently prescribed opioid in 2015 (Table 5.3Error! Reference source not found.).

Annual numbers of tramadol prescriptions peaked in 2013 (180.9 prescriptions per 1,000 population) (Appendix DError! Reference source not found.) at which point there had been a 90.2\% increase from 2005 (95.1 prescriptions per 1,000 population) (Appendix D). Between 2013 and 2015, there was a 13.6\% (from 180.9 to 156.3 prescriptions per 1,000 population) reduction in the annual 
number of tramadol prescriptions. Oxycodone prescriptions increased by $371.5 \%$ over the eleven years examined (Table 5.3Error! Reference source not found.). In 2015, oxycodone was the second most frequently prescribed 'strong' opioid although four times as many prescriptions for morphine were issued in the same year (Table 5.3Error! Reference source not found.).

Analysis of the medicines included in the 'other' group are included in Appendix D.

\subsubsection{Prescribing trends by oral morphine equivalence dose (OMEQ $)$}

The total annual oral morphine equivalent dose issued in the form of prescriptions examined for this study doubled (from 37,662,651 milligrams to $76,428,768$ milligrams per day) over the 11 years observed (Appendix D). This was $94.1 \%$ when adjusted to the population (Table 5.4Error! Reference source not found.).

Table 5.4: Daily oral morphine equivalent dose (milligrams) issued on prescription, adjusted to 1000 population, stratified by drug

\begin{tabular}{rcccc}
\hline \multicolumn{5}{c}{ Estimated oral morphine equivalent dose (milligrams) per 1,000 population } \\
\hline & $\mathbf{2 0 0 5}$ & $\mathbf{2 0 1 5}$ & $\begin{array}{c}\text { Rate change (\%) } \\
\mathbf{2 0 0 5 - 2 0 1 5}\end{array}$ & $\begin{array}{c}\text { Spearman's r, } \\
\text { p-value* }\end{array}$ \\
\hline Buprenorphine & 422 & 1,107 & 162.2 & $0.936, \mathrm{p}<.001^{*}$ \\
\hline Codeine & 5,795 & 10,449 & 80.3 & $>.999, \mathrm{p}<.001^{*}$ \\
\hline Dihydrocodeine & 1,996 & 1,488 & -25.4 & $-0.973, \mathrm{p}<.001^{*}$ \\
\hline Fentanyl & 1,168 & 2,691 & 130.4 & $0.809, \mathrm{p}<0.01^{*}$ \\
\hline Morphine & 1,419 & 7,081 & 399.2 & $>.999, \mathrm{p}<.001^{*}$ \\
\hline Oxycodone & 580 & 2,554 & 340.3 & $0.927, \mathrm{p}<.001^{*}$ \\
\hline Tramadol & 3,395 & 5,905 & 73.9 & $0.882, \mathrm{p}<.001^{*}$ \\
\hline Other & 1,493 & 291 & -80.5 & $-0.291, \mathrm{p}=0.385$ \\
\hline Total & 16,268 & 31,568 & 94.1 & \\
\hline
\end{tabular}

*p-value $<0.05$ = statistically significant. Annual data Appendix D

Codeine, the most prescribed opioid in Wales, had the highest OMEQ per day

(Figure 5.3Error! Reference source not found.) and the $\mathrm{OMEQ}_{\mathrm{e}}$ per 1,000

population increased by just over $80 \%$ (Table 5.4). Over the same period, dihydrocodeine, which has equivalent potency to codeine and is often used as the alternative 'weak' opioid, reduced in OMEQ $_{\text {e }}$ per day by $25.4 \%$. Tramadol had an overall increase of $74 \%$ over the study period, although the annual total 
$\mathrm{OMEQ}_{\mathrm{e}}$ started to reduce from 2014 (Appendix DError! Reference source not found.).

The four main 'strong' opioid being prescribed in Wales, (buprenorphine, fentanyl, morphine and oxycodone) were noted to have large increases in OMEQ between 2005 and 2015 (Error! Reference source not found.Figure

5.3Error! Reference source not found.). Morphine daily dose increased by nearly five times over the eleven years examined. By 2015, morphine was being prescribed at around three times the equivalent dose of either oxycodone or fentanyl and just over six times the $\mathrm{OMEQ}_{\mathrm{e}}$ of buprenorphine (Table 5.4).

Consequently, in terms of $\mathrm{OMEQ}_{e}$, codeine accounted for $35 \%$ of the opioid burden in Wales, tramadol for $22 \%$ and morphine for $14 \%$, resulting in $71 \%$ of the opioid burden between 2005 and 2015 being due to three drugs (Figure 5.3).

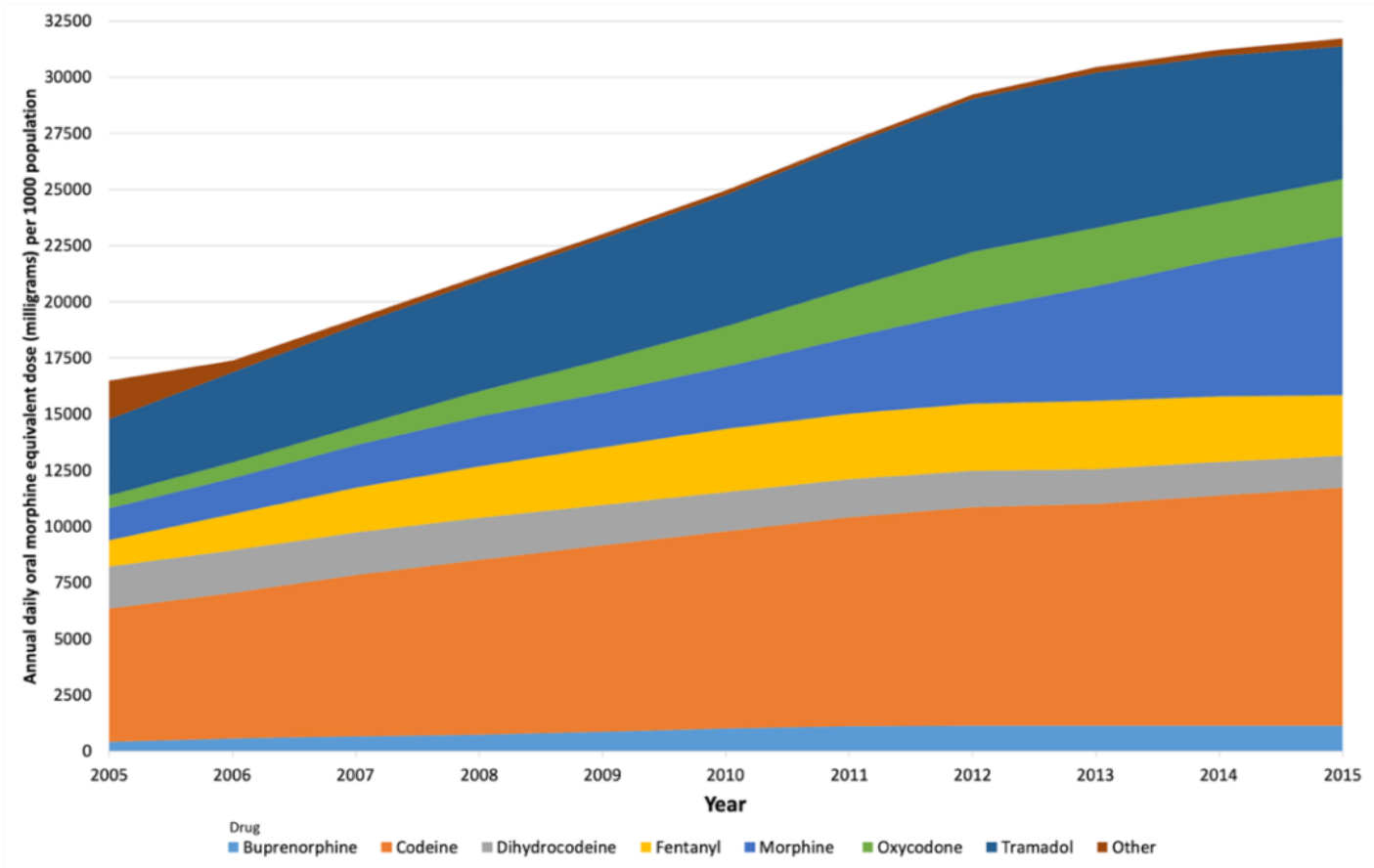

Figure 5.3: Trend in annual total daily oral morphine equivalent dose (milligrams) per 1,000 population, stratified by drug

A significant difference (Kruskal-Wallis, $p<.001, \eta^{2}=0.837$, $\mathrm{d}_{\text {cohen }}=4.54$ ) between the total $\mathrm{OMEQ}_{\mathrm{e}}$ for each named opioid group listed was found. Pairwise comparisons confirmed codeine $O M E Q_{e}$ were significantly higher than the $\mathrm{OMEQ}_{\mathrm{e}}$ for buprenorphine, dihydrocodeine, oxycodone and 'other' opioid 
analgesics but not fentanyl, morphine, or tramadol. The same comparisons were noted for 'other' opioids. Tramadol OMEQ was significantly greater than buprenorphine and oxycodone also (Appendix D).

\subsubsection{Gender differences in oral morphine equivalent dose (OMEQ $)$}

Over the study period, women received $53 \%$ more in terms of oral morphine equivalence dose than men (388,851,661 milligrams and 254,585,182 milligrams respectively) (Figure 5.4). The difference between the total $\mathrm{OMEQ}_{\mathrm{e}} \mathrm{s}$ was statistically significant $\left(p=.001, \eta^{2}=0.424, d_{\text {cohen }}=1.72\right)$ when examined using a Mann-Whitney test. Both genders demonstrated large increases in the OMEQ prescribed (Appendix D), with male $\mathrm{OMEQ}_{\mathrm{e}}$ rising by $111.5 \%$ (from $14,486,770$ to $30,635,826$ milligrams, Spearman's r=>.999, $p<.001$ ) and female OMEQ by 96.5\% (from 23,180,854 to 45,557,834 milligrams, Spearman's $r=>.999, p<.001$ ).

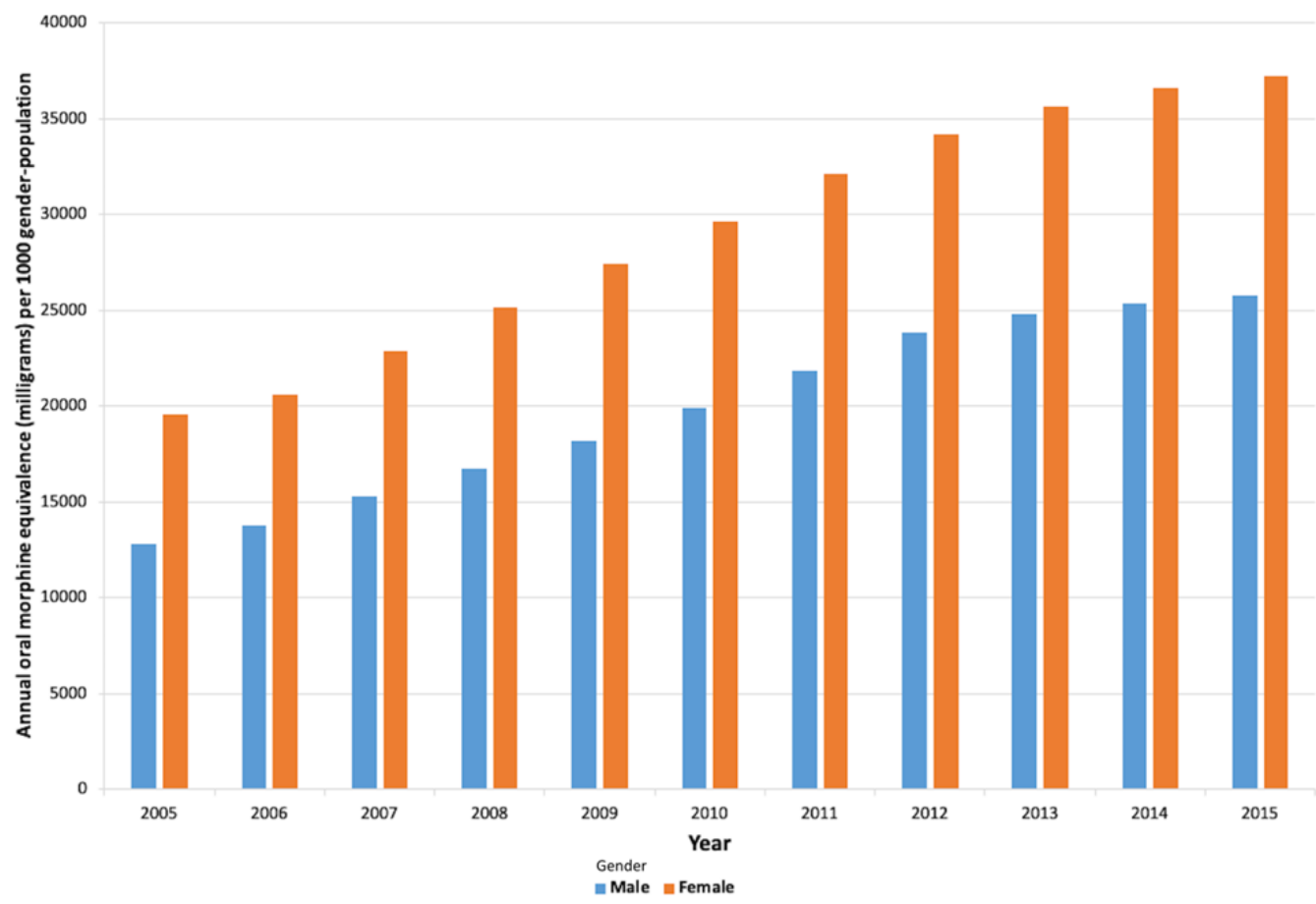

Figure 5.4: Trends in annualised $O M E Q_{e}$ (milligrams) per 1,000 population stratified by gender

\subsubsection{Gender differences in $\mathrm{OMEQ}_{\mathrm{e}}$ by drug}

When examined by drug prescribed, there were statistically significant

differences between genders in terms of total annual OMEQ prescribed (Table 5.5). There was no statistical difference between the OMEQ for morphine and 
oxycodone issued to men and women when examined by total milligrams prescribed or by milligrams per 1,000 gender-adjusted population (Table 5.5).

Women had higher percentage changes in $\mathrm{OMEQ}_{\mathrm{e}}$ than men for most opioids, except codeine and tramadol (Table 5.5). However, women received overall, total higher $\mathrm{OMEQ}_{\mathrm{e}}$ than men for every opioid prescribed, whether examined by total milligrams prescribed (Appendix D) or $\mathrm{OMEQ}_{\mathrm{e}}$ per 1,000 population (Table 5.5). 
Table 5.5: Trends in Total Annualised Oral Morphine Equivalent Doses (milligrams) per 1,000 population issued by gender. P-values calculated using Mann-Whitney tests for each drug

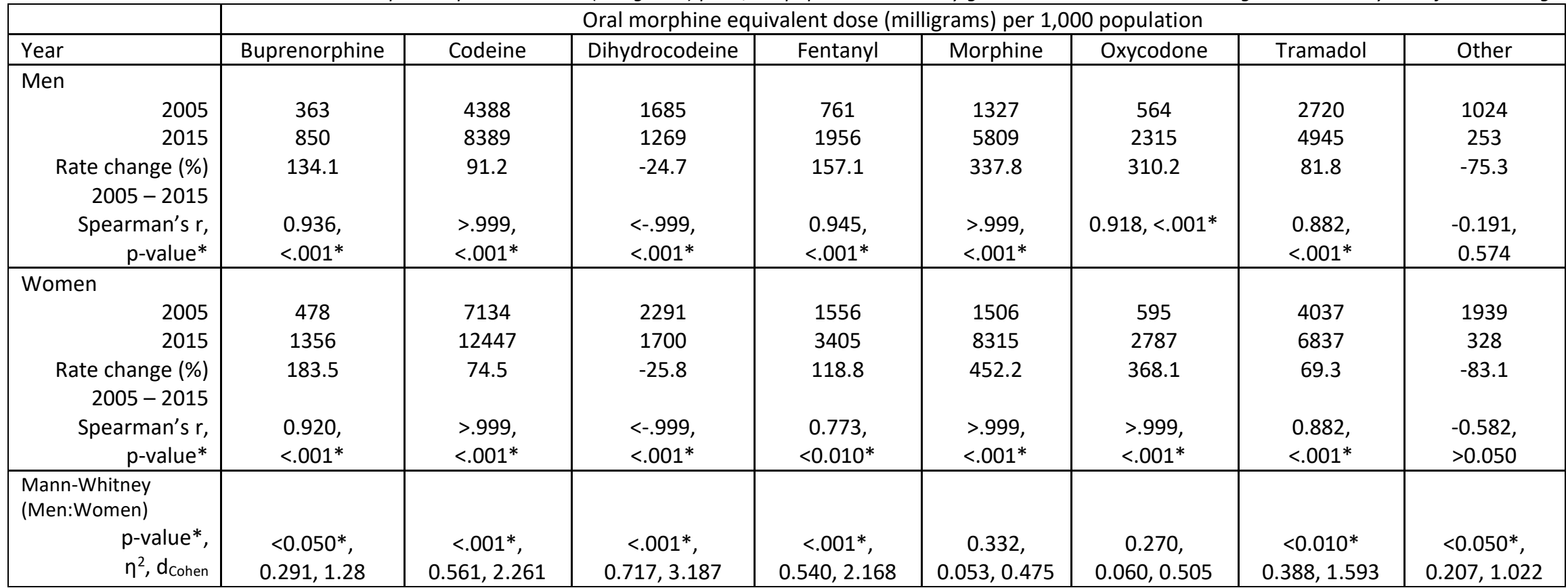

$*$ p-value $<.05=$ statistically significant. Annual data Appendix D 
Total fentanyl OMEQ e per 1,000 population (gender-adjusted) was $91 \%$ greater for women than men $(35,188$ versus 18,435 milligrams per 1,000 population respectively). The difference in the total $\mathrm{OMEQ}_{\mathrm{e}}$ of morphine prescribed was not statistically different over the 11 years examined. Although very large percentage increases were seen in both genders, women had 35\% more OMEQ prescribed than men in that time $(44,051$ versus 32,556 milligrams per 1,000 genderadjusted population respectively) (Appendix D). Codeine $\mathrm{OMEQ}_{\mathrm{e}}$ per 1,000 population was the highest of all opioids for both genders (Figure 5.5). Women received 53\% higher $\mathrm{OMEQ}_{\mathrm{e}}$ per 1,000 population than men between 2005 and 2015 (Table 5.5Error! Reference source not found.).

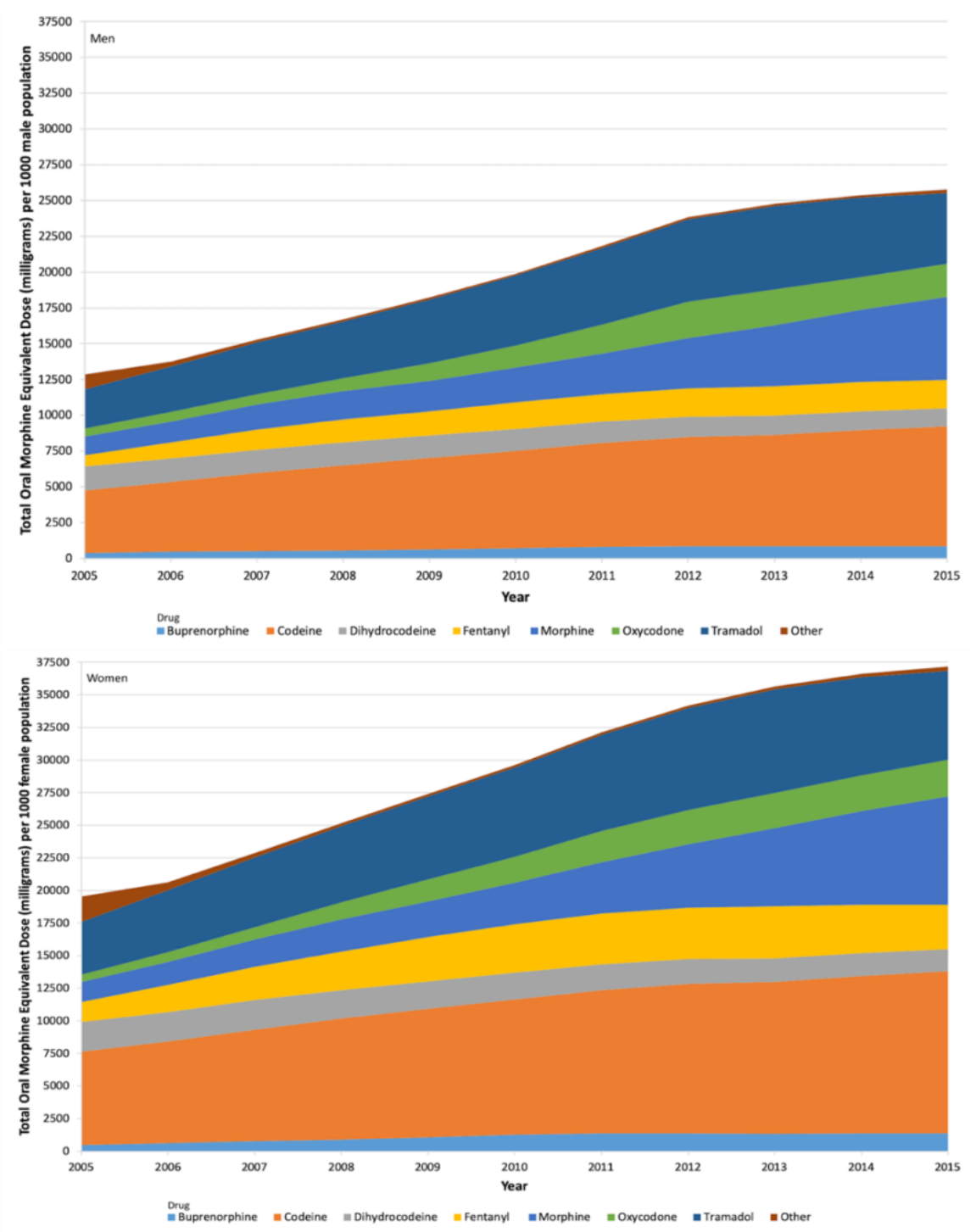

Figure 5.5: Trends in total Oral Morphine Equivalent Dose (milligrams) per 1,000 population, stratified by drug prescribed and examined by gender (Top - men, bottom - women) 
Statistically significant differences were noted for the total $\mathrm{OMEQ}_{\mathrm{e}}$ of each drug prescribed to men (Kruskal-Wallis, $p<.001, \eta^{2}=0.827, \mathrm{~d}_{\text {cohen }}=4.37$ ). Codeine was the drug for which $O M E Q_{e}$ prescribed was significantly different to all but morphine and tramadol (Appendix D).

The differences in $\mathrm{OMEQ}_{\mathrm{e}}$ per drug prescribed to women, were shown to be statistically significant (Kruskal-Wallis, $\mathrm{p}<.001, \eta^{2}=0.816$, $\mathrm{d}_{\text {cohen }}=4.211$ ). Post-hoc analysis confirmed a slightly different pattern of variance compared to men (Appendix D). For example, the $\mathrm{OMEQ}_{e}$ of codeine were not confirmed to be statistically different to those of morphine and fentanyl, two strong opioids which are much less frequently prescribed than codeine (Appendix D).

\subsubsection{Gender differences in $\mathrm{OMEQ}_{\mathrm{e}}$ per prescription}

The trends in the number of prescriptions issued by gender have been previously discussed (Section 3.5.3). Gender did not determine a statistically significant difference between the average total $\mathrm{OMEQ}_{\mathrm{e}}$ per prescription when all prescriptions were examined using a Mann-Whitney test (Appendix D). However, the $\mathrm{OMEQ}_{\mathrm{e}}$ per prescription issued was higher for men, for each of the major opioids prescribed (Error! Reference source not found.Table 5.6).

Statistically significant differences in the $\mathrm{OMEQ}_{\mathrm{e}}$ per prescription by gender were noted using Mann-Whitney tests for buprenorphine, dihydrocodeine, fentanyl, morphine and oxycodone (Table 5.6Error! Reference source not found.). Drugs generally considered 'weak' opioids (codeine, dihydrocodeine and tramadol) all demonstrated modest increases in $\mathrm{OMEQ}_{\mathrm{e}}$ per prescription for both genders (Table 5.6Error! Reference source not found.). 
Table 5.6: Trends in oral morphine equivalent dose (milligrams) per prescription issued and by drug, stratified by gender. P-values calculated using Mann-Whitney tests

\begin{tabular}{|c|c|c|c|c|c|c|c|c|c|}
\hline & \multicolumn{9}{|c|}{ Oral morphine equivalent dose (milligrams) per prescription issued } \\
\hline & Buprenorphine & Codeine & Dihydrocodeine & Fentanyl & Morphine & Oxycodone & Tramadol & Other & Total \\
\hline \multicolumn{10}{|l|}{ Men } \\
\hline 2005 & 98 & 17 & 13 & 187 & 89 & 113 & 36 & 27 & 24 \\
\hline 2015 & 46 & 20 & 14 & 169 & 71 & 110 & 38 & 60 & 33 \\
\hline $\begin{array}{r}\text { Percentage change } \\
(\%) \text { 2005-2015 }\end{array}$ & -53.0 & 14.9 & 4.7 & -9.4 & -20.0 & -2.8 & 6.1 & 124.0 & 34.3 \\
\hline Spearman's r, & -0.926 & 0.958 & 0.671 & -0.932 & -0.970 & 0.032 & 0.858 & 0.995 & 0.998 \\
\hline p-value* & $<.001 *$ & $<.001^{*}$ & $<0.050 *$ & $<.001^{*}$ & $<.001^{*}$ & 0.925 & $<0.010^{*}$ & $<.001^{*}$ & $<.001^{*}$ \\
\hline \multicolumn{10}{|l|}{ Women } \\
\hline 2005 & 97 & 16 & 12 & 187 & 83 & 102 & 36 & 26 & 23 \\
\hline 2015 & 32 & 19 & 13 & 137 & 66 & 93 & 38 & 56 & 31 \\
\hline $\begin{array}{r}\text { Percentage change } \\
(\%) \text { 2005-2015 }\end{array}$ & -67.0 & 17.2 & 6.2 & -26.8 & -19.9 & -9.1 & 5.5 & 113.2 & 35.4 \\
\hline Spearman's r, & -0.998 & 0.963 & 0.866 & $<-.999$ & -0.970 & -0.546 & 0.905 & 0.991 & 0.995 \\
\hline p-value* & $<.001^{*}$ & $<.001^{*}$ & $<0.010^{*}$ & $<.001^{*}$ & $<.001 *$ & 0.082 & $<.001^{*}$ & $<.001^{*}$ & $<.001 *$ \\
\hline \multicolumn{10}{|l|}{ Mann-Whitney } \\
\hline p-value* & $<0.050 *$ & 0.217 & $<0.050 *$ & $<0.010^{*}$ & $<0.050 *$ & $<.001^{*}$ & 0.797 & 0.652 & \\
\hline$\eta^{2}$ & 0.233 & 0.071 & 0.283 & 0.362 & 0.194 & 0.671 & 0.004 & 0.01 & \\
\hline $\mathbf{d}_{\text {Cohen }}$ & 1.103 & 0.552 & 1.257 & 1.508 & 0.983 & 2.855 & 0.126 & 0.197 & \\
\hline
\end{tabular}

*p-value $<0.05=$ statistically significant. Annual data in Appendix D 
Strong opioids all appeared to reduce in $\mathrm{OMEQ}_{\mathrm{e}}$ per prescription for both genders (Table 5.6), most notably for buprenorphine where although prescription numbers increased, they were predominantly for low $\mathrm{OMEQ}_{\mathrm{e}}$ products (Figure 5.6Error! Reference source not found. and Appendix D). The 'other' category of drugs was the drug category with the greatest OMEQ increase over the study period (Figure 5.6Error! Reference source not found. and Figure 5.7). An initial reduction in $\mathrm{OMEQ}_{\mathrm{e}}$ per prescription in this group was due to removal of low $\mathrm{OMEQ}_{\mathrm{e}}$ co-proxamol (dextropropoxyphene) from the UK market in 2005 , following safety concerns. The rise seen towards the end of the study period is attributed to the introduction of tapentadol, a drug which has a high oral morphine equivalence. It is notable that since 2011, when the drug was released in the UK, OMEQ per prescription for 'other' opioid analgesics began to increase quite rapidly for both genders (Figure 5.6 and Figure 5.7Error!

\section{Reference source not found.).}

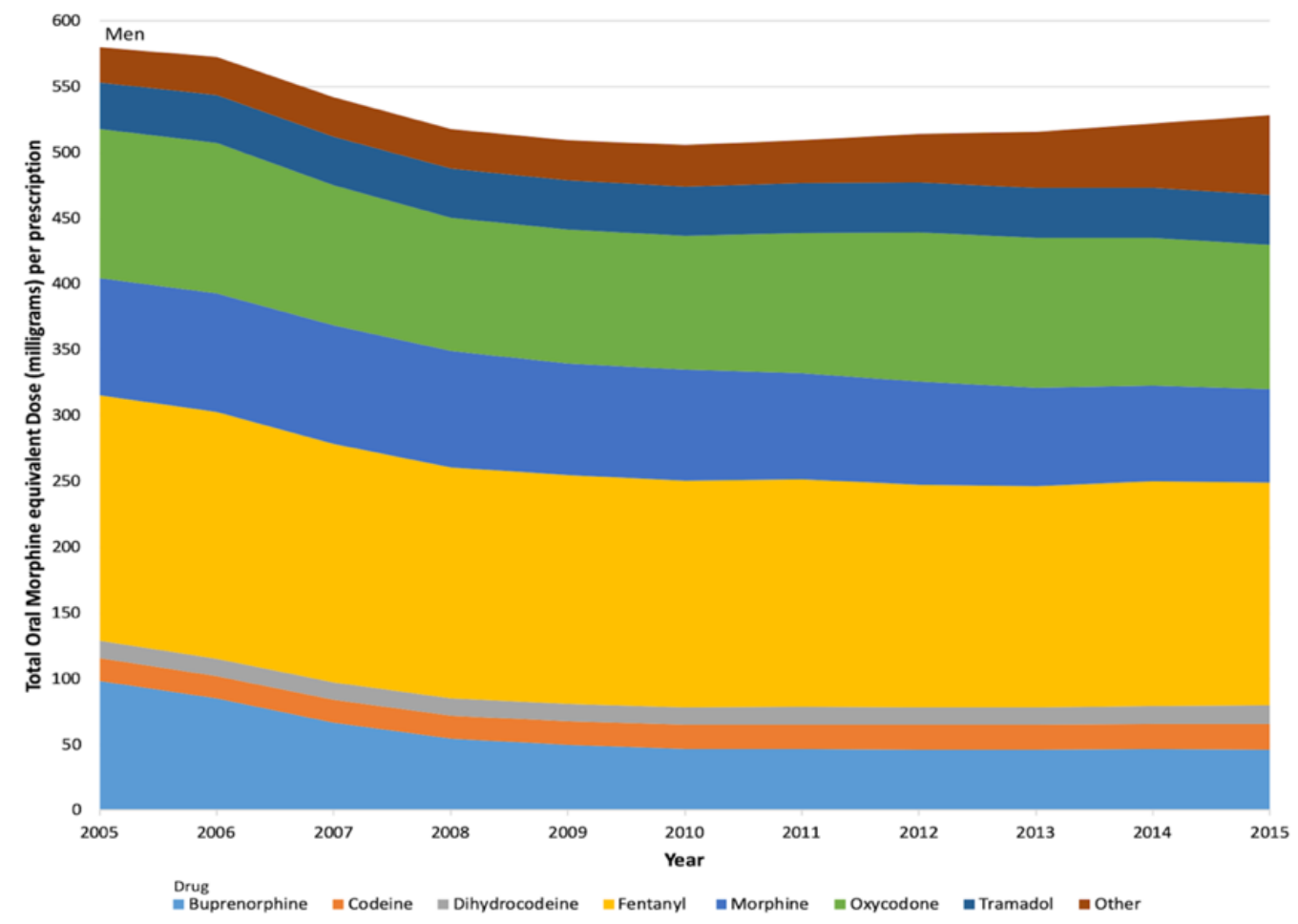

Figure 5.6: Trends in Oral Morphine Equivalent Dose (milligrams) per prescription issued to men and stratified by drug 


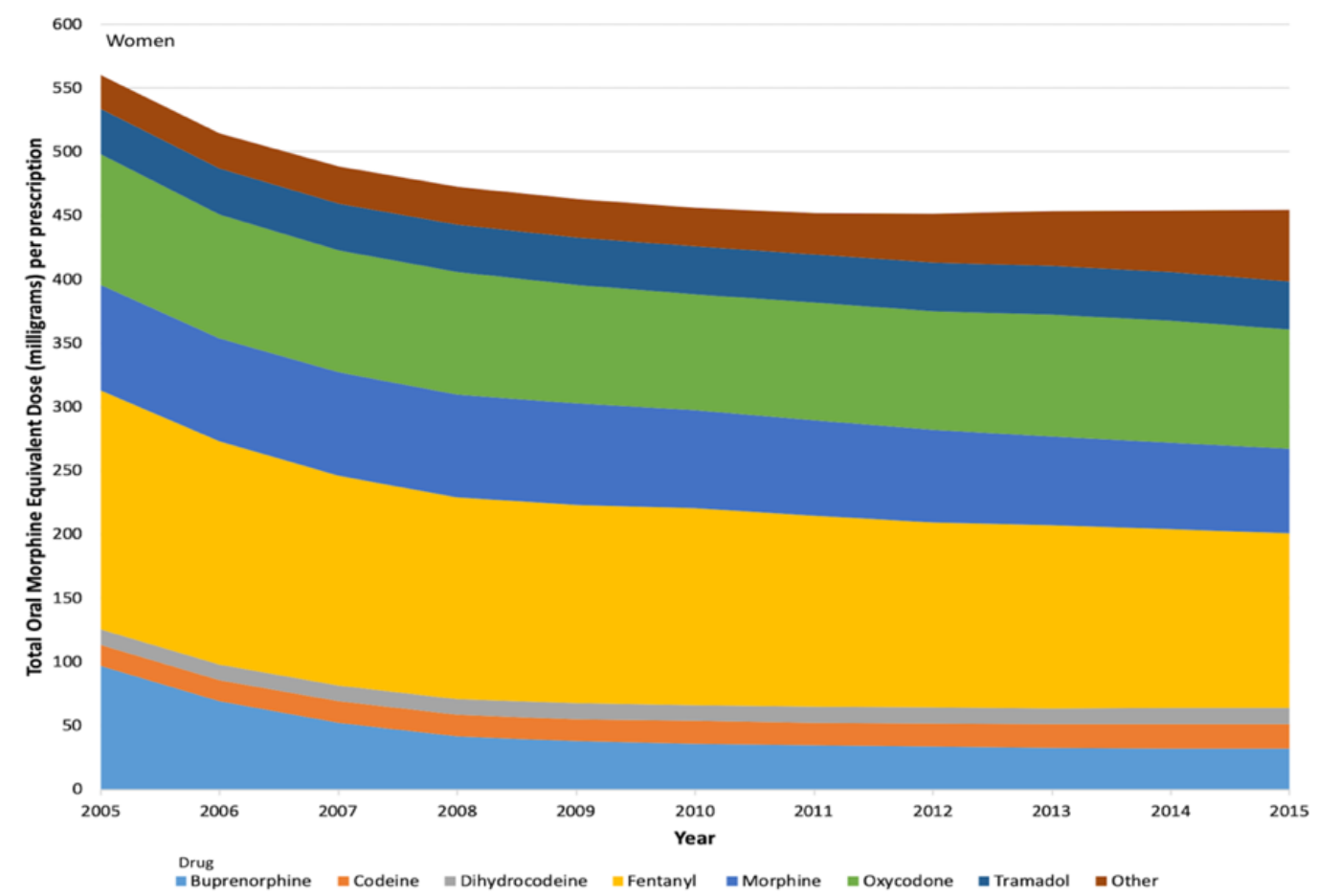

Figure 5.7: Trends in Oral Morphine Equivalent Dose (milligrams) per prescription issued to women and stratified by drug

Statistically significant difference was demonstrated in the $O M E Q_{e}$ per prescription of the different medicines prescribed to men (Kruskal-Wallis, $\left.p<.001, \eta^{2}=0.958, d_{\text {cohen }}=9.525\right)$. OMEQ per prescription for weak opioids codeine, dihydrocodeine and tramadol were significantly lower than for strong opioids morphine, oxycodone and fentanyl (Appendix D).

There were also statistically significant differences in the $\mathrm{OMEQ}_{\mathrm{e}}$ per prescription issued by drug to women (Kurskal Wallis, $p<.001, \eta^{2}=0.919$, $d_{\text {Cohen }}=6.753$ ). Codeine and dihydrocodeine had significantly lower $\mathrm{OMEQ}_{\mathrm{e}}$ per prescription compared to strong opioids, confirmed by post-hoc testing (Appendix D).

High dose opioid analgesic products (>120mg OMEQ $)$ were also analysed by gender (Appendix D).

\subsubsection{Differences in oral morphine equivalent dose by deprivation}

The most deprived areas of Wales received 100,711,696 milligrams more OMEQ compared to the least deprived between 2005 and 2015 (Table 5.7Error!

Reference source not found.Error! Reference source not found.). The most 
deprived areas in Wales had prescriptions issued totalling $28.4 \%$ of the total OMEQ issued in the country compared to $12.2 \%$ of the total $\mathrm{OMEQ}_{\mathrm{e}}$ being issued in the least deprived areas. Total $\mathrm{OMEQ}_{\mathrm{e}}$ issued in the most deprived areas remained more than twice that in the least deprived areas throughout the 11 years examined (Figure 5.8Error! Reference source not found.).

Table 5.7: Trends in total oral morphine equivalent dose prescribed stratified by deprivation (Welsh Index of Multiple Deprivation) and adjusted to population

\begin{tabular}{rccccc}
\hline Year & \multicolumn{3}{c}{ Estimated oral morphine equivalent dose (milligrams) per 1,000 population } \\
\cline { 2 - 6 } & WIMD1 & WIMD2 & WIMD3 & WIMD4 & WIMD5 \\
\hline $\begin{array}{r}\text { Total prescribed } \\
\mathbf{2 0 0 5 - 2 0 1 5}\end{array}$ & $176,824,265$ & $146,459,878$ & $129,880,669$ & $93,691,687$ & $76,112,569$ \\
\hline $\mathbf{2 0 0 5}$ & 21,757 & 18,203 & 17,108 & 12,242 & 9,381 \\
$\mathbf{2 0 1 5}$ & 43,176 & 35,475 & 32,564 & 23,534 & 17,557 \\
\hline Rate change & 98.4 & 94.9 & 90.3 & 92.2 & 87.2 \\
(\%) 2005-2015 & & & & & \\
\hline Spearman's r, & $>.999$, & $>.999$, & $>.999$, & $>.999$, & $>.999$, \\
p-value* & $<.001^{*}$ & $<.001^{*}$ & $<.001^{*}$ & $<.001^{*}$ & $<.001^{*}$ \\
\hline
\end{tabular}

WIMD1 = most deprived, WIMD5 = least deprived. ${ }^{*}$ p-value $<0.05=$ statistically significant. Annual results in Appendix D

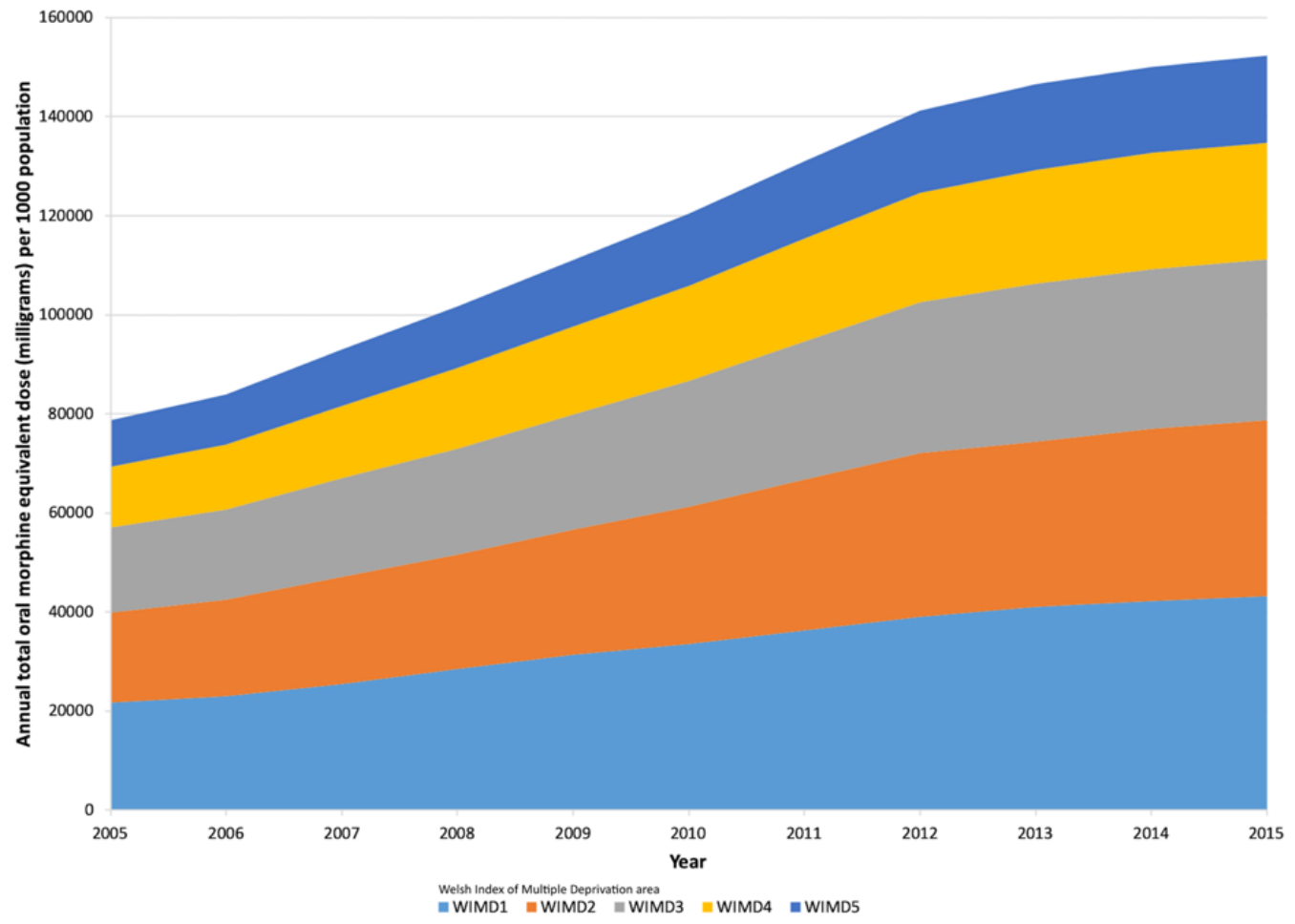

Figure 5.8: Trends in $\mathrm{OMEQ}_{e}$ (milligrams) per 1,000 population, stratified by deprivation using the Welsh Index of Multiple Deprivation 2011 (WIMD2011)

WIMD1 = most deprived, $W I M D 5=$ least deprived

All areas were noted to have had large increases in $\mathrm{OMEQ}_{\mathrm{e}}$ per 1,000 population prescribed over the 11 years examined with near doubling in annual totals by 2015 (Table 5.7Error! Reference source not found.Error! Reference source not 
found.). An inverse relationship between deprivation quintile and percentage increase in opioid burden (OMEQ e per 1,000 population) was noted (Table

\section{7)Error! Reference source not found.}

A Kruskal-Wallis analysis demonstrated a statistically significant difference $\left(p<.001, \eta^{2}=0.601 d_{\text {cohen }}=2.456\right)$ in the total OMEQ $_{e}$ (milligrams) prescribed across the five areas of deprivation in Wales. Post-hoc testing confirmed statistically significant differences between the three most deprived areas (WIMD1 to WIMD3) and the least deprived (WIMD5) and WIMD4 and the most deprived area (Appendix D). Similar differences were noted between quintiles with an overall statistically significant difference by Kruskal-Wallis analysis $\left(p<.001, \eta^{2}=0.61, d_{\text {cohen }}=2.503\right)$ between OMEQ $_{e}$ per 1,000 population (Appendix D).

$\mathrm{OMEQ}_{\mathrm{e}}$ per prescription were statistically the same across all levels of deprivation (Table 5.8) between 2005 and 2015 (Kruskal-Wallis $p=0.262$ ). Even so, the greatest percentage change in $\mathrm{OMEQ}_{\mathrm{e}}$ per prescription was seen in WIMD1 areas. As with earlier presented results, increasing deprivation was associated with greater percentage increase in $\mathrm{OMEQ}_{\mathrm{e}}$ per prescription and per person (Table 5.8).

Table 5.8: Trends in oral morphine equivalence stratified by deprivation (Welsh Index of Multiple Deprivation WIMD2011) and examined by dose per prescription and dose per person

\begin{tabular}{|c|c|c|c|c|c|}
\hline \multicolumn{6}{|c|}{ Oral morphine equivalent dose (milligrams) per prescription } \\
\hline Year & WIMD1 & WIMD2 & WIMD3 & WIMD4 & WIMD5 \\
\hline 2005 & 22 & 24 & 24 & 25 & 24 \\
\hline 2015 & 31 & 32 & 33 & 33 & 30 \\
\hline $\begin{array}{r}\text { Rate change (\%) } \\
2005-2015\end{array}$ & 41.0 & 36.3 & 33.1 & 28.9 & 27.9 \\
\hline $\begin{array}{r}\text { Spearman's r, p- } \\
\text { value* }\end{array}$ & $0.998,<.001^{*}$ & $0.993,<.001^{*}$ & $0.977,<.001^{*}$ & $0.995,<.001^{*}$ & $0.986,<.001^{*}$ \\
\hline \multicolumn{6}{|c|}{ Oral morphine equivalent dose (milligrams) per person receiving prescriptions } \\
\hline 2005 & 96 & 99 & 98 & 93 & 80 \\
\hline 2015 & 173 & 169 & 161 & 151 & 128 \\
\hline $\begin{array}{r}\text { Rate change (\%) } \\
2005-2015\end{array}$ & 79.2 & 70.2 & 64.7 & 61.3 & 60.2 \\
\hline $\begin{array}{r}\text { Spearman's r, } p- \\
\text { value }\end{array}$ & $>.999,<.001^{*}$ & $>.999,<.001 *$ & $>.999,<.001 *$ & $>.999,<.001^{*}$ & $>.999,<.001^{*}$ \\
\hline
\end{tabular}

WIMD1 = most deprived, WIMD5 = least deprived. ${ }^{*}$-value $<0.05=$ statistically significant. Annual data in Appendix D.

Differences between quintiles were statistically significant (Kruskal-Wallis $p<0.05, \eta^{2}=0.141 d_{\text {cohen }}=0.811$ ) for OMEQ $_{e}$ per person (Table 5.8). Post-hoc 
analysis only confirmed statistically greater $\mathrm{OMEQ}_{\mathrm{e}}$ per person in the two most deprived quintiles, compared to the least deprived (Appendix D).

Oral morphine equivalent dose per person appeared similar in 2005 , with the highest figure seen in WIMD2 areas and the lowest in the least deprived, at that time (Table 5.8). By the end of the study, the highest $O M E Q_{e}$ per person was noted in the most deprived areas due to a $79.2 \%$ increase over the 11 years. In 2015, there was $35 \%$ less $\mathrm{OMEQ}_{\mathrm{e}}$ prescribed per person in WIMD5 areas, compared to WIMD1 areas of Wales (Table 5.8Error! Reference source not found.).

\subsubsection{Differences in high dose oral morphine equivalent dose by deprivation}

Prescription opioid products which, based on the recommended dose, provide an $\mathrm{OMEQ}_{\mathrm{e}}$ of 120mg or higher totaled 146,289,419 milligrams between 2005 and 2015. This equates to $23.5 \%$ of the total $O M E Q_{e}$ prescribed ( total $=622,969,068$ milligrams) in that time. Spearman's $r$ correlations were very strong for all quintiles, confirming the increases in $\mathrm{OMEQ}_{e}$ over the study period (Appendix D).

Table 5.9: Trends in total oral morphine equivalent dose of 120 milligrams or higher prescribed stratified by deprivation (Welsh Index of Multiple Deprivation) and adjusted to deprivation quintile population.

\begin{tabular}{|c|c|c|c|c|c|}
\hline \multirow[t]{2}{*}{ Year } & \multicolumn{5}{|c|}{$\begin{array}{l}\text { Total oral morphine equivalent dose per } 1,000 \text { population for } \\
\text { products of over } 120 \mathrm{mg} \mathrm{OMEQ}\end{array}$} \\
\hline & WIMD1 & WIMD2 & WIMD3 & WIMD4 & WIMD5 \\
\hline $\begin{array}{r}\begin{array}{r}\text { Total } \\
\text { prescribed }\end{array} \\
(\mathrm{mg}) 2005- \\
2015 \\
\end{array}$ & $37,943,319$ & $32,622,635$ & $33,344,376$ & $25,420,454$ & $16,958,635$ \\
\hline 2005 & 3092 & 2561 & 3058 & 2515 & 1459 \\
\hline 2015 & 11574 & 9863 & 9853 & 7171 & 4487 \\
\hline $\begin{array}{r}\text { Rate change } \\
\text { (\%) } 2005- \\
2015\end{array}$ & 274.3 & 285.0 & 222.2 & 185.1 & 207.6 \\
\hline $\begin{array}{r}\text { Spearman's r, } \\
\text { p-value* }\end{array}$ & $\begin{array}{l}>.999 \\
<.001^{*}\end{array}$ & $\begin{array}{c}0.973 \\
<.001^{*}\end{array}$ & $\begin{array}{l}>.999 \\
<.001 *\end{array}$ & $\begin{array}{c}0.864 \\
<0.010^{*}\end{array}$ & $\begin{array}{l}>.999 \\
<.001^{*}\end{array}$ \\
\hline
\end{tabular}

WIMD1 = most deprived, WIMD5 = least deprived. ${ }^{*} p$-value $<0.05=$ statistically significant. Annual data in Appendix D

The total $\mathrm{OMEQ}_{\mathrm{e}}$ prescribed from high dose products were markedly different in the different areas of deprivation (Figure 5.9). The most deprived (WIMD1) areas of Wales had $27.4 \%$ of the total $\mathrm{OMEQ}_{\mathrm{e}}$ from $\geq 120 \mathrm{mg}$ products over the study period, similar to the distributions seen for all opioid prescribing (Chapter 4). 
There was a significant difference between $\mathrm{OMEQ}_{\mathrm{e}}$ from products with $\geq 120 \mathrm{mg}$ $\mathrm{OMEQ}_{\mathrm{e}}$ noted between quintiles (Kruskal-Wallis, $\mathrm{p}<0.01, \eta^{2}=0.262$, $\mathrm{d}_{\text {cohen }}=$ 1.191), although post-hoc analysis confirmed only statistically lower $O_{M E Q_{e}}$ in the least deprived quintile (WIMD5) compared to the three most deprived (WIMD1 to WIMD3) (Appendix D). Large percentage increases in prescribing rates of high dose products were confirmed by very strong Spearman's correlations between $\mathrm{OMEQ}_{\mathrm{e}}$ and time (Table 5.9Error! Reference source not found.).

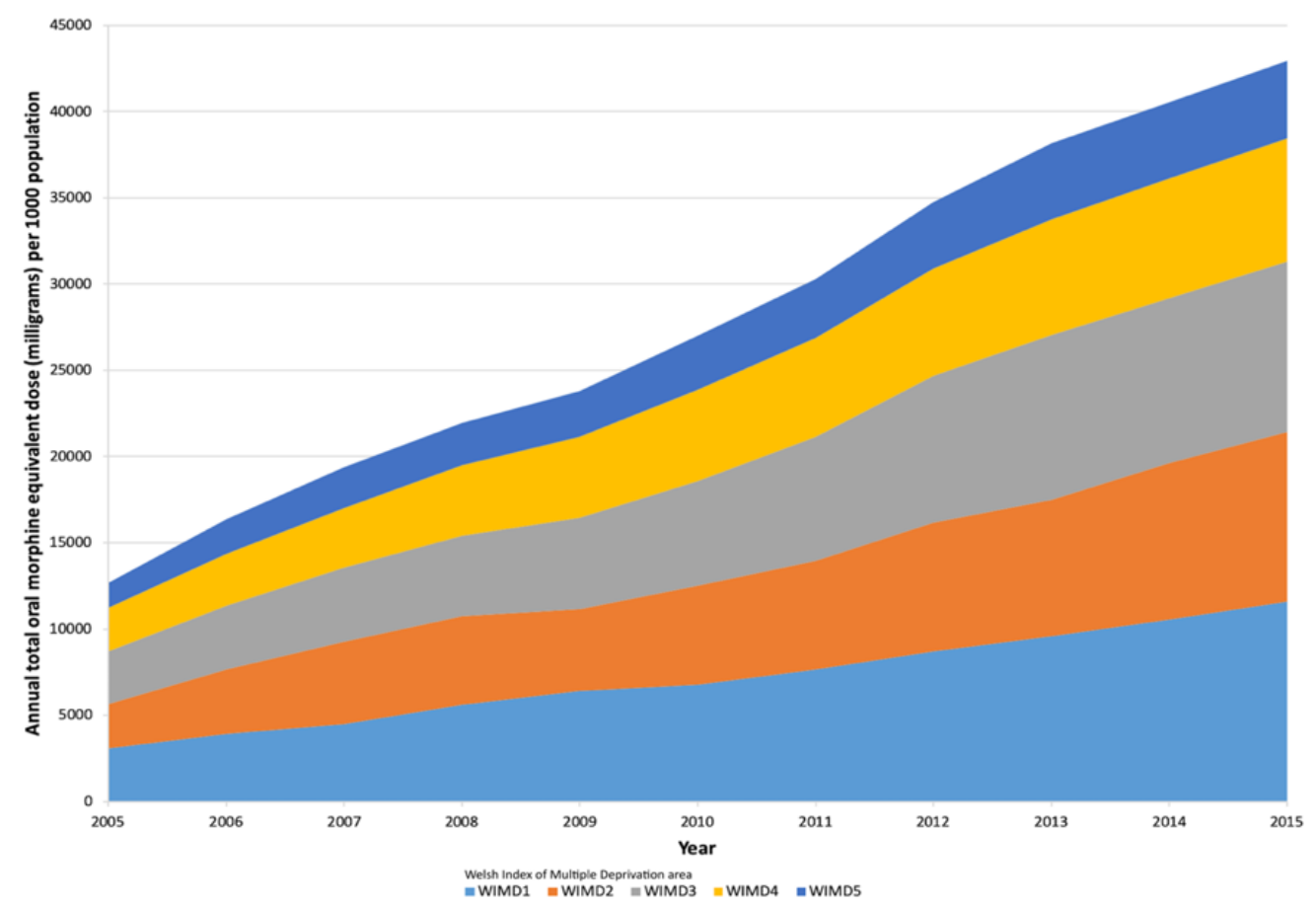

Figure 5.9: Trends in $\mathrm{OMEQ}_{e}$ of 120 milligrams or higher per 1,000 population, stratified by deprivation using the Welsh Index of Multiple Deprivation 2011 (WIMD2011)

WIMD1 = most deprived, $W I M D 5=$ least deprived

As with the totals of $\mathrm{OMEQ}_{\mathrm{e}}$ for any opioids, the highest rates of prescribing per 1,000 population were noted in the most deprived areas of Wales (Table 5.9). Oral morphine equivalent doses from products with a daily dose of $\geq 120 \mathrm{mg}$, in WIMD1 areas were more than double those seen in WIMD5 areas, throughout the 11 years examined with the difference between those two groups increasing slightly over that time (Figure 5.9Error! Reference source not found.). As for total $O M E Q_{e}$ from high dose products, there were significant differences between totals in each quintile (Kruskal-Wallis $p<0.01, \eta^{2}=0.29$, $d_{\text {cohen }}=1.279$ ), 
although statistically, OMEQ in WIMD1 to WIMD3 were significantly higher than in WIMD5 quintile areas, but no other differences were detected (Appendix D).

\subsubsection{Comparison of prescription numbers and $\mathrm{OMEQ}_{\mathrm{e}}$}

When percentage contributions of prescriptions and OMEQ $Q_{e}$ were compared, discrepancies were revealed. Codeine prescriptions accounted for over half of all prescriptions per 1,000 population over the study period. This translated however, to around $35 \%$ of opioid burden measured by $\mathrm{OMEQ}_{\mathrm{e}}$ (Figure 5.10Error! Reference source not found.). Similarly, dihydrocodeine took a greater percentage of prescription numbers than opioid burden. Tramadol, which in this study was classified as a weak opioid, (Section 2.6.3) had a higher opioid burden by $\mathrm{OMEQ}_{\mathrm{e}}$ than implied by the number of prescriptions per 1,000 population. All strong opioids and 'other' opioids were disproportionately represented in terms of prescription numbers compared to opioid burden (Figure 5.10Error! Reference source not found.).

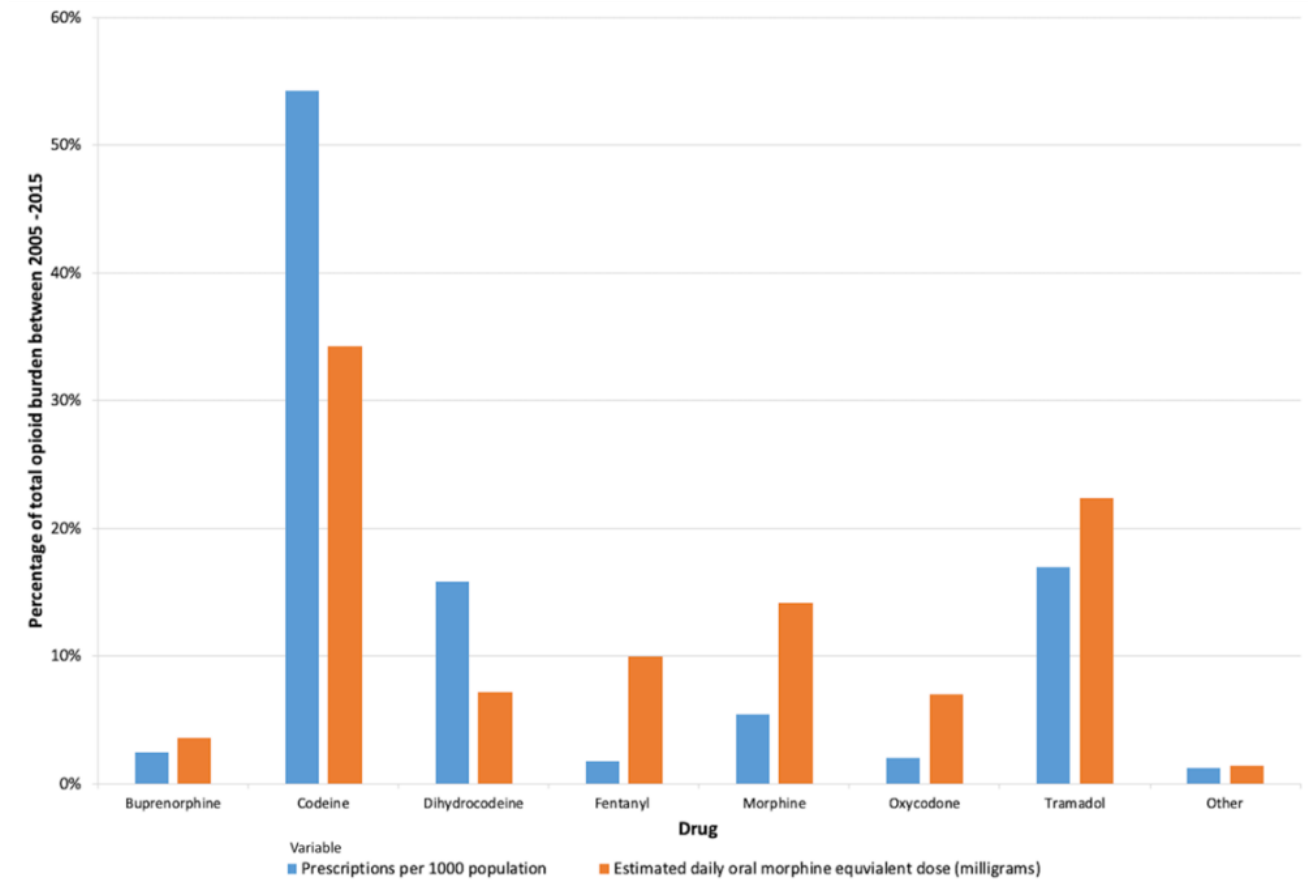

Figure 5.10: Comparison of the percentage contribution of each opioid prescribed by total prescriptions issued and total daily OMEQ dose (mg) in Wales between 2005 and 2015 
Examining the shape of trend graphs for the 11 years of the study, demonstrated for all opioids, a continuing but slowing increase in $\mathrm{OMEQ}_{e}$ despite a plateauing of prescriptions being issued (Figure 5.11Error! Reference source not found.).
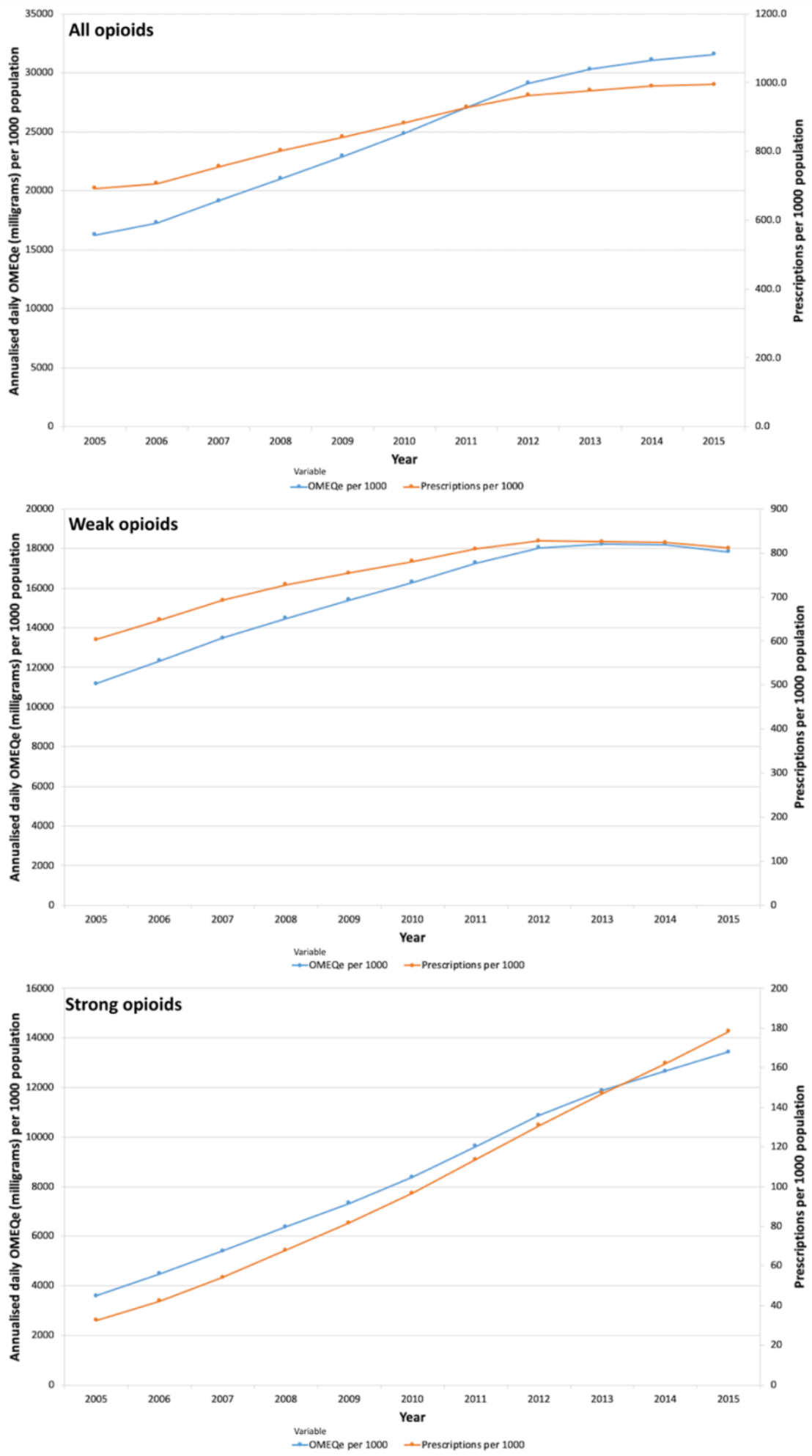

Figure 5.11: Trends in oral morphine equivalence compared to number of prescriptions issued per 1,000 population between $2005-2015$ 
Strong opioid $\mathrm{OMEQ}_{\mathrm{e}}$ increases were starting to decelerate toward the end of the study compared to a continued increasing trend in prescription numbers (Figure 5.11Error! Reference source not found.). In contrast, weak opioids trends looked the same for prescriptions and $\mathrm{OMEQ}_{\mathrm{e}}$, both seemingly peaking between 2012 and 2013 and starting to reduce at the end of the study period.

\subsection{Discussion}

\subsubsection{Summary and reflection on findings}

The results presented in this chapter reinforced the trends in prescribing already discussed (Section 3.6.1 and Section 4.6.1). Namely, a large increase in prescriptions issued between 2005 and 2015. What this part of the study added was the detail that more than half of those prescriptions were for a single drug, codeine. This finding reveals prescribing is dominated by weak opioid prescriptions. Whilst codeine increased both in the number of prescriptions and $\mathrm{OMEQ}_{\mathrm{e}}$ over the study period, there was little difference in the dose per prescription. This was perhaps expected, given codeine is so widely prescribed, prescribers are likely to be confident about 'normal' dosing ranges. A similar pattern was seen with tramadol, where a small reduction in prescriptions issued was noted, followed by a reduction in $\mathrm{OMEQ}_{\mathrm{e}}$ towards the end of the study period. Unlike many other opioid analgesics, both drugs have recommended maximum daily doses stated in reference sources such as the BNF (BNF: British National Formulary - NICE. 2021). Strong opioids all showed large percentage increases in prescriptions and $\mathrm{OMEQ}_{\mathrm{e}}$ doses. Morphine was by far the most burdensome strong opioid although it had lower average doses per prescription than other strong opioids, such as oxycodone and fentanyl. Influence on prescribing choices is discussed in more detail in Chapter 6.

The reasons for high dose opioid prescribing could not be elucidated from data available for this study but is certainly something which warrants further investigation. In this chapter, increasing numbers of prescriptions were associated with a small reduction in the $\mathrm{OMEQ}_{\mathrm{e}}$ overall, although the number of higher dosed, strong opioids increased significantly. This was perhaps expected 
from previous results (Section 3.6.7 and Appendix G), where weak opioid analgesics such as codeine and tramadol predominated at all durations. However, data also signaled a trend of strong opioids being increasingly represented as prescribing persistence rose (Appendix G). An example of this would be fentanyl, a drug that is not commonly prescribed. When it is, however, it appears to be prescribed at higher doses compared to other strong opioids, as demonstrated by a disproportionate $\mathrm{OMEQ}_{\mathrm{e}}$ compared to the number of prescriptions issued. This might point towards a lack of familiarity with dosing of non-morphine strong opioids and therefore, could support the wider use of OMEQ to illustrate prescribing data, where prescribers might contextualise dose choices more effectively. There was an increasing trend in $\geq 120 \mathrm{mg} \mathrm{OMEQ}_{\mathrm{e}}$ dosing of morphine and oxycodone, despite mounting evidence of the harms caused when that limit is exceeded.

Differences in prescription numbers for each drug analysed and subsequent $\mathrm{OMEQ}_{\mathrm{e}}$ were predictable, when examined by gender, with women receiving more prescriptions and being exposed to a greater $\mathrm{OMEQ}_{\mathrm{e}}$ burden overall. However, men received higher doses per prescription, which, whilst not statistically significant for all drugs analysed, was still perhaps unexpected. Added to this was the finding that again, overall prescription durations were slightly longer for men than women. A reflection on these results is based on the perception that men are less likely to present with pain, than women. Is there an underlying assumption on the part of prescribers, that, when men present, it must be significant and therefore warrants higher doses or 'stronger' analgesics? Conversely, is it suggestive women are expected to tolerate higher levels of pain? Gender differences in opioid prescribing have been discussed already in this thesis (Section 1.8, Section 3.6.5 and Section 4.6.3) and the discrepancies raised by the results in this chapter reinforce the need for more research.

The major finding from comparing prescription numbers to $O M E Q_{e}$ is the potential underestimation of opioid burden if only prescription numbers are used. Especially pertinent are products such as transdermal fentanyl or 
buprenorphine, where prescribers may be less familiar with the units of dosing. The result appears to be a disproportionate contribution to overall opioid burden based on relatively small prescription numbers. It perhaps suggests OMEQ is a more relatable measure of opioid burden and the added context it provides would be better understood by prescribers. The likelihood, however, is a combination of different measures are needed to provide the most complete picture of prescribing, over time. It is necessary to undertake further research on the measures of prescribing used in practice. As prescription numbers appear to be reducing, this study has demonstrated an increase in longer-term, higher dosed opioids, which are associated with much higher risks of harm. It could be too easy to assume that lowering the number of prescriptions lowers to burden on individuals or communities, but this may not be the case at all.

\subsubsection{Comparison with the literature}

\subsubsection{Choice of opioid}

Patterns of opioid use vary throughout the UK (Zin et al. 2014; Mordecai et al. 2018). Analgesic prescriptions dispensed in England between 1998 and 2017 revealed patterns of prescribing that this study upheld (Curtis et al. 2019). As in this study, codeine containing products were the most frequently prescribed analgesics, more so following the removal of co-proxamol/dextropropoxyphene from the UK market in 2005. Bedson et al. (2013) described a rapid increase in the prescribing of weak opioids (from 831 to 1021 prescriptions per10,000 people) such as co-codamol 8/500 following MHRA recommendations to stop coproxamol and selective-anti-inflammatory analgesics in 2004 and 2005 (Bedson et al. 2013). During the same period, a reduction in moderate analgesics (from 614 to 393 prescriptions per 10,000 people) including codeine up to $20 \mathrm{mg}$ per dose, was described.

The results of TOPAS and Bedson's work has been corroborated by a large analysis where codeine prescribing increased by 5 times between 2006 and 2017 (Jani et al. 2020). Unlike TOPAS, Curtis separated co-products (where an opioid is combined with a non-opioid, commonly paracetamol) from prescriptions for the 
opioid alone, revealing co-codamol (codeine and paracetamol) as the most frequently prescribed product. Scottish data showed a large increase in opioid analgesic prescriptions between 1995 and 2012 (Ruscitto et al. 2015). In 2010, codeine, tramadol and dihydrocodeine were the three most frequently prescribed medicines, as in the results presented here, with morphine by far the most frequently prescribed strong opioid (Ruscitto et al. 2015). Morphine was also shown to be prescribed more than other strong opioids between 2000 and 2010 in England and Wales, although oxycodone had a much higher percentage increase over that time (Zin et al. 2014).

TOPAS data would have demonstrated the same outcome, had data been presented separately. However, the aim was to analyse which opioid drugs were contributing to analgesic burden, regardless of the product used. Welsh data claimed tramadol accounted for up to 43\% of opioid prescribing in 2012 (Welsh Analytical Prescribing Support Unit 2013) leading to a big campaign to reduce tramadol prescribing. However, data used to write the report, did not include coproducts such as co-codamol, thus hugely underestimating the contribution of codeine to the overall prescribing burden in the country. Co-codamol was the $9^{\text {th }}$ most frequently prescribed medicine in Wales, with more than 1.8 million prescriptions issued in 2019-20 (Welsh Government 2020), clearly demonstrating the importance of it, in terms of prescribing burden, analgesic or otherwise. Codeine prescribing has been shown to predominate prescribing in the UK generally (Ashaye et al. 2018; Curtis et al. 2019). The COPERS trial (2018) examined opioid prescribing to people living in England with chronic musculoskeletal pain as a result of conditions such as osteoarthritis, back pain and fibromyalgia (Ashaye et al. 2018). Of the 703 participants recruited, 59\% (413 individuals) were prescribed opioid analgesics over a year period. Of those, 53\% (1768 of 3319 prescriptions) were for strong opioids like morphine or oxycodone. As in Wales, the most frequent prescription was for codeine (47\%), tramadol accounted for $26 \%$ and morphine for $9 \%$ of prescriptions issued in that time (Ashaye et al. 2018) giving a similar picture to that described in the TOPAS study. 
Different choices of opioids prevail outside of the UK. For example, hydromorphone is commonly prescribed in North America. One analysis of commercially insured patients noted $78.8 \%$ of all new prescriptions were for hydromorphone (Kern et al. 2015), compared to less than $0.01 \%$ of prescriptions examined during the 11 years of this study. Oxycodone, has predominated north American opioid prescribing for many years now (Fischer et al. 2014; Gomes et al. 2014) and has generally been considered the main source of the resulting problems of high prescribing and overdoses (Dhalla et al. 2011; Mulvihill et al. 2016). A comparison of oxycodone prescribing trends in the USA and Europe demonstrated over 10 years, oxycodone increased from $60 \mathrm{mg}$ to $175 \mathrm{mg}$ per capita in the USA, compared to 0 to $10 \mathrm{mg}$ per capita in Europe (Meyer et al. 2020). Prescribing in Australia is more similar to the UK, with codeine the most common opioid overall, but oxycodone is more regularly used than morphine (Karanges et al. 2016). Scandinavia uses several opioids not available in the UK, such as ketobemidone and nicomorphine which have accounted for a reasonable portion of their prescribing (Jarlbaek et al. 2005). What has been shown commonly throughout Europe however, is a rising use of oxycodone, fentanyl and tramadol (Hamunen et al. 2012; Palmaro and Lapeyre-Mestre 2015; Musazzi et al. 2018; Bosetti et al. 2019). This is often flagged as a concern due to the links of oxycodone and fentanyl with the opioid crisis in north America (Cicero et al. 2007; Kenan et al. 2012; Gomes et al. 2017). Whilst this study and others in the UK have also observed substantial increases in oxycodone prescribing in particular (Zin et al. 2014; Mordecai et al. 2018; Jani et al. 2020), levels have not reached those of morphine, as has been observed elsewhere.

\subsubsection{Comparison of trends in opioid burden}

To date, the largest UK-based examination of opioid prescribing by OMEQ suggested opioid burden was under-represented when only prescription numbers were used (Curtis et al. 2019). Curtis and colleagues demonstrated that a 34\% increase in prescriptions issued between 1998 and 2016 resulted in a $127 \%$ increase in OMEQ burden (Curtis et al. 2019). Although different methods 
of calculating OMEQ were used in the analysis presented here, a similar disparity was noted. A $44 \%$ increase in prescription numbers correlated to a $95 \%$ increase in OMEQ, albeit over a shorter timescale.

Analysis of 43 months prescribing data in England revealed tramadol to be responsible for the highest level of prescribing in OMEQ terms. Tramadol accounted for around 34\% of opioid burden (Mordecai et al. 2018), which, is similar to the proportion of opioid burden codeine contributes in Wales. Curtis et al. (2019) did not provide data on each drug or products' OMEQ increase over time, however, the shape of trend graphs implied codeine, tramadol and morphine OMEQs increased until 2015 before starting to slowly reduce (Curtis et al. 2019). The proportions of these three opioids appeared like those noted in Wales.

Fentanyl was discovered to account for the highest proportion of high dose opioid prescribing (>120mg OMEQ) in England between 1998 and 2017 (Curtis et al. 2019), which followed a trend first described in 2014 (Zin et al. 2014). The TOPAS study demonstrated a different outcome, with morphine responsible for the largest $\mathrm{OMEQ}_{e}$, which continued to increase throughout the period of analysis.

Jani and colleagues (2020) examined opioid dosing changes over two years, at individual patient level (Jani et al. 2020). An increase in OMEQ over a year was reported in a relatively small proportion of people previously started on $<50 \mathrm{mg}$ OMEQ with the majority (88.6\%) stopped. Of those initiated on doses of 50 $119 \mathrm{mg}$ OMEQ, reportedly $13.4 \%$ had a dose escalation to $>120 \mathrm{mg}$ OMEQ within a year, although the majority (86.7\%) were reduced or stopped. Of people commenced on $>200 \mathrm{mg}$ OMEQ, whilst a small proportion of the total $(1,446$ of $1,925,944$ people), had $45.4 \%$ remaining on that level of dosing at 6 months, although the percentage remaining on it, fell to $18.7 \%$ at 2 years (Jani et al. 2020b). This particular aspect of prescribing was not examined here. However, the implication of Jani et al.'s findings in addition to those presented here, 
intimates the rising annual trend in $\mathrm{OMEQ}_{\mathrm{e}}$, is less likely due to individuals having doses increased and more likely due to an increasing number of people being started on higher doses (Jani et al. 2020).

Mordecai et al. (2018) included buprenorphine and methadone in their analysis, resulting in them being marked as major contributors to opioid burden (Mordecai et al. 2018). Both drugs are used at high OMEQ, primarily for substance misuse management rather than analgesia. Methadone and buprenorphine products used in substance misuse, were removed from the $\mathrm{OMEQ}_{\mathrm{e}}$ analysis, to try to form the best reflection of analgesic opioids. Methadone tablets were included in the analysis but contributed very little to overall burden, as would be expected in analgesic terms.

Increases in opioid burden have been reported across Europe. A 187\% increase (from 36.0mg to $103.4 \mathrm{mg}$ OMEQ per capita) was reported between 2000 and 2015 in Poland. This was substantially less than estimated in this study using $\mathrm{OMEQ}_{\mathrm{e}}$ between 2005 and 2015 (estimated 16mg to 32mg over the period). However, the values are likely to be underestimated due to the lack of complete data. When opioid prescribing was compared between seven European countries using a DDD per 1,000 inhabitants measure, the UK was shown to have twice the rate of weak opioid prescribing and at least three times the rate of strong opioid prescribing than the others (Hider-Mlynarz et al. 2018). Based on the data presented, DDD per 1,000 inhabitants in the UK were reportedly almost equal for weak and strong opioids (approximately 51 DDD per person per day for weak and 48 DDD per person per day for strong opioids) (Hider-Mlynarz et al. 2018). While there are difficulties in directly comparing studies where different measures are used importantly is similar trends have been seen in many countries, with strong opioid consumption rising more quickly than weak opioids over time (Dzierżanowski and Ciałkowska-Rysz 2017).

Australia has seen large increases in opioid prescribing also. In 2013, an estimated 481mg OMEQ per person was sold (Degenhardt et al. 2016). Trend 
analysis between 1990 and 2014, demonstrated large percentage increases although using a non-OMEQ measure, the rise was from around 5 to 17 DDD per 1,000 population over that time (Karanges et al. 2016). As different opioids were included, it is not possible to calculate the OMEQ doses or compare to this study's findings with any accuracy. The trends noted however, are consistent with these findings and those already discussed (Section 3.5.2 and Section 4.5.1.1).

Previous studies have observed gender, age and socio-economic deprivation to be implicated for higher OMEQ doses of opioids (Spooner 2016; Todd et al. 2018; Macfarlane et al. 2020; Nowakowska et al. 2020; Richards et al. 2020). Studies were not found which examined those trends over time, in the way presented here. A systematic review of factors associated with high dose opioid prescribing (>90mg OMEQ per day) included being male (RR 1.21) and being unemployed (RR 1.44) which is often used as a proxy-measure of socioeconomic deprivation (Richards et al. 2020a).

This study substantiated the results, as increasing deprivation was demonstrated to be associated with higher $\mathrm{OMEQ}_{\mathrm{e}}$ burden and a greater percentage of high dose prescribing (>120mg OMEQ $)$. Empirically, overall OMEQ burden was significantly higher for women, according to the findings, other than for morphine and oxycodone. OMEQ $Q_{e}$ per prescription for strong opioids were significantly higher for men in this study, however. Higher $O M E Q_{e}$ was used to define 'high dose', but it appears correct to suggest men were more likely to receive a prescription for a strong opioid or to receive a higher dose. Prescribing in rural, less affluent areas of Australia with large male populations, has been observed to yield higher OMEQs (Degenhardt et al. 2016), which further corroborates the TOPAS results presented in this chapter and elsewhere (Section 3.5.3 and Section 4.5.2).

Substantial increases in opioid prescribing, with higher levels in more deprived populations have been reported in other parts of the UK (Mordecai et al. 2018) 
and internationally (Joynt et al. 2013; Wagemaakers et al. 2017). Increased levels of prescribing in areas of high socio-economic deprivation has been linked to greater reported pain intensity (Todd et al. 2018). However, limited evidence supports the notion that opioids are effective at reducing pain, particularly in the longer term (Furlan et al. 2006; Chaparro et al. 2014). High-dose opioids (above 120mg OMEQ) have been associated with increased levels of pain (Cohen et al. 2008). In the context of this and previous studies (Mordecai et al. 2018; Todd et al. 2018), the implications of increased opioid prescribing in more deprived areas are concerning. It exposes the most vulnerable people to higher levels of medicines, that may be ineffective at best and could cause additional health and well-being complications (Els et al. 2017).

However, similarities were demonstrated in the trends seen in age-group prescribing, where people aged 45 years and over had a significantly higher incidence of long-term opioid prescribing (Chapter 3 and Appendix G). Lalic et al. (2018) found people aged over 75 years were at least twice as likely to be persistently prescribed opioids, corroborating the findings presented in this thesis, that overall prescribing persistence increased with rising age (Lalic et al. 2018).

Few studies have examined gender trends in opioid use duration, despite it being widely acknowledged women are more likely to receive long-term opioid treatment (Darnall et al. 2012). One study, however, found women of all age groups had consistently higher levels of long-term prescribing than men (Bedson et al. 2016), which reflects this study's findings.

This study demonstrated weak opioids and codeine especially, were used most frequently at all durations of prescribing analysed, although the use of strong opioids increased with rising prescribing persistence (Chapter 3 and Appendix G). A previous review of prescribing in the UK and Germany, demonstrated codeine to be the most prescribed opioid in the UK (Chevalier et al. 2014). This was consistent with the findings of Kern et al. (2015), who observed weak opioids 
were prescribed chronically, twice as often as strong opioids. The choice of opioids in either category was different to those in this study however, with hydrocodone (not available in the UK) rather than codeine being the most frequently prescribed weak opioid. Oxycodone was prescribed long term, twice as often as any other strong opioid in the same study (Kern et al. 2015), whereas morphine was most used in Wales. Durations were not significantly different between different strong opioids in this study. Bedson and colleagues (2016), examining prescribing duration, noted $20 \%$ of people receiving opioid prescriptions for more than 2 years, received modified release strong opioids (Bedson et al. 2016). This confirms the results of TOPAS, showing an increased likelihood of receiving strong opioids as total duration of opioid prescribing lengthens (Appendix $G)$.

\subsection{Conclusion}

This is the first study that has examined burden in $\mathrm{OMEQ}_{\mathrm{e}}$ terms in Wales. Increasing numbers of prescriptions between 2005 and 2015, betrays the full extent of the opioid burden placed upon the Welsh population. Few UK based studies have studied OMEQ trends; although it is becoming more common place as researchers realise it is an effective means of providing contextualised data that can be used more easily in practice.

Whilst it was known that strong opioid prescribing has increased significantly in Wales since the beginning of the 2000's, this study reveals more about the nature of that increase. Whilst opioid analgesics of all strengths have increased, the implication of high levels of strong opioids, which carry a greater OMEQ burden per dose, is considerable. This is especially notable in areas of deprivation and in older people (results in Appendix D), both variables demonstrating large increases during the study period.

There is not a single measure of prescribing that provides sufficient information of risk. OMEQ is an estimated measure and so caution is required when applying it in clinical practise. The data used in this study was not wholly precise but 
demonstrates that OMEQ offers an easily interpreted measure for assessing dose burden in practise, given its ability to place all opioids on an 'equal' footing. Caution does need to be applied, when using OMEQ within guidelines to ensure it is not assumed to be a completely accurate measure of effectiveness and safety. It seems sensible to suggest that in Wales, a method of combining OMEQ and prescribing persistence as a measure of monitoring opioid prescribing in real time should be sought. In addition, clinicians would benefit from clear guidance on initiating and reviewing opioids, considering the risks of both dose and duration to each individual. Whilst prescription numbers may have started to slowly decrease, data presented here, suggests the overall burden posed by opioids was still increasing in 2015. Prescribers and policymakers must not assume that a fall in prescription numbers alone is sufficient to reduce the risks of opioids. 
Chapter 6

Time Series Analysis examining the effect of legislation and prescribing guidance on opioid prescribing in Wales

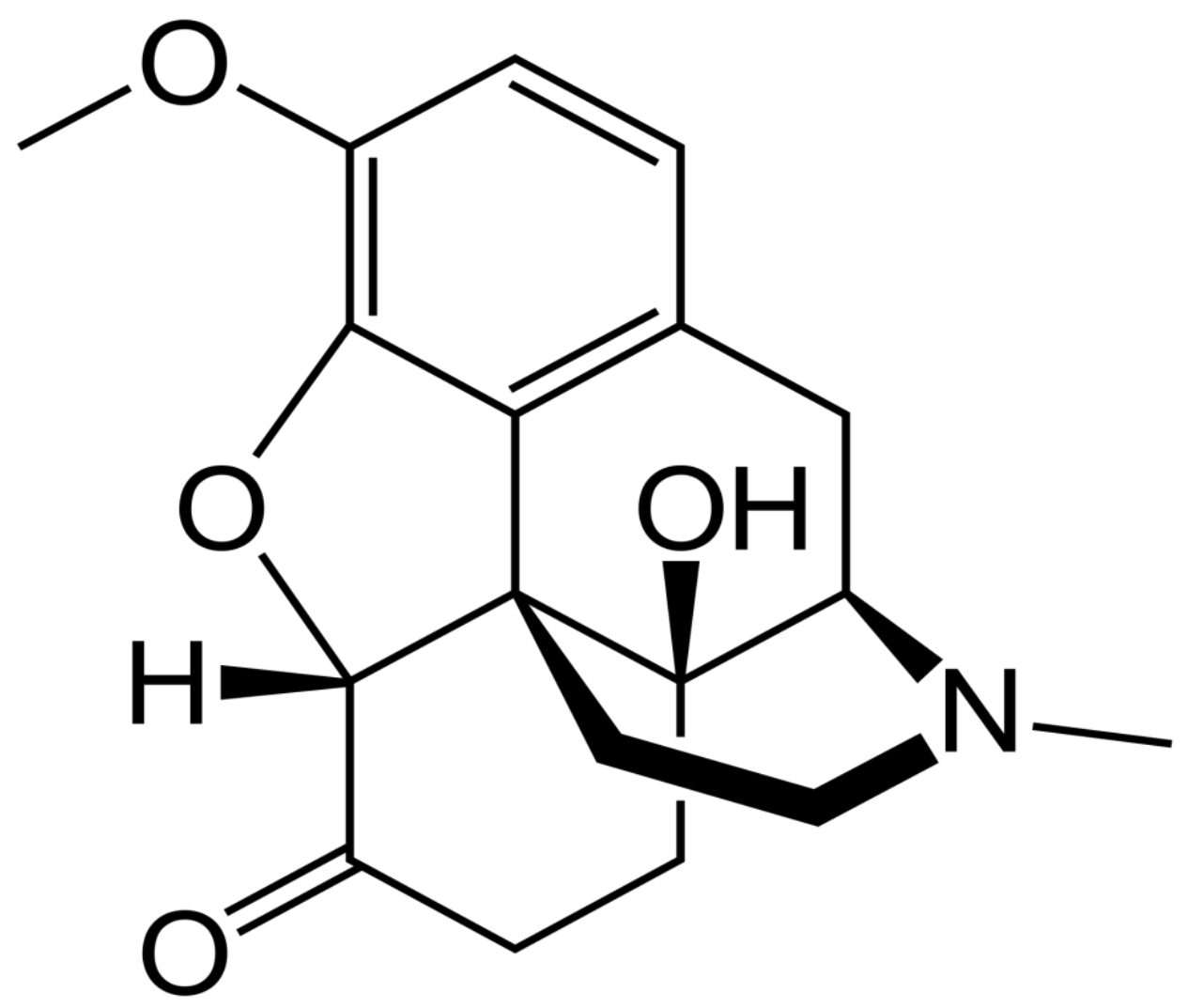

"They always say time changes things, but you actually have to change them yourself."

Andy Warhol 


\section{Chapter 6 - Time Series Analysis examining the effect of legislation and prescribing guidance on opioid prescribing in Wales}

\subsection{Chapter overview}

Chapters 1 and 2 discussed the background to the research and explanation of the methods adopted in order to extract and analyse the data, respectively. Chapters 3 and 4 presented trend data for opioid prescribing in Wales, revealing large increases in rates between 2005 and 2015, disproportionately affecting women and people living in the most socioeconomically deprived areas of Wales. Chapter 5 examined trends by $\mathrm{OMEQ}_{\mathrm{e}}$ which demonstrated three drugs, codeine, tramadol and morphine were responsible for $70 \%$ of opioid burden in the study population. Again, gender and deprivation predominated in terms of associations with greater burden. Whilst overall opioid burden was higher in women, data suggested that men received higher doses of prescribed opioid analgesics. Chapter 6 builds on the previous chapters, by using time series analysis (TSA) to investigate the effect of legislative changes and the development of National Prescribing Indicators in Wales on the trends in opioid analgesic prescribing between 2005 and 2015.

\subsection{Study objective to be addressed in this chapter}

- To determine if general opioid prescribing trends were influenced by legislative or clinical guidance changes during the study period using time series analysis

\subsection{Background}

Time Series Analysis (TSA) is an established method in healthcare research and has been used to examine a range of issues related to analgesics (Chang et al. 2016; Gomes et al. 2017; Musazzi et al. 2018; Alexander et al. 2019;). Ranging from predicting prescription choice within an electronic prescribing system (Helgason 2008) to predicting whether an individual will respond to treatment (Alexander et al. 2019), TSA can be a useful tool on its own or in combination 
with other analysis methods. The outcome of introducing new prescribing legislation and monitoring requirements has been studied using interrupted TSA as part of a trend analysis from Canada (Gomes et al. 2014) over a similar time span to TOPAS. Studies investigating the introduction of new opioid formulations of opioid medicines, specifically oxycodone have also used TSA, demonstrating changes in the choices being made by prescribers (Gomes et al. 2017; Musazzi et al. 2018). Interrupted TSA was also used to determine the effect of rescheduling hydrocodone on illicit opioid purchases in the USA in 2014 (Martin et al. 2018). It was planned as part of this study, to use TSA to create predictions of how prescribing might progress after the end of the study period (from 2016 to 2018) if no further changes were made to guidance or policy around the prescription of opioid medicines. No literature on using TSA to predict future opioid prescribing trends was found. However, TSA has been used to analyse changes in prescribing following the introduction of new products (Musazzi et al. 2018; Gomes et al. 2017; Puenpatom et al. 2012) with the actual trend noted compared to that predicted by the TSA.

As discussed in Section 1.11, guidelines and legislation introduced into the UK over the last 15 years, were designed to improve access to data and the quality and safety of prescribing practice (Baker 2004; National Institute for Health and Clinical Excellence 2008; Centre for Clinical Practice at NICE 2013; All Wales Medicines Strategy Group 2013). Despite this, as has been demonstrated in results presented in Chapter 3, Chapter 4 and Chapter 5, opioid prescribing trends in Wales appeared to be on an upwards trajectory for the majority of the study period. This chapter presents TSA as a method to analyse whether introducing legislation and guidelines which acted to manage prescribing of and rational use of opioid analgesics, affected prescribing in practice.

\subsection{Method}

\subsubsection{Forms of time series analysis}

Prescribing trend data can be considered a form of time series. Time series analysis (TSA) is a method of forecasting future values based on the known 
pattern of past data (Beard et al. 2019). Interrupted TSA is a quasi-experimental means of examining the influence of an intervention on the time series being tracked. Time series analysis uses observations, in the case of TOPAS, the number of prescriptions issued each month, over successive time periods (each month) which allows a trend (the time series) to be seen. The high volume of data available from the SAIL databank potentially lends itself to TSA as each data point (month) contains a large amount of data on which to base the prediction for the next.

Two forms of TSA were used. The first was a continuous TSA, where monthly data points were plotted for the whole study period (Section 6.4.2) and an assessment was then made of whether the trend described had been affected by any of a number of 'interventions', which for this study, were legislative or prescribing advisories.

An interrupted TSA was also performed, to assess the impact of a specific intervention, namely the introduction of a national prescribing indicator for 2012 to 2013, which encouraged morphine as the strong opioid of choice (Section 1.11). Effects of the intervention on the number of strong opioid prescriptions issued were examined using the changes in the level and slope of the plotted data-series and their calculated statistical significance (Bernal et al. 2017; Hebert et al. 2021).

The interrupted TSA model used was:

$Y=\beta_{0}+\beta_{1} *$ Time $_{1}+\beta_{2} *$ Intervention $+\beta_{3} *$ Intervention $*$ Time 2

Where $Y$ is the number of prescriptions issued (prescribing rate) and $\beta_{0}$ is the intercept (the number of prescriptions issued at the beginning of the study). ß1 represents the number of prescriptions issued per time unit (prescribing rate) prior to the intervention, $B 2$ is the level change that follows the intervention and $\beta_{3}$ is the change in the trend (rate of prescribing) after the intervention. Time in 
this equation refers to the elapsed time from the start of the study (Time 1 ) and after the intervention ( Time $_{2}$ ) respectively.

\subsubsection{Calculating the moving mean and centred-moving mean for TSA}

There is inherent random variation in data collected over time and methods are therefore, adopted in order to reduce or cancel the effects. An oft-used technique is 'smoothing', which, when properly applied, can clearly reveal the underlying trends, seasonal and cyclical components of the data. There are two general methods for smoothing, namely averaging methods and exponential smoothing methods. Simple averaging or using the mean of all past observations is effective for forecasting only in the absence of trends. Where trends are present, smoothing methods need to take them into account. To do this, smaller sets of data are averaged.

For this TSA, the moving means (MM) were calculated using 6 months of data (number of prescriptions issued) per set (Appendix E). The first set to be averaged were months one to six from 2005 (Figure 6.1). Six months of prescriptions were averaged, with the outcome being placed at the month-4 mark on the spreadsheet. The next MM was calculated from prescription numbers for months two to seven, with the outcome placed at month-5. This process was repeated for the rest of the eleven years of data, with the final value calculated at month-132.

This study, covering 11 full years of data, had an even number of datapoints (132 months). Using MM requires the calculated average to be placed in the middle of the time period being covered in order to produce the smooth plot required. Where there are an uneven number of data points, this is straightforward (e.g., if there are three timepoints, then the moving mean is placed at time point two). For this TSA, an additional smoothing technique was needed to take account of the even number of datapoints. Centred moving means (CMM) were calculated using pairs of consecutive moving means (Figure 6.1). The first CMM was 
calculated as the average of $\mathrm{MM} 1$ and $\mathrm{MM} 2$, the second $\mathrm{CMM}$ was the average of $\mathrm{MM} 2$ and $\mathrm{MM} 3$ and so on.

\begin{tabular}{|c|c|c|c|c|c|c|}
\hline \multicolumn{2}{|c|}{ E5 } & \multirow[t]{2}{*}{$\boldsymbol{A}$} & \multirow{2}{*}{$\frac{f x}{c}$} & \multicolumn{3}{|c|}{$=$ AVERAGE(D2:D7) } \\
\hline 4 & A & & & D & E & $\mathrm{F}$ \\
\hline 1 & Year & Month & & Frequency & Moving means & $\begin{array}{l}\text { Centred } \\
\text { moving } \\
\text { mean }\end{array}$ \\
\hline 2 & 2005 & 1 & 1 & 135478 & & \\
\hline 3 & 2005 & 2 & 2 & 125974 & & \\
\hline 4 & 2005 & 5 & 3 & 138023 & & \\
\hline 5 & 2005 & 4 & 4 & 131876 & 133321 & 132807 \\
\hline 6 & 2005 & -5 & 5 & 132551 & 132294 & 133563 \\
\hline 7 & 2005 & 6 & 6 & 136021 & 134831 & 134669 \\
\hline 8 & 2005 & 7 & 7 & 129321 & 134507 & 134686 \\
\hline 9 & 2005 & 8 & 8 & 141194 & 134864 & 135364 \\
\hline 10 & 2005 & 5 & 9 & 136081 & 135865 & 135723 \\
\hline 11 & 2005 & 10 & 10 & 134017 & 135582 & 136487 \\
\hline 12 & 2005 & 51 & 11 & 138553 & 137392 & 136179 \\
\hline 13 & 2005 & 12 & 12 & 134328 & 134966 & 135499 \\
\hline 14 & 2006 & 1 & 13 & 140180 & 136032 & 135427 \\
\hline 15 & 2006 & 2 & 14 & 126636 & 134821 & 135253 \\
\hline 16 & 2006 & 3 & 15 & 142476 & 135685 & 136068 \\
\hline 17 & 2006 & 4 & 16 & 126755 & 136450 & 136027 \\
\hline 18 & 2006 & 5 & 17 & 143735 & 135603 & 137037 \\
\hline
\end{tabular}

\begin{tabular}{|c|c|c|c|c|c|c|}
\hline \multirow[t]{2}{*}{ F5 } & \multicolumn{2}{|r|}{$\mathbf{1}$} & $\times \vee f x$ & \multicolumn{2}{|c|}{$=$ AVERAGE(E5:E6) } & \multirow[b]{2}{*}{$\mathrm{F}$} \\
\hline & A & B & c & D & E & \\
\hline 1 & Year & Month & & Frequency & Moving means & $\begin{array}{l}\text { Centred } \\
\text { moving } \\
\text { mean }\end{array}$ \\
\hline 2 & 2005 & 4 & 1 & 135478 & & \\
\hline 3 & 2005 & 2 & 2 & 125974 & & \\
\hline 4 & 2005 & 5 & 3 & 138023 & & \\
\hline 5 & 2005 & 4 & 4 & 131876 & 133321 [ & 132807 \\
\hline 6 & 2005 & 5 & 5 & 132551 & 132294 & 133563 \\
\hline 7 & 2005 & 6 & 6 & 136021 & 134831 & 134669 \\
\hline B & 2005 & 5 & 7 & 129321 & 134507 & 134686 \\
\hline 9 & 2005 & 8 & 8 & 141194 & 134864 & 135364 \\
\hline .0 & 2005 & 75 & 9 & 136081 & 135865 & 135723 \\
\hline 1 & 2005 & 10 & 10 & 134017 & 135582 & 136487 \\
\hline 2 & 2005 & 51 & 11 & 138553 & 137392 & 136179 \\
\hline 3 & 2005 & 12 & 12 & 134328 & 134966 & 135499 \\
\hline 4 & 2006 & 1 & 13 & 140180 & 136032 & 135427 \\
\hline .5 & 2006 & 2 & 14 & 126636 & 134821 & 135253 \\
\hline .6 & 2006 & 3 & 15 & 142476 & 135685 & 136068 \\
\hline .7 & 2006 & 4 & 16 & 126755 & 136450 & 136027 \\
\hline 8 & 2006 & 5 & 17 & 143735 & 135603 & 137037 \\
\hline 29 & 2006 & 6 & 18 & 138920 & 138472 & 137898 \\
\hline
\end{tabular}

Figure 6.1: Screenshot of Excel spreadsheet showing calculation of moving means (1) and centred moving means (2) for opioid prescriptions as part of time series analysis

Once MM and CMM were calculated for each dataset, scatter plots were drawn using the raw data (frequency/number of prescriptions) and the moving mean values. A trend line was inserted using the centred moving mean values, which gave an indication of the trend over the period examined.

\subsubsection{Seasonal adjustment}

Seasonal variation can be due to specific events such as holidays or types of weather for example. It was examined for the TOPAS study in order to determine if there were any differences in the number of prescriptions issued, based on the time of year or the month of issue.

Individual seasonal effect (ISE) was calculated by subtracting the centred moving mean from the number of prescriptions issued in the corresponding month. Ten ISEs for each of the 12 months were used to calculate the average seasonal effect (ASE) for the number of prescriptions issued (Figure 6.2). Seasonally adjusted values (SAV) were calculated by subtracting the average seasonal effect from the actual number of prescriptions recorded. A determination of whether the SAV was above or below what might have been expected, based on previous prescribing trends, was made. If the value of subtracting the SAV from CMM was 
less than zero, then the number of prescriptions issued were marked as less than expected. If the value was greater than zero, then the number of prescriptions were marked as above what would have been expected on the basis of previous prescribing patterns (Figure 6.2).

\begin{tabular}{|c|c|c|c|c|c|c|c|c|c|c|}
\hline$\div$ & $\times \vee f x$ & $=A V E R A G E$ & $E(L 14, L 26, L 38, L 50, L 62, L 74, L$ & ,L86,L98,L110 & 0, L122) & & & & & \\
\hline H & 1 & $\mathrm{~J}$ & $\mathrm{k}$ & L & M & $\mathrm{N}$ & 0 & $\mathrm{P}$ & Q & $\mathrm{R}$ \\
\hline Month & & Frequency & Centred moving mean & Individual se & Seasonally a & Below/Above expectation & & Average seas & sonal effects & \\
\hline 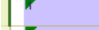 & 1 & 135478 & & & 133128.82 & & & January & 2349.1833 & \\
\hline 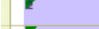 & ${ }^{2}$ & 125974 & & & 136992.11 & & & February & -11018.11 & \\
\hline 8 & 3 & 138023 & & & 131915.72 & & & March & 6107.2833 & \\
\hline 2 & 4 & 131876 & 132807.4167 & -931.4167 & 132503.2 & Below & & April & -627.197 & \\
\hline E & 5 & 132551 & 133562.6667 & -1011.667 & 132750.18 & Below & & May & -199.1818 & \\
\hline 8 & 6 & 136021 & 134669.1667 & 1351.8333 & 137213.87 & Above & & June & -1192.871 & \\
\hline 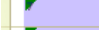 & 7 & 129321 & 134685.75 & -5364.75 & 125962.87 & Below & & July & 3358.1288 & \\
\hline 8 & 8 & 141194 & 135364.3333 & 5829.6667 & 143202.03 & Above & & August & -2008.03 & \\
\hline 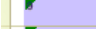 & ${ }^{9}$ & 136081 & 135723.4167 & 357.58333 & 136373.34 & Above & & September & -292.3409 & \\
\hline 60 & 10 & 134017 & 136487.25 & -2470.25 & 131101.32 & Below & & October & 2915.6833 & \\
\hline 1 & 11 & 138553 & 136179 & 2374 & 140146.24 & Above & & November & -1593.242 & \\
\hline$\sqrt{2}$ & 12 & 134328 & 135498.75 & -1170.75 & 131825.62 & Below & & December & 2502.3833 & \\
\hline$\pi$ & 13 & 140180 & 135426.5 & 4753.5 & 137830.82 & Above & & & & \\
\hline F & 14 & 126636 & 135253.1667 & -8617.167 & 137654.11 & Above & & & & \\
\hline 5 & 15 & 142476 & 136067.6667 & 6408.3333 & 136368.72 & Above & & & & \\
\hline $\bar{z}$ & 16 & 126755 & 136026.6667 & -9271.667 & 127382.2 & Below & & & & \\
\hline
\end{tabular}

\subsubsection{Predicting future issue of prescriptions}

A trendline was added to the scatter plot of values and centred moving mean using Excel graphing. The coefficient of determination $\left(R^{2}\right)$ was calculated for each trendline in order to decide how well the regression line might approximate the real values and consequently, the accuracy of predictions of future values. A coefficient of determination of $>.999$ means that a given month's number of prescriptions can explain $100 \%$ of the number of prescriptions issued in the following month. Consequently, the closer $\mathrm{R}^{2}$ is to $>.999$, the better ' $\mathrm{it}^{\prime}$ ' of the trendline and it can be assumed that the subsequent predictions are of greater accuracy.

Predicting future values for the number of prescriptions that might be issued is based on the equation that describes the trendline e.g., for a second order polynomial trendline:

$f(x)=a x^{2}+b x+c+A S E$ 
Where $\mathrm{x}$ is the time value, which in this study, is the month as a number based on January 2005 being equal to one and December 2015 being equal to 132 . Consequently, predictions after the end of the study period in 2015 will use time values from 133. ASE is the average seasonal effect and $a, b$ and $c$ are coefficients generated by Excel when determining the trendline.

Whilst predictions using existing data have a measure of accuracy based on the coefficient of determination, predicting future events is likely to be less accurate unless the time series remains stationary. TSA with trends or seasonality are not considered stationary, as those shifts can affect the value at different times in the analysis.

For this study, the chosen intervention for the interrupted TSA was the introduction of the 2012 to $2013 \mathrm{NPI}$ that aimed to increase the percentage of morphine as the total of all strong opioid prescribing. This was the first NPI that focussed on strong opioids, which were at the time, considered a bigger concern than overall opioid burden.

\subsubsection{Legislation changes in the UK between 2005 and 2015}

The major changes to legislation (Changes to the Misuse of Drugs Regulations 2001) as a result of the Shipman enquiry (NHS Prescription Services 2017) were (Figure 6.3):

1. 14 November 2005 - to allow computer generation of prescriptions (other than prescriber signature) and the computerisation of controlled drug registers for Schedule 1 and 2 drugs (Appendix E) (UK Government 2021).

2. 7 November 2006 - special forms for private controlled drug prescriptions (Schedule 2 and 3) and a central database to allow monitoring locally were introduced. Changes in collection arrangements of prescriptions, requiring a signature from the person collecting them from the dispensing pharmacy or on delivery if using a home-service. The validity of prescriptions for schedule 2, 3 and 4 controlled drugs was restricted to 28 days, meaning that 
prescriptions needed to be dispensed and collected within 28 days from issue. A requirement was introduced for standard operating procedures to be in place and complied with for any healthcare provider keeping stocks of controlled drugs. Further recommendations were issued although not legislated, that no more than 30 days of medicines should be prescribed at one time (for schedule 2, 3 and 4 controlled drugs). Professional guidance was also reinforced that doctors should only prescribe controlled drugs for themselves or their family in exceptional circumstances.

3. 1 January 2007 - Designated bodies, such as Health Trusts and Health Boards became required to appoint an Accountable Officer to monitor the use of controlled drugs within their organisations or across a wider area (in the case of Health Boards).

4. 1 September 2007 - a requirement was introduced that dispensing contractors (e.g., community pharmacies) no longer had to retain private controlled drug prescriptions (Schedule 2 and 3) for 2 years, in order to allow them to submit the original prescriptions for reimbursement. Controlled Drug Accountable Officers were also responsible for appointing authorised witnesses to oversee the destruction of controlled drugs.

5. January 2008 - Suppliers of schedule 2 and 3 controlled drugs became obliged to provide information in regard of supplies of controlled drugs, where there was no prescription to be submitted. This legislative change also introduced more flexible arrangements in regard of the formatting of controlled drug registers, although the requirement for verifying the identity of someone collecting a schedule 2 medicine was still necessary.

6. 23 April 2012 - Nurse and Pharmacist Independent Prescribers became able to prescribe opioid medicines (other than diamorphine, cocaine and dipipanone for addiction)

7. 1 April 2013 - the Controlled Drugs (Supervision and Use) regulations were updated to reflect organisational changes within the National Health Service. It reiterated the need for organisations to appoint Accountable Officers for 
Controlled Drugs but widened their potential 'reach' in terms of monitoring and investigating concerns.

8. 1 June 2014 - Tramadol was reclassified as a schedule 3 controlled drug although remained exempt from safe custody requirements.

9. 2 March 2015 - a new drug driving law came into force which made it a criminal offence to be in charge of a motorised vehicle with blood concentration levels above specified limits for named opioid analgesics and other prescribed and illicit drugs.

10. 1 June 2015 - Limited prescribing of scheduled opioid analgesics was allowed for independent physiotherapist and podiatrist prescribers. Under this legislation, it also became mandatory to use a standardised form for schedule 2 and 3 requisitions of controlled drugs.

11. July 2015 - a statutory instrument was introduced to allow electronic prescribing of schedule 2 and 3 controlled drugs under the Electronic Prescribing Service (EPS) structure in Primary and Community Care.

\subsubsection{Prescribing and condition guidance}

The National Prescribing Indicators for Wales and relevant NICE guidelines published within the study period 2005 to 2015 were (Figure 6.3):

12. 2008 - Osteoarthritis: National Clinical Guidelines for care and management in adults (NICE). Opioid analgesics were neither recommended for use or to be avoided. Prescribers were encouraged to consider paracetamol and NSAIDs (National Collaborating Centre for Chronic Conditions 2008).

13. 2009 - Low back pain: the acute management of patients with chronic (longer than 6 weeks) non-specific low back pain (NICE). The guideline included a recommendation for the short-term use of strong opioids to manage severe pain (National Institute for Health and Clinical Excellence 2008).

14. 2012-2013 - NPI Morphine as a percentage of strong opioid prescribing. Prescribers were encouraged to prescribe morphine first line where a strong opioid was required. 
15. 2013 - Neuropathic pain: the pharmacological management of neuropathic pain in adults in non-specialist settings (CG173 - NICE). Discouraged the use of opioid analgesics other than tramadol for short-term, flare up management (Centre for Clinical Practice at NICE 2013).

16. 2013-2014 - NPI from the previous year was repeated.

17. 2014 - Osteoarthritis: care and management (CG177 - NICE). Suggests prescribers consider opioids but does not specify particular categories of medicines to consider (National Institute for Health and Care Excellence 2014).

18. 2014-2015 - NPIs set out to encourage the appropriate prescribing of all opioid-containing analgesics and three measures were set out. The first was total opioid prescribing by the number of items (prescriptions) issued per 1,000 patient units (as adjusted measure based on population and demographic e.g., age). The second measure was a repeat of the previous two year's morphine indicator. A third indicator was directed specifically at tramadol prescribing with the aim of reducing the number of daily defined doses (Nielsen et al. 2017) of tramadol products per 1,000 patients in each Health Board area.

19. 2015-2016 - NPIs were maintained morphine as a percentage of strong opioid prescribing and to reduce tramadol prescriptions as for 2014 to 2015.

The launch of each prescribing indicator was plotted on a time-series analysis of total opioid prescribing as a marker for the timing of reinforcement of advice and guidance around opioid prescribing during the study period.

\subsubsection{Prescription charges}

20. Prescription charges for medicines dispensed in Wales were abolished in April 2007 (National Assembly Government 2010) (Figure 6.3). Prior to that, around $50 \%$ of the population were entitled to free prescriptions due to exemptions such as age, low income or having certain medical conditions (National Assembly Government 2010). 


\subsection{Results}

\subsubsection{Legislation and guidance changes}

Between 2005 and 2008, there was an 18\% increase (from 1,613,417 to $1,904,351$ prescriptions respectively) in the total number of opioid prescriptions issued annually. In the same period five pieces of legislation were introduced to Welsh law and prescription charges abandoned (Figure 6.3). Between 2008 and 2012, one further piece of legislation was introduced, and two NICE guidelines were published. Over the same period the number of prescriptions issued increased by $22 \%$ (from 1,904,351 to $2,318,407$ prescriptions per year). In the last four years of the study, six pieces of legislation were introduced which concerned opioid prescribing, including the reclassification of tramadol as a controlled substance in 2014 (Figure 6.3). 


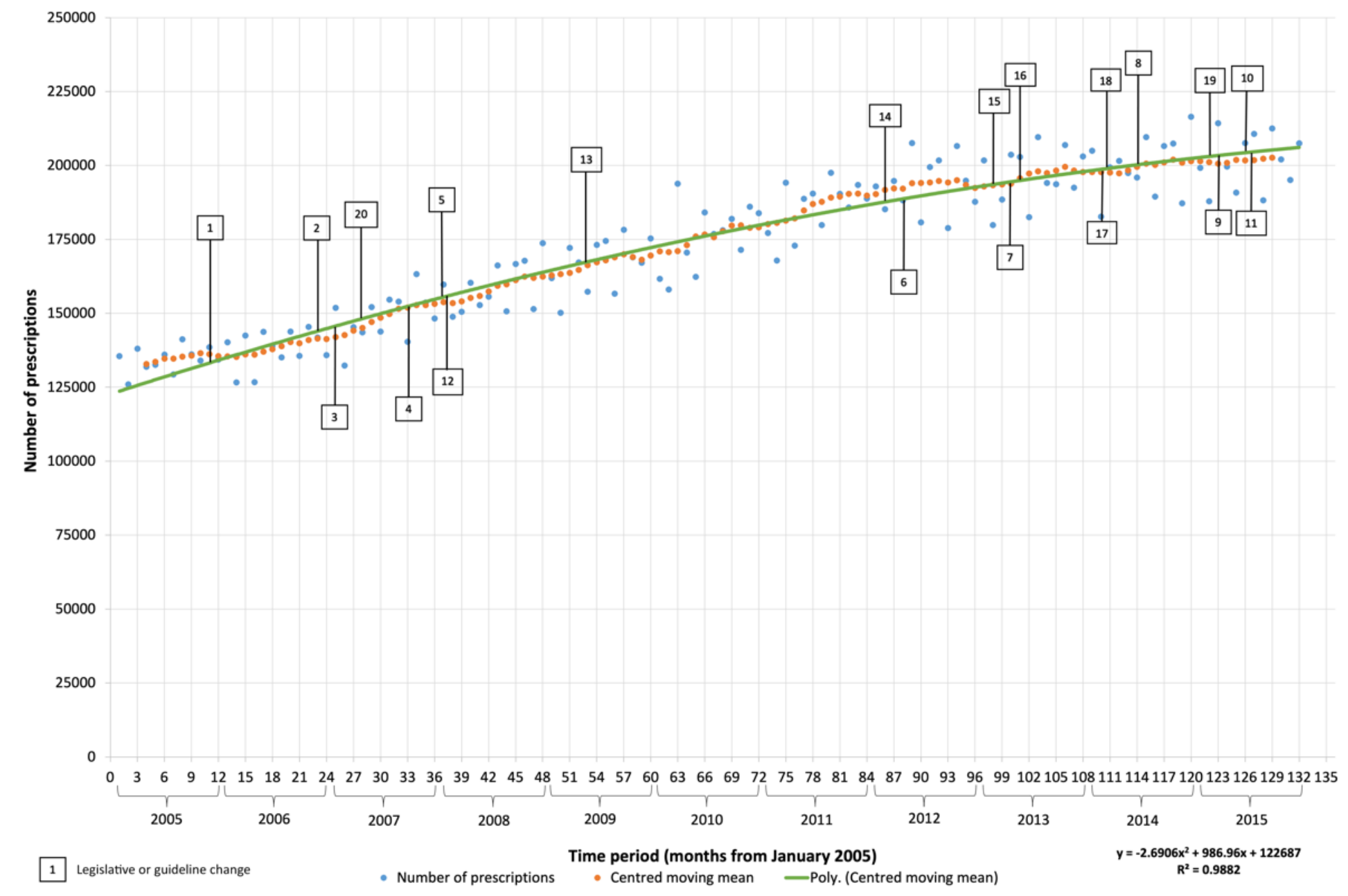

Figure 6.3: Monthly trend in the number of opioid prescriptions issued from Primary Care Practices who provide data to SAIL databank. Shown with a polynomial ( $\left.x^{2}\right)$ 'best fit' trendline, R2 $=0.9882$. Shown with time markers for legislation changes and National Prescribing indicators which included targets for reducing prescribing 
NPIs introduced in 2012 and repeated every year thereafter, included opioid prescribing markers. Two NICE guidelines which included recommendations on opioid analgesics were issued in the final three years of the study. Between 2012 and 2015, the increase in the annual number of opioids was $4 \%$ (from 2,318.407 to $2,415,374$ prescriptions per year, respectively).

\subsubsection{Interrupted Time Series Analysis}

A maximum likelihood (MLE) method or variable estimation was used rather than ordinary least squares (OLS) as MLE had a lower root mean squared error (RMSE) and corrected Akaike information criterion (AICc) and higher R-square (Table 6.1). That pattern of output indicates the MLE model was a better fit than the OLS.

Table 6.1: Output from testing for best-fit model of interrupted time series analysis

\begin{tabular}{llll}
\hline Maximum Likelihood Estimates & & \\
\hline MSE & 3491395401 & DFE & 125 \\
\hline SBC & 27931163 & Root MSE & 5285 \\
\hline MAE & 2668.7667 & AIC & 2648.58709 \\
\hline MAPE & 4149.3777 & AICC & 2649.49032 \\
\hline Log Likelihood & 2.45605683 & HQC & 2656.78716 \\
\hline Durbin-Watson & 1.9425 & Total R-Square & 0.9406 \\
\hline \multicolumn{5}{l}{} & & Observations & 0.9582 \\
\hline Ordinary Least Squares Estimates & & 132 \\
\hline SSE & 8355585466 & DFE & 128 \\
\hline SBC & 65278011 & Root MSE & 8079 \\
\hline MAE & 2765.29901 & AIC & 2753.7678 \\
\hline MAPE & 6683.04306 & AICC & 2754.08276 \\
\hline Log Likelihood & -1372.8839 & Total R-Square & 2758.45355 \\
\hline Durbin-Watson & 2.9599 & Observations & 0.9001 \\
\hline
\end{tabular}

Based on the parameter estimates derived, the model revealed a positive preintervention trend (Table 6.2). The intervention investigated (introduction of NPI for morphine as percentage of strong opioid prescribing, 2012 -2013), was followed by a non-significant step-up in the number of prescriptions issued. The step up was not unexpected, given the preceding trend. The post-intervention trend demonstrated a reduction in the number of prescriptions being issued (Table 6.2). 
Table 6.2: Results of interrupted time series analysis of opioid prescribing data

\begin{tabular}{|c|c|c|c|c|}
\hline \multicolumn{5}{|c|}{ Parameter estimates } \\
\hline Variable & $\begin{array}{l}\text { Estimate } \\
(95 \% \mathrm{Cl})\end{array}$ & $\begin{array}{c}\text { Standard } \\
\text { Error }\end{array}$ & t Value & $\begin{array}{l}\text { Approx } \\
\operatorname{Pr}>|t|\end{array}$ \\
\hline Intercept $\left(ß_{0}\right)$ & 127253 & 1266 & 100.51 & $<.0001$ \\
\hline $\begin{array}{r}\text { Pre-intervention } \\
\text { trend }\left(ß_{1}\right)\end{array}$ & $\begin{array}{c}738.5126 \\
(691.48-785.55)\end{array}$ & 23.9968 & 30.78 & $<.0001$ \\
\hline $\begin{array}{r}\text { Change in level } \\
\left(\beta_{2}\right)\end{array}$ & 750.9560 & 1659 & 0.45 & 0.6516 \\
\hline $\begin{array}{r}\text { Post-intervention } \\
\text { trend }\left(ß_{3}\right)\end{array}$ & $\begin{array}{c}-500.3484 \\
(-624.38--376.31)\end{array}$ & 63.2839 & -7.91 & $<.0001$ \\
\hline AR1 & 0.4526 & 0.0575 & 7.87 & $<.0001$ \\
\hline AR3 & -0.2928 & 0.0639 & -4.58 & $<.0001$ \\
\hline AR12 & -0.4037 & 0.0669 & -6.03 & $<.0001$ \\
\hline
\end{tabular}

Graphical representation reinforces the numeric trends. Whilst the number of prescriptions continued to increase after the introduction of the NPI 2012 -2013, the rate of increase significantly slowed (Figure 6.4).

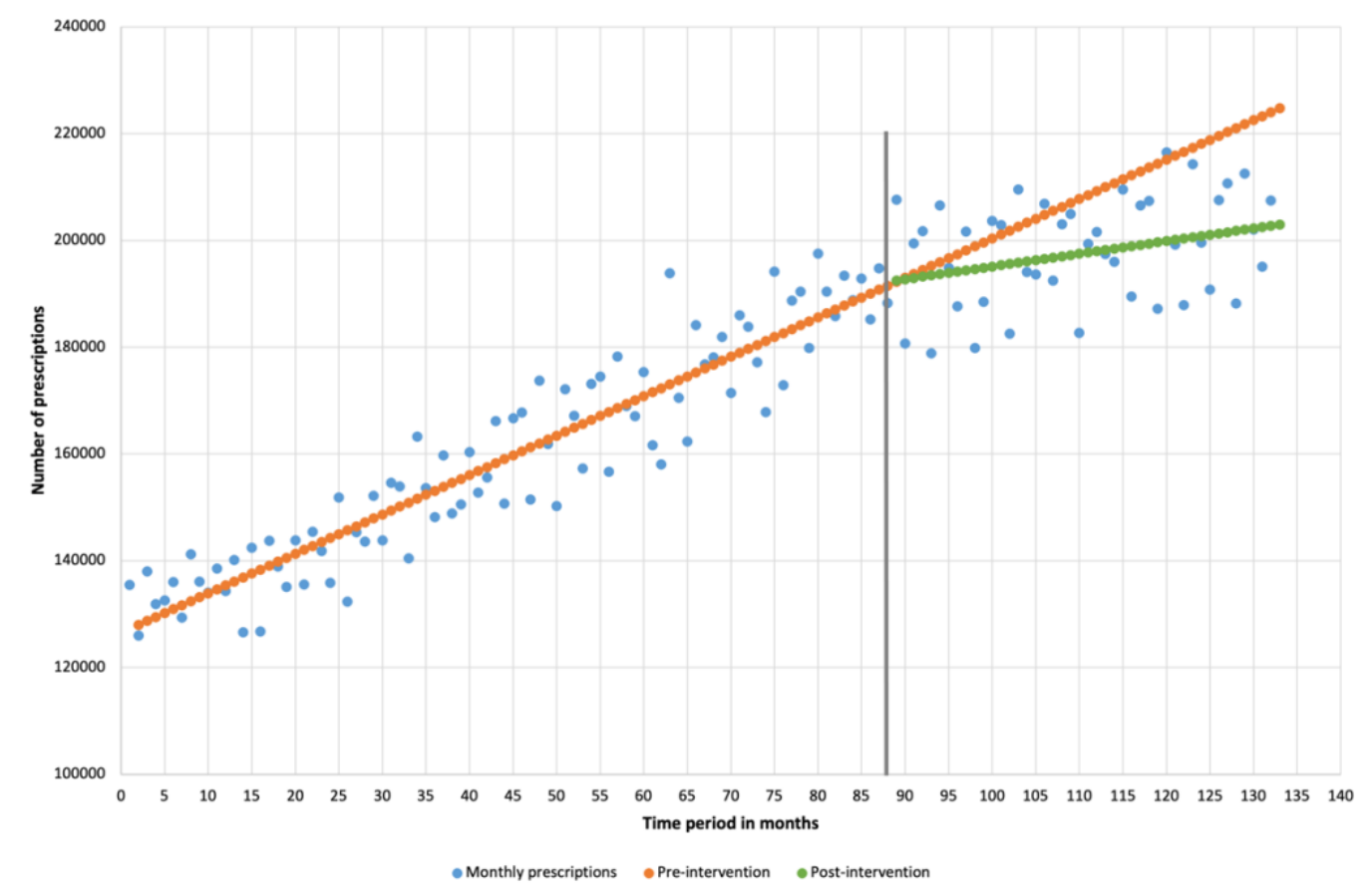

Figure 6.4: Plot of the number of opioid prescriptions between January 2005 (month 1) and December 2015 (month 132).

The solid grey line represents publication of National Prescribing Indicator 2012-13 which focussed on increasing the percentage of morphine as a total of all strong opioid prescribing

\subsubsection{Weak opioids}

Weak opioid prescribing was not the focus of the NPI 2012- 2013. However, following its introduction, the ITSA model demonstrated a non-significant step- 
down in prescribing and a subsequent trend of reduction in the number of monthly prescriptions issued (Table 6.3).

Table 6.3: Results of interrupted time series analysis of weak opioid prescribing data

\begin{tabular}{|c|c|c|c|c|}
\hline \multicolumn{5}{|c|}{ Parameter estimates } \\
\hline Variable & $\begin{array}{l}\text { Estimate } \\
(95 \% \mathrm{Cl})\end{array}$ & $\begin{array}{c}\text { Standard } \\
\text { Error }\end{array}$ & t Value & $\begin{array}{l}\text { Approx } \\
\operatorname{Pr}>|t|\end{array}$ \\
\hline Intercept ( $\left.ß_{0}\right)$ & 121148 & 1111 & 109.07 & $<.0001$ \\
\hline $\begin{array}{r}\text { Pre-intervention } \\
\text { trend }\left(ß_{1}\right)\end{array}$ & $\begin{array}{c}569.8058 \\
(528.62-610.99) \\
\end{array}$ & 21.0116 & 27.12 & $<.0001$ \\
\hline $\begin{array}{r}\text { Change in level } \\
\left(ß_{2}\right)\end{array}$ & -313.1082 & 1457 & -0.21 & 0.8302 \\
\hline $\begin{array}{r}\text { Post-intervention } \\
\text { trend }\left(\Omega_{3}\right)\end{array}$ & $\begin{array}{c}-594.2796 \\
(-705.29--483.27)\end{array}$ & 56.6371 & -10.49 & $<.0001$ \\
\hline AR1 & 0.4658 & 0.0570 & 8.17 & $<.0001$ \\
\hline AR3 & -0.2956 & 0.0632 & -4.68 & $<.0001$ \\
\hline AR12 & -0.4102 & 0.0662 & -6.20 & $<.0001$ \\
\hline
\end{tabular}

Graphical modelling of the ITSA reinforces the negative trend (Figure 6.5) noted in the number of weak opioid prescriptions issued each month in the study period following the intervention at month 88 (April 2012). The overall effect is to plateau the data for the remainder of the study period. This corroborates previous trends described in Chapter 3.

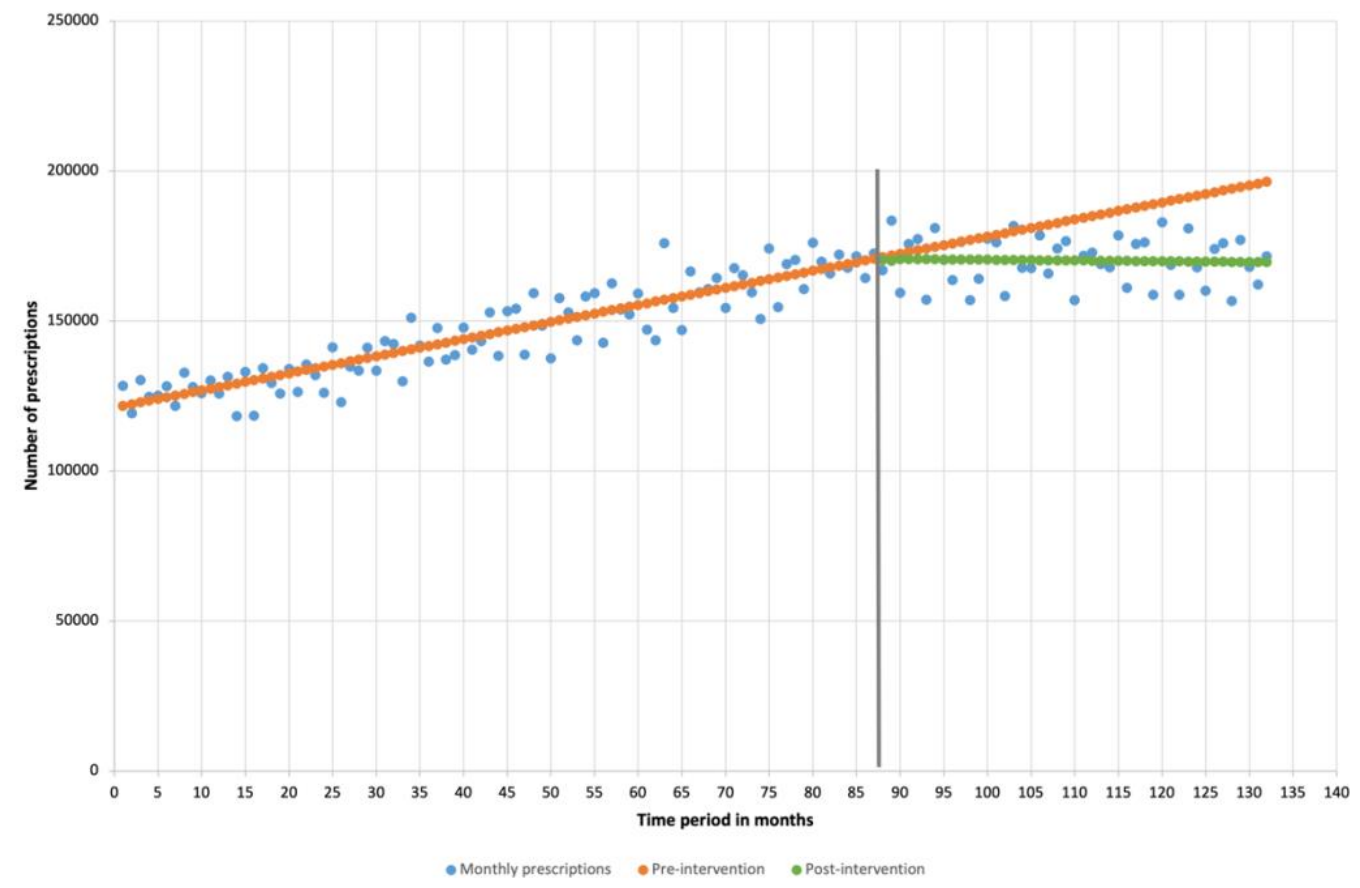

Figure 6.5: Plot of the number of weak opioid prescriptions between January 2005 (month 1) and December 2015 (month 132).

The solid grey line represents publication of National Prescribing Indicator 2012-13 which focussed on increasing the percentage of morphine as a total of all strong opioid prescribing 
As with the overall trends, AR3 and AR12 values suggest seasonal variation (Table 6.3) but this does not appear to have affected the overall pattern of prescribing noted in the model.

\subsubsection{Strong opioids}

Strong opioid prescribing prior to the intervention demonstrates a significant positive trend based on the ITSA model used and in line with the data already discussed (Chapter 3 and Chapter 4). There was a non-significant step-up in the model, following the intervention as there was with the overall prescribing ITSA (Table 6.2), followed by a continued positive trend. However, the rate of increase appears reduced post-intervention based on the model output (Table 6.4).

Table 6.4: Results of interrupted time series analysis of strong opioid prescribing data

\begin{tabular}{|c|c|c|c|c|}
\hline \multicolumn{5}{|c|}{ Parameter estimates } \\
\hline Variable & $\begin{array}{l}\text { Estimate } \\
(95 \% \mathrm{Cl})\end{array}$ & $\begin{array}{c}\text { Standard } \\
\text { Error }\end{array}$ & t Value & $\begin{array}{l}\text { Approx } \\
\operatorname{Pr}>|t|\end{array}$ \\
\hline Intercept $\left(ß_{0}\right)$ & 6253 & 453.8995 & 13.78 & $<.0001$ \\
\hline $\begin{array}{r}\text { Pre-intervention } \\
\text { trend }\left(ß_{1}\right)\end{array}$ & $\begin{array}{c}170.1021 \\
(152.11-188.09)\end{array}$ & 9.1780 & 18.53 & $<.0001$ \\
\hline $\begin{array}{r}\text { Change in level } \\
\left(\beta_{2}\right)\end{array}$ & 430.7013 & 351.6284 & 1.22 & 0.2230 \\
\hline $\begin{array}{r}\text { Post-intervention } \\
\text { trend }\left(B_{3}\right)\end{array}$ & $\begin{array}{c}112.3238 \\
(74.74-149.91)\end{array}$ & 19.1771 & 5.86 & $<.0001$ \\
\hline AR1 & 0.1361 & 0.0694 & 1.96 & 0.0521 \\
\hline AR3 & -0.3425 & 0.0676 & -5.07 & $<.0001$ \\
\hline AR12 & -0.3479 & 0.0867 & -4.01 & $<.0001$ \\
\hline
\end{tabular}

The trend post-intervention appears more rapid when depicted graphically (Figure 6.6). Previous trend analysis (Chapter 3) confirmed a slowing in annual percentage increase in the latter half of the study period which the ITSA model corroborates. 


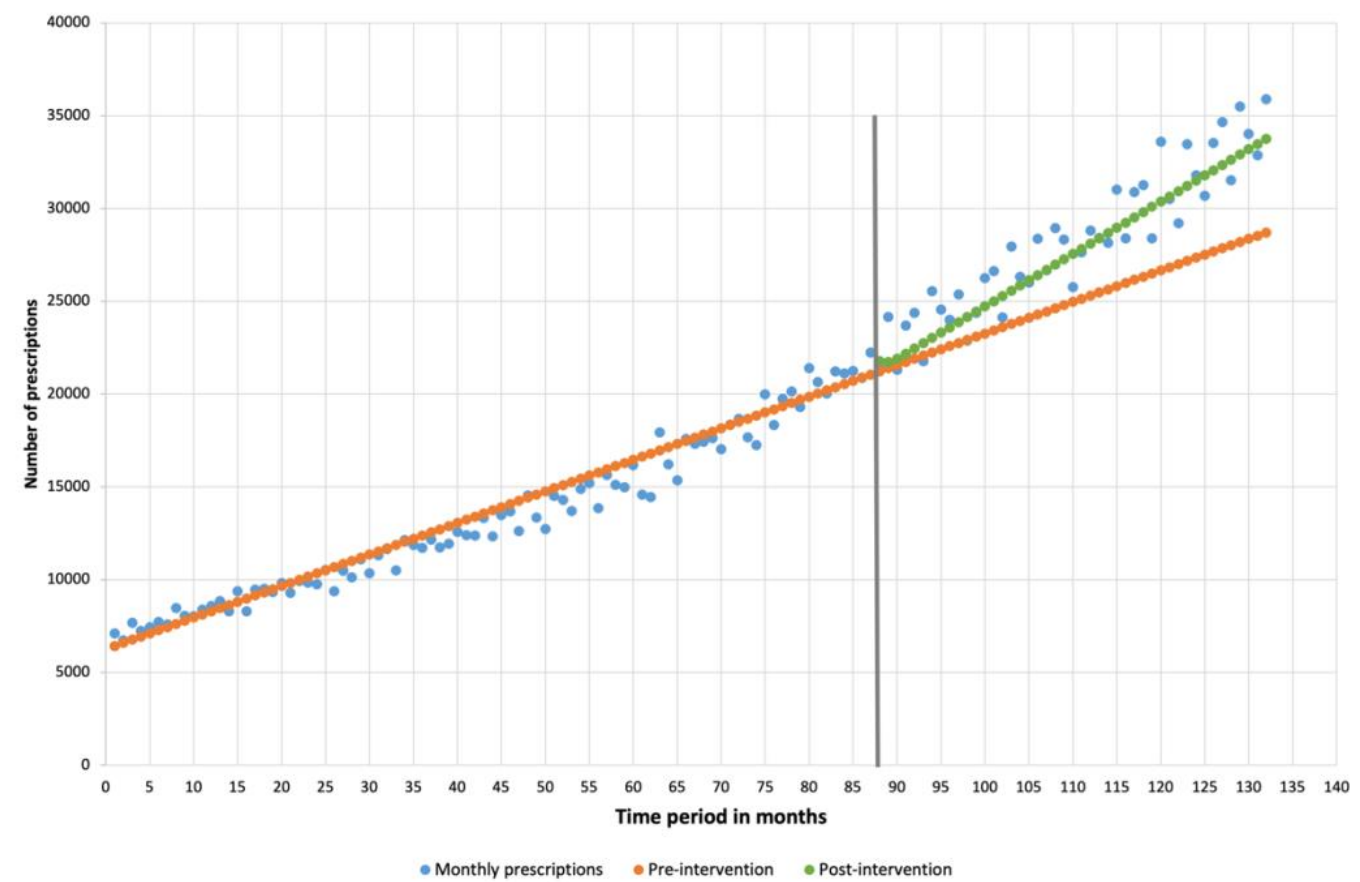

Figure 6.6: Plot of the number of strong opioid prescriptions between January 2005 (month 1) and December 2015 (month 132).

The solid grey line represents publication of National Prescribing Indicator 2012-13 which focussed on increasing the percentage of morphine as a total of all strong opioid prescribing

\subsubsection{Changes examined by gender}

ITSA by gender and examining all-type opioid prescribing clearly showed positive trends in monthly opioid prescriptions being issued prior to the intervention at month 88 (Table 6.5).

Table 6.5: Output from Intermittent Time Series Analysis by gender (full data in Error! Reference source not found.)

\begin{tabular}{rcccc}
$\begin{array}{l}\text { Estimate } \\
(\mathbf{9 5 \%} \mathrm{Cl})\end{array}$ & Intercept $\left(\boldsymbol{\beta}_{\mathbf{0}}\right)$ & $\begin{array}{c}\text { Pre-intervention } \\
\text { trend }\left(\boldsymbol{B}_{1}\right)\end{array}$ & $\begin{array}{c}\text { Change in level } \\
\left(\boldsymbol{B}_{2}\right)\end{array}$ & $\begin{array}{c}\text { Post- } \\
\text { intervention } \\
\text { trend }\left(\boldsymbol{B}_{3}\right)\end{array}$ \\
\hline Men & 47169 & 316.6277 & 573.3097 & -224.7378 \\
\hline Women & 80139 & 421.1498 & 186.3049 & -275.5532 \\
\hline
\end{tabular}

The model demonstrated the gradient for men was lower than for women, suggesting a slower rate of increase in monthly prescription numbers (Table 6.5). 


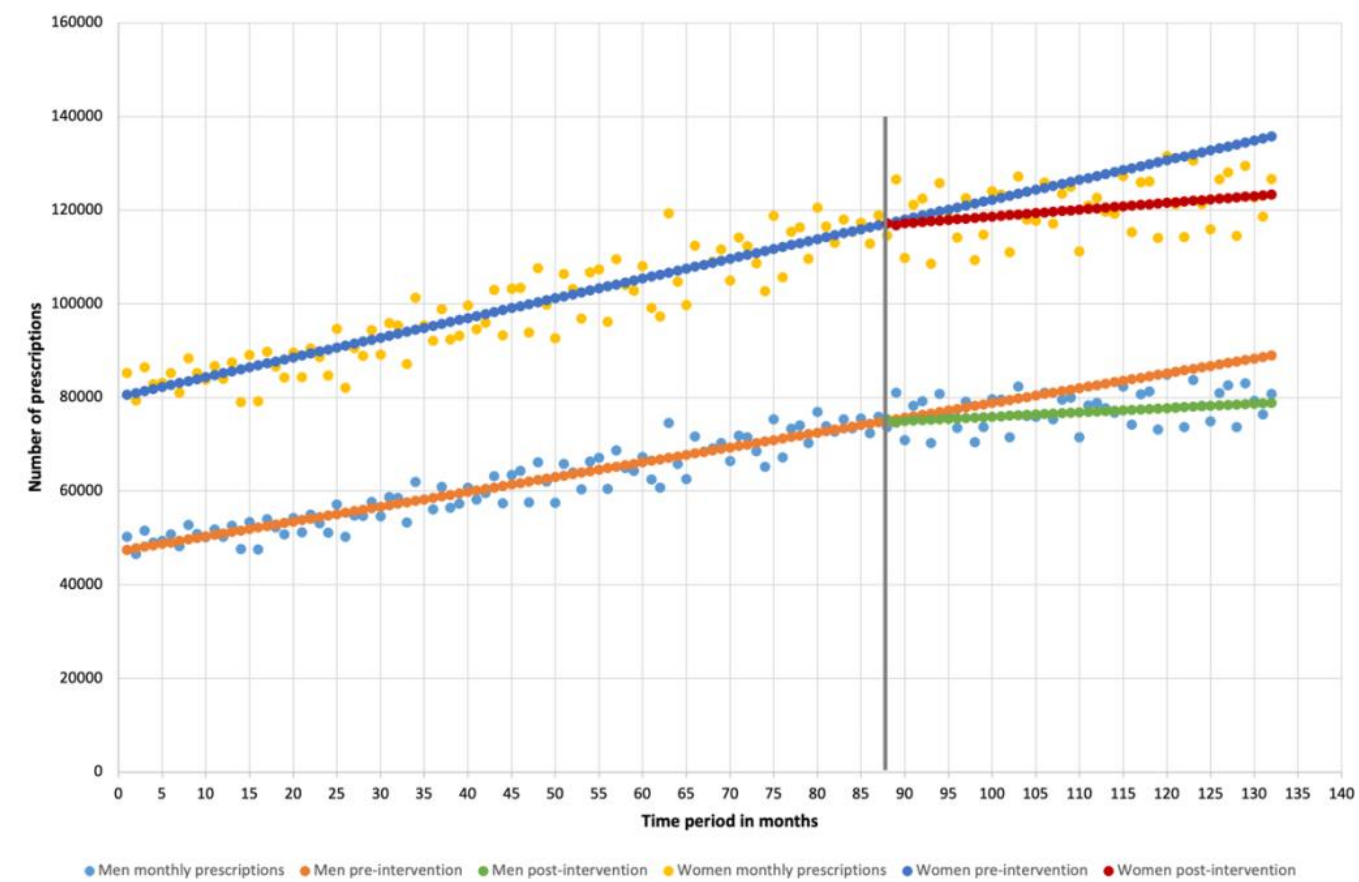

Figure 6.7: Plot of the number of opioid prescriptions issued by gender, between January 2005 (month 1) and December 2015 (month 132).

The black line is the intervention as previously described.

The intervention was followed by non-significant step-ups and then both genders were shown to have a reduction in overall numbers of prescriptions issued thereafter. As with previously described models (Section 6.5.2.1, Section 6.5.2.2 and Section 6.5.2.3) seasonal variation was demonstrated within both models but did not appear to influence the trends overall (Figure 6.7).

\subsubsection{Changes by socioeconomic deprivation}

ITSAs conducted for each socioeconomic deprivation quintile reinforced previously described differences between the most and least deprived areas (Section 4.5 and Section 5.5.4). All quintiles had positive trajectories prior to the 2012 intervention (Table 6.6).

Table 6.6: Output from Intermittent Time Series Analysis by socioeconomic deprivation quintile (full data in Appendix E)

\begin{tabular}{ccccc}
\hline Estimate & Intercept $\left(\boldsymbol{B}_{0}\right)$ & $\begin{array}{c}\text { Pre-intervention } \\
\text { trend }\left(\boldsymbol{B}_{1}\right)\end{array}$ & $\begin{array}{c}\text { Change in level } \\
\left(\boldsymbol{(}_{2}\right)\end{array}$ & $\begin{array}{c}\text { Post- } \\
\text { intervention } \\
\text { trend }\left(\boldsymbol{\beta}_{3}\right)\end{array}$ \\
\hline WIMD1 & 37163 & 194.9836 & 418.1434 & -135.0756 \\
\hline WIMD2 & 28836 & 167.1922 & 242.2058 & -128.7473 \\
\hline WIMD3 & 24929 & 142.9455 & 189.2449 & -92.1285 \\
\hline WIMD4 & 16719 & 118.6457 & -140.2672 & -87.9440 \\
\hline WIMD5 & 15083 & 95.1271 & 59.3972 & -73.9886 \\
\hline
\end{tabular}


Seasonal variation was detected, as with previous models, but again did not appear to influence the trends either pre- or post-intervention (Table 6.6).

Following the introduction of NPI 2012-2013, prescribing rates in all quintiles reduced, demonstrated by negative post-intervention trends.

\subsubsection{Seasonal variation}

Seasonal variation was observed throughout the year. The largest variation was seen between January, February and March (Table 6.7) but did not have an effect on the overall upward trend in prescription numbers over the study period.

Table 6.7: Average seasonal effects of opioid prescribing based on time series analysis and displayed by type of opioid prescribed

\begin{tabular}{rccc}
\multicolumn{1}{l}{ Month } & \multicolumn{3}{c}{ Average seasonal effects } \\
\cline { 2 - 4 } & Total & Weak & Strong \\
\hline January & 2349.183 & 2348.167 & 1.016667 \\
\hline February & -11018.1 & -9832.21 & -1185.9 \\
\hline March & 6107.283 & 5518.267 & 589.0167 \\
\hline April & -627.197 & -606.97 & -20.2273 \\
\hline May & -199.182 & -165.235 & -33.947 \\
\hline June & -1192.87 & -939.5 & -253.371 \\
\hline July & 3358.129 & 2836.568 & 521.5606 \\
\hline August & -2008.03 & -1831.77 & -176.265 \\
\hline September & -292.341 & -211.159 & -81.1818 \\
\hline October & 2915.683 & 2674.175 & 241.5083 \\
\hline November & -1593.24 & -1365.79 & -227.45 \\
\hline December & 2502.383 & 1850.45 & 651.9333 \\
\hline
\end{tabular}

The seasonal variation seen with weak opioid prescriptions mirrored the overall pattern (Figure 6.8), which was not unexpected given the proportion of weak opioids prescribed compared to strong opioid medicines. Strong opioid prescribing fluctuated in a similar vein to the overall trend, however, the degree of fluctuation was much less than seen for total and weak opioid data (Figure $6.8)$. 


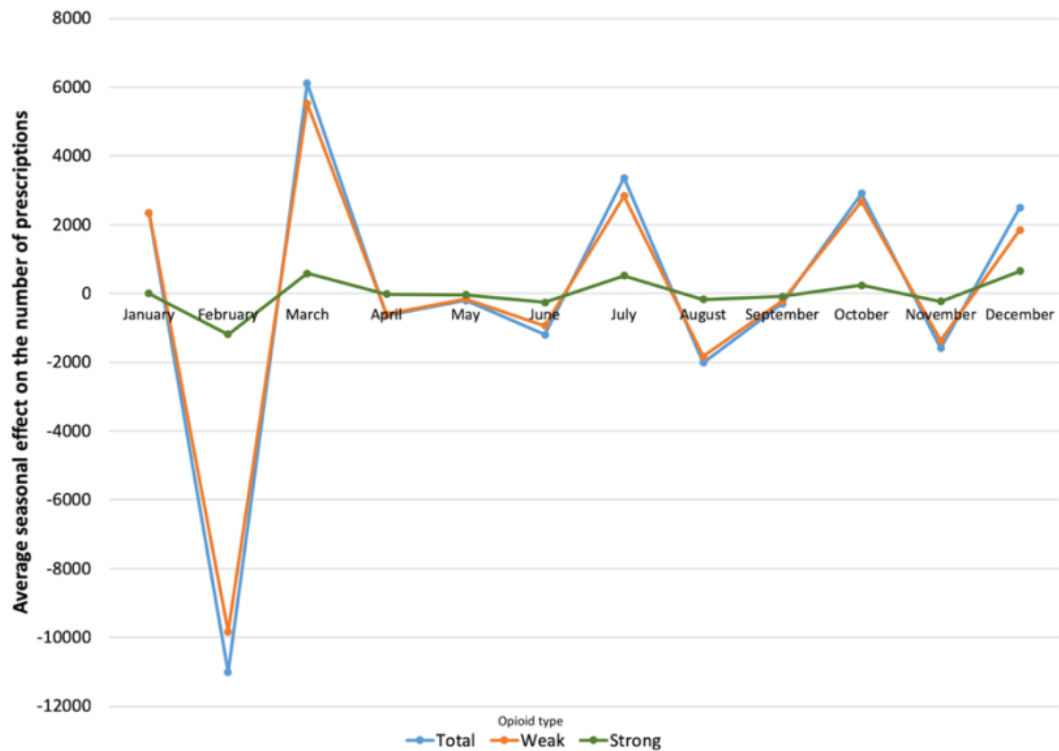

Figure 6.8: Graphical depiction of average seasonal variation in the number of opioid prescriptions, by type of opioid issued from time series analysis

\subsubsection{Predictions}

Predictions were made for the potential trends in future opioid prescribing with different models compared (Figure 6.9). Whilst all models had high coefficients of determination (Table 6.8), certain predictions appeared less likely from a clinical perspective. For example, the dramatic decline in prescribing predicted by the $3^{\text {rd }}$ order polynomial trendline would seem an improbable outcome based on clinical experience. Similarly, the linear prediction would seem to be less reflective of the slowing in the number of annual prescriptions noted towards the end of the study period.

Table 6.8: Comparison of predicted trends in opioid prescribing using trendline analysis

\begin{tabular}{rcccc}
\hline & \multicolumn{3}{c}{ Polynomial } & Linear \\
\cline { 2 - 4 } & $\mathrm{x}^{2}$ & $\mathrm{x}^{3}$ & $\mathrm{x}^{4}$ & \\
\hline $\mathbf{R}^{\mathbf{2}}$ & 0.9882 & 0.9956 & 0.9963 & 0.9695 \\
\hline Predicted values & & & & \\
January 2018 & 207067 & 185784 & 213668 & 232144 \\
January 2020 & 219378 & 150439 & 215529 & 247242 \\
\hline
\end{tabular}




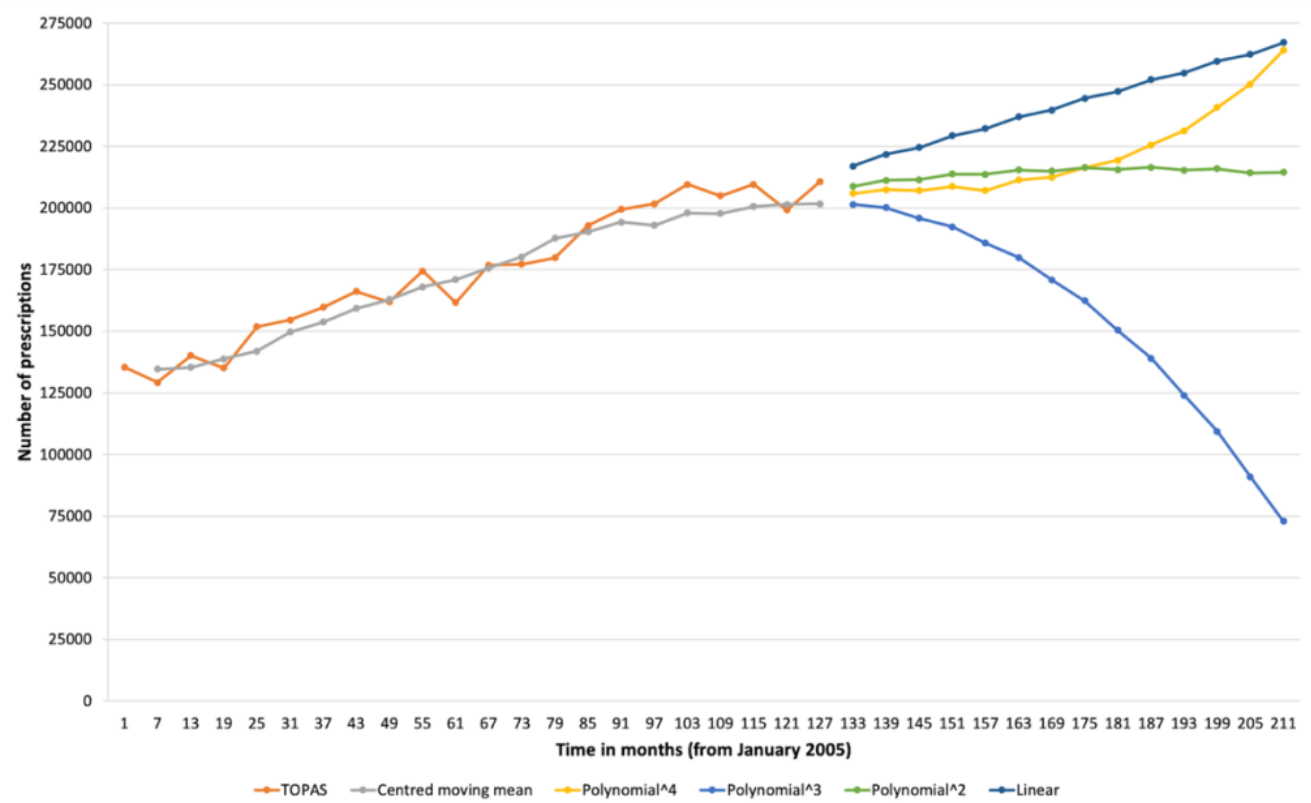

Figure 6.9: Predicted trends in the number of opioid prescriptions issued using different time-series trendline analysis

Predictions were made for all the TSAs conducted for this study. However, whilst the $\mathrm{R}^{2}$ values indicated a high degree of accuracy for the predictions made, the trends themselves were made from non-stationary data and so predictive quality was likely to actually be low. The predictions for overall opioid prescribing, weak and strong opioid prescribing are provided in Figure 6.9 and the rest are presented in Appendix E.

\subsection{Discussion}

\subsubsection{Summary and reflection on findings}

Time series analysis reinforced the findings from the trend analyses reported in Chapter 3 by confirming the large increases in the number of prescriptions issued between 2005 and 2015. Fluctuations in prescribing followed a similar pattern and did not strongly correlate to a seasonal pattern, although seasonal variation was detected. However, fluctuations were much less marked for strong opioid prescribing. It appears the prescribing data was sufficiently accurate to predict the progress of prescribing during the eleven years of the study.

Less certain was the influence of changes in controlled drug prescribing legislation over the period examined. Whilst the rate of overall prescribing was starting to slow from around 2013, this does not appear to be linked to 
legislative changes. The introduction of the NPI for $2012-2013$, focusing on increasing the percentage of morphine prescribed as a proportion of all strong opioid prescribing does appear to have influenced prescribing. Although strong opioid prescribing continued to increase after the NPI was launched (Figure 6.6), the rate of increase was tempered. This could be a result of drawing attention to the concerns about rising opioid levels in general, or a concerted effort to avoid strong opioids where possible, despite the issue of NICE guidance which could be seen as encouraging opioid use for some common non-cancer conditions. Weak opioid prescribing demonstrated a reduction following the intervention (Figure 6.5). Why this would be the case following an NPI focused on strong opioids is not clear. Around this time, concern was growing about opioid associated deaths, especially those associated with tramadol, which was classed as a weak opioid for this study (Office for National Statistics 2020). It is possible this was an influence on choice of opioid.

Predicting future changes in prescribing, on the basis of what was observed before, proved unsatisfactory. Whilst the models used to predict forthcoming trends appeared reliable in terms of their $\mathrm{R}^{2}$ values, a clinical 'sense-check' suggests some of the predictions would be extremely unlikely in practice. It is difficult to state with confidence whether TSA is a useful method of predicting future behaviour, not least as clinical decision making is an individual process which makes forecasting difficult (Mclntosh et al. 2016; Murshid and Mohaidin 2017). Further, this analysis seems to illustrate more work is needed to find an effective method of implementing change, whether through guidelines or other means. Despite the introduction of legislation designed to improve patient safety and governance of prescribers, as well as guidance to affect prescribing choices, opioid prescribing continued to increase. Determining the 'buttons' that need to be pushed to get professionals on board with the changing evidence base will be essential if meaningful change in practice is to occur. 


\subsubsection{Is TSA a useful means of predicting prescribing trends or changes?}

The most notable feature of the predictions made for opioid prescribing in Wales, from the TOPAS data, was their variance. It is more common to see TSA being used to compare actual versus predicted trends, rather than simply predicting forward, as attempted here. Helgason (2008) used TSA as a means of developing an algorithm for predicting drug selection based on prescribers' previous choices and combinations of medicines prescribed (Helgason 2008). The use of TSA in this instance was to reduce the time taken to choose medicines, as part of an electronic prescribing programme. There was up to $70 \%$ accuracy found in the predictions developed although it was noted that lack of data for less commonly prescribed medicines had the effect of reducing the reliability of the predictions (Helgason 2008).

Predicting the outcome of prescribing is difficult, due to the complex nature of pharmacokinetics and pharmacodynamics in each individual (Larney et al. 2018). For example, TSA combined with cluster analyses were shown to be helpful in predicting the response to pregabalin therapy in a group of patients with painful diabetic neuropathy, when compared to their real-time outcomes (Alexander et al. 2019). However, the model was not able to accurately predict which patients would develop adverse effects or discontinue treatment. Predictions, consequently, were binary, i.e., either someone would have a positive response or not (Alexander et al. 2019).

Studies examining the impact of legislation on prescribing trends have tended to use TSA as one part of the analysis (Gomes et al. 2014; Chang et al. 2016; Martin et al. 2018) suggesting the method may not have sufficient forecasting power on its own. The accuracy of predicting events declines the further into the future projections are attempted (Sherman et al. 2017). The complexity of decision making involved in prescribing decisions is difficult to account for in statistical modelling, as clinical decision making is rarely subject to only one or two static factors (Sherman et al. 2017). 
What effective TSA studies have in common is access to a large volume of data (Härdle et al. 1997; Bernal et al. 2017). The TSA presented here evaluated more than 22 million data points over the study period, which assists the precision of the analysis. Accuracy of TSA improves with data volume (Bernal et al. 2017) . Using national data sources such as the SAIL databank, where large datasets are available, are therefore suited to time series analyses of trends (Falk et al. 2012; Bernal et al. 2017), provided the limitations of the method for clinical decision making are taken into account.

\subsubsection{Effects of legislation on prescribing trends}

It was somewhat surprising that changes in UK law (Dyer 2004) designed to strengthen the checks and safety of prescribing opioid medicines appeared to have little effect on overall prescribing rates observed in this study. Jani et al. (2020) made the same observation following a large study examining opioid prescribing trends in the UK between 1998 and 2016. A reduction in tramadol, oxycodone and fentanyl was observed in primary care prescribing from 2012 onwards (Jani et al. 2020), as it was in Wales. Jani et al. suggests this may have been related to national regulations introduced in 2013, although, as with TOPAS data, it did not appear to affect prescribing rates of all opioid analgesics equally (Jani et al. 2020). This appears to be in contrast to earlier reported findings outside of the UK (Gomes et al. 2014) where a noticeable reduction in opioid prescribing followed Canadian law changes that increased prescription monitoring. Conversely, legislation changes introduced in Italy, increasing access to opioids for a wider range of conditions and consequent growth in the number of prescriptions for strong opioids in particular, was noted (Musazzi et al. 2018).

Whilst the two studies had different outcomes, the legislation and monitoring put in place both had the desired outcome. In Italy, for example, prior to the freeing up of prescribing, there was concern that pain was being undertreated (Musazzi et al. 2018). The change in the law and reimbursement allowed opioid analgesics to be prescribed and dispensed in more healthcare sectors, seemingly reducing inequality in pain management (Musazzi et al. 2018). The fear in 
Canada, however, was over-use and inappropriate prescribing of opioid analgesics, especially in the context of the opioid-crisis that was already hitting North America hard at that time (Dhalla et al. 2011; Gomes et al. 2014). The new legislation aimed to strengthen checks on prescribers and patients, by recording and monitoring who was issuing and receiving opioid analgesic prescriptions. Both interventions resulted in a reduction in prescribing (Gomes et al. 2014).

Legislation introduced in the UK over the years of this study was not focussed on the clinical use of opioid analgesics, unlike that in Italy and Canada during the same period (Gomes et al. 2014; Musazzi et al. 2018). Instead, their aim was to improve opioid analgesic prescribing and dispensing monitoring, enhanced record keeping and more clearly setting out how general practitioners in particular should be supervised and investigated in terms of concerns about their practice (Smith 2004; Department of Health 2013). Even when the Shipman inquiries (Section 1.11) were still fresh in the minds of practitioners, there was doubt how effective the new measures would be in preventing something similar again (Gallagher 2006). The over-riding aim of the changes was to prevent harm being caused to people subjected to opioid treatment and who were determined vulnerable on the basis of being frail or at the end of life (Dyer 2004; Baker 2004).

Relating this all back to prescribing trends in Wales, it seems reasonable to suggest that the limited effect of legislation might in part, be due to how it was implemented in practice. For example, changes to prescription writing requirements were mostly covered by general practice prescribing software (National Institute for Health and Care Excellence 2016). The more stringent documentation that pharmacists are required to complete and maintain (Royal Pharmaceutical Society of Great Britain 2021) has little impact on prescribers themselves, unless they are part of a dispensing practice. Perhaps changes in practice are more likely when prescribing becomes more inconvenient to the prescriber? 
The response to the opioid crisis in North America and the USA in particular, has been to impose and enforce fairly draconian laws and payment restrictions on prescribers (Ayres and Jalal 2018; Martin et al. 2018; Schatman and Shapiro 2019). Such changes have resulted in the sudden cessation of opioid prescriptions for thousands of people (Mundkur et al. 2017; Singer et al. 2019). Commentators have suggested such strict measures are directly responsible for people seeking out illicit opioids through cryptomarkets (Martin et al. 2018; Mundkur et al. 2018) and direct sales. Others have suggested stable prescribing rates, in tandem with rising synthetic opioid (fentanyl and diamorphine/heroin) deaths point more towards rising rates of illicit opioid use, rather than a problem of over-prescription (Singer et al. 2019). High rates of overdose-related deaths are still being seen in the USA, with 49,860 opioid associated deaths reported in 2019 (Centers for Disease Control and Prevention 2021).

\subsubsection{Abolition of prescription charges}

Prescription charges were abolished by the Welsh National Assembly Government in 2007, as a means of improving chronic condition management. The change did not appear to have a direct effect on the rate of opioid prescribing, based on the analysis. This confirms the findings of the (then) Welsh National Assembly Government who conducted a review of the changes 3 years after their introduction (National Assembly Government 2010). Prior to the removal of charges, $88 \%$ of all prescription items were dispensed without charge in Wales (National Assembly Government 2010). Consequently, the change is likely to have affected only a small proportion of prescribing and more likely to have been in the more affluent areas of Wales, where overall opioid prescribing levels were lower.

\subsubsection{Effects of national prescribing indicators and guidelines on prescribing trends}

On the basis of the interrupted-TSA conducted, there was some, albeit limited, influence of NPIs on overall opioid prescribing trends between 2005 and 2015. This contrasts with findings from an earlier study which found non-steroidal anti- 
inflammatory prescribing was influenced by the introduction of NICE guidelines for osteoarthritis (Bedson et al. 2013). The same study noted that the advice not to prescribe and subsequent withdrawal of co-proxamol, from the UK market in 2005 on safety grounds, led to a substantial reduction in its use (Bedson et al. 2013) (Appendix D). However, being unable to access a medicine is different to advising against its use whilst it remains available. The rapid reduction in coproxamol use in Wales and the UK in general, was aided by not being able to order it and its cost subsequently becoming prohibitive. This has not been the case with other opioids. As these TSA's demonstrate, whilst co-proxamol reduced in Wales, it was rapidly replaced and superseded by other analgesics. It may be hypothesised that the message around opioid safety was perhaps viewed as being a concern for co-proxamol but not extrapolated in the minds of prescribers much farther, given no indication of changes in prescribing rates were detected until 2012.

NICE guidance for osteoarthritis issued in 2008 did not make clear recommendations for or against the use of opioid analgesics (National Collaborating Centre for Chronic Conditions 2008). The 2009 guidance for persistent low back pain specifically recommended the short-term use of strong opioids (National Institute for Health and Clinical Excellence 2008). However, they did not provide information on monitoring effectiveness or stopping opioid analgesics, which were not of benefit. The TSA did not examine data by duration of prescribing, so it was not confirmed if the increase was in part due to more short-term prescriptions. However, the rise in prescribing noted up until 2012, was underway prior to the publishing of the NICE guidelines. Whilst analgesic related NPIs were in force throughout the study period, those related to opioids specifically, were not introduced until the 2012 to 2013 financial year (All Wales Medicines Strategy Group 2013). There was a slowing in the rate of increase after 2012, but it is not clear if it was stimulated by the NPIs themselves. Taking the results from all the trend analyses conducted for TOPAS (Chapter 3, Chapter 4 and Appendix E), a more nuanced picture emerges. Annual tramadol prescribing began to reduce between 2013 and 2014, although the NPI aimed at 
reducing tramadol prescribing introduced in response to a marked increase in tramadol-related deaths in Wales was not launched until the 2014 to 2015 financial year (All Wales Medicines Strategy Group 2013; Welsh Analytical Prescribing Support Unit 2013). Morphine was the most frequently prescribed strong opioid in Wales throughout the study period (Appendix D). Prescribing increased annually between 2005 and 2015 and whilst the rate of increase by prescriptions per 1,000 population did not change following the NPIs introduction, the oral morphine equivalent dose did rise towards the latter part of the study (Appendix D). The implication of this finding may be that prescribers felt confident to prescribe higher doses, as well as to more people. This was not likely to have been the hope of those who developed the indicator.

Neither Welsh NPIs nor NICE clinical guidelines have a legal basis and so are reliant on local arrangements, in terms of any enforcement, which may account for their varied impact on opioid prescribing and clinical practice. In contrast, regulatory standards and guidelines introduced to British Columbia, Canada in 2016 , led to an average reduction of $57 \mathrm{mg}$ oral morphine equivalent (OMEQ) per month in overall prescribing and reduced dosing for people receiving long-term prescriptions (Morrow et al. 2019). Similar reductions have been noted where prescription monitoring systems, which allow prescribers to see if patients are accessing prescriptions from other sources, have been implemented (Gomes et al. 2014; Chang et al. 2016). Legal enforcement of national guidance (Dowell et al. 2016) has also been used to address rising overdose deaths in the USA, where prescription opioids and the prescribers have been implicated (Weisberg and Stannard 2013; Coyle et al. 2018). Consequently, overall rates of prescribing in the USA have reduced by over $40 \%$ (from 81.3 to 46.7 prescriptions per 100 people) between 2012 and 2019. Reductions have not been seen in all areas, however. More rural areas or areas with higher levels of socioeconomic deprivation, continue to report prescribing rate six times higher than the average (Centers for Disease Control and Prevention 2021). 
Although, NPIs are used to benchmark prescribing trends, there is not an available measure for assessing whether analgesic prescribing complies with clinical guidelines from NICE or elsewhere.

\subsection{Conclusion}

Time series analysis is a useful method of determining the influence of changes in prescribing. Using TSA as a lone means of predicting future events has limitations but may be helpful in combination with other analyses. Reviewing how effective previous guidelines or legislative changes are in securing practice transformation should be seen as an essential part of developing new ones. Understanding the factors which combine to promote change should help cultivate more effective methods of supporting meaningful modifications in future.

Whilst modest, the changes in tramadol prescribing in Wales most closely echo other successful combinations of legislation changes, education and prescribing guidance elsewhere (Gomes et al. 2014; Chang et al. 2016; Musazzi et al. 2018). The combination of efforts likely increased the attention paid to the problem and therefore, acted as an encouragement and reminder for change. Similar influences were not used in Wales for other opioid-related prescribing issues however, and this may be why there was little effect on opioid prescribing overall.

Devising and introducing new legislation to control, or direct prescribing might be necessary to demonstrate a commitment to patient safety. However, using examples where it has led to a positive change (Gomes et al. 2014; Chang et al. 2016; Fink et al. 2018; Welsh Government 2019), it appears law changes may be best introduced in combination with clinical guidelines or other forms of monitoring that more noticeably affect daily practice. Regardless of the method adopted to improve prescribing, whether it is guidance or legislation, implementation is key. Examining methods for improving the uptake and 
compliance with prescribing recommendations and monitoring prescribers compliance with them would be a useful piece of further research. 


\section{Phase 2}

\section{Chapter 7}

Healthcare resource utilisation associated with opioid prescribing and associated cost-analysis
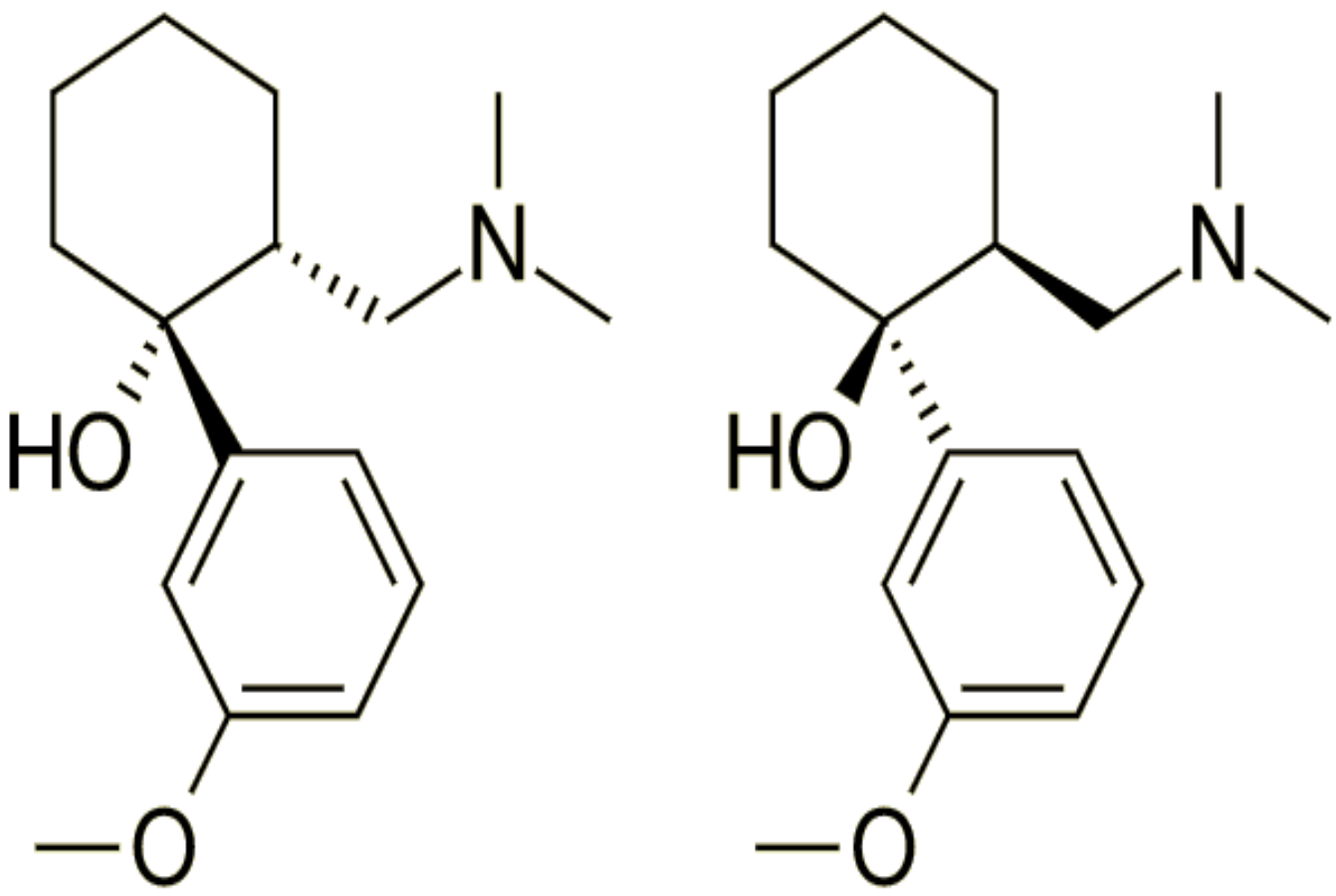

"It doesn't matter how many resources you have. If you don't know how to use them, it will never be enough."

Anon 


\section{Chapter 7 - Healthcare resource utilisation and cost- analysis associated with opioid prescribing}

\subsection{Chapter overview}

Phase 1 of the study reviewed the socioeconomic burden of pain and the use of opioid analgesics as part of its management. The results presented in Chapters 3 and 4 demonstrated large increases in the prescribing of all opioids in the 11 years examined, with women and older people receiving greater numbers of prescriptions. Prescribing in areas of greatest socioeconomic deprivation is much higher than in more affluent areas. Further, as set out in Chapter 5, marked increases in strong opioid prescriptions, added a disproportionate burden in oral morphine equivalent terms, in comparison to weak opioids. More people were receiving opioid prescriptions for longer periods of time by the end of the study duration, based on prescribing persistence data. As discussed in Chapter 6, the introduction of controlled drug legislation and to some extent NPIs in Wales, only limitedly tempered the rising rates of prescribing.

Phase 2 of the study now examines the combined effect of the trends reported so far, insomuch as it examines the impact of opioid prescribing on healthcare utilisation in Wales. For the duration each person received opioid prescriptions, their attendance at healthcare services in primary and secondary care were counted and compared to people with similar conditions but not receiving opioid prescriptions. A healthcare service-based cost-analysis was undertaken to provide an estimation of the potential impact of opioid analgesic use on healthcare budgets.

\subsection{Study objectives to be addressed in this chapter}

- To assess the frequency of primary and secondary healthcare attendance by patients with defined non-cancer pain conditions receiving opioid analgesic therapy

- To estimate healthcare service costs associated with the use of opioid analgesics 


\subsection{Background}

As time goes on, an increasing number of treatments, for a wider range of health conditions have become available. Simultaneously, people expect, and demand more say in what they want to receive in terms of healthcare. However, there will never be enough resources for everyone to have everything they want. This applies to healthcare as much as it applies in life.

\subsubsection{Efficiency}

Health economics attempts to maximise the output from a limited input. Two concepts, efficiency and equity form the central themes of health economic analysis (Phillips 2005). The most efficient system is one where changing the use of resources would not result in more benefit than is already being achieved. The implication is that all available resource is being used optimally and any reallocation, would require something to be given up elsewhere in the system (Phillips 2005). This phenomenon is also known as Pareto equilibrium (Kaplow and Shavell 2001) and could be argued is perhaps aspirational, rather than achievable in modern healthcare.

Efficiency can be derived in several ways. The NHS would appear to aim for efficiency saving most of the time. Commonly referred to as cost-effectiveness, the goal is to produce the same output for less money or to use the available resources to provide more services or treatments (Phillips and Thompson 2009). The reality, however, is that it is increasingly difficult to provide more without someone, somewhere losing out. Claxton et al. (2015) discussed this issue in relation to NICE changing its limits for cost-effectiveness of new therapies (Claxton et al. 2015). Claxton et al. suggested that, over time, the threshold for the acceptable cost per quality- adjusted life-year (QALY) considered by NICE had crept up. The maximum, previously considered to be a reasonable balance for healthcare services and society to bear, had in fact, become the minimum. It was postulated that the reason this had been allowed to happen, was due to the 
absence of those who truly bear the opportunity cost of the decision in decisionmaking processes (Claxton et al. 2015).

\subsubsection{Welfarism}

Welfare in economic terms can also be referred to as 'well-being'. Societal improvement is most likely to occur when an individual or organisation improves their own well-being, without that improvement being at the expense of another (Coast et al. 2008). Welfarism in health economic terms includes the notion that healthcare outcomes should be judged by their contribution to an individual's overall well-being (Brouwer et al. 2008; Coast et al. 2008). Positive health outcomes might be seen as equally important to good educational outcomes for example. Welfare theory would suggest therefore, that treating a person who manages their health condition and is able to generate good personal utility (well-being), is not an efficient use of resource. Instead, it is deemed more efficient to treat people who cope poorly with their health and whose utility is less. Cost-benefit analysis is used to aid welfare-based decisions (Kaplow and Shavell 2001; Shafrin 2008).

\subsubsection{Extra-welfarism}

Extra-welfarism centres on cost-effectiveness analysis. The theoretical basis of extra-welfarism assumes that people with the same condition value their health equally (Coast et al. 2008). Cost-effectiveness compares the cost and outcome of different interventions to determine which provides best value for money (The What is ...? series. 2014). Outcome measures in this approach are focused on health only and do not draw in non-health related utility (i.e., a treatment reduces reported pain intensity but there is not a measure of whether people are able to return to work). Extra-welfarism may also look to illicit a measure of willingness to pay from those who are providing or receiving the health intervention under examination (Gyrd-Hansen 2005). This is where the theory comes under strain as it is difficult to be assured a whole population values health equally (Gyrd-Hansen 2005; Brouwer et al. 2008; Bobinac et al. 2010). 


\subsubsection{What approach do we take to Heath Economic Analysis in Wales and the UK?}

In truth, there is no perfect approach to Health Economic Analysis (HEA). Nuance exists in every system, and it can be hard to measure outcomes across health and social care systems if data is not collected or available for analysis. On a theoretical basis, welfarism is often considered to be the preferable approach to decision making (Brouwer et al. 2008; Coast et al. 2008). Certainly, it seems sensible to view outcomes across a range of services and systems to truly understand their benefit. In reality however, it is not always realistic to pursue a welfarist approach in practice. Health economists are pragmatic and therefore, a range of measures are utilized, including cost-effectiveness (Coast et al. 2008).

\subsubsection{Equity and equality}

Equity differs from equality, insofar as it is based on need rather than giving all people the same. The International Monetary Fund (IMF), whilst not directly concerned with healthcare, have stated equity is ' $a$ worthy goal in and of itself because of its moral implication and its intimate link with fairness and social justice' (Expenditure Policy Division et al. 1998). Twenty years ago, when the need for equity in economic policy was being discussed, it was proposed that when equity is promoted, poverty is reduced and vice versa (Expenditure Policy Division et al. 1998; Braveman and Gruskin 2003). Braveman and Gruskin (2003) proposed health equity reduced the 'unequal opportunities to be healthy' commonly associated with being poor, disenfranchised racially, ethnically or religiously, being female or residing in rural locations (Braveman and Gruskin 2003). A welfare approach to healthcare could therefore be seen as the most equitable.

\subsubsection{Use of healthcare services}

It is accepted that people living with painful conditions access more healthcare resources than those without (Mehra et al. 2011; Bozic et al. 2012). Pain is often considered to be a symptom of an underlying condition; although increasingly, it 
can be considered as a condition in its own right (Raffaeli and Arnaudo 2017;

Treede et al. 2019; National Institute for Health and Care Excellence 2021).

People living with chronic pain especially, tend to have multiple co-morbidities (Macfarlane et al. 2020). For example, nearly $60 \%$ of people with neuropathic pain conditions were noted to have more than four co-morbid conditions (Berger et al. 2012). Conversely, over a third of people receiving at least one weak or strong opioid in a study of one year, did not have a defined diagnosis (Foy et al. 2016). Consequently, it can be difficult to determine whether reported healthcare use is linked to pain or another condition, unless specifically examined in the study.

\subsection{Method}

\subsubsection{Study design}

This phase of the study was designed as a retrospective, longitudinal casecontrolled study that examined data from people aged 18 years and over and who did not have a recorded diagnosis of cancer at any time between 2004 and 2015 on their Primary Care General Practice medical record.

\subsubsection{Cohort identification}

The cohort for Phase 2 were identified initially from the WLGP event database. ALFs for individuals with read-codes for at least one of six over-arching chronic pain diagnoses, namely back pain, neck pain, osteo or rheumatoid arthritis, fibromyalgia and neuropathic pain (Appendix F), on their Primary Care medical record were used to trace their interactions with other healthcare services through the previously discussed data linkage processes used by SAIL (Chapter 2).

\subsubsection{Case Subject group}

Case subjects were identified by having an opioid prescription issued within the study period (2005 to 2015 ). They were further stratified by the prescribing persistence (from less than 6 months up to 11 years) (Section 3.4.2.2 and Appendix F). 


\subsubsection{Control subject group}

SAIL analysts specified the method for creating the control-group, based on the criteria provided in the research protocol (Appendix A) and using methodology previously used by that organisation (Akbari and Torabi 2017). The control group was composed of people who had a recorded diagnosis of one of the six chronic pain diagnoses but no record of receiving an opioid within the time period they were controlled against the subject group.

Controls were matched to case subjects by age within the age-groups previously defined (Section 2.6.4), gender, WIMD, and availability of medical records in the period between 2005 and 2015. Medical records for control subjects were matched to case subjects for the duration (years) that the case subject received prescriptions for opioid analgesics.

Control subjects were matched to multiple case subjects based on the criteria described. For example, if a control subject had 6 years of medical records without receiving an opioid prescription, they could be matched to case subjects who had received prescriptions for any period up to 6 years. This method of matching, analysis allowed four controls for each case subject in the cohort study but also resulted in each control being used up to 20 times.

Healthcare service utilisation was measured for the duration each case subject was in receipt of opioid analgesic prescriptions. Control subject healthcare service use was measured for the same time duration as the case subjects to which they were matched.

\subsection{Data source}

As per the first study phase, split files were used to transfer person level data into the SAIL databank. The Phase 2 data sources comprised:

- Welsh Demographic Service Dataset (WDSD) which maintains a register of demographic detail for every Welsh resident who is registered with a Welsh 
GP practice. Information includes name, address, date of birth, GP practice and NHS number (Observatory 2020b).

- Welsh Longitudinal General Practice (WLGP) data (GP event data) which is extracted directly using Audit+, from the electronic health records maintained by each GP practice in Wales. Includes appointments and contacts between the practice and patient, symptoms, test results, diagnoses, and prescribed treatment (Secure Anonymised Information Linkage Databank 2021).

- Annual District Death Extract (ADDE) is taken directly from the Office for National Statistics' register of all deaths relating to people residing in Wales, even if their death occurred outside of the country (Secure Anonymised Information Linkage Databank 2021).

- Patient Episode Database Wales (PEDW) which can provide a record of all inpatient and day-case hospital activity. From this database, it is possible to extract admission and discharge dates, length of stay (LoS), diagnoses, operative and investigative procedures undertaken as well as outcome data such as discharge destination (Secure Anonymised Information Linkage Databank 2021).

- Emergency Department data which provides date of attendance, method of arrival (e.g., walk-in, ambulance, etc.), injury and accident details and whether the person is admitted to hospital from the emergency room. Data is collected and coded at each hospital, extracted, and initially encrypted by the NHS Wales Informatics Service (Secure Anonymised Information Linkage Databank 2021).

- Outpatient data which can provide dates of attendance, whether it was a first or repeat attendance and which specialty the person was attending to see. Data is extracted from NHS Wales Informatics Service who, in turn, use data collected and coded at each hospital in Wales through the Patient Administrative System (Secure Anonymised Information Linkage Databank 2021). 


\subsubsection{Data identification}

Data extraction specification was requested based on the study protocol (Appendix A). Read-codes (NHS Digital 2015) for prescribable opioid preparations were the same as for Phase 1 of the study. Identification codes for in-patient specialties were taken from the International Statistical Classification of Diseases and Related Health Problems, $10^{\text {th }}$ version (ICD-10), used internationally and defined by the World Health Organisation. Codes were also taken from the Systemised Nomenclature of Medicine - Clinical Terms (SNOMED-CT), an international standardized vocabulary of clinical terms used throughout the National Health Service for collecting activity and intervention data (NHS Digital 2021).

Extensive lists of codes for diagnoses, tests, investigations, adverse events (ADE), specialty referrals, admissions and procedures were developed from the Clinical Terminology Browser, SNOMED-CT, and the ICD-10 lists. However, due to constraints in extraction capacity within SAIL, detailed analysis was deemed unfeasible. Consequently, extensive code lists for diagnoses, tests and investigations had to be reduced to a concise list of overarching title codes for referral to secondary care, blood tests and imaging (Appendix F). This resulted in far fewer 'flags' for these items within the extracted dataset than would be expected for the number of subjects included. Analysis was still performed but with caveats applied.

Demographic data collated for each study individual included gender, age, and deprivation data (WIMD2011), using the anonymised linkage systems of SAIL (Chapter 2).

The data was subjected to repeated cross-sectional sampling to determine the number of highlighted events in all relevant Healthcare sectors. Costings were applied to all units of healthcare utilisation that could be attributed from the extracted data. 


\subsection{Cost Analysis}

\subsubsection{Costs included in the analysis}

The analysis of total healthcare costs associated with opioid use included:

- Costs of primary care general practice attendances (including staff time, tests, investigations, and imaging that resulted from appointment, where recorded)

- Costs of emergency department and out-patient attendances

- Costs of in-patient attendances including day/night costs and excess day charges

Insufficient data was available to allow costing of medicines or productivity losses. Furthermore, no quality-of-life data was recorded. Data regarding interventions made during attendance in any sector and detail of whether hospital admission was planned or unplanned could not be extracted from SAIL. Consequently, weighted average costs were assigned for each type of attendance.

Costs are expressed as 2014/15 UK pound sterling (f), which avoided the need to calculate inflation over the study period. Costs were not discounted as the data analysed was historical and a period of follow-up after the study end was not included.

\subsubsection{Cost of healthcare resource use}

Healthcare resource use, including Primary Care General Practice consultations, emergency department and out-patient attendances and in-patient stays, were collated from the different datasets available within the SAIL databank. Due to limitations in coding, only tests, investigations and imaging recorded using core codes (overarching codes for e.g., referral) were flagged. Consequently, this limited the number of people for whom those applied, although it applied equally to case and control. 
Each anonymised individual, linked by the methods previously described

(Chapter 2), was traced and every recorded attendance at any healthcare service 'flagged'. A final count for each of those attendances was included in a data table which had one line per individual. In-patient stays were recorded in more detail on a second table. This provided data on the admitting and discharging specialty, but interventions made during each admission were not recorded.

For Primary Care practice attendance, an average, weighted cost based on General Practitioner and Nursing costs (Curtis and Burns 2015) was calculated (Table 7.1). Weighting was based on published data which attributed $66 \%$ of Primary Care consultations to GPs or allied health professionals and $34 \%$ to nurses (Hippisley-Cox and Vinogradova 2009). Further, costs for investigations, blood tests, pathology and imaging were weighted and then averaged, based on the data available from National Schedules (Department of Health and Social Care 2015; Curtis and Burns 2015) (Appendix F).

Table 7.1: Unit costs for calculating Healthcare utilisation

\begin{tabular}{|c|c|c|}
\hline Healthcare Unit & $\begin{array}{l}\text { Unit } \\
\text { Cost (f) }\end{array}$ & Notes \\
\hline \multicolumn{3}{|l|}{ Primary Care } \\
\hline General Practitioner & 45.00 & Includes allied health professional costing \\
\hline Primary Care Nurse & 14.00 & $\begin{array}{l}\text { Based on } 15.5 \text { minutes per appointment, } 60 \\
\text { appointment per week, } £ 45 \text { ( } £ 56 \text { ) per hour }\end{array}$ \\
\hline Weighted Primary Care appointment & 34.46 & Based on $66 \% \mathrm{GP}, 34 \%$ nursing time \\
\hline Tests and investigations & 4.84 & $\begin{array}{l}\text { Weighted by recorded numbers of blood tests, } \\
\text { biochemistry and pathology, phlebotomy, and } \\
\text { imaging }\end{array}$ \\
\hline Imaging & 87.20 & $\begin{array}{l}\text { Imaging data provided in addition to all } \\
\text { investigations. Not included in total tests and } \\
\text { investigations costings }\end{array}$ \\
\hline \multicolumn{3}{|l|}{ Secondary Care } \\
\hline Out-patient appointment & 110.04 & $\begin{array}{l}\text { National costings give same cost for consultant- } \\
\text { led and non-Consultant led appointments }\end{array}$ \\
\hline Emergency Department attendance & 131.92 & Includes basic investigations \\
\hline Day case acute care attendance & 707.61 & $\begin{array}{l}\text { Includes minor operations, radiological } \\
\text { interventions, administration of chemotherapy } \\
\text { etc. }\end{array}$ \\
\hline Elective In-patient admission & 673.56 & $\begin{array}{l}\text { Based on } £ 3641.24 \text { for a weighted average } 5.4 \\
\text { days admission }\end{array}$ \\
\hline $\begin{array}{l}\text { Elective in-patient admission excess } \\
\text { days }\end{array}$ & 342.64 & For every day of an admission $\geq 6$ days \\
\hline $\begin{array}{l}\text { Non-elective/unplanned in-patient } \\
\text { admission }\end{array}$ & 353.20 & $\begin{array}{l}\text { Based on } \mathrm{f} 2969.07 \text { for weighted average } 8.4 \\
\text { days average excess days admission }\end{array}$ \\
\hline Non-elective in-patient excess days & 287.13 & For every day of an admission $\geq 9$ days \\
\hline $\begin{array}{l}\text { Weighted combined in-patient } \\
\text { admission }\end{array}$ & 522.99 & $\begin{array}{l}\text { Based on weighting from Welsh Providers 53\% } \\
\text { elective admissions, } 47 \% \text { non-elective } \\
\text { admissions }\end{array}$ \\
\hline
\end{tabular}




\begin{tabular}{|c|c|c|}
\hline $\begin{array}{l}\text { Weighted combined in-patient excess } \\
\text { days }\end{array}$ & 316.55 & \\
\hline In-patient day/night costs & 57.86 & $\begin{array}{l}\text { Applied to all admissions, based on } £ 347.93 \text { for } \\
\text { a weighted average } 6 \text { days. Administrative cost } \\
\text { of admission. }\end{array}$ \\
\hline Total weighted in-patient admission & 580.86 & $\begin{array}{l}\text { Weighted average cost for first } 5 \text { days of } \\
\text { admission }\end{array}$ \\
\hline $\begin{array}{l}\text { Total weighted in-patient excess days } \\
\text { admission }\end{array}$ & 374.42 & $\begin{array}{l}\text { Weighted average cost for each day } \geq 6 \text { days } \\
\text { admission }\end{array}$ \\
\hline
\end{tabular}

(Curtis and Burns 2015). Example of more detailed unit costs in Appendix F. Unit cost = cost per GP visit, per test, per OPD or ED attendance or IP admission per day

Secondary care costs, emergency department and out-patient attendances and in-patient stays were calculated from National Schedules for 2014-15 (Table 7.1) (Department of Health and Social Care 2015; Curtis and Burns 2015). All costs associated with paediatric services were removed from the costings lists. Weighted out-patient, day-case, in-patient and excess in-patient costs were calculated from the unit costs provided (Appendix $\mathrm{F}$ for example of weighting calculation). Data from Welsh Providers was used to calculate a weighting for elective and non-elective admissions (53\% versus $47 \%$ respectively) (Digital Health and Care Wales 2021), as this information was not made available in the data tables from SAIL.

Costs were assigned to each individual based on the total number of attendances for Primary Care or out-patient appointments. In-patient stay was provided by number of days. The national schedule was based on an average stay of 5.4 days. Consequently, in-patient stays between one and five days were costed at the weighted average (elective and non-elective) for standard in-patient days (allocated on a daily basis). If an in-patient admission continued for 6 or more days, any days over the first 5 , were classed as 'excess days' and the weighted average excess days cost was allocated. Total in-patient costs were, consequently, the combined total of standard and excess days.

Any secondary care admission of less than one full day, where admission and discharge date were the same, were classed and costed as a day case and the weighted day-case cost used. 


\subsection{Statistical analysis}

All statistical analysis was undertaken using SPSS version 26 (IBM Corporation 2021). Descriptive statistics, mean values, standard deviation, and standard error, were used to compare case and control groups.

Central limit theorem suggests when sample size is large, distribution tends to normal even if the population itself is not normally distributed (Lumley et al. 2002). Due to the large sample size in both arms of the study (case and control), it was determined parametric tests could provide accurate analysis.

To ensure the decision to adopt central limit theorem did not affect the analysis, non-parametric tests (Mann-Whitney and Kruskal-Wallis as per Chapter 2) were also performed between each factor, to compare cases and controls (Appendix F).

\subsubsection{Student's t-test}

Student's t-tests are used to contrast the means of two samples of normally distributed data (Field 2014). It works to a null hypothesis there is no difference between the groups being compared. Where differences are detected, the $p$ value provides an indication of whether they are due to chance or not, so a pvalue of .05 means there is a $5 \%$ chance the difference between groups occurred by chance, or that out of 100 repetitions of the same situation or experiment, the result would be replicated 95 times. For this study, they were used to assess the magnitude of difference between healthcare utilisation and costs.

\subsubsection{One-way analysis of variance (ANOVA)}

One-way ANOVA is used to compare the means of two or more groups of data, although most commonly for three or more. The analysis tests the null hypothesis that all three (or more) groups have the same mean value i.e., the groups are not different to each other. For this study, one-way ANOVA was used in Chapter 7 to analyse differences in healthcare utilisation within e.g., case subjects stratified into 6 groups, depending on age. 


\subsubsection{Two-way ANOVA}

A two-way ANOVA is used to test whether there is an interaction between two categorical variables (e.g., male or female), that combine to affect a dependent variable, or the outcome being examined. In this study, two-way ANOVA was used as a preliminary check of for example, whether receipt of opioid prescriptions had a greater effect on attendance in primary care for women than for men. The interaction effect between factors was deemed significant at a level of $5 \%$. Where the interaction term was not significant, univariate tests (t-test or one-way ANOVA) were also conducted, in order to take account of the relationship and provide a more accurate comparison. For independent t-tests, Levene's test for equality of variance was checked and if significant (at 5\%), unequal variance was assumed. Comparison of means using t-tests and one-way ANOVA also had a 5\% significance level.

Two-way ANOVA was used to examine the interactions between gender, agegroup, and socioeconomic deprivation (WIMD2011) (Appendix F).

\subsubsection{Multiple linear regression}

Regression analyses study the form and direction of the relationship between two or more variables. Regression analysis enables prediction of the value of one variable or the 'outcome' e.g., number of attendances in primary care, based on the values of other variables or 'predictors' e.g., gender, deprivation, age (Laerd Statistics 2021).

Regression analysis uses observations (data) to predict and quantify the relationship between a 'dependent' variable e.g., an outcome such as 'prescription for a strong opioid' and one or more 'independent' variables e.g., gender, age, deprivation status.

The basic equation for linear regression is

$y_{i}=\left(b_{0}+b_{1} X_{i}\right)+e_{i}$ 
where $y_{i}$ is the outcome being predicted; $X$ is the predictor variable i.e. the independent variable being used to make the prediction of outcome; $b_{1}$ is a parameter which quantifies the relationship between the predictor and outcomes variables; $b_{0}$ is the parameter that provides the outcome variable when the predictor is zero and $\mathrm{e}_{\mathrm{I}}$ is the error associated with the calculation (Field 2014).

In the case of this study, an example regression might be:

Number of prescriptions $=b_{0}+\left(b_{1} \times\right.$ male gender $)+e i$

If the value of $b_{1}$ is negative, it would imply the variable e.g., male gender, had a negative effect on the outcome being predicted i.e., makes the outcome less likely.

In clinical situations, it is unlikely that any one factor on its own, will account entirely for the outcome of interest. In these situations, the use of multiple regression allows prediction of how different variables might affect the outcome of interest. Multiple linear regressions were performed to investigate whether there were relationships between healthcare utilisation and opioid use (including duration of use for those who received them), age, deprivation status and gender. Regressions were run with different predictors to determine the model which provided the 'best fit' or highest $R$ and $R^{2}$ values. $A$ higher $R$ or $R^{2}$ value provides an indication of the accuracy of the model in making the predictions.

The equation for a multiple regression model is:

$y_{i}=\left(b_{0}+b_{1} X_{i}+b_{2} X_{2 i}+b_{3} X_{3 i}+b_{4} X_{4 i}+b_{n} X_{n}\right)+e_{i}$

where $b_{n}$ is the coefficient for the predictor variable $X_{n}$ i.e., as many variables as are of interest could be added. However, it does not always follow that more variables added in, lead to a better model. 


\subsubsection{Bonferroni-Holm correction}

In any statistical hypothesis testing, there is a risk of mistakenly accepting errors. In simple terms, the null hypothesis states that any difference between comparator groups is due to chance rather than the effect of the intervention being tested. In most situations, efforts to reduce the risk of type-1 errors include setting the significance level at either $5 \%$ or $1 \%$ ( $p$-values of $<.05$ or $<.01$ ). If those levels are met, it can be concluded the likelihood that the difference between groups is only $1-5 \%$ due to chance.

Type-1 errors are often referred to as 'false-positives'. The error results in the rejection of the null hypothesis when it should have been accepted. An alternative explanation is that a type-1 error will lead to a conclusion that a statistically significant difference exists between comparators when it does not. Conversely, failing to reject a false null hypothesis or accepting a 'false negative' is a type-2 error. In this case, it would be concluded that no statistically significant difference existed between comparators, when in truth, there was.

Claiming a chance difference as the outcome of an experimental intervention has potentially problematic implications. For this reason, type-1 errors are considered a risk that must be minimised. A potential risk for introducing type-1 errors occurs when multiple comparisons are made, as they have been to examine healthcare utilisation and analyse the costs involved in this part of the study. The probability of drawing at least one incorrect conclusion (type-1 error) from a series of hypothesis tests is known as the 'familywise error rate' (FWER). The likelihood of an FWER increases, as the number of comparisons is made (Olejnik et al. 1997; Glenn 2021).

Holm's Sequential Bonferroni Procedure (Holm-Bonferroni correction) is a method of managing type-1 errors when a series of hypothesis tests are undertaken (Holm 1979). Holm's method has greater power than the usual Bonferroni correction and compares the $p$-value for each of the comparisons conducted, in rank order i.e., the lowest $p$-value is compared first, the highest $p$ - 
value compared last, to the value of the desired p-value (e.g., 0.5 , referred to as $\alpha)$ divided by the number of comparisons being tested $(m)$. If first $p$-value being compared is less than $\alpha / m$, the null hypothesis is rejected, and the next comparison is made $(k+1$, where $k$ is the ranking of the $p$-value in the sorted list). The comparisons are made until the result exceeds $\alpha$. When that happens, all remaining hypotheses are accepted.

Holm-Bonferroni correction equation

$\alpha_{\text {Holm-Bonferroni }}=\alpha / \mathrm{m}-\mathrm{k}+1$

Holm-Bonferroni corrections were applied to all statistical output in this phase of the study to ensure hypothesis testing was accurately reported.

\subsubsection{Sensitivity analysis}

Sensitivity analysis is used to reduce uncertainty in health economic evaluations (Taylor and Filby 2014). Changing one or more parameters allows consideration of which of them most influences the outcome being examined e.g., total cost of healthcare utilisation. One-way and two-way sensitivity analysis was undertaken on healthcare cost data.

Control subject costs were increased by between 25 and $100 \%$ for one-way analyses. A two-way analysis was also undertaken where both case and control subject costs were adjusted. In addition to examining cost changes, t-tests were repeated (with a 5\% significance level) and Holm-Bonferroni corrections on the adjusted data.

\subsubsection{Threshold analysis}

Threshold analysis was used to determine how much case subject costs would need to decrease in order to come into line with the control group. Case and control subject costs were recalculated by different percentages. Independent ttests with a significance level of 5\%, were re-run with Holm-Bonferroni correction. 


\subsection{Results}

The Phase 2 study examined the records of 3,286,215 individuals aged 18 years and over. The case group included 657,243 individuals, who had a recorded diagnosis of a non-cancer pain condition and received prescriptions for opioids for a period of time between 2005 and 2015 (Table 7.2). The control group contained 2,628,972 subjects, matched by condition, age, gender and deprivation, who did not have a record of prescriptions for opioids being issued during the study period. The results of statistical analyses between case and control groups are presented in the tables throughout the rest of this chapter. Where comparisons were made within with case or control subject groups (e.g., comparing differences between control subjects by socioeconomic deprivation), they will be presented in the text.

Table 7.2: Demographic data for healthcare utilisation between cases and controls.

\begin{tabular}{|c|c|c|}
\hline \multirow[t]{2}{*}{ Number of subjects } & Case & Control \\
\hline & 657,243 & $2,628,972$ \\
\hline \multicolumn{3}{|l|}{ Gender (\% of total) } \\
\hline Male & $273,057(41.5)$ & $1,092,228(41.5)$ \\
\hline Female & $384,186(58.5)$ & $1,536,744(58.5)$ \\
\hline \multicolumn{3}{|l|}{ Age } \\
\hline Mean (SEM) & $57.0(0.02)$ & $57.1(0.01)$ \\
\hline Median & 57.0 & 57.0 \\
\hline Standard deviation & 18.1 & 18.7 \\
\hline \multicolumn{3}{|l|}{ Deprivation quintile* (\% of total) } \\
\hline WIMD1 & $153,649(23.4)$ & $614,596(23.4)$ \\
\hline WIMD2 & $136,752(20.8)$ & $547,008(20.8)$ \\
\hline WIMD3 & $137,653(20.9)$ & $550,612(20.9)$ \\
\hline WIMD4 & $113,083(17.2)$ & $452,332(17.2)$ \\
\hline WIMD5 & $116,106(17.7)$ & $464,424(17.7)$ \\
\hline \multirow{2}{*}{\multicolumn{3}{|c|}{$\begin{array}{l}\text { *Deprivation quintile based on Welsh Index of Multiple Deprivation 2011. WIMD1=most deprived, } \\
\text { least deprived Cases=people receiving opioid prescriptions, Control=not receiving opioid prescripti } \\
\text { The proportions of men and women were the same in each group (Table }\end{array}$}} \\
\hline & & \\
\hline \multicolumn{3}{|c|}{ 7.2Error! Reference source not found.) confirmed using a Chi-square test, $\chi^{2}$} \\
\hline \multicolumn{3}{|c|}{$(\mathrm{df}=1, \mathrm{~N}=3286215)=.00, \mathrm{p}>.999$. Mean average ages for cases and controls } \\
\hline \multicolumn{3}{|c|}{ appeared the same, although a statistically significant difference was found } \\
\hline \multicolumn{3}{|c|}{ when analysed using an independent t-test, $t(d f=1039533)=-2.163, p<0.05$, } \\
\hline \multicolumn{3}{|c|}{$\mathrm{d}_{\text {Cohen }}=0.003$. The small statistical difference in age seen between the two } \\
\hline
\end{tabular}




\subsubsection{Primary Care attendance}

Between 2005 and 2015, 190,984,317 general practice appointments were recorded for the 3.2 million people included in the study, with a mean average of 58.1 (SD=97.4) appointments per person. Opioid users accounted for $55 \%$ ( $n=105,457,258)$ of appointments. Case subjects had significantly more GP visits than control subjects (Table 7.3).

Presentations to General Practice, due to symptoms which could be associated with opioid use (e.g., nausea, vomiting, sedation), were relatively low in number (Table 7.3) but statistically significantly higher for cases than for controls.

Table 7.3: Comparison of Primary Care General Practice utilisation depending on receipt of opioid prescriptions (case versus control)

\begin{tabular}{|c|c|c|c|}
\hline Total, mean \pm (standard deviation) & Case & Control & $\mathrm{p}$-value $* *\left(\mathrm{~d}_{\text {cohen }}\right)$ \\
\hline Number of GP attendances & $105,457,258$ & $85,527,059$ & \\
\hline Mean (SD) & $160.5(146.3)$ & $32.53(56.8)$ & $<.001(0.96)$ \\
\hline Median & 121.0 & 10.0 & \\
\hline \multicolumn{4}{|l|}{ Prescribing persistence (cases only) } \\
\hline$<6$ months & $67.7(77.6)$ & & \\
\hline$>6$ months & $198.8(150.8)$ & & $<.001(1.3)$ \\
\hline \multicolumn{4}{|l|}{ Gender } \\
\hline Female & $175.2(149.1)$ & $36.6(59.2)$ & $<.001(1.02)$ \\
\hline Male & $139.7(139.6)$ & $26.8(52.7)$ & $<.001(0.89)$ \\
\hline \multicolumn{4}{|l|}{ Deprivation quintile* } \\
\hline WIMD1 & $167.2(149.7)$ & $36.2(57.7)$ & $<.001(0.96)$ \\
\hline WIMD2 & $164.7(148.6)$ & $33.7(58.9)$ & $<.001(0.97)$ \\
\hline WIMD3 & $162.3(147.5)$ & $31.9(55.8)$ & $<.001(0.97)$ \\
\hline WIMD4 & $154.9(144.5)$ & $30.3(55.5)$ & $<.001(0.95)$ \\
\hline WIMD5 & $149.8(137.8)$ & $29.2(55.3)$ & $<.001(0.96)$ \\
\hline \multicolumn{4}{|l|}{ Age group (years) } \\
\hline $18-24$ & $41.4(45.7)$ & $14.9(21.2)$ & $<.001(0.62)$ \\
\hline $25-44$ & $93.2(99.8)$ & $17.9(31.2)$ & $<.001(0.83)$ \\
\hline $45-64$ & $144.3(133.9)$ & $25.9(44.2)$ & $<.001(0.98)$ \\
\hline $65-74$ & $207.3(149.0)$ & $42.3(64.7)$ & $<.001(1.22)$ \\
\hline $75-84$ & $250.1(162.1)$ & $52.6(79.4)$ & $<.001(1.32)$ \\
\hline$\geq 85$ & $250.2(163.5)$ & $65.0(86.9)$ & $<.001(1.21)$ \\
\hline \multicolumn{4}{|l|}{ GP visits for ADEs possibly associated } \\
\hline with opioids & 444,050 & 450,510 & \\
\hline Mean \pm SD & $0.68(2.0)$ & $0.17(0.7)$ & $<.001(0.27)$ \\
\hline \multicolumn{4}{|l|}{ Gender } \\
\hline Female & $0.82(2.2)$ & $0.21(0.8)$ & $<.001(0.30)$ \\
\hline Male & $0.47(1.7)$ & $0.11(0.6)$ & $<.001(0.23)$ \\
\hline \multicolumn{4}{|l|}{ Deprivation quintile* } \\
\hline WIMD1 & $0.87(2.5)$ & $0.22(0.8)$ & $<.001(0.28)$ \\
\hline WIMD2 & $0.68(2.0)$ & $0.18(0.7)$ & $<.001(0.28)$ \\
\hline WIMD3 & $0.60(1.7)$ & $0.16(0.7)$ & $<.001(0.28)$ \\
\hline WIMD4 & $0.60(2.0)$ & $0.15(0.7)$ & $<.001(0.24)$ \\
\hline WIMD5 & $0.57(1.6)$ & $0.14(0.7)$ & $<.001(0.30)$ \\
\hline All tests and referrals from primary care & 206,747 & 158,290 & \\
\hline Mean \pm SD & $0.31(0.9)$ & $0.06(0.3)$ & $<.001(0.33)$ \\
\hline $\begin{array}{l}\text { Recorded imaging (X-ray, CT, MRI etc.) } \\
\text { Mean } \pm \text { SD }\end{array}$ & 90,700 & 74,756 & \\
\hline
\end{tabular}


*Deprivation quintile based on Welsh Index of Multiple Deprivation 2011. WIMD1 =most deprived, WIMD5 $=$ least deprived $* *$ p-value $<0.05=$ significant

Holm-Bonferroni confirmed it was correct to reject the null hypothesis

Significantly more primary care appointments were used by case subjects with prescribing persistence of more than 6 months than those with under 6 months persistence (Table 7.3).

Female case subjects had $25 \%$ more primary care appointments than men (t-test, $\left.p<.001, d_{\text {Cohen }}=0.25\right)$. Thirty-five percent more visits were recorded for women than men in the control group (t-test, $p<.001, d_{\text {Cohen }}=0.20$ ).

However, female case subjects had nearly 5 times as many appointments as their controls. The difference between the means of the two groups was statistically significant (Table 7.3). A similar difference was noted between men in the case and control groups in terms of number of primary care appointments (Table 7.3).

Amongst case subjects, the number of primary care attendances was $12 \%$ higher for those living in the most deprived areas of Wales (WIMD1) compared to the least deprived (WIMD5) (Table 7.3). There was a greater difference (24\%) in the mean number of attendances between the most deprived and the least deprived in the control group, who did not receive prescriptions for opioids. Statistically significant differences were found between all quintiles of both the case subjects (ANOVA, $p<.001, h^{2}{ }_{p}=.002$ ) and controls (ANOVA, $p<.001, h^{2}{ }_{p}=.002$ ) which were confirmed between all quintiles by post-hoc analysis.

However, in the most deprived areas (WIMD1), 360\% more primary care attendances were recorded in the case group than in the control group (Table 7.3). In the least deprived areas (WIMD5), case subjects visited their GP practice $412 \%$ more often than controls.

Primary Care attendance increased with age for cases and controls. In each agegroup, however, attendances were significantly higher for people receiving opioid prescriptions (Table 7.3). 
ANOVA confirmed statistically significant differences in primary care attendance between age-groups in the case subject group $\left(\mathrm{p}<0.001, \mathrm{~h}_{\mathrm{p}}{ }_{\mathrm{p}}=.160\right)$, although this was not confirmed for the two oldest groups (75-84 years and $85+$ years, $p>0.05$ ) when compared in pairs. Average primary care attendance numbers were significantly different between case subjects in the youngest age-group (age 18 24 years) and the oldest (age 85 years and over) ( $p<.001)$. The oldest age group had 6 times more attendances in Primary Care than the youngest.

The oldest control age group had 4 times more attendances than the youngest (Table 7.3). ANOVA demonstrated significant differences between the mean attendances of all control subject age-groups $\left(p<0.001, h^{2}{ }_{p}=.072\right)$ and were confirmed between all age-groups when compared pairwise.

Due to coding restrictions, relatively few tests, referrals and requests for imaging were collected from Primary Care General Practice records. However, based on those made available for analysis, case subjects received 5 times as many tests and imaging appointments than controls (Table 7.3).

\subsubsection{Out-Patient attendance}

In total 22,239,332 out-patient appointments were recorded for individuals whose data was analysed for this study. Case subjects accounted for $41 \%$ $(n=9,140,922)$ of those appointments in absolute terms, despite there being 4 times more people in the control group. However, the average number of outpatient appointments was 2.8 times greater for people receiving opioid prescriptions compared to those who did not (Table 7.4).

Three times more out-patient attendances were noted in case subjects with a prescribing persistence of more than 6 months. It was significantly more than noted in cases with less than 6 months of opioid prescribing persistence.

Differences were maintained between cases and controls when compared by gender (Table 7.4). Female case subjects had on average $25 \%$ more out-patient appointments than men ( $\mathrm{t}$-test, $\mathrm{p}<.001, \mathrm{~d}_{\text {cohen }}=0.25$ ). In the control group, 
women had $30 \%$ more out-patient appointments than men (t-test, $p<.001$, $\mathrm{d}_{\text {Cohen }}=0.22$ ).

Table 7.4: Comparison of out-patient resource utilisation depending on receipt of opioid prescriptions (case versus control)

\begin{tabular}{|c|c|c|c|}
\hline Total, mean \pm standard deviation & Case & Control & $\begin{array}{c}\text { p-value** } \\
\text { (d } \text { cohen })\end{array}$ \\
\hline Number of Outpatient attendances & $9,140,922$ & $13,098,410$ & \\
\hline Mean \pm SD & $13.9(19.4)$ & $5.0(10.0)$ & $<.001(0.50)$ \\
\hline \multicolumn{4}{|l|}{ Prescribing persistence (cases only) } \\
\hline$<6$ months & $5.6(10.1)$ & & \\
\hline$>6$ months & $17.4(21.2)$ & & $<.001(0.88)$ \\
\hline \multicolumn{4}{|l|}{ Gender } \\
\hline Female & $15.2(19.9)$ & $5.5(10.2)$ & $<.001(0.52)$ \\
\hline Male & $12.2(18.5)$ & $4.2(9.7)$ & $<.001(0.46)$ \\
\hline \multicolumn{4}{|l|}{ Deprivation quintile**** } \\
\hline WIMD1 & $15.2(20.7)$ & $5.3(10.0)$ & $<.001(0.52)$ \\
\hline WIMD2 & $14.4(19.9)$ & $5.1(10.4)$ & $<.001(0.50)$ \\
\hline WIMD3 & $13.5(19.0)$ & $5.0(9.7)$ & $<.001(0.49)$ \\
\hline WIMD4 & $13.0(18.4)$ & $4.6(9.3)$ & $<.001(0.50)$ \\
\hline WIMD5 & $13.0(18.4)$ & $4.8(10.7)$ & $<0001(0.48)$ \\
\hline \multicolumn{4}{|l|}{ Age group (years) ${ }^{* * *}$} \\
\hline $18-24$ & $4.6(8.1)$ & $1.7(5.7)$ & $<.001(0.37)$ \\
\hline $25-44$ & $9.9(15.0)$ & $3.4(7.3)$ & $<.001(0.48)$ \\
\hline $45-64$ & $12.7(18.7)$ & $4.3(9.0)$ & $<.001(0.49)$ \\
\hline $65-74$ & $17.2(21.9)$ & 7.3 (12.9) & $<.001(0.49)$ \\
\hline $75-84$ & $20.7(23.3)$ & 7.9 (12.9) & $<.001(0.59)$ \\
\hline$\geq 85$ & $17.4(20.5)$ & $5.7(9.8)$ & $<.001(0.61)$ \\
\hline
\end{tabular}

*Deprivation quintile based on Welsh Index of Multiple Deprivation 2011. WIMD1 =most deprived, WIMD5 $=$ least deprived $* *$ p-value $<0.05=$ significant $* * *$ Holm-Bonferroni confirmed it was correct to reject the null hypothesis

Cases from the most deprived areas of Wales (WIMD1) had $17 \%$ more outpatient appointments than those in the least deprived (WIMD5) over the study period. Differences between attendance by case subjects were statistically different when compared by quintile (ANOVA, $\mathrm{p}<.001, \mathrm{~h}_{\mathrm{p}}^{2}=.002$ ). Post-hoc analysis confirmed those differences in all but WIMD4 and WIMD5 quintiles ( $p>0.5$ ) when attendance was directly compared.

In the control group, that difference was $10 \%$. Comparison of out-patient attendance by deprivation quintile was found to be significant (ANOVA, $p<.001$, $\left.\mathrm{h}_{\mathrm{p}}^{2}=.001\right)$, which was confirmed by pairwise comparisons.

When quintiles were directly compared, differences in out-patient attendance between cases and controls was maintained at around 2.7 to 2.8 times more, regardless of deprivation quintile (Table 7.4). 
Average number of out-patient appointments per person were greatest in people aged 75 to 84 years, in both case and control groups (Table 7.4). Cases in the 75 to 84 years age-group had 4.5 times more out-patient attendances on average than seen in people aged 18 - 24 years (Table 7.4). Comparisons of outpatient attendances between case subjects of all age-groups were statistically significant (ANOVA, $\mathrm{p}<.001, \mathrm{~h}_{\mathrm{p}}{ }_{\mathrm{p}}=.04$ ) and confirmed by pairwise comparisons.

Differences in out-patient attendances groups in control subjects were similar to those seen in cases (Table 7.4). Statistically significant differences in average outpatient attendances were noted between all 6 age-groups (ANOVA, $p<.001, h^{2}{ }_{p}=$ $.30)$, confirmed by pairwise comparison.

Observed differences in out-patient attendance between case and control subjects in the same age-groups were around the average noted between cases and controls overall. Three times more out-patient appointments were noted in cases than controls in the $\geq 85$ years age group (Table 7.4 ).

\subsubsection{Emergency Department Utilisation}

Between 2005 and 2015, 2,810,268 Emergency Department (ED) attendances were recorded for the $3,286,215$ individuals included in the study, averaging 0.9 \pm 2.2 appointments per person.

On average, more than 3 times as many emergency department (ED) attendances per person (Table 7.5Error! Reference source not found.), and for any reason, were observed in the case subject group than the control group during the study period.

Table 7.5: Comparison of Emergency department resource utilisation depending on receipt of opioid prescriptions (case versus control)

\begin{tabular}{|c|c|c|c|}
\hline Total, mean (standard deviation) & Case & Control & $\mathrm{p}$-value** $\left(\mathrm{d}_{\text {Cohen }}\right)$ \\
\hline \multirow[t]{2}{*}{$\begin{array}{l}\text { Number of Emergency Department } \\
\text { attendances }\end{array}$} & $1,243,641$ & $1,566,627$ & \\
\hline & $1.89(3.4)$ & $0.6(1.8)$ & $<.001(0.42)$ \\
\hline \multicolumn{4}{|l|}{ Prescribing persistence (cases only) } \\
\hline$<6$ months & $0.96(1.9)$ & & \\
\hline$>6$ months & $2.3(3.8)$ & & $<.001(0.00 .51)$ \\
\hline \multicolumn{4}{|l|}{ Gender } \\
\hline Female & $1.9(3.4)$ & $0.58(1.7)$ & $<.001(0.42)$ \\
\hline
\end{tabular}




\begin{tabular}{lcccc}
\hline & Male & $1.9(3.4)$ & $0.61(1.8)$ & $<.001(0.40)$ \\
\hline Deprivation quintile* & WIMD1 & $2.3(3.9)$ & $0.77 \pm 2.1$ & $<.001(0.42)$ \\
& WIMD2 & $2.4(3.8)$ & $0.65 \pm 1.7$ & $<.001(0.40)$ \\
& WIMD3 & $1.8(3.2)$ & $0.55 \pm 1.9$ & $<.001(0.42)$ \\
& WIMD4 & $1.64 \pm 2.9$ & $0.53 \pm 1.4$ & $<.001(0.42)$ \\
& WIMD5 & $1.5 \pm 2.6$ & $0.41 \pm 1.2$ & $<.001(0.46)$ \\
\hline Age group (years) & & & & \\
& $\mathbf{1 8 - 2 4}$ & $2.0 \pm 3.9$ & $0.86 \pm 2.2$ & $<.001(0.32)$ \\
& $\mathbf{2 5 - 4 4}$ & $2.2 \pm 3.8$ & $0.61 \pm 1.5$ & $<.001(0.47)$ \\
& $\mathbf{4 5 - 6 4}$ & $1.7 \pm 3.2$ & $0.56 \pm 1.7$ & $<.001(0.37)$ \\
& $\mathbf{6 5 - 7 4}$ & $1.5 \pm 3.1$ & $0.56 \pm 2.2$ & $<.001(0.33)$ \\
& $\mathbf{7 5 - 8 4}$ & $2.0 \pm 3.1$ & $0.58 \pm 1.6$ & $<.001(0.48)$ \\
$\mathbf{2 8 5}$ & $2.5(3.3)$ & $0.67(1.6)$ & $<.001(0.61)$ \\
\hline
\end{tabular}

*Deprivation quintile based on Welsh Index of Multiple Deprivation 2011. WIMD1 =most deprived, WIMD5

$=$ least deprived ${ }^{* *}$ p-value $<0.05=$ significant

Holm-Bonferroni confirmed it was correct to reject the null hypothesis

Case subjects receiving opioid prescriptions for more than 6 months attended emergency departments 2.4 times more often than those with shorter durations of prescribing (Table 7.5 ).

Female and male case subjects had over 3 times more ED attendances compared to their respective controls (Table 7.5Error! Reference source not found.).

However, no statistical difference was found between male and female case subjects $\left(p=0.534, d_{\text {cohen }}=0.002\right)$, in terms of average number of attendances. However, female controls had significantly more ED attendances than male controls $\left(p<.001, \mathrm{~d}_{\text {Cohen }}=0.004\right)$.

Case subjects average attendance at ED had little numerical difference in the 2 most deprived quintiles (WIMD1 and WIMD2) although pairwise comparisons derived a statistically significant difference between them $(p<.001)$ (Table 7.5Error! Reference source not found.). Around 50\% more ED attendances were recorded in the two most deprived quintiles than the least deprived (WIMD5) for case subjects. Mean attendances increased as deprivation increased and the differences between quintiles were statistically significant (ANOVA, $p<.001$, $\left.\mathrm{h}_{\mathrm{p}}^{2}=.007\right)$.

There was slightly more difference (18\%) in the control group, in terms of average number of attendances between the lowest two quintiles (WIMD1 and WIMD2) (Table 7.5). However, 88\% more ED attendances happened in the most 
deprived quintile of controls, compared to the least (WIMD5). As with cases, there was a significant difference between control subjects' average attendances between quintiles. Greater levels of deprivation were associated with higher numbers of ED attendance (ANOVA, $\left.p<.001, h^{2}=0.005\right)$. Differences in attendance numbers were significant between all quintiles when control subjects were compared in pairs.

Overall, 3 times more ED appointments were recorded for case subjects in the most deprived quintile compared to controls (Table 7.5Error! Reference source not found.). This rose to 3.7 times more appointments by cases compared to controls in the least deprived quintile.Two-way ANOVA demonstrated however, that deprivation was not a lone influence for attendance (Appendix F).

The average number of emergency department attendances were lowest in the age group 65 to 74 years for both case and control subjects (Table 7.5Error! Reference source not found.).

In the case subject group, the highest average number of attendances was noted in the oldest age-group ( $\geq 85$ years). This was $66 \%$ higher than in the lowest age group. Comparisons of mean attendances determined significant differences between each age-group when analysed as a group and in pairs (ANOVA, $p<.001$, $\left.h^{2}{ }_{p}=.009\right)$.

In the control group, the highest number of average ED attendances were observed in 18 - to 24 -year-olds, $53 \%$ more than seen in the 65 to 74 years agegroup. Statistically significant differences in mean attendances were noted between all age-groups of control subjects (ANOVA, $p<.001, h^{2}{ }_{p}=.001$ ). Pairwise comparison confirmed differences between age groups other than the $45-64$ years and $\geq 85$ years groups where a statistical difference was not found $(p>0.1)$.

\subsubsection{In-Patient attendance and Length of Stay}

Case subjects had on average twice as many hospital admissions as controls, which was statistically significant ( 
Table 7.6). Length of Stay (LoS) was however, on average, $6 \%$ longer for control subjects and was statistically significant, albeit with a smaller effect size than noted for number of admissions.

Table 7.6: Comparison of In-patient admission and length of stay depending on receipt of opioid prescriptions (case versus control)

\begin{tabular}{|c|c|c|c|}
\hline Total, mean (standard deviation) & Case & Control & $\mathrm{p}$-value** ( $\left.\mathrm{d}_{\text {cohen }}\right)$ \\
\hline Number of In-patient admissions & $3,021,645$ & $5,676,577$ & \\
\hline Mean \pm SD & $4.6(8.5)$ & $2.2(6.3)$ & $<.001(0.30)$ \\
\hline \multicolumn{4}{|l|}{ Prescribing persistence (cases only) } \\
\hline$<6$ months & $2.9(6.2)$ & & \\
\hline$>6$ months & $5.3(9.2)$ & & $<.001(0.47)$ \\
\hline \multicolumn{4}{|l|}{ Gender } \\
\hline Female & $5.0(8.1)$ & $2.3(6.0)$ & $<.001(0.34)$ \\
\hline Male & $4.1(9.0)$ & $2.0(6.7)$ & $<.001(0.25)$ \\
\hline \multicolumn{4}{|l|}{ Deprivation quintile } \\
\hline WIMD1 & $4.9(8.1)$ & $2.3(4.8)$ & $<.001(0.35)$ \\
\hline WIMD2 & $4.8(8.7)$ & $2.2(4.7)$ & $<.001(0.32)$ \\
\hline WIMD3 & $4.5(8.3)$ & $2.3(8.3)$ & $<.001(0.26)$ \\
\hline WIMD4 & $4.4(9.0)$ & $2.0(6.4)$ & $<.001(0.29)$ \\
\hline WIMD5 & $4.2(8.3)$ & $1.9(6.9)$ & $<.001(0.29)$ \\
\hline \multicolumn{4}{|l|}{ Age group (years) } \\
\hline $18-24$ & $3.5(6.5)$ & $0.5(2.1)$ & $<.001(0.49)$ \\
\hline $25-44$ & $3.9(7.4)$ & $1.0(2.7)$ & $<.001(0.44)$ \\
\hline $45-64$ & $3.5(7.8)$ & $1.2(4.8)$ & $<.001(0.30)$ \\
\hline $65-74$ & $5.0(9.3)$ & $3.1(10.0)$ & $<.001(0.20)$ \\
\hline $75-84$ & $6.9(10.1)$ & $4.6(6.0)$ & $<.001(0.25)$ \\
\hline$\geq 85$ & $8.3(8.6)$ & $5.1(9.3)$ & $<.001(0.37)$ \\
\hline Length of stay (days) & 10758522 & 45482557 & \\
\hline Mean \pm SD & $16.4(54.7)$ & $17.3(64.9)$ & $<.001(-0.02)$ \\
\hline \multicolumn{4}{|l|}{ Prescribing persistence (cases only) } \\
\hline$<6$ months & $10.4(46.7)$ & & \\
\hline$>6$ months & $18.8(57.5)$ & & $<.001(0.37)$ \\
\hline \multicolumn{4}{|l|}{ Gender } \\
\hline Female & $18.0(57.0)$ & $19.3(68.2)$ & $<.001(-0.02)$ \\
\hline Male & $14.1(51.3)$ & $14.5(60.0)$ & $<.001(-0.01)$ \\
\hline \multicolumn{4}{|l|}{ Deprivation quintile } \\
\hline WIMD1 & $17.6(58.6)$ & $17.9(65.8)$ & $<.001(-0.006)$ \\
\hline WIMD2 & $16.9(56.4)$ & 18.0 (72.9) & $<.001(-0.02)$ \\
\hline WIMD3 & $16.5(53.6)$ & $18.9(62.1)$ & $<.001(-0.04)$ \\
\hline WIMD4 & $15.0(49.6)$ & $15.1(55.8)$ & $0.710(-.001)$ \\
\hline WIMD5 & $15.3(53.4)$ & $15.9(65.4)$ & $<.001(-0.01)$ \\
\hline \multicolumn{4}{|l|}{ Age group (years) } \\
\hline $18-24$ & $5.1(22.4)$ & $0.8(7.0)$ & $<.001(0.20)$ \\
\hline $25-44$ & $6.8(33.4)$ & $1.8(21.1)$ & $<.001(0.16)$ \\
\hline $45-64$ & $8.1(40.0)$ & $5.0(37.7)$ & $<.001(0.08)$ \\
\hline $65-74$ & $15.5(50.9)$ & $20.5(66.9)$ & $<.001(-0.09)$ \\
\hline $75-84$ & $34.6(78.8)$ & $50.3(108.2)$ & $<.001(-0.18)$ \\
\hline$\geq 85$ & $67.7(96.0)$ & $70.3(110.1)$ & $<.001(-0.03)$ \\
\hline
\end{tabular}


Case subjects who received opioid prescriptions persistently for more than 6 months had significantly more hospital admissions than those with less persistent prescribing. Both average number of admissions and average LoS were $80 \%$ higher in those with longer prescription persistence (

Table 7.6).

Female case subjects had $22 \%$ more hospital admissions per person than were noted for male cases. The difference in mean number of admissions was statistically significant ( $\mathrm{t}$-test, $\mathrm{p}<.001, \mathrm{~d}_{\text {cohen }}=0.10$ ). LoS was significantly longer for female than male cases, with an average of 4 days greater duration (t-test, $\mathrm{p}<.001, \mathrm{~d}_{\text {Cohen }}=0.07$ ) (

Table 7.6).

Amongst controls, females had an average 15\% more hospital admissions, which, was significantly more than for male controls but with a small effect size (t-test, $\left.p<.001, d_{\text {Cohen }}=0.05\right)$. Female controls also had significantly greater lengths of stay) than men (t-test, $\left.p<.001, \mathrm{~d}_{\text {Cohen }}=0.08\right)$.

Comparing cases and controls, female and male case subjects had on average, twice as many admissions per person as for their comparators in the control groups (

Table 7.6). These statistically significant differences in admissions were reversed when analysing LoS. Female controls stayed on average 1.3 days longer than female case subjects. Male controls had a smaller difference in LoS compared to their matched case subjects. It was still considered statistically significant, however (

Table 7.6).

Greater socio-economic deprivation was associated with a higher number of inpatient admissions and a longer duration of stay ( 
Table 7.6). Case subjects in the most deprived areas of Wales (WIMD1) had an average $16 \%$ more admissions than those in the least (WIMD5) deprived. Mean average number of case subject admissions were significantly higher with increasing deprivation (ANOVA, $\mathrm{p}<.001, \mathrm{~h}_{\mathrm{p}}^{2}=.001$ ) and confirmed by pairwise comparison. Case subject length of in-patient stay was also longer for those in more deprived areas (

Table 7.6) Duration of admission had an average $15 \%$ difference between most and least deprived areas. Comparisons determined statistically significant differences between LoS in each quintile and confirmed by pairwise comparisons (ANOVA, $\mathrm{p}<.001, \mathrm{~h}_{\mathrm{p}}^{2}=.0003$ ).

Higher numbers of admissions and LoS were also noted with increasing deprivation for the control group (

Table 7.6). A 21\% difference in number of in-patient admissions was noted between WIMD1 and WIMD5 quintiles and deemed statistically significant (ANOVA, $\mathrm{p}<.001, \mathrm{~h}_{\mathrm{p}}{ }_{\mathrm{p}}=.001$ ). The longest LoS in the control group was seen in the middle quintile (WIMD3) (

Table 7.6). There was a difference of 3 days between shortest and longest average LoS in the control group. Average LoS was determined to be significantly different between all quintiles and confirmed by pairwise comparisons (ANOVA, $\left.p<.001, h_{p}^{2}=.002\right)$.

When quintiles were compared by case versus control, average numbers of admissions were greater in all case subjects (

Table 7.6). The third quintile (WIMD3) revealed a 15\% longer average admission for controls compared to case subjects. However, all comparisons demonstrated significantly longer duration of admission in the control group compared to the case subjects, regardless of quintile. 
Both case and control subjects demonstrated an overall increase in number of inpatient admissions and LoS with advancing age (

Table 7.6). Amongst case subjects, there were 2.4 times as many admissions on average in the $85+$ years group compared to the $18-24$ years group. There were the same average number of admissions however, for 18-24 years and 45-64 years age groups. Statistically significant differences were confirmed between all case subject age-groups (ANOVA, $\mathrm{p}<.001, \mathrm{~h}^{2} \mathrm{p}=.031$ ) other than 18-24 years and 45-64 years groups where pairwise comparison demonstrated no statistical difference in the number of admissions.

Thirteen times longer average lengths of in-patient admission were observed in the oldest case subjects compared to the youngest. Three days was the difference in average admission length (

Table 7.6) between the three-younger age-groups (from 18-64 years). Admission duration then doubled with each advancing age-group. The changes in LoS between age-groups were considered significant when compared between case subject age-groups (ANOVA, $\mathrm{p}<.001, \mathrm{~h}_{\mathrm{p}}^{2}=.092$ ).

For control subjects, ten times more admissions were noted on average, for individuals aged 85 and above compared to the youngest group. As with case subjects, the control group showed a similar pattern of similar average number of admissions in the younger age groups with a widening gap as age increased. Differences between age-group ranked admissions were statistically significant and confirmed by direct pairwise comparisons (ANOVA, $p<.001, h^{2}=.058$ ).

Control group lengths of stay spanned 69.5 days on average, between the youngest and oldest. Eighty-eight times more days on average were spent in hospital by control subjects aged 85 years and over, compared to those aged 1824 years (

Table 7.6). Differences in average LoS by age-group were statistically significant (ANOVA, $\mathrm{p}<.001, \mathrm{~h}_{\mathrm{p}}^{2}=.116$ ) and confirmed by pairwise comparison. 
Analysing case and control subjects within the same age groups revealed interesting differences. Between 18 and 64 years, case subjects had significantly more admissions and a longer in-patient stay than their equivalent controls (

Table 7.6). Case subjects aged 18-24 years had 7 times more admissions and 6.4 times longer average admission duration compared to their controls for example

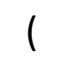

Table 7.6).

After 65 years old however, the pattern changed. Case subjects had a significantly higher average number of admissions per person, although the duration of stay was longer in the control group. For example, the age group 7584 control subjects had $50 \%$ more admissions on average than their controls but with $30 \%$ shorter average LoS (

Table 7.6).

\subsection{Regression Analysis}

Multiple linear regression analyses were used to predict which, if any, variables affected attendance in Primary Care (number of GP visits), out-patient, emergency department or in-patient attendances. The factors used to make the predictions were opioid prescription, age, gender, deprivation status (WIMD2011), recorded diagnosis of depression and/or anxiety and whether opioids were prescribed, and if they were prescribed for more or less than 6 months.

\subsubsection{Primary Care General Practice attendance}

Multiple linear regressions results indicated that the model was a significant predictor of the number of attendances in Primary Care (GP), F (10, 3286204) = 276498.2, $p<.001$. An $R^{2}$ of $0.457, S E=71.8(R=0.676)$ meant $45.7 \%$ of the variation in the original data could be explained by this model. The $ß_{0}$ (constant) for the regression equation was $-26.24, \mathrm{SE}=.138, \mathrm{p}<.001$. 
Based on the regression output (Table 7.7), the strongest predictors for attendance in Primary Care (GP) were prescription duration of more than 6 months $\left(B_{n}=143.50, S E=.121, p<.001\right)$, which increased GP attendance by 143.5 visits compared to an increase of 34.3 visits for less than 6 months $\left(\beta_{n}=34.33\right.$, $\mathrm{SE}=.171, \mathrm{p}<.001)$. Having a recorded diagnosis of depression $\left(\beta_{n}=22.979, \mathrm{SE}=.123\right.$, $p<.001$ ) increased primary care attendance by 23 visits. However, living in the least deprived areas of Wales (WIMD5) decreased GP attendance by 7.2 visits $\left(B_{n}=-7.18, S E=.126, p<.001\right)$, compared to living in the most deprived areas (WIMD1). Being male reduced likelihood of GP attendance by 10.4 visits ( $\beta_{n}=-$ $10.42, \mathrm{SE}=.081, \mathrm{p}<.001)$. 
Table 7.7: Output from multiple linear regression to predict Healthcare utilisation by visits or attendances

\begin{tabular}{|c|c|c|c|c|c|}
\hline \multirow[t]{2}{*}{ Variable $\beta_{n}(S E)$} & \multicolumn{2}{|c|}{ GP visits } & \multirow{2}{*}{$\begin{array}{c}\text { Outpatient visits } \\
1.399(.005)\end{array}$} & \multirow{2}{*}{$\begin{array}{c}\text { ED visits } \\
.007(.001) \\
\end{array}$} & \multirow{2}{*}{$\begin{array}{c}\text { Inpatient admission } \\
.786(.002)\end{array}$} \\
\hline & $17.698(.031)$ & $17.208(.033)$ & & & \\
\hline \multicolumn{6}{|l|}{ Deprivation quintile* } \\
\hline WIMD2 & $-2.122(.119)$ & $-3.789(.125)$ & $-.342(.020)$ & $-.128(.004)$ & $-.173(.009)$ \\
\hline WIMD3 & $-3.780(.119)$ & $-6.983(.125)$ & $-.717(.020)$ & $-.241(.004)$ & $-.156(.009)$ \\
\hline WIMD4 & $-5.845(.126)$ & $-9.456(.132)$ & $-1.087(.021)$ & $-.275(.004)$ & $-.394(.010)$ \\
\hline WIMD5 & $-7.180(.126)$ & $-12.017(.132)$ & $-.974(.021)$ & $-.382(.004)$ & $-.530(.010)$ \\
\hline Gender (male) & $-10.419(.081)$ & $-11.298(.084)$ & $-1.240(.014)$ & $.068(.002)$ & $-.227(.006)$ \\
\hline \multicolumn{6}{|l|}{ Duration of prescribing } \\
\hline Under six months & $34.329(.171)$ & $40.671(.178)$ & $.777(.029)$ & $.300(.005)$ & $-.392(.013)$ \\
\hline Over six months & $143.501(.121)$ & $163.975(.119)$ & $11.649(.020)$ & $1.540(.004)$ & $1.614(.009)$ \\
\hline Depression & $22.979(.123)$ & & $2.430(.021)$ & $.633(.004)$ & $.737(.009)$ \\
\hline ED Visits & $9.282(.018)$ & & & & \\
\hline p-value** & $<.001$ & $<.001$ & $<.001$ & $<.001$ & $<.001$ \\
\hline
\end{tabular}

*Deprivation quintile based on Welsh Index of Multiple Deprivation 2011. WIMD1 =most deprived, WIMD5 $=$ least deprived $* * p$-value $<0.05=\operatorname{significant}$ 
The regression was repeated, removing attendance at the emergency department and diagnosis of depression and/or anxiety as a predictive variable (Table 7.7). Emergency department attendance numbers appeared quite low for the number of subjects in the study. Therefore, from a clinical perspective, it was assumed removing ED attendance from the model would not lead to substantial change. Depression and/or anxiety might be a reason to visit the GP in itself, so it was interesting to review the impact of removing it from the model. The resulting regression equation remained significant, $F(8,3286206)=280702.3$, $p<.001, R^{2}=0.406, S E=75.0(R=0.627)$. In this case, $40.6 \%$ of the variance in the data was explained by the model. The $ß_{0}$ (constant) for the regression equation was -14.1, SE=.142. Consequently, removing ED attendance and depression/anxiety from consideration weakened the model slightly.

In the second model, receiving opioid prescriptions and for longer than 6 months $\left(\beta_{n}=163.97, S E=.119, p<.001\right)$ remained the most influential factors for having an appointment at in Primary Care, adding 164 visits (Table 7.7). Receiving opioid prescriptions for less than six months $\left(\beta_{n}=40.67, S E=.178, p<.001\right)$ added 40 visits compared to controls. Deprivation quintile had an inverse effect on GP attendance i.e., lower quintile increased attendance. Age was positively associated with primary care visits $\left(\beta_{n}=17.21, S E=.033, p<.001\right)$, with an increase of 17 visits per year, from age 18 years onwards.

\subsubsection{Out-patient attendance}

The multiple linear regression equation for predicting out-patient attendance was deemed significant $F(9,3286205)=56629.0, p<.001, R^{2}=0.134, S E=12.1(R$ $=0.366$ ) (Table 7.7$)$. However, this model only accounted for $13.4 \%$ of the variables that might predict the number of out-patient attendances of an individual. The $\beta_{0}$ (constant) for the out-patient regression equation was 1.2, $\mathrm{SE}=.023$.

In this equation, being prescribed opioids for more than 6 months $\left(\beta_{n}=11.65\right.$, $\mathrm{SE}=.020, \mathrm{p}<.001$ ) was demonstrated to be significant in increasing the likelihood 
of out-patient appointments. In this model, being resident in the most deprived areas (WIMD1) was taken as baseline, whilst all other quintiles reduced the likelihood of having an outpatient appointment (Table 7.7). A diagnosis of depression and/or anxiety had a greater positive effect $\left(\beta_{n}=2.4, S E=.021, p<.001\right)$ on outpatient visits than receiving opioid prescription for less than six months $\left(\beta_{n}=.78, S E=.029, p<.001\right)$ although both were considered statistically significant.

\subsubsection{Emergency Department attendance}

Multiple linear regression to predict the number of Emergency Department attendances gave a statistically significant result (Table 7.7) but was only reliable for $8.1 \%$ of the variance within the model, $F(9,3286205)=32121.3, p<.001, R^{2}=$ $0.081, \mathrm{SE}=2.14(\mathrm{R}=0.284)$. In this equation, the constant $\beta_{0}$ was $0.68, \mathrm{SE}=.004$.

In the model, recorded diagnosis of depression and/or anxiety $\left(\beta_{n}=.633, \mathrm{SE}=.004\right.$, $p<.001)$ and receiving opioid prescriptions for more than 6 months $\left(\beta_{n}=1.540\right.$, $\mathrm{SE}=.004, \mathrm{p}<.001)$ appeared to have more impact on ED attendance although none of the predictive variables were notable.

\subsubsection{Number of In-patient admissions}

The multiple linear regression equation for predicting the number of in-patient admissions was not effective (Table 7.7). Whilst a statistically significant result was obtained, the model was only able to predict around $7 \%$ of the variance within it, $F(9,3286205)=18090.5, p<.001, R^{2}=0.047(R=0.217), \beta_{0}=-.628$, $\mathrm{SE}=.011$.

As with predicting out-patient attendances, a diagnosis of depression and/or anxiety $\left(\beta_{n}=.737, S E=.009, p<.001\right)$ and being prescribed opioids for more than 6 months were the variables which appeared to exert most influence on the number of in-patient admissions. Increasing levels of deprivation had an inverse influence on the likelihood of in-patient admission. Male gender was significant factor albeit negative influence for predicting hospital admission. 


\subsection{Cost Analysis}

\subsubsection{Total Healthcare costs}

Taking the costings from the 3.2 million subjects included in this study, the average cost of Healthcare utilisation (Primary and Secondary Care) was estimated to be $£ 11,096.49 \pm 15.03$ per person (Table 7.8Error! Reference source not found.) over the 11-year study period. Costs for the case group receiving opioid prescriptions ( $f 16,453.35 \pm 33.08$ per person) were estimated to be $£ 6696.08$ per person ( $\left.\mathrm{Cl} 95 \% £ 6,623.39-£ 6,768.77, p<.001, d_{\text {Cohen's }}=.25\right)$ more than for people in the control group $(£ 9,757.27 \pm 16.77)$. There was a $68 \%$. difference in costs between the two groups (Table 7.8)

Using actual subject numbers (Section 7.4.2) estimated that the total healthcare cost for all subjects whether case or control, with recorded diagnoses of osteoarthritis, rheumatoid arthritis, neck pain, back pain, fibromyalgia, or neuropathic pain was $\mathrm{f} 11.8$ billion, between 2005 and 2015. That averaged just under $£ 1.1$ billion per year. Healthcare costs for people with those conditions and receiving opioid analgesics (case subjects) averaged $£ 0.9$ billion per year between 2005-2015, without factoring in medication costs. The data used for this study was representative of $78 \%$ of the Welsh population, so assuming this was a representative population, annual healthcare costs could be as much as $£ 1.3$ billion for those with the listed diagnoses with $£ 1.2$ billion allocated to those also receiving opioid medicines.

\subsubsection{Costs for specified services}

The average cost of total Primary Care attendance including tests and investigations, by individuals included in this study was estimated to be $£ 2,003.23 \pm £ 1.85$ (SEM) per person (Table 7.8Error! Reference source not

found.). The average cost of Primary Care attendance in the opioid group was 4.9 times per person higher than in the control group. The differences in all Primary Care costs between the two groups were statistically significant (Table 7.8Error! Reference source not found.). 
Table 7.8: Average costings per person for healthcare utilisation comparing all subjects within the study, cases and controls. Variable Mean average costs per person (f) (standard error of the mean)

\begin{tabular}{|c|c|c|c|c|c|}
\hline & $\begin{array}{c}\text { All subjects } \\
(n=3286215)\end{array}$ & $\begin{array}{c}\text { Cases } \\
(n=657243)\end{array}$ & $\begin{array}{c}\text { Controls } \\
(n=2628972)\end{array}$ & Difference $(95 \% \mathrm{Cl})$ & $\begin{array}{l}\text { p-value** } \\
\left.\text { (d } \mathrm{d}_{\text {Cohen's }}\right)\end{array}$ \\
\hline \multicolumn{6}{|l|}{ Primary care } \\
\hline GP appointments & $2002.71(1.85)$ & $5529.24(6.22)$ & 1121.07 (1.21) & 4408.17 (4395.76-4420.59) & $<.001(0.96)$ \\
\hline Potential opioid ADEs* & $9.38(0.02)$ & $23.28(0.09)$ & $5.91(0.02)$ & $17.37(17.21-17.55)$ & $<.001(0.27)$ \\
\hline Tests and investigations & $0.52(.001)$ & $1.52(0.01)$ & $0.27(.001)$ & $1.25(1.24-1.26)$ & $<.001(0.33)$ \\
\hline Imaging & $4.39(0.01)$ & $12.03(0.05)$ & $2.48(0.01)$ & $9.55(9.45-9.66)$ & $<.001(0.24)$ \\
\hline Total Primary Care & $2003.23(1.85)$ & $5530.77(6.22)$ & $1121.34(1.21)$ & $4409.43(4397.01-4421.84)$ & $<.001(0.96)$ \\
\hline \multicolumn{6}{|l|}{ Emergency department } \\
\hline Any ED attendance & $112.81(0.16)$ & $249.62(0.55)$ & $78.61(0.14)$ & $171.01(169.89-172.12)$ & $<.001(0.42)$ \\
\hline ED attendance for pain & $0.63(0.01)$ & $0.89(0.02)$ & $0.57(0.01)$ & $0.32(0.29-0.35)$ & $<.001(0.03)$ \\
\hline ED attendance for opioid ADEs* & $0.56(0.01)$ & $1.00(0.02)$ & $0.45(0.01)$ & $0.55(0.52-0.59)$ & $<.001(0.04)$ \\
\hline \multicolumn{6}{|l|}{ Secondary Care } \\
\hline Outpatient attendance & $744.69(0.79)$ & $1530.43(2.34)$ & $548.26(0.68)$ & $982.18(976.84-987.52)$ & $<.001(0.50)$ \\
\hline Day case acute care attendance & $779.64(2.01)$ & $1605.80(5.46)$ & $573.10(2.09)$ & $1032.70(1021.25-1044.51)$ & $<.001(0.24)$ \\
\hline In-patient admission - standard & $5676.42(6.73)$ & $5073.20(12.43)$ & 5935.49 (7.98) & $-862.29(891.25-833.34)$ & $<.001(0.16)$ \\
\hline In-patient admission - excess days & $8669.82(21.76)$ & $4579.52(26.81)$ & $10426.56(28.75)$ & $-5847.04(5924.09-5768.99)$ & $<.001(-0.06)$ \\
\hline Total in-patient & $7456.11(13.94)$ & $7536.73(27.97)$ & $7435.96(15.96)$ & $100.77(32.20-37.66)$ & $<.001(0.004)$ \\
\hline Total secondary care & $8235.76(14.27)$ & $9142.53(29.25)$ & $8009.06(16.27)$ & $1133.47(1067.88-1199.07)$ & $<.001(0.05)$ \\
\hline Total healthcare costs ner nerson & 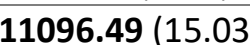 & 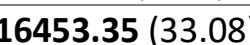 & 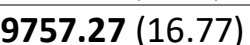 & 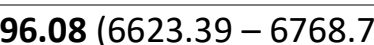 & $<.001(0.25)$ \\
\hline
\end{tabular}

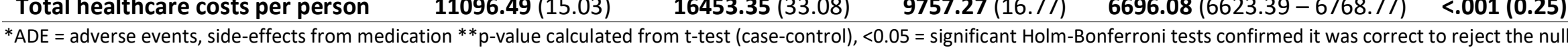
hypothesis 
Whilst the numbers of attendances for potential opioid adverse events were low for both cases and controls, costs for the opioid group were 3.9 times per person higher than for the control group (Table 7.8). Similarly, whilst only limited data was available on tests and investigations, 5.6 times higher costs per person were incurred by the opioid group compared to the control group. Imaging costs averaged at $£ 4.39 \pm £ 0.01$ per person, with case subjects incurring 4.9 times the costs of their controls (Table 7.8).

Costs associated with ED attendance incurred by those receiving opioid prescriptions were twice those of the control group (Table 7.8). Costs for ED attendances for possible opioid adverse events were, on average, lower than those for pain. The cost of pain-related attendances and adverse events that may be associated with opioid use, were significantly higher for case subjects than for the control group (Table 7.8Error! Reference source not found.).

Outpatient attendance costs for those in receipt of opioid prescriptions were 2.8 times higher than for people in the control group which was a statistically significant difference (Table 7.8). Average day case attendance costs per person in the study group were $£ 744.69 \pm £ 0.79$. Costs for case subjects were 2.8 times higher than for the control group (Table 7.8Error! Reference source not found.).

In-patient attendance was divided into standard admission, up to 5 days in a single admission and excess days, costed from day of admission onwards. Standard admission costs averaged $£ 5676.42 \pm £ 6.73$ per person and in a reversal of previous results, the costs were significantly higher in the control group than for the case (Table 7.8Error! Reference source not found.). In-patient excess days had a total average cost of $£ 8669.82 \pm £ 21.76$ per person. Control subjects incurred 2.3 times more excess days costs than cases (Table 7.8Error! Reference source not found.). 


\subsubsection{Examining costs by demographic factors}

\subsubsection{Gender}

Healthcare costs were $66 \%$ higher in female case subjects compared to their controls. (Table 7.9). Average male expenditure was $30 \%$ less than for female subjects. Male case subjects had on average $73 \%$ greater total healthcare costs compared to their controls (Table 7.9).

Table 7.9: Average costings by gender for healthcare utilisation, comparing cases and controls

\begin{tabular}{|c|c|c|c|c|c|}
\hline & \multicolumn{3}{|c|}{ Mean average costs $(\mathrm{f})$ (standard error of the mean) } & \multirow[b]{2}{*}{ Difference $(95 \% \mathrm{Cl})$} & \multirow[b]{2}{*}{ p-value* $\left(d_{\text {Cohen's }}\right)$} \\
\hline & All subjects & Cases & Controls & & \\
\hline Female & $\mathrm{n}=1920930$ & $\mathrm{n}=384186$ & $n=1536744$ & & \\
\hline Primary care & $2216.46(2.52)$ & 6037.93 (8.29) & $1261.10(1.65)$ & $4778.30(4761.74-4794.87)$ & $<.001(1.02)$ \\
\hline Emergency Department & $111.68(0.21)$ & $249.91(0.72)$ & $77.13(0.18)$ & $172.78(171.34-174.23)$ & $<.001(0.42)$ \\
\hline Secondary Care & $9093.64(19.44)$ & $9985.20(39.46)$ & $8870.75(22.21)$ & $1114.46(1-25.70-1203.21)$ & $<.001(0.04)$ \\
\hline Total & $12243.43(20.42)$ & $17942.03(44.45)$ & $10818.78(22.84)$ & $7123.25(7025.31-7221.18)$ & $<.001(0.26)$ \\
\hline Male & $\mathrm{n}=1365285$ & $n=273057$ & $\mathrm{n}=1092228$ & & \\
\hline Primary care & $1701.95(2.66)$ & $4813.54(9.21)$ & $924.06(1.74)$ & $3890.42(3872.05-3908.78)$ & $<.001(0.89)$ \\
\hline Emergency Department & $114.41(0.26)$ & $249.21(0.86)$ & $80.71(0.23)$ & $168.51(166.76-170.26)$ & $<.001(0.40)$ \\
\hline Secondary Care & $7028.73(20.74)$ & 7956.91 (43.17) & $6796.68(23.57)$ & $1160.22(1063.83-1256.62)$ & $<.001(0.05)$ \\
\hline Total & $9482.76(21.91)$ & $14358.81(49.01)$ & $8263.74(24.35)$ & $6095.07(5987.81-6202.33)$ & $<.001(0.24)$ \\
\hline
\end{tabular}

*p-value calculated from t-test (case-control), $<0.05$ = significant Holm-Bonferroni confirmed it was correct to reject the null hypothesis

\subsubsection{Deprivation}

Increased costs were associated with increased levels of socioeconomic deprivation in primary care, emergency department and overall healthcare costs (Table 7.10) within case and control groups. Examining costs by deprivation quintiles revealed significantly higher average costs in case subjects than controls in every measure examined. 
Table 7.10: Average costings per person by deprivation for healthcare utilisation comparing cases and controls

\begin{tabular}{|c|c|c|c|c|c|}
\hline \multicolumn{6}{|c|}{ Mean average costs per person $(f) \pm$ SEM } \\
\hline & $\begin{array}{c}\text { All subjects } \\
(n=3286215)\end{array}$ & $\begin{array}{c}\text { Cases } \\
(n=657243)\end{array}$ & $\begin{array}{c}\text { Controls } \\
(\mathrm{n}=2628972)\end{array}$ & Difference $(95 \% \mathrm{Cl})$ & p-value* $\left(d_{\text {Cohen's }}\right)$ \\
\hline \multicolumn{6}{|l|}{ Total Primary care } \\
\hline WIMD1 & $2149.60(3.91)$ & $17509.49(72.18)$ & $10283.28(35.11)$ & $4516.81(4500.53-4533.10)$ & $<.001(0.96)$ \\
\hline WIMD2 & $2064.27(4.15)$ & $16953.81(74.62)$ & $10068.36(40.24)$ & $4513.87(4486.19-4541.55)$ & $<.001(0.97)$ \\
\hline WIMD3 & $1998.26(4.07)$ & $16489.79(71.41)$ & $10475.45(36.00)$ & $4494.55(4467.15-4521.94)$ & $<.001(0.97)$ \\
\hline WIMD4 & $1902.76(4.38)$ & $15530.13(74.56)$ & $8680.84(35.40)$ & $4294.40(4264.84-4323.96)$ & $<.001(0.95)$ \\
\hline WIMD5 & $1838.42(4.19)$ & $15322.23(75.78)$ & 8891.71 (39.82) & $4155.40(4127.54-4183.26)$ & $<.001(0.96)$ \\
\hline \multicolumn{6}{|l|}{ Emergency department } \\
\hline WIMD1 & $142.06(0.40)$ & $303.46(1.32)$ & $101.71(0.36)$ & $201.75(199.06-204.44)$ & $<.001(0.42)$ \\
\hline WIMD2 & $122.78(0.38)$ & $269.27(1.35)$ & $86.07(0.31)$ & $183.52(180.81-186.22)$ & $<.001(0.40)$ \\
\hline WIMD3 & $105.45(0.36)$ & $236.78(1.14)$ & $72.61(0.34)$ & $164.17(161.84-166.49)$ & $<.001(0.42)$ \\
\hline WIMD4 & $99.79(0.33)$ & $216.99(1.13)$ & $70.49(0.28)$ & $146.50(144.22-148.79)$ & $<.001(0.46)$ \\
\hline WIMD5 & $83.80(0.28)$ & $201.86(1.02)$ & $54.29(0.29)$ & $147.57(145.52-149.62)$ & $<.001(0.46)$ \\
\hline \multicolumn{6}{|l|}{ Total Secondary Care } \\
\hline WIMD1 & $8634.71(30.06)$ & $9775.70(64.20)$ & 8349.47 (33.96) & $1426.23(1283.88-1568.58)$ & $<.001(0.6)$ \\
\hline WIMD2 & $8487.79(34.11)$ & $9421.72(66.13)$ & 8254.31 (39.29) & $1167.40(1016.65-1318.16)$ & $<.001(0.05)$ \\
\hline WIMD3 & $8839.61(30.63)$ & $9174.16(62.92)$ & 8755.97 (34.91) & $418.19(277.11-559.22)$ & $<.001(0.02)$ \\
\hline WIMD4 & $7358.20(30.32)$ & $8542.16(65.20)$ & $7062.20(34.20)$ & $1479.96(1335.65-1624.27)$ & $<.001(0.07)$ \\
\hline WIMD5 & $7549.73(33.75)$ & $8523.04(67.18)$ & 7306.40 (38.69) & $1216.64(1064.69-1368.58)$ & $<.001(0.05)$ \\
\hline \multicolumn{6}{|l|}{ Total costs } \\
\hline WIMD1 & $11728.52(31.75)$ & $17509.49(72.18)$ & $10283.28(35.11)$ & $7226.21(7068.89-7383.53)$ & $<.001(0.26)$ \\
\hline WIMD2 & $11445.45(35.64)$ & $16953.81(74.62)$ & $10068.36(40.24)$ & $6885.45(6719.28-7051.62)$ & $<.001(0.25)$ \\
\hline WIMD3 & $11678.32(32.28)$ & $16489.79(71.41)$ & $10475.45(36.00)$ & $6014.34(5857.60-6171.08)$ & $<.001(0.23)$ \\
\hline WIMD4 & $10050.70(32.21)$ & $15530.13(74.56)$ & 8680.84 (35.40) & $6849.29(6687.53-7011.05)$ & $<.001(0.28)$ \\
\hline WIMD5 & $10177.81(35.44)$ & $15322.23(75.78)$ & 8891.71 (39.82) & $6430.52(6262.73-6598.31)$ & $<.001(0.25)$ \\
\hline
\end{tabular}

*p-value calculated from t-test (case-control), $<0.05=$ significant.

Holm-Bonferroni confirmed it was correct to reject the null hypothesis 


\subsubsection{Prescribing Persistence}

Significantly greater costs were noted for people receiving more than 6 months of opioid prescriptions for all healthcare resources examined (Table 7.11). Average total healthcare costs were estimated to be $122 \%$ higher when opioid prescriptions persisted for more than 6 months.

\begin{tabular}{|c|c|c|c|c|}
\hline \multirow[t]{2}{*}{ Healthcare resource } & \multicolumn{2}{|c|}{ Opioid prescribing persistence } & \multirow[b]{2}{*}{ Difference $(95 \% \mathrm{Cl})$} & \multirow[b]{2}{*}{ p-value* $\left(d_{\text {Cohen's }}\right)$} \\
\hline & $\begin{array}{l}<6 \text { months } \\
(n=192281)\end{array}$ & $\begin{array}{l}>6 \text { months } \\
(\mathrm{n}=464962)\end{array}$ & & \\
\hline Primary care & 2333.25 (6.09) & $6850.92(7.62)$ & 4518.94 (4499.82 - 4538.07) & $<.001(1.25)$ \\
\hline Emergency department & $126.47(0.58)$ & $300.55(0.73)$ & $174.07(172.25-175.90)$ & $<.001(0.51)$ \\
\hline Secondary Care & $5760.49(44.52)$ & $10541.14(36.82)$ & 4780.65 (4667.42 - 4893.89) & $<.001(0.22)$ \\
\hline Total costs & 8835.89 (46.94) & $19603.49(41.68)$ & $10767.60(10644.57-10890.62)$ & $<.001(0.47)$ \\
\hline
\end{tabular}

*p-value calculated from t-test (case-control), $<0.05=$ significant

Holm-Bonferroni confirmed it was correct to reject the null hypothesis

\subsubsection{One-way sensitivity analysis}

Increasing control group costs for Primary care attendance, up to double of those observed in the study, did not result in a non-significant difference (acceptance of the null hypothesis). Doubling of control group primary care costs, resulted in a 2.5 times lower costs per person, which remained significantly less than for the unchanged case group. Doubling of emergency department attendance costs also failed to lead to acceptance of the null hypothesis. 
Secondary care and consequently total healthcare costs, when increased by $75 \%$ and above, became higher in the control group than for case subjects, with the difference being statistically significant.

Overall, the sensitivity analyses demonstrate that increases of more than $50 \%$ in healthcare attendances and therefore, costs, would be needed in the control group in order to bring the totals in that group, close to those noted in case subjects.

Table 7.12: Comparison of costs per person for sensitivity analysis: study estimates of case subject costs compared to re-calculated control subject costs

\begin{tabular}{|c|c|c|c|c|}
\hline \multirow[b]{2}{*}{$\begin{array}{l}\text { Increase to control subjects' } \\
\text { costs }\end{array}$} & \multicolumn{4}{|c|}{ Mean average costs per person $(f) \pm$ standard error of the mean } \\
\hline & $\begin{array}{c}\text { Cases } \\
(n=657243)\end{array}$ & $\begin{array}{c}\text { Controls } \\
(n=2628972)\end{array}$ & Difference $(95 \% \mathrm{Cl})$ & p-value* $\left(\mathrm{d}_{\text {Cohen's }}\right)$ \\
\hline \multicolumn{5}{|l|}{ Total Primary care } \\
\hline $25 \%$ & $5530.77(6.21)$ & $1401.68(1.51)$ & $4129.09(4116.54-4141.63)$ & $<.001(0.89)$ \\
\hline $50 \%$ & $5530.77(6.21)$ & $1682.02(1.81)$ & $3848.75(3836.06-3861.45)$ & $<.001(0.82)$ \\
\hline $75 \%$ & $5530.77(6.21)$ & $1962.35(2.11)$ & $3568.42(3555.54-3581.29)$ & $<.001(0.75)$ \\
\hline $100 \%$ & $5530.77(6.21)$ & $2242.69(2.42)$ & $3288.08(3275.00-3301.16)$ & $<.001(0.68)$ \\
\hline \multicolumn{5}{|l|}{ Emergency department } \\
\hline $25 \%$ & $249.62(0.55)$ & $98.27(0.18)$ & $151.36(150.22-152.49)$ & $<.001(0.36)$ \\
\hline $50 \%$ & $249.62(0.55)$ & $117.92(0.21)$ & $131.70(130.54-132.86)$ & $<.001(0.31)$ \\
\hline $75 \%$ & $249.62(0.55)$ & $137.57(0.25)$ & $112.05(110.86-113.23)$ & $<.001(0.26)$ \\
\hline $100 \%$ & $249.62(0.55)$ & $157.23(0.28)$ & $92.40(91.18-93.61)$ & $<.001(0.0 .20)$ \\
\hline \multicolumn{5}{|l|}{ Total Secondary Care } \\
\hline $25 \%$ & $9142.53(29.25)$ & $10011.33(20.34)$ & $-868.80(-953.52--784.07)$ & $<.001(-0.03)$ \\
\hline $50 \%$ & $9142.53(29.25)$ & $12013.59(24.41)$ & $-2871.06(-2945.72--2896.40)$ & $<.001(-0.10)$ \\
\hline $75 \%$ & $9142.53(29.25)$ & $14015.86(28.48)$ & $-4873.33(-4953.33--4793.32)$ & $<.001(-0.17)$ \\
\hline $100 \%$ & $9142.53(29.25)$ & $16018.12(32.55)$ & $-6875.59(-6961.35--6789.83)$ & $<.001(-0.22$ \\
\hline \multicolumn{5}{|l|}{ Total costs } \\
\hline $25 \%$ & $16453.35(33.08)$ & $12196.59(20.96)$ & $4256.76(4180.01-4333.52)$ & $<.001(0.15)$ \\
\hline $50 \%$ & $16453.35(33.08)$ & $14635.91(25.15)$ & $1817.45(1736.00-1898.89)$ & $<.001(0.06)$ \\
\hline
\end{tabular}




\begin{tabular}{|c|c|c|c|c|}
\hline $75 \%$ & $16453.35(33.08)$ & $17075.22(29.34)$ & $-621.87(-708.54--535.21)$ & $<.001(-0.02)$ \\
\hline $100 \%$ & $16453.35(33.08)$ & $19514.54(33.53)$ & $-3061.19(-3153.51--2968.87)$ & $<.001(-0.09)$ \\
\hline
\end{tabular}

*p-value calculated from t-test (case-control), $<0.05$ = significant Holm-Bonferroni confirmed it was correct to reject the null hypothesis

\subsubsection{Two-way sensitivity analysis}

Alterations made to both case and control subject costs, did not result in a non-significant difference between the groups (Table 7.13). When primary care costs were adjusted in order to produce a difference of $£ 18.99$. The difference was still considered statistically significant - likely due to the large numbers of subjects, as the effect size was negligible (Table 7.13).

Table 7.13: Comparison of costs per person for sensitivity analysis: study estimates of re-calculated case and control subject costs

\begin{tabular}{|c|c|c|c|c|}
\hline \multicolumn{5}{|c|}{ Mean average costs per person ( $\mathrm{f}$ ) (standard error of the mean) } \\
\hline Change to subjects' costs & Cases $(n=657243)$ & Controls ( $n=2628972)$ & Difference $(95 \% \mathrm{Cl})$ & p-value* $\left(d_{\text {Cohen's }}\right)$ \\
\hline \multicolumn{5}{|l|}{ Total Primary care } \\
\hline Case $\mathbf{2 5 \%}$ Control $\mathbf{1 2 5 \%}$ & $1382.69(1.56)$ & $1401.68(1.51)$ & $-18.99(-23.23--14.74)$ & $<.001(0.01)$ \\
\hline Case $50 \%$ Control $150 \%$ & $2765.38(3.11)$ & $1682.02(1.81)$ & $1083.37(1076.32-1090.42)$ & $<.001(0.42)$ \\
\hline Case $75 \%$ Control $175 \%$ & $4148.08(4.67)$ & $1962.35(2.11)$ & $2185.72(2175.69-2195.76)$ & $<.001(0.59)$ \\
\hline Case $50 \%$ Control $125 \%$ & $2765.38(3.11)$ & $1401.68(1.51)$ & $1363.71(1356.93-1370.48)$ & $<.001(0.54)$ \\
\hline Case $75 \%$ Control $150 \%$ & $4148.08(4.67)$ & $1682.02(1.81)$ & $2466.06(2456.25-2475 / 88)$ & $<.001(0.68)$ \\
\hline Case $\mathbf{2 5 \%}$ Control $\mathbf{1 7 5 \%}$ & $1382.69(1.56)$ & $1962.35(2.11)$ & $-579.66(-584.80--574.52)$ & $<.001(-0.31)$ \\
\hline \multicolumn{5}{|l|}{ Total Secondary Care } \\
\hline Case $25 \%$ Control $125 \%$ & $2285.63(7.31)$ & $10011.33(20.34)$ & $-7725.69(-7768--7683.33)$ & $<.001(-0.49)$ \\
\hline Case $50 \%$ Control $150 \%$ & $4571.27(14.62)$ & $12013.59(24.41)$ & $-7442.33(-7498.09--7386.56)$ & $<.001(-0.36)$ \\
\hline Case $75 \%$ Control $175 \%$ & $6856.90(21.93)$ & $14015.86(28.48)$ & $-7158.96(-7229.41--7088.51)$ & $<.001(-0.28)$ \\
\hline Case $50 \%$ Control $125 \%$ & $4571.27(14.62)$ & $10011.33(20.34)$ & $-5540.06(-5489.16--5390.96)$ & $<.001(-0.30)$ \\
\hline Case $75 \%$ Control $150 \%$ & $6856.90(21.93)$ & $12013.59(24.41)$ & $-5156.69(-5221.01--5092.37)$ & $<.001(-0.22)$ \\
\hline Case $\mathbf{2 5 \%}$ Control $\mathbf{1 7 5 \%}$ & $2285.63(7.31)$ & $14015.86(28.48)$ & $-11730.22(-11787.85--11672.60)$ & $<.001(-0.55)$ \\
\hline \multicolumn{5}{|l|}{ Total costs } \\
\hline Case $25 \%$ Control $125 \%$ & $4113.34(8.27)$ & $12196.59(20.96)$ & $-8083.25(-8127.41--8039.09)$ & $<.001(-0.50)$ \\
\hline
\end{tabular}




\begin{tabular}{|c|c|c|c|c|}
\hline Case $50 \%$ Control $150 \%$ & $8226.68(16.54)$ & $14635.91(25.15)$ & $-6409.23(-6468.23--6350.23)$ & $<.001(-0.29)$ \\
\hline Case $75 \%$ Control $175 \%$ & $12340.01(24.81)$ & $17075.22(29.34)$ & $-4735.21(-4810.52--4659.90)$ & $<.001(-0.17)$ \\
\hline Case $50 \%$ Control $125 \%$ & $8226.68(16.54)$ & 12196.59 (20.96) & $-3969.91(-4022.24--3917.59)$ & $<.001(-0.21)$ \\
\hline Case $75 \%$ Control $150 \%$ & $12340.01(24.81)$ & $14635.91(25.15)$ & $-2295.89(-2365.13--2226.65)$ & $<.001(-0.09)$ \\
\hline Case $25 \%$ Control $175 \%$ & $4113.34(8.27)$ & $17075.22(29.34)$ & $-12961.89(-13021.65--12902.14)$ & $<.001(-0.60)$ \\
\hline
\end{tabular}

*p-value calculated from t-test (case-control), $<0.05=$ significant Holm-Bonferroni confirmed it was correct to reject the null hypothesis 


\subsection{Discussion}

\subsubsection{Summary and reflections on findings}

People with pain-related diagnoses and receiving prescriptions for opioids accessed significantly more healthcare resources than those with similar diagnoses but not prescribed opioids from primary care. Increased healthcare resource utilisation and consequent costs were associated with female gender, higher levels of deprivation and prescribing persistence of six months or more.

The results demonstrated primary care bore the major burden of increased healthcare utilisation by those receiving opioid prescriptions. General practice remains the first port of call and gatekeeper of the rest of NHS access (Loudon 2008; Greenfield et al. 2016), so it is not surprising the majority of transactions take place in that sector. There is a link between primary care and ED attendance, noted by the small reduction in the percentage variance predicted in the regression models, when ED attendance was removed from the model. The obvious explanation is that increased ED attendance is indicative of greater incidence of illness, health concerns or difficulty coping with the same. ED attendance was a greater predictor of primary care interaction than gender in the regression model (Table 7.7), which might fit with that understanding.

Regression modelling was not very reliable for predicting attendance or admission to secondary care services. This is likely reflective of the complexity of decision making leading to referral or the decision of an individual to go to ED if for anything other than a genuine medical emergency, for example. Reasons for attending or referral were not available to this study and therefore, only postulations of cause can be made. People using opioids for long-term pain conditions may have greater health concerns or anxiety attached to their condition, leading them to seek support. The use of opioids may cause additional health problems leading to secondary care healthcare utilisation.

Greater healthcare utilisation by women compared to their male counterparts, in case and control groups, was a probable outcome given the prescribing trends noted in Phase 1 of the study (Section 3.5.3, Section 4.5.2 and Section 5.5.3) and 
the results of the regressions described (Table 7.7). Less expected was the degree of difference between case and control subjects. Whilst in primary care some of this variance might be accounted for by issued prescriptions being counted as an interaction, the reasons for the differences in outpatient attendance and inpatient admission require more investigation. Given the known adverse effects of opioids, it is not unreasonable to suspect that some increase in attendance is due to that, whether or not they are identified. It is difficult to make any definitive statement about possible causation without knowing reasons for attendance, but it is worthy of further investigation.

Similarly, equal rates of attendance at ED by male and female case subjects was an interesting finding, especially as there were gender differences in the control group. It is understood women present more frequently to healthcare services and they report pain more frequently than men (Rhudy and Williams 2005; Zhang et al. 2021). It is unlikely this alone would be sufficient explanation to account for the differences in secondary care interactions. However, more frequent primary care attendance is perhaps likely to result in more out-patient appointments. Primary care practitioners refer for specialist opinion and treatment of serious pathology or when they have exhausted treatment and support options (Ringberg et al. 2014; Tzartzas et al. 2019). As previously discussed, (Section 3.6.5 and Section 4.6.3), women report difficulty accessing treatment and support for painful conditions, especially those unique to females (Williams 2021). Further research to investigate differences in the outcome of appointments, between genders would be helpful. For example, examining whether there are gender differences in the proportion of primary care appointments that result in referral for specialist opinion.

Differences in healthcare utilisation based on age-group were heavily weighted towards case subjects in all aspects other than LoS. People under 65 years old, receiving opioid prescriptions had more hospital admissions than their control group and longer LoS per admission. Whilst older opioid users had more admissions than their controls, their admissions were on average shorter. 
Reasons for admission in the different groups were not available for this study. It would be valuable to determine how differences in LoS were linked to opioid prescribing. Younger patients accessing acute care services may perhaps be expected to be more unwell than their control subjects who, whilst having similar conditions, were not using opioid analgesics. Being unwell, may take longer to resolve, leading to greater LoS. In older people, admission could sometimes be due to opioid-induced effects which might be more quickly resolved in an acute care setting (e.g., constipation, sedation). However, whilst this may account for LoS, it does not easily explain the differences in out-patient or ED attendance that were also noted.

Receiving opioid prescriptions for 6 months or more was the strongest predictor for increased healthcare utilisation and consequently, costs in all sectors (Section 7.9). If each prescription issue was counted as an appointment in primary care, this may have skewed the model in favour of the variable. However, receipt of any opioid prescription was also a positive predictor of attendance. Significant differences in utilisation and consequent healthcare costs were demonstrated between groups of less than and more than six months prescription persistence. Duration of opioid use has been previously shown to be connected to adverse effects (Cohen et al. 2008; Mundkur et al. 2017; Bialas et al. 2020) and this would likely necessitate additional healthcare input. People requiring long-term prescribing of opioids may reasonably be presumed to be in worse health than those not receiving opioids despite having similar diagnoses. However, the evidence to use opioids in the conditions highlighted in this study is not supportive (Els et al. 2017; Faculty of Pain Medicine 2021). TOPAS affirms a link between prescribing persistence and healthcare use, even if the nature of the connection cannot be fathomed at this stage.

Stratification of utilisation and costs by deprivation showed some interesting patterns. Empirically, differences between quintiles in either case or control subject groups did not appear particularly large. Differences between groups were statistically significant for every measure, however. Regression analysis 
suggested greater deprivation was a better predictor of healthcare utilisation than lower deprivation. Perhaps notable is that healthcare utilisation in the least deprived quintiles was not as different to the most deprived as might have been expected. ADE data was not extensive, but there was a clear difference between case and control quintiles. ADEs of opioids often present as other health conditions or the potentiation of existing poor health. It is possible that too often, the contribution of opioids to the individual's presentation is missed as a result.

Prescribing trends described in Phase 1 of this study noted prescribing in WIMD5 areas had a larger percentage increase than WIMD1 areas during the study (Chapter 4). The gap in prescribing rates of strong opioids especially was narrowing towards the end of the study period and the implications for population health must be considered. Are the limited differences and increase in prescribing, an indication that the prevalence of pain is increasing in all sections of the Welsh population? If so, does the increase in opioid prescribing and subsequent increased use of healthcare services indicate a failure to provide timely and effective support to people living with painful conditions?

People living in less deprived areas would likely be considered to have easier access to services in most cases. A greater percentage may be able to self-fund pain management support or alternative treatments than their counterparts in more deprived areas. Despite that, opioid prescribing continued to increase, and more healthcare interactions occurred over the eleven years of the study.

The simplest explanation for the differences between case and control subjects noted in this study would be that people are receiving opioids because their condition is worse or more difficult to treat than their comparator's. However, this is likely a massive oversimplification. With the evidence of the harms of opioids growing all the time, opioid analgesics are no longer recommended for a number of the conditions examined in this study such as back pain (National Institute for Health and Care Excellence 2016), neuropathic pain (Centre for 
Clinical Practice at NICE 2013) and chronic primary pain (National Institute for Health and Care Excellence 2021), particularly for long-term use.

The concern is that without taking any action to change opioid prescribing, Wales could end up having an even greater percentage of the population harmed by opioids than might be implied by the data presented here. Certainly, the evidence does not support the notion that opioids are leading to improvement (Harrison and Cormack 2018; All Wales Medicines Strategy Group 2021).

\subsubsection{Comparison with other literature}

No previous studies from the UK, examining the association between opioid prescribing for chronic pain and healthcare utilisation were found. However, increased healthcare utilisation following the initiation of opioids has been reported across the world (Kern et al. 2015).

Chang and colleagues (2018) examined healthcare utilisation in American people deemed to be receiving 'high risk' prescription opioid use in 2012 (Chang et al. 2018). The study examined data from nearly 900,000 people, of whom just over $21 \%$ had received an opioid prescription. Comparisons were made between all people included in the study, including those receiving opioids and the specified user groups rather than as a case-controlled study (Chang et al. 2018). Total healthcare utilisation was examined by cost rather than attendances but were approximately twice as high in the opioid user group as the combined group's total across the study period.

Kern and colleagues (2015) conducted an analysis of healthcare resource utilisation by opioid users in the USA (Kern et al. 2015). Unlike this study, the comparisons made were between acute and chronic opioid use, rather than a case-control study. The Kern study looked at office visits (near equivalent to primary care in the UK), outpatient visits, inpatient admissions and length of stay. They observed an increase in all variables in the six months following opioid initiation, the largest percentage increases noted in people with weak opioids 
(Kern et al. 2015). After the initial large increase in healthcare utilisation, levels of use started to decline although did not return to pre-opioid levels after a further six months (Kern et al. 2015). Therefore, as seen in this study, extended prescription of opioids resulted in greater healthcare utilisation.

It is difficult to compare costs between the US and UK due to differences in healthcare systems and how tariffs are determined. The increased healthcare utilisation and associated costs demonstrated in this study are, however, consistent with the findings of other studies (Kern et al. 2015; Kay et al. 2017). Chang's study (2018) supports the TOPAS findings that there is a correlation between prescription opioid use, increased healthcare utilisation and, consequently, costs.

\subsubsection{Acute care}

Chang et al. demonstrated hospitalisation was three times higher in those receiving opioids than the combined group ( $9.28 \%$ versus $2.98 \%$ respectively) in 2012, when opioids were first prescribed (Chang et al. 2018). In this study, healthcare utilisation was examined during the whole period that opioids were being prescribed. Hospital admission was twice as frequent in opioid users in TOPAS case subjects as controls. Different measures were examined in the two studies, but the findings are mutually supportive. Both suggest people using prescription opioids are more likely to be hospitalised for any cause than people not using those medicines (Chang et al. 2018). Opioid users who undergo inpatient surgery have been shown to have an increased likelihood of further hospitalisations than nonopioid users. In a group of commercially insured patients for example, $17 \%$ of those prescribed opioids after one surgery had subsequent admissions compared to $12 \%$ who did not (Brummett et al. 2019).

A study from Germany observed a significant increase in outpatient consultations, following the initiation of opioids. As with other studies, the increase in resource utilisation did not persist, even when opioids continued (Bruggenjurgen et al. 2007). The study stratified results by drug and noted 
fentanyl was associated with additional resource utilisation compared to oxycodone and morphine (Bruggenjurgen et al. 2007).

\subsubsection{Emergency department utilisation}

Attendance at emergency departments was significantly more common by people receiving opioid medicines than control subjects. Data on reasons for attendance was not complete on extraction from the databank, so was not explored in this study. However, the National Pain Audit (2012) collated responses from people using chronic pain services across the UK in regard to healthcare utilisation. Twenty percent of respondents stated they had attended the emergency department in the previous six months, specifically looking for help with pain (Price et al. 2012).

Literature comparing ED attendance in people prescribed opioids with controls from the UK was not found. Braden et al. analysed emergency department attendance by people, receiving opioid prescriptions for 90 days or more, under one of two insurance schemes (federal and private), for non-cancer pain (Braden et al. 2010). Women composed $72 \%$ of Medicaid patients and $59 \%$ of privately insured people, with the average $62 \%$, slightly higher than noted in this study. The most common painful diagnosis was back pain, present in around $40 \%$ of cases. Emergency department visits were recorded in $25 \%$ of study participants, with an average of 0.8 attendances per person during a twelve-month study period. Greater numbers of ED visits were not correlated to dose or over 6month duration of use, unlike to what was noted in TOPAS (Braden et al. 2010). Increased visits to ED were associated however, with the receipt of more than one form of opioid medicine, e.g., modified release and standard release and the additional use of benzodiazepines (Braden et al. 2010).

Whilst polypharmacy was not examined in this study, the use of combinations of medicines have been an increasing cause of concern in the UK, due to higher risk of harm, dependence, and death (Torrance et al. 2018; Taylor et al. 2019). Of 9,940 people receiving 3 or more opioid prescriptions within 90 days, 51 suffered 
overdose and 6 died (Dunn et al. 2010). The risk of overdose increased with rising dose. Estimations suggest a 1.8\% annual overdose risk for people receiving opioid doses of 100mg OMEQ and above (Dunn et al. 2010).

\subsubsection{Prescription persistence and dose}

Kern et al. (2015) defined chronic opioid use as 183 or more days, and with repeated prescriptions filled within 30 days of the previous one (Kern et al. 2015), which was the same as the definition used in this study. Primary care visits and other outpatient-type appointments were noted to increase by 3 and 5 times, respectively in the first six months of treatment (Kern et al. 2015). After 6 months of opioid treatment, utilisation and costs reduced significantly with the largest reduction observed for in-patient admissions.

The use of opioids was associated with greater healthcare utilisation in another American study (Kay et al. 2017). Doses of greater than 200mg OMEQ were not associated with a higher number of ED visits or clinic visits than people receiving lower daily doses, however. Interestingly more calls to out of hours services were made by people prescribed the highest OMEQ doses compared to lower doses (Kay et al. 2017). These results differed from Chang et al. (2018), who defined chronic opioid use as receiving 100mg OMEQ per day for more than 90 consecutive days (Chang et al. 2018) and demonstrated chronic use as defined was associated with twice as much healthcare use compared to people on lower doses or no opioid. Even higher use of healthcare resources was observed when opioids were combined with other high-risk medicines such as benzodiazepines or in the presence of opioid misuse disorder (Chang et al. 2018). Prescribing persistence was shown to be lengthier in the areas of Wales with highest levels of deprivation (Section 3.5.5). Increased deprivation was also correlated to greater healthcare utilisation and consequent costs.

\subsubsection{Comparisons of costs}

Studies examining costs associated with opioid analgesics are generally focussed on adverse effects or misuse, rather than whether use results in additional 
healthcare problems. In Wales, the cost of medicines classified as opioid analgesics (not including co-products such as co-codamol) in the BNF (BNF: British National Formulary - NICE. 2021) increased 46.3\% from f10.4 million in the year 2004-2005 to $f 19.2$ million in $2014-2015$, totalling $£ 177.7$ million over the TOPAS study period (Welsh Government 2021). Opioid analgesic costs peaked in 2015-2016 and have reduced each year since, reflecting the small reduction in prescription numbers reported (Section 6.5) and falling costs of some medicines as they come off patent or Welsh contracts are negotiated. Prescribing accounts for around one-third of primary care spending (Auditor General for Wales 2018) in Wales. Health boards spent $£ 1.39$ billion on primary care services in 2016-2017, a 5\% reduction in real terms from 2010-2011 when figures were previously reported (Shared Services Partnership 2021). Overall, health board spending in 2016-2017 was recorded as $£ 6.32$ billion, so primary care accounted for $22 \%$ of the total (Auditor General for Wales 2018). Based on the calculations used for this study, the cost of healthcare use for people living with the six major chronic pain types is upwards of $f 1.3$ billion per year in Wales. This is the first estimate of healthcare costs for this patient group to be made in the country.

Kern and colleagues (2015) calculated the average all-cause costs which more than doubled (from $\$ 13,459$ to $\$ 31,695$ ) in the first six months of opioid treatment (Kern et al. 2015). Whilst there was a reduction in overall costs after six months for chronic opioid users, they did not return to pre-opioid levels. A difference was noted for in-patient stays, however, where a return to below pretreatment costs was noted after six months (Kern et al. 2015). This was an interesting finding in light of the results from this study, where in-patient length of stay and subsequently cost per admission was, on average, lower for people prescribed opioids than not. However, prescribing persistence greater than six months was associated with a much higher number of admissions, length of stay and therefore, overall associated costs than those with shorter duration of prescribing or none at all. 
The cost of back pain to the UK economy is often quoted as $f 12$ billion per year, although that estimate is now 20 years old (Maniadakis and Gray 2000). Healthcare costs, excluding medicines, comparable to those used in this study were estimated to be $\mathbf{f 9 7 4}$ million by Maniadakis and Gray (Maniadakis and Gray 2000). This demonstrates the healthcare costs of back pain and, in fact, all chronic pain conditions, are relatively small relative to the overall economic impact (Phillips 2008).

Studies which have examined the cost-effectiveness of using opioid analgesics from the perspective of only healthcare utilisation are rare. However, studies comparing the cost-effectiveness of opioid analgesics to other treatment strategies have been conducted. Takura et al. (2021) used a Markov model to calculate the incremental cost-effectiveness ratio (ICER) for people with hip or knee osteoarthritis (OA) and who received either opioids such as codeine or tramadol or non-opioid strategies including surgery or other medicines such as non-steroidal anti-inflammatory drugs, paracetamol or steroids, in line with treatment guidelines used in Japan (Takura et al. 2021). In people eligible for surgery, non-opioid treatment strategies had higher efficacy and were less expensive. QALYs for the knee OA, non-opioid group were 11.53 compared to 11.50 for the opioid group over 30 years (Takura et al. 2021). The analysis suggested that opioids were dominated for both hip and knee OA, leading the authors to conclude that non-opioid strategies were cost-effective compared to opioids for surgery-eligible patients with knee or hip OA (Takura et al. 2021). These conclusions concurred with those of Katz et al. (2016) who demonstrated regimens including tramadol were dominated by those using ibuprofen or naproxen (Katz et al. 2016). Also, Smith et al. (2017) observed that people who received tramadol or tramadol followed by oxycodone for knee OA had surgery delayed for seven or nine years, respectively. However, both opioid-containing strategies increased costs and decreased QALYs compared to opioid sparing regimens (Smith et al. 2017). 
A UK study examined the cost-effectiveness of tapentadol compared to oxycodone for people with chronic pain who had not responded to or could not tolerate morphine (Ikenberg et al. 2012). Over one-year or thirteen 4-week cycles of Markov model, tapentadol was associated with fewer health care visits and additional medicines for managing side-effects than oxycodone. However, the difference in QALYs between tapentadol (0.6371) and oxycodone (0.6237) was small. A second UK study undertook a cost-utility analysis using a cohort model to calculate cost differences between two oxycodone products, one including naloxone and designed to reduce the incidence of opioid-induced constipation. The 12-week study period generated a ICER of $£ 5,841.56$ per QALY for the naloxone containing product (Dunlop et al. 2012). The model demonstrated a reduction in healthcare resource use such as primary care visits in addition to fewer prescriptions for laxatives when the oxycodone-naloxone product was used. Quality of life gains were suggested by the authors, although differences were not demonstrated to be statistically significant (at week twelve, adjusted means were 0.5029 compared to $0.4640, p=.0185$ for the oxycodone/naloxone group and oxycodone group, respectively) (Dunlop et al. 2012). Whilst these studies, both run by the pharmaceutical companies marketing the medicines being trialled, demonstrated cost-effectiveness of different opioid analgesics or differences in healthcare use and associated costs, none examine specifically the difference in utilisation in the same way as the TOPAS study has attempted. Further research is warranted to examine, in detail, the impact of opioid prescribing for non-cancer pain on healthcare utilisation with a focus on less commonly studied adverse effects such as depression and anxiety or illness resulting from reduced immunity.

\subsubsection{Healthcare use associated with pain, opioid analgesics, and demographic factors}

Chronic pain is a difficult condition to manage and to definitively diagnose (National Institute for Health and Care Excellence 2021). The general perception of pain is that it is connected to an underlying problem, injury or illness (Section 1.3). Therefore, it perhaps makes sense from a clinical perspective to see more 
healthcare attendances by people using opioids, as it could be assumed they are experiencing more difficult symptoms than their study controls (McGorm et al. 2010; Johnston 2019; Tzartzas et al. 2019).

Whilst there is limited literature on the impact of opioids per se on healthcare utilisation, reasons for frequent attendance across sectors has been examined (Neal et al. 1998; McGorm et al. 2010; Ringberg et al. 2014; Patel et al. 2015; Welzel et al. 2017; Tzartzas et al. 2019). It has been estimated that $15 \%$ of primary care attendances are made by $3 \%$ of patients (Neal et al. 1998). Characteristics of frequent attenders appears to vary depending on the setting (Neal et al. 1998; Byrne et al. 2003; Hunt et al. 2006) and the conditions they are seeking support for (Menchetti et al. 2009; Lewer et al. 2020). Primary care frequent attenders are considered more likely to be female and attendance appears to increase with age (Neal et al. 1998; Welzel et al. 2017), which corroborates the findings of primary care attenders in TOPAS (Section 7.8.1). The contribution of chronic pain to presentation in primary care has also been observed to result in twice as many visits compared to non-pain related attendances with men and women almost equally represented (Mann et al. 2016).

Characteristics of frequent ED attenders appear different to those in other sectors (Byrne et al. 2003; Hunt et al. 2006; Greenfield et al. 2020). The most recent study of ED attendances in the UK demonstrated that $10 \%$ of people were frequent attenders and accounted for $25 \%$ of ED visits (Greenfield et al. 2020). Whilst that study did not discuss gender characteristics, others have revealed that men are likely to attend ED more frequently than women, and that attendance increased with age and was more common in people who lived alone (Hull et al. 1998; Byrne et al. 2003; Moore et al. 2009). A study of ED attendance, with pain as a driver for attendance had a similar gender split to this study, with $52 \%$ of attendees being male (Daniels et al. 2017), although attendance declined with age in that case. In line with previous studies, men in the control group of this study had more ED attendances than their female counterparts. 
Frequent ED attendance has been noted to be connected to recurrent presentation to primary care (Hull et al. 1998; Moore et al. 2009). This would fit with the results of the linear regression (Section 7.9.1), which showed a correlation between GP visits and ED attendance. Even in healthcare systems where insurance costs are incurred, greater attendance for emergency care has been linked to frequent attendance with family doctors (Byrne et al. 2003; Hunt et al. 2006). A 2012 study of secondary care healthcare use in England revealed around twice as many ED attendances in the most deprived deciles compared to the least. Further, deprivation was associated with lower conversion to hospital admission. This implies that ED attendance by people from more deprived deciles was more likely due to minor health problems (McCormick et al. 2012). Living in areas of higher socio-economic deprivation and having long-term health conditions have also been noted as predictors for more frequent attendance at ED (Scantlebury et al. 2015; Giebel et al. 2019). It appears perhaps that whilst there is greater health need in areas of socioeconomic deprivation, there is also more inappropriate use of services. Scantlebury et al.'s (2015) study noted 73.6\% of subjects in the $90^{\text {th }}$ centile (less deprived) reported knowing how to contact out of hours primary care services $(\mathrm{OOH})$ compared to $48 \%$ in the $10^{\text {th }}$ centile. It is not surprising therefore, that even minor health issues arising outside of normal working hours, might result in a visit to ED.

Referrals to secondary care were not fully coded for in this study (Section 8.6), although from the data available, case subjects received more and also more tests and radiology contacts (Section 7.8.1). Referrals into secondary care have been associated with less experienced doctors (Tzartzas et al. 2019), female primary care doctors (Ringberg et al. 2013), and previous experience or familiarity with the specialist being referred to (Kinchen 2004). More referrals were observed from ED for people from areas of higher deprivation (McCormick et al. 2012). It has been demonstrated, however, that deprivation is associated with lower rates of attendance and cancellation of booked appointments but increased likelihood of attending emergency departments (McCormick et al. 2012). This is potentially important as healthcare services of most benefit to 
people living with pain or using opioid analgesics are generally provided as outpatient clinics and require multiple attendances over extended durations.

An Australian study examined health service utilisation by 1,243 people living with chronic pain (Nielsen et al. 2016), specifically looking at the use of nonopioid pain treatments by people also using opioids. There was use of complementary and alternative medicines and specialist pain treatments by participants in all groups. However, the likelihood of accessing them was linked to the ability to pay or having insurance policies that covered it (Nielsen et al. 2016). As all participants were in receipt of opioid medicines, those who were unemployed or did not or could not have inclusive insurance policies, were more likely to have only opioids or other medicines to manage their pain (Nielsen et al. 2016). A partly insurance-based healthcare system is an additional barrier noted in the Australian study, rather than the only barrier considered to prevent people using alternatives to opioids (Nielsen et al. 2016). Remuneration for services including physiotherapy and psychology is problematic in countries where health insurers dominate decision making (Finestone et al. 2016). The NHS in Wales and the rest of the UK, reduces ability to pay as a cause of non-attendance or accessing some supportive services. Alternative therapies, which people with pain might want to access are still subject to payment however, thus introducing barriers for people with limited funds.

In the UK, pain management clinics are commissioned by CCGs or operated by Health Boards and, based on standards set by the Faculty of Pain Management should provide access to evidence-based, multi-disciplinary support (Faculty of Pain Medicine 2021). Price (2019) sets out the development of pain services in the UK, which has been heavily criticised by a number of different Government reports over the years (Price 2019). Since 2008, when the Chief Medical Officer's reports focussed on chronic pain (Chief Medical Officer of England 2009), more focus has been paid to pain management and service development. The approach taken in Scotland is set out in Section 8.4.2. The National Pain Audit (2012) highlighted that gaps remained in services throughout the UK (Price et al. 
2012). Services in Wales did not fully participate with the audit and consequently, were not able to be benchmarked against the outcomes examined (Price et al. 2012). Only 40\% of services were shown to operate multi-disciplinary services. The audit did not examine service provision based on socioeconomic deprivation, despite the overwhelming evidence that chronic pain is disproportionately associated with deprivation (Engel 1959; Saariaho et al. 2011; Newman et al. 2017; Gulliford 2020). Future reviews of service provision would benefit from examining not just the make-up of services but also their accessibility to different patient groups. This is especially important given the higher rates of opioid analgesic prescribing in more deprived areas demonstrated in this study (Chapter 4 and Section 5.5.4) and by others (Mordecai et al. 2018; Torrance et al. 2018; Chen et al. 2019) and the harms associated (Chapter 4).

\subsection{Conclusion}

There was a clear association between the receipt of prescription opioids and healthcare utilisation. Increased healthcare utilisation was associated with female gender, age 65 years and over, living in more socio-economically deprived areas and receiving opioid medications for more than 6 months. Length of hospital admission was the only variable where receiving opioids appeared to have a negative association, although opioid use was associated with more hospital admissions than control subjects.

The costs of healthcare utilisation were clearly greater in people receiving opioids, with an average annual cost of just under f1billion, based on the variables used in this study. Whilst not inclusive, this conservative estimate is the first to be made for healthcare costs linked to opioid use in Wales. All study subjects had recorded pain-related diagnoses and varied only by the receipt of opioid prescription. This study has also provided the first estimation of annual healthcare costs associated with six major pain-related diagnoses in Wales, namely osteoarthritis, rheumatoid arthritis, back pain, neck pain, fibromyalgia, and neuropathic pain. The estimation of 1.3 billion per year is near equivalent to 
the total primary care spend in Wales, which perhaps puts into perspective the overall burden that pain places upon the NHS.

Further analysis is needed to determine more accurately if there are differences in the services accessed by people prescribed opioids compared to non-users. Moreover, investigation whether differences are associated with particular opioid medicines or duration of use is warranted. As the evidence to support the use of opioids for long-term conditions weakens, there has been some reduction in prescribing. However, as discussed elsewhere in this thesis (Section 4.6), evidence suggests that people who are most susceptible to the harms of opioids, are more likely to be receiving them, at higher doses and for prolonged periods of time. The same factors were also associated with increased healthcare utilisation and consequent costs, which could be an indication of harm.

Policy makers and healthcare leaders will need to reconsider the reasons for opioid prescribing, particularly that which continues for extended periods and may be causative for other health concerns, from the perspective of patients and practitioners. It appears the cost of opioid prescribing to NHS Wales could be significant and related more to harms than help. Defining who is likely to benefit from opioid treatment without inflicting greater damage and who and where other options need to be provided and promoted, is emerging as a necessary next step. It is hoped this work is a useful contribution to improving and preserving the health and wellbeing of the people of Wales. It illustrates the need to urgently address health inequalities, iatrogenic harm and to ensure the best use of fragile NHS resources. 


\section{Chapter 8}

Discussion

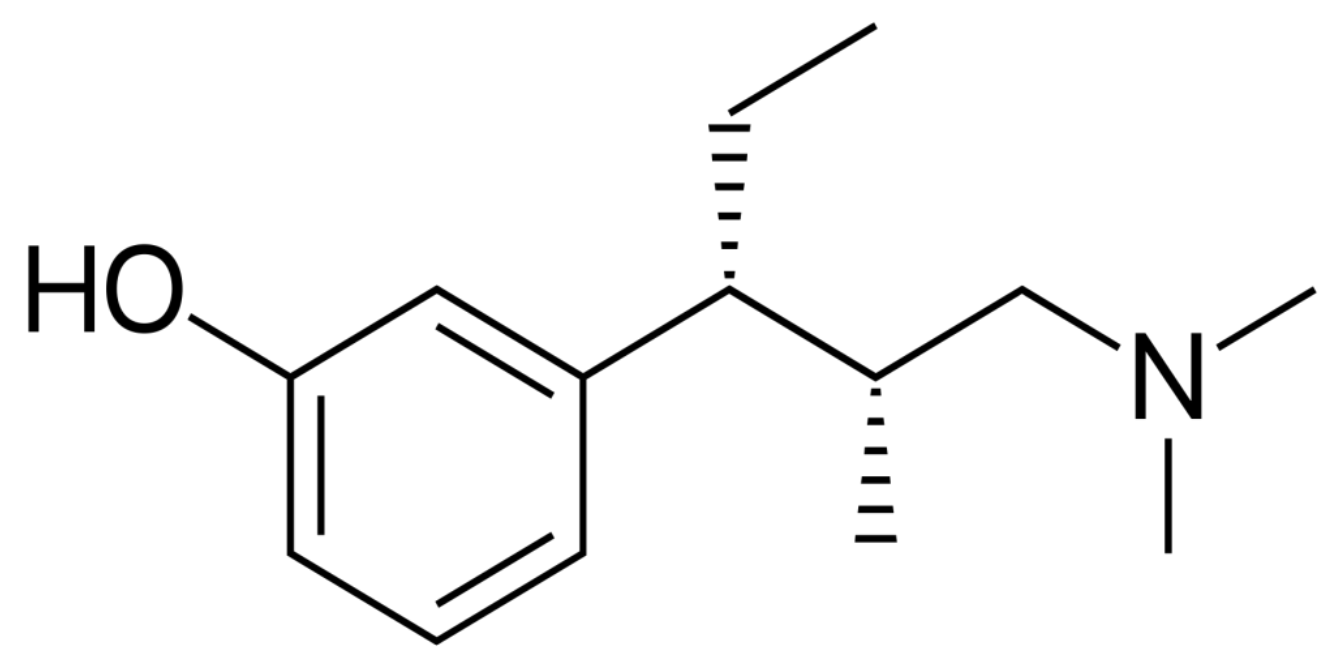

"'Finished last' will always be better than 'did not finish' which always trumps 'did not start'"

Anon 


\section{Chapter 8 - Discussion}

\subsection{Chapter overview}

This thesis sought to examine how opioid analgesic prescribing had changed over time in Wales and whether factors such as gender, age or socioeconomic status affected it. Further, the influence of guidelines and legislation on opioid prescribing and ultimately, how opioid prescribing might be associated with healthcare service use and its associated costs was examined.

The research objectives were:

1. To examine prescribing trends of opioid analgesics between 2005 and 20015 and scrutinise whether trends differ between gender or different age-groups (Phase 1 - Chapter 3)

2. To determine if opioid analgesic prescribing trends are affected by socioeconomic deprivation status (Phase 1 - Chapter 4)

3. To analyse trends in oral morphine equivalent doses and prescribing duration using estimated measures for each (Phase 1 - Chapter 5)

4. To determine if general opioid prescribing trends appear affected by legislative or clinical guidance changes during the study period using time series analysis (Phase 1 - Chapter 6)

5. To assess the frequency of primary and secondary healthcare attendance by patients with defined non-cancer pain conditions receiving opioid analgesic therapy (Phase 2 - Chapter 7)

6. To estimate healthcare service costs associated with the use of opioid analgesics (Phase 2 - Chapter 7)

This final chapter now seeks to draw together the threads from those that have gone before, to discuss factors of influence not previously covered and to set out the potential future direction of health care policy to address opioid prescribing and pain management more generally, in Wales. 


\subsection{Summary of study results}

The results of this study demonstrate large increases in prescribing of all opioid analgesics (Section 3.5.1), but especially of strong opioids such as morphine, oxycodone and fentanyl, which place a disproportionate burden on the population (Chapter 5). The highest levels of prescribing and consequent opioid burden were observed in the most socioeconomically deprived areas of Wales (Chapter 4 and Section 5.5.4) which, has implications for the overall health and wellbeing of those communities. Women received around $50 \%$ more prescriptions than men between 2005 and 2015 (Section 3.5.3), although there were signals that men may receive higher doses of opioids per prescription (Section 5.5.3.2). National prescribing indicators appeared to have a small effect on prescribing trends but legislation less so (Chapter 6).

People receiving prescriptions for opioid analgesics had 5 times more attendances in Primary Care (Section7.8.1), around 3 times as many out-patient (Section 7.8.2) and emergency department visits (Section 7.8.3) and twice as many in-patient attendances (Section 7.8.4) as those with similar pain-related diagnoses but who did not take opioid analgesics. Higher rates of attendance led to significantly higher healthcare costs associated with opioid analgesic use, compared to control subjects (Section 7.10.1).

Concerns about increasing rates of opioid prescribing in Wales have been borne out by this research. In particular, the use of large, linked datasets are an important tool in monitoring prescribing trends and allow targeted analyses of demographic groups who may be at greater risk of harm from changes in opioid prescribing. The TOPAS study demonstrates that expanding on the relatively simplistic view provided by standard dispensing data available to Health Board Medicines Management teams and national organisations setting prescribing policy, will be important in addressing the high rates of opioid prescribing that continue in Wales and reducing variation within the population. Means of accessing the kind of detailed data used in this study, in a more straightforward 
and timely manner need to be invested in, to improve the effectiveness of interventions made in practise.

The increases in prescribing rates were not necessarily unexpected, given previous prescribing data (All Wales Medicines Strategy Group 2013). Examination by different demographic categories has, however, brought issues into focus which have not been so widely discussed in Wales in relation to prescribing or addressed in initiatives aimed at changing prescribing habits (Welsh Government 2020; All Wales Therapeutics and Toxicology Centre 2021). Disproportionate prescribing to women and in areas of greatest deprivation are clearly issues that need to be addressed. Studies from other areas of the UK have also shown these disparities (Macfarlane et al. 2009; Zin et al. 2014; Ruscitto et al. 2015; Bedson et al. 2016; Mordecai et al. 2018; Todd et al. 2018; Torrance et al. 2018; Macfarlane et al. 2020).

Higher prevalence of pain, and consequently opioid prescribing, in women is acknowledged (Dao and LeResche 2000; Rhudy and Williams 2005; Pieretti et al. 2016), even if the reasons for it are less well understood (Terplan 2017; Mazure and Fiellin 2018). Higher levels of ill-health or pain in areas of greater socioeconomic deprivation are often used as the explanation for higher rates of opioid prescribing (Todd et al. 2018; Nowakowska et al. 2020; Schifanella et al. 2020) whilst acknowledging that this alone is not justification enough. Of particular concern is the harms of opioids medicines are likely to have greater impact in those areas where health is already worse.

Little data is available in Wales regarding specialist pain service access or how that might vary in areas of different socioeconomic deprivation. When the demographics of people accessing a chronic pain service in Glasgow were examined, it was notable that a higher proportion of attendees came from wealthier areas (Moore et al. 2020). People from less affluent areas of the city were less likely to attend the initial assessment and attended fewer sessions once accepted to the service. Given pain reporting appears to correlate with 
deprivation, this was surprising. The authors suggest multiple factors are likely to account for the difference, including poorer support seeking behaviour in people more socioeconomically deprived, lower referral rates from areas of higher deprivation and poor health activation (Moore et al. 2020).

\subsection{Why are opioids prescribed?}

Over the study period (2005 to 2015), evidence that opioids have poor effect for most people using them, particularly in chronic pain conditions, continued to emerge (Trescot et al. 2008; Els et al. 2017; Stannard 2018a). This begs the question of why opioids continue to be prescribed, when so much is known about the harms (Baldini et al. 2012; Blanch et al. 2014; Els et al. 2017; Salas et al. 2020) and limited benefit in reducing pain or improving function (Baldini et al. 2012; Krebs et al. 2018; Richards et al. 2018).

\subsubsection{Waiting for help}

Prescribing opioids has been suggested to be a surrogate for access to alternative pain management support (Finestone et al. 2016). Pain service provision in Wales was not mapped as part of this study but the National Pain Audit (2012) collected data demonstrating every health board in Wales had chronic pain service provision, with $80 \%$ claiming to have multi-disciplinary teams (Price et al. 2012). Average waiting time for these services at the time of the audit was 33 weeks (Price et al. 2012). Official figures on waiting times for pain services were not available during the composition of this thesis.

The impact of long waiting times on the people waiting was examined by the Community Health Councils of Wales (CHC) (Board of Community Health Councils in Wales 2018). It was acknowledged that being in pain whilst awaiting orthopaedic and other types of surgery was problematic, especially as some pain services do not see people on waiting lists for interventions from other specialities (Board of Community Health Councils in Wales 2018). People responding to the $\mathrm{CHC}$ consultation revealed concerns about continuing 'strong painkillers' due to worries "about side-effects, over reliance and reduced 
effectiveness." The report went on to highlight delays to surgery were coupled with "long waiting times for pain management clinics, up to 2 years in some cases." (Board of Community Health Councils in Wales 2018).

IASP gave recommendations on waiting times for pain services globally in 2011, with a suggested 8 weeks for persistent long-term pain without significant progression (Collett et al. 2011). A systematic review of the effects on people waiting for chronic pain treatment suggested that waiting for six months led to significant deterioration in health-related quality of life and psychological wellbeing (Lynch et al. 2008). An investigation by the Pharmaceutical Journal (2020) requested data directly from pain service providers and found waiting times ranged from 6 to 111 weeks, with the longest waits in Wales (Connelly 2020).

During the Covid-19 pandemic, many specialist services, including pain services, were temporarily suspended. Most English pain services reported continuation of some pain physician work direct to patients. However, the range of services available, inevitably reduced (Faculty of Pain Medicine 2020). No equivalent data for Wales was found. The Faculty of Pain Management (FPM) gave guidance to its members (anaesthetists) in relation to returning services post-pandemic (Faculty of Pain Medicine 2020). Whilst understandably the focus of advice was on prioritising workload and stratification processes for people referred, there appeared to be no consideration of the impact of changing services on people waiting and colleagues working in Primary Care (Faculty of Pain Medicine 2020). It may be assumed that people who are unable to access specialist interventions will more likely access whatever services are available.

Primary Care, unlike Acute, Secondary or Tertiary services, does not have the option of discharging people. Primary Care has to continue to offer support and treatment to people, even if they are waiting for or receiving input from specialist providers. This may lead to GPs and other practitioners in primary care resorting to prescribing or maintaining opioid prescribing in the face of poor 
outcomes, simply because they feel they have nothing else to offer (Finestone et al. 2016). McCrorie et al. (2015) support this notion, with GPs explaining during interviews that continuing to prescribe opioids was often due to long waits for other treatments or support, or patients not being ready to consider alternatives to medicines (McCrorie et al. 2015).

An examination of routinely collected longitudinal data from primary care practices in England noted increasing numbers of primary care appointments were more likely to result in the prescription of a strong opioid. An odds ratio of 3.04 (range 2.48 - 3.73) was observed for more than 12 visits per year (Foy et al. 2016). Increased attendance has been associated with greater self-perceived illhealth and the need for support (Neal et al. 1998; Morriss et al. 2012; Daniels et al. 2017) (Section 7.11). So, it is perhaps understandable that, in the absence of acceptable or timely alternatives and when people present with increasing need for support, prescribing becomes an acceptable option to all parties.

However, there is some evidence that even once seen by 'specialists', opioid prescribing remains a frequent intervention. Foy et al.'s study (2016) demonstrated referral to chronic pain services also resulted in an increased likelihood of strong opioid prescribing that was then likely to persist (OR 5.74, range 5.09-6.47) (Foy et al. 2016). McCrorie's study (2015) reported a GP statement, "...every time I send somebody to chronic pain [clinic] they come out with more medication, or injections.' [GP, female; Leeds; 319]" (McCrorie et al. 2015). If this is the case, then whilst prescribing sits predominantly in primary care (Auditor General for Wales 2018), it would seem influences on prescribing could be greater than simply prescriber preference or lack of alternative.

It is difficult to trace the source of analgesic medication using the data sources currently available in Wales. Whilst it may appear that an opioid was initiated by the GP, it could be on the recommendation of a practitioner from another sector. Tracing the relationship between prescription and request would require 
extracting out-patient communication data and the initiation of a prescription within a designated period afterwards, for example.

\subsubsection{Does 'ability to pay' affect prescribing?}

A major finding of this study was that opioid prescribing of all types is disproportionately higher in areas of higher deprivation. Some reasons for this observation have been previously discussed in Section 4.6. This may not be the case everywhere, however. A study from Germany, found indebtedness increased the likelihood of not receiving or taking pain medicines (Warth et al. 2019). Germany operates insurance or co-payment-based health services with potential that the observed reduction in medication use is linked to the inability to pay (Turunen \& Hiilamo 2014; Warth et al. 2019). Prescription charges were abolished in Wales in 2007 (National Assembly Government 2010). However, from the data observed in this study, it is clear that prescribing was already higher in more deprived areas, prior to the elimination of Welsh prescribing charges (Figure 4.2). It was estimated that at least $50 \%$ of the Welsh population were entitled to free prescriptions prior to removing charges, equating to $88 \%$ of items dispensed (National Assembly Government 2010).

The implications of the findings of this research are that removing prescribing charges was not associated with the rise in opioid prescribing noted. However, whether lack of payment encourages prescribing is a matter of dispute (Groves et al. 2010; National Assembly Government 2010; Carlisle 2017). In Wales, annual increases in prescribing slowed following the removal of prescribing charges. Pharmacy leaders in Wales deny that prescribers are encouraged by free prescriptions for their patients. Instead, they insist that higher levels of prescribing reflect the greater prevalence of poor health in the country (Carlisle 2017).

\subsubsection{Why might the legislation not have 'worked' in the UK?}

Although the results shared here (Chapter 6) imply the introduction of opioid focused NPIs may have contributed to a degree of reduction in opioid 
prescribing, legislation appeared to have less effect. Examining the literature raises the consideration that changes in practice may depend on the type of legislation introduced.

At the time, the murders committed by the GP Harold Shipman (Section 1.11), shook the healthcare fraternity. There was discussion of how healthcare would change as a consequence, including rebuilding trust between practitioners and patients and how competency in practice would be reviewed (Baker 2004). The TSA reported (Chapter 6) and the notable upward trends across the UK (Zin et al. 2014; Ruscitto et al. 2015; Davies et al. 2018; Curtis et al. 2019), however, implies that the impact of Shipman's behaviour and the subsequent legal changes did not impact prescribing directly. A possible explanation for this might be the area of practise that Shipman was found to have abused (Smith 2005). Most victims in the Shipman case were elderly people, some deemed to be receiving palliative care. The vast majority of opioid prescribing in Wales and the rest of the UK (Zin et al. 2014; Todd et al. 2018; Torrance et al. 2018 Curtis et al. 2019) however, was and remains, for non-cancer related pain (Section 3.5.1). Interestingly, the rates of opioid prescribing for people with a recorded diagnosis of cancer reduced in Wales between 2005 and 2015 (Appendix J).

Gomes and colleagues (2014) were able to demonstrate a combination of legislation strengthening requirements for patient identification and introducing a prescription monitoring (PMS) programme led to the number of opioid prescriptions reducing (Gomes et al. 2014). Whilst the Canadian changes were like the Shipman law changes, they appear to have been effective. In the UK, the focus of the benefits of the changes have concentrated on end-of-life care (Baker 2004; Pocock et al. 2018) and deterring rogue practitioners. In the early 2000's, when the main legal changes occurred, opioid prescribing was relatively modest and, particularly for chronic pain, prescribers worldwide were being encouraged to prescribe more (Kotecha and Sites 2013; Shapiro 2015; McGreal 2018). By the time Canada introduced PMS and legal enforcement, the opioid crisis was acknowledged in North America (Gomes et al. 2011; Franklin et al. 2012; Gomes 
et al. 2014). Also, evidence of potential limitations of opioids in non-cancer pain were becoming better known (Ballantyne and Shin 2008; Campbell et al. 2015; Els et al. 2017). In these situations, perhaps the legal requirements and accompanying guidance make sense in the clinical context the prescribers are working in. This could lead to improved compliance and so the desired outcome of reducing prescribing or improving monitoring.

In Wales, changes that could be connected to the NPIs began from around 2012 and were predominantly associated with a reduction in tramadol prescribing (Chapter 6). An indicator was introduced in the 2013-14 financial year, in combination with an education pack and practice audit. This was in response to a doubling of tramadol-associated deaths over five years (from 83 in 2008 to 175 in 2012) in England and Wales (Hawkes 2013, Office for National Statistics 2019). Drug associated deaths disproportionately affect Wales compared to England and in 2012, the difference amounted to 20 deaths per million ( 45.8 deaths per million in Wales compared to 25.4 in England) (Office for National Statistics 2013).

As illustrated in the TOPAS data (Chapter 5), tramadol was the second most prescribed opioid in Wales between 2005 and 2015. However, it was reported to be the most prescribed opioid in a number of regions of England between 2010 and 2014 when examined by oral morphine equivalence (Mordecai et al. 2018). Anxiety in regard of the leap upwards in tramadol associated deaths led to recategorisation of tramadol as a schedule 3 controlled drug in 2014, garnering attention from healthcare and mainstream media. Subsequent reductions in tramadol prescriptions issued in England were noted (Chen et al. 2019), corresponding with the reduction in prescription numbers noted in this study (Chapter 5).

\subsubsection{Are current methods of monitoring prescribing fit for purpose?}

OMEQ is a useful measure of opioid utilisation in the general population and an individual basis (Nielsen et al. 2017). This study has demonstrated differences 
between assumed burden of opioid prescribing using $\mathrm{OMEQ}_{e}$ and prescriptions issued, which may have important clinical implications. Evaluating opioid prescribing using OMEQ would provide easily comparable data that better reflects clinical practice.

It is important, however, to draw the distinction between using OMEQ as a means of familiarising prescribers with the concept of opioid burden, and assuming it guarantees equianalgesia in all people (Schatman et al. 2016). Whilst guidelines have adopted OMEQ as a way of setting a dose limit (Harrison and Cormack 2018) it should not be seen as something to aim for. As with any other drug, many factors affect an individual's response to opioids (Robert 2006; Trescot et al. 2008; Rennick et al. 2016; Arul and John 2020). Consequently, OMEQ, whilst a useful clinical guide, should probably not be used to assume levels of risk in an individual without also taking account of patient-specific factors such as renal and liver function, polypharmacy, age, comorbidities and so on. It is worth bearing in mind that opioid overdoses can occur at any dose, as can any adverse effects (Schatman et al. 2016). In simple terms, there is no such thing as a 'safe' dose of opioid, only the right dose for the right person at the right time for the right reasons.

The available literature on prescribing persistence indicates that duration is just as important as dose, in terms of increasing risk of harm from opioid analgesics (Cohen et al. 2008; Jain et al. 2018; Salas et al. 2020). Whilst symptoms of dependence have been demonstrated in people receiving 24 to 48 hours of opioids in acute settings (Shah et al. 2017; Liberman et al. 2019), other serious problems such as depression are associated with longer durations of use (Scherrer et al. 2016; Salas et al. 2020). Consequently, in the same vein as OMEQ, there may be no safe duration of use that suits all people. Much clearer guidance about the risks of treatment be it dose or duration, is required for both prescribers and people prescribed opioids. As Scottish prescribing guidelines have clearly explained, regular monitoring and review of people being prescribed opioids is essential (Harrison and Cormack 2018). Those reviews should not 
simply focus on relief of pain, but also be used as an opportunity to appraise the balance of benefit and risk for everyone. It would seem prudent to suggest continuing opioids without evidence of benefit to the patient is only likely to cause harm (Harrison and Cormack 2018; Taylor et al. 2019)

\subsubsection{Is there benefit to using OMEQ to monitor opioid prescribing burden?}

TOPAS results imply that the way prescribing data is currently examined does not provide sufficient insight into opioid burden in communities. Although this study used an estimated measurement due to the lack of prescribing information, it does hint that using oral morphine equivalence is more reflective of what is going on in practice than simply prescription numbers or DDDs. When OMEQ was used to compare prescribing in four Nordic countries, it demonstrated noteworthy differences in patterns of opioid consumption compared to those seen with DDDs (Svendsen et al. 2011). 'Weak' opioids such as codeine, carry higher DDD values than 'strong' opioids like morphine. Countries where codeine predominated appeared to have high overall opioid prescribing, which was reversed when OMEQ was used and the contribution of 'strong' opioids accounted for (Svendsen et al. 2011). Codeine, which is commonly prescribed but often not regarded as a 'problem', contributes significantly to opioid burden in Wales. The failure to acknowledge the dominance of codeine prescribing in all its forms until recently (All Wales Medicines Strategy Group 2019) has possibly resulted in an underestimate of the potential harm being caused. Prescribers' understanding of OMEQ is poor (Rennick et al. 2016). This is particularly pertinent to products with less familiar doses. Fentanyl and buprenorphine are dosed in micrograms rather than milligrams as for most other analgesics for example (BNF: British National Formulary - NICE. 2021). The study results showed a disproportionate burden by $\mathrm{OMEQ}_{e}$ compared to the number of prescriptions issued for both. Use of OMEQ as a measure of prescribing might improve comprehension of opioid equivalence and lead to safer prescribing.

Whilst overall it seems the tide is turning and opioid prescribing is starting to fall in Wales, the UK and further afield (DataLab 2017; Centers for Disease Control 
and Prevention 2021; All Wales Therapeutics and Toxicology Centre 2021) attention needs to turn to 'what' and 'for how long' rather than 'how much' is being prescribed. North American data is sometimes presented as numbers of units or tablets (McGreal 2018). Whilst often very large numbers, it is not in itself an explanation of the burden as it provides little explanation of dose or duration of use. Data for OMEQ gives a much more relatable explanation of the changes in burden. Further, there is evidence from several developed countries that people are remaining on opioid prescriptions for longer periods of time (Bedson et al. 2016; Shah et al. 2017; Jani et al. 2020; Black-Tiong et al. 2021). In the UK, 18.7\% of people started on $>200 \mathrm{mg}$ OMEQ doses, were observed to remain on that level of intake for at least 2 years (Jani et al. 2020). The incidence of long-term opioid prescribing, defined by receipt of three or more prescriptions within 90 days with no more than 6 months between episodes, increased from $5.5 \%$ in 2012 to $9.1 \%$ in 2018 in an Australian population (Black-Tiong et al. 2021). Perhaps to assess whether meaningful change is being made, in addressing the concerns about opioid use and long-term harms, a measure that takes account of dose (OMEQ) and duration is needed. The ideal use of opioids appears to be lower doses for shorter periods of time (Faculty of Pain Medicine 2021).

\subsubsection{Are the trends in resource utilisation and costs a sign of equality in healthcare?}

In conjunction with the first phase prescribing trend results (Section 3.5 and Section 5.5), perhaps the most concerning finding from the TOPAS study are the significant disparities between areas of high and low deprivation. Higher levels of prescribing and healthcare utilisation are observed in the most socioeconomically deprived areas of Wales, with high levels of poor general health and well-being (Section 4.5 and Section 7.8).

The high levels of opioid prescribing in the most deprived areas of Wales, might be interpreted by some as a perverse sign of equality. We are 'lucky' that receipt of medicines is not always related to the ability to pay in the UK (Section 8.3.2). In many parts of the world, opioids are not available, even for end of life care 
(Richards et al. 2020). In Wales, in theory, anyone with access to a GP can access an opioid prescription. Is that necessarily a good thing, however?

The concerns about opioid use have already been discussed (Section 1.7), as have those about the susceptibility of people living in the most deprived areas being exposed to higher OMEQ and longer prescribing persistence than in more socioeconomically well-off areas (Section 5.6). Phase 2 results reveal that the burden placed upon health services may, in part, be associated with the use of opioids. It is far from clear that opioids benefit many people who take them (Els et al. 2017; Taylor et al. 2019). May these results perhaps point towards people developing new or worsening health problems because of their use? If so, those problems appear likely to be felt more keenly in the most deprived areas of Wales, where services are more limited or less accessible to those who would most benefit.

\subsubsection{Will guidelines change prescribing in the future?}

In Chapter 6, the influence of NPIs on opioid prescribing was examined. At the time of writing, Wales does not have national guidelines for either opioid prescribing or the management of pain more widely. Since the end of the TOPAS study period NICE, who are regarded as an influential source of guidance in England and Wales, have increasingly recommended against the use of opioids for some common, long-term, painful conditions including low back pain (National Institute for Health and Care Excellence 2016), neuropathic pain (Centre for Clinical Practice at NICE 2013) and recently chronic primary pain (National Institute for Health and Care Excellence 2021). NICE validate recommendations against opioids on the basis of a lack of evidence suggesting benefit and significant evidence of harm (National Institute for Health and Care Excellence 2016; National Institute for Health and Care Excellence 2021). ITSA demonstrated some reduction in prescribing associated with an opioid focused $\mathrm{NPI}$, but this does not demonstrate or provide assurance that opioid analgesics are being used appropriately. 
In Scotland, introducing national prescribing guidelines for pain (SIGN 136) in 2013 has subsequently been associated with an $18.8 \%$ reduction in opioid prescribing over the following six years (Hebert et al. 2021). An update to their 2013 chronic pain guidelines in 2019, reflected the changes in advice around opioid analgesics. Unlike NICE, the Scottish Intercollegiate Guideline Network (SIGN) continue to include opioids as a short- to medium-term treatment option, albeit with caveats about assessing for suitability and ensuring outcomes are regularly monitored. Where evidence of poor effect or harm is noted, dose reduction and, if necessary, cessation is supported (Harrison and Cormack 2018; Scottish Intercollegiate Guideline Network SIGN 2019). SIGN suggest doses above 50mg OMEQ need to be reviewed at least annually and for specialist pain advice to be sought when people are prescribed $>90 \mathrm{mg}$ OMEQ per day (Scottish Intercollegiate Guideline Network 2019). Wales has no similar guidance, although practitioners working in Wales are supposed to follow NICE guidance, which should also be incorporated into Welsh guidelines where available (Welsh Government 2021). Clinical guidelines are developed within Health Boards, but the degree to which they are evidence based potentially depends on the scrutiny they are given under local arrangements. It is highly unlikely that processes as thorough as SIGN or NICE are used in those cases, resulting in variability in practise across the country.

Both NICE and SIGN have protocols for developing their guidance. SIGN consider a wider range of literature than is permitted by NICE strategies. In practise, this can result in differences in guidance issued. Opioids are an example of this, where SIGN continue to suggest they can be used albeit with caveats around monitoring and review (Scottish Intercollegiate Guideline Network 2019), whereas new NICE guidance for back pain and chronic primary pain have specifically recommended that opioid analgesics should not be offered (National Institute for Health and Care Excellence 2016; National Institute for Health and Care Excellence 2021). If the methodologies used are not well understood or explained, it can lead to misunderstanding and concerns about the implications of following the advice without context (Faculty of Pain Medicine 2021; Smith et 
al. 2021). It seems each time new guidance is issued, concerns are raised that people already prescribed opioid analgesics for long-term pain conditions, who benefit in terms of pain reduction and improved function, will have them stopped (Faculty of Pain Medicine 2021) despite there being no statements to encourage that. Organisational or individual's interpretation and implementation of guidance is out of the control of the publishers, however.

NHS England have published a directed enhanced service (DES) for structured medication reviews (SMR) to target people prescribed drugs associated with dependence and withdrawal as per the PHE review (2019) (Taylor et al. 2019; NHS England Primary Care Group 2021). This should be an opportunity to enhance care and safety for, people living with pain. However, in some organisations, it could be seen as a green light for undertaking reductions of medicines even when people believe they are benefiting from taking them. If anything is to be learned from the experience of North America, in terms of their handling of the opioid crisis, it must be that stringently applying rules or restrictions on prescribers and patients alike, is unlikely to be helpful as a lone strategy to address concerns about high levels of prescribing (Schatman and Shapiro 2019; Singer et al. 2019; Sacks et al. 2021).

All influences associated with opioid prescribing need to be addressed if meaningful change in prescribing is to be achieved in time. This study has shown that, in Wales, the main factors associated with receiving an opioid prescription and in using higher levels of healthcare are being socioeconomically deprived, being female, and receiving prescriptions for more than 6 months. However, it is unlikely that guidance would be able to direct prescribers to not prescribe for people based on their socioeconomic status or gender. It is possible to highlight where risks are greatest, however, and to encourage greater scrutiny of prescribing and provide support to reduce it in areas where prescribing is highest. Clearly, of greater importance is to address the factors underlying those differences. Improving the availability of women's health services and addressing socioeconomic deprivation for example, are vital, but likely to take a long time 
for change to occur. In the meantime, changing the duration of prescribing or the advice issued to people living with pain are changes that can be more easily legislated and would bring more rapid benefit to the population.

\subsection{Policy direction for pain management and analgesic stewardship in Wales}

If opioid prescribing is, as suggested, a surrogate for adequate pain management support (Finestone et al. 2016) and if pain experience is influenced by things other than physical pathology (Engel 1977; Gatchel et al. 2007; Stilwell and Harman 2019), it is likely that more than prescribing guidelines are needed for change to occur. Attempts have been made to address the growing health burden of pain in Wales. In 2008, the Welsh Assembly Government published service development and commissioning directives aimed at improving pain management services across the country (Welsh Assembly Government 2008). The major aim at that time was to change service delivery to improve integration, ensuring people received appropriate, evidence-based support where and when it was needed (Welsh Assembly Government 2008). In particular, the directive acknowledged the complexity of chronic pain and the need to reduce the risks of its development by improving overall health and wellbeing of the population (Welsh Assembly Government 2008).

The National Pain Audit for England and Wales (2012) had limited response from Welsh pain services beyond the first phase, which examined the composition and allocation of services (Price et al. 2012). Based on self-report, $60 \%(n=9)$ of services in Wales met the criteria for being multi-disciplinary (presence of a psychologist, a physiotherapist and a physician). The audit reported $90 \%$ of Welsh services had a consultant to undertake medication management and $30 \%$ had access to a pharmacist (Price et al. 2012). The audit did not examine how many services took responsibility for prescribing either by issuing prescriptions directly or providing follow-up support to monitor outcomes. The likelihood is that Welsh pain services make prescribing recommendations with the expectation they will be fulfilled by primary care colleagues. 
It is hard to determine how the 2008 directive had influenced the provision of service only 2 years later and no validated data on service provision in Wales, appears to have been collated since. The research presented in this thesis suggests, however, that regardless of specialist service provision, the use of opioid analgesics significantly increased after the 2008 directive. If opioid prescribing is inversely associated with pain management support (Finestone et al. 2016), the implication of the data is either that investment in secondary care services had minimal impact in primary care or the prevalence of pain was outrunning any expansion in support over the duration of the study.

In 2019, WG launched new guidance, 'Living with persistent pain in Wales' (Welsh Government 2019). The basis of the new guidance to Health Boards, professionals and people living with pain was new approaches to healthcare in Wales (Welsh Government 2019). Reducing the burden of long-term conditions in Wales by improving integration of health and social care whilst also addressing issues such as educational and housing disparities are at the heart of recent WG public health policies (Atherton and Welsh Government 2019; Welsh Government 2019). Currently, however, pain as a long-term condition does not have the same level of political interest as other long-term conditions, despite the concerns expressed about the consequences of failing to improve its management (Welsh Government 2019).

Trying to reduce opioid prescribing without addressing the reasons why people experience pain, why they feel unable to manage it or how physical and emotional conditions predispose people to it, seems to some extent a futile exercise. Significant investment will be required to reduce the current inequalities seen in areas of significant deprivation (Section 1.10), including providing employment opportunities, increasing social cohesion and normalising pain as something that does not always need medical intervention. 


\subsubsection{Drug monitoring and prescribing databases}

It is important to improve life opportunities and reduce socioeconomic disparity in order to reduce the pain prevalence over an extended duration. However, the risks of continuing opioid prescribing at the current levels noted in Wales (All Wales Therapeutics and Toxicology Centre 2021, Welsh Government and Stats Wales 2021) and described here, are a significant burden on public health and wellbeing in themselves. Despite there being multiple sources of data on medicines and prescribing here in the UK, it is not clear they are being used optimally to impact policy and practise in a timely manner or to pre-empt changes in variables, which could signify a concern. Examining the sources of information used in the UK and how prescribing data are used elsewhere is helpful to highlight where systems here could be improved for the benefit of the wider population.

The UK has the Yellow Card scheme to report adverse events related to medicines and medical devices (Medicines and Healthcare products Regulatory Agency 2021). Overseen by the Medicines and Healthcare products Regulatory Agency (MHRA), professional or patient-reported incidents are collected, and warnings issued when patterns of concern are noted (Medicines and Healthcare products Regulatory Agency 2021). Whilst overdose might be deemed by some as an adverse effect of medication, incidence data for England and Wales is not collected by MHRA but the Office for National Statistics (ONS) (Office for National Statistics 2019). Data on people accessing services for substance misuse are collated and analysed separately in Wales, Scotland and England (NWIS Information Services 2019, NHS Digital 2021; Public Health Scotland 2021).

In terms of prescription data, the largest source in the UK is in England, where the majority of the UK population reside (Office for National Statistics 2021). Since 2015, the OpenPrescribing project has provided an interface to search English prescribing data to practice level, some of it dating back to 1998. It claims to be capable of interrogating drug data to dose level, although on closer inspection, this refers to product dose rather than the dose prescribed per 
individual (Curtis \& Goldacre 2018). Whilst allowing easy trend analysis, OpenPrescribing is not a linked dataset and examining trends within different demographics still requires data from other sources. An example of this would be the paper produced by Curtis and colleagues (2019) examining opioid prescribing trends where socio-economic deprivation status was based on location of the prescriber rather than recipient, with freely available data taken from Public Health England. Further, age-based analysis was based on published proportions of registered patients in each practice (Curtis et al. 2019). So, whilst providing a comprehensive way of looking at prescribing trends, OpenPrescribing still lacks detail that would be helpful to provide insight of correlation (OpenPrescribing 2020).

Wales operates a reasonably similar system, allowing scrutiny of prescriber level prescribing data, but only as far as drug formulation and strength (Welsh Government and StatsWales 2021). More detail is available for registered NHS employees, from the Shared Services Partnership by downloading individual scanned prescriptions, where some demographic data can be sought. Also, dose, form and quantity of each prescribed medicine can be taken from the scanned prescription, although it is not a realistic means of research as prescriptions can only be viewed one at a time (Shared Services Partnership 2021). Similarly to English data (OpenPrescribing 2020), there is a lag in data release due to prescriptions needing to be physically received and remunerated before the data is confirmed (Shared Services Partnership 2021).

In Scotland, prescribing data are securely stored by the Information Services Division (ISD) of NHS National Services Scotland (Public Health Scotland 2021). This allows analysis to individual patient level of all prescriptions issued, including the date, dose, formulation, strength and quantity of all medicines dispensed (Torrance et al. 2018). A unique identifier, assigned to each person whose data is stored (Community Health Index number $(\mathrm{CHI})$, provides demographic data including gender and postcode and is also used by other datasets, allowing linkage (Torrance et al. 2018; Public Health Scotland 2021). 
The Information Services Division appears to operate the most complete prescribing review system in Great Britain at the moment and also has a shorter time to publishing than the English or Welsh data, although it still remains a retrospective system (Public Health Scotland 2021).

Unlike the UK, in America, Canada and Australia, national real-time prescribing monitoring is in place. Large datasets are created and added to, which are used to examine prescribing trends (Sproule 2015; Department of Health, Australian Government 2019; Centers for Disease Control and Prevention 2021). The idea was for opioid prescribing monitoring to capture incidents of doctor shopping and duplication prescriptions, which may point towards misuse (Ayres \& Jalal 2018). Sharing data within and between states was seen as a method of tracking the crisis, including whether the demographics of people affected changed. In theory, this would provide opportunities for targeted interventions (Martinez 2018; Ayres \& Jalal 2018). It is mandated in the majority of States, prescribers must check the prescription monitoring database before issuing certain controlled drug prescriptions. Public health officials and law enforcement are also encouraged to access the data to identify rogue prescribers and dispensers, targeting so-called 'pill mills' which have been associated with high levels of misuse and deaths in some communities (Chang et al. 2016; Butler et al. 2018; McGreal 2018). The Canadian Province of British Columbia introduced real-time monitoring (PharmaNet), which linked pharmacies and hospitals to a central database in 1995 (Wilsey et al. 2009; Dormuth et al. 2012; Wilsey \& Prasad 2012). A similar system was launched in Australia in 2019, with both systems providing real-time prescription monitoring producing alerts to regulators and healthcare professionals to patients who may be at risk of harm or could be misusing prescribed opioids (Department of Health, Australian Government 2019). Effectiveness of prescription monitoring has been reviewed and shown to be associated with reductions prescribing (Dormuth et al. 2012; Chang et al. 2016; Lachance \& Frey 2019; Wilson et al. 2019; Dobbin \& Liew 2020; Fetene et al. 2020). Initially following the introduction of monitoring programmes, large reductions in prescribing were noted. For example, British Columbia observed a 
$32.8 \%$ reduction in inappropriate opioid prescriptions (where inappropriate was defined as a prescription within 7 days of a prescription for 30 tablets or more) within six months of PharmaNet implementation (Dormuth et al. 2012). The reduction was sustained over the subsequent two years, with a further small reduction towards the end of the second year. A similar reduction and sustained lower level of prescribing was noted for benzodiazepines within the same system. The authors do not provide information about the impact on individuals' pain management experience or healthcare services (Dormuth et al. 2012).

In Florida in the late 2000 's, $4 \%$ of registered prescribers were responsible for $67 \%$ of opioid volume, equivalent to $40 \%$ of prescriptions dispensed. The percentages were unchanged following the introduction of prescribing monitoring in 2010, although reductions in overall patient and prescription numbers were observed. The system did not lead to people highlighted as highrisk prescribers (high volume, high dose, high patient numbers) becoming lower risk however (Chang et al. 2016). These examples imply prescription monitoring systems may contribute to a reduction in high-risk prescribing. A systematic review from 2019, using predominantly North American data, examined the effectiveness of prescribing monitoring systems to change opioid prescribing volume, use of multiple providers, inappropriate prescribing and non-medical opioid use (Wilson et al. 2019). Whilst some studies were able to demonstrate a reduction in opioid prescribing, overall, the outcomes appeared inconsistent. Whilst some states with prescribing monitoring reported significant reductions in prescribing, others were unable to demonstrate any changes. Similarly disparate outcomes were noted for different schedules of opioids, although 2 of 3 studies reported decreases for schedule II opioids such as oxycodone and hydromorphone (Wilson et al. 2019), which have been most closely associated with misuse and overdose related deaths in the USA (McGreal 2018). Both studies included in the review which examined the incidence of inappropriate prescribing, demonstrated a reduction. A decrease in nonmedical prescription opioid use, which has been associated with many of the deaths in North America (McGreal 2018) was not noted in either included study (Wilson et al. 2019). 
Further, none of this work has examined the personal impact on people living with pain.

Of course, not all opioid prescribing is considered high-risk, either by the prescriber, the patient or perhaps even by society. As the Florida study highlighted, a small minority of prescribers are likely responsible for the majority of the 'worst' practice (Chang et al. 2016). Consequently, protections are needed for monitoring systems, to ensure legitimate use of opioids is not affected or negative repercussions are inflicted upon prescribers or patients if data is misinterpreted or not analysed in context.

Whilst an abundance of prescribing and dispensing data is available in the UK (Curtis and Goldacre 2018; All Wales Therapeutics and Toxicology Centre 2021; Public Health Scotland 2021), it is not currently used as a monitoring system for active prescriptions as seen elsewhere. It has been suggested the UK adopts prospective prescribing monitoring system (Stewart \& Basler 2013). Given the experience of other nations, such a system could be initially useful at least. Longterm outcomes of prescribing monitoring schemes are less clear but there is perhaps, an argument to be had that some record of who is receiving potentially harmful medicines is better than nothing.

TOPAS has demonstrated the value of examining prescribing patterns in demographic groups and how it may allow more targeted interventions to reduce harm. This would be even more valuable if the data were available rapidly after collection. A deep learning model to classify patients receiving opioid analgesics and stratify their risk of developing misuse was designed and shown to be robust by a group in America (Che et al. 2017). The researchers were able to link prescriptions to diagnostic records in order to determine criteria for short and long-term prescribing, opioid-related adverse events and use them to predict the likelihood of future outcomes such as long-term opioid use (Che et al. 2017). How realistic such a complex system could be used in clinical practice and 
what advantages it confers over established prescription monitoring systems is unclear, however and warrants further research.

It is likely more cost-effective to prevent disease than treat it when it occurs. However, it is common that greater investment is made to treat illnesses within a healthcare system than taking a more societal view and looking at the role of non-medical interventions (Lyons et al. 2014). It is especially pertinent when deprivation is a key driver of poor health and poor health outcomes will not be solved by medical treatment alone (Marmot \& Bell 2012; Marmot 2017). Joining seemingly disparate data from different public services (e.g., healthcare, and social services) at an individual level should allow greater insight into how services interact, and people interact with them. Combining information may enable relationships to be identified between factors that would not be evident from a single source (Green et al. 2015). It is perhaps one of the greatest potentials of big data. In the UK, public services are incredibly important personally and politically. Using data that is being collected routinely seems logical, to determine outcomes, value for money and to inform plans for future development.

\subsubsection{Learning from our Celtic cousins}

Wales has much in common with Scotland, in respect to population levels of poor health, historically high levels of socioeconomic deprivation (Bambra 2016) and a high incidence of drug misuse relative to the general population (Liddell 2019; Scottish Affairs Select Committee 2019). Like Wales, concerns about opioid prescribing in Scotland have been expressed but, unlike Wales, research groups have been investigating pain and analgesic prescribing for some years already (Torrance et al. 2014; Ruscitto et al. 2015; Torrance et al. 2018; Torrance et al. 2020). Policy development to address chronic pain and prescribing is underway in Wales (Francis 2021) and it seems sensible and prudent to take lessons from the work already undertaken in Scotland, to assist that process. 
Work to improve the way pain was managed and, consequently, address associated issues including rising opioid prescribing started in Scotland in 1994 with a Scottish Office review of the support available to people with chronic pain (NHS Quality Improvement Scotland 2007). It suggested there was a good base on which to build. Further reviews in 2002 and then by McEwen in 2004 observed that whilst chronic pain services were accessible to people all over Scotland, they were not physically present in all areas (McEwen 2004). Due to the way services had developed, often down to the goodwill and tenacity of local professionals, it had led to piecemeal provision, not always supported by adequate funding and facilities (McEwen 2004). McEwen observed mounting waiting times compared to the data from the early 1990s although this was likely a reflection of increasing demand for support. Sixteen recommendations were made to every health board to develop either integrated acute and chronic pain services or a lone chronic pain service. This included an urgent review of pain service provision, ensuring it was adequate for the population, had an identified budget and could provide accurate and timely data. There was also a recommendation for health boards to communicate effectively between sectors, in regard of the services available and improve seamless care. Furthermore, formal links with community services and vocational rehabilitation support were to be established in each service (McEwen 2004). Of real importance in the McEwen review were recommendations on the need to take a national approach to improve quality and collaboration, in part to develop comparative data. Emphasis was given to properly evaluating new developments, effectively disseminating good practice, and building a culture of research. Finally, adding to the national approach, agreeing on a system which provided timely access to routine data from chronic pain services and a co-ordinated national education and training programme was encouraged (McEwen 2004).

Like Scotland, the 2008 directive in Wales acknowledged the complexity of pain, acute and chronic, and the need for widespread change to improve access to services. As with the McEwen recommendations, key actions included an assessment of local needs and developing local pathways to improve access in 
primary and secondary care. Also, regular periodic monitoring of medicines for chronic pain and development of guidance and support on prescribing (Welsh Assembly Government 2008). What the Welsh directive lacked however, was the academic monitoring incorporated in Scotland. Whilst laudable, the actions suggested in Wales did not include detail of how they might be reviewed, relying on health boards, the Welsh Pain Society (a small membership group which is not an official chapter of the British Pain Society), local authorities and Welsh Government to self-monitor. Research in respect of examining the burden of pain in Wales or the impact of introducing new services, trends in prescribing and so on, were not included at all. In researching this thesis, no data was available from Welsh Government on current levels of service provision or whether or how issues around pain and its management had been included in research programmes or funding streams in the intervening years (Francis 2021). Given the burden on health services, as demonstrated in this thesis, the social and wider economic implications of pain, lack of Wales-specific research and formal service review could be a problem. Without baseline data, how will improvement be measured and how will service users and providers know when 'good' is achieved?

In contrast, Scotland has undertaken successive reviews of pain services since 1994, assisting in maintaining focus on pain as an important health and social issue. Getting relevant information on Pain Services (GRIPS) became a highly influential benchmarking exercise in Scotland (NHS Quality Improvement Scotland 2007). An important outcome from the review was that, in Scotland, chronic pain became recognised as a condition in its own right and a national lead clinician for chronic pain appointed (Gilbert et al. 2014). Whilst this could appear simply a paper exercise, Scotland's experience demonstrates that, by drawing focus and providing strong clinical and political leadership, progress can be made.

A national steering group was created for Scotland in 2009 and developed a service model for chronic pain, recognising the majority of people receive pain 
management care and support in primary care rather than specialist centres (Breivik et al. 2012; Gilbert et al. 2014). Similar recognition of increasing support to primary care, was given in both 2008 and 2019 Welsh guidance on pain service development. Admitting the difficulty access support for pain management and the shortcomings of practitioners knowledge and importantly, time needed to undertake complex case work in primary care is vital. Not least, as most prescribing takes place in that sector and tends to be main intervention that can be easily made when someone presents with pain (McCrorie et al. 2015; Mills et al. 2016; Gordon et al. 2017).

As already discussed, (Section 8.3.6), national prescribing guidelines were introduced in Scotland in 2013 (Scottish Intercollegiate Guideline Network SIGN 2019) and were used as a means of educating practitioners and raising awareness of pain. This was accompanied by the launch of a dedicated website providing quality assured information accessible to people living with pain as well as those supporting them (Gilbert et al. 2014). Some information is available from national websites in Wales (All Wales Medicines Strategy Group and All Wales Therapeutics and Toxicology Centre 2020). However, developing a single site with nationally agreed resources, would help practitioners and patients alike, as there is a huge amount of information available on the internet, which can be difficult to navigate or be confident is up to date and clinically accurate.

Despite all the work which has been undertaken in Scotland to drive improvements in allocation of pain services, reduce disparity between different geographical areas and develop a national approach to pain management, more recent reviews still found areas requiring attention. Some variation in care continued in 2018. Data from Health Boards on service provision (Mellor and Chronic Pain Project Group 2018), despite core data measures being agreed (NHS Quality Improvement Scotland 2007) remained difficult to obtain.

Scottish quality prescribing guidelines for chronic pain were launched in 2018, again, including data measures to provide comparison across the country 
(Harrison and Cormack 2018). Like having national clinical leads to drive improvement forward and keep pain on the political agenda, the quality prescribing guidance is promoted in each health board by clinical staff, one of whom tends to be a pharmacist but without needing to be a pain specialist per se (Harrison 2018). As pain is ubiquitous to all sectors and specialities, wide promotion of good practice in prescribing is likely to become a meaningful intervention. The guidance gave a three year timescale to implement change, which does not preclude annual reviews but, gives a more realistic duration over which alterations in practice are likely to take place (Harrison and Cormack 2018).

In Wales, no currently agreed measures or standards exist for health boards to benchmark services. As discussed, (Section 6.6.5), national prescribing indicators may have had some effect on the number of opioid prescriptions being issued in Wales but does not appear to have impacted the overall burden of opioids (Section 5.5). Introducing quality prescribing guidelines, adapted from the Scottish document, has recently been agreed by the All Wales Prescribing Advisory Group (AWPAG) in addition to national prescribing advice (All Wales Prescribing Advisory Group 2021). Nationally agreed guidelines for a range of clinical conditions are becoming commonplace in Wales (All Wales Therapeutics and Toxicology Centre 2021). There are benefits for reducing country-wide variation and gaining local agreement to implement. Differences in individual practice and opinion can drive local guidelines or practice, even if it is not in line with the evidence base for the condition (Scott-Thomas 2020).

What is clear from the experience of colleagues in Scotland is meaningful and lasting change in practise takes time and a huge amount of determined and coordinated work. Whilst Wales has started on the process, a massive amount of work is needed still. Scotland had developed a national strategy, benchmarked services, and determined to appoint a national clinical lead in 2008, when Wales was politically, only just recognising the need to address chronic pain. Wales is 10 years behind, in terms of developing a nationally agreed approach to 
supporting people living with pain. Whilst some progress is being made in terms of reducing opioid prescribing (Welsh Government and Statistics for Wales 2020; All Wales Medicines Strategy Group 2021) pain education for practitioners and people with pain remains widely variable, service provision is patchy and links with third sector organisations and social care are not well developed in all services. Hence, prescribing initiatives alone will not improve pain management as a whole.

There is a pressing need to develop high quality data and research in Wales in all aspects of pain, management, and prescribing. The regular review of Health Board level services and prescribing, as seen in Scotland, seems to have contributed to maintaining motivation and attention for making improvements. Policy change without an accompanying research plan would seem to limit all parties' ability to determine what progress is made or how it affects the lives of people living with pain.

As a starting point, investigating why prescribers continue to prescribe opioid analgesics and why people with pain seek them, in preference to other management methods, within the Welsh context would be useful for shaping future policy and guidance. Data drives many healthcare developments nowadays. It is possible the SAIL databank may be able to extract relevant data to provide some sort of baseline measure of pain prevalence in Wales. Details of services can be requested from Health Boards and compared to nationally agreed standards set out by the Faculty of Pain Medicine (Faculty of Pain Medicine 2021) as used by the National Pain Audit (Price et al. 2012).

Measures to effectively monitor opioid prescribing will need some thought. As presented in this thesis (Section 1.11.1), what constitutes high population levels of prescribing is not necessarily agreed. Whilst an agreed maximum oral morphine equivalent dose might be accepted, (Faculty of Pain Medicine 2021) it is difficult to determine who is most at risk from the data currently available. It would be prudent to determine a national measure of opioid risk, that could be 
used to highlight individuals or areas most at need of intervention. Incentive schemes or funding opportunities need to be directed more towards people and communities who face the highest risk if opioid analgesics are continued. This thesis provides evidence of factors which could guide data identification including gender, age, duration of prescribing and socioeconomic deprivation.

\subsection{Strengths of this research}

This thesis contains the first large scale examination of opioid prescribing using linked healthcare data in Wales. It provides a new perspective on the trends noted from other sources (Welsh Government and Statistics for Wales 2020), which focus on numbers of prescriptions or DDDs (Section 8.3.4.1).

The results of the first part of the study (Section 2.4) presented here have examined gender (Section 3.5.3), age (Section 3.5.4) and drug-type (Section 3.5.2) associations with prescribing and set a baseline for future research into opioid analgesic prescribing in Wales. The methods adopted could be used to look at prescribing trends of other analgesics and the relationships between different medicines and the people receiving them. More detailed examination of prescribing by socioeconomic deprivation (Section 4.5) points to healthdivides between areas of Wales. Social determinants of health are increasingly important for setting health policy (Marmot 2017) but are currently not included in standard reporting of prescribing in Wales. Sharing the findings of this research with Welsh Government will be a crucial step in improving the quality of data used to understand prescribing trends. Knowing where there are elements of higher risk should also better direct resources to support changes in practice.

This is also the first examination of possible associations between opioid prescribing and healthcare resource utilisation in Wales (Section 7.8). The study was able to demonstrate clear differences in resource utilisation between people receiving opioid prescriptions and those who did not. Whilst the exact costs are likely to be underestimated, due to the limitations of data extraction, the differences between groups are notable (Section 7.10.1). Whilst not undertaken 
for this study, the data should allow inquiry of differences in resource utilisation in different areas of Wales. It would be valuable to undertake such analysis as part of ongoing research in this area. Linked data from the SAIL databank allows the opportunity to examine a range of health and social care interactions for individuals, communities, and the wider population. The choice to use the SAIL databank for this research was deliberate and entirely due to the ability to examine data to an individual level and across a range of healthcare services. It is hoped this study demonstrates the value and normalizes the use of large, linked datasets to determine prescribing trends, ideally in real-time.

The SAIL databank allows access to a substantial proportion of the population's data, thus reducing selection bias. The results are highly likely to be genuinely reflective of the Welsh population as there are good levels of representation of people living in all Health Board areas of Wales.

\subsubsection{Impact and influence of this research}

Research findings have been shared with Welsh Government and NHS Wales organisations in order to inform practice and policy development, during the course of thesis development. Locality specific data has been sent to Medicines Management Units in each of the seven Health Boards, to enable them to target opioid stewardship strategies as part of work to rationalise prescribing in line with NPIs. The outputs of the study, with particular reference to socioeconomic deprivation were shared with the Chief Pharmaceutical Officer for Wales and the Lead Civil Servant for long-term health conditions, to inform policy discussions within Welsh Government.

Based on the work examining $\mathrm{OMEQ}_{\mathrm{e}}$ burden presented in this thesis, AWTTC has adopted the category of high-dose opioid prescribing for the 2022-2023 NPIs. The $\mathrm{OMEQ}_{\mathrm{e}}$ lists developed for this study have been used to guide audits of prescribing for Health Boards and shared with the software company who provide Primary Care General Practice support as part of work to develop more effective, timely monitoring of prescribing. 


\subsection{Limitations of the research presented 8.6.1 Limits of data extraction}

The data presented in this thesis is formed from prescribing information from Primary Care in Wales, until the end of 2015. At the time of data extraction (2017 to 2019), data for 2016 onwards was incomplete and therefore, not released as part of this research. Although provision was made within the original protocol to run a subsequent extraction towards the end of the research period, circumstances did not allow it. This resulted in the data being five years out of date at the time of thesis preparation. It would have been informative to examine trends since 2015, to see how they have changed and whether more recent guidance and the increased publicity around opioid prescribing in Wales and the UK in general has had an effect.

Analysis of data stratified by Health Board and deprivation quintiles has been undertaken as part of this study (Appendix I). Geospatial analysis using LSOA level data (Schifanella et al. 2020) would provide greater insight to more precise areas of high prescribing which could then be linked to other criteria such as employment status, receipt of benefits and geographical location (e.g. urban or rural) and would allow more precisely targeted interventions from policymakers, healthcare and social support services. The study was designed to provide baseline analysis as no similar research had previously been undertaken into opioid prescribing in Wales. However, given the significant differences between deprivation quintiles which have been revealed at national and Health Board level (Appendix I), more detailed analyses are warranted.

For the prescribing persistence analyses, annual trend data were adapted from data originally extracted from the SAIL databank. Initially, the way the data were coded was unclear, reducing confidence to track concurrent prescriptions as had been planned. Extracted data were recoded as a double check, but results demonstrate a large increase in the final year of trend analysis. This is because data was counted from the end of the prescribing period backwards, rather than prospectively from the start of the period. If repeating this work or undertaking 
new analysis, consideration of how best to record persistence trends is needed, to avoid skewing the data. Using a 6-month cut off, regardless how long an individual remains on the opioid, may be one such way.

As discussed, there are limitations to using time series analysis alone for the purposes of forecasting changes in the complex process of prescribing. It would have been preferable to access data beyond 2015, to determine the accuracy of predictions made using the models developed. At the time of data extraction, SAIL was only able to provide full datasets up until the end of 2015. The study protocol and IGRP with SAIL included provision for data up until 2019 but this was not possible in the event.

Figures were drawn using opioid data from NHS Wales Shared Services, comparative analysis system for prescribing audit (CASPA) data (Shared Services Partnership 2021), the national repository for medication dispensing records in Wales (Figure 8.1). The data, at the time of this study, could not be linked with other databases in the SAIL databank. Consequently, it is not possible to link to individuals to determine the number of people to whom the prescriptions were issued. As the data is not the same as used for the TOPAS study analysis, it is included to demonstrate the shape of the trend that has been noted in the country since the end of the TOPAS study in December 2015 rather than an accurate comparison with the predictions made. 


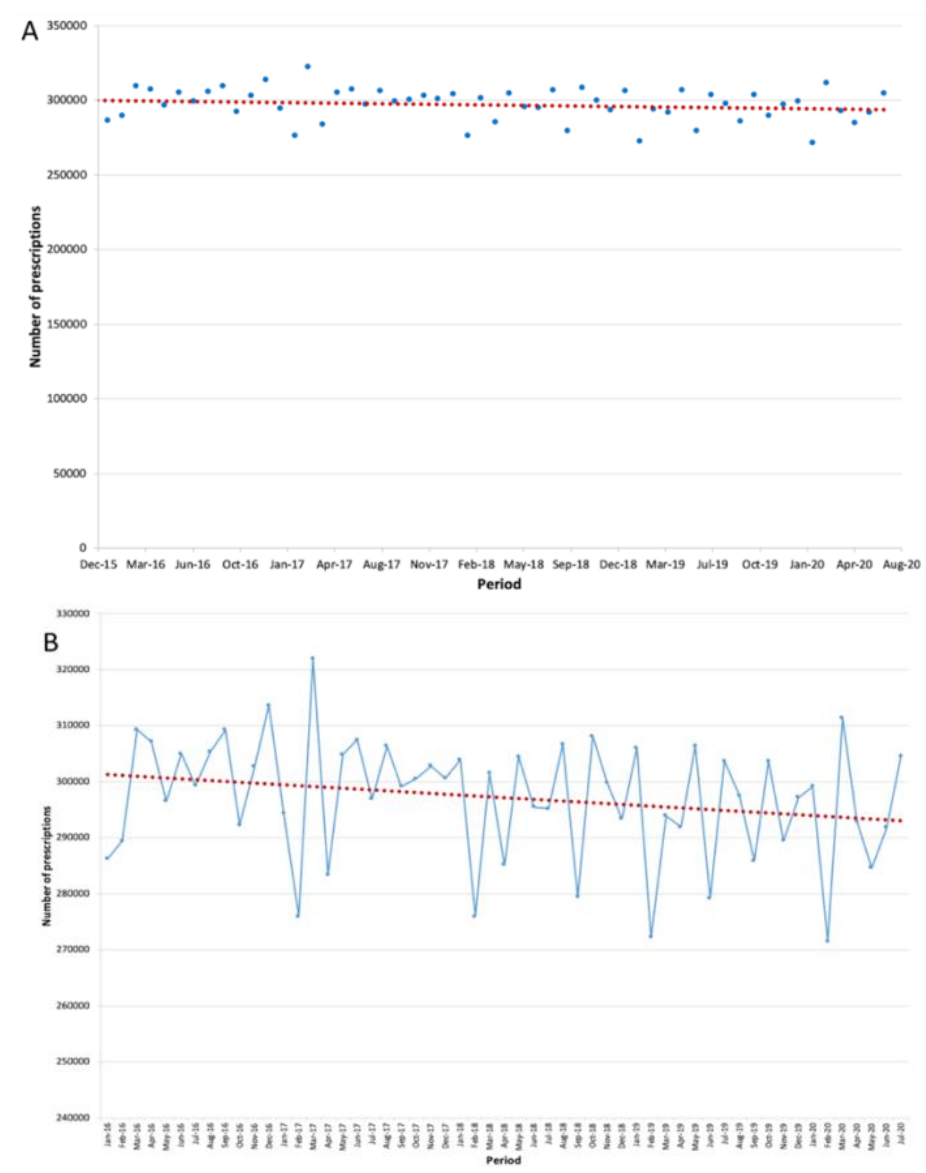

Figure 8.1: Trend of opioid prescribing in Wales from January 2016 - July 2020 using prescription dispensing data taken from CASPA. Linear trend lines added. A - Monthly number of prescriptions with linear trend line. $B$ - Close up of monthly trends that shows similarity with seasonally adjusted data noted from TOPAS study results.

NB. Data includes all prescriptions dispensed by community pharmacies and dispensing practices in Wales and therefore, may include prescriptions issued elsewhere in the UK

\subsubsection{Absence of dispensing data}

Drug costs were not included in the estimations presented here. At the time of the data extraction, the SAIL databank did not have access to dispensing data or all of the detail of what was prescribed at source. This meant that it was not possible to calculate exactly what dose or what quantity of medicine each individual received. Consequently, it was not possible to calculate what was spent on medicines during the study period. Whilst national prescribing data is available, it is not possible to assign each prescription to any of the anonymised individuals whose data was examined for the TOPAS study. When dispensing data becomes available to the SAIL databank, it should be used in place of prescribing records to link to other datasets and continue to regularly analyse changes in opioid use and associated healthcare utilisation. 
This study examined total opioid exposure over time. Due to research constraints, it was not possible to analyse OMEQ changes in combination with prescribing persistence as other researchers from the UK have done (Jani et al. 2020). As more evidence becomes available in regard of risks of opioid dose and duration, a Welsh perspective would be helpful and should be considered as a piece of future work.

\subsubsection{Defining variables}

The oral morphine equivalence and prescribing persistence measures used in this study were estimations borne of necessity due to limitations in accessible data. The results presented here are therefore likely an underestimation of both opioid burden and longevity of prescribing. As prescription dispensing data becomes available through the anonymous linkage systems of the SAIL databank, it will allow more accurate analysis of both variables by using dosing instructions and quantity prescribed to calculate OMEQ and duration (Zin et al. 2014). The data presented here was not able to accurately assess OMEQ per person or track that trend over time for example. Whilst other UK studies have looked at those variables, there is not currently, good quality Welsh data available. It is important for policymakers and clinicians to understand individual as well as population data, particularly as overall prescription numbers appear to be reducing.

For Phase 2, matching of case and control subjects used a method advocated by SAIL but was hampered by the disproportionate number of individuals with the diagnoses of interest also receiving opioid analgesic prescriptions. Further research is needed in Wales to provide more detailed analysis and controlling for non-pain comorbidities, to accurately determine the impact of prescribed opioid use on the type of healthcare accessed.

In relation to healthcare utilisation, it was not possible to retrieve the level of data necessary to make definitive statements about attendance rates. Reliance on a third party for data extraction necessitated compromise and led to less 
complete data than would have been ideal. For example, this study was not able to differentiate between primary care visits in person and interactions which might have been made by telephone, might have been a prescription request or a request for an appointment. Similarly, some data was available on the specialty that individuals attended for outpatient appointments, but it was not complete. Linking of attendance in different healthcare sectors would be beneficial in furthering understanding of how people use health services.

Only a small number of possibly pain related attendances were flagged as reasons for attendance at ED. This was determined as unreliable and further analysis using all possible attendance or diagnosis codes would provide greater insight. It is likely some admissions are not recognised by clinicians at the time as associated with opioid use and therefore, would not be picked up by the coding used for data extraction. Having access to all diagnosis codes would allow investigators to make a judgment on whether presentation could have been influenced by opioid use in some way.

The admitting and discharging specialty were recorded for in-patient admissions. However, data on interventions and investigations were not provided.

Determining the possible contribution of opioids to admissions for case subjects when compared to the control group was not possible. There was also no record of whether admission was planned (elective) or not (emergency), which would have cost implications. Based on unit costings used in this study (Curtis and Burns 2015; Department of Health and Social Care 2015), elective admissions were more costly than emergencies. This seems counterintuitive, as emergency admission conjures an impression of a person being more unwell and needing greater input, initially at least.

\subsubsection{Absence of qualitative data}

At the outset of this research study in 2015 , it was hoped to include an element of qualitative research, building on the work of McCrorie et al (2015) and others in examining the thoughts of patients and practitioners receiving and prescribing 
opioid analgesics. It was considered that initial data extraction would guide development of such work by revealing particular areas of interest that could then be examined through interviews and focus groups. Due to delays in the release of extracted data and its subsequent analysis, it was not possible to pursue the qualitative element of the study. It should form, however, an important element of future research, as detailed in the following section.

\subsection{Ideas for future research}

\subsubsection{Integrating prescribing and dispensing data}

This study is based on prescribing data, e.g., what a GP, pharmacist or nurse writes on a prescription in primary care. This does not always result in the prescription being dispensed and the intended patient receiving and taking the medicines as prescribed. In truth, it is not possible to examine what is taken by people who are prescribed medicines, certainly not at population level.

Dispensing data is the closest estimate of what is taken but at the time this study was conducted, was not available to be anonymously linked within the SAIL databank. As that data becomes available to SAIL, it would be preferable to use linked dispensing data to examine opioid trends as it provides a more detailed picture of individual and population use.

Detail of the doses and quantities prescribed would also lead to clearer understanding of prescribing persistence and OMEQ prescribing. More timely access to prescribing and dispensing data would allow in-time and in-depth monitoring of changes in opioid prescribing, the opioid burden in the population and responses to guidelines and legislation that may be introduced in future. It would enable policy makers and clinicians to react more quickly to changes and better target resource to areas of concern.

\subsubsection{Professional differences in prescribing}

The SAIL databank was unable to provide data on the profession of the prescriber for this study. As part of understanding reasons for such wide discrepancies in prescribing by deprivation quintile, it would be pertinent to find 
out more about the prescribers and their motivations for choosing to prescribe opioids to different patient types. Previous research to examine motivation has taken a qualitative approach (McCrorie et al. 2015; McIntosh et al. 2016) and this should be replicated amongst health care practitioners and people with lived experience of opioid analgesic use, in Wales. Better understanding of what underlies decision making, by prescribers and patients (McCrorie et al. 2015), is also helpful for developing strategy to address the issues raised and provide more targeted and appropriate interventions in different areas and amongst different populations. Wales has seen a large increase in the number of nonmedical prescribers (professionals other than medical doctors) (Alghamdi et al. 2020). Representatives of all healthcare professionals who are legally able to prescribe opioids, should be included in any such research in future.

\subsubsection{Socioeconomic deprivation}

Pain intensity is reported to be higher in areas of greater socio-economic deprivation (Todd et al. 2018) but measures are not routinely collected in clinical practice (Stanos et al. 2016; Bertakis et al. 2004). Whilst people may report having pain, it is not consistently coded in primary care. For this research, the primary care general practice database was scrutinised for codes related to known pain conditions such as osteoarthritis or back pain. The data was used to define groups for the Phase 2 study which examines resource utilisation (Section 7.4.2). However, the presence of a potentially painful condition does not necessarily correlate with presentation due to pain (Tracey 2016). A decision was made not to use diagnosis alone for the purpose of assessing changes in pain presentation over time. Further research to examine correlates between prescribing and pain reporting would need to access alternative data sources. Other studies have used data from the Health Survey for England (Todd et al. 2018) which includes questions about individual's experience of pain and analgesic use. The Welsh Health Survey does not currently include questions specific to pain intensity or analgesics (Perks and Roberts 2016).The UK Biobank has been used to collect self-reported data relating to pain experiences and 
medication use (Macfarlane et al. 2020). Extracting Welsh data from the UK Biobank and linking to other existing sources using the SAIL databank linkage systems should be investigated as an option for future research.

Reasons for disparities in opioid burden between areas of deprivation need further investigation. Lack of availability and acceptability of nonpharmacological management and services have been suggested among reasons why prescribing is favoured (McCrorie et al. 2015; Finestone et al. 2016). As discussed earlier in this chapter, provision of pain management support services across Wales, with particular reference to areas of greater deprivation, is also needed.

\subsubsection{Gender differences in opioid prescribing}

It was clear from the results presented here that women receive significantly more prescriptions for opioid analgesics than men. However, there were signals in the data indicating men might receive higher doses than women, which warrants further examination. Reasons for differences both in pain presentation and analgesic use in men and women have been considered in this thesis (Section 1.8, Section 3.6.5 and Section 4.6.3). Further investigation into the reasons for presentation and what differences exist between genders, in that respect, would enable researchers and clinicians to better understand the quality of management currently being provided. It would also be interesting to examine associations between pain presentation, opioid prescribing and the availability of gender-specific services such as women's health or gynaecology services. This research could use linked data available through the SAIL databank, in relation to service use and prescribing. However, it is also important to capture qualitative data from women and men accessing services and how their experiences differ, as well as practitioners interacting with them.

\subsubsection{Race and intersectional differences in prescribing}

There is building evidence of racial bias in both access to pain management support and in analgesic prescribing (Trawalter et al. 2012; Hoffman et al. 2016; 
Ghoshal et al. 2020). An editorial from the USA highlighted Latinix patients were only half as likely to receive opioid analgesia when presenting with long-bone fractures as non-Latinix white patients. Black patients were more likely to be denied opioids at all in those circumstances (Ghoshal et al. 2020). Race or ethnicity data was not available from the SAIL databank for this study although it is possible to access it. It is fair to say that Wales as a country, is not particularly ethnically diverse. People who are black, Asian or part of another minority ethnic group comprise just under 6\% of the Welsh population in 2020 (StatsWales 2021). The three largest cities in south Wales (Cardiff, Swansea, and Newport) have the majority black, Asian and ethnic minority representation. Swansea and Newport are also significant areas of deprivation (StatsWales 2019). It would be valid to undertake further research examining whether racial differences existed in opioid prescribing and how it might compare with the deprivation data presented in this study and the implications for service provision.

\subsubsection{Determining effects of long-term opioid prescribing}

Researchers have used different criteria to determine chronic opioid prescribing. For example, ten prescriptions in a 12-month period have been used as a measure of persistence in a number of studies (Boudreau et al. 2009; All Wales Medicines Strategy Group 2015; Bedson et al. 2016; Thielke et al. 2017). Jani et al. (2020) and Black-Tiong et al. (2021) both defined long-term prescribing as being in receipt of three prescriptions within a 90-day period (Jani et al. 2020; Black-Tiong et al. 2021). Further research should assess which measures produce more clinically meaningful data, by reflecting what happens in practice. Comparing data for persistent but intermittent opioid use and persistent prescribing as defined for the TOPAS study, could also shed new light on the impact of long-term opioid use. It appears exposure duration may be more clinically significant than dose for a number of adverse effects, including depression. 


\subsubsection{Cost-effectiveness studies}

All health care interactions take time, use resources, and have an associated cost, so it is still necessary to be aware of their impact. It can be confidently stated from TOPAS results, however, that interactions were significantly higher between people using opioids and primary care practices, regardless of the reason for them. Further research, which includes quality of life measures would provide an indication of the cost-effectiveness of opioid analgesics within a wider healthcare context and provide comparison with other methods of pain management. This would be especially interesting to examine over the mid to long-term given the persistent nature of pain for many people.

\subsubsection{Examining outcomes}

This study has examined prescribing and associated healthcare utilisation. What it has not been able to examine is the outcome for an individual or demographically defined group. The far higher healthcare utilisation by people receiving opioids may, in part, be due to people being seen as more unwell, hence the prescription. However, opioid-induced poor health is another possible explanation. This study examined healthcare utilisation during the period of opioid prescription. Future studies examining whether there is a change in healthcare interactions following the initiation of opioids in this group of people should be considered. That would provide some insight into whether opioid prescribing appeared to improve or worsen health. Expanding on McCrorie's (2015) qualitative work (McCrorie et al. 2015), examining reasons for prescribing would also improve understanding of why opioid prescribing starts or continues despite the growing evidence of the harms caused (Baldini et al. 2012; Els et al. 2017; Stannard 2018; Taylor et al. 2019).

\subsection{Study Conclusion}

This study, which is the first of its kind in Wales, has revealed that opioid analgesic prescribing for non-cancer pain significantly increased in in the country between 2005 and 2015. Whilst weak opioid medicines accounted for most 
prescriptions, prescribing rates for strong opioid analgesics such as morphine, oxycodone and fentanyl quadrupled over the period. Women received $50 \%$ more prescriptions than men and gender disparities were noted in overall opioid burden as well. Prescribing rates also rose with increasing age, which although seemingly expected due to higher levels of pain reporting with age, carries significant risk of harm in that population.

Prior to this study being undertaken, it was known the number of prescriptions for opioid analgesics being issued in Wales had hugely increased. However, the analyses presented here give much more detail on where and towards whom prescribing is directed. Primary care practice level data allows examination of prescribing trends but without the age, gender, and socioeconomic deprivation stratification this study presents. It is crucial to track prescribing trends, but the clinical application of the data is limited without being able to better identify those people most at risk, either due to dose, duration or associated demographic factors as has been done in this study.

This thesis proposes that current measures of prescribing are likely to underestimate the impact of opioid analgesic prescribing on the populace, either as a whole or specific sections of it. Estimated measures of oral morphine equivalence and prescribing persistence revealed a significant increase in opioid burden in the population over the 11 years of the study, which was not accounted for by prescribing rates alone. There is unlikely to be a perfect measure of prescribing, certainly not one that covers all medicines in all disease states. However, oral morphine equivalence measures are increasingly used in research and prescribing guidance, so finding a method of incorporating it into timely analysis would be prudent. The introduction of a variety of legislation and guidance during the study period did little to temper the rates of increase. It points towards a problem of dissemination and implementation of clinical guidelines. A lot of time is invested in developing policies and guidance but much less on how they will be adopted into practice or how compliance with them is measured. Welsh Government and Health Boards should exploit Wales being a 
relatively small country as an advantage to developing better systems for sharing practice. There is no good reason that national pathways of care, timely monitoring systems easily accessed by practitioners and researchers and professional and public awareness campaigns cannot be used to raise the profile of pain and the problems with opioid analgesics.

Opioid prescribing has previously been associated with an increase in healthcare utilisation, but this thesis gives the first indication of how that affects people living in Wales. Prescribing is one of the main activities in primary care. This study observed 5 times more primary care interactions with people with painrelated diagnoses receiving opioid analgesic prescriptions compared to those with similar diagnosis but not receiving opioids. Even assuming some of the difference in numbers of visits in primary care were for the prescription itself, this does not explain the 3 times greater use of healthcare in other sectors also noted. Whilst pain services are available across Wales, the majority of people living with pain will not access them. Investment in training and education of healthcare professionals from all disciplines, moving services into Primary Care and working more closely with third sector organisations to improve access to non-medical support is likely to have more benefit to the population than just expanding existing pain service models.

From a public health perspective, what is likely to be of most concern, is the disproportionate opioid prescribing and consequent healthcare utilisation observed in areas of Wales with the highest levels of socioeconomic deprivation and predominating in the working age population. This points towards far greater pain prevalence in the most deprived areas, where higher than average levels of ill-health, obesity and low levels of activity are also observed. Strong opioid analgesics like morphine, oxycodone and fentanyl rose particularly sharply in these areas during the study period. The harms associated with opioid analgesics are likely to have a disproportionately negative effect over time in deprived areas, worsening prognosis and potentially maintaining the health and well-being disparities noted between them and wealthier areas. What seems 
clear, having completed this research, is that guidelines alone are likely to be insufficient to reduce the use of opioid analgesics in Wales. The problem of opioid prescribing is linked to the problem of pain and how it is understood and managed within, and without healthcare. The perception that pain management is a specialty pursuit when, in fact, it is ubiquitous to all sections of healthcare, may be responsible in part for this.

Arising from this thesis are several suggestions for policy development to address opioid prescribing. Welsh Government policies are frequently aimed at reducing societal and health inequality but currently, prescribing guidelines, where available, do not differentiate between populations with disparate risks. Further research is also needed across the country to examine people's attitudes to pain and the use of opioid analgesics in that context. Factors which influence prescribing were not examined here, but based on previous studies, are often associated with a seeming lack of other safer, timely options to educate and support people presenting with pain. What must be guarded against however is using the lack of alternatives as a reason to continue prescribing opioid analgesics when they are not evidenced or there is no demonstrable benefit to those receiving them. Prescribers must be supported not to prescribe or to reduce and stop opioid analgesics which are causing harm, even when there is no alternative to offer. The sad truth is that for many long-term pain conditions, the 'right' answer, in terms of 'what makes this better?" is not known. It is clear however, that for many people, opioids are likely to be the wrong answer. Health Boards and other organisations including the Welsh Government must invest in public awareness campaigns to bring attention to the poor evidence for using opioid analgesics, especially in the long term and the additional health risks they can cause.

The reasons for disproportionate levels of opioid prescribing in areas with greater deprivation and the high rates of healthcare utilisation are complex and affect all countries in the UK. The research presented here, whilst focussed on Wales, corroborates the findings from around the UK where consistently 
opioid analgesic prescribing is observed to be higher in areas of greater socioeconomic deprivation. Given the large dataset used for this study, it is not unreasonable to suggest the results could be generalised and a similar pattern of increased healthcare utilisation would be likely observed across the UK more widely, with significant cost implications to the NHS as a whole. The observation that prescribing rates increased rapidly in the least deprived areas of Wales also suggests a wider problem of growing pain prevalence and opioid prescribing that is less frequently discussed and warrants further investigation. There is no doubt that in the long-term, reducing socioeconomic disparity would have a great effect on general health and well-being, but it appears that opioid analgesic use is widespread throughout the population. Actions taken to improve the quality of prescribing and support services in one demographic would benefit the whole population. Greater levels of resource are undoubtedly required to address the concerns in the most deprived areas of Wales and the UK more widely. However, no population demographic should be assumed to be untouched by over-prescribing of opioids. Adding to the problem by continuing to prescribe medicines which are not enabling people to live better and more effective lives, or which result in greater healthcare need should be avoided regardless of individual circumstances.

It is hoped this thesis provides a base on which to build further research into the prescribing of opioids and other analgesics. It highlights the need to investigate what might underly disparities in prescribing whether due to geographical location, gender, age or socioeconomic deprivation. It suggests methods of monitoring prescribing opioid trends which may be more meaningful to prescribers and help practitioners and policy makers to get to grips with the burden opioids place upon the population. This research also signals that healthcare use might be influenced by opioid prescribing, which warrants more detailed exploration. Although only a small country, Wales looks to have quite a big problem with pain and prescribing. The advantage of being small however, is 
that even a few people, willing to engage in the changes required, could make a big difference to population health. 
"It will be alright in the end and, if it's not alright, it's not the end." Mark Kermode 


\section{References}

Abdallah, C.G. and Geha, P. 2017. Chronic Pain and Chronic Stress: Two Sides of the Same Coin? Chronic Stress 1, pp. 247054701770476-10. Available at: http://journals.sagepub.com/doi/10.1177/2470547017704763.

Abel, G.A. et al. 2016. Adjusted indices of multiple deprivation to enable comparisons within and between constituent countries of the UK including an illustration using mortality rates. BMJ Open 6(11), p. e012750. Available at: https://bmjopen.bmj.com/content/6/11/e012750.full.pdf.

Addis, S. et al. 2019. Implementing Prudent Healthcare in the NHS in Wales; what are the barriers and enablers for clinicians? Journal of Evaluation in Clinical Practice 25(1), pp. 104-110. Available at:

http://eutils.ncbi.nlm.nih.gov/entrez/eutils/elink.fcgi?dbfrom=pubmed\&id=30144251\&retmode= $\underline{\text { ref\&cmd=prlinks. }}$

Administrative Data Research UK 2021. Administrative Data Research UK. Available at: https://www.adruk.org [Accessed: 16 April 2021].

Aguera-Ortiz, L. et al. 2013. Unexplained pain complaints and depression in older people in primary care. The Journal of Nutrition, Health \& Aging 17(6), pp. 574-577. Available at: http://eutils.ncbi.nlm.nih.gov/entrez/eutils/elink.fcgi?dbfrom=pubmed\&id=23732555\&retmode= ref\&cmd=prlinks.

Akbari, A. and Torabi, F. 2017. Personal communication - explanation of study matching. 21 March 2017

Alexander, J. et al. 2019. Assessing the Value of Time Series Real-World and Clinical Trial Data vs. Baseline-Only Data in Predicting Responses to Pregabalin Therapy for Patients with Painful Diabetic Peripheral Neuropathy. Clinical Drug Investigation 39(8), pp. 775-786. Available at: https://doi.org/10.1007/s40261-019-00812-6.

Alghamdi, S.S.A. et al. 2020. Prescribing trends over time by non-medical independent prescribers in primary care settings across Wales (2011-2018): a secondary database analysis. BMJ open 10(10), pp. e036379-8. Available at: https://bmjopen.bmj.com/lookup/doi/10.1136/bmjopen-2019-036379.

Alho, H. et al. 2020. Opioid-related deaths in Europe: Strategies for a comprehensive approach to address a major public health concern. The International journal on drug policy 76, p. 102616. Available at: https://linkinghub.elsevier.com/retrieve/pii/S0955395919303305.

Ali, A. et al. 2018. Managing Chronic Pain in the Elderly: An Overview of the Recent Therapeutic Advancements. Cureus 10(9), p. e3293. doi: 10.7759/cureus.3293.

All Wales Medicines Strategy Group 2013. National Prescribing Indicators 2013-2014. Available at: https://awmsg.nhs.wales/medicines-appraisals-and-guidance/medicinesoptimisation/national-prescribing-indicators/2013-2014/.

All Wales Medicines Strategy Group 2014. Polypharmacy: Guidance for Prescribing. Available at: https://awmsg.nhs.wales/files/guidelines-and-pils/polypharmacy-guidance-for-prescribing-pdf/ [Accessed: 14 November 2020]. 
All Wales Medicines Strategy Group 2021. All Wales Medicines Strategy Group: Prescribing Guidance. Available at: https://awmsg.nhs.wales/medicines-appraisals-and-guidance/medicinesoptimisation/prescribing-guidance/ [Accessed 24 July 2021]

All Wales Medicines Strategy Group and All Wales Therapeutics and Toxicology Centre 2020. Persistent Pain Resources. Available at: https://awttc.org/news/awmsg-resources-use-opioidsupdated-account-mhra-drug-safety-updates.

All Wales Medicines Strategy Group. 2015. Polypharmacy: Guidance for Prescribing., pp. 1-36. Available at: https://awmsg.nhs.wales/files/guidelines-and-pils/polypharmacy-guidance-forprescribing-pdf/.

All Wales Medicines Strategy Group. 2020. Persistent Pain Resources. Available at: https://awttc.org/news/awmsg-resources-use-opioids-updated-account-mhra-drug-safetyupdates.

All Wales Prescribing Advisory Group 2021. AWPAG minutes 17 Mar 21 - Final. Available at: https://awmsg.nhs.wales/files/awpag-meetings/awpag-minutes-17-mar-21-pdf/.

All Wales Therapeutics and Toxicology Centre 2018. National prescribing indicators 2019-2020. Available at: https://www.awttc.org/national-prescribing-indicators-2019-2020 [Accessed: 12 August 2021].

All Wales Therapeutics and Toxicology Centre 2021. All Wales Therapeutics and Toxicology Centre. Available at: https://www.awttc.org [Accessed: 8 June 2021].

Amsterdam, J. van and Brink, W. van den 2015. The Misuse of Prescription Opioids: A Threat for Europe? Current drug abuse reviews 8(1), pp. 3-14. Available at:

http://eutils.ncbi.nlm.nih.gov/entrez/eutils/elink.fcgi?dbfrom=pubmed\&id=26084418\&retmode= ref\&cmd=prlinks.

Andersson, H.I. 1994. The epidemiology of chronic pain in a Swedish rural area. Quality of life research : an international journal of quality of life aspects of treatment, care and rehabilitation 3 Suppl 1(Suppl 1), pp. S19-26. Available at:

http://eutils.ncbi.nlm.nih.gov/entrez/eutils/elink.fcgi?dbfrom=pubmed\&id=7866366\&retmode=r ef\&cmd=prlinks.

Andreu-Perez, J. et al. 2015. Big Data for Health. IEEE Journal of Biomedical and Health Informatics 19(4), pp. 1193-1208. doi: 10.1109/jbhi.2015.2450362.

Arul, J. and John, W. 2020. Basic Opioid Pharmacology - An Update. British Journal of Pain 14(2), pp. 115-121. Available at: https://pubmed.ncbi.nlm.nih.gov/32537150.

Ashaye, T. et al. 2018. Opioid prescribing for chronic musculoskeletal pain in UK primary care: results from a cohort analysis of the COPERS trial. BMJ Open 8(6), p. e019491. doi: 10.1136/bmjopen-2017-019491.

Asmundson, G.J.G. and Katz, J. 2009. Understanding the co-occurrence of anxiety disorders and chronic pain: state-of-the-art. Depression and anxiety 26(10), pp. 888-901. Available at: http://eutils.ncbi.nIm.nih.gov/entrez/eutils/elink.fcgi?dbfrom=pubmed\&id=19691031\&retmode= ref\&cmd=prlinks. 
Atherton, F. and Welsh Government. 2019. Valuing our health: Chief Medical Officer's Annual Report 2018/19. Available at: https://gov.wales/sites/default/files/publications/2019-05/valuingour-health.pdf.

Auditor General for Wales 2018. A picture of primary care in Wales. Wales Audit Office. Available at: https://www.audit.wales/system/files/publications/picture-of-primary-care-2018-

english 0.pdf.

Australian Government Department of Health 2019. National Real Time Prescription Monitoring (RTPM). Available at: https://www.health.gov.au/initiatives-and-programs/national-real-timeprescription-monitoring-rtpm.

Aylward, M. et al. 2013. Simply Prudent Healthcare - achieving better care and value for money in Wales - discussion paper. Cardiff: Bevan Commission., p. 10. Available at:

https://ctrtraining.co.uk/documents/BevanCommissionSimplyPrudentHealthcarev104122013.pdf

Ayres, I. and Jalal, A. 2018. The Impact of Prescription Drug Monitoring Programs on U.S. Opioid Prescriptions. The Journal of law, medicine \& ethics : a journal of the American Society of Law, Medicine I\& Ethics 46(2), pp. 387-403. Available at:

http://eutils.ncbi.nlm.nih.gov/entrez/eutils/elink.fcgi?dbfrom=pubmed\&id=30146997\&retmode= ref\&cmd=prlinks

Azevedo, L.F. et al. 2013. Chronic Pain and Health Services Utilization. Medical care 51(10), pp. 859-869. Available at:

http://search.ebscohost.com/login.aspx?direct=true\&db=ccm\&AN=107970643\&site=ehost$\underline{\text { live } \& \text { scope }=\text { site } \& \text { authtype }=\text { shib } \& \text { custid }=\text { s } 8000044}$

Aziz, Q. et al. 2019. The IASP classification of chronic pain for ICD-11: chronic secondary visceral pain. PAIN 160(1), pp. 69-76. Available at:

https://journals.Iww.com/pain/Fulltext/2019/01000/The IASP classification of chronic pain fo r.9.aspx.

Baker, R. 2004. Implications of Harold Shipman for general practice. Postgraduate Medical Journal 80(944), pp. 303-6-- discussion 307--8. Available at:

http://eutils.ncbi.nIm.nih.gov/entrez/eutils/elink.fcgi?dbfrom=pubmed\&id=15192157\&retmode= ref\&cmd=prlinks.

Baldini, A. et al. 2012. A Review of Potential Adverse Effects of Long-Term Opioid Therapy: A Practitioner's Guide. The primary care companion for CNS disorders 14(3). Available at: https://www.ncbi.nlm.nih.gov/pmc/articles/PMC3466038/.

Ballantyne, J.C. 2003. Chronic pain following treatment for cancer: the role of opioids. The oncologist 8(6), pp. 567-575. doi: 10.1634/theoncologist.8-6-567.

Ballantyne, J.C. 2017. Opioids for the Treatment of Chronic Pain: Mistakes Made, Lessons Learned, and Future Directions. Anesthesia and Analgesia 125(5), pp. 1769-78. doi: 10.1213/ane<.0010000000002500.

Ballantyne, J.C. and Shin, N.S. 2008. Efficacy of Opioids for Chronic Pain: A Review of the Evidence. Clinical Journal of Pain 24(6), pp. 469-478.

Bambra, C. 2016. Health Divides - Where you live can kill you. Bristol: Bristol: Policy Press. Available at: https://policy.bristoluniversitypress.co.uk/health-divides. 
Barnard, H. 2018. Poverty in Wales 2018., pp. 1-34. Available at: https://www.jrf.org.uk. [Accessed 9 September 2020]

BBC News 2018. NHS data breach affects 150,000 patients in England. Available at: https://www.bbc.co.uk/news/technology-44682369.

Bedard, N.A. et al. 2020. Optimum Designs for Large Database Research in Musculoskeletal Pain Management. Journal of Bone and Joint Surgery 102(Suppl 1), pp. 54-58. Available at: https://journals.Iww.com/10.2106/JBJS.20<.00101.

Bedson, J. et al. 2016. Trends in long-term opioid prescribing in primary care patients with musculoskeletal conditions: an observational database study. Pain 157(7), pp. 1525-1531. Available at:

http://eutils.ncbi.nIm.nih.gov/entrez/eutils/elink.fcgi?dbfrom=pubmed\&id=27003191\&retmode= ref\&cmd=prlinks.

Bekkering, G.E. et al. 2011. Can morphine still be considered to be the standard for treating chronic pain? A systematic review including pair-wise and network meta-analyses. Current medical research and opinion 27(7), pp. 1477-1491. Available at: http://www.tandfonline.com/doi/full/10.1185/03007995.2011.586332.

Bennett, M.I. et al. 2019. The IASP classification of chronic pain for ICD-11: chronic cancer-related pain. Pain 160(1), p. 38. Available at:

https://journals.Iww.com/pain/Fulltext/2019/01000/The IASP classification of chronic pain fo r.5.aspx.

Benoliel, R. et al. 2019. The IASP classification of chronic pain for ICD-11: chronic secondary headache or orofacial pain. Pain 160(1). Available at:

https://journals.Iww.com/pain/Fulltext/2019/01000/The IASP classification of chronic pain fo r.8.aspx.

Benyamin, R. et al. 2008. Opioid complications and side effects. Pain physician 11(2 Suppl), pp. S105-20.

Berger, A. et al. 2012. Clinical characteristics and patterns of healthcare utilization in patients with painful neuropathic disorders in UK general practice: a retrospective cohort study. BMC Neurology 12(1), p. 8. Available at: https://doi.org/10.1186/1471-2377-12-8.

Bernal, J.L. et al. 2017. Interrupted time series regression for the evaluation of public health interventions: a tutorial. International journal of epidemiology 46(1), pp. 348-355. Available at: http://eutils.ncbi.nlm.nih.gov/entrez/eutils/elink.fcgi?dbfrom=pubmed\&id=27283160\&retmode= ref\&cmd=prlinks.

Bevan, G. et al. 2014. The Four Health Systems of the United Kingdom. Health Foundation. Available at:

http://books.google.co.uk/books?id=jszPvQEACAAJ\&dq=intitle:The+four+health+systems+of+the +United+Kingdom+how+do+they+compare \&hl=\&cd=1\&source=gbs api.

Bialas, P. et al. 2020. Efficacy and harms of long-term opioid therapy in chronic non-cancer pain: Systematic review and meta-analysis of open-label extension trials with a study duration $\geq 26$ weeks. European Journal of Pain 24(2), pp. 265-278. doi: 10.1002/ejp.1496.

Black-Tiong, S. et al. 2021. Trends in long-term opioid prescriptions for musculoskeletal conditions in Australian general practice: a national longitudinal study using Medicinelnsight, 
2012-2018. BMJ Open 11(4), p. e045418. Available at:

https://bmjopen.bmj.com/content/11/4/e045418.

Black, N. 1999. High-quality clinical databases: breaking down barriers. The Lancet 353(9160), pp. 1205-1206. Available at: https://linkinghub.elsevier.com/retrieve/pii/S0140673699001087.

Blanch, B. et al. 2014. An overview of the patterns of prescription opioid use, costs and related harms in Australia. British Journal of Clinical Pharmacology 78(5), pp. 1159-1166. Available at: http://doi.wiley.com/10.1111/bcp.12446.

Blozik, E. et al. 2009. Depression and anxiety as major determinants of neck pain: a crosssectional study in general practice. BMC Musculoskeletal Disorders 10(1), p. 13. doi: 10.1186/1471-2474-10-13.

Blyth, F.M. and Schneider, C.H. 2018. Global burden of pain and global pain policy-creating a purposeful body of evidence. Pain 159 Suppl 1, pp. S43-S48. doi: 10.1097/j.pain<.0010000000001311.

Blyth, F.M. et al. 2019. The Global Burden of Musculoskeletal Pain-Where to From Here? American Journal of Public Health 109(1), pp. 35-40. Available at:

http://eutils.ncbi.nlm.nih.gov/entrez/eutils/elink.fcgi?dbfrom=pubmed\&id=30495997\&retmode= ref\&cmd=prlinks.

Board of Community Health Councils in Wales. 2018. Our lives on hold... Cardiff: Community Health Councils of Wales. Available at:

http://www.wales.nhs.uk/sitesplus/documents/899/Our\%20lives\%20on\%20hold\%20\%20ENGLISH\%2010.05.20181.pdf.

Bobinac, A. et al. 2010. Willingness to pay for a quality-adjusted life-year: the individual perspective. Value in health : the journal of the International Society for Pharmacoeconomics and Outcomes Research 13(8), pp. 1046-1055. Available at: https://linkinghub.elsevier.com/retrieve/pii/S1098301511718381.

Bohensky, M.A. 2010. Data Linkage: A powerful research tool with potential problems. BMC Health Services Research 10(1), pp. 1-7. doi: 10.1186/1472-6963-10-346.

Bosetti, C. et al. 2019. Trends in the consumption of opioids for the treatment of severe pain in Europe, 1990-2016. European Journal of Pain 23(4), pp. 697-707. Available at:

https://onlinelibrary.wiley.com/doi/full/10.1002/ejp.1337.

Boudreau, D. et al. 2009. Trends in long-term opioid therapy for chronic non-cancer pain. Pharmacoepidemiology and Drug Safety 18(12), pp. 1166-1175. Available at:

http://doi.wiley.com/10.1002/pds.1833.

Bourgeais, V. and Krueger, A. 2017. Eurostat newsrelease: 2015 GDP per capita in 276 EU regions. Eurostat Press Office. Available at:

https://ec.europa.eu/eurostat/documents/2995521/7962764/1-30032017-AP-EN.pdf/4e9c09e5c743-41a5-afc8-eb4aa89913f6.

Bowsher, D. et al. 1991. Prevalence of chronic pain in the British population : a telephone survey of 1037 households. Pain Clinic 4, pp. 223-230. 
Bozic, K.J. et al. 2012. Resource utilization and costs before and after total joint arthroplasty. BMC Health Services Research 12(73). Available at:

https://link.springer.com/content/pdf/10.1186/1472-6963-12-73.pdf.

Braden, J.B. et al. 2010. Emergency Department Visits Among Recipients of Chronic Opioid Therapy. Archives of Internal Medicine 170(16), pp. 1425-1432. doi:

10.1001/archinternmed.2010.273.

Bradley, P. et al. 2014. Achieving prudent healthcare in NHS Wales. Public Health Wales. Available at:

https://pureadmin.uhi.ac.uk/ws/portalfiles/portal/1921669/Achieving_prudent healthcare in N HS Wales paper Revised version FINAL .pdf.

Brady, J.E. et al. 2017. Risk markers for fatal and non-fatal prescription drug overdose: a metaanalysis. Injury epidemiology 4(1), p. 24. Available at:

http://eutils.ncbi.nlm.nih.gov/entrez/eutils/elink.fcgi?dbfrom=pubmed\&id=28762157\&retmode= ref\&cmd=prlinks.

Braveman, P. and Gottlieb, L. 2014. The social determinants of health: it's time to consider the causes of the causes. Public health reports (Washington, D.C. : 1974) 129 Suppl 2(1_suppl2), pp. 19-31. Available at: https://www.ncbi.nlm.nih.gov/pmc/articles/PMC3863696/\# ffn sectitle.

Breivik, H. and Stubhaug, A. 2014. Burden of disease is often aggravated by opioid treatment of chronic pain patients: Etiology and prevention. Pain 155(12), pp. 2441-2443. Available at: http://journals.Iww.com/00006396-201412000-00002.

Breivik, H. et al. 2006. Survey of chronic pain in Europe: Prevalence, impact on daily life, and treatment. European Journal of Pain 10(4), pp. 287-287. doi: 10.1016/j.ejpain.2005.06.009.

Breivik, H. et al. 2013. The individual and societal burden of chronic pain in Europe: the case for strategic prioritisation and action to improve knowledge and availability of appropriate care. $B M C$ Public Health 13(1), pp. 1-29. Available at:

http://bmcpublichealth.biomedcentral.com/articles/10.1186/1471-2458-13-1229.

Brena, S.F. and Sanders, S.H. 1992. Chronic pain: fix or manage? Clinical Journal of Pain 8(2), pp. $172-7$.

Brew, B. et al. 2016. The health and wellbeing of Australian farmers: a longitudinal cohort study. BMC Public Health 16(1), pp. 1-11. Available at: http://dx.doi.org/10.1186/s12889-016-3664-y.

Brouwer, W.B.F. et al. 2008. Welfarism vs. extra-welfarism. Journal of health economics 27(2), pp. 325-338. Available at:

http://eutils.ncbi.nlm.nih.gov/entrez/eutils/elink.fcgi?dbfrom=pubmed\&id=18179835\&retmode= ref\&cmd=prlinks.

Bruggenjurgen, B. et al. 2007. Utilisation of medical resources of patients with pain undergoing an outpatient opioid therapy. Gesundheitswesen (Bundesverband der Arzte des Offentlichen Gesundheitsdienstes (Germany)) 69(6), pp. 353-358. doi: 10.1055/s-2007-981679.

Brummett, C.M. et al. 2019. Health Care Burden Associated with Outpatient Opioid Use Following Inpatient or Outpatient Surgery. Journal of Managed Care \& Specialty Pharmacy 25(9), pp. 973983. doi: 10.18553/jmcp.2019.19055. 
Buchbinder, R. et al. 2013. Placing the global burden of low back pain in context. Best Practice \& Research Clinical Rheumatology 27(5), pp. 575-589. doi: 10.1016/j.berh.2013.10.007.

Butler, J.M. et al. 2018. Big Data and the Opioid Crisis: Balancing Patient Privacy with Public Health. The Journal of law, medicine \& ethics : a journal of the American Society of Law, Medicine I\& Ethics 46(2), pp. 440-453. Available at:

http://eutils.ncbi.nlm.nih.gov/entrez/eutils/elink.fcgi?dbfrom=pubmed\&id=30146994\&retmode= ref\&cmd=prlinks.

Byrne, M. et al. 2003. Frequent attenders to an emergency department: A study of primary healthcare use, medical profile, and psychosocial characteristics. Annals of emergency medicine 41(3), pp. 309-318. doi: 10.1067/mem.2003.68.

Calderwood, C. 2017. Realising Realistic Medicine: Chief Medical Officer for Scotland annual report 2015-2016. Edinburgh: Healthcare Quality and Improvement Directorate, Scottish Government. Available at: https://www.gov.scot/publications/chief-medical-officer-scotlandannual-report-2015-16-realising-realistic-9781786526731/.

Campbell, G. et al. 2015. The Pain and Opioids IN Treatment study: characteristics of a cohort using opioids to manage chronic non-cancer pain. Pain 156(2), pp. 231-242. Available at: http://eutils.ncbi.nlm.nih.gov/entrez/eutils/elink.fcgi?dbfrom=pubmed\&id=25599444\&retmode= $\underline{\text { ref\&cmd=prlinks. }}$.

Carlisle, D. 2017. Prescription charges backfire on UK health and wealth. Available at: https://www.pharmaceutical-journal.com/news-and-analysis/prescription-charges-backfire-onuk-health-andnbspwealth/20203213.article?firstPass=false.

Carr, D.B. and Goudas, L.C. 1999. Acute Pain. The Lancet 353(9169), pp. 2051-2058. Available at: https://www.thelancet.com/journals/lancet/article/PIIS0140-6736(99)03313-9/fulltext.

Centers for Disease Control and Prevention 2016. CDC guideline for prescribing opioids for chronic pain-United States, 2016. Available at: http://scholar.google.comjavascript:void(0).

Centers for Disease Control and Prevention 2020. America's Drug Overdose Epidemic: Putting data to action. Available at: https://www.cdc.gov/injury/features/prescription-drugoverdose/index.html [Accessed: December 2020].

Centers for Disease Control and Prevention 2021. Opioid overdose: US Opioid Dispensing Rate Maps. Available at: https://www.cdc.gov/drugoverdose/maps/rxrate-maps.html.

Centers for Disease Control and Prevention 2021. Opioid Overdose. Available at: https://www.cdc.gov/drugoverdose/epidemic/index.html [Accessed: 26 June 2021].

Centers for Disease Control and Prevention 2021. Prescription Drug Monitoring Programs (PDMPs). Available at: https://www.cdc.gov/drugoverdose/pdmp/states.html [Accessed: January 2021].

Centre for Clinical Practice at National Institute for Health and Care Excellence . 2013.

Neuropathic Pain: The Pharmacological Management of Neuropathic Pain in Adults in Nonspecialist Settings. Available at:

http://eutils.ncbi.nlm.nih.gov/entrez/eutils/elink.fcgi?dbfrom=pubmed\&id=25577930\&retmode= ref\&cmd=prlinks. 
Chan, B.T. et al. 2005. Factors influencing family physicians to enter rural practice: Does rural or urban background make a difference? Canadian Family Physician 51, pp. 1246-1247. Available at: https://www.ncbi.nlm.nih.gov/pmc/articles/PMC1479469/pdf/jCFP v051 pg1247.pdf.

Chang, H.-Y. et al. 2016. Impact of prescription drug monitoring programs and pill mill laws on high-risk opioid prescribers: A comparative interrupted time series analysis. Drug and alcohol dependence 165, pp. 1-8. doi: 10.1016/j.drugalcdep.2016.04.033.

Chang, H.-Y. et al. 2018. Healthcare costs and utilization associated with high-risk prescription opioid use: a retrospective cohort study. BMC Medicine 16(69). doi:

https://doi.org/10.1186/s12916-018-1058-y.

Chaparro, L.E. et al. 2013. Opioids compared to placebo or other treatments for chronic low-back pain. The Cochrane Database of Systematic Reviews . Available at:

http://onlinelibrary.wiley.com/doi/10.1002/14651858.CD004959.pub4/pdf.

Chaparro, L.E. et al. 2014. Opioids Compared with Placebo or Other Treatments for Chronic Low Back Pain. Spine 39(7), pp. 556-563. doi: 10.1097/brs<.0010000000000249.

Chapman, C.R. et al. 2010. Opioid Pharmacotherapy for Chronic Non-Cancer Pain in the United States: A Research Guideline for Developing an Evidence-Base. Journal of pain : official journal of the American Pain Society. 11(9).

Che, Z. et al. 2017. Deep Learning Solutions for Classifying Patients on Opioid Use. AMIA ... Annual Symposium proceedings. AMIA Symposium 2017, pp. 525-534. Available at: http://eutils.ncbi.nlm.nih.gov/entrez/eutils/elink.fcgi?dbfrom=pubmed\&id=29854117\&retmode= ref\&cmd=prlinks.

Chen, T.-C. et al. 2019. Impact of tramadol classification on the prescribing of other pain medicines commonly related to drug-misuse deaths in UK. Pharmacoepidemiology and Drug Safety 28, pp. 253-253. Available at:

http://gateway.webofknowledge.com/gateway/Gateway.cgi?GWVersion=2\&SrcAuth=mekentosj \&SrcApp=Papers\&DestLinkType=FullRecord\&DestApp=WOS\&KeyUT=000481785601272.

Chevalier, P. et al. 2014. A description of clinical characteristics and treatment patterns observed within prescribed opioid users in Germany and the UK. Pain Management 4(4), pp. 267-76. doi: https://doi.org/10.2217/pmt.14.26.

Chief Medical Officer of England 2009. 150 years of the Annual Report of the Chief Medical Officer. London: Department of Health (England)., p. 70. Available at: https://webarchive.nationalarchives.gov.uk/ukgwa/20130107105354/http:/www.dh.gov.uk/prod consum dh/groups/dh_digitalassets/documents/digitalasset/dh_096231.pdf.

Chou, R. et al. 2009. Clinical guidelines for the use of chronic opioid therapy in chronic noncancer pain. The Journal of Pain 10(2), pp. 113-130. Available at: https://pubmed.ncbi.nlm.nih.gov/19187889.

Cicero, T.J. et al. 2007. Trends in the use and abuse of branded and generic extended-release oxycodone and fentanyl products in the United States. Drug and alcohol dependence 91(2-3), pp. 115-120. Available at: http://eutils.ncbi.nlm.nih.gov/entrez/eutils/elink.fcgi?dbfrom=pubmed\&id=17590285\&retmode= ref\&cmd=prlinks. 
Claxton, K. et al. 2015. Causes for concern: Is NICE failing to uphold its responsibilities to all NHS patients? Health Economics 24, pp. 1-7. Available at:

https://onlinelibrary.wiley.com/doi/pdfdirect/10.1002/hec.3130.

Clinical Practice Research Datalink (CPRD). 2021. Available at: https://www.cprd.com/ [Accessed: February 2021].

Coast, J. et al. 2008. Welfarism, extra-welfarism and capability: the spread of ideas in health economics. Social Science \& Medicine 67(7), pp. 1190-1198. Available at:

http://eutils.ncbi.nlm.nih.gov/entrez/eutils/elink.fcgi?dbfrom=pubmed\&id=18657346\&retmode= ref\&cmd=prlinks.

Cohen, S.P. et al. 2008. The Effect of Opioid Dose and Treatment Duration on the Perception of a Painful Standardized Clinical Stimulus. Regional anesthesia and pain medicine 33(3), pp. 199-206. Available at: https://rapm.bmj.com/lookup/doi/10.1016/j.rapm.2007.10.009.

Coll, M.-P. et al. 2012. The role of gender in the interaction between self-pain and the perception of pain in others. The Journal of Pain 13(7), pp. 695-703. Available at: https://linkinghub.elsevier.com/retrieve/pii/S1526590012006207.

Collett, B. et al. 2011. IASP Task force on wait times: Summary and recommendations. International Association for the Study of Pain. Available at: https://www.iasp-pain.org/wpcontent/uploads/2021/07/IASP-Recommendations-for-Wait-Times-for-Treatment-of-Pain.pdf.

Collett, B.J. 2001. Chronic opioid therapy for non-cancer pain. BJA: British Journal of Anaesthesia 87(1), pp. 133-143. doi: 10.1093/bja/87.1.133.

Colvin, L.A. et al. 2019. Postoperative pain management and opioids 3 Perioperative opioid analgesia-when is enough too much? A review of opioid-induced tolerance and hyperalgesia. The Lancet 393(10180), pp. 1558-1568. Available at: http://dx.doi.org/10.1016/S01406736(19)30430-1.

Compton, W.M. et al. 2015. Prescription opioid abuse: Problems and responses. Preventive Medicine 80, pp. 5-9. Available at:

https://linkinghub.elsevier.com/retrieve/pii/S0091743515001036.

Connelly, D. 2020. Some patients with chronic pain face waiting years to see a specialist - The Pharmaceutical Journal. The Pharmaceutical Journal February 2020. Available at: https://pharmaceutical-journal.com/article/news/some-patients-with-chronic-pain-face-waitingyears-to-see-a-specialist.

Cooney, M.A. et al. 2013. Current Knowledge of Pain After Breast Cancer Treatment: A Systematic Review. Pain Management Nursing 14(2), p. 110. Available at: https://doi.org/10.1016/j.pmn.2010.09.002.

Corran, T.M. et al. 1997. The classification of patients with chronic pain: age as a contributing factor. The Clinical Journal of Pain 13(3), pp. 207-214. Available at: http://journals.Iww.com/00002508-199709000-00005.

Coyle, D.T. et al. 2018. Opioid analgesic dose and the risk of misuse, overdose, and death: A narrative review. Pharmacoepidemiology and Drug Safety 27(5), pp. 464-472. Available at: http://eutils.ncbi.nlm.nih.gov/entrez/eutils/elink.fcgi?dbfrom=pubmed\&id=29243305\&retmode= ref\&cmd=prlinks. 
Crisp, R. et al. 2009. Work and worklessness in deprived neighbourhoods: Policy assumptions and personal experiences. Joseph Rowntree Foundation., p. 45. Available at: https://www.basw.co.uk/system/files/resources/basw 10124-1 0.pdf.

Cross, M. et al. 2014. The global burden of rheumatoid arthritis: estimates from the Global Burden of Disease 2010 study. Annals of the Rheumatic Diseases 73(7), pp. 1316-1322. doi: 10.1136/annrheumdis-2013-204627.

Currow, D.C. et al. 2016. Using opioids in general practice for chronic non-cancer pain: an overview of current evidence. The Medical Journal of Australia 204(8), pp. 1-7. Available at: http://dx.doi.org/10.5694/mja16<.00166.

Curtis, H.J. and Goldacre, B. 2018. OpenPrescribing: normalised data and software tool to research trends in English NHS primary care prescribing 1998-2016. BMJ open 8(2), pp. e01992110. Available at: https://bmjopen.bmj.com/lookup/doi/10.1136/bmjopen-2017-019921.

Curtis, H.J. et al. 2019. Opioid prescribing trends and geographical variation in England, 19982018: a retrospective database study. The Lancet Psychiatry 6(2), pp. 140-150. Available at: http://eutils.ncbi.nlm.nih.gov/entrez/eutils/elink.fcgi?dbfrom=pubmed\&id=30580987\&retmode= ref\&cmd=prlinks.

Curtis, L. and Burns, A. 2015. Unit Costs of Health and Social Care 2015. Canterbury: Personal Social Services Research Unit, University of Kent. Available at: https://www.pssru.ac.uk/pub/uc/uc2015/full.pdf.

Dance, A. 2019. THE PAIN GAP. Nature 567, pp. 448-450.

Daniels, J. et al. 2017. Better safe than sorry? Frequent attendance in a hospital emergency department: an exploratory study. British Journal of Pain 12(1), pp. 10-19. doi: $10.1177 / 2049463717720635$.

Dao, T.T. and LeResche, L. 2000. Gender differences in pain. Journal of Orofacial Pain 14(3), pp. 169-84-- discussion 184--95. Available at: http://eutils.ncbi.nlm.nih.gov/entrez/eutils/elink.fcgi?dbfrom=pubmed\&id=11203754\&retmode= ref\&cmd=prlinks.

Darnall, B.D. et al. 2012. Medical and Psychological Risks and Consequences of Long-Term Opioid Therapy in Women. Pain Medicine 13(9), pp. 1181-1211. Available at: https://academic.oup.com/painmedicine/article-pdf/13/9/1181/5276347/13-9-1181.pdf.

Dash, S. et al. 2019. Big data in healthcare: management, analysis and future prospects. Journal of Big Data 6(1), pp. 1-25. Available at: https://doi.org/10.1186/s40537-019-0217-0.

Datapharm Ltd 2021. Electronic Medicines Compendium. Available at: https://www.medicines.org.uk/emc [Accessed: 2021].

Davies, E. et al. 2018. Examining patterns in opioid prescribing for non-cancer related pain in wales: preliminary data from a retrospective cross-sectional study using large datasets. British Journal of Pain 13(3), pp. 145-158. doi: 10.1177/2049463718800737.

De Mauro, A. et al. 2016. A formal definition of Big Data based on its essential features. Library Review 65(3), pp. 122-135. Available at:

https://www.emerald.com/insight/content/doi/10.1108/LR-06-2015-0061/full/html. 
Degenhardt, L. et al. 2016. The extent and correlates of community-based pharmaceutical opioid utilisation in Australia. Pharmacoepidemiology and Drug Safety 25(5), pp. 521-538. Available at: http://eutils.ncbi.nlm.nih.gov/entrez/eutils/elink.fcgi?dbfrom=pubmed\&id=26781123\&retmode= ref\&cmd=prlinks.

Delamothe, T. 2008. Founding principles. British Medical Journal 336(7655), pp. 1216-1218. Available at: https://www.bmj.com/lookup/doi/10.1136/bmi.39582.501192.94.

Demos, K. et al. 2013. Does Farming Have an Effect on Health Status? A Comparison Study in West Greece. International Journal of Environmental Research and Public Health 10(3), pp. 776792. Available at: http://www.mdpi.com/1660-4601/10/3/776.

Department of Health 2012. Long Term Conditions Compendium: Third Edition. Department of Health., pp. 1-53. Available at: http://www.innspub.net/wp-content/uploads/2013/12/IJBV4No1-p158-166.pdf.

Department of Health 2021. The Misuse of Drugs and the Misuse of Drugs (Supply to Addicts) (Amendment) Regulations 2005. Available at:

http://webarchive.nationalarchives.gov.uk/+/www.dh.gov.uk/en/Healthcare/Medicinespharmac yandindustry/Prescriptions/DH 4123320.

Department of Health and Social Care. 2015. NHS reference costs 2014 to 2015. Available at: https://www.gov.uk/government/publications/nhs-reference-costs-2014-to-2015.

Desai, R.J. et al. 2015. Exposure to prescription opioid analgesics in utero and risk of neonatal abstinence syndrome: population based cohort study. BMJ 350(may14 1), p. h2102. Available at: https://www.bmj.com/content/350/bmj.h2102.

Devulder, J. et al. 2005. Impact of long-term use of opioids on quality of life in patients with chronic, non-malignant pain. Current medical research and opinion 21(10), pp. 1555-1568. Available at: https://www.tandfonline.com/doi/abs/10.1185/030079905X65321.

Dhalla, I.A. et al. 2011. Facing up to the prescription opioid crisis. BMJ 343(aug23 1), p. d5142. Available at: https://www.bmj.com/content/343/bmj.d5142.

Digital Health and Care Wales 2021. PEDW Data Online. Available at: https://dhcw.nhs.wales/information-services/welsh-data-hub/pedw-data-online/ [Accessed: 25 April 2020].

Dillie, K.S. et al. 2008. Quality of Life Associated with Daily Opioid Therapy in a Primary Care Chronic Pain Sample. Journal of American Board of Family Medicine 21(2), pp. 108-117. doi: 10.3122/jabfm.2008.02.070144.

Dobbin, M. and Liew, D.F. 2020. Real-time prescription monitoring: helping people at risk of harm. Australian Prescriber 43(5), pp. 164-167. Available at: https://www.nps.org.au/australianprescriber/articles/real-time-prescription-monitoring-helping-people-at-risk-of-harm.

Docking, R.E. et al. 2011. Epidemiology of back pain in older adults: prevalence and risk factors for back pain onset. Rheumatology (Oxford, England) 50(9), pp. 1645-1653. Available at: http://www.rheumatology.oxfordjournals.org/cgi/doi/10.1093/rheumatology/ker175.

Doidge, J. et al. 2020. Quality assessment in data linkage. Available at: https://www.gov.uk/government/publications/joined-up-data-in-government-the-future-ofdata-linking-methods/quality-assessment-in-data-linkage. 
Donovan, O. 2018. State of Wales. Available at: https://stateofwales.com/2018/10/the-welsheconomy-ii-an-economic-history-of-wales-in-brief/ [Accessed: 2 January 2021].

Dormuth, C.R. et al. 2012. Effect of a centralized prescription network on inappropriate prescriptions for opioid analgesics and benzodiazepines. Canadian Medical Association Journal 184(16), pp. E852-E856. Available at: https://www.cmaj.ca/content/184/16/E852.short.

Dowell, D. et al. 2016. CDC Guideline for Prescribing Opioids for Chronic Pain - United States, 2016. MMWR. Recommendations and Reports 65(1), pp. 1-49. doi: 10.15585/mmwr.rr6501e1er.

Dowell, D. et al. 2016. CDC guideline for prescribing opioids for chronic pain-United States, 2016. JAMA 315(15), pp. 1624-1645.

Dunlop, W. et al. 2012. Quality of life benefits and cost impact of prolonged release oxycodone/naloxone versus prolonged release oxycodone in patients with moderate-to-severe non-malignant pain and opioid-induced constipation: a UK cost-utility analysis. Journal of Medical Economics 15(3), pp. 564-575. Available at: https://doi.org/10.3111/13696998.2012.665279.

Dunn, K.M. et al. 2010. Overdose and prescribed opioids: Associations among chronic non-cancer pain patients. Annals of Internal Medicine 152(2), pp. 85-92. Available at: https://www.ncbi.nlm.nih.gov/pmc/articles/PMC3000551/pdf/ukmss-32216.pdf.

Dyer, C. 2004. Shipman inquiry recommends tighter rules on controlled drugs. BMJ 329(7459), p. 188. Available at: https://www.bmj.com/content/329/7459/188.3.

Dzierżanowski, T. and Ciałkowska-Rysz, A. 2017. Accessibility of opioid analgesics and barriers to optimal chronic pain treatment in Poland in 2000-2015. Supportive Care in Cancer 25(3), pp. 1-7. Available at: http://dx.doi.org/10.1007/s00520-016-3460-3.

EBM DataLab. 2017. OpenPrescribing.net 4.7.2: Opioid analgesics. Available at: https://openprescribing.net/bnf/040702/ [Accessed: 17 August 2021].

Eccleston, C. 2011. A normal psychology of chronic pain. Psychologist 24(6), pp. 422-425. Available at:

https://www.scopus.com/inward/record.uri?partnerID=HzOxMe3b\&scp=79959187272\&origin=i nward.

Eccleston, C. et al. 2021. Pragmatic but flawed: the NICE guideline on chronic pain. Lancet 397. Available at: www.thelancet.com.

Economics Observatory2020. How is coronavirus affecting the Welsh economy? - Economics Observatory. Available at: https://www.economicsobservatory.com/how-coronavirus-affectingwelsh-economy [Accessed: April 2021].

El-Ansary, M.S. and Galiongy, P. 1984. The history of pain relief by the ancient Egyptians. Pain 18(2), p. S34. doi: 10.1016/0304-3959(84)90184-2.

Elliott, A.M. et al. 2002. The course of chronic pain in the community: results of a 4-year followup study. Pain 99(1-2), pp. 299-307. Available at:

http://eutils.ncbi.nIm.nih.gov/entrez/eutils/elink.fcgi?dbfrom=pubmed\&id=12237208\&retmode= ref\&cmd=prlinks.

Els, C. et al. 2017. Adverse events associated with medium- and long-term use of opioids for chronic non-cancer pain: an overview of Cochrane Reviews. The Cochrane database of systematic reviews 10(10), p. CD012509. Available at: 
http://eutils.ncbi.nlm.nih.gov/entrez/eutils/elink.fcgi?dbfrom=pubmed\&id=29084357\&retmode= ref\&cmd=prlinks.

Engel, G.L. 1959. Psychogenic pain and pain-prone patient. The American journal of medicine 26(6), pp. 899-918. Available at: https://www.amjmed.com/article/0002-9343(59)90212-8/pdf.

Engel, G.L. 1977. The need for a new medical model: A challenge for biomedicine. Science 196(4286), pp. 1-9.

Enke, O. et al. 2018. Anticonvulsants in the treatment of low back pain and lumbar radicular pain: A systematic review and meta-analysis. Canadian Medical Association Journal 190(26), pp. E786E793. Available at:

http://eutils.ncbi.nlm.nih.gov/entrez/eutils/elink.fcgi?dbfrom=pubmed\&id=29970367\&retmode= $\underline{\text { ref\&cmd=prlinks. }}$

European Commission 2018. The EU Data Protection Reform and Big Data. Available at: https://op.europa.eu/en/publication-detail/-/publication/51fc3ba6-e601-11e7-974901aa75ed71a1.

European Monitoring Centre for Drugs and Drug Addiction. 2020. European Drug Report 2020: Trends and Developments. Luxembourg: Publications Office of the European Union. Available at: https://www.emcdda.europa.eu/system/files/publications/13236/TDAT20001ENN web.pdf.

Everdingen, M.H.J. van den B. et al. 2013. Prolonged QT interval by methadone: Relevance for daily practice? A prospective study in patients with cancer and noncancer pain. Journal of Opioid Management 9(4). doi: https://doi.org/10.5055/jom.2013.0167.

Expenditure Policy Division et al. 1998. IMF Conference on Economic Policy and Equity--Issues Paper.pdf. Washington, USA: International Monetary Fund. Available at:

https://www.imf.org/external/np/fad/equity/issues.htm.

Faculty of Pain Medicine of the Royal College of Anaesthetists 2021. Opioids Aware: A resource for patients and healthcare professionals to support prescribing of opioid medicines for pain. Available at: https://www.fpm.ac.uk/faculty-of-pain-medicine/opioids-aware [Accessed: 24 January 2021].

Faculty of Pain Medicine of the Royal College of Anaesthetists 2020. FPM guidance on resumption of pain services following disruption by the COVID-19 pandemic. Available at: https://fpm.ac.uk/covid-19-fpm-guidance-resumption-pain-services.

Faculty of Pain Medicine of the Royal College of Anaesthetists 2021. FPM concerns regarding new NICE Chronic Pain Guidelines. Available at: https://fpm.ac.uk/fpm-concerns-regarding-new-nicechronic-pain-guidelines. [Accessed: 3 May 2021].

Faculty of Pain Medicine of the Royal College of Anaesthetists 2021. Core Standards for Pain Management Services in the UK. London: RCoA. Available at:

https://fpm.ac.uk/sites/fpm/files/documents/2021-07/FPM-Core-Standards-2021 1.pdf.

Falk, M. et al. 2012. A first course on Time Series Analysis: Examples with SAS. Berlin: Druck und Verlag: epubli $\mathrm{GmbH}$. Available at:

http://books.google.co.uk/books?id=kTjQMRla0ssC\&printsec=frontcover\&dq=intitle:A+First+Cou rse+on+Time+Series+Analysis\&hl=\&cd=1\&source=gbs api [Accessed: 4 February 2020]. 
Fasick, V. et al. 2015. The hippocampus and TNF: Common links between chronic pain and depression. Neuroscience and biobehavioral reviews 53, pp. 139-159. Available at:

https://linkinghub.elsevier.com/retrieve/pii/S0149763415000883.

Favaretto, M. et al. 2020. What is your definition of Big Data? Researchers' understanding of the phenomenon of the decade. PloS one 15(2), pp. e0228987-20. Available at:

https://dx.plos.org/10.1371/journal.pone.0228987.

Fayaz, A. et al. 2016. Prevalence of chronic pain in the UK: a systematic review and meta-analysis of population studies. BMJ open 6(6), p. e010364. doi: 10.1136/bmjopen-2015-010364. Available at: https://bmjopen.bmj.com/content/6/6/e010364.abstract.

Fehrmann, E. et al. 2019. The impact of age and gender on the ICF-based assessment of chronic low back pain. Disability and Rehabilitation 41(10), pp. 1190-1199. Available at: https://doi.org/10.1080/09638288.2018.1424950.

Fetene, D.M. et al. 2020. The impact of Victoria's real time prescription monitoring system (SafeScript) on a cohort of people who inject drugs. The Medical Journal of Australia 213(3), pp. 141-2. Available at: https://onlinelibrary.wiley.com/doi/abs/10.5694/mja2.50659.

Field, A. 2014. Discovering Statistics using IBM SPSS Statistics 5e + SPSS 24. SAGE Publications, Incorporated. Available at:

http://books.google.co.uk/books?id=JQhSvQEACAAJ\&dq=inauthor:andy+field+discovering+statist ics\&hl=\&cd=7\&source=gbs api.

Fillingim, R. and Gear, R. (2004) "Sex differences in opioid analgesia: clinical and experimental findings," European Journal of Pain, 8(5), pp. 413-425. doi:10.1016/j.ejpain.2004.01.007. Available at: https://pubmed.ncbi.nlm.nih.gov/15324773/.

Fillingim, R.B. et al. 2005. Morphine responses and experimental pain: sex differences in side effects and cardiovascular responses but not analgesia. The Journal of Pain 6(2), pp. 116-124. Available at:

http://eutils.ncbi.nlm.nih.gov/entrez/eutils/elink.fcgi?dbfrom=pubmed\&id=15694878\&retmode= ref\&cmd=prlinks.

Finestone, H.M. et al. 2016. Opioid prescribing is a surrogate for inadequate pain management resources. Canadian Family Physician 62(6), pp. 465-468. Available at: http://www.cfp.ca/content/62/6/465.full.

Fink, D.S. et al. 2018. Association Between Prescription Drug Monitoring Programs and Nonfatal and Fatal Drug Overdoses. Annals of internal medicine 168(11), pp. 783-790. Available at: https://www.acpjournals.org/doi/10.7326/M17-3074.

Fischer, B. et al. 2014. Non-medical use of prescription opioids and prescription opioid-related harms: Why so markedly higher in North America compared to the rest of the world? Addiction (Abingdon, England) 109(2), pp. 177-181. Available at: http://doi.wiley.com/10.1111/add.12224.

Fischer, B. et al. 2014. Trends and changes in prescription opioid analgesic dispensing in Canada 2005-2012: an update with a focus on recent interventions. BMC Health Services Research 14(1), pp. 1-8.

Fischer, B. et al. 2016. Depression - A major but neglected consequence contributing to the health toll from prescription opioids? Psychiatry research 243, pp. 331-334. doi: 10.1016/j.psychres.2016.06.053. 
Fischer, L. et al. 2007. The protective role of testosterone in the development of temporomandibular joint pain. The Journal of Pain 8(5), pp. 437-442. Available at: http://eutils.ncbi.nlm.nih.gov/entrez/eutils/elink.fcgi?dbfrom=pubmed\&id=17360240\&retmode= ref\&cmd=prlinks.

Ford, D.V. et al. 2009. The SAIL Databank: building a national architecture for e-health research and evaluation. BMC Health Services Research 9, p. 157. doi: 10.1186/1472-6963-9-157.

Foy, R. et al. 2016. Prescribed opioids in primary care: cross-sectional and longitudinal analyses of influence of patient and practice characteristics. BMJ Open 6(5), p. e010276. Available at: https://bmjopen.bmj.com/content/6/5/e010276.

Francis, K. 2021. Personal Communication. Pain service provision data and plans for the future. August 2021.

Franklin, G.M. and American Association of Neurology 2014. Opioids for chronic noncancer pain: A position paper of the American Academy of Neurology. Neurology 83(14), pp. 1277-1284. doi: $10.1212 / \mathrm{wn} \mid<.0010000000000839$.

Franklin, G.M. et al. 2012. Bending the prescription opioid dosing and mortality curves: Impact of the Washington State opioid dosing guideline. American Journal of Industrial Medicine 55(4), pp. 325-331. doi: 10.1002/ajim.21998.

Fredheim, O.M.S. et al. 2010. Increasing use of opioids from 2004 to 2007 -

pharmacoepidemiological data from a complete national prescription database in Norway.

European Journal of Pain 14(3), pp. 289-294. Available at:

http://doi.wiley.com/10.1016/j.ejpain.2009.05.006.

Fullerton, E.F. et al. 2018. Impact of sex on pain and opioid analgesia: a review. Current opinion in behavioral sciences 23, pp. 183-190. Available at:

http://eutils.ncbi.nlm.nih.gov/entrez/eutils/elink.fcgi?dbfrom=pubmed\&id=30906823\&retmode= ref\&cmd=prlinks.

Furlan, A.D. et al. 2006. Opioids for chronic noncancer pain: a meta-analysis of effectiveness and side effects. Canadian Medical Association Journal 174(11), pp. 1589-1594. doi: 10.1503/cmaj.051528.

Furlan, A.D. et al. 2011. A Comparison between Enriched and Nonenriched Enrolment Randomized Withdrawal Trials of Opioids for Chronic Noncancer Pain. Pain Research and Management 16(5), pp. 337-351. doi: 10.1155/2011/465281.

Galama, T.J. and Kippersluis, H. van 2018. A Theory of Socio-economic Disparities in Health over the Life Cycle. The Economic Journal 129(617), pp. 338-374. Available at: https://doi.org/10.1111/ecoj.12577.

Gallagher, R.M. 2006. Empathy: A Timeless Skill for the Pain Medicine Toolbox. Pain Medicine 7(3), pp. 213-214. doi: 10.1111/j.1526-4637.2006.00163.x.

Gatchel, R.J. et al. 2007. The biopsychosocial approach to chronic pain: scientific advances and future directions. Psychological bulletin 133(4), pp. 581-624. Available at: http://eutils.ncbi.nlm.nih.gov/entrez/eutils/elink.fcgi?dbfrom=pubmed\&id=17592957\&retmode= ref\&cmd=prlinks. 
Gazelka, H.M. et al. 2020. Opioids in Older Adults: Indications, Prescribing, Complications, and Alternative Therapies for Primary Care. Mayo Clinic proceedings 95(4), pp. 793-800. Available at: https://linkinghub.elsevier.com/retrieve/pii/S0025619620301452.

Ghoshal, M. et al. 2020. Chronic Noncancer Pain Management and Systemic Racism: Time to Move Toward Equal Care Standards. Journal of pain research 13, pp. 2825-2836. Available at: https://www.dovepress.com/chronic-noncancer-pain-management-and-systemic-racism-timeto-move-tow-peer-reviewed-fulltext-article-JPR.

Gibson, S.J. and Farrell, M. 2004. A review of age differences in the neurophysiology of nociception and the perceptual experience of pain. The Clinical journal of pain 20(4), pp. 227239. Available at: http://journals.Iww.com/00002508-200407000-00004.

Gibson, S.J. and Helme, R.D. 2001. Age-related differences in pain perception and report. Clinics in geriatric medicine 17(3), pp. 433-456. Available at:

https://linkinghub.elsevier.com/retrieve/pii/S0749069005700793.

Gibson, S.J. and Lussier, D. 2012. Prevalence and relevance of pain in older persons. Pain medicine (Malden, Mass.) 13 Suppl 2(suppl 2), pp. S23-6. Available at: https://academic.oup.com/painmedicine/article-lookup/doi/10.1111/j.1526-4637.2012.01349.x.

Gifford, L.S. and Butler, D.S. 1997. The Integration of Pain Sciences in Clinical Practice. Journal of Hand Therapy 10(2), pp. 86-95. Available at: https://reader.elsevier.com/reader/sd/pii/S0894113097800634?token=29D597C6677F73C9DEE3 0A0B44ACB066573115BDFDEF6365046AA8FD69D4AA2909DE13DA3A40B6E6C576DD49EB6CCAB 6\&originRegion=eu-west-1\&originCreation=20210626102920.

Gilbert, S. et al. 2014. The Scottish model for chronic pain management services. British Journal of Healthcare Management 20(12), pp. 1-11.

Gisev, N. et al. 2016. Initiation of strong prescription opioids in Australia: cohort characteristics and factors associated with the type of opioid initiated. British Journal of Clinical Pharmacology 82(4), pp. 1-40. Available at: http://doi.wiley.com/10.1111/bcp.13026.

Glenn, S. 2021. Familywise Error Rate. Available at: https://www.statisticshowto.com/familywiseerror-rate/ [Accessed: 9 March 2020].

Gomes, T. et al. 2011. Opioid dose and drug-related mortality in patients with non-malignant pain. Archives of Internal Medicine 171(7), pp. 686-691. Available at:

http://eutils.ncbi.nIm.nih.gov/entrez/eutils/elink.fcgi?dbfrom=pubmed\&id=21482846\&retmode= ref\&cmd=prlinks.

Gomes, T. et al. 2014. Impact of legislation and a prescription monitoring program on the prevalence of potentially inappropriate prescriptions for monitored drugs in Ontario: a time series analysis. CMAJ open 2(4), pp. E256-61. Available at:

http://eutils.ncbi.nlm.nih.gov/entrez/eutils/elink.fcgi?dbfrom=pubmed\&id=25485251\&retmode= ref\&cmd=prlinks. doi: $10.9778 /$ cmajo.20140027

Gomes, T. et al. 2017. Changes in the dispensing of opioid medications in Canada following the introduction of a tamper-deterrent formulation of long-acting oxycodone: a time series analysis. CMAJ open 5(4), pp. E800-E807. Available at:

http://eutils.ncbi.nlm.nih.gov/entrez/eutils/elink.fcgi?dbfrom=pubmed\&id=29167237\&retmode= ref\&cmd=prlinks. 
González-Rold́an, A.M. et al. 2020. Age-Related Changes in Pain Perception Are Associated with Altered Functional Connectivity During Resting State. Frontiers in Aging Neuroscience 12, pp. 1110. Available at: https://www.frontiersin.org/article/10.3389/fnagi.202.00116/full.

Gordon, K. et al. 2017. Barriers to self-management of chronic pain in primary care: a qualitative focus group study. The British journal of general practice : the journal of the Royal College of General Practitioners 67(656), pp. e209-e217. Available at:

http://eutils.ncbi.nlm.nih.gov/entrez/eutils/elink.fcgi?dbfrom=pubmed\&id=27993899\&retmode= ref\&cmd=prlinks.

Gosport Independent Panel 2018. The Panel Report - 20th June 2018. Available at: https://www.gosportpanel.independent.gov.uk/panel-report/ [Accessed: 11 September 2020].

Green, K. et al. 2019. Prescribing trends of gabapentin, pregabalin, and oxycodone: a secondary analysis of primary care prescribing patterns in England. bjgpopen.org 3(3), p.

bjgpopen19X101662-10. doi: 10.3399/bjgpopen19x101662.

Greenfield, G. et al. 2016. Rethinking primary care's gatekeeper role. British Medical Journal 354, p. i4803. Available at: https://www.bmj.com/content/354/bmj.i4803.abstract.

Greenfield, G. et al. 2020. Frequent attendances at emergency departments in England. Emergency medicine journal : EMJ 37(10), pp. 597-599. doi: 10.1136/emermed-2018-208189.

Greenspan, J.D. et al. 2007. Studying sex and gender differences in pain and analgesia: A consensus report. PAIN 132(Supplement 1), pp. S26-S45. doi: 10.1016/j.pain.2007.10.014.

Grichnik, K.P. and Ferrante, F.M. 1991. The difference between acute and chronic pain. The Mount Sinai journal of medicine, New York 58(3), pp. 217-220. Available at:

http://eutils.ncbi.nlm.nih.gov/entrez/eutils/elink.fcgi?dbfrom=pubmed\&id=1875958\&retmode=r ef\&cmd=prlinks.

Groves, S. et al. 2010. Abolition of prescription charges in Wales: the impact on medicines use in those who used to pay. The International journal of pharmacy practice 18(6), pp. 332-340.

Available at:

http://eutils.ncbi.nlm.nih.gov/entrez/eutils/elink.fcgi?dbfrom=pubmed\&id=21054593\&retmode= ref\&cmd=prlinks.

Gulliford, M. 2020. Opioid use, chronic pain and deprivation. EClinicalMedicine 21, p. 100341. Available at: https://linkinghub.elsevier.com/retrieve/pii/S2589537020300857.

Gureje, O. et al. 1998. Persistent Pain and Well-being. JAMA 280(2), pp. 147-151. Available at: https://jamanetwork.com/journals/jama/articlepdf/187723/joc72070.pdf.

Gustavsson, A. et al. 2012. Pharmaceutical treatment patterns for patients with a diagnosis related to chronic pain initiating a slow-release strong opioid treatment in Sweden. Pain 153(12), pp. 2325-2331. Available at:

http://eutils.ncbi.nlm.nih.gov/entrez/eutils/elink.fcgi?dbfrom=pubmed\&id=22944610\&retmode= ref\&cmd=prlinks.

Gustavsson, A. et al. 2012. Socio-economic burden of patients with a diagnosis related to chronic pain - Register data of 840,000 Swedish patients. European Journal of Pain 16(2), pp. 289-299. Available at: http://doi.wiley.com/10.1016/j.ejpain.2011.07.006. 
Gyrd-Hansen, D. 2005. Willingness to pay for a QALY: theoretical and methodological issues. PharmacoEconomics 23(5), pp. 423-432. Available at:

http://link.springer.com/10.2165/00019053-200523050-00002.

Hamunen, K. et al. 2012. Trends in opioid consumption in the Nordic countries 2002-2006. European Journal of Pain 13(9), pp. 954-962. Available at: http://doi.wiley.com/10.1016/i.ejpain.2008.11.006.

Hanna, L. et al. 2020. "My life is under control with these medications": an interpretative phenomenological analysis of managing chronic pain with opioids. BMC Musculoskeletal Disorders 21(1), p. 61. Available at: https://doi.org/10.1186/s12891-020-3055-5.

Hanna, M. 2012. Pain in Europe: A Public Health Priority. Journal of pain \& palliative care pharmacotherapy 26(2), pp. 182-184 3p. Available at:

http://search.ebscohost.com/login.aspx?direct=true \&db=ccm\&AN=104471393\&site=ehostlive\&scope=site\&authtype=shib\&custid=s8000044.

Hansen, A.B. et al. 2015. Consumption of and satisfaction with healthcare among opioid users with chronic non-malignant pain. Acta anaesthesiologica Scandinavica 59(10), pp. 1355-1366. Available at:

http://eutils.ncbi.nIm.nih.gov/entrez/eutils/elink.fcgi?dbfrom=pubmed\&id=26113028\&retmode= ref\&cmd=prlinks.

Härdle, W. et al. 1997. A Review of Nonparametric Time Series Analysis. International Statistical Review 65(1), pp. 49-72. Available at: https://onlinelibrary.wiley.com/doi/full/10.1111/j.17515823.1997.tb00367.x.

Harrison, H. 2018. Personal Communication. Discussion about quality prescribing guidelines.

Harrison, H. and Cormack, J. 2018. Quality Prescribing for Chronic Pain: A guide for improvement 2018-2021. Available at: https://www.therapeutics.scot.nhs.uk/wpcontent/uploads/2018/03/Strategy-Chronic-Pain-Quality-Prescribing-for-Chronic-Pain-2018.pdf [Accessed: 4 June 2019].

Hart, J.T. 1971. The health of coal mining communities. Journal of the Royal College of General Practitioners 21, pp. 517-528.

Häuser, W. et al. 2021. European* clinical practice recommendations on opioids for chronic noncancer pain - Part 1: Role of opioids in the management of chronic noncancer pain. European Journal of Pain 25(5), pp. 949-968. doi: 10.1002/ejp.1736.

Hawkes, N. 2013. Deaths from tramadol and legal highs reach new highs in England and Wales. BMJ 347(aug30 2), pp. f5336-f5336. Available at:

http://www.bmj.com/cgi/doi/10.1136/bmj.f5336.

Hayhurst, C.J. et al. 2018. Pain and Its Long-term Interference of Daily Life After Critical Illness. Anesthesia and analgesia 127(3), pp. 690-697. Available at:

https://journals.Iww.com/anesthesia-

analgesia/Fulltext/2018/09000/Pain and Its Long term Interference of Daily Life.19.aspx.

Healthcare Improvement Scotland 2014. Chronic Pain Services in Scotland: Where are we now? Available at:

https://www.healthcareimprovementscotland.org/our work/long term conditions/chronic pai n/where are we now.aspx [Accessed: July 9, 2021]. 
Hebert, H.L. et al. 2021. Assessing the impact of SIGN 136 on opioid prescribing rates in Scotland: An interrupted time series analysis. medRxiv , p. 2021.02.19.21251770-18. Available at: https://www.medrxiv.org/content/10.1101/2021.02.19.21251770v1.

Heins, S.E. et al. 2018. High-Risk Prescribing to Medicaid Enrolees Receiving Opioid Analgesics: Individual- and County-Level Factors. Substance use \& misuse 53(10), pp. 1591-1601. Available at:

http://eutils.ncbi.nlm.nih.gov/entrez/eutils/elink.fcgi?dbfrom=pubmed\&id=29303393\&retmode= ref\&cmd=prlinks.

Helgason, I.S. 2008. Predicting Prescription Patterns. Available at: https://dspace.mit.edu/bitstream/handle/1721.1/43873/263428528MIT.pdf?sequence $=2 \&$ isAllowed $=y$

Henriksen, G. and Willoch, F. 2008. Imaging of opioid receptors in the central nervous system. Brain 131, pp. 1171-1196. Available at: https://www.ncbi.nlm.nih.gov/pmc/articles/PMC2367693/.

Henschke, N. et al. 2015. The epidemiology and economic consequences of pain. Mayo Clinic proceedings $90(1)$, pp. 139-147. Available at:

http://eutils.ncbi.nlm.nih.gov/entrez/eutils/elink.fcgi?dbfrom=pubmed\&id=25572198\&retmode= ref\&cmd=prlinks.

Hider-Mlynarz, K. et al. 2018. Trends in analgesic consumption in France over the last 10 years and comparison of patterns across Europe. British Journal of Clinical Pharmacology 84, pp. 13241334. Available at: https://bpspubs.onlinelibrary.wiley.com/doi/pdf/10.1111/bcp.13564.

Higgins, C. et al. 2019. Evidence of opioid-induced hyperalgesia in clinical populations after chronic opioid exposure: a systematic review and meta-analysis. British journal of anaesthesia 122(6), pp. e114-e126. Available at: https://doi.org/10.1016/j.bja.2018.09.019.

Hilderink, P.H. et al. 2012. The Temporal Relation Between Pain and Depression: Results from the Longitudinal Aging Study Amsterdam. Psychosomatic medicine 74(9), pp. 945-951. Available at: https://journals.Iww.com/psychosomaticmedicine/Fulltext/2012/11000/The Temporal Relation Between_Pain and Depression_.12.aspx.

Hoffman, K.M. et al. 2016. Racial bias in pain assessment and treatment recommendations, and false beliefs about biological differences between blacks and whites. Proceedings of the National Academy of Sciences of the United States of America 113(16), pp. 4296-4301. doi: 10.1073/pnas.1516047113.

Holloway, K. et al. 2018. Fatal and non-fatal overdose among opiate users in South Wales: A qualitative study of peer responses. The International journal on drug policy 56, pp. 56-63. Available at:

http://eutils.ncbi.nlm.nih.gov/entrez/eutils/elink.fcgi?dbfrom=pubmed\&id=29605706\&retmode= $\underline{\text { ref\&cmd=prlinks. }}$.

Home Office 2019. Drugs Misuse: Findings from the 2018/19 Crime Survey for England and Wales. Available at: https://www.gov.uk/government/statistics/drug-misuse-findings-from-the-2018-to2019-csew [Accessed: May 14, 2021].

Hong, J. et al. 2013. Costs associated with treatment of chronic low back pain: an analysis of the UK General Practice Research Database. Spine 38(1), pp. 75-82. Available at:

https://journals.Iww.com/spinejournal/Abstract/2013/01010/Costs Associated With Treatment of Chronic Low.13.aspx. 
Hong, L. et al. 2019. Big Data in Health Care: Applications and Challenges. Data and Information Management 2(3), pp. 175-197. Available at: https://content.sciendo.com/doi/10.2478/dim$\underline{2018-0014}$.

Hoy, D. et al. 2014. The global burden of low back pain: estimates from the Global Burden of Disease 2010 study. Annals of the Rheumatic Diseases 73(6), pp. 968-974. Available at: http://ard.bmj.com/cgi/doi/10.1136/annrheumdis-2013-204428.

Huang, A.R. and Mallet, L. 2013. Prescribing opioids in older people. Maturitas 74(2), pp. 123129. Available at: http://dx.doi.org/10.1016/j.maturitas.2012.11.002.

Hughes, O. 2018. Researchers say analysis of prescribing hampered by "poor quality" NHS data. Available at: https://www.digitalhealth.net/2018/08/researchers-say-prescribing-analysishampered-poor-quality-data/ [Accessed: 6 May 2021].

Hull, S. et al. 1998. The use and overlap of AED and general practice services by patients registered at two inner London general practices. British Journal of General Practice 46, pp. 1575-1579.

Hunt, K.A. et al. 2006. Characteristics of Frequent Users of Emergency Departments. Annals of emergency medicine 48(1), pp. 1-8. doi: 10.1016/j.annemergmed.2005.12.030.

Husain, M.J. et al. 2013. Linked routine data to enhance health-economics analysis. Farmeconomia. Health economics and therapeutic pathways 14(2), pp. 47-49. Available at: http://test-journals-3.1.2.seedmedicalpublishers.com/index.php/FE/article/view/677/769.

Husky, M.M. et al. 2018. Chronic back pain and its association with quality of life in a large French population survey. Health and Quality of Life Outcomes 16(1), pp. 1-9. doi: 10.1186/s12955-0181018-4.

IBM Corp. Released 2019. IBM SPSS Statistics for Windows, Version 26.0. Armonk, NY: IBM Corp.

Ikenberg, R. et al. 2012. Cost-effectiveness of tapentadol prolonged release compared with oxycodone controlled release in the UK in patients with severe non-malignant chronic pain who failed 1st line treatment with morphine. Journal of Medical Economics 15(4), pp. 724-736. Available at: https://doi.org/10.3111/13696998.2012.670174.

Information Commissioner 2021. Information Commissioners Office. Available at: ico.org.uk [Accessed: 6 February 2021].

Institute for Health Metrics and Evaluation. Available at: http://www.healthdata.org [Accessed: 3 April 2021].

International Association for the Study of Pain (IASP) 2019. Chronic Pain has arrived in the ICD-11. Available at: $\underline{\text { https://www.iasp-pain.org/PublicationsNews/NewsDetail.aspx? ItemNumber }=8340}$

International Narcotics Control Board 2016. Availability of Internationally Controlled Drugs: Ensuring Adequate Access for Medical and Scientific Purposes. Vienna: United Nations. Available at: https://www.unodc.org/documents/drug-prevention-andtreatment/INCB Access Supplement-AR15 availability English.pdf.

IQVIA 2020. Prescription opioid use in the US has declined by $60 \%$ from 2011 peak, according to new report from the IQVUA Institute for human data science. Available at: 
https://www.iqvia.com/newsroom/2020/12/prescription-opioid-use-in-the-us-has-declined-by60-from-2011-peak-according-to-new-report-from-the [Accessed: 9 April 2021].

Iroju, O.G. and Olaleke, J.O. 2015. A Systematic Review of Natural Language Processing in Healthcare. International Journal of Information Technology and Computer Science 7(8), pp. 4450. Available at: http://www.mecs-press.org/ijitcs/ijitcs-v7-n8/v7n8-7.html.

Islam, M. M., \& Wollersheim, D. (2019). A comparison of opioids and benzodiazepines dispensing in Australia. PloS One, 14(8), e0221438-13. https://doi.org/10.1371/journal.pone.0221438

Jain, N. et al. 2018. Chronic preoperative opioid use is a risk factor for increased complications, resource use, and costs after cervical fusion. The spine journal : official journal of the North American Spine Society 18(11), pp. 1989-1998. Available at: https://linkinghub.elsevier.com/retrieve/pii/S1529943018301268.

Janevic, M.R. et al. 2017. Racial and Socioeconomic Disparities in Disabling Chronic Pain: Findings from the Health and Retirement Study. The Journal of Pain 18(12), pp. 1459-1467. doi: 10.1016/j.jpain.2017.07.005.

Jani, M. et al. 2020. Time trends and prescribing patterns of opioid drugs in UK primary care patients with non-cancer pain: A retrospective cohort study. PLoS Medicine 17(10), pp. e1003270-16. doi: 10.1371/journal.pmed.1003270. doi: 10.1101/2020.04.07.20049015.

Jarlbaek, L. et al. 2005. Use of Opioids in a Danish Population-Based Cohort of Cancer Patients. Journal of Pain and Symptom Management 29(4), pp. 336-343. Available at: https://linkinghub.elsevier.com/retrieve/pii/S0885392405000540.

Jee, K. and Kim, G.-H. 2013. Potentiality of Big Data in the Medical Sector: Focus on How to Reshape the Healthcare System. Healthcare Informatics Research 19(2), pp. 79-8. Available at: http://e-hir.org/journal/view.php?id=10.4258/hir.2013.19.2.79.

Jennings, P.A. et al. 2009. Measuring acute pain in the prehospital setting. Emergency medicine journal : EMJ 26(8), pp. 552-555. Available at:

http://eutils.ncbi.nlm.nih.gov/entrez/eutils/elink.fcgi?dbfrom=pubmed\&id=19625547\&retmode= ref\&cmd=prlinks.

Jensen, M.K. et al. 2006. 10-year follow-up of chronic non-malignant pain patients: Opioid use, health related quality of life and health care utilization. European Journal of Pain 10(5), pp. 423433. Available at: https://www.sciencedirect.com/science/article/pii/S109038010500073X.

Jesulola, E. et al. 2018. Understanding the pathophysiology of depression: From monoamines to the neurogenesis hypothesis model - are we there yet? Behavioural Brain Research 341, pp. 7990. Available at:

https://www.sciencedirect.com/science/article/pii/S0166432817318521?via\%3Dihub.

Jimenez, N. et al. 2011. A review of the experience, epidemiology, and management of pain among American Indian, Alaska Native, and Aboriginal Canadian peoples. The Journal of Pain 12(5), pp. 511-522. Available at:

http://eutils.ncbi.nlm.nih.gov/entrez/eutils/elink.fcgi?dbfrom=pubmed\&id=21330217\&retmode= $\underline{\text { ref\&cmd=prlinks. }}$.

Johnson, Q. et al. 2013. A Review of Management of Acute Pain. MIssouri Medicine 110(1), pp. 74-79. 
Johnston, D.A. et al. 2019. The relationship between depression symptoms, absenteeism and presenteeism. Journal of Affective Disorders 256, pp. 536-540. Available at:

https://www.sciencedirect.com/science/article/pii/S016503271930028X.

Joint Formulary Committee. 2021. BNF: British National Formulary - NICE. Available at: https://bnf.nice.org.uk/guidance/controlled-drugs-and-drug-dependence.html.

Jones, C.A. et al. 2009. Health status and health care access of farm and rural populations. Available at: https://www.ers.usda.gov/webdocs/publications/44424/9371_eib57_1_.pdf.

Jones, K.F. et al. 2020. Exploring factors associated with long-term opioid therapy in cancer survivors: an integrative review. Journal of Pain and Symptom Management . doi: 10.1016/j.jpainsymman.2020.08.015.

Jones, K.H. et al. 2014. A case study of the Secure Anonymous Information Linkage (SAIL) Gateway: A privacy-protecting remote access system for health-related research and evaluation. Journal of Biomedical Informatics 50, pp. 196-204. Available at:

http://linkinghub.elsevier.com/retrieve/pii/S1532046414000045.

Joseph Rowntree Foundation 2013. Monitoring poverty and social exclusion 2013. Available at: https://www.jrf.org.uk/report/monitoring-poverty-and-social-exclusion-2013 [Accessed: 5 March 2020].

Joynt, M. et al. 2013. The Impact of Neighborhood Socioeconomic Status and Race on the Prescribing of Opioids in Emergency Departments Throughout the United States. Journal of General Internal Medicine 28(12), pp. 1604-1610. doi: 10.1007/s11606-013-2516-z.

Justins, D.M. 1996. Management strategies for chronic pain. Annals of the Rheumatic Diseases 55(9), p. 588. Available at: https://ard.bmj.com/content/annrheumdis/55/9/588.full.pdf.

Kaplow, L. and Shavell, S. 2001. kaplow-shavell_(jpe01).pdf. The Journal of Political Economy 109(2), pp. 281-286. Available at: http://links.jstor.org/sici?sici=00223808\%28200104\%29109\%3A2\%3C281\%3AANMOPA\%3E2.0.CO\%3B2-R.

Kapoor, S. and Thorn, B.E. 2014; Healthcare use and prescription of opioids in rural residents with pain. Rural and Remote Health. 14: 2879. https://doi.org/10.22605/RRH2879

Karanges, E.A. et al. 2016. Twenty-five years of prescription opioid use in Australia: a whole-ofpopulation analysis using pharmaceutical claims. British Journal of Clinical Pharmacology 82(1), pp. 255-267. doi: 10.1111/bcp.12937.

Katz, J.N. et al. 2016. Cost-effectiveness of nonsteroidal anti-inflammatory drugs and opioids in the treatment of knee osteoarthritis in older patients with multiple comorbidities. Osteoarthritis and Cartilage 24(3), pp. 409-418. doi: 10.1016/j.joca.2015.10.006.

Katz, N. and Mazer, N.A. 2009. The impact of opioids on the endocrine system. The Clinical journal of pain 25(2), pp. 170-175. Available at:

http://eutils.ncbi.nIm.nih.gov/entrez/eutils/elink.fcgi?dbfrom=pubmed\&id=19333165\&retmode= ref\&cmd=prlinks.

Kaufman, A.R. and Hersh, E.D. 2020. The political consequences of opioid overdoses. PloS one 15(8), p. e0236815. Available at:

http://eutils.ncbi.nlm.nih.gov/entrez/eutils/elink.fcgi?dbfrom=pubmed\&id=32750079\&retmode= ref\&cmd=prlinks. 
Kawulich, B. 2012. Selecting a research approach: paradigm, methodology and methods. In: Doing social research: A global context. McGraw-Hill Higher Education, pp. 51-61. Available at: https://www.researchgate.net/profile/Barbara-

Kawulich/publication/257944787 Selecting a research_approach Paradigm methodology and methods/links/56166fc308ae37cfe40910fc/Selecting-a-research-approach-Paradigmmethodology-and-methods.pdf [Accessed: 16 February 2021].

Kay, C. et al. 2017. Utilization of Healthcare Services and Ambulatory Resources Associated with Chronic Noncancer Pain. Pain Medicine 18(7), pp. 1236-1246. Available at: https://academic.oup.com/painmedicine/article-lookup/doi/10.1093/pm/pnw336.

Keefe, F.J. et al. 2000. The relationship of gender to pain, pain behavior, and disability in osteoarthritis patients: the role of catastrophizing. Pain 87(3), pp. 325-334. Available at: http://content.wkhealth.com/linkback/openurl?sid=WKPTLP:landingpage\&an=00006396200008020-00010.

Kenan, K. et al. 2012. Trends in prescriptions for oxycodone and other commonly used opioids in the United States, 2000-2010. Open Medicine 6(2), pp. 41-47-2547. doi:

10.2105/ajph.2009.180646.

Kern, D.M. et al. 2015. Treatment patterns, healthcare utilization, and costs of chronic opioid treatment for non-cancer pain in the United States. The American Journal of Managed Care 21(3), pp. e222-34. Available at: https://www.ajmc.com/view/treatment-patterns-healthcareutilization-and-costs-of-chronic-opioid-treatment-for-non-cancer-pain-in-the-united-states.

Keyes, K.M. et al. 2014. Understanding the rural-urban differences in nonmedical prescription opioid use and abuse in the United States. American Journal of Public Health 104(2), pp. e52-9. Available at: http://ajph.aphapublications.org/doi/10.2105/AJPH.2013.301709.

Khaldi, K. 2017. Quantitative, Qualitative or Mixed Research: Which Research Paradigm to Use? Journal of Educational and Social Research 7(2), pp. 15-24. doi: 10.5901/jesr.2017.v7n2p15.

Kinnaird, E. et al. 2019. From pain treatment to opioid dependence: a qualitative study of the environmental influence on codeine use in UK adults. BMJ open 9(4), pp. e025331-10. Available at: https://bmjopen.bmj.com/lookup/doi/10.1136/bmjopen-2018-025331.

Kluwer, W. 2021. Morphine Equivalent Dosing. Available at:

https://www.wolterskluwercdi.com/sites/default/files/documents/ebooks/morphine-equivalentdosing-ebook.pdf?v3 [Accessed: 5 September 2021].

Knights, D. et al. 2018. 'Please make comfortable': prescribing opioids in the wake of Gosport. British Journal of General Practice 68(675), pp. 462-463. Available at: https://bigp.org/content/68/675/462.

Kolodny, A. and Frieden, T.R. 2017. Ten Steps the Federal Government Should Take Now to Reverse the Opioid Addiction Epidemic. JAMA 318(16), pp. 1537-1538. Available at: http://eutils.ncbi.nlm.nih.gov/entrez/eutils/elink.fcgi?dbfrom=pubmed\&id=29049522\&retmode= ref\&cmd=prlinks.

Korff, M.V. et al. 2008. De Facto Long-term Opioid Therapy for Noncancer Pain. The Clinical Journal of Pain 24(6), pp. 521-527. doi: 10.1097/ajp.0b013e318169d03b. 
Kotecha, M.K. and Sites, B.D. 2013. Pain policy and abuse of prescription opioids in the USA: a cautionary tale for Europe. Anaesthesia 68(12), pp. 1210-1215. Available at:

http://doi.wiley.com/10.1111/anae.12450.

Krebs, E.E. et al. 2018. Effect of Opioid vs Nonopioid Medications on Pain-Related Function in Patients with Chronic Back Pain or Hip or Knee Osteoarthritis Pain: The SPACE Randomized Clinical Trial. JAMA 319(9), pp. 872-882. Available at:

http://eutils.ncbi.nIm.nih.gov/entrez/eutils/elink.fcgi?dbfrom=pubmed\&id=29509867\&retmode= ref\&cmd=prlinks.

Kruse, C.S. et al. 2016. Challenges and Opportunities of Big Data in Health Care: A Systematic Review. JMIR medical informatics 4(4), p. e38. Available at: http://eutils.ncbi.nIm.nih.gov/entrez/eutils/elink.fcgi?dbfrom=pubmed\&id=27872036\&retmode= ref\&cmd=prlinks.

Kurani, S. et al. 2020. Place, poverty and prescriptions: a cross-sectional study using Area Deprivation Index to assess opioid use and drug-poisoning mortality in the USA from 2012 to 2017. BMJ open 10(5), pp. e035376-7. doi: 10.1136/bmjopen-2019-035376.

Lachance, C. and Frey, N. 2019. Prescription Monitoring Programs for Optimizing Medication Use and Preventing Harm. Available at: http://books.google.co.uk/books?id=q85dzQEACAAJ\&dq=prescription+monitoring\&hl=\&cd=3\&so urce=gbs api.

Lalic, S. et al. 2018. Predictors of persistent prescription opioid analgesic use among people without cancer in Australia. British Journal of Clinical Pharmacology 84(6), pp. 1267-1278. doi: 10.1111/bcp.13556.

Lambeek, L.C. et al. 2011. The trend in total cost of back pain in The Netherlands in the period 2002 to 2007. Spine 36(13), pp. 1050-1058. Available at:

http://eutils.ncbi.nIm.nih.gov/entrez/eutils/elink.fcgi?dbfrom=pubmed\&id=21150697\&retmode= ref\&cmd=prlinks.

Laney, D. (2001) 3D Data Management: Controlling Data Volume, Velocity and Variety. META Group Research Note, 6. Available at: http://blogs.gartner.com/doug-laney/files/2012/01/ad9493D-Data-Management-Controlling-Data-Volume-Velocity-and-Variety.pdf

Lange, L.L. and Jacox, A. 1993. Using large data bases in nursing and health policy research. Journal of professional nursing : official journal of the American Association of Colleges of Nursing 9(4), pp. 204-211. Available at: https://linkinghub.elsevier.com/retrieve/pii/875572239390037D.

Large, R.G. and Schug, S.A. 1995. Opioids for chronic pain of non-malignant origin-Caring or crippling. Healthcare Analysis 3(1), p. 5. Available at: https://doi.org/10.1007/BF02197187.

Larney, S. et al. 2018. Using routinely collected data to understand and predict adverse outcomes in opioid agonist treatment: Protocol for the Opioid Agonist Treatment Safety (OATS) Study. BMJ open 8(8), pp. e025204-6. Available at: http://bmjopen.bmj.com/lookup/doi/10.1136/bmjopen2018-025204.

Latham, J. and Davis, B.D. 1994. The socioeconomic impact of chronic pain. Disability and rehabilitation 16(1), pp. 39-44. Available at:

http://eutils.ncbi.nIm.nih.gov/entrez/eutils/elink.fcgi?dbfrom=pubmed\&id=8136509\&retmode=r ef\&cmd=prlinks. 
Lautenbacher, S. et al. 2017. Age changes in pain perception: A systematic-review and metaanalysis of age effects on pain and tolerance thresholds. Neuroscience and biobehavioral reviews 75, pp. 104-113. Available at: http://dx.doi.org/10.1016/j.neubiorev.2017.01.039.

Lavrakas, P. 2008. Encyclopedia of Survey Research Methods. doi: 10.4135/9781412963947.

Lawton, S. and Miles, G. 2019. Occupational skin and lung disease in coalfield communities. Nursing Times 115(7), pp. 58-60. Available at: https://insights.ovid.com/nursingtimes/nrtm/2019/07/000/occupational-skin-lung-disease-coalfield/44/00006203.

Lee, M. et al. 2011. A comprehensive review of opioid-induced hyperalgesia. Pain Physician 14, pp. 145-161. Available at: https://www.painphysicianjournal.com/current/pdf?article=MTQ0Ng\%3D\%3D\&journal=60.

legislation.gov.uk and UK Government 2021. Misuse of Drugs Regulations 2001. Available at: https://www.legislation.gov.uk/uksi/2001/3998/schedule/1/made [Accessed: 5 September 2021].

LeResche, L. et al. 2005. Relationship of pain and symptoms to pubertal development in adolescents. Pain 118(1-2), pp. 201-209. doi: 10.1016/j.pain.2005.08.011.

Levy, B. et al. 2015. Trends in Opioid Analgesic-Prescribing Rates by Specialty, U.S., 2007-2012. American Journal of Preventive Medicine 49(3), pp. 409-413. doi: 10.1016/j.amepre.2015.02.020.

Levy, N. et al. 2021. An international multidisciplinary consensus statement on the prevention of opioid-related harm in adult surgical patients. Available at:

http://eutils.ncbi.nlm.nih.gov/entrez/eutils/elink.fcgi?dbfrom=pubmed\&id=33027841\&retmode= ref\&cmd=prlinks [Accessed: 1 June 2021].

Lewer, D. et al. 2020. Frequency of health-care utilization by adults who use illicit drugs: a systematic review and meta-analysis. Addiction (Abingdon, England) 115(6), pp. 1011-1023. Available at:

http://eutils.ncbi.nlm.nih.gov/entrez/eutils/elink.fcgi?dbfrom=pubmed\&id=31705770\&retmode= ref\&cmd=prlinks.

Liberman, J.S. et al. 2019. Opioid prescriptions at hospital discharge are associated with more post discharge healthcare utilization. Am Heart Assoc 8(3), p. e010664. Available at: https://www.ahajournals.org/doi/abs/10.1161/jaha.118.010664.

Liddell, D. 2019. Poverty is the root cause of Scotland's fatal drug overdose crisis. Available at: https://www.povertyalliance.org/blog-poverty-is-the-root-of-scotlands-fatal-drug-overdosecrisis/.

Lin, D.H. et al. 2017. Financial Conflicts of Interest and the Centers for Disease Control and Prevention's 2016 Guideline for Prescribing Opioids for Chronic Pain. JAMA Internal Medicine 177(3), p. 427. doi: 10.1001/jamainternmed.2016.8471.

Loudon, I. 2008. The principle of referral: the gatekeeping role of the GP. The British journal of general practice : the journal of the Royal College of General Practitioners 58(547), pp. 128-30-discussion 130. Available at: https://bjgp.org/content/58/547/128.abstract.

Loyland, B. 2016. The co-occurrence of chronic pain and psychological distress and its associations with salient socio-demographic characteristics among long-term social assistance 
recipients in Norway. Scandinavian Journal of Pain 11(1), pp. 65-72. Available at:

https://www.degruyter.com/view/journals/sjpain/11/1/article-p65.xml.

Lozano-Ondoua, A. et al. 2013. Mechanisms of cancer-induced bone pain. Neuroscience Letters 0 0(557), pp. 52-59. Available at:

https://www.ncbi.nlm.nih.gov/pmc/articles/PMC4542064/pdf/nihms527830.pdf.

Lumley, T. et al. 2002. The importance of the normality assumption in large public health data sets. Annual review of public health 23(1), pp. 151-169. Available at:

http://eutils.ncbi.nlm.nih.gov/entrez/eutils/elink.fcgi?dbfrom=pubmed\&id=11910059\&retmode= ref\&cmd=prlinks.

Lusa, S. et al. 2015. Sleep disturbances predict long-term changes in low back pain among Finnish firefighters: 13-year follow-up study. International Archives of Occupational and Environmental Health 88(3), pp. 369-379. Available at: https://doi.org/10.1007/s00420-014-0968-z.

Lutz, P.-E. and Kieffer, B.L. 2013. The multiple facets of opioid receptor function: implications for addiction. Current Opinion in Neurobiology 23(4), pp. 473-479. doi:

https://doi.org/10.1016/j.conb.2013.02.005.

Lynch, M.E. et al. 2008. A systematic review of the effect of waiting for treatment for chronic pain. Pain 136(1-2), pp. 97-116. Available at:

https://journals.Iww.com/pain/Fulltext/2008/05000/A systematic review of the effect of wai ting for.14.aspx.

Lyons, R.A. et al. 2009. The SAIL databank: linking multiple health and social care datasets. BMC Medical Informatics and Decision Making 9(1), p. 3. Available at:

http://bmcmedinformdecismak.biomedcentral.com/articles/10.1186/1472-6947-9-3.

Ma, C. et al. 2015. Big data in pharmacy practice: current use, challenges, and the future.

Integrated Pharmacy Research and Practice Volume 4, pp. 91-9. Available at:

http://www.dovepress.com/big-data-in-pharmacy-practice-current-use-challenges-and-the-

future-peer-reviewed-article-IPRP.

Macfarlane, G.J. et al. 2009. The influence of socioeconomic status on the reporting of regional and widespread musculoskeletal pain: results from the 1958 British Birth Cohort Study. Annals of the Rheumatic Diseases 68(10), pp. 1591-1595. doi: 10.1136/ard.2008.093088.

Macfarlane, G.J. et al. 2020. The epidemiology of regular opioid use and its association with mortality: Prospective cohort study of 466486 UK biobank participants., pp. 1-8. doi:

10.1016/j.eclinm.2020.100321.

Mackes, N.K. et al. 2020. Early childhood deprivation is associated with alterations in adult brain structure despite subsequent environmental enrichment. ["Sonuga-Barke et al. eds. Proceedings of the National Academy of Sciences 117(1), pp. 641-649. Available at: https://www.pnas.org/content/117/1/641.

Mackey, M.-C.K.S. 2019. Managing twin crises in chronic pain and prescription opioids. BMJ 364, pp. 1-2. Available at: http://dx.doi.org/doi:10.1136/bmj.1917.

MacQueen, I.T. et al. 2018. Recruiting rural healthcare providers today: a systematic review of training program success and determinants of geographic choices. Journal of General and Internal Medicine 33(2), pp. 191-199. Available at:

https://www.ncbi.nlm.nih.gov/pmc/articles/PMC5789104/pdf/11606 2017 Article 4210.pdf. 
Maniadakis, N. and Gray, A. 2000. The economic burden of back pain in the UK. Pain 84(1), pp. 95-103. Available at:

http://search.ebscohost.com/login.aspx?direct=true\&db=ccm\&AN=104718901\&site=ehostlive\&scope=site \&authtype=shib\&custid=s8000044.

Mann, E.G., et al. 2016. Frequency and characteristics of healthcare visits associated with chronic pain: results from a population-based Canadian study. Canadian Journal of Anesthesiology 63, pp. 411-441. Available at: https://link.springer.com/content/pdf/10.1007/s12630-015-0578-6.pdf.

Marks, J.H. 2020. Lessons from Corporate Influence in the Opioid Epidemic: Toward a Norm of Separation. Journal of Bioethical Inquiry 17(2), pp. 1-17. doi: 10.1007/s11673-020-09982-x.

Marmot, M. 2005. Social determinants of health inequalities. The Lancet 365(9464), pp. 10991104. Available at: https://www.sciencedirect.com/science/article/pii/S0140673605711466.

Marmot, M. 2017. Closing the health gap. Scandinavian journal of public health 45(7), pp. 723731. Available at:

http://eutils.ncbi.nlm.nih.gov/entrez/eutils/elink.fcgi?dbfrom=pubmed\&id=29162013\&retmode= ref\&cmd=prlinks.

Marmot, M. and Bell, R. 2012. Fair society, healthy lives. Public Health 126, pp. S4-S10. Available at: https://www.sciencedirect.com/science/article/pii/S0033350612001862.

Marouf, R. et al. 2014. Reduced pain inhibition is associated with reduced cognitive inhibition in healthy aging. Pain 155(3), pp. 494-502. Available at:

http://dx.doi.org/10.1016/j.pain.2013.11.011.

Marsh, S. 2017. "Unnecessary" painkillers could leave thousands addicted, doctors warn. The Guardian . Available at: http://www.theguardian.com/science/2017/may/05/unnecessaryopioid-painkiller-prescriptions-thousands-addicted-nhs-doctors-warn.

Marsh, S. 2021. "I was told to live with it": women tell of doctors dismissing their pain. The Guardian 16 April. Available at: https://www.theguardian.com/society/2021/apr/16/painkillerswomen-tell-of-doctors-dismissing-their-pain.

Martin, J. et al. 2018. Effect of restricting the legal supply of prescription opioids on buying through online illicit marketplaces: Interrupted time series analysis. BMJ 361, p. k2270. Available at:

http://eutils.ncbi.nlm.nih.gov/entrez/eutils/elink.fcgi?dbfrom=pubmed\&id=29899119\&retmode= ref\&cmd=prlinks.

Martinez, C. 2018. Cracking the Code: Using Data to Combat the Opioid Crisis. The Journal of law, medicine \& ethics : a journal of the American Society of Law, Medicine I\& Ethics 46(2), pp. 454471. Available at:

http://eutils.ncbi.nlm.nih.gov/entrez/eutils/elink.fcgi?dbfrom=pubmed\&id=30146995\&retmode= ref\&cmd=prlinks.

Matthias, M.S. 2020. Opioid Tapering and the Patient-Provider Relationship. Journal of General Internal Medicine 35(1), pp. 8-9. doi: 10.1007/s11606-019-05337-4.

Matthias, M.S. et al. 2013. "I'm not abusing or anything": patient-physician communication about opioid treatment in chronic pain. Patient Education and Counseling 93(2), pp. 197-202. doi: 10.1016/j.pec.2013.06.021. 
Matthias, M.S. et al. 2014. Communicating about opioids for chronic pain: a qualitative study of patient attributions and the influence of the patient-physician relationship. European Journal of Pain 18(6), pp. 835-843. Available at: https://onlinelibrary.wiley.com/doi/full/10.1002/j.15322149.2013.00426.x.

Mauro, A.D. et al. 2016. A formal definition of Big Data based on its essential features. Library Review 65(3), pp. 122-135. Available at:

https://www.emerald.com/insight/content/doi/10.1108/LR-06-2015-0061/full/html.

Mazereeuw, G. et al. 2018. Depression in chronic pain: might opioids be responsible? Pain 159(11), pp. 2142-2145. doi: 10.1097/j.pain<.0010000000001305.

Mazure, C.M. and Fiellin, D.A. 2018. Women and opioids: something different is happening here. The Lancet 392(10141), pp. 9-11. Available at: http://dx.doi.org/10.1016/S0140-6736(18)31203$\underline{0}$.

McAreavey, R. and Brown, D.L. 2019. Comparative analysis of rural poverty and inequality in the UK and the US. Palgrave Communications 5(1), pp. 1-10. Available at:

http://dx.doi.org/10.1057/s41599-019-0332-8.

McCormick, B. et al. 2012. Are hospital services used differently in deprived areas? Evidence to identify commissioning challenges. Working Paper No 2. Oxford: Centre for Health Service Economics and Organisation . Available at: https://www.chseo.org.uk/downloads/wp2hospitalservices-deprivedareas.pdf.

McCrorie, C. et al. 2015. Understanding long-term opioid prescribing for non-cancer pain in primary care: a qualitative study. BMC Family Practice 16(1), pp. 1-9. Available at: http://dx.doi.org/10.1186/s12875-015-0335-5.

McEwen, J. 2004. Chronic Pain Services in Scotland: Report by Professor James McEwen. Edinburgh: Scottish Executive Health Department., pp. 1-25. Available at: https://www.sehd.scot.nhs.uk/mels/hdl2004 48.pdf.

McGorm, K. et al. 2010. Patients repeatedly referred to secondary care with symptoms unexplained by organic disease: prevalence, characteristics and referral pattern. Family practice 27(5), pp. 479-486. doi: 10.1093/fampra/cmq053.

McGreal, C., 2018. American Overdose, The Opioid Tragedy in Three Acts. Guardian Faber Publishing, London. ISBN: 1783351683

McIntosh, T. et al. 2016. Influences on prescribing decision-making among non-medical prescribers in the United Kingdom: systematic review. Family practice 33(6), pp. 572-579. Available at:

http://eutils.ncbi.nlm.nih.gov/entrez/eutils/elink.fcgi?dbfrom=pubmed\&id=27543795\&retmode= ref\&cmd=prlinks.

McLaughlin, K.A. et al. 2014. Childhood adversity and neural development: Deprivation and threat as distinct dimensions of early experience. Neuroscience \& Biobehavioral Reviews 47, pp. 578-591. doi: 10.1016/j.neubiorev.2014.10.012.

McMillan, M. et al. 2015. Prevalence of Musculoskeletal Disorders Among Saskatchewan Farmers. Journal of Agromedicine 20(3), pp. 292-301. Available at: https://doi.org/10.1080/1059924X.2015.1042611. 
Medicines and Healthcare products Regulatory Agency 2021. Yellow Card. Available at: https://www.gov.uk/report-problem-medicine-medical-device [Accessed: 15 June 2021].

Mehra, M. et al. 2011. The burden of chronic low back pain with and without a neuropathic component: a healthcare resource use and cost analysis. Journal of Medical Economics 15(2), pp. 245-252. doi: 10.3111/13696998.2011.642090.

Mehta, N. and Pandit, A. 2018. Concurrence of big data analytics and healthcare: A systematic review. International journal of medical informatics 114(1), pp. 57-65. Available at: https://linkinghub.elsevier.com/retrieve/pii/S1386505618302466.

Meints, S.M. and Edwards, R.R. 2018. Evaluating psychosocial contributions to chronic pain outcomes. Progress in Neuropsychopharmacology \& Biological Psychiatry 87(Part B), pp. 168182. Available at: https://doi.org/10.1016/j.pnpbp.2018.01.017.

Meldrum, M.L. 2003. A capsule history of pain management. JAMA 290(18), pp. 2470-5. Available at: https://pubmed.ncbi.nIm.nih.gov/14612484/.

Mellor, R. and Chronic Pain Policy Group 2018. Scottish Public Health Network (ScotPHN) report: Health Care Needs Assessment of Adult Chronic Pain Services in Scotland. Glasgow: Scottish Public Health Network. Available at: $\underline{\text { www.scotphn.net. }}$

Melzack, R. and Wall, P.D. 1965. Pain mechanisms: a new theory. Science 150(3699), pp. 971979. Available at:

http://scholar.google.com/scholar?q=related:pYHDAy030AAJ:scholar.google.com/\&hl=en\&num= 20\&as sdt $=0,5$.

Menchetti, M. et al. 2009. Recognition and treatment of depression in primary care: effect of patients' presentation and frequency of consultation. 66(4), pp. 335-341. doi: 10.1016/j.jpsychores.2008.10.008.

Mercadante, S. et al. 2019. Opioid-Induced Tolerance and Hyperalgesia. CNS Drugs 33(10), pp. 943-955. doi: 10.1007/s40263-019-00660-0.

Merrill, T. and Kitson, L. 2017. End of Coal Mining in South Wales: Lessons learned from industrial transformation. Geneva: International Institute for Sustainable Development. Available at: https://www.iisd.org/system/files/publications/end-of-coal-mining-south-wales-lessonslearned.pdf.

Merriwether, E.N. et al. 2018. Physical activity is related to function and fatigue but not pain in women with fibromyalgia: baseline analyses from the Fibromyalgia Activity Study with TENS (FAST). Arthritis research \& therapy 20(1), pp. 199-13. Available at: https://arthritisresearch.biomedcentral.com/articles/10.1186/s13075-018-1671-3.

Meyer, A. et al. 2020. Prescription Opioid Prescribing in Western Europe and the United States. Rhode Island medical journal (2013) 103(2), pp. 45-48. Available at: http://www.rimed.org/rimedicaljournal/2020/03/2020-03-45-contribution-meyer.pdf.

Michael, P.F. 2008. Public Health in Wales (1800-2000): A brief history. Available at: http://scholar.google.comjavascript:void(0).

Microsoft Corporation 2018. Microsoft Excel. Available at: $\underline{\text { https://office.microsoft.com/excel }}$ [Accessed: 8 January 2021]. 
Mills, S. et al. 2016. Identification and Management of Chronic Pain in Primary Care: a Review. Current Psychiatry Reports 18(2), pp. 1-9. Available at:

http://eutils.ncbi.nlm.nih.gov/entrez/eutils/elink.fcgi?dbfrom=pubmed\&id=26820898\&retmode= ref\&cmd=prlinks.

Ministry of Housing 2019. English indices of deprivation 2019. Available at: https://www.gov.uk/government/statistics/english-indices-of-deprivation-2019 [Accessed: 7 September 2018].

Mogil, J.S. and Chanda, M.L. 2005. The case for the inclusion of female subjects in basic science studies of pain. Pain 117(1-2), pp. 1-5. doi: 10.1016/j.pain.2005.06.020.

Molton, I.R. and Terrill, A.L. 2014. Overview of persistent pain in older adults. American Psychologist 69(2), pp. 197-207. Available at: http://doi.apa.org/getdoi.cfm?doi=10.1037/a0035794.

Moore, F.R. et al. 2020. Sociodemographic predictors of attendance at a Scottish pain management programme. British Journal of Pain , pp. 1-8. Available at: https://doi.org/10.1177/2049463720970579.

Moore, L. et al. 2009. Characteristics of frequent attenders in an emergency department: analysis of 1-year attendance data. Emergency medicine journal : EMJ 26(4), pp. 263-267. doi: 10.1136/emj.2008.059428.

Mordecai, L. et al. 2018. Patterns of regional variation of opioid prescribing in primary care in England: a retrospective observational study. The British journal of general practice : the journal of the Royal College of General Practitioners 68(668), pp. e225-e233. doi: 10.3399/bjgp18x695057. Available at: https://bjgp.org/content/68/668/e225.abstract.

Morgan, C.L. et al. 2011. The relationship between self-reported severe pain and measures of socio-economic disadvantage. European journal of pain (London, England) 15(10), pp. 11071111. doi: 10.1016/j.ejpain.2011.04.010.

Morley, K.I. et al. 2017. Polysubstance use and misuse or abuse of prescription opioid analgesics: a multi-level analysis of international data. Pain 158(6), pp. 1138-1144. Available at: http://Insights.ovid.com/crossref?an=00006396-201706000-00020.

Morriss, R. et al. 2012. Persistent frequent attenders in primary care: costs, reasons for attendance, organisation of care and potential for cognitive behavioural therapeutic intervention. BMC Family Practice 13(1), pp. 39-14. doi: 10.1186/1471-2296-13-39.

Moseley, G.L. 2007. Reconceptualising pain according to modern pain science. Physical Therapy Reviews 12(3), pp. 169-178. Available at: https://doi.org/10.1179/108331907X223010.

Moseley, L.G. and Vlaeyen, J.W. 2015. Beyond nociception: the imprecision hypothesis of chronic pain. Pain 156(1), pp. 35-38. Available at: https://journals.Iww.com/pain/Citation/2015/01000/Beyond nociception the imprecision hy pothesis of.7.aspx.

Mulvihill, G. et al. 2016. Drugmakers fight state opioid limits amid crisis. Kitsap Sun 18 September, p. 7A. Available at: http://web.kitsapsun.com/webdot/PDFArchive/0918A007BCKS1.PDF. 
Mundkur, M.L. et al. 2017. Will strict limits on opioid prescription duration prevent addiction? Advocating for evidence-based policymaking. Substance Abuse 38(3), pp. 237-238. Available at: http://eutils.ncbi.nlm.nih.gov/entrez/eutils/elink.fcgi?dbfrom=pubmed\&id=28632485\&retmode= ref\&cmd=prlinks.

Mundkur, M.L. et al. 2018. Patterns of opioid initiation at first visits for pain in United States primary care settings. Pharmacoepidemiology and Drug Safety 27(5), pp. 495-503. Available at: http://eutils.ncbi.nlm.nih.gov/entrez/eutils/elink.fcgi?dbfrom=pubmed\&id=28971545\&retmode= ref\&cmd=prlinks.

Murshid, M.A. and Mohaidin, Z. 2017. Models and theories of prescribing decisions: A review and suggested a new model. Pharmacy practice 15(2), p. 990. Available at:

http://eutils.ncbi.nlm.nih.gov/entrez/eutils/elink.fcgi?dbfrom=pubmed\&id=28690701\&retmode= ref\&cmd=prlinks.

Musazzi, U.M. et al. 2018. Do laws impact opioids consumption? A breakpoint analysis based on Italian sales data. Journal of pain research 11, pp. 1665-1672. Available at:

http://eutils.ncbi.nlm.nih.gov/entrez/eutils/elink.fcgi?dbfrom=pubmed\&id=30214276\&retmode= ref\&cmd=prlinks.

National Assembly Government 2010. Helping to improve Wales' health: free prescriptions three years on. Available at: http://www.wales.nhs.uk/documents/prescriptions-report-threeyears.pdf.

National Audit of Cardiac Rehabilitation. 2021. Available at: https://digital.nhs.uk/data-andinformation/clinical-audits-and-registries/national-audit-of-cardiac-rehabilitation [Accessed: 9 February 2021].

National Collaborating Centre for Chronic Conditions 2008. Osteoarthritis: National clinical guidelines for care and management in adults. Available at:

https://books.google.co.uk/books?hl=en\&|r=\&id=aBD4O4raN2UC\&oi=fnd\&pg=PR5\&dq=NICE+ost eoarthritis $+2008 \&$ ots $=\mathrm{L}-$

FP35d7SJ\&sig=FCPG0Johk707WcHnsT7guP6VohU\#v=onepage\&q=NICE\%20osteoarthritis\%20200 8\&f=false [Accessed: 5 February 2021].

National Institute for Health and Care Excellence (NICE) 2021. Chronic pain (primary and secondary) in over 16s: assessment of all chronic pain and management of chronic primary pain (NG193). Available at: www.nice.org.uk/guidance/ng193.

National Institute for Health and Care Excellence 2016. Controlled drugs: safe use and management. Available at: https://www.nice.org.uk/guidance/ng46/evidence/full-guideline-pdf2427186353.

National Institute for Health and Care Excellence 2016. Low back pain and sciatica in over 16s: assessment and management. Available at: www.nice.org.uk/guidance/ng59.

National Institute for Health and Care Excellence 2021. Chronic pain (primary and secondary) in over 16s: assessment of all chronic pain and management of chronic primary pain (NG193). Available at: www.nice.org.uk/guidance/ng193.

National Joint Registry. 2021. Available at: www.njrcentre.org.uk/njrcentre/default.aspx [Accessed: 10 March 2021].

National Office for Statistics 2013. General Health in England and Wales: 2011 and comparison with 2001. UK Government. Available at:

https://www.ons.gov.uk/peoplepopulationandcommunity/healthandsocialcare/healthandwellbei 
ng/articles/generalhealthinenglandandwales/2013-01-30\#general-health-across-the-englishregions-and-wales.

National Office for Statistics 2019 Deaths related to drug poisoning in England and Wales: 2018 registrations. Office for National Statistics. Available at:

https://www.ons.gov.uk/peoplepopulationandcommunity/birthsdeathsandmarriages/deaths/bul letins/deathsrelatedtodrugpoisoninginenglandandwales/2018registrations.

National Office for Statistics 2020. Deaths related to drug poisoning in England and Wales: 2019 registrations. Available at:

https://www.ons.gov.uk/peoplepopulationandcommunity/birthsdeathsandmarriages/deaths/bul letins/deathsrelatedtodrugpoisoninginenglandandwales/2019registrations [Accessed: 2 August 2021].

National Office for Statistics 2021. Population estimates. Available at:

https://www.ons.gov.uk/peoplepopulationandcommunity/populationandmigration/populatione stimates [Accessed: March 2021].

National Records of Scotland Web Team 2019. National Records of Scotland. National Records of Scotland . Available at: https://www.nrscotland.gov.uk/statistics-and-data/statistics/statistics-bytheme/vital-events/deaths/drug-related-deaths-in-scotland.

National Science Foundation 2014. Critical Techniques and Technologies for Advancing Big Data Science \& Engineering (BIGDATA) (nsf14543). Available at:

https://www.nsf.gov/pubs/2014/nsf14543/nsf14543.htm [Accessed: 21 May 2021].

National Vascular Registry. 2021. Available at: https://www.vsqip.org.uk [Accessed: 10 February 2021].

Natusch, D. 2012. Equianalgesic doses of opioids - their use in clinical practice. British Journal of Pain 6(1), pp. 43-46. doi: 10.1177/2049463712437628.

Neal, R.D. et al. 1998. Frequency of patients' consulting in general practice and workload generated by frequent attenders: comparisons between practices. The British journal of general practice : the journal of the Royal College of General Practitioners 48(426), pp. 895-898.

Nelson, L.S. et al. 2015. Addressing the Opioid Epidemic. JAMA 314(14), pp. 1453-1454. Available at: https://jamanetwork.com/journals/jama/articlepdf/2456149/jed150093.pdf.

Newman, A.K. et al. 2017. The relationship of sociodemographic and psychological variables with chronic pain variables in a low-income population. Pain 158(9), pp. 1687-1696. Available at: http://eutils.ncbi.nlm.nih.gov/entrez/eutils/elink.fcgi?dbfrom=pubmed\&id=28570481\&retmode= ref\&cmd=prlinks.

Newton, J.N. et al. 2015. Changes in health in England, with analysis by English regions and areas of deprivation, 1990-2013: a systematic analysis for the Global Burden of Disease Study 2013. Lancet 386(10010), pp. 2257-2274. Available at: https://linkinghub.elsevier.com/retrieve/pii/S0140673615001956.

NHS Digital. 2015. UK Read Code. Available at: https://data.gov.uk/dataset/f262aa32-9c4e-44f199eb-4900deada7a4/uk-read-code [Accessed: 2 February 2017]. 
NHS Digital. 2021. Protecting patient data. Available at: https://digital.nhs.uk/services/nationaldata-opt-out/understanding-the-national-data-opt-out/protecting-patient-data [Accessed: 9 April 2021].

NHS Digital. 2021. SNOMED CT. Available at: https://digital.nhs.uk/services/terminology-andclassifications/snomed-ct [Accessed: 7 February 2017].

NHS England Primary Care Group 2021. Network Contracted Direct Enhanced Service. Available at: https://www.england.nhs.uk/wp-content/uploads/2021/03/B0431-network-contract-desspecification-pcn-requirements-and-entitlements-21-22.pdf [Accessed: 4 August 2021].

NHS Quality Improvement Scotland 2007. Getting to GRIPS with Chronic Pain in Scotland. Available at:

https://www.healthcareimprovementscotland.org/our work/long term conditions/programme resources/getting to grips with chronic.aspx [Accessed: 6 July 2021].

NHS Wales 2009. NHS in Wales: Why we are changing the structure. Available at:

http://www.wales.nhs.uk/documents/nhs\%20reform\%20leaflet october\%202009.pdf [Accessed: 16 May 2020].

NHS Wales 2021. Health in Wales. Available at: https://www.wales.nhs.uk/statisticsanddata [Accessed: 2 September 2021].

Nicholas, M. et al. 2019. The IASP classification of chronic pain for ICD-11: chronic primary pain. Pain 160(1), pp. 28-37. doi: 10.1097/j.pain<.0010000000001390.

Nicholas, M. et al. 2019. The IASP classification of chronic pain for ICD-11: chronic primary pain. Pain 160(1), pp. 28-37. Available at:

https://journals.Iww.com/pain/Fulltext/2019/01000/The IASP classification of chronic pain fo r.4.aspx.

Nichols, V.P. et al. 2020. Experiences of people taking opioid medication for chronic nonmalignant pain: a qualitative evidence synthesis using meta-ethnography. BMJ open 10(2), p. e032988. Available at: https://bmjopen.bmj.com/content/10/2/e032988.abstract.

Nielsen, S. et al. 2016. Health service utilisation by people living with chronic non-cancer pain: findings from the Pain and Opioids IN Treatment (POINT) study. Australian Health Review 40(5), pp. 490-499. Available at: https://www.publish.csiro.au/ah/pdf/AH15047.

Nielsen, S. et al. 2017. Defined daily doses (DDD) do not accurately reflect opioid doses used in contemporary chronic pain treatment. Pharmacoepidemiology and Drug Safety 26(5), pp. 587591. Available at:

http://eutils.ncbi.nlm.nih.gov/entrez/eutils/elink.fcgi?dbfrom=pubmed\&id=28101968\&retmode= ref\&cmd=prlinks.

Niesters, M. et al. 2010. Do sex differences exist in opioid analgesia? A systematic review and meta-analysis of human experimental and clinical studies. Pain 151(1), pp. 61-68. Available at: http://eutils.ncbi.nlm.nih.gov/entrez/eutils/elink.fcgi?dbfrom=pubmed\&id=20692097\&retmode= ref\&cmd=prlinks.

Noble, M. et al. 2008. Long-Term Opioid Therapy for Chronic Noncancer Pain: A Systematic Review and Meta-Analysis of Efficacy and Safety. Journal of Pain and Symptom Management 35(2), pp. 214-228. doi: 10.1016/j.jpainsymman.2007.03.015. 
Noble, M. et al. 2010. Long-term opioid management for chronic noncancer pain. Cochrane Database of Systematic Reviews (1), p. CD006605. Available at:

https://www.ncbi.nlm.nih.gov/pmc/articles/PMC6494200/pdf/CD006605.pdf.

Norman, P. et al. 2014. The pits may be closed but adverse effects on health may still be evident: exploring the links between coal mine locations, socioeconomic deprivation and self $\sim$ dots.

researchgate.net. Available at:

https://www.researchgate.net/profile/Paul Norman/publication/339626827 The pits may be closed but adverse effects on health may still be evident exploring the links between co al mine locations socioeconomic deprivation and selfreported health in Great Britain/links/5e5d01ea4585152ce8ff9d48/The-pits-may-be-closedbut-adverse-effects-on-health-may-still-be-evident-exploring-the-links-between-coal-minelocations-socioeconomic-deprivation-and-self-reported-health-in-Great-Britain.pdf.

Northern Ireland Statistics Research Agency. Multiple Deprivation Measure 2017. Available at: https://www.nisra.gov.uk/statistics/deprivation/northern-ireland-multiple-deprivation-measure2017-nimdm2017 [Accessed: November 2020].

Nowakowska, M. et al. 2020. Association of socioeconomic deprivation with opioid prescribing in primary care in England: a spatial analysis. Journal of epidemiology and community health 11(2), p. jech-2020--214676--9. Available at: https://jech.bmj.com/lookup/doi/10.1136/jech-2020$\underline{214676 .}$

Nugraha, B. et al. 2019. The IASP classification of chronic pain for ICD-11: functioning properties of chronic pain. Pain 160(1), p. 88. Available at:

https://journals.Iww.com/pain/Fulltext/2019/01000/The IASP classification of chronic pain fo r.12.aspx.

O'Brien, M.D.C. and Wand, A.P.F. 2020. A systematic review of the evidence for the efficacy of opioids for chronic non-cancer pain in community-dwelling older adults. Age and ageing 49(2), pp. 175-183. doi: 10.1093/ageing/afz175.

OECD Health Division 2019. Using Routinely Collected Data to Inform Pharmaceutical Policies. Available at: https://www.oecd.org/health/health-systems/routinely-collected-data-to-informpharmaceutical-policies.htm [Accessed: 3 May 2021].

Office for National Statistics 2013. General Health in England and Wales: 2011 and comparison with 2001. Available at:

https://www.ons.gov.uk/peoplepopulationandcommunity/healthandsocialcare/healthandwellbei ng/articles/generalhealthinenglandandwales/2013-01-30 [Accessed: 19 July 2021].

Office for National Statistics 2021. Deaths related to drug poisoning in England and Wales: 2018 registrations. Available at:

https://www.ons.gov.uk/peoplepopulationandcommunity/birthsdeathsandmarriages/deaths/bul letins/deathsrelatedtodrugpoisoninginenglandandwales/2018registrations [Accessed: 26 August 2021].

Oliver, J. and Carlson, C. 2020. Misperceptions about the Opioid Epidemic: Exploring the Facts. Pain management nursing : official journal of the American Society of Pain Management Nurses 21(1), pp. 100-109. Available at: https://doi.org/10.1016/i.pmn.2019.05.004.

OpenPrescribing.net (2020) "Open Prescribing - Opioid Analgesic Data." Available at: https://openprescribing.net/bnf/040702/ [Accessed: June 2020] 
Palmaro, A. and Lapeyre-Mestre, M. 2015. Trends in opioid analgesics use in Europe: a ten-year perspective. Clinical Therapeutics 37(8), p. e4. Available at:

http://www.clinicaltherapeutics.com/article/S0149291815003288/fulltext.

Palmaro, A. and Lapeyre-Mestre, M. 2015. Trends in opioid analgesics use in Europe: a ten-year perspective. Clinical Therapeutics 37(8), p. e4. doi: 10.1016/j.clinthera.2015.05.021.

Patel, S. et al. 2015. Clinical characteristics of persistent frequent attenders in primary care: casecontrol study. Family practice 48, pp. cmv076-7. doi: 10.1093/fampra/cmv076.

Patient Knows Best 2014. NHS in Wales. Available at: https://patientsknowbest.com/wales/ [Accessed: 1 September 2021].

Pergolizzi, J.V. et al. 2017. The Basic Pharmacology of Opioids Informs the Opioid Discourse about Misuse and Abuse: A Review. Pain and therapy 6(1), pp. 1-16. Available at:

http://eutils.ncbi.nlm.nih.gov/entrez/eutils/elink.fcgi?dbfrom=pubmed\&id=28341939\&retmode= ref\&cmd=prlinks.

Perks, W. and Roberts, C. 2016. Welsh Health Survey 2015: Health Service use results. Cardiff: Welsh Government. Available at: https://gov.wales/welsh-health-survey.

Perrot, S. et al. 2019. The IASP classification of chronic pain for ICD-11: chronic secondary musculoskeletal pain. Pain 160(1), pp. 77-82. Available at:

https://journals.Iww.com/pain/Fulltext/2019/01000/The IASP classification of chronic pain fo r.10.aspx.

Phillips, C.J. 2005. Health Economics: an introduction for health professionals. Oxford: Blackwell Publishing Ltd and BMJ Books.

Phillips, C.J. 2008. The real cost of pain management. Anaesthesia 56(11), pp. 1031-1033.

Available at: http://doi.wiley.com/10.1111/i.1365-2044.2001.02367.x.

Phillips, C.J. and Harper, C. 2011. The economics associated with persistent pain. Current opinion in Supportive and Palliative Care 5(2), pp. 127-130. Available at:

http://eutils.ncbi.nlm.nih.gov/entrez/eutils/elink.fcgi?dbfrom=pubmed\&id=21430542\&retmode= ref\&cmd=prlinks.

Pieretti, S. et al. 2016. Gender differences in pain and its relief. Annali dell'Istituto Superiore di Sanita 52(2), pp. 184-189. Available at:

http://eutils.ncbi.nlm.nih.gov/entrez/eutils/elink.fcgi?dbfrom=pubmed\&id=27364392\&retmode= $\underline{\text { ref\&cmd=prlinks. }}$

Pocock, L. et al. 2018. Now Gosport: what next? British Journal of General Practice 68(675), pp. 460-461. Available at: https://bjgp.org/content/68/675/460.

Portenoy, R.K. and Foley, K.M. 1986. Chronic use of opioid analgesics in non-malignant pain: report of 38 cases. Pain 25(2), pp. 171-186. doi: 10.1016/0304-3959(86)90091-6.

Powell, G.A. 2018. An Assessment of the Use of Routinely Recorded Data in the UK in a Randomised Controlled Trial. Available at: https://livrepository.liverpool.ac.uk/3026084/1/200161058 Apr2018.pdf.

Price, C. et al. 2012. National Pain Audit Final Report 2010-2012. Dr Foster. Available at: https://www.hqip.org.uk/wp-content/uploads/2018/02/EBfl8i.pdf. 
Price, C. et al. 2019. The National Pain Audit for specialist pain services in England and Wales 2010-2014. British Journal of Pain 13(3), pp. 185-193. Available at:

http://eutils.ncbi.nlm.nih.gov/entrez/eutils/elink.fcgi?dbfrom=pubmed\&id=31308943\&retmode= ref\&cmd=prlinks.

Prunuske, J. P. et al. 2014. Opioid prescribing patterns for non-malignant chronic pain for rural versus non-rural US adults: a population-based study using 2010 NAMCS data. BMC health services research, 14, 563. https://doi.org/10.1186/s12913-014-0563-8

Pryor, D.B. et al. 1985. Clinical data bases. Accomplishments and unrealized potential. Medical care 23(5), pp. 623-647. Available at: http://journals.Iww.com/00005650-198505000-00020.

Public Health England 2017. Chronic pain in adults 2017: Health Survey for England. Available at: https://assets.publishing.service.gov.uk/government/uploads/system/uploads/attachment data /file/940858/Chronic Pain Report.pdf.

Public Health England. 2017. Chapter 6: Social Determinants of Health. Public Health England., pp. 60-79. Available at: https://www.gov.uk/government/publications/health-profile-forengland/chapter-6-social-determinants-of-health.

Public Health England. 2017. Chronic pain in adults 2017: Health Survey for England. London: Public Health England. Available at:

https://assets.publishing.service.gov.uk/government/uploads/system/uploads/attachment data /file/940858/Chronic Pain Report.pdf.

Public Health Scotland 2021. Data \& intelligence from PHS. Available at: https://beta.isdscotland.org/ [Accessed: March 2021].

Public Health Scotland 2021. Prescriptions in the Community. Available at: https://www.opendata.nhs.scot/dataset/84393984-14e9-4b0d-a797-b288db64d088 [Accessed: March 2021].

Public Health Wales Observatory 2018. Health and its determinants in Wales. Available at: https://www2.nphs.wales.nhs.uk/PubHObservatoryProjDocs.nsf/61c1e930f9121fd080256f2a004 937ed/99a9490d2e6d05268025820b005851de/\$FILE/Health\&determinantsinWales Report Eng .pdf [Accessed: 4 August 2021].

Public Health Wales Observatory 2020. Public Health Wales Observatory. Available at: http://www.publichealthwalesobservatory.wales.nhs.uk/measuring-inequalities-2016-files.

Puenpatom RA. Et al. 2012. Daily average consumption of 2 long-acting opioids: an interrupted time series analysis. Am Health Drug Benefits. 2012 Jan;5(1):52-60. PMID: 24991311; PMCID: PMC4046462.

Quality Watch et al. 2020. Poorest get worse quality of NHS care in England, new research finds. Available at: https://www.nuffieldtrust.org.uk/news-item/poorest-get-worse-quality-of-nhs-carein-england-new-research-finds [Accessed: 13 March 2020].

Raffaeli, W. and Arnaudo, E. 2017. Pain as a disease: an overview. Journal of pain research 10, pp. 2003-2008. Available at: https://www.dovepress.com/pain-as-a-disease-an-overview-peerreviewed-article-JPR.

Raftery, M.N. et al. 2011. Chronic pain in the Republic of Ireland--community prevalence, psychosocial profile and predictors of pain-related disability: results from the Prevalence, Impact 
and Cost of Chronic Pain (PRIME) study, part 1. Pain 152(5), pp. 1096-1103. Available at: https://www.jpain.org/article/S1526-5900(11)00816-9/fulltext.

Raghavan, S. et al. 2011. New opioid side effects and implications for long-term therapy. Trends in Anaesthesia and Critical Care 1(1), pp. 18-21. doi: 10.1016/j.cacc.2010.06.003.

Rathbone, T. et al. 2020. Sex and gender considerations in low back pain clinical practice guidelines: a scoping review. BMJ Open Sport \& Exercise Medicine 6(1), p. e000972. doi: 10.1136/bmjsem-2020-000972.

Reddy, K.S.K. et al. 2012. Human experimental pain models: A review of standardized methods in drug development. Journal of research in medical sciences : the official journal of Isfahan University of Medical Sciences 17(6), pp. 587-595. Available at: http://eutils.ncbi.nlm.nih.gov/entrez/eutils/elink.fcgi?dbfrom=pubmed\&id=23626642\&retmode= ref\&cmd=prlinks.

Rennick, A. et al. 2016. Variability in opioid equivalence calculations. Pain medicine (Malden, Mass.) 17(5), pp. 892-898. Available at: https://academic.oup.com/painmedicine/articlelookup/doi/10.1111/pme.12920.

Rhudy, J.L. and Williams, A.E. 2005. Gender differences in pain: do emotions play a role? Gender medicine 2(4), pp. 208-226. Available at:

https://linkinghub.elsevier.com/retrieve/pii/S1550857905800518.

Rice, A.S.C. et al. 2016. Pain and the global burden of disease. Pain 157(4), pp. 791-796. Available at:

http://eutils.ncbi.nIm.nih.gov/entrez/eutils/elink.fcgi?dbfrom=pubmed\&id=26670465\&retmode= ref\&cmd=prlinks.

Richard, D.J.G.P. and Richard, P. 2012. The Economic Costs of Pain in the United States. The Journal of Pain 13(8), pp. 715-724. doi: 10.1016/j.jpain.2012.03.009.

Richards, G.C. et al. 2018. Effects of long-term opioid analgesics on cognitive performance and plasma cytokine concentrations in patients with chronic low back pain: a cross-sectional pilot study. PAIN Reports 3(4), pp. e669-7413.

Richards, G.C. et al. 2020a. Factors associated with the prescribing of high-dose opioids in primary care: a systematic review and meta-analysis. BMC medicine 18(1), p. 68. doi: 10.1186/s12916-020-01528-7.

Richards, G.C. et al. 2020b. Relation between opioid consumption and inclusion of opioids in 137 national essential medicines lists. BMJ global health 5(11). doi: 10.1136/bmjgh-2020-003563.

Riley, J.L. et al. 2001. Sex differences in negative emotional responses to chronic pain. The Journal of Pain 2(6), pp. 354-359. Available at:

https://www.sciencedirect.com/science/article/abs/pii/\$1526590001862611.

Ringberg, U. et al. 2014. Examining the variation in GPs' referral practice: a cross-sectional study of GPs' reasons for referral. The British journal of general practice : the journal of the Royal College of General Practitioners 64(624), pp. e426-33. Available at:

http://eutils.ncbi.nlm.nih.gov/entrez/eutils/elink.fcgi?dbfrom=pubmed\&id=24982495\&retmode= ref\&cmd=prlinks. 
Rivat, C. and Ballantyne, J. 2016. The dark side of opioids in pain management: basic science explains clinical observation. PAIN Reports e570(1), pp. 1-9. doi:

$10.1097 / \mathrm{pr} 9<.0010000000000570$.

Rocha, V. et al. 2017. Neighbourhood socioeconomic deprivation and health-related quality of life: A multilevel analysis. PloS one 12(12), pp. e0188736-16. Available at:

https://dx.plos.org/10.1371/journal.pone.0188736.

Roberts, H. 2016. Research Briefing: Misuse of prescription and over-the-counter medications. Cardiff: National Assembly for Wales Research Service., p. 12. Available at:

https://senedd.wales/media/irghgjnw/16-039-web-english.pdf.

Rodriguez, J. 2020. Covid-19 and the Welsh economy: shutdown sectors and key workers. Cardiff: Cardiff University. Available at:

https://www.cardiff.ac.uk/ data/assets/pdf file/0009/2409921/Covid economy report 1 Fina I rep.pdf.

Rosner, B. et al. 2019. Opioid prescription patterns in Germany and the global opioid epidemic: Systematic review of available evidence. PLOS ONE 14(8), p. e0221153. Available at: https://doi.org/10.1371/journal.pone.0221153.

Routledge P. 2013. Personal Communication during Advisory Panel on Substance Misuse Evidence Gathering. Welsh Assembly Government.

Rovner, G.S. et al. 2017. Chronic pain and sex-differences; women accept and move, while men feel blue. PLOS ONE 12(4), p. e0175737. doi: 10.1371/journal.pone.0175737.

Royal Pharmaceutical Society of Great Britain. 2017. Medicines, Ethics, and Practice. Available at: http://books.google.co.uk/books?id=VhdtAAAAMAAJ\&q=intitle:Medicines+Ethics+and+Practice\& $\underline{\mathrm{dq}}=$ intitle:Medicines+Ethics+and+Practice $\& \mathrm{hl}=\& \mathrm{~cd}=1 \&$ source=gbs api.

Royal Pharmaceutical Society of Great Britain. 2021. Medicines, Ethics, and Practice. London: Pharmaceutical Press. Available at: https://www.rpharms.com/publications/the-mep.

Ruau, D. et al. 2012. Sex Differences in Reported Pain Across 11,000 Patients Captured in Electronic Medical Records. The Journal of Pain 13(3), pp. 228-234. Available at: http://dx.doi.org/10.1016/j.jpain.2011.11.002.

Ruscitto, A. et al. 2015. Changes in opioid and other analgesic use 1995-2010: Repeated crosssectional analysis of dispensed prescribing for a large geographical population in Scotland. European Journal of Pain 19(1), pp. 59-66. doi: 10.1002/ejp.520.

Saariaho, T.H. et al. 2011. Early maladaptive schemas in Finnish adult chronic pain patients and a control sample. Scandinavian journal of psychology 52(2), pp. 146-153. Available at:

http://doi.wiley.com/10.1111/j.1467-9450.2010.00849.x.

Sabatowski, R. et al. 2004. Pain Treatment: A Historical Overview. Current Pharmaceutical Design 10(7), pp. 701-716. doi: 10.2174/1381612043452974.

Sacks, D.W. et al. 2021. Can policy affect initiation of addictive substance use? Evidence from opioid prescribing. Journal of health economics 76, p. 102397. Available at: https://doi.org/10.1016/j.jhealeco.2020.102397. 
Salas-Vega, S. et al. 2016. Big Data and Health Care: Challenges and Opportunities for Coordinated Policy Development in the EU. Health Systems \& Reform 1(4), pp. 285-300. Available at: https://www.tandfonline.com/doi/full/10.1080/23288604.2015.1091538.

Salas, J. et al. 2017. New-onset depression following stable, slow, and rapid rate of prescription opioid dose escalation. Pain 158(2), pp. 306-312. doi: 10.1097/j.pain<.0010000000000763.

Salas, J. et al. 2020. The association of opioid use duration and new depression episode among patients with and without insomnia. Journal of opioid management 16(5), pp. 317-328. doi: 10.5055/jom.2020.0587.

SAS Institute Inc 2021. SAS 9.4 Ink. Available at: https://www.sas.com/en gb/home.html?gclid=CjOKCQjwssyJBhDXARIsAK98ITTEFxlhVtulGrJOHIx bouBdIYi556GES Id8FfmuAKSj7c7sLkKPN4aAkMKEALw wcB [Accessed: 2 June 2021].

Schatman, M.E. and Shapiro, H. 2019. Damaging State Legislation Regarding Opioids: The Need to Scrutinize Sources of Inaccurate Information Provided to Lawmakers. Journal of pain research 12, pp. 3049-3053. Available at: https://www.dovepress.com/damaging-state-legislation-regardingopioids-the-need-to-scrutinize-so-peer-reviewed-article-JPR.

Schatman, M.E. et al. 2016. The MEDD myth: the impact of pseudoscience on pain research and prescribing-guideline development. Journal of pain research Volume 9, pp. 153-4. Available at: https://www.dovepress.com/the-medd-myth-the-impact-of-pseudoscience-on-pain-researchand-prescri-peer-reviewed-article-JPR.

Scherrer, J.F. et al. 2016. Increased Risk of Depression Recurrence After Initiation of Prescription Opioids in Noncancer Pain Patients. The Journal of Pain 17(4), pp. 473-482. Available at: https://www.sciencedirect.com/science/article/pii/S1526590015010007.

Scherrer, J.F. et al. 2016. Prescription Opioid Duration, Dose, and Increased Risk of Depression in 3 Large Patient Populations. The Annals of Family Medicine 14(1), pp. 54-62. doi: 10.1370/afm.1885.

Schieber, L.Z. et al. 2020. Variation in Adult Outpatient Opioid Prescription Dispensing by Age and Sex - United States, 2008-2018. MMWR. Morbidity and Mortality Weekly Report 69(11), pp. 298-302. doi: 10.15585/mmwr.mm6911a5.

Schifanella, R. et al. 2020. Spatial heterogeneity and socioeconomic determinants of opioid prescribing in England between 2015 and 2018. BMC Medicine 18(1), pp. 127-13. Available at: https://bmcmedicine.biomedcentral.com/articles/10.1186/s12916-020-01575-0.

Schofferman, J. 2006. Restoration of Function: The Missing Link in Pain Medicine? Pain medicine (Malden, Mass.) 7, pp. S159-S165. Available at: https://onlinelibrary.wiley.com/doi/pdf/10.1111/j.1526-4637.2006.00131.x.

Schofield, P. 2012. Pain in Older Adults: Epidemiology, Impact and Barriers to Management. Reviews in Pain 1(1), pp. 12-14.

Schofield, P. 2017. Pain management in older adults. Medicine 45(1), pp. 41-45. Available at: http://dx.doi.org/10.1016/j.mpmed.2016.10.005.

Schofield, P. and Abdulla, A. 2018. Pain assessment in the older population: what the literature says. Age and ageing 47(3), pp. 324-327. Available at: https://academic.oup.com/ageing/article/47/3/324/4951960. 
Scholz, J. et al. 2019. The IASP classification of chronic pain for ICD-11: chronic neuropathic pain. Pain 160(1), pp. 53-59. Available at:

https://journals.Iww.com/pain/Fulltext/2019/01000/The IASP classification of chronic pain fo r.7.aspx.

Schug, S.A. et al. 2016. Acute pain management: scientific evidence, fourth edition, 2015. The Medical Journal of Australia 204(8), pp. 315-317. doi: 10.5694/mja16.00133.

Schug, S.A. et al. 2019. The IASP classification of chronic pain for ICD-11. PAIN 160(1), pp. 45-52. doi: $10.1097 /$ j.pain<.0010000000001413.

Scioli-Salter, E.R. et al. 2015. The shared neuroanatomy and neurobiology of comorbid chronic pain and PTSD: therapeutic implications. The Clinical journal of pain 31(4), pp. 363-374. Available at:

http://eutils.ncbi.nIm.nih.gov/entrez/eutils/elink.fcgi?dbfrom=pubmed\&id=24806468\&retmode= ref\&cmd=prlinks.

Scott-Thomas, S. 2020. Personal Communication. Discussion about national and local guideline implementation.

Scottish Affairs Select Committee 2019. Problem drug use in Scotland. UK Parliament. Available at:

https://publications.parliament.uk/pa/cm201919/cmselect/cmscotaf/44/4405.htm\#_idTextAnch or006.

Scottish Government 2021. Policy - Social care. Available at:

https://www.gov.scot/policies/social-care/health-and-social-care-integration/ [Accessed: 3 August 2021].

Scottish Intercollegiate Guideline Network SIGN 2019. Management of Chronic Pain. Available at: https://www.sign.ac.uk/media/1108/sign136 2019.pdf [Accessed: 15 September 2020].

Secure Anonymised Information Linkage (SAIL) Databank. 2021. The SAIL databank. Available at: https://saildatabank.com/. [Accessed: 24 August 2021].

Secure Anonymised Information Linkage Databank 2021. The SAIL databank. Available at: https://saildatabank.com/ [Accessed: 16 June 2021].

Sellick, J. 2018. How can we build a rural health and care workforce. Available at: https://www.rsnonline.org.uk/how-can-we-build-a-rural-health-and-care-workforce.

Seyfried, O. and Hester, J. 2012. Opioids and endocrine dysfunction. British Journal of Pain 6(1), pp. 17-24. doi: 10.1177/2049463712438299.

Shafrin, J. 2008. Welfarists vs. Extra-welfarists. Available at: https://www.healthcareeconomist.com/2008/02/13/welfarists-vs-extra-welfarists/ [Accessed: 29 January 2021].

Shah, A. et al. 2017. Characteristics of Initial Prescription Episodes and Likelihood of Long-Term Opioid Use - United States, 2006-2015. MMWR. Morbidity and Mortality Weekly Report 66(10), pp. 265-269. doi: 10.15585/mmwr.mm6610a1.

Shaheen, P.E. et al. 2009. Opioid Equianalgesic Tables: Are They All Equally Dangerous? Journal of Pain and Symptom Management 38(3), pp. 409-417. doi: 10.1016/j.jpainsymman.2009.06.004. 
Shared Services Partnership, NHS Wales 2021. General Practice Prescribing Data Extract. Available at: https://nwssp.nhs.wales/ourservices/primary-care-services/generalinformation/data-and-publications/general-practice-prescribing-data-extract/ [Accessed: August 2021].

Shared Services Partnership, NHS Wales. CASPA data. Available at: https://nwssp.nhs.wales/ourservices/primary-care-services/our-latest-news/caspa-datadecember-2020/ [Accessed: August 2021].

Sharma, S. et al. 2019. Health system strengthening is needed to respond to the burden of pain in low- and middle-income countries and to support healthy ageing. Journal of global health 9(2), p. 020317. Available at:

http://eutils.ncbi.nlm.nih.gov/entrez/eutils/elink.fcgi?dbfrom=pubmed\&id=33274068\&retmode= ref\&cmd=prlinks.

Shaw, K. and Fudin, J. 2013. Evaluation and comparison of online equianalgesic opioid dose conversion calculators. paindr.com , pp. 61-66.

Sicular, S. 2013. Gartner's Big Data Definition Consists of Three Parts, Not to Be Confused with Three "V"s. Available at: https://www.forbes.com/sites/gartnergroup/2013/03/27/gartners-bigdata-definition-consists-of-three-parts-not-to-be-confused-with-three-vs/ [Accessed: January 2021].

Silversides, A. 2011. Use of strong opioids in Canada rose sharply between 2005 and 2010. British Medical Journal 343(aug25 2), p. d5441. doi: 10.1136/bmj.d5441.

Singer, J.A. et al. 2019. Today's nonmedical opioid users are not yesterday's patients; implications of data indicating stable rates of nonmedical use and pain reliever use disorder. Journal of pain research 12, pp. 617-620. Available at: https://www.dovepress.com/todays-nonmedical-opioidusers-are-not-yesterdays-patients-implication-peer-reviewed-article-JPR.

Smith, B.H. and Torrance, N. 2012. Epidemiology of Neuropathic Pain and Its Impact on Quality of Life. Current pain and headache reports 16(3), pp. 191-198. Available at: http://link.springer.com/10.1007/s11916-012-0256-0.

Smith, B.H. et al. 2019. The IASP classification of chronic pain for ICD-11: applicability in primary care. PAIN 160(1), pp. 1-5. doi: 10.1097/j.pain<.0010000000001360. Available at: https://journals.Iww.com/pain/Fulltext/2019/01000/The_IASP classification of chronic pain fo r.11.aspx.

Smith, B.H. et al. 2021. Drugs for chronic pain - we still need them. Available at: https://bjgplife.com/2021/02/02/drugs-for-chronic-pain-we-still-need-them/.

Smith, E. et al. 2014. The global burden of other musculoskeletal disorders: estimates from the Global Burden of Disease 2010 study. Annals of the Rheumatic Diseases 73(8), pp. 1462-1469. Available at: http://ard.bmj.com/cgi/doi/10.1136/annrheumdis-2013-204680.

Smith, J. 2005. The Shipman Inquiry. Available at:

https://webarchive.nationalarchives.gov.uk/ukgwa/20090808155005/http://www.the-shipmaninquiry.org.uk/home.asp. [Accessed: 24 January 2018].

Smith, S.R. et al. 2017. Cost-Effectiveness of Tramadol and Oxycodone in the Treatment of Knee Osteoarthritis. Arthritis Care \& Research 69(2), pp. 234-242. doi: 10.1002/acr.22916. 
Solà, I. et al. 2019. Quality of life and well-being from the perspective of patients on opioid agonist maintenance treatment: study protocol for a systematic review of qualitative research and a scoping review of measures. Systematic Reviews 8(1), pp. 1-7. doi: 10.1186/s13643-0191237-8.

Sorge, R.E. and Strath, L.J. 2018. Sex differences in pain responses. Current Opinion in Physiology 6, pp. 75-81. Available at: https://linkinghub.elsevier.com/retrieve/pii/S2468867318300786.

Spooner,.L. et al. 2016. "High-Dose Opioid Prescribing and Opioid- Related Hospitalization: A Population-Based Study," pp. 1-12. doi:https://doi.org/10.1371/journal.pone.0170834. Accessed at: https://journals.plos.org/plosone/article?id=10.1371/journal.pone.0167479.

Sproule, B. 2015. Prescription monitoring programs in Canada: best practice and program review. Available at: http://nperesource.casn.ca/wp-content/uploads/2017/01/CCSA-PrescriptionMonitoring-Programs-in-Canada-Report-2015-en.pdf.

Stannard, C. 2018a. Where now for opioids in chronic pain? Drug and therapeutics bulletin 56(10), pp. 118-122. Available at:

http://eutils.ncbi.nIm.nih.gov/entrez/eutils/elink.fcgi?dbfrom=pubmed\&id=30254063\&retmode= ref\&cmd=prlinks.

Stannard, C.F. 2018b. Pain and pain prescribing: what is in a number? British journal of anaesthesia 120(6), pp. 1147-1149. Available at:

https://linkinghub.elsevier.com/retrieve/pii/S0007091218301806.

Stanos,S. 2016. Rethinking chronic pain in a primary care setting, Postgraduate Medicine, 128:5, 502-515, doi: 10.1080/00325481.2016.1188319

StatsWales 2010. Welsh Index of Multiple Deprivation - LSOA maps 2011. Available at: https://statswales.gov.wales/Catalogue/Community-Safety-and-Social-Inclusion/Welsh-Index-ofMultiple-Deprivation/Archive/LSOA-Maps [Accessed: July 26, 2019].

StatsWales 2010. Welsh Index of Multiple Deprivation 2011 by rank and local super output area. Available at: https://statswales.gov.wales/Catalogue/Community-Safety-and-SocialInclusion/Welsh-Index-of-Multiple-Deprivation/Archive/WIMD-2011/wimd2011 [Accessed: 12 May 2021].

StatsWales 2019. Population estimates by local authority and year. Available at: https://statswales.gov.wales/Catalogue/Population-and-Migration/Population/Estimates/LocalAuthority/populationestimates-by-localauthority-year.

StatsWales 2019. WIMD 2019. Available at: https://statswales.gov.wales/Catalogue/CommunitySafety-and-Social-Inclusion/Welsh-Index-of-Multiple-Deprivation/WIMD-maps-2019. [Accessed: 24 July 2021].

StatsWales 2020. Ethnicity by area and ethnic group. Available at: https://statswales.gov.wales/Catalogue/Equality-and-Diversity/Ethnicity/ethnicity-by-areaethnicgroup [Accessed: November 2020].

StatsWales 2020. General health and illness by year. Available at: https://statswales.gov.wales/catalogue/National-Survey-for-Wales/Population-Health/Adultgeneral-health-and-illness/genhealthillness-by-year [Accessed: December 2020]. 
Steel, N. et al. 2018. Changes in health in the countries of the UK and 150 English Local Authority areas 1990-2016: a systematic analysis for the Global Burden of Disease Study 2016. The Lancet 392(10158), pp. 1647-1661. doi: 10.1016/s0140-6736(18)32207-4.

Stewart, G. and Basler, M.H. 2013. Wanted: national monitoring system for long term opioids. British Medical Journal 347(f5586), p. 22.

Stilwell, P. and Harman, K. 2019. An enactive approach to pain: beyond the biopsychosocial model. Phenomenology and the Cognitive Sciences 18(4), pp. 1-29. doi: 10.1007/s11097-01909624-7.

Stoicea, N. et al. 2019. Current perspectives on the opioid crisis in the US healthcare system. Medicine 98(20), pp. e15425-8. Available at: http://journals.Iww.com/00005792-201905170$\underline{00010}$.

Stubbs, B. et al. 2014a. Pain and the Risk for Falls in Community-Dwelling Older Adults: Systematic Review and Meta-Analysis. Archives of Physical Medicine and Rehabilitation 95(1), pp. 175-187.e9. doi: 10.1016/j.apmr.2013.08.241.

Stubbs, B. et al. 2014b. Pain is associated with recurrent falls in community-dwelling older adults: evidence from a systematic review and meta-analysis. Pain Medicine 15(7), pp. 1115-1128. doi: 10.1111/pme.12462.

Sullivan, M.D. and Ballantyne, J.C. 2016. Must we reduce pain intensity to treat chronic pain? PAIN 157(1), pp. 65-69. doi: 10.1097/j.pain<.0010000000000336.

Sullivan, M.D. et al. 2008. Trends in use of opioids for non-cancer pain conditions 2000-2005 in Commercial and Medicaid insurance plans: The TROUP study. Pain 138(2), pp. 440-449. Available at: http://content.wkhealth.com/linkback/openurl?sid=WKPTLP:landingpage\&an=00006396200808310-00022.

Sullivan, M.J.L. et al. 2000. Gender differences in pain and pain behavior: The role of catastrophizing. Cognitive Therapy and Research 24(1), pp. 121-134. Available at: http://en.wikipedia.org/wiki/Anxiety.

Sutherland, K. and Coyle, N. 2009. Quality in Healthcare in England, Wales, Scotland and Northern Ireland: an intra-UK chartbook. London: The Health Foundation. Available at: www.health.org.uk.

Svendsen, K. et al. 2011. Choosing the unit of measurement counts: the use of oral morphine equivalents in studies of opioid consumption is a useful addition to defined daily doses. Palliative medicine 25(7), pp. 725-732. Available at:

http://eutils.ncbi.nlm.nih.gov/entrez/eutils/elink.fcgi?dbfrom=pubmed\&id=21378066\&retmode= ref\&cmd=prlinks.

Takura, T. et al. 2015. The societal burden of chronic pain in Japan: an internet survey. Journal of orthopaedic science : official journal of the Japanese Orthopaedic Association 20(4), pp. 750-760. Available at:

http://eutils.ncbi.nlm.nih.gov/entrez/eutils/elink.fcgi?dbfrom=pubmed\&id=25963609\&retmode= ref\&cmd=prlinks.

Takura, T. et al. 2021. Cost-Effectiveness Analysis of the Treatment Strategies with or without Opioid Medications in Surgery-Eligible Patients with Osteoarthritis in Japan. PharmacoEconomics - Open , pp. 1-13. doi: 10.1007/s41669-021-00292-5. 
Tan, E.C. et al. 2015. Analgesic Use and Daytime Sleepiness in Residents with and Without Dementia in Residential Aged Care Facilities. Drugs \& Aging 32(12), pp. 1045-1053. Available at: https://doi.org/10.1007/s40266-015-0325-2.

Taylor, M. and Filby, A. 2014. What is sensitivity analysis? Available at: https://www.whatisseries.co.uk/what-is-sensitivity-analysis/.

Taylor, S. et al. 2019. Dependence and withdrawal associated with some prescribed medicines: an evidence review. London: Public Health England. Available at:

https://assets.publishing.service.gov.uk/government/uploads/system/uploads/attachment data /file/940255/PHE PMR report Dec2020.pdf.

Techtarget 2021. SQL definition. Available at: http://searchsqlserver.techtarget.com/definition/SQL [Accessed: 11 July 2017].

Terplan, M. 2017. Women and the opioid crisis: historical context and public health solutions. Fertility and sterility 108(2), pp. 195-199. Available at: https://linkinghub.elsevier.com/retrieve/pii/S0015028217304314.

Thakral, M. et al. 2018. Comparing Pain and Depressive Symptoms of Chronic Opioid Therapy Patients Receiving Dose Reduction and Risk Mitigation Initiatives with Usual Care. The Journal of Pain 19(1), pp. 111-120. doi: 10.1016/j.jpain.2017.09.006.

The Joseph Rowntree Foundation Analysis Unit 2017. UK Poverty 2017: A comprehensive analysis of poverty trends and figures. York: Joseph Rowntree Foundation, p. 113. Available at: https://www.jrf.org.uk/report/uk-poverty-2017.

The What is ...? series. 2014. Available at: www.whatisseries.co.uk. [Accessed: September 2020]

Thielke, S.M. et al. 2017. A Prospective Study of Predictors of Long-term Opioid Use Among Patients with Chronic Noncancer Pain. The Clinical journal of pain 33(3), p. 198. Available at: http://journals.Iww.com/clinicalpain/Fulltext/2017/03000/A Prospective Study of Predictors o f Long term.2.aspx.

Thiru, K. et al. 2003. Systematic review of scope and quality of electronic patient record data in primary care. British Medical Journal 326(7398), p. 1070. Available at:

http://eutils.ncbi.nlm.nih.gov/entrez/eutils/elink.fcgi?dbfrom=pubmed\&id=12750210\&retmode= ref\&cmd=prlinks.

Todd, A. et al. 2018. The Pain Divide: a cross-sectional analysis of chronic pain prevalence, pain intensity and opioid utilisation in England. BMJ open 8(7), p. e023391. doi: 10.1136/bmjopen2018-023391.

Torrance, N. et al. 2014. Estimating the burden of disease in chronic pain with and without neuropathic characteristics: Does the choice between the EQ-5D and SF-6D matter? Pain 155(10), pp. 1996-2004. Available at:

http://content.wkhealth.com/linkback/openurl?sid=WKPTLP:landingpage\&an=00006396201410000-00016.

Torrance, N. et al. 2018. Association of opioid prescribing practices with chronic pain and benzodiazepine co-prescription: a primary care data linkage study. British journal of anaesthesia 120(6), pp. 1345-1355. Available at:

https://linkinghub.elsevier.com/retrieve/pii/S000709121830134X. 
Torrance, N. et al. 2020. Trends in gabapentinoid prescribing, co-prescribing of opioids and benzodiazepines, and associated deaths in Scotland. British journal of anaesthesia 125(2), pp. 159-167. doi: 10.1016/j.bja.2020.05.017.

Tracey, I. 2016. Finding the Hurt in Pain. Cerebrum (cer-15-16), pp. 1-12. Available at: https://www.ncbi.nlm.nih.gov/pmc/articles/PMC5501013/.

Tracey, I. and Mantyh, P.W. 2007. The cerebral signature for pain perception and its modulation. Neuron 55(3), pp. 377-391. Available at:

http://eutils.ncbi.nlm.nih.gov/entrez/eutils/elink.fcgi?dbfrom=pubmed\&id=17678852\&retmode= ref\&cmd=prlinks.

Trawalter, S. et al. 2012. Racial bias in perceptions of others' pain. PloS one 7(11), p. e48546. Available at: https://journals.plos.org/plosone/article?id=10.1371/journal.pone.0048546.

Treede, R.-D. et al. 2015. A classification of chronic pain for ICD-11. Pain 156(6), pp. 1003-1007. doi: 10.1097/j.pain<.0010000000000160.

Treede, R.-D. et al. 2019. Chronic pain as a symptom or a disease: the IASP Classification of Chronic Pain for the International Classification of Diseases (ICD-11). Pain 160(1), pp. 19-27. Available at:

http://eutils.ncbi.nIm.nih.gov/entrez/eutils/elink.fcgi?dbfrom=pubmed\&id=30586067\&retmode= ref\&cmd=prlinks.

Trescot, A.M. et al. 2008. Effectiveness of opioids in the treatment of chronic non-cancer pain. Pain physician, 11(2 Suppl), pp. S181-200. Available at:

http://eutils.ncbi.nlm.nih.gov/entrez/eutils/elink.fcgi?dbfrom=pubmed\&id=18443639\&retmode= ref\&cmd=prlinks.

Tsang, A. et al. 2008. Common Chronic Pain Conditions in Developed and Developing Countries: Gender and Age Differences and Comorbidity with Depression-Anxiety Disorders. Journal of Pain 9(10), pp. 883-891. Available at:

https://linkinghub.elsevier.com/retrieve/pii/S1526590008005750.

Turk, D.C. et al. 2016. Assessment of Psychosocial and Functional Impact of Chronic Pain. The Journal of Pain 17(9 Suppl), pp. T21-49. Available at:

http://eutils.ncbi.nlm.nih.gov/entrez/eutils/elink.fcgi?dbfrom=pubmed\&id=27586830\&retmode= ref\&cmd=prlinks.

Turner, D. et al. 2019. Harm Reduction Database Wales: Drug related mortality Annual Report 2018-19. Cardiff, Wales: Public Health Wales. Available at: https://phw.nhs.wales/news/drugdeaths-at-their-highest-ever-levels-in-wales/harm-reduction-database-wales-drug-relatedmortality-annual-report-2018-19/.

Turunen, E. and Hiilamo, H. 2014. Health effects of indebtedness: a systematic review. BMC Public Health 14(1), pp. 1323-8. Available at:

http://bmcpublichealth.biomedcentral.com/articles/10.1186/1471-2458-14-489.

Tzartzas, K. et al. 2019. General practitioners referring patients to specialists in tertiary healthcare: a qualitative study., pp. 1-9. doi: 10.1186/s12875-019-1053-1.

van den Beuken-van Everdingen, M.H.J. et al. 2013. Prolonged QT interval by methadone: Relevance for daily practice? A prospective study in patients with cancer and noncancer pain. Journal of Opioid Management 9(4). doi: https://doi.org/10.5055/jom.2013.0167. 
Velayudhan, A. et al. 2014. Opioid-induced hyperalgesia. Continuing Education in Anaesthesia, Critical Care and Pain 14(3), pp. 125-129. Available at:

https://www.scopus.com/inward/record.uri?partnerID=HzOxMe3b\&scp=84900564622\&origin=i nward.

Verhamme, K.M.C. and Bohnen, A.M. 2019. Comment Are we facing an opioid crisis in Europe? The Lancet Public Health 4 KW-(10), pp. e483-e484.

Vincent, K. and Tracey, I. 2008. Hormones and their Interaction with the Pain Experience. Reviews in pain 2(2), pp. 20-24. Available at:

http://journals.sagepub.com/doi/10.1177/204946370800200206.

Vokinger, K.N. 2018. Opioid Crisis in the US - Lessons from Western Europe. The Journal of law, medicine \& ethics : a journal of the American Society of Law, Medicine \& Ethics 46(1), pp. 189190. doi: $10.1177 / 1073110518766033$.

Vos, T. et al. 2017. Global, regional, and national incidence, prevalence, and years lived with disability for 328 diseases and injuries for 195 countries, 1990-2016: a systematic analysis for the Global Burden of Disease Study 2016. The Lancet 390(10100), pp. 1211-1259. Available at: http://www.thelancet.com/article/S0140673617321542/fulltext.

Vos, T. et al. 2020. Global burden of 369 diseases and injuries in 204 countries and territories, 1990 - 2019: a systematic analysis for the Global Burden of Disease Study 2019. The Lancet 396(10258), pp. 1204-1222. Available at: http://dx.doi.org/10.1016/S0140-6736(20)30925-9.

Wagemaakers, F.N. et al. 2017. Opioid analgesic use in Australia and The Netherlands: a crosscountry comparison. International Journal of Clinical Pharmacy 39(4), pp. 874-880. Available at: http://link.springer.com/10.1007/s11096-017-0492-9.

Walley, T. and Roberts, D. 2000. Average daily quantities: a tool for measuring prescribing volume in England. Pharmacoepidemiology and Drug Safety 9(1), pp. 55-8. Available at: https://onlinelibrary.wiley.com/doi/pdf/10.1002/\%28SICI\%2910991557\%28200001/02\%299\%3A1\%3C55\%3A\%3AAID-PDS467\%3E3.0.CO\%3B2-H.

Walsh, E. and National Welsh Informatics Service (NWIS) - Information Services, 2019. Treatment Data - Substance Misuse in Wales 2018-19. Available at: https://gov.wales/sites/default/files/publications/2019-10/treatment-data-substance-misuse-inwales-2018-19.pdf.

Wang, W. and Krishnan, E. 2014. Big Data and Clinicians: A Review on the State of the Science. JMIR medical informatics 2(1), pp. e1-11. Available at: http://www.medinform.jmir.org/2014/1/e1/.

Warraich, H.J. 2021. Why men and women feel pain differently. The Washington Post 15 May. Available at: https://www.washingtonpost.com/health/women-and-pain/2021/05/14/98cb41169d55-11eb-9d05-ae06f4529ece story.html.

Warth, J. et al. 2019. Over-indebtedness and its association with pain and pain medication use. Preventive Medicine Reports 16, p. 100987. Available at: https://www.sciencedirect.com/science/article/pii/S2211335519301585.

Weesie, Y.M. et al. 2020. Use of Opioids Increases with Age in Older Adults: An Observational Study (2005\&ndash;2017)., pp. 1-8. doi: 10.3389/fphar.2020.00648/full. 
Weisberg, D. and Stannard, C. 2013. Lost in translation? Learning from the opioid epidemic in the USA. Anaesthesia 68(12), pp. 1215-1219. doi: 10.1111/anae.12503.

Welsh Analytical Prescribing Support Unit (2013) NHS Wales Primary Care Prescribing Analysis: Tramadol. Cardiff: All Wales Therapeutics and Toxicology Centre. Available at:

https://awmsg.nhs.wales/files/prescribing-analysis/primary-care-prescribing-analysis-tramadolpdf/.

Welsh Assembly Government 2008. Designed for People with Chronic Conditions. Cardiff: Welsh Assembly Government. Available at: http://www.wales.nhs.uk/documents/chronicpaine.pdf.

Welsh Government 2011. Welsh Index of Multiple deprivation 2011., pp. 1-15. Available at: https://gov.wales/welsh-index-multiple-deprivation-full-index-update-ranks-2011.

Welsh Government 2016. Key Industries. Available at: https://tradeandinvest.wales/keyindustries [Accessed: 5 November 2020].

Welsh Government 2019. Welsh Index of Multiple Deprivation 2019. Available at:

https://statswales.gov.wales/Catalogue/Community-Safety-and-Social-Inclusion/Welsh-Index-ofMultiple-Deprivation/WIMD-2019.

Welsh Government 2019a. A Healthier Wales. Welsh Government. Available at: https://gov.wales/sites/default/files/publications/2019-10/a-healthier-wales-action-plan.pdf [Accessed 19 March 2021]

Welsh Government 2019b. Living with Persistent Pain in Wales. Welsh Government. Available at: https://gov.wales/sites/default/files/publications/2019-05/living-with-persistent-pain-inwales.pdf [Accessed: 24 February 2021].

Welsh Government 2021. National Institute for Health and Care Excellence (NICE) guidelines. Available at: https://gov.wales/national-institute-health-and-care-excellence-nice-guidelines [Accessed: 10 August 2021].

Welsh Government and NHS Wales 2016. Prudent Healthcare. Available at: https://gov.wales/sites/default/files/publications/2019-04/securing-health-and-well-being-forfuture-generations.pdf.

Welsh Government and Statistics for Wales 2020. Prescriptions in Wales, 2019-20. Available at: https://gov.wales/sites/default/files/statistics-and-research/2020-09/prescriptions-wales-april2019-march-2020-610.pdf.

Welsh Government and Statistics for Wales 2021. Prescriptions in Wales: interactive dashboard. Available at: https://gov.wales/prescriptions-wales-interactive-dashboard.

Welzel, F.D. et al. 2017. Frequent attenders in late life in primary care: a systematic review of European studies., pp. 1-14. doi: 10.1186/s12875-017-0700-7.

Wen, L.S. and Sadeghi, N.B. 2020. The opioid crisis and the 2020 US election: crossroads for a national epidemic. Lancet 396(10259), pp. 1316-1318. Available at: https://linkinghub.elsevier.com/retrieve/pii/S0140673620321139.

Wenig, C.M. et al. 2009. Costs of back pain in Germany. European Journal of Pain 13(3), pp. 280286. Available at: 
http://eutils.ncbi.nlm.nih.gov/entrez/eutils/elink.fcgi?dbfrom=pubmed\&id=18524652\&retmode= ref\&cmd=prlinks.

Whitehead, M. et al. 2021. Poverty, health, and covid-19. British Medical Journal 372(n376), pp. n376-2. Available at: https://www.bmj.com/content/372/bmj.n376.abstract.

WHO Collaborating Centre for Drug Statistics Methodology 2021. International language for drug utilization research. Available at: https://www.whocc.no [Accessed: 2 September 2021].

Whynes, D.K. 2008. Deprivation and self-reported health: are there "Scottish effects" in England and Wales? Journal of Public Health 31(1), pp. 147-153. doi: 10.1093/pubmed/fdn089.

Wiffen, P.J. et al. 2017. Opioids for cancer pain - an overview of Cochrane reviews. Pain, C. et al. eds. Cochrane Database of Systematic Reviews 168(10), pp. 1102-23. doi: 10.1002/14651858.cd012592.pub2.

Wilkerson, R.G. et al. 2016. The Opioid Epidemic in the United States. Emergency medicine clinics of North America 34(2), pp. e1-e23. Available at:

http://linkinghub.elsevier.com/retrieve/pii/S073386271500108X.

Williams, A.C. de C. 2021. Women's pain is routinely underestimated, and gender stereotypes are to blame - new research. Available at: https://theconversation.com/womens-pain-is-routinelyunderestimated-and-gender-stereotypes-are-to-blame-new-research-158599 [Accessed: 7 July 2021].

Wilsey, B. and Prasad, H. 2012. Real-time access to prescription drug monitoring databases. Canadian Medical Association Journal 184(16), pp. 1767-1768. Available at: http://www.cmaj.ca/cgi/doi/10.1503/cmaj.121495.

Wilson, M.N. et al. 2019. Effectiveness of Prescription Monitoring Programs in Reducing Opioid Prescribing, Dispensing, and Use Outcomes: A Systematic Review. The Journal of Pain 20(12), pp. 1383-1393. Available at: https://doi.org/10.1016/j.jpain.2019.04.007.

Wolf, A. et al. 2019. Data resource profile: Clinical Practice Research Datalink (CPRD) Aurum. International journal of epidemiology 48(6), pp. 1740-1740g. Available at: https://academic.oup.com/ije/article/48/6/1740/5374844.

World Health Organisation 2019. WHO Model Lists of Essentials Medicines. Available at: https://www.who.int/groups/expert-committee-on-selection-and-use-of-essentialmedicines/essential-medicines-lists [Accessed: 2 April 2021].

World Health Organization WHO 2021. WHO's cancer pain ladder for adults. WHO. Available at: http://www.who.int/cancer/palliative/painladder/en/.

Wu, A. et al. 2020. Global low back pain prevalence and years lived with disability from 1990 to 2017: estimates from the Global Burden of Disease Study 2017. Annals of translational medicine 8(6), pp. 299-299. Available at: https://atm.amegroups.com/article/view/38037/html.

Yarborough, B.J.H. et al. 2016. Understanding opioid overdose characteristics involving prescription and illicit opioids: A mixed methods analysis. Drug and alcohol dependence 167, pp. 49-56. Available at: http://linkinghub.elsevier.com/retrieve/pii/S0376871616302216.

Zhang, L. et al. 2021. Gender biases in estimation of others' pain. The Journal of Pain . doi: 10.1016/j.jpain.2021.03.001. 
Zin, C.S. et al. 2014. Changes in trends and pattern of strong opioid prescribing in primary care. European Journal of Pain 18(9), pp. 1343-1351. Available at: https://nottinghamrepository.worktribe.com/output/1100471.

Zingmond, D.S. et al. 2004. Linking hospital discharge and death records-accuracy and sources of bias. Journal of Clinical Epidemiology 57(1), pp. 21-29. Available at: https://linkinghub.elsevier.com/retrieve/pii/S0895435603002506.

Zis, P. et al. 2017. Depression and chronic pain in the elderly: links and management challenges. Clinical Interventions in Aging Volume 12, pp. 709-720. Available at:

https://www.dovepress.com/depression-and-chronic-pain-in-the-elderly-links-and-managementchalle-peer-reviewed-article-CIA 


\section{Appendices}

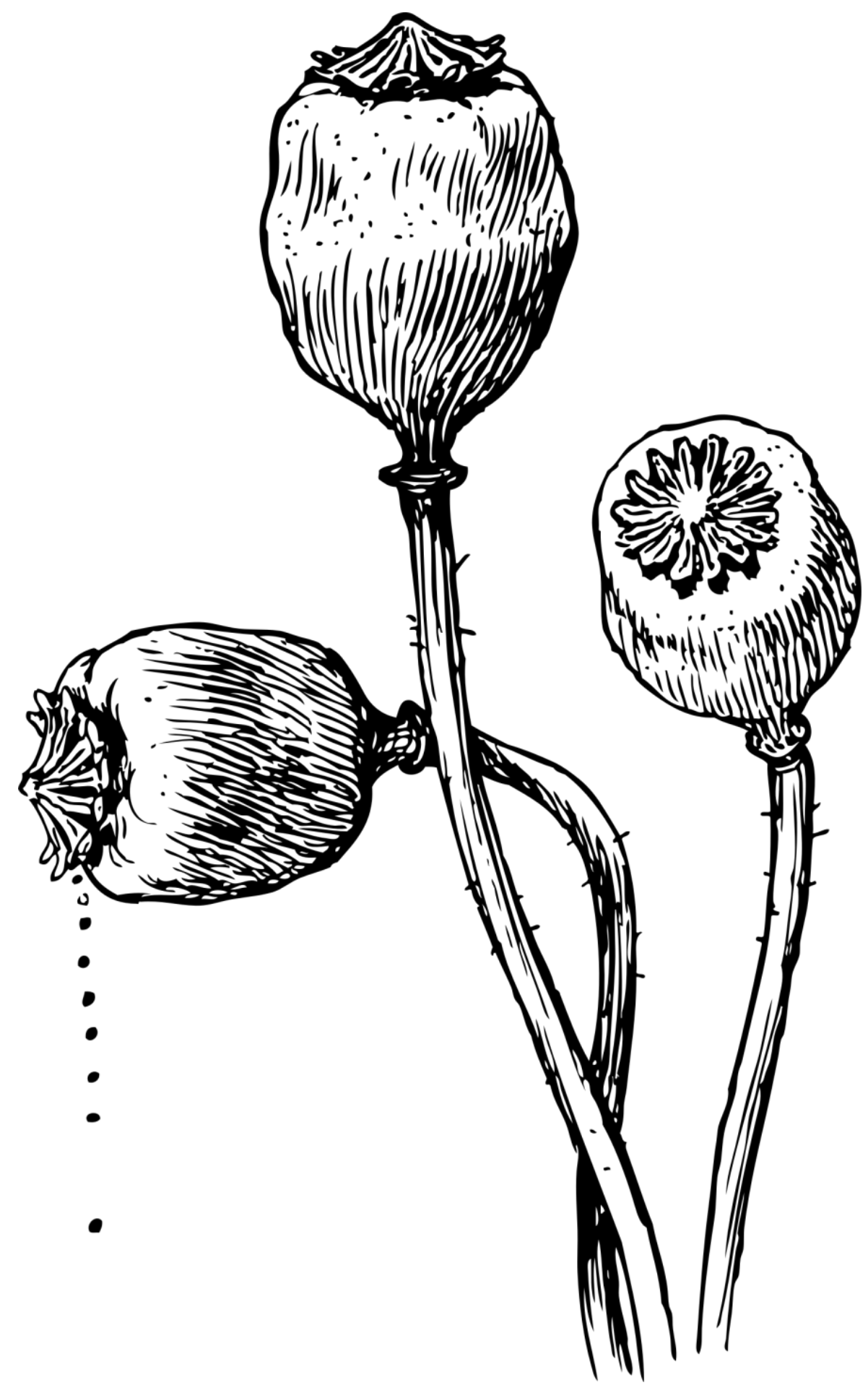




\section{Table of Contents}

List of Appendix tables $\quad 365$

$\begin{array}{ll}\text { List of Appendix figures } & 374\end{array}$

$\begin{array}{ll}\text { Appendix A Methods } & 379\end{array}$

$\begin{array}{ll}\text { A.1 SAIL Application form } & 379\end{array}$

A.2 TOPAS Project specification $\quad 385$

$\begin{array}{ll}\text { A.3 Original study protocol } & 387\end{array}$

A.4 Read codes for opioid analgesics 398

$\begin{array}{ll}\text { A.5 Population estimates } & 408\end{array}$

Appendix B General trends $\quad 412$

B.1.1 Trends in number of people $\quad 412$

B.1.2 Prevalence of people prescribed opioids in SAIL databank population 414

B.2 Prescribing by drug-type $\quad 415$

B.2.1 Trends in number of people by drug-type $\quad 415$

B.3 Trends in prescribing by gender $\quad 418$

B.3.1 Trends in numbers of people by gender $\quad 418$

B.3.2 Trends in numbers of prescriptions by gender $\quad 423$

B.4 Trends in prescribing by age $\quad 424$

B.4.1 Trends in numbers of prescriptions by age-group 433

Appendix C Trends in prescribing by socioeconomic deprivation 436

C.1 Data preparation 436

$\begin{array}{ll}\text { C.2 Results } & 439\end{array}$

C.2.1 Trends in the number of people receiving prescriptions by deprivation $\quad 439$

C.2.2 Trends in number of people receiving prescriptions by opioid-type 443

C.2.3 Number of prescriptions per 1000 quintile-population 447

C.2.4 Gender differences in the number of people receiving prescriptions by deprivation 450

C.2.4.1 All Opioids $\quad 450$

C.2.4.2 Weak opioids $\quad 451$

C.2.4.3 Strong opioids $\quad 454$

C.2.5 Gender differences in the number of prescriptions by deprivation 455

C.2.5.1 Weak opioids $\quad 456$

$\begin{array}{ll}\text { C.2.5.2 Strong opioids } & 458\end{array}$

Appendix D Prescribing trends by drug, oral morphine equivalence 461

D.1 Number of people receiving prescriptions, stratified by drug 461

D.1.1 Annual number of prescriptions by drug $\quad 462$

D.1.2 Annual number of prescriptions per 1000 population by drug 462

D.1.3 Analysis of prescribing rates for opioid analgesics classed as 'other' 464

$\begin{array}{ll}D .2 \text { Oral morphine equivalence } & 467\end{array}$

D.2.1 $\mathrm{OMEQ}_{\mathrm{e}}$ per prescription $\quad 468$

D.2.2 $\mathrm{OMEQ}_{\mathrm{e}}$ for products of greater than $120 \mathrm{mg} \quad 470$

D.3 Gender differences in OMEQ

D.3.1 Gender differences in total OMEQe $\quad 473$

D.3.2 Gender differences in OMEQe per 1000 population $\quad 474$

D.3.3 Gender differences in OMEQe per prescription $\quad 476$

D.3.4 Gender differences for high dose opioid prescription 478 
D.4 $\mathrm{OMEQ}_{e}$ in areas of socioeconomic deprivation 483

D.4.1 Total OMEQe by quintile of deprivation 483

D.4.2 OMEQe per 1000 population and by deprivation quintile 483

D.4.3 OMEQe per prescription and per person $\quad 484$

D.4.4 High dose prescribing by deprivation quintile $\quad 485$

Appendix E Time Series Analysis examining the effect of legislation and prescribing guidance on $\begin{array}{ll}\text { opioid prescribing in Wales } & 487\end{array}$

E.1 Schedules of controlled drugs in the UK

$\begin{array}{ll}\text { E.1.1 Schedule } 1 \text { controlled drugs } & 487\end{array}$

E.1.2 Schedule 2 controlled drugs $\quad 489$

E.1.3 Schedule 3 controlled drugs $\quad 491$

E.1.4 Schedule 4 Part I controlled drugs $\quad 491$

Part ii controlled drugs $\quad 492$

E.1.5 Schedule 5 controlled drugs $\quad 494$

E.2 Interrupted time series $\quad 494$

E.2.1 Output by gender $\quad 494$

E.2.2 Continuous time series analysis $\quad 495$

E.2.2.1 Weak opioids $\quad 496$

$\begin{array}{ll}\text { E.2.2.2 Strong opioids } & 497\end{array}$

E.2.2.3 Gender 498

$\begin{array}{ll}\text { E.2.3 Deprivation } & 499\end{array}$

$\begin{array}{ll}\text { E.3 Predictions } & 507\end{array}$

$\begin{array}{ll}\text { E.3.1 Predictions for weak opioid prescribing } & 507\end{array}$

E.3.2 Predictions for strong opioid prescribing $\quad 508$

E.3.3 Predictions by gender $\quad 509$

E.3.4 Predictions by deprivation $\quad 511$

E.3.5 Prescribing by Health Board 513

E.4 Predictions for Health Board prescribing $\quad 517$

Appendix $\mathrm{F}$ Healthcare resource utilisation associated with opioid prescribing and associated cost analysis

F.1 Metadata $\quad 520$

F.2 Readcodes $\quad 524$

F.3 Two-way ANOVA for Healthcare utilisation 546

F.4 Mann-Whitney and Kruskall-Wallis results $\quad 548$

$\begin{array}{ll}\text { F.4.1 Primary care general practice } & 548\end{array}$

F.4.1.1 Primary care general practice attendance by prescribing persistence $\quad 548$

F.4.1.2 Primary care general practice attendance by gender $\quad 548$

F.4.1.3 Primary Care General Practice attendance by deprivation status $\quad 549$

F.4.1.4 Primary care General Practice attendance by age-group $\quad 549$

F.4.1.5 Tests and imaging from Primary Care $\quad 550$

$\begin{array}{ll}\text { F.4.2 Out-Patient attendance } & 550\end{array}$

F.4.2.1 Out-patient attendance by prescribing persistence $\quad 550$

F.4.2.2 Out-patient attendance by gender $\quad 550$

F.4.2.3 Out-patient attendance by deprivation $\quad 550$

F.4.2.4 Out-patient attendance by age-group $\quad 551$

F.4.3 Emergency Department Utilisation $\quad 551$

F.4.3.1 Emergency department attendance by prescription persistence $\quad 551$

F.4.3.2 Emergency department attendance by gender 551

F.4.3.3 Emergency Department attendance by deprivation $\quad 551$

F.4.3.4 Emergency Department attendance by age-group $\quad 552$

F.4.4 Inpatient attendance $\quad 552$ 
F.4.4.1 In-Patient attendance and length of stay 552

F.4.4.2 In-patient utilisation by prescription persistence $\quad 552$

F.4.4.3 In-patient utilisation by gender $\quad 552$

F.4.4.4 In-patient utilisation by deprivation $\quad 553$

F.4.4.5 In-patient utilisation by age-group 553

F.5 Example of outpatient unit costs for cost-analysis $\quad 555$

$\begin{array}{ll}\text { F.6 Threshold analysis } & 558\end{array}$

Appendix G Prescribing Persistence $\quad 560$

G.1 Descriptive annual data for prescribing persistence $\quad 560$

G.1.1 Annual descriptive data for prescribing persistence greater than 31 days 562

G.2 Prescribing persistence by gender $\quad 565$

G.2.1 Analysis by gender differences $\quad 565$

G.2.2 Extended durations of prescribing persistence by gender 566

G.3 Analysis by age-group $\quad 567$

G.3.1.1 Extended durations of prescribing persistence by age-group $\quad 574$

G.4 Prescribing persistence by deprivation $\quad 575$

G.4.1.1 Extended durations of prescribing persistence by deprivation 576

G.5 Prescribing persistence by opioid type $\quad 581$

G.5.1.1 Extended durations of prescribing persistence by drug 587

Appendix H Prescribing trends stratified by recorded diagnosis of depression and anxiety $\mathbf{5 9 0}$

H.1 Read codes for depression and anxiety 590

$\begin{array}{ll}H .2 \text { Results } & 606\end{array}$

H.2.1 Trends in the number of people receiving prescriptions 606

H.2.2 Trends in the number of prescriptions $\quad 609$

H.2.3 Trends in the number of people receiving prescriptions by gender 612

H.2.4 Trends in the number of prescriptions by gender 616

Appendix I Results stratified by Health Board $\quad 621$

$\begin{array}{ll}\text { l.1 Health Board level data } & 621\end{array}$

I.2 Opioid prescribing by Health Board $\quad 621$

$\begin{array}{ll}\text { I.2.1 Total opioid prescribing } & 621\end{array}$

$\begin{array}{ll}\text { I.2.2 Number of prescriptions issued } & 623\end{array}$

$\begin{array}{ll}\text { I.2.3 Types of opioids being prescribed } & 628\end{array}$

I.3 Opioid prescribing by deprivation per Health Board 630

$\begin{array}{ll}\text { I.3.1 Prescriptions by deprivation area } & 638\end{array}$

I.3.2 Weak opioid prescribing by deprivation $\quad 644$

I.3.3 Strong opioid prescribing by deprivation $\quad 651$

$\begin{array}{ll}\text { I.3.4 Log-linear regression } & 658\end{array}$

I.4 Abertawe Bro Morgannwg University Health Board 660

I.5 Aneurin Bevan Local Health Board 662

I.6 Betsi Cadwaladr University Health Board $\quad 665$

I.7 Cardiff and Vale University Health Board 667

I.8 Cwm Taf University Health Board $\quad 670$

I.9 Hywel Dda Local Health Board $\quad 672$

$\begin{array}{ll}\text { I.10 Powys Teaching Health Board } & 675\end{array}$ 


\section{List of Appendix tables}

Table A.2: Read codes for weak opioid analgesics including the original list shared from Nottingham University

Table A.3: Read codes for strong opioid analgesics including the original list shared from

Nottingham University

401

Table A.4: Calculating population estimates per Health Board

Table A.5: Percentage of each Health Board primary care population represented within SAIL

databank

Table A.6: Calculated annual SAIL databank population per Health Board 408

Table A.7: Health Board populations per 1000 population

Table A.8: SAIL population - based on $78 \%$ of Welsh primary care population represented within the Databank

Table A.9: SAIL population by gender

Table A.10: Percentage gender split by Health Board

Table A.11: Health Board populations (aged 18 years and over) by gender and age-group 410

Table A.12: SAIL databank population per 1000 by age-group

Table A.13: Annual populations by gender and by Health Board, based on percentage gender split

Table B.1: Trends in the number of people receiving opioid prescriptions, 2005 - 2015 and stratified by opioid type

Table B.2: Determining prevalence of opioid prescribing in the SAIL databank population

Table B.3: Annual changes in opioid prescribing, stratified by opioid group

Table B.4: Trends in the number of men receiving opioid prescriptions 2005 - 2015

Table B.5: Trends in the number of women receiving opioid prescriptions $2005-2015$

Table B.6: Trends in the number of prescriptions issued to men and by opioid type

Table B.7: Trends in the number of prescriptions issued to women and by opioid type

Table B.8: Trends in the number of people receiving opioid prescriptions stratified by age group

Table B.9: Dunn's pairwise comparisons and Bonferroni correction output for people per 1000 population receiving opioid prescriptions, stratified by age-group

Table B.10: Trends in the number of people receiving weak opioid prescriptions, stratified by age group

Table B.11: Dunn's pairwise comparisons and Bonferroni correction output for people per 1000 population receiving weak opioid prescriptions, stratified by age-group

Table B.12: Trends in the number of people receiving strong opioid prescriptions, stratified by age group

Table B.13: Dunn's pairwise comparisons and Bonferroni correction output for people per 1000

population receiving strong opioid prescriptions, stratified by age-group

Table B.14: Trends in the total number of opioid prescriptions stratified by age group

Table B.15: Dunn's pairwise comparisons and Bonferroni correction output for all opioid prescriptions per 1000 population, stratified by age-group

Table B.16: Trends in the number of weak opioid prescriptions, stratified by age group

Table B.17: Dunn's pairwise comparisons and Bonferroni correction output for weak opioid prescriptions per 1000 population, stratified by age-group

Table B.18: Trends in the number strong opioid prescriptions, stratified by age group

Table B.19: Dunn's pairwise comparisons and Bonferroni correction output for strong opioid prescriptions per 1000 population, stratified by age-group

Table C.1: Example of LSOA data used to determine Health Board WIMD population estimates 436 Table C.2: Welsh Country population data for 2011. Designated Health Board indicated Table C.3: Population calculations for each Health Board using mid-year estimates and adjusted to SAIL percentage representation per Health Board

Table C.4: Estimated populations per 1000 WIMD quintile per Health Board and total for Wales based on SAIL population estimates 
Table C.5: Annual number of people receiving opioid prescriptions per socio-economic deprivation quintile between 2005 -2015

Table C.6: Comparison of quintile-population adjusted numbers of people receiving prescriptions for all opioid medicines by Welsh Index of Multiple Deprivation quintiles across Wales between 2005 and 2015

Table C.7: Dunn's pairwise comparison and Bonferroni post-hoc analysis of difference between the number people receiving opioid prescriptions in the 5 different areas of deprivation as defined by the Welsh Index of Multiple Deprivation (WIMD)

Table C.8: Dunn's pairwise comparison and Bonferroni post-hoc analysis of difference between the number people per 1000 population receiving opioid prescriptions in the 5 different areas of deprivation as defined by the Welsh Index of Multiple Deprivation (WIMD)

Table C.9: Annual number of people receiving opioid prescriptions per socio-economic deprivation quintile and opioid-type between 2005 -2015

Table C.10: Comparison of annual numbers of people receiving weak opioid prescriptions between 2005 and 2015 by deprivation area (Population adjusted data)

Table C.11: Dunn's pairwise comparison and Bonferroni post-hoc analysis of difference between the number people per 1000 receiving weak opioid prescriptions in the 5 different areas of deprivation as defined by the Welsh Index of Multiple Deprivation (WIMD)

Table C.12: Comparison of annual numbers of people receiving strong opioid prescriptions between 2005 and 2015 by deprivation area (Population adjusted data)

Table C.13: Dunn's pairwise comparison and Bonferroni post-hoc analysis of difference between the number people per 1000 receiving strong opioid prescriptions in the 5 different areas of deprivation as defined by the Welsh Index of Multiple Deprivation (WIMD)

Table C.14: Comparison of numbers of prescriptions for all opioid medicines by Welsh Index of

Multiple Deprivation quintiles across Wales between 2005 and 2015

Table C.15: Comparison of quintile-population adjusted numbers of prescriptions for all opioid medicines by Welsh Index of Multiple Deprivation quintiles across Wales between 2005 and 2015

Table C.16: Dunn's pairwise comparison and Bonferroni post-hoc analysis of difference between the number of opioid prescriptions per 1000 population in the 5 different areas of deprivation as defined by the Welsh Index of Multiple Deprivation (WIMD)

Table C.17: Comparison of numbers of prescriptions for weak opioid medicines by Welsh Index of Multiple Deprivation quintiles across Wales between 2005 and 2015

Table C.18: Comparison of quintile-population adjusted numbers of prescriptions for weak opioid medicines by Welsh Index of Multiple Deprivation quintiles across Wales between 2005 and 2015

Table C.19: Dunn's pairwise comparison and Bonferroni post-hoc analysis of difference between the number of weak opioid prescriptions per 1000 population in the 5 different areas of deprivation as defined by the Welsh Index of Multiple Deprivation (WIMD)

Table C.20: Comparison of numbers of prescriptions for strong opioid medicines by Welsh Index

of Multiple Deprivation quintiles across Wales between 2005 and 2015

Table C.21: Comparison of quintile-population adjusted numbers of prescriptions for strong opioid medicines by Welsh Index of Multiple Deprivation quintiles across Wales between 2005 and 2015

Table C.22: Dunn's pairwise comparison and Bonferroni post-hoc analysis of difference between the number of strong opioid prescriptions per 1000 population in the 5 different areas of deprivation as defined by the Welsh Index of Multiple Deprivation (WIMD)

Table C.23: Comparison of annual numbers of people by gender receiving strong opioid prescriptions between 2005 and 2015 by deprivation area (Population adjusted data)

Table C.24: Annual number of people per 1000 population receiving weak opioid prescriptions per deprivation quintile and gender

Table C.25: Annual number of people per 1000 population receiving strong opioid prescriptions per deprivation quintile and gender

Table C.26: Comparison of numbers of prescriptions for all opioid medicines by Welsh Index of Multiple Deprivation quintiles and gender across Wales between 2005 and 2015 
Table C.27: Comparison of quintile-population adjusted numbers of prescriptions for all opioid medicines by Welsh Index of Multiple Deprivation quintiles and gender across Wales between 2005 and 2015

Table C.28: Dunn's pairwise comparison and Bonferroni post-hoc analysis of difference between the number of all opioid prescriptions per 1000 population, issued to men in the 5 different areas of deprivation as defined by the Welsh Index of Multiple Deprivation (WIMD)

Table C.29: Dunn's pairwise comparison and Bonferroni post-hoc analysis of difference between the number of all opioid prescriptions per 1000 population, issued to women in the 5 different areas of deprivation as defined by the Welsh Index of Multiple Deprivation (WIMD)

Table C.30: Annual weak opioid prescriptions issued per socioeconomic deprivation quintile and gender

Table C.31: Comparison of quintile-population adjusted numbers of prescriptions for weak opioid medicines by Welsh Index of Multiple Deprivation quintiles and gender across Wales between 2005 and 2015

Table C.32: Dunn's pairwise comparison and Bonferroni post-hoc analysis of difference between the number of weak opioid prescriptions per 1000 population, issued to men in the 5 different areas of deprivation as defined by the Welsh Index of Multiple Deprivation (WIMD)

Table C.33: Dunn's pairwise comparison and Bonferroni post-hoc analysis of difference between the number of weak opioid prescriptions per 1000 population, issued to women in the 5 different areas of deprivation as defined by the Welsh Index of Multiple Deprivation (WIMD) 458 Table C.34: Annual strong opioid prescriptions issued per deprivation quintile and gender 458 Table C.35: Comparison of quintile-population adjusted numbers of prescriptions for strong opioid medicines by Welsh Index of Multiple Deprivation quintiles and gender across Wales between 2005 and 2015

Table C.36: Dunn's pairwise comparison and Bonferroni post-hoc analysis of difference between the number of strong opioid prescriptions per 1000 population, issued to men in the 5 different areas of deprivation as defined by the Welsh Index of Multiple Deprivation (WIMD) 460 Table C.37: Dunn's pairwise comparison and Bonferroni post-hoc analysis of difference between the number of strong opioid prescriptions per 1000 population, issued to women in the 5 different areas of deprivation as defined by the Welsh Index of Multiple Deprivation (WIMD) 460

Table D.1: Trends in the number of people receiving prescriptions 2005 - 2015, stratified by drug 461

Table D.2: Trends in the number of people per 1000 population, receiving opioid analgesic prescriptions 2005 - 2015, stratified by drug

Table D.3: Trends in the number of prescriptions issued 2005 - 2015, stratified by drug

Table D.4: Trends in the number of opioid prescriptions per 1000 population $2005-2015$, stratified by drug

Table D.5: Dunn's pairwise tests with Bonferroni corrections demonstrating relationship between the number of opioid prescriptions, examined by which drug was prescribed 464 Table D.6: Trends in the number of prescriptions issued for drugs classified as 'other', 2005-2015

Table D.7: Trends in the annual number of prescriptions for 'other' opioids 2005 - 2015. Data adjusted to number per 1000 population

Table D.8: Dunn's pairwise tests with Bonferroni corrections demonstrating relationship between the number of opioid prescriptions, examined by which drug was prescribed Table D.9: Daily oral morphine equivalent dose (milligrams) issued on prescription, given as annual totals, and stratified by drug

Table D.10: Daily oral morphine equivalent dose (milligrams) issued on prescription, adjusted to 1000population, stratified by drug

Table D.11: Oral morphine equivalent dose per prescription issued for stated drug and subsequent percentage change in OMEQe per prescription between 2005 and 2015, in addition to the number of prescriptions issued per 1000 population and percentage change over the study period 468

Table D.12: Dunn's pairwise tests with Bonferroni corrections demonstrating relationships between the daily oral morphine equivalent doses per prescription, by drug 
Table D.13: Trends in annual total oral morphine equivalent dose per 1000 population, stratified by drug and rate change for 2005 - 2015

Table D.14: Dunn's pairwise tests with Bonferroni corrections demonstrating relationships between the daily oral morphine equivalent doses per prescription for high-dose prescriptions (>120 milligrams OMEQe), by drug

Table D.15: Trends in annual totals for preparations of $120 \mathrm{mg}$ or greater oral morphine equivalent dose in milligrams per prescription, stratified by drug and with rate change for 2005 2015

Table D.16: Trends in Total Annualised Oral Morphine Equivalent Doses (milligrams) issued by gender. $p$-values calculated using Mann-Whitney tests for each drug

Table D.17: Dunn's pairwise tests with Bonferroni corrections demonstrating relationships between the annualised daily oral morphine equivalent doses prescribed to men, by drug Table D.18: Dunn's pairwise tests with Bonferroni corrections demonstrating relationships between the annualised daily oral morphine equivalent doses prescribed to women, by drug 474 Table D.19: Trends in Total Annualised Oral Morphine Equivalent Doses (milligrams) per 1000 population issued by gender. p-values calculated using Mann-Whitney tests for each drug Table D.20: Dunn's pairwise tests with Bonferroni corrections demonstrating relationships between the annualised daily oral morphine equivalent doses prescribed to men per 1000 population, by drug

Table D.21: Dunn's pairwise tests with Bonferroni corrections demonstrating relationships between the annualised daily oral morphine equivalent doses prescribed to women per 1000 population, by drug

Table D.22: Trends in oral morphine equivalent dose (milligrams) per prescription issued and by drug, stratified by gender. p-values calculated using Mann-Whitney tests

Table D.23: Dunn's pairwise tests with Bonferroni corrections demonstrating relationships between the annualised daily oral morphine equivalent doses prescribed per prescription to men, by drug

Table D.24: Dunn's pairwise tests with Bonferroni corrections demonstrating relationships between the annualised daily oral morphine equivalent doses prescribed per prescription to women, by drug

Table D.25: Gender trends for preparations of $120 \mathrm{mg}$ or greater oral morphine equivalent dose in milligrams

Table D.26: Gender trends in annual totals for preparations of $120 \mathrm{mg}$ or greater oral morphine equivalent dose in milligrams and adjusted to oral morphine equivalent dose per 1000 genderadjusted population, stratified by drug and with percentage and rate change for 2005 - 2015479 Table D.27: Gender trends for preparations of $120 \mathrm{mg}$ or greater oral morphine equivalent dose per 1000 gender-adjusted population, stratified by drug and with rate change

Table D.28: Trends in total oral morphine equivalent dose prescribed stratified by deprivation (Welsh Index of Multiple Deprivation) and adjusted to population.

Table D.29: Dunn's pairwise comparison and Bonferroni post-hoc analysis of difference between the oral morphine equivalent doses prescribed in the 5 different areas of deprivation as defined by the Welsh Index of Multiple Deprivation (WIMD)

Table D.30: Trends in total oral morphine equivalent dose prescribed stratified by deprivation (Welsh Index of Multiple Deprivation) and adjusted to population

Table D.31: Dunn's pairwise comparison and Bonferroni post-hoc analysis of difference between the oral morphine equivalent doses per 1000 population prescribed in the 5 different areas of deprivation as defined by the Welsh Index of Multiple Deprivation (WIMD)

Table D.32: Trends in oral morphine equivalence stratified by deprivation (Welsh Index of Multiple Deprivation WIMD2011) and examined by dose per prescription and dose per person

Table D.33: Dunn's pairwise comparison and Bonferroni post-hoc analysis of difference between the number people receiving opioid prescriptions in the 5 different areas of deprivation as defined by the Welsh Index of Multiple Deprivation (WIMD)

Table D.34: Trends in total oral morphine equivalent dose of 120 milligrams or higher prescribed stratified by deprivation (Welsh Index of Multiple Deprivation) 
Table D.35: Dunn's pairwise comparison and Bonferroni post-hoc analysis of difference between OMEQe from products with $>120 \mathrm{mg}$ OMEQe daily dose, in the 5 different areas of deprivation as defined by the Welsh Index of Multiple Deprivation (WIMD)

Table D.36: Trends in total oral morphine equivalent dose of 120 milligrams or higher prescribed stratified by deprivation (Welsh Index of Multiple Deprivation) and adjusted to deprivation quintile population

Table D.37: Dunn's pairwise comparison and Bonferroni post-hoc analysis of difference between OMEQe from products with $>120 \mathrm{mg}$ OMEQe daily dose per 1000 population, in the 5 different areas of deprivation as defined by the Welsh Index of Multiple Deprivation (WIMD)

Table E.1: Output from testing for best-fit model of interrupted time series analysis for men (all opioids)

Table E.2: Results of interrupted time series analysis of opioid prescribing data for men 495 Table E.3: Output from testing for best-fit model of interrupted time series analysis for women (all opioids)

Table E.4: Results of interrupted time series analysis of opioid prescribing data for women 495

Table E.5: Example Time Series Analysis calculations

Table E.6: Comparison of predicted trends in weak opioid prescribing using trendline analysis 508 Table E.7: Comparison of predicted trends in strong opioid prescribing using trendline analysis

Table E.8: Comparison of predicted trends in opioid prescribing for female patients using trendline analysis

Table E.9: Comparison of predicted trends in opioid prescribing for male patients using trendline analysis

Table E.10: Comparison of predicted trends in opioid prescribing for Health Boards using trendline analysis

Table E.11: Comparison of predicted trends in opioid prescribing for Health Boards using trendline analysis

Table F.1: Metadata used to extract data from main table of Phase 2 study using SQL coding or identifying columns in table

Table F.2: Read codes for pain-related conditions used to extract case and control subjects $\quad 522$ Table F.3: Comparison of Primary Care General Practice utilisation depending on receipt of opioid prescriptions (case versus control)

Table F.4: Comparison of out-patient resource utilisation depending on receipt of opioid prescriptions (case versus control)

Table F.5: Comparison of Emergency department resource utilisation depending on receipt of opioid prescriptions (case versus control)

Table F.6: Comparison of In-patient admission and length of stay depending on receipt of opioid prescriptions (case versus control)

Table F.7: Example calculation for weighting unit costs using National Unit costs for Emergency

Department attendance in 2015

Table F.8: Output of threshold analysis for healthcare services costs using changes in case subject costs. p-values attained from Mann-Whitney analysis case versus control subjects.

Table G.1: Annualised prescribing persistence data, full breakdown

Table G.2: Descriptive data with all single, not repeated within 31-day events removed 560

Table G.3: Trends in prescription persistence (in days), stratified by gender per 1000 gender-

adjusted population

Table G.4: Trends in prescribing persistence (in days), stratified by age group.

Table G.5: Dunn's pairwise comparisons and Bonferroni correction output for average prescribing duration of all prescribing events $2005-2015$, stratified by age-group

Table G.6: Dunn's pairwise comparisons and Bonferroni correction output for average prescribing duration for events with at least two prescriptions issued per individual, 2005 - 2015, stratified 
Table G.7: Dunn's pairwise comparisons and Bonferroni correction output for numbers of events with prescribing persistence between 3 and 6 months per 1000 population, $2005-2015$, stratified by age-group

Table G.8: Combined prescribing persistence descriptive data 2005 - 2015, stratified by age group

Table G.9: Dunn's pairwise comparisons and Bonferroni correction output for numbers of events with prescribing persistence greater than 6 months, 2005 - 2015, stratified by age-group 573 Table G.10: Dunn's pairwise comparisons and Bonferroni correction output for numbers of events with prescribing persistence greater than 6 months per 1000 population, $2005-2015$, stratified by age-group

Table G.11: Dunn's pairwise comparison and Bonferroni post-hoc analysis of mean prescribing persistence (days), in the 5 different areas of deprivation as defined by the Welsh Index of Multiple Deprivation (WIMD)

Table G.12: Dunn's pairwise comparison and Bonferroni post-hoc analysis of mean prescribing persistence (days) when events not repeated within 31 days were removed from analysis, in the 5 different areas of deprivation as defined by the Welsh Index of Multiple Deprivation (WIMD)

Table G.13: Dunn's pairwise comparison and Bonferroni post-hoc analysis of average number of events with a prescribing persistence between $3-6$ months, in the 5 different areas of deprivation as defined by the Welsh Index of Multiple Deprivation (WIMD)

Table G.14: Dunn's pairwise comparison and Bonferroni post-hoc analysis for number of events per 1000 population with prescribing persistence between 3 - 6 months, in the 5 different areas of deprivation as defined by the Welsh Index of Multiple Deprivation (WIMD)

Table G.15: Trends in prescribing persistence between 2005 and 2015, stratified by Welsh Index of Multiple Deprivation (WIMD) quintile where WIMD1 = most deprived and WIMD5 = least deprived

Table G.16: Dunn's pairwise comparison and Bonferroni post-hoc analysis of average number of events with a prescribing persistence greater than 6 months, in the 5 different areas of deprivation as defined by the Welsh Index of Multiple Deprivation (WIMD)

Table G.17: Dunn's pairwise comparison and Bonferroni post-hoc analysis for number of events per 1000 population with prescribing persistence greater than 6 months, in the 5 different areas of deprivation as defined by the Welsh Index of Multiple Deprivation (WIMD) 579

Table G.18: Prescribing persistence stratified by drug, totalled for 2005 - 2015

Table G.19: Dunn's pairwise tests with Bonferroni corrections demonstrating relationships between average prescribing persistence (days) by drug for all prescriptions

Table G.20: Dunn's pairwise tests with Bonferroni corrections demonstrating relationships between average prescribing persistence (days) by drug where events not repeated within 31 days were removed from analysis

Table G.21: Trends in prescription persistence between 2005 and 2015, stratified by drug group

Table G.22: Dunn's pairwise tests with Bonferroni corrections demonstrating relationships between average prescribing persistence (days) by drug and where prescribing persistence was between 3 and 6 months

Table G.23: Dunn's pairwise tests with Bonferroni corrections demonstrating relationships between average prescribing persistence (days) by drug and where prescribing persistence was over 6 months

Table G.24: Dunn's pairwise tests with Bonferroni corrections demonstrating relationships between average prescribing persistence (days) by drug and where prescribing persistence was between 3 and 6 months

Table G.25: Dunn's pairwise tests with Bonferroni corrections demonstrating relationships between average prescribing persistence (days) by drug and where prescribing persistence was over 6 months 
Table H.3: Annual trends in the number of opioid prescriptions with or without a recorded diagnosis of depression and/or anxiety. Data adjusted to number per 1000 sex-adjusted population

Table H.4: Sex differences in prescribing of weak and strong opioids in people with and without recorded diagnoses of depression/anxiety. Data adjusted to people per 1000 sex-adjusted population

Table H.5: Gender differences in prescription trends of weak and strong opioids with and without recorded diagnoses of depression/anxiety. Data adjusted to prescriptions per 1000 sex-adjusted population

Table I.1: Dunn's pairwise comparison and Bonferroni post-hoc analysis of difference between the number of people receiving opioid prescriptions by Welsh Health Board between 2005 and 2015

Table I.2: Trends in the annual number of people receiving opioid prescriptions in each Health Board in Wales and compared to the National Average (All Wales). Percentage change over the 11 year study period included. Data population adjusted per Health Board (number of people per 1000 population)

Table I.3: Dunn's pairwise comparison and Bonferroni post-hoc analysis of difference between the number of opioid prescriptions being issued by Welsh Health Board between 2005 and 2015

Table I.4: Trends in the annual number of opioid prescriptions in each Health Board in Wales and compared to the National Average (All Wales). Rate change over the 11 year study period included. Data population adjusted per Health Board (number of people per 1000 population)

Table I.5: Trends in the number of opioid prescriptions per 1000 population, issued in each Health Board. Adjusted to annual Health Board population

Table I.6: Dunn's pairwise comparison and Bonferroni post-hoc analysis of difference between the number of weak opioid prescriptions being issued by Welsh Health Board between 2005 and 2015

Table I.7: Dunn's pairwise comparison and Bonferroni post-hoc analysis of difference between the number of people receiving opioid prescriptions being issued in WIMD1 areas (most deprived) by Welsh Health Board between 2005 and 2015

Table I.8: Dunn's pairwise comparison and Bonferroni post-hoc analysis of difference between the number of people receiving opioid prescriptions being issued in WIMD2 areas by Welsh Health Board between 2005 and 2015

Table I.9: Trends in the annual number of people receiving opioid prescriptions in each Health Board in Wales by Welsh Index of Multiple Deprivation (WIMD) area

Table I.10: Dunn's pairwise comparison and Bonferroni post-hoc analysis of difference between the number of people receiving opioid prescriptions being issued in WIMD3 areas by Welsh Health Board between 2005 and 2015

Table I.11: Dunn's pairwise comparison and Bonferroni post-hoc analysis of difference between the number of people receiving opioid prescriptions being issued in WIMD4 areas by Welsh Health Board between 2005 and 2015

Table I.12: Dunn's pairwise comparison and Bonferroni post-hoc analysis of difference between the number of people receiving opioid prescriptions being issued in WIMD4 areas by Welsh Health Board between 2005 and 2015

Table I.13: Dunn's pairwise comparison and Bonferroni post-hoc analysis of difference between the number of opioid prescriptions being issued in WIMD1 areas by Welsh Health Board between 2005 and 2015

Table I.14: Dunn's pairwise comparison and Bonferroni post-hoc analysis of difference between the number of opioid prescriptions being issued in WIMD2 areas by Welsh Health Board between 2005 and 2015

Table I.15: Trends in the annual number of opioid prescriptions in each Health Board in Wales by Welsh Index of Multiple Deprivation (WIMD) area. WIMD1=most deprived and WIMD5=least deprived area. Rate change over the 11-year study period included. Data population adjusted per Health Board (number of prescriptions per 1000 population) 
Table I.16: Dunn's pairwise comparison and Bonferroni post-hoc analysis of difference between the number of opioid prescriptions being issued in WIMD3 areas by Welsh Health Board between 2005 and 2015

Table I.17: Dunn's pairwise comparison and Bonferroni post-hoc analysis of difference between the number of opioid prescriptions being issued in WIMD4 areas by Welsh Health Board between 2005 and 2015

Table I.18: Dunn's pairwise comparison and Bonferroni post-hoc analysis of difference between the number of opioid prescriptions being issued in WIMD5 areas by Welsh Health Board between 2005 and 2015

Table I.19: Trends in the annual number of weak opioid prescriptions in each Health Board in Wales by Welsh Index of Multiple Deprivation (WIMD) area

Table I.20: Dunn's pairwise comparison post-hoc analysis of difference between the number of weak opioid prescriptions being issued in WIMD1 areas by Welsh Health Board between 2005 and 2015

Table I.21: Dunn's pairwise comparison post-hoc analysis of difference between the number of weak opioid prescriptions being issued in WIMD2 areas by Welsh Health Board between 2005 and 2015

Table I.22: Dunn's pairwise comparison post-hoc analysis of difference between the number of weak opioid prescriptions being issued in WIMD3 areas by Welsh Health Board between 2005 and 2015

Table I.23: Dunn's pairwise comparison post-hoc analysis of difference between the number of weak opioid prescriptions being issued in WIMD4 areas by Welsh Health Board between 2005 and 2015

Table I.24: Dunn's pairwise comparison post-hoc analysis of difference between the number of weak opioid prescriptions being issued in WIMD5 areas by Welsh Health Board between 2005 and 2015

Table 1.25: Trends in the annual number of weak opioid prescriptions in each Health Board in Wales by Welsh Index of Multiple Deprivation (WIMD) area. WIMD1=most deprived and WIMD5=least deprived area. Rate change over the 11-year study period included

Table I.26: Dunn's pairwise comparison post-hoc analysis of difference between the number of strong opioid prescriptions being issued in WIMD1 areas by Welsh Health Board between 2005 and 2015

Table I.27: Dunn's pairwise comparison post-hoc analysis of difference between the number of strong opioid prescriptions being issued in WIMD2 areas by Welsh Health Board between 2005 and 2015

Table I.28: Dunn's pairwise comparison post-hoc analysis of difference between the number of strong opioid prescriptions being issued in WIMD3 areas by Welsh Health Board between 2005 and 2015

Table I.29: Dunn's pairwise comparison post-hoc analysis of difference between the number of strong opioid prescriptions being issued in WIMD4 areas by Welsh Health Board between 2005 and 2015

Table I.30: Dunn's pairwise comparison post-hoc analysis of difference between the number of strong opioid prescriptions being issued in WIMD5 areas by Welsh Health Board between 2005 and 2015

Table I.31: Logistic regression for strong opioid prescribing in ABMUHB 656

Table I.32: Logistic regression for strong opioid prescribing in ABUHB 656

Table I.33: Logistic regression for strong opioid prescribing in BCUHB 656

Table I.34: Logistic regression for strong opioid prescribing in CVUHB 657

Table I.35: Logisitic regression for strong opioid prescribing in CTUHB 657

Table I.36: Logistic regression for strong opioid prescribing in PTHB 658

Table 1.37: Annual change in the number of people per 1000 population receiving opioid prescriptions and annual change rate (percentage change) in Abertawe Bro Morgannwg University Health Board (ABMUHB)

Table I.38: Changes in the number of opioid prescriptions issued by deprivation area within Abertawe Bro Morgannwg University Health Board between 2005 and 2015 
Table I.39: Annual change in the number of people per 1000 population receiving opioid prescriptions and annual change rate (percentage change) in Aneurin Bevan University Health Board (ABUHB)

Table I.40: Changes in the number of opioid prescriptions issued by deprivation area within Aneurin Bevan University Health Board between 2005 and 2015

Table I.41: Annual change in the number of people per 1000 population receiving opioid prescriptions and annual change rate (percentage change) in Betsi Cadwaladr University Health Board (BCUHB)

Table I.42: Changes in the number of opioid prescriptions issued by deprivation area within Betsi

Cadwaladr University Health Board between 2005 and 2015

Table I.43: Annual change in the number of people per 1000 population receiving opioid prescriptions and annual change rate (percentage change) in Cardiff and Vale University Health Board (CVUHB)

Table I.44: Changes in the number of opioid prescriptions issued by deprivation area within Cardiff and Vale University Health Board between 2005 and 2015

Table 1.45: Annual change in the number of people per 1000 population receiving opioid prescriptions and annual change rate (percentage change) in Cwm Taf University Health Board (CTUHB) 668

Table I.46: Changes in the number of opioid prescriptions issued by deprivation area within Cwm Taf University Health Board between 2005 and 2015

Table 1.47: Annual change in the number of people per 1000 population receiving opioid prescriptions and annual change rate (percentage change) in Hywel Dda University Health Board (HDUHB)

Table 1.48: Changes in the number of opioid prescriptions issued by deprivation area within Cwm Taf University Health Board between 2005 and 2015

able I.49: Annual change in the number of people per 1000 population receiving opioid prescriptions and annual change rate (percentage change) in Powys Teaching Health Board (PTHB) 673

Table I.50: Changes in the number of opioid prescriptions issued by deprivation area within Powys Teaching Board between 2005 and 2015 


\section{List of Appendix figures}

Figure B.1: Number of people receiving prescriptions for opioids between 2005 - 2015 and prescribing prevalence

Figure B.2: Annual change rate of the number of people receiving opioid prescriptions and the Welsh population between $2006-2015$

Figure B.3: Trends in the number of people receiving weak and strong opioid prescribing20052015

Figure B.4: Percentage of people receiving prescriptions for weak or strong opioids as a percentage of total number of people

Figure B.5: Gender differences in the number of people receiving weak opioid prescriptions. Data presented adjusted for gender-population

Figure B.6: Trends in the number of prescriptions for opioid analgesics displayed by annual, gender-adjusted population

Figure B.7: Gender differences in the number of people receiving strong opioid prescriptions.

Data presented adjusted for gender-population

Figure B.8: Left: Trends in the number of people issued with opioid prescriptions between 2005 and 2015, stratified by age-group. Right: Data displayed by number of people per 1000 age-group adjusted population per year

Figure B.9: Comparison of trends in population and number of people receiving opioid prescriptions based on SAIL databank populations 2005 to 2015

Figure B.10: Trends in number of people receiving weak opioid analgesic prescriptions, stratified

by age group and adjusted to age-group population

Figure B.11: Trends in the number of people receiving strong opioid prescriptions between 2005 and 2015 and stratified by age-group. Data adjusted to age-group adjusted population

Figure C.1: Trends in the annual number of people receiving any opioid prescriptions presented by deprivation area and adjusted to population of each area of deprivation in Wales (WIMD = Welsh Index of Multiple Deprivation where WIMD1 = most socio-economically deprived and WIMD5 = least socio-economically deprived)

Figure C.2: Annual percentage change in the number of people prescribed opioids by quintiles of deprivation in Wales (WIMD = Welsh Index of Multiple Deprivation where WIMD1 = most deprived and WIMD5 = least deprived)

Figure C.3: Trends in the annual number of people receiving weak opioid prescriptions presented by deprivation area and adjusted to population of each area of deprivation in Wales $\quad 444$ Figure C.4: Annual percentage change in the number of people prescribed weak opioids by area of deprivation in Wales

Figure C.5: Trends in the annual number of people receiving strong opioid prescriptions presented by deprivation area and adjusted to population of each area of deprivation in Wales

Figure C.6: Annual percentage change in the number of people prescribed strong opioids by each area of deprivation in Wales

Figure C.7: Trends in the number of people receiving opioid prescriptions, stratified by gender and socioeconomic deprivation quintile

Figure C.8: Trends in the number of people per 1000 population receiving strong opioid by gender and socioeconomic deprivation quintile (WIMD)

Figure C.9: Annual percentage change in the number of prescriptions weak opioids by gender and socioeconomic deprivation quintile

Figure C.10: Trends in the number of people per 1000 population receiving strong opioid by gender and socioeconomic deprivation quintile (WIMD)

Figure C.11: Annual percentage change in the number of people receiving strong opioid prescriptions by gender and socioeconomic deprivation quintile

Figure D.1: Trends in number of prescriptions per 1000 population, stratified by drug between 2005 - 2015 
Figure D.2: Trend in the number of prescriptions of named opioids by percentage of the total number of people issued opioid prescriptions categorised as 'other' in the study protocol 466 Figure D.3: Trends in the daily oral morphine equivalent dose per prescription issued for stated drug, between $2005-2015$

Figure D.D.4: Trends in oral morphine equivalent dose for preparations of 120 milligrams or more per day between $2005-2015$

Figure D.5: Trends in Oral Morphine Equivalent Dose (milligrams) per prescription issued with products with $120 \mathrm{mg}$ OME and greater and stratified by drug. Examined by gender (Top - men, bottom - women)

Figure E.1: Monthly trend in the number of opioid prescriptions issued from Primary Care Practices who provide data to SAIL databank. Shown with a polynomial (x4) 'best fit' trendline, R2 $=0.996$

Figure E.2: Monthly trend in the number of weak opioid prescriptions issued from Primary Care Practices who provide data to SAIL databank. Shown with a polynomial 4th order 'best fit' trendline, R2=0.9935

Figure E.3: Monthly trend in the number of strong opioid prescriptions issued from Primary Care Practices who provide data to SAIL databank. Shown with a polynomial trendline, R2=0.9992 497 Figure E.4: Monthly trend in the number of opioid prescriptions issued to female patients from Primary Care Practices who provide data to SAIL databank. Shown with a polynomial trendline, R2=0.996

Figure E.5: Monthly trend in the number of opioid prescriptions issued to male patients from Primary Care Practices who provide data to SAIL databank. Shown with a polynomial trendline, R2 $=0.997$

Figure E.6: Monthly trend in the number of opioid prescriptions issued to in WIMD1 (most deprived) areas. Shown with a 3rd order polynomial trendline, R2=0.996

Figure E.7: Monthly trend in the number of opioid prescriptions issued to in WIMD2 areas. Shown with a 4th order polynomial trendline, R2 $=0.994$

Figure E.8: Monthly trend in the number of opioid prescriptions issued to in WIMD3. Shown with a 4th order polynomial trendline, R2 $=0.996$

Figure E.9: Monthly trend in the number of opioid prescriptions issued to in WIMD4. Shown with a 4th order polynomial trendline, R2 $=0.996$

Figure E.11: Example of calculation for seasonal variation (Top) and example TSA plot from which the seasonal variations were calculated

Figure E.12: Predicted trends in the number of weak opioid prescriptions issued using different time-series trendline analysis

Figure E.13: Predicted trends in the number of strong opioid prescriptions issued using different time-series trendline analysis

Figure E.14: Predicted trends in the number of opioid prescriptions issued to women using different time-series trendline analysis

Figure E.15: Predicted trends in the number of opioid prescriptions issued to men using different time-series trendline analysis

Figure E.16: Predicted trends in the number of opioid prescriptions issued to people in the different areas of deprivation (WIMD2011) (4th order polynomial prediction)

Figure E.17: Predicted trends in the number of opioid prescriptions issued to people in the different areas of deprivation (WIMD2011) (2nd order polynomial prediction)

Figure E.18: Monthly trend in the number of opioid prescriptions issued to in Abertawe Bro Morgannwg University Health Board. Shown with a 4th order polynomial trendline, R2=0.990 513 Figure E.19: Monthly trend in the number of opioid prescriptions issued to in Aneurin Bevan University Health Board. Shown with a 4th order polynomial trendline, R2 $=0.996$

Figure E.20: Monthly trend in the number of opioid prescriptions issued to in Betsi Cadwaladr University Health Board. Shown with a 4th order polynomial trendline, R2 $=0.997$ Figure E.21: Monthly trend in the number of opioid prescriptions issued to in Cardiff and Vale University Health Board. Shown with a 4th order polynomial trendline, R2 $=0.992$ 
Figure E.22: Monthly trend in the number of opioid prescriptions issued to in Cwm Taf University Health Board. Shown with a 4th order polynomial trendline, R2 $=0.993$

Figure E.23: Monthly trend in the number of opioid prescriptions issued to in Hywel Dda University Health Board. Shown with a 4th order polynomial trendline, R2 $=0.990$

Figure E.24: Monthly trend in the number of opioid prescriptions issued to in Powys Teaching

Health Board. Shown with a 4th order polynomial trendline, R2=0.995

Figure E.25: Graphical depiction of average seasonal variation in the number of opioid prescriptions issued by Health Board, from time series analysis

Figure E.26: Predicted trends in the number of opioid prescriptions issued to people in the 7 Health Boards (4th order polynomial prediction)

Figure E.27: Predicted trends in the number of opioid prescriptions issued to people in the 7 Health Boards (2nd order polynomial prediction)

Figure F.1: Example two-way ANOVA to establish interaction between factors prior to full analysis of healthcare utilisation

Figure G.1: Trends in prescribing persistence for all prescriptions and prescribing events with 2 or more consecutive prescriptions, stratified by gender

Figure G.2: Trends in the number of prescribing events with persistence of 3-6 months and over 6 months, stratified by gender

Figure G.3: Trends in average prescription persistence 2005 - 2015, stratified by age-group 566 Figure G.4: Trends in prescription persistence 2005 - 2015 using data from people who received more than 1 prescription within a 31-day period, stratified by age group

Figure G.5: Trends in the number of events with a prescribing persistence of between 3 and 6 months, between 2005 and 2015 and stratified by age-group

Figure G.6: Trends in the number of events with prescribing persistence of over 6 months, between 2005 and 2015 and stratified by age-group per 1000 age-adjusted population

Figure G.7: Trends in average annual prescribing persistence (days) stratified by Welsh Index of

Multiple Deprivation quintiles. WIMD1=most deprived, WIMD5 = least deprived

Figure G.8: Trends in average annual prescribing persistence (days) for events of two or more prescriptions issued within 32 days, stratified by Welsh Index of Multiple Deprivation quintiles.

Figure G.9: Trends in prescription persistence of 3-6months in events per 1000 deprivation adjusted population. WIMD1 $=$ most deprived, WIMD5 $=$ least deprived

Figure-G.10: Trends in prescription persistence of over 6 months in events per 1000 deprivation adjusted population. WIMD1 $=$ most deprived, WIMD5 $=$ least deprived

Figure G.11: Percentage contribution to prescribing persistence by drug, stratified by number of continuous days

Figure G.12: Trends in average annual prescribing persistence between 2005 - 2015, stratified by drug group

Figure G.13: Trends in average annual prescribing persistence, stratified by drug group and with

>31-day repeats removed

Figure G.14: Trends in prescribing persistence with duration of 3-6 months, between 2005 and

2015, stratified by drug group

Figure-G.15: Trends in persistence of prescribing between 2005 and 2015, stratified by nominated duration

Figure H.1: Trends in the number of people per 1000 population, receiving prescriptions for opioids and with or without a recorded diagnosis of depression and/or anxiety

Figure H.2: Trends in the annual number of people per 1000 population receiving strong opioid prescriptions and whether they have a recorded diagnosis of depression and/or anxiety 606 Figure H.3: Annual number of people per 1000 population receiving weak opioid prescriptions and whether they have a recorded diagnosis of depression and/or anxiety

Figure H.4: Trends in the number of prescriptions per 1000 population with or without a recorded diagnosis of depression and/or anxiety 
Figure H.5: Trends in the number of strong opioid prescriptions per 1000 population with or without a recorded diagnosis of depression and/or anxiety

Figure H.6: Trends in the number of weak opioid prescriptions per 1000 population with or without a recorded diagnosis of depression and/or anxiety

Figure H.7: Trends in opioid prescribing by sex and presence of a recorded diagnosis of depression/anxiety. Data presented by number of people per 1000 sex-adjusted population 612 Figure H.8: Trends in strong opioid prescribing by sex and presence of a recorded diagnosis of depression/anxiety. Data presented by number of people per 1000 sex-adjusted population 613 Figure H.9: Trends in weak opioid prescribing by sex and presence of a recorded diagnosis of depression/anxiety. Data presented by number of people per 1000 sex-adjusted population Figure H.10: Trends in the number opioid prescribing by gender and presence of a recorded diagnosis of depression/anxiety. Data presented by number of people per 1000 sex-adjusted population

Figure H.11: Trends in the number of strong opioid prescriptions by sex and presence of a recorded diagnosis of depression/anxiety. Data presented by number of prescriptions per 1000 sex-adjusted population

Figure H.12: Trends in the number of weak opioid prescribing by gender and presence of a recorded diagnosis of depression/anxiety. Data presented by number of prescriptions per 1000 gender-adjusted population

Figure I.1: Trend in the annual number of people per 1000 population receiving opioid prescriptions in each Health Board in Wales and compared to the National average. Data population adjusted to each Health Board

Figure I.2: Trend in the annual number of prescriptions per 1000 population in each Health Board in Wales and compared to the National trend. Data population adjusted to each Health Board 623 Figure I.3: Trend in the annual number of strong opioid prescriptions in each Health Board and compared to the National trend. Data population adjusted to Health Board

Figure 1.4: Trend in the annual number of weak opioid prescriptions in each Health Board and compared to the National trend. Data population adjusted to Health Board

Figure 1.5: Trend in the number of people receiving prescriptions for opioid medicines in the most deprived areas (as per Welsh Index of Multiple Deprivation (WIMD)) in each Health Board in Wales. Data adjusted to population of WIMD1 areas in each Health Board Figure I.6: Trend in the number of people receiving prescriptions for opioid medicines in the 2nd most deprived areas (as per Welsh Index of Multiple Deprivation (WIMD)) in each Health Board in Wales. Data adjusted to population of WIMD2 areas in each Health Board

Figure 1.7: Trend in the number of people receiving prescriptions for opioid medicines in the WIMD3 areas (as per Welsh Index of Multiple Deprivation (WIMD)) in each Health Board in Wales. Data adjusted to population of WIMD3 areas in each Health Board

Figure 1.8: Trend in the number of people receiving prescriptions for opioid medicines in the WIMD4 areas (as per Welsh Index of Multiple Deprivation (WIMD)) in each Health Board in Wales. Data adjusted to population of WIMD4 areas in each Health Board

Figure 1.9: Trend in the number of people receiving prescriptions for opioid medicines in the least deprived areas (as per Welsh Index of Multiple Deprivation (WIMD)) in each Health Board in Wales. Data adjusted to population of WIMD5 areas in each Health Board

Figure I.10: Trend in the number of opioid prescriptions in the most deprived areas (as per Welsh Index of Multiple Deprivation (WIMD)) in each Health Board in Wales. Data adjusted to population of WIMD1 areas in each Health Board

Figure 1.11: Trend in the number of prescriptions for opioid medicines in the 2nd deprived areas (as per Welsh Index of Multiple Deprivation (WIMD)) in each Health Board in Wales. Data adjusted to population of WIMD2 areas in each Health Board

Figure I.12: Trend in the number of prescriptions for opioid medicines in the 3rd most deprived areas (as per Welsh Index of Multiple Deprivation (WIMD)) in each Health Board in Wales. Data adjusted to population of WIMD3 areas in each Health Board Figure I.13: Trend in the number of prescriptions for opioid medicines in the less deprived areas (WIMD4 as per Welsh Index of Multiple Deprivation (WIMD)) in each Health Board in Wales. Data adjusted to population of WIMD4 areas in each Health Board 
Figure I.14: Trend in the number of prescriptions for opioid medicines in the least deprived areas (as per Welsh Index of Multiple Deprivation (WIMD)) in each Health Board in Wales. Data adjusted to population of WIMD5 areas in each Health Board

Figure I.15: Trend in the number of people receiving prescriptions for opioid medicines in the most deprived areas (as per Welsh Index of Multiple Deprivation (WIMD)) in each Health Board in Wales. Data adjusted to population of WIMD1 areas in each Health Board

Figure I.16: Trend in the number of people receiving prescriptions for weak opioid medicines in the 2 nd most deprived areas (as per Welsh Index of Multiple Deprivation (WIMD)) in each Health Board in Wales

Figure 1.17: Trend in the number of people receiving prescriptions for weak opioid medicines in the 3rd most deprived areas (as per Welsh Index of Multiple Deprivation (WIMD)) in each Health Board in Wales

Figure I.18: Trend in the number of people receiving prescriptions for weak opioid medicines in the lesser deprived areas (as per Welsh Index of Multiple Deprivation (WIMD)) in each Health Board in Wales

Figure I.19: Trend in the number of people receiving prescriptions for weak opioid medicines in the least deprived areas (as per Welsh Index of Multiple Deprivation (WIMD)) in each Health Board in Wales

Figure 1.20: Trend in the number of people receiving prescriptions for strong opioid medicines in the most deprived areas (as per Welsh Index of Multiple Deprivation (WIMD)) in each Health Board in Wales

Figure I.21: Trend in the number of people receiving prescriptions for strong opioid medicines in the 2 nd most deprived areas (as per Welsh Index of Multiple Deprivation (WIMD)) in each Health Board in Wales

Figure 1.22: Trend in the number of people receiving prescriptions for strong opioid medicines in the 3rd most deprived areas (as per Welsh Index of Multiple Deprivation (WIMD)) in each Health Board in Wales

Figure 1.23: Trend in the number of people receiving prescriptions for strong opioid medicines in the lesser deprived areas (as per Welsh Index of Multiple Deprivation (WIMD)) in each Health Board in Wales

Figure 1.24: Trend in the number of people receiving prescriptions for strong opioid medicines in the least deprived areas (as per Welsh Index of Multiple Deprivation (WIMD)) in each Health Board in Wales

Figure I.25: Trends in the number of weak or strong opioid prescriptions issued per 1000 population in Abertawe Bro Morgannwg University Health Board by deprivation area (WIMD) 660 Figure I.26: Trends in the number of weak or strong opioid prescriptions issued per 1000 population in Aneurin Bevan University Health Board by deprivation area (WIMD)

Figure I.27: Trends in the number of weak or strong opioid prescriptions issued per 1000 population in Betsi Cadwaladr University Health Board by deprivation area (WIMD) Figure 1.28: Trends in the number of weak or strong opioid prescriptions issued per 1000 population in Cardiff and Vale University Health Board by deprivation area (WIMD)

Figure I.29: Trends in the number of weak or strong opioid prescriptions issued per 1000 population in Cwm Taf University Health Board by deprivation area (WIMD)

Figure I.30: Trends in the number of weak or strong opioid prescriptions issued per 1000 population in Hywel Dda University Health Board by deprivation area (WIMD) 
Appendix A Methods

A.1 SAIL Application form

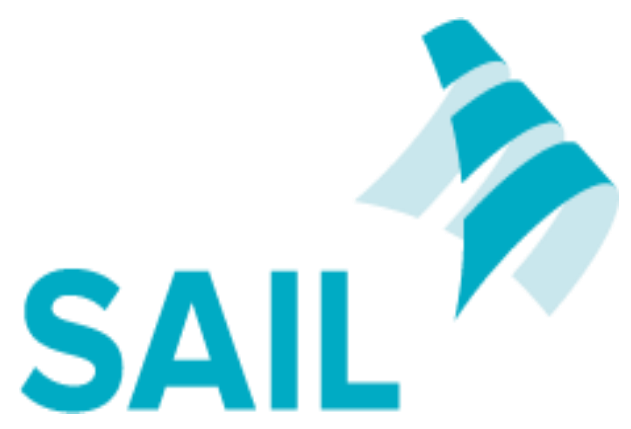

Secure Anonymised Information Linkage (SAIL) Information Governance Review Panel (IGRP) Application Form 


\section{SAIL IGRP Application Form}

The following form has been designed to collect the information needed for the information governance approval process for work involving the SAIL databank. The information you provide will facilitate consideration of your enquiry. Guidance notes on completing this form can be found at:

http://www.saildatabank.com/media/25300/Guidance Notes for SAIL IGRP Application.docx

\section{SAIL Feasibility Agreement}

All projects require a SAIL Feasibility Agreement to be completed and signed before proceeding to IGRP. This agreement will have been developed as part of the initial project scoping process with a senior SAIL analyst. Do not continue with this form until you have had your project scoping discussion.

Please provide the agreement number: $\quad 0507$

\section{1a. Provide contact details of project lead:}

Name: $\quad$ Emma Davies

Job title: Advanced Pharmacy Practitioner in Pain Management / PhD student

Organisation: Abertawe Bro Morgannwg University Health Board / Swansea University

Address:

, College of Human and Health Sciences, Swansea

Tel:

Fax:

Email:

1b. Provide contact details of the lead contact from any other organisation who will be accessing the data:

\begin{tabular}{|l|l|l|l|}
\hline & Name & Job title & Organisation \\
\hline 1 & & & \\
\hline 2 & & & \\
\hline 3 & & & \\
\hline 4 & & & \\
\hline
\end{tabular}

2. Provide full title of the project: Trends in Opioid Prescribing and Associated Resource Utilisation in Wales (TOPAS)

3. Provide details on who is commissioning the project:

PhD project - Swansea University, SCHE - PhD fellowship is funded by Swansea University and $\mathrm{RCBC}$

Research monies funded by a personal research grant from Pharmacy Research UK

\section{Provide the aim of the project, including anticipated outcomes:}

Please include a copy of the protocol/plan for the proposed work with SAIL, including the contact details of any co-applicants when you return your completed form.

This study aims to:

- Describe the trends of opioid prescribing in the Welsh Primary Care Population, over a 10-year period (2005 - 2015)

- Determine any causal or potential association between opioid use, non-pain morbidities and the utilisation of other health care services, in primary, community or acute care settings

- Develop an economic evaluation that enables the full costs of opioid use in chronic, non-cancer pain to be more accurately determined.

\section{Provide a lay summary of the project:}


Opioids are drugs, derived directly from or related to the naturally occurring alkaloids found in the opium poppy (papaver somniferum). Traditionally, opioids have been used to treat pain following trauma and surgery and also in the management of pain due to cancer and at the end of life.

In the last twenty to thirty years, there has been increasing use of opioids for the longterm treatment of chronic, non-cancer pain (CNCP), arising from conditions such as osteoarthritis, low back pain and fibromyalgia. This is despite the medical evidence being limited to short-term benefits and side effects of these drugs. Much less is known about the effectiveness and safety of opioids when given over longer treatment periods and in high doses for non-cancer pain.

Scientific literature and national media in the United Kingdom have expressed concern about whether the known increase in opioid prescribing will lead to increased incidence of dependence and misuse. Studies from North America suggest that patients taking opioids use more healthcare resources than other patients(Kern et al. 2015; Wilkerson et al. 2016) and may have a higher death rate than non-users (Yarborough et al. 2016).

In Wales, there are similar concerns following several years of increasing numbers of deaths involving opioid medications and a general upwards trend in opioid prescribing. Insight into the consequences of chronic non-cancer pain, particularly focussed on the use of opioids, has been little studied in the general public or 'patients'. Numerous studies have enquired into clinician's perceptions of chronic pain, how it is best managed and the prescribing and safety of opioid medication within that context. However, to date, there has not been a large-scale, observational study assessing the actual patterns of opioid use in clinical practice over extended periods of time nor if any link exists between the use of these drugs and other health care utilisation.

This study, therefore, aims to describe the use of opioids in all Welsh patients prescribed them and whose data is available via a national repository. The prescribing patterns and clinical outcomes, including all interactions with health care services across sectors will be evaluated in order to determine if there is an association between opioid use and health care utilisation.

From this, the intention is to establish an economic estimation that of the real costs of opioid use in chronic, non-cancer pain.

6. Provide an outline of the public engagement strategy for the study, or a brief explanation why there is not public engagement:

This study evaluates established medical interventions requiring no patient user group involvement in this instance. It is envisaged that further work will be developed from this project, and which would require patient and public involvement.

\section{Provide information on the relevant permissions you have obtained or that are being sought: \\ Obtained \\ Being sought}

Not required

Research ethics

[ \]
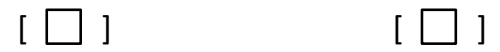

Please state the name of the committee that is being applied to/ has given approval, as applicable:

Research ethics committee: 
If you have ticked 'not required' please specify the reasons:

$\square$ The project will use only anonymised data, and therefore research ethics review is not required.

Other:

required

Obtained

Being sought

Not

Independent peer review

[ 凶 ]

$[\square]$

[ $\square$ ]

Please state the name of the peer reviewer that is being applied to/ has given approval, as applicable:

Peer reviewer: The research protocol underwent peer review by Pharmacy Research UK as part of the grant application process

If you have ticked 'not required' please specify the reasons:

$\triangle$ The project will use only anonymised data, and therefore independent review is not required. Other:

Permission from data-holding

Obtained

Being sought Not

required

Organisation to use their datasets

[ $]$

[ $\square] \quad[\square]$

Please state the name of the data provider that is being applied to/ has given approval, as applicable:

Data organisation:

If you have ticked 'not required' please specify the reasons.

$\bigotimes$ The project uses only SAIL unrestricted core datasets and/or data held by the project.

Other:

Please note that it is the responsibility of the project lead to ensure that the relevant permissions are obtained.

8a. Provide a prospective start date for the work involving SAIL: 01/11/2016

8b. Provide anticipated end date of the project: (End date OR time duration after approval) 01/10/2020 (proposed date for thesis submission)

9a. Provide details of data you require access to for the proposed work with SAIL?

Please list:

The SAIL datasets you require information from

Welsh Demographic Service

GP Event data

Patient Episode Database Wales

Accident and Emergency room data

Outpatient data

ONS Mortality data

The information needed from each dataset

\section{Welsh Demographic Service}

Patient week of birth, gender code, date of death, address and registration history for all Wales population from 2005 to 2015, (to establish event recording completeness for a case cohort and matched controls). To Include LSOA derived Welsh Index of Multiple Deprivation scores and Health Board of residence for each residency period. 


\section{GP Event data}

Event code, event date, event value

\section{Patient Episode Database Wales}

All in patient and day-case hospital activity for the opiate cohort and suitably defined control group including admission dates, discharge dates, lengths of stay, diagnoses, operative procedures, method of admission, elective or non-elective flag, outcomes in terms of discharge destination. Including all fields required to establish linkage between the spell, diagnosis and episode tables, and to establish episode and diagnostic sequencing. Also, to include FRG based costing information for each item of activity (This could be provided after the establishment of suitable cases and matched control cohorts rather than providing the whole of Wales dataset).

\section{Accident and Emergency room data}

Date of attendance, date of incidence, method of arrival, injury and accident detail, discharged to home or admitted to hospital flag (to be used for costing purposes).

\section{Outpatients data}

Dates of attendance, first or repeat attendance flag, specialty of attendance, non-attendance

\section{ONS Mortality data}

Date of death, primary cause of death, secondary cause of death.

Please indicate the time period for which data is requested

We will be analysing a cohort from 2005 - 2015 - there will be a subsequent shorter review of the main points of interest towards the end of thesis preparation to examine if there have been recent changes. We have requested earlier data to establish cohort stability in 2005.

Please indicate the geographic area for which data is requested

Wales - all Health Boards

Please indicate demographic criteria for the data requested (age, gender, etc.)

18 years and above, all people accessing primary/ secondary/tertiary care

9b. Will you be providing any other dataset(s) to be incorporated into the SAIL databank?

Yes [ $\square]$ No [ $]$

If yes:

Provide the name of the dataset(s):

Provide details of the contents of the dataset(s):

9c. Provide an outline of your analysis plan including the anticipated outputs: See attached protocol

\section{9d. Are the results/methods developed likely to have other potential applications?}

Yes [ $]$ ] No [ $\square]$

If yes, please specify:

This methodology may be used to examine the impact of prescribing within other specialities / morbidities in future.

The results will be shared with Welsh Government organisations e.g., All Wales Medicines Strategy Group as part of a policy development.

10a. Please indicate your plans for publishing the results of your project, e.g.,, target journal or intended recipients of report:

The work will form part of a PhD thesis and will also be used to develop articles for publishing in pain and economics journals e.g.,, European Journal of Pain. 
Results will be disseminated to national and international conferences and journals for publishing.

10b. What are the potentially sensitive issues that need to be taken into account when publicising the findings of the project?

Please outline the issues and your proposed solutions:

Issues over prescribing are often deemed sensitive, should they reveal certain patterns that could be attributed to individual prescribers. In this case, however, all data is anonymised. Further, certain aspects e.g.,, Health Board where the prescribing took place, is already in the public domain and therefore, it is not expected that any issues arising from this study will be particularly sensitive. Opiate addiction sometimes manifests certain very apparent signs: dependency, physical withdrawal symptoms, financial problems and behavioural change. Due care and attention will be paid to the presentation of data to ensure that no small cell counts are generated or can be derived from the outputs. This will ensure that any reader of the research could not attempt, in conjunction with other data or observations known to them, to deduce the identity of any individual.

\section{What to do next}

Please return your completed form and supporting documents by email to Cynthia McNerney, Information Governance Coordinator $q$ Thank you 


\section{A.2 TOPAS Project specification}

\section{Prerequisite requirements}

We have only been given project encrypted LSOA codes, so determination of Health Board area of residence is not possible with the current dataset. Submit an IGRP amendment to obtain unencrypted LSOA code.

\section{Stepwise data processing.}

\section{Determine read codes for opiate prescribing}

1a. Produce a list of medication read codes for opiate drugs/ all pain management medications with indication of usage in SAIL by year 2005 to 2015 to provide to Emma for confirmation of project inclusion

1b. Establish a cohort of patients with any prescription of opioids from the list of 1.a

2. Determine a project cohort

2a. Import list of cancers from Nottingham to be provided by Emma, for exclusion criteria, and perform any code mapping required to interpret it. $2 \mathrm{~b}$. Produce data table of opioid prescribing pattern per patient. To include start opioid prescribing, end of prescribing, data available over the interim period in the study period. Flag up cancer patients (using Nottingham list), other chronic pain patients, and non-chronic pain patients

2c. Establish the inclusion / exclusion criterion from the Opioid prescribed patients that the project wishes to include and exclude (e.g.,, chronic pain vs single Co-codamol prescription for short term recovery, time and data available in the study period)

2d. Finalise the "Opioid users pool" cohort.

3. Establish non opioid comparator group

3a. Establish if a cohort of people exist with chronic pain who are not prescribed opioids or very few opioids

3b. Create a comparator group, excluding cancer patients

4. Create comorbidity and health service utilisation data for the previously defined opioid and non-opioid cohorts

4a Establish patient profiles with age, gender, long term conditions, regular medications etc., date of death where applicable from GP data. Include information on the completeness of patient's records in the primary care data during the study period (i.e., periods registered with participating GPS). Should include geographical marker, i.e., Health Board for area comparator. Should include number of Primary Care events.

4b Linkage to PEDW to establish day case and inpatient hospitalisation during the study period, including admission method, dates, diagnoses, operative procedure, length of stay and costing data. 
4c Linkage to EDDS data to include all attendances at A\&E in the study period, including reason for attendance, dates, injuries, outcomes.

4d Linkage to Outpatient data to include all outpatient attendances during the study period, consultant specialty, clinic, specialty, dates, attendance information

4e Linkage to ADDE mortality data to include date and primary and secondary causes of death where applicable. 


\section{A.3 Original study protocol}

\section{Trends in Opioid Prescribing and ASsociated resource utilisation in Wales (TOPAS)}

Student: Emma Davies

Supervisors: Professor Ceri Phillips, Dr Jaynie Rance, Dr Bernadette Sewell, Professor Roger Knaggs (Nottingham), Professor Damon Berridge

Lay summary of research

Opioids are drugs, derived directly from or related to the naturally occurring alkaloids found in the opium poppy (papaver somniferum). The terms 'opiate' and 'opioid' are often used interchangeably although they technically denote a subtle difference in that opiates are 'naturally occurring' i.e., derived directly from opium and opioids are partially or wholly synthetic, with their chemistry based on the compounds derived from opium. Traditionally, opioids have been used to treat pain following trauma and surgery and also in the management of pain due to cancer and at the end of life.

In the last twenty to thirty years, there has been increasing use of opioids for the long-term treatment of chronic, non-cancer pain (CNCP), arising from conditions such as osteoarthritis, low back pain and fibromyalgia. This is despite the medical evidence being limited to short-term benefits and side effects of these drugs. Much less is known about the effectiveness and safety of opioids when given over longer treatment periods and in high doses for non-cancer pain.

Scientific literature and national media in the United Kingdom have expressed concern about whether the known increase in opioid prescribing will lead to increased incidence of dependence and misuse. Studies from North America suggest that patients taking opioids use more healthcare resources than other patients and may have a higher death rate than non-users.

In Wales, there are similar concerns following several years of increasing numbers of deaths involving opioid medications and a general upwards trend in opioid prescribing. However, to date, there has not been a large-scale, observational study assessing the actual patterns of opioid use in clinical practice over extended periods of time nor if any link exists between the use of these drugs and other health care utilisation.

Insight into the consequences of chronic non-cancer pain, particularly focussed on the use of opioids, has been little studied in the general public or 'patients'. Numerous studies have enquired into clinician's perceptions of chronic pain, how it is best managed and the prescribing and safety of opioid medication within that context. There appears to be less literature examining patients' thoughts on the best ways to manage their pain or if they share similar concerns to practitioners and researchers about the rapid rise of opioids as part of that management.

This study, therefore, aims to describe the use of opioids in all Welsh patients prescribed them and whose data is available via a national repository. The 
prescribing patterns and clinical outcomes, including all interactions with health care services across sectors will be evaluated in order to determine if there is an association between opioid use and health care utilisation.

From this, the intention is to establish an economic estimation of the costs of opioid use in chronic, non-cancer pain and which will allow services to develop more meaningful models of care and management to benefit patients in the long-term.

\section{Research Hypotheses}

- That opioid utilisation has increased in Wales in the last 10 years and varies between different Health Boards, population demographic and type of prescriber

- That there is association between individual patients' use of opioids and their use of health care resources and that this relationship can be determined by the opioid being used, persistence of use and the doses prescribed

\section{Research Aims}

This study aims to:

- Describe the trends of opioid prescribing in the Welsh Primary Care Population, over a 10-year period (2005 - 2015)

- Determine any causal or potential association between opioid use, non-pain morbidities and the utilisation of other health care services, in primary, community or acute care settings

- Develop an economic evaluation that enables the full costs of opioid use in chronic, non-cancer pain to be more accurately determined, in order to inform the development of more effective services for pain management and improved use of medicines

\section{Research objectives}

To describe the trends of opioid prescribing

1. Opioid utilisation and prescribing patterns evaluation
a. To evaluate the prescribing data of opioid prescriptions and to explore geographic (Health Board), patient age and deprivation level variations
b. To investigate the impact of major clinical guidance and recent legislative changes on opioid prescribing patterns

2. Opioid prescribing persistence in chronic, non-malignant pain management
a. To determine the persistence of opioid prescribing and assess whether the persistence and pattern of prescribing can be a proxy for measuring adherence, drug diversion and misuse
b. To determine the potential factors contributing to persistence of long-term opioid prescribing including the nature of $\mathrm{CP}$ being treated

3. Clinical outcomes related to long-term opioid utilisation 
a. To assess adverse effects which are associated with short-term and long-term opioid use

b. To compare the mortality rate between opioid and non-opioid users in patients with a specific chronic, non-malignant pain condition

To determine associations between opioid use, morbidity and health care utilisation:

4. Association between opioid use and health care utilisation

a. To assess the frequency of primary and secondary healthcare referral or attendance by patients on long-term opioids therapy compared to the general population (patients not receiving opioids)

b. To assess if any association is determined by the type of opioid medication (e.g.,, weak or strong opioid) being prescribed, the doses administered or the duration of use

To evaluate the cost of opioid use in chronic, non-cancer pain:

5. Estimation of the 'true cost' of opioid prescribing

a. To develop economic descriptions of the impact of health care utilisation associated with long-term opioid therapy which may then be used in the development of strategies to change how opioid prescribing is used in $\mathrm{CP}$

Background

Pain is generally defined as 'an unpleasant sensory and emotional experience, associated with actual or potential tissue damage, or is described in such terms. (Merskey H, Bogduk N, 1994) Chronic pain (CP) is accepted to be pain that has persisted for longer than three months and can be more accurately described as chronic, non-malignant pain (CNMP) or chronic non-cancer pain (CNCP), which helps to further differentiate it from pain associated with end-of-life morbidities, tumours or chemotherapy.

Chronic pain may result from nociceptive or neuropathic aetiologies and some people will display elements of both. People living with CP will commonly have comorbidities such as sleep disturbance, anxiety and depression in addition to physical debilitation. (Martelli MF et al. 2004) Severe CP also adversely affects employment, relationships and people's general health, for example daily back pain is associated with a higher incidence of coronary events (National Pain Audit, 2012(National Pain Audit Final Report,2012). The complex interplay of bio-psychosocial factors results in CP causing a reduction in the quality of life of sufferers more than almost any other condition (Chief Medical Officer, 2009(READING BETWEEN THE LINES: The annual profile for substance misuse 2014-15, 2015).

The reported prevalence of $\mathrm{CP}$ varies considerably depending on the type of pain being recorded and the population being studied. The Chief Medical Officer's report 2008 gave an estimate of 7.8 million people in the United Kingdom being affected by moderate to severe pain of 6 months duration or more. This pertains 
to over a third of households having someone in pain at any given time (READING BETWEEN THE LINES: The annual profile for substance misuse 2014$15,2015)$. The high economic burden of CP in addition to poor quality of life has resulted in increasing attention being paid to its treatment over the last 10 years.

In Wales as in the rest of the UK, estimates of around $13 \%$ of the population are likely affected by chronic pain (Breivik $\mathrm{H}$ et al, 2006). The Welsh Government published a commissioning directive in 2008 , aimed at improving access to multidisciplinary, multi-modal pain services the intimation being at that time, that services were developed around the skills and experience of the professionals running the services rather than patient need (Welsh Government, 2008).

The use of opioids to treat pain has been established for thousands of years and commonly accepted for the treatment of cancer and acute pain e.g.,, pain associated with trauma or surgery (Kalso E et al, 2004). Over more recent times, the use of opioids in the treatment of $\mathrm{CP}$ has increased. Some attribute the change in attitude to opioids to a sentinel paper by Portenoy and Foley in the late 1980's that appeared to open the door to a more liberal approach to prescribing in non-cancer pain (Portenoy, 1986) despite the available evidence being limited to short-term efficacy and side-effects. It is thought that opioids are likely to have less effect over time periods longer than 12 weeks and beyond certain doses the harms of continuation outweigh any therapeutic benefits (Opioids Aware, 2015).

Concerns over the risk of dependence, tolerance and addiction have been raised (Chou et al, 2009; Franklin, GM, 2014).

Despite this, over the last 10 to 15 year period, Wales has seen an increase in analgesic prescribing, which fits with general trends in the UK, Europe and the United States. ((Zin, Chen, \& Knaggs, 2014); (Ruscitto, Smith, \& Guthrie, 2014); (Wright, Katz, Abrams, Solomon, \& Losina, 2014); (Fredheim, 2010); (Olsen, Daumit, \& Ford, 2006) In the UK, a doubling of opioid prescriptions was seen in the five-year period to 2011, (NTA, 2011) Although the exact reasons for th trend have still to be fully elucidated more recent studies have demonstrated the increase to be associated with use in CP. (Zin et al., 2014)

Patient's adherence to opioids, the incidence of adverse-effects, risks of dependence and addiction and the impact on patient's general health are not clearly defined in a Welsh or UK-wide population. The British Pain Society initially published good practice guidelines for prescribing opioids in persistent pain in 2004 (BPS, 2004) and this is now updated to an online, evidence-based resource (Opioids Aware, 2015). The effect in practice of such initiatives is unclear beyond what can be elucidated from prescribing data.

The current study attempts to begin to address the overall use of opioids in the Welsh population and to develop an economic model to examine the impact of existing patterns and potential benefits of future change. The effectiveness and 
safety of long-term opioid use in the management of chronic pain will be explored as well as any association with differences in other health care utilisation or clinical morbidity in those receiving opioids for CP compared to those who do not.

\section{Study type}

This study is in two parts; firstly, an observational study to analyse opioid utilisation and patient characteristics as well as to assess the association between long-term opioid exposure and clinical outcomes including the use of non-pain related health care services in Wales.

Secondly, the development of an economic evaluation that accounts for all facets of opioid prescribing and which will allow a realistic estimation of cost-benefits of any proposed future changes to prescribing or the allocation of services.

Prescribing trends study

Cross sectional study design: This will be achieved via a quantitative, observational study conducted using a cross-sectional (with repeated crosssections) and longitudinal cohort study designs, during a ten-year period from 2005 - 2015. Data will be accessed via the Secured Anonymised Information Linkage (SAIL) Databank, which provides hospital episode statistic (HES) data in addition to primary care prescribing and other health care interactions.

All patients aged 18 years or over (adult), at any point in the study period (2005 2015) and prescribed any of the identified opioid drugs during that period, will be included in the cross-sectional study. Identification of patients will be solely by the prescription of opioids, using specific drug product codes from the SAIL datasets.

The commonly prescribed opioid or opioid-containing drugs selected as study drugs for this purpose include; codeine phosphate, codeine and paracetamol (cocodamol), dihydrocodeine, dihydrocodeine and paracetamol (co-dydramol), buprenorphine, dextropropoxyphene, dextropropoxyphene and paracetamol (co-proxamol), tramadol, tramadol and paracetamol, morphine, oxycodone, hydromorphone, fentanyl, tapentadol, meptazinol and pethidine. Prescriptions will be identified by read code and then duration data will be calculated. The outcome measures will be summed and repeatedly calculated to generate monthly and annual data.

Included patients' opioid prescription records will be measured repeatedly during the study period (2005 - 2015) and then collated to generate a range of prescribing measures in aggregated time-series and monthly/annual time-series data in order to undertake trend analysis.

Measures on utilisation of study drugs include the number of prescriptions, the strength of dosage form and the cost of opioids described. Costs will be ascribed using data from national tariffs for prescription medication. 
In this study, there is no particular selection of comparison and instead the data will be stratified by Health Board. Aggregated utilisation and time-series data will be stratified according to Health Board and demographic of the local population to determine any variation.

Various legislative changes e.g., Controlled drug legislation post-Shipman, change in controlled drug scheduling of tramadol in 2014, introduction of new drugdriving legislation in $\mathbf{2 0 1 5}$ will be used as marker points. Any changes in opioid prescribing after those dates may then be attributed to the guidance or legislation changes.

Cohort study design: All patients aged 18 or over (adult) at any point in the study period (2005 - 2015) and with a specified coded chronic pain condition e.g., low back pain will be included in the cohort study. All patients with a cancer diagnosis at any point in their medical history will be excluded from this arm of the study. (Due to the potential problems with coding in primary care in particular, in order to ensure that patients receiving opioids for chronic, noncancer pain only are included in the cohort study, it will be necessary to identify all patients who have ever received a cancer diagnosis and then remove their data from this section of analysis).

Chronic pain conditions include back pain, chronic low back pain, spinal pain, neck pain, osteoarthritis, inflammatory arthritis (ankylosing spondylitis, psoriatic arthropathy, rheumatoid arthritis), painful diabetic neuropathy, post-herpetic neuralgia, trigeminal neuralgia and phantom limb pain. For clinical outcomes, only patients with complete records including hospital episode statistics (HES) will be included in the final analysis.

Adult patients with specific chronic, non-cancer pain conditions will be selected from 2005 at the beginning of the study period and grouped into either nonopioid users (control group), short-term opioid users ( $<6$ months) or long-term opioid users ( $>6$ months).

In order to control confounding for clinical outcomes, the intervention and control groups will be matched by age, gender, Charlson Comorbidity Index (CCl) (Quan, 2011), inclusion day, opioid history (2004-2005) and deprivation score.

Patients will be followed from the date of the first prescription of any of the study drugs - within the study period to six months after the final prescription covering date or the end of the study period. This mirrors the selections of Zin et al and would allow comparison with combined data for Wales and England at a later time (Zin et al., 2014).

Prescribing persistence is defined as, 'the duration of time from initiation to discontinuation of therapy' (Cramer et al., 2008). Prescription of study drugs, prescribed dose and duration of prescribing will be analysed for individual 
patients. The duration of prescribing will be used to calculate exposure days, prescription covering days and to then estimate the medication possession ratio (MPR). The MPR will be further adjusted by covariates such as sociodemographic characteristics (e.g., age at diagnosis, sex, deprivation scores), smoking and alcohol use, co-morbid conditions, number of drugs prescribed and disease-related pain conditions.

Adult patients with a specified chronic pain indication (low back pain) are included and the corresponding opioid drug utilisation data will be analysed. Evaluating prescribing persistence and medication possession ratio (MPR) will identify patients who have continuous short-term or long-term exposure to any of the study drugs. Patients identified with a specified CP indication but without an opioid prescription will be entered into the control group.

Continuous use is assumed when the gap between each opioid prescriptioncovering period is less than 30 days. Short-term use refers to the accumulated period of opioid prescription covering less than 180 days ( 6 months). Long-term use refers to the accumulated prescription period covering more than 180 days (6 months). The intervention (long-term users) and control groups will be selected by $1: 1$ matching in age, gender, $\mathrm{CCl}$, inclusion day, opioid use history (2004 - 2005) and deprivation score.

Prescription data for the study opioid drugs for individual patients will be collected; number of items and where possible, dose data and duration of prescribing will be used to assess prescribing persistence. The medication possession ratio (MPR)(calculated from number of days of medication supplied within the refill period) for different opioids will be compared between different types of CP.

Clinical outcomes will be analysed using read codes for the most common adverse effects (ADE) of opioids (constipation, nausea, dizziness and drowsiness and vomiting) will be used to search the records of all adult patients with a $\mathrm{CP}$ indication and opioid prescription. Proxy measures such as anti-emetic and laxative prescriptions will be evaluated. Comparison will be made of ADEs and their frequency between long-term and short-term opioid users and further comparison made between those and the control group in order to determine $A D E$ rates between the groups and if an association can be made between opioid use and duration of use.

Mortality rates of long-term and short-term opioid users will be calculated and compared to each other and to the mortality rate within the control group.

Association between opioid use, morbidity and health care utilisation Study design: Health care utilisation in all sectors including those related to managing pain or opioid related outcomes will be captured using ICD-10 and OPCS-4 coding in according with the data recording methods for the Patient Episode Database for Wales (PEDW), which collects hospital data. Long-term 
ADE such as endocrine, immune dysfunction, sexual dysfunction and infertility will be examined, by proxy measures if necessary, such as GP and outpatient attendances. Time to events will also be measured. Data will be grouped according to long-term or short-term opioid use and compared to the control group of patients not receiving opioid prescriptions.

Health care utilisation data in patients receiving opioid prescriptions will be further analysed by categorising into 'weak' and 'strong' opioids as per the World Health Organisation (WHO) analgesic ladder \{Anonymous:wj\} and then further by dose banding of <20mg, 20-49mg, 50-99mg, 100-199mg and $>200 \mathrm{mg}$ daily MEQ and as described in previous work (Dunn, Saunders, \& Rutter, 2010, Gomes, Mamdani, Dhalla, Paterson, \& Juurlink, 2011). Duration of prescribing will be a secondary measure to analyse the association between prescribed opioid dose and healthcare interactions.

To Estimate the cost of opioid use in chronic, non-cancer pain:

1. Estimation of the cost of opioid prescribing

a. To develop economic descriptions of the impact of health care utilisation associated with long-term opioid therapy which may then be used in the development of strategies to change how opioid prescribing is used in $\mathrm{CP}$

Sample size / Power calculations

It has been estimated that $13 \%$ of the UK population suffer with moderate to severe pain (Breivik, 2006), which, equates to 400,000 people in Wales, a country with a population of around 3 million (Statistics Wales, 2015). In the European Union (EU), $13 \%$ of patients with persistent pain appear to be prescribed weak opioids and 3\% strong opioids (Langley, 2011).

Using the estimated prevalence of chronic pain above, this suggests 52000 patients receive prescriptions for weak opioids and 12000 patients' strong opioids in Wales. However, there has been a marked increase in prescribing of opioid analgesics within primary care in Wales from 1 million items in 2007 to 1.4 million items in 2013 , an increase of nearly $40 \%$, meaning that these figures could be an underestimate.

The SAIL database in Wales as a whole represents over $70 \%$ of Primary Care Practices (General Practitioners). Therefore, the Law of Large Numbers and Central Limit Theorem dictate that it should be expected to estimate these effects accurately. In fact, the Law of Truly Large Numbers may be used even to detect differences in rare events, if it is found to be relevant to the study. In clinical trials of short duration, over half (51\%) of patients taking opioids within clinical trials experience at least one treatment emergent adverse effect. (Moore, 2005) Recent data from the United States (US) suggest doses of morphine 200mg (or equivalent dose of an alternative opioid), were associated with a nearly 3-fold increase in the risk of opioid-related mortality. (Gomes, 2011) 
Since long-term opioid exposure information is limited in Wales, there are currently no estimates with which to determine power-based sample sizes for detecting the association between long-term opioid exposure and clinical outcomes. Similarly, no estimates can be made for power-based sample sizes for quantifying any apparent causal relationship.

\section{Data / Statistical Analysis}

Data management

Records with missing dates of birth, diagnosis and drug, which consequently, are unable to identify age, disease conditions and drug utilisation, will be excluded from the analysis. However, as appropriate, then a progressive procedure will be in place to either incorporate or exclude missing data.

Missing data will be included in the sensitivity analysis; firstly, it will be flagged and then used to compare analyses in which missing data is dropped with analyses relying on multiple imputations. This will enable the establishment of randomness and leverage of any missing data.

All datasets will be stored on SAIL use authenticated, password protected servers. Although access can be gained remotely, data extraction from the server is not possible without written authority from SAIL. The database mainframe is situated in a locked and highly secured building. Initial data extraction can only be performed by a trained coder with the appropriate level of security clearance from SAIL. All data is anonymised.

Extracted data will be double encrypted before being made available to the researcher in order to maintain its security. Individual SAIL records will not be divulged outside of the research group (researcher, supervisors and informatics support).

Information processing and data analysis will be performed on mainstream applications such as IBM SPSS ${ }^{\odot}$, in addition to specialised statistical and econometric packages like STATA 14 (Stats Corp LP, 2014. USA).

Statistical analysis

Baseline descriptive analysis will be undertaken for all variables in the study, in order to ensure data and variables are included and analysed appropriately. Simple statistical analysis methods (e.g., Analysis of Variance (ANOVA)) will be used to compare average drug utilisation across different groups. Linear trend analysis will be used on time-series data before further time-series analysis is undertaken.

For the longitudinal data, either static (cross-sectional modelling for correlation and treatment effects, including generalised linear-regression modelling) or dynamic (repeated cross-sectional and longitudinal methods including generalised estimating equations), seemingly unrelated regression and panel 
data methods will be used. The Cox proportional hazard model will be used to compare outcome events and time to events between groups and the results reported as hazard ratio $(\mathrm{HR})$ and $95 \%$ confidence interval $(95 \% \mathrm{CI})$.

Patient or User-group involvement

This study evaluates an established medical intervention requiring no patient user group involvement in this instance.

Predictable limitations of the Prescribing Trends study design, data sources and analytic methods

- The study uses secondary data where the investigator has not witnessed the care provided or collected the data directly. Therefore, an assumption is made that the data is accurately recorded and the interventions have actually taken place as described.

- Measuring opioid use on the basis of prescriptions issued may not reflect the actual utilisation as the drugs may not have been dispensed by a pharmacy or taken by the patient.

- Measuring medication adherence using prescription issue also means it is not possible to determine if the patient actually had the prescription dispensed or consumed the dispensed medication themselves. Some people may order a prescription but not get it refilled. Some people may get prescriptions dispensed more often than they take the medication, or they may store medication for future use or in some cases, give or sell it to others.

\section{Dissemination}

The findings from this study will be prepared for publication in refereed international journals. The findings will also be communicated at conferences, seminars, research workshops and meetings.

With the extensive links to patients and professional stakeholder that Swansea University has, the results will also be made available to them for comment. This work contributes to a programme of research based in the Swansea Centre for Health Economics that focuses on using large patient databases to assess drug utilisation, prescribing patterns, medicines adherence, clinical outcomes and economic impact related to the studied drugs and diseases. Therefore, this work will also contribute to comparative analysis of the methods and results across disease types and patient groups, and dissemination is likely to include this aspect of the work.

\section{References}

- $\quad$ Breivik, H., Collett, B., Ventafridda, V., Cohen, R., \& Gallacher, D. (2012). Survey of chronic pain in Europe: Prevalence, impact on daily life, and treatment. European Journal of Pain, 10(4), 287-287.

- $\quad$ British Pain Society. Opioids for persistent pain: Good practice. 2004

- $\quad$ Chief Medical Officer. Pain: Breaking through the barrier. Department of Health, 2009; 1 - 8.

- Chou R, Ballantyne JC, Fanciullo GJ, Fine PG, Miaskowski C. Research gaps on use of opioids for chronic non-cancer pain: Findings from a review of the evidence for an American Pain Society and American Academy of Pain Medicine Clinical Practice Guideline. Journal of Pain. 2009; 10 (2); $147-59$

- $\quad$ Cramer, J. A., Roy, A., Burrell, A., Fairchild, C. J., Fuldeore, M. J., Ollendorf, D. A., \& Wong, P. K. (2008). Medication Compliance and Persistence: Terminology and Definitions. Value in Health, 11(1), 44-47. http://doi.org/10.1111/i.15244733.2007.00213.x

- Dunn, K. M., Saunders, K. W., \& Rutter, C. M. (2010). Overdose and prescribed opioids: Associations among chronic noncancer pain patients. Annals of Internal .... http://doi.org/10.1059/0003-4819-152-2-201001190-00006 
- $\quad$ Fairman, K., \& Motheral, B. Evaluating Medication Adherence: which measure is right for your program? Journal of Managed Care Pharmacy, 2000. 6 (6), 499-506.

- Franklin GM. Opioids for chronic non-cancer pain: A position statement of the American Academy of Neurology. Neurology. 2014; 83 (September); $1277-1284$

- $\quad$ Fredheim, O. M. S., Skurtveit, S., Breivik, H., \& Borchgrevink, P. C. (2010). Increasing use of opioids from 2004 to 2007 \&\#8211; Pharmacoepidemiological data from a complete national prescription database in Norway. European Journal of Pain, 14(3), 289-294. http://doi.org/10.1016/i.ejpain.2009.05.006

- Furlan AD, Sandoval JA, Mailis-Gagnon A, Tunks E. Opioids for chronic non-cancer pain: a meta-analysis of effectiveness and side-effects. Canadian Medical Association Journal. 2006; 174 (11): 1589 - 94.

- $\quad$ Gomes, T., Mamdani, M. M., Dhalla, I. A., Paterson, J. M., \& Juurlink, D. N. (2011). Opioid Dose and Drug-Related Mortality in Patients With Nonmalignant Pain. Archives of ..., 171(7), 686-691. http://doi.org/10.1001/archinternmed.2011.117

- $\quad$ https://www.rcoa.ac.uk/faculty-of-pain-medicine/opioids-aware/clinical-use-of-opioids/effectiveness-for-long-term-pain (Accessed November 2015)

- $\quad$ Kalso, E, Edwards JE, Moore RA, McQuay HJ. Opioids in non-cancer pain: systematic review of efficacy and safety. Pain. 2004; 112 (3); $372-80$ )

- $\quad$ Langley PC. The prevalence, correlates and treatment of pain in the European Union. Current Medical Research and Opinion. 2011; 27 (2): $463-80$

- Martelli MF, Zasler ND, Bender MC, Nicholson K. Psychological, neuropsychological and medical considerations in assessment and management of pain. Journal of Head Trauma Rehabilitation. 2004; 19 (1); $10-28$.

- Merskey H, Bogduk N. Classification of chronic pain - descriptions of chronic pain syndromes and definitions of pain terms. 2 ed. Seattle: International Association for Study of Pain; 1994

- Moore RA, McQuay HJ. Prevalence of opioid adverse effects in chronic, non-malignant pain: systematic review of randomised trials of oral opioids. Arthritis Research and Therapy. 2005; 7 (5): R1046 - 51.

- National Pain AuditFinal Report. (2012). National Pain AuditFinal Report, 1-66.

- Olsen, Y., Daumit, G. L., \& Ford, D. E. (2006). Opioid Prescriptions by U.S. Primary Care Physicians From 1992 to 2001. Journal of Pain, 7(4), 225-235. http://doi.org/10.1016/j.jpain.2005.11.006

- Portenoy RK, Foley KM. Chronic use of opioid analgesics in non-malignant pain: report of 38 cases. Pain. $1986 ; 25$ (2); 171 86

- $\quad$ Quan, H., Li, B., Couris, C. M., Fushimi, K., Graham, P., Hider, P., et al. (2011). Updating and Validating the Charlson Comorbidity Index and Score for Risk Adjustment in Hospital Discharge Abstracts Using Data From 6 Countries. American Journal of Epidemiology, 173(6), 676-682. http://doi.org/10.1093/aje/kwq433

- $\quad$ READING BETWEEN THE LINES: The annual profile for substance misuse 2014-15. (2015). READING BETWEEN THE LINES: The annual profile for substance misuse 2014-15, 1-86.

- $\quad$ Ruscitto, A., Smith, B. H., \& Guthrie, B. (2014). Changes in opioid and other analgesic use 1995-2010: Repeated crosssectional analysis of dispensed prescribing for a large geographical population in Scotland. European Journal of Pain, 19(1), 59-66. http://doi.org/10.1002/ejp.520

- Welsh Assembly Government. Designed for people with chronic conditions; Service development and commissioning directives; Chronic non-malignant pain. 2008, $1-46$.

- $\quad$ Welsh Government \& Statistics Wales. (2015). 2014 Mid-year Population Estimates for Wales (No. SB 34/2015) (1-8). Cardiff.

- Wright, E. A., Katz, J. N., Abrams, S., Solomon, D. H., \& Losina, E. (2014). Trends in Prescription of Opioids From 2003-2009 in Persons with Knee Osteoarthritis. Arthritis Care \& Research, 66(10), 1489-1495. http://doi.org/10.1002/acr.22360

- $\quad$ Zin, C. S., Chen, L. C., \& Knaggs, R. D. (2014). Changes in trends and pattern of strong opioid prescribing in primary care. European Journal of Pain, 18(9), 1343-1351. http://doi.org/10.1002/j.1532-2149.2014.496. 


\section{A.4 Read codes for opioid analgesics}

Table A.1: Read codes for weak opioid analgesics including the original list shared from Nottingham University

\begin{tabular}{|c|c|c|}
\hline $\begin{array}{l}\text { Read code (Version } \\
\text { 3) }\end{array}$ & Term ID & Term details \\
\hline \multicolumn{3}{|l|}{ Dextropropoxyphene } \\
\hline dia7. & y029g & Co-proxamol $32.5 \mathrm{mg} / 325 \mathrm{mg}$ tablet \\
\hline diax. & y07bs & Co-proxamol $32.5 \mathrm{mg} / 325 \mathrm{mg} / 5 \mathrm{~mL} \mathrm{~s} / \mathrm{f}$ suspension \\
\hline diaw. & y029s & Cosalgesic tablet \\
\hline dj61. & y02BC & Dextropropoxyphene hydrochloride $65 \mathrm{mg}$ capsule \\
\hline diad. & y029i & Distalgesic tablet \\
\hline dibh. & y00IU & Paxalgesic tablet \\
\hline \multicolumn{3}{|l|}{ Dipipanone } \\
\hline $\mathrm{x} 01 \mathrm{KW}$ & y047z & Dipipanone \\
\hline dj92. & $\mathrm{y} 02 \mathrm{Bc}$ & Dipipanone+cyclizine $10 \mathrm{mg} / 30 \mathrm{mg}$ tablet \\
\hline dj91. & $\mathrm{y} 02 \mathrm{Bb}$ & Diconal tablet \\
\hline \multicolumn{3}{|l|}{ Buprenorphine } \\
\hline dj3p. & yOGpG & Buprenorphine 10micrograms/hour patch \\
\hline dj3q. & y0GpH & Buprenorphine 20micrograms/hour patch \\
\hline dj3o. & y0GpF & Buprenorphine 5micrograms/hour patch \\
\hline dj3B. & y0GpK & BuTrans 10micrograms/hour patch \\
\hline dj3C. & y0GpL & BuTrans 20micrograms/hour patch \\
\hline dj3A. & y0GpJ & BuTrans 5micrograms/hour patch \\
\hline \multicolumn{3}{|l|}{ Codeine } \\
\hline dia1. & y0015 & Aspirin/paracetamol/codeine tablets \\
\hline x00et & y03SK & Aspirin+codeine $300 \mathrm{mg} / 8 \mathrm{mg}$ tablet \\
\hline diaq. & y029o & Co-codamol 8mg/500mg capsule \\
\hline dia3. & y029c & Co-codamol 8mg/500mg dispersible tablet \\
\hline diap. & y029n & Co-codamol 8mg/500mg effervescent tablet \\
\hline dia2. & y029b & Co-codamol $8 \mathrm{mg} / 500 \mathrm{mg}$ tablet \\
\hline dia5. & y029e & Co-codaprin $8 \mathrm{mg} / 500 \mathrm{mg}$ dispersible tablet \\
\hline dia4. & y029d & Co-codaprin $8 \mathrm{mg} / 500 \mathrm{mg}$ tablet \\
\hline diaO. & yOEdF & Co-codaprin $8 \mathrm{mg} / 500 \mathrm{mg}$ dispersible tablet \\
\hline dj41. & y02Ay & Codeine phosphate $15 \mathrm{mg}$ tablet \\
\hline cg11. & y01vd & Codeine phosphate $15 \mathrm{mg} / 5 \mathrm{~mL}$ linctus \\
\hline cg12. & yOAVG & Codeine phosphate $15 \mathrm{mg} / 5 \mathrm{~mL} \mathrm{~s} / \mathrm{f}$ linctus \\
\hline dj44. & y02B1 & Codeine phosphate $25 \mathrm{mg} / 5 \mathrm{~mL}$ syrup \\
\hline dj42. & $\mathrm{y} 02 \mathrm{Az}$ & Codeine phosphate $30 \mathrm{mg}$ tablet \\
\hline dj43. & y02BO & Codeine phosphate $60 \mathrm{mg}$ tablet \\
\hline dj45. & y02B2 & Codeine phosphate $60 \mathrm{mg} / 1 \mathrm{~mL}$ injection \\
\hline dica. & yz4v2 & CODIPAR $15 \mathrm{mg} / 500 \mathrm{mg}$ effervescent tablets \\
\hline diaT. & y0F5Q & Codipar Caplet \\
\hline diaP. & y0Edl & Codis 500 dispersible tablet \\
\hline diab. & y0018 & Codis dispersible tablet \\
\hline cg14. & y01vg & Galcodine $15 \mathrm{mg} / 5 \mathrm{~mL} \mathrm{~s} / \mathrm{f}$ linctus \\
\hline j280. & y0844 & Ibuprofen+codeine phosphate $200 \mathrm{mg} / 12.5 \mathrm{mg}$ tablet \\
\hline j28V. & y09u6 & Ibuprofen+codeine phosphate $200 \mathrm{mg} / 12.8 \mathrm{mg}$ tablet \\
\hline j28z. & $y 02 x j$ & Ibuprofen+codeine phosphate $300 \mathrm{mg} / 20 \mathrm{mg} \mathrm{m} / \mathrm{r}$ tablet \\
\hline dicN. & y0Fw0 & Kapake $30 / 500$ effervescent tablet \\
\hline diao. & yOCrA & Kapake capsule \\
\hline diaL. & yOCYe & Kapake Insts $1000 \mathrm{mg} / 60 \mathrm{mg} / \mathrm{sachet}$ powder \\
\hline diaJ. & yOCRd & Kapake Insts $500 \mathrm{mg} / 30 \mathrm{mg} / \mathrm{sachet}$ powder \\
\hline diam. & y01Ez & Kapake tablet \\
\hline dib3. & $\mathrm{yOOIH}$ & Medocodeine tablet \\
\hline dibW. & yODDg & Medocodene $30 / 500$ capsule \\
\hline dicR. & y0Gom & Medocodene 30/500 effervescent tablet \\
\hline dl13. & $\mathrm{y} 02 \mathrm{CL}$ & Migraleve yellow tablet \\
\hline dl1a. & y00JK & Migraleve yellow tablets $\times 48$ \\
\hline j28I. & y0845 & Nurofen Plus tablet \\
\hline dib9. & yOOIN & Panadeine Forte tablet \\
\hline diba. & y00IO & Panadeine soluble tablet \\
\hline dib8. & y00IM & Panadeine tablet \\
\hline dibN. & y09zp & Panadol Ultra tablet \\
\hline diaK. & yOCYd & Paracetamol+codeine phosphate $1000 \mathrm{mg} / 60 \mathrm{mg} / \mathrm{sachet}$ powder \\
\hline dia3. & y08Gi & Paracetamol+codeine phosphate $500 \mathrm{mg} / 8 \mathrm{mg}$ dispersible tablet \\
\hline dia2. & y08Gh & Paracetamol+codeine phosphate $500 \mathrm{mg} / 8 \mathrm{mg}$ tablet \\
\hline
\end{tabular}




\begin{tabular}{|c|c|c|}
\hline dibQ. & y0CJ1 & Paracetamol+codeine phosphate $500 \mathrm{mg} / 12.8 \mathrm{mg}$ tablet \\
\hline dibM. & y09zo & Paracetamol+codeine phosphate $500 \mathrm{mg} / 13.5 \mathrm{mg}$ tablet \\
\hline dicv. & yz4v3 & $\begin{array}{l}\text { PARACETAMOL+CODEINE PHOSPHATE } 500 \mathrm{mg} / 15 \mathrm{mg} \text { effervescent } \\
\text { tablet }\end{array}$ \\
\hline diaU. & y0F5P & Paracetamol+codeine phosphate $500 \mathrm{mg} / 15 \mathrm{mg}$ tablet \\
\hline dibD. & y029w & Paracetamol+codeine phosphate $500 \mathrm{mg} / 30 \mathrm{mg}$ capsule \\
\hline dibB. & y029u & Paracetamol+codeine phosphate $500 \mathrm{mg} / 30 \mathrm{mg}$ effervescent tablet \\
\hline dian. & y01M5 & Paracetamol+codeine phosphate $500 \mathrm{mg} / 30 \mathrm{mg}$ tablet \\
\hline dial. & yOCRc & Paracetamol+codeine phosphate $500 \mathrm{mg} / 30 \mathrm{mg} / \mathrm{sach}$ t powder \\
\hline diar. & y09aF & Paracodol capsule \\
\hline dibb. & y029v & Paracodol soluble tablet \\
\hline dibc. & y00IP & Paradeine tablet \\
\hline dibe. & y00IR & Parake tablet \\
\hline dibo. & y00la & Solpadeine Forte dispersible tablet \\
\hline dibR. & y0CJ2 & Solpadeine Max tablet \\
\hline j2pT. & $\mathrm{yOH} 2 \mathrm{p}$ & Solpadeine Migraine Ibuprofen \& Codeine tablet \\
\hline x04uo & y0Fj8 & Solpadeine Plus capsule \\
\hline dibn. & yOFjA & Solpadeine Plus Soluble effervescent tablet \\
\hline dibz. & y02A4 & Solpadol Caplet \\
\hline dibS. & yOCLZ & Solpadol capsule \\
\hline dibu. & y02A1 & Solpadol effervescent tablet \\
\hline dibp. & y00Ib & Syndol tablet \\
\hline dibt. & y02A0 & Tylex capsule \\
\hline dibG. & y08J2 & Tylex Effervescent soluble tablet \\
\hline dicM. & yOFoo & Ultramol capsule \\
\hline dicL. & yOFon & Ultramol tablet \\
\hline dibX. & yODWj & Ultramol soluble tablet \\
\hline dibs. & y00le & Veganin tablet \\
\hline dic3. & yODZy & Zapain Caplet \\
\hline dic4. & yODZz & Zapain capsule \\
\hline \multicolumn{3}{|c|}{ Dihydrocodeine } \\
\hline dj85. & y00J4 & DF118 10mg $/ 5 \mathrm{~mL}$ elixir \\
\hline dj84. & y00J3 & DF118 30mg tablet \\
\hline dj86. & y00J5 & DF118 50mg/1mL injection \\
\hline dj8d. & y01lc & DF118 Forte 40mg tablet \\
\hline dj8a. & y02BX & DHC Continus $120 \mathrm{mg} \mathrm{m} / \mathrm{r}$ tablet \\
\hline dj87. & y02BU & DHC Continus $60 \mathrm{mg} \mathrm{m} / \mathrm{r}$ tablet \\
\hline dj89. & y02BW & DHC Continus $90 \mathrm{mg} \mathrm{m} / \mathrm{r}$ tablet \\
\hline dj8.. & $\mathrm{y} 02 \mathrm{BQ}$ & Dihydrocodeine tartrate \\
\hline dj82. & y02BS & Dihydrocodeine tartrate $10 \mathrm{mg} / 5 \mathrm{~mL}$ elixir \\
\hline dj8c. & y02BZ & Dihydrocodeine tartrate $120 \mathrm{mg} \mathrm{m} / \mathrm{r}$ tablet \\
\hline dj81. & y02BR & Dihydrocodeine tartrate $30 \mathrm{mg}$ tablet \\
\hline dj8e. & y01Id & Dihydrocodeine tartrate $40 \mathrm{mg}$ tablet \\
\hline dj83. & y02BT & Dihydrocodeine tartrate $50 \mathrm{mg} / 1 \mathrm{~mL}$ injection \\
\hline dj88. & y02BV & Dihydrocodeine tartrate $60 \mathrm{mg} \mathrm{m} / \mathrm{r}$ tablet \\
\hline dj8b. & y02BY & Dihydrocodeine tartrate $90 \mathrm{mg} \mathrm{m} / \mathrm{r}$ tablet \\
\hline dia6. & y029f & Co-dydramol 10mg/500mg tablet \\
\hline dicy. & y0Gpm & $\begin{array}{l}\text { Paracetamol 500mg / dihydrocodeine tartrate } 7.46 \mathrm{mg} \\
\text { effervescent tablet }\end{array}$ \\
\hline dibH. & y09nz & $\begin{array}{l}\text { Paracetamol+dihydrocodeine tartrate } 250 \mathrm{mg} / 20 \mathrm{mg} \text { effervescent } \\
\text { tablet }\end{array}$ \\
\hline dibl. & y09na & $\begin{array}{l}\text { Paracetamol+dihydrocodeine tartrate } 250 \mathrm{mg} / 30 \mathrm{mg} \text { effervescent } \\
\text { tablet }\end{array}$ \\
\hline dibE. & $y 029 x$ & Paracetamol+dihydrocodeine tartrate $500 \mathrm{mg} / 20 \mathrm{mg}$ tablet \\
\hline dibF. & y029y & Paracetamol+dihydrocodeine tartrate $500 \mathrm{mg} / 30 \mathrm{mg}$ tablet \\
\hline dibf. & y00IS & Paramol 10/500mg tablet \\
\hline dics. & yOGpQ & Paramol Soluble tablet \\
\hline dibx. & y01Aw & Paramol tablet \\
\hline dibK. & y09ne & Remedeine effervescent tablet \\
\hline dibJ. & y09nc & Remedeine Forte effervescent tablet \\
\hline dibw. & y02A3 & Remedeine Forte tablet \\
\hline dibv. & y02A2 & Remedeine tablet \\
\hline \multicolumn{3}{|c|}{ Tramadol } \\
\hline djAj. & yz5EN & ACEON $100 \mathrm{mg} \mathrm{m} / \mathrm{r}$ tablets \\
\hline djAk. & yz5EO & ACEON $150 \mathrm{mg} \mathrm{m} / \mathrm{r}$ tablets \\
\hline djAl. & yz5EP & ACEON $200 \mathrm{mg} \mathrm{m} / \mathrm{r}$ tablets \\
\hline djiw. & y0D7m & Dromadol SR $100 \mathrm{mg} \mathrm{m} / \mathrm{r}$ tablet \\
\hline djix. & y0D7n & Dromadol SR $150 \mathrm{mg} \mathrm{m} / \mathrm{r}$ tablet \\
\hline
\end{tabular}




\begin{tabular}{|c|c|c|}
\hline djiY. & y0D7o & Dromadol SR 200mg m/r tablet \\
\hline djiz. & y0D7q & Dromadol SR 75mg m/r tablet \\
\hline djia. & yODQK & Dromadol XL 150mg m/r tablet \\
\hline djib. & yODQL & Dromadol XL 200mg m/r tablet \\
\hline djic. & yODQM & Dromadol XL 300mg m/r tablet \\
\hline djid. & yODQN & Dromadol XL 400mg m/r tablet \\
\hline djif. & y0GZd & Larapam SR $100 \mathrm{mg} \mathrm{m} / \mathrm{r}$ tablet \\
\hline djig. & y0GZe & Larapam SR $150 \mathrm{mg} \mathrm{m} / \mathrm{r}$ tablet \\
\hline djih. & yOGZf & Larapam SR 200mg m/r tablet \\
\hline djA1. & $\mathrm{yOH} 8 \mathrm{q}$ & MABRON 100mg $\mathrm{m} / \mathrm{r}$ tablets \\
\hline djA2. & yOH8r & MABRON 150mg m/r tablets \\
\hline djA3. & $\mathrm{yOH} 8 \mathrm{~s}$ & MABRON $200 \mathrm{mg} \mathrm{m} / \mathrm{r}$ tablets \\
\hline djAf. & $\mathrm{yz} 4 \mathrm{v} 4$ & MAROL $100 \mathrm{mg} \mathrm{m} / \mathrm{r}$ tablets \\
\hline djAg. & yz4v5 & MAROL $150 \mathrm{mg} \mathrm{m} / \mathrm{r}$ tablets \\
\hline djAh. & yz4v6 & MAROL $200 \mathrm{mg} \mathrm{m} / \mathrm{r}$ tablets \\
\hline djAc. & yz3FD & MAXITRAM SR 100mg m/r capsules \\
\hline djAd. & yz3FE & MAXITRAM SR 150mg m/r capsules \\
\hline djAe. & yz3FF & MAXITRAM SR $200 \mathrm{mg} \mathrm{m} / \mathrm{r}$ capsules \\
\hline djAb. & yz3FC & MAXITRAM SR 50mg m/r capsules \\
\hline djA7. & yOIFm & NOBLIGAN RETARD $100 \mathrm{mg} \mathrm{m} / \mathrm{r}$ tablets \\
\hline djA8. & yOJHo & OLDARAM $100 \mathrm{mg} \mathrm{m} / \mathrm{r}$ tablets \\
\hline djA9. & yOJHp & OLDARAM $150 \mathrm{mg} \mathrm{m} / \mathrm{r}$ tablets \\
\hline djAa. & $\mathrm{yOJHq}$ & OLDARAM $200 \mathrm{mg} \mathrm{m} / \mathrm{r}$ tablets \\
\hline dicz. & yOFyn & Paracetamol 325mg / tramadol hydrochloride $37.5 \mathrm{mg}$ tablet \\
\hline x05sn & yOFym & Paracetamol+tramadol \\
\hline dicx. & yz4ki & $\begin{array}{l}\text { PARACETAMOL+TRAMADOL HCL } 325 \mathrm{mg} / 37.5 \mathrm{mg} \text { effervescent } \\
\text { tablets }\end{array}$ \\
\hline djAm. & yz7Ci & TILODOL SR $100 \mathrm{mg} \mathrm{m} / \mathrm{r}$ tablets \\
\hline djAn. & $y z 7 C j$ & TILODOL SR $150 \mathrm{mg} \mathrm{m} / \mathrm{r}$ tablets \\
\hline djAo. & yz7Ck & TILODOL SR 200mg $\mathrm{m} / \mathrm{r}$ tablets \\
\hline djim. & yOHR3 & TRADOREC XL $100 \mathrm{mg} \mathrm{m} / \mathrm{r}$ tablets \\
\hline djin. & yOHR4 & TRADOREC XL 200mg $\mathrm{m} / \mathrm{r}$ tablets \\
\hline djio. & y0HR5 & TRADOREC XL 300mg $\mathrm{m} / \mathrm{r}$ tablets \\
\hline dicY. & yz4kf & TRAMACET $325 \mathrm{mg} / 37.5 \mathrm{mg}$ effervescent tablets \\
\hline dicO. & yOFyo & Tramacet $325 \mathrm{mg} / 37.5 \mathrm{mg}$ tablet \\
\hline dicO. & y0Fyo & Tramacet $325 \mathrm{mg} / 37.5 \mathrm{mg}$ tablet \\
\hline djAi. & yz53f & TRAMADOL $100 \mathrm{mg} / 1 \mathrm{~mL}$ oral drops solution \\
\hline djiG. & y0AZ4 & Tramadol hydrochloride $100 \mathrm{mg} \mathrm{m} / \mathrm{r}$ capsule \\
\hline dji5. & y0803 & Tramadol hydrochloride $100 \mathrm{mg} \mathrm{m} / \mathrm{r}$ tablet \\
\hline dji1. & y01LN & Tramadol hydrochloride $100 \mathrm{mg} / 2 \mathrm{~mL}$ injection \\
\hline djiP. & y0CE7 & Tramadol hydrochloride $100 \mathrm{mg} /$ sachet powder \\
\hline djiH. & y0AZ5 & Tramadol hydrochloride $150 \mathrm{mg} \mathrm{m} / \mathrm{r}$ capsule \\
\hline dji6. & y0804 & Tramadol hydrochloride $150 \mathrm{mg} \mathrm{m} / \mathrm{r}$ tablet \\
\hline djil. & y0AZ6 & Tramadol hydrochloride $200 \mathrm{mg} \mathrm{m} / \mathrm{r}$ capsule \\
\hline dji7. & y0805 & Tramadol hydrochloride $200 \mathrm{mg} \mathrm{m} / \mathrm{r}$ tablet \\
\hline djiy. & y0Cuo & Tramadol hydrochloride $300 \mathrm{mg} \mathrm{m} / \mathrm{r}$ tablet \\
\hline djiz. & y0Cup & Tramadol hydrochloride $400 \mathrm{mg} \mathrm{m} / \mathrm{r}$ tablet \\
\hline dji2. & y01LO & Tramadol hydrochloride 50mg capsule \\
\hline djiF. & y0AZ3 & Tramadol hydrochloride $50 \mathrm{mg} \mathrm{m} / \mathrm{r}$ capsule \\
\hline djiv. & yOHvm & TRAMADOL HYDROCHLORIDE 50mg $\mathrm{m} / \mathrm{r}$ tablets \\
\hline djiw. & y0Fh8 & Tramadol hydrochloride 50mg oro-dispersible tablet \\
\hline djic. & y09uV & Tramadol hydrochloride $50 \mathrm{mg}$ soluble tablet \\
\hline djio. & YOCE6 & Tramadol hydrochloride $50 \mathrm{mg} /$ sachet powder \\
\hline djix. & y0D7p & Tramadol hydrochloride $75 \mathrm{mg} \mathrm{m} / \mathrm{r}$ tablet \\
\hline djiB. & y09kU & Tramake 50mg capsule \\
\hline djiR. & y0CE9 & Tramake Insts $100 \mathrm{mg} /$ sachet powder \\
\hline djiQ. & y0CE8 & Tramake Insts 50mg/sachet powder \\
\hline djiq. & yOHet & TRAMQUEL SR $100 \mathrm{mg} \mathrm{m} / \mathrm{r}$ capsules \\
\hline djir. & yOHeu & TRAMQUEL SR $150 \mathrm{mg} \mathrm{m} / \mathrm{r}$ capsules \\
\hline djis. & yOHev & TRAMQUEL SR $200 \mathrm{mg} \mathrm{m} / \mathrm{r}$ capsules \\
\hline djip. & yOHes & TRAMQUEL SR 50mg m/r capsules \\
\hline djiN. & y0c8U & Zamadol $100 \mathrm{mg} / 2 \mathrm{~mL}$ injection \\
\hline djii. & y0Gop & Zamadol $24 \mathrm{hr} 150 \mathrm{mg} \mathrm{m} / \mathrm{r}$ tablet \\
\hline djij. & y0Goq & Zamadol 24hr 200mg m/r tablet \\
\hline djik. & yOGor & Zamadol 24hr 300mg m/r tablet \\
\hline djil. & y0Gos & Zamadol $24 \mathrm{hr} 400 \mathrm{mg} \mathrm{m} / \mathrm{r}$ tablet \\
\hline djie. & y09xP & Zamadol 50mg capsule \\
\hline djie. & y0Fh9 & Zamadol Melt 50mg oro-dispersible tablet \\
\hline
\end{tabular}




\begin{tabular}{|c|c|c|}
\hline djik. & y0AZ8 & Zamadol SR $100 \mathrm{mg} \mathrm{m} / \mathrm{r}$ capsule \\
\hline djiL. & y0AZ9 & Zamadol SR $150 \mathrm{mg} \mathrm{m} / \mathrm{r}$ capsule \\
\hline djiM. & yOAZA & Zamadol SR $200 \mathrm{mg} \mathrm{m} / \mathrm{r}$ capsule \\
\hline djiJ. & y0AZ7 & Zamadol SR 50mg m/r capsule \\
\hline djA4. & y0IC3 & ZERIDAME SR $100 \mathrm{mg} \mathrm{m} / \mathrm{r}$ tablets \\
\hline djA5. & y0IC4 & ZERIDAME SR $150 \mathrm{mg} \mathrm{m} / \mathrm{r}$ tablets \\
\hline djA6. & y0IC5 & ZERIDAME SR 200mg m/r tablets \\
\hline dji3. & y01LP & Zydol $100 \mathrm{mg} / 2 \mathrm{~mL}$ injection \\
\hline dji4. & y01LQ & Zydol 50mg capsule \\
\hline djiD. & y09uW & Zydol Soluble 50mg tablet \\
\hline dji8. & y0806 & Zydol SR $100 \mathrm{mg} \mathrm{m} / \mathrm{r}$ tablet \\
\hline dji9. & y0807 & Zydol SR $150 \mathrm{mg} \mathrm{m} / \mathrm{r}$ tablet \\
\hline djiA. & y0808 & Zydol SR 200mg m/r tablet \\
\hline djit. & $\mathrm{yOHvl}$ & ZYDOL SR $50 \mathrm{mg} \mathrm{m} / \mathrm{r}$ tablets \\
\hline djis. & y0Cum & Zydol XL $150 \mathrm{mg} \mathrm{m} / \mathrm{r}$ tablet \\
\hline djit. & yocun & Zydol XL 200mg $\mathrm{m} / \mathrm{r}$ tablet \\
\hline djiU. & yoCuq & Zydol XL 300mg m/r tablet \\
\hline djiv. & yocur & Zydol XL $400 \mathrm{mg} \mathrm{m} / \mathrm{r}$ tablet \\
\hline \multicolumn{3}{|c|}{ Meptazinol } \\
\hline 0443. & y03J4 & Meptazinol 200mg tablet \\
\hline 0441. & y03J2 & Meptid 200mg tablet \\
\hline djb1. & y00JC & Meptid [analg] 200mg tablets \\
\hline $044 z$. & y03J5 & Meptazinol $100 \mathrm{mg} / 1 \mathrm{~mL}$ injection \\
\hline 0442. & y03J3 & Meptid 100mg/1mL injection \\
\hline djb2. & yOOJD & Meptid [analg] 100mg/1mL injection \\
\hline \multicolumn{3}{|c|}{ Paracetamol and Pentazocine } \\
\hline diav. & y029r & Paracetamol+pentazocine $500 \mathrm{mg} / 15 \mathrm{mg}$ tablet \\
\hline diah. & y029l & Fortagesic tablet \\
\hline
\end{tabular}

Table A.2: Read codes for strong opioid analgesics including the original list shared from Nottingham University

\begin{tabular}{|c|c|c|}
\hline Read code (version 3 ) & Term ID & Term details \\
\hline \multicolumn{3}{|l|}{ Fentanyl } \\
\hline o4d7. & yOJGc & ABSTRAL 100micrograms sublingual tablets \\
\hline o4d9. & yOJGe & ABSTRAL 200micrograms sublingual tablets \\
\hline o4db. & yOJGg & ABSTRAL 300micrograms sublingual tablets \\
\hline o4dd. & yOJGi & ABSTRAL 400micrograms sublingual tablets \\
\hline o4df. & yOJGk & ABSTRAL 600micrograms sublingual tablets \\
\hline o4dh. & y0JGm & ABSTRAL 800micrograms sublingual tablets \\
\hline o42G. & yODIA & Actiq 1200micrograms lozenge \\
\hline 042H. & yODIB & Actiq 1600micrograms lozenge \\
\hline o42C. & y0DI6 & Actiq 200micrograms lozenge \\
\hline 042D. & y0DI7 & Actiq 400micrograms lozenge \\
\hline o42E. & y0DI8 & Actiq 600micrograms lozenge \\
\hline o42F. & y0DI9 & Actiq 800 micrograms lozenge \\
\hline o4dG. & yz7SO & BREAKYL 200micrograms buccal film \\
\hline o4dl. & yz7SQ & BREAKYL 400micrograms buccal film \\
\hline o4dK. & yz7SS & BREAKYL 800micrograms buccal film \\
\hline o42B. & y07tK & Durogesic 100micrograms/hour patch \\
\hline 0428. & $\mathrm{y} 07 \mathrm{tH}$ & Durogesic 25micrograms/hour patch \\
\hline 0429. & $\mathrm{y} 07 \mathrm{tl}$ & Durogesic 50micrograms/hour patch \\
\hline 042A. & $\mathrm{y} 07 \mathrm{tJ}$ & Durogesic 75micrograms/hour patch \\
\hline 042L. & yOGXC & Durogesic DTrans 100micrograms/hour patch \\
\hline $042 Q$. & y0Guy & Durogesic DTrans 12micrograms/hour patch \\
\hline 0421. & yOGXB & Durogesic DTrans 25micrograms/hour patch \\
\hline 042J. & yOGXA & Durogesic DTrans 50micrograms/hour patch \\
\hline 042K. & y0GX9 & Durogesic DTrans 75micrograms/hour patch \\
\hline 042l. & YOJGR & EFFENTORA 100micrograms buccal tablets \\
\hline $042 n$. & yOJGT & EFFENTORA 200micrograms buccal tablets \\
\hline 04d1. & yOJGW & EFFENTORA 400micrograms buccal tablets \\
\hline o4d3. & yOJGY & EFFENTORA 600micrograms buccal tablets \\
\hline o4d5. & yOJGa & EFFENTORA 800micrograms buccal tablets \\
\hline o4dF. & yz5bd & FENCINO 100micrograms/hr patches \\
\hline o4dB. & $y z 5 b z$ & FENCINO 12micrograms/hr patches \\
\hline o4dC. & yz5ba & FENCINO 25micrograms/hr patches \\
\hline o4dD. & yz5bb & FENCINO 50micrograms/hr patches \\
\hline o4dE. & yz5bc & FENCINO 75micrograms/hr patches \\
\hline
\end{tabular}




\begin{tabular}{|c|c|c|}
\hline o42h. & yOIND & FENTALIS RESERVOIR 100micrograms/hr patches \\
\hline o42i. & yOIVN & FENTALIS RESERVOIR 25micrograms/hr patches \\
\hline 042j. & yOIVO & FENTALIS RESERVOIR 50micrograms/hr patches \\
\hline 042k. & yOIVP & FENTALIS RESERVOIR 75micrograms/hr patches \\
\hline $042 z$. & $\mathrm{y} 018 \mathrm{r}$ & Fentanyl 100micrograms injection \\
\hline 042m. & & FENTANYL 100mcg buccal tablets \\
\hline o4dv. & yz3Qs & FENTANYL 100micrograms nasal spray \\
\hline 04d8. & yOJGd & FENTANYL 100micrograms sublingual tablets \\
\hline 042x. & y03Iz & Fentanyl 100micrograms/2mL injection \\
\hline 0427. & $\mathrm{y} 07 \mathrm{tG}$ & Fentanyl 100micrograms/hour patch \\
\hline o42r. & y0DI4 & Fentanyl 1200micrograms lozenge \\
\hline o42R. & y0Gux & Fentanyl 12micrograms/hour patch \\
\hline o4dP. & yz7UP & FENTANYL 133micrograms sublingual tablets \\
\hline o42q. & y0DI5 & Fentanyl 1600micrograms lozenge \\
\hline 04dH. & yz7SP & FENTANYL 200micrograms buccal film \\
\hline 0420. & yOJGU & FENTANYL 200micrograms buccal tablets \\
\hline 042v. & yODIO & Fentanyl 200micrograms lozenge \\
\hline o4dx. & yz3Qu & FENTANYL 200micrograms nasal spray \\
\hline o4da. & yOJGf & FENTANYL 200micrograms sublingual tablets \\
\hline 0424. & $\mathrm{y} 07 \mathrm{tC}$ & Fentanyl 25micrograms/hour patch \\
\hline o4dR. & yz7UR & FENTANYL 267micrograms sublingual tablets \\
\hline o4dc. & yOJGh & FENTANYL 300micrograms sublingual tablets \\
\hline o4e3. & yz7WL & FENTANYL 37.5micrograms/hour transdermal patches \\
\hline o4dJ. & yz7SR & FENTANYL 400micrograms buccal film \\
\hline o4d2. & yOJGX & FENTANYL 400micrograms buccal tablets \\
\hline $042 u$. & y0DI1 & Fentanyl 400micrograms lozenge \\
\hline 04dA. & yz4jp & FENTANYL 400micrograms nasal spray \\
\hline o4de. & y0JGj & FENTANYL 400micrograms sublingual tablets \\
\hline 042y. & y03J0 & Fentanyl 500micrograms/10mL injection \\
\hline o4dt. & yz3Qq & FENTANYL 50micrograms nasal spray \\
\hline 0425. & $\mathrm{y} 07 \mathrm{tD}$ & Fentanyl 50micrograms/hour patch \\
\hline o4dU. & yz7UU & FENTANYL 533micrograms sublingual tablets \\
\hline 04d4. & yOJGZ & FENTANYL 600micrograms buccal tablets \\
\hline 042t. & yODI2 & Fentanyl 600micrograms lozenge \\
\hline o4dg. & yOJGI & FENTANYL 600micrograms sublingual tablets \\
\hline o4dN. & yz7UN & FENTANYL 67micrograms sublingual tablets \\
\hline 0426. & $\mathrm{y} 07 \mathrm{tF}$ & Fentanyl 75micrograms/hour patch \\
\hline o4dL. & yz7ST & FENTANYL 800micrograms buccal film \\
\hline o4d6. & yOJGb & FENTANYL 800micrograms buccal tablets \\
\hline 042s. & y0DI3 & Fentanyl 800micrograms lozenge \\
\hline o4di. & yOJGn & FENTANYL 800micrograms sublingual tablets \\
\hline o4du. & yz3ar & INSTANYL 100micrograms nasal spray \\
\hline o4dw. & yz3Qt & INSTANYL 200micrograms nasal spray \\
\hline o4ds. & yz3Qp & INSTANYL 50micrograms nasal spray \\
\hline o42W. & yOHUw & MATRIFEN 100micrograms/hr patches \\
\hline 042S. & yOHUs & MATRIFEN 12micrograms/hr patches \\
\hline 042T. & yOHUt & MATRIFEN 25micrograms/hr patches \\
\hline o42U. & yOHUu & MATRIFEN 50micrograms/hr patches \\
\hline 042V. & yOHUv & MATRIFEN 75micrograms/hr patches \\
\hline o42b. & yOIGc & MEZOLAR MATRIX 100micrograms/hour patches \\
\hline o42X. & yOIGY & MEZOLAR MATRIX 12micrograms/hour patches \\
\hline o42Y. & yOIGZ & MEZOLAR MATRIX 25micrograms/hour patches \\
\hline o4e2. & yz7WK & MEZOLAR MATRIX 37.5micrograms/hour patches \\
\hline $042 Z$. & yOIGa & MEZOLAR MATRIX 50micrograms/hour patches \\
\hline 042a. & yOIGb & MEZOLAR MATRIX 75micrograms/hour patches \\
\hline o4e1. & yz7WJ & OPIODUR 100micrograms/hour transdermal patches \\
\hline o4dW. & yz7WE & OPIODUR 12micrograms/hour transdermal patches \\
\hline o4dX. & yz7WF & OPIODUR 25micrograms/hour transdermal patches \\
\hline o4dY. & yz7WG & OPIODUR 50micrograms/hour transdermal patches \\
\hline o4dz. & yz7WH & OPIODUR 75micrograms/hour transdermal patches \\
\hline o42g. & y0IK1 & OSMACH 100micrograms/hour patches \\
\hline o42d. & y0IJy & OSMACH 25micrograms/hour patches \\
\hline o42e. & yOIJz & OSMACH 50micrograms/hour patches \\
\hline o42f. & yOIKO & OSMACH 75micrograms/hour patches \\
\hline o4dm. & y0J19 & OSMANIL 100micrograms/hr patches \\
\hline o4dr. & yz3Qo & OSMANIL 12micrograms/hr patches \\
\hline o4dj. & y0JI6 & OSMANIL 25micrograms/hr patches \\
\hline o4dk. & y0JI7 & OSMANIL 50micrograms/hr patches \\
\hline
\end{tabular}




\begin{tabular}{|c|c|c|}
\hline o4dl. & y0J18 & OSMANIL 75micrograms/hr patches \\
\hline o4dy. & yz4jq & PECFENT 100micrograms nasal spray \\
\hline o4dz. & yz4jr & PECFENT 400micrograms nasal spray \\
\hline o4dO. & yz7uO & RECIVIT 133micrograms sublingual tablets \\
\hline o4dQ. & yz7UQ & RECIVIT 267micrograms sublingual tablets \\
\hline o4dS. & yz7US & RECIVIT 400micrograms sublingual tablets \\
\hline o4dT. & yz7UT & RECIVIT 533micrograms sublingual tablets \\
\hline o4dM. & yz7UM & RECIVIT 67micrograms sublingual tablets \\
\hline o4dV. & yz7UV & RECIVIT 800micrograms sublingual tablets \\
\hline 0421. & y03Iv & Sublimaze 100 micrograms $/ 2 \mathrm{~mL}$ injection \\
\hline 0422. & y03Iw & Sublimaze 500 micrograms $/ 10 \mathrm{~mL}$ injection \\
\hline 042P. & y0Gmv & Tilofyl 100micrograms/hour patch \\
\hline o42M. & y0Gms & Tilofyl 25micrograms/hour patch \\
\hline 042N. & y0Gmt & Tilofyl 50micrograms/hour patch \\
\hline 0420. & y0Gmu & Tilofyl 75micrograms/hour patch \\
\hline o4dq. & $y z 3 C f$ & VICTANYL 100micrograms/hour patches \\
\hline o4dn. & $\mathrm{yz} 3 \mathrm{Cc}$ & VICTANYL 25micrograms/hour patches \\
\hline o4do. & $y z 3 C d$ & VICTANYL 50micrograms/hour patches \\
\hline o4dp. & yz3Ce & VICTANYL 75micrograms/hour patches \\
\hline $04 \mathrm{e} 8$. & yz83K & YEMEX 100micrograms/hour transdermal patches \\
\hline 04e4. & yz83G & YEMEX 12micrograms/hour transdermal patches \\
\hline o4e5. & yz83H & YEMEX 25micrograms/hour transdermal patches \\
\hline o4e6. & yz83I & YEMEX 50micrograms/hour transdermal patches \\
\hline o4e7. & yz83J & YEMEX 75micrograms/hour transdermal patches \\
\hline \multicolumn{3}{|c|}{ Pentazocine } \\
\hline djf7. & $\mathrm{y} 02 \mathrm{Bv}$ & Fortral $25 \mathrm{mg}$ tablet \\
\hline djf8. & y02Bw & Fortral 30mg/1mL injection \\
\hline djf6. & y02Bu & Fortral 50mg capsule \\
\hline djfa. & y02By & Fortral 50mg suppository \\
\hline djf9. & $\mathrm{y} 02 \mathrm{Bx}$ & Fortral $60 \mathrm{mg} / 2 \mathrm{~mL}$ injection \\
\hline djf3. & $\mathrm{y} 02 \mathrm{Br}$ & Pentazocine $30 \mathrm{mg} / 1 \mathrm{~mL}$ injection \\
\hline djf5. & y02Bt & Pentazocine 50mg suppository \\
\hline xOORT & y03Sx & Pentazocine $60 \mathrm{mg} / 1 \mathrm{~mL}$ injection \\
\hline djf2. & $\mathrm{y} 02 \mathrm{~Bq}$ & Pentazocine hydrochloride $25 \mathrm{mg}$ tablet \\
\hline djf1. & y02Bp & Pentazocine hydrochloride 50mg capsule \\
\hline \multicolumn{3}{|c|}{ Dipipanone } \\
\hline dj91. & $\mathrm{y} 02 \mathrm{Bb}$ & Diconal tablet \\
\hline dj92. & $\mathrm{y} 02 \mathrm{Bc}$ & Dipipanone+cyclizine $10 \mathrm{mg} / 30 \mathrm{mg}$ tablet \\
\hline \multicolumn{3}{|c|}{ Buprenorphine } \\
\hline dj3x. & y02Av & Buprenorphine 200micrograms sublingual tablet \\
\hline dj3u. & yOChF & Buprenorphine $2 \mathrm{mg}$ sublingual tablet \\
\hline dj3y. & y02Aw & Buprenorphine 300micrograms $/ 1 \mathrm{~mL}$ injection \\
\hline dj3t. & yOEcR & Buprenorphine 35micrograms/hour transdermal patch \\
\hline dj35. & y02Au & Buprenorphine 400micrograms sublingual tablet \\
\hline dj3s. & yOEcT & Buprenorphine $52.5 \mathrm{micrograms} /$ hour transdermal patch \\
\hline dj3z. & y00ly & Buprenorphine 600 micrograms $/ 2 \mathrm{~mL}$ injection \\
\hline dj3r. & yOEcV & Buprenorphine 70micrograms/hour transdermal patch \\
\hline dj3v. & yOChG & Buprenorphine 8mg sublingual tablet \\
\hline dj3s. & $y z 7 R n$ & HAPOCTASIN 35micrograms/hour patches \\
\hline dj3ı. & yz7Rm & HAPOCTASIN 52.5micrograms/hour patches \\
\hline dj3H. & yz7RI & HAPOCTASIN 70micrograms/hour patches \\
\hline dj3L. & yz7sz & NATZON $2 \mathrm{mg}$ sublingual tablets \\
\hline dj3k. & yz7sy & NATZON 400micrograms sublingual tablets \\
\hline dj3M. & yz7sa & NATZON 8mg sublingual tablets \\
\hline dj3d. & yz5pv & PREFIBIN 2mg sublingual tablets \\
\hline dj3c. & yz5pu & PREFIBIN 400micrograms sublingual tablets \\
\hline dj3e. & yz5pw & PREFIBIN 8mg sublingual tablets \\
\hline dj37. & yOChH & Subutex $2 \mathrm{mg}$ sublingual tablet \\
\hline dj36. & yOChE & Subutex 400micrograms sublingual tablet \\
\hline dj38. & yOChl & Subutex $8 \mathrm{mg}$ sublingual tablet \\
\hline dj31. & y02Ar & Temgesic $200 \mathrm{mcg}$ sublingual tablet \\
\hline dj32. & y02As & Temgesic 300micrograms $/ 1 \mathrm{~mL}$ injection \\
\hline dj34. & y02At & Temgesic $400 \mathrm{mcg}$ sublingual tablet \\
\hline dj33. & y001x & Temgesic 600micrograms $/ 2 \mathrm{~mL}$ injection \\
\hline dj3f. & $y z 5 p x$ & TEPHINE 200micrograms sublingual tablets \\
\hline dj3g. & yz5py & TEPHINE 400micrograms sublingual tablets \\
\hline dj39. & yOEcS & Transtec 35micrograms/hour transdermal patch \\
\hline dj3a. & yOEcU & Transtec 52.5micrograms/hour transdermal patch \\
\hline
\end{tabular}




\begin{tabular}{|c|c|c|}
\hline dj3b. & yOEcW & Transtec 70micrograms/hour transdermal patch \\
\hline \multicolumn{3}{|c|}{ Diamorphine } \\
\hline dj7A. & y02BO & Diagesil 100mg injection (pdr for recon) \\
\hline dj78. & $\mathrm{y} 02 \mathrm{BM}$ & Diagesil 10mg injection (pdr for recon) \\
\hline dj79. & y02BN & Diagesil 30mg injection (pdr for recon) \\
\hline dj7B. & y02BP & Diagesil 500mg injection (pdr for recon) \\
\hline dj77. & y02BL & Diagesil 5mg injection (pdr for recon) \\
\hline cg31. & yOODR & Diamorphine $3 \mathrm{mg} / 5 \mathrm{~mL}$ linctus \\
\hline dj75. & y02BJ & Diamorphine hydrochloride $100 \mathrm{mg}$ injection \\
\hline dj73. & y02BH & Diamorphine hydrochloride $10 \mathrm{mg}$ injection \\
\hline dj71. & y02BF & Diamorphine hydrochloride $10 \mathrm{mg}$ tablet \\
\hline dj74. & $\mathrm{y} 02 \mathrm{BI}$ & Diamorphine hydrochloride $30 \mathrm{mg}$ injection \\
\hline dj76. & y02BK & Diamorphine hydrochloride 500mg injection \\
\hline dj72. & y02BG & Diamorphine hydrochloride 5mg injection \\
\hline dj22. & y00ls & Diamorphine simple elixir \\
\hline dj7F. & y07bN & Diaphine $100 \mathrm{mg}$ injection (pdr for recon) \\
\hline dj7D. & y07bL & Diaphine 10mg injection (pdr for recon) \\
\hline dj7E. & y07bM & Diaphine 30mg injection (pdr for recon) \\
\hline dj7G. & y07bO & Diaphine 500mg injection (pdr for recon) \\
\hline dj7C. & y07bK & Diaphine $5 \mathrm{mg}$ injection (pdr for recon) \\
\hline \multicolumn{3}{|c|}{ Hydromorphone } \\
\hline djj1. & yOADO & Hydromorphone hydrochloride $1.3 \mathrm{mg}$ capsule \\
\hline djj8. & yOADW & Hydromorphone hydrochloride $16 \mathrm{mg} \mathrm{m} / \mathrm{r}$ capsule \\
\hline djj3. & yOADQ & Hydromorphone hydrochloride $2.6 \mathrm{mg}$ capsule \\
\hline djj9. & yOADX & Hydromorphone hydrochloride $24 \mathrm{mg} \mathrm{m} / \mathrm{r}$ capsule \\
\hline djj5. & yOADT & Hydromorphone hydrochloride $2 \mathrm{mg} \mathrm{m} / \mathrm{r}$ capsule \\
\hline djj6. & yOADU & Hydromorphone hydrochloride $4 \mathrm{mg} \mathrm{m} / \mathrm{r}$ capsule \\
\hline djj7. & yOADV & Hydromorphone hydrochloride $8 \mathrm{mg} \mathrm{m} / \mathrm{r}$ capsule \\
\hline djj2. & yOADP & Palladone $1.3 \mathrm{mg}$ capsule \\
\hline djj4. & yOADR & Palladone $2.6 \mathrm{mg}$ capsule \\
\hline djjD. & yOADb & Palladone-SR $16 \mathrm{mg} \mathrm{m} / \mathrm{r}$ capsule \\
\hline djjE. & yOADc & Palladone-SR $24 \mathrm{mg} \mathrm{m} / \mathrm{r}$ capsule \\
\hline djjA. & yOADY & Palladone-SR $2 \mathrm{mg} \mathrm{m} / \mathrm{r}$ capsule \\
\hline djjB. & yOADZ & Palladone-SR $4 \mathrm{mg} \mathrm{m} / \mathrm{r}$ capsule \\
\hline djjC. & yOADa & Palladone-SR $8 \mathrm{mg} \mathrm{m} / \mathrm{r}$ capsule \\
\hline \multicolumn{3}{|c|}{ Morphine } \\
\hline x025k & y02AH & Cyclimorph 10 injection \\
\hline $\mathrm{x} 025 \mathrm{I}$ & y02AJ & Cyclimorph 15 injection \\
\hline dj1c. & y07BJ & Cyclimorph-10 injection $1 \mathrm{~mL}$ \\
\hline dj1d. & y07BK & Cyclimorph-15 injection $1 \mathrm{~mL}$ \\
\hline dj1e. & y00li & Duromorph $64 \mathrm{mg} / 1 \mathrm{~mL} \mathrm{~m} / \mathrm{r}$ injection \\
\hline djy9. & yOEIT & Filnarine SR $100 \mathrm{mg} \mathrm{m} / \mathrm{r}$ tablet \\
\hline djy6. & YOEIQ & Filnarine SR $10 \mathrm{mg} \mathrm{m} / \mathrm{r}$ tablet \\
\hline djy7. & YOEIR & Filnarine SR $30 \mathrm{mg} \mathrm{m} / \mathrm{r}$ tablet \\
\hline djy8. & yOEIS & Filnarine SR $60 \mathrm{mg} \mathrm{m} / \mathrm{r}$ tablet \\
\hline dj1q. & y02AY & Min-I-Jet Morphine sulphate $20 \mathrm{mg} / 2 \mathrm{~mL}$ prefilled syringe \\
\hline djzY. & y09wG & Morcap SR 100mg m/r capsule \\
\hline djzb. & y09wK & Morcap SR $20 \mathrm{mg} \mathrm{m} / \mathrm{r}$ capsule \\
\hline djzc. & y09wL & Morcap SR 50mg m/r capsule \\
\hline djyD. & yOFOi & Morphgesic SR $100 \mathrm{mg} \mathrm{m} / \mathrm{r}$ tablet \\
\hline djyA. & yOFOf & Morphgesic SR $10 \mathrm{mg} \mathrm{m} / \mathrm{r}$ tablet \\
\hline djyB. & yOFOg & Morphgesic SR 30mg m/r tablet \\
\hline djyC. & yOFOh & Morphgesic SR $60 \mathrm{mg} \mathrm{m} / \mathrm{r}$ tablet \\
\hline dj2.. & y00lq & Morphine analgesic elixirs \\
\hline $\mathbf{x} 00 \mathrm{Xt}$ & y03SL & Morphine hydrochloride $10 \mathrm{mg} / \mathrm{mL}$ oral solution \\
\hline dj1S. & y02Ad & Morphine hydrochloride $15 \mathrm{mg}$ suppository \\
\hline dj1T. & y02Af & Morphine hydrochloride $30 \mathrm{mg}$ suppository \\
\hline dj21. & y00Ir & Morphine simple elixir \\
\hline dj11. & y08D1 & Morphine sulph [CNS] 10mg injection \\
\hline dj12. & y08D2 & Morphine sulph [CNS] 15mg injection \\
\hline dj13. & y08D3 & Morphine sulphate [CNS] 20mg/1ml injection \\
\hline dj15. & y08D5 & Morphine sulphate [CNS] 20mg/2ml injection \\
\hline dj14. & y08D4 & Morphine sulphate [CNS] 30mg/1ml injection \\
\hline dj16. & y08D6 & Morphine sulphate [CNS] 30mg/2ml injection \\
\hline dj17. & y08EF & Morphine sulphate [CNS] $40 \mathrm{mg} / 2 \mathrm{ml}$ injection \\
\hline dj18. & y08D7 & Morphine sulphate [CNS] $60 \mathrm{mg} / 2 \mathrm{ml}$ injection \\
\hline djzG. & y08PI & Morphine sulphate $100 \mathrm{mg} \mathrm{m} / \mathrm{r}$ capsule \\
\hline djz8. & $\mathrm{y} 02 \mathrm{CF}$ & Morphine sulphate $100 \mathrm{mg} \mathrm{m} / \mathrm{r}$ tablet \\
\hline
\end{tabular}




\begin{tabular}{|c|c|c|}
\hline djzi. & yOAPM & Morphine sulphate $100 \mathrm{mg} / 50 \mathrm{~mL}$ injection \\
\hline djze. & y0A5o & Morphine sulphate $100 \mathrm{mg} / 50 \mathrm{~mL}$ prefilled syringe \\
\hline dj1M. & y02AV & Morphine sulphate $100 \mathrm{mg} / 5 \mathrm{~mL}$ oral solution \\
\hline dj1K. & $\mathrm{y} 01 \mathrm{CE}$ & Morphine sulphate $100 \mathrm{mg} / 5 \mathrm{~mL}$ oral unit dose vial \\
\hline djzB. & y01la & Morphine sulphate $100 \mathrm{mg} / \mathrm{sachet}$ granules for suspension \\
\hline 0451. & $\mathrm{y} 018 \mathrm{v}$ & Morphine sulphate $10 \mathrm{mg}$ injection \\
\hline djzD. & $\mathrm{y08Pi}$ & Morphine sulphate $10 \mathrm{mg} \mathrm{m} / \mathrm{r}$ capsule \\
\hline djz4. & y02CB & Morphine sulphate $10 \mathrm{mg} \mathrm{m} / \mathrm{r}$ tablet \\
\hline dj1D. & y02AK & Morphine sulphate $10 \mathrm{mg}$ suppository \\
\hline djz1. & y02C8 & Morphine sulphate $10 \mathrm{mg}$ tablet \\
\hline $\mathrm{x} 02 \mathrm{LZ}$ & y02A7 & Morphine sulphate $10 \mathrm{mg} / 1 \mathrm{~mL}$ injection \\
\hline dj10. & yOEbR & Morphine sulphate $10 \mathrm{mg} / 1 \mathrm{~mL}$ prefilled syringe \\
\hline dj1L. & y02AU & Morphine sulphate $10 \mathrm{mg} / 5 \mathrm{~mL}$ oral solution \\
\hline dj1G. & y01CD & Morphine sulphate $10 \mathrm{mg} / 5 \mathrm{~mL}$ oral unit dose vial \\
\hline djzP. & y09ll & Morphine sulphate $120 \mathrm{mg} \mathrm{m} / \mathrm{r}$ capsule \\
\hline djzQ. & y09lJ & Morphine sulphate $150 \mathrm{mg} \mathrm{m} / \mathrm{r}$ capsule \\
\hline 0452. & y018w & Morphine sulphate $15 \mathrm{mg}$ injection \\
\hline djz5. & y02CC & Morphine sulphate $15 \mathrm{mg} \mathrm{m} / \mathrm{r}$ tablet \\
\hline dj19. & y02AD & Morphine sulphate $15 \mathrm{mg}$ suppository \\
\hline X02La & y02A8 & Morphine sulphate $15 \mathrm{mg} / 1 \mathrm{~mL}$ injection \\
\hline djyl. & y0Fk1 & Morphine sulphate $1 \mathrm{mg} / \mathrm{mL}$ Minijet $10 \mathrm{~mL}$ prefilled syringe \\
\hline djzR. & y09IK & Morphine sulphate $200 \mathrm{mg} \mathrm{m} / \mathrm{r}$ capsule \\
\hline djz9. & y02CG & Morphine sulphate $200 \mathrm{mg} \mathrm{m} / \mathrm{r}$ tablet \\
\hline djzC. & y01lb & Morphine sulphate $200 \mathrm{mg} / \mathrm{sachet}$ granules for suspension \\
\hline djzZ. & y09wl & Morphine sulphate $20 \mathrm{mg} \mathrm{m} / \mathrm{r}$ capsule \\
\hline dj1E. & $\mathrm{y} 02 \mathrm{AL}$ & Morphine sulphate $20 \mathrm{mg}$ suppository \\
\hline djz2. & $\mathrm{y} 02 \mathrm{C9}$ & Morphine sulphate $20 \mathrm{mg}$ tablet \\
\hline 0453. & y02A9 & Morphine sulphate $20 \mathrm{mg} / 1 \mathrm{~mL}$ injection \\
\hline 0455. & $y 018 x$ & Morphine sulphate $20 \mathrm{mg} / 2 \mathrm{~mL}$ injection \\
\hline X02Ld & y02AB & Morphine sulphate $20 \mathrm{mg} / 2 \mathrm{~mL}$ prefilled syringe \\
\hline djzs. & yOCZh & Morphine sulphate $20 \mathrm{mg} / \mathrm{mL}$ oral solution \\
\hline dj1Q. & y02AZ & Morphine sulphate $20 \mathrm{mg} /$ sachet granules for suspension \\
\hline djzE. & $\mathrm{y} 08 \mathrm{Pj}$ & Morphine sulphate $30 \mathrm{mg} \mathrm{m} / \mathrm{r}$ capsule \\
\hline djz6. & y02CD & Morphine sulphate $30 \mathrm{mg} \mathrm{m} / \mathrm{r}$ tablet \\
\hline dj1a. & y02AE & Morphine sulphate $30 \mathrm{mg}$ suppository \\
\hline 0454. & y02AA & Morphine sulphate $30 \mathrm{mg} / 1 \mathrm{~mL}$ injection \\
\hline 0456. & y00If & Morphine sulphate $30 \mathrm{mg} / 2 \mathrm{~mL}$ injection \\
\hline djy5. & yOERh & Morphine sulphate $30 \mathrm{mg} / 30 \mathrm{~mL}$ injection \\
\hline dj11. & y02AS & Morphine sulphate $30 \mathrm{mg} / 5 \mathrm{~mL}$ oral unit dose vial \\
\hline dj1R. & y02Ab & Morphine sulphate $30 \mathrm{mg} /$ sachet granules for suspension \\
\hline 0457. & y00Ig & Morphine sulphate $40 \mathrm{mg} / 2 \mathrm{~mL}$ injection \\
\hline djza. & y09wJ & Morphine sulphate $50 \mathrm{mg} \mathrm{m} / \mathrm{r}$ capsule \\
\hline djzL. & y09br & Morphine sulphate $50 \mathrm{mg}$ tablet \\
\hline djzh. & yOAPL & Morphine sulphate $50 \mathrm{mg} / 50 \mathrm{~mL}$ injection \\
\hline djzd. & y0A5m & Morphine sulphate $50 \mathrm{mg} / 50 \mathrm{~mL}$ prefilled syringe \\
\hline djz3. & y02CA & Morphine sulphate $5 \mathrm{mg} \mathrm{m} / \mathrm{r}$ tablet \\
\hline djzF. & y08Pk & Morphine sulphate $60 \mathrm{mg} \mathrm{m} / \mathrm{r}$ capsule \\
\hline djz7. & $\mathrm{y} 02 \mathrm{CE}$ & Morphine sulphate $60 \mathrm{mg} \mathrm{m} / \mathrm{r}$ tablet \\
\hline dj18. & y02AC & Morphine sulphate $60 \mathrm{mg} / 2 \mathrm{~mL}$ injection \\
\hline djzA. & y01Iz & Morphine sulphate $60 \mathrm{mg} /$ sachet granules for suspension \\
\hline djzO. & y09lH & Morphine sulphate $90 \mathrm{mg} \mathrm{m} / \mathrm{r}$ capsule \\
\hline x0081 & y03TP & Morphine tartrate+cyclizine \\
\hline dj1i. & y02AR & MST Continus $100 \mathrm{mg} \mathrm{m} / \mathrm{r}$ tablet \\
\hline dj1X. & y02An & MST Continus $100 \mathrm{mg} /$ sachet granules for suspension \\
\hline dj1f. & y02AM & MST Continus $10 \mathrm{mg} \mathrm{m} / \mathrm{r}$ tablet \\
\hline dj1z. & y02Ap & MST Continus $15 \mathrm{mg} \mathrm{m} / \mathrm{r}$ tablet \\
\hline dj1t. & $\mathrm{y} 02 \mathrm{Ae}$ & MST Continus $200 \mathrm{mg} \mathrm{m} / \mathrm{r}$ tablet \\
\hline dj1Y. & y01IY & MST Continus $200 \mathrm{mg} /$ sachet granules for suspension \\
\hline dj1N. & y02AW & MST Continus 20mg/sachet granules for suspension \\
\hline dj1g. & y02AO & MST Continus 30mg $\mathrm{m} / \mathrm{r}$ tablet \\
\hline dj1P. & y02AX & MST Continus 30mg/sachet granules for suspension \\
\hline dj1y. & y02Ao & MST Continus $5 \mathrm{mg} \mathrm{m} / \mathrm{r}$ tablet \\
\hline dj1h. & y02AP & MST Continus $60 \mathrm{mg} \mathrm{m} / \mathrm{r}$ tablet \\
\hline dj1W. & y02Al & MST Continus $60 \mathrm{mg} / \mathrm{sachet}$ granules for suspension \\
\hline djzV. & y0910 & MXL $120 \mathrm{mg} \mathrm{m} / \mathrm{r}$ capsule \\
\hline djzW. & y09IP & MXL $150 \mathrm{mg} \mathrm{m} / \mathrm{r}$ capsule \\
\hline $\operatorname{djz} X$. & y09IQ & MXL 200mg m/r capsule \\
\hline djzS. & y09IL & MXL 30mg m/r capsule \\
\hline
\end{tabular}




\begin{tabular}{|c|c|c|}
\hline djzT. & y09IM & MXL $60 \mathrm{mg} \mathrm{m} / \mathrm{r}$ capsule \\
\hline djzU. & y09IN & MXL $90 \mathrm{mg} \mathrm{m} / \mathrm{r}$ capsule \\
\hline dj1k. & y00lk & Nepenthe $4.2 \mathrm{mg} / 0.5 \mathrm{~mL}$ injection \\
\hline dj1j. & y00lj & Nepenthe $8.4 \mathrm{mg} / \mathrm{mL}$ elixir \\
\hline dj1b. & y00Ih & Opium $10 \mathrm{mg} / \mathrm{mL}$ tincture \\
\hline dj1J. & y02AT & Oramorph $100 \mathrm{mg} / 5 \mathrm{~mL}$ oral unit dose vial \\
\hline dj1l. & y00Il & Oramorph $10 \mathrm{mg} / 5 \mathrm{~mL}$ liquid $100 \mathrm{~mL}$ \\
\hline dj1p. & y00Ip & Oramorph $10 \mathrm{mg} / 5 \mathrm{~mL}$ liquid $250 \mathrm{~mL}$ \\
\hline dj1m. & y00Im & Oramorph $10 \mathrm{mg} / 5 \mathrm{~mL}$ liquid $500 \mathrm{~mL}$ \\
\hline dj12. & y0CP9 & Oramorph $10 \mathrm{mg} / 5 \mathrm{~mL}$ oral solution $300 \mathrm{~mL}$ \\
\hline dj1F. & y02AN & Oramorph $10 \mathrm{mg} / 5 \mathrm{~mL}$ oral unit dose vial \\
\hline dj1H. & $\mathrm{y} 02 \mathrm{AQ}$ & Oramorph $30 \mathrm{mg} / 5 \mathrm{~mL}$ oral unit dose vial \\
\hline dj10. & y00lo & Oramorph concentrated $20 \mathrm{mg} / \mathrm{mL}$ liquid $120 \mathrm{~mL}$ \\
\hline dj1n. & y00In & Oramorph concentrated $20 \mathrm{mg} / \mathrm{mL}$ liquid $30 \mathrm{~mL}$ \\
\hline dj1x. & y02Am & Oramorph SR $100 \mathrm{mg} \mathrm{m} / \mathrm{r}$ tablet \\
\hline dj1u. & y02Ag & Oramorph SR $10 \mathrm{mg} \mathrm{m} / \mathrm{r}$ tablet \\
\hline dj1v. & $\mathrm{y} 02 \mathrm{Ai}$ & Oramorph SR 30mg m/r tablet \\
\hline dj1w. & y02Ak & Oramorph SR $60 \mathrm{mg} \mathrm{m} / \mathrm{r}$ tablet \\
\hline djzp. & y0c8f & $\begin{array}{l}\text { Pharma-Ject Morphine sulphate } 100 \mathrm{mg} / 50 \mathrm{~mL} \text { prefilled } \\
\text { syringe }\end{array}$ \\
\hline djzo. & y0c8e & Pharma-Ject Morphine sulphate $50 \mathrm{mg} / 50 \mathrm{~mL}$ prefilled syringe \\
\hline djzg. & y0A5q & Rapiject Morphine sulphate $100 \mathrm{mg} / 50 \mathrm{~mL}$ prefilled syringe \\
\hline djzf. & y0A5p & Rapiject Morphine sulphate $50 \mathrm{mg} / 50 \mathrm{~mL}$ prefilled syringe \\
\hline djyH. & yOFOp & Rhotard SR $100 \mathrm{mg} \mathrm{m} / \mathrm{r}$ tablet \\
\hline djyE. & yOFOm & Rhotard SR $10 \mathrm{mg} \mathrm{m} / \mathrm{r}$ tablet \\
\hline djyF. & yOFOn & Rhotard SR 30mg m/r tablet \\
\hline djyG. & yOFOo & Rhotard SR 60mg m/r tablet \\
\hline dj1A. & $\mathrm{y} 02 \mathrm{AF}$ & Sevredol 10mg suppository \\
\hline dj1r. & y02Aa & Sevredol 10mg tablet \\
\hline djzq. & yOCai & Sevredol $10 \mathrm{mg} / 5 \mathrm{~mL}$ oral solution $100 \mathrm{~mL}$ \\
\hline djzt. & yOCaj & Sevredol $10 \mathrm{mg} / 5 \mathrm{~mL}$ oral solution $300 \mathrm{~mL}$ \\
\hline djzu. & y0Cak & Sevredol $10 \mathrm{mg} / 5 \mathrm{~mL}$ oral solution $500 \mathrm{~mL}$ \\
\hline dj1B. & y02AG & Sevredol 20mg suppository \\
\hline dj1s. & $\mathrm{y} 02 \mathrm{Ac}$ & Sevredol 20mg tablet \\
\hline djzv. & yOCam & Sevredol $20 \mathrm{mg} / \mathrm{mL}$ concentrated oral solution $120 \mathrm{~mL}$ \\
\hline djzr. & yoCal & Sevredol $20 \mathrm{mg} / \mathrm{mL}$ concentrated oral solution $30 \mathrm{~mL}$ \\
\hline dj1C. & $\mathrm{y} 02 \mathrm{Al}$ & Sevredol 30mg suppository \\
\hline djzM. & y09bs & Sevredol 50mg tablet \\
\hline djzm. & yOAVM & Zomorph $100 \mathrm{mg} \mathrm{m} / \mathrm{r}$ capsule \\
\hline djzj. & yOAVJ & Zomorph $10 \mathrm{mg} \mathrm{m} / \mathrm{r}$ capsule \\
\hline djzn. & yOAVN & Zomorph $200 \mathrm{mg} \mathrm{m} / \mathrm{r}$ capsule \\
\hline djzk. & yOAVK & Zomorph $30 \mathrm{mg} \mathrm{m} / \mathrm{r}$ capsule \\
\hline djzl. & yOAVL & Zomorph $60 \mathrm{mg} \mathrm{m} / \mathrm{r}$ capsule \\
\hline \multicolumn{3}{|c|}{ Papavertum } \\
\hline 0474. & y018y & Omnopon 10mg tablet \\
\hline 0479. & $\mathrm{y} 01 \mathrm{HL}$ & Omnopon $15.4 \mathrm{mg} / 1 \mathrm{~mL}$ injection \\
\hline o4b2. & y04F1 & Omnopon Paediatric $7.84 \mathrm{mg} / 1 \mathrm{~mL}$ injection \\
\hline 047y. & y0192 & Papaveretum $10 \mathrm{mg}$ tablet \\
\hline 0478. & y01HK & Papaveretum BP $15.4 \mathrm{mg} / 1 \mathrm{~mL}$ injection \\
\hline o47A. & y080f & Papaveretum BP 7.7mg/1mL injection \\
\hline \multicolumn{3}{|c|}{ Oxycodone } \\
\hline djm4. & yz7wY & DOLOCODON PR $10 \mathrm{mg} \mathrm{m} / \mathrm{r}$ tablets \\
\hline djm5. & $y z 7 w z$ & DOLOCODON PR $20 \mathrm{mg} \mathrm{m} / \mathrm{r}$ tablets \\
\hline djm6. & yz7wa & DOLOCODON PR 40mg $\mathrm{m} / \mathrm{r}$ tablets \\
\hline djm3. & $y z 7 w X$ & DOLOCODON PR $5 \mathrm{mg} \mathrm{m} / \mathrm{r}$ tablets \\
\hline djkM. & yz60c & LONGTEC $10 \mathrm{mg} \mathrm{m} / \mathrm{r}$ tablets \\
\hline djkN. & yz60d & LONGTEC $20 \mathrm{mg} \mathrm{m} / \mathrm{r}$ tablets \\
\hline djkO. & yz60e & LONGTEC $40 \mathrm{mg} \mathrm{m} / \mathrm{r}$ tablets \\
\hline djkL. & $y z 60 b$ & LONGTEC $5 \mathrm{mg} \mathrm{m} / \mathrm{r}$ tablets \\
\hline djkP. & yz60f & LONGTEC $80 \mathrm{mg} \mathrm{m} / \mathrm{r}$ tablets \\
\hline djkw. & yz7GB & LYNLOR 10mg capsules \\
\hline djkX. & yz7GC & LYNLOR 20mg capsules \\
\hline djkV. & yz7GA & LYNLOR $5 \mathrm{mg}$ capsules \\
\hline djki. & $y z 3 L x$ & OXYCODONE HCL+NALOXONE HCL 40mg/20mg $\mathrm{m} / \mathrm{r}$ tablets \\
\hline djkj. & yz3Ly & OXYCODONE HCL+NALOXONE HCL $5 \mathrm{mg} / 2.5 \mathrm{mg} \mathrm{m} / \mathrm{r}$ tablets \\
\hline djku. & y0D6x & Oxycodone hydrochloride $10 \mathrm{mg}$ capsule \\
\hline djkw. & y0D6M & Oxycodone hydrochloride $10 \mathrm{mg} \mathrm{m} / \mathrm{r}$ tablet \\
\hline djks. & y0D6d & Oxycodone hydrochloride $10 \mathrm{mg} / \mathrm{mL}$ concentrate $\mathrm{s} / \mathrm{f}$ liquid \\
\hline
\end{tabular}




\begin{tabular}{|c|c|c|}
\hline djko. & yOFPs & $\begin{array}{l}\text { Oxycodone hydrochloride } 10 \mathrm{mg} / \mathrm{mL} \text { injection solution } 1 \mathrm{~mL} \\
\text { ampoule }\end{array}$ \\
\hline djkn. & y0FQ1 & $\begin{array}{l}\text { Oxycodone hydrochloride } 10 \mathrm{mg} / \mathrm{mL} \text { injection solution } 2 \mathrm{~mL} \\
\text { ampoule }\end{array}$ \\
\hline djke. & $\mathrm{yz} 4 \mathrm{vQ}$ & OXYCODONE HYDROCHLORIDE $120 \mathrm{mg} \mathrm{m} / \mathrm{r}$ tablets \\
\hline djkh. & yz4vT & OXYCODONE HYDROCHLORIDE $15 \mathrm{mg} \mathrm{m} / \mathrm{r}$ tablets \\
\hline djkv. & y0D6Y & Oxycodone hydrochloride $20 \mathrm{mg}$ capsule \\
\hline djkx. & y0D6N & Oxycodone hydrochloride $20 \mathrm{mg} \mathrm{m} / \mathrm{r}$ tablet \\
\hline djkg. & yz4vS & OXYCODONE HYDROCHLORIDE $30 \mathrm{mg} \mathrm{m} / \mathrm{r}$ tablets \\
\hline djky. & y0D60 & Oxycodone hydrochloride $40 \mathrm{mg} \mathrm{m} / \mathrm{r}$ tablet \\
\hline djkk. & yz315 & $\begin{array}{l}\text { OXYCODONE HYDROCHLORIDE } 50 \mathrm{mg} / 1 \mathrm{~mL} \text { soln for injection } \\
\text { ampoules }\end{array}$ \\
\hline djkt. & y0D6W & Oxycodone hydrochloride $5 \mathrm{mg}$ capsule \\
\hline djkq. & yOEix & Oxycodone hydrochloride $5 \mathrm{mg} \mathrm{m} / \mathrm{r}$ tablet \\
\hline djkr. & y0D6c & Oxycodone hydrochloride $5 \mathrm{mg} / 5 \mathrm{~mL} \mathrm{~s} / \mathrm{f}$ liquid \\
\hline djkf. & yz4vR & OXYCODONE HYDROCHLORIDE $60 \mathrm{mg} \mathrm{m} / \mathrm{r}$ tablets \\
\hline djkz. & y0D6P & Oxycodone hydrochloride $80 \mathrm{mg} \mathrm{m} / \mathrm{r}$ tablet \\
\hline djkm. & yOJFp & $\begin{array}{l}\text { OXYCODONE HYDROCHLORIDE+NALOXONE HYDROCHLORIDE } \\
10 \mathrm{mg} / 5 \mathrm{mg} \mathrm{m} / \mathrm{r} \text { tablets }\end{array}$ \\
\hline djkl. & yOJFo & $\begin{array}{l}\text { OXYCODONE HYDROCHLORIDE+NALOXONE HYDROCHLORIDE } \\
20 \mathrm{mg} / 10 \mathrm{mg} \mathrm{m} / \mathrm{r} \text { tablets }\end{array}$ \\
\hline djk4. & y0D6Q & OxyContin $10 \mathrm{mg} \mathrm{m} / \mathrm{r}$ tablet \\
\hline djkk. & yz4vP & OXYCONTIN $120 \mathrm{mg} \mathrm{m} / \mathrm{r}$ tablets \\
\hline djkH. & yz4vM & OXYCONTIN $15 \mathrm{mg} \mathrm{m} / \mathrm{r}$ tablets \\
\hline djk5. & y0D6R & OxyContin $20 \mathrm{mg} \mathrm{m} / \mathrm{r}$ tablet \\
\hline djkl. & $\mathrm{yz} 4 \mathrm{vN}$ & OXYCONTIN 30mg $\mathrm{m} / \mathrm{r}$ tablets \\
\hline djk6. & y0D6S & OxyContin $40 \mathrm{mg} \mathrm{m} / \mathrm{r}$ tablet \\
\hline djkp. & yOEiY & OxyContin $5 \mathrm{mg} \mathrm{m} / \mathrm{r}$ tablet \\
\hline djkJ. & $\mathrm{yz} 4 \mathrm{vO}$ & OXYCONTIN $60 \mathrm{mg} \mathrm{m} / \mathrm{r}$ tablets \\
\hline djk7. & y0D6T & OxyContin $80 \mathrm{mg} \mathrm{m} / \mathrm{r}$ tablet \\
\hline djkR. & yz61P & OXYLAN $10 \mathrm{mg} \mathrm{m} / \mathrm{r}$ tablets \\
\hline djkS. & yz61Q & OXYLAN $20 \mathrm{mg} \mathrm{m} / \mathrm{r}$ tablets \\
\hline djkT. & yz61R & OXYLAN 40mg $\mathrm{m} / \mathrm{r}$ tablets \\
\hline djkQ. & yz610 & OXYLAN $5 \mathrm{mg} \mathrm{m} / \mathrm{r}$ tablets \\
\hline djkU. & yz61S & OXYLAN $80 \mathrm{mg} \mathrm{m} / \mathrm{r}$ tablets \\
\hline djk2. & y0D6a & OxyNorm $10 \mathrm{mg}$ capsule \\
\hline djk9. & y0D6f & OxyNorm $10 \mathrm{mg} / \mathrm{mL}$ concentrate liquid \\
\hline djkA. & yOFPw & OxyNorm $10 \mathrm{mg} / \mathrm{mL}$ injection solution $1 \mathrm{~mL}$ ampoule \\
\hline djkB. & y0FQ4 & OxyNorm $10 \mathrm{mg} / \mathrm{mL}$ injection solution $2 \mathrm{~mL}$ ampoule \\
\hline djk3. & y0D6b & OxyNorm 20mg capsule \\
\hline djkE. & $y z 314$ & OXYNORM $50 \mathrm{mg} / 1 \mathrm{~mL}$ solution for injection ampoules \\
\hline djk1. & y0D6Z & OxyNorm 5mg capsule \\
\hline djk8. & y0D6e & OxyNorm $5 \mathrm{mg} / 5 \mathrm{~mL}$ liquid \\
\hline djkc. & yz7sc & RELTEBON $10 \mathrm{mg} \mathrm{m} / \mathrm{r}$ tablets \\
\hline djkd. & yz7sd & RELTEBON $20 \mathrm{mg} \mathrm{m} / \mathrm{r}$ tablets \\
\hline djm1. & yz7sf & RELTEBON 40mg $\mathrm{m} / \mathrm{r}$ tablets \\
\hline djkb. & yz7sb & RELTEBON $5 \mathrm{mg} \mathrm{m} / \mathrm{r}$ tablets \\
\hline djm2. & yz7sg & RELTEBON $80 \mathrm{mg} \mathrm{m} / \mathrm{r}$ tablets \\
\hline djkZ. & $y z 7 Z 1$ & SHORTEC $10 \mathrm{mg}$ capsules \\
\hline djka. & $y z 7 Z m$ & SHORTEC $20 \mathrm{mg}$ capsules \\
\hline djkY. & yz7Zk & SHORTEC $5 \mathrm{mg}$ capsules \\
\hline djkC. & yOJFm & TARGINACT $10 \mathrm{mg} / 5 \mathrm{mg} \mathrm{m} / \mathrm{r}$ tablets \\
\hline djkD. & yOJFn & TARGINACT $20 \mathrm{mg} / 10 \mathrm{mg} \mathrm{m} / \mathrm{r}$ tablets \\
\hline djkG. & yz3Lw & TARGINACT $40 \mathrm{mg} / 20 \mathrm{mg} \mathrm{m} / \mathrm{r}$ tablets \\
\hline djkF. & yz3Lv & TARGINACT $5 \mathrm{mg} / 2.5 \mathrm{mg} \mathrm{m} / \mathrm{r}$ tablets \\
\hline \multicolumn{3}{|c|}{ Pethidine } \\
\hline djg1. & y00JF & Pethidine $25 \mathrm{mg}$ paediatric tablet \\
\hline djg2. & $\mathrm{y} 02 \mathrm{CO}$ & Pethidine hydrochloride $50 \mathrm{mg}$ tablet \\
\hline 0483. & $\mathrm{y} 02 \mathrm{C} 1$ & Pethidine hydrochloride $50 \mathrm{mg} / 1 \mathrm{~mL}$ injection \\
\hline djg6. & yOOJG & Pethilorfan 50mg/1mL injection \\
\hline djg3. & y08DA & Pethidine [analg] 50mg/1ml injection \\
\hline 0484. & y07a4 & Pethidine hydrochloride $100 \mathrm{mg} / 2 \mathrm{~mL}$ injection \\
\hline 0481. & y03SV & Pethidine hydrochloride $50 \mathrm{mg} / 5 \mathrm{~mL}$ injection \\
\hline 0482. & y03SW & Pethidine hydrochloride $100 \mathrm{mg} / 10 \mathrm{~mL}$ injection \\
\hline djg8. & y02C4 & $\begin{array}{l}\text { Pethidine hydrochloride+promethazine hydrochloride } \\
100 \mathrm{mg} / 50 \mathrm{mg} / 2 \mathrm{~mL} \text { injection }\end{array}$ \\
\hline djg5. & $\mathrm{y} 02 \mathrm{C3}$ & Pamergan P100 injection \\
\hline djg7. & yOOJH & Pethilorfan $100 \mathrm{mg} / 2 \mathrm{~mL}$ injection \\
\hline
\end{tabular}




\begin{tabular}{|c|c|c|}
\hline \multicolumn{3}{|c|}{ Methadone } \\
\hline djcy. & $\mathrm{y} 02 \mathrm{Bh}$ & Methadone hydrochloride $5 \mathrm{mg}$ tablet \\
\hline djc1. & $\mathrm{y} 02 \mathrm{Be}$ & Physeptone $5 \mathrm{mg}$ tablet \\
\hline \multicolumn{3}{|c|}{ Tapentadol } \\
\hline & yz4vL & TAPENTADOL $50 \mathrm{mg} \mathrm{m} / \mathrm{r}$ tablets \\
\hline & $\mathrm{yz} 4 \mathrm{vH}$ & TAPENTADOL $250 \mathrm{mg} \mathrm{m} / \mathrm{r}$ tablets \\
\hline & $\mathrm{yz} 4 \mathrm{vl}$ & TAPENTADOL $200 \mathrm{mg} \mathrm{m} / \mathrm{r}$ tablets \\
\hline & yz4vK & TAPENTADOL $100 \mathrm{mg} \mathrm{m} / \mathrm{r}$ tablets \\
\hline & yz4vG & TAPENTADOL 50mg tablets \\
\hline & yz7Tu & TAPENTADOL $20 \mathrm{mg} / \mathrm{mL}$ oral solution \\
\hline & yz4vJ & TAPENTADOL $150 \mathrm{mg} \mathrm{m} / \mathrm{r}$ tablets \\
\hline & $\mathrm{yz} 4 \mathrm{vF}$ & TAPENTADOL $75 \mathrm{mg}$ tablets \\
\hline & yz4v9 & PALEXIA SR $100 \mathrm{mg} \mathrm{m} / \mathrm{r}$ tablets \\
\hline & $y z 4 v B$ & PALEXIA SR $200 \mathrm{mg} \mathrm{m} / \mathrm{r}$ tablets \\
\hline & $\mathrm{yz} 4 \mathrm{vC}$ & PALEXIA SR $250 \mathrm{mg} \mathrm{m} / \mathrm{r}$ tablets \\
\hline & yz4vA & PALEXIA SR $150 \mathrm{mg} \mathrm{m} / \mathrm{r}$ tablets \\
\hline & yz4v8 & PALEXIA SR 50mg $\mathrm{m} / \mathrm{r}$ tablets \\
\hline & $\mathrm{yz} 4 \mathrm{vD}$ & PALEXIA 50mg tablets \\
\hline & yz4vE & PALEXIA 75mg tablets \\
\hline & yz7Tt & PALEXIA $20 \mathrm{mg} / \mathrm{mL}$ oral solution \\
\hline
\end{tabular}

\section{A.5 Population estimates}

Table A.3: Calculating population estimates per Health Board

\begin{tabular}{|l|l|l|l|l|l|l|l|l|}
\hline $\begin{array}{l}\text { \% of total } \\
\text { Welsh } \\
\text { populatio } \\
\mathrm{n}\end{array}$ & $\begin{array}{l}\text { Wales } \\
\text { Total }\end{array}$ & ABMUHB & ABUHB & BCUHB & CVUHB & CTUHB & HDUHB & PTHB \\
\hline 2005 & 2969300 & 504,781 & 564,167 & 653246 & 475088 & 296930 & 356316 & 118772 \\
\hline 2006 & 2985700 & 507,569 & 567,283 & 656854 & 477712 & 298570 & 358284 & 119428 \\
\hline 2007 & 3006300 & 511,071 & 571,197 & 661386 & 481008 & 300630 & 360756 & 120252 \\
\hline 2008 & 3025900 & 514,403 & 574,921 & 665698 & 484144 & 302590 & 363108 & 121036 \\
\hline 2009 & 3038872 & 512,921 & 572,518 & 684,575 & 463,818 & 292,899 & 379,051 & 133,090 \\
\hline 2010 & 3049971 & 515,420 & 574,778 & 685,911 & 467,837 & 292,952 & 380,195 & 132,878 \\
\hline 2011 & 3063758 & 517,981 & 577,077 & 688,417 & 472,121 & 293,224 & 381,867 & 133,071 \\
\hline 2012 & 3074067 & 519,481 & 577,981 & 690,434 & 475,324 & 294,497 & 383,398 & 132,952 \\
\hline 2013 & 3082412 & 520,710 & 579,101 & 691,986 & 478,869 & 295,135 & 383,906 & 132,705 \\
\hline 2014 & 3092036 & 523,001 & 580,401 & 694,038 & 481,979 & 295,953 & 383,989 & 132,675 \\
\hline 2015 & 3099086 & 525,466 & 581,789 & 694,473 & 484,752 & 296,735 & 383,229 & 132,642 \\
\hline
\end{tabular}

$\mathrm{ABMUHB}=$ Abertawe Bro Morgannwg University Health Board, $\mathrm{ABUHB}=$ Aneurin Bevan University Health Board, $\mathrm{BCUHB}=$ Betsi Cadwaladr University Health Board, $\mathrm{CVUHB}=$ Cardiff and Vale University Health Board, $\mathrm{CTUHB}=\mathrm{Cwm}$ Taf University Health Board, HDUHB $=$ Hywel Dda University Health Board, PTHB = Powys Teaching Health Board

Table A.4: Percentage of each Health Board primary care population represented within SAIL databank Health Board

\section{Percentage}

represented in SAIL

databank

\begin{tabular}{ll}
\hline Abertawe Bro Morgannwg University Health Board & $96 \%$ \\
\hline Aneurin Bevan University Health Board & $70 \%$ \\
\hline Betsi Cadwaladr University Health Board & $75 \%$ \\
\hline Cardiff and Vale University Health Board & $80 \%$ \\
\hline Cwm Taf University Health Board & $77 \%$ \\
\hline Hywel Dda University Health Board & $81 \%$ \\
\hline Powys Teaching Health Board & $41 \%$
\end{tabular}

Table A.5: Calculated annual SAIL databank population per Health Board

\begin{tabular}{|c|c|c|c|c|c|c|c|}
\hline SAIL & ABMUHB & ABUHB & BCUHB & CVUHB & CTUHB & HDUHB & PTHB \\
\hline 2005 & 484590 & 394917 & 489935 & 380070 & 228636 & 288616 & 48697 \\
\hline 2006 & 487266 & 397098 & 492641 & 382170 & 229899 & 290210 & 48965 \\
\hline 2007 & 490628 & 399838 & 496040 & 384806 & 231485 & 292212 & 49303 \\
\hline
\end{tabular}




\begin{tabular}{|l|l|l|l|l|l|l|l|}
\hline 2008 & 493827 & 402445 & 499274 & 387315 & 232994 & 294117 & 49625 \\
\hline 2009 & 492404 & 400763 & 513431 & 371054 & 225532 & 307031 & 54567 \\
\hline 2010 & 494803 & 402345 & 514433 & 374270 & 225573 & 307958 & 54480 \\
\hline 2011 & 497262 & 403954 & 516313 & 377697 & 225782 & 309312 & 54559 \\
\hline 2012 & 498702 & 404587 & 517826 & 380259 & 226763 & 310552 & 54510 \\
\hline 2013 & 499882 & 405371 & 518990 & 383095 & 227254 & 310964 & 54409 \\
\hline 2014 & 502081 & 406281 & 520529 & 385583 & 227884 & 311031 & 54397 \\
\hline 2015 & 504447 & 407252 & 520855 & 387802 & 228486 & 310415 & 54383 \\
\hline
\end{tabular}

Table A.6: Health Board populations per 1000 population

\begin{tabular}{|r|c|c|c|c|c|c|c|}
\hline SAIL/1000 & ABMUHB & ABUHB & BCUHB & CVUHB & CTUHB & HDUHB & PTHB \\
\hline 2005 & 484.6 & 394.9 & 489.9 & 380.1 & 228.6 & 288.6 & 48.7 \\
\hline 2006 & 487.3 & 397.1 & 492.6 & 382.2 & 229.9 & 290.2 & 49.0 \\
\hline 2007 & 490.6 & 399.8 & 496.0 & 384.8 & 231.5 & 292.2 & 49.3 \\
\hline 2008 & 493.8 & 402.4 & 499.3 & 387.3 & 233.0 & 294.1 & 49.6 \\
\hline 2009 & 492.4 & 400.8 & 513.4 & 371.1 & 225.5 & 307.0 & 54.6 \\
\hline 2010 & 494.8 & 402.3 & 514.4 & 374.3 & 225.6 & 308.0 & 54.5 \\
\hline 2011 & 497.3 & 404.0 & 516.3 & 377.7 & 225.8 & 309.3 & 54.6 \\
\hline 2012 & 498.7 & 404.6 & 517.8 & 380.3 & 226.8 & 310.6 & 54.5 \\
\hline 2013 & 499.9 & 405.4 & 519.0 & 383.1 & 227.3 & 311.0 & 54.4 \\
\hline 2014 & 502.1 & 406.3 & 520.5 & 385.6 & 227.9 & 311.0 & 54.4 \\
\hline 2015 & 504.4 & 407.3 & 520.9 & 387.8 & 228.5 & 310.4 & 54.4 \\
\hline
\end{tabular}

Table A.7: SAIL population - based on 78\% of Welsh primary care population represented within the Databank

\begin{tabular}{|l|c|c|c|}
\hline Year & All Wales & SAIL & Population/1000 \\
\hline 2005 & 2969309 & 2315460 & 2315.46014 \\
\hline 2006 & 2985668 & 2328249 & 2328.24886 \\
\hline 2007 & 3006299 & 2344313 & 2344.31274 \\
\hline 2008 & 3025867 & 2359597 & 2359.59682 \\
\hline 2009 & 3038872 & 2364783 & 2364.78285 \\
\hline 2010 & 3049971 & 2373862 & 2373.86162 \\
\hline 2011 & 3063758 & 2384879 & 2384.87907 \\
\hline 2012 & 3074067 & 2393199 & 2393.19855 \\
\hline 2013 & 3082412 & 2399964 & 2399.96386 \\
\hline 2014 & 3092036 & 2407785 & 2407.78501 \\
\hline 2015 & 3099086 & 2413641 & 2413.64067 \\
\hline
\end{tabular}

Table A.8: SAIL population by gender

Table A.8: SAIL population by gender
\begin{tabular}{|l|c|c|c|c|c|c|c|c|}
\hline & Male & Female & Male\% & Female\% & MaleSAIL & $\begin{array}{c}\text { Female } \\
\text { SAIL }\end{array}$ & $\begin{array}{c}\text { MaleSAIL } \\
/ 1000\end{array}$ & $\begin{array}{c}\text { FemaleSA } \\
\text { IL/1000 }\end{array}$ \\
\hline 2005 & 1447755 & 1521554 & $48.8 \%$ & $51.2 \%$ & 1128956 & 1186504 & 1129.0 & 1186.5 \\
\hline 2006 & 1455660 & 1530008 & $48.8 \%$ & $51.2 \%$ & 1135136 & 1193113 & 1135.1 & 1193.1 \\
\hline 2007 & 1468535 & 1537764 & $48.8 \%$ & $51.2 \%$ & 1145164 & 1199149 & 1145.2 & 1199.1 \\
\hline 2008 & 1479395 & 1546472 & $48.9 \%$ & $51.1 \%$ & 1153645 & 1205952 & 1153.6 & 1206.0 \\
\hline 2009 & 1487848 & 1551024 & $49.0 \%$ & $51.0 \%$ & 1157810 & 1206973 & 1157.8 & 1207.0 \\
\hline 2010 & 1495493 & 1554478 & $49.0 \%$ & $51.0 \%$ & 1163976 & 1209885 & 1164.0 & 1209.9 \\
\hline 2011 & 1504475 & 1559283 & $49.1 \%$ & $50.9 \%$ & 1171108 & 1213771 & 1171.1 & 1213.8 \\
\hline 2012 & 1509936 & 1564131 & $49.1 \%$ & $50.9 \%$ & 1175504 & 1217695 & 1175.5 & 1217.7 \\
\hline 2013 & 1515227 & 1567185 & $49.2 \%$ & $50.8 \%$ & 1179755 & 1220209 & 1179.8 & 1220.2 \\
\hline 2014 & 1521315 & 1570721 & $49.2 \%$ & $50.8 \%$ & 1184656 & 1223129 & 1184.7 & 1223.1 \\
\hline 2015 & 1525561 & 1573525 & $49.2 \%$ & $50.8 \%$ & 1188143 & 1225498 & 1188.1 & 1225.5 \\
\hline
\end{tabular}

Table A.9: Percentage gender split by Health Board

\begin{tabular}{|l|c|c|}
\hline & Female & Male \\
\hline ABMUHB & $50.5 \%$ & $49.5 \%$ \\
\hline ABUHB & $51.0 \%$ & $49.0 \%$ \\
\hline
\end{tabular}




\begin{tabular}{|l|l|l|}
\hline BCUHB & $50.7 \%$ & $49.3 \%$ \\
\hline CTUHB & $51.0 \%$ & $49.0 \%$ \\
\hline CVUHB & $50.9 \%$ & $49.1 \%$ \\
\hline HDUHB & $50.8 \%$ & $49.2 \%$ \\
\hline PTHB & $50.5 \%$ & $49.5 \%$ \\
\hline
\end{tabular}

Table A.10: Health Board populations (aged 18 years and over) by gender and age-group

\begin{tabular}{|c|c|c|c|c|c|c|c|}
\hline \multicolumn{8}{|l|}{2015 data } \\
\hline Women & $18-24$ & $25-44$ & $45-64$ & 65 to 74 & 75 to 84 & $85+$ & Total \\
\hline BCUHB & 27,170 & 77,742 & 95,580 & 43,692 & 26,454 & 12,978 & 351,485 \\
\hline PTHB & 4,220 & 12,914 & 19,726 & 9,473 & 5,669 & 2,905 & 67,025 \\
\hline HDUHB & 16,795 & 39,885 & 54,080 & 25,230 & 15,147 & 7,432 & 194,519 \\
\hline ABMUHB & 23,379 & 64,372 & 70,355 & 29,650 & 18,752 & 8,528 & 265,264 \\
\hline CTUHB & 13,361 & 38,153 & 39,168 & 16,154 & 9,597 & 4,209 & 151,187 \\
\hline$A B \cup H B$ & 23,853 & 72,094 & 79,627 & 32,656 & 19,742 & 8,775 & 296,777 \\
\hline CVUHB & 32,704 & 64,627 & 58,379 & 21,095 & 14,135 & 7,112 & 247,268 \\
\hline Men & $18-24$ & $25-44$ & $45-64$ & 65 to 74 & 75 to 84 & $85+$ & Total \\
\hline BCUHB & 29,627 & 78,107 & 91,253 & 42,142 & 22,250 & 6,908 & 341,875 \\
\hline PTHB & 4,839 & 12,888 & 19,048 & 9,363 & 5,104 & 1,643 & 65,705 \\
\hline HDUHB & 19,089 & 38,662 & 51,019 & 24,442 & 13,283 & 4,115 & 188,610 \\
\hline ABMUHB & 27,386 & 65,814 & 67,107 & 26,980 & 14,722 & 4,485 & 260,257 \\
\hline CTUHB & 14,143 & 36,545 & 37,914 & 15,245 & 7,805 & 2,117 & 145,438 \\
\hline$A B \cup H B$ & 25,040 & 68,748 & 77,346 & 30,638 & 16,308 & 4,600 & 285,468 \\
\hline CVUHB & 31,389 & 67,026 & 55,251 & 19,337 & 10,407 & 3,494 & 238,208 \\
\hline
\end{tabular}

Table A.11: SAIL databank population per 1000 by age-group

\begin{tabular}{|l|l|l|l|l|l|l|}
\hline \multirow{2}{*}{ Year } & \multicolumn{7}{l}{ Population/1000 per age-group (years) } \\
\cline { 2 - 7 } & $\mathbf{1 8 - 2 4}$ & $\mathbf{2 5 - 4 4}$ & $\mathbf{4 5 - 6 4}$ & $\mathbf{6 5 - 7 4}$ & $\mathbf{7 5 - 8 4}$ & $\mathbf{8 5 +}$ \\
\hline 2005 & 268.312 & 771.909 & 762.367 & 272.062 & 185.366 & 62.324 \\
\hline 2006 & 273.651 & 771.538 & 773.165 & 273.704 & 184.364 & 65.875 \\
\hline 2007 & 280.233 & 771.48 & 783.638 & 277.502 & 183.512 & 68.86 \\
\hline 2008 & 284.388 & 770.558 & 792.625 & 283.815 & 183.985 & 70.601 \\
\hline 2009 & 288.48 & 766.376 & 800.144 & 290.886 & 184.576 & 72.135 \\
\hline 2010 & 292.336 & 759.521 & 807.755 & 297.052 & 186.469 & 73.734 \\
\hline 2011 & 295.923 & 754.452 & 814.557 & 302.703 & 188.359 & 75.331 \\
\hline 2012 & 300.246 & 745.539 & 811.498 & 318.14 & 190.806 & 76.932 \\
\hline 2013 & 298.455 & 742.365 & 810.751 & 329.493 & 193.839 & 77.298 \\
\hline 2014 & 295.506 & 738.476 & 813.698 & 338.448 & 197.433 & 78.866 \\
\hline 2015 & 292.995 & 737.577 & 815.853 & 346.097 & 199.375 & 79.301 \\
\hline
\end{tabular}

Table A.12: Annual populations by gender and by Health Board, based on percentage gender split

\begin{tabular}{|c|c|c|c|c|c|c|c|}
\hline \multicolumn{7}{|l|}{ Female } \\
\hline SAIL & ABMUHB & ABUHB & BCUHB & CVUHB & CTUHB & HDUHB & PTHB \\
\hline 2005 & 244.6 & 201.3 & 248.4 & 193.7 & 116.5 & 146.5 & 24.6 \\
\hline 2006 & 246.0 & 202.4 & 249.7 & 194.8 & 117.1 & 147.3 & 24.7 \\
\hline 2007 & 247.7 & 205.1 & 251.5 & 196.1 & 117.9 & 148.4 & 24.9 \\
\hline 2008 & 249.3 & 205.1 & 253.1 & 197.4 & 118.7 & 149.3 & 25.1 \\
\hline 2009 & 248.5 & 204.3 & 260.3 & 189.1 & 114.9 & 155.9 & 27.6 \\
\hline 2010 & 249.8 & 205.1 & 260.8 & 190.8 & 114.9 & 156.4 & 27.5 \\
\hline 2011 & 251.0 & 205.9 & 261.7 & 192.5 & 115.0 & 157.0 & 27.6 \\
\hline 2012 & 251.7 & 206.2 & 262.5 & 193.8 & 115.5 & 157.7 & 27.5 \\
\hline 2013 & 252.3 & 206.6 & 263.1 & 195.3 & 115.7 & 157.9 & 27.5 \\
\hline 2014 & 253.4 & 207.1 & 263.9 & 196.5 & 116.1 & 157.9 & 27.5 \\
\hline 2015 & 254.6 & 207.6 & 264.0 & 197.7 & 116.4 & 157.6 & 27.5 \\
\hline \multicolumn{7}{|c|}{ Male } & \\
\hline SAIL & ABMUHB & ABUHB & BCUHB & CVUHB & CTUHB & HDUHB & PTHB \\
\hline 2005 & 240.0 & 193.6 & 241.6 & 186.4 & 112.2 & 142.1 & 24.1 \\
\hline 2006 & 241.3 & 194.7 & 242.9 & 187.4 & 112.8 & 142.9 & 24.2 \\
\hline
\end{tabular}




\begin{tabular}{|l|l|l|l|l|l|l|l|}
\hline 2007 & 243.0 & 196.0 & 244.6 & 188.7 & 113.6 & 143.9 & 24.4 \\
\hline 2008 & 244.6 & 197.3 & 246.2 & 189.9 & 114.3 & 144.8 & 24.6 \\
\hline 2009 & 243.9 & 196.5 & 253.2 & 181.9 & 110.7 & 151.1 & 27.0 \\
\hline 2010 & 245.0 & 197.3 & 253.7 & 183.5 & 110.7 & 151.6 & 27.0 \\
\hline 2011 & 246.3 & 198.1 & 254.6 & 185.2 & 110.8 & 152.3 & 27.0 \\
\hline 2012 & 247.0 & 198.4 & 255.3 & 186.4 & 111.3 & 152.9 & 27.0 \\
\hline 2013 & 247.6 & 198.7 & 255.9 & 187.8 & 111.5 & 153.1 & 26.9 \\
\hline 2014 & 248.6 & 199.2 & 256.7 & 189.1 & 111.8 & 153.1 & 26.9 \\
\hline 2015 & 249.8 & 199.7 & 256.8 & 190.1 & 112.1 & 152.8 & 26.9 \\
\hline
\end{tabular}




\section{Appendix B General trends}

\section{B.1.1 Trends in number of people}

The number of people receiving opioid prescriptions increased by $19.4 \%$ (from 126.2 to 150.6 per 1000 population) between 2005 and 2015 (Figure B.1). Spearman's Rho confirmed the positive trend $(r=0.827, p<0.01)$. The trend in population adjusted number of people, was similar to the empirical trend in numbers (Table B.1).

Table B.1: Trends in the number of people receiving opioid prescriptions, 2005 - 2015 and stratified by opioid type

\begin{tabular}{|c|c|c|c|c|c|c|c|c|c|}
\hline \multirow[b]{3}{*}{ Year } & \multicolumn{6}{|c|}{ Non-cancer $n=1,099,026$} & \multirow{2}{*}{\multicolumn{3}{|c|}{ Strong opioids }} \\
\hline & \multicolumn{3}{|c|}{ Total } & \multicolumn{3}{|c|}{ Weak opioids } & & & \\
\hline & $\begin{array}{c}\text { Number of } \\
\text { people }\end{array}$ & $\begin{array}{c}\text { People per } \\
1000 \\
\text { population }\end{array}$ & $\begin{array}{c}\text { Annual } \\
\text { change } \\
*(\%)\end{array}$ & $\begin{array}{c}\text { Number of } \\
\text { people }\end{array}$ & $\begin{array}{c}\text { People per } \\
1000 \\
\text { population }\end{array}$ & $\begin{array}{c}\text { Annual } \\
\text { change* } \\
(\%)\end{array}$ & $\begin{array}{c}\text { Number of } \\
\text { people }\end{array}$ & $\begin{array}{c}\text { People per } \\
1000 \\
\text { population }\end{array}$ & $\begin{array}{c}\text { Annual } \\
\text { change* } \\
(\%)\end{array}$ \\
\hline 2005 & 292,209 & 126.2 & & 281,978 & 121.8 & & 10,231 & 4.4 & \\
\hline 2006 & 298,838 & 128.4 & 1.7 & 287,449 & 123.5 & 1.4 & 11,389 & 4.9 & 10.7 \\
\hline 2007 & 321,430 & 137.1 & 6.8 & 308,614 & 131.6 & 6.6 & 12,816 & 5.5 & 11.8 \\
\hline 2008 & 336,377 & 142.6 & 4.0 & 322,315 & 136.6 & 3.8 & 14,062 & 6.0 & 9.0 \\
\hline 2009 & 348,136 & 147.2 & 3.3 & 332,543 & 140.6 & 2.9 & 15,593 & 6.6 & 10.6 \\
\hline 2010 & 356199 & 150.1 & 1.9 & 339,198 & 142.9 & 1.6 & 17,001 & 7.2 & 8.6 \\
\hline 2011 & 363,861 & 152.6 & 1.7 & 345,031 & 144.7 & 1.2 & 18,830 & 7.9 & 10.2 \\
\hline 2012 & 368,869 & 154.1 & 1.0 & 347,299 & 145.1 & 0.3 & 21,570 & 9.0 & 14.2 \\
\hline 2013 & 366,047 & 152.5 & -1.0 & 341,700 & 142.4 & -1.9 & 24,347 & 10.1 & 12.6 \\
\hline 2014 & 366,903 & 152.4 & -0.1 & 338,652 & 140.6 & -1.2 & 28,251 & 11.7 & 15.7 \\
\hline 2015 & 363,560 & 150.6 & -1.2 & 331,555 & 137.4 & -2.3 & 32,005 & 13.3 & 13.0 \\
\hline Percentage change (\%) 2005 - 2015 & 24.4 & 19.3 & & 17.6 & 12.8 & & 212.8 & 202.3 & \\
\hline Spearman's r, p-value** & $\begin{array}{c}0.882 \\
\mathrm{p}<.001^{* *}\end{array}$ & $\begin{array}{c}0.827 \\
p<0.01^{*}\end{array}$ & & $\begin{array}{c}0.945 \\
p<.001 * *\end{array}$ & $\begin{array}{c}0.909 \\
p<.001 * *\end{array}$ & & $\begin{array}{c}>.999 \\
\mathrm{p}<.001^{* *}\end{array}$ & $\begin{array}{c}>.999 \\
\mathrm{p}<.001 * *\end{array}$ & \\
\hline
\end{tabular}




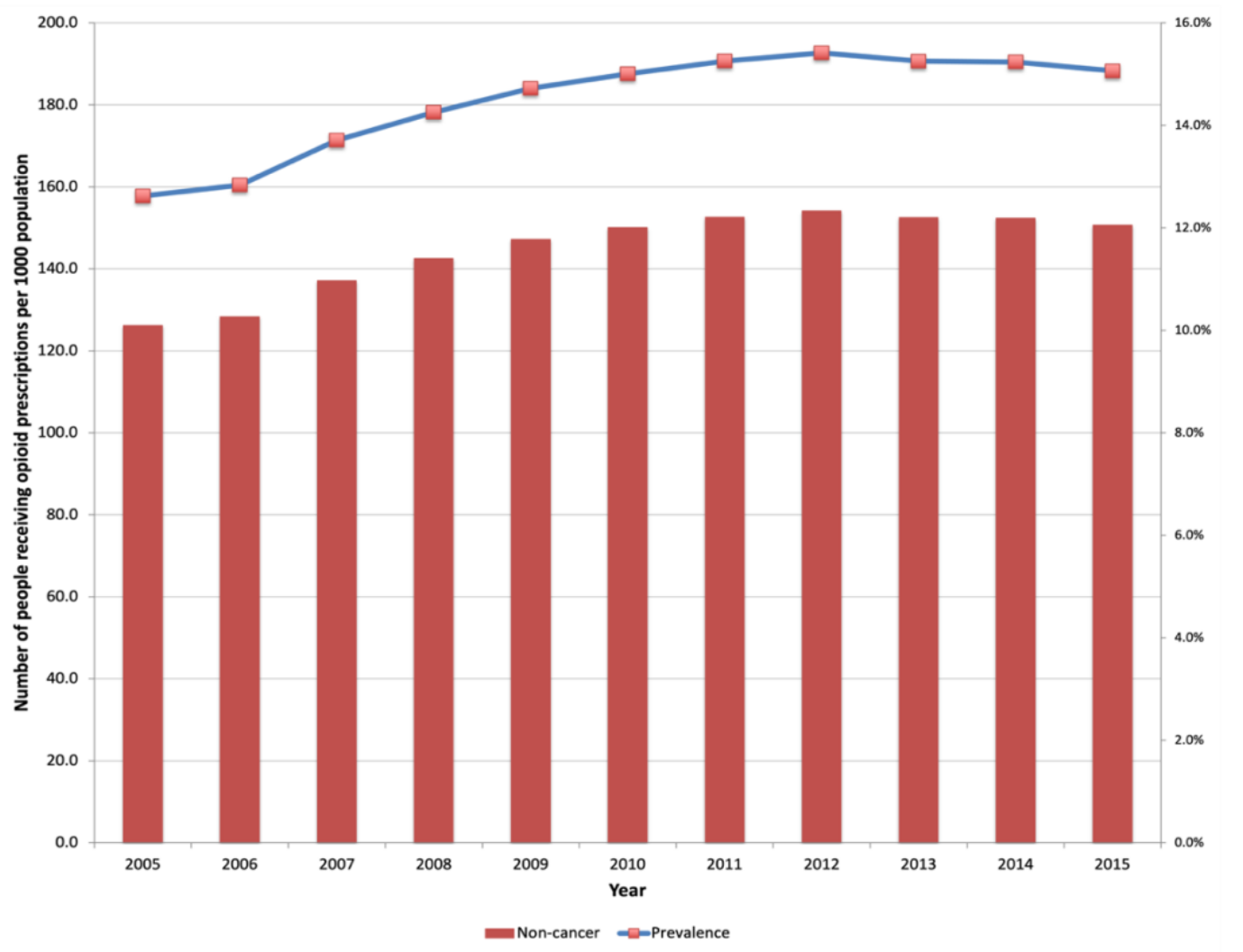

Figure B.1: Number of people receiving prescriptions for opioids between 2005 - 2015 and prescribing prevalence 


\section{B.1.2 Prevalence of people prescribed opioids in SAIL databank population}

The prevalence of non-cancer related opioid prescribing in the SAIL databank population was calculated at 12.6\% in 2005 and peaked in 2012 at 15.4\% (Figure B.1). After that, a small annual reduction led to a non-cancer opioid prescribing prevalence of $15.1 \%$ in 2015 (Table B.2). Over the same period, the Welsh population increased by $4.4 \%$ (Figure B.2)

The largest annual increases in the number of people receiving opioid prescriptions were observed between 2005 and 2010 (Figure B.2). In the second half of the study period, the annual percentage increase in numbers slowed and then started to reduce between 2012 and 2015 (Table B.1).

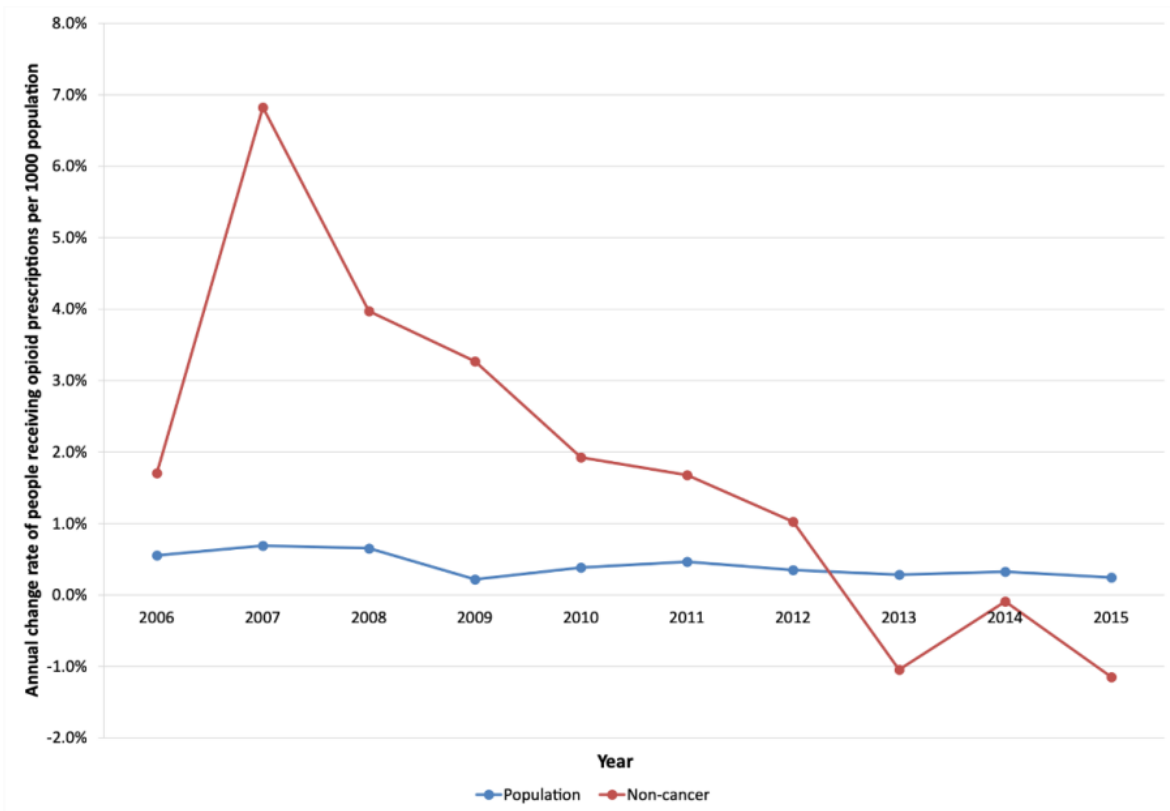

Figure B.2: Annual change rate of the number of people receiving opioid prescriptions and the Welsh population between $2006-2015$

Table B.2: Determining prevalence of opioid prescribing in the SAIL databank population

\begin{tabular}{ccccc} 
Year & $\begin{array}{c}\text { SAIL Databank } \\
\text { population }\end{array}$ & $\begin{array}{c}\text { Number of people } \\
\text { prescribed opioid } \\
\text { analgesics }\end{array}$ & $\begin{array}{c}\text { Prevalence in } \\
\text { SAIL Databank } \\
\text { (\%) }\end{array}$ & $\begin{array}{c}\text { Annual percentage } \\
\text { change in prevalence } \\
\text { (\%) }\end{array}$ \\
\hline $\mathbf{2 0 0 5}$ & $2,315,460$ & 292,209 & 12.6 & 1.7 \\
$\mathbf{2 0 0 6}$ & $2,328,249$ & 298,838 & 12.8 & 6.8 \\
$\mathbf{2 0 0 7}$ & $2,344,313$ & 321,430 & 13.7 & 4.0 \\
$\mathbf{2 0 0 8}$ & $2,359,597$ & 336,377 & 14.3 & 3.3 \\
$\mathbf{2 0 0 9}$ & $2,364,783$ & 348,136 & 14.7 & 1.9 \\
$\mathbf{2 0 1 0}$ & $2,373,862$ & 356,199 & 15.0 & 1.7 \\
$\mathbf{2 0 1 1}$ & $2,384,879$ & 363,861 & 15.3 & 1.0 \\
$\mathbf{2 0 1 2}$ & $2,393,199$ & 368,869 & 15.4 & -1.0 \\
$\mathbf{2 0 1 3}$ & $2,399,964$ & 366,047 & 15.3 & -0.1 \\
$\mathbf{2 0 1 4}$ & $2,407,785$ & 366,903 & 15.2 & -1.2 \\
$\mathbf{2 0 1 5}$ & $2,413,641$ & 363,560 & 15.1 & \\
\hline
\end{tabular}




\section{B.2 Prescribing by drug-type}

\section{B.2.1 Trends in number of people by drug-type}

From 2005 until 2015, the number of people per 1000 population receiving strong opioid prescriptions, tripled (Table B.1). In contrast to weak opioid prescribing, a faster increase in the rate was observed in the second half of the study period (Table B.3). Numbers of people receiving strong opioids rose $85.2 \%$ between 2010 and 2015 compared to $62.1 \%$ between 2005 and 2010 (Figure B.3).

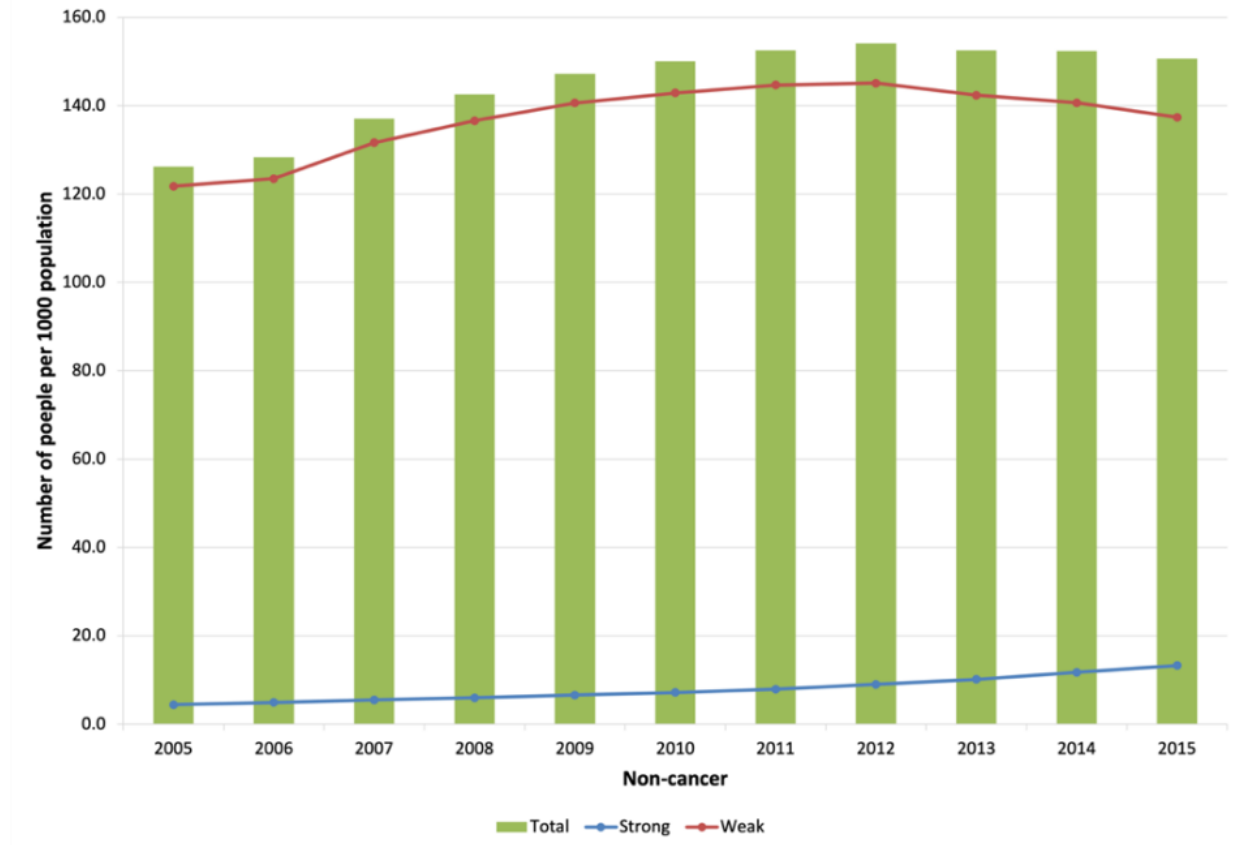

Figure B.3: Trends in the number of people receiving weak and strong opioid prescribing2005-2015

Between 2005 and 2015, strong opioid analgesic prescriptions were issued to $5.4 \%$ of all those who received prescriptions in that time. The percentage of people receiving strong opioids as a percentage of all people receiving opioid prescriptions each year, rose $151.4 \%$ (from $3.5 \%$ to $8.8 \%$ of all prescribing) in the 11-year period (Figure B.4). 


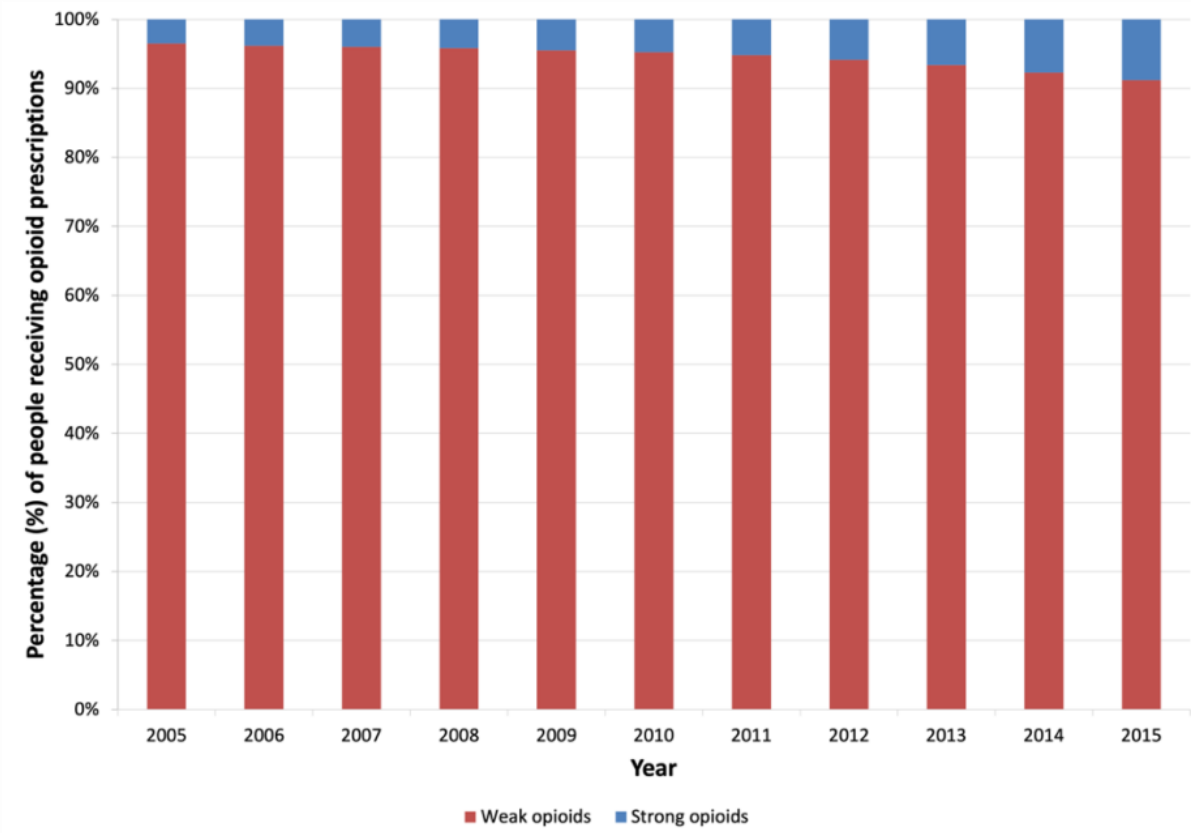

Figure B.4: Percentage of people receiving prescriptions for weak or strong opioids as a percentage of total number of people 


\begin{tabular}{|c|c|c|c|c|c|c|c|c|c|}
\hline \multicolumn{10}{|c|}{ Non-cancer $n=22,786,565$} \\
\hline \multirow[b]{2}{*}{ Year } & \multicolumn{3}{|c|}{ Total } & \multicolumn{3}{|c|}{ Weak opioids } & \multicolumn{3}{|c|}{ Strong opioids } \\
\hline & $\begin{array}{c}\text { Number of } \\
\text { prescriptions }\end{array}$ & $\begin{array}{c}\text { Prescriptions } \\
\text { per } 1000 \\
\text { population }\end{array}$ & $\begin{array}{c}\text { Annual } \\
\text { change* } \\
(\%)\end{array}$ & $\begin{array}{c}\text { Number of } \\
\text { prescriptions }\end{array}$ & $\begin{array}{l}\text { Prescriptions } \\
\text { per } 1000 \\
\text { population }\end{array}$ & $\begin{array}{c}\text { Annual } \\
\text { change* } \\
(\%)\end{array}$ & $\begin{array}{c}\text { Number of } \\
\text { prescriptions }\end{array}$ & $\begin{array}{l}\text { Prescriptions } \\
\text { per } 1000 \\
\text { population }\end{array}$ & $\begin{array}{c}\text { Annual } \\
\text { change* } \\
(\%)\end{array}$ \\
\hline 2005 & $1,613,417$ & 696.8 & & $1,520,441$ & 656.6 & & 92,976 & 40.2 & \\
\hline 2006 & $1,656,332$ & 711.4 & 2.1 & $1,544,592$ & 663.4 & 1.0 & 111,740 & 48.0 & 19.5 \\
\hline 2007 & $1,783,100$ & 760.6 & 6.9 & $1,651,974$ & 704.7 & 6.2 & 131,126 & 55.9 & 16.5 \\
\hline 2008 & $1,904,351$ & 807.1 & 6.1 & $1,751,223$ & 742.2 & 5.3 & 153,128 & 64.9 & 16.0 \\
\hline 2010 & $2,108,572$ & 888.2 & 4.9 & $1,906,075$ & 802.9 & 3.9 & 202,497 & 85.3 & 15.7 \\
\hline 2011 & $2,227,037$ & 933.8 & 5.1 & $1,990,199$ & 834.5 & 3.9 & 236,838 & 99.3 & 16.4 \\
\hline 2012 & $2,318,407$ & 968.7 & 3.7 & $2,043,247$ & 853.8 & 2.3 & 275,160 & 115.0 & 15.8 \\
\hline 2013 & $2,358,706$ & 982.8 & 1.5 & $2,044,810$ & 852.0 & -0.2 & 313,896 & 130.8 & 13.8 \\
\hline 2014 & $2,398,718$ & 996.2 & 1.4 & $2,048,026$ & 850.6 & -0.2 & 350,692 & 145.6 & 11.4 \\
\hline 2015 & $2,415,374$ & 1000.7 & 0.5 & $2,021,677$ & 837.6 & -1.5 & 393,697 & 163.1 & 12.0 \\
\hline $\begin{array}{r}\text { Percentage } \\
\text { change (\%) } 2005 \\
-2015 \\
\end{array}$ & 49.7 & 43.6 & & 33.0 & 27.6 & & 323.4 & 306.2 & \\
\hline $\begin{array}{r}\text { Spearman's r, } \\
\text { p-value** }\end{array}$ & $\begin{array}{c}>.999 \\
p<.001^{* *}\end{array}$ & $\begin{array}{c}>.999 \\
\mathrm{p}<.001^{* *}\end{array}$ & & $\begin{array}{c}0.945 \\
p<.001 * *\end{array}$ & $\begin{array}{c}0.909 \\
p<.001 * *\end{array}$ & & $\begin{array}{c}>.999 \\
p<.001^{* *}\end{array}$ & $>.999, \mathrm{p}<.001^{* *}$ & \\
\hline
\end{tabular}




\section{B.3 Trends in prescribing by gender}

\section{B.3.1 Trends in numbers of people by gender}

Over the 11 years examined, significantly more women received weak opioid prescriptions compared to men (Mann-Whitney $U$ test, $U=121.00, S E=15.23$, $\left.p<.001, \eta^{2}=0.72, d_{\text {cohen }}=3.19\right)$. Women composed $60.1 \%(2,147,966$ women from $3,576,966$ total) of the total number of people receiving prescriptions for weak opioids between 2005 and 2015 (Figure B.5). As previously observed, the difference in the number of men and women receiving prescriptions each year slightly reduced over the 11 years examined. The gap between annual numbers reduced by $11.1 \%$ (from $52.9 \%$ to $41.8 \%$ more women than men per 1000 population per year) by the end of the study period (Table B.4 and Error! Reference source not found.).

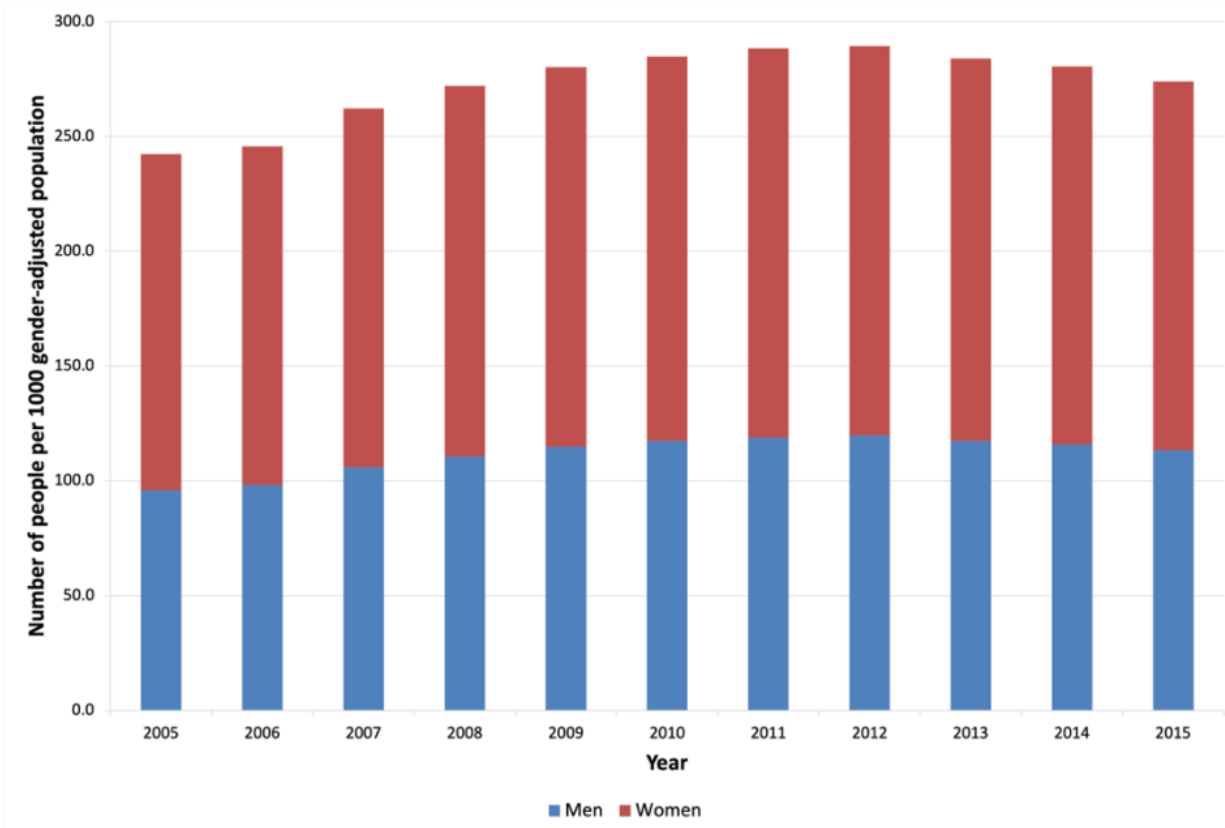

Figure B.5: Gender differences in the number of people receiving weak opioid prescriptions. Data presented adjusted for gender-population

Numbers of men and women receiving weak opioids peaked in 2012 (Figure B.5) as with the trend in all prescribing (Figure B.1). Women were observed to have an increase of $9.7 \%$ (from 146.5 to 160.7 women per 1000 gender-adjusted population) over the 11 years analysed (Spearman's $r=0.745, p<0.01$ ). An average $45 \%$ fewer men than women received weak opioid prescriptions each year (Table B.5). The increase in the number of men, however, was $18.3 \%$ (from 95.8 to 113.3 men per 1000 gender-adjusted population) (Table B.4) (Spearman's $r=0.764, p<0.01)$. 
Table B.4: Trends in the number of men receiving opioid prescriptions 2005 - 2015

\begin{tabular}{|c|c|c|c|c|c|c|c|c|c|c|}
\hline \multicolumn{11}{|c|}{ Men } \\
\hline \multirow[b]{2}{*}{ Year } & & \multicolumn{3}{|c|}{ Total } & \multicolumn{3}{|c|}{ Weak } & \multicolumn{3}{|c|}{ Strong } \\
\hline & & $\begin{array}{c}\text { Number of } \\
\text { people }\end{array}$ & $\begin{array}{c}\text { People per } \\
1000 \\
\text { population }\end{array}$ & $\begin{array}{c}\text { Annual } \\
\text { change* } \\
(\%)\end{array}$ & $\begin{array}{l}\text { Number of } \\
\text { people }\end{array}$ & $\begin{array}{c}\text { People per } \\
1000 \\
\text { population }\end{array}$ & $\begin{array}{c}\text { Annual } \\
\text { change* } \\
(\%)\end{array}$ & $\begin{array}{l}\text { Number } \\
\text { of people }\end{array}$ & $\begin{array}{c}\text { People per } \\
1000 \\
\text { population }\end{array}$ & $\begin{array}{c}\text { Annual } \\
\text { change* } \\
(\%)\end{array}$ \\
\hline & 2005 & 112,388 & 99.6 & & 108,165 & 95.8 & & 4,223 & 3.7 & \\
\hline & 2006 & 116,055 & 102.2 & 2.7 & 111,497 & 98.2 & 2.5 & 4,558 & 4.0 & 7.3 \\
\hline & 2007 & 126,294 & 110.3 & 7.9 & 121,287 & 105.9 & 7.8 & 5,007 & 4.4 & 8.9 \\
\hline & 2008 & 132,916 & 115.2 & 4.5 & 127,581 & 110.6 & 4.4 & 5,335 & 4.6 & 5.8 \\
\hline & 2009 & 138,875 & 119.9 & 4.1 & 133,033 & 114.9 & 3.9 & 5,842 & 5.0 & 9.1 \\
\hline & 2010 & 143,193 & 123.0 & 2.6 & 136,706 & 117.4 & 2.2 & 6,487 & 5.6 & 10.5 \\
\hline & 2011 & 146,295 & 124.9 & 1.5 & 139,165 & 118.8 & 1.2 & 7,130 & 6.1 & 9.2 \\
\hline & 2012 & 148,838 & 126.6 & 1.4 & 140,708 & 119.7 & 0.7 & 8,130 & 6.9 & 13.6 \\
\hline & 2013 & 147,604 & 125.1 & -1.2 & 138,410 & 117.3 & -2.0 & 9,194 & 7.8 & 12.7 \\
\hline & 2014 & 147,869 & 124.8 & -0.2 & 137,188 & 115.8 & -1.3 & 10,681 & 9.0 & 15.7 \\
\hline & 2015 & 146,629 & 123.4 & -1.1 & 134,628 & 113.3 & -2.2 & 12,001 & 10.1 & 12.0 \\
\hline Percent & $15-2015$ & 24.5 & 19.2 & & 24.5 & 18.3 & & 184.2 & 173.0 & \\
\hline \multicolumn{2}{|c|}{ Spearman's r, p-value** } & $\begin{array}{c}0.918 \\
\mathrm{p}<.001^{* *}\end{array}$ & $\begin{array}{c}0.845 \\
p<0.01^{* *}\end{array}$ & & $\begin{array}{c}0.782 \\
p<0.01^{* *}\end{array}$ & $\begin{array}{c}0.664 \\
p<0.05^{* *}\end{array}$ & & $\begin{array}{c}>.999 \\
p<.001^{* *}\end{array}$ & $\begin{array}{c}>.999 \\
p<.001^{* *}\end{array}$ & \\
\hline
\end{tabular}

*based on people per thousand ${ }^{* *} p<0.05=$ statistically significant

Table B.5: Trends in the number of women receiving opioid prescriptions 2005 - 2015

\begin{tabular}{|c|c|c|c|c|c|c|c|c|c|}
\hline \multicolumn{10}{|c|}{ Women } \\
\hline & \multicolumn{3}{|c|}{ Total } & \multicolumn{3}{|c|}{ Weak } & \multicolumn{3}{|c|}{ Strong } \\
\hline Year & $\begin{array}{c}\text { Number of } \\
\text { people }\end{array}$ & $\begin{array}{c}\text { People per } \\
1000 \\
\text { population }\end{array}$ & $\begin{array}{c}\text { Annual } \\
\text { change* } \\
(\%)\end{array}$ & $\begin{array}{c}\text { Number of } \\
\text { people }\end{array}$ & $\begin{array}{c}\text { People per } \\
1000 \\
\text { population }\end{array}$ & $\begin{array}{c}\text { Annual } \\
\text { change* } \\
(\%)\end{array}$ & $\begin{array}{c}\text { Number of } \\
\text { people }\end{array}$ & $\begin{array}{c}\text { People per } \\
1000 \\
\text { population }\end{array}$ & $\begin{array}{c}\text { Annual } \\
\text { change* } \\
(\%)\end{array}$ \\
\hline
\end{tabular}




\begin{tabular}{|c|c|c|c|c|c|c|c|c|c|}
\hline 2005 & 179,821 & 151.6 & & 173,813 & 146.5 & & 6,008 & 5.1 & \\
\hline 2006 & 182,783 & 153.2 & 1.1 & 175,952 & 147.5 & 0.7 & 6,831 & 5.7 & 13.1 \\
\hline 2007 & 195,136 & 162.7 & 6.2 & 187,327 & 156.2 & 5.9 & 7,809 & 6.5 & 13.7 \\
\hline 2008 & 203,461 & 168.7 & 3.7 & 194,734 & 161.5 & 3.4 & 8,727 & 7.2 & 11.1 \\
\hline 2009 & 209,261 & 173.4 & 2.8 & 199,510 & 165.3 & 2.4 & 9,751 & 8.1 & 11.6 \\
\hline 2010 & 213,006 & 176.1 & 1.5 & 202,492 & 167.4 & 1.3 & 10,514 & 8.7 & 7.6 \\
\hline 2011 & 217,566 & 179.2 & 1.8 & 205,866 & 169.6 & 1.3 & 11,700 & 9.6 & 10.9 \\
\hline 2012 & 220,031 & 180.7 & 0.8 & 206,591 & 169.7 & 0.0 & 13,440 & 11.0 & 14.5 \\
\hline 2013 & 218,443 & 179.0 & -0.9 & 203,290 & 166.6 & -1.8 & 15,153 & 12.4 & 12.5 \\
\hline 2014 & 219,034 & 179.1 & 0.0 & 201,464 & 164.7 & -1.1 & 17,570 & 14.4 & 15.7 \\
\hline 2015 & 216,931 & 177.0 & -1.2 & 196,927 & 160.7 & -2.4 & 20,004 & 16.3 & 13.6 \\
\hline Percentage change (\%) 2005 - 2015 & 20.6 & 16.8 & & 13.3 & 9.7 & & 233.0 & 222.4 & \\
\hline Spearman's r, p-value ${ }^{* *}$ & $0.882, \mathrm{p}<.001 * *$ & $\begin{array}{c}0.836 \\
p<0.01^{* *}\end{array}$ & & $\begin{array}{c}0.691 \\
p<0.05^{* *}\end{array}$ & $\begin{array}{c}0.555 \\
p=0.077\end{array}$ & & $\begin{array}{c}>.999 \\
p<.001^{* *}\end{array}$ & $\begin{array}{c}>.999 \\
p<.001^{* *}\end{array}$ & \\
\hline
\end{tabular}

*based on people per thousand ${ }^{* *} p<0.05$ = statistically significant

The annual number of men per 1000 population receiving opioid prescriptions increased 19.3\% (from 99.6 to 123.4 people per 1000 genderadjusted population) between 2005 and 2015 (Table B.4). A 16.8\% (from 151.6 to 177.0 people per 1000 population) rise in the number of women receiving prescriptions was observed over the same period (Table B.5). Strong correlation between time and the change in number of people prescribed opioids, was significant for both genders based on Spearman's Rho results (Table B.4 and Table B.5). A Mann-Whitney U test confirmed the number of women receiving prescriptions was significantly greater than the number of men, over the 11 years analysed

$\left(U=121.00, S D=15.23, p<.001^{*}, \eta^{2}=0.72, d_{\text {cohen }}=3.19\right)$. 


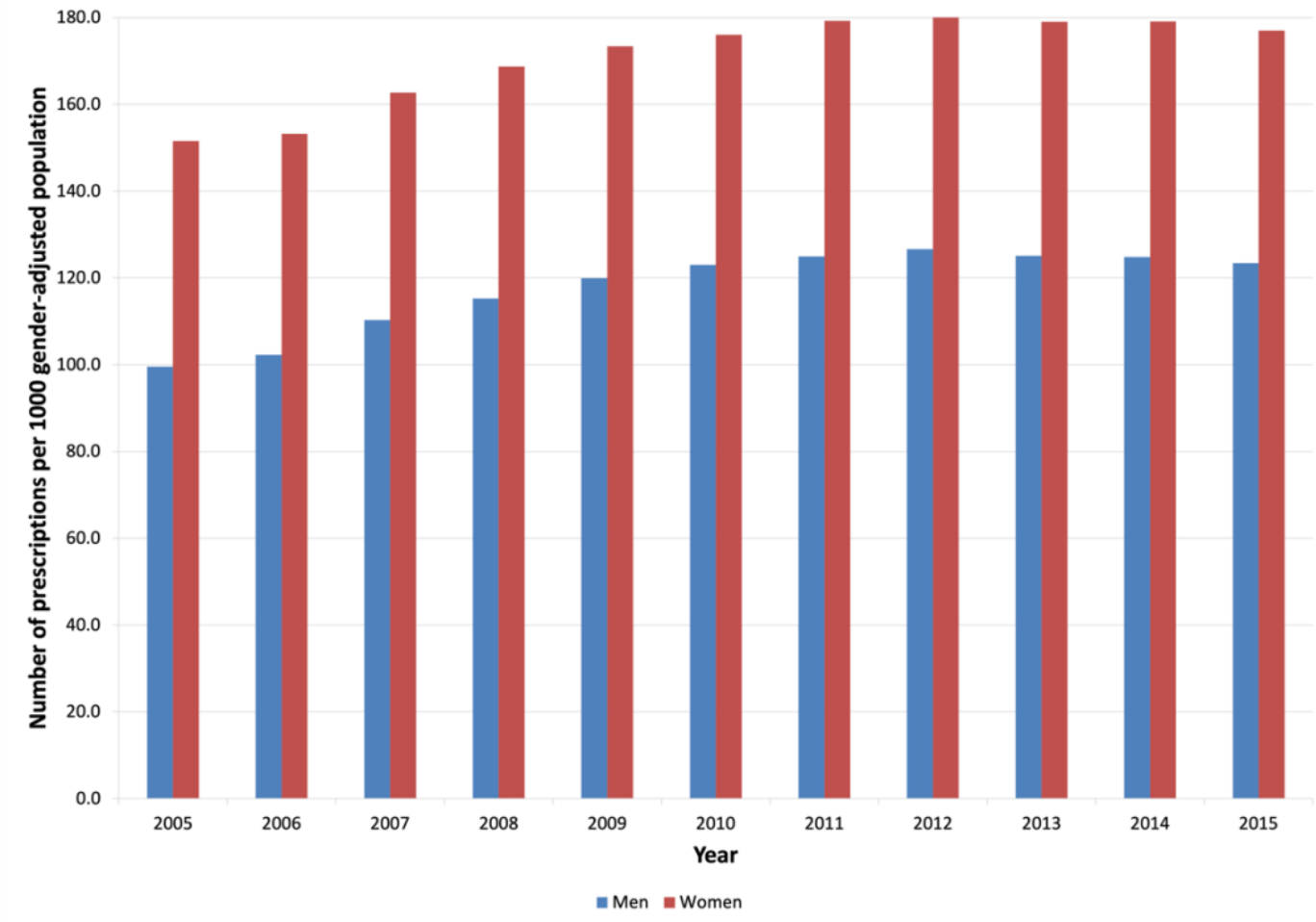

Figure B.6: Trends in the number of prescriptions for opioid analgesics displayed by annual, gender-adjusted population

Peak numbers of men and women were observed in 2012 and then began to slowly decline (Figure B.6). The difference between the number of men and women receiving opioid analgesic prescriptions each year grew proportionately less over time. In 2005, 52\% more women than men received opioid prescriptions, but this reduced to 43\% in 2015 (Table B.4 and Table B.5). 
Large percentage increases in the number of men and women receiving strong opioid prescriptions were noted between 2005 and 2015 (Table B.4 and Table B.5). The number of women increased $222.4 \%$ (from 5.1 to 16.3 women per 1000 population) with very strong correlation observed by Spearman's Rho test $(r=>.999, p<.001$ ). Similarly, men had a very strongly correlated $170 \%$ (from 3.7 to 10.1 men per 1000 population) increase in numbers receiving strong opioids between 2005 and 2015 (Table B.4).

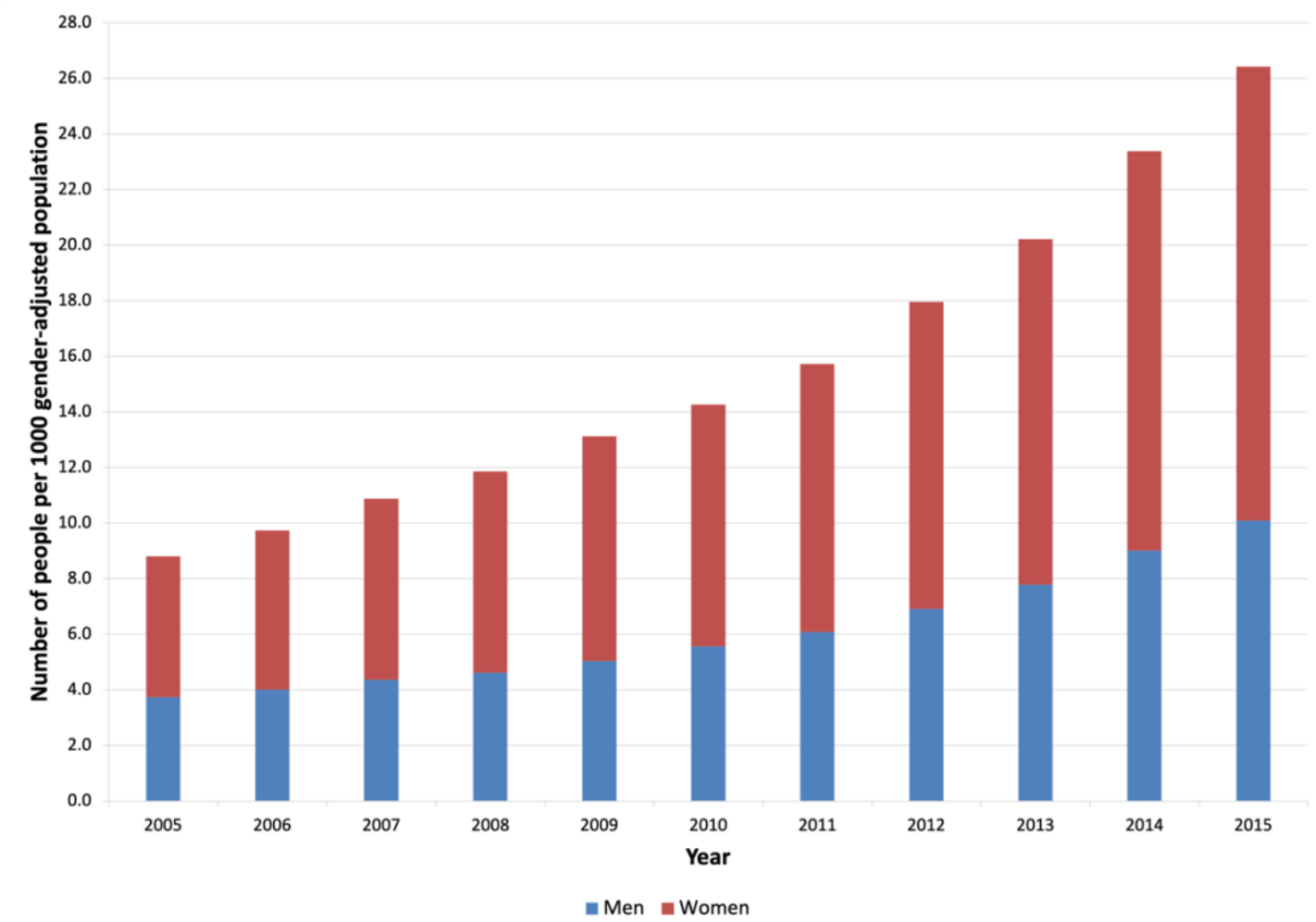

Figure B.7: Gender differences in the number of people receiving strong opioid prescriptions. Data presented adjusted for gender-population

Over the study period, significantly more women than men received strong opioid analgesics (Mann-Whitney $\mathrm{U}, \mathrm{U}=98.00, \mathrm{SE}=15.23, \mathrm{p}<0.05, \eta^{2}=0.28$, $\mathrm{d}_{\text {cohen }}=$ 1.23). Women made up $62 \%$ (127,507 of 206,095 total number of people) of all people receiving strong opioid prescriptions between 2005 and 2015. Whilst both genders were observed to have large increases in number, the gap between men and women receiving prescriptions widened (Figure B.7). At the beginning of the study period in 2005, there were $35.4 \%$ (5.1 women per 1000 population versus 3.7 men per 1000 population) more women than men receiving strong opioid prescriptions. In 2015, the difference was 61.6\% (16.3 women per 1000 population versus 10.1 men per 1000 population) (Table B.4 and Table B.5). 


\section{B.3.2 Trends in numbers of prescriptions by gender}

Table B.6: Trends in the number of prescriptions issued to men and by opioid type

\begin{tabular}{|c|c|c|c|c|c|c|c|c|c|}
\hline \multirow[b]{3}{*}{ Year } & \multicolumn{6}{|c|}{ Men } & \multirow{2}{*}{\multicolumn{3}{|c|}{ Strong }} \\
\hline & \multicolumn{3}{|c|}{ Total } & \multicolumn{3}{|c|}{ Weak } & & & \\
\hline & $\begin{array}{c}\text { Number of } \\
R x\end{array}$ & $\begin{array}{l}\text { Rx per } 1000 \\
\text { population }\end{array}$ & $\begin{array}{c}\text { Annual } \\
\text { change* } \\
(\%)\end{array}$ & $\begin{array}{c}\text { Number of } \\
R x\end{array}$ & $\begin{array}{l}\text { Rx per } 1000 \\
\text { population }\end{array}$ & $\begin{array}{c}\text { Annual } \\
\text { change* } \\
(\%)\end{array}$ & $\begin{array}{l}\text { Number } \\
\text { of } R x\end{array}$ & $\begin{array}{l}\text { Rx per } 1000 \\
\text { population }\end{array}$ & $\begin{array}{c}\text { Annual } \\
\text { change* }(\%)\end{array}$ \\
\hline 2005 & 601,797 & 533.1 & & 560,449 & 496.4 & & 41,348 & 36.6 & \\
\hline 2006 & 623,000 & 548.8 & 3.0 & 575,246 & 506.8 & 2.1 & 47,754 & 42.1 & 14.9 \\
\hline 2007 & 675,953 & 590.3 & 7.5 & 620,623 & 542.0 & 6.9 & 55,330 & 48.3 & 14.9 \\
\hline 2008 & 725,495 & 628.9 & 6.5 & 662,071 & 573.9 & 5.9 & 63,424 & 55.0 & 13.8 \\
\hline 2009 & 769,076 & 664.3 & 5.6 & 699,022 & 603.7 & 5.2 & 70,054 & 60.5 & 10.1 \\
\hline 2010 & 815,391 & 700.5 & 5.5 & 735,080 & 631.5 & 4.6 & 80,311 & 69.0 & 14.0 \\
\hline 2011 & 866,271 & 739.7 & 5.6 & 772,484 & 659.6 & 4.4 & 93,787 & 80.1 & 16.1 \\
\hline 2012 & 907,710 & 772.2 & 4.4 & 796,944 & 678.0 & 2.8 & 110,766 & 94.2 & 17.7 \\
\hline 2013 & 924,056 & 783.3 & 1.4 & 799,842 & 678.0 & 0.0 & 124,214 & 105.3 & 11.7 \\
\hline 2014 & 939,578 & 793.1 & 1.3 & 802,384 & 677.3 & -0.1 & 137,194 & 115.8 & 10.0 \\
\hline 2015 & 945,303 & 795.6 & 0.3 & 790,866 & 665.6 & -1.7 & 154,437 & 130.0 & 12.2 \\
\hline Percentage change (\%) 2005 - 2015 & 57.1 & 49.3 & & 41.1 & 34.1 & & 273.5 & 255.2 & \\
\hline $\begin{array}{r}\text { Spearman's r, } \\
\text { p -value** }\end{array}$ & $\begin{array}{c}>.999 \\
p<.001^{* *}\end{array}$ & $\begin{array}{c}>.999 \\
p<.001^{* *}\end{array}$ & & $\begin{array}{c}0.945 \\
p<0.005^{* *}\end{array}$ & $\begin{array}{c}0.909 \\
p<.001^{* *}\end{array}$ & & $\begin{array}{c}>.999 \\
p<.001^{* *}\end{array}$ & $\begin{array}{c}>.999 \\
p<.001^{* *}\end{array}$ & \\
\hline
\end{tabular}

$\mathrm{Rx}=$ prescriptions

Table B.7: Trends in the number of prescriptions issued to women and by opioid type

\begin{tabular}{|c|c|c|c|c|c|c|c|c|c|}
\hline \multicolumn{10}{|c|}{ Women } \\
\hline & \multicolumn{3}{|c|}{ Total } & \multicolumn{3}{|c|}{ Weak } & \multicolumn{3}{|c|}{ Strong } \\
\hline Year & $\begin{array}{c}\text { Number of } \\
\text { Rx }\end{array}$ & $\begin{array}{c}\text { Rx per } 1000 \\
\text { population }\end{array}$ & $\begin{array}{c}\text { Annual } \\
\text { change* } \\
(\%)\end{array}$ & $\begin{array}{c}\text { Number of } \\
\operatorname{Rx}\end{array}$ & $\begin{array}{c}\text { Rx per } 1000 \\
\text { population }\end{array}$ & $\begin{array}{c}\text { Annual } \\
\text { change* } \\
(\%)\end{array}$ & $\begin{array}{c}\text { Number of } \\
\text { Rx }\end{array}$ & $\begin{array}{c}\text { Rx per } 1000 \\
\text { population }\end{array}$ & $\begin{array}{c}\text { Annual } \\
\text { change* } \\
(\%)\end{array}$ \\
\hline
\end{tabular}




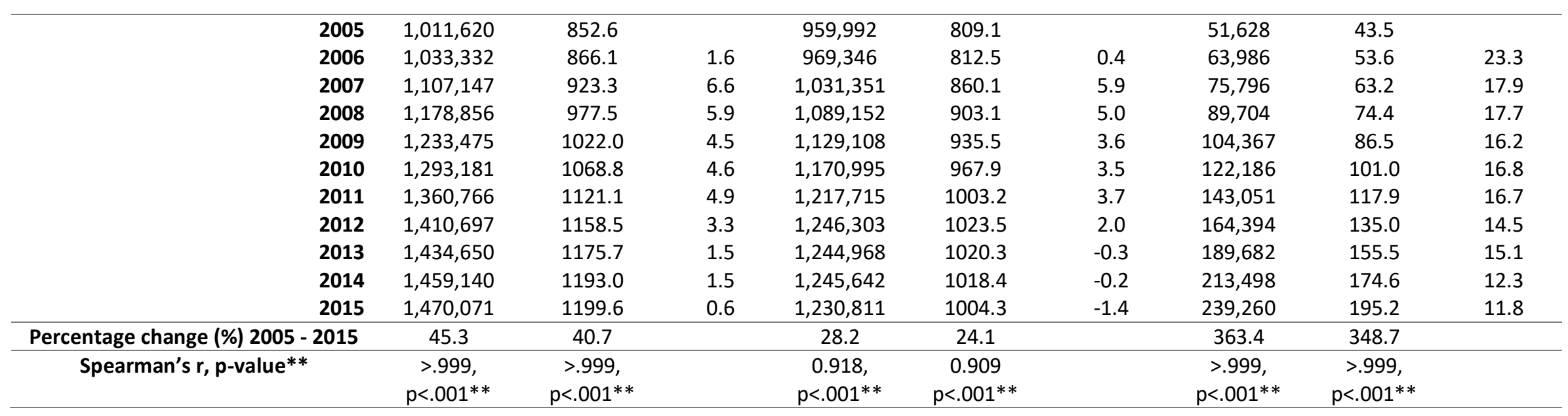

\section{B.4 Trends in prescribing by age}

Increases in the number of people receiving opioid analgesic prescriptions were observed in all but the youngest age group over the period observed (Table B.8). When data was adjusted to age-group population, it was demonstrated people aged 65 years and over, had higher numbers of prescriptions issued than younger people (Figure B.8).

Table B.8: Trends in the number of people receiving opioid prescriptions stratified by age group

\begin{tabular}{|c|c|c|c|c|c|c|c|c|c|c|c|c|}
\hline \multirow[b]{3}{*}{ Year } & \multicolumn{12}{|c|}{ Age-group } \\
\hline & \multicolumn{2}{|c|}{$18-24$} & \multicolumn{2}{|c|}{$25-44$} & \multicolumn{2}{|c|}{$45-64$} & \multicolumn{2}{|c|}{$65-74$} & \multicolumn{2}{|c|}{$75-84$} & \multicolumn{2}{|c|}{$85+$} \\
\hline & $\begin{array}{l}\text { Number } \\
\text { of people }\end{array}$ & $\begin{array}{l}\text { People } \\
\text { per } 1000\end{array}$ & $\begin{array}{l}\text { Number } \\
\text { of people }\end{array}$ & $\begin{array}{c}\text { People } \\
\text { per } 1000\end{array}$ & $\begin{array}{l}\text { Number } \\
\text { of people }\end{array}$ & $\begin{array}{c}\text { People } \\
\text { per } 1000\end{array}$ & $\begin{array}{l}\text { Number } \\
\text { of people }\end{array}$ & $\begin{array}{l}\text { People } \\
\text { per } 1000\end{array}$ & $\begin{array}{l}\text { Number } \\
\text { of people }\end{array}$ & $\begin{array}{c}\text { People } \\
\text { per } 1000\end{array}$ & $\begin{array}{l}\text { Number } \\
\text { of people }\end{array}$ & $\begin{array}{c}\text { People per } \\
1000\end{array}$ \\
\hline 2005 & 17,555 & 65.4 & 89,804 & 116.3 & 150,091 & 196.9 & 76,472 & 281.1 & 53,982 & 291.2 & 18,374 & 294.8 \\
\hline 2006 & 18,065 & 66.0 & 93,444 & 121.1 & 155,944 & 201.7 & 76,352 & 279.0 & 51,894 & 281.5 & 19,013 & 288.6 \\
\hline 2007 & 19,604 & 70.0 & 102,151 & 132.4 & 169,499 & 216.3 & 80,038 & 288.4 & 53,639 & 292.3 & 20,091 & 291.8 \\
\hline 2008 & 20,245 & 71.2 & 107,708 & 139.8 & 179,826 & 226.9 & 83,308 & 293.5 & 54,845 & 298.1 & 21,061 & 298.3 \\
\hline 2009 & 21,098 & 73.1 & 111,133 & 145.0 & 187,579 & 234.4 & 86,308 & 296.7 & 56,213 & 304.6 & 21,515 & 298.3 \\
\hline
\end{tabular}




\begin{tabular}{|c|c|c|c|c|c|c|c|c|c|c|c|c|}
\hline 2010 & 21,086 & 72.1 & 112,319 & 147.9 & 193,826 & 240.0 & 88,802 & 298.9 & 58,288 & 312.6 & 22,358 & 303.2 \\
\hline 2011 & 20,990 & 70.9 & 114,367 & 151.6 & 199,636 & 245.1 & 91,595 & 302.6 & 60,253 & 319.9 & 23,030 & 305.7 \\
\hline 2012 & 20,570 & 68.5 & 114,420 & 153.5 & 202,684 & 249.8 & 95,185 & 299.2 & 61,759 & 323.7 & 24,311 & 316.0 \\
\hline 2013 & 19,779 & 66.3 & 112,348 & 151.3 & 200,999 & 247.9 & 97,203 & 295.0 & 62,869 & 324.3 & 24,685 & 319.3 \\
\hline 2014 & 18,896 & 63.9 & 110,409 & 149.5 & 201,750 & 247.9 & 99,065 & 292.7 & 64,780 & 328.1 & 25,693 & 325.8 \\
\hline 2015 & 17,763 & 60.6 & 107,077 & 145.2 & 200,222 & 245.4 & 100,641 & 290.8 & 65,267 & 327.4 & 25,859 & 326.1 \\
\hline $\begin{array}{r}\text { Rate change } \\
(\%)\end{array}$ & 1.2 & -7.3 & 19.2 & 24.8 & 33.4 & 24.7 & 31.6 & 3.5 & 20.9 & 12.4 & 40.7 & 10.6 \\
\hline $\begin{array}{r}\text { Spearman's } \\
r, p \text {-value* }\end{array}$ & $\begin{array}{c}0.109 \\
p=0.750\end{array}$ & $\begin{array}{c}-0.300 \\
p=0.370\end{array}$ & $\begin{array}{c}0.582 \\
p=0.060\end{array}$ & $\begin{array}{c}0.782 \\
p<0.05^{*}\end{array}$ & $\begin{array}{c}0.918 \\
p<.001 *\end{array}$ & $\begin{array}{c}0.916 \\
p<.001 *\end{array}$ & $\begin{array}{c}0.991 \\
p<.001^{*}\end{array}$ & $\begin{array}{c}0.445 \\
p=0.170\end{array}$ & $\begin{array}{c}0.973 \\
p<.001 *\end{array}$ & $\begin{array}{c}0.982 \\
p<.001^{*}\end{array}$ & $\begin{array}{c}>.999 \\
\mathrm{p}<.001^{*}\end{array}$ & $\begin{array}{c}0.973 \\
p<.001 *\end{array}$ \\
\hline
\end{tabular}




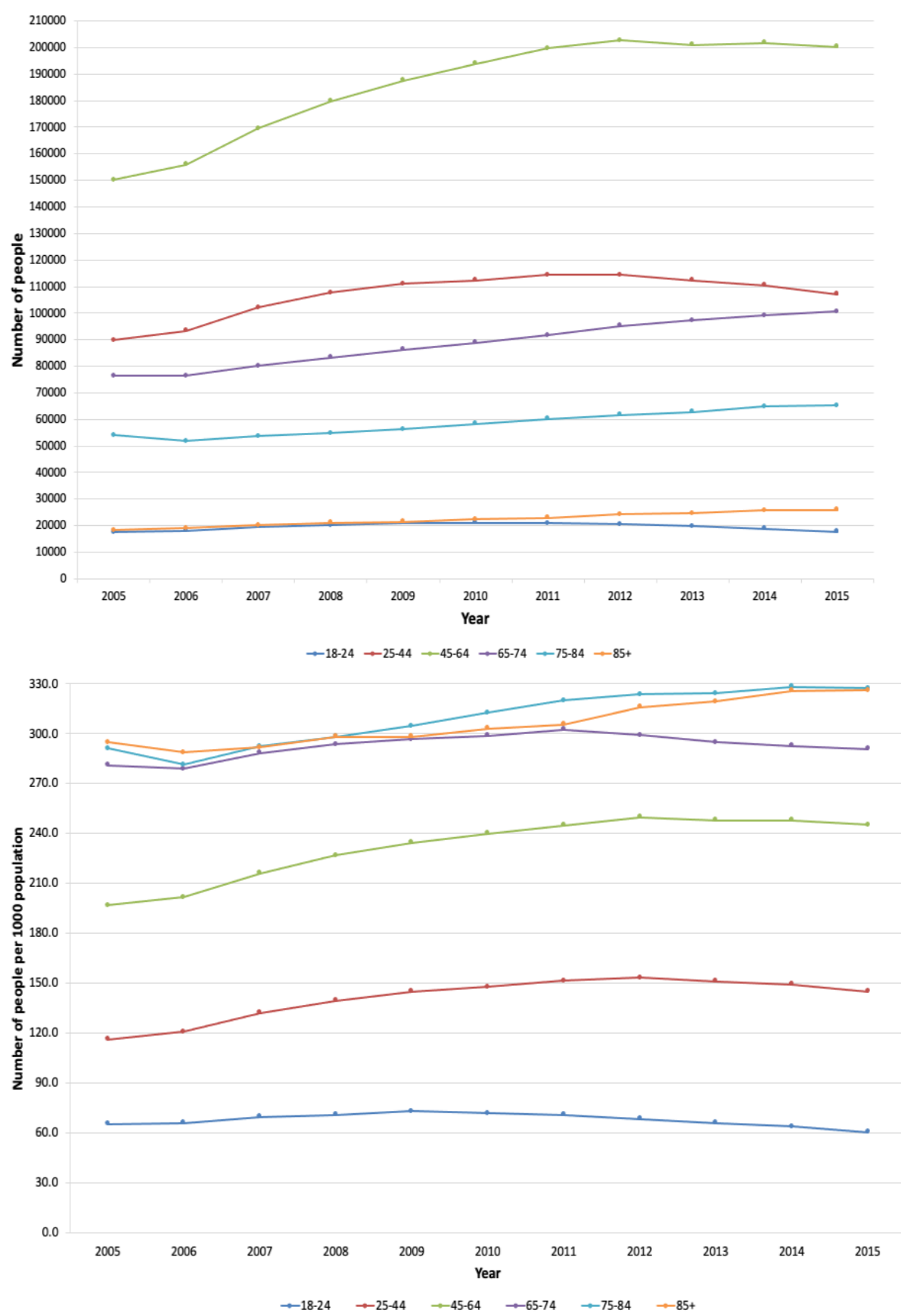

Figure B.8: Left: Trends in the number of people issued with opioid prescriptions between 2005 and 2015, stratified by age-group. Right: Data displayed by number of people per 1000 age-group adjusted population per year

The larger increases in numbers of people per 1000 population receiving prescriptions were of $24.8 \%$ (from 116.3 to 145.2 people per 1000 population, Spearman's $r=0.782, p<0.05$ ) in age 25 to 44 years and $24.7 \%$ (from 196.9 to 245.4 people per 1000 population, Spearman's $r=0.916, p<.001)$ in 45 - to 64year-olds. Strong correlations, indicating an increasing trend over time, were noted in 4 age groups (Table B.8). A moderate increase was noted in the number of people aged 45 to 64 years. A reduction was observed in the number of people per 1000 population aged 18-to 24 years receiving opioid which had a weak correlation based on Spearman's r (Table B.8). 
Kruskal-Wallis test output revealed a statistically significant difference in the number of people receiving opioid prescriptions over the study period $(\mathrm{H}=56.75$, $\left.p<.001, \eta^{2}=0.86, d_{\text {cohen }}=5.01\right)$. Dunn's pairwise comparisons and Bonferroni corrections confirmed significantly more people aged 65 years and over received prescriptions than those aged 18 to 24 years or 25 to 44 years. Significantly more people aged 85 years and over had prescriptions than those aged 45 to 64 years old. No statistical differences were noted in the remaining pairs compared (Table B.9 and Figure B.9).

Table B.9: Dunn's pairwise comparisons and Bonferroni correction output for people per 1000 population receiving opioid prescriptions, stratified by age-group

\begin{tabular}{rccccc}
\hline Age group (years) & $\mathbf{1 8 - 2 4}$ & $\mathbf{2 5 - 4 4}$ & $\mathbf{4 5 - 6 4}$ & $\mathbf{6 5 - 7 4}$ & $\mathbf{7 5 - 8 4}$ \\
\hline $\mathbf{2 5 - 4 4}$ & $>.999$ & & & & \\
\hline $\mathbf{4 5 - 6 4}$ & 0.094 & $>.999$ & & & \\
\hline $\mathbf{6 5 - 7 4}$ & $<.001^{*}$ & $<0.050^{*}$ & 0.684 & & \\
\hline $\mathbf{7 5 - 8 4}$ & $<.001^{*}$ & $<.001^{*}$ & 0.069 & $>.999$ & \\
\hline $85+$ & $<.001^{*}$ & $<.001^{*}$ & $<0.050^{*}$ & $>.999$ & $>.999$ \\
\hline
\end{tabular}

$*_{p}<0.05=$ statistically significant

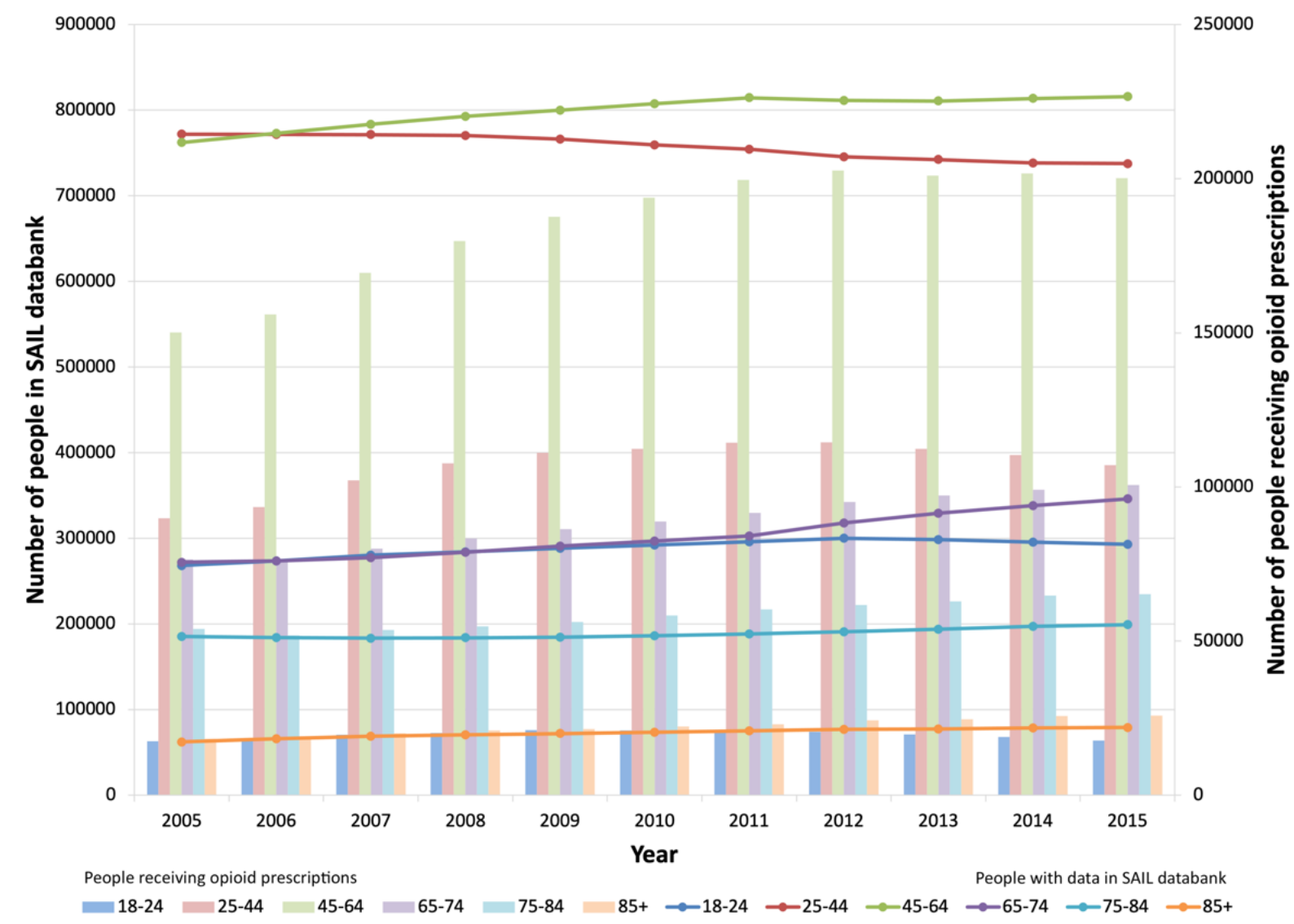

Figure B.9: Comparison of trends in population and number of people receiving opioid prescriptions based on SAIL databank populations 2005 to 2015

The trends in the number of people receiving weak opioid prescriptions between 2005 and 2015 varied, depending on the age-group examined (Figure B.10). The annual number of people receiving weak opioid prescriptions reduced in 3 of the 6 age-groups, which was in contrast to actual age-adjusted populations over the same time, when over the same time period, the only reduction in annual 
number of people was in the 25-44 years age-group (Table B.10). There was a significant difference (Kruskal-Wallis $\mathrm{H}=57.73, \mathrm{p}<.001, \eta^{2}=0.88, \mathrm{~d}_{\text {cohen }}=5.41$ ) in the number of people receiving weak opioid prescriptions when the data was examined by age-group. As with previous pairwise comparisons, statistically more people age 65 years and over, were confirmed to receive weak opioid prescriptions when compared to people age 18-44 years. Statistically more people age 75-84 years, but not those age 65-74 or 85+ year received prescriptions when compared to the $45-64$ years age-group (Table B.11).

The annual number of people in the $18-24$ years age group reduced by $8.6 \%$ (from 64.4 to 58.9 people per 1000 age-adjusted population, Spearman's $r=$ $0.364, p>0.05$ ) between 2005 and 2015, despite an increase of 12\% (from 64.4 to 72.1 people per 1000 age-adjusted population) from the start of the study to 2009 (Error! Reference source not found.).

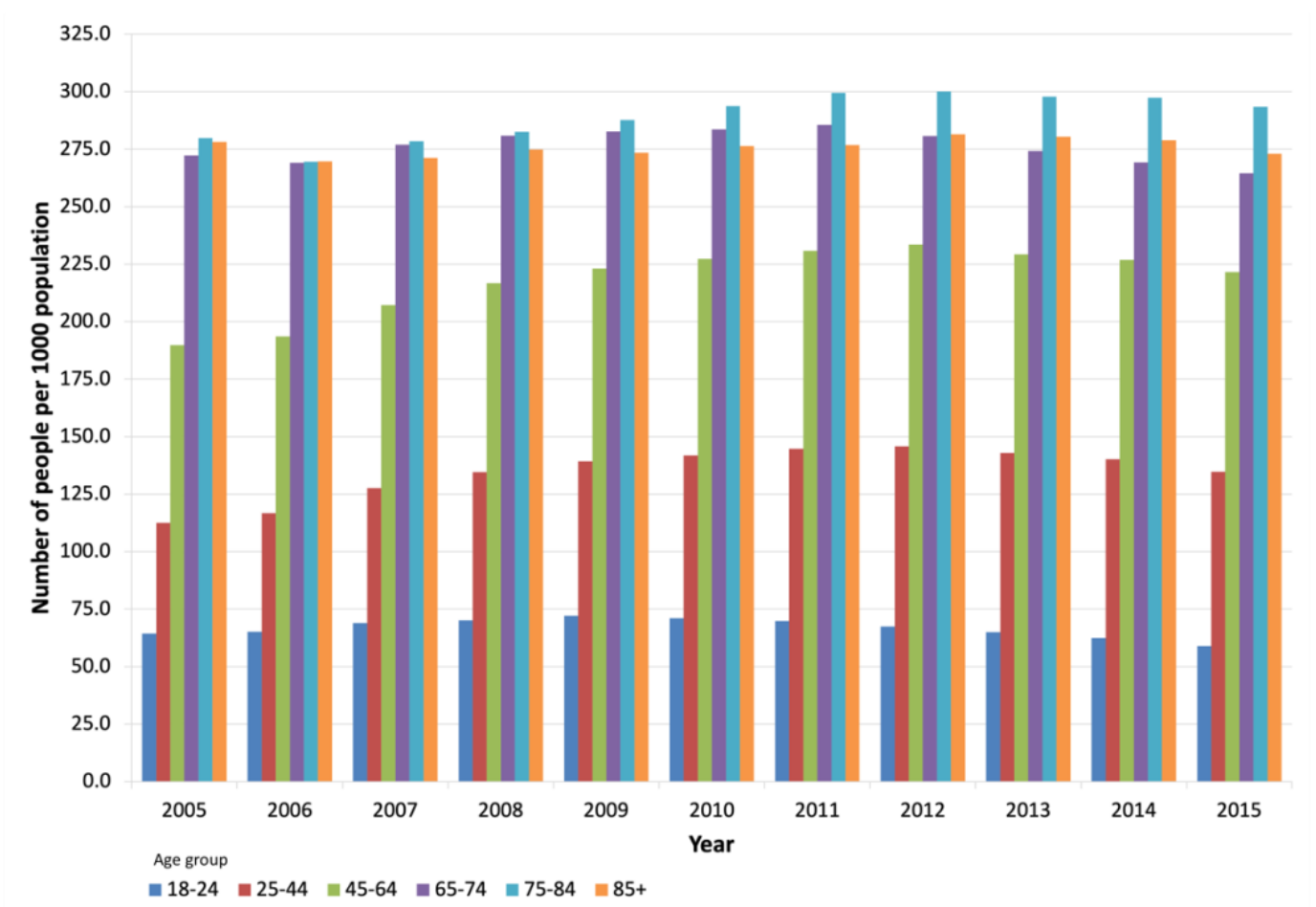

Figure B.10: Trends in number of people receiving weak opioid analgesic prescriptions, stratified by age group and adjusted to age-group population 
Table B.10: Trends in the number of people receiving weak opioid prescriptions, stratified by age group

\begin{tabular}{|c|c|c|c|c|c|c|c|c|c|c|c|c|}
\hline & \multicolumn{12}{|c|}{ Age-group } \\
\hline & \multicolumn{2}{|c|}{$18-24$} & \multicolumn{2}{|c|}{$25-44$} & \multicolumn{2}{|c|}{$45-64$} & \multicolumn{2}{|c|}{$65-74$} & \multicolumn{2}{|c|}{$75-84$} & \multicolumn{2}{|c|}{$85+$} \\
\hline & $\begin{array}{l}\text { Number } \\
\text { of people }\end{array}$ & $\begin{array}{l}\text { People } \\
\text { per } 1000\end{array}$ & $\begin{array}{l}\text { Number } \\
\text { of people }\end{array}$ & $\begin{array}{l}\text { People } \\
\text { per } 1000\end{array}$ & $\begin{array}{l}\text { Number } \\
\text { of people }\end{array}$ & $\begin{array}{l}\text { People } \\
\text { per } 1000\end{array}$ & $\begin{array}{l}\text { Number } \\
\text { of people }\end{array}$ & $\begin{array}{l}\text { People } \\
\text { per } 1000\end{array}$ & $\begin{array}{l}\text { Number } \\
\text { of people }\end{array}$ & $\begin{array}{l}\text { People } \\
\text { per } 1000\end{array}$ & $\begin{array}{l}\text { Number } \\
\text { of people }\end{array}$ & $\begin{array}{c}\text { People } \\
\text { per } 1000\end{array}$ \\
\hline 2005 & 17,287 & 64.4 & 86,835 & 112.5 & 144,712 & 189.8 & 74,085 & 272.3 & 51,873 & 279.8 & 17,337 & 278.2 \\
\hline 2006 & 17,811 & 65.1 & 90,064 & 116.7 & 149,698 & 193.6 & 73,664 & 269.1 & 49,688 & 269.5 & 17,762 & 269.6 \\
\hline 2007 & 19,327 & 69.0 & 98,417 & 127.6 & 162,404 & 207.2 & 76,864 & 277.0 & 51,116 & 278.5 & 18,671 & 271.1 \\
\hline 2008 & 19,957 & 70.2 & 103,736 & 134.6 & 171,752 & 216.7 & 79,722 & 280.9 & 51,989 & 282.6 & 19,404 & 274.8 \\
\hline 2009 & 20,799 & 72.1 & 106,736 & 139.3 & 178,496 & 223.1 & 82,258 & 282.8 & 53,104 & 287.7 & 19,733 & 273.6 \\
\hline 2010 & 20,784 & 71.1 & 107,706 & 141.8 & 183,606 & 227.3 & 84,244 & 283.6 & 54,786 & 293.8 & 20,372 & 276.3 \\
\hline 2011 & 20,683 & 69.9 & 109,239 & 144.8 & 188,023 & 230.8 & 86,452 & 285.6 & 56,411 & 299.5 & 20,857 & 276.9 \\
\hline 2012 & 20,223 & 67.4 & 108,719 & 145.8 & 189,501 & 233.5 & 89,312 & 280.7 & 57,252 & 300.1 & 21,655 & 281.5 \\
\hline 2013 & 19,399 & 65.0 & 106,116 & 142.9 & 185,901 & 229.3 & 90,361 & 274.2 & 57,737 & 297.9 & 21,680 & 280.5 \\
\hline 2014 & 18,457 & 62.5 & 103,480 & 140.1 & 184,579 & 226.8 & 91,146 & 269.3 & 58,715 & 297.4 & 21,998 & 278.9 \\
\hline 2015 & 17,256 & 58.9 & 99,401 & 134.8 & 180,833 & 221.6 & 91,567 & 264.6 & 58,511 & 293.5 & 21,652 & 273.0 \\
\hline $\begin{array}{r}\text { Rate change } \\
(\%)\end{array}$ & -0.2 & -8.6 & 14.5 & 19.8 & 25.0 & 16.8 & 23.6 & -2.8 & 12.8 & 4.9 & 24.9 & -1.8 \\
\hline $\begin{array}{r}\text { Spearman's } \\
r, p \text {-value* }\end{array}$ & $\begin{array}{l}0.018 \\
p=0.60\end{array}$ & $\begin{array}{l}-0.364 \\
p=0.272\end{array}$ & $\begin{array}{c}0.455 \\
p=0.160\end{array}$ & $\begin{array}{c}0.691 \\
p<0.05^{*}\end{array}$ & $\begin{array}{c}0.782 \\
p<0.05^{*}\end{array}$ & $\begin{array}{c}0.691 \\
p<0.05^{*}\end{array}$ & $\begin{array}{c}\text { 0.991, } \\
p<.001 *\end{array}$ & $\begin{array}{l}-0.127 \\
p=0.709\end{array}$ & $\begin{array}{c}0.964 \\
p<.001 *\end{array}$ & $\begin{array}{c}0.755 \\
p<0.01 *\end{array}$ & $\begin{array}{c}0.945 \\
p<.001^{*}\end{array}$ & $\begin{array}{c}0.418 \\
p=0.201\end{array}$ \\
\hline
\end{tabular}

${ }^{*} p<0.05$ = statistically significant

Table B.11: Dunn's pairwise comparisons and Bonferroni correction output for people per 1000 population receiving weak opioid prescriptions, stratified by age-group

\begin{tabular}{rccccc}
\hline Age group (years) & $\mathbf{1 8 - 2 4}$ & $\mathbf{2 5 - 4 4}$ & $\mathbf{4 5 - 6 4}$ & $\mathbf{6 5 - 7 4}$ & $\mathbf{7 5 - 8 4}$ \\
\hline $\mathbf{2 5}-\mathbf{4 4}$ & $>.999$ & & & & \\
\hline $\mathbf{4 5 - 6 4}$ & 0.108 & $>.999$ & & & \\
\hline $\mathbf{6 5 - 7 4}$ & $<.001^{*}$ & $<0.050^{*}$ & 0.799 & & \\
\hline $\mathbf{7 5 - 8 4}$ & $<.001^{*}$ & $<.001^{*}$ & $<0.050^{*}$ & $>.999$ & \\
\hline $\mathbf{8 5 +}$ & $<.001^{*}$ & $<.001^{*}$ & $<0.050^{*}$ & $>.999$ & $>.999$ \\
\hline
\end{tabular}

${ }^{*} p<0.05=$ statistically significant 
The greatest percentage increase in the number of people receiving weak opioid prescriptions, was of $19.8 \%$ (from 112.5 to 134.8 people per 1000 age-adjusted population, Spearman's $r=0.691, p<0.05$ ) in people aged $24-44$ years. There was a peak of 145.8 people per 1000 population in 2012 (29.6\% increase) before the annual number of people in that age-group began to decline (Figure B.11).

The trends in the number of people receiving strong opioid prescriptions demonstrated large increases in all age-groups other than those aged $18-24$ years (Table B.12), where the numbers increased 73.2 (from 1.0 to 1.7 people per 1000 population, Spearman's $r=0.882, p<.001$ ) (Table B.12).

The largest percentage increase in the annual number of people receiving strong opioid prescriptions was noted in the $45-64$ years age-group. The $236.8 \%$ (from 7.1 to 23.8 people per 1000 age-adjusted population, Spearman's $r=>.999$, $p<.001$ ) increase, was fairly steady over the 11 years. However, from 2011, there was a faster rate of annual increase, notably in the age groups 65 years and above (Table B.12).

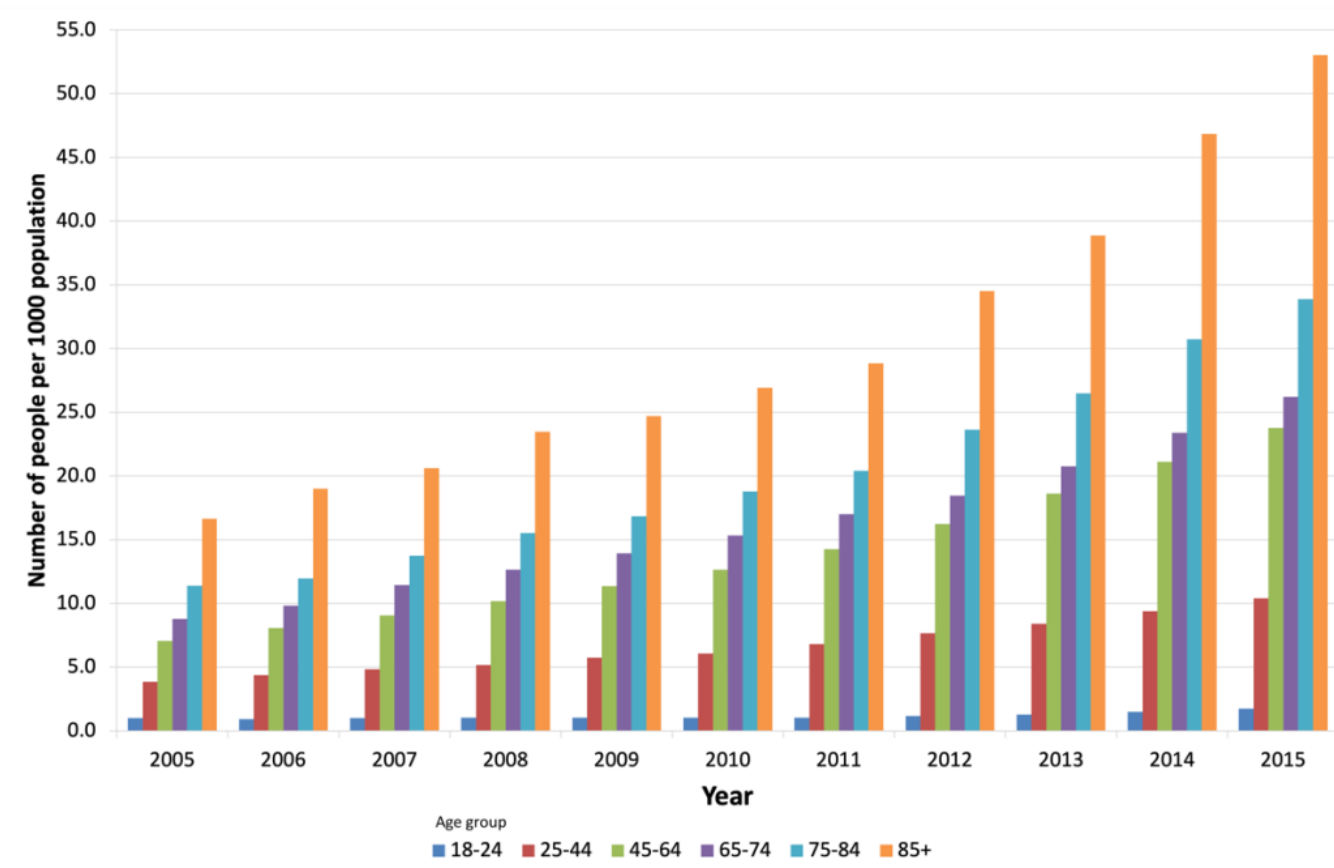

Figure B.11: Trends in the number of people receiving strong opioid prescriptions between 2005 and 2015 and stratified by age-group. Data adjusted to age-group adjusted population

The group with the highest number of people receiving strong opioid prescriptions was 85 years and over (Figure B.11). Based on median values, $43 \%$ more people aged 85 and above, were issued with strong opioid prescriptions than the number in the next highest recipients, aged 75 to 84 years (26.9 and 18.8 people per 1000 age-adjusted population respectively).

Very similar increases in the number of people receiving strong opioid prescriptions were noted in the $65-74$ years age-group $(198.8 \%$, from 8.8 to 26.2 people per 1000 age-adjusted population) and the $75-84$ years age-group (197.8\%, from 11.4 to 33.9 people per 1000 age-adjusted population) (Table 
B.12). More people in the older of the two groups were issued with prescriptions however, with $26 \%$ more people aged $74-84$ years receiving prescriptions based on median values (223.3 versus 177.8 people per 1000 age-adjusted population respectively) (Table B.12).

Differences between all the groups were calculated to be statistically significant (Kruskal-Wallis $\mathrm{H}=37.84, \mathrm{p}<.001, \eta^{2}=0.55$, $\mathrm{d}_{\text {cohen }}=2.20$ ). Pairwise comparisons confirmed significantly fewer people aged 18 to 25 years received opioid prescriptions than all age groups other than 25 to 44 years (Table B.13). There was also confirmation significantly fewer people aged 25 to 44 years, received opioids than people in the oldest age group. No other statistical differences were detected (Table B.13). 
Table B.12: Trends in the number of people receiving strong opioid prescriptions, stratified by age group

\begin{tabular}{|c|c|c|c|c|c|c|c|c|c|c|c|c|}
\hline \multirow[b]{3}{*}{ Year } & \multicolumn{12}{|c|}{ Age-group } \\
\hline & \multicolumn{2}{|c|}{$18-24$} & \multicolumn{2}{|c|}{$25-44$} & \multicolumn{2}{|c|}{$45-64$} & \multicolumn{2}{|c|}{$65-74$} & \multicolumn{2}{|c|}{$75-84$} & \multicolumn{2}{|c|}{$85+$} \\
\hline & $\begin{array}{l}\text { Number } \\
\text { of people }\end{array}$ & $\begin{array}{l}\text { People } \\
\text { per } 1000\end{array}$ & $\begin{array}{l}\text { Number } \\
\text { of people }\end{array}$ & $\begin{array}{l}\text { People } \\
\text { per } 1000\end{array}$ & $\begin{array}{l}\text { Number } \\
\text { of people }\end{array}$ & $\begin{array}{l}\text { People } \\
\text { per } 1000\end{array}$ & $\begin{array}{l}\text { Number } \\
\text { of people }\end{array}$ & $\begin{array}{l}\text { People } \\
\text { per } 1000\end{array}$ & $\begin{array}{l}\text { Number } \\
\text { of people }\end{array}$ & $\begin{array}{l}\text { People } \\
\text { per } 1000\end{array}$ & $\begin{array}{l}\text { Number } \\
\text { of people }\end{array}$ & $\begin{array}{c}\text { People per } \\
1000\end{array}$ \\
\hline 2005 & 268 & 1.0 & 2,969 & 3.8 & 5,379 & 7.1 & 2,387 & 8.8 & 2,109 & 11.4 & 1,037 & 16.6 \\
\hline 2006 & 254 & 0.9 & 3,380 & 4.4 & 6,246 & 8.1 & 2,688 & 9.8 & 2,206 & 12.0 & 1,251 & 19.0 \\
\hline 2007 & 277 & 1.0 & 3,734 & 4.8 & 7,095 & 9.1 & 3,174 & 11.4 & 2,523 & 13.7 & 1,420 & 20.6 \\
\hline 2008 & 288 & 1.0 & 3,972 & 5.2 & 8,074 & 10.2 & 3,586 & 12.6 & 2,856 & 15.5 & 1,657 & 23.5 \\
\hline 2009 & 299 & 1.0 & 4,397 & 5.7 & 9,083 & 11.4 & 4,050 & 13.9 & 3,109 & 16.8 & 1,782 & 24.7 \\
\hline 2010 & 302 & 1.0 & 4,613 & 6.1 & 10,220 & 12.7 & 4,558 & 15.3 & 3,502 & 18.8 & 1,986 & 26.9 \\
\hline 2011 & 307 & 1.0 & 5,128 & 6.8 & 11,613 & 14.3 & 5,143 & 17.0 & 3,842 & 20.4 & 2,173 & 28.8 \\
\hline 2012 & 347 & 1.2 & 5,701 & 7.6 & 13,183 & 16.2 & 5,873 & 18.5 & 4,507 & 23.6 & 2,656 & 34.5 \\
\hline 2013 & 380 & 1.3 & 6,232 & 8.4 & 15,098 & 18.6 & 6,842 & 20.8 & 5,132 & 26.5 & 3,005 & 38.9 \\
\hline 2014 & 439 & 1.5 & 6,929 & 9.4 & 17,171 & 21.1 & 7,919 & 23.4 & 6,065 & 30.7 & 3,695 & 46.9 \\
\hline 2015 & 507 & 1.7 & 7,676 & 10.4 & 19,389 & 23.8 & 9,074 & 26.2 & 6,756 & 33.9 & 4,207 & 53.1 \\
\hline Rate change (\%) & 89.2 & 73.2 & 158.5 & 170.6 & 260.5 & 236.8 & 280.1 & 198.8 & 220.3 & 197.8 & 305.7 & 218.8 \\
\hline $\begin{array}{l}\text { Spearman's } \\
\text { r, p-value* }\end{array}$ & $\begin{array}{c}0.991 \\
p<.001 *\end{array}$ & $\begin{array}{c}0.882 \\
p<.001^{*}\end{array}$ & $\begin{array}{c}>.999 \\
p<.001^{*}\end{array}$ & $\begin{array}{c}>.999 \\
p<.001^{*}\end{array}$ & $\begin{array}{c}>.999 \\
p<.001^{*}\end{array}$ & $\begin{array}{c}>.999 \\
p<.001^{*}\end{array}$ & $\begin{array}{c}>.999 \\
p<.001^{*}\end{array}$ & $\begin{array}{c}>.999 \\
p<.001^{*}\end{array}$ & $\begin{array}{c}>.999 \\
p<.001^{*}\end{array}$ & $\begin{array}{c}>.999 \\
p<.001^{*}\end{array}$ & $\begin{array}{c}>.999 \\
p<.001^{*}\end{array}$ & $\begin{array}{c}>.999 \\
p<.001^{*}\end{array}$ \\
\hline
\end{tabular}

${ }^{*} p<0.05$ = statistically significant

Table B.13: Dunn's pairwise comparisons and Bonferroni correction output for people per 1000 population receiving strong opioid prescriptions, stratified by age-group

Age group

18-24

25-44

45-64

65-74

75-84

\begin{tabular}{rccccc}
\hline $\mathbf{2 5 - 4 4}$ & 0.373 & & & & \\
\hline $\mathbf{4 5 - 6 4}$ & $<0.005^{*}$ & $>.999$ & & & \\
\hline $\mathbf{6 5 - 7 4}$ & $<0.005^{*}$ & $>.999$ & $>.999$ & & \\
\hline $\mathbf{7 5 - 8 4}$ & $<.001^{*}$ & 0.442 & $>.999$ & $>.999$ & $>.999$ \\
\hline $85+$ & $<.001^{*}$ & $<0.050^{*}$ & $>.999$ & $>.999$ & \\
\hline
\end{tabular}

${ }^{*} p<0.05=$ statistically significant 


\section{B.4.1 Trends in numbers of prescriptions by age-group}

Table B.14: Trends in the total number of opioid prescriptions stratified by age group

\begin{tabular}{|c|c|c|c|c|c|c|c|c|c|c|c|c|}
\hline \multirow[b]{3}{*}{ Year } & \multicolumn{12}{|c|}{ Age-group } \\
\hline & \multicolumn{2}{|c|}{$18-24$} & \multicolumn{2}{|c|}{$25-44$} & \multicolumn{2}{|c|}{$45-64$} & \multicolumn{2}{|c|}{$65-74$} & \multicolumn{2}{|c|}{$75-84$} & \multicolumn{2}{|c|}{$85+$} \\
\hline & $\begin{array}{c}\text { Number } \\
\text { of } R x\end{array}$ & $\begin{array}{c}\text { Rx per } \\
1000\end{array}$ & $\begin{array}{c}\text { Number } \\
\text { of } R x\end{array}$ & $\begin{array}{c}\text { Rx per } \\
1000\end{array}$ & $\begin{array}{c}\text { Number of } \\
R x\end{array}$ & $\begin{array}{c}\text { Rx per } \\
1000\end{array}$ & $\begin{array}{c}\text { Number } \\
\text { of } R x\end{array}$ & $\begin{array}{c}\text { Rx per } \\
1000\end{array}$ & $\begin{array}{c}\text { Number } \\
\text { of } R x\end{array}$ & $\begin{array}{c}\text { Rx per } \\
1000\end{array}$ & $\begin{array}{c}\text { Number } \\
\text { of } R x\end{array}$ & $\begin{array}{c}\text { Rx per } \\
1000\end{array}$ \\
\hline 2005 & 31,950 & 119.1 & 304,699 & 394.7 & 626,760 & 822.1 & 329,694 & 1211.8 & 235,612 & 1271.1 & 84,702 & 1359.1 \\
\hline 2006 & 32,457 & 118.6 & 318,389 & 412.7 & 654,666 & 846.7 & 333,848 & 1219.7 & 229,671 & 1245.7 & 87,301 & 1325.3 \\
\hline 2007 & 35,726 & 127.5 & 345,851 & 448.3 & 715,204 & 912.7 & 354,076 & 1275.9 & 238,786 & 1301.2 & 93,457 & 1357.2 \\
\hline 2008 & 36,641 & 128.8 & 369,762 & 479.9 & 778,707 & 982.4 & 374,467 & 1319.4 & 247,010 & 1342.6 & 97,764 & 1384.7 \\
\hline 2009 & 38,386 & 133.1 & 390,721 & 509.8 & 822,823 & 1028.3 & 390,644 & 1342.9 & 258,730 & 1401.8 & 101,247 & 1403.6 \\
\hline 2011 & 39,955 & 135.0 & 432,110 & 572.7 & 926,426 & 1137.3 & 429,556 & 1419.1 & 285,696 & 1516.8 & 113,294 & 1503.9 \\
\hline 2012 & 40,162 & 133.8 & 443,172 & 594.4 & 962,289 & 1185.8 & 454,683 & 1429.2 & 297,987 & 1561.7 & 120,114 & 1561.3 \\
\hline 2013 & 39,055 & 130.9 & 443,657 & 597.6 & 976,109 & 1204.0 & 470,904 & 1429.2 & 305,635 & 1576.7 & 123,346 & 1595.7 \\
\hline 2014 & 38,466 & 130.2 & 440,743 & 596.8 & 994,740 & 1222.5 & 483,118 & 1427.5 & 315,598 & 1598.5 & 126,053 & 1598.3 \\
\hline 2015 & 36,275 & 123.8 & 441,430 & 598.5 & $1,002,718$ & 1229.0 & 491,974 & 1421.5 & 318,235 & 1596.2 & 124,742 & 1573.0 \\
\hline $\begin{array}{r}\text { Rate change } \\
(\%)\end{array}$ & 13.5 & 4.0 & 44.9 & 51.6 & 60.0 & 49.5 & 49.2 & 17.3 & 35.1 & 25.6 & 47.3 & 15.7 \\
\hline $\begin{array}{l}\text { Spearman's } \\
\text { r, p-value* }\end{array}$ & $\begin{array}{c}0.627 \\
p<0.05^{*}\end{array}$ & $\begin{array}{c}0.418 \\
p=0.201\end{array}$ & $\begin{array}{c}0.927 \\
p<.001 *\end{array}$ & $\begin{array}{c}\text { 0.991, } \\
p<.001 *\end{array}$ & $\begin{array}{c}>.999 \\
p<.001 *\end{array}$ & $\begin{array}{c}>.999 \\
\mathrm{p}<.001^{*}\end{array}$ & $\begin{array}{c}>.999 \\
p<.001 *\end{array}$ & $\begin{array}{c}0.909 \\
p<.001 *\end{array}$ & $\begin{array}{c}\text { 0.991, } \\
p<.001 *\end{array}$ & $\begin{array}{c}0.982 \\
\mathrm{p}<.001^{*}\end{array}$ & $\begin{array}{c}\text { 0.991, } \\
p<.001 *\end{array}$ & $\begin{array}{c}0.945 \\
p<.001 *\end{array}$ \\
\hline
\end{tabular}

$\mathrm{Rx}=$ prescriptions $* \mathrm{p}<0.05=$ statistically significant

Table B.15: Dunn's pairwise comparisons and Bonferroni correction output for all opioid prescriptions per 1000 population, stratified by age-group

\begin{tabular}{rccccc}
\hline Age group (years) & $\mathbf{1 8 - 2 4}$ & $\mathbf{2 5 - 4 4}$ & $\mathbf{4 5 - 6 4}$ & $\mathbf{6 5 - 7 4}$ & $\mathbf{7 5 - 8 4}$ \\
\hline $\mathbf{2 5 - 4 4}$ & $>.999$ & & & & \\
\hline $\mathbf{4 5 - 6 4}$ & 0.108 & $>.999$ & & & \\
\hline $\mathbf{6 5 - 7 4}$ & $<.001^{*}$ & $<0.050$ & 0.820 & & \\
\hline $\mathbf{7 5 - 8 4}$ & $<.001^{*}$ & $.001^{*}$ & $<0.050^{*}$ & $>.999$ & \\
\hline $\mathbf{8 5 +}$ & $<.001^{*}$ & $<.001^{*}$ & $<0.050^{*}$ & $>.999$ & $>.999$
\end{tabular}

${ }^{*} p<0.050=$ statistically significant 


\begin{tabular}{|c|c|c|c|c|c|c|c|c|c|c|c|c|}
\hline \multirow[b]{3}{*}{ Year } & \multicolumn{12}{|c|}{ Age-group } \\
\hline & \multicolumn{2}{|c|}{$18-24$} & \multicolumn{2}{|c|}{$25-44$} & \multicolumn{2}{|c|}{$45-64$} & \multicolumn{2}{|c|}{$65-74$} & \multicolumn{2}{|c|}{$75-84$} & \multicolumn{2}{|c|}{$85+$} \\
\hline & $\begin{array}{c}\text { Number } \\
\text { of } R x\end{array}$ & $\begin{array}{c}\text { Rx per } \\
1000\end{array}$ & $\begin{array}{c}\text { Number } \\
\text { of } R x\end{array}$ & $\begin{array}{c}\text { Rx per } \\
1000\end{array}$ & $\begin{array}{l}\text { Number } \\
\text { of } R x\end{array}$ & $\begin{array}{c}\text { Rx per } \\
1000\end{array}$ & $\begin{array}{c}\text { Number } \\
\text { of } R x\end{array}$ & $\begin{array}{c}\text { Rx per } \\
1000\end{array}$ & $\begin{array}{c}\text { Number } \\
\text { of Rx }\end{array}$ & $\begin{array}{c}\text { Rx per } \\
1000\end{array}$ & $\begin{array}{c}\text { Number } \\
\text { of } R x\end{array}$ & $\begin{array}{c}\text { Rx per } \\
1000\end{array}$ \\
\hline 2005 & 29,766 & 110.9 & 279,581 & 362.2 & 589,893 & 773.8 & 316,152 & 1162.1 & 224,977 & 1213.7 & 80,072 & 1284.8 \\
\hline 2006 & 30,275 & 110.6 & 287,318 & 372.4 & 610,343 & 789.4 & 317,689 & 1160.7 & 217,872 & 1181.7 & 81,095 & 1231.0 \\
\hline 2007 & 33,436 & 119.3 & 311,769 & 404.1 & 661,935 & 844.7 & 334,453 & 1205.2 & 224,313 & 1222.3 & 86,068 & 1249.9 \\
\hline 2008 & 34,136 & 120.0 & 331,408 & 430.1 & 714,588 & 901.5 & 351,318 & 1237.8 & 230,592 & 1253.3 & 89,181 & 1263.2 \\
\hline 2009 & 36,045 & 124.9 & 348,405 & 454.6 & 747,893 & 934.7 & 364,008 & 1251.4 & 240,223 & 1301.5 & 91,556 & 1269.2 \\
\hline 2010 & 36,467 & 124.7 & 358,869 & 472.5 & 785,957 & 973.0 & 378,349 & 1273.7 & 250,777 & 1344.9 & 95,656 & 1297.3 \\
\hline 2011 & 37,673 & 127.3 & 375,571 & 497.8 & 823,998 & 1011.6 & 392,528 & 1296.7 & 260,365 & 1382.3 & 100,064 & 1328.3 \\
\hline 2012 & 37,840 & 126.0 & 379,447 & 509.0 & 843,144 & 1039.0 & 411,095 & 1292.2 & 267,238 & 1400.6 & 104,483 & 1358.1 \\
\hline 2013 & 36,816 & 123.4 & 374,078 & 503.9 & 837,467 & 1033.0 & 419,585 & 1273.4 & 270,753 & 1396.8 & 106,111 & 1372.8 \\
\hline 2014 & 35,789 & 121.1 & 368,118 & 498.5 & 836,574 & 1028.1 & 425,053 & 1255.9 & 276,218 & 1399.0 & 106,274 & 1347.5 \\
\hline 2015 & 33,447 & 114.2 & 361,235 & 489.8 & 824,065 & 1010.1 & 425,647 & 1229.8 & 274,092 & 1374.8 & 103,191 & 1301.3 \\
\hline $\begin{array}{r}\text { Rate } \\
\text { change (\%) }\end{array}$ & 12.4 & 2.9 & 29.2 & 35.2 & 39.7 & 30.5 & 34.6 & 5.8 & 21.8 & 13.3 & 28.9 & 1.3 \\
\hline $\begin{array}{l}\text { Spearman's } \\
\text { r, p-value* }\end{array}$ & $\begin{array}{l}0.582 \\
p=0.60\end{array}$ & $\begin{array}{c}0.418 \\
p=0.201\end{array}$ & $\begin{array}{c}0.827 \\
p<0.01 *\end{array}$ & $\begin{array}{c}0.873 \\
p<.001^{*}\end{array}$ & $\begin{array}{c}0.909 \\
p<.001^{*}\end{array}$ & $\begin{array}{c}0.873 \\
p<.001^{*}\end{array}$ & $\begin{array}{c}>.999 \\
p<.001^{*}\end{array}$ & $\begin{array}{c}0.582 \\
p=0.060\end{array}$ & $\begin{array}{c}0.964 \\
p<.001^{*}\end{array}$ & $\begin{array}{c}0.873 \\
p<.001^{*}\end{array}$ & $\begin{array}{c}0.945 \\
p<.001^{*}\end{array}$ & $\begin{array}{c}0.791 \\
p<0.01^{*}\end{array}$ \\
\hline
\end{tabular}

$\mathrm{Rx}=$ prescriptions $* \mathrm{p}<0.05=$ statistically significant

Table B.17: Dunn's pairwise comparisons and Bonferroni correction output for weak opioid prescriptions per 1000 population, stratified by age-group

\begin{tabular}{rccccc}
\hline Age group (years) & $\mathbf{1 8 - 2 4}$ & $\mathbf{2 5 - 4 4}$ & $\mathbf{4 5 - 6 4}$ & $\mathbf{6 5 - 7 4}$ & $\mathbf{7 5 - 8 4}$ \\
\hline $\mathbf{2 5 - 4 4}$ & $>.999$ & & & & \\
\hline $\mathbf{4 5 - 6 4}$ & 0.108 & $>.999$ & & & \\
\hline $\mathbf{6 5 - 7 4}$ & $<.001^{*}$ & $<0.010^{*}$ & 0.318 & & \\
\hline $\mathbf{7 5 - 8 4}$ & $<.001^{*}$ & $<.001^{*}$ & $<0.010^{*}$ & $>.999$ & \\
\hline $\mathbf{8 5 +}$ & $<.001^{*}$ & $<0.010^{*}$ & 0.495 & $>.999$ & $>.999$ \\
\hline
\end{tabular}

${ }^{*} p<0.05=$ statistically significant 
Table B.18: Trends in the number strong opioid prescriptions, stratified by age group

\begin{tabular}{|c|c|c|c|c|c|c|c|c|c|c|c|c|}
\hline & \multicolumn{12}{|c|}{ Age-group } \\
\hline & \multicolumn{2}{|c|}{$18-24$} & \multicolumn{2}{|c|}{$25-44$} & \multicolumn{2}{|c|}{$45-64$} & \multicolumn{2}{|c|}{$65-74$} & \multicolumn{2}{|c|}{$75-84$} & \multicolumn{2}{|c|}{$85+$} \\
\hline & $\begin{array}{c}\text { Number } \\
\text { of Rx }\end{array}$ & $\begin{array}{c}\text { Rx per } \\
1000\end{array}$ & $\begin{array}{l}\text { Number } \\
\text { of Rx }\end{array}$ & $\begin{array}{c}\text { Rxper } \\
1000\end{array}$ & $\begin{array}{c}\text { Number } \\
\text { of } R x\end{array}$ & $\begin{array}{c}\text { Rx per } \\
1000\end{array}$ & $\begin{array}{c}\text { Number } \\
\text { of Rx }\end{array}$ & $\begin{array}{c}\text { Rxper } \\
1000\end{array}$ & $\begin{array}{c}\text { Number } \\
\text { of Rx }\end{array}$ & $\begin{array}{c}\text { Rx per } \\
1000\end{array}$ & $\begin{array}{c}\text { Number } \\
\text { of Rx }\end{array}$ & $\begin{array}{c}\text { Rxper } \\
1000\end{array}$ \\
\hline 2005 & 2,184 & 8.1 & 25,118 & 32.5 & 36,867 & 48.4 & 13,542 & 49.8 & 10,635 & 57.4 & 4,630 & 74.3 \\
\hline 2006 & 2,182 & 8.0 & 31,071 & 40.3 & 44,323 & 57.3 & 16,159 & 59.0 & 11,799 & 64.0 & 6,206 & 94.2 \\
\hline 2007 & 2,290 & 8.2 & 34,082 & 44.2 & 53,269 & 68.0 & 19,623 & 70.7 & 14,473 & 78.9 & 7,389 & 107.3 \\
\hline 2008 & 2,505 & 8.8 & 38,354 & 49.8 & 64,119 & 80.9 & 23,149 & 81.6 & 16,418 & 89.2 & 8,583 & 121.6 \\
\hline 2009 & 2,341 & 8.1 & 42,316 & 55.2 & 74,930 & 93.6 & 26,636 & 91.6 & 18,507 & 100.3 & 9,691 & 134.3 \\
\hline 2010 & 2,294 & 7.8 & 49,095 & 64.6 & 86,431 & 107.0 & 31,565 & 106.3 & 22,157 & 118.8 & 10,955 & 148.6 \\
\hline 2011 & 2,282 & 7.7 & 56,539 & 74.9 & 102,428 & 125.7 & 37,028 & 122.3 & 25,331 & 134.5 & 13,230 & 175.6 \\
\hline 2012 & 2,322 & 7.7 & 63,725 & 85.5 & 119,145 & 146.8 & 43,588 & 137.0 & 30,749 & 161.2 & 15,631 & 203.2 \\
\hline 2013 & 2,239 & 7.5 & 69,579 & 93.7 & 138,642 & 171.0 & 51,319 & 155.8 & 34,882 & 180.0 & 17,235 & 223.0 \\
\hline 2014 & 2,677 & 9.1 & 72,625 & 98.3 & 158,166 & 194.4 & 58,065 & 171.6 & 39,380 & 199.5 & 19,779 & 250.8 \\
\hline 2015 & 2,828 & 9.7 & 80,195 & 108.7 & 178,653 & 219.0 & 66,327 & 191.6 & 44,143 & 221.4 & 21,551 & 271.8 \\
\hline Rate change (\%) & 29.5 & 18.6 & 219.3 & 234.1 & 384.6 & 352.8 & 389.8 & 285.0 & 315.1 & 285.9 & 365.5 & 265.8 \\
\hline $\begin{array}{l}\text { Spearman's } \\
\text { r, p-value* }\end{array}$ & $\begin{array}{c}0.609 \\
p<0.05^{*}\end{array}$ & $\begin{array}{c}0.041 \\
p=0.905\end{array}$ & $\begin{array}{c}>.999 \\
p<.001^{*}\end{array}$ & $\begin{array}{c}>.999 \\
p<.001^{*}\end{array}$ & $\begin{array}{c}>.999 \\
p<.001^{*}\end{array}$ & $\begin{array}{c}>.999 \\
p<.001^{*}\end{array}$ & $\begin{array}{c}>.999 \\
p<.001^{*}\end{array}$ & $\begin{array}{c}>.999 \\
p<.001^{*}\end{array}$ & $\begin{array}{c}>.999 \\
p<.001^{*}\end{array}$ & $\begin{array}{c}>.999 \\
p<.001^{*}\end{array}$ & $\begin{array}{c}>.999 \\
p<.001^{*}\end{array}$ & $\begin{array}{c}>.999 \\
p<.001^{*}\end{array}$ \\
\hline
\end{tabular}

$\mathrm{Rx}=$ prescriptions $* \mathrm{p}<0.05=$ statistically significant

Table B.19: Dunn's pairwise comparisons and Bonferroni correction output for strong opioid prescriptions per 1000 population, stratified by age-group

\begin{tabular}{rccccc}
\hline $\begin{array}{r}\text { Age group } \\
\text { (years) }\end{array}$ & $\mathbf{1 8 - 2 4}$ & $\mathbf{2 5 - 4 4}$ & $\mathbf{4 5 - 6 4}$ & $\mathbf{6 5 - 7 4}$ & $\mathbf{7 5 - 8 4}$ \\
\hline $\mathbf{2 5 - 4 4}$ & $>.999$ & & & & \\
\hline $\mathbf{4 5 - 6 4}$ & $<0.010^{*}$ & 0.630 & & & \\
\hline $\mathbf{6 5 - 7 4}$ & $<0.005^{*}$ & 0.149 & $>.999$ & & \\
\hline $\mathbf{7 5 - 8 4}$ & $<.001^{*}$ & $<0.050^{*}$ & $>.999$ & $>.999$ & \\
\hline $\mathbf{8 5 +}$ & $<.001^{*}$ & $<.001^{*}$ & 0.115 & 0.507 & $>.999$
\end{tabular}

${ }^{*} \mathrm{p}<0.05$ = statistically significant 


\section{Appendix C Trends in prescribing by socioeconomic deprivation \\ C.1 Data preparation}

Table C.1: Example of LSOA data used to determine Health Board WIMD population estimates

\begin{tabular}{|c|c|c|c|c|}
\hline LSOA & $\begin{array}{l}\text { National } \\
\text { Rank }\end{array}$ & LSOA number & County & $\begin{array}{l}\text { Population } \\
\text { estimate }\end{array}$ \\
\hline W01000001: Aberffraw \& Rhosneigr 1 & 731 & W01000001 & Isle of Anglesey 007A & 1,113 \\
\hline W01000002: Aberffraw \& Rhosneigr 2 & 852 & W01000002 & Isle of Anglesey 007B & 1,266 \\
\hline W01000003: Amlwch Port & 527 & W01000003 & Isle of Anglesey 001A & 2,519 \\
\hline W01000004: Amlwch Rural & 768 & W01000004 & Isle of Anglesey 001B & 1,281 \\
\hline W01000005: Beaumaris & 1,032 & W01000005 & Isle of Anglesey 005A & 1,936 \\
\hline W01000006: Bodffordd & 917 & W01000006 & Isle of Anglesey 006A & 1,534 \\
\hline W01000007: Bodorgan & 870 & W01000007 & Isle of Anglesey 009A & 1,696 \\
\hline W01000008: Braint & 1,811 & W01000008 & Isle of Anglesey 008A & 1,522 \\
\hline W01000009: Bryngwran & 486 & W01000009 & Isle of Anglesey 007C & 1,908 \\
\hline W01000010: Brynteg & 1,500 & W01000010 & Isle of Anglesey 002A & 1,868 \\
\hline W01000011: Cadnant (Isle of Anglesey) & 1,602 & W01000011 & Isle of Anglesey 008B & 1,229 \\
\hline W01000012: Cefni & 1,649 & W01000012 & Isle of Anglesey 006B & 1,487 \\
\hline W01000013: Cwm Cadnant & 1,651 & W01000013 & Isle of Anglesey 005B & 2,260 \\
\hline W01000014: Cyngar & 1,115 & W01000014 & Isle of Anglesey 006C & 2,062 \\
\hline W01000015: Gwyngyll & 1,758 & W01000015 & Isle of Anglesey $008 \mathrm{C}$ & 1,581 \\
\hline W01000016: Holyhead Town & 246 & W01000016 & Isle of Anglesey 003A & 1,201 \\
\hline W01000017: Kingsland & 377 & W01000017 & Isle of Anglesey 003B & 1,532 \\
\hline W01000018: Llanbadrig & 890 & W01000018 & Isle of Anglesey $001 \mathrm{C}$ & 1,358 \\
\hline W01000019: Llanbedrgoch & 1,318 & W01000019 & Isle of Anglesey $005 \mathrm{C}$ & 1,505 \\
\hline W01000020: Llanddyfnan & 1,335 & W01000020 & Isle of Anglesey 002B & 1,314 \\
\hline W01000021: Llaneilian & 826 & W01000021 & Isle of Anglesey $002 \mathrm{C}$ & 2,262 \\
\hline W01000022: Llanfaethlu & 953 & W01000022 & Isle of Anglesey 004A & 1,650 \\
\hline W01000023: Llanfair-yn-Neubwll 1 & 878 & W01000023 & Isle of Anglesey 007D & 1,411 \\
\hline W01000024: Llanfair-yn-Neubwll 2 & 1,058 & W01000024 & Isle of Anglesey 007E & 1,606 \\
\hline W01000025: Llanfihangel Ysgeifiog & 1,121 & W01000025 & Isle of Anglesey 009B & 2,025 \\
\hline W01000026: Llangoed & 986 & W01000026 & Isle of Anglesey 005D & 1,226 \\
\hline W01000027: Llanidan & 736 & W01000027 & Isle of Anglesey 009C & 1,849 \\
\hline W01000028: Llannerch-y-medd & 644 & W01000028 & Isle of Anglesey 001D & 1,947 \\
\hline W01000029: London Road & 341 & W01000029 & Isle of Anglesey $003 \mathrm{C}$ & 1,499 \\
\hline W01000030: Maeshyfryd & 320 & W01000030 & Isle of Anglesey 003D & 2,306 \\
\hline W01000031: Mechell & 773 & W01000031 & Isle of Anglesey 001E & 1,551 \\
\hline W01000032: Moelfre & 932 & W01000032 & Isle of Anglesey 002D & 1,063 \\
\hline W01000033: Morawelon & 146 & W01000033 & Isle of Anglesey 003E & 1,507 \\
\hline W01000034: Parc a'r Mynydd & 1,066 & W01000034 & Isle of Anglesey 004B & 1,156 \\
\hline W01000097: Ogwen 2 & 765 & W01000097 & Gwynedd 003D & 1,107 \\
\hline W01000098: Peblig (Caernarfon) & 119 & W01000098 & Gwynedd 006C & 2,300 \\
\hline W01000099: Penisarwaun & 1,321 & W01000099 & Gwynedd 005C & 1,762 \\
\hline W01000100: Penrhyndeudraeth 1 & 1,194 & W01000100 & Gwynedd 010B & 1,057 \\
\hline W01000101: Penrhyndeudraeth 2 & 1,347 & W01000101 & Gwynedd 013D & 1,530 \\
\hline W01000918: Crynant & 991 & W01000918 & $\begin{array}{l}\text { Neath Port Talbot } \\
002 \mathrm{~A}\end{array}$ & 1,906 \\
\hline W01000919: Cwmllynfell & 716 & W01000919 & $\begin{array}{l}\text { Neath Port Talbot } \\
020 \mathrm{~A}\end{array}$ & 1,175 \\
\hline $\begin{array}{l}\text { W01000920: Cymmer (Neath Port Talbot) } \\
1\end{array}$ & 446 & W01000920 & $\begin{array}{l}\text { Neath Port Talbot } \\
011 \mathrm{~A}\end{array}$ & 1,254 \\
\hline $\begin{array}{l}\text { W01000921: Cymmer (Neath Port Talbot) } \\
2\end{array}$ & 25 & W01000921 & $\begin{array}{l}\text { Neath Port Talbot } \\
\text { 011B }\end{array}$ & 1,574 \\
\hline
\end{tabular}




\begin{tabular}{|c|c|c|c|c|}
\hline W01000922: Dyffryn 1 & 551 & W01000922 & $\begin{array}{l}\text { Neath Port Talbot } \\
007 \mathrm{C}\end{array}$ & 1,687 \\
\hline W01000923: Dyffryn 2 & 1,315 & W01000923 & $\begin{array}{l}\text { Neath Port Talbot } \\
\text { 007D }\end{array}$ & 1,475 \\
\hline W01000924: Glyncorrwg & 168 & W01000924 & $\begin{array}{l}\text { Neath Port Talbot } \\
011 \mathrm{C}\end{array}$ & 1,100 \\
\hline W01000925: Glynneath 1 & 268 & W01000925 & $\begin{array}{l}\text { Neath Port Talbot } \\
\text { 003B }\end{array}$ & 1,565 \\
\hline W01000926: Glynneath 2 & 1,160 & W01000926 & $\begin{array}{l}\text { Neath Port Talbot } \\
003 \mathrm{C}\end{array}$ & 1,860 \\
\hline W01000927: Godre'r graig & 642 & W01000927 & $\begin{array}{l}\text { Neath Port Talbot } \\
020 \mathrm{~B}\end{array}$ & 1,655 \\
\hline W01000928: Gwaun-Cae-Gurwen 1 & 497 & W01000928 & $\begin{array}{l}\text { Neath Port Talbot } \\
020 \mathrm{C}\end{array}$ & 1,602 \\
\hline W01000929: Gwaun-Cae-Gurwen 2 & 475 & W01000929 & $\begin{array}{l}\text { Neath Port Talbot } \\
\text { 020D }\end{array}$ & 1,299 \\
\hline W01000930: Gwynfi & 193 & W01000930 & $\begin{array}{l}\text { Neath Port Talbot } \\
\text { 011D }\end{array}$ & 1,365 \\
\hline W01000931: Lower Brynamman & 627 & W01000931 & $\begin{array}{l}\text { Neath Port Talbot } \\
020 \mathrm{E}\end{array}$ & 1,332 \\
\hline W01000932: Margam 1 & 583 & W01000932 & $\begin{array}{l}\text { Neath Port Talbot } \\
\text { 019A }\end{array}$ & 1,044 \\
\hline W01000933: Margam 2 & 1,429 & W01000933 & $\begin{array}{l}\text { Neath Port Talbot } \\
\text { 019B }\end{array}$ & 1,981 \\
\hline W01000934: Neath East 1 & 167 & W01000934 & $\begin{array}{l}\text { Neath Port Talbot } \\
\text { 008A }\end{array}$ & 1,263 \\
\hline W01000935: Neath East 2 & 141 & W01000935 & $\begin{array}{l}\text { Neath Port Talbot } \\
\text { 008B }\end{array}$ & 1,572 \\
\hline W01000936: Neath East 3 & 201 & W01000936 & $\begin{array}{l}\text { Neath Port Talbot } \\
\text { 012A }\end{array}$ & 1,525 \\
\hline W01000937: Neath East 4 & 196 & W01000937 & $\begin{array}{l}\text { Neath Port Talbot } \\
\text { 013E }\end{array}$ & 2,057 \\
\hline W01000938: Neath North 1 & 1,465 & W01000938 & $\begin{array}{l}\text { Neath Port Talbot } \\
008 \mathrm{C}\end{array}$ & 1,390 \\
\hline W01000939: Neath North 2 & 92 & W01000939 & $\begin{array}{l}\text { Neath Port Talbot } \\
\text { 008D }\end{array}$ & 1,262 \\
\hline W01000940: Neath North 3 & 271 & W01000940 & $\begin{array}{l}\text { Neath Port Talbot } \\
008 \mathrm{E}\end{array}$ & 1,291 \\
\hline W01000941: Neath South 1 & 364 & W01000941 & $\begin{array}{l}\text { Neath Port Talbot } \\
\text { 012B }\end{array}$ & 1,545 \\
\hline W01000942: Neath South 2 & 176 & W01000942 & $\begin{array}{l}\text { Neath Port Talbot } \\
012 \mathrm{C}\end{array}$ & 1,891 \\
\hline W01000943: Neath South 3 & 1,765 & W01000943 & $\begin{array}{l}\text { Neath Port Talbot } \\
\text { 012D }\end{array}$ & 1,555 \\
\hline
\end{tabular}

Using WIMD2011 data (StatsWales 2010)

Table C.2: Welsh Country population data for 2011. Designated Health Board indicated

\begin{tabular}{|c|c|c|c|}
\hline County code & County & $\begin{array}{l}\text { Estimated } \\
\text { population }\end{array}$ & Health Board \\
\hline W06000001 & Ise of Anglesey / Ynys Mon & 69,913 & BCUHB \\
\hline W06000002 & Gwynedd / Gwynedd & 121,523 & BCUHB \\
\hline W06000003 & Conwy /Conwy & 115,326 & BCUHB \\
\hline W06000004 & Denbighshire / Sir Ddinbych & 93,919 & BCUHB \\
\hline W06000005 & Flintshire / Sir y Fflint & 152,666 & BCUHB \\
\hline W06000006 & Wrexham / Wrecsam & 135,070 & BCUHB \\
\hline W06000008 & Ceredigion / Ceredigion & 75,293 & HDUHB \\
\hline W06000009 & Pembrokeshire / Sir Benfro & 122,613 & HDUHB \\
\hline W06000010 & Carmarthenshire / Sir Gaerfyrddin & 183,961 & HDUHB \\
\hline W06000011 & Swansea / Abertawe & 238,691 & ABMUHB \\
\hline W06000012 & $\begin{array}{l}\text { Neath Port Talbot / Castell-nedd Port } \\
\text { Talbot }\end{array}$ & 139,880 & ABMUHB \\
\hline W06000013 & Bridgend / Pen-y-bont ar Ogwr & 139,410 & ABMUHB \\
\hline W06000014 & The Vale of Glamorgan / Bro Morgannwg & 126,679 & CVUHB \\
\hline W06000015 & Cardiff / Caerdydd & 345,442 & CVUHB \\
\hline W06000016 & Rhondda Cynon Taf / Rhondda Cynon Taf & 234,373 & CTUHB \\
\hline W06000018 & Caerphilly / Caerffili & 178,782 & CTUHB \\
\hline W06000019 & Blaenau Gwent / Blaenau Gwent & 69,812 & ABUHB \\
\hline W06000020 & Torfaen / Tor-faen & 91,190 & $A B \cup H B$ \\
\hline
\end{tabular}




\begin{tabular}{llll}
\hline W06000021 & Monmouthshire / Sir Fynwy & 91,508 & ABUHB \\
\hline W06000022 & Newport / Casnewydd & 145,785 & ABUHB \\
\hline W06000023 & Powys / Powys & 133,071 & PTHB \\
\hline W06000024 & Merthyr Tydfil / Merthyr Tudful & 58,851 & CTUHB \\
\hline & Total population & $3,063,758$ & \\
\hline
\end{tabular}

(StatsWales 2010)

Table C.3: Population calculations for each Health Board using mid-year estimates and adjusted to SAIL percentage representation per Health Board

\begin{tabular}{|c|c|c|c|c|c|c|c|c|}
\hline & ABMUHB & ABUHB & BCUHB & CVUHB & CTUHB & HDUHB & PTHB & $\begin{array}{c}\text { Total } \\
\text { Welsh } \\
\text { population }\end{array}$ \\
\hline $\begin{array}{c}\% \text { of } \\
\text { total }\end{array}$ & $17 \%$ & $19 \%$ & $22 \%$ & $16 \%$ & $10 \%$ & $12 \%$ & $4 \%$ & \\
\hline 2005 & 504,781 & 564,167 & 653,246 & 475,088 & 296,930 & 356316 & 118772 & $2,969,300$ \\
\hline 2006 & 507,569 & 567,283 & 656,854 & 477,712 & 298,570 & 358284 & 119428 & $2,985,700$ \\
\hline 2007 & 511,071 & 571,197 & 661,386 & 481,008 & 300,630 & 360756 & 120252 & $3,006,300$ \\
\hline 2008 & 514,403 & 574,921 & 665,698 & 484,144 & 302,590 & 363108 & 121036 & $3,025,900$ \\
\hline 2009 & 512,921 & 572,518 & 684,575 & 463,818 & 292,899 & 379,051 & 133,090 & $3,038,872$ \\
\hline 2010 & 515,420 & 574,778 & 685,911 & 467,837 & 292,952 & 380,195 & 132,878 & $3,049,971$ \\
\hline 2011 & 517,981 & 577,077 & 688,417 & 472,121 & 293,224 & 381,867 & 133,071 & $3,063,758$ \\
\hline 2012 & 519,481 & 577,981 & 690,434 & 475,324 & 294,497 & 383,398 & 132,952 & $3,074,067$ \\
\hline 2013 & 520,710 & 579,101 & 691,986 & 478,869 & 295,135 & 383,906 & 132,705 & $3,082,412$ \\
\hline 2014 & 523,001 & 580,401 & 694,038 & 481,979 & 295,953 & 383,989 & 132,675 & $3,092,036$ \\
\hline 2015 & 525,466 & 581,789 & 694,473 & 484,752 & 296,735 & 383,229 & 132,642 & $3,099,086$ \\
\hline $\begin{array}{r}\text { SAlL } \\
\text { population }\end{array}$ & ABMUHB & ABUHB & BCUHB & CVUHB & CTUHB & HDUHB & PTHB & Total \\
\hline 2005 & 484,590 & 394,917 & 489,935 & 380,070 & 228,636 & 288,616 & 48,697 & $2,315,460$ \\
\hline 2006 & 487,266 & 397,098 & 492,641 & 382,170 & 229,899 & 290,210 & 48,965 & $2,328,249$ \\
\hline 2007 & 490,628 & 399,838 & 496,040 & 384,806 & 231,485 & 292,212 & 49,303 & $2,344,313$ \\
\hline 2008 & 493,827 & 402,445 & 499,274 & 387,315 & 232,994 & 294,117 & 49,625 & $2,359,597$ \\
\hline 2009 & 492,404 & 400,763 & 513,431 & 371,054 & 225,532 & 307,031 & 54,567 & $2,364,783$ \\
\hline 2010 & 494,803 & 402,345 & 514,433 & 374,270 & 225,573 & 307,958 & 54,480 & $2,373,862$ \\
\hline 2011 & 497,262 & 403,954 & 516,313 & 377,697 & 225,782 & 309,312 & 54,559 & $2,384,879$ \\
\hline 2012 & 498,702 & 404,587 & 517,826 & 380,259 & 226,763 & 310,552 & 54,510 & $2,393,199$ \\
\hline 2013 & 499,882 & 405,371 & 518,990 & 383,095 & 227,254 & 310,964 & 54,409 & $2,399,964$ \\
\hline 2014 & 502,081 & 406,281 & 520,529 & 385,583 & 227,884 & 311,031 & 54,397 & $2,407,785$ \\
\hline 2015 & 504,447 & 407,252 & 520,855 & 387,802 & 228,486 & 310,415 & 54,383 & $2,413,641$ \\
\hline
\end{tabular}

(StatsWales 2010)

Table C.4: Estimated populations per 1000 WIMD quintile per Health Board and total for Wales based on SAIL population estimates

\begin{tabular}{|c|c|c|c|c|c|c|c|c|c|c|c|}
\hline ALLWale & WIMD & WIMD & WIMD & WIMD & WIMD & CTLHB & WIMD & WIMD & WIMD & WIMD & WIMD \\
\hline$s$ & 1 & 2 & 3 & 4 & 5 & & 1 & 2 & 3 & 4 & 5 \\
\hline 2005 & 474.3 & 471.9 & 449.2 & 439.0 & 478.2 & 2005 & 68.6 & 80.0 & 34.3 & 18.3 & 27.4 \\
\hline 2006 & 476.9 & 474.5 & 451.6 & 441.4 & 480.8 & 2006 & 69.0 & 80.5 & 34.5 & 18.4 & 27.6 \\
\hline 2007 & 480.2 & 477.8 & 454.8 & 444.5 & 484.2 & 2007 & 69.4 & 81.0 & 34.7 & 18.5 & 27.8 \\
\hline 2008 & 483.4 & 480.9 & 457.7 & 447.4 & 487.3 & 2008 & 69.9 & 81.5 & 34.9 & 18.6 & 28.0 \\
\hline 2009 & 479.4 & 481.3 & 462.9 & 454.1 & 484.6 & 2009 & 67.7 & 78.9 & 33.8 & 18.0 & 27.1 \\
\hline 2010 & 481.4 & 482.9 & 464.4 & 455.6 & 486.9 & 2010 & 67.7 & 79.0 & 33.8 & 18.0 & 27.1 \\
\hline 2011 & 483.7 & 484.9 & 466.5 & 457.6 & 489.6 & 2011 & 67.7 & 79.0 & 33.9 & 18.1 & 27.1 \\
\hline 2012 & 485.4 & 486.6 & 468.0 & 459.1 & 491.5 & 2012 & 68.0 & 79.4 & 34.0 & 18.1 & 27.2 \\
\hline 2013 & 486.9 & 488.0 & 469.1 & 460.2 & 493.3 & 2013 & 68.2 & 79.5 & 34.1 & 18.2 & 27.3 \\
\hline 2014 & 488.7 & 489.3 & 470.4 & 461.5 & 495.2 & 2014 & 68.4 & 79.8 & 34.2 & 18.2 & 27.3 \\
\hline 2015 & 490.3 & 490.5 & 471.2 & 462.3 & 496.7 & 2015 & 68.5 & 80.0 & 34.3 & 18.3 & 27.4 \\
\hline ABLHB & $\begin{array}{c}\text { WIMD } \\
1\end{array}$ & $\begin{array}{c}\text { WIMD } \\
2\end{array}$ & $\underset{3}{\text { WIMD }}$ & $\begin{array}{c}\text { WIMD } \\
4\end{array}$ & $\begin{array}{c}\text { WIMD } \\
5\end{array}$ & HDLHB & $\begin{array}{c}\text { WIMD } \\
1\end{array}$ & $\begin{array}{c}\text { WIMD } \\
2\end{array}$ & $\begin{array}{c}\text { WIMD } \\
\mathbf{3}\end{array}$ & $\begin{array}{c}\text { WIMD } \\
4\end{array}$ & $\begin{array}{c}\text { WIMD } \\
5\end{array}$ \\
\hline 2005 & 102.7 & 86.9 & 75.0 & 55.3 & 71.1 & 2005 & 20.2 & 54.8 & 98.1 & 72.2 & 43.3 \\
\hline 2006 & 103.2 & 87.4 & 75.4 & 55.6 & 71.5 & 2006 & 20.3 & 55.1 & 98.7 & 72.6 & 43.5 \\
\hline 2007 & 104.0 & 88.0 & 76.0 & 56.0 & 72.0 & 2007 & 20.5 & 55.5 & 99.4 & 73.1 & 43.8 \\
\hline 2008 & 104.6 & 88.5 & 76.5 & 56.3 & 72.4 & 2008 & 20.6 & 55.9 & 100.0 & 73.5 & 44.1 \\
\hline 2009 & 104.2 & 88.2 & 76.1 & 56.1 & 72.1 & 2009 & 21.5 & 58.3 & 104.4 & 76.8 & 46.1 \\
\hline 2010 & 104.6 & 88.5 & 76.4 & 56.3 & 72.4 & 2010 & 21.6 & 58.5 & 104.7 & 77.0 & 46.2 \\
\hline 2011 & 105.0 & 88.9 & 76.8 & 56.6 & 72.7 & 2011 & 21.7 & 58.8 & 105.2 & 77.3 & 46.4 \\
\hline 2012 & 105.2 & 89.0 & 76.9 & 56.6 & 72.8 & 2012 & 21.7 & 59.0 & 105.6 & 77.6 & 46.6 \\
\hline 2013 & 105.4 & 89.2 & 77.0 & 56.8 & 73.0 & 2013 & 21.8 & 59.1 & 105.7 & 77.7 & 46.6 \\
\hline 2014 & 105.6 & 89.4 & 77.2 & 56.9 & 73.1 & 2014 & 21.8 & 59.1 & 105.8 & 77.8 & 46.7 \\
\hline
\end{tabular}




\begin{tabular}{|c|c|c|c|c|c|c|c|c|c|c|c|}
\hline 2015 & 105.9 & 89.6 & 77.4 & 57.0 & 73.3 & 2015 & 21.7 & 59.0 & 105.5 & 77.6 & 46.6 \\
\hline ABMUHB & $\begin{array}{c}\text { WIMD } \\
1\end{array}$ & $\begin{array}{c}\text { WIMD } \\
2\end{array}$ & $\begin{array}{c}\text { WIMD } \\
3\end{array}$ & $\begin{array}{c}\text { WIMD } \\
4\end{array}$ & $\begin{array}{c}\text { WIMD } \\
5\end{array}$ & PTHB & $\begin{array}{c}\text { WIMD } \\
1\end{array}$ & $\begin{array}{c}\text { WIMD } \\
2\end{array}$ & $\begin{array}{c}\text { WIMD } \\
3\end{array}$ & $\begin{array}{c}\text { WIMD } \\
4\end{array}$ & $\begin{array}{c}\text { WIMD } \\
5\end{array}$ \\
\hline 2005 & 126.0 & 106.6 & 72.7 & 63.0 & 116.3 & 2005 & 1.9 & 4.9 & 10.7 & 26.3 & 4.9 \\
\hline 2006 & 126.7 & 107.2 & 73.1 & 63.3 & 116.9 & 2006 & 2.0 & 4.9 & 10.8 & 26.4 & 4.9 \\
\hline 2007 & 127.6 & 107.9 & 73.6 & 63.8 & 117.8 & 2007 & 2.0 & 4.9 & 10.8 & 26.6 & 4.9 \\
\hline 2008 & 128.4 & 108.6 & 74.1 & 64.2 & 118.5 & 2008 & 2.0 & 5.0 & 10.9 & 26.8 & 5.0 \\
\hline 2009 & 128.0 & 108.3 & 73.9 & 64.0 & 118.2 & 2009 & 2.2 & 5.5 & 12.0 & 29.5 & 5.5 \\
\hline 2010 & 128.6 & 108.9 & 74.2 & 64.3 & 118.8 & 2010 & 2.2 & 5.4 & 12.0 & 29.4 & 5.4 \\
\hline 2011 & 129.3 & 109.4 & 74.6 & 64.6 & 119.3 & 2011 & 2.2 & 5.5 & 12.0 & 29.5 & 5.5 \\
\hline 2012 & 129.7 & 109.7 & 74.8 & 64.8 & 119.7 & 2012 & 2.2 & 5.5 & 12.0 & 29.4 & 5.5 \\
\hline 2013 & 130.0 & 110.0 & 75.0 & 65.0 & 120.0 & 2013 & 2.2 & 5.4 & 12.0 & 29.4 & 5.4 \\
\hline 2014 & 130.5 & 110.5 & 75.3 & 65.3 & 120.5 & 2014 & 2.2 & 5.4 & 12.0 & 29.4 & 5.4 \\
\hline 2015 & 131.2 & 111.0 & 75.7 & 65.6 & 121.1 & 2015 & 2.2 & 5.4 & 12.0 & 29.4 & 5.4 \\
\hline BCUHB & $\begin{array}{c}\text { WIMD } \\
1\end{array}$ & $\begin{array}{c}\text { WIMD } \\
2\end{array}$ & $\begin{array}{c}\text { WIMD } \\
3\end{array}$ & $\begin{array}{c}\text { WIMD } \\
4\end{array}$ & $\begin{array}{c}\text { WIMD } \\
5\end{array}$ & CVUHB & $\begin{array}{c}\text { WIMD } \\
1\end{array}$ & $\begin{array}{c}\text { WIMD } \\
2\end{array}$ & $\begin{array}{c}\text { WIMD } \\
3\end{array}$ & $\begin{array}{c}\text { WIMD } \\
4\end{array}$ & $\begin{array}{c}\text { WIMD } \\
5\end{array}$ \\
\hline 2005 & 63.7 & 93.1 & 112.7 & 147.0 & 78.4 & 2005 & 91.2 & 45.6 & 45.6 & 57.0 & 136.8 \\
\hline 2006 & 64.0 & 93.6 & 113.3 & 147.8 & 78.8 & 2006 & 91.7 & 45.9 & 45.9 & 57.3 & 137.6 \\
\hline 2007 & 64.5 & 94.2 & 114.1 & 148.8 & 79.4 & 2007 & 92.4 & 46.2 & 46.2 & 57.7 & 138.5 \\
\hline 2008 & 64.9 & 94.9 & 114.8 & 149.8 & 79.9 & 2008 & 93.0 & 46.5 & 46.5 & 58.1 & 139.4 \\
\hline 2009 & 66.7 & 97.6 & 118.1 & 154.0 & 82.1 & 2009 & 89.1 & 44.5 & 44.5 & 55.7 & 133.6 \\
\hline 2010 & 66.9 & 97.7 & 118.3 & 154.3 & 82.3 & 2010 & 89.8 & 44.9 & 44.9 & 56.1 & 134.7 \\
\hline 2011 & 67.1 & 98.1 & 118.8 & 154.9 & 82.6 & 2011 & 90.6 & 45.3 & 45.3 & 56.7 & 136.0 \\
\hline 2012 & 67.3 & 98.4 & 119.1 & 155.3 & 82.9 & 2012 & 91.3 & 45.6 & 45.6 & 57.0 & 136.9 \\
\hline 2013 & 67.5 & 98.6 & 119.4 & 155.7 & 83.0 & 2013 & 91.9 & 46.0 & 46.0 & 57.5 & 137.9 \\
\hline 2014 & 67.7 & 98.9 & 119.7 & 156.2 & 83.3 & 2014 & 92.5 & 46.3 & 46.3 & 57.8 & 138.8 \\
\hline 2015 & 67.7 & 99.0 & 119.8 & 156.3 & 83.3 & 2015 & 93.1 & 46.5 & 46.5 & 58.2 & 139.6 \\
\hline
\end{tabular}

\section{C.2 Results}

\section{C.2.1 Trends in the number of people receiving prescriptions by deprivation}

Over the study period, 1.7 times more people in WIMD1 (most deprived) quintiles across Wales, received prescriptions for opioid analgesics than those in WIMD5 (least deprived) quintiles (Figure C.1). Greater percentage increases in the annual number of people receiving prescriptions, were observed in the less socio-economic deprived quintiles, the largest being in WIMD4 areas $(28.7 \%$ increase, from 98.8 to 120.8 people per 1000 population). Spearman's $r$ tests revealed strong correlations between time and the number of people receiving prescriptions, for all quintiles (Table C.5). 


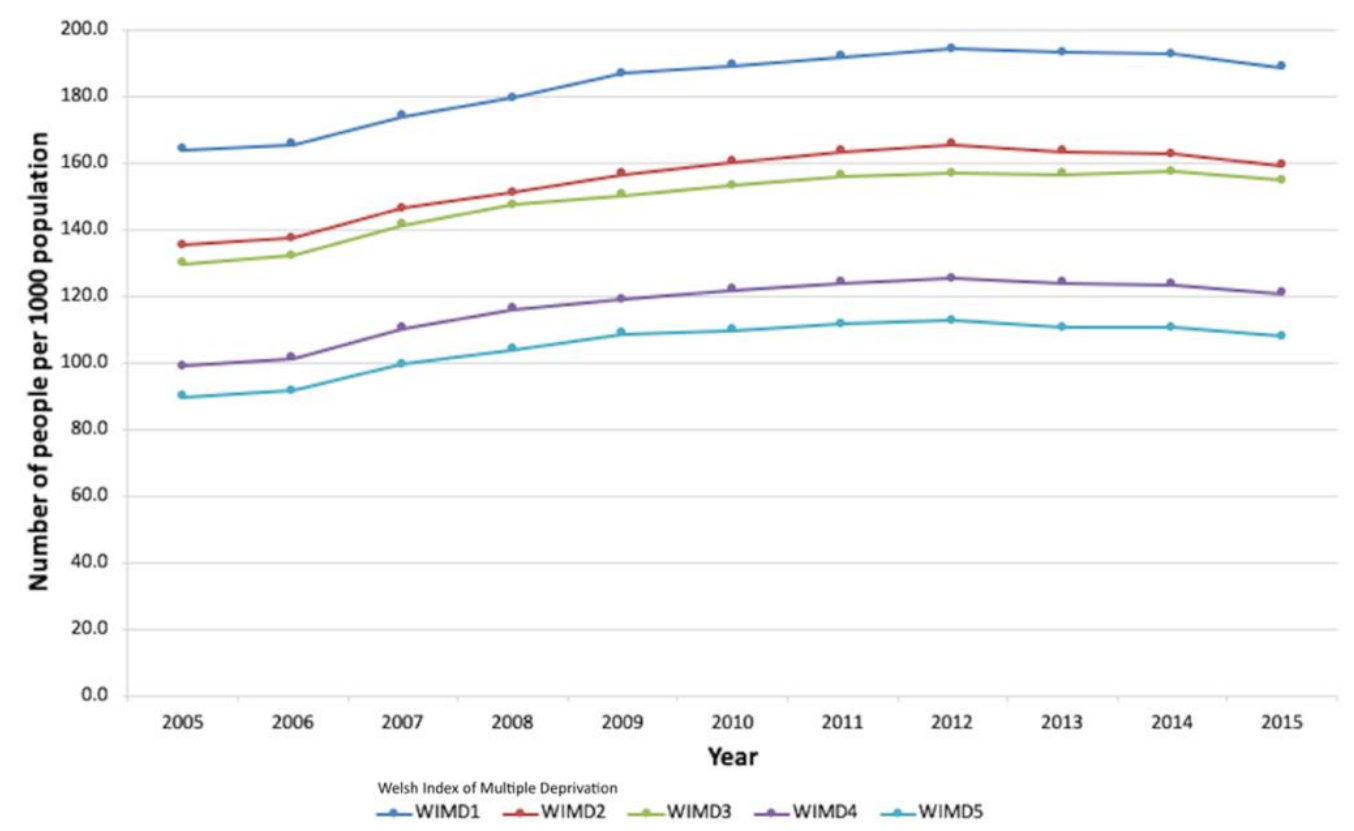

Figure C.1: Trends in the annual number of people receiving any opioid prescriptions presented by deprivation area and adjusted to population of each area of deprivation in Wales (WIMD $=$ Welsh Index of Multiple Deprivation where WIMD1 = most socio-economically deprived and WIMD5 = least socioeconomically deprived)

There was little difference between patient numbers in the $2^{\text {nd }}$ and $3^{\text {rd }}$ quintiles, which narrowed in the final two years of the examination and was confirmed by statistical analysis (Figure C.1).

Comparison of the number of people living in each quintile over the study period demonstrated significantly more received opioid prescriptions as deprivation increased $\left(p<.001, \eta^{2}=0.883, d_{\text {cohen }}=5.494\right)$ (Table C.5). Pairwise comparison confirmed significantly more people in WIMD1-3 quintiles received prescriptions compared to the least deprived (WIMD5) $(p<.001, p<.001$ and $p<0.01$ respectively) (Table C.7). Statistically more people living in WIMD1 and WIMD2 areas received prescriptions when compared to the number in WIMD4 quintiles $(p<.001$ and $p<0.05$ respectively). Empirically greater numbers of people living in the most deprived quintiles received opioid prescriptions than any other quintile for the duration of the study. However, the difference in numbers between WIMD1, WIMD2 and WIMD3 were not confirmed to be statistically significant (Table C.7).

Table C.5: Annual number of people receiving opioid prescriptions per socio-economic deprivation quintile between $2005-2015$

\begin{tabular}{cccccc}
\hline & \multicolumn{5}{c}{ Number of people } \\
\cline { 2 - 6 } Year & WIMD1 & WIMD2 & WIMD3 & WIMD4 & WIMD5 \\
\hline $\mathbf{2 0 0 5}$ & 77,785 & 63,835 & 58,211 & 43,386 & 42,861 \\
$\mathbf{2 0 0 6}$ & 78,989 & 65,228 & 59,633 & 44,728 & 43,963 \\
$\mathbf{2 0 0 7}$ & 83,539 & 69,836 & 64,206 & 48,987 & 48,145 \\
$\mathbf{2 0 0 8}$ & 86,800 & 72,643 & 67,392 & 51,919 & 50,608 \\
$\mathbf{2 0 0 9}$ & 89,502 & 75,331 & 69,589 & 53,993 & 52,683 \\
$\mathbf{2 0 1 0}$ & 91,047 & 77,406 & 71,150 & 55,442 & 53,472 \\
$\mathbf{2 0 1 1}$ & 92,810 & 79,297 & 72,837 & 56,674 & 54,655 \\
$\mathbf{2 0 1 2}$ & 94,293 & 80,532 & 73,435 & 57,535 & 55,398 \\
$\mathbf{2 0 1 3}$ & 94,037 & 79,771 & 73,439 & 57,073 & 54,516 \\
\hline
\end{tabular}




\begin{tabular}{|c|c|c|c|c|c|c|}
\hline 2014 & 94,091 & 79,541 & 73,957 & 56,887 & 54,750 & Welsh Index of \\
\hline 2015 & 92,541 & 78,122 & 72,911 & 55,852 & 53,618 & Multiple \\
\hline $\begin{array}{r}\text { Change rate (\%) } \\
2005-2015\end{array}$ & 19.0 & 22.4 & 25.3 & 28.7 & 25.1 & $\begin{array}{l}\text { Deprivation } \\
\text { (WIMD) where }\end{array}$ \\
\hline $\begin{array}{r}\text { Spearman's } r, p- \\
\text { value }\end{array}$ & $\begin{array}{l}0.827 \\
p<0.010 *\end{array}$ & $\begin{array}{l}0.793 \\
p<0.010^{*}\end{array}$ & $0.900,<.001^{*}$ & $\begin{array}{l}0.800 \\
p<0.010^{*}\end{array}$ & $\begin{array}{l}0.736 \\
p<0.050 *\end{array}$ & $\begin{array}{l}\text { WIMD1 = most } \\
\text { socio- }\end{array}$ \\
\hline
\end{tabular}

economically deprived, WIMD5 = least socio-economically deprived. ${ }^{*}$ p-value $<0.05=$ statistically significant

Across the whole study period, $26.4 \%(975,434 n=3,700,881)$ of all people who received any opioid prescription were living in the most deprived areas of Wales (WIMD1). Conversely, people living in the least deprived areas comprised $15.3 \%$ $(564,669)$ of people who received opioid prescriptions. In all areas, there was a peak in the annual number of people receiving prescriptions in 2012 with subsequent reductions over the three years that followed (Figure C.2).

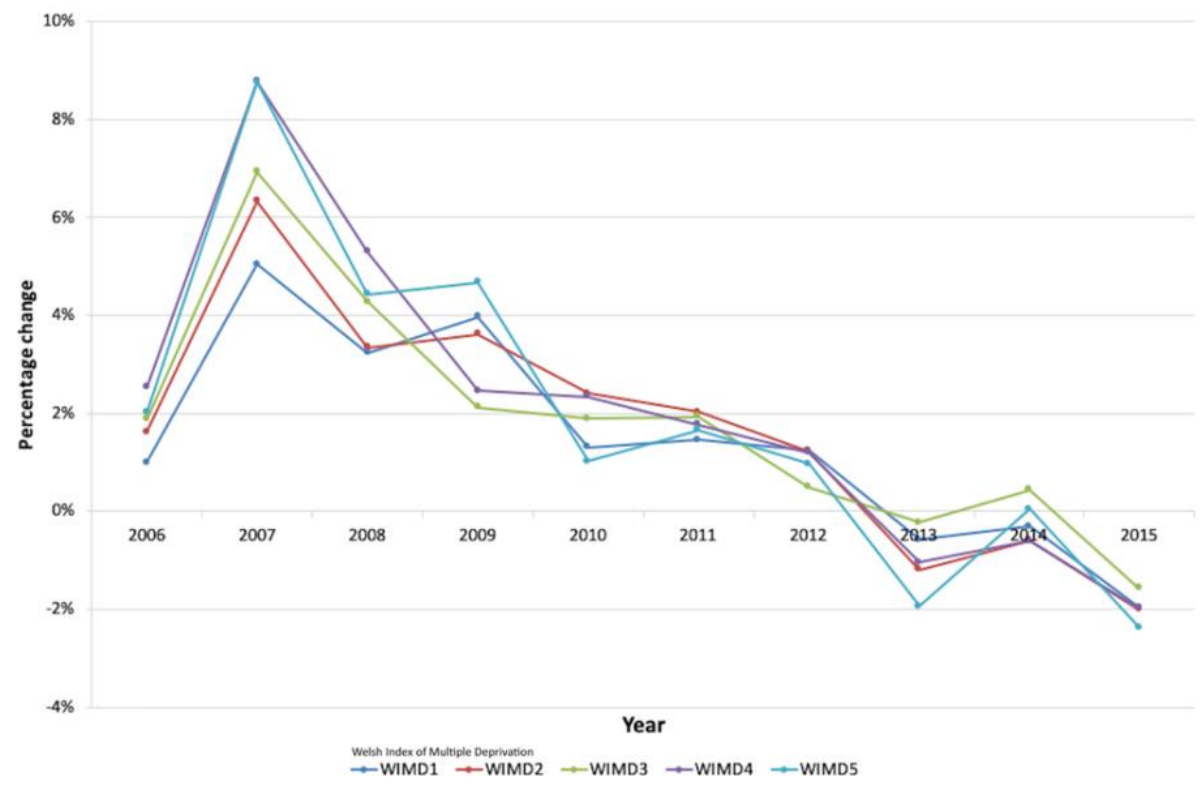

Figure C.2: Annual percentage change in the number of people prescribed opioids by quintiles of deprivation in Wales (WIMD = Welsh Index of Multiple Deprivation where WIMD1 = most deprived and WIMD5 = least deprived)

Table C.6: Comparison of quintile-population adjusted numbers of people receiving prescriptions for all opioid medicines by Welsh Index of Multiple Deprivation quintiles across Wales between 2005 and 2015

\begin{tabular}{rccccc}
\hline & \multicolumn{5}{c}{ Number of people per 1000 population } \\
\cline { 2 - 6 } Year & WIMD1 & WIMD2 & WIMD3 & WIMD4 & WIMD5 \\
\hline $\mathbf{2 0 0 5}$ & 164.0 & 135.3 & 129.6 & 98.8 & 89.6 \\
$\mathbf{2 0 0 6}$ & 165.6 & 137.5 & 132.0 & 101.3 & 91.4 \\
$\mathbf{2 0 0 7}$ & 174.0 & 146.2 & 141.2 & 110.2 & 99.4 \\
$\mathbf{2 0 0 8}$ & 179.6 & 151.1 & 147.2 & 116.0 & 103.9 \\
$\mathbf{2 0 0 9}$ & 186.7 & 156.5 & 150.4 & 118.9 & 108.7 \\
$\mathbf{2 0 1 0}$ & 189.1 & 160.3 & 153.2 & 121.7 & 109.8 \\
$\mathbf{2 0 1 1}$ & 191.9 & 163.5 & 156.2 & 123.9 & 111.6 \\
$\mathbf{2 0 1 2}$ & 194.3 & 165.5 & 156.9 & 125.3 & 112.7 \\
$\mathbf{2 0 1 3}$ & 193.1 & 163.5 & 156.5 & 124.0 & 110.5 \\
$\mathbf{2 0 1 4}$ & 192.5 & 162.6 & 157.2 & 123.3 & 110.6 \\
$\mathbf{2 0 1 5}$ & 188.7 & 159.3 & 154.7 & 120.8 & 107.9 \\
\hline Change rate (\%) & 19.0 & 22.4 & 25.3 & 28.7 & 25.1 \\
\hline $\mathbf{2 0 0 5}-\mathbf{2 0 1 5}$ & & & & & \\
\hline
\end{tabular}

Welsh Index of Multiple Deprivation (WIMD) where WIMD1 = most socio-economically deprived, WIMD5 = least socio-economically deprived. ${ }^{*}$ p-value $<0.05=$ statistically significant 
Table C.7: Dunn's pairwise comparison and Bonferroni post-hoc analysis of difference between the number people receiving opioid prescriptions in the 5 different areas of deprivation as defined by the Welsh Index of Multiple Deprivation (WIMD)

\section{Welsh Index of Multiple Deprivation}

\begin{tabular}{ccccc}
\hline & 1 & 2 & 3 & 4 \\
\hline $\mathbf{2}$ & .404 & & & \\
\hline $\mathbf{3}$ & .061 & $>.999$ & & \\
\hline $\mathbf{4}$ & $<.001^{*}$ & $.023^{*}$ & .188 & $>.999$
\end{tabular}

${ }^{*} p<0.05$ = statistically significant. WIMD1=most socio-economically deprived, WIMD5=least socioeconomically deprived

Table C.8: Dunn's pairwise comparison and Bonferroni post-hoc analysis of difference between the number people per 1000 population receiving opioid prescriptions in the 5 different areas of deprivation as defined by the Welsh Index of Multiple Deprivation (WIMD)

\begin{tabular}{ccccc}
\hline \multicolumn{5}{c}{ Welsh Index of Multiple Deprivation } \\
\hline $\mathbf{2}$ & 1 & 2 & 3 & 4 \\
\hline $\mathbf{3}$ & .813 & & & \\
\hline $\mathbf{4}$ & $<.064$ & $>.999$ & & \\
\hline $\mathbf{5}$ & $<.001^{*}$ & $.005^{*}$ & .128 & $>.999$
\end{tabular}

$*_{p}<0.05=$ statistically significant. WIMD1=most socio-economically deprived, WIMD5=least socioeconomically deprived 


\section{C.2.2 Trends in number of people receiving prescriptions by opioid-type}

Examining prescribing trends by different opioid types, there were differences in the patterns of people receiving weak and strong opioid prescriptions, although the proportions of people within each area of deprivation were similar in all groups (Table C.9). However, the proportion of total weak opioid prescribing that was for people in the most deprived areas decreased by 3.1\% between 2005 and 2015 (from $26.6 \%$ to $25.8 \%$ of people receiving weak opioids). The proportion of people in the most deprived areas of Wales receiving strong opioids however, increased by $5 \%$ over the same time.

Table C.9: Annual number of people receiving opioid prescriptions per socio-economic deprivation quintile and opioid-type between 2005 -2015

\begin{tabular}{|c|c|c|c|c|c|c|c|c|c|c|}
\hline \multirow[t]{2}{*}{ Year } & \multicolumn{5}{|c|}{ Weak opioids } & \multicolumn{5}{|c|}{ Strong opioids } \\
\hline & WIMD1 & WIMD2 & WIMD3 & WIMD4 & WIMD5 & WIMD1 & WIMD2 & WIMD3 & WIMD4 & WIMD5 \\
\hline 2005 & 75271 & 61630 & 56015 & 41750 & 41383 & 2514 & 2205 & 2196 & 1636 & 1478 \\
\hline 2006 & 76255 & 62794 & 57218 & 42832 & 42284 & 2734 & 2434 & 2415 & 1896 & 1679 \\
\hline 2007 & 80465 & 67039 & 61567 & 46874 & 46246 & 3074 & 2797 & 2639 & 2113 & 1899 \\
\hline 2008 & 83323 & 69693 & 64458 & 49592 & 48503 & 3477 & 2950 & 2934 & 2327 & 2105 \\
\hline 2009 & 85643 & 72055 & 66311 & 51363 & 50423 & 3859 & 3276 & 3278 & 2630 & 2260 \\
\hline 2010 & 86849 & 73784 & 67570 & 52571 & 51115 & 4198 & 3622 & 3580 & 2871 & 2357 \\
\hline 2011 & 88056 & 75269 & 68899 & 53573 & 51974 & 4754 & 4028 & 3938 & 3101 & 2681 \\
\hline 2012 & 88921 & 75912 & 68953 & 53956 & 52318 & 5372 & 4620 & 4482 & 3579 & 3080 \\
\hline 2013 & 87847 & 74559 & 68274 & 53129 & 51073 & 6190 & 5212 & 5165 & 3944 & 3443 \\
\hline 2014 & 86969 & 73404 & 67987 & 52282 & 50860 & 7122 & 6137 & 5970 & 4605 & 3890 \\
\hline 2015 & 84443 & 71248 & 66173 & 50830 & 49311 & 8098 & 6874 & 6738 & 5022 & 4307 \\
\hline $\begin{array}{l}\text { Change } \\
\text { rate (\%) }\end{array}$ & 12.2 & 15.6 & 18.1 & 21.7 & 19.2 & 222.1 & 211.7 & 206.8 & 207.0 & 191.4 \\
\hline $\begin{array}{r}2005- \\
2015\end{array}$ & & & & & & & & & & \\
\hline
\end{tabular}

Table C.10: Comparison of annual numbers of people receiving weak opioid prescriptions between 2005 and 2015 by deprivation area (Population adjusted data)

\begin{tabular}{|c|c|c|c|c|c|}
\hline \multirow[t]{2}{*}{ Year } & \multicolumn{5}{|c|}{ Number of people per 1000} \\
\hline & WIMD1 & WIMD2 & WIMD3 & WIMD4 & WIMD5 \\
\hline 2005 & 158.7 & 130.6 & 124.7 & 95.1 & 86.5 \\
\hline 2006 & 159.9 & 132.3 & 126.7 & 97.0 & 87.9 \\
\hline 2007 & 167.6 & 140.3 & 135.4 & 105.5 & 95.5 \\
\hline 2008 & 172.4 & 144.9 & 140.8 & 110.8 & 99.5 \\
\hline 2009 & 178.7 & 149.7 & 143.3 & 113.1 & 104.0 \\
\hline 2010 & 180.4 & 152.8 & 145.5 & 115.4 & 105.0 \\
\hline 2011 & 182.1 & 155.2 & 147.7 & 117.1 & 106.2 \\
\hline 2012 & 183.2 & 156.0 & 147.3 & 117.5 & 106.4 \\
\hline 2013 & 180.4 & 152.8 & 145.5 & 115.4 & 103.5 \\
\hline 2014 & 178.0 & 150.0 & 144.5 & 113.3 & 102.7 \\
\hline 2015 & 172.2 & 145.3 & 140.4 & 110.0 & 99.3 \\
\hline Change rate (\%) & 12.2 & 15.6 & 18.1 & 21.7 & 19.2 \\
\hline
\end{tabular}

*Welsh Index of Multiple Deprivation (WIMD) where WIMD1 = most socio-economically deprived, WIMD5 = least socio-economically deprived

In the least deprived areas, the proportion of people receiving weak opioids increased $2.4 \%$ (from $14.5 \%$ to $14.9 \%$ ) whereas the proportion of people receiving strong opioids in those areas reduced by 5.5\% (from $14.3 \%$ to $13.5 \%$ ).

Over the 11 years of the study period, the number of people living in the most deprived areas and receiving weak opioid prescriptions, increased $8.5 \%$ (from 
158.7 to 172.2 people per 1000). Those in the least deprived areas, receiving weak opioid prescriptions increased by $14.7 \%$ (from 86.5 to 99.3 people per 1000 population) over that period. Whilst there were overall increases in the numbers of people receiving weak opioid prescriptions each year; the annual number of people in every area of Wales peaked in 2012 and subsequently reduced each year (Figure C.3).

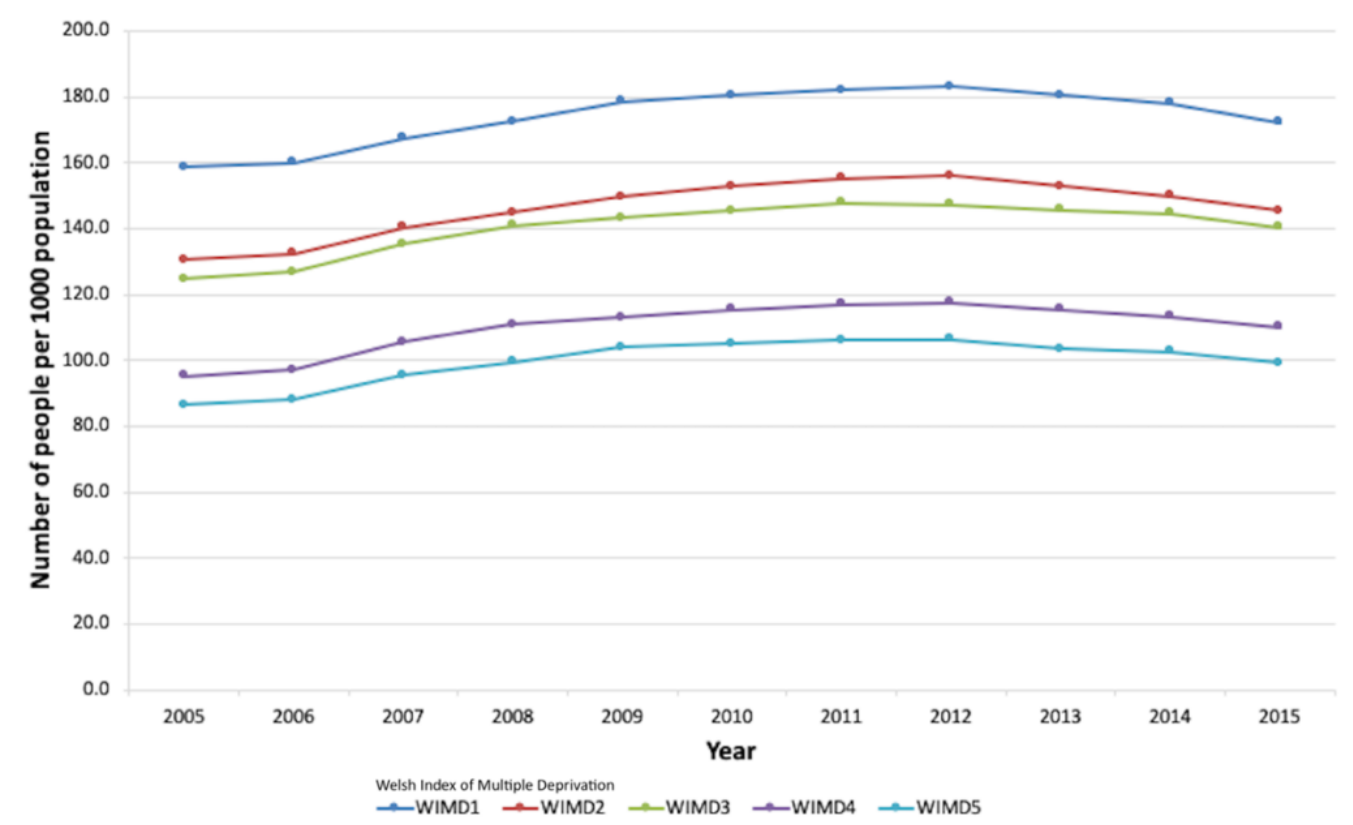

Figure C.3: Trends in the annual number of people receiving weak opioid prescriptions presented by deprivation area and adjusted to population of each area of deprivation in Wales

$(\mathrm{WIMD}=$ Welsh Index of Multiple Deprivation where WIMD1 = most deprived and WIMD5 =least deprived

There were statistically significant differences between each deprivation area for the number of people receiving weak opioid prescriptions, $(p<.001)$. However, post-hoc Dunn's pairwise and Bonferroni testing demonstrated significant differences between half of the compared pairs of deprivation areas (Table C.11).

Table C.11: Dunn's pairwise comparison and Bonferroni post-hoc analysis of difference between the number people per 1000 receiving weak opioid prescriptions in the 5 different areas of deprivation as defined by the Welsh Index of Multiple Deprivation (WIMD)

\begin{tabular}{ccccc}
\hline \multicolumn{5}{c}{ Welsh Index of Multiple Deprivation } \\
\hline & 1 & 2 & 3 & 4 \\
\hline $\mathbf{2}$ & .391 & & & \\
\hline $\mathbf{3}$ & .056 & $>.999$ & & \\
\hline $\mathbf{4}$ & $<.001^{*}$ & $.025^{*}$ & .206 & $>.999$ \\
\hline $\mathbf{5}$ & $<.001^{*}$ & $<.001^{*}$ & $.006^{*}$ & \\
\hline
\end{tabular}

${ }^{*} p<0.05=$ statistically significant. WIMD1=most socio-economically deprived, WIMD5=least socioeconomically deprived 


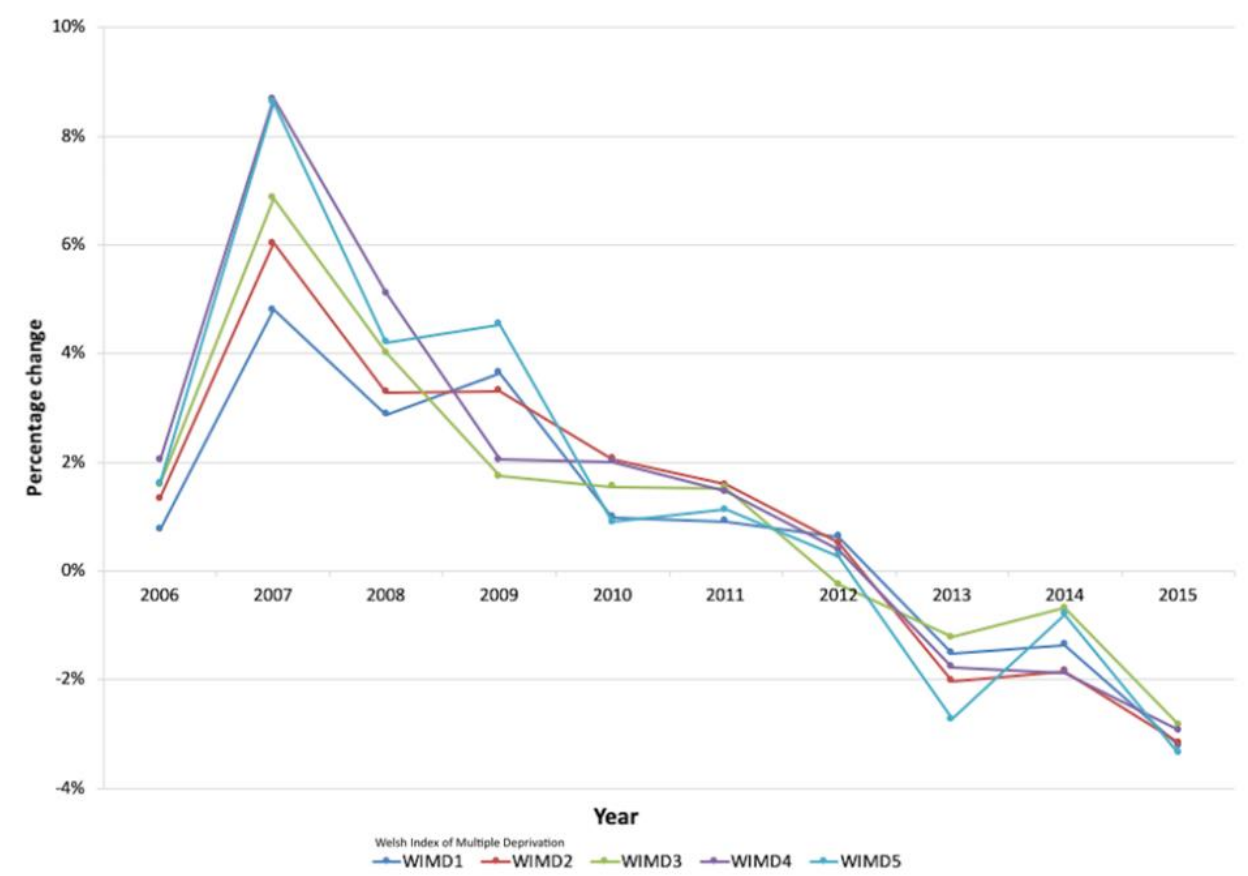

Figure C.4: Annual percentage change in the number of people prescribed weak opioids by area of deprivation in Wales

$($ WIMD $=$ Welsh Index of Multiple Deprivation where WIMD1 = most deprived and WIMD5 = least deprived $)$

Table C.12: Comparison of annual numbers of people receiving strong opioid prescriptions between 2005 and 2015 by deprivation area (Population adjusted data)

\begin{tabular}{rccccc}
\hline Year & \multicolumn{5}{c}{ Number of people per 1000 population } \\
\cline { 2 - 6 } & WIMD1 & WIMD2 & WIMD3 & WIMD4 & WIMD5 \\
\hline $\mathbf{2 0 0 5}$ & 5.3 & 4.7 & 4.9 & 3.7 & 3.1 \\
$\mathbf{2 0 0 6}$ & 5.7 & 5.1 & 5.3 & 4.3 & 3.5 \\
$\mathbf{2 0 0 7}$ & 6.4 & 5.9 & 5.8 & 4.8 & 3.9 \\
$\mathbf{2 0 0 8}$ & 7.2 & 6.1 & 6.4 & 5.2 & 4.3 \\
$\mathbf{2 0 0 9}$ & 8.1 & 6.8 & 7.1 & 5.8 & 4.7 \\
$\mathbf{2 0 1 0}$ & 8.7 & 7.5 & 7.7 & 6.3 & 4.8 \\
$\mathbf{2 0 1 1}$ & 9.8 & 8.3 & 8.4 & 6.8 & 5.5 \\
$\mathbf{2 0 1 3}$ & 11.1 & 9.5 & 9.6 & 7.8 & 6.3 \\
$\mathbf{2 0 1 4}$ & 12.7 & 10.7 & 11.0 & 8.6 & 7.0 \\
$\mathbf{2 0 1 5}$ & 14.6 & 12.5 & 12.7 & 10.0 & 7.9 \\
\hline Change rate & 211.6 & 14.0 & 14.3 & 10.9 & 8.7 \\
(\%) 2005 - 2015 & & 200.0 & 192.5 & 191.5 & 180.5 \\
\hline
\end{tabular}

*Welsh Index of Multiple Deprivation (WIMD) where WIMD1 = most socio-economically deprived, WIMD5 = least socio-economically deprived

The number of people receiving strong opioids increased in all areas between 2005 and 2015. Conversely to the weak opioid trends however, the greatest increase in the number of people receiving strong opioids was in the areas of highest deprivation ( $211.6 \%$, from 5.3 to 16.5 people per 1000 population) and the areas of least deprivation saw a significant albeit smaller increase $(180.5 \%$, from 3.1 to 8.7 people per 1000 population) (Figure C.5). 


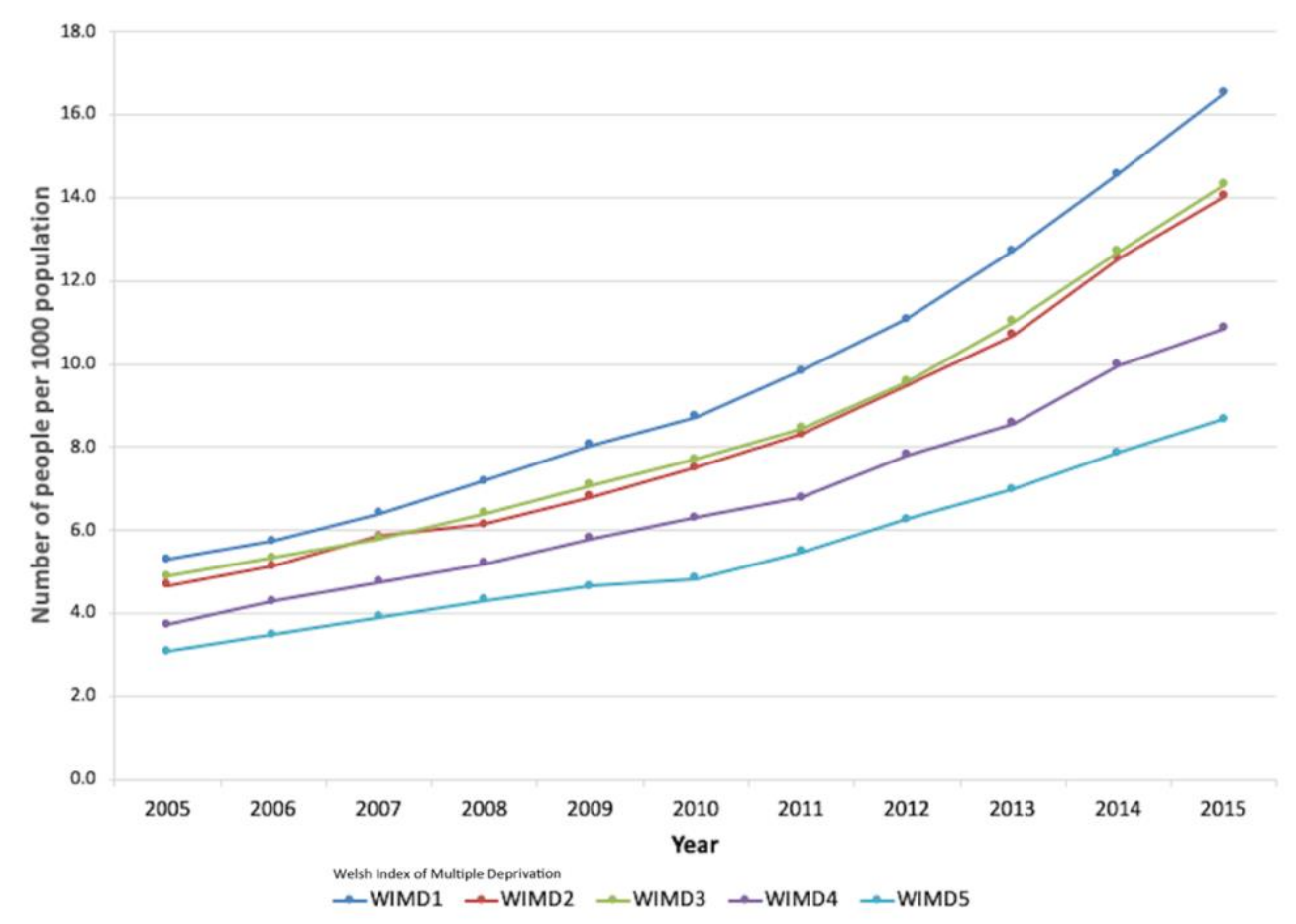

Figure C.5: Trends in the annual number of people receiving strong opioid prescriptions presented by deprivation area and adjusted to population of each area of deprivation in Wales (WIMD = Welsh Index of Multiple Deprivation where WIMD1 = most deprived and WIMD5)

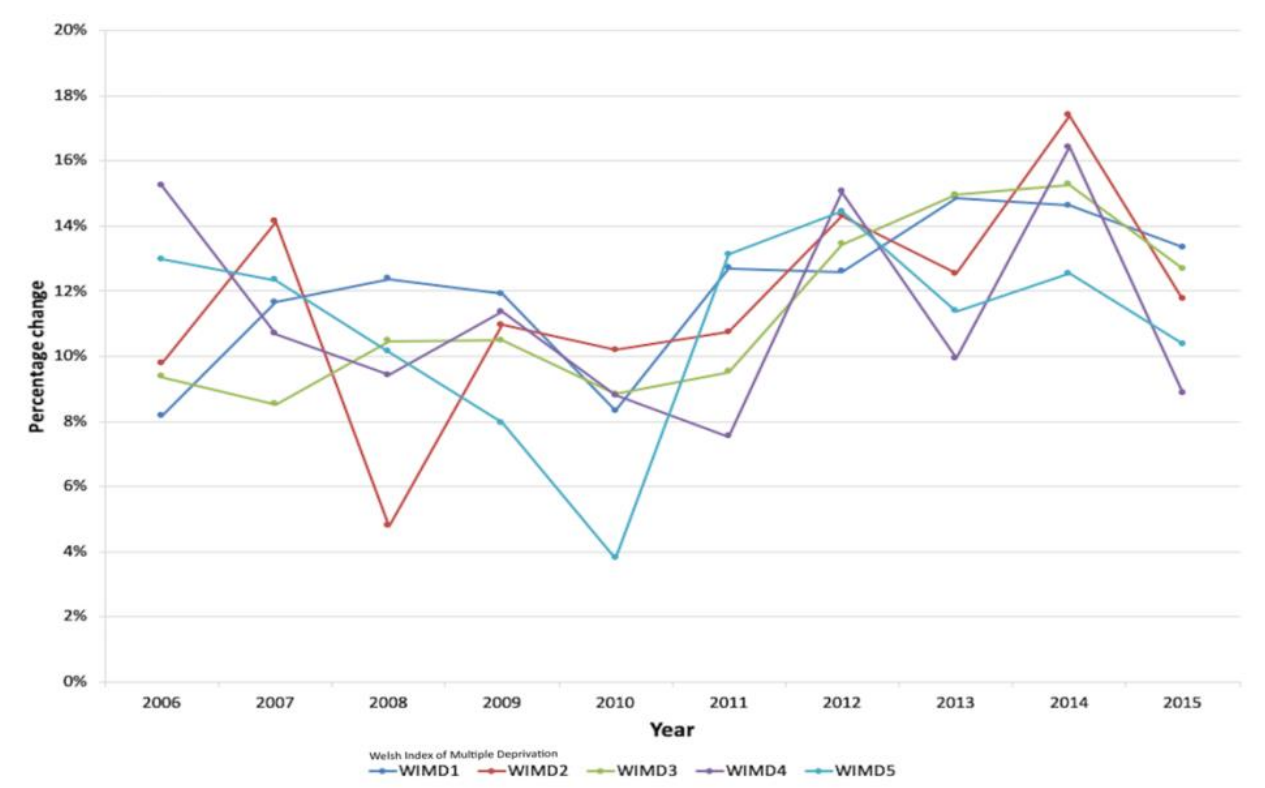

Figure C.6: Annual percentage change in the number of people prescribed strong opioids by each area of deprivation in Wales

$(W I M D=$ Welsh Index of Multiple Deprivation where WIMD1 = most deprived and WIMD5 = least deprived $)$

A Kruskal-Wallis test to examine whether there was any difference between the number of people receiving in each of the WIMD areas receiving strong opioid prescriptions was statistically significant to $95 \%(p=.012)$. Post-hoc Dunn's pairwise and Bonferroni tests however, revealed that statistically significant difference ( $p=.013)$ only existed between the most deprived (WIMD1) (median=8.72) and least deprived (WIMD5) (median=4.84) areas in Wales (Table C.13). 
Table C.13: Dunn's pairwise comparison and Bonferroni post-hoc analysis of difference between the number people per 1000 receiving strong opioid prescriptions in the 5 different areas of deprivation as defined by the Welsh Index of Multiple Deprivation (WIMD)

\begin{tabular}{ccccc}
\hline \multicolumn{5}{c}{ Welsh Index of Multiple Deprivation } \\
\hline & 1 & 2 & 3 & 4 \\
\hline $\mathbf{2}$ & $>.999$ & & & \\
& $>.999$ & $>.999$ & & \\
\hline $\mathbf{4}$ & .437 & $>.999$ & .999 & $>.999$ \\
\hline $\mathbf{5}$ & $<.013^{*}$ & .224 & .117 & \\
\hline
\end{tabular}

$*_{p}<0.05=$ statistically significant. WIMD1=most deprived, WIMD5=least deprived

\section{C.2.3 Number of prescriptions per 1000 quintile-population}

Table C.14: Comparison of numbers of prescriptions for all opioid medicines by Welsh Index of Multiple Deprivation quintiles across Wales between 2005 and 2015

\begin{tabular}{|c|c|c|c|c|c|}
\hline & \multicolumn{5}{|c|}{ Prescriptions } \\
\hline & WIMD1 & WIMD2 & WIMD3 & WIMD4 & WIMD5 \\
\hline 2005 & 468,209 & 365,651 & 315,481 & 213,808 & 191,128 \\
\hline 2006 & 479,395 & 377,245 & 326,237 & 220,888 & 197,495 \\
\hline 2007 & 511,937 & 404,814 & 348,966 & 242,883 & 214,680 \\
\hline 2008 & 546,873 & 428,625 & 369,954 & 263,033 & 232,032 \\
\hline 2009 & 573,347 & 450,463 & 389,448 & 279,393 & 244,183 \\
\hline 2010 & 599,109 & 477,062 & 410,357 & 295,375 & 256,554 \\
\hline 2011 & 629,842 & 505,328 & 435,569 & 311,598 & 270,458 \\
\hline 2012 & 655,685 & 526,799 & 453,808 & 323,142 & 280,578 \\
\hline 2013 & 669,980 & 531,688 & 461,424 & 328,837 & 286,035 \\
\hline 2014 & 677,949 & 538,450 & 471,503 & 336,231 & 288,436 \\
\hline 2015 & 681,386 & 541,863 & 473,611 & 336,065 & 290,619 \\
\hline Percentage change (\%) & 45.5 & 48.2 & 50.1 & 57.2 & 52.1 \\
\hline
\end{tabular}

WIMD1 = most socio=economically deprived, WIMD5 = least socio-economically deprived

Table C.15: Comparison of quintile-population adjusted numbers of prescriptions for all opioid medicines by Welsh Index of Multiple Deprivation quintiles across Wales between 2005 and 2015

\begin{tabular}{rccccc}
\hline & \multicolumn{5}{c}{ Prescriptions per 1000 population } \\
\cline { 2 - 5 } $\mathbf{2 0 0 5}$ & WIMD1 & WIMD2 & WIMD3 & WIMD4 & WIMD5 \\
$\mathbf{2 0 0 6}$ & 1005.1 & 774.8 & 702.4 & 487.0 & 399.7 \\
$\mathbf{2 0 0 7}$ & 1066.0 & 795.0 & 722.3 & 500.4 & 410.7 \\
$\mathbf{2 0 0 8}$ & 1131.4 & 847.3 & 767.4 & 546.4 & 443.4 \\
$\mathbf{2 0 0 9}$ & 1196.1 & 891.3 & 808.3 & 587.9 & 476.1 \\
$\mathbf{2 0 1 0}$ & 1244.6 & 935.9 & 841.4 & 615.3 & 503.9 \\
$\mathbf{2 0 1 1}$ & 1302.3 & 1042.0 & 883.6 & 648.4 & 526.9 \\
$\mathbf{2 0 1 2}$ & 1350.9 & 1082.7 & 933.8 & 680.9 & 552.4 \\
$\mathbf{2 0 1 3}$ & 1376.0 & 1090.0 & 969.7 & 703.9 & 570.9 \\
$\mathbf{2 0 1 4}$ & 1387.3 & 1100.4 & 1002.4 & 714.6 & 579.9 \\
$\mathbf{2 0 1 5}$ & 1389.8 & 1104.8 & 1005.2 & 728.5 & 582.5 \\
$\mathbf{2 0 0 5}-\mathbf{2 0 1 5}$ & 40.8 & 42.6 & 43.1 & 727.0 & 585.1 \\
\hline Change rate (\%) & & & 49.3 & 46.4
\end{tabular}

*Welsh Index of Multiple Deprivation (WIMD) where WIMD1 = most socio-economically deprived, WIMD5 $=$ least socio-economically deprived

Table C.16: Dunn's pairwise comparison and Bonferroni post-hoc analysis of difference between the number of opioid prescriptions per 1000 population in the 5 different areas of deprivation as defined by the Welsh Index of Multiple Deprivation (WIMD)

\begin{tabular}{ccccc}
\hline \multicolumn{5}{c}{ Welsh Index of Multiple Deprivation } \\
\hline $\mathbf{2}$ & 1 & 2 & 3 & 4 \\
\hline $\mathbf{3}$ & .999 & & \\
\hline $\mathbf{4}$ & $<.001^{*}$ & $>.999$ & .291 \\
\hline
\end{tabular}




$$
5 \quad<.001 * \quad<.001^{*} \quad .008^{*} \quad>.999
$$

$* p<0.05=$ statistically significant WIMD1=most deprived, WIMD5=least deprived

Table C.17: Comparison of numbers of prescriptions for weak opioid medicines by Welsh Index of Multiple Deprivation quintiles across Wales between 2005 and 2015

\begin{tabular}{rccccc}
\hline & \multicolumn{5}{c}{ Prescriptions } \\
\cline { 2 - 6 } $\mathbf{2 0 0 5}$ & WIMD1 & WIMD2 & WIMD3 & WIMD4 & WIMD5 \\
\hline $\mathbf{2 0 0 6}$ & 444,471 & 344,748 & 296,048 & 199,945 & 180,355 \\
$\mathbf{2 0 0 7}$ & 477,884 & 353,396 & 302,192 & 204,078 & 183,923 \\
$\mathbf{2 0 0 8}$ & 506,624 & 376,692 & 321,873 & 222,569 & 198,465 \\
$\mathbf{2 0 0 9}$ & 527,574 & 396,352 & 339,457 & 238,008 & 212,901 \\
$\mathbf{2 0 1 0}$ & 546,577 & 413,939 & 354,211 & 250,354 & 223,225 \\
$\mathbf{2 0 1 1}$ & 567,653 & 434,307 & 369,303 & 261,486 & 232,104 \\
$\mathbf{2 0 1 2}$ & 582,792 & 465,245 & 386,452 & 273,258 & 242,180 \\
$\mathbf{2 0 1 3}$ & 585,591 & 465,853 & 395,403 & 280,183 & 248,146 \\
$\mathbf{2 0 1 4}$ & 583,292 & 462,801 & 394,873 & 281,625 & 248,513 \\
$\mathbf{2 0 1 5}$ & 571,761 & 455,600 & 393,310 & 284,188 & 247,871 \\
(\%) 2005-2015 & 28.6 & 32.2 & 32.9 & 279,830 & 246,765 \\
\hline Percentage change & & & 40.0 & 36.8
\end{tabular}

WIMD1 = most socio=economically deprived, WIMD5 = least socio-economically deprived

Table C.18: Comparison of quintile-population adjusted numbers of prescriptions for weak opioid medicines by Welsh Index of Multiple Deprivation quintiles across Wales between 2005 and 2015

\begin{tabular}{rccccc}
\hline & \multicolumn{5}{c}{ Number of prescriptions per 1000 population } \\
\cline { 2 - 6 } $\mathbf{2 0 0 5}$ & WIMD1 & WIMD2 & WIMD3 & WIMD4 & WIMD5 \\
$\mathbf{2 0 0 6}$ & 937.1 & 730.5 & 659.1 & 455.4 & 377.2 \\
$\mathbf{2 0 0 7}$ & 995.1 & 744.7 & 669.1 & 462.3 & 382.5 \\
$\mathbf{2 0 0 8}$ & 1048.1 & 788.4 & 707.8 & 500.7 & 409.9 \\
$\mathbf{2 0 0 9}$ & 1100.6 & 824.2 & 741.6 & 532.0 & 436.9 \\
$\mathbf{2 0 1 0}$ & 1135.5 & 860.0 & 765.3 & 551.4 & 460.6 \\
$\mathbf{2 0 1 1}$ & 1173.7 & 899.3 & 795.2 & 574.0 & 476.7 \\
$\mathbf{2 0 1 2}$ & 1200.7 & 938.8 & 828.5 & 597.2 & 494.7 \\
$\mathbf{2 0 1 3}$ & 1202.7 & 955.0 & 844.9 & 610.3 & 504.9 \\
$\mathbf{2 0 1 4}$ & 1193.6 & 945.8 & 841.7 & 612.0 & 503.8 \\
$\mathbf{2 0 1 5}$ & 1166.2 & 928.9 & 848.0 & 615.8 & 500.6 \\
\hline (\%) $2005-2015$ & 24.5 & 27.2 & 834.8 & 605.3 & 496.8 \\
\hline
\end{tabular}

(\%) 2005 - 2015

WIMD1 = most socio=economically deprived, WIMD5 = least socio-economically deprived

Table C.19: Dunn's pairwise comparison and Bonferroni post-hoc analysis of difference between the number of weak opioid prescriptions per 1000 population in the 5 different areas of deprivation as defined by the Welsh Index of Multiple Deprivation (WIMD)

\begin{tabular}{ccccc}
\hline \multicolumn{5}{c}{ Welsh Index of Multiple Deprivation } \\
\hline $\mathbf{2}$ & 1 & 2 & 3 & 4 \\
\hline $\mathbf{3}$ & .767 & & & \\
\hline $\mathbf{4}$ & $<.054$ & $>.999$ & & \\
\hline $\mathbf{5}$ & $<.001^{*}$ & $.015^{*}$ & .311 & $>.999$ \\
\hline
\end{tabular}

${ }^{*} \mathrm{p}<0.05$ = statistically significant WIMD1=most deprived, WIMD5-least deprived

Table C.20: Comparison of numbers of prescriptions for strong opioid medicines by Welsh Index of Multiple Deprivation quintiles across Wales between 2005 and 2015

\begin{tabular}{lllll}
\hline \multicolumn{5}{c}{ Prescriptions } \\
\cline { 2 - 5 } \\
WIMD1 & WIMD2 & WIMD3 & WIMD4 & WIMD5 \\
\hline
\end{tabular}




\begin{tabular}{rccccc}
\hline $\mathbf{2 0 0 5}$ & 23,738 & 20,903 & 19,433 & 13,863 & 10,773 \\
$\mathbf{2 0 0 6}$ & 28,851 & 23,849 & 24,045 & 16,810 & 13,572 \\
$\mathbf{2 0 0 7}$ & 34,053 & 28,122 & 27,093 & 20,314 & 16,215 \\
$\mathbf{2 0 0 8}$ & 40,249 & 32,273 & 30,497 & 25,025 & 19,131 \\
$\mathbf{2 0 0 9}$ & 45,773 & 36,524 & 35,237 & 29,039 & 20,958 \\
$\mathbf{2 0 1 0}$ & 52,532 & 42,755 & 41,054 & 33,889 & 24,450 \\
$\mathbf{2 0 1 1}$ & 62,189 & 50,083 & 49,117 & 38,340 & 28,278 \\
$\mathbf{2 0 1 2}$ & 72,893 & 57,878 & 58,405 & 42,959 & 32,432 \\
$\mathbf{2 0 1 3}$ & 84,389 & 65,835 & 66,551 & 47,212 & 37,522 \\
$\mathbf{2 0 1 4}$ & 94,657 & 75,649 & 72,592 & 52,043 & 40,565 \\
$\mathbf{2 0 1 5}$ & 109,625 & 86,263 & 80,301 & 56,235 & 43,854 \\
\hline Percentage change & 361.8 & 312.7 & 313.2 & 305.6 & 307.1 \\
(\%) 2005-2015 & & & &
\end{tabular}

WIMD1 = most socio=economically deprived, WIMD5 = least socio-economically deprived

Table C.21: Comparison of quintile-population adjusted numbers of prescriptions for strong opioid medicines by Welsh Index of Multiple Deprivation quintiles across Wales between 2005 and 2015

\begin{tabular}{|c|c|c|c|c|c|}
\hline & \multicolumn{5}{|c|}{ Prescriptions per 1000 population } \\
\hline & WIMD1 & WIMD2 & WIMD3 & WIMD4 & WIMD5 \\
\hline 2005 & 50.0 & 44.3 & 43.3 & 31.6 & 22.5 \\
\hline 2006 & 60.5 & 50.3 & 53.2 & 38.1 & 28.2 \\
\hline 2007 & 70.9 & 58.9 & 59.6 & 45.7 & 33.5 \\
\hline 2008 & 83.3 & 67.1 & 66.6 & 55.9 & 39.3 \\
\hline 2009 & 95.5 & 75.9 & 76.1 & 64.0 & 43.2 \\
\hline 2010 & 109.1 & 88.5 & 88.4 & 74.4 & 50.2 \\
\hline 2011 & 128.6 & 103.3 & 105.3 & 83.8 & 57.8 \\
\hline 2012 & 150.2 & 119.0 & 124.8 & 93.6 & 66.0 \\
\hline 2013 & 173.3 & 135.0 & 141.9 & 102.6 & 76.1 \\
\hline 2014 & 193.7 & 154.6 & 154.3 & 112.8 & 81.9 \\
\hline 2015 & 223.6 & 175.9 & 170.4 & 121.6 & 88.3 \\
\hline Change rate (\%) & 346.8 & 297.1 & 293.9 & 285.2 & 291.9 \\
\hline
\end{tabular}

WIMD1 = most socioeconomically deprived, WIMD5 = least socio-economically deprived

Table C.22: Dunn's pairwise comparison and Bonferroni post-hoc analysis of difference between the number of strong opioid prescriptions per 1000 population in the 5 different areas of deprivation as defined by the Welsh Index of Multiple Deprivation (WIMD)

\begin{tabular}{ccccc}
\hline \multicolumn{5}{c}{ Welsh Index of Multiple Deprivation } \\
\hline $\mathbf{2}$ & 1 & 2 & 3 & 4 \\
\hline $\mathbf{3}$ & $>.999$ & & & \\
\hline $\mathbf{4}$ & .999 & $>.999$ & & \\
\hline $\mathbf{5}$ & $.006^{*}$ & $>.999$ & $>.999$ & $>.999$ \\
\hline
\end{tabular}

${ }^{*} p<0.05$ = statistically significant. WIMD1=most deprived, WIMD5 = least deprived 
C.2.4 Gender differences in the number of people receiving prescriptions by deprivation

\section{C.2.4.1 All Opioids}

Table C.23: Comparison of annual numbers of people by gender receiving strong opioid prescriptions between 2005 and 2015 by deprivation area (Population adjusted data)

\begin{tabular}{|c|c|c|c|c|c|c|c|c|c|c|}
\hline \multirow[t]{2}{*}{ Year } & \multicolumn{5}{|c|}{ Men } & \multicolumn{5}{|c|}{ Women } \\
\hline & WIMD1 & WIMD2 & WIMD3 & WIMD4 & WIMD5 & WIMD1 & WIMD2 & WIMD3 & WIMD4 & WIMD5 \\
\hline 2005 & 64.6 & 52.5 & 49.1 & 37.6 & 33.3 & 99.4 & 82.8 & 80.5 & 61.3 & 56.3 \\
\hline 2006 & 65.5 & 53.6 & 51.2 & 38.8 & 34.2 & 100.1 & 83.8 & 80.9 & 62.5 & 57.1 \\
\hline 2007 & 69.0 & 57.9 & 55.2 & 43 & 38 & 105 & 88.2 & 85.9 & 67.1 & 61.5 \\
\hline 2008 & 72.0 & 59.7 & 58.1 & 45.4 & 40 & 107.6 & 91.3 & 89.1 & 70.7 & 63.9 \\
\hline 2009 & 75.8 & 62.6 & 60.3 & 47 & 41.9 & 110.9 & 94 & 90.1 & 71.9 & 66.8 \\
\hline 2010 & 76.8 & 64.7 & 61.3 & 48.6 & 43.2 & 112.3 & 95.6 & 91.9 & 73.1 & 66.6 \\
\hline 2011 & 77.9 & 66.2 & 62.5 & 49.5 & 43.8 & 114 & 97.3 & 93.7 & 74.3 & 67.8 \\
\hline 2012 & 79.6 & 67.1 & 62.9 & 50.2 & 44.3 & 114.7 & 98.3 & 94 & 75.1 & 68.4 \\
\hline 2013 & 79.1 & 66.3 & 62.5 & 49.6 & 43.3 & 114 & 97.1 & 94.1 & 74.5 & 67.3 \\
\hline 2014 & 78.3 & 65.9 & 63.2 & 49.4 & 43.3 & 114.2 & 96.6 & 94 & 73.9 & 67.3 \\
\hline 2015 & 76.8 & 64.5 & 61.8 & 48.2 & 42.9 & 112 & 94.7 & 92.9 & 72.6 & 65.1 \\
\hline Change rate (\%) & 18.9 & 22.9 & 25.9 & 28.2 & 28.8 & 12.7 & 14.4 & 15.4 & 18.4 & 15.6 \\
\hline 2005-2015 & & & & & & & & & & \\
\hline
\end{tabular}



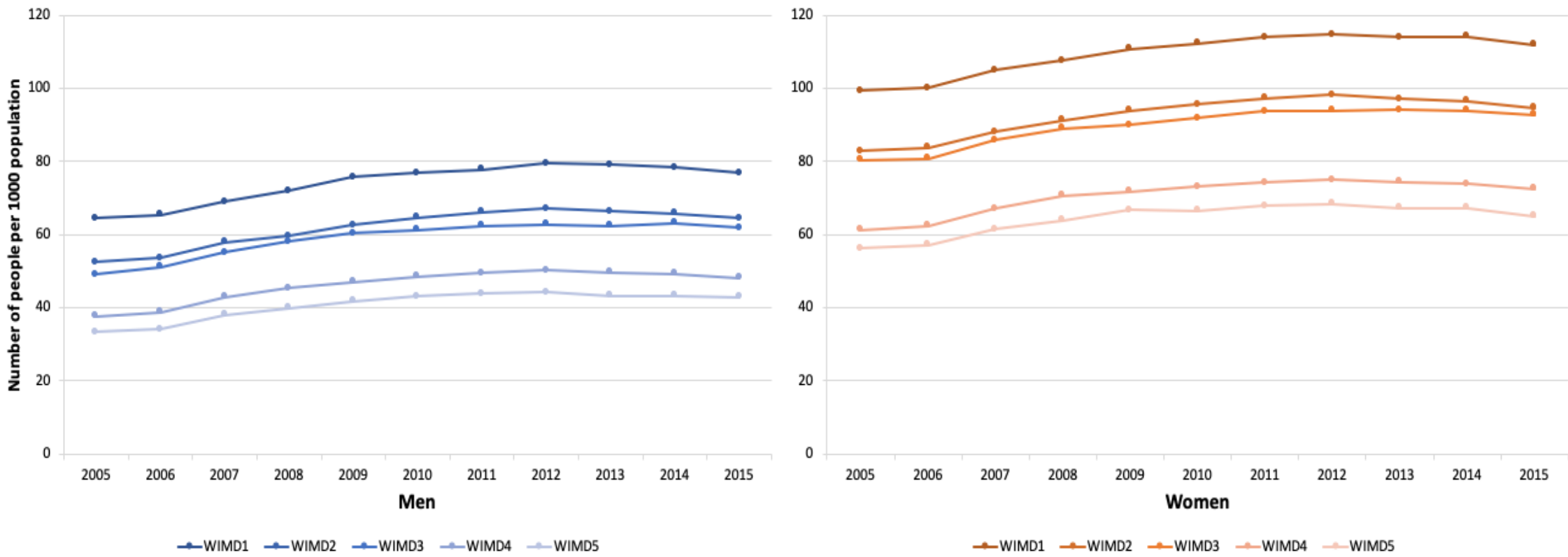

Figure C.7: Trends in the number of people receiving opioid prescriptions, stratified by gender and socioeconomic deprivation quintile WIMD - Welsh Index of Multiple Deprivation, WIMD1=most deprived, WIMD5=least deprived

\section{C.2.4.2 Weak opioids}

Table C.24: Annual number of people per 1000 population receiving weak opioid prescriptions per deprivation quintile and gender Year Number of prescriptions per 1000 population

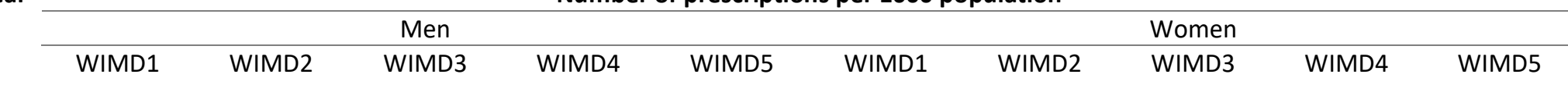




\begin{tabular}{|c|c|c|c|c|c|c|c|c|c|c|}
\hline 2005 & 62.2 & 50.6 & 47.1 & 36.1 & 32.1 & 96.5 & 80 & 77.6 & 59.1 & 54.4 \\
\hline 2006 & 63.1 & 51.5 & 49 & 37.1 & 33 & 96.8 & 80.8 & 77.7 & 59.9 & 54.9 \\
\hline 2007 & 66.4 & 55.6 & 52.9 & 41.2 & 36.6 & 101.2 & 84.7 & 82.4 & 64.2 & 59 \\
\hline 2008 & 69.1 & 57.3 & 55.7 & 43.5 & 38.5 & 103.3 & 87.6 & 85.1 & 67.4 & 61.1 \\
\hline 2010 & 73.4 & 61.8 & 58.4 & 46.2 & 41.5 & 107 & 91 & 87.1 & 69.2 & 63.5 \\
\hline 2011 & 74.1 & 63 & 59.3 & 47 & 41.9 & 108 & 92.2 & 88.4 & 70.1 & 64.3 \\
\hline 2012 & 75.2 & 63.5 & 59.3 & 47.3 & 42.1 & 108 & 92.5 & 88 & 70.2 & 64.3 \\
\hline 2013 & 74 & 62.3 & 58.4 & 46.4 & 40.8 & 106.4 & 90.5 & 87.2 & 69.1 & 62.8 \\
\hline 2014 & 72.6 & 61.2 & 58.4 & 45.6 & 40.5 & 105.3 & 88.8 & 86.1 & 67.7 & 62.2 \\
\hline 2015 & 70.4 & 59.3 & 56.5 & 44.2 & 39.8 & 101.9 & 85.9 & 83.9 & 65.8 & 59.5 \\
\hline $\begin{array}{r}\text { Change rate (\%) } \\
2005-2015\end{array}$ & 13.2 & 17.2 & 20.0 & 22.4 & 24.0 & 5.6 & 7.4 & 8.1 & 11.3 & 9.4 \\
\hline
\end{tabular}

WIMD - Welsh Index of Multiple Deprivation, WIMD1=most deprived, WIMD5=least deprived
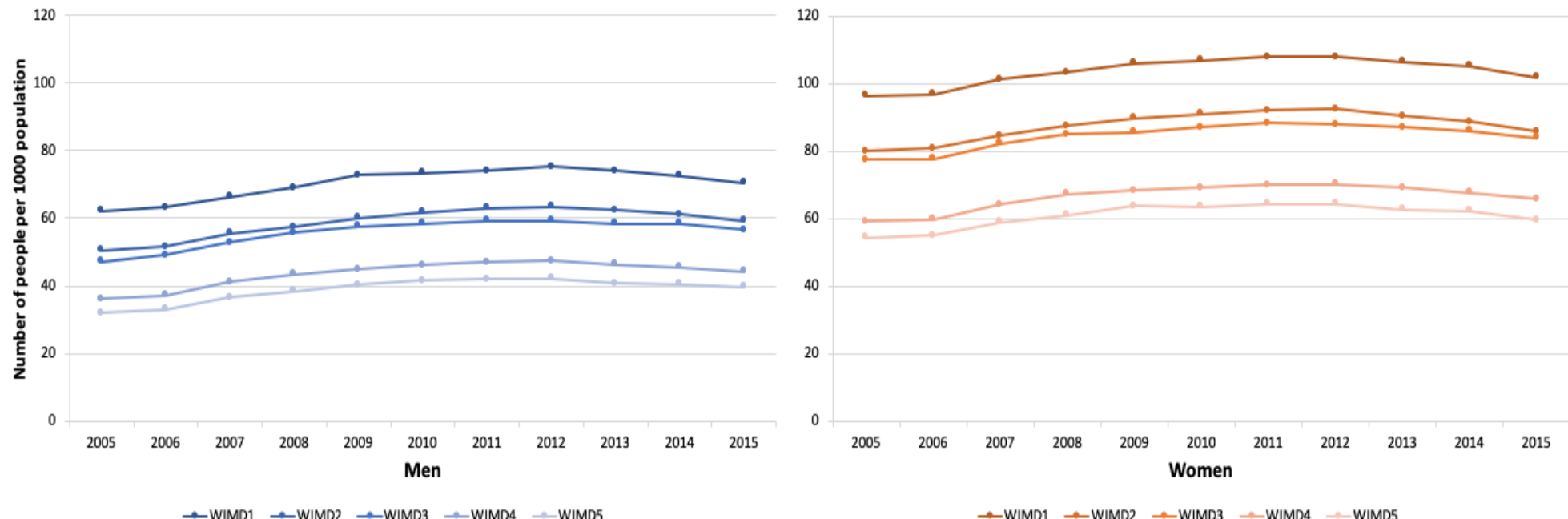

Figure C.8: Trends in the number of people per 1000 population receiving strong opioid by gender and socioeconomic deprivation quintile (WIMD) $(\mathrm{WIMD}=$ Welsh Index of Multiple Deprivation where WIMD1 = most deprived and WIMD5 = least deprived $)$ 

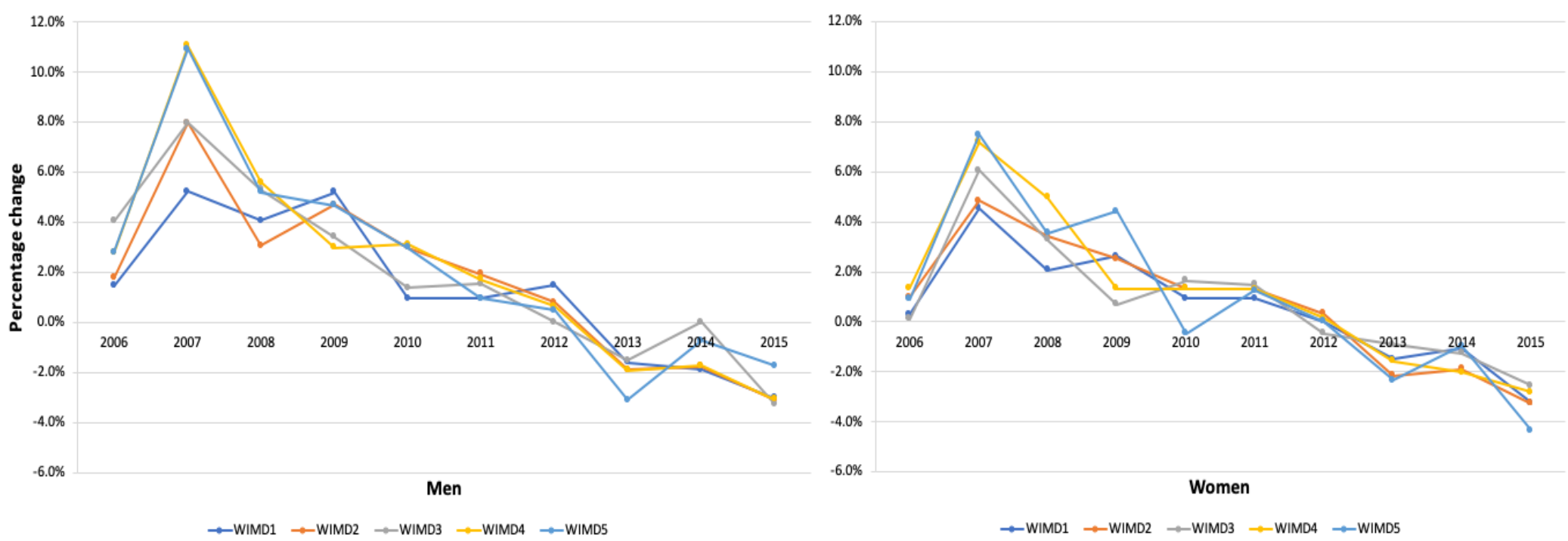

$-6.0 \%$

Women

Figure C.9: Annual percentage change in the number of prescriptions weak opioids by gender and socioeconomic deprivation quintile $(W I M D=$ Welsh Index of Multiple Deprivation where WIMD1 = most deprived and WIMD5 = least deprived $)$ 


\section{C.2.4.3 Strong opioids}

Table C.25: Annual number of people per 1000 population receiving strong opioid prescriptions per deprivation quintile and gender

\begin{tabular}{rcccccccccc}
\hline Year & \multicolumn{3}{c}{ Men } \\
\cline { 2 - 10 } & WIMD1 & WIMD2 & WIMD3 & WIMD4 & WIMD5 & WIMD1 & WIMD2 & WIMD3 & WIMD4 & WIMD5 \\
\hline $\mathbf{2 0 0 5}$ & 2.4 & 1.9 & 2 & 1.5 & 1.2 & 2.9 & 2.8 & 2.9 & 2.2 & 1.9 \\
$\mathbf{2 0 0 6}$ & 2.4 & 2.1 & 2.2 & 1.7 & 1.2 & 3.3 & 3 & 3.2 & 2.6 & 2.2 \\
$\mathbf{2 0 0 7}$ & 2.6 & 2.3 & 2.3 & 1.8 & 1.4 & 3.8 & 3.5 & 3.5 & 2.9 & 2.5 \\
$\mathbf{2 0 0 8}$ & 2.9 & 2.4 & 2.4 & 1.9 & 1.5 & 4.3 & 3.7 & 4 & 3.3 & 2.8 \\
$\mathbf{2 0 0 9}$ & 3.1 & 2.6 & 2.7 & 2.2 & 1.6 & 4.9 & 4.2 & 4.4 & 3.6 & 3 \\
$\mathbf{2 0 1 0}$ & 3.4 & 2.9 & 2.9 & 2.4 & 1.7 & 5.3 & 4.6 & 4.8 & 3.9 & 3.1 \\
$\mathbf{2 0 1 1}$ & 3.8 & 3.2 & 3.2 & 2.5 & 1.9 & 6 & 5.1 & 5.3 & 4.2 & 3.5 \\
$\mathbf{2 0 1 2}$ & 4.4 & 3.6 & 3.6 & 2.9 & 2.2 & 6.7 & 5.8 & 6 & 4.9 & 4.1 \\
$\mathbf{2 0 1 3}$ & 5.1 & 4 & 4.1 & 3.2 & 2.5 & 7.6 & 6.6 & 6.9 & 5.4 & 4.5 \\
$\mathbf{2 0 1 4}$ & 5.7 & 4.7 & 4.8 & 3.8 & 2.8 & 8.9 & 7.8 & 7.9 & 6.2 & 5.1 \\
$\mathbf{2 0 1 5}$ & 6.4 & 5.2 & 5.3 & 4 & 3.1 & 10.1 & 8.8 & 9 & 6.8 & 5.6 \\
\hline Change & 166.7 & 173.7 & 165.0 & 166.7 & 158.3 & 248.3 & 214.3 & 210.3 & 209.1 & 194.7 \\
rate (\%) & & & & & & & & & & \\
$\mathbf{2 0 0 5 - 2 0 1 5}$ & & & & & & & & &
\end{tabular}

WIMD - Welsh Index of Multiple Deprivation, WIMD1=most deprived, WIMD5=least deprived

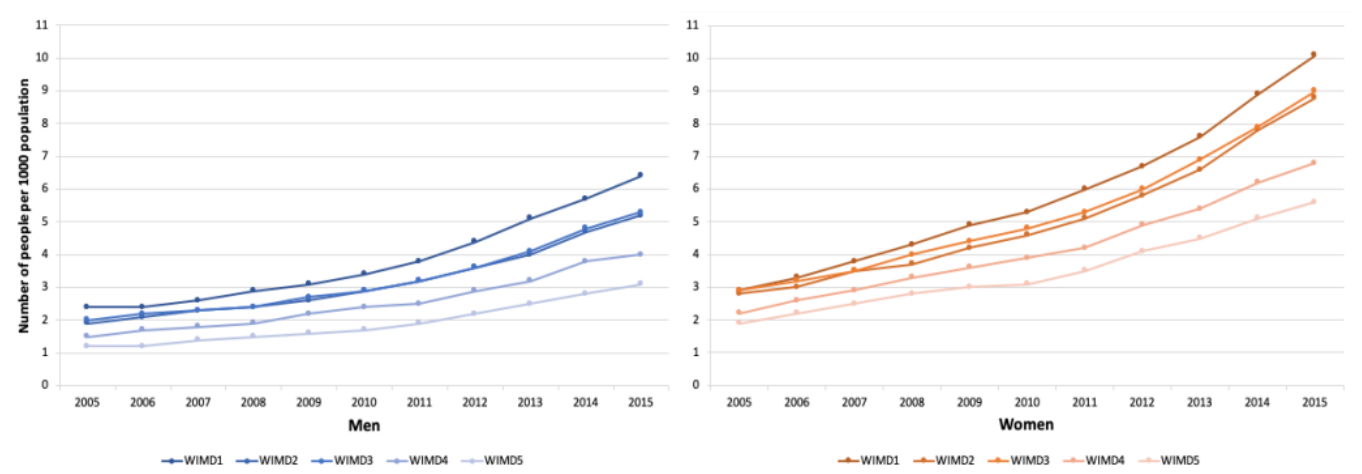

Figure C.10: Trends in the number of people per 1000 population receiving strong opioid by gender and socioeconomic deprivation quintile (WIMD)

WIMD = Welsh Index of Multiple Deprivation, WIMD1=most deprived, WIMD5=least deprived
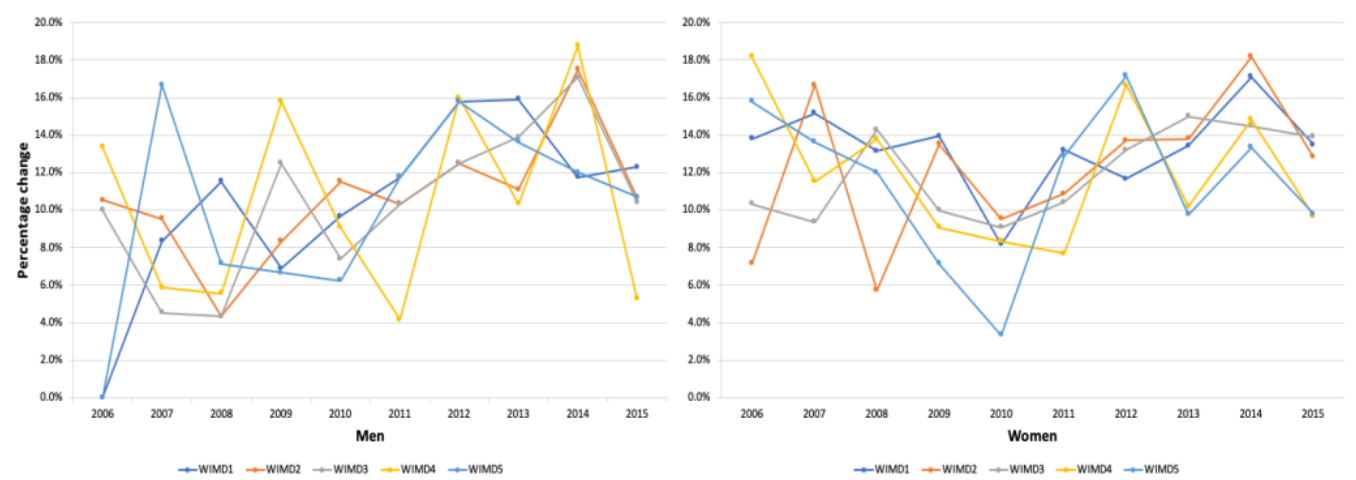

Figure C.11: Annual percentage change in the number of people receiving strong opioid prescriptions by gender and socioeconomic deprivation quintile

(WIMD = Welsh Index of Multiple Deprivation where WIMD1 = most deprived and WIMD5 = least deprived) 


\section{C.2.5 Gender differences in the number of prescriptions by deprivation}

Table C.26: Comparison of numbers of prescriptions for all opioid medicines by Welsh Index of Multiple Deprivation quintiles and gender across Wales between 2005 and 2015

\begin{tabular}{|c|c|c|c|c|c|c|c|c|c|c|}
\hline & \multicolumn{10}{|c|}{ Number of prescriptions per 1000 population } \\
\hline & \multicolumn{2}{|c|}{ WIMD1 } & \multicolumn{2}{|c|}{ WIMD2 } & \multicolumn{2}{|c|}{ WIMD3 } & \multicolumn{2}{|c|}{ WIMD4 } & \multicolumn{2}{|c|}{ WIMD5 } \\
\hline & Male & Female & Male & Female & Male & Female & Male & Female & Male & Female \\
\hline 2005 & 180,485 & 287,724 & 137,739 & 227,912 & 115,711 & 199,770 & 77,661 & 136,147 & 66,543 & 124,585 \\
\hline 2006 & 186,937 & 292,458 & 143,091 & 234,154 & 120,974 & 205,263 & 80,922 & 139,966 & 69,375 & 128,120 \\
\hline 2007 & 201,146 & 310,791 & 154,878 & 249,936 & 128,994 & 219,972 & 90,455 & 152,428 & 76,600 & 138,080 \\
\hline 2008 & 215,423 & 331,450 & 164,964 & 263,661 & 137,332 & 232,622 & 98,359 & 164,674 & 83,167 & 148,865 \\
\hline 2009 & 227,674 & 345,673 & 175,166 & 275,297 & 146,322 & 243,126 & 104,464 & 174,929 & 88,422 & 155,758 \\
\hline 2010 & 239,540 & 359,569 & 187,131 & 289,931 & 155,761 & 254,596 & 111,290 & 184,085 & 92,972 & 163,582 \\
\hline 2011 & 252,648 & 377,194 & 199,022 & 306,306 & 167,704 & 267,865 & 118,837 & 192,761 & 98,056 & 172,402 \\
\hline 2012 & 265,923 & 389,762 & 207,338 & 319,461 & 176,429 & 277,379 & 123,102 & 200,040 & 102,365 & 178,213 \\
\hline 2013 & 271,650 & 398,330 & 209,655 & 322,033 & 179,137 & 282,287 & 124,535 & 204,302 & 105,626 & 180,409 \\
\hline 2014 & 273,306 & 404,643 & 211,997 & 326,453 & 183,512 & 287,991 & 128,256 & 207,975 & 106,618 & 181,818 \\
\hline 2015 & 272,779 & 408,607 & 214,563 & 327,300 & 184,211 & 289,400 & 127,415 & 208,650 & 107,876 & 182,743 \\
\hline $\begin{array}{r}\text { Change rate (\%) } \\
2005-2015\end{array}$ & 51.1 & 42.0 & 55.8 & 43.6 & 59.2 & 44.9 & 64.1 & 53.3 & 62.1 & 46.7 \\
\hline
\end{tabular}

WIMD1 = most socioeconomically deprived, WIMD5 = least socioeconomically deprived

Table C.27: Comparison of quintile-population adjusted numbers of prescriptions for all opioid medicines by Welsh Index of Multiple Deprivation quintiles and gender across Wales between 2005 and 2015

Number of prescriptions per 1000 population

\begin{tabular}{|c|c|c|c|c|c|c|c|c|c|c|}
\hline & \multicolumn{2}{|c|}{ WIMD1 } & \multicolumn{2}{|c|}{ WIMD2 } & \multicolumn{2}{|c|}{ WIMD3 } & \multicolumn{2}{|c|}{ WIMD4 } & \multicolumn{2}{|c|}{ WIMD5 } \\
\hline & Male & Female & Male & Female & Male & Female & Male & Female & Male & Female \\
\hline 2005 & 380.5 & 606.6 & 290.4 & 480.5 & 244.0 & 421.2 & 163.7 & 287.0 & 140.3 & 262.7 \\
\hline 2006 & 394.1 & 616.6 & 301.7 & 493.7 & 255.0 & 432.8 & 170.6 & 295.1 & 146.3 & 270.1 \\
\hline 2007 & 424.1 & 655.2 & 326.5 & 526.9 & 272.0 & 463.8 & 190.7 & 321.4 & 161.5 & 291.1 \\
\hline 2008 & 454.2 & 698.8 & 347.8 & 555.9 & 289.5 & 490.4 & 207.4 & 347.2 & 175.3 & 313.8 \\
\hline 2009 & 480.0 & 728.8 & 369.3 & 580.4 & 308.5 & 512.6 & 220.2 & 368.8 & 186.4 & 328.4 \\
\hline 2010 & 505.0 & 758.1 & 394.5 & 611.3 & 328.4 & 536.8 & 234.6 & 388.1 & 196.0 & 344.9 \\
\hline 2011 & 532.7 & 795.2 & 419.6 & 645.8 & 353.6 & 564.7 & 250.5 & 406.4 & 206.7 & 363.5 \\
\hline
\end{tabular}




\begin{tabular}{|c|c|c|c|c|c|c|c|c|c|c|}
\hline 2012 & 560.6 & 821.7 & 437.1 & 673.5 & 372.0 & 584.8 & 259.5 & 421.7 & 215.8 & 375.7 \\
\hline 2013 & 572.7 & 839.8 & 442.0 & 678.9 & 377.7 & 595.1 & 262.6 & 430.7 & 222.7 & 380.4 \\
\hline 2014 & 576.2 & 853.1 & 446.9 & 688.3 & 386.9 & 607.2 & 270.4 & 438.5 & 224.8 & 383.3 \\
\hline 2015 & 575.1 & 861.5 & 452.4 & 690.0 & 388.4 & 610.1 & 268.6 & 439.9 & 227.4 & 385.3 \\
\hline Change rate (\%)2005-2015 & 51.1 & 42.0 & 55.8 & 43.6 & 59.2 & 44.9 & 64.1 & 53.3 & 62.1 & 46.7 \\
\hline $\begin{array}{r}\text { Mann-Whitney } \\
\text { (between genders in same } \\
\text { quintile) }\end{array}$ & \multicolumn{2}{|c|}{$\begin{array}{c}\mathrm{p}<.001^{*}, \mathrm{n}^{2}=0.717 \\
\mathrm{~d}_{\text {Cohen }}=3.187\end{array}$} & \multicolumn{2}{|c|}{$\begin{array}{c}p<.001^{*}, \eta^{2}=0.717 \\
d_{\text {Cohen }}=3.187\end{array}$} & \multicolumn{2}{|c|}{$\begin{array}{c}p<.001^{*}, \eta^{2}=0.717 \\
d_{\text {Cohen }}=3.187\end{array}$} & \multicolumn{2}{|c|}{$\begin{array}{c}\mathrm{p}<.001^{*}, \mathrm{n}^{2}=0.717 \\
\mathrm{~d}_{\text {Cohen }}=3.187\end{array}$} & \multicolumn{2}{|c|}{$\begin{array}{c}\mathrm{p}<.001^{*}, \mathrm{n}^{2}=0.717 \\
\mathrm{~d}_{\text {Cohen }}=3.187\end{array}$} \\
\hline
\end{tabular}

${ }^{*} \mathrm{p}<0.05$ = statistically significant WIMD1 = most socioeconomically deprived, WIMD5 = least socio-economically deprived

Table C.28: Dunn's pairwise comparison and Bonferroni post-hoc analysis of difference between the number of all opioid prescriptions per 1000 population, issued to men in the 5 different areas of deprivation as defined by the Welsh Index of Multiple Deprivation (WIMD)

\begin{tabular}{ccccc}
\multicolumn{5}{c}{ Welsh Index of Multiple Deprivation } \\
\hline $\mathbf{2}$ & 1 & 2 & 3 & 4 \\
\hline $\mathbf{3}$ & .999 & & & \\
\hline $\mathbf{4}$ & $<.001^{*}$ & $>.999$ & & \\
\hline $\mathbf{5}$ & $<.001^{*}$ & $.019^{*}$ & .391 & $>.999$ \\
\hline
\end{tabular}

$* \mathrm{p}<0.05=$ statistically significant WIMD1=most deprived, WIMD5=least deprived

Table C.29: Dunn's pairwise comparison and Bonferroni post-hoc analysis of difference between the number of all opioid prescriptions per 1000 population, issued to women in the 5 different areas of deprivation as defined by the Welsh Index of Multiple Deprivation (WIMD)

\begin{tabular}{|c|c|c|c|c|}
\hline \multicolumn{5}{|c|}{ Welsh Index of Multiple Deprivation } \\
\hline & 1 & 2 & 3 & 4 \\
\hline 2 & 910 & & & \\
\hline 3 & 128 & $>.999$ & & \\
\hline 4 & $<.001^{*}$ & $.017^{*}$ & .192 & \\
\hline 5 & $<.001^{*}$ & $<.001^{*}$ & $.005 *$ & $>.995$ \\
\hline
\end{tabular}

$* \mathrm{p}<0.05=$ statistically significant. WIMD1=most deprived, WIMD5=least deprived

\section{C.2.5.1 Weak opioids}

Table C.30: Annual weak opioid prescriptions issued per socioeconomic deprivation quintile and gender

$$
\text { Year }
$$

Men

WIMD4

WIMD5

WIMD1

WIMD2




\begin{tabular}{|c|c|c|c|c|c|c|c|c|c|c|}
\hline 2005 & 169330 & 128356 & 107158 & 71727 & 62262 & 275141 & 216392 & 188890 & 128218 & 118093 \\
\hline 2006 & 173510 & 132820 & 110733 & 74198 & 64477 & 277034 & 220576 & 191459 & 129880 & 119446 \\
\hline 2007 & 185700 & 143001 & 117561 & 82139 & 70785 & 292184 & 233691 & 204312 & 140430 & 127680 \\
\hline 2008 & 197585 & 151315 & 125221 & 87979 & 76615 & 309039 & 245037 & 214236 & 150029 & 136286 \\
\hline 2009 & 208274 & 159860 & 132766 & 93001 & 81018 & 319300 & 254079 & 221445 & 157353 & 142207 \\
\hline 2010 & 217625 & 169633 & 139628 & 97839 & 84848 & 328952 & 264674 & 229675 & 163647 & 147256 \\
\hline 2011 & 227459 & 178409 & 147587 & 103679 & 88828 & 340194 & 276836 & 238865 & 169579 & 153352 \\
\hline 2012 & 235539 & 183803 & 151927 & 106026 & 91338 & 347253 & 285118 & 243476 & 174157 & 156808 \\
\hline 2013 & 237391 & 183623 & 151611 & 106719 & 91840 & 348200 & 282230 & 243262 & 174906 & 156673 \\
\hline 2014 & 235446 & 181973 & 154159 & 108129 & 92517 & 347846 & 280828 & 244752 & 176059 & 155354 \\
\hline 2015 & 229046 & 180077 & 151425 & 105933 & 93037 & 342715 & 275523 & 241885 & 173897 & 153728 \\
\hline Total & 2316905 & 1792870 & 1489776 & 1037369 & 897565 & 3527858 & 2834984 & 2462257 & 1738155 & 1566883 \\
\hline
\end{tabular}

WIMD - Welsh Index of Multiple Deprivation, WIMD1=most deprived, WIMD5=least deprived

Table C.31: Comparison of quintile-population adjusted numbers of prescriptions for weak opioid medicines by Welsh Index of Multiple Deprivation quintiles and gender across Wales between 2005 and 2015

\begin{tabular}{|c|c|c|c|c|c|c|c|c|c|c|}
\hline \multirow{3}{*}{ Year } & \multicolumn{10}{|c|}{ Number of prescriptions per 1000 population } \\
\hline & \multicolumn{2}{|c|}{ WIMD1 } & \multicolumn{2}{|c|}{ WIMD2 } & \multicolumn{2}{|c|}{ WIMD3 } & \multicolumn{2}{|c|}{ WIMD4 } & \multicolumn{2}{|c|}{ WIMD5 } \\
\hline & Male & Female & Male & Female & Male & Female & Male & Female & Male & Female \\
\hline 2005 & 357.0 & 580.1 & 270.6 & 456.2 & 225.9 & 398.2 & 151.2 & 270.3 & 131.3 & 249.0 \\
\hline 2006 & 365.8 & 584.1 & 280.0 & 465.0 & 233.5 & 403.6 & 156.4 & 273.8 & 135.9 & 251.8 \\
\hline 2007 & 391.5 & 616.0 & 301.5 & 492.7 & 247.9 & 430.7 & 173.2 & 296.1 & 149.2 & 269.2 \\
\hline 2008 & 416.6 & 651.5 & 319.0 & 516.6 & 264.0 & 451.7 & 185.5 & 316.3 & 161.5 & 287.3 \\
\hline 2009 & 439.1 & 673.2 & 337.0 & 535.7 & 279.9 & 466.9 & 196.1 & 331.7 & 170.8 & 299.8 \\
\hline 2010 & 458.8 & 693.5 & 357.6 & 558.0 & 294.4 & 484.2 & 206.3 & 345.0 & 178.9 & 310.5 \\
\hline 2011 & 479.5 & 717.2 & 376.1 & 583.6 & 311.2 & 503.6 & 218.6 & 357.5 & 187.3 & 323.3 \\
\hline 2012 & 496.6 & 732.1 & 387.5 & 601.1 & 320.3 & 513.3 & 223.5 & 367.2 & 192.6 & 330.6 \\
\hline 2013 & 500.5 & 734.1 & 387.1 & 595.0 & 319.6 & 512.9 & 225.0 & 368.7 & 193.6 & 330.3 \\
\hline 2014 & 496.4 & 733.4 & 383.6 & 592.1 & 325.0 & 516.0 & 228.0 & 371.2 & 195.1 & 327.5 \\
\hline 2015 & 482.9 & 722.5 & 379.7 & 580.9 & 319.2 & 510.0 & 223.3 & 366.6 & 196.1 & 324.1 \\
\hline Change rate (\%) & 35.3 & 24.6 & 40.3 & 27.3 & 41.3 & 28.1 & 47.7 & 35.6 & 49.4 & 30.2 \\
\hline \multicolumn{11}{|l|}{$2005-2015$} \\
\hline $\begin{array}{r}\text { Mann-Whitney } \\
\text { (between genders in } \\
\text { same quintile) }\end{array}$ & \multicolumn{2}{|c|}{$\begin{array}{c}\mathrm{p}<.001^{*}, \mathrm{\eta}^{2}=0.717 \\
\mathrm{~d}_{\text {Cohen }}=3.187\end{array}$} & \multicolumn{2}{|c|}{$\begin{array}{c}\mathrm{p}<.001^{*}, \mathrm{\eta}^{2}=0.717 \\
\mathrm{~d}_{\text {Cohen }}=3.187\end{array}$} & \multicolumn{2}{|c|}{$\begin{array}{c}\mathrm{p}<.001^{*}, \mathrm{n}^{2}=0.717 \\
\mathrm{~d}_{\text {Cohen }}=3.187\end{array}$} & \multicolumn{2}{|c|}{$\begin{array}{c}\mathrm{p}<.001^{*}, \mathrm{\eta}^{2}=0.717 \\
\mathrm{~d}_{\text {Cohen }}=3.187\end{array}$} & \multicolumn{2}{|c|}{$\begin{array}{c}\mathrm{p}<.001^{*}, \mathrm{n}^{2}=0.717 \\
\mathrm{~d}_{\text {Cohen }}=3.187\end{array}$} \\
\hline
\end{tabular}


${ }^{*} p<0.05$ = statistically significant. WIMD1 = most socioeconomically deprived, WIMD5 = least socioeconomically deprived

Table C.32: Dunn's pairwise comparison and Bonferroni post-hoc analysis of difference between the number of weak opioid prescriptions per 1000 population, issued to men in the 5 different areas of deprivation as defined by the Welsh Index of Multiple Deprivation (WIMD)

\begin{tabular}{ccccc}
\hline \multicolumn{5}{c}{ Welsh Index of Multiple Deprivation } \\
\hline $\mathbf{2}$ & 1 & 2 & 3 & 4 \\
\hline $\mathbf{3}$ & .910 & & & \\
\hline $\mathbf{4}$ & .061 & $>.999$ & & \\
\hline $\mathbf{5}$ & $<.001^{*}$ & $.012^{*}$ & .291 & $>.999$ \\
\hline
\end{tabular}

${ }^{*} \mathrm{p}<0.05$ = statistically significant. WIMD1=most deprived, WIMD5=least deprived

Table C.33: Dunn's pairwise comparison and Bonferroni post-hoc analysis of difference between the number of weak opioid prescriptions per 1000 population, issued to women in the 5 different areas of deprivation as defined by the Welsh Index of Multiple Deprivation (WIMD)

\begin{tabular}{ccccc}
\hline \multicolumn{5}{c}{ Welsh Index of Multiple Deprivation } \\
\hline $\mathbf{2}$ & 1 & 2 & 3 & 4 \\
\hline $\mathbf{3}$ & .553 & & & \\
\hline $\mathbf{4}$ & $<.046^{*}$ & $>.999$ & & \\
\hline $\mathbf{5}$ & $<.001^{*}$ & $.020^{*}$ & .301 & $>.999$ \\
\hline
\end{tabular}

$* \mathrm{p}<0.05$ = statistically significant. WIMD1=most deprived, WIMD5=least deprived

\section{C.2.5.2 Strong opioids}

Table C.34: Annual strong opioid prescriptions issued per deprivation quintile and gender

\begin{tabular}{|c|c|c|c|c|c|c|c|c|c|c|}
\hline \multirow[t]{2}{*}{ Year } & \multicolumn{5}{|c|}{ Men } & \multicolumn{5}{|c|}{ Women } \\
\hline & WIMD1 & WIMD2 & WIMD3 & WIMD4 & WIMD5 & WIMD1 & WIMD2 & WIMD3 & WIMD4 & WIMD5 \\
\hline 2005 & 11,155 & 9,383 & 8,553 & 5,934 & 4,281 & 12,583 & 11,520 & 10,880 & 7,929 & 6,492 \\
\hline 2006 & 13,427 & 10,271 & 10,241 & 6,724 & 4,898 & 15,424 & 13,578 & 13,804 & 10,086 & 8,674 \\
\hline 2007 & 15,446 & 11,877 & 11,433 & 8,316 & 5,815 & 18,607 & 16,245 & 15,660 & 11,998 & 10,400 \\
\hline 2008 & 17,838 & 13,649 & 12,111 & 10,380 & 6,552 & 22,411 & 18,624 & 18,386 & 14,645 & 12,579 \\
\hline 2009 & 19,400 & 15,306 & 13,556 & 11,463 & 7,404 & 26,373 & 21,218 & 21,681 & 17,576 & 13,551 \\
\hline 2010 & 21,915 & 17,498 & 16,133 & 13,451 & 8,124 & 30,617 & 25,257 & 24,921 & 20,438 & 16,326 \\
\hline 2011 & 25,189 & 20,613 & 20,117 & 15,158 & 9,228 & 37,000 & 29,470 & 29,000 & 23,182 & 19,050 \\
\hline
\end{tabular}




\begin{tabular}{|c|c|c|c|c|c|c|c|c|c|c|}
\hline 2012 & 30,384 & 23,535 & 24,502 & 17,076 & 11,027 & 42,509 & 34,343 & 33,903 & 25,883 & 21,405 \\
\hline 2013 & 34,259 & 26,032 & 27,526 & 17,816 & 13,786 & 50,130 & 39,803 & 39,025 & 29,396 & 23,736 \\
\hline 2014 & 37,860 & 30,024 & 29,353 & 20,127 & 14,101 & 56,797 & 45,625 & 43,239 & 31,916 & 26,464 \\
\hline 2015 & 43,733 & 34,486 & 32,786 & 21,482 & 14,839 & 65,892 & 51,777 & 47,515 & 34,753 & 29,015 \\
\hline Total & 270,606 & 212,674 & 206,311 & 147,927 & 100,055 & 378,343 & 307,460 & 298,014 & 227,802 & 187,692 \\
\hline
\end{tabular}

WIMD - Welsh Index of Multiple Deprivation, WIMD1=most deprived, WIMD5=least deprived

Table C.35: Comparison of quintile-population adjusted numbers of prescriptions for strong opioid medicines by Welsh Index of Multiple Deprivation quintiles and gender across Wales between 2005 and 2015

\begin{tabular}{|c|c|c|c|c|c|c|c|c|c|c|}
\hline & \multicolumn{10}{|c|}{ Number of prescriptions per 1000 population } \\
\hline & \multicolumn{2}{|c|}{ WIMD1 } & \multicolumn{2}{|c|}{ WIMD2 } & \multicolumn{2}{|c|}{ WIMD3 } & \multicolumn{2}{|c|}{ WIMD4 } & \multicolumn{2}{|c|}{ WIMD5 } \\
\hline & Male & Female & Male & Female & Male & Female & Male & Female & Male & Female \\
\hline 2005 & 23.5 & 26.5 & 19.8 & 24.3 & 18.0 & 22.9 & 12.5 & 16.7 & 9.0 & 13.7 \\
\hline 2006 & 28.3 & 32.5 & 21.7 & 28.6 & 21.6 & 29.1 & 14.2 & 21.3 & 10.3 & 18.3 \\
\hline 2007 & 32.6 & 39.2 & 25.0 & 34.2 & 24.1 & 33.0 & 17.5 & 25.3 & 12.3 & 21.9 \\
\hline 2008 & 37.6 & 47.2 & 28.8 & 39.3 & 25.5 & 38.8 & 21.9 & 30.9 & 13.8 & 26.5 \\
\hline 2009 & 40.9 & 55.6 & 32.3 & 44.7 & 28.6 & 45.7 & 24.2 & 37.1 & 15.6 & 28.6 \\
\hline 2010 & 46.2 & 64.5 & 36.9 & 53.2 & 34.0 & 52.5 & 28.4 & 43.1 & 17.1 & 34.4 \\
\hline 2011 & 53.1 & 78.0 & 43.5 & 62.1 & 42.4 & 61.1 & 32.0 & 48.9 & 19.5 & 40.2 \\
\hline 2013 & 72.2 & 105.7 & 54.9 & 83.9 & 58.0 & 82.3 & 37.6 & 62.0 & 29.1 & 50.0 \\
\hline 2014 & 79.8 & 119.7 & 63.3 & 96.2 & 61.9 & 91.2 & 42.4 & 67.3 & 29.7 & 55.8 \\
\hline 2015 & 92.2 & 138.9 & 72.7 & 109.2 & 69.1 & 100.2 & 45.3 & 73.3 & 31.3 & 61.2 \\
\hline $\begin{array}{r}\text { Change rate (\%) } \\
2005-2015\end{array}$ & 292.0 & 423.7 & 267.5 & 349.5 & 283.3 & 336.7 & 262.0 & 338.3 & 246.6 & 346.9 \\
\hline $\begin{array}{l}\text { Mann-Whitney ( } \\
\text { between genders in same } \\
\text { quintile) }\end{array}$ & \multicolumn{2}{|c|}{$\begin{array}{c}\mathrm{p}=.193, \mathrm{n}^{2}=0.082 \\
\mathrm{~d}_{\text {Cohen }}=0.599\end{array}$} & \multicolumn{2}{|c|}{$\begin{array}{c}\mathrm{p}=.116, \mathrm{n}^{2}=0.118 \\
\mathrm{~d}_{\text {Cohen }}=0.73\end{array}$} & \multicolumn{2}{|c|}{$\begin{array}{c}\mathrm{p}=.101, \eta^{2}=0.127 \\
\mathrm{~d}_{\text {Cohen }}=0.764\end{array}$} & \multicolumn{2}{|c|}{$\begin{array}{c}\mathrm{p}=.056, \eta^{2}=0.165 \\
\mathrm{~d}_{\text {Cohen }}=0.889\end{array}$} & \multicolumn{2}{|c|}{$\begin{array}{c}\mathrm{p}<.01^{*}, \mathrm{\eta}^{2}=0.298 \\
\mathrm{~d}_{\text {Cohen }}=1.303\end{array}$} \\
\hline
\end{tabular}

${ }^{*} p<0.05$ = statistically significant. WIMD1 = most socioeconomically deprived, WIMD5 = least socioeconomically deprived 
Table C.36: Dunn's pairwise comparison and Bonferroni post-hoc analysis of difference between the number of strong opioid prescriptions per 1000 population, issued to men in the 5 different areas of deprivation as defined by the Welsh Index of Multiple Deprivation (WIMD)

\begin{tabular}{ccccc}
\multicolumn{5}{c}{ Welsh Index of Multiple Deprivation } \\
\hline $\mathbf{2}$ & $>.999$ & 2 & 3 & 4 \\
\hline $\mathbf{3}$ & $>.999$ & & & \\
\hline $\mathbf{4}$ & .229 & $>.999$ & $>.999$ & \\
\hline $\mathbf{5}$ & $<.001^{*}$ & $>.999$ & $.021^{*}$ & .570 \\
\hline
\end{tabular}

${ }^{*} \mathrm{p}<0.05=$ statistically significant. WIMD1=most deprived, $\mathrm{WIMD5}=$ =east deprived

Table C.37: Dunn's pairwise comparison and Bonferroni post-hoc analysis of difference between the number of strong opioid prescriptions per 1000 population, issued to women in the 5 different areas of deprivation as defined by the Welsh Index of Multiple Deprivation (WIMD)

\begin{tabular}{ccccc}
\hline \multicolumn{5}{c}{ Welsh Index of Multiple Deprivation } \\
\hline $\mathbf{2}$ & $>.999$ & 2 & 3 & 4 \\
\hline $\mathbf{3}$ & $>.999$ & $>.999$ & & \\
\hline $\mathbf{4}$ & .885 & $>.999$ & $>.999$ & \\
\hline $\mathbf{5}$ & $.048^{*}$ & .391 & .291 & $>.999$
\end{tabular}

${ }^{*} \mathrm{p}<0.05$ = statistically significant. WIMD1=most deprived, WIMD5=least deprived 


\section{Appendix $D$ Prescribing trends by drug, oral morphine equivalence}

\section{D.1 Number of people receiving prescriptions, stratified by drug}

Table D.1: Trends in the number of people receiving prescriptions 2005 - 2015, stratified by drug

\begin{tabular}{|c|c|c|c|c|c|c|c|c|}
\hline \multirow{2}{*}{ Year } & \multicolumn{8}{|c|}{ Number of people receiving prescriptions } \\
\hline & Buprenorphine & Codeine & Dihydrocodeine & Fentanyl & Morphine & Oxycodone & Tramadol & Total \\
\hline 2005 & 2421 & 187808 & 76482 & 2377 & 4552 & 1285 & 47452 & 354570 \\
\hline 2006 & 3893 & 202378 & 73947 & 2993 & 4828 & 1461 & 52226 & 350641 \\
\hline 2007 & 5303 & 222913 & 73904 & 3535 & 5557 & 1864 & 56792 & 374383 \\
\hline 2008 & 6766 & 237076 & 71433 & 3889 & 6342 & 2380 & 59393 & 390504 \\
\hline 2009 & 7824 & 245827 & 69187 & 3967 & 6981 & 3301 & 63746 & 403653 \\
\hline 2010 & 8696 & 252226 & 65879 & 4202 & 8092 & 3679 & 67435 & 412776 \\
\hline 2011 & 9262 & 258633 & 62507 & 4234 & 9919 & 3822 & 69840 & 420564 \\
\hline 2012 & 9032 & 261372 & 58319 & 4304 & 12608 & 4090 & 72153 & 424301 \\
\hline 2013 & 8643 & 257740 & 54940 & 4319 & 15756 & 3744 & 69703 & 417424 \\
\hline 2014 & 8421 & 261062 & 50673 & 4254 & 19531 & 3711 & 62621 & 413451 \\
\hline 2015 & 8485 & 260646 & 46827 & 4040 & 22818 & 3989 & 54325 & 404938 \\
\hline $\begin{array}{r}\text { Percentage change } \\
\text { (\%) 2005-2015 }\end{array}$ & 250.5 & 38.8 & -38.8 & 70.0 & 401.3 & 210.4 & 14.5 & 14.2 \\
\hline
\end{tabular}

Table D.2: Trends in the number of people per 1000 population, receiving opioid analgesic prescriptions 2005 - 2015, stratified by drug

\begin{tabular}{|c|c|c|c|c|c|c|c|c|}
\hline \multirow[t]{2}{*}{ Year } & \multicolumn{8}{|c|}{ Number of people per 1000 population } \\
\hline & Buprenorphine & Codeine & Dihydrocodeine & Fentanyl & Morphine & Oxycodone & Tramadol & Other \\
\hline 2005 & 1.0 & 81.1 & 33.0 & 1.0 & 2.0 & 0.6 & 20.5 & 13.9 \\
\hline 2006 & 1.7 & 86.9 & 31.8 & 1.3 & 2.1 & 0.6 & 22.4 & 3.8 \\
\hline 2007 & 2.3 & 95.1 & 31.5 & 1.5 & 2.4 & 0.8 & 24.2 & 1.9 \\
\hline 2008 & 2.9 & 100.5 & 30.3 & 1.6 & 2.7 & 1.0 & 25.2 & 1.4 \\
\hline 2009 & 3.3 & 104.0 & 29.3 & 1.7 & 3.0 & 1.4 & 27.0 & 1.2 \\
\hline 2010 & 3.7 & 106.3 & 27.8 & 1.8 & 3.4 & 1.5 & 28.4 & 1.1 \\
\hline 2011 & 3.9 & 108.4 & 26.2 & 1.8 & 4.2 & 1.6 & 29.3 & 1.0 \\
\hline 2012 & 3.8 & 109.2 & 24.4 & 1.8 & 5.3 & 1.7 & 30.1 & 1.0 \\
\hline 2013 & 3.6 & 107.4 & 22.9 & 1.8 & 6.6 & 1.6 & 29.0 & 1.1 \\
\hline
\end{tabular}




\begin{tabular}{|c|c|c|c|c|c|c|c|c|}
\hline 2014 & 3.5 & 108.4 & 21.0 & 1.8 & 8.1 & 1.5 & 26.0 & 1.3 \\
\hline 2015 & 3.5 & 108.0 & 19.4 & 1.7 & 9.5 & 1.7 & 22.5 & 1.6 \\
\hline $\begin{array}{r}\text { Rate change } \\
\text { (\%)2005-2015 }\end{array}$ & 236.2 & 33.1 & -41.3 & 63.0 & 380.9 & 197.8 & 9.8 & -88.7 \\
\hline
\end{tabular}

\section{D.1.1 Annual number of prescriptions by drug}

Table D.3: Trends in the number of prescriptions issued 2005 - 2015, stratified by drug

\begin{tabular}{|c|c|c|c|c|c|c|c|c|c|}
\hline \multirow[t]{2}{*}{ Year } & \multicolumn{9}{|c|}{ Number of prescriptions } \\
\hline & Buprenorphine & Codeine & Dihydrocodeine & Fentanyl & Morphine & Oxycodone & Tramadol & Other & Total \\
\hline 2005 & 19,059 & 812,403 & 364,488 & 14,462 & 39,175 & 12,623 & 220,149 & 131,058 & $1,613,417$ \\
\hline 2006 & 27,139 & 888,403 & 361,947 & 20,932 & 44,987 & 15,738 & 257,652 & 39,534 & $1,656,332$ \\
\hline 2007 & 36,905 & 973,514 & 362,142 & 27,539 & 53,259 & 19,734 & 287,450 & 22,557 & $1,783,100$ \\
\hline 2008 & 47,808 & $1,050,084$ & 354,498 & 33,249 & 62,808 & 26,940 & 312,421 & 16,543 & $1,904,351$ \\
\hline 2009 & 58,591 & $1,100,773$ & 341,850 & 37,522 & 70,967 & 36,036 & 341,727 & 15,085 & $2,002,551$ \\
\hline 2010 & 70,809 & $1,152,691$ & 330,325 & 41,749 & 84,417 & 44,492 & 369,896 & 14,193 & $2,108,572$ \\
\hline 2011 & 79,097 & $1,207,202$ & 322,668 & 44,561 & 106,043 & 53,938 & 400,437 & 13,091 & $2,227,037$ \\
\hline 2012 & 82,179 & $1,245,621$ & 307,635 & 46,852 & 135,234 & 60,719 & 427,030 & 13137 & $2,318,407$ \\
\hline 2013 & 8,1934 & $1,252,940$ & 293,774 & 48,274 & 173,286 & 60,602 & 434,130 & 13,766 & $2,358,706$ \\
\hline 2014 & 83,036 & $1,286,777$ & 281,823 & 46,723 & 211,894 & $59,, 524$ & 414,720 & 14,221 & $2,398,718$ \\
\hline 2015 & 84,200 & $1,311,224$ & 268,230 & 44,221 & 251,467 & 62,036 & 377,323 & 16,673 & $2,415,374$ \\
\hline Percentage change & $341.8 \%$ & $61.4 \%$ & $-26.4 \%$ & $205.8 \%$ & $541.9 \%$ & $391.5 \%$ & $71.4 \%$ & $-87.3 \%$ & $49.7 \%$ \\
\hline
\end{tabular}

\section{D.1.2 Annual number of prescriptions per 1000 population by drug}

Table D.4: Trends in the number of opioid prescriptions per 1000 population 2005 - 2015, stratified by drug

\begin{tabular}{|c|c|c|c|c|c|c|c|c|c|}
\hline \multirow[t]{2}{*}{ Year } & \multicolumn{9}{|c|}{ Prescriptions per 1000 population } \\
\hline & Buprenorphine & Codeine & Dihydrocodeine & Fentanyl & Morphine & Oxycodone & Tramadol & Other & Total \\
\hline 2005 & 8.2 & 350.9 & 157.4 & 6.2 & 16.9 & 5.5 & 95.1 & 56.6 & 696.8 \\
\hline 2006 & 11.7 & 381.6 & 155.5 & 9.0 & 19.3 & 6.8 & 110.7 & 17.0 & 711.4 \\
\hline 2007 & 15.7 & 415.3 & 154.5 & 11.7 & 22.7 & 8.4 & 122.6 & 9.6 & 760.6 \\
\hline 2008 & 20.3 & 445.0 & 150.2 & 14.1 & 26.6 & 11.4 & 132.4 & 7.0 & 807.1 \\
\hline
\end{tabular}




\begin{tabular}{|c|c|c|c|c|c|c|c|c|c|}
\hline 2009 & 24.8 & 465.5 & 144.6 & 15.9 & 30.0 & 15.2 & 144.5 & 6.4 & 846.8 \\
\hline 2010 & 29.8 & 485.6 & 139.2 & 17.6 & 35.6 & 18.7 & 155.8 & 6.0 & 888.2 \\
\hline 2011 & 33.2 & 506.2 & 135.3 & 18.7 & 44.5 & 22.6 & 167.9 & 5.5 & 933.8 \\
\hline 2012 & 34.3 & 520.5 & 128.5 & 19.6 & 56.5 & 25.4 & 178.4 & 5.5 & 968.7 \\
\hline 2013 & 34.1 & 522.1 & 122.4 & 20.1 & 72.2 & 25.3 & 180.9 & 5.7 & 982.8 \\
\hline 2014 & 34.5 & 534.4 & 117.0 & 19.4 & 88.0 & 24.7 & 172.2 & 5.9 & 996.2 \\
\hline 2015 & 34.9 & 543.3 & 111.1 & 18.3 & 104.2 & 25.7 & 156.3 & 6.9 & 1000.7 \\
\hline $\begin{array}{r}\text { Percentage change } \\
(\%) 2005-2015\end{array}$ & 323.8 & 54.8 & -29.4 & 193.3 & 515.8 & 371.5 & 64.4 & -87.8 & 43.6 \\
\hline
\end{tabular}

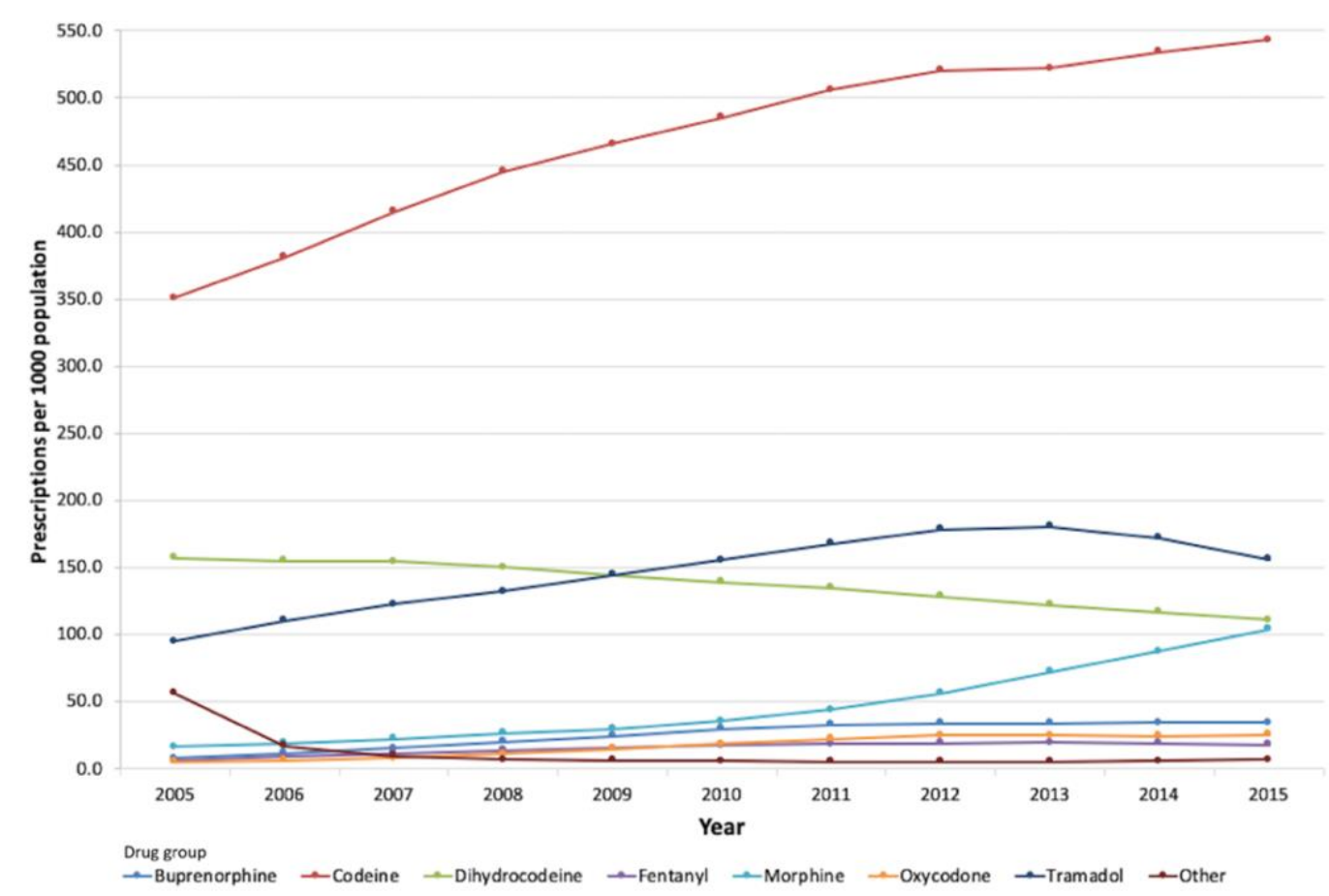

Figure D.1: Trends in number of prescriptions per 1000 population, stratified by drug between 2005 - 2015 
Table D.5: Dunn's pairwise tests with Bonferroni corrections demonstrating relationship between the number of opioid prescriptions, examined by which drug was prescribed

\begin{tabular}{lccccccc}
\hline Drug name & Buprenorphine & Codeine & Dihydrocodeine & Fentanyl & Morphine & Oxycodone & Tramadol \\
\hline Codeine & $.001^{*}$ & & & & & & \\
\hline Dihydrocodeine & .199 & $>.999$ & & & & & \\
\hline Fentanyl & $>.999$ & $<.001^{*}$ & $.001^{*}$ & & & & \\
\hline Morphine & $>.999$ & $.010^{*}$ & .858 & $>.999$ & & & \\
\hline Oxycodone & .657 & $<.001^{*}$ & $<.001^{*}$ & $>.999$ & .145 & & \\
\hline Tramadol & .382 & $>.999$ & $>.999$ & $.003^{*}$ & $>.999$ & $<.001^{*}$ & $.001^{*}$ \\
\hline Other & $>.999$ & $<.001^{*}$ &. $.001^{*}$ & $>.999$ & .781 & $>.999$ & \\
\hline
\end{tabular}

${ }^{*} p<0.05$ is significant

\section{D.1.3 Analysis of prescribing rates for opioid analgesics classed as 'other'}

Table D.6: Trends in the number of prescriptions issued for drugs classified as 'other', 2005-2015

\begin{tabular}{|c|c|c|c|c|c|c|c|c|c|}
\hline \multirow[t]{2}{*}{ Year } & \multicolumn{9}{|c|}{ Number of prescriptions issues } \\
\hline & Dextropropoxyphene & Diamorphine & Dipipanone & Hydromorphone & Meptazinol & Methadone & Pentazocine & Pethidine & Tapentadol \\
\hline 2005 & 120842 & 1004 & 1137 & 186 & 2289 & 528 & 640 & 4432 & 252 \\
\hline 2006 & 28413 & 998 & 1331 & 197 & 2138 & 603 & 626 & 5228 & 1047 \\
\hline 2007 & 12009 & 963 & 1206 & 210 & 1874 & 569 & 569 & 5157 & 2059 \\
\hline 2008 & 6149 & 1162 & 1283 & 166 & 1793 & 594 & 392 & 5004 & 2724 \\
\hline 2009 & 5048 & 1071 & 1203 & 192 & 1700 & 567 & 395 & 4909 & 4904 \\
\hline 2010 & 4424 & 1083 & 1208 & 168 & 1574 & 452 & 437 & 4847 & \\
\hline 2011 & 3228 & 1269 & 945 & 142 & 1391 & 558 & 438 & 4868 & \\
\hline 2012 & 2632 & 1676 & 696 & 222 & 1286 & 532 & 393 & 4653 & \\
\hline 2013 & 2296 & 2124 & 649 & 315 & 1202 & 473 & 337 & 4311 & \\
\hline 2014 & 2204 & 3580 & 503 & 211 & 1105 & 550 & 287 & 3057 & \\
\hline 2015 & 1963 & 4471 & 358 & 141 & 990 & 571 & 235 & 3040 & \\
\hline $\begin{array}{l}\text { Percentage change } \\
\text { (\%) 2005-2015 }\end{array}$ & -98.4 & 345.3 & -68.5 & -24.2 & -56.7 & 8.1 & -63.3 & -31.4 & 1846.0 \\
\hline
\end{tabular}

Table D.7: Trends in the annual number of prescriptions for 'other' opioids 2005 - 2015. Data adjusted to number per 1000 population Prescriptions per 1000 population receiving named opioid prescriptions 


\begin{tabular}{|c|c|c|c|c|c|c|c|c|c|}
\hline & Dextropropoxyphene & Diamorphine & Dipipanone & Hydromorphone & Meptazinol & Methadone & Pentazocine & Pethidine & Tapentadol \\
\hline 2005 & 52.189 & 0.434 & 0.491 & 0.080 & 0.989 & 0.228 & 0.276 & 1.914 & $0<.001$ \\
\hline 2006 & 12.204 & 0.429 & 0.572 & 0.085 & 0.918 & 0.259 & 0.269 & 2.245 & $0<.001$ \\
\hline 2007 & 5.123 & 0.411 & 0.514 & 0.090 & 0.799 & 0.243 & 0.243 & 2.200 & $0<.001$ \\
\hline 2008 & 2.606 & 0.492 & 0.544 & 0.070 & 0.760 & 0.252 & 0.166 & 2.121 & $0<.001$ \\
\hline 2009 & 2.135 & 0.453 & 0.509 & 0.081 & 0.719 & 0.240 & 0.167 & 2.076 & $0<.001$ \\
\hline 2010 & 1.864 & 0.456 & 0.509 & 0.071 & 0.663 & 0.190 & 0.184 & 2.042 & $0<.001$ \\
\hline 2011 & 1.354 & 0.532 & 0.396 & 0.060 & 0.583 & 0.234 & 0.184 & 2.041 & 0.106 \\
\hline 2012 & 1.100 & 0.700 & 0.291 & 0.093 & 0.537 & 0.222 & 0.164 & 1.944 & 0.437 \\
\hline 2013 & 0.957 & 0.885 & 0.270 & 0.131 & 0.501 & 0.197 & 0.140 & 1.796 & 0.858 \\
\hline 2014 & 0.915 & 1.487 & 0.209 & 0.088 & 0.459 & 0.228 & 0.119 & 1.270 & 1.131 \\
\hline 2015 & 0.813 & 1.852 & 0.148 & 0.058 & 0.410 & 0.237 & 0.097 & 1.260 & 2.032 \\
\hline \multicolumn{10}{|l|}{ Percentage change } \\
\hline (\%) 2005-2015 & -98.4 & 327.2 & -69.8 & -27.3 & -58.5 & 3.7 & -64.8 & -34.2 & 1822.8 \\
\hline $\begin{array}{r}\text { Spearman's r, } \\
\text { p value* }\end{array}$ & $<-.999, p<.001^{*}$ & $0.968, p<.001^{*}$ & $\begin{array}{l}-0.818 \\
p<0.01^{*}\end{array}$ & $0.064, p=0.853$ & $\begin{array}{c}<-.999 \\
p<.001 *\end{array}$ & $\begin{array}{l}-0.227 \\
p=0.502\end{array}$ & $-0.873, p<.001 *$ & $\begin{array}{c}-0.733 \\
p<0.05 *\end{array}$ & $\begin{array}{c}\text { Not } \\
\text { calculated }\end{array}$ \\
\hline
\end{tabular}

*p-value $<0.05=$ statistically significant

Table D.8: Dunn's pairwise tests with Bonferroni corrections demonstrating relationship between the number of opioid prescriptions, examined by which drug was prescribed

\begin{tabular}{|c|c|c|c|c|c|c|c|c|}
\hline Drug name & Dextropropoxyphene & Diamorphine & Dipipanone & Hydromorphone & Meptazinol & Methadone & Pentazocine & Pethidine \\
\hline Diamorphine & $>.999$ & & & & & & & \\
\hline Dipipanone & .086 & $>.999$ & & & & & & \\
\hline Hydromorphone & $<.001 *$ & $.003^{*}$ & .106 & & & & & \\
\hline Meptazinol & $>.999$ & $>.999$ & $>.999$ & $.001 *$ & & & & \\
\hline Methadone & $.001 *$ & $>.999$ & $>.999$ & $>.999$ & .587 & & & \\
\hline Pentazocine & $<.001 *$ & .256 & $>.999$ & $>.999$ & .118 & $>.999$ & & \\
\hline Pethidine & $>.999$ & .924 & $.050^{*}$ & $<.001 *$ & $>.999$ & $<.001^{*}$ & $<.001^{*}$ & \\
\hline Tapentadol & $<.001^{*}$ & .442 & $>.999$ & $>.999$ & .202 & $>.999$ & $>.999$ & $<.001 *$ \\
\hline
\end{tabular}

$* p<0.05$ is significant 
There was a significant difference (Kruskal-Wallis $p<.001, \eta^{2}=0.778 . d_{\text {cohen }}=3.75$ ) in the number of prescriptions issued of the different opioids classified as 'other' in this study as determined by a Kruskal-Wallis test. Post-hoc Dunn's pairwise tests, and Bonferroni adjustments demonstrated significantly fewer prescriptions for hydromorphone compared to dextropropoxyphene, diamorphine, meptazinol and pethidine (Table D.8). Other differences were noted in pairwise comparison although given the lower prescribing rates for all these medicines, the clinical significance of these findings is likely to be limited. Six of the nine medicines included in the 'other' group of opioid analgesics were observed to have reductions in prescribing between 2005 and 2015, confirmed by negative Spearman's $r$ results (Table D.7).

Dextropropoxyphene had a $98.4 \%$ (from 52.2 to 0.8 prescriptions per 1000 population) reduction in the number of annual prescriptions issued between 2005 and 2015. In 2005, the dextropropoxyphene prescriptions was more than 650 times greater than the least prescribed opioid, hydromorphone (Figure D.2). In 2015, tapentadol was the most frequently prescribed 'other' opioid accounting for 2 prescriptions per 1000 people, which equated to $0.2 \%$ of the total of all opioid prescriptions examined in the study (Figure D.2).

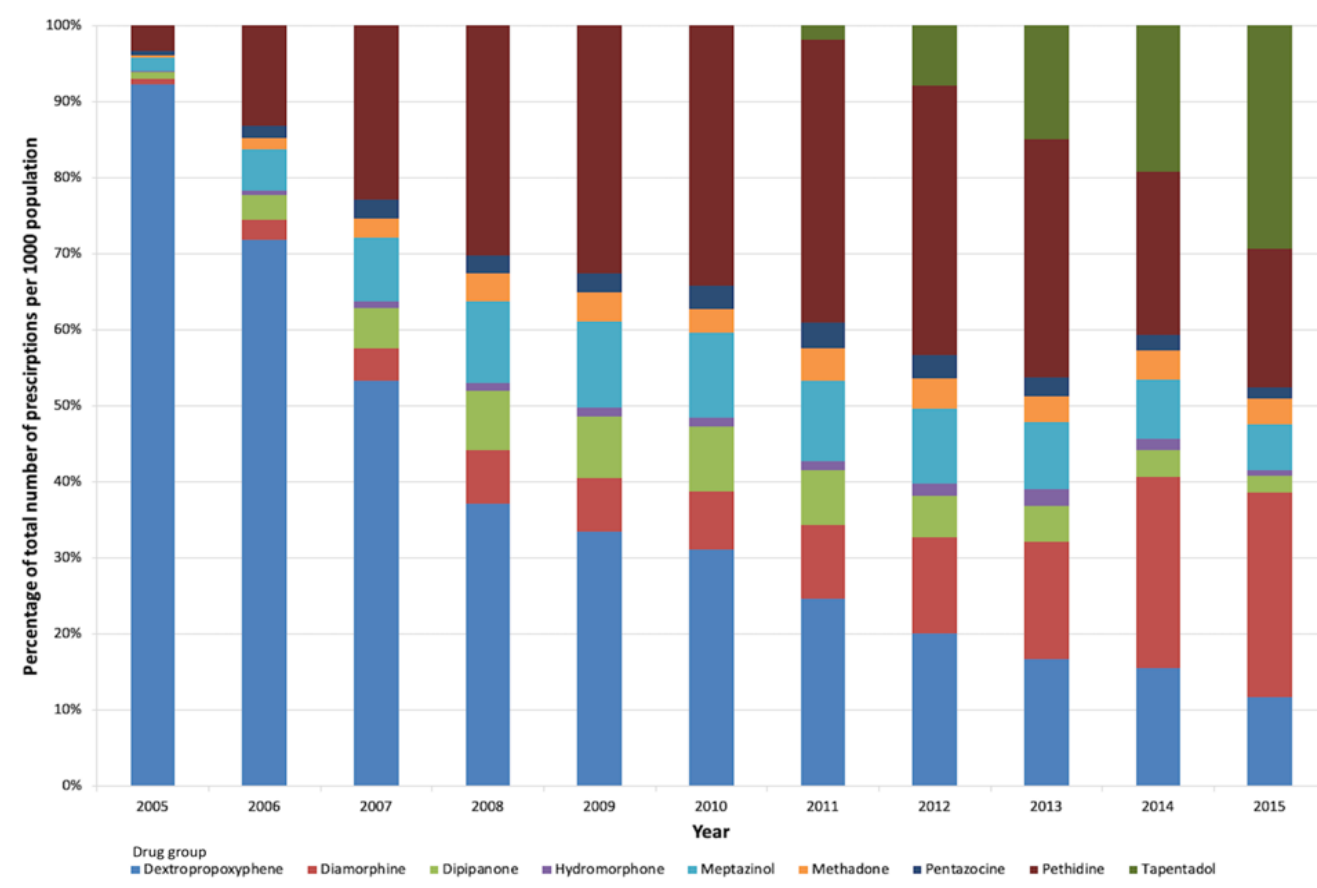

Figure D.2: Trend in the number of prescriptions of named opioids by percentage of the total number of people issued opioid prescriptions categorised as 'other' in the study protocol 


\section{D.2 Oral morphine equivalence}

Table D.9: Daily oral morphine equivalent dose (milligrams) issued on prescription, given as annual totals, and stratified by drug

\begin{tabular}{|c|c|c|c|c|c|c|c|c|c|}
\hline \multirow{2}{*}{ Year } & \multicolumn{9}{|c|}{ Oral morphine equivalent dose (milligrams) prescribed } \\
\hline & Buprenorphine & Codeine & Dihydrocodeine & Fentanyl & Morphine & Oxycodone & Tramadol & Other & Total \\
\hline 2005 & 977,677 & $13,418,363$ & $4,620,630$ & $2,704,640$ & $3,284,610$ & $1,343,350$ & $7,861,525$ & $3,456,828$ & $37,667,623$ \\
\hline 2006 & $1,297,958$ & $14,812,688$ & $4,565,578$ & $3,748,545$ & $3,755,130$ & $1,633,260$ & $9,310,730$ & $1,077,115$ & $40,201,004$ \\
\hline 2007 & $1,528,732$ & $16,472,069$ & $4,584,342$ & $4,676,595$ & $4,513,110$ & $1,960,460$ & $10,546,155$ & 620,756 & $44,902,219$ \\
\hline 2008 & $1,717,634$ & $18,008,755$ & $4,510,564$ & $5,437,230$ & $5,242,780$ & $2,636,820$ & $11,615,165$ & 448,050 & $49,616,998$ \\
\hline 2009 & $2,027,878$ & $19,279,480$ & $4,357,848$ & $6,045,195$ & $5,785,330$ & $3,456,140$ & $12,800,175$ & 410,189 & $54,162,235$ \\
\hline 2010 & $2,339,484$ & $20,508,656$ & $4,221,454$ & $6,677,120$ & $6,667,420$ & $4,224,140$ & $13,938,800$ & 393,050 & $58,970,124$ \\
\hline 2011 & $2,596,580$ & $21,828,568$ & $4,142,234$ & $6,965,715$ & $8,113,590$ & $5,279,440$ & $15,193,800$ & 375,278 & $64,495,205$ \\
\hline 2012 & $2,671,866$ & $22,931,778$ & $3,974,680$ & $7,124,570$ & $10,083,060$ & $6,164,520$ & $16,280,490$ & 425,250 & $69,656,214$ \\
\hline 2014 & $2,683,534$ & $24,327,146$ & $3,723,046$ & $6,982,015$ & $14,775,830$ & $6,047,200$ & $15,789,745$ & 508,316 & $74,836,832$ \\
\hline 2015 & $2,672,408$ & $25,220,520$ & $3,591,820$ & $6,496,270$ & $17,091,470$ & $6,165,400$ & $14,253,325$ & 702,447 & $76,193,660$ \\
\hline \multicolumn{10}{|l|}{ Percentage change } \\
\hline (\%) $2005-2015$ & 182.0 & 86.2 & -20.5 & 141.0 & 417.7 & 368.3 & 81.2 & -79.7 & 102.9 \\
\hline
\end{tabular}

Table D.10: Daily oral morphine equivalent dose (milligrams) issued on prescription, adjusted to 1000population, stratified by drug

\begin{tabular}{|c|c|c|c|c|c|c|c|c|c|}
\hline \multirow[t]{2}{*}{ Year } & \multicolumn{9}{|c|}{ Estimated oral morphine equivalent dose (milligrams) per 1000 population } \\
\hline & Buprenorphine & Codeine & Dihydrocodeine & Fentanyl & Morphine & Oxycodone & Tramadol & Other & Total \\
\hline 2005 & 422 & 5,795 & 1,996 & 1,168 & 1,419 & 580 & 3,395 & 1,493 & 16,268 \\
\hline 2006 & 557 & 6,362 & 1,961 & 1,610 & 1,613 & 701 & 3,999 & 463 & 17,267 \\
\hline 2007 & 652 & 7,026 & 1,956 & 1,995 & 1,925 & 836 & 4,499 & 265 & 19,154 \\
\hline 2008 & 728 & 7,632 & 1,912 & 2,304 & 2,222 & 1,117 & 4,923 & 190 & 21,028 \\
\hline 2009 & 858 & 8,153 & 1,843 & 2,556 & 2,446 & 1,462 & 5,413 & 173 & 22,904 \\
\hline 2010 & 986 & 8,639 & 1,778 & 2,813 & 2,809 & 1,779 & 5,872 & 166 & 24,841 \\
\hline 2011 & 1,089 & 9,153 & 1,737 & 2,921 & 3,402 & 2,214 & 6,371 & 157 & 27,043 \\
\hline 2012 & 1,116 & 9,582 & 1,661 & 2,977 & 4,213 & 2,576 & 6,803 & 178 & 29,106 \\
\hline 2013 & 1,098 & 9,738 & 1,590 & 3,037 & 5,138 & 2,599 & 6,901 & 205 & 30,307 \\
\hline 2014 & 1,115 & 10,104 & 1,546 & 2,900 & 6,137 & 2,512 & 6,558 & 211 & 31,081 \\
\hline 2015 & 1,107 & 10,449 & 1,488 & 2,691 & 7,081 & 2,554 & 5,905 & 291 & 31,568 \\
\hline
\end{tabular}




\begin{tabular}{llllllll}
\hline $\begin{array}{l}\text { Rate change (\%) } \\
\begin{array}{l}2005-2015 \\
* p<0.05 \text { is statistically significant }\end{array}\end{array}$ & 162.2 & 80.3 & -25.4 & 130.4 & 399.2 & 340.3 & 73.9 \\
\hline
\end{tabular}

\section{D.2.1 OMEQ per prescription}

When each drug was stratified by $\mathrm{OMEQ}_{e}$ per prescription issued, there was an overall increase of $35.5 \%$ (from 23 milligrams to 32 milligrams $\mathrm{OMEQ}_{\mathrm{e}}$ per prescription) between 2005 and 2015 (Table D.11). The four drugs classified as 'strong' opioids for this study, all demonstrated reductions in $\mathrm{OMEQ}_{\mathrm{e}}$ per prescription issued over the period examined (Figure D.3). Buprenorphine OMEQ $\mathrm{e}_{\mathrm{e}}$ reduced by $61.7 \%$ and this appeared to be due to a reduction in high-dose transdermal delivery systems (TDS) (patches) and oral preparations (preparations not being used for the treatment of drug misuse). However, the number of prescriptions issued for buprenorphine hugely increased due to the use of low-dose TDS (Table D.3).

Table D.11: Oral morphine equivalent dose per prescription issued for stated drug and subsequent percentage change in OMEQe per prescription between 2005 and 2015 , in addition to the number of prescriptions issued per 1000 population and percentage change over the study period

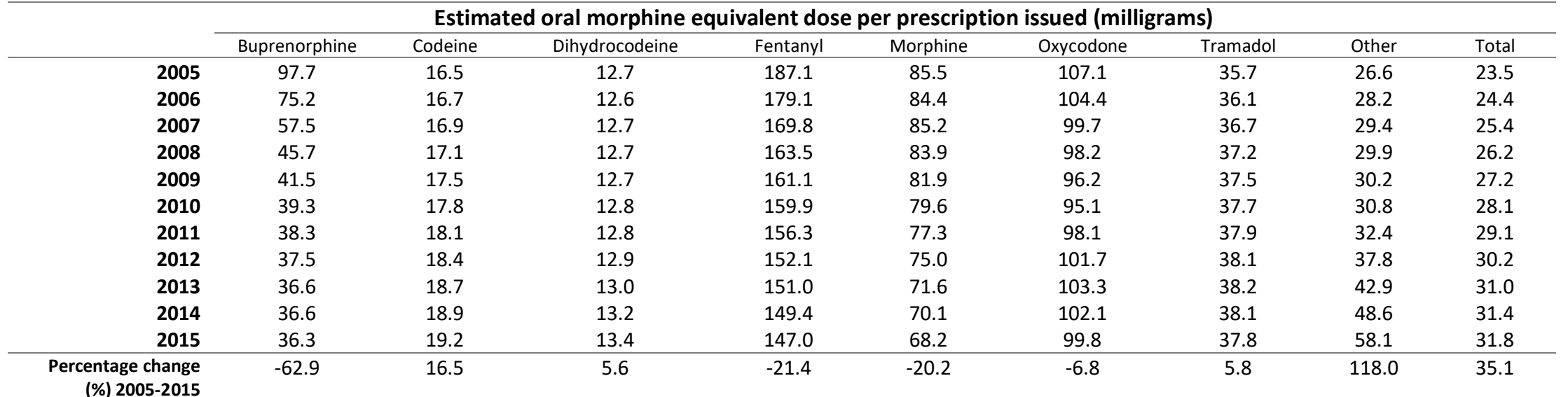


Whilst the number of prescriptions for 'other' opioids reduced, primarily due to the removal of dextropropoxyphene containing medicines from the UK market, an increase in $\mathrm{OMEQ}_{\mathrm{e}}$ was seen towards the end of the study period (Figure D.3). This appears to be a consequence of tapentadol prescribing, a drug introduced in the UK in 2011 and which has a high morphine equivalent dose.

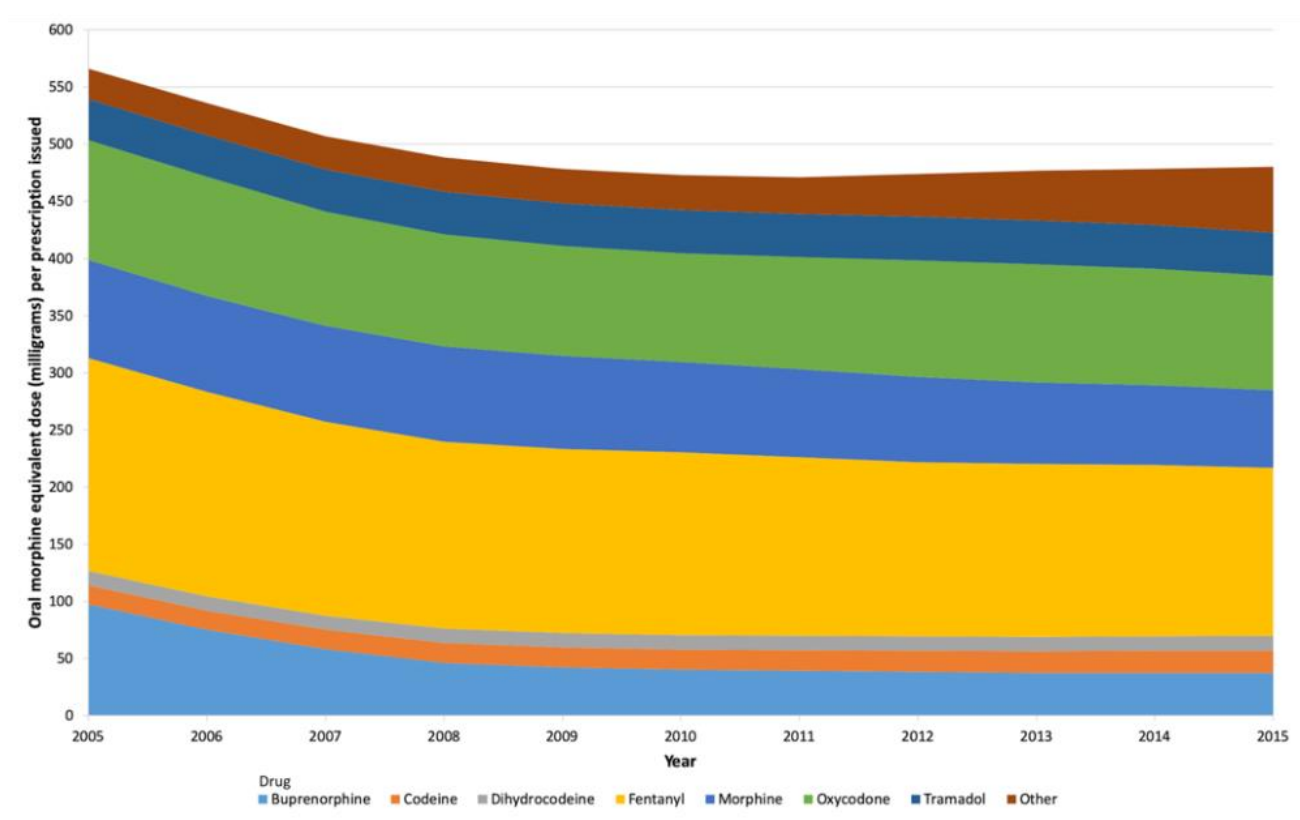

Figure D.3: Trends in the daily oral morphine equivalent dose per prescription issued for stated drug, between $2005-2015$

Fentanyl had the highest daily $\mathrm{OMEQ}_{\mathrm{e}}$ per prescription of all opioids being issued (Table D.11) with oxycodone the next highest (Figure D.3). Whilst the number of morphine prescriptions increased by nearly six times over the 11 years, examined, the $\mathrm{OMEQ}_{\mathrm{e}}$ per prescription reduced over the same time by $20.6 \%$ (from 86 to 68 milligrams per prescription) (Table D.11). The differences in $\mathrm{OMEQ}_{\mathrm{e}}$ per prescription when stratified by the drug prescribed, were significantly different (Kruskal-Wallis $p<.001, \eta^{2}=0.943 . d_{\text {cohen }}=8.10$ ). Significantly lower $\mathrm{OMEQ}_{\mathrm{e}}$ per prescription were observed for codeine compared to the three most prescribed strong opioids, fentanyl, morphine, and oxycodone. This was also the case for dihydrocodeine, which also had lower $\mathrm{OMEQ}_{\mathrm{e}}$ than buprenorphine. Tramadol OMEQe per prescription was significantly lower than seen for fentanyl and oxycodone (.

Table D.12).

Table D.12: Dunn's pairwise tests with Bonferroni corrections demonstrating relationships between the daily oral morphine equivalent doses per prescription, by drug

\begin{tabular}{|c|c|c|c|c|c|c|c|}
\hline Drug & Buprenorphine & Codeine & Dihydrocodeine & Fentanyl & Morphine & Oxycodone & Tramado \\
\hline Codeine & 0.121 & & & & & & \\
\hline Dihydrocodeine & $0.003 *$ & $>.999$ & & & & & \\
\hline Morphine & $>.999$ & $.002 *$ & $<.001^{*}$ & .899 & & & \\
\hline Oxycodone & 0.827 & $<.001^{*}$ & $<.001^{*}$ & $>.999$ & $>.999$ & & \\
\hline
\end{tabular}


${ }^{*} p<0.05$ is statistically significant 


\section{D.2.2 OMEQ for products of greater than $120 \mathrm{mg}$}

Examining the trend in prescriptions for doses of opioids that were equivalent or more than 120mg morphine per day, (based on the strength of the preparation prescribed), demonstrated increases in all 4 strong opioids commonly prescribed (Table D.13). Whilst there were similar percentage increases in the OMEQ for morphine (337.6\%, from 1098 to 4805 milligrams per 1000 population) and oxycodone (321.5\%, from 393to 1659 milligrams per 1000 population) (Table D.13), the total OMEQ for high-dose morphine was nearly three times that of high-dose oxycodone throughout the study period (Figure D.5). There was a large percentage increase $(485.6 \%$, from 25 to 146 milligrams per 1000 population) in the $\mathrm{OMEQ}_{\mathrm{e}}$ of 'other' opioids from 2011 which was due to prescribing of tapentadol.

Table D.13: Trends in annual total oral morphine equivalent dose per 1000 population, stratified by drug and rate change for 2005 - 2015

\begin{tabular}{|c|c|c|c|c|c|}
\hline \multirow[t]{2}{*}{ Year } & \multicolumn{5}{|c|}{ Annual total daily oral morphine equivalent dose (milligrams) per 1000 population } \\
\hline & Buprenorphine & Fentanyl & Morphine & Oxycodone & Other \\
\hline 2005 & 205 & 952 & 1098 & 393 & 25 \\
\hline 2006 & 276 & 1284 & 1236 & 468 & 24 \\
\hline 2007 & 296 & 1559 & 1470 & 534 & 16 \\
\hline 2008 & 264 & 1769 & 1683 & 703 & 14 \\
\hline 2009 & 292 & 1949 & 1822 & 913 & 13 \\
\hline 2010 & 321 & 2150 & 2068 & 1106 & 15 \\
\hline 2011 & 337 & 2202 & 2450 & 1400 & 20 \\
\hline 2012 & 337 & 2209 & 3009 & 1683 & 44 \\
\hline 2013 & 330 & 2240 & 3580 & 1737 & 64 \\
\hline 2014 & 345 & 2133 & 4214 & 1674 & 84 \\
\hline 2015 & 339 & 1952 & 4805 & 1659 & 146 \\
\hline Rate change (\%) & 65.3 & 105.0 & 337.6 & 321.5 & 485.6 \\
\hline 2005-2015 & & & & & \\
\hline
\end{tabular}


Table D.14: Dunn's pairwise tests with Bonferroni corrections demonstrating relationships between the daily oral morphine equivalent doses per prescription for high-dose prescriptions (>120 milligrams OMEQe), by drug

\begin{tabular}{rcccc}
\hline Drug & Buprenorphine & Fentanyl & Morphine & Oxycodone \\
\hline Fentanyl & $.007^{*}$ & & & \\
\hline Morphine & .542 & $<.001^{*}$ & & \\
\hline Oxycodone & .689 & $>.999$ & $.002^{*}$ & \\
\hline Other & .197 & $<.001^{*}$ & $>.999$ & $<.001^{*}$ \\
\hline
\end{tabular}

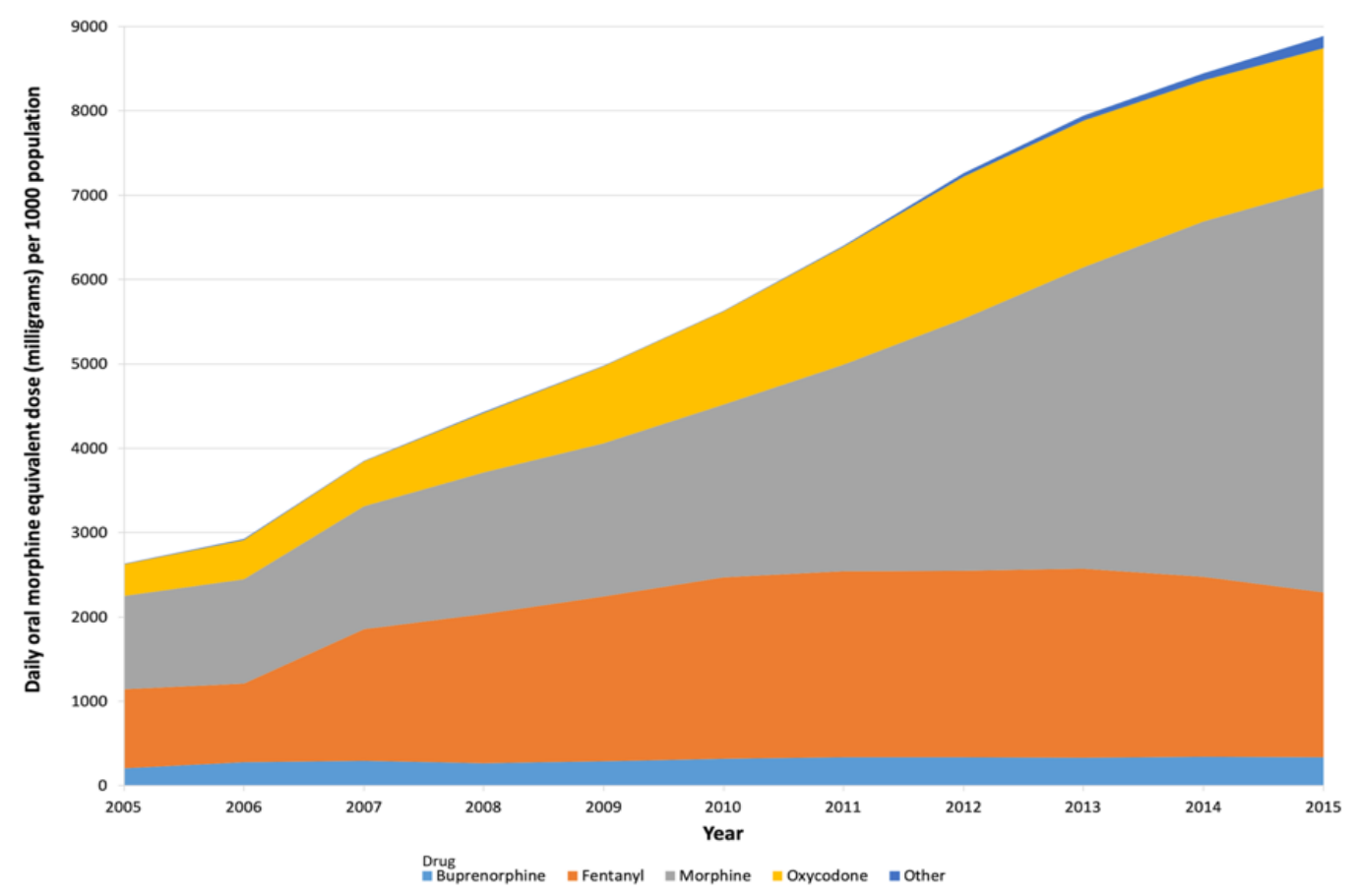

Figure D.D.4: Trends in oral morphine equivalent dose for preparations of 120 milligrams or more per day between $2005-2015$

The differences between all 5 groups of opioids being prescribed at doses of 120 milligram $\mathrm{OMEQ}_{\mathrm{e}}$ per 1000 population or higher, were deemed significantly different overall (Kruskal-Wallis $p<.001, \eta^{2}=0.835$. $d_{\text {cohen }}=4.49$ ). Post-hoc tests confirmed morphine, fentanyl and oxycodone were prescribed at higher $\mathrm{OMEQ}_{\mathrm{e}}$ pe 1000 population than was seen for 'other' opioids. Buprenorphine OMEQ was significantly lower than observed for morphine and fentanyl (Table D.14).

There was little change in the $\mathrm{OMEQ}_{\mathrm{e}}$ per prescription for most products being prescribed at $120 \mathrm{mg} \mathrm{OMEQ}_{\mathrm{e}}$ per day or higher during the study period (Table D.15Whilst morphine $\mathrm{OMEQ}_{\mathrm{e}}$ above 120 milligrams per day increased in total, the $\mathrm{OMEQ}_{\mathrm{e}}$ per prescription reduced by $4.9 \%$ over the 11 years examined (from 134 to 127 milligrams per prescription) (Error! Reference source not found.).

Table D.15: Trends in annual totals for preparations of $120 \mathrm{mg}$ or greater oral morphine equivalent dose in milligrams per prescription, stratified by drug and with rate change for 2005 - 2015

\begin{tabular}{cccccc}
\hline \multirow{2}{*}{ Year } & \multicolumn{4}{c}{ Annual total daily oral morphine equivalent dose (milligrams) per prescription } \\
\cline { 2 - 6 } & Buprenorphine & Fentanyl & Morphine & Oxycodone & Other \\
\hline $\mathbf{2 0 0 5}$ & 136 & 247 & 134 & 186 & 133 \\
$\mathbf{2 0 0 6}$ & 137 & 254 & 132 & 187 & 132 \\
$\mathbf{2 0 0 7}$ & 138 & 256 & 133 & 179 & 137 \\
$\mathbf{2 0 0 8}$ & 139 & 257 & 132 & 181 & 139 \\
\hline
\end{tabular}




\begin{tabular}{rrrrrr}
$\mathbf{2 0 0 9}$ & 139 & 259 & 132 & 178 & 139 \\
$\mathbf{2 0 1 0}$ & 139 & 258 & 132 & 178 & 135 \\
$\mathbf{2 0 1 1}$ & 139 & 256 & 130 & 182 & 134 \\
$\mathbf{2 0 1 2}$ & 138 & 257 & 130 & 184 & 138 \\
$\mathbf{2 0 1 3}$ & 138 & 260 & 129 & 183 & 137 \\
$\mathbf{2 0 1 4}$ & 132 & 259 & 129 & 183 & 141 \\
$\mathbf{2 0 1 5}$ & 139 & 259 & 127 & 184 & 142 \\
\hline Rate change (\%) & 2.5 & 5.0 & -4.9 & -1.6 & 6.5 \\
\hline
\end{tabular}




\section{D.3 Gender differences in $\mathrm{OMEQ}_{\mathrm{e}}$}

\section{D.3.1 Gender differences in total OMEQe}

Table D.16: Trends in Total Annualised Oral Morphine Equivalent Doses (milligrams) issued by gender. p-values calculated using Mann-Whitney tests for each drug

\begin{tabular}{|c|c|c|c|c|c|c|c|c|c|}
\hline \multirow[t]{2}{*}{ Year } & \multicolumn{9}{|c|}{ Oral morphine equivalent dose (milligrams) prescribed } \\
\hline & Buprenorphine & Codeine & Dihydrocodeine & Fentanyl & Morphine & Oxycodone & Tramadol & Other & Total \\
\hline \multicolumn{10}{|l|}{ Men } \\
\hline 2005 & 410156 & 4953639 & 1902306 & 858765 & 1497950 & 636990 & 3071205 & 1155759 & 14486770 \\
\hline 2006 & 553876 & 5526777 & 1862508 & 1252790 & 1672290 & 736160 & 3627890 & 385029 & 15617320 \\
\hline 2007 & 615768 & 6208104 & 1875812 & 1615830 & 1985740 & 831980 & 4139380 & 223934 & 17496548 \\
\hline 2008 & 649328 & 6826073 & 1866868 & 1862430 & 2278280 & 1049740 & 4566795 & 173936 & 19273450 \\
\hline 2009 & 734270 & 7392070 & 1822380 & 1948750 & 2480120 & 1428840 & 5118830 & 163333 & 21088593 \\
\hline 2010 & 831594 & 7926231 & 1758474 & 2162280 & 2842270 & 1796480 & 5674415 & 154301 & 23146045 \\
\hline 2011 & 951130 & 8509375 & 1723444 & 2242295 & 3328020 & 2370360 & 6256045 & 151642 & 25532311 \\
\hline 2012 & 1006858 & 8967529 & 1647382 & 2355650 & 4129530 & 2986980 & 6761750 & 168242 & 28023921 \\
\hline 2013 & 993264 & 9180250 & 1596988 & 2432115 & 5027900 & 2945700 & 6872275 & 179737 & 29228229 \\
\hline 2014 & 1008324 & 9589378 & 1561302 & 2450740 & 5968060 & 2715820 & 6565515 & 197031 & 30056170 \\
\hline 2015 & 1010378 & 9966759 & 1508154 & 2323820 & 6901450 & 2750220 & 5874980 & 300065 & 30635826 \\
\hline $\begin{array}{r}\text { Percentage change } \\
\text { (\%) 2005-2015 }\end{array}$ & 146.3 & 101.2 & -20.7 & 170.6 & 360.7 & 331.8 & 91.3 & -74.0 & 111.5 \\
\hline \multicolumn{10}{|l|}{ Women } \\
\hline 2005 & 567,521 & $8,464,725$ & 2718324 & 1845875 & 1786660 & 706360 & 4790320 & 2301069 & 23180854 \\
\hline 2006 & 744,082 & 9285911 & 2703070 & 2495755 & 2082840 & 897100 & 5682840 & 692086 & 24583684 \\
\hline 2007 & 912,964 & 10263965 & 2708530 & 3060765 & 2527370 & 1128480 & 6406775 & 396822 & 27405671 \\
\hline 2008 & $1,068,306$ & 11182682 & 2643696 & 3574800 & 2964500 & 1587080 & 7048370 & 274114 & 30343548 \\
\hline 2009 & $1,293,608$ & 11887410 & 2535468 & 4096445 & 3305210 & 2027300 & 7681345 & 246856 & 33073642 \\
\hline 2010 & $1,507,890$ & 12582425 & 2462980 & 4514840 & 3825150 & 2427660 & 8264385 & 238749 & 35824079 \\
\hline 2011 & $1,645,450$ & 13319193 & 2418790 & 4723420 & 4785570 & 2909080 & 8937755 & 223636 & 38962894 \\
\hline 2012 & $1,665,008$ & 13964249 & 2327298 & 4768920 & 5953530 & 3177540 & 9518740 & 257008 & 41632293 \\
\hline 2013 & $1,641,454$ & 14191389 & 2219490 & 4857705 & 7303890 & 3291120 & 9689350 & 312101 & 43506499 \\
\hline 2014 & $1,675,210$ & 14737768 & 2161744 & 4531275 & 8807770 & 3331380 & 9224230 & 311285 & 44780662 \\
\hline 2015 & $1,662,030$ & 15253761 & 2083666 & 4172450 & 10190020 & 3415180 & 8378345 & 402382 & 45557834 \\
\hline $\begin{array}{r}\text { Percentage change } \\
\text { (\%) 2005-2015 }\end{array}$ & 192. & 80.2 & -23.3 & 126.0 & 470.3 & $383 . .5$ & 74.9 & -82.5 & 96.5 \\
\hline $\begin{array}{l}\text { Mann-Whitney } p- \\
\text { value*, } \eta^{2}, d_{\text {cohen }}\end{array}$ & $<0.010^{*}, 0.321,1.377$ & $\begin{array}{c}<.001^{*}, 0.561 \\
2.261\end{array}$ & $<.001 *, 0.717,3.187$ & $\begin{array}{c}<.001^{*} \\
0.540,2.168\end{array}$ & $\begin{array}{c}0.217,0.075 \\
0.586\end{array}$ & $\begin{array}{c}0.243,0.067 \\
0.536\end{array}$ & $\begin{array}{c}<0.010^{*} \\
0.406,1.653\end{array}$ & $\begin{array}{c}<0.050^{*} \\
0.261,1.189\end{array}$ & \\
\hline
\end{tabular}


${ }^{*}$ p-value $<0.05=$ statistically significant

Table D.17: Dunn's pairwise tests with Bonferroni corrections demonstrating relationships between the annualised daily oral morphine equivalent doses prescribed to men, by drug

\begin{tabular}{|c|c|c|c|c|c|c|c|}
\hline Drug & Buprenorphine & Codeine & Dihydrocodeine & Fentanyl & Morphine & Oxycodone & Tramadol \\
\hline Codeine & $<.001^{*}$ & & & & & & \\
\hline Dihydrocodeine & $>.999$ & $.003^{*}$ & & & & & \\
\hline Fentanyl & .570 & $.017^{*}$ & $>.999$ & & & & \\
\hline Morphine & $.009 *$ & .877 & $>.999$ & $>.999$ & & & \\
\hline Oxycodone & $>.999$ & $.004^{*}$ & $>.999$ & $>.999$ & $>.999$ & & \\
\hline Tramadol & $<.001^{*}$ & $>.999$ & .065 & .291 & $>.999$ & .093 & \\
\hline Other & $>.999$ & $<.001^{*}$ & .408 & $.027^{*}$ & $<.001^{*}$ & .095 & $<.001^{*}$ \\
\hline
\end{tabular}

*p-value $<.05$ = statistically significant

Table D.18: Dunn's pairwise tests with Bonferroni corrections demonstrating relationships between the annualised daily oral morphine equivalent doses prescribed to women, by drug Drug Buprenorphin

Codeine

Dihydrocodein Fentany

Morphine

Oxycodone

Tramadol

Codeine $\quad<.001^{*}$

\begin{tabular}{|c|c|c|c|c|c|c|c|}
\hline Codeine & $<.001^{*}$ & & & & & & \\
\hline Dihydrocodeine & $>.999$ & $.001^{*}$ & & & & & \\
\hline Fentanyl & .061 & .161 & $>.999$ & & & & \\
\hline Morphine & $.038^{*}$ & .246 & $>.999$ & $>.999$ & & & \\
\hline Oxycodone & $>.999$ & $<.001^{*}$ & $>.999$ & $>.999$ & $>.999$ & & \\
\hline Tramadol & $<.001^{*}$ & $>.999$ & .067 & $>.999$ & $>.999$ & .023 & \\
\hline Other & $>.999$ & $<.001^{*}$ & .212 & $.002 *$ & $.001^{*}$ & .509 & $<.001^{*}$ \\
\hline
\end{tabular}

${ }^{*}$ p-value $<.05$ = statistically significant

\section{D.3.2 Gender differences in OMEQe per 1000 population}

Table D.19: Trends in Total Annualised Oral Morphine Equivalent Doses (milligrams) per 1000 population issued by gender. p-values calculated using Mann-Whitney tests for each drug Year

Oral morphine equivalent dose (milligrams) per 1000 population

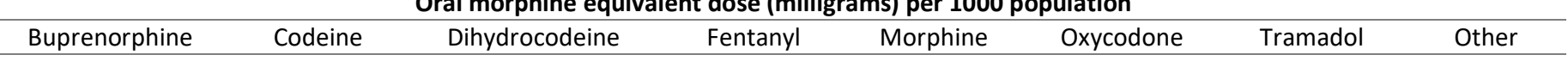




\begin{tabular}{|c|c|c|c|c|c|c|c|c|}
\hline Men & & & & & & & & \\
\hline 2005 & 363 & 4388 & 1685 & 761 & 1327 & 564 & 2720 & 1024 \\
\hline 2006 & 488 & 4869 & 1641 & 1104 & 1473 & 649 & 3196 & 339 \\
\hline 2007 & 538 & 5421 & 1638 & 1411 & 1734 & 727 & 3615 & 196 \\
\hline 2008 & 563 & 5917 & 1618 & 1614 & 1975 & 910 & 3959 & 151 \\
\hline 2009 & 634 & 6385 & 1574 & 1683 & 2142 & 1234 & 4421 & 141 \\
\hline 2010 & 714 & 6810 & 1511 & 1858 & 2442 & 1543 & 4875 & 133 \\
\hline 2011 & 812 & 7266 & 1472 & 1915 & 2842 & 2024 & 5342 & 129 \\
\hline 2012 & 857 & 7629 & 1401 & 2004 & 3513 & 2541 & 5752 & 143 \\
\hline 2013 & 842 & 7781 & 1354 & 2062 & 4262 & 2497 & 5825 & 152 \\
\hline 2014 & 851 & 8095 & 1318 & 2069 & 5038 & 2292 & 5542 & 166 \\
\hline 2015 & 850 & 8389 & 1269 & 1956 & 5809 & 2315 & 4945 & 253 \\
\hline \multicolumn{9}{|l|}{ Rate change (\%) } \\
\hline $2005-2015$ & 134.1 & 91.2 & -24.7 & 157.1 & 337.8 & 310.2 & 81.8 & -75.3 \\
\hline \multicolumn{9}{|l|}{ Women } \\
\hline 2005 & 478 & 7134 & 2291 & 1556 & 1506 & 595 & 4037 & 1939 \\
\hline 2006 & 624 & 7783 & 2266 & 2092 & 1746 & 752 & 4763 & 580 \\
\hline 2007 & 761 & 8559 & 2259 & 2552 & 2108 & 941 & 5343 & 331 \\
\hline 2008 & 886 & 9273 & 2192 & 2964 & 2458 & 1316 & 5845 & 227 \\
\hline 2009 & 1072 & 9849 & 2101 & 3394 & 2738 & 1680 & 6364 & 205 \\
\hline 2010 & 1246 & 10400 & 2036 & 3732 & 3162 & 2007 & 6831 & 197 \\
\hline 2011 & 1356 & 10973 & 1993 & 3892 & 3943 & 2397 & 7364 & 184 \\
\hline 2012 & 1367 & 11468 & 1911 & 3916 & 4889 & 2609 & 7817 & 211 \\
\hline 2013 & 1345 & 11630 & 1819 & 3981 & 5986 & 2697 & 7941 & 256 \\
\hline 2014 & 1370 & 12049 & 1767 & 3705 & 7201 & 2724 & 7542 & 254 \\
\hline 2015 & 1356 & 12447 & 1700 & 3405 & 8315 & 2787 & 6837 & 328 \\
\hline \multicolumn{9}{|l|}{ Rate change (\%) } \\
\hline $2005-2015$ & 183.5 & 74.5 & -25.8 & 118.8 & 452.2 & 368.1 & 69.3 & -83.1 \\
\hline lue (Mann- & $.010^{*}$ & $<.001^{*}$ & $<.001^{*}$ & $<.001^{*}$ & .332 & .270 & $.004^{*}$ & $.019 *$ \\
\hline
\end{tabular}

${ }^{*} \mathrm{p}$-value $<.05=$ statistically significant

Table D.20: Dunn's pairwise tests with Bonferroni corrections demonstrating relationships between the annualised daily oral morphine equivalent doses prescribed to men per 1000 population, by drug

Drug Buprenorphine Codeine Dihydrocodeine Fentanyl Morphine Oxycodone Tramadol




\begin{tabular}{|c|c|c|c|c|c|c|c|}
\hline Codeine & $<.001^{*}$ & & & & & & \\
\hline Dihydrocodeine & $>.999$ & $0.003^{*}$ & & & & & \\
\hline Fentanyl & 0.570 & $0.017^{*}$ & $>.999$ & & & & \\
\hline Morphine & $0.009 *$ & 0.877 & $>.999$ & $>.999$ & & & \\
\hline Oxycodone & $>.999$ & $0.004 *$ & $>.999$ & $>.999$ & $>.999$ & & \\
\hline Tramadol & $<.001 *$ & $>.999$ & 0.065 & 0.291 & $>.999$ & 0.093 & \\
\hline Other & $>.999$ & $<.001 *$ & 0.408 & $0.027^{*}$ & $<.001^{*}$ & 0.095 & $<.001^{*}$ \\
\hline
\end{tabular}

$*_{p}$-value $<.05$ = statistically significant

Table D.21: Dunn's pairwise tests with Bonferroni corrections demonstrating relationships between the annualised daily oral morphine equivalent doses prescribed to women per 1000 population, by drug

\begin{tabular}{|c|c|c|c|c|c|c|c|}
\hline Drug & Buprenorphine & Codeine & Dihydrocodeine & Fentanyl & Morphine & Oxycodone & Tramadol \\
\hline Codeine & $<.001^{*}$ & & & & & & \\
\hline Dihydrocodeine & $>.999$ & $<.001^{*}$ & & & & & \\
\hline Fentanyl & 0.061 & 0.161 & $>.999$ & & & & \\
\hline Morphine & $0.038^{*}$ & 0.246 & $>.999$ & $>.999$ & & & \\
\hline Oxycodone & $>.999$ & $<.001^{*}$ & $>.999$ & $>.999$ & $>.999$ & & \\
\hline Tramadol & $<.001 *$ & $>.999$ & 0.067 & $>.999$ & $>.999$ & 0.023 & \\
\hline Other & $>.999$ & $<.001^{*}$ & 0.212 & $0.002^{*}$ & $.001 *$ & 0.509 & $<.001^{*}$ \\
\hline
\end{tabular}

${ }^{*} p$-value $<.05=$ statistically significant

\section{D.3.3 Gender differences in OMEQe per prescription}

Table D.22: Trends in oral morphine equivalent dose (milligrams) per prescription issued and by drug, stratified by gender. p-values calculated using Mann-Whitney tests

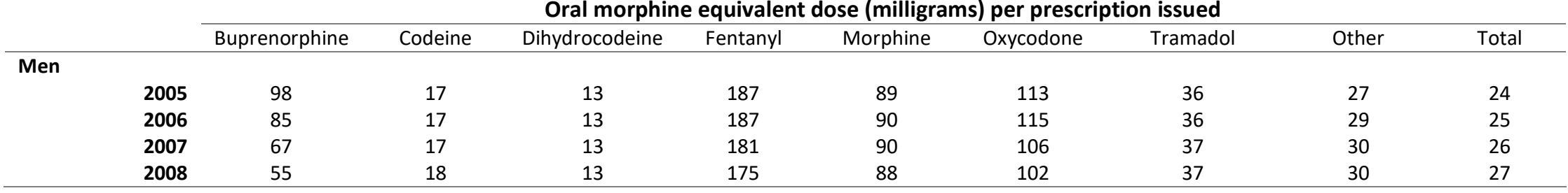




\begin{tabular}{|c|c|c|c|c|c|c|c|c|c|c|}
\hline & 2009 & 50 & 18 & 13 & 174 & 85 & 101 & 37 & 31 & 28 \\
\hline & 2010 & 47 & 18 & 13 & 172 & 84 & 102 & 38 & 31 & 29 \\
\hline & 2011 & 47 & 18 & 13 & 173 & 81 & 106 & 38 & 33 & 30 \\
\hline & 2012 & 46 & 19 & 13 & 169 & 78 & 113 & 38 & 37 & 31 \\
\hline & 2013 & 46 & 19 & 13 & 168 & 75 & 114 & 38 & 42 & 32 \\
\hline & 2014 & 47 & 19 & 14 & 171 & 73 & 112 & 38 & 49 & 32 \\
\hline & 2015 & 46 & 20 & 14 & 169 & 71 & 110 & 38 & 60 & 33 \\
\hline $\begin{array}{l}\text { Percentage cha } \\
\text { (\%) } 2005-2\end{array}$ & $\begin{array}{l}\text { ange } \\
2015\end{array}$ & -53.0 & 14.9 & 4.7 & -9.4 & -20.0 & -2.8 & 6.1 & 124.0 & 34.3 \\
\hline \multicolumn{11}{|c|}{ Women } \\
\hline & 2005 & 97 & 16 & 12 & 187 & 83 & 102 & 36 & 26 & 23 \\
\hline & 2006 & 69 & 16 & 12 & 175 & 80 & 97 & 36 & 28 & 24 \\
\hline & 2007 & 53 & 17 & 12 & 164 & 81 & 95 & 37 & 29 & 25 \\
\hline & 2008 & 42 & 17 & 12 & 158 & 81 & 96 & 37 & 30 & 26 \\
\hline & 2009 & 38 & 17 & 12 & 156 & 79 & 93 & 37 & 30 & 27 \\
\hline & 2010 & 36 & 18 & 12 & 155 & 76 & 91 & 38 & 30 & 28 \\
\hline & 2011 & 35 & 18 & 13 & 149 & 75 & 92 & 38 & 32 & 29 \\
\hline & 2012 & 34 & 18 & 13 & 145 & 73 & 93 & 38 & 38 & 30 \\
\hline & 2013 & 33 & 18 & 13 & 144 & 69 & 95 & 38 & 43 & 30 \\
\hline & 2014 & 32 & 19 & 13 & 140 & 68 & 95 & 38 & 48 & 31 \\
\hline & 2015 & 32 & 19 & 13 & 137 & 66 & 93 & 38 & 56 & 31 \\
\hline $\begin{array}{r}\text { Percentage cha } \\
\text { (\%) 2005-2 }\end{array}$ & $\begin{array}{l}\text { ange } \\
2015\end{array}$ & -67.0 & 17.2 & 6.2 & -26.8 & -19.9 & -9.1 & 5.5 & 113.2 & 35.4 \\
\hline $\begin{array}{l}\text { Mann-Whitney } p \\
\text { value }\end{array}$ & & $.023 *$ & .217 & $.010^{*}$ & $.003^{*}$ & $.040^{*}$ & $<.001$ & .797 & .652 & .365 \\
\hline
\end{tabular}

${ }^{*} p$-value $<.05$ = statistically significant

Table D.23: Dunn's pairwise tests with Bonferroni corrections demonstrating relationships between the annualised daily oral morphine equivalent doses prescribed per prescription to men, by drug

\begin{tabular}{|c|c|c|c|c|c|c|c|}
\hline Drug & Buprenorphine & Codeine & Dihydrocodeine & Fentanyl & Morphine & Oxycodone & Tramadol \\
\hline Codeine & .062 & & & & & & \\
\hline Dihydrocodeine & $.001 *$ & $>.999$ & & & & & \\
\hline Fentanyl & .075 & $<.001^{*}$ & $<.001^{*}$ & & & & \\
\hline Morphine & $>.999$ & $.003 *$ & $<.001^{*}$ & .845 & & & \\
\hline Oxycodone & $>.999$ & $<.001^{*}$ & $<.001^{*}$ & $>.999$ & $>.999$ & & \\
\hline
\end{tabular}




\begin{tabular}{rrrrrrrr}
\hline Tramadol & $>.999$ & $>.999$ & .179 & $<.001^{*}$ & .819 & $.024^{*}$ & \\
\hline Other & $>.999$ & $>.999$ & .408 & $<.001^{*}$ & .385 & $.008^{*}$ & $>.999$ \\
\hline
\end{tabular}

${ }^{*}$ p-value $<.05=$ statistically significant

Table D.24: Dunn's pairwise tests with Bonferroni corrections demonstrating relationships between the annualised daily oral morphine equivalent doses prescribed per prescription to women, by drug

\begin{tabular}{|c|c|c|c|c|c|c|c|}
\hline Drug & Buprenorphine & Codeine & Dihydrocodeine & Fentanyl & Morphine & Oxycodone & Tramadol \\
\hline Codeine & .0479 & & & & & & \\
\hline Dihydrocodeine & $.019 *$ & $>.999$ & & & & & \\
\hline Fentanyl & $.007^{*}$ & $<.001^{*}$ & $<.001^{*}$ & & & & \\
\hline Morphine & $>.999$ & $.002 *$ & $<.001^{*}$ & .920 & & & \\
\hline Oxycodone & .269 & $<.001 *$ & $<.001 *$ & $>.999$ & $>.999$ & & \\
\hline Tramadol & $>.999$ & .690 & $.031^{*}$ & $.004^{*}$ & $>.999$ & .179 & \\
\hline Other & $>.999$ & $>.999$ & .236 & $<.001^{*}$ & .592 & $.023 *$ & $>.999$ \\
\hline
\end{tabular}

${ }^{*}$ p-value $<.05$ = statistically significant

\section{D.3.4 Gender differences for high dose opioid prescription}

Table D.25: Gender trends for preparations of 120mg or greater oral morphine equivalent dose in milligrams

\begin{tabular}{|c|c|c|c|c|c|c|}
\hline & & \multicolumn{5}{|c|}{ Annual total daily oral morphine equivalent dose (milligrams) } \\
\hline & & Buprenorphine & Fentanyl & Morphine & Oxycodone & Other \\
\hline \multicolumn{7}{|l|}{ Men } \\
\hline & 2005 & 208064 & 716295 & 1190320 & 455090 & 31560 \\
\hline & 2006 & 293336 & 1026620 & 1308560 & 526360 & 29908 \\
\hline & 2007 & 312280 & 1303620 & 1562600 & 559480 & 17760 \\
\hline & 2008 & 273544 & 1489020 & 1745000 & 686200 & 15656 \\
\hline & 2009 & 299160 & 1558840 & 1880740 & 943880 & 16212 \\
\hline & 2010 & 329280 & 1731300 & 2127520 & 1189600 & 18500 \\
\hline & 2011 & 368088 & 1784600 & 2453950 & 1607760 & 22788 \\
\hline & 2012 & 389216 & 1842920 & 2983340 & 2116360 & 37516 \\
\hline & 2013 & 392024 & 1885260 & 3553150 & 2108560 & 55292 \\
\hline & 2014 & 420168 & 1927465 & 4169900 & 1953280 & 81072 \\
\hline
\end{tabular}




\begin{tabular}{|c|c|c|c|c|c|}
\hline 2015 & 412976 & 1812410 & 4741300 & 1933280 & 160644 \\
\hline $\begin{array}{r}\text { Percentage change (\%) } 2005- \\
2015\end{array}$ & 153.0 & 153.0 & 298.3 & 324.8 & 409.0 \\
\hline \multicolumn{6}{|l|}{ Women } \\
\hline 2005 & 266744 & 1488620 & 1352540 & 456000 & 26264 \\
\hline 2006 & 350040 & 1963720 & 1568920 & 563640 & 25560 \\
\hline 2007 & 382624 & 2352240 & 1882450 & 692840 & 20776 \\
\hline 2008 & 349024 & 2685600 & 2226160 & 972920 & 16768 \\
\hline 2009 & 390176 & 3051200 & 2427070 & 1215360 & 13472 \\
\hline 2010 & 431760 & 3373640 & 2780800 & 1436160 & 18232 \\
\hline 2011 & 435664 & 3467620 & 3388520 & 1731760 & 26040 \\
\hline 2012 & 416688 & 3443100 & 4218870 & 1912560 & 67476 \\
\hline 2013 & 400888 & 3490920 & 5038100 & 2059480 & 98016 \\
\hline 2014 & 410800 & 3207780 & 5976730 & 2076560 & 120632 \\
\hline 2015 & 404928 & 2899880 & 6857400 & 2070120 & 192352 \\
\hline $\begin{array}{r}\text { Percentage change (\%) } 2005- \\
2015\end{array}$ & 51.8 & 94.8 & 407.0 & 354.0 & 632.4 \\
\hline p-value (Mann-Whitney) & .088 & $<.001 *$ & .217 & .748 & .847 \\
\hline
\end{tabular}

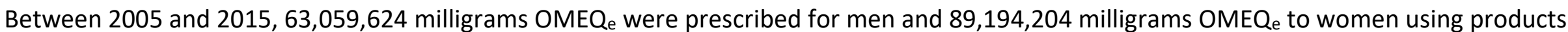
with a total daily dose of $120 \mathrm{mg} \mathrm{OMEQ}_{\mathrm{e}}$ or higher (when prescribed at recommended doses). This equates to $24.8 \%$ of OMEQ $\mathrm{Prescribed}$ over the study period, coming from formulations with a potential daily dose of $120 \mathrm{mg}$ morphine-equivalent or higher.

Table D.26: Gender trends in annual totals for preparations of $120 \mathrm{mg}$ or greater oral morphine equivalent dose in milligrams and adjusted to oral morphine equivalent dose per 1000 genderadjusted population, stratified by drug and with percentage and rate change for 2005 - 2015

\begin{tabular}{|c|c|c|c|c|c|c|}
\hline \multirow[b]{2}{*}{ Year } & & \multicolumn{5}{|c|}{ Annual total daily oral morphine equivalent dose (milligrams) per 1000 population } \\
\hline & & Buprenorphine & Fentanyl & Morphine & Oxycodone & Other \\
\hline \multicolumn{7}{|l|}{ Men } \\
\hline & 2005 & 184 & 634 & 1054 & 403 & 28 \\
\hline & 2006 & 258 & 904 & 1153 & 464 & 26 \\
\hline & 2007 & 273 & 1138 & 1365 & 489 & 16 \\
\hline
\end{tabular}




\begin{tabular}{|c|c|c|c|c|c|}
\hline 2008 & 237 & 1291 & 1513 & 595 & 14 \\
\hline 2009 & 258 & 1346 & 1624 & 815 & 14 \\
\hline 2010 & 283 & 1487 & 1828 & 1022 & 16 \\
\hline 2011 & 314 & 1524 & 2095 & 1373 & 19 \\
\hline 2012 & 331 & 1568 & 2538 & 1800 & 32 \\
\hline 2013 & 332 & 1598 & 3012 & 1787 & 47 \\
\hline 2014 & 355 & 1627 & 3520 & 1649 & 68 \\
\hline 2015 & 348 & 1525 & 3991 & 1627 & 135 \\
\hline Rate change (\%)2005 - 2015 & 89.1 & 140.4 & 278.5 & 303.7 & 383.7 \\
\hline \multicolumn{6}{|l|}{ Women } \\
\hline 2005 & 225 & 1255 & 1140 & 384 & 22 \\
\hline 2006 & 293 & 1646 & 1315 & 472 & 21 \\
\hline 2007 & 319 & 1962 & 1570 & 578 & 17 \\
\hline 2008 & 289 & 2227 & 1846 & 807 & 14 \\
\hline 2009 & 323 & 2528 & 2011 & 1007 & 11 \\
\hline 2010 & 357 & 2788 & 2298 & 1187 & 15 \\
\hline 2011 & 359 & 2857 & 2792 & 1427 & 21 \\
\hline 2012 & 342 & 2828 & 3465 & 1571 & 55 \\
\hline 2013 & 329 & 2861 & 4129 & 1688 & 80 \\
\hline 2014 & 336 & 2623 & 4886 & 1698 & 99 \\
\hline 2015 & 330 & 2366 & 5596 & 1689 & 157 \\
\hline Rate change (\%) $2005-2015$ & 47.0 & 88.6 & 390.9 & 339.5 & 609.1 \\
\hline Mann-Whitney p-value* & 0.193 & $<.001^{*}$ & 0.332 & $>.999$ & 0.949 \\
\hline
\end{tabular}

${ }^{*}$ p-value $<.05$ = statistically significant 
The total daily $\mathrm{OMEQ}_{\mathrm{e}}$ of $120 \mathrm{mg}$ or above derived increased for both genders during the study period (Figure D.5). The smallest increase was noted from buprenorphine products (Table D.27) although still nearly doubled for men (from 208,064 to 412,976 milligrams, $98.5 \%$ increase) and increased by just over half for women (from 266,744 to 404,928 milligrams, $51.8 \%$ increase).

Whilst not statistically significant (Table D.27), the difference between the $\mathrm{OMEQ}_{\mathrm{e}}$ of morphine over $120 \mathrm{mg}$ daily received by women and men increased over the 11 years examined. In 2005, women received prescriptions of approximately $14 \%$ more OMEQ $_{e}$ than men $(1,352,540$ versus $1,190,320$ milligrams respectively). In 2015 , that had increased to a $45 \%$ difference in milligrams morphine prescribed $(6,857,400$ versus $4,741,300$ milligrams respectively) (Figure D.5). The average difference between women and men in the OMEQ from preparations of $120 \mathrm{mg}$ daily $\mathrm{OMEQ}_{e}$ and above, over the study period was $36 \%(27,716,380$ versus $37,717,560$ milligrams respectively).

Table D.27: Gender trends for preparations of $120 \mathrm{mg}$ or greater oral morphine equivalent dose per 1000 gender-adjusted population, stratified by drug and with rate change

Annual total daily oral morphine equivalent dose (milligrams) per prescription

\begin{tabular}{|c|c|c|c|c|c|}
\hline Year & Buprenorphine & Fentanyl & Morphine & Oxycodone & Other \\
\hline \multicolumn{6}{|l|}{ Men } \\
\hline 2005 & 136 & 237 & 135 & 186 & 126 \\
\hline 2006 & 138 & 258 & 136 & 195 & 126 \\
\hline 2007 & 139 & 261 & 139 & 187 & 127 \\
\hline 2008 & 139 & 262 & 136 & 182 & 127 \\
\hline 2009 & 139 & 262 & 136 & 181 & 130 \\
\hline 2010 & 138 & 261 & 137 & 180 & 128 \\
\hline 2011 & 138 & 266 & 134 & 184 & 135 \\
\hline 2012 & 138 & 272 & 133 & 187 & 137 \\
\hline 2013 & 139 & 274 & 132 & 185 & 137 \\
\hline 2014 & 126 & 278 & 131 & 183 & 141 \\
\hline 2015 & 139 & 282 & 130 & 185 & 141 \\
\hline $\begin{array}{l}\text { Percentage change } \\
\text { (\%) } 2005-2015\end{array}$ & 2.2 & 19.0 & -3.7 & -0.9 & 12.2 \\
\hline \multicolumn{6}{|l|}{ Women } \\
\hline 2005 & 135 & 252 & 133 & 187 & 144 \\
\hline 2006 & 136 & 251 & 129 & 180 & 140 \\
\hline 2007 & 137 & 254 & 128 & 173 & 146 \\
\hline 2008 & 139 & 254 & 129 & 181 & 152 \\
\hline 2009 & 139 & 257 & 129 & 177 & 151 \\
\hline 2010 & 139 & 257 & 129 & 176 & 142 \\
\hline 2011 & 139 & 251 & 128 & 179 & 133 \\
\hline 2012 & 138 & 250 & 128 & 182 & 138 \\
\hline 2013 & 138 & 253 & 127 & 182 & 137 \\
\hline 2014 & 138 & 248 & 127 & 183 & 140 \\
\hline 2015 & 139 & 247 & 126 & 182 & 143 \\
\hline $\begin{array}{r}\text { Rate change (\%) } \\
2005-2015\end{array}$ & 2.7 & -2.1 & -5.5 & -2.3 & -0.6 \\
\hline $\begin{array}{r}\text { p-value (Mann- } \\
\text { Whitney) }\end{array}$ & 0.847 & $<.001^{*}$ & $<.001^{*}$ & 0.171 & $<.001^{*}$ \\
\hline
\end{tabular}

${ }^{*} p$-value $<.05=$ statistically significant 

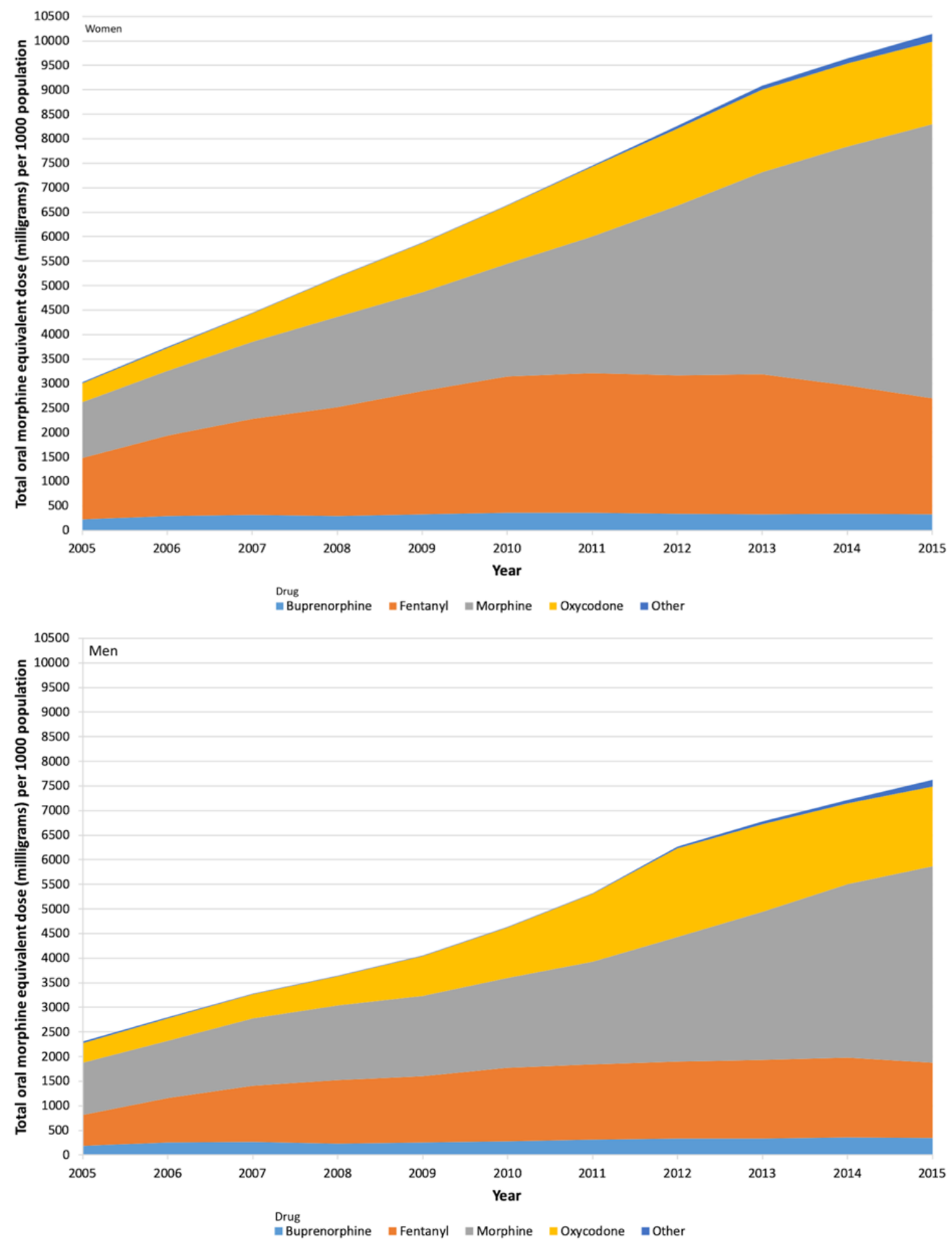

Figure D.5: Trends in Oral Morphine Equivalent Dose (milligrams) per prescription issued with products with $120 \mathrm{mg}$ OME and greater and stratified by drug. Examined by gender (Top - men, bottom - women)

Fentanyl $\mathrm{OMEQ}_{\mathrm{e}}$ increased for both genders (Table C.28) and whilst not the largest increase seen over the study period, was the only $\mathrm{OMEQ}_{\mathrm{e}}$ category with a statistically significant difference between genders $(p<.001)$, when subjected to Mann-Whitney analysis.

Oral morphine equivalent dose per prescription appeared Fentanyl and morphine $\mathrm{OMEQ}_{\mathrm{e}} \mathrm{s}$ per prescription were higher for men $(p<.005$ and $p<.001$ respectively) and 'other' opioid $\mathrm{OMEQ}_{\mathrm{e}} \mathrm{s}$ were higher for women $\left(\mathrm{p}<.001^{*}\right)$. Buprenorphine demonstrated a small increase in $O M E Q_{e}$ per prescription for high-dose preparations for men and women. High-dose $\mathrm{OMEQ}_{\mathrm{e}}$ per prescription 
reduced for all other categories for women although increased for fentanyl and 'other' opioids for men over the same time period (Table D.27)

\section{D.4 $\mathrm{OMEQ}_{\mathrm{e}}$ in areas of socioeconomic deprivation}

\section{D.4.1 Total OMEQe by quintile of deprivation}

Table D.28: Trends in total oral morphine equivalent dose prescribed stratified by deprivation (Welsh Index of Multiple Deprivation) and adjusted to population.

\begin{tabular}{rccccc}
\hline \multicolumn{5}{c}{ Year } & \multicolumn{5}{c}{ Oral morphine equivalent dose (milligrams) prescribed } \\
\cline { 2 - 6 } & WIMD1 & WIMD2 & WIMD3 & WIMD4 & WIMD5 \\
\hline $\mathbf{2 0 0 5}$ & $10,319,636$ & $8,590,375$ & $7,684,060$ & $5,374,595$ & $4,486,035$ \\
$\mathbf{2 0 0 6}$ & $11,002,416$ & $9,234,855$ & $8,203,320$ & $5,758,390$ & $4,925,110$ \\
$\mathbf{2 0 0 7}$ & $12,215,442$ & $10,384,487$ & $9,027,524$ & $6,499,993$ & $5,474,191$ \\
$\mathbf{2 0 0 8}$ & $13,795,845$ & $11,098,955$ & $9,780,820$ & $7,300,944$ & $6,032,501$ \\
$\mathbf{2 0 0 9}$ & $15,060,880$ & $12,151,599$ & $10,743,046$ & $8,084,842$ & $6,469,233$ \\
$\mathbf{2 0 1 0}$ & $16,120,158$ & $13,438,172$ & $11,745,722$ & $8,774,296$ & $7,069,176$ \\
$\mathbf{2 0 1 1}$ & $17,575,890$ & $14,766,808$ & $12,985,192$ & $9,525,368$ & $7,586,660$ \\
$\mathbf{2 0 1 2}$ & $18,971,106$ & $16,079,570$ & $14,242,512$ & $10,133,897$ & $8,162,570$ \\
$\mathbf{2 0 1 3}$ & $19,953,808$ & $16,327,490$ & $14,955,099$ & $10,546,332$ & $8,563,467$ \\
$\mathbf{2 0 1 4}$ & $20,641,165$ & $16,988,538$ & $15,170,432$ & $10,814,134$ & $8,622,456$ \\
$\mathbf{2 0 1 5}$ & $21,167,919$ & $17,399,026$ & $15,342,942$ & $10,878,897$ & $8,721,170$ \\
\hline Percentage change & 105.1 & 102.5 & 99.7 & 102.4 & 94.4 \\
(\%) 2005-2015 & & & & & \\
\hline Total prescribed & $176,824,265$ & $146,459,878$ & $129,880,669$ & $93,691,687$ & $76,112,569$ \\
$\mathbf{2 0 0 5 - 2 0 1 5}$ & & & & &
\end{tabular}

WIMD1 $=$ most deprived, WIMD5 $=$ least deprived

Table D.29: Dunn's pairwise comparison and Bonferroni post-hoc analysis of difference between the oral morphine equivalent doses prescribed in the 5 different areas of deprivation as defined by the Welsh Index of Multiple Deprivation (WIMD)

\begin{tabular}{ccccc}
\hline \multicolumn{5}{c}{ Welsh Index of Multiple Deprivation } \\
\hline $\mathbf{2}$ & $>.999$ & 2 & 3 & 4 \\
\hline $\mathbf{3}$ & 0.537 & $>.999$ & \\
\hline $\mathbf{4}$ & $.001^{*}$ & 0.052 & 0.504 & \\
\hline $\mathbf{5}$ & $<.001^{*}$ & $<.001^{*}$ & $0.017^{*}$ & $>.999$ \\
\hline
\end{tabular}

${ }^{*} \mathrm{p}$-value $<.05=$ statistically significant, WIMD1 = most deprived, WIMD5 = least deprived

\section{D.4.2 OMEQe per 1000 population and by deprivation quintile}

Table D.30: Trends in total oral morphine equivalent dose prescribed stratified by deprivation (Welsh Index of Multiple Deprivation) and adjusted to population

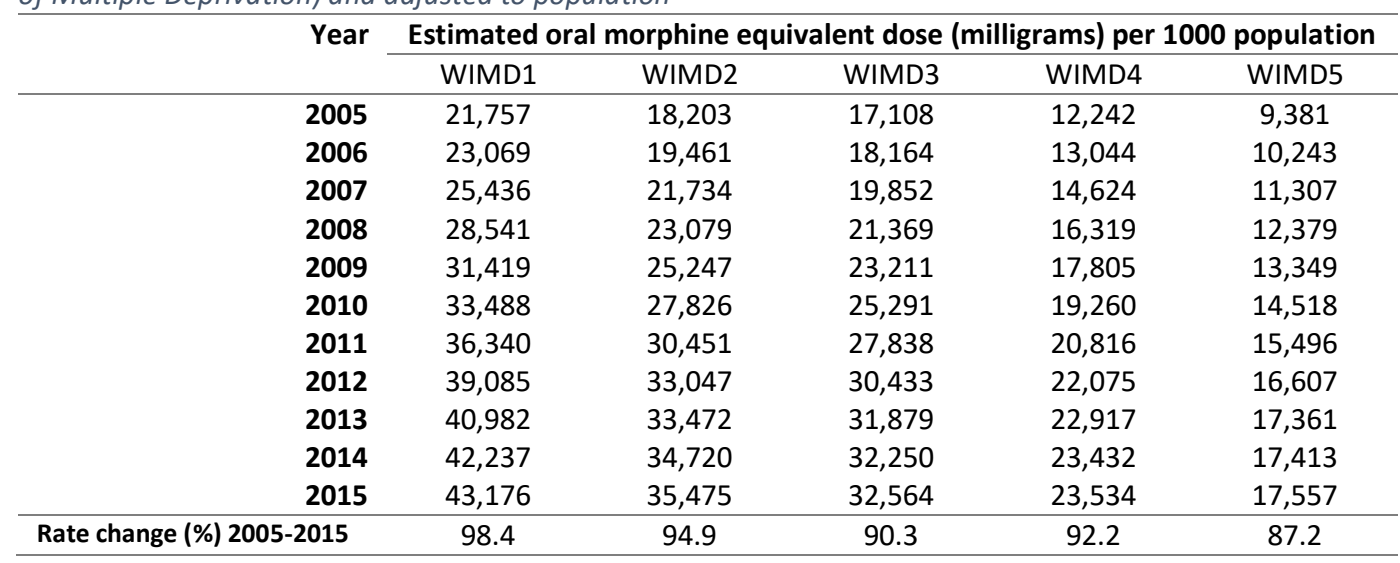

WIMD1 = most deprived, WIMD5 = least deprived 
Table D.31: Dunn's pairwise comparison and Bonferroni post-hoc analysis of difference between the oral morphine equivalent doses per 1000 population prescribed in the 5 different areas of deprivation as defined by the Welsh Index of Multiple Deprivation (WIMD)

\begin{tabular}{lllll}
\hline \multicolumn{5}{c}{ Welsh Index of Multiple Deprivation } \\
\hline $\mathbf{2}$ & 1 & 2 & 3 & 4 \\
\hline $\mathbf{3}$ & $>.999$ & & & \\
\hline $\mathbf{4}$ & 0.790 & $>.999$ & & \\
\hline $\mathbf{5}$ & $0.003^{*}$ & 0.119 & 0.570 & $>.999$ \\
\hline${ }^{*} \mathrm{p}$-value $<.05=$ statistically significant, WIMD1 = most deprived, WIMD5 = least deprived
\end{tabular}

\section{D.4.3 OMEQe per prescription and per person}

Table D.32: Trends in oral morphine equivalence stratified by deprivation (Welsh Index of Multiple Deprivation WIMD2011) and examined by dose per prescription and dose per person

\begin{tabular}{rccccc}
\hline Year & \multicolumn{5}{c}{ Oral morphine equivalent dose (milligrams) per prescription } \\
\cline { 2 - 6 } & WIMD1 & WIMD2 & WIMD3 & WIMD4 & WIMD5 \\
\hline $\mathbf{2 0 0 5}$ & 22 & 24 & 24 & 25 & 24 \\
$\mathbf{2 0 0 6}$ & 23 & 25 & 25 & 26 & 25 \\
$\mathbf{2 0 0 7}$ & 24 & 26 & 26 & 27 & 26 \\
$\mathbf{2 0 0 8}$ & 25 & 26 & 27 & 28 & 26 \\
$\mathbf{2 0 0 9}$ & 26 & 27 & 28 & 29 & 27 \\
$\mathbf{2 0 1 1}$ & 27 & 28 & 29 & 30 & 28 \\
$\mathbf{2 0 1 2}$ & 28 & 29 & 30 & 31 & 28 \\
$\mathbf{2 0 1 3}$ & 30 & 31 & 32 & 31 & 29 \\
$\mathbf{2 0 1 4}$ & 31 & 31 & 33 & 32 & 30 \\
$\mathbf{2 0 1 5}$ & 31 & 32 & 32 & 32 & 30 \\
\hline $\mathbf{2 0 0 5}-\mathbf{2 0 1 5}$ & 41.0 & 32 & 33 & 33 & 30 \\
\hline $\mathbf{2 0 0 5}$ & Oral morphine equivalent dose (milligrams) per person receiving prescriptions \\
\hline $\mathbf{2 0 0 6}$ & 104 & 99 & 98 & 93 & 80 \\
$\mathbf{2 0 0 7}$ & 109 & 107 & 104 & 99 & 87 \\
$\mathbf{2 0 0 8}$ & 119 & 113 & 107 & 102 & 89 \\
$\mathbf{2 0 0 9}$ & 126 & 116 & 110 & 108 & 93 \\
$\mathbf{2 0 1 0}$ & 131 & 123 & 118 & 116 & 96 \\
$\mathbf{2 0 1 1}$ & 141 & 131 & 123 & 120 & 102 \\
$\mathbf{2 0 1 2}$ & 150 & 141 & 134 & 128 & 107 \\
$\mathbf{2 0 1 3}$ & 160 & 151 & 147 & 136 & 115 \\
$\mathbf{2 0 1 4}$ & 166 & 157 & 156 & 144 & 124 \\
$\mathbf{2 0 1 5}$ & 173 & 164 & 158 & 148 & 125 \\
\hline $\mathbf{2 0 0 5}-\mathbf{2 0 1 5}$ & 79.2 & 70.2 & 64.7 & 61.3 & 60.2 \\
\hline Rate & & & & & \\
\hline
\end{tabular}

WIMD1 = most deprived, WIMD5 = least deprived

Table D.33: Dunn's pairwise comparison and Bonferroni post-hoc analysis of difference between the number people receiving opioid prescriptions in the 5 different areas of deprivation as defined by the Welsh Index of Multiple Deprivation (WIMD)

\begin{tabular}{ccccc}
\hline \multicolumn{5}{c}{ Welsh Index of Multiple Deprivation } \\
\hline $\mathbf{2}$ & 1 & 2 & 3 & 4 \\
\hline $\mathbf{3}$ & $>.999$ & & & \\
\hline $\mathbf{4}$ & $>.999$ & $>.999$ & & \\
\hline $\mathbf{5}$ & $.046^{*}$ & $>.999$ & $>.999$ & .745 \\
\hline
\end{tabular}

*p-value $<.05$ = statistically significant WIMD1=most deprived, WIMD5-least deprived 


\section{D.4.4 High dose prescribing by deprivation quintile}

Table D.34: Trends in total oral morphine equivalent dose of 120 milligrams or higher prescribed stratified by deprivation (Welsh Index of Multiple Deprivation)

\begin{tabular}{|c|c|c|c|c|c|}
\hline \multirow[t]{2}{*}{ Year } & \multicolumn{5}{|c|}{ Oral morphine equivalent dose for doses of $\mathbf{1 2 0}$ milligrams per day and over } \\
\hline & WIMD1 & WIMD2 & WIMD3 & WIMD4 & WIMD5 \\
\hline 2005 & $1,466,652$ & $1,208,800$ & $1,373,446$ & $1,104,120$ & 697,496 \\
\hline 2006 & $1,860,742$ & $1,779,300$ & $1,674,320$ & $1,319,344$ & 969,624 \\
\hline 2007 & $2,157,344$ & $2,285,544$ & $1,941,096$ & $1,537,720$ & $1,140,146$ \\
\hline 2008 & $2,718,216$ & $2,470,438$ & $2,128,180$ & $1,822,578$ & $1,201,084$ \\
\hline 2009 & $3,072,296$ & $2,288,286$ & $2,440,202$ & $2,133,356$ & $1,289,852$ \\
\hline 2010 & $3,263,196$ & $2,759,170$ & $2,829,734$ & $2,405,498$ & $1,525,984$ \\
\hline 2011 & $3,691,928$ & $3,071,124$ & $3,338,804$ & $2,639,240$ & $1,670,600$ \\
\hline 2012 & $4,223,200$ & $3,622,164$ & $4,000,144$ & $2,841,846$ & $1,895,168$ \\
\hline 2013 & $4,665,156$ & $3,858,312$ & 4,476,098 & $3,099,268$ & $2,162,012$ \\
\hline 2014 & $5,150,352$ & $4,442,212$ & 4,499,933 & $3,202,667$ & $2,178,040$ \\
\hline 2015 & $5,674,237$ & $4,837,285$ & $4,642,419$ & $3,314,817$ & $2,228,629$ \\
\hline $\begin{array}{r}\text { Percentage change } \\
(\%) 2005-2015\end{array}$ & 286.9 & 300.2 & 238.0 & 200.2 & 219.5 \\
\hline $\begin{array}{r}\text { Total prescribed } \\
2005-2015\end{array}$ & $37,943,319$ & $32,622,635$ & $33,344,376$ & $25,420,454$ & $16,958,635$ \\
\hline
\end{tabular}

Table D.35: Dunn's pairwise comparison and Bonferroni post-hoc analysis of difference between OMEQe from products with $>120 \mathrm{mg}$ OMEQe daily dose, in the 5 different areas of deprivation as defined by the Welsh Index of Multiple Deprivation (WIMD)

\begin{tabular}{lcccc}
\hline \multicolumn{5}{c}{ Welsh Index of Multiple Deprivation } \\
\hline $\mathbf{2}$ & 1 & 2 & 3 & 4 \\
\hline $\mathbf{3}$ & $>.999$ & & & \\
\hline $\mathbf{4}$ & $>.999$ & $>.999$ & \\
\hline $\mathbf{5}$ & .663 & $>.999$ & $>.999$ & .643 \\
\hline
\end{tabular}

${ }^{*} \mathrm{p}$-value $<.05$ = statistically significant, WIMD1=most deprived, WIMD5=least deprived

Table D.36: Trends in total oral morphine equivalent dose of 120 milligrams or higher prescribed stratified by deprivation (Welsh Index of Multiple Deprivation) and adjusted to deprivation quintile population

\begin{tabular}{|c|c|c|c|c|c|}
\hline \multirow[t]{2}{*}{ Year } & \multicolumn{5}{|c|}{$\begin{array}{c}\text { Total oral morphine equivalent dose per } 1000 \text { population for products of over } 120 \mathrm{mg} \\
\mathrm{OMEQ}_{\mathrm{e}}\end{array}$} \\
\hline & WIMD1 & WIMD2 & WIMD3 & WIMD4 & WIMD5 \\
\hline 2005 & 3,092 & $2,, 561$ & 3,058 & 2,515 & 1,459 \\
\hline 2006 & 3,901 & 3,750 & 3,707 & 2,989 & 2,017 \\
\hline 2007 & 4,492 & 4,784 & 4,268 & 3,460 & 2,355 \\
\hline 2008 & 5,624 & 5,137 & 4,650 & 4,074 & 2,465 \\
\hline 2009 & 6,409 & 4,754 & 5,272 & 4,698 & 2,662 \\
\hline 2010 & 6,779 & 5,713 & 6,093 & 5,280 & 3,134 \\
\hline 2011 & 7,633 & 6,333 & 7,158 & 5,768 & 3,412 \\
\hline 2012 & 8,701 & 7,444 & 8,547 & 6,190 & 3,856 \\
\hline 2013 & 9,581 & 7,910 & 9,541 & 6,735 & 4,383 \\
\hline 2014 & 10,539 & 9,079 & 9,566 & 6,940 & 4,399 \\
\hline 2015 & 11,574 & 9,863 & 9,853 & 7,171 & 4,487 \\
\hline $\begin{array}{r}\text { Rate change } \\
(\%)\end{array}$ & 274.3 & 285.0 & 222.2 & 185.1 & 207.6 \\
\hline $2005-2015$ & & & & & \\
\hline
\end{tabular}

Table D.37: Dunn's pairwise comparison and Bonferroni post-hoc analysis of difference between OMEQe from products with $>120 \mathrm{mg}$ OMEQe daily dose per 1000 population, in the 5 different areas of deprivation as defined by the Welsh Index of Multiple Deprivation (WIMD)

Welsh Index of Multiple Deprivation

123

34 


\begin{tabular}{ccccc}
\hline $\mathbf{2}$ & $>.999$ & & & \\
\hline $\mathbf{3}$ & $>.999$ & $>.999$ & & \\
\hline $\mathbf{4}$ & $>.999$ & $>.999$ & $>.999$ & .099 \\
\hline $\mathbf{5}$ & $<.001^{*}$ & $.013^{*}$ & $.009^{*}$ & .098
\end{tabular}

${ }^{*} \mathrm{p}$-value $<.05$ = statistically significant WIMD1=most deprived, WIMD5-least deprived 


\section{Appendix E Time Series Analysis examining the effect of legislation and prescribing guidance on opioid prescribing in Wales}

\section{E.1 Schedules of controlled drugs in the UK}

\section{E.1.1 Schedule 1 controlled drugs}

Subject to the requirements of regulations $14,15,16,18,19,20,23,26$ and 27 (legislation.gov.uk and UK Government 2021)

1. The following substances and products, namely -

(a)Bufotenine

- Cannabinol

- Cannabinol derivatives not being dronabinol or its stereoisomers

- Cannabis and cannabis resin

- Cathinone

- Coca leaf

- Concentrate of poppy-straw

- Eticyclidine

- Etryptamine

- Lysergamide

- $\quad$ Lysergide and other $\mathrm{N}$-alkyl derivatives of lysergamide

- Mescaline

- Methcathinone

- Psilocin

- Raw opium

- Rolicyclidine

- Tenocyclidine

- 4-Bromo-2,5-dimethoxy-a-methylphenethylamine

- $\quad N, N$-Diethyltryptamine

- $\quad \mathrm{N}, \mathrm{N}$-Dimethyltryptamine

- 2,5-Dimethoxy- $\alpha, 4$-dimethylphenethylamine

- $\quad \mathrm{N}$-Hydroxy-tenamphetamine

- 4-Methyl-aminorex

(b)any compound (not being a compound for the time being specified in sub-paragraph (a) above) structurally derived from tryptamine or from a ring-hydroxy tryptamine by substitution at the nitrogen atom of the sidechain with one or more alkyl substituents but no other substituent;

(c)the following phenethylamine derivatives, namely-

- Allyl(a-methyl-3,4-methylenedioxyphenethyl)amine

- 2-Amino-1-(2,5-dimethoxy-4-methylphenyl)ethanol

- 2-Amino-1-(3,4-dimethoxyphenyl)ethanol

- Benzyl( $\alpha$-methyl-3,4-methylenedioxyphenethyl)amine

- 4-Bromo-8,2,5-trimethoxyphenethylamine

- $\quad \mathrm{N}$-(4-sec-Butylthio-2,5-dimethoxyphenethyl)hydroxylamine

- Cyclopropylmethyl( $\alpha$-methyl-3,4-methylenedioxyphenethyl)amine

- 2-(4,7-Dimethoxy-2,3-dihydro-1H-indan-5-yl)ethylamine

- 2-(4,7-Dimethoxy-2,3-dihydro-1H-indan-5-yl)-1-methylethylamine

- 2-(2,5-Dimethoxy-4-methylphenyl)cyclopropylamine

- 2-(1,4-Dimethoxy-2-naphthyl)ethylamine

- 2-(1,4-Dimethoxy-2-naphthyl)-1-methylethylamine

- $\quad N-(2,5$-Dimethoxy-4-propylthiophenethyl)hydroxylamine

- 2-(1,4-Dimethoxy-5,6,7,8-tetrahydro-2-naphthyl)ethylamine

- 2-(1,4-Dimethoxy-5,6,7,8-tetrahydro-2-naphthyl)-1-methylethylamine 
- $\quad \alpha, \alpha$-Dimethyl-3,4-methylenedioxyphenethylamine

- $\alpha, \alpha$-Dimethyl-3,4-methylenedioxyphenethyl(methyl)amine

- Dimethyl( $\alpha$-methyl-3,4-methylenedioxyphenethyl)amine

- $\quad \mathrm{N}$-(4-Ethylthio-2,5-dimethoxyphenethyl)hydroxylamine

- 4-lodo-2,5-dimethoxy-a-methylphenethyl(dimethyl)amine

- 2-(1,4-Methano-5,8-dimethoxy-1,2,3,4-tetrahydro-6-naphthyl)ethylamine

- 2-(1,4-Methano-5,8-dimethoxy-1,2,3,4-tetrahydro-6-naphthyl)-1-methylethylamine

- 2-(5-Methoxy-2,2-dimethyl-2,3-dihydrobenzo[b]furan-6-yl)-1-methylethylamine

- 2-Methoxyethyl( $\alpha$-methyl-3,4-methylenedioxyphenethyl)amine

- 2-(5-Methoxy-2-methyl-2,3-dihydrobenzo[b]furan-6-yl)-1-methylethylamine

- $\quad \beta$-Methoxy-3,4-methylenedioxyphenethylamine

- 1-(3,4-Methylenedioxybenzyl)butyl(ethyl)amine

- 1-(3,4-Methylenedioxybenzyl)butyl(methyl)amine

- 2-(a-Methyl-3,4-methylenedioxyphenethylamino)ethanol

- $\quad \alpha-M e t h y l-3,4-m e t h y l e n e d i o x y p h e n e t h y l(p r o p-2-y n y l) a m i n e$

- $\quad N$-Methyl- $N$-( $\alpha$-methyl-3,4-methylenedioxyphenethyl)hydroxylamine

- $\quad$-Methyl- $N$-( $\alpha$-methyl-3,4-methylenedioxyphenethyl)hydroxylamine

- $\alpha$-Methyl-4-(methylthio)phenethylamine

- $\quad \beta, 3,4,5-$ Tetramethoxyphenethylamine

- $\quad$ 3,2,5-Trimethoxy-4-methylphenethylamine

(d)any compound (not being methoxyphenamine or a compound for the time being specified in subparagraph (a) above) structurally derived from phenethylamine, an $N$-alkylphenethylamine, $\alpha$ methylphenethylamine, an $N$-alkyl- $\alpha$-methylphenethylamine, $\alpha$-ethylphenethylamine, or an $N$-alkyl- $\alpha$ ethylphenethylamine by substitution in the ring to any extent with alkyl, alkoxy, alkylenedioxy or halide substitutents, whether or not further substituted in the ring by one or more other univalent substituents;

(e)any compound (not being a compound for the time being specified in Schedule 2) structurally derived from fentanyl by modification in any of the following ways, that is to say -

(i)by replacement of the phenyl portion of the phenethyl group by any heteromonocycle whether or not further substituted in the heterocycle;

(ii)by substitution in the phenethyl group with alkyl, alkenyl, alkoxy, hydroxy, halogeno, haloalkyl, amino or nitro groups;

(iii)by substitution in the piperidine ring with alkyl or alkenyl groups;

(iv)by substitution in the aniline ring with alkyl, alkoxy, alkylenedioxy, halogeno or haloalkyl groups; (v)by substitution at the 4-position of the piperidine ring with any alkoxycarbonyl or alkoxyalkyl or acyloxy group;

(vi)by replacement of the $N$-propionyl group by another acyl group;

(f)any compound (not being a compound for the time being specified in Schedule 2) structurally derived from pethidine by modification in any of the following ways, that is to say-

(i)by replacement of the l-methyl group by an acyl, alkyl whether or not unsaturated, benzyl or phenethyl group, whether or not further substituted;

(ii)by substitution in the piperidine ring with alkyl or alkenyl groups or with a propano bridge, whether or not further substituted;

(iii)by substitution in the 4-phenyl ring with alkyl, alkoxy, aryloxy, halogeno or haloalkyl groups;

(iv)by replacement of the 4-ethoxycarbonyl by any other alkoxycarbonyl or any alkoxyalkyl or acyloxy group;

(v)by formation of an $\mathrm{N}$-oxide or of a quaternary base.

2. Any stereoisomeric form of a substance specified in paragraph 1.

3. Any ester or ether of a substance specified in paragraph 1 or 2.

4. Any salt of a substance specified in any of paragraphs 1 to 3 .

5. Any preparation or other product containing a substance or product specified in any of paragraphs 1 to 4 , not being a preparation specified in Schedule 5. 


\section{E.1.2 Schedule 2 controlled drugs}

\section{Subject to the requirements of regulations $14,15,16,18,19,20,21,23,26$ and}

\section{7}

1. The following substances and products, namely-

Acetorphine

Alfentanil

Allylprodine

Alphacetylmethadol

Alphameprodine

Alphamethadol

Alphaprodine

Anileridine

Benzethidine

Benzylmorphine (3-benzylmorphine)

Betacetylmethadol

Betameprodine

Betamethadol

Betaprodine

Bezitramide

Carfentanil

Clonitazene

Cocaine

Desomorphine

Dextromoramide

Diamorphine

Diampromide

Diethylthiambutene

Difenoxin

Dihydrocodeinone

O-carboxymethyloxime

Dihydromorphine

Dimenoxadole
Levomoramide

Levophenacylmorphan

Levorphanol

Lofentanil

Medicinal opium

Metazocine

Methadone

Methadyl acetate

Methyldesorphine

Methyldihydromorphine

(6-methyldihydromorphine)

Metopon

Morpheridine

Morphine

Morphine methobromide, morphine $\mathrm{N}$-oxide and other pentavalent nitrogen morphine derivatives

Myrophine

Nicomorphine

Noracymethadol

Norlevorphanol

Normethadone

Normorphine

Norpipanone

Oxycodone

Oxymorphone

Pethidine

Phenadoxone

Phenampromide 


\begin{tabular}{|c|c|}
\hline Dimepheptanol & Phenazocine \\
\hline Dimethylthiambutene & Phencyclidine \\
\hline Dioxaphetyl butyrate & Phenomorphan \\
\hline Diphenoxylate & Phenoperidine \\
\hline Dipipanone & Piminodine \\
\hline Dronabinol & Piritramide \\
\hline Drotebanol & Proheptazine \\
\hline Ecgonine, and any derivative of & Properidine \\
\hline ecgonine which is convertible to & Racemethorphan \\
\hline ecgonine or to cocaine & Racemoramide \\
\hline Ethylmethylthiambutene & Racemorphan \\
\hline Etonitazene & Sufentanil \\
\hline Etorphine & Thebacon \\
\hline Etoxeridine & Thebaine \\
\hline Fentanyl & Tilidate \\
\hline Furethidine & Trimeperidine \\
\hline Hydrocodone & Zipeprol \\
\hline Hydromorphinol & 4-Cyano-2-dimethylamino-4,4- \\
\hline Hydromorphone & diphenylbutane \\
\hline Hydroxypethidine & 4-Cyano-1-methyl-4- \\
\hline Isomethadone & phenylpiperidine \\
\hline Ketobemidone & 2-Methyl-3-morpholino-1,1-diphenylpropane- \\
\hline \multirow[t]{4}{*}{ Levomethorphan } & carboxylic acid \\
\hline & $\alpha$-Methylphenethylhydroxylamine \\
\hline & 1-Methyl-4-phenylpiperidine-4-carboxylic acid \\
\hline & 4-Phenylpiperidine-4-carboxylic acid ethyl ester \\
\hline
\end{tabular}

2. Any stereoisomeric form of a substance specified in paragraph 1 not being dextromethorphan or dextrorphan.

3. Any ester or ether of a substance specified in paragraph 1 or 2 , not being a substance specified in paragraph 6.

4. Any salt of a substance specified in any of paragraphs 1 to 3.

5. Any preparation or other product containing a substance or product specified in any of paragraphs 1 to 4 , not being a preparation specified in Schedule 5 .

6. The following substances and products, namely- 


\begin{tabular}{|c|c|}
\hline Amphetamine & Methylamphetamine \\
\hline Codeine & Methylphenidate \\
\hline Dextropropoxyphene & Nicocodine \\
\hline Dihydrocodeine & Nicodicodine (6-nicotinoyldihydrocodeine) \\
\hline Ethylmorphine (3-ethylmorphine) & Norcodeine \\
\hline Fenethylline & Phenmetrazine \\
\hline Glutethimide & Pholcodine \\
\hline Lefetamine & Propiram \\
\hline Mecloqualone & Quinalbarbitone \\
\hline \multicolumn{2}{|c|}{$\begin{array}{l}\text { 7. Any stereoisomeric form of a substance specified in paragraph } 6 . \\
\text { 8. Any salt of a substance specified in paragraph } 6 \text { or } 7 \text {. } \\
\text { 9. Any preparation or other product containing a substance or product specified in any of paragraphs }\end{array}$} \\
\hline
\end{tabular}

\section{E.1.3 Schedule 3 controlled drugs}

\section{Subject to the requirements of regulations 14, 15 (except temazepam), 16, 18,}

\section{$22,23,24,26$ and 27}

1. The following substances, namely-

$\begin{array}{ll}\text { Benzphetamine } & \text { Mephentermine } \\ \text { Buprenorphine } & \text { Meprobamate } \\ \text { Cathine } & \text { Methylphenobarbitone } \\ \text { Chlorphentermine } & \text { Methyprylone } \\ \text { Diethylpropion } & \text { Pentazocine } \\ \text { Ethchlorvynol } & \text { Phendimetrazine } \\ \text { Ethinamate } & \text { Phentermine } \\ \text { Flunitrazepam } & \text { Pipradrol } \\ \text { Mazindol } & \text { Temazepam }\end{array}$

(b)any 5, 5 disubstituted barbituric acid not being quinalbarbitone.

2. Any stereoisomeric form of a substance specified in paragraph 1 not being phenylpropanolamine.

3. Any salt of a substance specified in paragraph 1 or 2 .

4. Any preparation or other product containing a substance specified in any of paragraphs 1 to 3 , not being a preparation specified in Schedule 5 .

\section{E.1.4 Schedule 4 Part I controlled drugs}

Subject to the requirements of regulations $22,23,26$ and 27

1. The following substances and products, namely- 


\begin{tabular}{|c|c|}
\hline Alprazolam & Ketazolam \\
\hline Aminorex & Loprazolam \\
\hline Bromazepam & Lorazepam \\
\hline Brotizolam & Lormetazepam \\
\hline Camazepam & Medazepam \\
\hline Chlordiazepoxide & Mefenorex \\
\hline Clobazam & Mesocarb \\
\hline Clonazepam & Midazolam \\
\hline Clorazepic acid & Nimetazepam \\
\hline Clotiazepam & Nitrazepam \\
\hline Cloxazolam & Nordazepam \\
\hline Delorazepam & Oxazepam \\
\hline Diazepam & Oxazolam \\
\hline Estazolam & Pemoline \\
\hline Ethyl loflazepate & Pinazepam \\
\hline Fencamfamin & Prazepam \\
\hline Fenproporex & Pyrovalerone \\
\hline Fludiazepam & Tetrazepam \\
\hline Flurazepam & Triazolam \\
\hline Halazepam & $N$-Ethylamphetamine \\
\hline
\end{tabular}

Haloxazolam

2. Any stereoisomeric form of a substance specified in paragraph 1.

3. Any salt of a substance specified in paragraph 1 or 2 .

4. Any preparation or other product containing a substance or product specified in any of paragraphs 1 to 3 , not being a preparation specified in Schedule 5 .

Part ii controlled drugs excepted from the prohibition on possession when in the form of a medicinal product; excluded from the application of offences arising from the prohibition on importation and exportation when imported or exported in the form of a medicinal product by any person for administration to himself; and subject to the requirements of regulations 22, 23, 26 and 27

1. The following substances, namely-

$\begin{array}{ll}\text { Atamestane } & \text { Methenolone } \\ \text { Bolandiol } & \text { Methyltestosterone } \\ \text { Bolasterone } & \text { Metribolone } \\ \text { Bolazine } & \text { Mibolerone }\end{array}$




\begin{tabular}{ll} 
Boldenone & Nandrolone \\
Bolenol & Norboletone \\
Bolmantalate & Norclostebol \\
Calusterone & Norethandrolone \\
4-Chloromethandienone & Ovandrotone \\
Clostebol & Oxabolone \\
Drostanolone & Oxandrolone \\
Enestebol & Oxymesterone \\
Epitiostanol & Oxymetholone \\
Ethyloestrenol & Prasterone \\
Fluoxymesterone & Propetandrol \\
Formebolone & Thiomesterone \\
Furazabol & Trenbolone \\
Mebolazine & Stenbolone \\
Mepitiostane & Stanbolone \\
Mesabolone & Roxibolone \\
Mestanolone & Standrone \\
\hline
\end{tabular}

2. Any compound (not being Trilostane or a compound for the time being specified in paragraph 1 of this Part of this Schedule) structurally derived from 17-hydroxyandrostan-3-one or from 17hydroxyestran-3-one by modification in any of the following ways, that is to say -

(a)by further substitution at position 17 by a methyl or ethyl group;

(b)by substitution to any extent at one or more of positions $1,2,4,6,7,9,11$ or 16, but at no other position;

(c)by unsaturation in the carbocyclic ring system to any extent, provided that there are no more than two ethylenic bonds in any one carbocyclic ring;

(d)by fusion of ring A with a heterocyclic system.

3. Any substance which is an ester or ether (or, where more than one hydroxyl function is available, both an ester and an ether) of a substance specified in paragraph 1 or described in paragraph 2 of this Part of this Schedule.

4. The following substances, namely-

- Chorionic Gonadotrophin (HCG)

- Clenbuterol

- Non-human chorionic gonadotrophin

- Somatotropin

- Somatrem

- Somatropin

5. Any stereoisomeric form of a substance specified or described in any of paragraphs 1 to 4 of this Part of this Schedule. 
6. Any salt of a substance specified or described in any of paragraphs 1 to 5 of this Part of this Schedule.

7. Any preparation or other product containing a substance or product specified or described in any of paragraphs 1 to 6 of this Part of this Schedule, not being a preparation specified in Schedule 5.

\section{E.1.5 Schedule 5 controlled drugs}

\section{Excepted from the prohibition on importation, exportation and possession and subject to the requirements of regulations 24 and 26}

1.-(1) Any preparation of one or more of the substances to which this paragraph applies, not being a preparation designed for administration by injection, when compounded with one or more other active or inert ingredients and containing a total of not more than 100 milligrams of the substance or substances (calculated as base) per dosage unit or with a total concentration of not more than $2.5 \%$ (calculated as base) in undivided preparations.

(2) The substances to which this paragraph applies are acetyldihydrocodeine, codeine, dihydrocodeine, ethylmorphine, nicocodine, nicodicodine (6-nicotinoyldihydrocodeine), norcodeine and pholcodine and their respective salts.

2. Any preparation of cocaine containing not more than $0.1 \%$ of cocaine calculated as cocaine base, being a preparation compounded with one or more other active or inert ingredients in such a way that the cocaine cannot be recovered by readily applicable means or in a yield which would constitute a risk to health.

3. Any preparation of medicinal opium or of morphine containing (in either case) not more than $0.2 \%$ of morphine calculated as anhydrous morphine base, being a preparation compounded with one or more other active or inert ingredients in such a way that the opium or, as the case may be, the morphine cannot be recovered by readily applicable means or in a yield which would constitute a risk to health.

4. Any preparation of dextropropoxyphene, being a preparation designed for oral administration, containing not more than 135 milligrams of dextropropoxyphene (calculated as base) per dosage unit or with a total concentration of not more than $2.5 \%$ (calculated as base) in undivided preparations.

5. Any preparation of difenoxin containing, per dosage unit, not more than 0.5 milligrams of difenoxin and a quantity of atropine sulphate equivalent to at least $5 \%$ of the dose of difenoxin.

6. Any preparation of diphenoxylate containing, per dosage unit, not more than 2.5 milligrams of diphenoxylate calculated as base, and a quantity of atropine sulphate equivalent to at least $1 \%$ of the dose of diphenoxylate.

7. Any preparation of propiram containing, per dosage unit, not more than 100 milligrams of propiram calculated as base and compounded with at least the same amount (by weight) of methylcellulose.

8. Any powder of ipecacuanha and opium comprising-

- $10 \%$ opium, in powder,

- $10 \%$ ipecacuanha root, in powder, well mixed with

- $80 \%$ of any other powdered ingredient containing no controlled drug.

9. Any mixture containing one or more of the preparations specified in paragraphs 1 to 8 , being a mixture of which none of the other ingredients is a controlled drug.

\section{E.2 Interrupted time series}

\section{E.2.1 Output by gender}

Table E.1: Output from testing for best-fit model of interrupted time series analysis for men (all opioids)

\begin{tabular}{llll}
\hline Maximum Likelihood Estimates & & \\
\hline SSE & 495392365 & DFE & 124 \\
\hline MSE & 3995100 & Root MSE & 1999 \\
\hline SBC & 2415.99783 & AIC & 2392.93541 \\
\hline MAE & 1560.32384 & AICC & 2394.10614 \\
\hline MAPE & 2.40060036 & HQC & 2402.30692 \\
\hline Log Likelihood & -1188.4677 & Transformed Regression R-Square & 0.9472 \\
\hline
\end{tabular}




\begin{tabular}{llll}
\hline Maximum Likelihood Estimates & & \\
\hline Durbin-Watson & 1.8623 & Total R-Square & 0.9672 \\
\hline & Observations & 132 \\
\hline
\end{tabular}

Table E.2: Results of interrupted time series analysis of opioid prescribing data for men

\begin{tabular}{lllll}
\hline Parameter estimates & & & \\
\hline Variable & $\begin{array}{l}\text { Estimate } \\
(95 \% \mathrm{Cl})\end{array}$ & Standard Error & t Value & Approx Pr $>|\mathrm{t}|$ \\
\hline Intercept $\left(\boldsymbol{B}_{0}\right)$ & 47169 & 509.7996 & 92.52 & $<<.0011$ \\
\hline $\begin{array}{l}\text { Pre-intervention } \\
\text { trend }\left(\boldsymbol{B}_{1}\right)\end{array}$ & $\begin{array}{l}316.6277 \\
(297.81-335.45)\end{array}$ & 9.6027 & 32.97 & $<<.0011$ \\
\hline Change in level $\left(\boldsymbol{B}_{2}\right)$ & 573.3097 & 635.2972 & 0.90 & 0.3686 \\
\hline Post-intervention & -224.7378 & 24.9513 & -9.01 & $<<.0011$ \\
trend $\left(\boldsymbol{B}_{3}\right)$ & $(-273.64--175.83))$ & & & \\
\hline AR1 & 0.4456 & 0.0566 & 7.87 & $<<.0011$ \\
\hline AR3 & -0.2648 & 0.0634 & -4.18 & $<<.0011$ \\
\hline AR12 & -0.3425 & 0.0728 & -4.70 & $<<.0011$ \\
\hline
\end{tabular}

Table E.3: Output from testing for best-fit model of interrupted time series analysis for women (all opioids)

\begin{tabular}{|c|c|c|c|}
\hline \multicolumn{4}{|c|}{ Maximum Likelihood Estimates } \\
\hline SSE & 1337137308 & DFE & 125 \\
\hline MSE & 10697098 & Root MSE & 3271 \\
\hline SBC & 2541.99381 & AIC & 2521.8142 \\
\hline MAE & 2577.76903 & AICC & 2522.71742 \\
\hline MAPE & 2.48072689 & HQC & 2530.01426 \\
\hline Log Likelihood & -1253.9071 & Transformed Regression R-Square & 0.9316 \\
\hline \multirow[t]{2}{*}{ Durbin-Watson } & 1.9518 & Total R-Square & 0.9517 \\
\hline & & Observations & 132 \\
\hline
\end{tabular}

Table E.4: Results of interrupted time series analysis of opioid prescribing data for women

\begin{tabular}{lllll}
\hline Parameter estimates & & & & \\
\hline Variable & $\begin{array}{l}\text { Estimate } \\
(95 \% \mathrm{Cl})\end{array}$ & Standard Error & t Value & Approx $\operatorname{Pr}|\mathrm{t}|$ \\
\hline Intercept $\left(\boldsymbol{B}_{0}\right)$ & 80139 & 779.0447 & 102.87 & $<<.0011$ \\
\hline $\begin{array}{l}\text { Pre-intervention } \\
\text { trend }\left(\boldsymbol{B}_{1}\right)\end{array}$ & $\begin{array}{l}421.1498 \\
(392.20-450.45))\end{array}$ & 14.7691 & 28.52 & $<<.0011$ \\
\hline Change in level $\left(\boldsymbol{B}_{2}\right)$ & 186.3049 & 1025 & 0.18 & 0.8560 \\
\hline $\begin{array}{l}\text { Post-intervention } \\
\text { trend }\left(\boldsymbol{B}_{3}\right)\end{array}$ & -275.5532 & 39.0088 & -7.06 & $<<.0011$ \\
\hline AR1 & $(-352.01--199.10)$ & & & \\
\hline AR3 & 0.4542 & 0.0579 & 7.85 & $<<.0011$ \\
\hline AR12 & -0.2920 & 0.0642 & -4.55 & $<<.0011$ \\
\hline & -0.3999 & 0.0671 & -5.96 & $<<.0011$ \\
\hline
\end{tabular}

\section{E.2.2 Continuous time series analysis}

The shape of the centred moving mean (CMM) graph was the same as the plot of annual prescriptions issued by the practices providing data to the SAIL databank. It demonstrated a steady monthly increase from January 2005 (Figure E.1) which appeared to be slowing and possible nearing plateau following the end of the study period (December 2015). Trendlines were fitted to the data with 'best fit' $\left(R^{2}=0.9968\right)$ demonstrated by a fourth order polynomial line, although the trendlines trialled all had high coefficients of determination. 


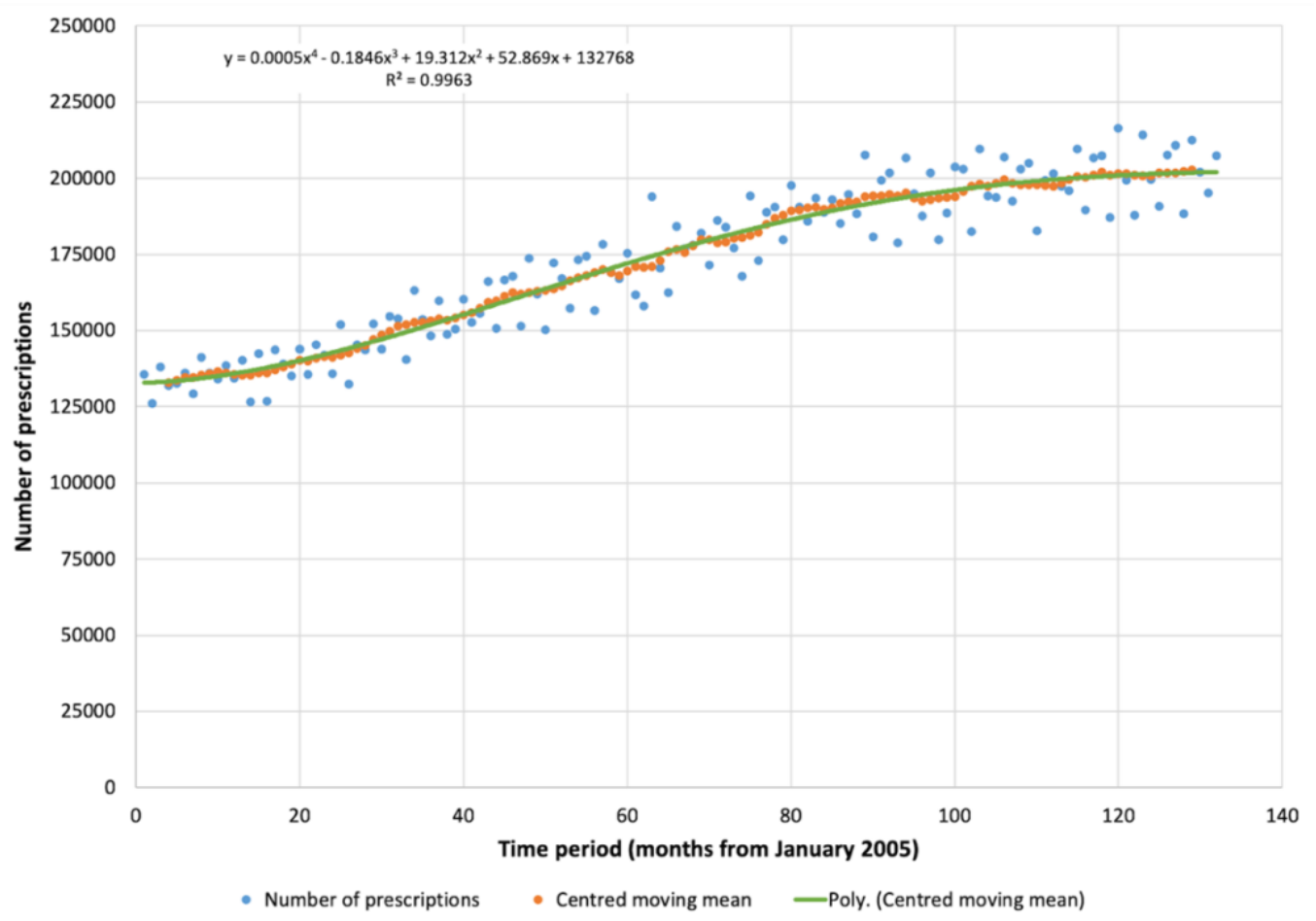

Figure E.1: Monthly trend in the number of opioid prescriptions issued from Primary Care Practices who provide data to SAIL databank. Shown with a polynomial (x4) 'best fit' trendline, $R 2=0.996$

\section{E.2.2.1 Weak opioids}

A fourth polynomial trendline demonstrated the highest coefficient of determination for the time-series of prescription data for weak opioids over the 11-year period examined (Figure E.2). The trendline followed the expected pattern, with a slow decline in the annual number of weak opioid prescriptions issued from around month 100 (2013) to the end of the study period. 


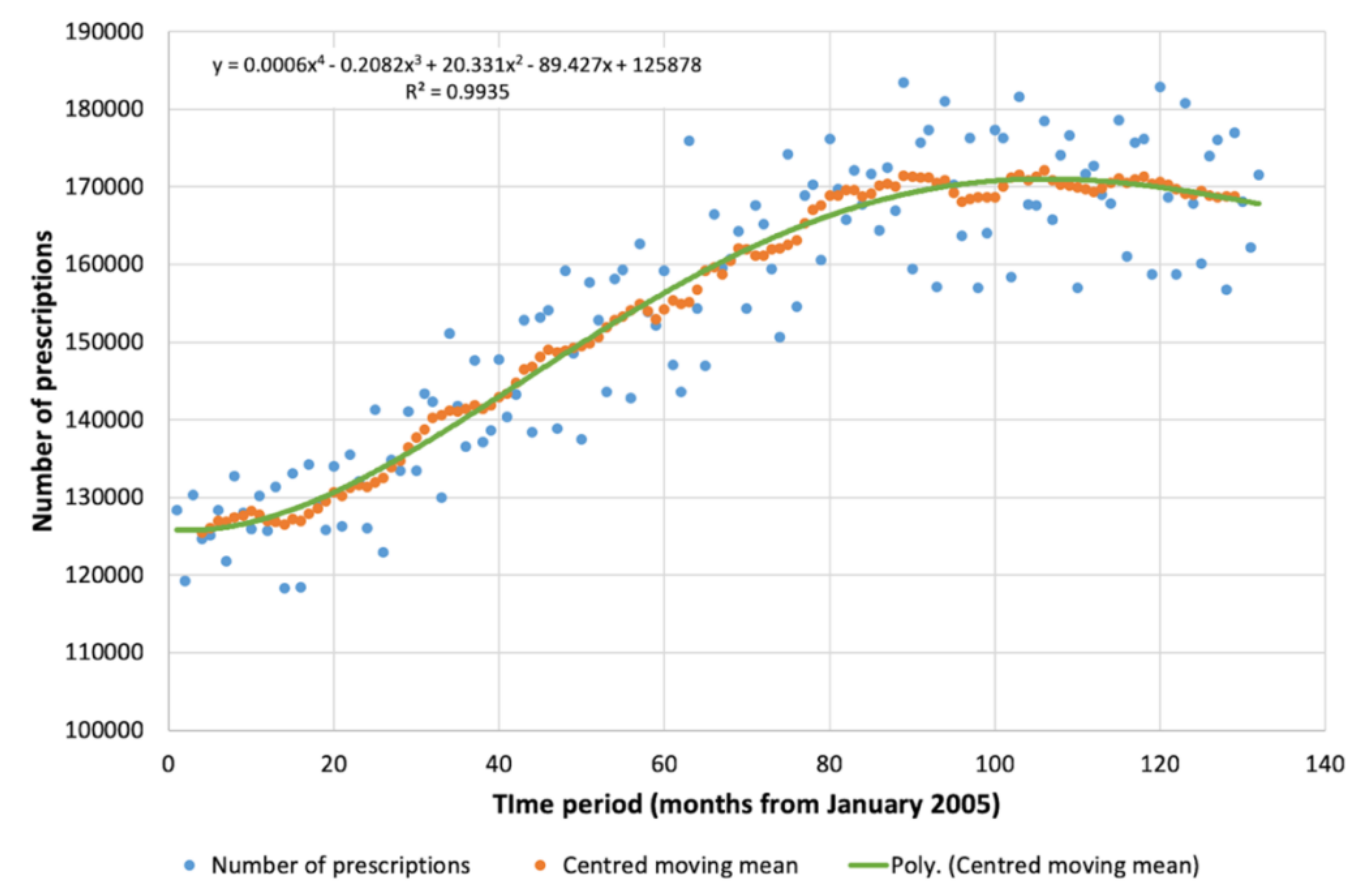

Figure E.2: Monthly trend in the number of weak opioid prescriptions issued from Primary Care Practices who provide data to SAIL databank. Shown with a polynomial 4th order 'best fit' trendline, $R 2=0.9935$

\section{E.2.2.2 Strong opioids}

The number of strong opioid prescriptions increased steadily throughout the 11 years examined and showed no discernible pattern of seasonal variation (Figure E.3). The monthly variation was noted to mirror that seen with weak opioids, inasmuch as positive and negative effects were seen in the same months. Coefficients of determination $\left(\mathrm{R}^{2}\right)$ were good for a number of trendline models, with a fourth order polynomial trendline having the 'best fit' although the third order polynomial trendline produced a similar $\mathrm{R}^{2}$ value.

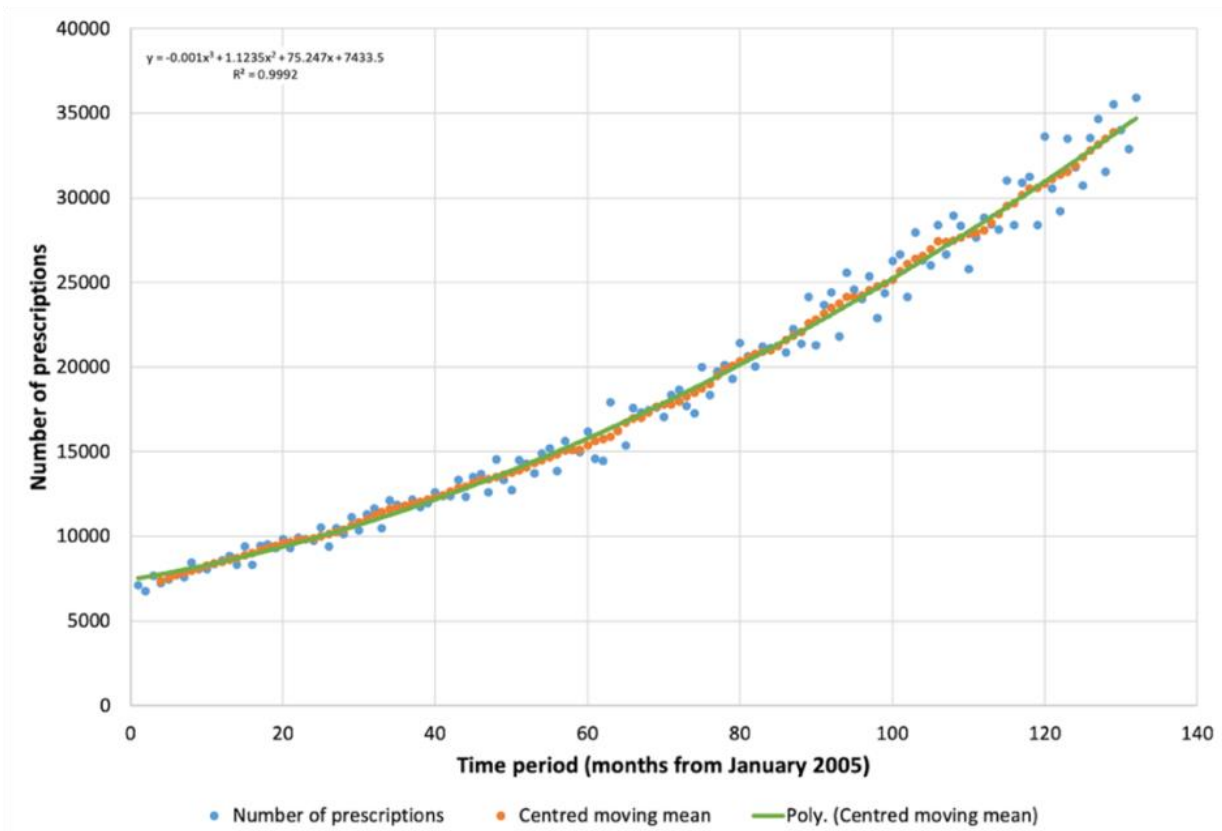

Figure E.3: Monthly trend in the number of strong opioid prescriptions issued from Primary Care Practices who provide data to SAIL databank. Shown with a polynomial trendline, $R 2=0.9992$ 


\section{E.2.2.3 Gender}

Time series analysis by gender confirmed the trend in the number of prescriptions issued over the 11-year study period, seen in previous analysis). The patterns of prescribing and monthly variation were the same for the number of prescriptions issued to men and women (Figure E.4 and Figure E.5). The pattern of average seasonal effect was similar to that seen for opioids overall, with the largest variation seen, for both genders, between January and March (Error! Reference source not found.). The monthly variation did not appear to have any effect on the upward trend in numbers of prescriptions issued, however.

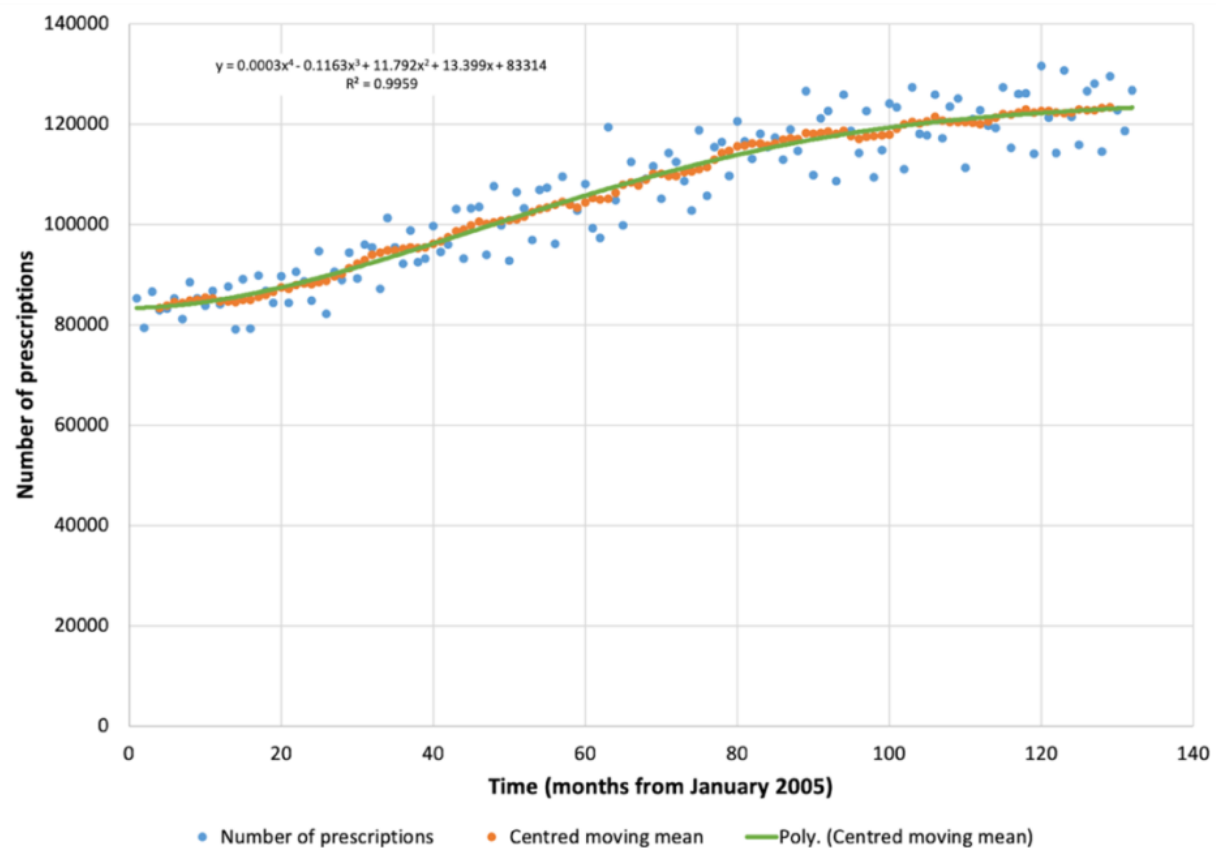

Figure E.4: Monthly trend in the number of opioid prescriptions issued to female patients from Primary Care Practices who provide data to SAIL databank. Shown with a polynomial trendline, $R 2=0.996$

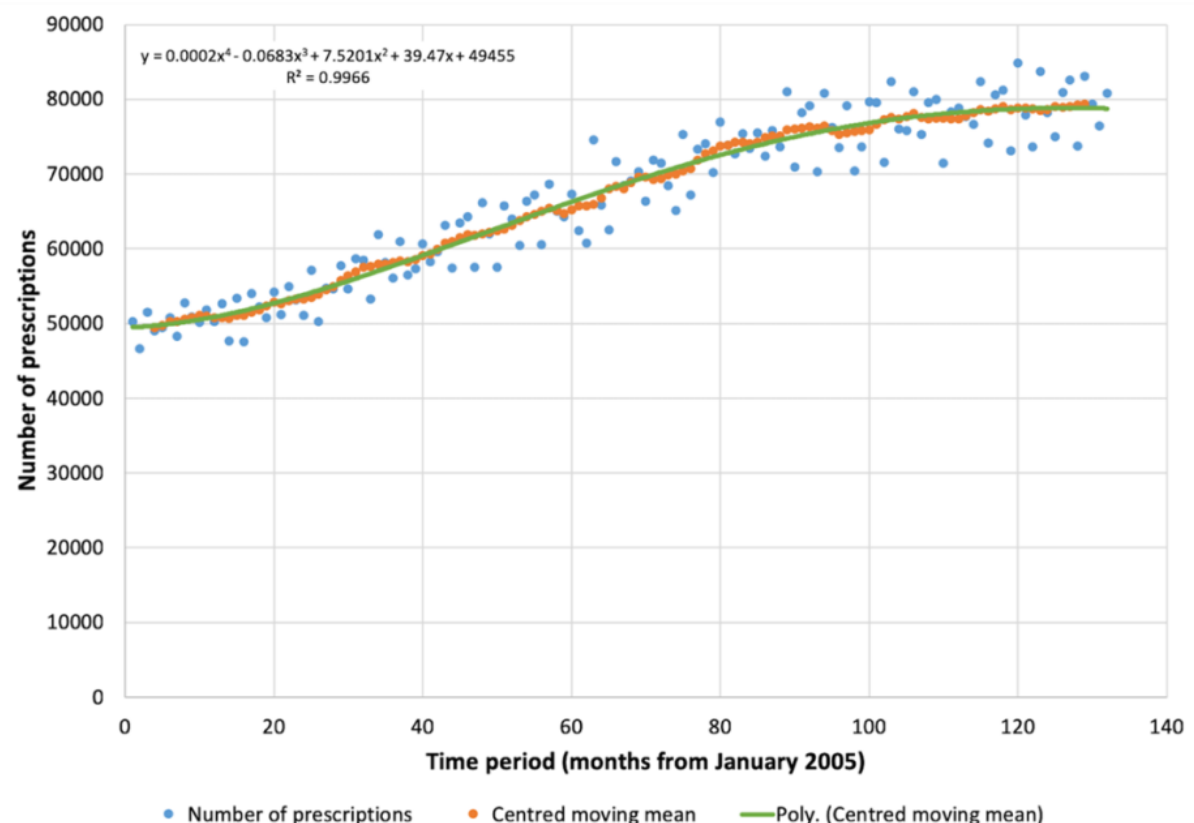

Figure E.5: Monthly trend in the number of opioid prescriptions issued to male patients from Primary Care Practices who provide data to SAIL databank. Shown with a polynomial trendline, $R 2=0.997$ 
Fourth order polynomial trendlines had strong coefficients of determination $\left(R^{2}\right)$ for both women and men and could therefore be seen as strong trends (Table 5 and 6).

\section{E.2.3 Deprivation}

The time series analyses for the different areas of deprivation in Wales, reflected for the most part, the trends noted in the previous analysis of populationadjusted annual prescription numbers (Figure E.6 to Figure E.10). The $4^{\text {th }}$ order polynomial trendline for WIMD1 (most deprived) areas, however, was showing an upturn in $2015\left(R^{2}=0.99\right)$ whereas the other 4 quintiles appeared to be plateauing or starting to reduce.

The trendline for WIMD1 areas (Figure E.6) when fitted with a $3^{\text {rd }}$ order polynomial line, demonstrates the rise in numbers that is already reported (Chapter 4). The accuracy of fit of the trendlines, using $3^{\text {rd }}$ or $4^{\text {th }}$ order polynomial equations, were very good $\left(R^{2}>0.990\right)$ for all areas of deprivation.

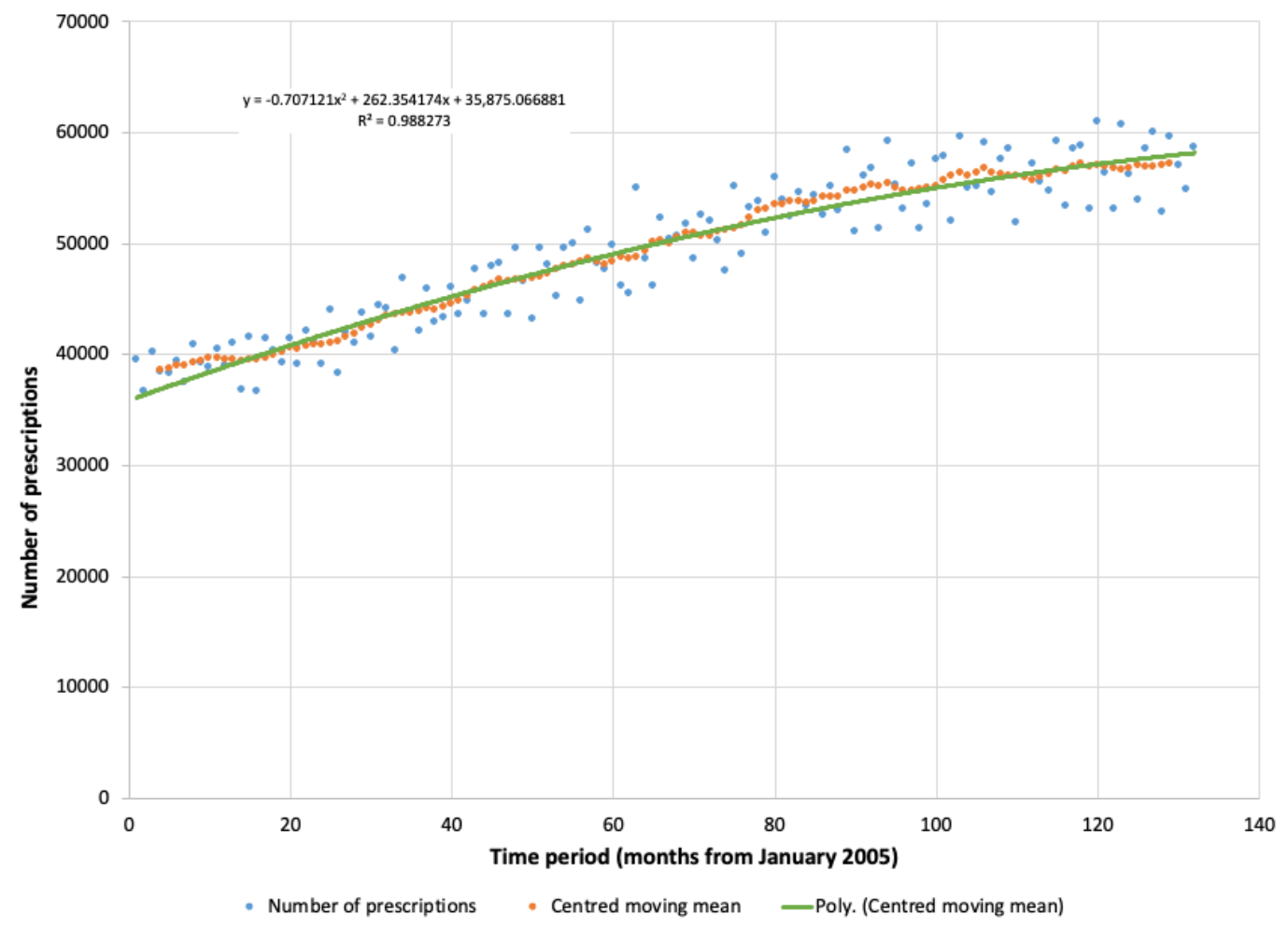

Figure E.6: Monthly trend in the number of opioid prescriptions issued to in WIMD1 (most deprived) areas. Shown with a 3rd order polynomial trendline, $R 2=0.996$ 


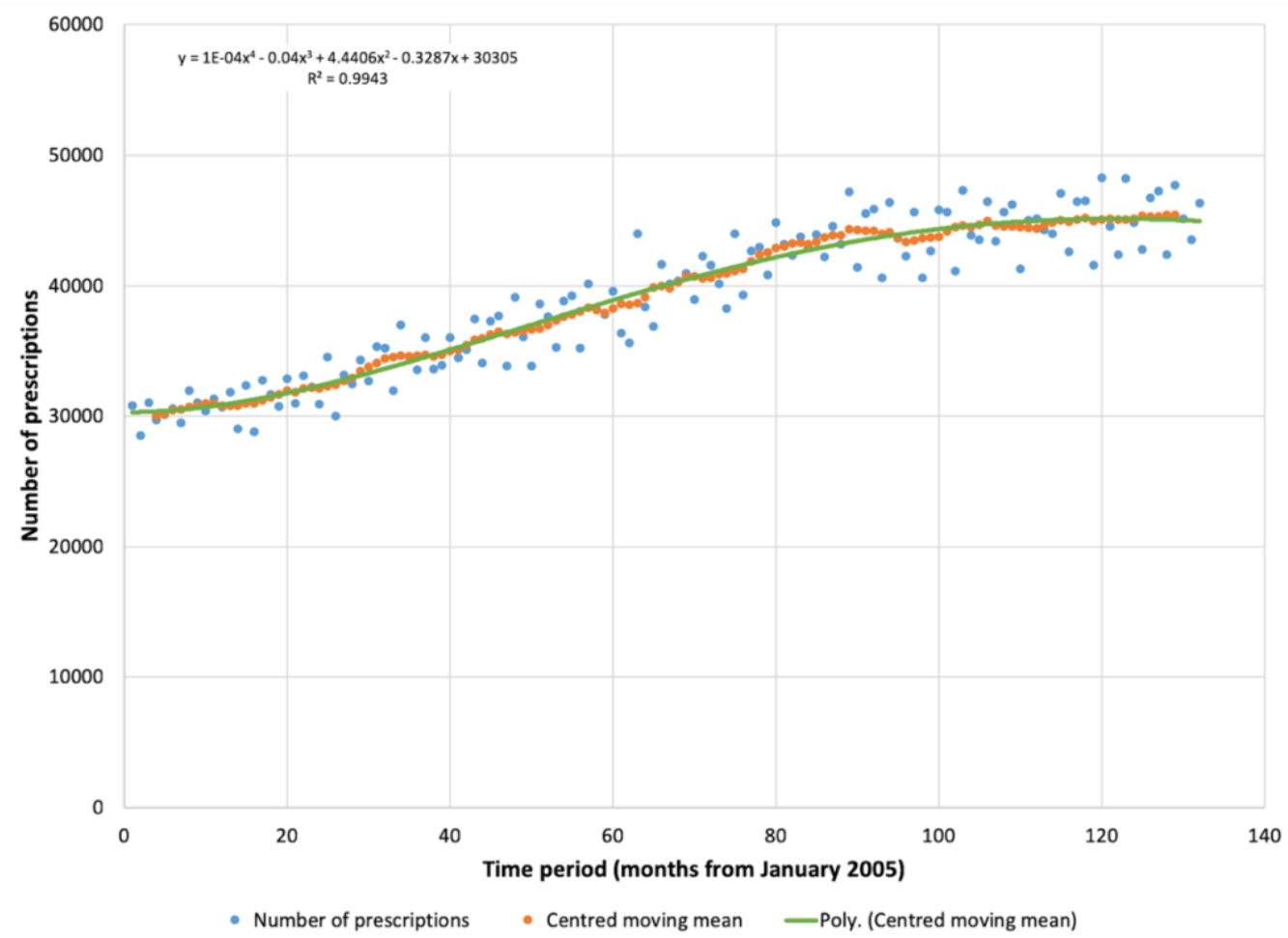

Figure E.7: Monthly trend in the number of opioid prescriptions issued to in WIMD2 areas. Shown with a 4th order polynomial trendline, $R 2=0.994$

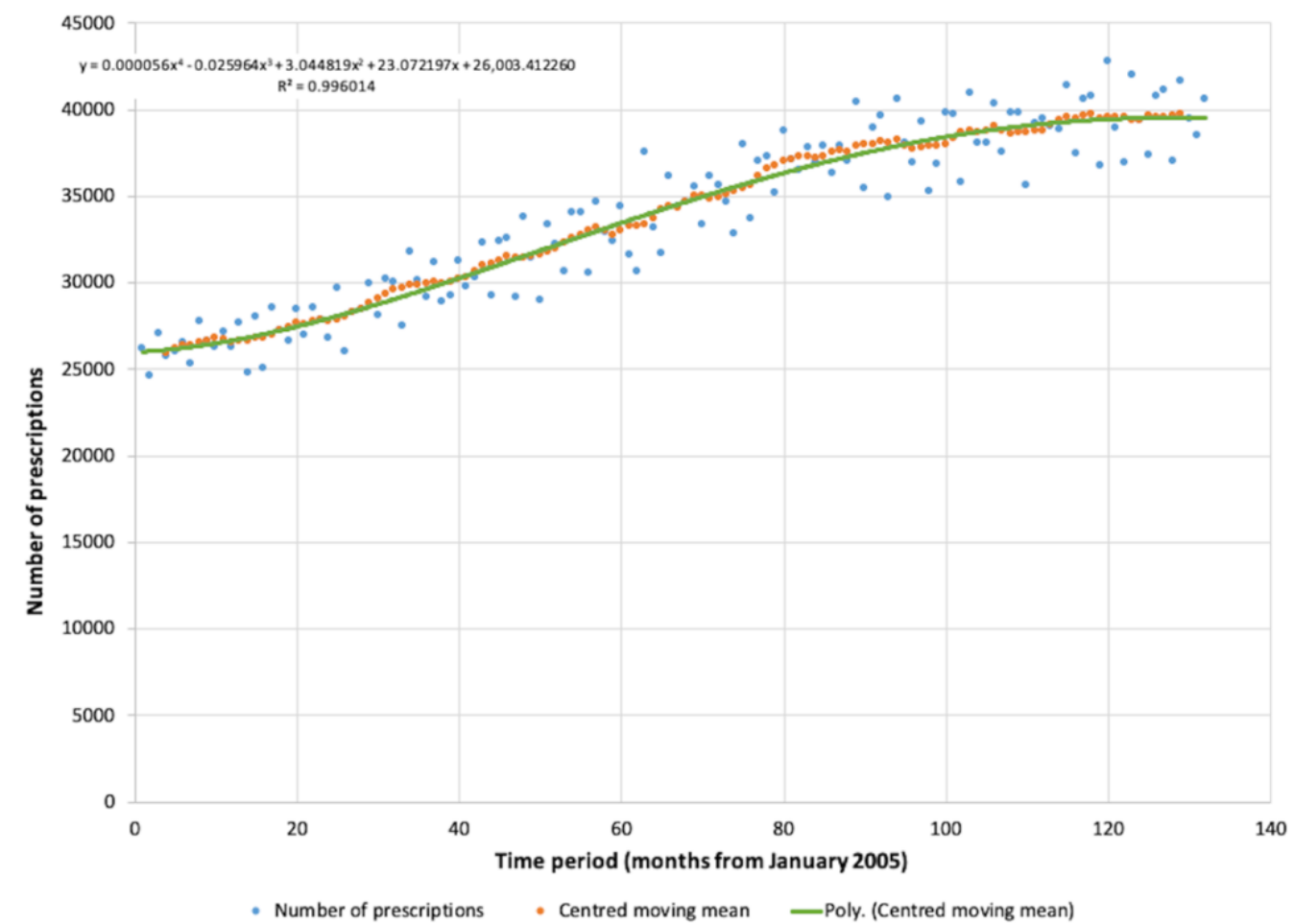

Figure E.8: Monthly trend in the number of opioid prescriptions issued to in WIMD3. Shown with a 4th order polynomial trendline, $R 2=0.996$ 


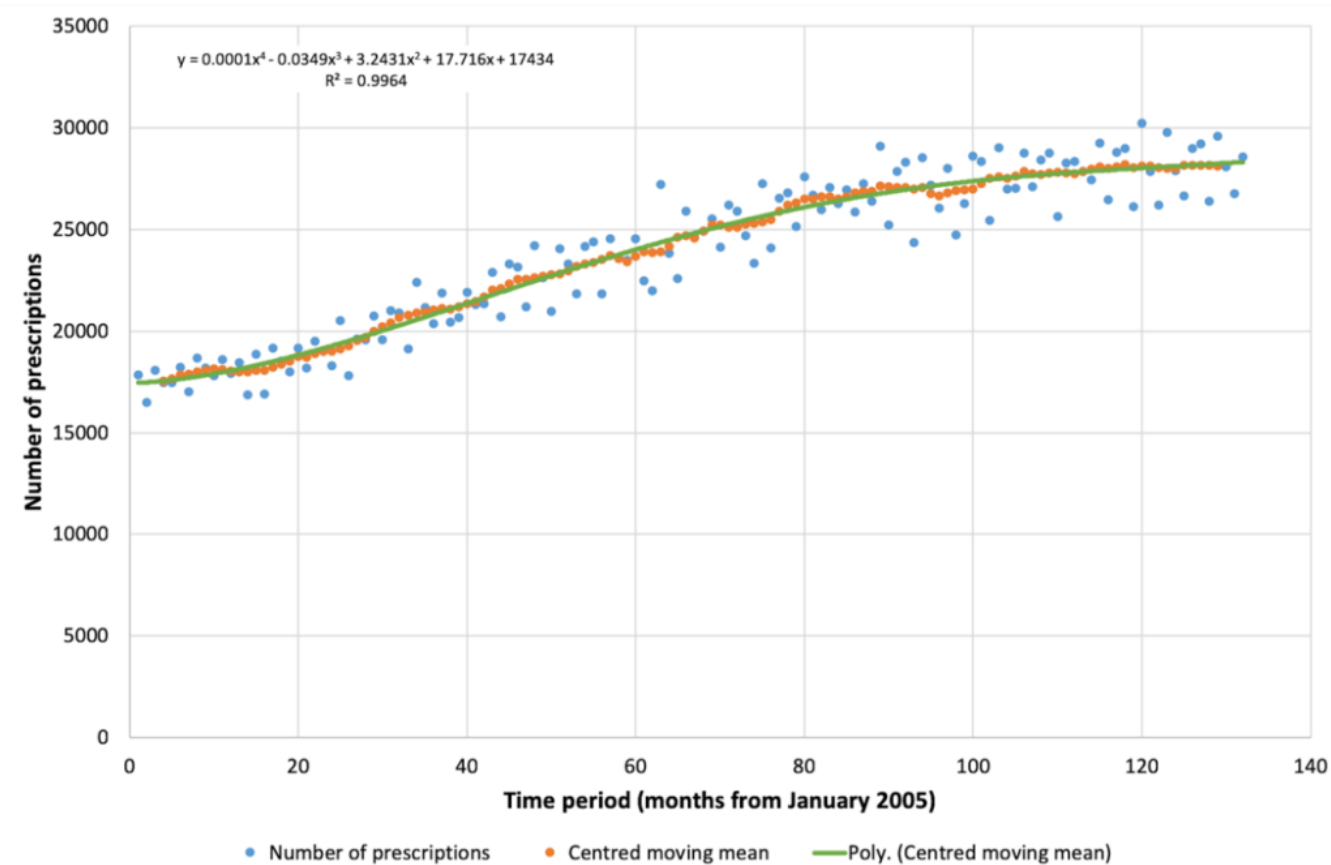

Figure E.9: Monthly trend in the number of opioid prescriptions issued to in WIMD4. Shown with a 4th order polynomial trendline, $R 2=0.996$

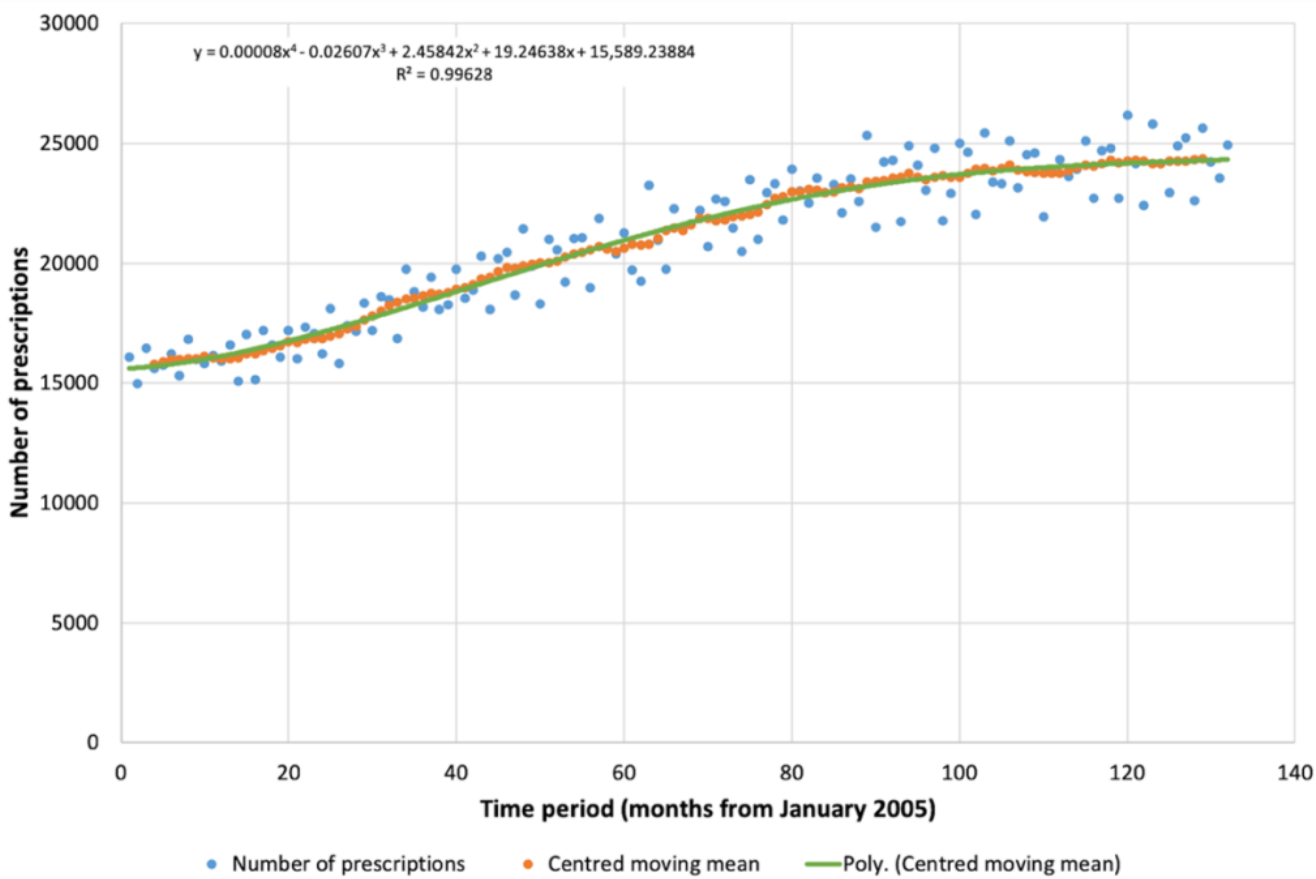

Figure E.10: Monthly trend in the number of opioid prescriptions issued to in WIMD5 (least deprived) areas. Shown with a 4 th order polynomial trendline, $R 2=0.996$ 
Table E.5: Example Time Series Analysis calculations

\begin{tabular}{|c|c|c|c|c|c|c|c|c|c|c|c|c|}
\hline Year & Month & & Frequency & moving means & $\begin{array}{l}\text { centred } \\
\text { moving } \\
\text { mean }\end{array}$ & Month & & Frequency & $\begin{array}{l}\text { centred } \\
\text { moving } \\
\text { mean }\end{array}$ & ISE & SAV & $\begin{array}{l}\text { Below/Above } \\
\text { expectation }\end{array}$ \\
\hline 2005 & 1 & 1 & 5105 & & & 1 & 1 & 5105 & & & 5061.642 & \\
\hline 2005 & 2 & 2 & 4698 & & & 2 & 2 & 4698 & & & 5092.808 & \\
\hline 2005 & 3 & 3 & 5254 & & & 3 & 3 & 5254 & & & 5000.575 & \\
\hline 2005 & 4 & 4 & 4977 & 5018 & 4988 & 4 & 4 & 4977 & 4988 & -10.9167 & 5013.545 & Above \\
\hline 2005 & 5 & 5 & 4976 & 4958 & 4988 & 5 & 5 & 4976 & 4988 & -11.75 & 4984.795 & Below \\
\hline 2005 & 6 & 6 & 5099 & 5018 & 4994 & 6 & 6 & 5099 & 4994 & 105.25 & 5164.273 & Above \\
\hline 2005 & 7 & 7 & 4742 & 4970 & 4965 & 7 & 7 & 4742 & 4965 & -222.75 & 4625.826 & Below \\
\hline 2005 & 8 & 8 & 5059 & 4960 & 4947 & 8 & 8 & 5059 & 4947 & 112.5 & 5153.856 & Above \\
\hline 2005 & 9 & 9 & 4965 & 4933 & 4886 & 9 & 9 & 4965 & 4886 & 79.16667 & 4965.235 & Above \\
\hline 2005 & 10 & 10 & 4918 & 4839 & 4832 & 10 & 10 & 4918 & 4832 & 85.83333 & 4789.875 & Below \\
\hline 2005 & 11 & 11 & 4816 & 4826 & 4747 & 11 & 11 & 4816 & 4747 & 69.25 & 4847.2 & Above \\
\hline 2005 & 12 & 12 & 4531 & 4668 & 4650 & 12 & 12 & 4531 & 4650 & -118.667 & 4454.217 & Below \\
\hline 2006 & 1 & 13 & 4666 & 4632 & 4577 & 1 & 13 & 4666 & 4577 & 89.25 & 4622.642 & Above \\
\hline 2006 & 3 & 15 & 4749 & 4508 & 4517 & 3 & 15 & 4749 & 4517 & 231.5833 & 4495.575 & Below \\
\hline 2006 & 4 & 16 & 4259 & 4527 & 4510 & 4 & 16 & 4259 & 4510 & -251.167 & 4295.545 & Below \\
\hline 2006 & 5 & 17 & 4734 & 4494 & 4548 & 5 & 17 & 4734 & 4548 & 185.75 & 4742.795 & Above \\
\hline 2006 & 6 & 18 & 4642 & 4603 & 4575 & 6 & 18 & 4642 & 4575 & 67.08333 & 4707.273 & Above \\
\hline 2006 & 7 & 19 & 4468 & 4547 & 4600 & 7 & 19 & 4468 & 4600 & -132.083 & 4351.826 & Below \\
\hline 2006 & 8 & 20 & 4765 & 4653 & 4657 & 8 & 20 & 4765 & 4657 & 107.5833 & 4859.856 & Above \\
\hline 2006 & 9 & 21 & 4414 & 4662 & 4657 & 9 & 21 & 4414 & 4657 & -242.833 & 4414.235 & Below \\
\hline 2006 & 10 & 22 & 4896 & 4652 & 4700 & 10 & 22 & 4896 & 4700 & 196.25 & 4767.875 & Above \\
\hline 2006 & 11 & 23 & 4785 & 4748 & 4726 & 11 & 23 & 4785 & 4726 & 58.66667 & 4816.2 & Above \\
\hline 2006 & 12 & 24 & 4584 & 4705 & 4746 & 12 & 24 & 4584 & 4746 & -162.333 & 4507.217 & Below \\
\hline 2007 & 1 & 25 & 5041 & 4788 & 4783 & 1 & 25 & 5041 & 4783 & 258.25 & 4997.642 & Above \\
\hline
\end{tabular}




\begin{tabular}{|c|c|c|c|c|c|c|c|c|c|c|c|c|}
\hline 2007 & 2 & 26 & 4511 & 4778 & 4802 & 2 & 26 & 4511 & 4802 & -290.667 & 4905.808 & Above \\
\hline 2007 & 3 & 27 & 4908 & 4825 & 4839 & 3 & 27 & 4908 & 4839 & 68.58333 & 4654.575 & Below \\
\hline 2007 & 4 & 28 & 4839 & 4854 & 4867 & 4 & 28 & 4839 & 4867 & -27.6667 & 4875.545 & Above \\
\hline 2007 & 5 & 29 & 5069 & 4880 & 4941 & 5 & 29 & 5069 & 4941 & 128.1667 & 5077.795 & Above \\
\hline 2007 & 6 & 30 & 4753 & 5002 & 4983 & 6 & 30 & 4753 & 4983 & -230.417 & 4818.273 & Below \\
\hline 2007 & 7 & 31 & 5199 & 4965 & 5018 & 7 & 31 & 5199 & 5018 & 180.8333 & 5082.826 & Above \\
\hline 2007 & 8 & 32 & 5243 & 5071 & 5082 & 8 & 32 & 5243 & 5082 & 161.5 & 5337.856 & Above \\
\hline 2007 & 9 & 33 & 4687 & 5092 & 5104 & 9 & 33 & 4687 & 5104 & -417.083 & 4687.235 & Below \\
\hline 2007 & 10 & 34 & 5477 & 5117 & 5129 & 10 & 34 & 5477 & 5129 & 348.1667 & 5348.875 & Above \\
\hline 2007 & 11 & 35 & 5191 & 5141 & 5118 & 11 & 35 & 5191 & 5118 & 72.66667 & 5222.2 & Above \\
\hline 2007 & 12 & 36 & 4902 & 5096 & 5135 & 12 & 36 & 4902 & 5135 & -232.5 & 4825.217 & Below \\
\hline 2008 & 1 & 37 & 5347 & 5174 & 5167 & 1 & 37 & 5347 & 5167 & 179.5833 & 5303.642 & Above \\
\hline 2008 & 2 & 38 & 4969 & 5161 & 5155 & 2 & 38 & 4969 & 5155 & -186.167 & 5363.808 & Above \\
\hline 2008 & 3 & 39 & 5155 & 5149 & 5170 & 3 & 39 & 5155 & 5170 & -15.3333 & 4901.575 & Below \\
\hline 2008 & 4 & 40 & 5404 & 5192 & 5217 & 4 & 40 & 5404 & 5217 & 187.0833 & 5440.545 & Above \\
\hline 2008 & 5 & 41 & 5117 & 5242 & 5251 & 5 & 41 & 5117 & 5251 & -134.25 & 5125.795 & Below \\
\hline 2008 & 7 & 43 & 5650 & 5328 & 5350 & 7 & 43 & 5650 & 5350 & 299.8333 & 5533.826 & Above \\
\hline 2008 & 8 & 44 & 5078 & 5372 & 5364 & 8 & 44 & 5078 & 5364 & -286.417 & 5172.856 & Below \\
\hline 2008 & 9 & 45 & 5562 & 5357 & 5402 & 9 & 45 & 5562 & 5402 & 160 & 5562.235 & Above \\
\hline 2008 & 10 & 46 & 5668 & 5447 & 5421 & 10 & 46 & 5668 & 5421 & 246.6667 & 5539.875 & Above \\
\hline 2008 & 11 & 47 & 5024 & 5395 & 5385 & 11 & 47 & 5024 & 5385 & -361 & 5055.2 & Below \\
\hline 2008 & 12 & 48 & 5702 & 5375 & 5389 & 12 & 48 & 5702 & 5389 & 313.1667 & 5625.217 & Above \\
\hline 2009 & 1 & 49 & 5338 & 5403 & 5387 & 1 & 49 & 5338 & 5387 & -48.9167 & 5294.642 & Below \\
\hline 2009 & 2 & 50 & 4954 & 5371 & 5384 & 2 & 50 & 4954 & 5384 & -429.75 & 5348.808 & Below \\
\hline 2009 & 3 & 51 & 5732 & 5397 & 5388 & 3 & 51 & 5732 & 5388 & 344.4167 & 5478.575 & Above \\
\hline 2009 & 4 & 52 & 5475 & 5379 & 5414 & 4 & 52 & 5475 & 5414 & 60.83333 & 5511.545 & Above \\
\hline 2009 & 5 & 53 & 5179 & 5450 & 5468 & 5 & 53 & 5179 & 5468 & -289.417 & 5187.795 & Below \\
\hline
\end{tabular}




\begin{tabular}{|c|c|c|c|c|c|c|c|c|c|c|c|c|}
\hline 2009 & 6 & 54 & 5593 & 5487 & 5498 & 6 & 54 & 5593 & 5498 & 95 & 5658.273 & Above \\
\hline 2009 & 7 & 55 & 5766 & 5509 & 5511 & 7 & 55 & 5766 & 5511 & 254.6667 & 5649.826 & Above \\
\hline 2009 & 8 & 56 & 5177 & 5514 & 5535 & 8 & 56 & 5177 & 5535 & -357.833 & 5271.856 & Below \\
\hline 2009 & 9 & 57 & 5864 & 5556 & 5565 & 9 & 57 & 5864 & 5565 & 298.8333 & 5864.235 & Above \\
\hline 2009 & 10 & 58 & 5503 & 5574 & 5532 & 10 & 58 & 5503 & 5532 & -28.75 & 5374.875 & Below \\
\hline 2009 & 11 & 59 & 5433 & 5489 & 5490 & 11 & 59 & 5433 & 5490 & -57 & 5464.2 & Below \\
\hline 2009 & 12 & 60 & 5703 & 5491 & 5576 & 12 & 60 & 5703 & 5576 & 126.5833 & 5626.217 & Above \\
\hline 2010 & 1 & 61 & 5255 & 5662 & 5671 & 1 & 61 & 5255 & 5671 & -415.667 & 5211.642 & Below \\
\hline 2010 & 2 & 62 & 5187 & 5679 & 5668 & 2 & 62 & 5187 & 5668 & -480.667 & 5581.808 & Below \\
\hline 2010 & 3 & 63 & 6891 & 5656 & 5676 & 3 & 63 & 6891 & 5676 & 1214.75 & 6637.575 & Above \\
\hline 2010 & 4 & 64 & 5607 & 5697 & 5738 & 4 & 64 & 5607 & 5738 & -131.167 & 5643.545 & Below \\
\hline 2010 & 5 & 65 & 5293 & 5780 & 5842 & 5 & 65 & 5293 & 5842 & -548.583 & 5301.795 & Below \\
\hline 2010 & 6 & 66 & 5946 & 5903 & 5833 & 6 & 66 & 5946 & 5833 & 113.5 & 6011.273 & Above \\
\hline 2010 & 7 & 67 & 5755 & 5762 & 5777 & 7 & 67 & 5755 & 5777 & -21.8333 & 5638.826 & Below \\
\hline 2010 & 8 & 68 & 5928 & 5792 & 5867 & 8 & 68 & 5928 & 5867 & 60.58333 & 6022.856 & Above \\
\hline 2010 & 9 & 69 & 6041 & 5943 & 5966 & 9 & 69 & 6041 & 5966 & 74.91667 & 6041.235 & Above \\
\hline 2010 & 10 & 70 & 5789 & 5989 & 6004 & 10 & 70 & 5789 & 6004 & -214.75 & 5660.875 & Below \\
\hline 2010 & 11 & 71 & 6198 & 6018 & 5985 & 11 & 71 & 6198 & 5985 & 212.9167 & 6229.2 & Above \\
\hline 2010 & 12 & 72 & 6225 & 5952 & 5981 & 12 & 72 & 6225 & 5981 & 243.5833 & 6148.217 & Above \\
\hline 2011 & 1 & 73 & 5928 & 6011 & 6006 & 1 & 73 & 5928 & 6006 & -78.1667 & 5884.642 & Below \\
\hline 2011 & 2 & 74 & 5531 & 6002 & 6007 & 2 & 74 & 5531 & 6007 & -475.5 & 5925.808 & Below \\
\hline 2011 & 3 & 75 & 6394 & 6012 & 6019 & 3 & 75 & 6394 & 6019 & 374.6667 & 6140.575 & Above \\
\hline 2011 & 4 & 76 & 5733 & 6027 & 6029 & 4 & 76 & 5733 & 6029 & -295.5 & 5769.545 & Below \\
\hline 2011 & 5 & 77 & 6258 & 6030 & 6116 & 5 & 77 & 6258 & 6116 & 142.3333 & 6266.795 & Above \\
\hline 2011 & 6 & 78 & 6319 & 6202 & 6208 & 6 & 78 & 6319 & 6208 & 110.9167 & 6384.273 & Above \\
\hline 2011 & 7 & 79 & 5944 & 6215 & 6249 & 7 & 79 & 5944 & 6249 & -305.417 & 5827.826 & Below \\
\hline 2011 & 8 & 80 & 6561 & 6284 & 6317 & 8 & 80 & 6561 & 6317 & 244.25 & 6655.856 & Above \\
\hline 2011 & 9 & 81 & 6473 & 6349 & 6348 & 9 & 81 & 6473 & 6348 & 125.0833 & 6473.235 & Above \\
\hline
\end{tabular}




\begin{tabular}{|c|c|c|c|c|c|c|c|c|c|c|c|c|}
\hline 2011 & 10 & 82 & 6150 & 6347 & 6395 & 10 & 82 & 6150 & 6395 & -244.667 & 6021.875 & Below \\
\hline 2011 & 11 & 83 & 6649 & 6443 & 6408 & 11 & 83 & 6649 & 6408 & 241.3333 & 6680.2 & Above \\
\hline 2011 & 12 & 84 & 6302 & 6373 & 6376 & 12 & 84 & 6302 & 6376 & -74.4167 & 6225.217 & Below \\
\hline 2012 & 1 & 85 & 6522 & 6380 & 6389 & 1 & 85 & 6522 & 6389 & 132.5833 & 6478.642 & Above \\
\hline 2012 & 2 & 86 & 6139 & 6399 & 6440 & 2 & 86 & 6139 & 6440 & -301.25 & 6533.808 & Above \\
\hline 2012 & 3 & 87 & 6520 & 6482 & 6475 & 3 & 87 & 6520 & 6475 & 45.08333 & 6266.575 & Below \\
\hline 2012 & 4 & 88 & 6259 & 6468 & 6495 & 4 & 88 & 6259 & 6495 & -235.917 & 6295.545 & Below \\
\hline 2012 & 5 & 89 & 7150 & 6522 & 6582 & 5 & 89 & 7150 & 6582 & 568.4167 & 7158.795 & Above \\
\hline 2012 & 6 & 90 & 6217 & 6641 & 6595 & 6 & 90 & 6217 & 6595 & -378.417 & 6282.273 & Below \\
\hline 2012 & 7 & 91 & 6847 & 6550 & 6609 & 7 & 91 & 6847 & 6609 & 238.25 & 6730.826 & Above \\
\hline 2012 & 8 & 92 & 6854 & 6668 & 6622 & 8 & 92 & 6854 & 6622 & 232.3333 & 6948.856 & Above \\
\hline 2012 & 9 & 93 & 5971 & 6576 & 6587 & 9 & 93 & 5971 & 6587 & -615.75 & 5971.235 & Below \\
\hline 2012 & 10 & 94 & 6968 & 6598 & 6589 & 10 & 94 & 6968 & 6589 & 378.9167 & 6839.875 & Above \\
\hline 2012 & 11 & 95 & 6596 & 6580 & 6522 & 11 & 95 & 6596 & 6522 & 73.58333 & 6627.2 & Above \\
\hline 2012 & 12 & 96 & 6352 & 6465 & 6487 & 12 & 96 & 6352 & 6487 & -135.417 & 6275.217 & Below \\
\hline 2013 & 1 & 97 & 6740 & 6510 & 6502 & 1 & 97 & 6740 & 6502 & 238.0833 & 6696.642 & Above \\
\hline 2013 & 2 & 98 & 6161 & 6494 & 6511 & 2 & 98 & 6161 & 6511 & -350.25 & 6555.808 & Above \\
\hline 2013 & 3 & 99 & 6244 & 6529 & 6516 & 3 & 99 & 6244 & 6516 & -272.083 & 5990.575 & Below \\
\hline 2013 & 4 & 100 & 6869 & 6503 & 6539 & 4 & 100 & 6869 & 6539 & 329.5833 & 6905.545 & Above \\
\hline 2013 & 5 & 101 & 6807 & 6576 & 6624 & 5 & 101 & 6807 & 6624 & 183.1667 & 6815.795 & Above \\
\hline 2013 & 6 & 102 & 6199 & 6672 & 6710 & 6 & 102 & 6199 & 6710 & -510.583 & 6264.273 & Below \\
\hline 2013 & 7 & 103 & 7173 & 6747 & 6778 & 7 & 103 & 7173 & 6778 & 395.5 & 7056.826 & Above \\
\hline 2013 & 8 & 104 & 6741 & 6808 & 6803 & 8 & 104 & 6741 & 6803 & -61.9167 & 6835.856 & Above \\
\hline 2013 & 9 & 105 & 6693 & 6798 & 6876 & 9 & 105 & 6693 & 6876 & -182.75 & 6693.235 & Below \\
\hline 2013 & 10 & 106 & 7235 & 6954 & 6948 & 10 & 106 & 7235 & 6948 & 287.5 & 7106.875 & Above \\
\hline 2013 & 11 & 107 & 6746 & 6941 & 6909 & 11 & 107 & 6746 & 6909 & -163.083 & 6777.2 & Below \\
\hline 2013 & 12 & 108 & 7134 & 6877 & 6902 & 12 & 108 & 7134 & 6902 & 232 & 7057.217 & Above \\
\hline 2014 & 1 & 109 & 7099 & 6927 & 6920 & 1 & 109 & 7099 & 6920 & 179.25 & 7055.642 & Above \\
\hline
\end{tabular}




\begin{tabular}{|c|c|c|c|c|c|c|c|c|c|c|c|c|}
\hline 2014 & 2 & 110 & 6354 & 6912 & 6942 & 2 & 110 & 6354 & 6942 & -587.667 & 6748.808 & Below \\
\hline 2014 & 3 & 111 & 6995 & 6971 & 6961 & 3 & 111 & 6995 & 6961 & 34.25 & 6741.575 & Below \\
\hline 2014 & 4 & 112 & 7146 & 6951 & 6985 & 4 & 112 & 7146 & 6985 & 160.75 & 7182.545 & Above \\
\hline 2014 & 5 & 113 & 7098 & 7020 & 7057 & 5 & 113 & 7098 & 7057 & 40.91667 & 7106.795 & Above \\
\hline 2014 & 6 & 114 & 7011 & 7094 & 7142 & 6 & 114 & 7011 & 7142 & -131.417 & 7076.273 & Below \\
\hline 2014 & 7 & 115 & 7516 & 7191 & 7230 & 7 & 115 & 7516 & 7230 & 286 & 7399.826 & Above \\
\hline 2014 & 8 & 116 & 6799 & 7269 & 7255 & 8 & 116 & 6799 & 7255 & -456.417 & 6893.856 & Below \\
\hline 2014 & 9 & 117 & 7574 & 7242 & 7325 & 9 & 117 & 7574 & 7325 & 249.4167 & 7574.235 & Above \\
\hline 2014 & 10 & 118 & 7618 & 7408 & 7392 & 10 & 118 & 7618 & 7392 & 226.0833 & 7489.875 & Above \\
\hline 2014 & 11 & 119 & 6931 & 7376 & 7390 & 11 & 119 & 6931 & 7390 & -459.333 & 6962.2 & Below \\
\hline 2014 & 12 & 120 & 8008 & 7405 & 7432 & 12 & 120 & 8008 & 7432 & 575.8333 & 7931.217 & Above \\
\hline 2015 & 1 & 121 & 7327 & 7460 & 7428 & 1 & 121 & 7327 & 7428 & -100.667 & 7283.642 & Below \\
\hline 2015 & 2 & 122 & 6969 & 7396 & 7410 & 2 & 122 & 6969 & 7410 & -441.167 & 7363.808 & Below \\
\hline 2015 & 3 & 123 & 7906 & 7425 & 7398 & 3 & 123 & 7906 & 7398 & 508.3333 & 7652.575 & Above \\
\hline 2015 & 4 & 124 & 7232 & 7371 & 7420 & 4 & 124 & 7232 & 7420 & -187.917 & 7268.545 & Below \\
\hline 2015 & 5 & 125 & 7107 & 7469 & 7469 & 5 & 125 & 7107 & 7469 & -361.5 & 7115.795 & Below \\
\hline 2015 & 6 & 126 & 7682 & 7468 & 7505 & 6 & 126 & 7682 & 7505 & 177.3333 & 7747.273 & Above \\
\hline 2015 & 7 & 127 & 7920 & 7542 & 7615 & 7 & 127 & 7920 & 7615 & 304.9167 & 7803.826 & Above \\
\hline 2015 & 8 & 128 & 6959 & 7689 & 7759 & 8 & 128 & 6959 & 7759 & -799.583 & 7053.856 & Below \\
\hline 2015 & 9 & 129 & 8350 & 7829 & 7882 & 9 & 129 & 8350 & 7882 & 468.4167 & 8350.235 & Above \\
\hline 2015 & 10 & 130 & 8113 & 7935 & & 10 & 130 & 8113 & & & 7984.875 & Above \\
\hline 2015 & 11 & 131 & 7948 & & & 11 & 131 & 7948 & & & 7979.2 & Above \\
\hline 2015 & 12 & 132 & 8317 & & & 12 & 132 & 8317 & & & 8240.217 & Above \\
\hline 2016 & 1 & 133 & 27287 & & & & & & & & & \\
\hline 2016 & 2 & 134 & & & & & & & & & & \\
\hline
\end{tabular}


Average seasonal

effects

$\begin{array}{ll}\text { January } & 43.35833 \\ \text { February } & -394.808 \\ \text { March } & 253.425 \\ & \\ \text { April } & -36.5455 \\ \text { May } & -8.79545 \\ \text { June } & -65.2727 \\ & \\ \text { July } & 116.1742 \\ & \\ \text { August } & -94.8561 \\ \text { September } & -0.23485 \\ \text { October } & 128.125 \\ \text { November } & -31.2 \\ \text { December } & 76.78333\end{array}$

*When the seasonal effect is positive this means that this month you would expect more patients than another month.

To get a prediction, substitute the time value into the equation on the graph and add the seasonal effect

E.g.,

Predicted value for January 2019 would be:

$y=629.1 x \quad$ Seasonal effect $=$

$x=169+131026$

2349.183

Prediction for Jan 2019

237387.3

Predicted value for February 2019 would be:

$\begin{array}{lll} & y=629.1 x & \text { Seasonal effect }=\text { - } \\ x=170 \quad+131026 & 11018.1 \\ \text { Prediction for Feb } 2019 & \\ \text { 237578.2 } & \end{array}$

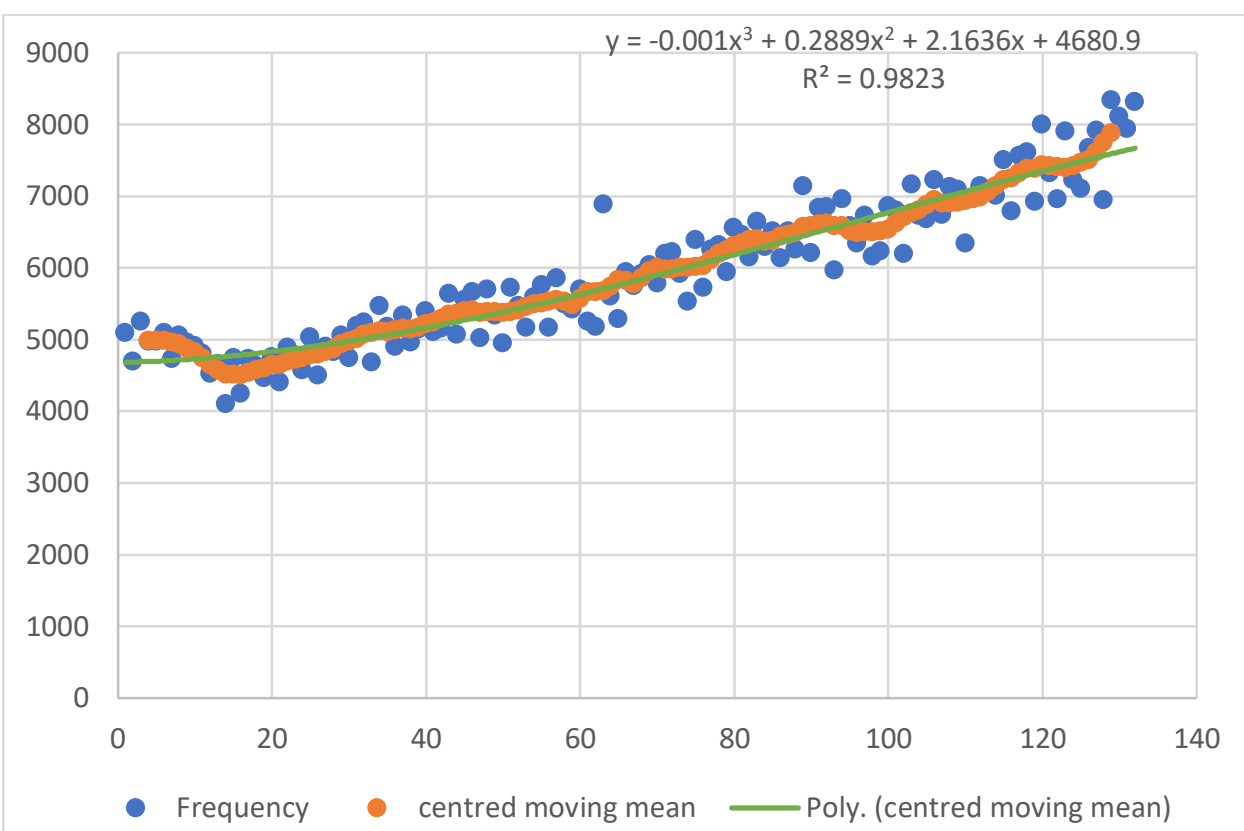

Figure E.11: Example of calculation for seasonal variation (Top) and example TSA plot from which the seasonal variations were calculated

\section{E.3 Predictions}

\section{E.3.1 Predictions for weak opioid prescribing}

The predicted trends in the number of weak opioid prescriptions using the equations generated from the trendline analyses, were very similar to those seen for all opioid prescribing (Figure E.12). Two predictions were for prescribing to continue to prescribe, albeit following the quadratic polynomial prediction, there 
would be an initial plateauing prior to a further increase. As with the total opioid predictions, the $3^{\text {rd }}$ order polynomial equation suggests there could be a dramatic reduction in the number of weak opioid prescriptions issued. Whilst that trendline had a high $\mathrm{R}^{2}$ value (Table E.6) at prediction hints weak opioid prescribing could reduce to zero by the end of 2022.

Table E.6: Comparison of predicted trends in weak opioid prescribing using trendline analysis

\begin{tabular}{|c|c|c|c|c|}
\hline & \multicolumn{3}{|c|}{ Polynomial } & \multirow[t]{2}{*}{ Linear } \\
\hline & $x^{2}$ & $x^{3}$ & $x^{4}$ & \\
\hline $\mathbf{R}^{2}$ & 0.9767 & 0.9916 & 0.9935 & 0.9054 \\
\hline \multicolumn{5}{|l|}{ Predicted values } \\
\hline January 2018 & 169996 & 142712 & 174157 & 194848 \\
\hline January 2020 & 162264 & 98508 & 187502 & 204921 \\
\hline
\end{tabular}

The linear trendline had the lowest coefficient of determination of any of the lines (Table 3 ) and was suggestive of a continued increase in the annual number of weak opioid prescriptions issued.

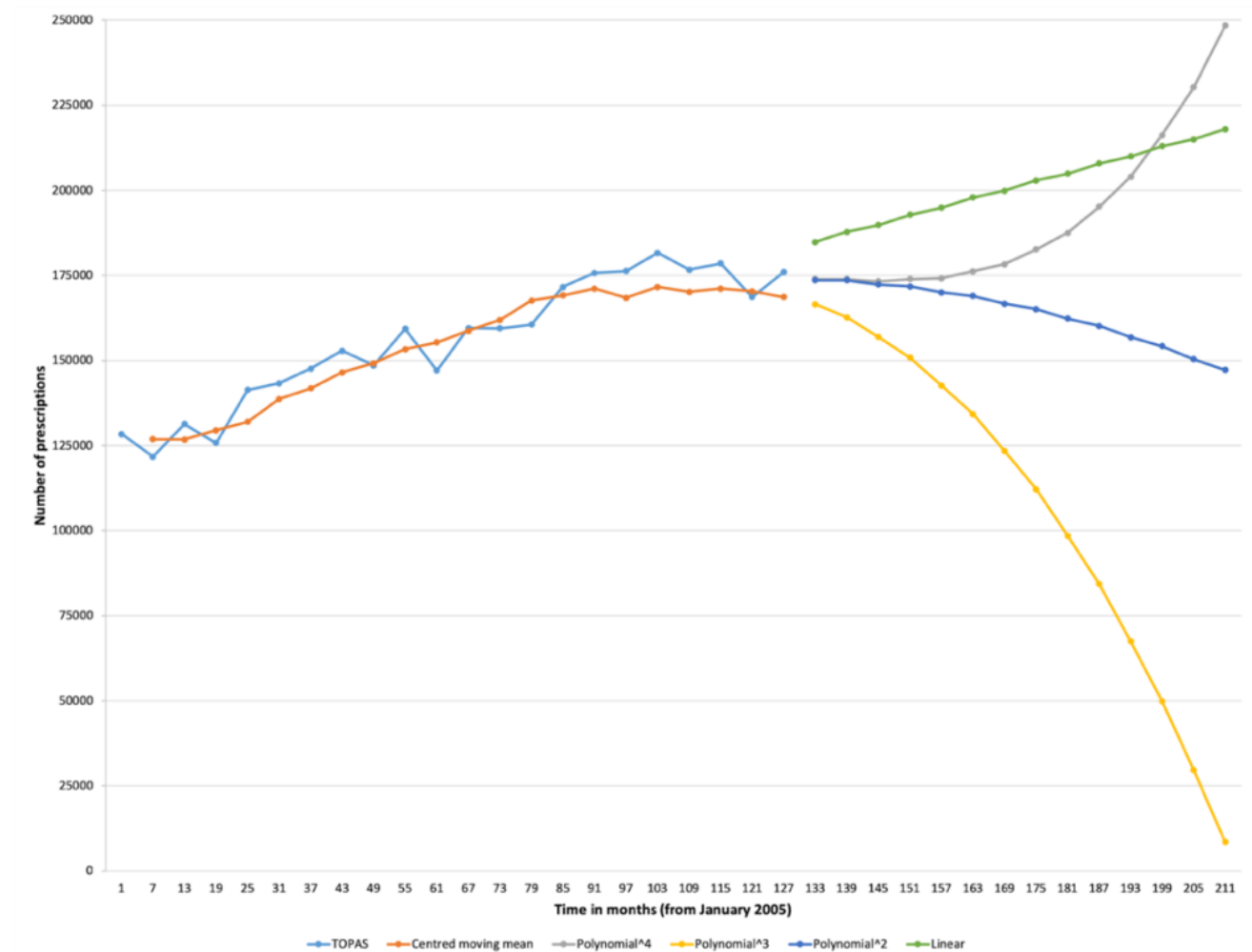

Figure E.12: Predicted trends in the number of weak opioid prescriptions issued using different time-series trendline analysis

\section{E.3.2 Predictions for strong opioid prescribing}

Three of the four predictions for strong opioid prescribing anticipated continued increases in the number of prescriptions issued in the time following the end of the study period (Figure E.13). The trendline of 'best fit' ( th $^{\text {th }}$ order polynomial), predicted a further increase in strong opioid prescriptions being issued until mid 2019 before downturn in the number of prescriptions. 
Table E.7: Comparison of predicted trends in strong opioid prescribing using trendline analysis

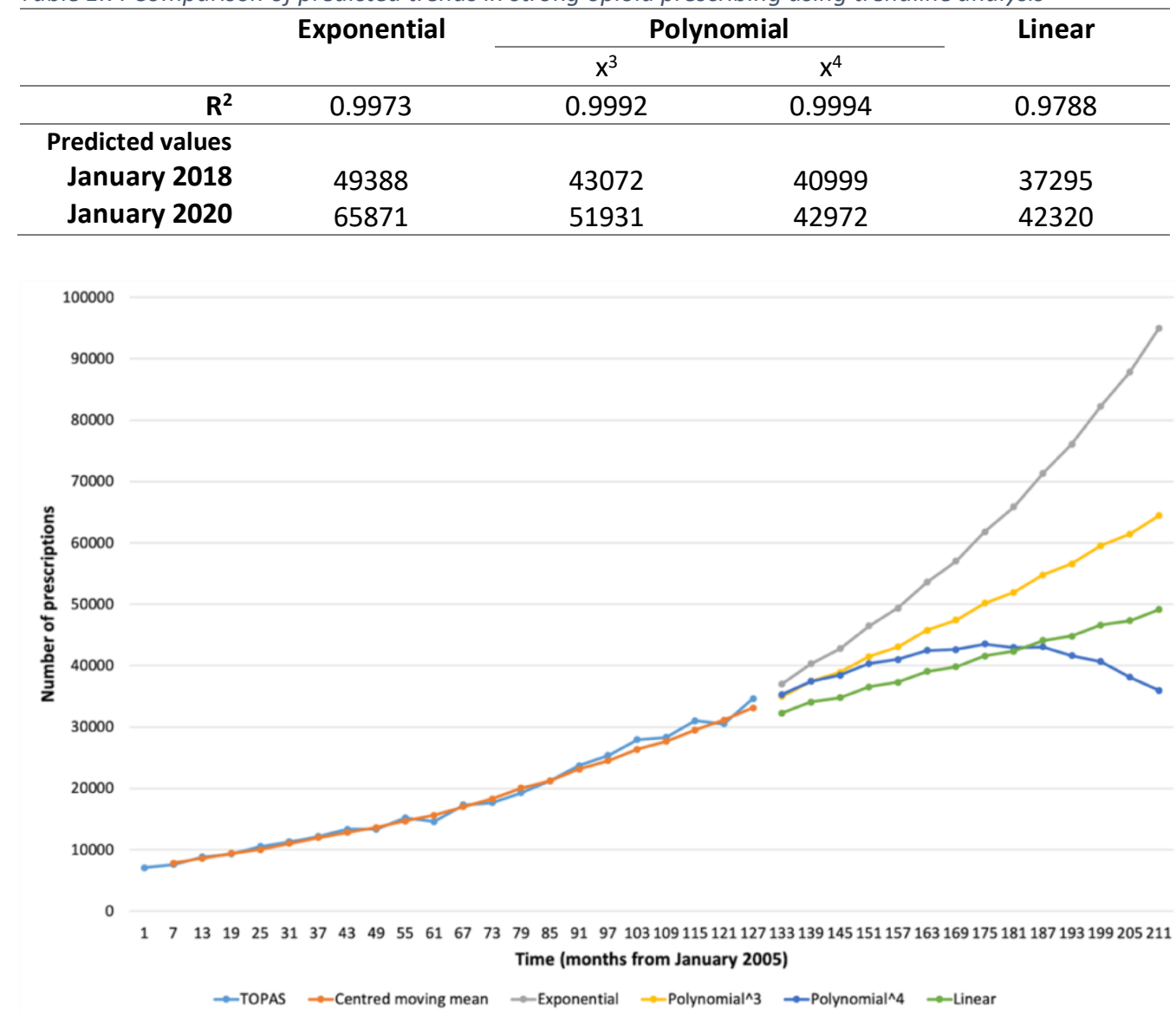

Figure E.13: Predicted trends in the number of strong opioid prescriptions issued using different time-series trendline analysis

\section{E.3.3 Predictions by gender}

In the female group, the trendline predictions (Figure E.14) mooted the annual number of opioid prescriptions could increase in 2 scenarios and decrease in another 2, assuming no changes were made to any factors which could influence prescribing.

The number of prescriptions for men appeared to increase in 3 of 4 predictions (Figure E.15), the difference being how quickly change could occur if nothing was done to intervene.

Table E.8: Comparison of predicted trends in opioid prescribing for female patients using trendline analysis

\begin{tabular}{rcccc}
\hline & \multicolumn{3}{c}{ Polynomial } & Linear \\
\cline { 2 - 4 } & $\mathrm{x}^{2}$ & $\mathrm{x}^{3}$ & $\mathrm{x}^{4}$ & \\
\hline $\mathbf{R}^{\mathbf{2}}$ & 0.988 & 0.995 & 0.996 & 0.970 \\
\hline Predicted values & 129517 & 114521 & 109677 & 140026 \\
January 2018 & 130606 & 95577 & 105808 & 148644 \\
January 2020 & & & & \\
\hline
\end{tabular}




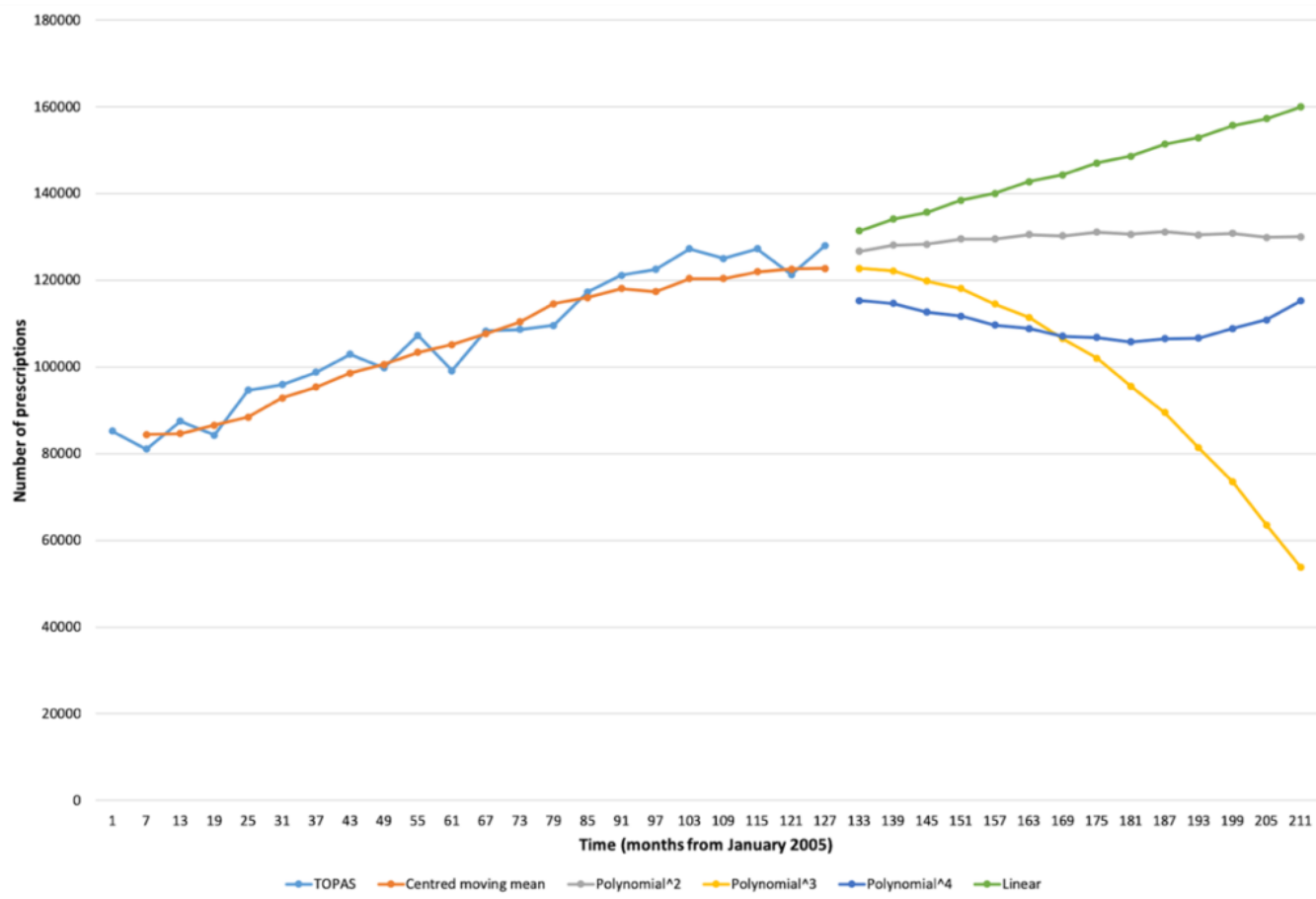

Figure E.14: Predicted trends in the number of opioid prescriptions issued to women using different timeseries trendline analysis

Table E.9: Comparison of predicted trends in opioid prescribing for male patients using trendline analysis

\begin{tabular}{rcccc}
\hline & \multicolumn{3}{c}{ Polynomial } & Linear \\
\cline { 2 - 4 } & $\mathbf{x}^{2}$ & $\mathrm{x}^{3}$ & $\mathrm{x}^{4}$ & \\
\hline Redicted values & 0.988 & 0.996 & 0.997 & 0.969 \\
\hline January 2018 & 84150 & 71263 & 99170 & 92118 \\
January 2020 & 84921 & 54863 & 113574 & 98598 \\
\hline
\end{tabular}

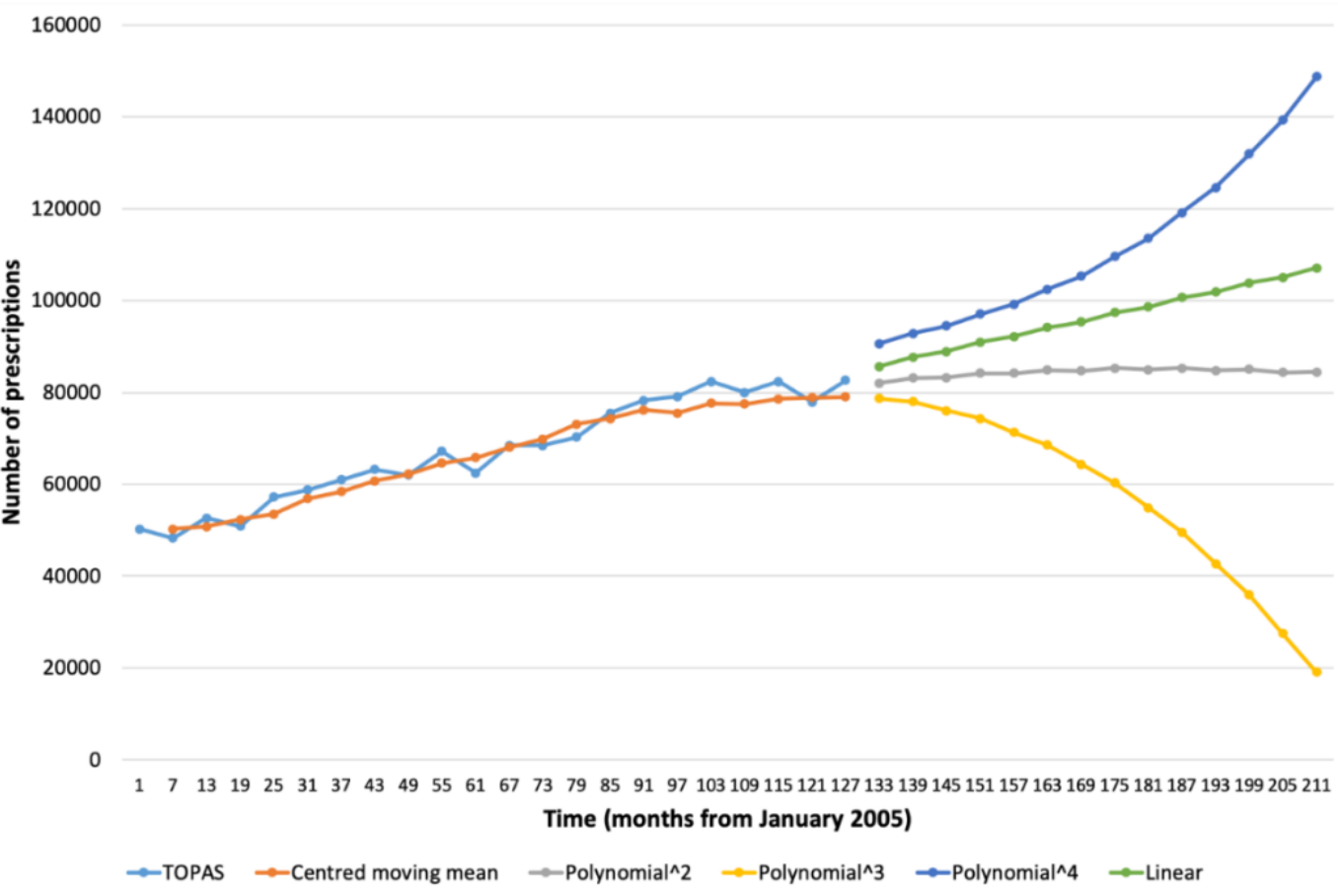

Figure E.15: Predicted trends in the number of opioid prescriptions issued to men using different time-series trendline analysis 


\section{E.3.4 Predictions by deprivation}

Continuation of the $4^{\text {th }}$ order polynomial trendlines for each area of deprivation appeared to predict, that should those trends continue, by 2020 (Table E.10) the difference in the number of opioid prescriptions issued in each area of deprivation throughout Wales would be smaller than in 2015 (Figure E.16).

Table E.10: Comparison of predicted trends in opioid prescribing for Health Boards using trendline analysis

\begin{tabular}{rccccc} 
& \multicolumn{5}{c}{ Welsh Index of Multiple Deprivation (WIMD) } \\
\cline { 2 - 6 } & $\mathbf{1}$ & $\mathbf{2}$ & $\mathbf{3}$ & $\mathbf{4}$ & $\mathbf{5}$ \\
\hline $\begin{array}{r}\text { Polynomial } \\
\mathbf{R}^{\mathbf{2}}\left(\mathbf{x}^{\mathbf{2}}\right)\end{array}$ & 0.988 & 0.983 & 0.988 & 0.990 & 0.990 \\
$\begin{array}{r}\text { Predicted values } \\
\text { January 2018 }\end{array}$ & 60413 & 47704 & 42433 & 29201 & 25126 \\
January 2020 & 60974 & 47856 & 43218 & 28872 & 24798 \\
\hline $\begin{array}{r}\text { Polynomial } \\
\mathbf{R}^{\mathbf{2}}\left(\mathbf{x}^{\mathbf{4}}\right)\end{array}$ & 0.996 & 0.994 & 0.996 & 0.996 & 0.996 \\
Predicted values & & & & & \\
January 2018 & 46970 & 45581 & 40668 & 26135 & 27217 \\
January 2020 & 45307 & 45793 & 40315 & 27550 & 31178 \\
\hline
\end{tabular}

Based on those predictions, there would be a reduction in the number of prescriptions issued in the areas with the greatest deprivation (WIMD1). The change seen from 2016, based on the polynomial prediction $\left(R^{2}=0.99\right)$ but would undergo an upturn in around 2020. The WIMD3 areas were predicted to maintain prescribing at consistent levels after $2015\left(R^{2}=0.996\right)$, whereas the remaining three areas (WIMD2, WIMD4 and WIMD5) were predicted to have an increase in opioid prescription numbers, most notably in the least deprived areas (Table E.10).

The result of the predicted changes in prescribing from $4^{\text {th }}$ order polynomial equations, is that over the time period predicted, the difference in the number of prescriptions issued in each of the deprivation areas, looked to reduce. If borne out, that would lead to similar numbers of opioid prescriptions being issued in all five areas of deprivation across Wales. 


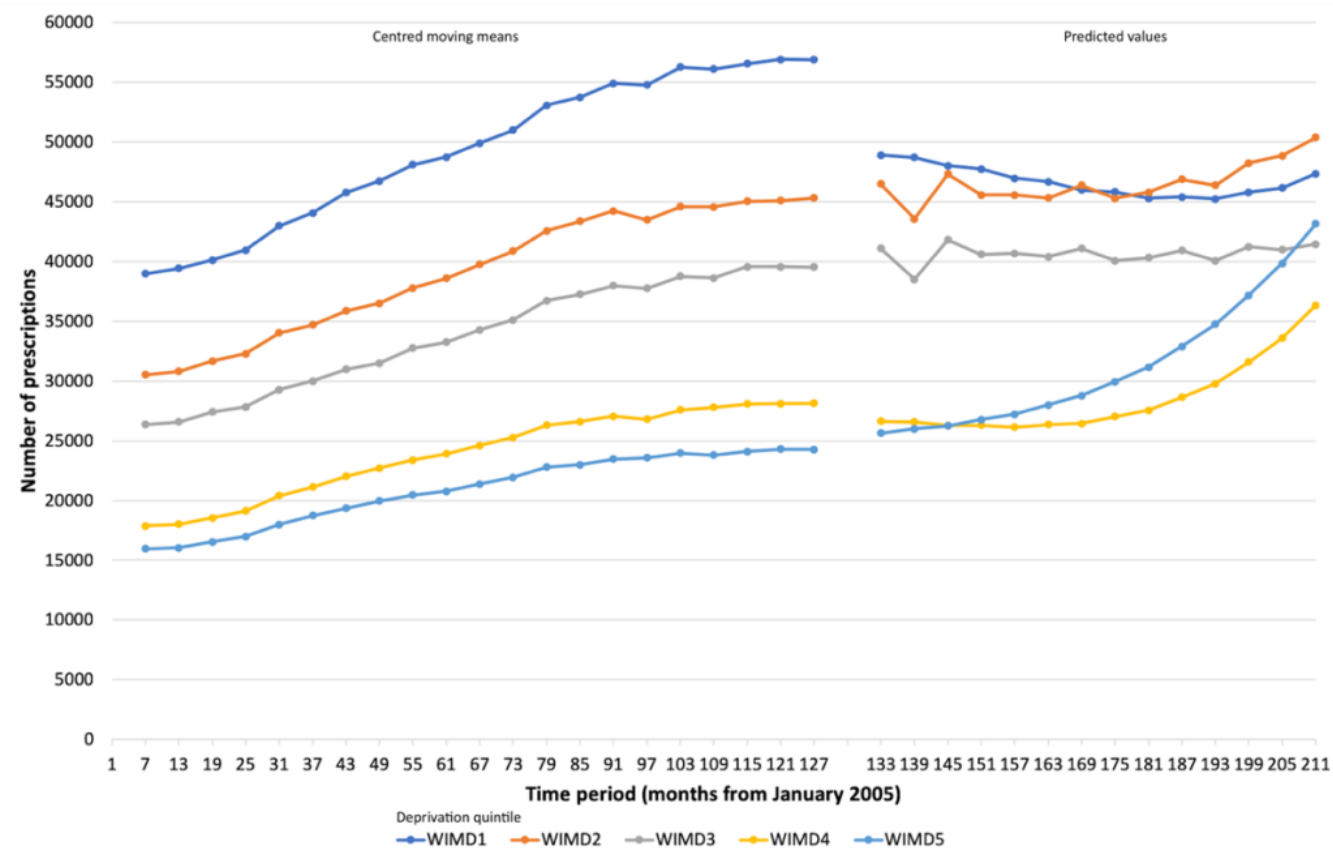

Figure E.16: Predicted trends in the number of opioid prescriptions issued to people in the different areas of deprivation (WIMD2011) (4th order polynomial prediction)

WIMD1 = most deprived, WIMD5 = least deprived

The second order polynomial predictions appeared to follow on from the trends noted in the study period. Although the predictions seem to show a continued increase in the number of prescriptions issued in each area overall; the trendlines show a slowdown in the rate of increase.

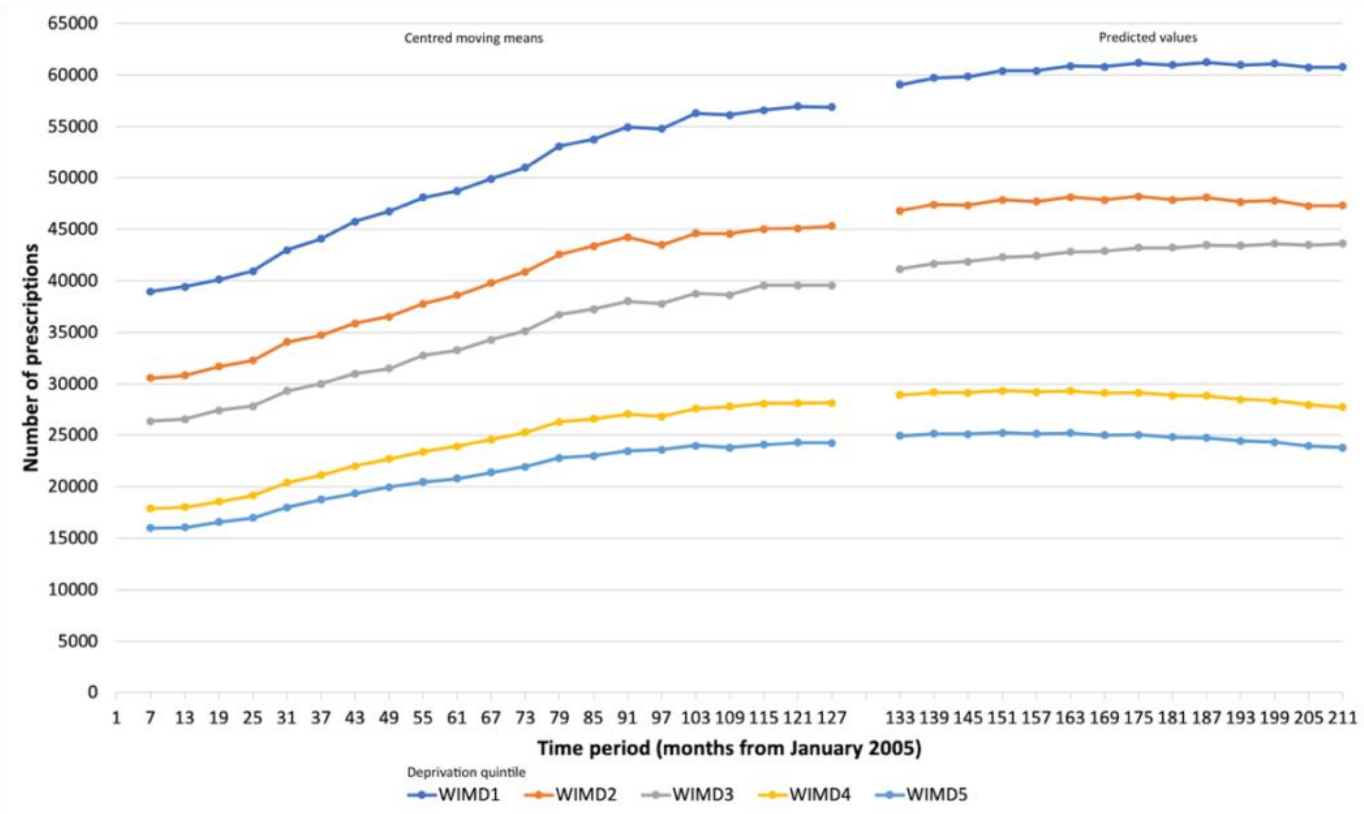

Figure E.17: Predicted trends in the number of opioid prescriptions issued to people in the different areas of deprivation (WIMD2011) (2nd order polynomial prediction)

WIMD1 = most deprived, WIMD5 = least deprived

In the least deprived areas (WIMD4 and WIMD5), there were predicted reductions towards the end of the period (Figure E.17). The predicted change from the second order polynomial trendlines demonstrate a further widening in 
the difference between prescribing of opioids in the most and least deprived areas.

\section{E.3.5 Prescribing by Health Board}

Time series analyses for each Health Board (HB) was conducted using the original data (not population adjusted) from the SAIL datasets. However, the $4^{\text {th }}$ order polynomial trendlines for each $\mathrm{HB}$ were the same as those seen for the population adjusted prescribing trend graphs (Figure E.18 to Figure E.24).

Based on the trendlines, two Health Boards (ABMUHB $R^{2}=0.99$, Figure E.18 and HDUHB $R^{2}=0.99$, Figure E.19) appeared to be demonstrating a continued, albeit slowing increase in the number of prescriptions issued. The other five HBs appeared to be showing a turnaround in prescription numbers towards the end of the study period in 2015 (Figure E.20 to Figure E.24).

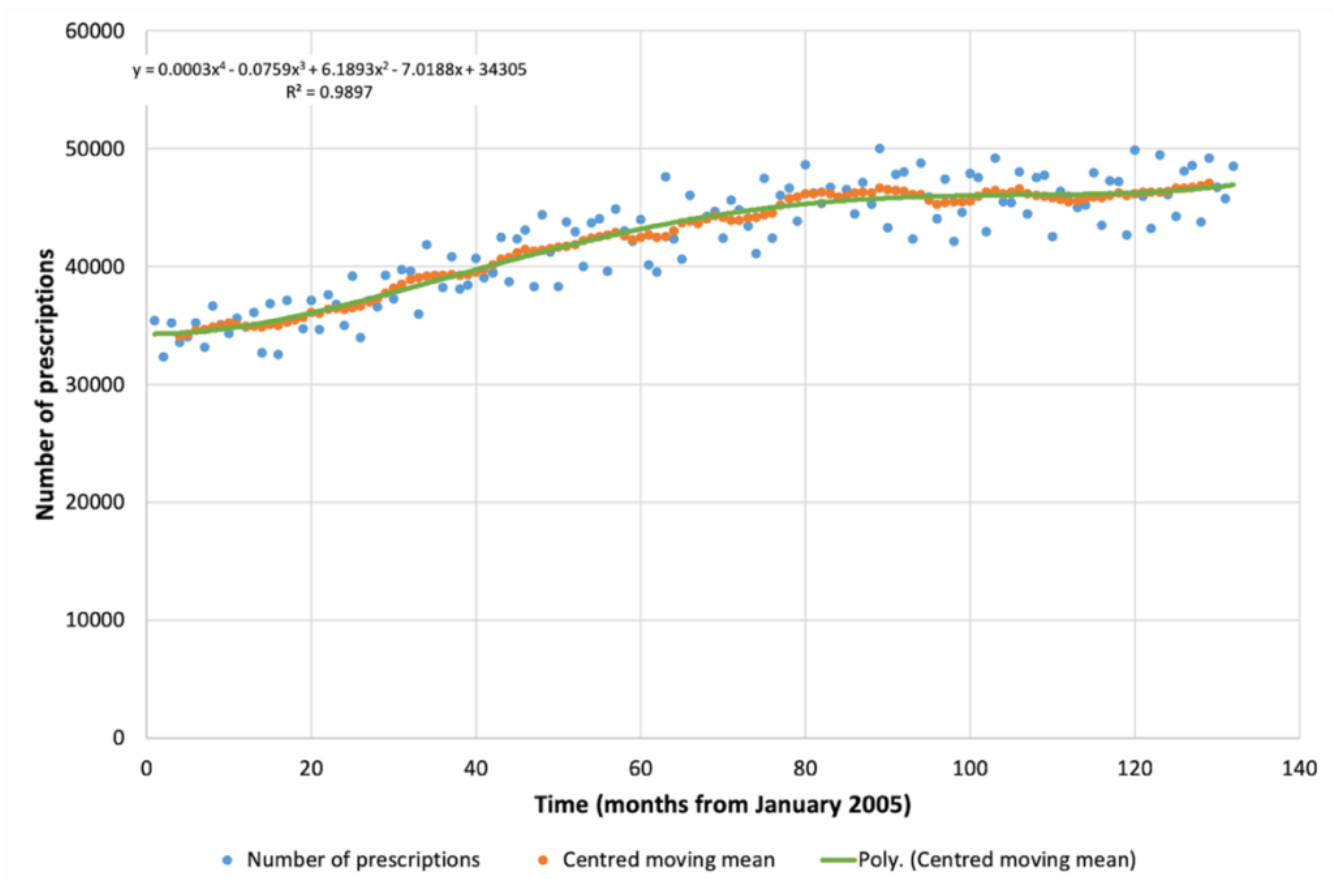

Figure E.18: Monthly trend in the number of opioid prescriptions issued to in Abertawe Bro Morgannwg University Health Board. Shown with a 4 th order polynomial trendline, $R 2=0.990$ 


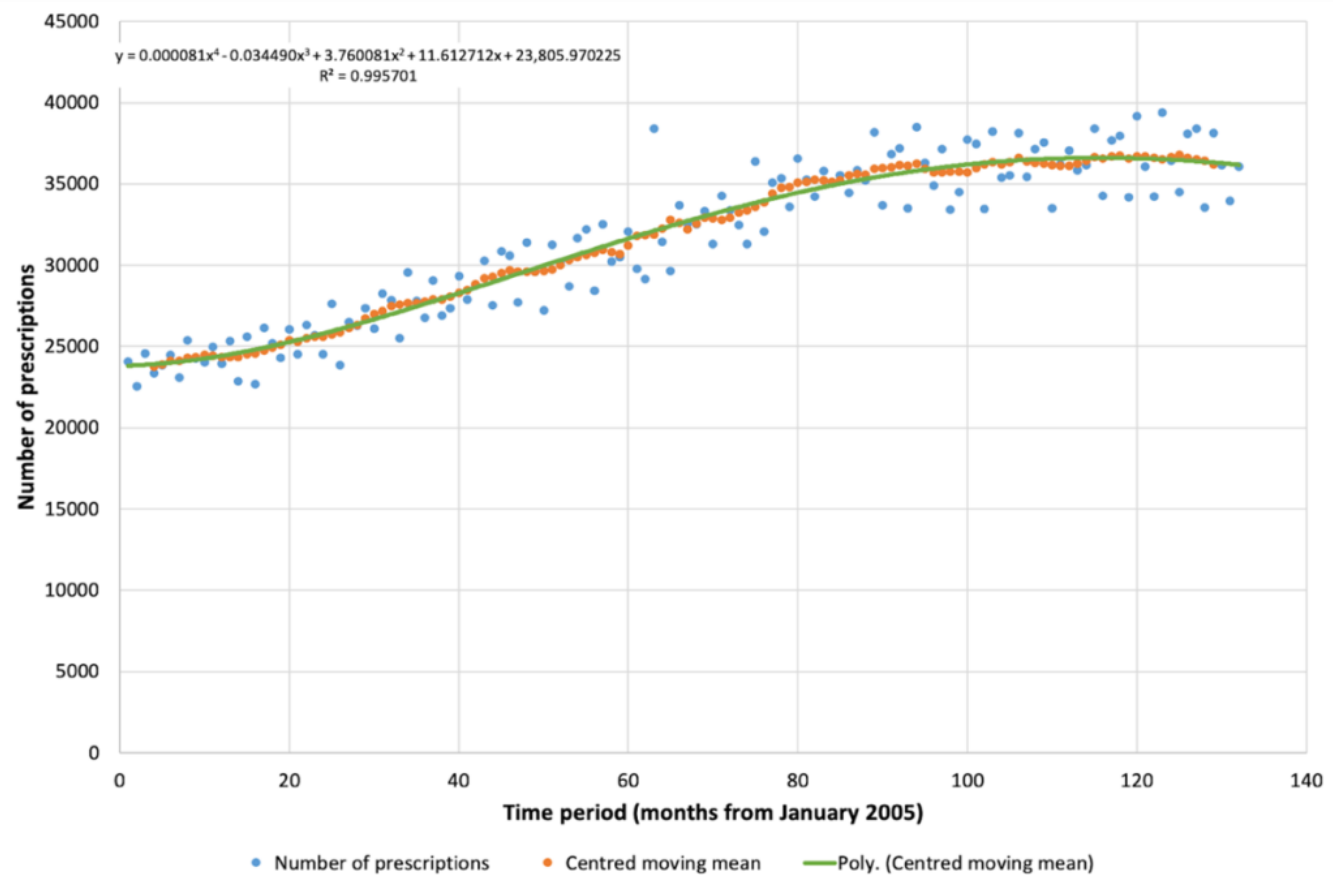

Figure E.19: Monthly trend in the number of opioid prescriptions issued to in Aneurin Bevan University Health Board. Shown with a 4 th order polynomial trendline, $R 2=0.996$

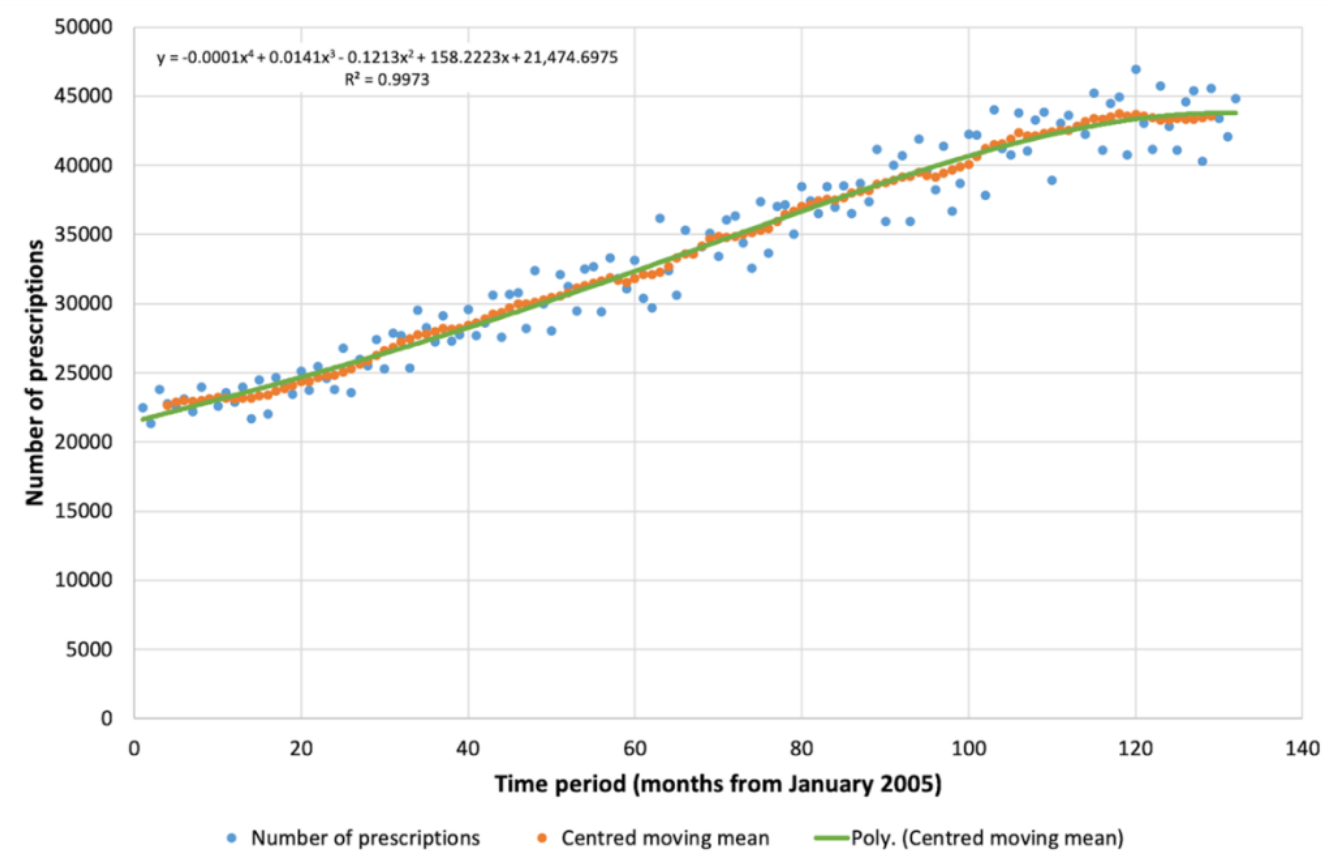

Figure E.20: Monthly trend in the number of opioid prescriptions issued to in Betsi Cadwaladr University Health Board. Shown with a 4 th order polynomial trendline, $R 2=0.997$ 


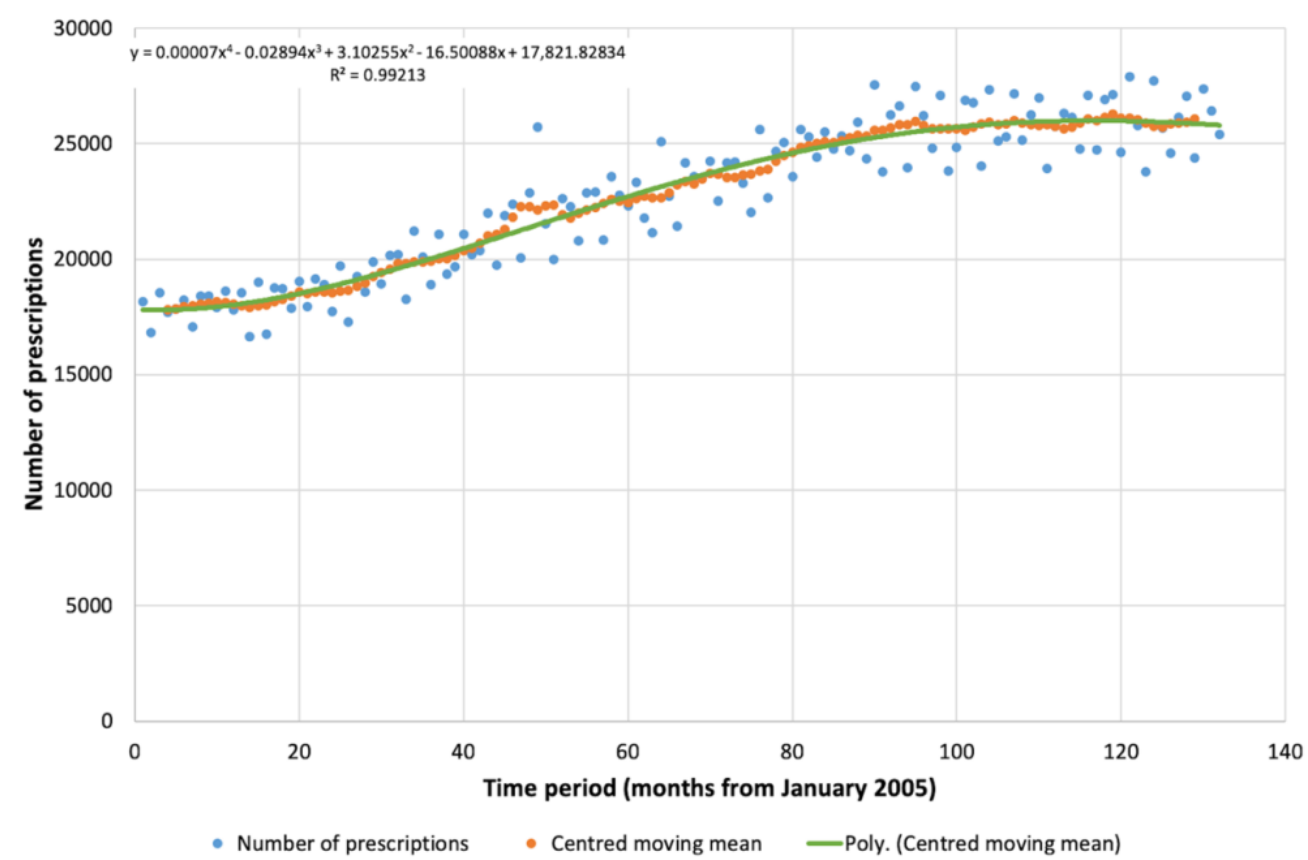

Figure E.21: Monthly trend in the number of opioid prescriptions issued to in Cardiff and Vale University Health Board. Shown with a 4th order polynomial trendline, $R 2=0.992$

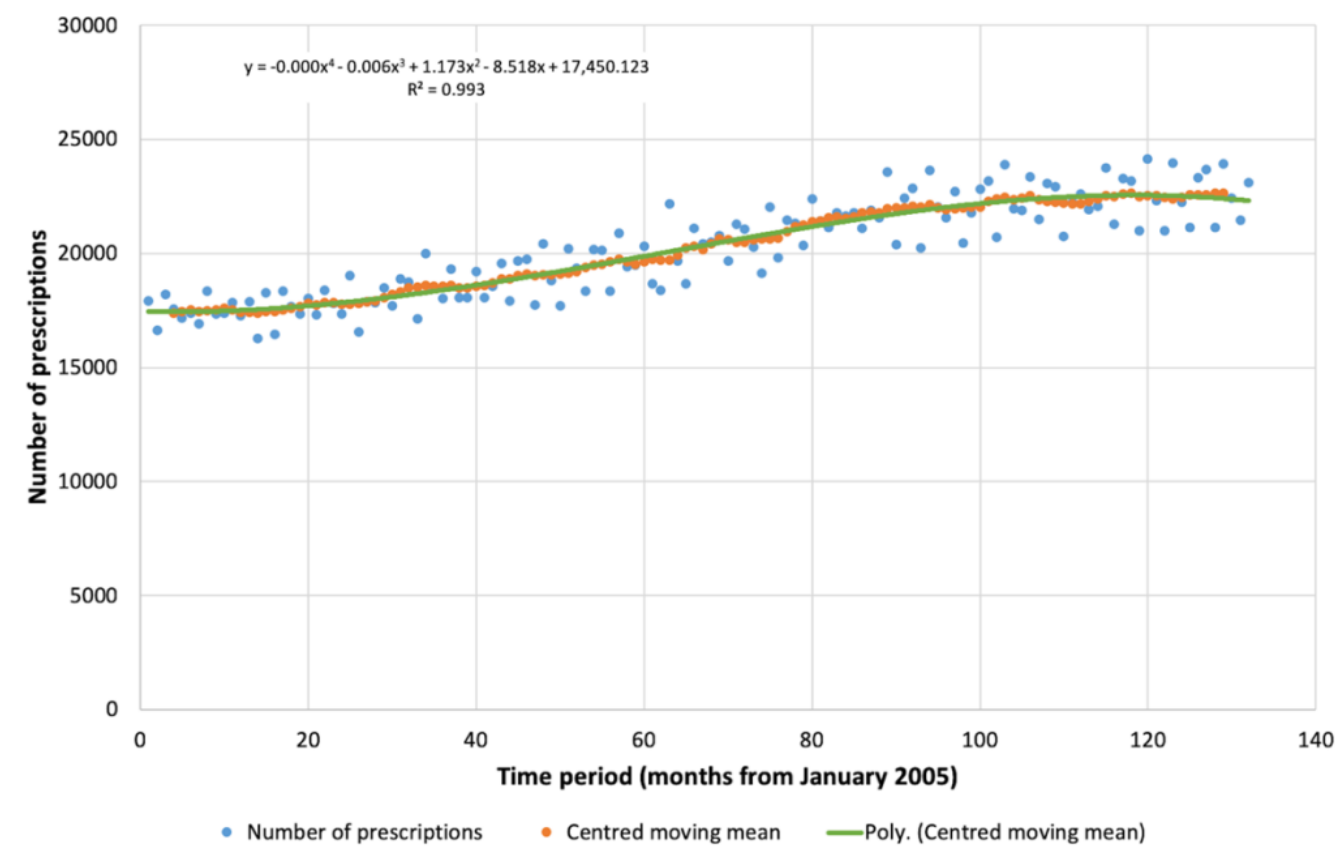

Figure E.22: Monthly trend in the number of opioid prescriptions issued to in Cwm Taf University Health Board. Shown with a 4 th order polynomial trendline, $R 2=0.993$ 


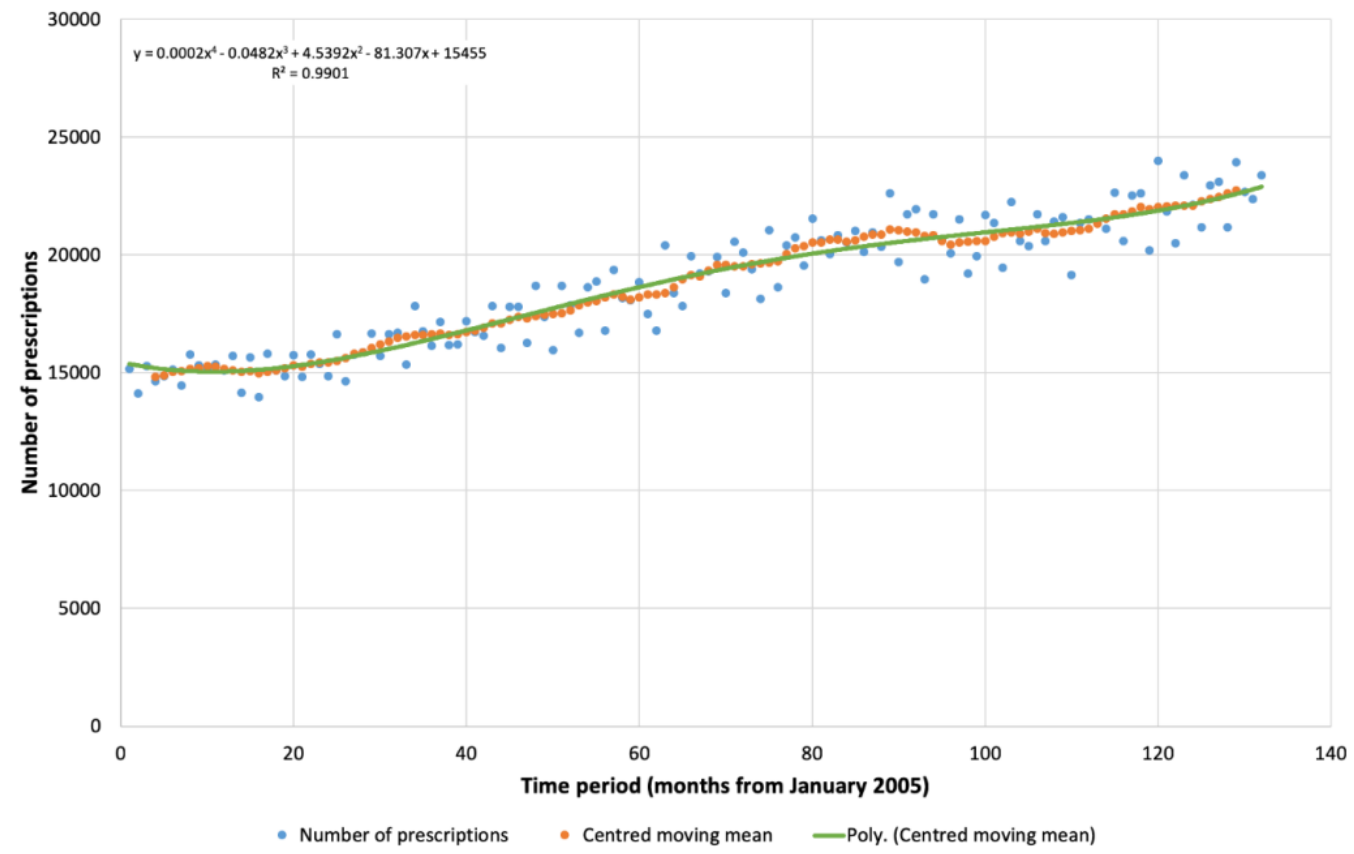

Figure E.23: Monthly trend in the number of opioid prescriptions issued to in Hywel Dda University Health Board. Shown with a 4 th order polynomial trendline, $R 2=0.990$

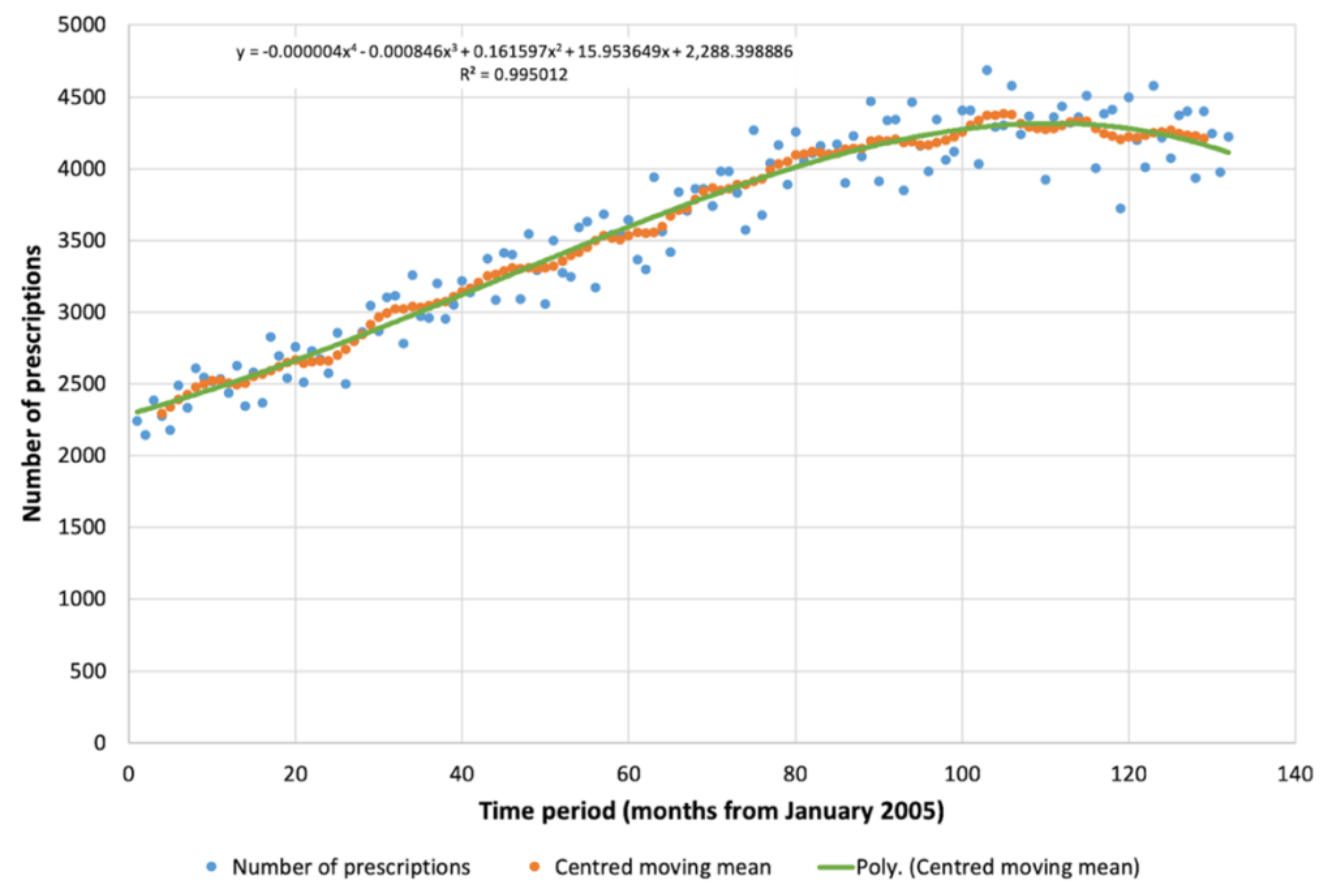

Figure E.24: Monthly trend in the number of opioid prescriptions issued to in Powys Teaching Health Board. Shown with a 4th order polynomial trendline, $R 2=0.995$

Average seasonal effects were similar in pattern as were seen with the total opioid prescribing trends (Figure E.25). The exception was CVUHB $\left(R^{2}=0.992\right)$ which had a different pattern in the average seasonal effect to the other HBs although there was still no clear seasonal trend in any Health Board. 


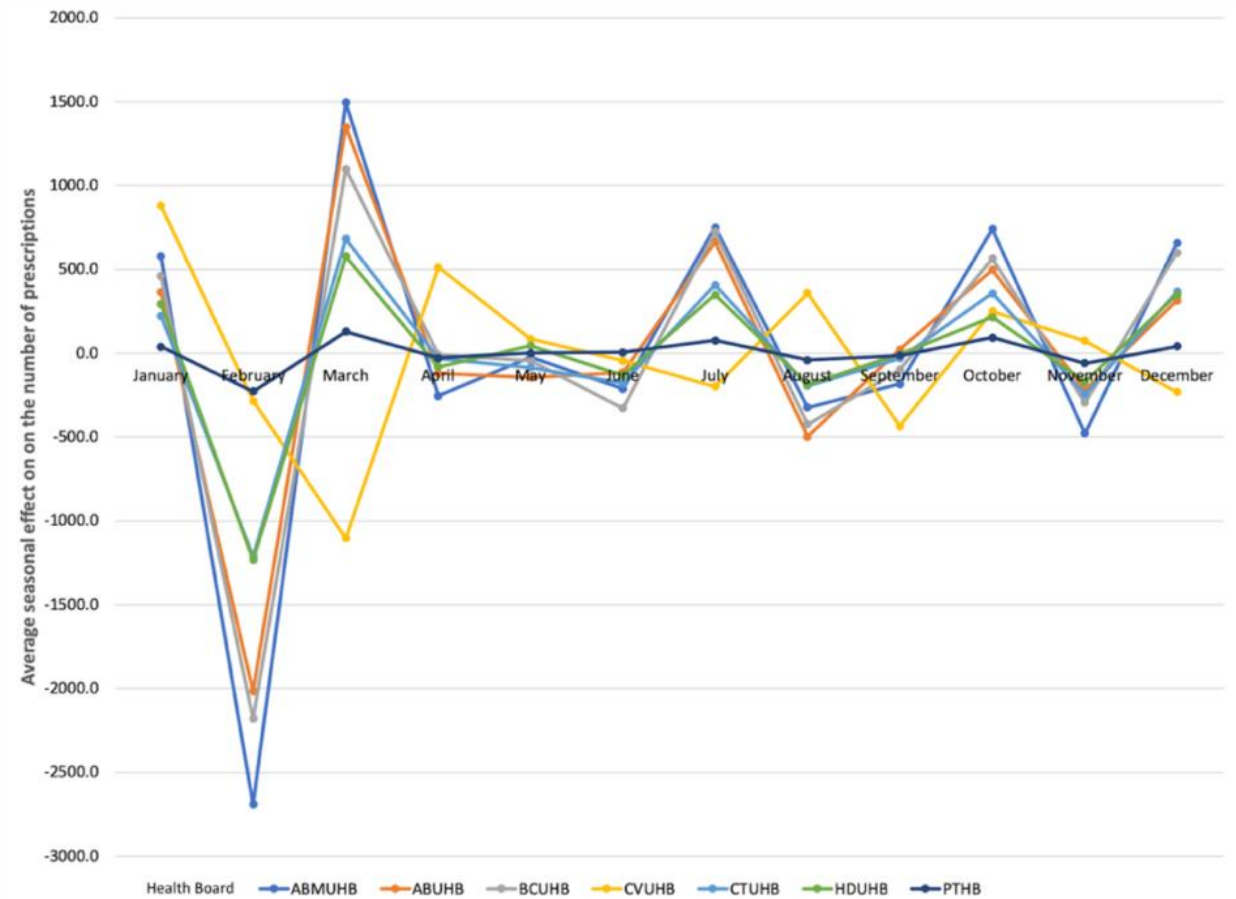

Figure E.25: Graphical depiction of average seasonal variation in the number of opioid prescriptions issued by Health Board, from time series analysis

$A B M U H B=$ Abertawe Bro Morgannwg University Health Board; $A B U H B=$ Aneurin Bevan University Health Board; $B C U H B=$ Betsi Cadwallader University Health Board; $C V U H B=$ Cardiff and Vale University Health Board; CTUHB = Cwm Taf University Health Board; HDUHB = Hywel Dda University Health Board; PTHB = Powys Teaching Health Board

\section{E.4 Predictions for Health Board prescribing}

The polynomial trendlines were continued via calculation to predict possible changes in number of prescriptions that could be issued in each Health Board following the end of the study period in 2015 (Table E.11 and Figure E.26).Fourth order polynomial equations predicted dramatic increases in the number of prescriptions issued after 2015 in ABMUHB and HDUHB (Figure E.26). The changes appeared remote from the end of the trendlines in 2015 but due to the nature of $4^{\text {th }}$ order polynomial trendlines sudden changes in either direction is possible. Interestingly however, the 5 other Health Boards were predicted to demonstrate reductions in prescriptions numbers issued.

Table E.11: Comparison of predicted trends in opioid prescribing for Health Boards using trendline analysis

\begin{tabular}{rccccccc}
\hline & \multicolumn{7}{c}{ Health Board } \\
\hline & ABMUHB & ABUHB & BCUHB & CVUHB & CTUHB & HDUHB & PTHB \\
\hline $\begin{array}{r}\text { Polynomial } \\
\mathbf{R}^{\mathbf{2}}\left(\mathbf{x}^{\mathbf{2}}\right)\end{array}$ & 0.981 & 0.983 & 0.993 & 0.980 & 0.974 & 0.980 & 0.983 \\
$\begin{array}{r}\text { Predicted values } \\
\text { January 2018 }\end{array}$ & 45488 & 38020 & 50277 & 27676 & 24285 & 23950 & 4360 \\
January 2020 & 42961 & 37479 & 54066 & 27405 & 25014 & 24784 & 4180 \\
\hline $\begin{array}{r}\text { Polynomial } \\
\mathbf{R}^{\mathbf{2}}\left(\mathbf{x}^{\mathbf{4}}\right)\end{array}$ & 0.990 & 0.996 & 0.997 & 0.992 & 0.993 & 0.990 & 0.995 \\
$\begin{array}{r}\text { Predicted values } \\
\text { January 2018 }\end{array}$ & 74887 & 30772 & 37593 & 23120 & 20759 & 49856 & 3110 \\
January 2020 & 108297 & 27673 & 22880 & 20879 & 16467 & 78584 & 1199 \\
\hline
\end{tabular}




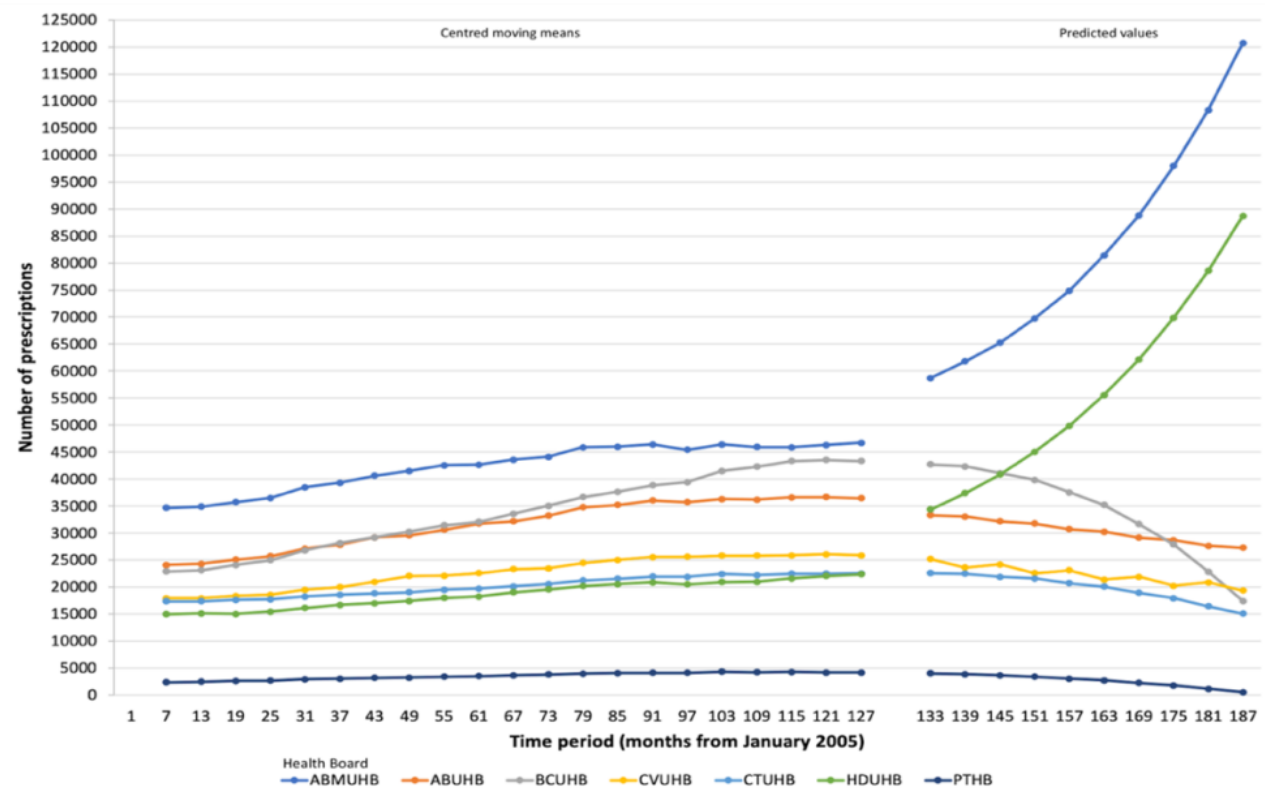

Figure E.26: Predicted trends in the number of opioid prescriptions issued to people in the 7 Health Boards (4th order polynomial prediction)

$\mathrm{ABMUHB}=\mathrm{Abertawe}$ Bro Morgannwg University Health Board; $\mathrm{ABUHB}=$ Aneurin Bevan University Health Board; $\mathrm{BCUHB}=$ Betsi Cadwaladr University Health Board; $\mathrm{CVUHB}=$ Cardiff and Vale University Health Board; CTUHB = Cwm Taf University Health Board; HDUHB = Hywel Dda University Health Board; PTHB = Powys Teaching Health Board

Second order polynomial equations predicted future trends appeared to follow the previous patterns of prescribing more realistically (Figure E.27). Using those predictions, BCUHB could have the largest continued increase in the number of opioid prescriptions issued monthly after the end of the TOPAS study period in 2015. ABMUHB prediction was of a reduction in the number of prescriptions being issued after 2015 (Figure E.27). The other HBs were predicted to have much slower increases or plateau in prescription numbers after 2015 (Figure E.27). 


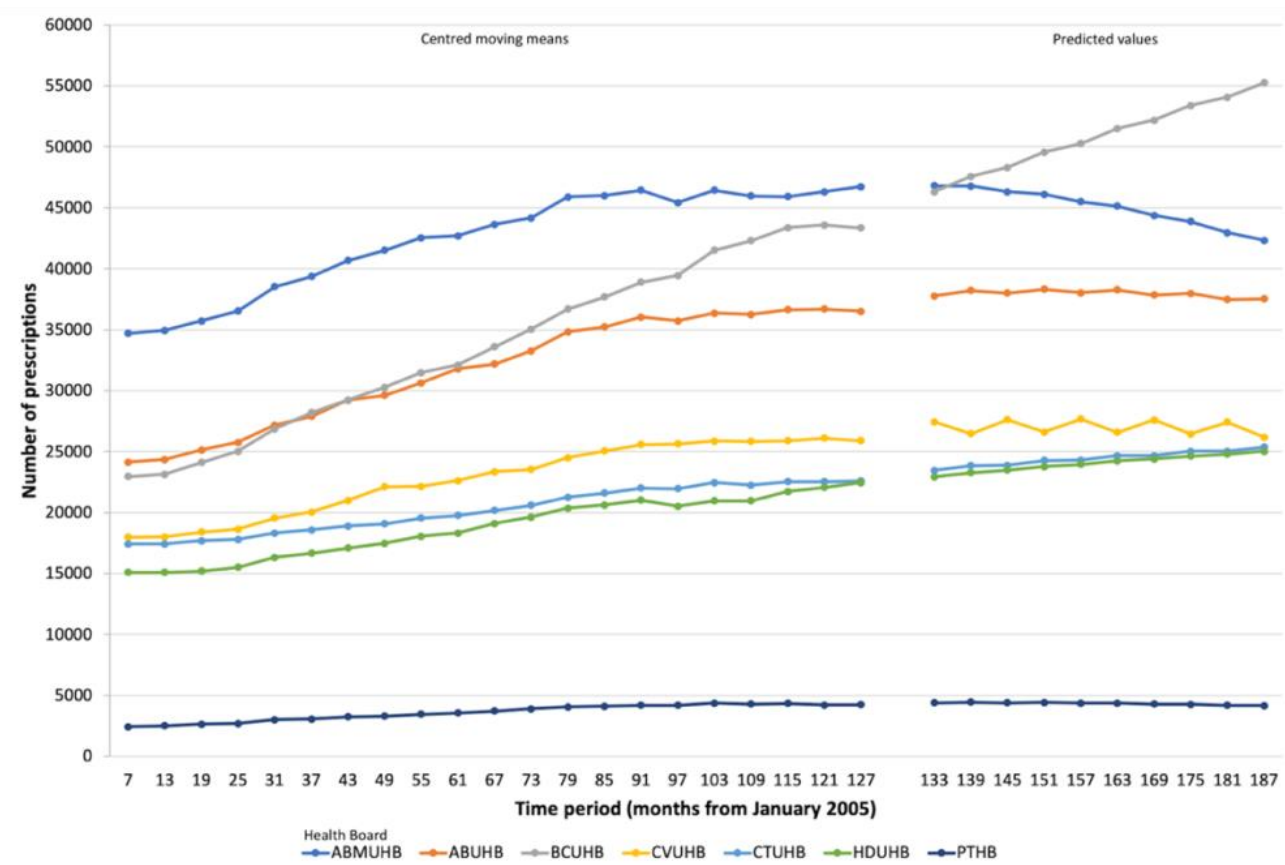

Figure E.27: Predicted trends in the number of opioid prescriptions issued to people in the 7 Health Boards (2nd order polynomial prediction)

$\mathrm{ABMUHB}=$ Abertawe Bro Morgannwg University Health Board; $\mathrm{ABUHB}=$ Aneurin Bevan University Health Board; $\mathrm{BCUHB}=$ Betsi Cadwaladr University Health Board; $\mathrm{CVUHB}=$ Cardiff and Vale University Health Board; $\mathrm{CTUHB}=\mathrm{Cwm}$ Taf University Health Board; HDUHB = Hywel Dda University Health Board; PTHB = Powys Teaching Health Board 


\section{Appendix F Healthcare resource utilisation associated with opioid prescribing and associated cost analysis}

\section{F.1 Metadata}

Table F.1: Metadata used to extract data from main table of Phase 2 study using SQL coding or identifying columns in table

\begin{tabular}{|c|c|c|c|}
\hline & COLUMN NAME & DESCRIPTION & Project Specific Detail \\
\hline 1 & ALF_E & Anonymized linkage field & $\begin{array}{l}\text { INTEGER: Including cases and } \\
\text { their corresponding controls }\end{array}$ \\
\hline 2 & GROUPS & $\begin{array}{l}\text { Assigning a group number } \\
\text { to each set of ALFS }\end{array}$ & $\begin{array}{l}\text { INTEGER: Each set includes } 1 \\
\text { Case-ALF and } 4 \text { corresponding } \\
\text { controls }\end{array}$ \\
\hline 3 & TYPE & Type of ALF & $\begin{array}{l}\text { CASE } \\
\text { CONTROL }\end{array}$ \\
\hline 4 & AGE & Age at the 01-01-2016 & $>=18$ \\
\hline 5 & AGE_CAT & Age categories & $\begin{array}{l}\text { 1: }[18-30] \\
2:[31-40] \\
3:[41-50] \\
4:[51-60] \\
5:[61-80] \\
6: 80+\end{array}$ \\
\hline 6 & GNDR_CD & Gender code & $\begin{array}{l}\text { Each individual has a gender } \\
\text { code allocated: } 1=\text { Male, } \\
\text { 2=Female }\end{array}$ \\
\hline 7 & OPIOID & They've received an opioid & Only applies to cases \\
\hline 8 & $\begin{array}{l}\text { MAX_PR_PERIOD } \\
\text { _CAT }\end{array}$ & $\begin{array}{l}\text { Maximum prescription } \\
\text { period category for cases } \\
\text { who have had opioids } \\
\text { prescription in their WLGP } \\
\text { record. }\end{array}$ & 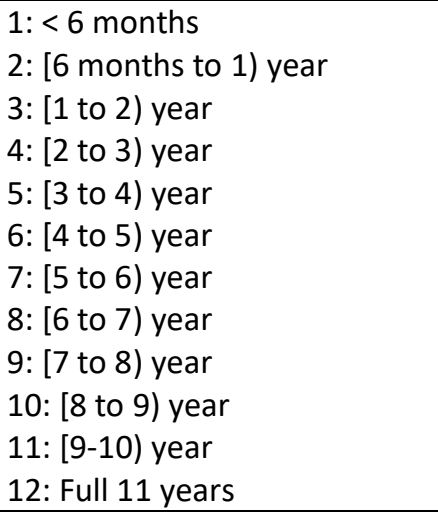 \\
\hline 9 & $\begin{array}{l}\text { WLGP_REC_DUR_ } \\
\text { CAT }\end{array}$ & $\begin{array}{l}\text { Maximum duration of } \\
\text { having continuous record } \\
\text { of WLGP in the SAIL } \\
\text { databank. }\end{array}$ & $\begin{array}{l}\text { 1: } 6 \\
\text { 2: } 6 \text { months to } 1 \text { year } \\
\text { 3: [1 to } 2 \text { ) year } \\
\text { 4: [2 to 3) year } \\
\text { 5: [3 to 4) year } \\
\text { 6: [4 to 5) year } \\
\text { 7: [5 to 6) year } \\
\text { 8: [6 to 7) year } \\
\text { 9: [7 to 8) year } \\
\text { 10: [8 to 9) year } \\
\text { 11: [9-10) year } \\
\text { 12: Full } 11 \text { years }\end{array}$ \\
\hline 10 & WIMD2011_5th & $\begin{array}{l}\text { 5th quintile for deprivation } \\
\text { score }\end{array}$ & Value $1=$ most deprived \\
\hline 11 & $\begin{array}{l}\text { FIRST_OP_EVENT } \\
\text { _DT }\end{array}$ & $\begin{array}{l}\text { The earliest date of opioid } \\
\text { prescription in the study } \\
\text { period for cases }\end{array}$ & DATE \\
\hline
\end{tabular}




\begin{tabular}{|c|c|c|c|}
\hline 12 & $\begin{array}{l}\text { EDDS_ALL_ATT_C } \\
\text { NT }\end{array}$ & $\begin{array}{l}\text { Total number of A\&E } \\
\text { attendances regardless } \\
\text { of the type of their } \\
\text { attendance }\end{array}$ & $\begin{array}{l}\text { Didn't use the code lists and } \\
\text { just followed cases and } \\
\text { controls to see how many } \\
\text { time they've been into A\&E } \\
\text { Note: If someone had more } \\
\text { than one of the codes on } \\
\text { the same date, then their } \\
\text { EDDS_ATTENDANCE_CNT } \\
\text { >= EDDS_ALL_ATT_CNT }\end{array}$ \\
\hline 13 & $\begin{array}{l}\text { EDDS_ATTENDAN } \\
\text { CE_CNT }\end{array}$ & $\begin{array}{l}\text { Number of A\&E } \\
\text { attendances for cases and } \\
\text { controls }\end{array}$ & $\begin{array}{l}\text { Using both ICD10 and EDDS } \\
\text { specified codes } \\
\text { Note: For cases the number of } \\
\text { attendances were count after } \\
\text { their first opioid prescription } \\
\text { all attendances for controls. } \\
\end{array}$ \\
\hline 14 & $\begin{array}{l}\text { OPDW_ATT_CN } \\
T\end{array}$ & $\begin{array}{l}\text { Total number of } \\
\text { attendances at the } \\
\text { outpatient }\end{array}$ & $\begin{array}{l}\text { didn't use the code lists and } \\
\text { just followed cases and } \\
\text { controls to see how many } \\
\text { time they've been into } \\
\text { outpatient } \\
\text { Note: For cases the number of } \\
\text { attendances were count after } \\
\text { their first opioid prescription } \\
\text { all attendances for controls. }\end{array}$ \\
\hline 15 & PEDW_ATT_CNT & $\begin{array}{l}\text { Total number of hospital } \\
\text { admissions }\end{array}$ & $\begin{array}{l}\text { Didn't use the code lists and } \\
\text { just followed cases and } \\
\text { controls to see how many } \\
\text { time they've been } \\
\text { hospitalized } \\
\text { Note: For cases the number of } \\
\text { attendances were count after } \\
\text { their first opioid prescription } \\
\text { all attendances for controls. } \\
\end{array}$ \\
\hline 16 & DEPRESSION & $\begin{array}{l}\text { Whether or not they } \\
\text { had depression }\end{array}$ & $\begin{array}{l}0=\text { no } \\
1=\text { yes }\end{array}$ \\
\hline 17 & WEAK_OP & $\begin{array}{l}\text { They've been } \\
\text { administered weak } \\
\text { opioid based on their GP } \\
\text { record }\end{array}$ & $\begin{array}{l}0=\text { no } \\
1=\text { yes }\end{array}$ \\
\hline 18 & STRONG_OP & $\begin{array}{l}\text { They've been } \\
\text { administered strong } \\
\text { opioid based on their GP } \\
\text { record }\end{array}$ & $\begin{array}{l}0=\text { no } \\
1=\text { yes }\end{array}$ \\
\hline \multicolumn{4}{|c|}{$\begin{array}{l}\text { Break down of EDDS attendance counts*(See final notes for instruction of how to use } \\
\text { underlying table for breakdown of counts on other conditions) } \\
\text { EDDS_ALL_ATT_CNT } \\
\text { EDDS_ATTENDANCE_CNT } \\
\text { Note: For cases the number of attendances were count after their first opioid } \\
\text { prescription all attendances for controls. }\end{array}$} \\
\hline 19 & $\begin{array}{l}\text { EDDS_AT_ADVE } \\
\text { RS_CNT }\end{array}$ & $\begin{array}{l}\text { Number of A\&E } \\
\text { attendances for adverse } \\
\text { reactions to drugs }\end{array}$ & $\begin{array}{l}\text { Using provided categories on } \\
\text { ICD10 codes }\end{array}$ \\
\hline
\end{tabular}




\begin{tabular}{|c|c|c|c|}
\hline 20 & $\begin{array}{l}\text { EDDS_AT_PAIN_ } \\
\text { CNT }\end{array}$ & $\begin{array}{l}\text { Number of } A \& E \\
\text { attendances for pain }\end{array}$ & $\begin{array}{l}\text { Using provided categories on } \\
\text { ICD10 codes }\end{array}$ \\
\hline 21 & $\begin{array}{l}\text { EDDS_AT_INFEC } \\
\text { CNT }\end{array}$ & $\begin{array}{l}\text { Number of } A \& E \\
\text { attendances for infection }\end{array}$ & $\begin{array}{l}\text { Using provided categories on } \\
\text { ICD10 codes }\end{array}$ \\
\hline \multicolumn{4}{|c|}{$\begin{array}{l}\text { Break down of EDDS attendance counts based on } 17 \text { categories of EDDS specific code } \\
\text { list } \\
\text { Note: For cases the number of attendances were count after their first opioid } \\
\text { prescription all attendances for controls. }\end{array}$} \\
\hline 22 & $\begin{array}{l}\text { EDDS_AT_ENDO } \\
\text { C }\end{array}$ & $\begin{array}{l}\text { Number of } A \& E \\
\text { attendances for } \\
\text { endocrinological } \\
\text { Conditions }\end{array}$ & $\begin{array}{l}\text { Using provided categories on } \\
\text { A\&E codes }\end{array}$ \\
\hline 23 & $\begin{array}{l}\text { EDDS_AT_FRAC } \\
T\end{array}$ & $\begin{array}{l}\text { Number of } A \& E \\
\text { attendances for Fracture }\end{array}$ & $\begin{array}{l}\text { Using provided categories on } \\
\text { A\&E codes }\end{array}$ \\
\hline 24 & EDDS_AT_GAST & $\begin{array}{l}\text { Number of A\&E } \\
\text { attendances for } \\
\text { Gastrointestinal } \\
\text { Conditions }\end{array}$ & $\begin{array}{l}\text { Using provided categories on } \\
\text { A\&E codes }\end{array}$ \\
\hline 25 & EDDS_AT_GUM & $\begin{array}{l}\text { Number of A\&E } \\
\text { attendances for Genito- } \\
\text { Urinary Medicine }\end{array}$ & $\begin{array}{l}\text { Using provided categories on } \\
\text { A\&E codes }\end{array}$ \\
\hline 26 & $\begin{array}{l}\text { EDDS_AT_HEAD } \\
\text { INJ }\end{array}$ & $\begin{array}{l}\text { Number of A\&E } \\
\text { attendances for Head } \\
\text { injury }\end{array}$ & $\begin{array}{l}\text { Using provided categories on } \\
\text { A\&E codes }\end{array}$ \\
\hline 27 & $\begin{array}{l}\text { EDDS_AT_JOINT } \\
\text { INJ }\end{array}$ & $\begin{array}{l}\text { Number of } A \& E \\
\text { attendances for Joint } \\
\text { Injury }\end{array}$ & $\begin{array}{l}\text { Using provided categories on } \\
\text { A\&E codes }\end{array}$ \\
\hline 28 & $\begin{array}{l}\text { EDDS_AT_LINFE } \\
\text { C }\end{array}$ & $\begin{array}{l}\text { Number of A\&E } \\
\text { attendances for local } \\
\text { infection }\end{array}$ & $\begin{array}{l}\text { Using provided categories on } \\
\text { A\&E codes }\end{array}$ \\
\hline 29 & EDDS_AT_NC & $\begin{array}{l}\text { Number of } A \& E \\
\text { attendances for } \\
\text { Neurological Conditions }\end{array}$ & $\begin{array}{l}\text { Using provided categories on } \\
\text { A\&E codes }\end{array}$ \\
\hline 30 & EDDS_AT_PAIN & $\begin{array}{l}\text { Number of } A \& E \\
\text { attendances for pain }\end{array}$ & $\begin{array}{l}\text { Using provided categories on } \\
\text { A\&E codes }\end{array}$ \\
\hline 31 & EDDS_AT_POIS & $\begin{array}{l}\text { Number of } A \& E \\
\text { attendances for poisoning } \\
\text { or overdose }\end{array}$ & $\begin{array}{l}\text { Using provided categories on } \\
\text { A\&E codes }\end{array}$ \\
\hline 32 & EDDS_AT_PSYC & $\begin{array}{l}\text { Number of } A \& E \\
\text { attendances for } \\
\text { Psychological/psychiatric } \\
\text { conditions }\end{array}$ & $\begin{array}{l}\text { Using provided categories on } \\
\text { A\&E codes }\end{array}$ \\
\hline 33 & EDDS_AT_RESP & $\begin{array}{l}\text { Number of A\&E } \\
\text { attendances for } \\
\text { Respiratory Conditions }\end{array}$ & $\begin{array}{l}\text { Using provided categories on } \\
\text { A\&E codes }\end{array}$ \\
\hline 34 & EDDS_AT_RHEU & $\begin{array}{l}\text { Number of A\&E } \\
\text { attendances for } \\
\text { Rheumatologically } \\
\text { Conditions }\end{array}$ & $\begin{array}{l}\text { Using provided categories on } \\
\text { A\&E codes }\end{array}$ \\
\hline 35 & EDDS_AT_SOC & $\begin{array}{l}\text { Number of A\&E } \\
\text { attendances for Social } \\
\text { Problems/Homelessness }\end{array}$ & $\begin{array}{l}\text { Using provided categories on } \\
\text { A\&E codes }\end{array}$ \\
\hline 36 & EDDS_AT_STINJ & $\begin{array}{l}\text { Number of A\&E } \\
\text { attendances for Soft Tissue } \\
\text { Injury }\end{array}$ & $\begin{array}{l}\text { Using provided categories on } \\
\text { A\&E codes }\end{array}$ \\
\hline
\end{tabular}




\begin{tabular}{|l|l|l|l|}
\hline 37 & $\begin{array}{l}\text { EDDS_AT_UROL } \\
\text { OG }\end{array}$ & $\begin{array}{l}\text { Number of A\&E } \\
\text { attendances for Urological } \\
\text { Conditions }\end{array}$ & $\begin{array}{l}\text { Using provided categories on } \\
\text { A\&E codes }\end{array}$ \\
\hline 38 & $\begin{array}{l}\text { EDDS_AT_WOU } \\
\text { ND }\end{array}$ & $\begin{array}{l}\text { Number of A\&E } \\
\text { attendances for Wound }\end{array}$ & $\begin{array}{l}\text { Using provided categories on } \\
\text { A\&E codes }\end{array}$ \\
\hline $\begin{array}{l}\text { Break down of PEDW attendance counts based on } 3 \text { categories of ICD10 code list } \\
\text { Note: For cases the number of attendances were count after their first opioid } \\
\text { prescription all attendances for controls. }\end{array}$ \\
\hline 39 & $\begin{array}{l}\text { PEDW_ADVERS } \\
\text { CNT }\end{array}$ & $\begin{array}{l}\text { Number of PEDW } \\
\text { attendances for adverse } \\
\text { reactions to drugs }\end{array}$ & $\begin{array}{l}\text { Using provided categories on } \\
\text { ICD10 codes }\end{array}$ \\
\hline 40 & $\begin{array}{l}\text { PEDW_PAIN_CN } \\
\text { T }\end{array}$ & $\begin{array}{l}\text { Number of PEDW } \\
\text { attendances for pain }\end{array}$ & $\begin{array}{l}\text { Using provided categories on } \\
\text { ICD10 codes }\end{array}$ \\
\hline 41 & $\begin{array}{l}\text { PEDW_INFEC_C } \\
\text { NT }\end{array}$ & $\begin{array}{l}\text { Number of PEDW } \\
\text { attendances for infection }\end{array}$ & $\begin{array}{l}\text { Using provided categories on } \\
\text { ICD10 codes }\end{array}$ \\
\hline
\end{tabular}




\section{F.2 Readcodes}

Table F.2: Read codes for pain-related conditions used to extract case and control subjects

\begin{tabular}{|c|c|c|c|c|c|c|}
\hline Medcode & Readcode & Readterm & cause & $\begin{array}{l}\text { Readcode- } \\
\text { V2-5dig }\end{array}$ & PREF_TERM_30 & PREF_TERM_60 \\
\hline 6736 & $16 \mathrm{~A} . .00$ & Stiff neck symptom & NECK & $16 A .$. & Stiff neck symptom & \\
\hline 1355 & $16 \mathrm{~A} 2.00$ & Stiff neck & NECK & $16 \mathrm{~A} 2$. & Stiff neck & \\
\hline 14882 & $16 \mathrm{~A} 3.00$ & Wry neck/torticollis & NECK & $16 \mathrm{~A}$. & Wry neck/torticollis & \\
\hline 16961 & $16 \mathrm{~A} 3.12$ & Wry neck symptom & NECK & $16 \mathrm{A3}$. & & \\
\hline 41031 & $16 \mathrm{AZ} .00$ & Stiff neck symptom NOS & NECK & $16 \mathrm{AZ}$. & Stiff neck symptom NOS & \\
\hline 16183 & $1 \mathrm{D} 21.00$ & Symptom: head/neck & NECK & $1 \mathrm{D} 21$. & Symptom: head/neck & \\
\hline 6188 & $1 \mathrm{D} 21.12$ & $\mathrm{C} / \mathrm{O}$ - a neck symptom & NECK & $1 \mathrm{D} 21$. & & \\
\hline 7033 & $2 \mathrm{H} 2 \mathrm{D} .00$ & O/E - neck joint abnormal & NECK & 2H2D. & O/E - neck joint abnormal & \\
\hline 97899 & 2121.00 & O/E - sign - head/neck & NECK & 2121. & O/E - sign - head/neck & \\
\hline 59990 & $7 \mathrm{G} 15.11$ & Sensory skin flap including to head or neck & NECK & $7 \mathrm{G} 15$. & Sensory skin flap & \\
\hline 94954 & 7G15200 & Local sensory skin flap to head or neck & NECK & 7G152 & $\begin{array}{l}\text { Local sensory skin flap } \\
\text { hd/nk }\end{array}$ & $\begin{array}{l}\text { Local sensory skin } \\
\text { flap to head or neck }\end{array}$ \\
\hline 61137 & 7G19.11 & $\begin{array}{l}\text { Local subcutaneous pedicle skin flap } \\
\text { including to head/neck }\end{array}$ & NECK & 7G19. & $\begin{array}{l}\text { Local subcut pedicle skin } \\
\text { flap }\end{array}$ & $\begin{array}{l}\text { Local subcutaneous } \\
\text { pedicle skin flap }\end{array}$ \\
\hline 59990 & $7 \mathrm{G} 15.11$ & Sensory skin flap including to head or neck & NECK & $7 \mathrm{G} 15$. & Sensory skin flap & \\
\hline 94954 & $7 G 15200$ & Local sensory skin flap to head or neck & NECK & $7 \mathrm{G} 152$ & $\begin{array}{l}\text { Local sensory skin flap } \\
\text { hd/nk }\end{array}$ & $\begin{array}{l}\text { Local sensory skin } \\
\text { flap to head or neck }\end{array}$ \\
\hline 61137 & $7 G 19.11$ & $\begin{array}{l}\text { Local subcutaneous pedicle skin flap } \\
\text { including to head/neck }\end{array}$ & NECK & 7G19. & $\begin{array}{l}\text { Local subcut pedicle skin } \\
\text { flap }\end{array}$ & $\begin{array}{l}\text { Local subcutaneous } \\
\text { pedicle skin flap }\end{array}$ \\
\hline 57534 & L331z00 & Cord tight round neck NOS & NECK & L331z & Cord tight round neck NOS & \\
\hline 123 & N131.00 & Cervicalgia - pain in neck & NECK & N131. & Cervicalgia - pain in neck & \\
\hline 28823 & $\mathrm{~N} 135.11$ & Contracture of neck & NECK & N135. & Torticollis unspecified & \\
\hline 9683 & $\mathrm{~N} 135 \mathrm{z} 11$ & Stiff neck NOS & NECK & $\mathrm{N} 135 \mathrm{z}$ & Torticollis NOS & \\
\hline 2617 & $\mathrm{~N} 135 \mathrm{z} 12$ & Wry neck & NECK & N135z & & \\
\hline 20994 & N13y200 & Crick in neck & NECK & N13y2 & Crick in neck & \\
\hline 3640 & N13z.00 & Cervical and neck disorders NOS & NECK & N13z. & $\begin{array}{l}\text { Cervical/neck disorder } \\
\text { NOS }\end{array}$ & $\begin{array}{l}\text { Cervical and neck } \\
\text { disorders NOS }\end{array}$ \\
\hline 4698 & N240500 & Fibrositis of neck & NECK & N2405 & Fibrositis of neck & \\
\hline 65125 & PE1..11 & Congenital wry neck & NECK & PE1.. & $\begin{array}{l}\text { Congen.sternomastoid } \\
\text { torticol. }\end{array}$ & $\begin{array}{l}\text { Congenital } \\
\text { sternomastoid } \\
\text { torticollis }\end{array}$ \\
\hline 16664 & R04..00 & [D]Head and neck symptoms & NECK & R04.. & $\begin{array}{l}\text { [D]Head and neck } \\
\text { symptoms }\end{array}$ & \\
\hline 48124 & R04z.00 & [D]Other symptoms affecting head and neck & NECK & R04z. & $\begin{array}{l}\text { [D]Head and neck, other } \\
\text { sympt. }\end{array}$ & $\begin{array}{l}\text { [D]Other symptoms } \\
\text { affecting head and } \\
\text { neck }\end{array}$ \\
\hline 15178 & R04zz00 & [D]Head and neck symptoms NOS & NECK & $\mathrm{R} 04 \mathrm{zz}$ & \multicolumn{2}{|c|}{ [D]Head and neck symptoms NOS } \\
\hline 10252 & $\mathrm{~S} 1 \ldots 00$ & Fracture of neck and trunk & NECK & $\mathrm{S} 1 \ldots$ & Fracture of neck and trunk & \\
\hline 3288 & S10A.00 & Fracture of neck & NECK & S10A. & Fracture of neck & \\
\hline 25284 & S1z..00 & Fracture of neck and trunk NOS & NECK & S1z.. & $\begin{array}{l}\text { Fracture of neck and trunk } \\
\text { NOS }\end{array}$ & \\
\hline 99397 & S261600 & Open fracture thumb proximal phalanx, neck & NECK & S2616 & $\begin{array}{l}\text { Opn \# thumb prox phlnx, } \\
\text { neck }\end{array}$ & $\begin{array}{l}\text { Open fracture thumb } \\
\text { proximal phalanx, } \\
\text { neck }\end{array}$ \\
\hline 2225 & S30..00 & Fracture of neck of femur & NECK & s30.. & \#Neck of femur & $\begin{array}{l}\text { Fracture of neck of } \\
\text { femur }\end{array}$ \\
\hline 41001 & S490.12 & Closed dislocation of neck & NECK & S490. & Cls dslc cervical spine & $\begin{array}{l}\text { Closed dislocation } \\
\text { cervical spine }\end{array}$ \\
\hline 93829 & S491.12 & Open dislocation of neck & NECK & S491. & $\begin{array}{l}\text { Open dislocation cerv } \\
\text { spine }\end{array}$ & $\begin{array}{l}\text { Open dislocation } \\
\text { cervical spine }\end{array}$ \\
\hline 2463 & S570.00 & Neck sprain & NECK & S570. & Neck sprain & \\
\hline 15212 & S570000 & Neck sprain, unspecified & NECK & S5700 & Neck sprain, unspecified & \\
\hline 25576 & S570z00 & Neck sprain NOS & NECK & $\mathrm{S} 570 \mathrm{z}$ & Neck sprain NOS & \\
\hline 8812 & SD0y100 & Superficial injury of neck NOS & NECK & SD0y1 & $\begin{array}{l}\text { Superficial injury of neck } \\
\text { NOS }\end{array}$ & \\
\hline 69652 & SD0z100 & Superficial injury of neck NOS, infected & NECK & SDOz1 & $\begin{array}{l}\text { Superfic.inj.neck } \\
\text { NOS+infectn. }\end{array}$ & $\begin{array}{l}\text { Superficial injury of } \\
\text { neck NOS, infected }\end{array}$ \\
\hline 36121 & SDC0.00 & Superficial injuries involving head with neck & NECK & SDCO. & $\begin{array}{l}\text { Supfcl inj invlv head wth } \\
\text { neck }\end{array}$ & $\begin{array}{l}\text { Superficial injuries } \\
\text { involving head with } \\
\text { neck }\end{array}$ \\
\hline 20117 & SE08.00 & Other contusion neck & NECK & SE08. & Other contusion neck & \\
\hline 11724 & SF0..00 & Crush injury, face, scalp and neck & NECK & SFO.. & $\begin{array}{l}\text { Crush injury, face, } \\
\text { scalp+neck }\end{array}$ & $\begin{array}{l}\text { Crush injury, face, } \\
\text { scalp and neck }\end{array}$ \\
\hline 12864 & SF02.00 & Crush injury, neck & NECK & SF02. & Crush injury, neck & \\
\hline 66769 & SF02z00 & Crush injury, neck NOS & NECK & SF02z & Crush injury, neck NOS & \\
\hline 96413 & SFOz.00 & Crush injury, face, scalp and neck NOS & NECK & SFOz. & $\begin{array}{l}\text { Crush inj,face,scalp+neck } \\
\text { NOS }\end{array}$ & $\begin{array}{l}\text { Crush injury, face, } \\
\text { scalp and neck NOS }\end{array}$ \\
\hline 51916 & SH14900 & $\begin{array}{l}\text { Deep full thickness burn of neck without loss } \\
\text { of body part }\end{array}$ & NECK & SH149 & $\begin{array}{l}\text { Deep F/T burn-neck-no } \\
\text { BPL }\end{array}$ & $\begin{array}{l}\text { Deep full thickness } \\
\text { burn of neck without } \\
\text { loss of body part }\end{array}$ \\
\hline
\end{tabular}




\begin{tabular}{|c|c|c|c|c|c|c|}
\hline 44249 & SJ70.00 & Head and neck superficial nerve injury & NECK & SJ70. & $\begin{array}{l}\text { Head/neck superfic.nerve } \\
\text { inj. }\end{array}$ & $\begin{array}{l}\text { Head and neck } \\
\text { superficial nerve } \\
\text { injury }\end{array}$ \\
\hline 24486 & SJ70.11 & Head and neck superficial nerve injury & NECK & SJ70. & & \\
\hline 35864 & SJ70.12 & Neck superficial nerve injury & NECK & SJ70. & & \\
\hline 20930 & SJ71.00 & Injury of peripheral nerves of neck & NECK & SJ71. & $\begin{array}{l}\text { Injury/periphrl nerves of } \\
\text { neck }\end{array}$ & $\begin{array}{l}\text { Injury of peripheral } \\
\text { nerves of neck }\end{array}$ \\
\hline 23690 & SJ8..00 & Injury of nerves and spinal cord at neck level & NECK & SJ8.. & & \\
\hline 4446 & SK10.00 & Other face and neck injuries & NECK & SK10. & Other face/neck injuries & $\begin{array}{l}\text { Other face and neck } \\
\text { injuries }\end{array}$ \\
\hline 17360 & SK10800 & Injury of muscle and tendon at neck level & NECK & SK108 & $\begin{array}{l}\text { Injur/muscle+tendon/neck } \\
\text { level }\end{array}$ & $\begin{array}{l}\text { Injury of muscle and } \\
\text { tendon at neck level }\end{array}$ \\
\hline 1192 & SK10y00 & Other neck injuries & NECK & SK10y & Other neck injuries & \\
\hline 14799 & SK10z00 & Other face and neck injuries NOS & NECK & SK10z & $\begin{array}{l}\text { Other face/neck injuries } \\
\text { NOS }\end{array}$ & $\begin{array}{l}\text { Other face and neck } \\
\text { injuries NOS }\end{array}$ \\
\hline 54854 & SK1×100 & Multiple superficial injuries of neck & NECK & SK $1 \times 1$ & $\begin{array}{l}\text { Multipl superfic } \\
\text { injuries/neck }\end{array}$ & $\begin{array}{l}\text { Multiple superficial } \\
\text { injuries of neck }\end{array}$ \\
\hline 21101 & SK1×300 & Multiple injuries of neck & NECK & SK1×3 & Multiple injuries of neck & \\
\hline 32009 & SR10.00 & Fractures involving head with neck & NECK & SR10. & $\begin{array}{l}\text { Fracture involv head with } \\
\text { neck }\end{array}$ & $\begin{array}{l}\text { Fractures involving } \\
\text { head with neck }\end{array}$ \\
\hline 73786 & SR10000 & Closed fractures involving head with neck & NECK & SR100 & $\begin{array}{l}\text { Cls fract invol head with } \\
\text { neck }\end{array}$ & $\begin{array}{l}\text { Closed fractures } \\
\text { involving head with } \\
\text { neck }\end{array}$ \\
\hline 12327 & SR20.00 & $\begin{array}{l}\text { Dislocations, sprains and strains involving } \\
\text { head with neck }\end{array}$ & NECK & SR20. & $\begin{array}{l}\text { Disloc,sprns+strns inv } \\
\text { hd+neck }\end{array}$ & $\begin{array}{l}\text { Dislocations, sprains } \\
\text { and strains involving } \\
\text { head with neck }\end{array}$ \\
\hline 48651 & SR30.00 & Crushing injuries involving head with neck & NECK & SR30. & $\begin{array}{l}\text { Crush inj invlv head with } \\
\text { neck }\end{array}$ & $\begin{array}{l}\text { Crushing injuries } \\
\text { involving head with } \\
\text { neck }\end{array}$ \\
\hline 37326 & Syu1.00 & {$[X]$ Injuries to the neck } & NECK & Syu1. & {$[X]$ Injuries to the neck } & \\
\hline 58728 & Syu1100 & $\begin{array}{l}{[X] \text { Superficial injury of other parts of the }} \\
\text { neck }\end{array}$ & NECK & Syu11 & $\begin{array}{l}{[X] \text { Superfic inj oth part }} \\
\text { neck }\end{array}$ & $\begin{array}{l}{[X] \text { Superficial injury }} \\
\text { of other parts of the } \\
\text { neck }\end{array}$ \\
\hline 70825 & Syu1200 & {$[X]$ Superficial injury of neck, part unspecified } & NECK & Syu12 & $\begin{array}{l}{[X] \text { Superf inj neck, part }} \\
\text { unsp }\end{array}$ & $\begin{array}{l}\text { [X]Superficial injury } \\
\text { of neck, part } \\
\text { unspecified }\end{array}$ \\
\hline 99936 & Syu1600 & {$[\mathrm{X}]$ Fracture of other parts of neck } & NECK & Syu16 & $\begin{array}{l}{[X] \text { Fracture other parts }} \\
\text { neck }\end{array}$ & $\begin{array}{l}{[\mathrm{X}] \text { Fracture of other }} \\
\text { parts of neck }\end{array}$ \\
\hline 53850 & Syu1800 & $\begin{array}{l}{[X] \text { Sprain/strain of joint/ligam of oth \& unsp }} \\
\text { part of neck }\end{array}$ & NECK & Syu18 & $\begin{array}{l}{[\mathrm{X}] \mathrm{Spr} / \mathrm{str} \mathrm{jt} / \mathrm{lg} \text { ot/un pt }} \\
\text { neck }\end{array}$ & $\begin{array}{l}{[X] \text { Sprain/strain of }} \\
\text { joint/ligam of oth \& } \\
\text { unsp part of neck }\end{array}$ \\
\hline 102012 & Syu1A00 & $\begin{array}{l}{[X] \text { Injury of other and unspecified nerves of }} \\
\text { neck }\end{array}$ & NECK & Syu1A & $\begin{array}{l}{[X] \text { Inj oth unsp nerve of }} \\
\text { neck }\end{array}$ & $\begin{array}{l}{[X] \text { Injury of other }} \\
\text { and unspecified } \\
\text { nerves of neck }\end{array}$ \\
\hline 68839 & Syu1F00 & {$[X]$ Other specified injuries of neck } & NECK & Syu1F & {$[\mathrm{X}]$ Oth specif inj of neck } & $\begin{array}{l}{[X] \text { Other specified }} \\
\text { injuries of neck }\end{array}$ \\
\hline 97345 & SyuLCOO & $\begin{array}{l}{[X] \text { Sequelae of unspecified injury of neck and }} \\
\text { trunk }\end{array}$ & NECK & SyulC & $\begin{array}{l}{[X] \text { Seq unspec injury }} \\
\text { neck+trnk }\end{array}$ & $\begin{array}{l}{[\mathrm{X}] \text { Sequelae of }} \\
\text { unspecified injury of } \\
\text { neck and trunk }\end{array}$ \\
\hline 36875 & Z6G6200 & Neck stretching & NECK & Z6G62 & & \\
\hline 63752 & ZV48.00 & {$[\mathrm{V}]$ Head, neck or trunk problems } & NECK & ZV48. & $\begin{array}{l}{[\mathrm{V}] \text { Head, neck/ trunk }} \\
\text { problem }\end{array}$ & $\begin{array}{l}{[\mathrm{V}] \text { Head, neck or }} \\
\text { trunk problems }\end{array}$ \\
\hline 7116 & ZV48.12 & [V]Problems with neck & NECK & zV48. & & \\
\hline 56003 & ZV48500 & [V]Sensory problem of neck or trunk & NECK & ZV485 & $\begin{array}{l}{[\mathrm{V}] \text { Neck/trunk sensory }} \\
\text { problem }\end{array}$ & $\begin{array}{l}{[V] \text { Sensory problem }} \\
\text { of neck or trunk }\end{array}$ \\
\hline
\end{tabular}

\begin{tabular}{|l|l|l|l|l|l|}
\hline Medcode & Readcode & Readterm & cause & $\begin{array}{l}\text { Readcode-V2- } \\
\mathbf{5 d i g}\end{array}$ & PREF_TERM_30 \\
\hline 8120 & 14 N3000 & H/O Spinal surgery & SPINAL PAIN & $14 N 30$ & H/O Spinal surgery \\
\hline 73015 & 2 AD6.00 & O/E - ciliospinal reflex -ve. & SPINAL PAIN & 2 AD6. & O/E - ciliospinal reflex -ve. \\
\hline 71673 & 7040 & Partial extirpation of spinal cord & SPINAL PAIN & 7040 & \\
\hline 62660 & 7041000 & Stereotactic chordotomy of spinal cord & SPINAL PAIN & 70410 & Stereotac chordotomy spin cord \\
\hline 15218 & 7041100 & Open chordotomy of spinal cord NEC & SPINAL PAIN & 70411 & Open chordotomy spin cord NEC \\
\hline 57641 & 7041200 & Myelotomy of spinal cord & SPINAL PAIN & 70412 & Myelotomy of spinal cord \\
\hline 21088 & 7042100 & Percutaneous chordotomy of spinal cord & SPINAL PAIN & 70421 & Percut chordotomy spinal cord \\
\hline 5307 & 7043100 & Closure of spinal myelomeningocele & SPINAL PAIN & 70431 & Closure spin myelomeningocele \\
\hline 29633 & 7043200 & Closure of spinal meningocele & SPINAL PAIN & 70432 & Closure of spinal meningocele \\
\hline 1145 & 7047000 & Spinal myelography NEC & SPINAL PAIN & 70470 & Spinal myelography NEC \\
\hline 66902 & 7047100 & Spinal manometry & SPINAL PAIN & 70471 & Spinal manometry \\
\hline 67831 & 7048011 & Destruction of lesion of spinal nerve root & SPINAL PAIN & 70480 & Extirpatn lesion spinal $n$ root \\
\hline 21655 & 7048100 & Rhizotomy of spinal nerve root & SPINAL PAIN & 70481 & Rhizotomy spinal nerve root \\
\hline 60506 & 7048400 & Destruction of spinal nerve root NEC & SPINAL PAIN & 70484 & Destruct spinal nerve root NEC \\
\hline 33481 & 7049 & Other destruction of spinal cord & SPINAL PAIN & 7049 & \\
\hline
\end{tabular}




\begin{tabular}{|c|c|c|c|c|c|}
\hline 97105 & 7049y00 & Other specified destruction of spinal cord & SPINAL PAIN & $7049 y$ & Other destruct spinal cord OS \\
\hline 21288 & $7 \mathrm{JJ} . .11$ & Lumbar spinal cord operations & SPINAL PAIN & 7Ј3.. & Lumbar spine operations \\
\hline 63452 & $7 J 42300$ & $\begin{array}{l}\text { Spinal extension traction for fracture of } \\
\text { spine }\end{array}$ & SPINAL PAIN & $7 \mathrm{~J} 423$ & Spinal extension traction \# \\
\hline 15622 & $7 \mathrm{~J} 42500$ & Spinal traction for fracture of spine NEC & SPINAL PAIN & $7 \mathrm{~J} 425$ & Spinal traction \# spine NEC \\
\hline 42002 & $7 \mathrm{~J} 47900$ & Spinal probing & SPINAL PAIN & $7 \mathrm{~J} 479$ & Spinal probing \\
\hline 16885 & $7 J 49.00$ & Denervation of spinal facet joint of vertebra & SPINAL PAIN & $7 \mathrm{~J} 49$. & Denervat spinal facet vertebra \\
\hline 45679 & $7 \mathrm{~J} 49100$ & $\begin{array}{l}\text { Denervation of spinal facet joint of cervical } \\
\text { vertebra NEC }\end{array}$ & SPINAL PAIN & $7 \mathrm{~J} 491$ & Denerv cervic spinal facet NEC \\
\hline 65587 & 7J49300 & $\begin{array}{l}\text { Denervation of spinal facet joint of thoracic } \\
\text { vertebra NEC }\end{array}$ & SPINAL PAIN & $7 \mathrm{~J} 493$ & Denerv thoracic spin facet NEC \\
\hline 28261 & $7 J 49500$ & $\begin{array}{l}\text { Denervation of spinal facet joint of lumbar } \\
\text { vertebra NEC }\end{array}$ & SPINAL PAIN & $7 \mathrm{~J} 495$ & Denerv lumbar spin facet NEC \\
\hline 70138 & 7J49y00 & $\begin{array}{l}\text { Denervation of spinal facet joint of vertebra } \\
\text { OS }\end{array}$ & SPINAL PAIN & $7 \mathrm{~J} 49 \mathrm{y}$ & Denerv vertebr spinal facet OS \\
\hline 40516 & 7J49z00 & $\begin{array}{l}\text { Denervation of spinal facet joint of vertebra } \\
\text { NOS }\end{array}$ & SPINAL PAIN & $7 \mathrm{~J} 49 \mathrm{z}$ & Denerv verteb spinal facet NOS \\
\hline 69837 & A940.12 & Syphilitic posterior spinal sclerosis & SPINAL PAIN & A940. & Tabes dorsalis - neurosyphilis \\
\hline 54143 & B74y000 & Lipoma of spinal column & SPINAL PAIN & $\mathrm{B} 74 \mathrm{y} 0$ & Lipoma of spinal column \\
\hline 70427 & B74y100 & Lipoma of spinal canal - extradural & SPINAL PAIN & B74y 1 & Lipoma of spine - extradural \\
\hline 64310 & B74y200 & Lipoma of spinal canal - intradural & SPINAL PAIN & B74y2 & Lipoma of spine - intradural \\
\hline 70599 & В74уз00 & Lipoma of spinal cord & SPINAL PAIN & B74y3 & Lipoma of spinal cord \\
\hline 9544 & B7F4000 & Spinal meningioma & SPINAL PAIN & $B 7 F 40$ & Spinal meningioma \\
\hline 44774 & B925100 & $\begin{array}{l}\text { Neoplasm of uncertain behaviour of spinal } \\
\text { cord }\end{array}$ & SPINAL PAIN & B9251 & Uncert. neopl. spinal cord \\
\hline 28567 & F04..00 & Intracranial and intraspinal abscesses & SPINAL PAIN & F04.. & Intracranial / spinal abscess \\
\hline 15434 & F041.00 & Intraspinal abscess & SPINAL PAIN & F041. & Intraspinal abscess \\
\hline 73764 & F041200 & Subdural intraspinal abscess & SPINAL PAIN & F0412 & Intraspinal abscess-subdural \\
\hline 60611 & F041z00 & Intraspinal abscess NOS & SPINAL PAIN & F041z & Intraspinal abscess NOS \\
\hline 23466 & F04z.00 & Intracranial or intraspinal abscess NOS & SPINAL PAIN & Fo4z. & Intracranial / spinal absc.NOS \\
\hline 95615 & F150.11 & Infantile spinal muscular atrophy & SPINAL PAIN & F150. & Werdnig - Hoffmann disease \\
\hline 9179 & $\mathrm{~F} 151.00$ & Spinal muscular atrophy & SPINAL PAIN & F151. & Spinal muscular atrophy \\
\hline 70572 & F151000 & Unspecified spinal muscular atrophy & SPINAL PAIN & F1510 & Spinal muscular atrophy unspec \\
\hline 70109 & F151300 & X-linked bulbo-spinal atrophy & SPINAL PAIN & F1513 & X-linked bulbo-spinal atrophy \\
\hline 57632 & $\mathrm{~F} 151 \mathrm{z} 00$ & Spinal muscular atrophy NOS & SPINAL PAIN & F151z & Spinal muscular atrophy NOS \\
\hline 17194 & $\mathrm{~F} 16 . .00$ & Other diseases of spinal cord & SPINAL PAIN & F16.. & Other diseases of spinal cord \\
\hline 17216 & F161000 & $\begin{array}{l}\text { Myelopathy due to acute infarction of spinal } \\
\text { cord }\end{array}$ & SPINAL PAIN & F1610 & Myelopathy-infarct spine cord \\
\hline 65630 & F161100 & $\begin{array}{l}\text { Myelopathy due to arterial thrombosis of } \\
\text { spinal cord }\end{array}$ & SPINAL PAIN & F1611 & Myelopathy-thrombos spine cord \\
\hline 67980 & F161200 & Myelopathy due to oedema of spinal cord & SPINAL PAIN & $\mathrm{F} 1612$ & Myelopathy-oedema spine cord \\
\hline 33535 & F161500 & Anterior spinal artery thrombosis & SPINAL PAIN & F1615 & Ant spinal artery thrombosis \\
\hline 7736 & F162.00 & $\begin{array}{l}\text { Subacute combined degeneration of spinal } \\
\text { cord }\end{array}$ & SPINAL PAIN & F162. & Subacute combined cord degen. \\
\hline 69886 & F201.00 & Multiple sclerosis of the spinal cord & SPINAL PAIN & F201. & Multiple sclerosis-spinal cord \\
\hline 53396 & F290y00 & Other spinal puncture reaction & SPINAL PAIN & F290y & Other spinal puncture reaction \\
\hline 71885 & F292100 & Spinal meningeal adhesions & SPINAL PAIN & F2921 & Adhesions - spinal meninges \\
\hline 51667 & F292111 & Spinal meningeal adhesions & SPINAL PAIN & F2921 & \\
\hline 40237 & F292300 & Cyst of the spinal meninges & SPINAL PAIN & F2923 & Cyst - spinal meninges \\
\hline 62548 & Fyu0B00 & $\begin{array}{l}\text { [X]Intracranial+intraspinal } \\
\text { abscess+granuloma in diseases CE }\end{array}$ & SPINAL PAIN & Fyu0B & {$[\mathrm{X}]$ Intrcrn+intrspn $a b+g r n / d \mathrm{CE}$} \\
\hline 101525 & Fyu3200 & $\begin{array}{l}{[X] \text { Subacute combined degeneration/spinal }} \\
\text { cord in diseases } \mathrm{CE}\end{array}$ & SPINAL PAIN & Fyu32 & {$[\mathrm{X}]$ Subac cmb dgn/spn crd/ds CE } \\
\hline 53754 & FyuA700 & [X]Other specified diseases of spinal cord & SPINAL PAIN & FyuA7 & [X]Oth spcf disease/spinal crd \\
\hline 50241 & G769.00 & $\begin{array}{l}\text { Anterior spinal and vertebral artery } \\
\text { compression syndromes }\end{array}$ & SPINAL PAIN & G769. & Ant spin/vert art compr syndr \\
\hline 65804 & L384100 & $\begin{array}{l}\text { Spinal/epidural anaesth-induced headache } \\
\text { during puerp }\end{array}$ & SPINAL PAIN & L3841 & Sp/ep anes-ind hdache dur puer \\
\hline 42755 & L387.00 & $\begin{array}{l}\text { Spinal/epidural anesth-induced headache } \\
\text { dur labour/delivery }\end{array}$ & SPINAL PAIN & L387. & Sp/epi anes-ind hdache,lab/del \\
\hline 24200 & N101.00 & Spinal enthesopathy & SPINAL PAIN & N101. & Spinal enthesopathy \\
\hline 45246 & N12A.11 & Post spinal surgery syndrome & SPINAL PAIN & N12A. & Postlaminectomy syndrome \\
\hline
\end{tabular}




\begin{tabular}{|c|c|c|c|c|c|}
\hline 69388 & N130000 & Idiopathic cervical spinal stenosis & SPINAL PAIN & $\mathrm{N} 1300$ & Idiopathic $\mathrm{Cx}$ spinal stenosis \\
\hline 45296 & N130100 & Degenerative cervical spinal stenosis & SPINAL PAIN & N1301 & Degenerativ $\mathrm{Cx}$ spinal stenosis \\
\hline 15331 & N140.00 & Spinal stenosis, excluding cervical region & SPINAL PAIN & N140. & Spinal stenosis excl.cervical \\
\hline 3370 & N140.11 & Spinal stenosis & SPINAL PAIN & N140. & \\
\hline 41147 & N140000 & Spinal stenosis of unspecified region & SPINAL PAIN & $\mathrm{N} 1400$ & Spinal stenosis unspec.region \\
\hline 62612 & N140100 & Thoracic spinal stenosis & SPINAL PAIN & N1401 & Thoracic spinal stenosis \\
\hline 9912 & N140200 & Lumbar spinal stenosis & SPINAL PAIN & $\mathrm{N} 1402$ & Lumbar spinal stenosis \\
\hline 73730 & N140400 & Degenerative thoracic spinal stenosis & SPINAL PAIN & N1404 & Degenerativ th spinal stenosis \\
\hline 98630 & N140600 & $\begin{array}{l}\text { Thoracic spinal stenosis secondary to other } \\
\text { disease }\end{array}$ & SPINAL PAIN & N1406 & Th spin stenos due to oth dis \\
\hline 72614 & N140700 & Idiopathic lumbar spinal stenosis & SPINAL PAIN & N1407 & Idiopathic lu spinal stenosis \\
\hline 43577 & N140800 & Degenerative lumbar spinal stenosis & SPINAL PAIN & N1408 & Degenerativ lu spinal stenosis \\
\hline 93836 & N140900 & latrogenic lumbar spinal stenosis & SPINAL PAIN & N1409 & latrogenic lu spinal stenosis \\
\hline 97870 & N140A00 & $\begin{array}{l}\text { Lumbar spinal stenosis secondary to other } \\
\text { disease }\end{array}$ & SPINAL PAIN & $\mathrm{N} 140 \mathrm{~A}$ & Lu spin stenos due to oth dis \\
\hline 35117 & $\mathrm{~N} 140 \mathrm{z} 00$ & Spinal stenosis NOS & SPINAL PAIN & $\mathrm{N} 140 \mathrm{z}$ & Spinal stenosis NOS \\
\hline 18226 & $\mathrm{~N} 14 \mathrm{z} .12$ & Spinal disorder NOS & SPINAL PAIN & N14z. & Back disorders NOS \\
\hline 100102 & Nyu5B00 & {$[X]$ Spinal osteochondrosis, unspecified } & SPINAL PAIN & Nyu5B & {$[\mathrm{X}]$ Spin osteochondrosis, unsp } \\
\hline 53578 & P111.00 & Spinal hydromeningocele & SPINAL PAIN & P111. & Spinal hydromeningocele \\
\hline 6866 & P113.00 & Spinal meningocele & SPINAL PAIN & P113. & Spinal meningocele \\
\hline 53929 & P113000 & Spinal meningocele of unspecified site & SPINAL PAIN & P1130 & Spinal meningocele-unspec.site \\
\hline 91600 & P113200 & Thoracic spinal meningocele & SPINAL PAIN & P1132 & Spinal meningocele-thoracic \\
\hline 61862 & P113300 & Lumbar spinal meningocele & SPINAL PAIN & P1133 & Spinal meningocele-lumbar \\
\hline 50365 & P113z00 & Spinal meningocele NOS & SPINAL PAIN & P113z & Spinal meningocele NOS \\
\hline 70266 & P25..00 & Other specified spinal cord anomalies & SPINAL PAIN & P25.. & Other spec.spinal cord anomaly \\
\hline 48940 & P252.00 & Congenital tethering of spinal cord & SPINAL PAIN & P252. & Congen tethering spinal cord \\
\hline 95591 & P25y.00 & Other specified anomalies of spinal cord & SPINAL PAIN & P25y. & Spinal cord anomalies OS \\
\hline 45142 & P25y112 & Myelodysplasia of spinal cord & SPINAL PAIN & P25y1 & Atelomyelia \\
\hline 45918 & P25y200 & Congenital anomaly of spinal meninges & SPINAL PAIN & P25y2 & Spinal meninges congen.anomaly \\
\hline 73166 & $\mathrm{P} 25 \mathrm{y} 400$ & Spinal cord hypoplasia & SPINAL PAIN & $\mathrm{P} 25 \mathrm{y} 4$ & Spinal cord hypoplasia \\
\hline 16164 & $\mathrm{P} 25 z .00$ & Spinal cord anomalies NOS & SPINAL PAIN & P25z. & Spinal cord anomalies NOS \\
\hline 36974 & P2y1.00 & Congenital spinal cord anomaly & SPINAL PAIN & P2y1. & Congenital spinal cord anomaly \\
\hline 31857 & Q204.00 & $\begin{array}{l}\text { Spine or spinal cord injury due to birth } \\
\text { trauma }\end{array}$ & SPINAL PAIN & Q204. & Birth spine/spinal cord injury \\
\hline 100378 & Q204z00 & $\begin{array}{l}\text { Spine or spinal cord injury due to birth } \\
\text { trauma NOS }\end{array}$ & SPINAL PAIN & Q204z & Birth spine/cord injury NOS \\
\hline 8255 & $S 10 . .00$ & $\begin{array}{l}\text { Fracture of spine without mention of spinal } \\
\text { cord injury }\end{array}$ & SPINAL PAIN & S10.. & \#Spine - no cord lesion \\
\hline 30058 & S10..11 & $\begin{array}{l}\text { Fracture of transverse process spine - no } \\
\text { spinal cord lesion }\end{array}$ & SPINAL PAIN & S10.. & \\
\hline 4409 & S10..12 & $\begin{array}{l}\text { Fracture of vertebra without spinal cord } \\
\text { lesion }\end{array}$ & SPINAL PAIN & S10.. & \\
\hline 42561 & S100111 & $\begin{array}{l}\text { C1 vertebra closed fracture - no spinal cord } \\
\text { lesion }\end{array}$ & SPINAL PAIN & S1001 & Closed fracture atlas \\
\hline 33967 & S100211 & $\begin{array}{l}\text { C2 vertebra closed fracture without spinal } \\
\text { cord lesion }\end{array}$ & SPINAL PAIN & S1002 & Closed fracture axis \\
\hline 52699 & S100311 & $\begin{array}{l}\text { C3 vertebra closed fracture without spinal } \\
\text { cord lesion }\end{array}$ & SPINAL PAIN & $\mathrm{S} 1003$ & Clsd \# third cerv vertebra \\
\hline 67358 & S100411 & $\begin{array}{l}\text { C4 vertebra closed fracture without spinal } \\
\text { cord lesion }\end{array}$ & SPINAL PAIN & S1004 & Clsd \# fourth cerv vertebra \\
\hline 34873 & S100511 & $\begin{array}{l}\text { C5 vertebra closed fracture without spinal } \\
\text { cord lesion }\end{array}$ & SPINAL PAIN & S1005 & Clsd \# fifth cerv vertebra \\
\hline 33503 & S100611 & $\begin{array}{l}\text { C6 vertebra closed fracture without spinal } \\
\text { cord lesion }\end{array}$ & SPINAL PAIN & S1006 & Clsd \# sixth cerv vertebra \\
\hline 38053 & S100711 & $\begin{array}{l}\text { C7 vertebra closed fracture without spinal } \\
\text { cord lesion }\end{array}$ & SPINAL PAIN & S1007 & Clsd \# seventh cerv vertebra \\
\hline 69098 & S101111 & $\begin{array}{l}\text { C1 vertebra open fracture without spinal } \\
\text { cord lesion }\end{array}$ & SPINAL PAIN & S1011 & Open fracture atlas \\
\hline 97120 & S101211 & $\begin{array}{l}\text { C2 vertebra open fracture without spinal } \\
\text { cord lesion }\end{array}$ & SPINAL PAIN & S1012 & Open fracture axis \\
\hline 60382 & S101311 & $\begin{array}{l}\text { C3 vertebra open fracture without spinal } \\
\text { cord lesion }\end{array}$ & SPINAL PAIN & S1013 & Open \# third cerv vertebra \\
\hline 99151 & S101511 & $\begin{array}{l}\text { C5 vertebra open fracture without spinal } \\
\text { cord lesion }\end{array}$ & SPINAL PAIN & S1015 & Open \# fifth cerv vertebra \\
\hline 62719 & S101611 & $\begin{array}{l}\text { C6 vertebra open fracture without spinal } \\
\text { cord lesion }\end{array}$ & SPINAL PAIN & S1016 & Open \# sixth cerv vertebra \\
\hline
\end{tabular}




\begin{tabular}{|c|c|c|c|c|c|}
\hline 59996 & S101711 & $\begin{array}{l}\text { C7 vertebra open fracture without spinal } \\
\text { cord lesion }\end{array}$ & SPINAL PAIN & S1017 & Open \# seventh cerv vert \\
\hline 34166 & S10z.00 & $\begin{array}{l}\text { Fracture of spine without mention of spinal } \\
\text { cord lesion NOS }\end{array}$ & SPINAL PAIN & S10z. & \#Spine - no cord lesion - NOS \\
\hline 32063 & S11..00 & Fracture of spine with spinal cord lesion & SPINAL PAIN & S11.. & \#Spine + cord lesion \\
\hline 72324 & S11..11 & $\begin{array}{l}\text { Fracture of transverse process of spine }+ \\
\text { spinal cord lesion }\end{array}$ & SPINAL PAIN & S11.. & \\
\hline 43786 & S11..12 & Fracture of vertebra with spinal cord lesion & SPINAL PAIN & S11.. & \\
\hline 62337 & S110000 & $\begin{array}{l}\text { Cls spinal fracture with unspec cervical cord } \\
\text { lesion, C1-4 }\end{array}$ & SPINAL PAIN & $\mathrm{S} 1100$ & Clsd \# C1-C4 unspec cord les \\
\hline 100110 & S110500 & $\begin{array}{l}\text { Cls spinal \# with incomplete cervical cord } \\
\text { lesion, C1-4 NOS }\end{array}$ & SPINAL PAIN & S1105 & Clsd \# C1-C4 incomp cord les \\
\hline 72711 & S110600 & $\begin{array}{l}\text { Cls spinal fracture with unspec cervical cord } \\
\text { lesion, C5-7 }\end{array}$ & SPINAL PAIN & S1106 & Clsd \# C5-C7 unspec cord les \\
\hline 102735 & S110700 & $\begin{array}{l}\text { Cls spinal fracture with complete cervcl cord } \\
\text { lesion, C5-7 }\end{array}$ & SPINAL PAIN & S1107 & Clsd \# C5-C7 complete cord les \\
\hline 96514 & S110800 & $\begin{array}{l}\text { Cls spinal fracture with anterior cervcl cord } \\
\text { lesion, C5-7 }\end{array}$ & SPINAL PAIN & S1108 & Clsd \# C5-C7 ant cord lesion \\
\hline 101956 & S110B00 & $\begin{array}{l}\text { Cls spinal \# with incomplete cervical cord } \\
\text { lesion, C5-7 NOS }\end{array}$ & SPINAL PAIN & S110B & Clsd \# C5-C7 incomp cord les \\
\hline 69432 & $\$ 111.00$ & $\begin{array}{l}\text { Open fracture of cervical spine with spinal } \\
\text { cord lesion }\end{array}$ & SPINAL PAIN & S111. & Open cervical \#+cord lesion \\
\hline 35849 & S112.00 & $\begin{array}{l}\text { Closed fracture of thoracic spine with spinal } \\
\text { cord lesion }\end{array}$ & SPINAL PAIN & S112. & Closed thoracic \#+cord lesion \\
\hline 48958 & S112100 & $\begin{array}{l}\text { Cls spinal fracture wth complete thoracic } \\
\text { cord lesion,T1-6 }\end{array}$ & SPINAL PAIN & S1121 & 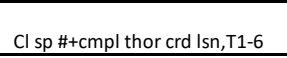 \\
\hline 43091 & S112600 & $\begin{array}{l}\text { Cls spinal fracture with unspec thoracic cord } \\
\text { lesion, T7-12 }\end{array}$ & SPINAL PAIN & S1126 & Cl sp \#+unsp thor cd Isn,T7-12 \\
\hline 31545 & S112700 & $\begin{array}{l}\text { Cls spinal fracture with complete thorac cord } \\
\text { lesion, } \mathrm{T7}-12\end{array}$ & SPINAL PAIN & S1127 & Cl sp \#+cmp thor crd Isn,T7-12 \\
\hline 102043 & S112A00 & $\begin{array}{l}\text { Cls spinal fracture with posterior thorac cord } \\
\text { lesion, } T 7-12\end{array}$ & SPINAL PAIN & S112A & $\mathrm{Cl}$ sp \#+pst thor crd Isn,T7-12 \\
\hline 70475 & S112B00 & $\begin{array}{l}\text { Cls spinal \# with incomplete thoracid cord } \\
\text { lesion, T7-12 NOS }\end{array}$ & SPINAL PAIN & S112B & Cl \# T7-12incomp cord Isn NOS \\
\hline 60615 & S113.00 & $\begin{array}{l}\text { Open fracture of thoracic spine with spinal } \\
\text { cord lesion }\end{array}$ & SPINAL PAIN & S113. & Open thoracic \#+cord lesion \\
\hline 49567 & S114000 & $\begin{array}{l}\text { Closed spinal fracture with unspecified } \\
\text { lumbar cord lesion }\end{array}$ & SPINAL PAIN & S1140 & Cls spn \# + unsp lumb crd lesn \\
\hline 95529 & S114100 & $\begin{array}{l}\text { Closed spinal fracture with complete lumbar } \\
\text { cord lesion }\end{array}$ & SPINAL PAIN & S1141 & Cls spn \#+ comp lumb crd lesn \\
\hline 73788 & S114500 & $\begin{array}{l}\text { Closed spinal fracture with cauda equina } \\
\text { lesion }\end{array}$ & SPINAL PAIN & S1145 & Cls spn \#+ cauda equina lesn \\
\hline 94189 & S115.00 & $\begin{array}{l}\text { Open fracture of lumbar spine with spinal } \\
\text { cord lesion }\end{array}$ & SPINAL PAIN & S115. & Open lumbar \# + cord lesion \\
\hline 96473 & S117.00 & $\begin{array}{l}\text { Open fracture of sacrum with spinal cord } \\
\text { lesion }\end{array}$ & SPINAL PAIN & S117. & Open sacral \# + cord lesion \\
\hline 94584 & S117300 & $\begin{array}{l}\text { Open fracture of sacrum with other spinal } \\
\text { cord injury }\end{array}$ & SPINAL PAIN & S1173 & Open sacral\#+other cord injury \\
\hline 55195 & S11z.00 & Fracture of spine with spinal cord lesion NOS & SPINAL PAIN & S11z. & \#Spine + cord lesion NOS \\
\hline 67389 & S492300 & $\begin{array}{l}\text { Closed spinal dislocation with complete } \\
\text { thoracic cord lesion }\end{array}$ & SPINAL PAIN & S4923 & Cls spnl dsI+comp thrc crd Isn \\
\hline 30160 & SJ...00 & Nerve and spinal cord injuries & SPINAL PAIN & SJ... & Nerve/spinal cord injuries \\
\hline 11853 & SJ...13 & Spinal cord injuries & SPINAL PAIN & SJ... & \\
\hline 34835 & SJ2..00 & $\begin{array}{l}\text { Spinal cord injury without evidence of spinal } \\
\text { bone injury }\end{array}$ & SPINAL PAIN & SJ2.. & Spinal cord inj.-no bone inj. \\
\hline 62286 & SJ21.00 & $\begin{array}{l}\text { Thoracic cord injury without spinal bone } \\
\text { injury }\end{array}$ & SPINAL PAIN & SJ21. & Thoracic cord inj.-no bone inj \\
\hline 59278 & SJ21.11 & Dorsal cord injury without spinal bone injury & SPINAL PAIN & SJ21. & \\
\hline 102775 & SJ21z00 & $\begin{array}{l}\text { Thoracic cord injury without spinal bone } \\
\text { injury, NOS }\end{array}$ & SPINAL PAIN & SJ21z & Thoracic cord inj.-no \#, NOS \\
\hline 71521 & SJ22.00 & $\begin{array}{l}\text { Lumbar cord injury without spinal bone } \\
\text { injury }\end{array}$ & SPINAL PAIN & SJ22. & Cord inj.lumbar-no bone inj. \\
\hline 58996 & SJ2z.00 & $\begin{array}{l}\text { Spinal cord injury without spinal bone injury } \\
\text { NOS }\end{array}$ & SPINAL PAIN & $\mathrm{SJ} 2 \mathrm{z}$. & Spinal cord inj.-no \# NOS \\
\hline 30093 & SJ3..00 & Nerve roots and spinal plexus injuries & SPINAL PAIN & SJ3.. & Nerve roots/spinal plexus inj. \\
\hline 26231 & SJ3..11 & Spinal plexus injury & SPINAL PAIN & SJ3.. & \\
\hline 53904 & SJ3z.00 & Nerve root and spinal plexus injuries NOS & SPINAL PAIN & SJ3z. & Nerv.root/spinal plex.inj.NOS \\
\hline 23690 & SJ8..00 & Injury of nerves and spinal cord at neck level & SPINAL PAIN & SJ8.. & Injur/nerv\&spinl cord/neck lev \\
\hline 41935 & SJ80.00 & $\begin{array}{l}\text { Concussion and oedema of cervical spinal } \\
\text { cord }\end{array}$ & SPINAL PAIN & SJ80. & Concuss\&oed/cervicl spinl cord \\
\hline 23778 & SJ9...00 & $\begin{array}{l}\text { Injury of nerves and spinal cord at thorax } \\
\text { level }\end{array}$ & SPINAL PAIN & SJg.. & Injur/nerv+spinl crd/thorx lev \\
\hline 100009 & SJ90.00 & $\begin{array}{l}\text { Concussion and oedema of thoracic spinal } \\
\text { cord }\end{array}$ & SPINAL PAIN & SJ90. & Concuss+oed/thoracic spinl crd \\
\hline 60687 & SJA0.00 & $\begin{array}{l}\text { Concussion and oedema of lumbar spinal } \\
\text { cord }\end{array}$ & SPINAL PAIN & SJAO. & Concuss+oed/lumbar spinal corc \\
\hline 3253 & SJz..00 & Nerve and spinal cord injury NOS & SPINAL PAIN & SJz.. & Nerve/spinal cord injury NOS \\
\hline 97861 & SP06300 & Infected spinal fixation device & SPINAL PAIN & SP063 & Infected spinal fixatn device \\
\hline 98874 & Syu1900 & $\begin{array}{l}{[X] \text { Other and unspecified injuries of cervical }} \\
\text { spinal cord }\end{array}$ & SPINAL PAIN & Syu19 & [X]Oth unsp inj cerv spin cord \\
\hline
\end{tabular}




\begin{tabular}{|c|c|c|c|c|c|}
\hline 98833 & Syu2B00 & $\begin{array}{l}{[X] \text { Other and unspecified injuries of thoracic }} \\
\text { spinal cord }\end{array}$ & SPINAL PAIN & Syu2B & [X]Oth unsp inj thor spin cord \\
\hline 71966 & Sуu3800 & {$[\mathrm{X}]$ Other injury of lumbar spinal cord } & SPINAL PAIN & Syu38 & {$[\mathrm{X}] \mathrm{Oth}$ inj of lumb spin cord } \\
\hline 52139 & Zw04300 & [Q] Central spinal stenosis & SPINAL PAIN & Zw043 & [Q] Central spinal stenosis \\
\hline 91625 & Zw04400 & [Q] Lateral spinal stenosis & SPINAL PAIN & Zw044 & [Q] Lateral spinal stenosis \\
\hline 94588 & Zw04500 & [Q] Central and lateral spinal stenosis & SPINAL PAIN & Zw045 & [Q] Central + lat spin stenos \\
\hline 24796 & $16 C 4.00$ & Back pain worse on sneezing & BACK PAIN & $16 C 4$. & Back pain worse on sneezing \\
\hline 3763 & $16 \mathrm{C} 5.00$ & C/O - low back pain & BACK PAIN & $16 \mathrm{C} 5$. & $\mathrm{C} / \mathrm{O}$ - low back pain \\
\hline 3324 & $16 C 6.00$ & Back pain without radiation NOS & BACK PAIN & $16 \mathrm{C} 6$. & Back pain without radiat NOS \\
\hline 10231 & $16 C 9.00$ & Chronic low back pain & BACK PAIN & $16 \mathrm{C} 9$. & Chronic low back pain \\
\hline 12189 & 16CA.00 & Mechanical low back pain & BACK PAIN & 16CA. & Mechanical low back pain \\
\hline 5476 & $\mathrm{~N} 12 . .13$ & Acute back pain - disc & BACK PAIN & $\mathrm{N} 12 .$. & Intervertebral disc disorders \\
\hline 5916 & N141.11 & Acute back pain - thoracic & BACK PAIN & N141. & Pain in thoracic spine \\
\hline 154 & N142.11 & Low back pain & BACK PAIN & N142. & Pain in lumbar spine \\
\hline 5023 & N142.13 & Acute back pain - lumbar & BACK PAIN & N142. & \\
\hline 5840 & N143.11 & Acute back pain with sciatica & BACK PAIN & N143. & Sciatica \\
\hline 5923 & N145.11 & Acute back pain - unspecified & BACK PAIN & N145. & Backache, unspecified \\
\hline 6704 & N145.12 & Back pain, unspecified & BACK PAIN & N145. & \\
\hline 51894 & A985300 & Gonococcal spondylitis & $\begin{array}{l}\text { ANKYLOSING } \\
\text { SPONDYLITIS } \\
\end{array}$ & A9853 & Gonococcal spondylitis \\
\hline 42405 & N045000 & Juvenile ankylosing spondylitis & $\begin{array}{l}\text { ANKYLOSING } \\
\text { SPONDYLITIS }\end{array}$ & N0450 & Juv ankylosing spondylitis \\
\hline 2184 & $\mathrm{~N} 100.00$ & Ankylosing spondylitis & $\begin{array}{l}\text { ANKYLOSING } \\
\text { SPONDYLITIS }\end{array}$ & N100. & Ankylosing spondylitis \\
\hline 40946 & N100.11 & Marie - Strumpell spondylitis & $\begin{array}{l}\text { ANKYLOSING } \\
\text { SPONDYLITIS }\end{array}$ & N100. & \\
\hline 1963 & N10z.00 & Spondylitis NOS & $\begin{array}{l}\text { ANKYLOSING } \\
\text { SPONDYLITIS } \\
\end{array}$ & N10z. & Spondylitis NOS \\
\hline 61090 & N11y000 & Brucella spondylitis & $\begin{array}{l}\text { ANKYLOSING } \\
\text { SPONDYLITIS }\end{array}$ & N11yo & Brucella spondylitis \\
\hline 49740 & N11y100 & Enterobacterial spondylitis & $\begin{array}{l}\text { ANKYLOSING } \\
\text { SPONDYLITIS }\end{array}$ & N11y1 & Enterobacterial spondylitis \\
\hline
\end{tabular}

\begin{tabular}{|c|c|c|c|c|c|c|}
\hline Medcode & Readcode & Readterm & cause & $\begin{array}{l}\text { Readcode- } \\
\text { V2-5dig } \\
\end{array}$ & PREF_TERM_30 & PREF_TERM_60 \\
\hline 9760 & $14 \mathrm{G} 2.00$ & $\mathrm{H} / \mathrm{O}$ : osteoarthritis & OSTEOARTHRITIS & $14 \mathrm{G} 2$. & $\mathrm{H} / \mathrm{O}$ : osteoarthritis & \\
\hline 3057 & N05..00 & $\begin{array}{l}\text { Osteoarthritis and allied } \\
\text { disorders }\end{array}$ & OSTEOARTHRITIS & N05.. & $\begin{array}{l}\text { Osteoarthritis+allied } \\
\text { disord. }\end{array}$ & $\begin{array}{l}\text { Osteoarthritis and allied } \\
\text { disorders }\end{array}$ \\
\hline 396 & N05..11 & Osteoarthritis & OSTEOARTHRITIS & N05.. & & \\
\hline 4353 & N050.00 & Generalised osteoarthritis - OA & OSTEOARTHRITIS & N050. & $\begin{array}{l}\text { Generalised } \\
\text { osteoarthritis-OA }\end{array}$ & $\begin{array}{l}\text { Generalised osteoarthritis } \\
\mathrm{OA}\end{array}$ \\
\hline 38631 & N050000 & $\begin{array}{l}\text { Generalised osteoarthritis of } \\
\text { unspecified site }\end{array}$ & OSTEOARTHRITIS & N0500 & $\begin{array}{l}\text { Generalised OA-site } \\
\text { unspecif. }\end{array}$ & $\begin{array}{l}\text { Generalised osteoarthritis } \\
\text { of unspecified site }\end{array}$ \\
\hline 36327 & N050100 & $\begin{array}{l}\text { Generalised osteoarthritis of the } \\
\text { hand }\end{array}$ & OSTEOARTHRITIS & N0501 & $\begin{array}{l}\text { Generalised OA- } \\
\text { hand }\end{array}$ & $\begin{array}{l}\text { Generalised osteoarthritis } \\
\text { of the hand }\end{array}$ \\
\hline 23676 & N050200 & $\begin{array}{l}\text { Generalised osteoarthritis of } \\
\text { multiple sites }\end{array}$ & OSTEOARTHRITIS & N0502 & $\begin{array}{l}\text { Generalised OA- } \\
\text { multiple sites }\end{array}$ & $\begin{array}{l}\text { Generalised osteoarthritis } \\
\text { of multiple sites }\end{array}$ \\
\hline 34867 & N050z00 & Generalised osteoarthritis NOS & OSTEOARTHRITIS & $\mathrm{N} 050 \mathrm{z}$ & $\begin{array}{l}\text { Generalised } \\
\text { osteoarthritis NOS }\end{array}$ & \\
\hline 32839 & N051.00 & Localised, primary osteoarthritis & OSTEOARTHRITIS & N051. & $\begin{array}{l}\text { Local.primary } \\
\text { osteoarthritis }\end{array}$ & $\begin{array}{l}\text { Localised, primary } \\
\text { osteoarthritis }\end{array}$ \\
\hline 54224 & N051000 & $\begin{array}{l}\text { Localised, primary osteoarthritis } \\
\text { of unspecified site }\end{array}$ & OSTEOARTHRITIS & N0510 & $\begin{array}{l}\text { Local.primary OA- } \\
\text { site unspec. }\end{array}$ & $\begin{array}{l}\text { Localised, primary } \\
\text { osteoarthritis of } \\
\text { unspecified site }\end{array}$ \\
\hline 24022 & N051100 & $\begin{array}{l}\text { Localised, primary osteoarthritis } \\
\text { of the shoulder region }\end{array}$ & OSTEOARTHRITIS & N0511 & $\begin{array}{l}\text { Local.primary OA- } \\
\text { shoulder regn }\end{array}$ & $\begin{array}{l}\text { Localised, primary } \\
\text { osteoarthritis of the } \\
\text { shoulder region }\end{array}$ \\
\hline 24217 & N051200 & $\begin{array}{l}\text { Localised, primary osteoarthritis } \\
\text { of the upper arm }\end{array}$ & OSTEOARTHRITIS & N0512 & $\begin{array}{l}\text { Local.primary OA- } \\
\text { upper arm }\end{array}$ & $\begin{array}{l}\text { Localised, primary } \\
\text { osteoarthritis of the upper } \\
\text { arm }\end{array}$ \\
\hline 34806 & N051300 & $\begin{array}{l}\text { Localised, primary osteoarthritis } \\
\text { of the forearm }\end{array}$ & OSTEOARTHRITIS & N0513 & $\begin{array}{l}\text { Local.primary OA- } \\
\text { forearm }\end{array}$ & $\begin{array}{l}\text { Localised, primary } \\
\text { osteoarthritis of the } \\
\text { forearm }\end{array}$ \\
\hline 21350 & N051400 & $\begin{array}{l}\text { Localised, primary osteoarthritis } \\
\text { of the hand }\end{array}$ & OSTEOARTHRITIS & N0514 & $\begin{array}{l}\text { Local.primary OA- } \\
\text { hand }\end{array}$ & $\begin{array}{l}\text { Localised, primary } \\
\text { osteoarthritis of the hand }\end{array}$ \\
\hline 15839 & N051500 & $\begin{array}{l}\text { Localised, primary osteoarthritis } \\
\text { of the pelvic region/thigh }\end{array}$ & OSTEOARTHRITIS & N0515 & $\begin{array}{l}\text { Local.primary OA- } \\
\text { pelvic/thigh }\end{array}$ & $\begin{array}{l}\text { Localised, primary } \\
\text { osteoarthritis of the pelvic } \\
\text { region/thigh }\end{array}$ \\
\hline 21159 & N051600 & $\begin{array}{l}\text { Localised, primary osteoarthritis } \\
\text { of the lower leg }\end{array}$ & OSTEOARTHRITIS & N0516 & $\begin{array}{l}\text { Local.primary OA- } \\
\text { lower leg }\end{array}$ & $\begin{array}{l}\text { Localised, primary } \\
\text { osteoarthritis of the lower } \\
\text { leg }\end{array}$ \\
\hline 25793 & N051700 & $\begin{array}{l}\text { Localised, primary osteoarthritis } \\
\text { of the ankle and foot }\end{array}$ & OSTEOARTHRITIS & N0517 & $\begin{array}{l}\text { Local.primary OA- } \\
\text { ankle/foot }\end{array}$ & $\begin{array}{l}\text { Localised, primary } \\
\text { osteoarthritis of the ankle } \\
\text { and foot }\end{array}$ \\
\hline
\end{tabular}




\begin{tabular}{|c|c|c|c|c|c|c|}
\hline 20472 & N051800 & $\begin{array}{l}\text { Localised, primary osteoarthritis } \\
\text { of other specified site }\end{array}$ & OSTEOARTHRITIS & N0518 & $\begin{array}{l}\text { Local.primary OA- } \\
\text { other specif }\end{array}$ & $\begin{array}{l}\text { Localised, primary } \\
\text { osteoarthritis of other } \\
\text { specified site }\end{array}$ \\
\hline 24958 & N051D00 & $\begin{array}{l}\text { Localised, primary osteoarthritis } \\
\text { of the wrist }\end{array}$ & OSTEOARTHRITIS & N051D & $\begin{array}{l}\text { Local prim } \\
\text { osteoarth wrist }\end{array}$ & $\begin{array}{l}\text { Localised, primary } \\
\text { osteoarthritis of the wrist }\end{array}$ \\
\hline 28908 & N051E00 & $\begin{array}{l}\text { Localised, primary osteoarthritis } \\
\text { of toe }\end{array}$ & OSTEOARTHRITIS & N051E & $\begin{array}{l}\text { Local prim } \\
\text { osteoarth toe }\end{array}$ & $\begin{array}{l}\text { Localised, primary } \\
\text { osteoarthritis of toe }\end{array}$ \\
\hline 18602 & N051F00 & $\begin{array}{l}\text { Localised, primary osteoarthritis } \\
\text { of elbow }\end{array}$ & OSTEOARTHRITIS & N051F & $\begin{array}{l}\text { Local prim } \\
\text { osteoarth elbow }\end{array}$ & $\begin{array}{l}\text { Localised, primary } \\
\text { osteoarthritis of elbow }\end{array}$ \\
\hline 20660 & N051z00 & $\begin{array}{l}\text { Localised, primary osteoarthritis } \\
\text { NOS }\end{array}$ & OSTEOARTHRITIS & N051z & $\begin{array}{l}\text { Localised primary } \\
\text { OA NOS }\end{array}$ & $\begin{array}{l}\text { Localised, primary } \\
\text { osteoarthritis NOS }\end{array}$ \\
\hline 42045 & N052.00 & $\begin{array}{l}\text { Localised, secondary } \\
\text { osteoarthritis }\end{array}$ & OSTEOARTHRITIS & N052. & $\begin{array}{l}\text { Local.secondary } \\
\text { osteoarthritis }\end{array}$ & $\begin{array}{l}\text { Localised, secondary } \\
\text { osteoarthritis }\end{array}$ \\
\hline 68712 & N052000 & $\begin{array}{l}\text { Localised, secondary } \\
\text { osteoarthritis of unspecified site }\end{array}$ & OSTEOARTHRITIS & N0520 & $\begin{array}{l}\text { Local.secondary OA- } \\
\text { site unsp. }\end{array}$ & $\begin{array}{l}\text { Localised, secondary } \\
\text { osteoarthritis of } \\
\text { unspecified site }\end{array}$ \\
\hline 33574 & N052100 & $\begin{array}{l}\text { Localised, secondary } \\
\text { osteoarthritis of the shoulder } \\
\text { region }\end{array}$ & OSTEOARTHRITIS & N0521 & $\begin{array}{l}\text { Local.secondary OA- } \\
\text { shoulder }\end{array}$ & $\begin{array}{l}\text { Localised, secondary } \\
\text { osteoarthritis of the } \\
\text { shoulder region }\end{array}$ \\
\hline 41088 & N052200 & $\begin{array}{l}\text { Localised, secondary } \\
\text { osteoarthritis of the upper arm }\end{array}$ & OSTEOARTHRITIS & N0522 & $\begin{array}{l}\text { Local.secondary OA- } \\
\text { upper arm }\end{array}$ & $\begin{array}{l}\text { Localised, secondary } \\
\text { osteoarthritis of the upper } \\
\text { arm }\end{array}$ \\
\hline 45815 & N052300 & $\begin{array}{l}\text { Localised, secondary } \\
\text { osteoarthritis of the forearm }\end{array}$ & OSTEOARTHRITIS & N0523 & $\begin{array}{l}\text { Local.secondary OA- } \\
\text { forearm }\end{array}$ & $\begin{array}{l}\text { Localised, secondary } \\
\text { osteoarthritis of the } \\
\text { forearm }\end{array}$ \\
\hline 23638 & N052400 & $\begin{array}{l}\text { Localised, secondary } \\
\text { osteoarthritis of the hand }\end{array}$ & OSTEOARTHRITIS & N0524 & $\begin{array}{l}\text { Local.secondary OA- } \\
\text { hand }\end{array}$ & $\begin{array}{l}\text { Localised, secondary } \\
\text { osteoarthritis of the hand }\end{array}$ \\
\hline 44041 & N052500 & $\begin{array}{l}\text { Localised, secondary } \\
\text { osteoarthritis of pelvic } \\
\text { region/thigh }\end{array}$ & OSTEOARTHRITIS & N0525 & $\begin{array}{l}\text { Local.secondary OA- } \\
\text { pelv./thigh }\end{array}$ & $\begin{array}{l}\text { Localised, secondary } \\
\text { osteoarthritis of pelvic } \\
\text { region/thigh }\end{array}$ \\
\hline 33479 & N052600 & $\begin{array}{l}\text { Localised, secondary } \\
\text { osteoarthritis of the lower leg }\end{array}$ & OSTEOARTHRITIS & N0526 & $\begin{array}{l}\text { Local.secondary OA- } \\
\text { lower leg }\end{array}$ & $\begin{array}{l}\text { Localised, secondary } \\
\text { osteoarthritis of the lower } \\
\text { leg }\end{array}$ \\
\hline 34035 & N052700 & $\begin{array}{l}\text { Localised, secondary } \\
\text { osteoarthritis of the ankle and } \\
\text { foot }\end{array}$ & OSTEOARTHRITIS & N0527 & $\begin{array}{l}\text { Local.secondary OA- } \\
\text { ankle/foot }\end{array}$ & $\begin{array}{l}\text { Localised, secondary } \\
\text { osteoarthritis of the ankle } \\
\text { and foot }\end{array}$ \\
\hline 32891 & N052800 & $\begin{array}{l}\text { Localised, secondary } \\
\text { osteoarthritis of other specified } \\
\text { site }\end{array}$ & OSTEOARTHRITIS & N0528 & $\begin{array}{l}\text { Local.secondary OA- } \\
\text { other spec. }\end{array}$ & $\begin{array}{l}\text { Localised, secondary } \\
\text { osteoarthritis of other } \\
\text { specified site }\end{array}$ \\
\hline 57912 & N052z00 & $\begin{array}{l}\text { Localised, secondary } \\
\text { osteoarthritis NOS }\end{array}$ & OSTEOARTHRITIS & N052z & $\begin{array}{l}\text { Localised secondary } \\
\text { OA NOS }\end{array}$ & $\begin{array}{l}\text { Localised, secondary } \\
\text { osteoarthritis NOS }\end{array}$ \\
\hline 34122 & N053.00 & $\begin{array}{l}\text { Localised osteoarthritis, } \\
\text { unspecified }\end{array}$ & OSTEOARTHRITIS & N053. & $\begin{array}{l}\text { Localised OA } \\
\text { unspecified }\end{array}$ & $\begin{array}{l}\text { Localised osteoarthritis, } \\
\text { unspecified }\end{array}$ \\
\hline 49545 & N053000 & $\begin{array}{l}\text { Localised osteoarthritis, } \\
\text { unspecified, of unspecified site }\end{array}$ & OSTEOARTHRITIS & N0530 & $\begin{array}{l}\text { Local.OA unsp.-site } \\
\text { unspecif. }\end{array}$ & $\begin{array}{l}\text { Localised osteoarthritis, } \\
\text { unspecified, of unspecified } \\
\text { site }\end{array}$ \\
\hline 15441 & N053100 & $\begin{array}{l}\text { Localised osteoarthritis, } \\
\text { unspecified, of shoulder region }\end{array}$ & OSTEOARTHRITIS & N0531 & $\begin{array}{l}\text { Local.OA unsp.- } \\
\text { shoulder region }\end{array}$ & $\begin{array}{l}\text { Localised osteoarthritis, } \\
\text { unspecified, of shoulder } \\
\text { region }\end{array}$ \\
\hline 59637 & N053200 & $\begin{array}{l}\text { Localised osteoarthritis, } \\
\text { unspecified, of the upper arm }\end{array}$ & OSTEOARTHRITIS & N0532 & $\begin{array}{l}\text { Local.OA unsp.- } \\
\text { upper arm }\end{array}$ & $\begin{array}{l}\text { Localised osteoarthritis, } \\
\text { unspecified, of the upper } \\
\text { arm }\end{array}$ \\
\hline 60537 & N053300 & $\begin{array}{l}\text { Localised osteoarthritis, } \\
\text { unspecified, of the forearm }\end{array}$ & OSTEOARTHRITIS & N0533 & $\begin{array}{l}\text { Local.OA unsp.- } \\
\text { forearm }\end{array}$ & $\begin{array}{l}\text { Localised osteoarthritis, } \\
\text { unspecified, of the forearm }\end{array}$ \\
\hline 16242 & N053400 & $\begin{array}{l}\text { Localised osteoarthritis, } \\
\text { unspecified, of the hand }\end{array}$ & OSTEOARTHRITIS & N0534 & $\begin{array}{l}\text { Local.OA unsp.- } \\
\text { hand }\end{array}$ & $\begin{array}{l}\text { Localised osteoarthritis, } \\
\text { unspecified, of the hand }\end{array}$ \\
\hline 20626 & N053500 & $\begin{array}{l}\text { Localised osteoarthritis, } \\
\text { unspecified, pelvic region/thigh }\end{array}$ & OSTEOARTHRITIS & N0535 & $\begin{array}{l}\text { Local.OA unsp.- } \\
\text { pelvic/thigh }\end{array}$ & $\begin{array}{l}\text { Localised osteoarthritis, } \\
\text { unspecified, pelvic } \\
\text { region/thigh }\end{array}$ \\
\hline 34804 & N053600 & $\begin{array}{l}\text { Localised osteoarthritis, } \\
\text { unspecified, of the lower leg }\end{array}$ & OSTEOARTHRITIS & N0536 & $\begin{array}{l}\text { Local.OA unsp.- } \\
\text { lower leg }\end{array}$ & $\begin{array}{l}\text { Localised osteoarthritis, } \\
\text { unspecified, of the lower } \\
\text { leg }\end{array}$ \\
\hline 1296 & N053611 & Patellofemoral osteoarthritis & OSTEOARTHRITIS & N0536 & & \\
\hline 4461 & N053700 & $\begin{array}{l}\text { Localised osteoarthritis, } \\
\text { unspecified, of the ankle and } \\
\text { foot }\end{array}$ & OSTEOARTHRITIS & N0537 & $\begin{array}{l}\text { Local.OA unsp.- } \\
\text { ankle/foot }\end{array}$ & $\begin{array}{l}\text { Localised osteoarthritis, } \\
\text { unspecified, of the ankle } \\
\text { and foot }\end{array}$ \\
\hline 18112 & N053800 & $\begin{array}{l}\text { Localised osteoarthritis, } \\
\text { unspecified, of other spec site }\end{array}$ & OSTEOARTHRITIS & N0538 & $\begin{array}{l}\text { Local.OA unsp.- } \\
\text { other specified }\end{array}$ & $\begin{array}{l}\text { Localised osteoarthritis, } \\
\text { unspecified, of other spec } \\
\text { site }\end{array}$ \\
\hline 31200 & N053z00 & $\begin{array}{l}\text { Localised osteoarthritis, } \\
\text { unspecified, NOS }\end{array}$ & OSTEOARTHRITIS & N053z & $\begin{array}{l}\text { Localised OA } \\
\text { unspecified NOS }\end{array}$ & $\begin{array}{l}\text { Localised osteoarthritis, } \\
\text { unspecified, NOS }\end{array}$ \\
\hline 21528 & N054.00 & $\begin{array}{l}\text { Oligoarticular osteoarthritis, } \\
\text { unspecified }\end{array}$ & OSTEOARTHRITIS & N054. & $\begin{array}{l}\text { Oligoarticular OA, } \\
\text { unspecified }\end{array}$ & $\begin{array}{l}\text { Oligoarticular } \\
\text { osteoarthritis, unspecified }\end{array}$ \\
\hline 48214 & N054000 & $\begin{array}{l}\text { Oligoarticular osteoarthritis, } \\
\text { unspec, of unspecified sites }\end{array}$ & OSTEOARTHRITIS & N0540 & $\begin{array}{l}\text { Oligoartic } \mathrm{OA} \text {, unsp- } \\
\text { unsp sites }\end{array}$ & $\begin{array}{l}\text { Oligoarticular } \\
\text { osteoarthritis, unspec, of } \\
\text { unspecified sites }\end{array}$ \\
\hline 52095 & N054100 & $\begin{array}{l}\text { Oligoarticular osteoarthritis, } \\
\text { unspecified, of shoulder }\end{array}$ & OSTEOARTHRITIS & N0541 & $\begin{array}{l}\text { Oligoartic OA, } \\
\text { unspec-shoulder }\end{array}$ & $\begin{array}{l}\text { Oligoarticular } \\
\text { osteoarthritis, unspecified, } \\
\text { of shoulder }\end{array}$ \\
\hline 97073 & N054200 & $\begin{array}{l}\text { Oligoarticular osteoarthritis, } \\
\text { unspecified, of upper arm }\end{array}$ & OSTEOARTHRITIS & N0542 & $\begin{array}{l}\text { Oligoartic } O A, \\
\text { unspec-upp arm }\end{array}$ & $\begin{array}{l}\text { Oligoarticular } \\
\text { osteoarthritis, unspecified, } \\
\text { of upper arm }\end{array}$ \\
\hline 59616 & N054400 & $\begin{array}{l}\text { Oligoarticular osteoarthritis, } \\
\text { unspecified, of hand }\end{array}$ & OSTEOARTHRITIS & N0544 & $\begin{array}{l}\text { Oligoartic OA, } \\
\text { unspec-hand }\end{array}$ & $\begin{array}{l}\text { Oligoarticular } \\
\text { osteoarthritis, unspecified, } \\
\text { of hand }\end{array}$ \\
\hline 68648 & N054500 & $\begin{array}{l}\text { Oligoarticular osteoarthritis, } \\
\text { unspecified, of pelvis/thigh }\end{array}$ & OSTEOARTHRITIS & N0545 & $\begin{array}{l}\text { Oligoartic OA, } \\
\text { unspec-pelv/thi }\end{array}$ & $\begin{array}{l}\text { Oligoarticular } \\
\text { osteoarthritis, unspecified, } \\
\text { of pelvis/thigh }\end{array}$ \\
\hline 41090 & N054600 & $\begin{array}{l}\text { Oligoarticular osteoarthritis, } \\
\text { unspecified, of lower leg }\end{array}$ & OSTEOARTHRITIS & N0546 & $\begin{array}{l}\text { Oligoartic OA, } \\
\text { unspec-leg }\end{array}$ & $\begin{array}{l}\text { Oligoarticular } \\
\text { osteoarthritis, unspecified, } \\
\text { of lower leg }\end{array}$ \\
\hline
\end{tabular}




\begin{tabular}{|c|c|c|c|c|c|c|}
\hline 72109 & N054700 & $\begin{array}{l}\text { Oligoarticular osteoarthritis, } \\
\text { unspecified, of ankle/foot }\end{array}$ & OSTEOARTHRITIS & N0547 & $\begin{array}{l}\text { Oligoartic OA, } \\
\text { unspec-ank/foot }\end{array}$ & $\begin{array}{l}\text { Oligoarticular } \\
\text { osteoarthritis, unspecified, } \\
\text { of ankle/foot }\end{array}$ \\
\hline 41985 & N054800 & $\begin{array}{l}\text { Oligoarticular osteoarthritis, } \\
\text { unspecified, other spec sites }\end{array}$ & OSTEOARTHRITIS & N0548 & $\begin{array}{l}\text { Oligoartic } \mathrm{OA} \text {, } \\
\text { unspec-oth site }\end{array}$ & $\begin{array}{l}\text { Oligoarticular } \\
\text { osteoarthritis, unspecified, } \\
\text { other spec sites }\end{array}$ \\
\hline 57267 & N054900 & $\begin{array}{l}\text { Oligoarticular osteoarthritis, } \\
\text { unspecified, multiple sites }\end{array}$ & OSTEOARTHRITIS & N0549 & $\begin{array}{l}\text { Oligoartic OA, } \\
\text { unspec-multiple }\end{array}$ & $\begin{array}{l}\text { Oligoarticular } \\
\text { osteoarthritis, unspecified, } \\
\text { multiple sites }\end{array}$ \\
\hline 53858 & N054z00 & $\begin{array}{l}\text { Osteoarthritis of more than one } \\
\text { site, unspecified, NOS }\end{array}$ & OSTEOARTHRITIS & N054z & $\begin{array}{l}\text { OA,1 site } \\
+, \text { unspecified NOS }\end{array}$ & $\begin{array}{l}\text { Osteoarthritis of more than } \\
\text { one site, unspecified, NOS }\end{array}$ \\
\hline 5776 & N05z.00 & Osteoarthritis NOS & OSTEOARTHRITIS & N05z. & Osteoarthritis NOS & \\
\hline 35527 & N05z000 & $\begin{array}{l}\text { Osteoarthritis NOS, of } \\
\text { unspecified site }\end{array}$ & OSTEOARTHRITIS & No5zo & $\begin{array}{l}\text { Osteoarthritis NOS- } \\
\text { site unspec }\end{array}$ & $\begin{array}{l}\text { Osteoarthritis NOS, of } \\
\text { unspecified site }\end{array}$ \\
\hline 3147 & N05z100 & $\begin{array}{l}\text { Osteoarthritis NOS, of shoulder } \\
\text { region }\end{array}$ & OSTEOARTHRITIS & N05z1 & $\begin{array}{l}\text { Osteoarthritis NOS- } \\
\text { shoulder }\end{array}$ & $\begin{array}{l}\text { Osteoarthritis NOS, of } \\
\text { shoulder region }\end{array}$ \\
\hline 50848 & N05z200 & $\begin{array}{l}\text { Osteoarthritis NOS, of the upper } \\
\text { arm }\end{array}$ & OSTEOARTHRITIS & N05z2 & $\begin{array}{l}\text { Osteoarthritis NOS- } \\
\text { upper arm }\end{array}$ & $\begin{array}{l}\text { Osteoarthritis NOS, of the } \\
\text { upper arm }\end{array}$ \\
\hline 639 & N05z211 & Elbow osteoarthritis NOS & OSTEOARTHRITIS & N05z2 & & \\
\hline 24152 & N05z300 & $\begin{array}{l}\text { Osteoarthritis NOS, of the } \\
\text { forearm }\end{array}$ & OSTEOARTHRITIS & No5z3 & $\begin{array}{l}\text { Osteoarthritis NOS- } \\
\text { forearm }\end{array}$ & $\begin{array}{l}\text { Osteoarthritis NOS, of the } \\
\text { forearm }\end{array}$ \\
\hline 15206 & N05z311 & Wrist osteoarthritis NOS & OSTEOARTHRITIS & N05z3 & & \\
\hline 658 & N05z400 & Osteoarthritis NOS, of the hand & OSTEOARTHRITIS & N05z4 & $\begin{array}{l}\text { Osteoarthritis NOS- } \\
\text { hand }\end{array}$ & $\begin{array}{l}\text { Osteoarthritis NOS, of the } \\
\text { hand }\end{array}$ \\
\hline 4490 & N05z411 & Finger osteoarthritis NOS & OSTEOARTHRITIS & N05z4 & & \\
\hline 1959 & N05z412 & Thumb osteoarthritis NOS & OSTEOARTHRITIS & N05z4 & & \\
\hline 4967 & N05z500 & $\begin{array}{l}\text { Osteoarthritis NOS, pelvic } \\
\text { region/thigh }\end{array}$ & OSTEOARTHRITIS & $\mathrm{N} 05 \mathrm{z} 5$ & $\begin{array}{l}\text { Osteoarthritis NOS- } \\
\text { pelv./thigh }\end{array}$ & $\begin{array}{l}\text { Osteoarthritis NOS, pelvic } \\
\text { region/thigh }\end{array}$ \\
\hline 2209 & N05z511 & Hip osteoarthritis NOS & OSTEOARTHRITIS & N05z5 & & \\
\hline 15144 & N05z600 & $\begin{array}{l}\text { Osteoarthritis NOS, of the lower } \\
\text { leg }\end{array}$ & OSTEOARTHRITIS & N05z6 & $\begin{array}{l}\text { Osteoarthritis NOS- } \\
\text { lower leg }\end{array}$ & $\begin{array}{l}\text { Osteoarthritis NOS, of the } \\
\text { lower leg }\end{array}$ \\
\hline 665 & N05z611 & Knee osteoarthritis NOS & OSTEOARTHRITIS & N05z6 & & \\
\hline 15447 & N05z700 & $\begin{array}{l}\text { Osteoarthritis NOS, of ankle and } \\
\text { foot }\end{array}$ & OSTEOARTHRITIS & N05z7 & $\begin{array}{l}\text { Osteoarthritis NOS- } \\
\text { ankle/foot }\end{array}$ & $\begin{array}{l}\text { Osteoarthritis NOS, of } \\
\text { ankle and foot }\end{array}$ \\
\hline 52897 & N05z711 & Ankle osteoarthritis NOS & OSTEOARTHRITIS & N05z7 & & \\
\hline 1312 & N05z712 & Foot osteoarthritis NOS & OSTEOARTHRITIS & N05z7 & & \\
\hline 4878 & N05z713 & Toe osteoarthritis NOS & OSTEOARTHRITIS & N05z7 & & \\
\hline 15052 & N05z800 & $\begin{array}{l}\text { Osteoarthritis NOS, other } \\
\text { specified site }\end{array}$ & OSTEOARTHRITIS & N05z8 & $\begin{array}{l}\text { Osteoarthritis NOS- } \\
\text { other spec }\end{array}$ & $\begin{array}{l}\text { Osteoarthritis NOS, other } \\
\text { specified site }\end{array}$ \\
\hline 5802 & N05z900 & Osteoarthritis NOS, of shoulder & OSTEOARTHRITIS & No5z9 & $\begin{array}{l}\text { Osteoarthritis NOS, } \\
\text { shoulder }\end{array}$ & $\begin{array}{l}\text { Osteoarthritis NOS, of } \\
\text { shoulder }\end{array}$ \\
\hline 3814 & N05zA00 & $\begin{array}{l}\text { Osteoarthritis NOS, of } \\
\text { sternoclavicular joint }\end{array}$ & OSTEOARTHRITIS & N05zA & $\begin{array}{l}\text { OA NOS- } \\
\text { sternoclavicular } \\
\text { joint }\end{array}$ & $\begin{array}{l}\text { Osteoarthritis NOS, of } \\
\text { sternoclavicular joint }\end{array}$ \\
\hline 2229 & N05zB00 & $\begin{array}{l}\text { Osteoarthritis NOS, of } \\
\text { acromioclavicular joint }\end{array}$ & OSTEOARTHRITIS & $\mathrm{N} 05 \mathrm{zB}$ & $\begin{array}{l}\text { OA NOS- } \\
\text { acromioclavicular } \\
\text { joint }\end{array}$ & $\begin{array}{l}\text { Osteoarthritis NOS, of } \\
\text { acromioclavicular joint }\end{array}$ \\
\hline 19713 & $\mathrm{~N} 05 z \mathrm{COO}$ & Osteoarthritis NOS, of elbow & OSTEOARTHRITIS & $\mathrm{N} 05 \mathrm{zC}$ & OA NOS-elbow & $\begin{array}{l}\text { Osteoarthritis NOS, of } \\
\text { elbow }\end{array}$ \\
\hline 65748 & N05zD00 & $\begin{array}{l}\text { Osteoarthritis NOS, of distal } \\
\text { radio-ulnar joint }\end{array}$ & OSTEOARTHRITIS & $\mathrm{N} 05 \mathrm{zD}$ & $\begin{array}{l}\text { OA NOS-dist radio- } \\
\text { ulnar joint }\end{array}$ & $\begin{array}{l}\text { Osteoarthritis NOS, of } \\
\text { distal radio-ulnar joint }\end{array}$ \\
\hline 9649 & N05zE00 & Osteoarthritis NOS, of wrist & OSTEOARTHRITIS & N05zE & OA NOS-wrist & Osteoarthritis NOS, of wrist \\
\hline 7866 & N05zF00 & Osteoarthritis NOS, of MCP joint & OSTEOARTHRITIS & $\mathrm{N} 05 \mathrm{zF}$ & OA NOS-MCP joint & $\begin{array}{l}\text { Osteoarthritis NOS, of MCP } \\
\text { joint }\end{array}$ \\
\hline 11032 & N05zG00 & $\begin{array}{l}\text { Osteoarthritis NOS, of PIP joint } \\
\text { of finger }\end{array}$ & OSTEOARTHRITIS & N05zG & $\begin{array}{l}\text { OA NOS-PIP joint of } \\
\text { finger }\end{array}$ & $\begin{array}{l}\text { Osteoarthritis NOS, of PIP } \\
\text { joint of finger }\end{array}$ \\
\hline 9681 & N05zHOO & $\begin{array}{l}\text { Osteoarthritis NOS, of DIP joint } \\
\text { of finger }\end{array}$ & OSTEOARTHRITIS & $\mathrm{N} 05 \mathrm{zH}$ & $\begin{array}{l}\text { OA NOS-DIP joint of } \\
\text { finger }\end{array}$ & $\begin{array}{l}\text { Osteoarthritis NOS, of DIP } \\
\text { joint of finger }\end{array}$ \\
\hline 6812 & N05zJ00 & Osteoarthritis NOS, of hip & OSTEOARTHRITIS & N05zJ & OA NOS-hip & Osteoarthritis NOS, of hip \\
\hline 34023 & N05zK00 & $\begin{array}{l}\text { Osteoarthritis NOS, of sacro-iliac } \\
\text { joint }\end{array}$ & OSTEOARTHRITIS & $\mathrm{N} 05 \mathrm{zK}$ & OA NOS-SI joint & $\begin{array}{l}\text { Osteoarthritis NOS, of } \\
\text { sacro-iliac joint }\end{array}$ \\
\hline 2487 & N05zL00 & Osteoarthritis NOS, of knee & OSTEOARTHRITIS & N05zL & $\begin{array}{l}\text { Osteoarthritis NOS, } \\
\text { of knee }\end{array}$ & \\
\hline 70425 & N05zM00 & $\begin{array}{l}\text { Osteoarthritis NOS, of tibio- } \\
\text { fibular joint }\end{array}$ & OSTEOARTHRITIS & N05zM & $\begin{array}{l}\text { OA NOS tibio-fibular } \\
\text { joint }\end{array}$ & $\begin{array}{l}\text { Osteoarthritis NOS, of } \\
\text { tibio-fibular joint }\end{array}$ \\
\hline 8202 & N05zN00 & Osteoarthritis NOS, of ankle & OSTEOARTHRITIS & $\mathrm{N} 05 \mathrm{zN}$ & OA NOS-ankle & $\begin{array}{l}\text { Osteoarthritis NOS, of } \\
\text { ankle }\end{array}$ \\
\hline 40972 & N05zP00 & $\begin{array}{l}\text { Osteoarthritis NOS, of subtalar } \\
\text { joint }\end{array}$ & OSTEOARTHRITIS & $\mathrm{N} 05 \mathrm{zP}$ & $\begin{array}{l}\text { OA NOS-subtalar } \\
\text { joint }\end{array}$ & $\begin{array}{l}\text { Osteoarthritis NOS, of } \\
\text { subtalar joint }\end{array}$ \\
\hline 55388 & N05zQ00 & $\begin{array}{l}\text { Osteoarthritis NOS, of } \\
\text { talonavicular joint }\end{array}$ & OSTEOARTHRITIS & $\mathrm{N} 05 \mathrm{zQ}$ & $\begin{array}{l}\text { OA NOS- } \\
\text { talonavicular joint }\end{array}$ & $\begin{array}{l}\text { Osteoarthritis NOS, of } \\
\text { talonavicular joint }\end{array}$ \\
\hline 54350 & N05zR00 & $\begin{array}{l}\text { Osteoarthritis NOS, of other } \\
\text { tarsal joint }\end{array}$ & OSTEOARTHRITIS & $\mathrm{N} 05 \mathrm{zR}$ & $\begin{array}{l}\text { OA NOS-other tarsal } \\
\text { joint }\end{array}$ & $\begin{array}{l}\text { Osteoarthritis NOS, of } \\
\text { other tarsal joint }\end{array}$ \\
\hline 6887 & N05zSOO & $\begin{array}{l}\text { Osteoarthritis NOS, of 1st MTP } \\
\text { joint }\end{array}$ & OSTEOARTHRITIS & N05zS & $\begin{array}{l}\text { OA NOS-1st MTP } \\
\text { joint }\end{array}$ & $\begin{array}{l}\text { Osteoarthritis NOS, of 1st } \\
\text { MTP joint }\end{array}$ \\
\hline 9010 & N05zT00 & $\begin{array}{l}\text { Osteoarthritis NOS, of lesser } \\
\text { MTP joint }\end{array}$ & OSTEOARTHRITIS & N05zT & $\begin{array}{l}\text { OA NOS-lesser MTP } \\
\text { joint }\end{array}$ & $\begin{array}{l}\text { Osteoarthritis NOS, of } \\
\text { lesser MTP joint }\end{array}$ \\
\hline 27834 & N05zU00 & $\begin{array}{l}\text { Osteoarthritis NOS, of IP joint of } \\
\text { toe }\end{array}$ & OSTEOARTHRITIS & $\mathrm{N} 05 \mathrm{zU}$ & $\begin{array}{l}\text { OA NOS-IP joint of } \\
\text { toe }\end{array}$ & $\begin{array}{l}\text { Osteoarthritis NOS, of IP } \\
\text { joint of toe }\end{array}$ \\
\hline
\end{tabular}




\begin{tabular}{|c|c|c|c|c|c|c|}
\hline 27972 & N05zz00 & Osteoarthritis NOS & OSTEOARTHRITIS & N05zz & Osteoarthritis NOS & \\
\hline 7429 & $\mathrm{~N} 11 . .12$ & Osteoarthritis of spine & OSTEOARTHRITIS & N11.. & $\begin{array}{l}\text { Spondylosis + allied } \\
\text { disorders }\end{array}$ & $\begin{array}{l}\text { Spondylosis and allied } \\
\text { disorders }\end{array}$ \\
\hline 17092 & N110.12 & Osteoarthritis cervical spine & OSTEOARTHRITIS & N110. & $\begin{array}{l}\text { Cervical spond.-no } \\
\text { myelopathy }\end{array}$ & $\begin{array}{l}\text { Cervical spondylosis } \\
\text { without myelopathy }\end{array}$ \\
\hline 18826 & N11D.00 & Osteoarthritis of spine & OSTEOARTHRITIS & N11D. & $\begin{array}{l}\text { Osteoarthritis of } \\
\text { spine }\end{array}$ & \\
\hline 41378 & N11D000 & Osteoarthritis of cervical spine & OSTEOARTHRITIS & N11D0 & $\begin{array}{l}\text { Osteoarthritis } \\
\text { cervical spine }\end{array}$ & $\begin{array}{l}\text { Osteoarthritis of cervical } \\
\text { spine }\end{array}$ \\
\hline 47024 & N11D100 & Osteoarthritis of thoracic spine & OSTEOARTHRITIS & N11D1 & $\begin{array}{l}\text { Osteoarthritis } \\
\text { thoracic spine }\end{array}$ & $\begin{array}{l}\text { Osteoarthritis of thoracic } \\
\text { spine }\end{array}$ \\
\hline 22452 & N11D200 & Osteoarthritis of lumbar spine & OSTEOARTHRITIS & N11D2 & $\begin{array}{l}\text { Osteoarthritis of } \\
\text { lumbar spine }\end{array}$ & \\
\hline 53184 & N11D300 & Osteoarthritis of spine NOS & OSTEOARTHRITIS & N11D3 & $\begin{array}{l}\text { Osteoarthritis of } \\
\text { spine NOS }\end{array}$ & \\
\hline 829 & N11z.11 & Osteoarthritis spine & OSTEOARTHRITIS & N11z. & Spondylosis NOS & \\
\hline
\end{tabular}

\begin{tabular}{|c|c|c|c|c|c|c|}
\hline Medcode & Readcode & Readterm & cause & $\begin{array}{l}\text { Readcode- } \\
\text { V2-5dig } \\
\end{array}$ & PREF_TERM_30 & PREF_TERM_60 \\
\hline 476 & M160.00 & Psoriatic arthropathy & $\begin{array}{l}\text { PSORIATIC } \\
\text { ARTHROPATHY }\end{array}$ & M160. & Psoriatic arthropathy & \\
\hline 96880 & M160.11 & Psoriatic arthritis & $\begin{array}{l}\text { PSORIATIC } \\
\text { ARTHROPATHY }\end{array}$ & M160. & & \\
\hline 32149 & M160100 & $\begin{array}{l}\text { Distal interphalangeal } \\
\text { psoriatic arthropathy }\end{array}$ & $\begin{array}{l}\text { PSORIATIC } \\
\text { ARTHROPATHY }\end{array}$ & M1601 & $\begin{array}{l}\text { Dist interphal psoriat } \\
\text { arthrop }\end{array}$ & $\begin{array}{l}\text { Distal interphalangeal psoriatic } \\
\text { arthropathy }\end{array}$ \\
\hline 12500 & M160z00 & Psoriatic arthropathy NOS & $\begin{array}{l}\text { PSORIATIC } \\
\text { ARTHROPATHY } \\
\end{array}$ & M160z & Psoriatic arthropathy NOS & \\
\hline 59107 & Nyu1300 & $\begin{array}{l}{[X] \text { Other psoriatic }} \\
\text { arthropathies }\end{array}$ & $\begin{array}{l}\text { PSORIATIC } \\
\text { ARTHROPATHY }\end{array}$ & Nyu13 & $\begin{array}{l}{[\mathrm{X}] \text { Oth psoriatic }} \\
\text { arthropathies }\end{array}$ & $\begin{array}{l}{[\mathrm{X}] \text { Other psoriatic }} \\
\text { arthropathies }\end{array}$ \\
\hline 6639 & $14 \mathrm{G} 1.00$ & $\mathrm{H} / \mathrm{O}$ : rheumatoid arthritis & $\begin{array}{l}\text { RHEUMATOID } \\
\text { ARTHRITIS } \\
\end{array}$ & $14 \mathrm{G} 1$. & $\mathrm{H} / \mathrm{O}$ : rheumatoid arthritis & \\
\hline 62401 & F371200 & $\begin{array}{l}\text { Polyneuropathy in } \\
\text { rheumatoid arthritis }\end{array}$ & $\begin{array}{l}\text { RHEUMATOID } \\
\text { ARTHRITIS } \\
\end{array}$ & F3712 & $\begin{array}{l}\text { Polyneuropathy+rheumatoid } \\
\text { arth }\end{array}$ & $\begin{array}{l}\text { Polyneuropathy in rheumatoid } \\
\text { arthritis }\end{array}$ \\
\hline 31209 & F396400 & $\begin{array}{l}\text { Myopathy due to } \\
\text { rheumatoid arthritis }\end{array}$ & $\begin{array}{l}\text { RHEUMATOID } \\
\text { ARTHRITIS } \\
\end{array}$ & F3964 & $\begin{array}{l}\text { Myopathy+rheumatoid } \\
\text { arthritis }\end{array}$ & $\begin{array}{l}\text { Myopathy due to rheumatoid } \\
\text { arthritis }\end{array}$ \\
\hline 27603 & N04..00 & $\begin{array}{l}\text { Rheumatoid arthritis and } \\
\text { other inflammatory } \\
\text { polyarthropathy }\end{array}$ & $\begin{array}{l}\text { RHEUMATOID } \\
\text { ARTHRITIS } \\
\end{array}$ & N04.. & Rheumatoid arthritis+similar & $\begin{array}{l}\text { Rheumatoid arthritis and other } \\
\text { inflammatory polyarthropathy }\end{array}$ \\
\hline 844 & N040.00 & Rheumatoid arthritis & $\begin{array}{l}\text { RHEUMATOID } \\
\text { ARTHRITIS } \\
\end{array}$ & N040. & Rheumatoid arthritis & \\
\hline 44743 & N040000 & $\begin{array}{l}\text { Rheumatoid arthritis of } \\
\text { cervical spine }\end{array}$ & $\begin{array}{l}\text { RHEUMATOID } \\
\text { ARTHRITIS } \\
\end{array}$ & N0400 & $\begin{array}{l}\text { Rheumatoid arthritis-Cx } \\
\text { spine }\end{array}$ & $\begin{array}{l}\text { Rheumatoid arthritis of } \\
\text { cervical spine }\end{array}$ \\
\hline 44203 & N040100 & $\begin{array}{l}\text { Other rheumatoid arthritis } \\
\text { of spine }\end{array}$ & $\begin{array}{l}\text { RHEUMATOID } \\
\text { ARTHRITIS } \\
\end{array}$ & N0401 & $\begin{array}{l}\text { Oth rheumatoid arthritis- } \\
\text { spine }\end{array}$ & $\begin{array}{l}\text { Other rheumatoid arthritis of } \\
\text { spine }\end{array}$ \\
\hline 21358 & N040200 & $\begin{array}{l}\text { Rheumatoid arthritis of } \\
\text { shoulder }\end{array}$ & $\begin{array}{l}\text { RHEUMATOID } \\
\text { ARTHRITIS } \\
\end{array}$ & N0402 & $\begin{array}{l}\text { Rheumatoid arthritis- } \\
\text { shoulder }\end{array}$ & $\begin{array}{l}\text { Rheumatoid arthritis of } \\
\text { shoulder }\end{array}$ \\
\hline 100914 & N040400 & $\begin{array}{l}\text { Rheumatoid arthritis of } \\
\text { acromioclavicular joint }\end{array}$ & $\begin{array}{l}\text { RHEUMATOID } \\
\text { ARTHRITIS } \\
\end{array}$ & N0404 & $\begin{array}{l}\text { Rheumatoid arthr- } \\
\text { acromioclav } j\end{array}$ & $\begin{array}{l}\text { Rheumatoid arthritis of } \\
\text { acromioclavicular joint }\end{array}$ \\
\hline 59738 & N040500 & $\begin{array}{l}\text { Rheumatoid arthritis of } \\
\text { elbow }\end{array}$ & $\begin{array}{l}\text { RHEUMATOID } \\
\text { ARTHRITIS } \\
\end{array}$ & N0405 & $\begin{array}{l}\text { Rheumatoid arthritis of } \\
\text { elbow }\end{array}$ & \\
\hline 63365 & N040600 & $\begin{array}{l}\text { Rheumatoid arthritis of } \\
\text { distal radio-ulnar joint }\end{array}$ & $\begin{array}{l}\text { RHEUMATOID } \\
\text { ARTHRITIS } \\
\end{array}$ & N0406 & $\begin{array}{l}\text { Rheumatoid arthritis-dist } \\
\text { RUJ }\end{array}$ & $\begin{array}{l}\text { Rheumatoid arthritis of distal } \\
\text { radio-ulnar joint }\end{array}$ \\
\hline 48832 & N040700 & $\begin{array}{l}\text { Rheumatoid arthritis of } \\
\text { wrist }\end{array}$ & $\begin{array}{l}\text { RHEUMATOID } \\
\text { ARTHRITIS } \\
\end{array}$ & N0407 & Rheumatoid arthritis of wrist & \\
\hline 42299 & N040800 & $\begin{array}{l}\text { Rheumatoid arthritis of } \\
\text { MCP joint }\end{array}$ & $\begin{array}{l}\text { RHEUMATOID } \\
\text { ARTHRITIS } \\
\end{array}$ & N0408 & $\begin{array}{l}\text { Rheumatoid arthritis-MCP } \\
\text { joint }\end{array}$ & $\begin{array}{l}\text { Rheumatoid arthritis of MCP } \\
\text { joint }\end{array}$ \\
\hline 41941 & N040900 & $\begin{array}{l}\text { Rheumatoid arthritis of PIP } \\
\text { joint of finger }\end{array}$ & $\begin{array}{l}\text { RHEUMATOID } \\
\text { ARTHRITIS } \\
\end{array}$ & N0409 & $\begin{array}{l}\text { Rheumatoid arthritis-PIPJ- } \\
\text { fing }\end{array}$ & $\begin{array}{l}\text { Rheumatoid arthritis of PIP } \\
\text { joint of finger }\end{array}$ \\
\hline 63198 & N040A00 & $\begin{array}{l}\text { Rheumatoid arthritis of DIP } \\
\text { joint of finger }\end{array}$ & $\begin{array}{l}\text { RHEUMATOID } \\
\text { ARTHRITIS }\end{array}$ & N040A & $\begin{array}{l}\text { Rheumatoid arthritis-DIPJ- } \\
\text { fing }\end{array}$ & $\begin{array}{l}\text { Rheumatoid arthritis of DIP } \\
\text { joint of finger }\end{array}$ \\
\hline 49067 & N040B00 & Rheumatoid arthritis of hip & $\begin{array}{l}\text { RHEUMATOID } \\
\text { ARTHRITIS } \\
\end{array}$ & NO40B & Rheumatoid arthritis of hip & \\
\hline 100776 & N040C00 & $\begin{array}{l}\text { Rheumatoid arthritis of } \\
\text { sacro-iliac joint }\end{array}$ & $\begin{array}{l}\text { RHEUMATOID } \\
\text { ARTHRITIS } \\
\end{array}$ & NO40C & Rheumatoid arthritis of SIJ & $\begin{array}{l}\text { Rheumatoid arthritis of sacro- } \\
\text { iliac joint }\end{array}$ \\
\hline 50863 & N040D00 & $\begin{array}{l}\text { Rheumatoid arthritis of } \\
\text { knee }\end{array}$ & $\begin{array}{l}\text { RHEUMATOID } \\
\text { ARTHRITIS } \\
\end{array}$ & NO40D & Rheumatoid arthritis of knee & \\
\hline 51239 & N040F00 & $\begin{array}{l}\text { Rheumatoid arthritis of } \\
\text { ankle }\end{array}$ & $\begin{array}{l}\text { RHEUMATOID } \\
\text { ARTHRITIS } \\
\end{array}$ & NO4OF & $\begin{array}{l}\text { Rheumatoid arthritis of } \\
\text { ankle }\end{array}$ & \\
\hline 73619 & NO40G00 & $\begin{array}{l}\text { Rheumatoid arthritis of } \\
\text { subtalar joint }\end{array}$ & $\begin{array}{l}\text { RHEUMATOID } \\
\text { ARTHRITIS } \\
\end{array}$ & NO40G & $\begin{array}{l}\text { Rheumatoid arthr-subtalar } \\
\text { jnt }\end{array}$ & $\begin{array}{l}\text { Rheumatoid arthritis of } \\
\text { subtalar joint }\end{array}$ \\
\hline 70658 & $\mathrm{NO4OHOO}$ & $\begin{array}{l}\text { Rheumatoid arthritis of } \\
\text { talonavicular joint }\end{array}$ & $\begin{array}{l}\text { RHEUMATOID } \\
\text { ARTHRITIS } \\
\end{array}$ & $\mathrm{NO4OH}$ & $\begin{array}{l}\text { Rheumatoid arthr-talonav } \\
\text { joint }\end{array}$ & $\begin{array}{l}\text { Rheumatoid arthritis of } \\
\text { talonavicular joint }\end{array}$ \\
\hline 71784 & N040J00 & $\begin{array}{l}\text { Rheumatoid arthritis of } \\
\text { other tarsal joint }\end{array}$ & $\begin{array}{l}\text { RHEUMATOID } \\
\text { ARTHRITIS } \\
\end{array}$ & N040J & $\begin{array}{l}\text { Rheumatoid arthr-oth tarsal } \\
\text { jt }\end{array}$ & $\begin{array}{l}\text { Rheumatoid arthritis of other } \\
\text { tarsal joint }\end{array}$ \\
\hline 51238 & N040K00 & $\begin{array}{l}\text { Rheumatoid arthritis of 1st } \\
\text { MTP joint }\end{array}$ & $\begin{array}{l}\text { RHEUMATOID } \\
\text { ARTHRITIS } \\
\end{array}$ & NO40K & $\begin{array}{l}\text { Rheumatoid arthr-1st MTP } \\
\text { joint }\end{array}$ & $\begin{array}{l}\text { Rheumatoid arthritis of 1st } \\
\text { MTP joint }\end{array}$ \\
\hline 99414 & N040L00 & $\begin{array}{l}\text { Rheumatoid arthritis of } \\
\text { lesser MTP joint }\end{array}$ & $\begin{array}{l}\text { RHEUMATOID } \\
\text { ARTHRITIS } \\
\end{array}$ & NO40L & $\begin{array}{l}\text { Rheumatoid arthr-lesser } \\
\text { MTP jt }\end{array}$ & $\begin{array}{l}\text { Rheumatoid arthritis of lesser } \\
\text { MTP joint }\end{array}$ \\
\hline 6916 & N040P00 & $\begin{array}{l}\text { Seronegative rheumatoid } \\
\text { arthritis }\end{array}$ & $\begin{array}{l}\text { RHEUMATOID } \\
\text { ARTHRITIS } \\
\end{array}$ & NO40P & $\begin{array}{l}\text { Seronegative rheumat } \\
\text { arthritis }\end{array}$ & $\begin{array}{l}\text { Seronegative rheumatoid } \\
\text { arthritis }\end{array}$ \\
\hline 31054 & N040S00 & $\begin{array}{l}\text { Rheumatoid arthritis - } \\
\text { multiple joint }\end{array}$ & $\begin{array}{l}\text { RHEUMATOID } \\
\text { ARTHRITIS } \\
\end{array}$ & NO40S & $\begin{array}{l}\text { Rheumat arthr - multiple } \\
\text { joint }\end{array}$ & $\begin{array}{l}\text { Rheumatoid arthritis - multiple } \\
\text { joint }\end{array}$ \\
\hline 8350 & N040T00 & $\begin{array}{l}\text { Flare of rheumatoid } \\
\text { arthritis }\end{array}$ & $\begin{array}{l}\text { RHEUMATOID } \\
\text { ARTHRITIS } \\
\end{array}$ & NO40T & Flare of rheumatoid arthritis & \\
\hline 4186 & N043.00 & $\begin{array}{l}\text { Juvenile rheumatoid } \\
\text { arthritis - Stills disease }\end{array}$ & $\begin{array}{l}\text { RHEUMATOID } \\
\text { ARTHRITIS } \\
\end{array}$ & N043. & Juvenile R.A.- Still's disease & $\begin{array}{l}\text { Juvenile rheumatoid arthritis - } \\
\text { Still's disease }\end{array}$ \\
\hline 47831 & N043100 & $\begin{array}{l}\text { Acute polyarticular juvenile } \\
\text { rheumatoid arthritis }\end{array}$ & $\begin{array}{l}\text { RHEUMATOID } \\
\text { ARTHRITIS } \\
\end{array}$ & N0431 & Acute polyartic.juvenile R.A. & $\begin{array}{l}\text { Acute polyarticular juvenile } \\
\text { rheumatoid arthritis }\end{array}$ \\
\hline
\end{tabular}




\begin{tabular}{|c|c|c|c|c|c|c|}
\hline 21533 & N043200 & $\begin{array}{l}\text { Pauciarticular juvenile } \\
\text { rheumatoid arthritis }\end{array}$ & $\begin{array}{l}\text { RHEUMATOID } \\
\text { ARTHRITIS } \\
\end{array}$ & N0432 & Pauciarticular juvenile R.A. & $\begin{array}{l}\text { Pauciarticular juvenile } \\
\text { rheumatoid arthritis }\end{array}$ \\
\hline 36276 & N043300 & $\begin{array}{l}\text { Monarticular juvenile } \\
\text { rheumatoid arthritis }\end{array}$ & $\begin{array}{l}\text { RHEUMATOID } \\
\text { ARTHRITIS } \\
\end{array}$ & N0433 & Monarticular juvenile R.A. & $\begin{array}{l}\text { Monarticular juvenile } \\
\text { rheumatoid arthritis }\end{array}$ \\
\hline 27557 & N043z00 & $\begin{array}{l}\text { Juvenile rheumatoid } \\
\text { arthritis NOS }\end{array}$ & $\begin{array}{l}\text { RHEUMATOID } \\
\text { ARTHRITIS } \\
\end{array}$ & N043z & $\begin{array}{l}\text { Juvenile rheumatoid } \\
\text { arthr.NOS }\end{array}$ & $\begin{array}{l}\text { Juvenile rheumatoid arthritis } \\
\text { NOS }\end{array}$ \\
\hline 31360 & N045500 & $\begin{array}{l}\text { Juvenile rheumatoid } \\
\text { arthritis }\end{array}$ & $\begin{array}{l}\text { RHEUMATOID } \\
\text { ARTHRITIS } \\
\end{array}$ & N0455 & Juvenile rheumatoid arthritis & \\
\hline 9707 & N047.00 & $\begin{array}{l}\text { Seropositive errosive } \\
\text { rheumatoid arthritis }\end{array}$ & $\begin{array}{l}\text { RHEUMATOID } \\
\text { ARTHRITIS }\end{array}$ & N047. & Seropositive errosive RA & $\begin{array}{l}\text { Seropositive errosive } \\
\text { rheumatoid arthritis }\end{array}$ \\
\hline 12019 & N04X.00 & $\begin{array}{l}\text { Seropositive rheumatoid } \\
\text { arthritis, unspecified }\end{array}$ & $\begin{array}{l}\text { RHEUMATOID } \\
\text { ARTHRITIS } \\
\end{array}$ & N04X. & Seroposit rheum arthr, unsp & $\begin{array}{l}\text { Seropositive rheumatoid } \\
\text { arthritis, unspecified }\end{array}$ \\
\hline 28853 & N04y012 & $\begin{array}{l}\text { Fibrosing alveolitis } \\
\text { associated with rheumatoid } \\
\text { arthritis }\end{array}$ & $\begin{array}{l}\text { RHEUMATOID } \\
\text { ARTHRITIS }\end{array}$ & $\mathrm{N} 04 \mathrm{y} 0$ & Rheumatoid lung & \\
\hline 70221 & Nyu1200 & $\begin{array}{l}{[\mathrm{X}] \text { Other specified }} \\
\text { rheumatoid arthritis }\end{array}$ & $\begin{array}{l}\text { RHEUMATOID } \\
\text { ARTHRITIS } \\
\end{array}$ & Nyu12 & $\begin{array}{l}{[\mathrm{X}] \mathrm{Oth} \text { spcf rheumatd }} \\
\text { arthritis }\end{array}$ & $\begin{array}{l}{[\mathrm{X}] \text { Other specified rheumatoid }} \\
\text { arthritis }\end{array}$ \\
\hline 56202 & Nyu1G00 & $\begin{array}{l}\text { [X]Seropositive rheumatoid } \\
\text { arthritis, unspecified }\end{array}$ & $\begin{array}{l}\text { RHEUMATOID } \\
\text { ARTHRITIS }\end{array}$ & Nyu1G & $\begin{array}{l}\text { [X]Seroposit rheum arthr, } \\
\text { unsp }\end{array}$ & $\begin{array}{l}{[\mathrm{X}] \text { Seropositive rheumatoid }} \\
\text { arthritis, unspecified }\end{array}$ \\
\hline
\end{tabular}

\begin{tabular}{|c|c|c|c|c|c|}
\hline $\begin{array}{l}\text { Readcode-V2- } \\
\text { 5dig }\end{array}$ & PREF_TERM_30 & PREF_TERM_60 & $\begin{array}{l}\text { Readcode- } \\
\text { V3-5dig }\end{array}$ & TERM_ID & TERM_30 \\
\hline $\mathrm{X} 75 \mathrm{rx}$ & Y7Coo & Fibromyalgia & N239. & Fibromyalgia & \\
\hline $\mathrm{N} 2401$ & Y7Cor & Fibrositis unspecified & N248. & Fibromyalgia & \\
\hline N2405 & YaY8a & Fibrositis of neck & $\mathrm{N} 2480$ & \multicolumn{2}{|c|}{ Myofascial pain syndrome } \\
\hline N2406 & YaY8b & Fibrositis arm & N2481 & $\begin{array}{l}\text { Piriformis } \\
\text { syndrome }\end{array}$ & \\
\hline $\mathrm{Xa01F}$ & YaOtZ & Chronic fatigue syndrome & F286. & \multicolumn{2}{|c|}{ Chronic fatigue syndrome } \\
\hline XaPoo & YasR0 & Severe chronic fatigue syndrome & F2860 & \multicolumn{2}{|c|}{ Mild chronic fatigue syndrome } \\
\hline XaPon & YasQz & $\begin{array}{l}\text { Moderate chronic fatigue } \\
\text { syndrome }\end{array}$ & F2861 & \multicolumn{2}{|c|}{ Moderate chronic fatigue syndrome } \\
\hline XaPom & YasQy & Mild chronic fatigue syndrome & F2862 & \multicolumn{2}{|c|}{ Severe chronic fatigue syndrome } \\
\hline XaPom & YasQy & Mild chronic fatigue syndrome & & & \\
\hline
\end{tabular}

\begin{tabular}{|c|c|c|c|c|c|}
\hline Medcode & Readcode & Readterm & cause & $\begin{array}{l}\text { Readcode- } \\
\text { V2-5dig }\end{array}$ & PREF_TERM_30 \\
\hline 16481 & 1475 & $\mathrm{H} / \mathrm{O}$ : trigeminal neuralgia & $\begin{array}{l}\text { TRIGEMINAL } \\
\text { NEURALGIA }\end{array}$ & 1475 & \\
\hline 11498 & A531200 & Postherpetic trigeminal neuralgia & $\begin{array}{l}\text { TRIGEMINAL } \\
\text { NEURALGIA }\end{array}$ & A5312 & $\begin{array}{l}\text { Postherpetic } \\
\text { trigem.neuralgia }\end{array}$ \\
\hline 7584 & F300.00 & Post-herpetic trigeminal neuralgia & $\begin{array}{l}\text { TRIGEMINAL } \\
\text { NEURALGIA }\end{array}$ & $\mathrm{F} 300$. & $\begin{array}{l}\text { Post-herpetic } \\
\text { trigem.neuralgia }\end{array}$ \\
\hline 1541 & F301.00 & Other specified trigeminal neuralgia & $\begin{array}{l}\text { TRIGEMINAL } \\
\text { NEURALGIA } \\
\end{array}$ & F301. & $\begin{array}{l}\text { Trigeminal } \\
\text { neuralgia OS }\end{array}$ \\
\hline 6581 & $\mathrm{~F} 301 \mathrm{z} 00$ & Trigeminal neuralgia NOS & $\begin{array}{l}\text { TRIGEMINAL } \\
\text { NEURALGIA }\end{array}$ & $\mathrm{F} 301 \mathrm{z}$ & $\begin{array}{l}\text { Trigeminal } \\
\text { neuralgia NOS }\end{array}$ \\
\hline 9193 & F336.00 & Phantom limb syndrome & PHANTOM LIMB PAIN & F336. & $\begin{array}{l}\text { Phantom limb } \\
\text { syndrome }\end{array}$ \\
\hline 18016 & $F 336000$ & Phantom limb syndrome with pain & PHANTOM LIMB PAIN & $\mathrm{F} 3360$ & $\begin{array}{l}\text { Phantom limb } \\
\text { syndrome wth } \\
\text { pain }\end{array}$ \\
\hline 54894 & $\mathrm{~F} 336100$ & Phantom limb syndrome without pain & PHANTOM LIMB PAIN & F3361 & $\begin{array}{l}\text { Phantom limb syn } \\
\text { w'out pain }\end{array}$ \\
\hline 1598 & A531.11 & Post-herpetic neuralgia & $\begin{array}{l}\text { POST HERPETIC } \\
\text { NEURALGIA }\end{array}$ & A531. & $\begin{array}{l}\text { Herpes } \\
\text { zoster+other CNS } \\
\text { compl. }\end{array}$ \\
\hline 17180 & A531500 & Postzoster neuralgia & $\begin{array}{l}\text { POST HERPETIC } \\
\text { NEURALGIA }\end{array}$ & A5315 & $\begin{array}{l}\text { Postzoster } \\
\text { neuralgia }\end{array}$ \\
\hline 10223 & A531511 & Postherpetic neuralgia & $\begin{array}{l}\text { POST HERPETIC } \\
\text { NEURALGIA }\end{array}$ & A5315 & \\
\hline 7795 & C106.12 & Diabetes mellitus with neuropathy & DIABETIC NEUROPATHY & C106. & $\begin{array}{l}\text { Diab.mell. with } \\
\text { neuropathy }\end{array}$ \\
\hline 16491 & C106.13 & Diabetes mellitus with polyneuropathy & DIABETIC NEUROPATHY & C106. & \\
\hline 24694 & С108B00 & Insulin dependent diabetes mellitus with mononeuropathy & DIABETIC NEUROPATHY & C108B & $\begin{array}{l}\text { IDDM with } \\
\text { mononeuropathy }\end{array}$ \\
\hline 99231 & C108B11 & Type I diabetes mellitus with mononeuropathy & DIABETIC NEUROPATHY & C108B & \\
\hline 41716 & $\mathrm{C} 108 \mathrm{C} 00$ & Insulin dependent diabetes mellitus with polyneuropathy & DIABETIC NEUROPATHY & C108C & $\begin{array}{l}\text { IDDM with } \\
\text { polyneuropathy }\end{array}$ \\
\hline 72320 & C109A00 & $\begin{array}{l}\text { Non-insulin dependent diabetes mellitus with } \\
\text { mononeuropathy }\end{array}$ & DIABETIC NEUROPATHY & C109A & $\begin{array}{l}\text { NIDDM with } \\
\text { mononeuropathy }\end{array}$ \\
\hline 50813 & C109A11 & Type II diabetes mellitus with mononeuropathy & DIABETIC NEUROPATHY & C109A & \\
\hline 45467 & С109B00 & $\begin{array}{l}\text { Non-insulin dependent diabetes mellitus with } \\
\text { polyneuropathy }\end{array}$ & DIABETIC NEUROPATHY & $\mathrm{C} 109 \mathrm{~B}$ & $\begin{array}{l}\text { NIDDM with } \\
\text { polyneuropathy }\end{array}$ \\
\hline 47409 & C109B11 & Type II diabetes mellitus with polyneuropathy & DIABETIC NEUROPATHY & $\mathrm{C} 109 \mathrm{~B}$ & \\
\hline 68105 & C10EB00 & Type 1 diabetes mellitus with mononeuropathy & DIABETIC NEUROPATHY & C10EB & $\begin{array}{l}\text { Type } 1 \text { diab mell + } \\
\text { mononeurop }\end{array}$ \\
\hline 46301 & C10EC00 & Type 1 diabetes mellitus with polyneuropathy & DIABETIC NEUROPATHY & $\mathrm{C} 10 \mathrm{EC}$ & $\begin{array}{l}\text { Type } 1 \text { diab mell + } \\
\text { polyneurop }\end{array}$ \\
\hline 91943 & C10EC11 & Type I diabetes mellitus with polyneuropathy & DIABETIC NEUROPATHY & C1OEC & \\
\hline 101311 & $\mathrm{C} 10 \mathrm{EC} 12$ & Insulin dependent diabetes mellitus with polyneuropathy & DIABETIC NEUROPATHY & C10EC & \\
\hline 62674 & C10FA00 & Type 2 diabetes mellitus with mononeuropathy & DIABETIC NEUROPATHY & C10FA & $\begin{array}{l}\text { Type } 2 \text { diab mell } \\
\text { mononeurop }\end{array}$ \\
\hline 95351 & C10FA11 & Type II diabetes mellitus with mononeuropathy & DIABETIC NEUROPATHY & C10FA & \\
\hline
\end{tabular}




\begin{tabular}{|l|l|l|l|l|l|}
\hline 18425 & C10FB00 & Type 2 diabetes mellitus with polyneuropathy & DIABETIC NEUROPATHY & C10FB & $\begin{array}{l}\text { Type 2 diab mell + } \\
\text { polyneurop }\end{array}$ \\
\hline 50527 & C10FB11 & Type II diabetes mellitus with polyneuropathy & DIABETIC NEUROPATHY & C10FB & $\begin{array}{l}\text { Autonomic } \\
\text { neuropathy- } \\
\text { diabetes }\end{array}$ \\
\hline 17067 & F171100 & Autonomic neuropathy due to diabetes & DIABETIC NEUROPATHY & F1711 \\
\hline 31790 & F372.00 & Polyneuropathy in diabetes & DIABETIC NEUROPATHY & F372. & $\begin{array}{l}\text { Polyneuropathy in } \\
\text { diabetes }\end{array}$ \\
\hline 5002 & F372.11 & Diabetic polyneuropathy & DIABETIC NEUROPATHY & F372. & \\
\hline 2342 & F372.12 & Diabetic neuropathy & DIABETIC NEUROPATHY & F372. & \\
\hline 48078 & F372000 & Acute painful diabetic neuropathy & DIABETIC NEUROPATHY & F3720 & $\begin{array}{l}\text { Acute painful diab } \\
\text { neuropathy }\end{array}$ \\
\hline 35785 & F372100 & Chronic painful diabetic neuropathy & DIABETIC NEUROPATHY & F3721 & $\begin{array}{l}\text { Chron painful diab } \\
\text { neuropathy }\end{array}$ \\
\hline 24571 & F372200 & Asymptomatic diabetic neuropathy & DIABETIC NEUROPATHY & F3722 & $\begin{array}{l}\text { Asymptomatic } \\
\text { diab neuropathy }\end{array}$ \\
\hline 37315 & F3y0.00 & Diabetic mononeuropathy & DIABETIC NEUROPATHY & F3y0. & $\begin{array}{l}\text { Diabetic } \\
\text { mononeuropathy }\end{array}$ \\
\hline
\end{tabular}

\begin{tabular}{|c|c|c|c|c|c|}
\hline READ_VRS & $\begin{array}{l}\text { PAIN_CA } \\
\mathrm{T}\end{array}$ & CODE_CAT & READ_CD & TERM_30 & TERM_60 \\
\hline V2 & NECK & NECK & $16 A .$. & Stiff neck symptom & \\
\hline V2 & NECK & NECK & $16 \mathrm{~A} 2$. & Stiff neck & \\
\hline V2 & NECK & NECK & $16 \mathrm{~A} 3$. & Wry neck/torticollis & \\
\hline $\mathrm{V} 2$ & NECK & NECK & $16 \mathrm{AZ}$. & Stiff neck symptom NOS & \\
\hline V2 & NECK & NECK & $1 \mathrm{D} 21$. & Symptom: head/neck & \\
\hline V2 & NECK & NECK & 2H2D. & $\mathrm{O} / \mathrm{E}$ - neck joint abnormal & \\
\hline $\mathrm{V} 2$ & NECK & NECK & 2121. & $\mathrm{O} / \mathrm{E}$ - sign - head/neck & \\
\hline V2 & NECK & NECK & $7 \mathrm{G} 15$. & Sensory skin flap & \\
\hline V2 & NECK & NECK & 7G152 & Local sensory skin flap hd/nk & $\begin{array}{l}\text { Local sensory skin flap to } \\
\text { head or neck }\end{array}$ \\
\hline $\mathrm{V} 2$ & NECK & NECK & 7G19. & Local subcut pedicle skin flap & $\begin{array}{l}\text { Local subcutaneous } \\
\text { pedicle skin flap }\end{array}$ \\
\hline V2 & NECK & NECK & L331z & Cord tight round neck NOS & \\
\hline V2 & NECK & NECK & N131. & Cervicalgia - pain in neck & \\
\hline $\mathrm{V} 2$ & NECK & NECK & N135. & Torticollis unspecified & \\
\hline V2 & NECK & NECK & N135z & Torticollis NOS & \\
\hline V2 & NECK & NECK & N13y2 & Crick in neck & \\
\hline V2 & NECK & NECK & N13z. & Cervical/neck disorder NOS & $\begin{array}{l}\text { Cervical and neck } \\
\text { disorders NOS }\end{array}$ \\
\hline V2 & NECK & NECK & N2405 & Fibrositis of neck & \\
\hline V2 & NECK & NECK & PE1.. & Congen.sternomastoid torticol. & $\begin{array}{l}\text { Congenital } \\
\text { sternomastoid torticollis }\end{array}$ \\
\hline V2 & NECK & NECK & R04.. & [D]Head and neck symptoms & \\
\hline V2 & NECK & NECK & R04z. & [D]Head and neck, other sympt. & $\begin{array}{l}\text { [D]Other symptoms } \\
\text { affecting head and neck }\end{array}$ \\
\hline V2 & NECK & NECK & $\mathrm{R} 04 \mathrm{zz}$ & [D]Head and neck symptoms NOS & \\
\hline V2 & NECK & NECK & $\mathrm{S} 1 \ldots$ & Fracture of neck and trunk & \\
\hline V2 & NECK & NECK & S10A. & Fracture of neck & \\
\hline V2 & NECK & NECK & S1z.. & Fracture of neck and trunk NOS & \\
\hline V2 & NECK & NECK & S2616 & Opn \# thumb prox phlnx, neck & $\begin{array}{l}\text { Open fracture thumb } \\
\text { proximal phalanx, neck }\end{array}$ \\
\hline $\mathrm{V} 2$ & NECK & NECK & S30.. & \#Neck of femur & $\begin{array}{l}\text { Fracture of neck of } \\
\text { femur }\end{array}$ \\
\hline V2 & NECK & NECK & S490. & Cls dslc cervical spine & $\begin{array}{l}\text { Closed dislocation } \\
\text { cervical spine }\end{array}$ \\
\hline V2 & NECK & NECK & S491. & Open dislocation cerv spine & $\begin{array}{l}\text { Open dislocation cervical } \\
\text { spine }\end{array}$ \\
\hline $\mathrm{V} 2$ & NECK & NECK & S570. & Neck sprain & \\
\hline V2 & NECK & NECK & S5700 & Neck sprain, unspecified & \\
\hline V2 & NECK & NECK & $\mathrm{S} 570 \mathrm{z}$ & Neck sprain NOS & \\
\hline V2 & NECK & NECK & SD0y1 & Superficial injury of neck NOS & \\
\hline
\end{tabular}




\begin{tabular}{|c|c|c|c|c|c|}
\hline V2 & NECK & NECK & SD0z1 & Superfic.inj.neck NOS+infectn. & $\begin{array}{l}\text { Superficial injury of neck } \\
\text { NOS, infected }\end{array}$ \\
\hline V2 & NECK & NECK & SDCO. & Supfcl inj invlv head wth neck & $\begin{array}{l}\text { Superficial injuries } \\
\text { involving head with neck }\end{array}$ \\
\hline V2 & NECK & NECK & SE08. & Other contusion neck & \\
\hline V2 & NECK & NECK & SFO.. & Crush injury, face, scalp+neck & $\begin{array}{l}\text { Crush injury, face, scalp } \\
\text { and neck }\end{array}$ \\
\hline V2 & NECK & NECK & SF02. & Crush injury, neck & \\
\hline V2 & NECK & NECK & $\mathrm{SF} 02 \mathrm{z}$ & Crush injury, neck NOS & \\
\hline V2 & NECK & NECK & SFOz. & Crush inj,face,scalp+neck NOS & $\begin{array}{l}\text { Crush injury, face, scalp } \\
\text { and neck NOS }\end{array}$ \\
\hline V2 & NECK & NECK & SH149 & Deep F/T burn-neck-no BPL & $\begin{array}{l}\text { Deep full thickness burn } \\
\text { of neck without loss of } \\
\text { body part }\end{array}$ \\
\hline V2 & NECK & NECK & SJ70. & Head/neck superfic.nerve inj. & $\begin{array}{l}\text { Head and neck } \\
\text { superficial nerve injury }\end{array}$ \\
\hline $\mathrm{V} 2$ & NECK & NECK & SJ71. & Injury/periphrl nerves of neck & $\begin{array}{l}\text { Injury of peripheral } \\
\text { nerves of neck }\end{array}$ \\
\hline V2 & NECK & NECK & SK10. & Other face/neck injuries & $\begin{array}{l}\text { Other face and neck } \\
\text { injuries }\end{array}$ \\
\hline V2 & NECK & NECK & SK108 & Injur/muscle+tendon/neck level & $\begin{array}{l}\text { Injury of muscle and } \\
\text { tendon at neck level }\end{array}$ \\
\hline V2 & NECK & NECK & SK10y & Other neck injuries & \\
\hline $\mathrm{V} 2$ & NECK & NECK & SK10z & Other face/neck injuries NOS & $\begin{array}{l}\text { Other face and neck } \\
\text { injuries NOS }\end{array}$ \\
\hline $\mathrm{V} 2$ & NECK & NECK & SK1x1 & Multipl superfic injuries/neck & $\begin{array}{l}\text { Multiple superficial } \\
\text { injuries of neck }\end{array}$ \\
\hline V2 & NECK & NECK & SK1×3 & Multiple injuries of neck & \\
\hline V2 & NECK & NECK & SR10. & Fracture involv head with neck & $\begin{array}{l}\text { Fractures involving head } \\
\text { with neck }\end{array}$ \\
\hline V2 & NECK & NECK & SR100 & Cls fract invol head with neck & $\begin{array}{l}\text { Closed fractures } \\
\text { involving head with neck }\end{array}$ \\
\hline V2 & NECK & NECK & SR20. & Disloc,sprns+strns inv hd+neck & $\begin{array}{l}\text { Dislocations, sprains and } \\
\text { strains involving head } \\
\text { with neck }\end{array}$ \\
\hline $\mathrm{V} 2$ & NECK & NECK & SR30. & Crush inj invlv head with neck & $\begin{array}{l}\text { Crushing injuries } \\
\text { involving head with neck }\end{array}$ \\
\hline $\mathrm{V} 2$ & NECK & NECK & Syu1. & {$[\mathrm{X}]$ Injuries to the neck } & \\
\hline V2 & NECK & NECK & Syu11 & {$[X]$ Superfic inj oth part neck } & $\begin{array}{l}{[X] \text { Superficial injury of }} \\
\text { other parts of the neck }\end{array}$ \\
\hline V2 & NECK & NECK & Syu12 & {$[\mathrm{X}]$ Superf inj neck, part unsp } & $\begin{array}{l}{[X] \text { Superficial injury of }} \\
\text { neck, part unspecified }\end{array}$ \\
\hline V2 & NECK & NECK & Syu16 & {$[\mathrm{X}]$ Fracture other parts neck } & $\begin{array}{l}{[X] \text { Fracture of other }} \\
\text { parts of neck }\end{array}$ \\
\hline V2 & NECK & NECK & Syu18 & {$[\mathrm{X}] \mathrm{Spr} / \mathrm{str} \mathrm{jt} / \mathrm{g}$ ot/un pt neck } & $\begin{array}{l}X] \text { Sprain/strain of } \\
\text { joint/ligam of oth \& unsp } \\
\text { part of neck }\end{array}$ \\
\hline V2 & NECK & NECK & Syu1A & {$[X] \operatorname{Inj}$ oth unsp nerve of neck } & $\begin{array}{l}{[\mathrm{X}] \text { Injury of other and }} \\
\text { unspecified nerves of } \\
\text { neck }\end{array}$ \\
\hline $\mathrm{V} 2$ & NECK & NECK & Syu1F & {$[X]$ th specif inj of neck } & $\begin{array}{l}{[X] \text { Other specified }} \\
\text { injuries of neck } \\
\end{array}$ \\
\hline V2 & NECK & NECK & SyuLC & {$[\mathrm{X}]$ Seq unspec injury neck+trnk } & $\begin{array}{l}{[X] \text { Sequelae of }} \\
\text { unspecified injury of } \\
\text { neck and trunk }\end{array}$ \\
\hline V2 & NECK & NECK & Z6G62 & & \\
\hline V2 & NECK & NECK & ZV48. & [V]Head, neck/ trunk problem & $\begin{array}{l}{[\mathrm{V}] \mathrm{Head}, \text { neck or trunk }} \\
\text { problems }\end{array}$ \\
\hline V2 & NECK & NECK & ZV485 & [V]Neck/trunk sensory problem & $\begin{array}{l}\text { [V]Sensory problem of } \\
\text { neck or trunk }\end{array}$ \\
\hline V2 & BACK & SPINAL PAIN & $14 \mathrm{~N} 30$ & H/O Spinal surgery & \\
\hline V2 & BACK & SPINAL PAIN & $2 \mathrm{AD6}$. & O/E - ciliospinal reflex -ve. & \\
\hline V2 & BACK & SPINAL PAIN & 7040 & & \\
\hline $\mathrm{V} 2$ & BACK & SPINAL PAIN & 70410 & Stereotac chordotomy spin cord & $\begin{array}{l}\text { Stereotactic chordotomy } \\
\text { of spinal cord }\end{array}$ \\
\hline V2 & BACK & SPINAL PAIN & 70411 & Open chordotomy spin cord NEC & $\begin{array}{l}\text { Open chordotomy of } \\
\text { spinal cord NEC }\end{array}$ \\
\hline V2 & BACK & SPINAL PAIN & 70412 & Myelotomy of spinal cord & \\
\hline V2 & BACK & SPINAL PAIN & 70421 & Percut chordotomy spinal cord & $\begin{array}{l}\text { Percutaneous } \\
\text { chordotomy of spinal } \\
\text { cord }\end{array}$ \\
\hline V2 & BACK & SPINAL PAIN & 70431 & Closure spin myelomeningocele & $\begin{array}{l}\text { Closure of spinal } \\
\text { myelomeningocele }\end{array}$ \\
\hline
\end{tabular}




\begin{tabular}{|c|c|c|c|c|c|}
\hline V2 & BACK & SPINAL PAIN & 70432 & Closure of spinal meningocele & \\
\hline V2 & BACK & SPINAL PAIN & 70470 & Spinal myelography NEC & \\
\hline V2 & BACK & SPINAL PAIN & 70471 & Spinal manometry & \\
\hline V2 & BACK & SPINAL PAIN & 70480 & Extirpatn lesion spinal $\mathrm{n}$ root & $\begin{array}{l}\text { Extirpation of lesion of } \\
\text { spinal nerve root }\end{array}$ \\
\hline V2 & BACK & SPINAL PAIN & 70481 & Rhizotomy spinal nerve root & $\begin{array}{l}\text { Rhizotomy of spinal } \\
\text { nerve root }\end{array}$ \\
\hline V2 & BACK & SPINAL PAIN & 70484 & Destruct spinal nerve root NEC & $\begin{array}{l}\text { Destruction of spinal } \\
\text { nerve root NEC }\end{array}$ \\
\hline V2 & BACK & SPINAL PAIN & 7049 & & \\
\hline V2 & BACK & SPINAL PAIN & $7049 y$ & Other destruct spinal cord OS & $\begin{array}{l}\text { Other specified } \\
\text { destruction of spinal } \\
\text { cord }\end{array}$ \\
\hline V2 & BACK & SPINAL PAIN & 7Ј3.. & Lumbar spine operations & \\
\hline V2 & BACK & SPINAL PAIN & $7 \mathrm{~J} 423$ & Spinal extension traction \# & $\begin{array}{l}\text { Spinal extension traction } \\
\text { for fracture of spine }\end{array}$ \\
\hline V2 & BACK & SPINAL PAIN & $7 \mathrm{~J} 425$ & Spinal traction \# spine NEC & $\begin{array}{l}\text { Spinal traction for } \\
\text { fracture of spine NEC }\end{array}$ \\
\hline V2 & BACK & SPINAL PAIN & $7 J 479$ & Spinal probing & \\
\hline V2 & BACK & SPINAL PAIN & $7 \mathrm{~J} 49$. & Denervat spinal facet vertebra & $\begin{array}{l}\text { Denervation of spinal } \\
\text { facet joint of vertebra }\end{array}$ \\
\hline V2 & BACK & SPINAL PAIN & $7 \mathrm{~J} 491$ & Denerv cervic spinal facet NEC & $\begin{array}{l}\text { Denervation of spinal } \\
\text { facet joint of cervical } \\
\text { vertebra NEC }\end{array}$ \\
\hline V2 & BACK & SPINAL PAIN & $7 \mathrm{~J} 493$ & Denerv thoracic spin facet NEC & $\begin{array}{l}\text { Denervation of spinal } \\
\text { facet joint of thoracic } \\
\text { vertebra NEC }\end{array}$ \\
\hline V2 & BACK & SPINAL PAIN & $7 \mathrm{~J} 495$ & Denerv lumbar spin facet NEC & $\begin{array}{l}\text { Denervation of spinal } \\
\text { facet joint of lumbar } \\
\text { vertebra NEC }\end{array}$ \\
\hline V2 & BACK & SPINAL PAIN & $7 \mathrm{~J} 49 \mathrm{y}$ & Denerv vertebr spinal facet OS & $\begin{array}{l}\text { Denervation of spinal } \\
\text { facet joint of vertebra } \\
\text { OS }\end{array}$ \\
\hline V2 & BACK & SPINAL PAIN & $7 \mathrm{~J} 49 \mathrm{z}$ & Denerv verteb spinal facet NOS & $\begin{array}{l}\text { Denervation of spinal } \\
\text { facet joint of vertebra } \\
\text { NOS }\end{array}$ \\
\hline V2 & BACK & SPINAL PAIN & A940. & Tabes dorsalis - neurosyphilis & \\
\hline V2 & BACK & SPINAL PAIN & $\mathrm{B} 74 \mathrm{y} 0$ & Lipoma of spinal column & \\
\hline V2 & BACK & SPINAL PAIN & B74y1 & Lipoma of spine - extradural & $\begin{array}{l}\text { Lipoma of spinal canal - } \\
\text { extradural }\end{array}$ \\
\hline V2 & BACK & SPINAL PAIN & B74y2 & Lipoma of spine - intradural & $\begin{array}{l}\text { Lipoma of spinal canal - } \\
\text { intradural }\end{array}$ \\
\hline $\mathrm{V} 2$ & BACK & SPINAL PAIN & $\mathrm{B} 74 \mathrm{y} 3$ & Lipoma of spinal cord & \\
\hline $\mathrm{V} 2$ & BACK & SPINAL PAIN & B7F40 & Spinal meningioma & \\
\hline V2 & BACK & SPINAL PAIN & B9251 & Uncert. neopl. spinal cord & $\begin{array}{l}\text { Neoplasm of uncertain } \\
\text { behaviour of spinal cord }\end{array}$ \\
\hline V2 & BACK & SPINAL PAIN & F04.. & Intracranial / spinal abscess & $\begin{array}{l}\text { Intracranial and } \\
\text { intraspinal abscesses }\end{array}$ \\
\hline $\mathrm{V} 2$ & BACK & SPINAL PAIN & F041. & Intraspinal abscess & \\
\hline V2 & BACK & SPINAL PAIN & F0412 & Intraspinal abscess-subdural & $\begin{array}{l}\text { Subdural intraspinal } \\
\text { abscess }\end{array}$ \\
\hline V2 & BACK & SPINAL PAIN & $\mathrm{F} 041 \mathrm{z}$ & Intraspinal abscess NOS & \\
\hline V2 & BACK & SPINAL PAIN & F04z. & Intracranial / spinal absc.NOS & $\begin{array}{l}\text { Intracranial or } \\
\text { intraspinal abscess NOS }\end{array}$ \\
\hline V2 & BACK & SPINAL PAIN & $\mathrm{F} 150$. & Werdnig - Hoffmann disease & \\
\hline V2 & BACK & SPINAL PAIN & F151. & Spinal muscular atrophy & \\
\hline V2 & BACK & SPINAL PAIN & F1510 & Spinal muscular atrophy unspec & $\begin{array}{l}\text { Unspecified spinal } \\
\text { muscular atrophy }\end{array}$ \\
\hline V2 & BACK & SPINAL PAIN & F1513 & X-linked bulbo-spinal atrophy & \\
\hline V2 & BACK & SPINAL PAIN & $\mathrm{F} 151 \mathrm{z}$ & Spinal muscular atrophy NOS & \\
\hline V2 & BACK & SPINAL PAIN & F16.. & Other diseases of spinal cord & \\
\hline V2 & BACK & SPINAL PAIN & F1610 & Myelopathy-infarct spine cord & $\begin{array}{l}\text { Myelopathy due to acute } \\
\text { infarction of spinal cord }\end{array}$ \\
\hline V2 & BACK & SPINAL PAIN & F1611 & Myelopathy-thrombos spine cord & $\begin{array}{l}\text { Myelopathy due to } \\
\text { arterial thrombosis of } \\
\text { spinal cord }\end{array}$ \\
\hline V2 & BACK & SPINAL PAIN & F1612 & Myelopathy-oedema spine cord & $\begin{array}{l}\text { Myelopathy due to } \\
\text { oedema of spinal cord }\end{array}$ \\
\hline
\end{tabular}




\begin{tabular}{|c|c|c|c|c|c|}
\hline V2 & BACK & SPINAL PAIN & F1615 & Ant spinal artery thrombosis & $\begin{array}{l}\text { Anterior spinal artery } \\
\text { thrombosis }\end{array}$ \\
\hline V2 & BACK & SPINAL PAIN & F162. & Subacute combined cord degen. & $\begin{array}{l}\text { Subacute combined } \\
\text { degeneration of spinal } \\
\text { cord }\end{array}$ \\
\hline V2 & BACK & SPINAL PAIN & F201. & Multiple sclerosis-spinal cord & $\begin{array}{l}\text { Multiple sclerosis of the } \\
\text { spinal cord }\end{array}$ \\
\hline V2 & BACK & SPINAL PAIN & F290y & Other spinal puncture reaction & \\
\hline V2 & BACK & SPINAL PAIN & F2921 & Adhesions - spinal meninges & $\begin{array}{l}\text { Spinal meningeal } \\
\text { adhesions }\end{array}$ \\
\hline V2 & BACK & SPINAL PAIN & F2923 & Cyst - spinal meninges & $\begin{array}{l}\text { Cyst of the spinal } \\
\text { meninges }\end{array}$ \\
\hline V2 & BACK & SPINAL PAIN & FyuOB & {$[\mathrm{X}]$ Intrcrn+intrspn ab+grn/d CE } & $\begin{array}{l}{[X] \text { Intracranial+intraspin }} \\
\text { al abscess+granuloma in } \\
\text { diseases } \mathrm{CE}\end{array}$ \\
\hline V2 & BACK & SPINAL PAIN & Fyu32 & [X]Subac cmb dgn/spn crd/ds CE & $\begin{array}{l}{[\mathrm{X}] \text { Subacute combined }} \\
\text { degeneration/spinal } \\
\text { cord in diseases } \mathrm{CE} \\
\end{array}$ \\
\hline V2 & BACK & SPINAL PAIN & FyuA7 & {$[\mathrm{X}]$ Oth spcf disease/spinal crd } & $\begin{array}{l}\mathrm{X}] \text { Other specified } \\
\text { diseases of spinal cord }\end{array}$ \\
\hline V2 & BACK & SPINAL PAIN & G769. & Ant spin/vert art compr syndr & $\begin{array}{l}\text { Anterior spinal and } \\
\text { vertebral artery } \\
\text { compression syndromes }\end{array}$ \\
\hline V2 & BACK & SPINAL PAIN & L3841 & Sp/ep anes-ind hdache dur puer & $\begin{array}{l}\text { Spinal/epidural anaesth- } \\
\text { induced headache } \\
\text { during puerp }\end{array}$ \\
\hline V2 & BACK & SPINAL PAIN & L387. & Sp/epi anes-ind hdache,lab/del & $\begin{array}{l}\text { Spinal/epidural anesth- } \\
\text { induced headache dur } \\
\text { labour/delivery }\end{array}$ \\
\hline $\mathrm{V} 2$ & BACK & SPINAL PAIN & N101. & Spinal enthesopathy & \\
\hline V2 & BACK & SPINAL PAIN & N12A. & Postlaminectomy syndrome & \\
\hline V2 & BACK & SPINAL PAIN & N1300 & Idiopathic Cx spinal stenosis & $\begin{array}{l}\text { Idiopathic cervical spinal } \\
\text { stenosis }\end{array}$ \\
\hline V2 & BACK & SPINAL PAIN & N1301 & Degenerativ $\mathrm{Cx}$ spinal stenosis & $\begin{array}{l}\text { Degenerative cervical } \\
\text { spinal stenosis }\end{array}$ \\
\hline V2 & BACK & SPINAL PAIN & N140. & Spinal stenosis excl.cervical & $\begin{array}{l}\text { Spinal stenosis, } \\
\text { excluding cervical region }\end{array}$ \\
\hline V2 & BACK & SPINAL PAIN & N1400 & Spinal stenosis unspec.region & $\begin{array}{l}\text { Spinal stenosis of } \\
\text { unspecified region }\end{array}$ \\
\hline $\mathrm{V} 2$ & BACK & SPINAL PAIN & N1401 & Thoracic spinal stenosis & \\
\hline V2 & BACK & SPINAL PAIN & $\mathrm{N} 1402$ & Lumbar spinal stenosis & \\
\hline V2 & BACK & SPINAL PAIN & $\mathrm{N} 1404$ & Degenerativ th spinal stenosis & $\begin{array}{l}\text { Degenerative thoracic } \\
\text { spinal stenosis }\end{array}$ \\
\hline V2 & BACK & SPINAL PAIN & N1406 & Th spin stenos due to oth dis & $\begin{array}{l}\text { Thoracic spinal stenosis } \\
\text { secondary to other } \\
\text { disease }\end{array}$ \\
\hline V2 & BACK & SPINAL PAIN & N1407 & Idiopathic lu spinal stenosis & $\begin{array}{l}\text { Idiopathic lumbar spinal } \\
\text { stenosis }\end{array}$ \\
\hline V2 & BACK & SPINAL PAIN & N1408 & Degenerativ lu spinal stenosis & $\begin{array}{l}\text { Degenerative lumbar } \\
\text { spinal stenosis }\end{array}$ \\
\hline V2 & BACK & SPINAL PAIN & N1409 & latrogenic lu spinal stenosis & $\begin{array}{l}\text { latrogenic lumbar spinal } \\
\text { stenosis }\end{array}$ \\
\hline V2 & BACK & SPINAL PAIN & $\mathrm{N} 140 \mathrm{~A}$ & Lu spin stenos due to oth dis & $\begin{array}{l}\text { Lumbar spinal stenosis } \\
\text { secondary to other } \\
\text { disease }\end{array}$ \\
\hline V2 & BACK & SPINAL PAIN & $\mathrm{N} 140 \mathrm{z}$ & Spinal stenosis NOS & \\
\hline $\mathrm{V} 2$ & BACK & SPINAL PAIN & $\mathrm{N} 14 \mathrm{z}$. & Back disorders NOS & \\
\hline $\mathrm{V} 2$ & BACK & SPINAL PAIN & Nyu5B & [X]Spin osteochondrosis, unsp & $\begin{array}{l}\mathrm{X}] \text { Spinal } \\
\text { osteochondrosis, } \\
\text { unspecified } \\
\end{array}$ \\
\hline V2 & BACK & SPINAL PAIN & P111. & Spinal hydromeningocele & \\
\hline V2 & BACK & SPINAL PAIN & P113. & Spinal meningocele & \\
\hline V2 & BACK & SPINAL PAIN & $\mathrm{P} 1130$ & Spinal meningocele-unspec.site & $\begin{array}{l}\text { Spinal meningocele of } \\
\text { unspecified site }\end{array}$ \\
\hline V2 & BACK & SPINAL PAIN & $\mathrm{P} 1132$ & Spinal meningocele-thoracic & $\begin{array}{l}\text { Thoracic spinal } \\
\text { meningocele }\end{array}$ \\
\hline V2 & BACK & SPINAL PAIN & P1133 & Spinal meningocele-lumbar & $\begin{array}{l}\text { Lumbar spinal } \\
\text { meningocele }\end{array}$ \\
\hline $\mathrm{V} 2$ & BACK & SPINAL PAIN & P113z & Spinal meningocele NOS & \\
\hline V2 & BACK & SPINAL PAIN & P25.. & Other spec.spinal cord anomaly & $\begin{array}{l}\text { Other specified spinal } \\
\text { cord anomalies }\end{array}$ \\
\hline V2 & BACK & SPINAL PAIN & P252. & Congen tethering spinal cord & $\begin{array}{l}\text { Congenital tethering of } \\
\text { spinal cord }\end{array}$ \\
\hline V2 & BACK & SPINAL PAIN & $\mathrm{P} 25 \mathrm{y}$. & Spinal cord anomalies OS & $\begin{array}{l}\text { Other specified } \\
\text { anomalies of spinal cord }\end{array}$ \\
\hline
\end{tabular}




\begin{tabular}{|c|c|c|c|c|c|}
\hline V2 & BACK & SPINAL PAIN & P25y1 & Atelomyelia & \\
\hline V2 & BACK & SPINAL PAIN & P25y2 & Spinal meninges congen.anomaly & $\begin{array}{l}\text { Congenital anomaly of } \\
\text { spinal meninges }\end{array}$ \\
\hline V2 & BACK & SPINAL PAIN & $\mathrm{P} 25 \mathrm{y} 4$ & Spinal cord hypoplasia & \\
\hline V2 & BACK & SPINAL PAIN & P25z. & Spinal cord anomalies NOS & \\
\hline V2 & BACK & SPINAL PAIN & P2y1. & Congenital spinal cord anomaly & \\
\hline V2 & BACK & SPINAL PAIN & Q204. & Birth spine/spinal cord injury & $\begin{array}{l}\text { Spine or spinal cord } \\
\text { injury due to birth } \\
\text { trauma }\end{array}$ \\
\hline V2 & BACK & SPINAL PAIN & Q204z & Birth spine/cord injury NOS & $\begin{array}{l}\text { Spine or spinal cord } \\
\text { injury due to birth } \\
\text { trauma NOS }\end{array}$ \\
\hline V2 & BACK & SPINAL PAIN & S10.. & \#Spine - no cord lesion & $\begin{array}{l}\text { Fracture of spine } \\
\text { without mention of } \\
\text { spinal cord injury }\end{array}$ \\
\hline $\mathrm{V} 2$ & BACK & SPINAL PAIN & S1001 & Closed fracture atlas & \\
\hline V2 & BACK & SPINAL PAIN & $\mathrm{S} 1002$ & Closed fracture axis & \\
\hline V2 & BACK & SPINAL PAIN & S1003 & Clsd \# third cerv vertebra & $\begin{array}{l}\text { Closed fracture of third } \\
\text { cervical vertebra }\end{array}$ \\
\hline V2 & BACK & SPINAL PAIN & S1004 & Clsd \# fourth cerv vertebra & $\begin{array}{l}\text { Closed fracture of fourth } \\
\text { cervical vertebra }\end{array}$ \\
\hline V2 & BACK & SPINAL PAIN & S1005 & Clsd \# fifth cerv vertebra & $\begin{array}{l}\text { Closed fracture of fifth } \\
\text { cervical vertebra }\end{array}$ \\
\hline V2 & BACK & SPINAL PAIN & S1006 & Clsd \# sixth cerv vertebra & $\begin{array}{l}\text { Closed fracture of sixth } \\
\text { cervical vertebra }\end{array}$ \\
\hline $\mathrm{V} 2$ & BACK & SPINAL PAIN & S1007 & Clsd \# seventh cerv vertebra & $\begin{array}{l}\text { Closed fracture of } \\
\text { seventh cervical } \\
\text { vertebra }\end{array}$ \\
\hline V2 & BACK & SPINAL PAIN & S1011 & Open fracture atlas & \\
\hline V2 & BACK & SPINAL PAIN & S1012 & Open fracture axis & \\
\hline V2 & BACK & SPINAL PAIN & S1013 & Open \# third cerv vertebra & $\begin{array}{l}\text { Open fracture of third } \\
\text { cervical vertebra }\end{array}$ \\
\hline V2 & BACK & SPINAL PAIN & S1015 & Open \# fifth cerv vertebra & $\begin{array}{l}\text { Open fracture of fifth } \\
\text { cervical vertebra }\end{array}$ \\
\hline V2 & BACK & SPINAL PAIN & S1016 & Open \# sixth cerv vertebra & $\begin{array}{l}\text { Open fracture of sixth } \\
\text { cervical vertebra }\end{array}$ \\
\hline V2 & BACK & SPINAL PAIN & S1017 & Open \# seventh cerv vert & $\begin{array}{l}\text { Open fracture of seventh } \\
\text { cervical vertebra }\end{array}$ \\
\hline V2 & BACK & SPINAL PAIN & S10z. & \#Spine - no cord lesion - NOS & $\begin{array}{l}\text { Fracture of spine } \\
\text { without mention of } \\
\text { spinal cord lesion NOS }\end{array}$ \\
\hline V2 & BACK & SPINAL PAIN & S11.. & \#Spine + cord lesion & $\begin{array}{l}\text { Fracture of spine with } \\
\text { spinal cord lesion }\end{array}$ \\
\hline V2 & BACK & SPINAL PAIN & $\$ 1100$ & Clsd \# C1-C4 unspec cord les & $\begin{array}{l}\text { Cls spinal fracture with } \\
\text { unspec cervical cord } \\
\text { lesion, C1-4 }\end{array}$ \\
\hline V2 & BACK & SPINAL PAIN & S1105 & Clsd \# C1-C4 incomp cord les & $\begin{array}{l}\text { Cls spinal \# with } \\
\text { incomplete cervical cord } \\
\text { lesion, C1-4 NOS }\end{array}$ \\
\hline V2 & BACK & SPINAL PAIN & S1106 & Clsd \# C5-C7 unspec cord les & $\begin{array}{l}\text { Cls spinal fracture with } \\
\text { unspec cervical cord } \\
\text { lesion, C5-7 }\end{array}$ \\
\hline V2 & BACK & SPINAL PAIN & S1107 & Clsd \# C5-C7 complete cord les & $\begin{array}{l}\text { Cls spinal fracture with } \\
\text { complete cervcl cord } \\
\text { lesion, C5-7 }\end{array}$ \\
\hline V2 & BACK & SPINAL PAIN & S1108 & Clsd \# C5-C7 ant cord lesion & $\begin{array}{l}\text { Cls spinal fracture with } \\
\text { anterior cervcl cord } \\
\text { lesion, C5-7 }\end{array}$ \\
\hline V2 & BACK & SPINAL PAIN & S110B & Clsd \# C5-C7 incomp cord les & $\begin{array}{l}\text { Cls spinal \# with } \\
\text { incomplete cervical cord } \\
\text { lesion, C5-7 NOS }\end{array}$ \\
\hline V2 & BACK & SPINAL PAIN & S111. & Open cervical \#+cord lesion & $\begin{array}{l}\text { Open fracture of cervical } \\
\text { spine with spinal cord } \\
\text { lesion }\end{array}$ \\
\hline V2 & BACK & SPINAL PAIN & S112. & Closed thoracic \#+cord lesion & $\begin{array}{l}\text { Closed fracture of } \\
\text { thoracic spine with } \\
\text { spinal cord lesion }\end{array}$ \\
\hline V2 & BACK & SPINAL PAIN & S1121 & $\mathrm{Cl} \mathrm{sp} \mathrm{\# +cmpl} \mathrm{thor} \mathrm{crd} \mathrm{Isn,T1-6}$ & $\begin{array}{l}\text { Cls spinal fracture wth } \\
\text { complete thoracic cord } \\
\text { lesion,T1-6 }\end{array}$ \\
\hline V2 & BACK & SPINAL PAIN & S1126 & $\mathrm{Cl}$ sp \#+unsp thor cd Isn,T7-12 & $\begin{array}{l}\text { Cls spinal fracture with } \\
\text { unspec thoracic cord } \\
\text { lesion, T7-12 }\end{array}$ \\
\hline V2 & BACK & SPINAL PAIN & S1127 & $\mathrm{Cl} \mathrm{sp} \mathrm{\# +cmp} \mathrm{thor} \mathrm{crd} \mathrm{Isn,T7-12}$ & $\begin{array}{l}\text { Cls spinal fracture with } \\
\text { complete thorac cord } \\
\text { lesion, T7-12 }\end{array}$ \\
\hline
\end{tabular}




\begin{tabular}{|c|c|c|c|c|c|}
\hline V2 & BACK & SPINAL PAIN & S112A & $\mathrm{Cl}$ sp \#+pst thor crd Isn,T7-12 & $\begin{array}{l}\text { Cls spinal fracture with } \\
\text { posterior thorac cord } \\
\text { lesion, } \mathrm{T7}-12\end{array}$ \\
\hline V2 & BACK & SPINAL PAIN & S112B & Cl \# T7-12incomp cord Isn NOS & $\begin{array}{l}\text { Cls spinal \# with } \\
\text { incomplete thoracid } \\
\text { cord lesion, T7-12 NOS }\end{array}$ \\
\hline V2 & BACK & SPINAL PAIN & S113. & Open thoracic \#+cord lesion & $\begin{array}{l}\text { Open fracture of } \\
\text { thoracic spine with } \\
\text { spinal cord lesion }\end{array}$ \\
\hline V2 & BACK & SPINAL PAIN & $\mathrm{S} 1140$ & Cls spn \#+ unsp lumb crd lesn & $\begin{array}{l}\text { Closed spinal fracture } \\
\text { with unspecified lumbar } \\
\text { cord lesion }\end{array}$ \\
\hline V2 & BACK & SPINAL PAIN & S1141 & Cls spn \# + comp lumb crd lesn & $\begin{array}{l}\text { Closed spinal fracture } \\
\text { with complete lumbar } \\
\text { cord lesion }\end{array}$ \\
\hline V2 & BACK & SPINAL PAIN & S1145 & Cls spn \# + cauda equina lesn & $\begin{array}{l}\text { Closed spinal fracture } \\
\text { with cauda equina lesion }\end{array}$ \\
\hline V2 & BACK & SPINAL PAIN & S115. & Open lumbar \# + cord lesion & $\begin{array}{l}\text { Open fracture of lumbar } \\
\text { spine with spinal cord } \\
\text { lesion }\end{array}$ \\
\hline V2 & BACK & SPINAL PAIN & S117. & Open sacral \# + cord lesion & $\begin{array}{l}\text { Open fracture of sacrum } \\
\text { with spinal cord lesion }\end{array}$ \\
\hline V2 & BACK & SPINAL PAIN & S1173 & Open sacral\#+other cord injury & $\begin{array}{l}\text { Open fracture of sacrum } \\
\text { with other spinal cord } \\
\text { injury }\end{array}$ \\
\hline $\mathrm{V} 2$ & BACK & SPINAL PAIN & S11z. & \#Spine + cord lesion NOS & $\begin{array}{l}\text { Fracture of spine with } \\
\text { spinal cord lesion NOS }\end{array}$ \\
\hline V2 & BACK & SPINAL PAIN & 54923 & Cls spnl dsl+comp thrc crd Isn & $\begin{array}{l}\text { Closed spinal dislocation } \\
\text { with complete thoracic } \\
\text { cord lesion }\end{array}$ \\
\hline V2 & BACK & SPINAL PAIN & SJ... & Nerve/spinal cord injuries & $\begin{array}{l}\text { Nerve and spinal cord } \\
\text { injuries }\end{array}$ \\
\hline V2 & BACK & SPINAL PAIN & $\mathrm{SJ} 2 .$. & Spinal cord inj.-no bone inj. & $\begin{array}{l}\text { Spinal cord injury } \\
\text { without evidence of } \\
\text { spinal bone injury }\end{array}$ \\
\hline V2 & BACK & SPINAL PAIN & SJ21. & Thoracic cord inj.-no bone inj & $\begin{array}{l}\text { Thoracic cord injury } \\
\text { without spinal bone } \\
\text { injury }\end{array}$ \\
\hline V2 & BACK & SPINAL PAIN & $\mathrm{SJ} 21 \mathrm{z}$ & Thoracic cord inj.-no \#, NOS & $\begin{array}{l}\text { Thoracic cord injury } \\
\text { without spinal bone } \\
\text { injury, NOS }\end{array}$ \\
\hline V2 & BACK & SPINAL PAIN & SJ22. & Cord inj.-lumbar-no bone inj. & $\begin{array}{l}\text { Lumbar cord injury } \\
\text { without spinal bone } \\
\text { injury }\end{array}$ \\
\hline V2 & BACK & SPINAL PAIN & SJ2z. & Spinal cord inj.-no \# NOS & $\begin{array}{l}\text { Spinal cord injury } \\
\text { without spinal bone } \\
\text { injury NOS }\end{array}$ \\
\hline V2 & BACK & SPINAL PAIN & SJ3.. & Nerve roots/spinal plexus inj. & $\begin{array}{l}\text { Nerve roots and spinal } \\
\text { plexus injuries }\end{array}$ \\
\hline V2 & BACK & SPINAL PAIN & SJ3z. & Nerv.root/spinal plex.inj.NOS & $\begin{array}{l}\text { Nerve root and spinal } \\
\text { plexus injuries NOS }\end{array}$ \\
\hline V2 & BACK & SPINAL PAIN & SJ8.. & Injur/nerv\&spinl cord/neck lev & $\begin{array}{l}\text { Injury of nerves and } \\
\text { spinal cord at neck level }\end{array}$ \\
\hline V2 & BACK & SPINAL PAIN & SJ80. & Concuss\&oed/cervicl spinl cord & $\begin{array}{l}\text { Concussion and oedema } \\
\text { of cervical spinal cord }\end{array}$ \\
\hline V2 & BACK & SPINAL PAIN & SJ9.. & Injur/nerv+spinl crd/thorx lev & $\begin{array}{l}\text { Injury of nerves and } \\
\text { spinal cord at thorax } \\
\text { level }\end{array}$ \\
\hline V2 & BACK & SPINAL PAIN & SJg0. & Concuss+oed/thoracic spinl crd & $\begin{array}{l}\text { Concussion and oedema } \\
\text { of thoracic spinal cord }\end{array}$ \\
\hline V2 & BACK & SPINAL PAIN & SJAO. & Concuss+oed/lumbar spinal cord & $\begin{array}{l}\text { Concussion and oedema } \\
\text { of lumbar spinal cord }\end{array}$ \\
\hline V2 & BACK & SPINAL PAIN & SJz.. & Nerve/spinal cord injury NOS & $\begin{array}{l}\text { Nerve and spinal cord } \\
\text { injury NOS }\end{array}$ \\
\hline V2 & BACK & SPINAL PAIN & SP063 & Infected spinal fixatn device & $\begin{array}{l}\text { Infected spinal fixation } \\
\text { device }\end{array}$ \\
\hline V2 & BACK & SPINAL PAIN & Syu19 & [X]Oth unsp inj cerv spin cord & $\begin{array}{l}{[\mathrm{X}] \text { Other and unspecified }} \\
\text { injuries of cervical spinal } \\
\text { cord }\end{array}$ \\
\hline V2 & BACK & SPINAL PAIN & Syu2B & [X]Oth unsp inj thor spin cord & $\begin{array}{l}{[X] \text { Other and unspecified }} \\
\text { injuries of thoracic spinal } \\
\text { cord }\end{array}$ \\
\hline V2 & BACK & SPINAL PAIN & Syu38 & [X]Oth inj of lumb spin cord & $\begin{array}{l}\mathrm{X}] \text { Other injury of } \\
\text { lumbar spinal cord }\end{array}$ \\
\hline V2 & BACK & SPINAL PAIN & Zw043 & [Q] Central spinal stenosis & \\
\hline V2 & BACK & SPINAL PAIN & Zw044 & [Q] Lateral spinal stenosis & \\
\hline V2 & BACK & SPINAL PAIN & Zw045 & [Q] Central + lat spin stenos & $\begin{array}{l}\text { [Q] Central and lateral } \\
\text { spinal stenosis }\end{array}$ \\
\hline $\mathrm{V} 2$ & BACK & BACK PAIN & $16 C 4$. & Back pain worse on sneezing & \\
\hline V2 & BACK & BACK PAIN & $16 \mathrm{C} 5$. & $\mathrm{C} / \mathrm{O}$ - low back pain & \\
\hline
\end{tabular}




\begin{tabular}{|c|c|c|c|c|c|}
\hline V2 & BACK & BACK PAIN & $16 \mathrm{C6}$. & Back pain without radiat NOS & $\begin{array}{l}\text { Back pain without } \\
\text { radiation NOS }\end{array}$ \\
\hline V2 & BACK & BACK PAIN & $16 \mathrm{C} 9$. & Chronic low back pain & \\
\hline $\mathrm{V} 2$ & BACK & BACK PAIN & $16 \mathrm{CA}$. & Mechanical low back pain & \\
\hline $\mathrm{V} 2$ & BACK & BACK PAIN & $\mathrm{N} 12 .$. & Intervertebral disc disorders & \\
\hline V2 & BACK & BACK PAIN & N141. & Pain in thoracic spine & \\
\hline V2 & BACK & BACK PAIN & N142. & Pain in lumbar spine & \\
\hline $\mathrm{V} 2$ & BACK & BACK PAIN & N143. & Sciatica & \\
\hline V2 & BACK & BACK PAIN & N145. & Backache, unspecified & \\
\hline V2 & BACK & ANKYLOSING SPONDYLITIS & A9853 & Gonococcal spondylitis & \\
\hline V2 & BACK & ANKYLOSING SPONDYLITIS & N0450 & Juv ankylosing spondylitis & $\begin{array}{l}\text { Juvenile ankylosing } \\
\text { spondylitis }\end{array}$ \\
\hline V2 & BACK & ANKYLOSING SPONDYLITIS & N100. & Ankylosing spondylitis & \\
\hline V2 & BACK & ANKYLOSING SPONDYLITIS & N10z. & Spondylitis NOS & \\
\hline V2 & BACK & ANKYLOSING SPONDYLITIS & N11yo & Brucella spondylitis & \\
\hline V2 & BACK & ANKYLOSING SPONDYLITIS & N11y1 & Enterobacterial spondylitis & \\
\hline V2 & $\mathrm{OA}$ & OSTEOARTHRITIS & $14 \mathrm{G} 2$. & $\mathrm{H} / \mathrm{O}$ : osteoarthritis & \\
\hline V2 & $\mathrm{OA}$ & OSTEOARTHRITIS & N05.. & Osteoarthritis+allied disord. & $\begin{array}{l}\text { Osteoarthritis and allied } \\
\text { disorders }\end{array}$ \\
\hline V2 & $\mathrm{OA}$ & OSTEOARTHRITIS & N050. & Generalised osteoarthritis-OA & $\begin{array}{l}\text { Generalised } \\
\text { osteoarthritis - OA }\end{array}$ \\
\hline V2 & $\mathrm{OA}$ & OSTEOARTHRITIS & N0500 & Generalised OA-site unspecif. & $\begin{array}{l}\text { Generalised } \\
\text { osteoarthritis of } \\
\text { unspecified site } \\
\end{array}$ \\
\hline V2 & $\mathrm{OA}$ & OSTEOARTHRITIS & N0501 & Generalised OA-hand & $\begin{array}{l}\text { Generalised } \\
\text { osteoarthritis of the } \\
\text { hand }\end{array}$ \\
\hline V2 & $\mathrm{OA}$ & OSTEOARTHRITIS & N0502 & Generalised OA-multiple sites & $\begin{array}{l}\text { Generalised } \\
\text { osteoarthritis of multiple } \\
\text { sites }\end{array}$ \\
\hline $\mathrm{V} 2$ & OA & OSTEOARTHRITIS & $\mathrm{N} 050 \mathrm{z}$ & Generalised osteoarthritis NOS & \\
\hline V2 & $\mathrm{OA}$ & OSTEOARTHRITIS & N051. & Local.primary osteoarthritis & $\begin{array}{l}\text { Localised, primary } \\
\text { osteoarthritis }\end{array}$ \\
\hline V2 & $\mathrm{OA}$ & OSTEOARTHRITIS & N0510 & Local.primary OA-site unspec. & $\begin{array}{l}\text { Localised, primary } \\
\text { osteoarthritis of } \\
\text { unspecified site } \\
\end{array}$ \\
\hline V2 & $\mathrm{OA}$ & OSTEOARTHRITIS & N0511 & Local.primary OA-shoulder regn & $\begin{array}{l}\text { Localised, primary } \\
\text { osteoarthritis of the } \\
\text { shoulder region }\end{array}$ \\
\hline V2 & $\mathrm{OA}$ & OSTEOARTHRITIS & N0512 & Local.primary OA-upper arm & $\begin{array}{l}\text { Localised, primary } \\
\text { osteoarthritis of the } \\
\text { upper arm }\end{array}$ \\
\hline V2 & $\mathrm{OA}$ & OSTEOARTHRITIS & N0513 & Local.primary OA-forearm & $\begin{array}{l}\text { Localised, primary } \\
\text { osteoarthritis of the } \\
\text { forearm }\end{array}$ \\
\hline V2 & $\mathrm{OA}$ & OSTEOARTHRITIS & N0514 & Local.primary OA-hand & $\begin{array}{l}\text { Localised, primary } \\
\text { osteoarthritis of the } \\
\text { hand }\end{array}$ \\
\hline V2 & $\mathrm{OA}$ & OSTEOARTHRITIS & N0515 & Local.primary OA-pelvic/thigh & $\begin{array}{l}\text { Localised, primary } \\
\text { osteoarthritis of the } \\
\text { pelvic region/thigh }\end{array}$ \\
\hline V2 & $\mathrm{OA}$ & OSTEOARTHRITIS & N0516 & Local.primary OA-lower leg & $\begin{array}{l}\text { Localised, primary } \\
\text { osteoarthritis of the } \\
\text { lower leg }\end{array}$ \\
\hline V2 & $\mathrm{OA}$ & OSTEOARTHRITIS & N0517 & Local.primary OA-ankle/foot & $\begin{array}{l}\text { Localised, primary } \\
\text { osteoarthritis of the } \\
\text { ankle and foot }\end{array}$ \\
\hline V2 & $\mathrm{OA}$ & OSTEOARTHRITIS & N0518 & Local.primary OA-other specif & $\begin{array}{l}\text { Localised, primary } \\
\text { osteoarthritis of other } \\
\text { specified site }\end{array}$ \\
\hline V2 & $\mathrm{OA}$ & OSTEOARTHRITIS & N051D & Local prim osteoarth wrist & $\begin{array}{l}\text { Localised, primary } \\
\text { osteoarthritis of the } \\
\text { wrist }\end{array}$ \\
\hline V2 & $\mathrm{OA}$ & OSTEOARTHRITIS & N051E & Local prim osteoarth toe & $\begin{array}{l}\text { Localised, primary } \\
\text { osteoarthritis of toe }\end{array}$ \\
\hline V2 & $\mathrm{OA}$ & OSTEOARTHRITIS & N051F & Local prim osteoarth elbow & $\begin{array}{l}\text { Localised, primary } \\
\text { osteoarthritis of elbow }\end{array}$ \\
\hline V2 & $\mathrm{OA}$ & OSTEOARTHRITIS & N051z & Localised primary OA NOS & $\begin{array}{l}\text { Localised, primary } \\
\text { osteoarthritis NOS }\end{array}$ \\
\hline V2 & $\mathrm{OA}$ & OSTEOARTHRITIS & N052. & Local.secondary osteoarthritis & $\begin{array}{l}\text { Localised, secondary } \\
\text { osteoarthritis }\end{array}$ \\
\hline
\end{tabular}




\begin{tabular}{|c|c|c|c|c|c|}
\hline V2 & $\mathrm{OA}$ & OSTEOARTHRITIS & N0520 & Local.secondary OA-site unsp. & $\begin{array}{l}\text { Localised, secondary } \\
\text { osteoarthritis of } \\
\text { unspecified site }\end{array}$ \\
\hline V2 & OA & OSTEOARTHRITIS & N0521 & Local.secondary OA-shoulder & $\begin{array}{l}\text { Localised, secondary } \\
\text { osteoarthritis of the } \\
\text { shoulder region }\end{array}$ \\
\hline V2 & OA & OSTEOARTHRITIS & N0522 & Local.secondary OA-upper arm & $\begin{array}{l}\text { Localised, secondary } \\
\text { osteoarthritis of the } \\
\text { upper arm }\end{array}$ \\
\hline V2 & OA & OSTEOARTHRITIS & N0523 & Local.secondary OA-forearm & $\begin{array}{l}\text { Localised, secondary } \\
\text { osteoarthritis of the } \\
\text { forearm }\end{array}$ \\
\hline V2 & $\mathrm{OA}$ & OSTEOARTHRITIS & N0524 & Local.secondary OA-hand & $\begin{array}{l}\text { Localised, secondary } \\
\text { osteoarthritis of the } \\
\text { hand }\end{array}$ \\
\hline V2 & OA & OSTEOARTHRITIS & N0525 & Local.secondary OA-pelv./thigh & $\begin{array}{l}\text { Localised, secondary } \\
\text { osteoarthritis of pelvic } \\
\text { region/thigh }\end{array}$ \\
\hline V2 & $\mathrm{OA}$ & OSTEOARTHRITIS & N0526 & Local.secondary OA-lower leg & $\begin{array}{l}\text { Localised, secondary } \\
\text { osteoarthritis of the } \\
\text { lower leg }\end{array}$ \\
\hline V2 & OA & OSTEOARTHRITIS & N0527 & Local.secondary OA-ankle/foot & $\begin{array}{l}\text { Localised, secondary } \\
\text { osteoarthritis of the } \\
\text { ankle and foot }\end{array}$ \\
\hline V2 & $\mathrm{OA}$ & OSTEOARTHRITIS & N0528 & Local.secondary OA-other spec. & $\begin{array}{l}\text { Localised, secondary } \\
\text { osteoarthritis of other } \\
\text { specified site }\end{array}$ \\
\hline V2 & $\mathrm{OA}$ & OSTEOARTHRITIS & N052z & Localised secondary OA NOS & $\begin{array}{l}\text { Localised, secondary } \\
\text { osteoarthritis NOS }\end{array}$ \\
\hline V2 & $\mathrm{OA}$ & OSTEOARTHRITIS & N053. & Localised OA unspecified & $\begin{array}{l}\text { Localised osteoarthritis, } \\
\text { unspecified }\end{array}$ \\
\hline V2 & $\mathrm{OA}$ & OSTEOARTHRITIS & N0530 & Local.OA unsp.-site unspecif. & $\begin{array}{l}\text { Localised osteoarthritis, } \\
\text { unspecified, of } \\
\text { unspecified site }\end{array}$ \\
\hline V2 & $\mathrm{OA}$ & OSTEOARTHRITIS & N0531 & Local.OA unsp.-shoulder region & $\begin{array}{l}\text { Localised osteoarthritis, } \\
\text { unspecified, of shoulder } \\
\text { region }\end{array}$ \\
\hline V2 & OA & OSTEOARTHRITIS & N0532 & Local.OA unsp.-upper arm & $\begin{array}{l}\text { Localised osteoarthritis, } \\
\text { unspecified, of the upper } \\
\text { arm }\end{array}$ \\
\hline V2 & OA & OSTEOARTHRITIS & N0533 & Local.OA unsp.-forearm & $\begin{array}{l}\text { Localised osteoarthritis, } \\
\text { unspecified, of the } \\
\text { forearm }\end{array}$ \\
\hline V2 & $\mathrm{OA}$ & OSTEOARTHRITIS & N0534 & Local.OA unsp.-hand & $\begin{array}{l}\text { Localised osteoarthritis, } \\
\text { unspecified, of the hand }\end{array}$ \\
\hline V2 & $\mathrm{OA}$ & OSTEOARTHRITIS & N0535 & Local.OA unsp.-pelvic/thigh & $\begin{array}{l}\text { Localised osteoarthritis, } \\
\text { unspecified, pelvic } \\
\text { region/thigh }\end{array}$ \\
\hline V2 & OA & OSTEOARTHRITIS & N0536 & Local.OA unsp.-lower leg & $\begin{array}{l}\text { Localised osteoarthritis, } \\
\text { unspecified, of the lower } \\
\text { leg }\end{array}$ \\
\hline V2 & $\mathrm{OA}$ & OSTEOARTHRITIS & N0537 & Local.OA unsp.-ankle/foot & $\begin{array}{l}\text { Localised osteoarthritis, } \\
\text { unspecified, of the ankle } \\
\text { and foot }\end{array}$ \\
\hline V2 & $\mathrm{OA}$ & OSTEOARTHRITIS & N0538 & Local.OA unsp.-other specified & $\begin{array}{l}\text { Localised osteoarthritis, } \\
\text { unspecified, of other } \\
\text { spec site }\end{array}$ \\
\hline V2 & $\mathrm{OA}$ & OSTEOARTHRITIS & N053z & Localised OA unspecified NOS & $\begin{array}{l}\text { Localised osteoarthritis, } \\
\text { unspecified, NOS }\end{array}$ \\
\hline V2 & OA & OSTEOARTHRITIS & N054. & Oligoarticular $\mathrm{OA}$, unspecified & $\begin{array}{l}\text { Oligoarticular } \\
\text { osteoarthritis, } \\
\text { unspecified }\end{array}$ \\
\hline V2 & $\mathrm{OA}$ & OSTEOARTHRITIS & N0540 & Oligoartic OA, unsp-unsp sites & $\begin{array}{l}\text { Oligoarticular } \\
\text { osteoarthritis, unspec, of } \\
\text { unspecified sites }\end{array}$ \\
\hline V2 & $\mathrm{OA}$ & OSTEOARTHRITIS & N0541 & Oligoartic OA, unspec-shoulder & $\begin{array}{l}\text { Oligoarticular } \\
\text { osteoarthritis, } \\
\text { unspecified, of shoulder }\end{array}$ \\
\hline V2 & $\mathrm{OA}$ & OSTEOARTHRITIS & N0542 & Oligoartic OA, unspec-upp arm & $\begin{array}{l}\text { Oligoarticular } \\
\text { osteoarthritis, } \\
\text { unspecified, of upper } \\
\text { arm }\end{array}$ \\
\hline V2 & $\mathrm{OA}$ & OSTEOARTHRITIS & N0544 & Oligoartic OA, unspec-hand & $\begin{array}{l}\text { Oligoarticular } \\
\text { osteoarthritis, } \\
\text { unspecified, of hand }\end{array}$ \\
\hline V2 & OA & OSTEOARTHRITIS & N0545 & Oligoartic OA, unspec-pelv/thi & $\begin{array}{l}\text { Oligoarticular } \\
\text { osteoarthritis, } \\
\text { unspecified, of } \\
\text { pelvis/thigh }\end{array}$ \\
\hline V2 & OA & OSTEOARTHRITIS & N0546 & Oligoartic $\mathrm{OA}$, unspec-leg & $\begin{array}{l}\text { Oligoarticular } \\
\text { osteoarthritis, } \\
\text { unspecified, of lower leg }\end{array}$ \\
\hline V2 & OA & OSTEOARTHRITIS & N0547 & Oligoartic OA, unspec-ank/foot & $\begin{array}{l}\text { Oligoarticular } \\
\text { osteoarthritis, }\end{array}$ \\
\hline
\end{tabular}




\begin{tabular}{|c|c|c|c|c|c|}
\hline & & & & & $\begin{array}{l}\text { unspecified, of } \\
\text { ankle/foot }\end{array}$ \\
\hline V2 & $\mathrm{OA}$ & OSTEOARTHRITIS & N0548 & Oligoartic $\mathrm{OA}$, unspec-oth site & $\begin{array}{l}\text { Oligoarticular } \\
\text { osteoarthritis, } \\
\text { unspecified, other spec } \\
\text { sites }\end{array}$ \\
\hline $\mathrm{V} 2$ & $\mathrm{OA}$ & OSTEOARTHRITIS & N0549 & Oligoartic OA, unspec-multiple & $\begin{array}{l}\text { Oligoarticular } \\
\text { osteoarthritis, } \\
\text { unspecified, multiple } \\
\text { sites }\end{array}$ \\
\hline $\mathrm{V} 2$ & $\mathrm{OA}$ & OSTEOARTHRITIS & N054z & $\mathrm{OA}, 1$ site + , unspecified NOS & $\begin{array}{l}\text { Osteoarthritis of more } \\
\text { than one site, } \\
\text { unspecified, NOS }\end{array}$ \\
\hline $\mathrm{V} 2$ & $\mathrm{OA}$ & OSTEOARTHRITIS & N05z. & Osteoarthritis NOS & \\
\hline V2 & $\mathrm{OA}$ & OSTEOARTHRITIS & $\mathrm{NO5zO}$ & Osteoarthritis NOS-site unspec & $\begin{array}{l}\text { Osteoarthritis NOS, of } \\
\text { unspecified site }\end{array}$ \\
\hline $\mathrm{V} 2$ & $\mathrm{OA}$ & OSTEOARTHRITIS & N05z1 & Osteoarthritis NOS-shoulder & $\begin{array}{l}\text { Osteoarthritis NOS, of } \\
\text { shoulder region }\end{array}$ \\
\hline $\mathrm{V} 2$ & $\mathrm{OA}$ & OSTEOARTHRITIS & N05z2 & Osteoarthritis NOS-upper arm & $\begin{array}{l}\text { Osteoarthritis NOS, of } \\
\text { the upper arm }\end{array}$ \\
\hline V2 & $\mathrm{OA}$ & OSTEOARTHRITIS & N05z3 & Osteoarthritis NOS-forearm & $\begin{array}{l}\text { Osteoarthritis NOS, of } \\
\text { the forearm }\end{array}$ \\
\hline V2 & $\mathrm{OA}$ & OSTEOARTHRITIS & N05z4 & Osteoarthritis NOS-hand & $\begin{array}{l}\text { Osteoarthritis NOS, of } \\
\text { the hand }\end{array}$ \\
\hline V2 & $\mathrm{OA}$ & OSTEOARTHRITIS & N05z5 & Osteoarthritis NOS-pelv./thigh & $\begin{array}{l}\text { Osteoarthritis NOS, } \\
\text { pelvic region/thigh }\end{array}$ \\
\hline $\mathrm{V} 2$ & $\mathrm{OA}$ & OSTEOARTHRITIS & N05z6 & Osteoarthritis NOS-lower leg & $\begin{array}{l}\text { Osteoarthritis NOS, of } \\
\text { the lower leg }\end{array}$ \\
\hline $\mathrm{V} 2$ & $\mathrm{OA}$ & OSTEOARTHRITIS & N05z7 & Osteoarthritis NOS-ankle/foot & $\begin{array}{l}\text { Osteoarthritis NOS, of } \\
\text { ankle and foot }\end{array}$ \\
\hline $\mathrm{V} 2$ & $\mathrm{OA}$ & OSTEOARTHRITIS & N05z8 & Osteoarthritis NOS-other spec & $\begin{array}{l}\text { Osteoarthritis NOS, } \\
\text { other specified site }\end{array}$ \\
\hline V2 & $\mathrm{OA}$ & OSTEOARTHRITIS & N05z9 & Osteoarthritis NOS, shoulder & $\begin{array}{l}\text { Osteoarthritis NOS, of } \\
\text { shoulder }\end{array}$ \\
\hline V2 & $O A$ & OSTEOARTHRITIS & N05zA & OA NOS-sternoclavicular joint & $\begin{array}{l}\text { Osteoarthritis NOS, of } \\
\text { sternoclavicular joint }\end{array}$ \\
\hline V2 & $O A$ & OSTEOARTHRITIS & N05zB & OA NOS-acromioclavicular joint & $\begin{array}{l}\text { Osteoarthritis NOS, of } \\
\text { acromioclavicular joint }\end{array}$ \\
\hline V2 & $\mathrm{OA}$ & OSTEOARTHRITIS & $\mathrm{NO5zC}$ & OA NOS-elbow & $\begin{array}{l}\text { Osteoarthritis NOS, of } \\
\text { elbow }\end{array}$ \\
\hline V2 & $\mathrm{OA}$ & OSTEOARTHRITIS & N05zD & OA NOS-dist radio-ulnar joint & $\begin{array}{l}\text { Osteoarthritis NOS, of } \\
\text { distal radio-ulnar joint }\end{array}$ \\
\hline $\mathrm{V} 2$ & $\mathrm{OA}$ & OSTEOARTHRITIS & N05zE & OA NOS-wrist & $\begin{array}{l}\text { Osteoarthritis NOS, of } \\
\text { wrist }\end{array}$ \\
\hline $\mathrm{V} 2$ & $\mathrm{OA}$ & OSTEOARTHRITIS & $\mathrm{NO5zF}$ & OA NOS-MCP joint & $\begin{array}{l}\text { Osteoarthritis NOS, of } \\
\text { MCP joint }\end{array}$ \\
\hline $\mathrm{V} 2$ & $\mathrm{OA}$ & OSTEOARTHRITIS & N05zG & OA NOS-PIP joint of finger & $\begin{array}{l}\text { Osteoarthritis NOS, of } \\
\text { PIP joint of finger }\end{array}$ \\
\hline $\mathrm{V} 2$ & $\mathrm{OA}$ & OSTEOARTHRITIS & $\mathrm{N} 05 \mathrm{zH}$ & OA NOS-DIP joint of finger & $\begin{array}{l}\text { Osteoarthritis NOS, of } \\
\text { DIP joint of finger }\end{array}$ \\
\hline V2 & $\mathrm{OA}$ & OSTEOARTHRITIS & N05zJ & OA NOS-hip & $\begin{array}{l}\text { Osteoarthritis NOS, of } \\
\text { hip }\end{array}$ \\
\hline $\mathrm{V} 2$ & $\mathrm{OA}$ & OSTEOARTHRITIS & N05zK & OA NOS-SI joint & $\begin{array}{l}\text { Osteoarthritis NOS, of } \\
\text { sacro-iliac joint }\end{array}$ \\
\hline $\mathrm{V} 2$ & $\mathrm{OA}$ & OSTEOARTHRITIS & N05zL & Osteoarthritis NOS, of knee & \\
\hline $\mathrm{V} 2$ & $\mathrm{OA}$ & OSTEOARTHRITIS & N05zM & OA NOS tibio-fibular joint & $\begin{array}{l}\text { Osteoarthritis NOS, of } \\
\text { tibio-fibular joint }\end{array}$ \\
\hline $\mathrm{V} 2$ & $\mathrm{OA}$ & OSTEOARTHRITIS & $\mathrm{N} 05 \mathrm{zN}$ & OA NOS-ankle & $\begin{array}{l}\text { Osteoarthritis NOS, of } \\
\text { ankle }\end{array}$ \\
\hline V2 & $\mathrm{OA}$ & OSTEOARTHRITIS & $\mathrm{N} 05 \mathrm{zP}$ & OA NOS-subtalar joint & $\begin{array}{l}\text { Osteoarthritis NOS, of } \\
\text { subtalar joint }\end{array}$ \\
\hline V2 & $O A$ & OSTEOARTHRITIS & N05zQ & OA NOS-talonavicular joint & $\begin{array}{l}\text { Osteoarthritis NOS, of } \\
\text { talonavicular joint }\end{array}$ \\
\hline V2 & $\mathrm{OA}$ & OSTEOARTHRITIS & N05zR & OA NOS-other tarsal joint & $\begin{array}{l}\text { Osteoarthritis NOS, of } \\
\text { other tarsal joint }\end{array}$ \\
\hline V2 & OA & OSTEOARTHRITIS & N05zS & OA NOS-1st MTP joint & $\begin{array}{l}\text { Osteoarthritis NOS, of } \\
\text { 1st MTP joint }\end{array}$ \\
\hline $\mathrm{V} 2$ & $\mathrm{OA}$ & OSTEOARTHRITIS & N05zT & OA NOS-lesser MTP joint & $\begin{array}{l}\text { Osteoarthritis NOS, of } \\
\text { lesser MTP joint }\end{array}$ \\
\hline $\mathrm{V} 2$ & $\mathrm{OA}$ & OSTEOARTHRITIS & $\mathrm{NO5zU}$ & OA NOS-IP joint of toe & $\begin{array}{l}\text { Osteoarthritis NOS, of IP } \\
\text { joint of toe }\end{array}$ \\
\hline $\mathrm{V} 2$ & $\mathrm{OA}$ & OSTEOARTHRITIS & N05zz & Osteoarthritis NOS & \\
\hline $\mathrm{V} 2$ & $\mathrm{OA}$ & OSTEOARTHRITIS & N11.. & Spondylosis + allied disorders & $\begin{array}{l}\text { Spondylosis and allied } \\
\text { disorders }\end{array}$ \\
\hline $\mathrm{V} 2$ & $\mathrm{OA}$ & OSTEOARTHRITIS & N110. & Cervical spond.-no myelopathy & $\begin{array}{l}\text { Cervical spondylosis } \\
\text { without myelopathy }\end{array}$ \\
\hline $\mathrm{V} 2$ & $\mathrm{OA}$ & OSTEOARTHRITIS & N11D. & Osteoarthritis of spine & \\
\hline V2 & $\mathrm{OA}$ & OSTEOARTHRITIS & N11D0 & Osteoarthritis cervical spine & $\begin{array}{l}\text { Osteoarthritis of cervical } \\
\text { spine }\end{array}$ \\
\hline
\end{tabular}




\begin{tabular}{|c|c|c|c|c|c|}
\hline V2 & $\mathrm{OA}$ & OSTEOARTHRITIS & N11D1 & Osteoarthritis thoracic spine & $\begin{array}{l}\text { Osteoarthritis of thoracic } \\
\text { spine }\end{array}$ \\
\hline V2 & $\mathrm{OA}$ & OSTEOARTHRITIS & $\mathrm{N} 11 \mathrm{D} 2$ & Osteoarthritis of lumbar spine & \\
\hline V2 & $\mathrm{OA}$ & OSTEOARTHRITIS & N11D3 & Osteoarthritis of spine NOS & \\
\hline V2 & $\mathrm{OA}$ & OSTEOARTHRITIS & N11z. & Spondylosis NOS & \\
\hline V2 & RA & PSORIATIC ARTHROPATHY & M160. & Psoriatic arthropathy & \\
\hline V2 & RA & PSORIATIC ARTHROPATHY & M1601 & Dist interphal psoriat arthrop & $\begin{array}{l}\text { Distal interphalangeal } \\
\text { psoriatic arthropathy }\end{array}$ \\
\hline V2 & RA & PSORIATIC ARTHROPATHY & $\mathrm{M} 160 \mathrm{z}$ & Psoriatic arthropathy NOS & \\
\hline V2 & RA & PSORIATIC ARTHROPATHY & Nyu13 & [X]Oth psoriatic arthropathies & $\begin{array}{l}{[\mathrm{X}] \text { Other psoriatic }} \\
\text { arthropathies }\end{array}$ \\
\hline V2 & $\mathrm{RA}$ & RHEUMATOID ARTHRITIS & $14 \mathrm{G} 1$. & $\mathrm{H} / \mathrm{O}$ : rheumatoid arthritis & \\
\hline V2 & RA & RHEUMATOID ARTHRITIS & F3712 & Polyneuropathy+rheumatoid arth & $\begin{array}{l}\text { Polyneuropathy in } \\
\text { rheumatoid arthritis }\end{array}$ \\
\hline V2 & RA & RHEUMATOID ARTHRITIS & F3964 & Myopathy+rheumatoid arthritis & $\begin{array}{l}\text { Myopathy due to } \\
\text { rheumatoid arthritis }\end{array}$ \\
\hline $\mathrm{V} 2$ & RA & RHEUMATOID ARTHRITIS & N04.. & Rheumatoid arthritis+similar & $\begin{array}{l}\text { Rheumatoid arthritis and } \\
\text { other inflammatory } \\
\text { polyarthropathy }\end{array}$ \\
\hline V2 & RA & RHEUMATOID ARTHRITIS & N040. & Rheumatoid arthritis & \\
\hline V2 & RA & RHEUMATOID ARTHRITIS & N0400 & Rheumatoid arthritis-Cx spine & $\begin{array}{l}\text { Rheumatoid arthritis of } \\
\text { cervical spine }\end{array}$ \\
\hline $\mathrm{V} 2$ & RA & RHEUMATOID ARTHRITIS & N0401 & Oth rheumatoid arthritis-spine & $\begin{array}{l}\text { Other rheumatoid } \\
\text { arthritis of spine }\end{array}$ \\
\hline V2 & RA & RHEUMATOID ARTHRITIS & N0402 & Rheumatoid arthritis-shoulder & $\begin{array}{l}\text { Rheumatoid arthritis of } \\
\text { shoulder }\end{array}$ \\
\hline V2 & RA & RHEUMATOID ARTHRITIS & N0404 & Rheumatoid arthr-acromioclav $\mathrm{j}$ & $\begin{array}{l}\text { Rheumatoid arthritis of } \\
\text { acromioclavicular joint }\end{array}$ \\
\hline V2 & $\mathrm{RA}$ & RHEUMATOID ARTHRITIS & N0405 & Rheumatoid arthritis of elbow & \\
\hline V2 & RA & RHEUMATOID ARTHRITIS & N0406 & Rheumatoid arthritis-dist RUJ & $\begin{array}{l}\text { Rheumatoid arthritis of } \\
\text { distal radio-ulnar joint }\end{array}$ \\
\hline V2 & RA & RHEUMATOID ARTHRITIS & N0407 & Rheumatoid arthritis of wrist & \\
\hline V2 & RA & RHEUMATOID ARTHRITIS & N0408 & Rheumatoid arthritis-MCP joint & $\begin{array}{l}\text { Rheumatoid arthritis of } \\
\text { MCP joint }\end{array}$ \\
\hline V2 & RA & RHEUMATOID ARTHRITIS & N0409 & Rheumatoid arthritis-PIPJ-fing & $\begin{array}{l}\text { Rheumatoid arthritis of } \\
\text { PIP joint of finger }\end{array}$ \\
\hline V2 & RA & RHEUMATOID ARTHRITIS & N040A & Rheumatoid arthritis-DIPJ-fing & $\begin{array}{l}\text { Rheumatoid arthritis of } \\
\text { DIP joint of finger }\end{array}$ \\
\hline V2 & $\mathrm{RA}$ & RHEUMATOID ARTHRITIS & NO40B & Rheumatoid arthritis of hip & \\
\hline V2 & RA & RHEUMATOID ARTHRITIS & NO40C & Rheumatoid arthritis of SIJ & $\begin{array}{l}\text { Rheumatoid arthritis of } \\
\text { sacro-iliac joint }\end{array}$ \\
\hline V2 & RA & RHEUMATOID ARTHRITIS & NO40D & Rheumatoid arthritis of knee & \\
\hline V2 & RA & RHEUMATOID ARTHRITIS & NO40F & Rheumatoid arthritis of ankle & \\
\hline V2 & RA & RHEUMATOID ARTHRITIS & NO40G & Rheumatoid arthr-subtalar jnt & $\begin{array}{l}\text { Rheumatoid arthritis of } \\
\text { subtalar joint }\end{array}$ \\
\hline V2 & RA & RHEUMATOID ARTHRITIS & $\mathrm{NO} 40 \mathrm{H}$ & Rheumatoid arthr-talonav joint & $\begin{array}{l}\text { Rheumatoid arthritis of } \\
\text { talonavicular joint }\end{array}$ \\
\hline V2 & RA & RHEUMATOID ARTHRITIS & N040J & Rheumatoid arthr-oth tarsal jt & $\begin{array}{l}\text { Rheumatoid arthritis of } \\
\text { other tarsal joint }\end{array}$ \\
\hline V2 & RA & RHEUMATOID ARTHRITIS & NO40K & Rheumatoid arthr-1st MTP joint & $\begin{array}{l}\text { Rheumatoid arthritis of } \\
\text { 1st MTP joint }\end{array}$ \\
\hline V2 & RA & RHEUMATOID ARTHRITIS & NO40L & Rheumatoid arthr-lesser MTP jt & $\begin{array}{l}\text { Rheumatoid arthritis of } \\
\text { lesser MTP joint }\end{array}$ \\
\hline V2 & RA & RHEUMATOID ARTHRITIS & NO40P & Seronegative rheumat arthritis & $\begin{array}{l}\text { Seronegative } \\
\text { rheumatoid arthritis }\end{array}$ \\
\hline V2 & $\mathrm{RA}$ & RHEUMATOID ARTHRITIS & NO40S & Rheumat arthr - multiple joint & $\begin{array}{l}\text { Rheumatoid arthritis - } \\
\text { multiple joint }\end{array}$ \\
\hline V2 & $\mathrm{RA}$ & RHEUMATOID ARTHRITIS & NO40T & Flare of rheumatoid arthritis & \\
\hline V2 & RA & RHEUMATOID ARTHRITIS & N043. & Juvenile R.A.- Still's disease & $\begin{array}{l}\text { Juvenile rheumatoid } \\
\text { arthritis - Still's disease }\end{array}$ \\
\hline V2 & RA & RHEUMATOID ARTHRITIS & N0431 & Acute polyartic.juvenile R.A. & $\begin{array}{l}\text { Acute polyarticular } \\
\text { juvenile rheumatoid } \\
\text { arthritis }\end{array}$ \\
\hline V2 & RA & RHEUMATOID ARTHRITIS & N0432 & Pauciarticular juvenile R.A. & $\begin{array}{l}\text { Pauciarticular juvenile } \\
\text { rheumatoid arthritis }\end{array}$ \\
\hline V2 & RA & RHEUMATOID ARTHRITIS & N0433 & Monarticular juvenile R.A. & $\begin{array}{l}\text { Monarticular juvenile } \\
\text { rheumatoid arthritis }\end{array}$ \\
\hline V2 & RA & RHEUMATOID ARTHRITIS & N043z & Juvenile rheumatoid arthr.NOS & $\begin{array}{l}\text { Juvenile rheumatoid } \\
\text { arthritis NOS }\end{array}$ \\
\hline V2 & $\mathrm{RA}$ & RHEUMATOID ARTHRITIS & N0455 & Juvenile rheumatoid arthritis & \\
\hline
\end{tabular}




\begin{tabular}{|c|c|c|c|c|c|}
\hline V2 & $\mathrm{RA}$ & RHEUMATOID ARTHRITIS & N047. & Seropositive errosive RA & $\begin{array}{l}\text { Seropositive errosive } \\
\text { rheumatoid arthritis }\end{array}$ \\
\hline V2 & RA & RHEUMATOID ARTHRITIS & N04X. & Seroposit rheum arthr, unsp & $\begin{array}{l}\text { Seropositive rheumatoid } \\
\text { arthritis, unspecified }\end{array}$ \\
\hline $\mathrm{V} 2$ & RA & RHEUMATOID ARTHRITIS & N04y0 & Rheumatoid lung & \\
\hline $\mathrm{V} 2$ & RA & RHEUMATOID ARTHRITIS & Nyu12 & [X]Oth spcf rheumatd arthritis & $\begin{array}{l}\mathrm{X}] \text { Other specified } \\
\text { rheumatoid arthritis }\end{array}$ \\
\hline $\mathrm{V} 2$ & $\mathrm{RA}$ & RHEUMATOID ARTHRITIS & Nyu1G & {$[\mathrm{X}]$ Seroposit rheum arthr, unsp } & $\begin{array}{l}\mathrm{X}] \text { Seropositive } \\
\text { rheumatoid arthritis, } \\
\text { unspecified }\end{array}$ \\
\hline V3 & FIBRO & FIBROMYALGIA & $\mathrm{X} 75 \mathrm{rx}$ & Fibromyalgia & \\
\hline V3 & FIBRO & FIBROMYALGIA & N2401 & Fibrositis unspecified & \\
\hline V3 & FIBRO & FIBROMYALGIA & N2405 & Fibrositis of neck & \\
\hline V3 & FIBRO & FIBROMYALGIA & N2406 & Fibrositis arm & \\
\hline V3 & FIBRO & FIBROMYALGIA & $\mathrm{Xa01F}$ & Chronic fatigue syndrome & \\
\hline V3 & FIBRO & FIBROMYALGIA & XaPoo & Severe chronic fatigue syndrome & \\
\hline V3 & FIBRO & FIBROMYALGIA & XaPon & $\begin{array}{l}\text { Moderate chronic fatigue } \\
\text { syndrome }\end{array}$ & \\
\hline V3 & FIBRO & FIBROMYALGIA & XaPom & Mild chronic fatigue syndrome & \\
\hline V3 & FIBRO & FIBROMYALGIA & XaPom & Mild chronic fatigue syndrome & \\
\hline V2 & FIBRO & FIBROMYALGIA & N239. & Fibromyalgia & \\
\hline V2 & FIBRO & FIBROMYALGIA & N248. & Fibromyalgia & \\
\hline $\mathrm{V} 2$ & FIBRO & FIBROMYALGIA & N2480 & Myofascial pain syndrome & \\
\hline $\mathrm{V} 2$ & FIBRO & FIBROMYALGIA & N2481 & Piriformis syndrome & \\
\hline V2 & FIBRO & FIBROMYALGIA & $\mathrm{F} 286$. & Chronic fatigue syndrome & \\
\hline V2 & FIBRO & FIBROMYALGIA & $\mathrm{F} 2860$ & Mild chronic fatigue syndrome & \\
\hline V2 & FIBRO & FIBROMYALGIA & $\mathrm{F} 2861$ & $\begin{array}{l}\text { Moderate chronic fatigue } \\
\text { syndrome }\end{array}$ & \\
\hline V2 & FIBRO & FIBROMYALGIA & F2862 & Severe chronic fatigue syndrome & \\
\hline V2 & NEP & TRIGEMINAL NEURALGIA & 1475 & & \\
\hline V2 & NEP & TRIGEMINAL NEURALGIA & A5312 & Postherpetic trigem.neuralgia & $\begin{array}{l}\text { Postherpetic trigeminal } \\
\text { neuralgia }\end{array}$ \\
\hline V2 & NEP & TRIGEMINAL NEURALGIA & F300. & Post-herpetic trigem.neuralgia & $\begin{array}{l}\text { Post-herpetic trigeminal } \\
\text { neuralgia }\end{array}$ \\
\hline V2 & NEP & TRIGEMINAL NEURALGIA & F301. & Trigeminal neuralgia OS & $\begin{array}{l}\text { Other specified } \\
\text { trigeminal neuralgia }\end{array}$ \\
\hline V2 & NEP & TRIGEMINAL NEURALGIA & $\mathrm{F} 301 \mathrm{z}$ & Trigeminal neuralgia NOS & \\
\hline V2 & NEP & PHANTOM LIMB PAIN & F336. & Phantom limb syndrome & \\
\hline V2 & NEP & PHANTOM LIMB PAIN & F3360 & Phantom limb syndrome wth pain & $\begin{array}{l}\text { Phantom limb syndrome } \\
\text { with pain }\end{array}$ \\
\hline V2 & NEP & PHANTOM LIMB PAIN & F3361 & Phantom limb syn w'out pain & $\begin{array}{l}\text { Phantom limb syndrome } \\
\text { without pain }\end{array}$ \\
\hline V2 & NEP & $\begin{array}{l}\text { POST HERPETIC } \\
\text { NEURALGIA }\end{array}$ & A531. & Herpes zoster+other CNS compl. & $\begin{array}{l}\text { Herpes zoster with other } \\
\text { central nervous system } \\
\text { complication }\end{array}$ \\
\hline V2 & NEP & $\begin{array}{l}\text { POST HERPETIC } \\
\text { NEURALGIA }\end{array}$ & A5315 & Postzoster neuralgia & \\
\hline V2 & NEP & DIABETIC NEUROPATHY & C106. & Diab.mell. with neuropathy & $\begin{array}{l}\text { Diabetes mellitus with } \\
\text { neurological } \\
\text { manifestation }\end{array}$ \\
\hline V2 & NEP & DIABETIC NEUROPATHY & C108B & IDDM with mononeuropathy & $\begin{array}{l}\text { Insulin dependent } \\
\text { diabetes mellitus with } \\
\text { mononeuropathy }\end{array}$ \\
\hline V2 & NEP & DIABETIC NEUROPATHY & $\mathrm{C} 108 \mathrm{C}$ & IDDM with polyneuropathy & $\begin{array}{l}\text { Insulin dependent } \\
\text { diabetes mellitus with } \\
\text { polyneuropathy }\end{array}$ \\
\hline V2 & NEP & DIABETIC NEUROPATHY & C109A & NIDDM with mononeuropathy & $\begin{array}{l}\text { Non-insulin dependent } \\
\text { diabetes mellitus with } \\
\text { mononeuropathy }\end{array}$ \\
\hline V2 & NEP & DIABETIC NEUROPATHY & C109B & NIDDM with polyneuropathy & $\begin{array}{l}\text { Non-insulin dependent } \\
\text { diabetes mellitus with } \\
\text { polyneuropathy }\end{array}$ \\
\hline V2 & NEP & DIABETIC NEUROPATHY & C10EB & Type 1 diab mell + mononeurop & $\begin{array}{l}\text { Type } 1 \text { diabetes mellitus } \\
\text { with mononeuropathy }\end{array}$ \\
\hline V2 & NEP & DIABETIC NEUROPATHY & C1OEC & Type 1 diab mell + polyneurop & $\begin{array}{l}\text { Type } 1 \text { diabetes mellitus } \\
\text { with polyneuropathy }\end{array}$ \\
\hline V2 & NEP & DIABETIC NEUROPATHY & C10FA & Type 2 diab mell mononeurop & $\begin{array}{l}\text { Type } 2 \text { diabetes mellitus } \\
\text { with mononeuropathy }\end{array}$ \\
\hline
\end{tabular}




\begin{tabular}{|l|l|l|l|l|l|}
\hline V2 & NEP & DIABETIC NEUROPATHY & C1OFB & Type 2 diab mell + polyneurop & $\begin{array}{l}\text { Type } 2 \text { diabetes mellitus } \\
\text { with polyneuropathy }\end{array}$ \\
\hline V2 & NEP & DIABETIC NEUROPATHY & F1711 & Autonomic neuropathy-diabetes & $\begin{array}{l}\text { Autonomic neuropathy } \\
\text { due to diabetes }\end{array}$ \\
\hline V2 & NEP & DIABETIC NEUROPATHY & F372. & Polyneuropathy in diabetes & \\
\hline V2 & NEP & DIABETIC NEUROPATHY & F3720 & Acute painful diab neuropathy & $\begin{array}{l}\text { Acute painful diabetic } \\
\text { neuropathy }\end{array}$ \\
\hline V2 & NEP & DIABETIC NEUROPATHY & F3721 & Chron painful diab neuropathy & $\begin{array}{l}\text { Chronic painful diabetic } \\
\text { neuropathy }\end{array}$ \\
\hline V2 & NEP & DIABETIC NEUROPATHY & F3722 & Asymptomatic diab neuropathy & $\begin{array}{l}\text { Asymptomatic diabetic } \\
\text { neuropathy }\end{array}$ \\
\hline V2 & NEP & DIABETIC NEUROPATHY & F3y0. & Diabetic mononeuropathy & \\
\hline
\end{tabular}


F.3 Two-way ANOVA for Healthcare utilisation
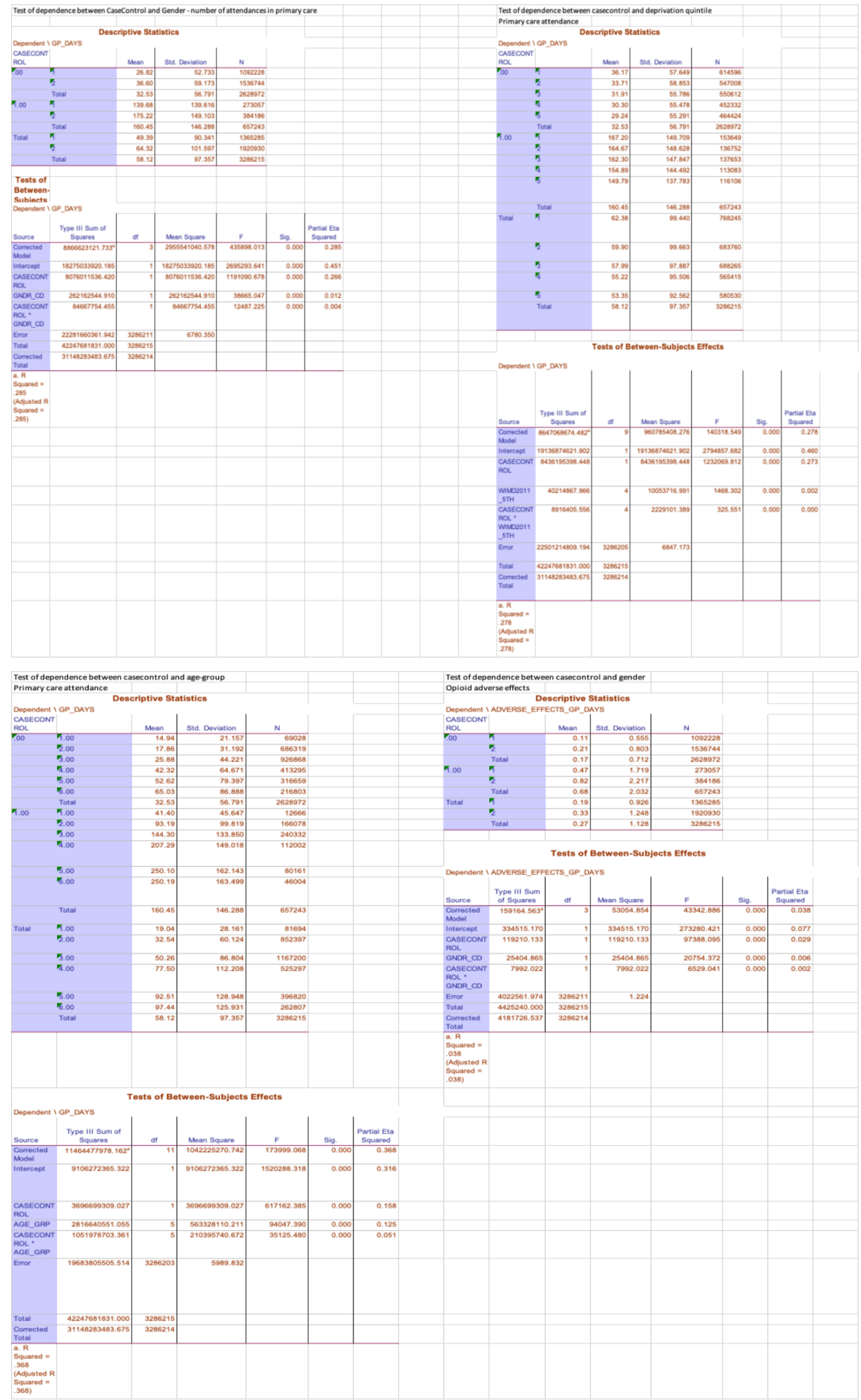


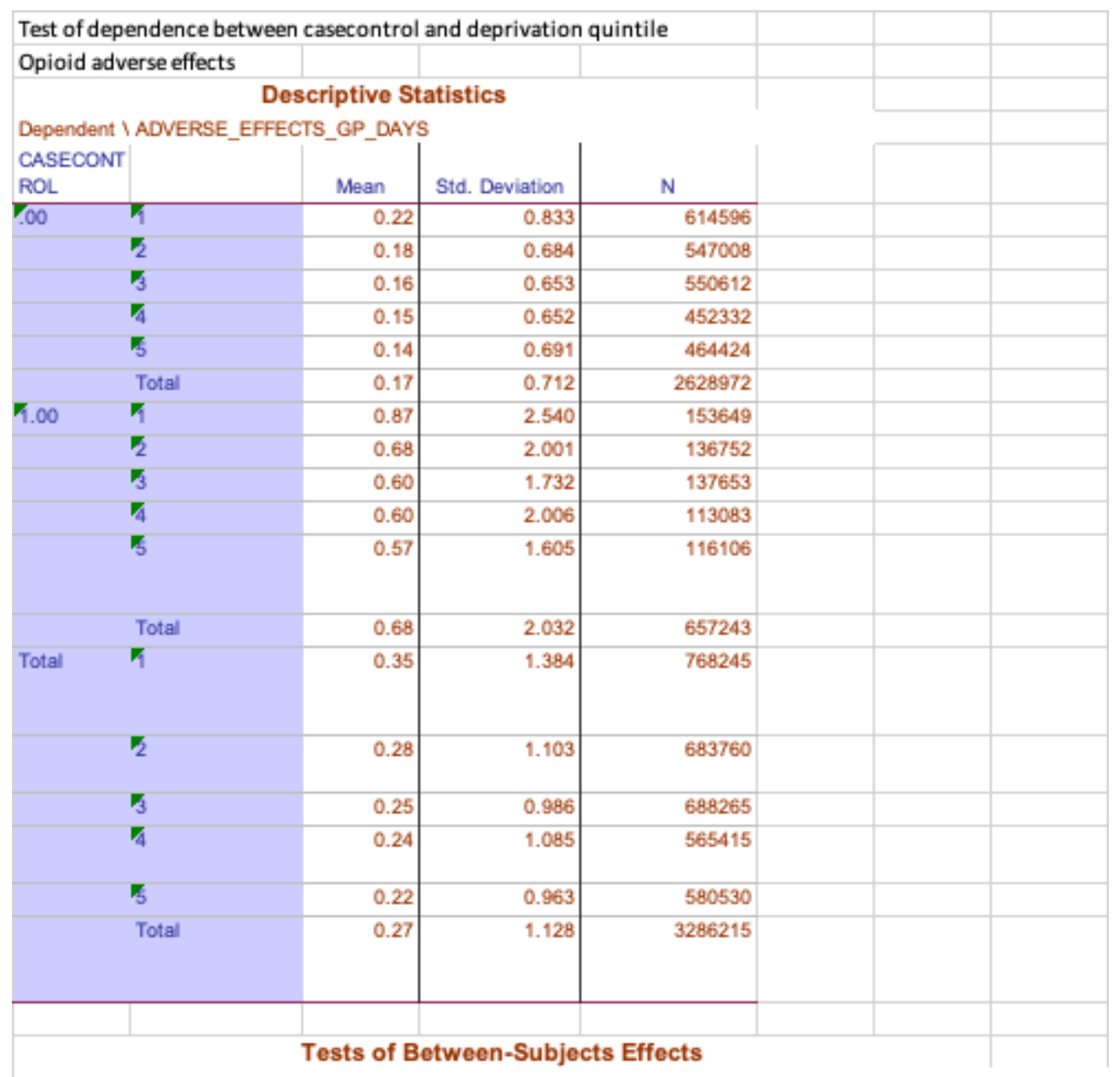

\begin{tabular}{|c|c|c|c|c|c|c|}
\hline $\begin{array}{l}\text { Dependent I } \\
\text { Source }\end{array}$ & ADVERSE_EFFEC & GP_DAY: & Mean Square & $\mathrm{F}$ & Sig. & $\begin{array}{l}\text { Partial Eta } \\
\text { Squared }\end{array}$ \\
\hline $\begin{array}{l}\text { Corrected } \\
\text { Model }\end{array}$ & $144244.587^{a}$ & 9 & 16027.176 & 13044.910 & 0.000 & 0.034 \\
\hline Intercept & 360661.645 & 1 & 360661.645 & 293551.306 & 0.000 & 0.082 \\
\hline $\begin{array}{l}\text { CASECONT } \\
\text { ROL }\end{array}$ & 127892.536 & 1 & 127892.536 & 104094.854 & 0.000 & 0.031 \\
\hline $\begin{array}{l}\text { WIMD2011 } \\
\text { _5TH }\end{array}$ & 10472.812 & 4 & 2618.203 & 2131.019 & 0.000 & 0.003 \\
\hline $\begin{array}{l}\text { CASECONT } \\
\text { ROL * } \\
\text { WIMD2011 } \\
\text {-5TH }\end{array}$ & 3935.332 & 4 & 983.833 & 800.766 & 0.000 & 0.001 \\
\hline Error & 4037481.950 & 3286205 & 1.229 & & & \\
\hline Total & 4425240.000 & 3286215 & & & & \\
\hline $\begin{array}{l}\text { Corrected } \\
\text { Total }\end{array}$ & 4181726.537 & 3286214 & & & & \\
\hline $\begin{array}{l}\text { a. R } \\
\text { Squared = } \\
.034 \\
\text { (Adjusted R } \\
\text { Squared = } \\
.034 \text { ) }\end{array}$ & & & & & & \\
\hline
\end{tabular}




\begin{tabular}{|c|c|c|c|c|c|c|c|}
\hline \multicolumn{7}{|c|}{ Casecontrol and gender } & \\
\hline \multicolumn{8}{|c|}{ Descriptive Statistics } \\
\hline \multicolumn{8}{|c|}{ Dependent \OPDW_ATT_CNT } \\
\hline $\begin{array}{l}\text { CASECONT } \\
\text { ROL }\end{array}$ & & Mean & Std. Deviation & $\mathrm{N}$ & & & \\
\hline .00 & 1 & 4.20 & 9.735 & 1092228 & & & \\
\hline r & 2 & 5.54 & 10.197 & 1536744 & & & \\
\hline & Total & 4.98 & 10.029 & 2628972 & & & \\
\hline 1.00 & 4 & 12.16 & 18.537 & 273057 & & & \\
\hline b & 2 & 15.15 & 19.933 & 384186 & & & \\
\hline & Total & 13.91 & 19.421 & 657243 & & & \\
\hline Total & 1 & 5.79 & 12.437 & 1365285 & & & \\
\hline r & 2 & 7.46 & 13.320 & 1920930 & & & \\
\hline & Total & 6.77 & 12.987 & 3286215 & & & \\
\hline & & & & & & & \\
\hline \multicolumn{7}{|c|}{ Tests of Between-Subjects Effects } & \\
\hline Dependent I & OPDW_ATT_CNT & & & & & & \\
\hline Source & $\begin{array}{l}\text { Type III Sum of } \\
\text { Squares }\end{array}$ & df & Mean Square & $\mathrm{F}$ & Sig. & $\begin{array}{c}\text { Partial Eta } \\
\text { Squared }\end{array}$ & \\
\hline $\begin{array}{l}\text { Corrected } \\
\text { Model }\end{array}$ & $44463335.318^{a}$ & 3 & 14821111.773 & 95541.453 & 0.000 & 0.080 & \\
\hline Intercept & 175264293.682 & 1 & 175264293.682 & 1129807.642 & 0.000 & 0.256 & \\
\hline $\begin{array}{l}\text { CASECONT } \\
\text { ROL }\end{array}$ & 39427551.464 & 1 & 39427551.464 & 254162.146 & 0.000 & 0.072 & \\
\hline GNDR_CD & 2395853.012 & 1 & 2395853.012 & 15444.407 & 0.000 & 0.005 & \\
\hline $\begin{array}{l}\text { CASECONT } \\
\text { ROL * } \\
\text { GNDR_CD }\end{array}$ & 348477.690 & 1 & 348477.690 & 2246.395 & 0.000 & 0.001 & \\
\hline Error & 509781867.691 & 3286211 & 155.128 & & & & \\
\hline Total & 704749016.000 & 3286215 & & & & & \\
\hline $\begin{array}{l}\text { Corrected } \\
\text { Total }\end{array}$ & 554245203.010 & 3286214 & & & & & \\
\hline \begin{tabular}{|l|} 
a. $R$ \\
Squared = \\
.080 \\
(Adjusted R \\
Squared = \\
.080 )
\end{tabular} & & & & & & & \\
\hline
\end{tabular}

Figure F.1: Example two-way ANOVA to establish interaction between factors prior to full analysis of healthcare utilisation

\section{F.4 Mann-Whitney and Kruskall-Wallis results}

\section{F.4.1 Primary care general practice}

\section{F.4.1.1 Primary care general practice attendance by prescribing persistence}

Case subjects with prescribing persistence of more than 6 months compared to those with under 6 months persistence, $p<.001, \eta^{2}=0.25$, $d_{\text {cohen }}=1.14$ (Table F.3).

\section{F.4.1.2 Primary care general practice attendance by gender}

Female case subjects compared to men $\mathrm{p}<.001, \eta^{2}=0.02, \mathrm{~d}_{\text {Cohen }}=0.30$.

Control group women compared to men $p<.001, \eta^{2}=0.01, d_{\text {Cohen }}=0.22$

Women case versus control $p<.001, \eta^{2}=0.25, d_{\text {Cohen }}=1.2$

Male cases versus control $p<.001, \eta^{2}=0.24, d_{\text {cohen }}=1.1$ 


\section{F.4.1.3 Primary Care General Practice attendance by deprivation status}

Comparison of case subjects by WIMD2011 quintile, $p<.001, \eta^{2}=0.05, \mathrm{~d}_{\text {cohen }}=$ 0.5. Comparison of controls by quintile, $p<.001, \eta^{2}=0.002$, $\mathrm{d}_{\text {cohen }}=0.09$ which were confirmed between all quintiles by post-hoc analysis.

Table F.3: Comparison of Primary Care General Practice utilisation depending on receipt of opioid prescriptions (case versus control)

\begin{tabular}{|c|c|c|c|}
\hline Total, mean \pm standard deviation & Case & Control & p-value $* *\left(\eta^{2}, d_{\text {cohen }}\right)$ \\
\hline Number of GP attendances & 105457258 & 85527059 & \\
\hline Mean (SD) & $160.5(146.3)$ & $32.53(56.8)$ & $<.001(0.24,1.13)$ \\
\hline Median & 121.0 & 10.0 & \\
\hline \multicolumn{4}{|l|}{ Prescribing persistence (cases only) } \\
\hline$<6$ months & $67.7(77.6)$ & & \\
\hline$>6$ months & $198.8(150.8)$ & & $<.001(0.25,1.14)$ \\
\hline \multicolumn{4}{|l|}{ Gender } \\
\hline Female & $175.2(149.1)$ & $36.6(59.2)$ & $<.001(0.25,1.2)$ \\
\hline Male & $139.7(139.6)$ & $26.8(52.7)$ & $<.001(0.24,1.1)$ \\
\hline \multicolumn{4}{|l|}{ Deprivation quintile* } \\
\hline WIMD1 & $167.2(149.7)$ & $36.2(57.7)$ & $<.001(0.23,1.1)$ \\
\hline WIMD2 & $164.7(148.6)$ & $33.7(58.9)$ & $<.001(0.24,1.1)$ \\
\hline WIMD3 & $162.3(147.5)$ & $31.9(55.8)$ & $<.001(0.25,1.2)$ \\
\hline WIMD4 & $154.9(144.5)$ & $30.3(55.5)$ & $<.001(0.25,1.2)$ \\
\hline WIMD5 & $149.8(137.8)$ & $29.2(55.3)$ & $<.001(0.26,1.2)$ \\
\hline \multicolumn{4}{|l|}{ Age group (years) } \\
\hline $18-24$ & $41.4(45.7)$ & $14.9(21.2)$ & $<.001(0.09,0.6)$ \\
\hline $25-44$ & $93.2(99.8)$ & $17.9(31.2)$ & $<.001(0.24,1.1)$ \\
\hline $45-64$ & $144.3(133.9)$ & $25.9(44.2)$ & $<.001(0.27,1.2)$ \\
\hline $65-74$ & $207.3(149.0)$ & $42.3(64.7)$ & $<.001(0.30,1.3)$ \\
\hline $75-84$ & $250.1(162.1)$ & $52.6(79.4)$ & $<.001(0.30,1.3)$ \\
\hline$\geq 85$ & $250.2(163.5)$ & $65.0(86.9)$ & $<.001(0.23,1.1)$ \\
\hline \multicolumn{4}{|l|}{$\begin{array}{l}\text { GP visits for ADEs possibly associated } \\
\text { with opioids }\end{array}$} \\
\hline $\begin{array}{l}\text { with opioids } \\
\qquad \text { Mean } \pm \text { SD }\end{array}$ & $\begin{array}{c}444050 \\
0.68(2.0)\end{array}$ & $\begin{array}{l}450510 \\
0.17(0.7)\end{array}$ & $<.001(0.02,0.28)$ \\
\hline \multicolumn{4}{|l|}{ Gender } \\
\hline Female & $0.82(2.2)$ & $0.21(0.8)$ & $<.001(0.03,0.33)$ \\
\hline Male & $0.47(1.7)$ & $0.11(0.6)$ & $<.001(0.01,0.21)$ \\
\hline \multicolumn{4}{|l|}{ Deprivation quintile* } \\
\hline WIMD1 & $0.87(2.5)$ & $0.22(0.8)$ & $<.001(0.03,0.3)$ \\
\hline WIMD2 & $0.68(2.0)$ & $0.18(0.7)$ & $<.001(0.02,0.3)$ \\
\hline WIMD3 & $0.60(1.7)$ & $0.16(0.7)$ & $<.001(0.02,0.3)$ \\
\hline WIMD4 & $0.6(2.0)$ & $0.15(0.7)$ & $<.001(0.02,0.25)$ \\
\hline WIMD5 & $0.57(1.6)$ & $0.14(0.7)$ & $<.001(0.02,0.26)$ \\
\hline \multirow{2}{*}{$\begin{array}{l}\text { All tests and referrals from primary } \\
\text { care }\end{array}$} & 206747 & 158290 & \\
\hline & $0.31(0.9)$ & $0.06(0.3)$ & $<.001(0.01,0.21)$ \\
\hline Mean \pm SD & & & \\
\hline Recorded imaging (X-ray, CT, MRI etc.) & 90700 & 74756 & \\
\hline Mean \pm SD & $0.14(0.5)$ & $0.03(0.2)$ & $<.001(0.003,0.10)$ \\
\hline
\end{tabular}

*Deprivation quintile based on Welsh Index of Multiple Deprivation 2011. WIMD1 =most deprived, WIMD5 $=$ least deprived ${ }^{*}$ p-value $<0.05=$ significant

\section{F.4.1.4 Primary care General Practice attendance by age-group}

Pairwise comparisons of the six age-groups of case subjects, $p<.001, \eta^{2}=0.19$, $\mathrm{d}_{\text {Cohen }}=0.97$, other than for the two oldest groups (75-84 years and $85+$ years, $\mathrm{p}>0.05$ ).

Differences between the mean attendances of all control subject age-groups $p<.001, \eta^{2}=0.03, d_{\text {cohen }}=0.32$. Differences in attendance were confirmed between all age-groups when compared pairwise $(p<.001)$. 


\section{F.4.1.5 Tests and imaging from Primary Care}

Case subjects versus controls for tests, $\mathrm{p}<.001, \eta^{2}=0.01, \mathrm{~d}_{\text {cohen }}=0.21$

and imaging appointments, $p<.001, \eta^{2}=0.003, d_{\text {cohen }}=0.1$ )

\section{F.4.2 Out-Patient attendance}

Cases versus control, $\mathrm{p}<.001, \eta^{2}=0.09, \mathrm{~d}_{\text {cohen }}=0.63$

\section{F.4.2.1 Out-patient attendance by prescribing persistence}

Case subjects with a prescribing persistence of more than 6 months compared to under 6 months, $\mathrm{p}<.001, \eta^{2}=0.16$, $\mathrm{d}_{\text {cohen }}=0.88$.

\section{F.4.2.2 Out-patient attendance by gender}

Case subject women compared to men, $p<.001, \eta^{2}=0.01$, $d_{\text {cohen }}=0.24$.

Control women compared to men, $p<.001, \eta^{2}=0.01, d_{\text {cohen }}=0.22$.

Table F.4: Comparison of out-patient resource utilisation depending on receipt of opioid prescriptions (case versus control)

\begin{tabular}{|c|c|c|c|}
\hline Total, mean \pm standard deviation & Case & Control & $\mathrm{p}$-value ${ }^{* *}\left(\eta^{2}, \mathrm{~d}_{\text {cohen }}\right)$ \\
\hline Number of Outpatient & 9140922 & 13098410 & \\
\hline attendances & $13.9(19.4)$ & $5.0(10.0)$ & $<.001(0.09,0.63)$ \\
\hline \multicolumn{4}{|l|}{ Mean \pm SD } \\
\hline \multicolumn{4}{|l|}{ Prescribing persistence (cases } \\
\hline \multirow[t]{3}{*}{ only) } & $5.6(10.1)$ & & \\
\hline & $17.4(21.2)$ & & $<.001(0.16,0.88)$ \\
\hline & & & \\
\hline \multicolumn{4}{|l|}{ Gender } \\
\hline Female & $15.2(19.9)$ & $5.5(10.2)$ & $<.001(0.10,0.65)$ \\
\hline Male & $12.2(18.5)$ & $4.2(9.7)$ & $<.001(0.09,0.62)$ \\
\hline \multicolumn{4}{|l|}{ Deprivation quintile* } \\
\hline WIMD1 & $\circ \quad(20.7)$ & $5.3(10.0)$ & $<.001(0.09,0.64)$ \\
\hline WIMD2 & 14.4 (19.9) & $5.1(10.4)$ & $<.001(0.09,0.64)$ \\
\hline WIMD3 & $13.5(19.0)$ & $5.0(9.7)$ & $<.001(0.09 .0 .62)$ \\
\hline WIMD4 & $13.0(18.4)$ & $4.6(9.3)$ & $<.001(0.09,0.63)$ \\
\hline WIMD5 & $13.0(18.4)$ & $4.8(10.7)$ & $<0001(0.09,0.64)$ \\
\hline \multicolumn{4}{|l|}{ Age group (years) } \\
\hline $18-24$ & $4.6(8.1)$ & $1.7(5.7)$ & $<.001(0.06,0.48)$ \\
\hline $25-44$ & $9.9(15.0)$ & $3.4(7.3)$ & $<.001(0.09,0.61)$ \\
\hline $45-64$ & $12.7(18.7)$ & $4.3(9.0)$ & $<.001(0.10,0.66)$ \\
\hline $65-74$ & 17.2 (21.9) & $7.3(12.9)$ & $<.001(0.09,0.63)$ \\
\hline $75-84$ & $20.7(23.3)$ & 7.9 (12.9) & $<.001(0.11,0.70)$ \\
\hline$\geq 85$ & $17.4(20.5)$ & $5.7(9.8)$ & $<.001(0.11,0.70)$ \\
\hline
\end{tabular}

\section{F.4.2.3 Out-patient attendance by deprivation}

Case subject differences in attendance by quintile, $p<.001, \eta^{2}=.002$, $d_{\text {cohen }}=$ 0.10 . Post-hoc analysis confirmed those differences in all but WIMD4 and WIMD5 quintiles ( $p>.5$ ) when attendance was directly compared.

Control group differences in attendance by deprivation quintile, $p<.001, \eta^{2}=$ $.002, \mathrm{~d}_{\text {Cohen }}=.09$, which was confirmed by pairwise comparisons. 


\section{F.4.2.4 Out-patient attendance by age-group}

Case subject comparisons of out-patient attendances between all age-groups, $\mathrm{p}<.001, \eta^{2}=.062, \mathrm{~d}_{\text {cohen }}=.51$ and were confirmed by pairwise comparisons. Control subject comparisons, $\mathrm{p}<.001, \eta^{2}=.055$, $\mathrm{d}_{\text {cohen }}=.48$ and again, confirmed by pairwise comparison.

\section{F.4.3 Emergency Department Utilisation}

Case subjects versus control subjects, $p<.001, \eta^{2}=0.07, \mathrm{~d}_{\text {cohen }}=.53$.

\section{F.4.3.1 Emergency department attendance by prescription persistence}

Case subjects receiving opioid prescriptions for more than 6 months compared to shorter durations of prescribing (Table F.5).

\section{F.4.3.2 Emergency department attendance by gender}

Male versus female case subjects, $p<.001, \eta^{2}=0$, $d_{\text {cohen }}=.004$.

Female versus male controls, $p<.001, \eta^{2}=0$, $\mathrm{d}_{\text {cohen }}=.004$.

Table F.5: Comparison of Emergency department resource utilisation depending on receipt of opioid prescriptions (case versus control)

\begin{tabular}{|c|c|c|c|}
\hline Total, mean (standard deviation) & Case & Control & p-value** $\left(\eta^{2}, d_{\text {cohen }}\right)$ \\
\hline $\begin{array}{l}\text { Number of Emergency } \\
\text { Department attendances }\end{array}$ & $\begin{array}{l}1243641 \\
1.89(3.4)\end{array}$ & $\begin{array}{c}1566627 \\
0.6(1.8)\end{array}$ & $<.001(0.07,0.53)$ \\
\hline \multicolumn{4}{|l|}{$\begin{array}{l}\text { Prescribing persistence (cases } \\
\text { only) }\end{array}$} \\
\hline $\begin{array}{l}<6 \text { months } \\
>6 \text { months }\end{array}$ & $\begin{array}{l}0.96(1.9) \\
2.3(3.8)\end{array}$ & & $<.001(0.06,0.51)$ \\
\hline \multicolumn{4}{|l|}{ Gender } \\
\hline Female & $1.9(3.4)$ & $0.58(1.7)$ & $<.001(0.06,0.52)$ \\
\hline Male & $1.9(3.4)$ & $0.61(1.8)$ & $<.001(0.07,0.53)$ \\
\hline \multicolumn{4}{|l|}{ Deprivation quintile* } \\
\hline WIMD1 & $2.3(3.9)$ & $0.77 \pm 2.1$ & $<.001(0.08,0.57)$ \\
\hline WIMD2 & $2.4(3.8)$ & $0.65 \pm 1.7$ & $<.001(0.07,0.55)$ \\
\hline WIMD3 & $1.8(3.2)$ & $0.55 \pm 1.9$ & $<.001(0.07,0.53)$ \\
\hline WIMD4 & $1.64 \pm 2.9$ & $0.53 \pm 1.4$ & $<.001(0.05,0.47)$ \\
\hline WIMD5 & $1.5 \pm 2.6$ & $0.41 \pm 1.2$ & $<.001(0.06,0.52)$ \\
\hline \multicolumn{4}{|l|}{ Age group (years) } \\
\hline $18-24$ & $2.0 \pm 3.9$ & $0.86 \pm 2.2$ & $<.001(0.03,0.37)$ \\
\hline $25-44$ & $2.2 \pm 3.8$ & $0.61 \pm 1.5$ & $<.001(0.08,0.59)$ \\
\hline $45-64$ & $1.7 \pm 3.2$ & $0.56 \pm 1.7$ & $<.001(0.06,0.48)$ \\
\hline $65-74$ & $1.5 \pm 3.1$ & $0.56 \pm 2.2$ & $<.001(0.05,0.47)$ \\
\hline $75-84$ & $2.0 \pm 3.1$ & $0.58 \pm 1.6$ & $<.001(0.08,0.59)$ \\
\hline$\geq 85$ & $2.5(3.3)$ & $0.67(1.6)$ & $<.001(0.10,0.66)$ \\
\hline
\end{tabular}

*Deprivation quintile based on Welsh Index of Multiple Deprivation 2011. WIMD1 =most deprived, WIMD5

$=$ least deprived ${ }^{*}$ p-value $<0.05=$ significant

\section{F.4.3.3 Emergency Department attendance by deprivation}

Case subject differences between quintiles, $p<.001, \eta^{2}=0.01, \mathrm{~d}_{\text {cohen }}=0.20$.

Control group differences by quintile, $p<.001, \eta^{2}=0.005$, $d_{\text {cohen }}=0.14$. 
Differences in attendance numbers were significant between all quintiles when compared in pairs. Case subjects versus controls in WIMD1 quintiles, $p<.001, \eta^{2}=$ $0.08, \mathrm{~d}_{\text {Cohen }}=0.57$ (Table F.5).

Cases compared to controls in WIMD5 quintile, $p<.001, \eta^{2}=0.06$, $d_{\text {cohen }}=0.52$.

\section{F.4.3.4 Emergency Department attendance by age-group}

Comparisons of mean attendances in case subjects by age-group $\left(p<.001, \eta^{2}=\right.$ $\left.0.02, \mathrm{~d}_{\text {Cohen }}=0.24\right)$.

Control group by age-group, $\mathrm{p}<.001, \eta^{2}=0.002, \mathrm{~d}_{\text {Cohen }}=0.09$. Pairwise comparison confirmed differences between age groups other than the $45-64$ and $\geq 85$ years groups where a statistical difference was not found ( $p>0.1$ ).

\section{F.4.4 Inpatient attendance}

\section{F.4.4.1 In-Patient attendance and length of stay}

Case subjects versus controls, $p<.001, \eta^{2}=0.07, d_{\text {Cohen }}=0.53$.

Length of stay case versus controls, $p<.001, \eta^{2}=0.02$, $\mathrm{d}_{\text {cohen }}=0.28$.

\section{F.4.4.2 In-patient utilisation by prescription persistence}

Case subjects with $>6$ months prescription persistence (Table F.6) compared to under 6 months use, $p<.001, \eta^{2}=0.005$, $d_{\text {cohen }}=0.47$.

\section{F.4.4.3 In-patient utilisation by gender}

In-patient admissions, female case subjects compared to male cases, $p<.001, \eta^{2}$ $=0.02, \mathrm{~d}_{\text {Cohen }}=0.26$.

Length of stay female case subjects versus male cases, $p<.001, \eta^{2}=0.012$, $d_{\text {cohen }}$ $=0.22$ (Table F.6).

Control subjects, in-patient admissions for females compared to male, $p<.001, \eta^{2}$ $=0.01, \mathrm{~d}_{\text {Cohen }}=0.18$.

Female controls length of stay versus men, $p<.001, \eta^{2}=0.06, d_{\text {cohen }}=0.16$.

Female controls compared to female case subjects' length of stay, $p<.001, \eta^{2}=$ 0.02, D $_{\text {Cohen }}=0.29$, (Table F.6).

Male controls versus male case subjects' length of stay, $p<.001, \eta^{2}=0.02$, D cohen $=0.27$.

Table F.6: Comparison of In-patient admission and length of stay depending on receipt of opioid prescriptions (case versus control)

\begin{tabular}{|c|c|c|c|}
\hline Total, mean (standard deviation) & Case & Control & p-value ${ }^{* *}\left(\eta^{2}, d_{\text {cohen }}\right)$ \\
\hline Number of In-patient admissions & 3021645 & 5676577 & \\
\hline Mean \pm SD & $4.6(8.5)$ & $2.2(6.3)$ & $<.001(0.07,0.53)$ \\
\hline \multicolumn{4}{|l|}{ Prescribing persistence (cases } \\
\hline $\begin{array}{l}<6 \text { months } \\
>6 \text { months }\end{array}$ & $5.3(9.2)$ & & $<.001(0.05,0.47)$ \\
\hline \multicolumn{4}{|l|}{ Gender } \\
\hline Female & $5.0(8.1)$ & $2.3(6.0)$ & $<.001(0.07,0.56)$ \\
\hline Male & $4.1(9.0)$ & $2.0(6.7)$ & $<.001(0.06,0.51)$ \\
\hline \multicolumn{4}{|l|}{ Deprivation quintile } \\
\hline WIMD1 & $4.9(8.1)$ & $2.3(4.8)$ & $<.001(0.07,0.53)$ \\
\hline WIMD2 & $4.8(8.7)$ & $2.2(4.7)$ & $<.001(0.07,0.55)$ \\
\hline
\end{tabular}




\begin{tabular}{|c|c|c|c|c|}
\hline & WIMD3 & $4.5(8.3)$ & $2.3(8.3)$ & $<.001(0.06,0.51)$ \\
\hline & WIMD4 & $4.4(9.0)$ & $2.0(6.4)$ & $<.001(0.07,0.55)$ \\
\hline & WIMD5 & $4.2(8.3)$ & $1.9(6.9)$ & $<.001(0.07,0.54)$ \\
\hline \multicolumn{5}{|l|}{ Age group (years) } \\
\hline & $18-24$ & $3.5(6.5)$ & $0.5(2.1)$ & $<.001(0.13,0.76)$ \\
\hline & $25-44$ & $3.9(7.4)$ & $1.0(2.7)$ & $<.001(0.12,0.74)$ \\
\hline & $45-64$ & $3.5(7.8)$ & $1.2(4.8)$ & $<.001(0.09,0.64)$ \\
\hline & $65-74$ & $5.0(9.3)$ & $3.1(10.0)$ & $<.001(0.05,0.47)$ \\
\hline & $75-84$ & $6.9(10.1)$ & $4.6(6.0)$ & $<.001(0.02,0.29)$ \\
\hline & $\geq 85$ & $8.3(8.6)$ & $5.1(9.3)$ & $<.001(0.04,0.39)$ \\
\hline \multirow[t]{2}{*}{ Length of stay (days) } & & 10758522 & 45482557 & \\
\hline & Mean \pm SD & $16.4(54.7)$ & $17.3(64.9)$ & $<.001(0.02,0.28)$ \\
\hline \multicolumn{5}{|c|}{ Prescribing persistence (cases } \\
\hline only) & $\begin{array}{l}<6 \text { months } \\
>6 \text { months }\end{array}$ & $18.8(57.5)$ & & $<.001(0.03,0.37)$ \\
\hline \multicolumn{5}{|l|}{ Gender } \\
\hline & Female & $18.0(57.0)$ & $19.3(68.2)$ & $<.001(0.02,0.29)$ \\
\hline & Male & $14.1(51.3)$ & $14.5(60.0)$ & $<.001(0.02,0.27)$ \\
\hline \multicolumn{5}{|l|}{ Deprivation quintile } \\
\hline & WIMD1 & $17.6(58.6)$ & $17.9(65.8)$ & $<.001(0.02,0.28)$ \\
\hline & WIMD2 & $16.9(56.4)$ & $18.0(72.9)$ & $<.001(0.02,0.31)$ \\
\hline & WIMD3 & $16.5(53.6)$ & $18.9(62.1)$ & $<.001(0.02,0.27)$ \\
\hline & WIMD4 & $15.0(49.6)$ & $15.1(55.8)$ & $<.001(0.02,0.30)$ \\
\hline & WIMD5 & $15.3(53.4)$ & $15.9(65.4)$ & $<.001(0.02,0.26)$ \\
\hline \multicolumn{5}{|l|}{ Age group (years) } \\
\hline & $18-24$ & $5.1(22.4)$ & $0.8(7.0)$ & $<.001(0.08,0.58)$ \\
\hline & $25-44$ & $6.8(33.4)$ & $1.8(21.1)$ & $<.001(0.07,0.57)$ \\
\hline & $45-64$ & $8.1(40.0)$ & $5.0(37.7)$ & $<.001(0.04,0.41)$ \\
\hline & $65-74$ & $15.5(50.9)$ & $20.5(66.9)$ & $<.001(0.01,0.20)$ \\
\hline & $75-84$ & $34.6(78.8)$ & $50.3(108.2)$ & $<.001(0.002,0.10)$ \\
\hline & $\geq 85$ & $67.7(96.0)$ & $70.3(110.1)$ & $<.001(.001,0.05)$ \\
\hline
\end{tabular}

*Deprivation quintile based on Welsh Index of Multiple Deprivation 2011. WIMD1 =most deprived, WIMD5 $=$ least deprived ${ }^{*}$ p-value $<0.05=$ significant

\section{F.4.4.4 In-patient utilisation by deprivation}

Case subjects mean average number of admissions by deprivation quintile, $p<$ $.001, \eta^{2}=0.002$, $D_{\text {cohen }}=0.09$, confirmed by pairwise comparison.

Length of in-patient stay for case subjects by deprivation quintile and confirmed by pairwise comparisons, $\mathrm{p}<.001, \eta^{2}=0.002$, $\mathrm{D}_{\text {Cohen }}=0.08$.

Control group admissions by deprivation, $\mathrm{p}<.001, \eta^{2}=0.002$, $\mathrm{D}_{\text {cohen }}=0.08$.

Length of stay for control group by deprivation quintile (WIMD3) (Table F.6) and confirmed by pairwise comparisons, $p<.001, \eta^{2}=.001$, $D_{\text {cohen }}=0.08$ )

\section{F.4.4.5 In-patient utilisation by age-group}

Average in-patient admissions between case-subject control groups was found to be statistically significant, $p<.001, \eta^{2}=0.08, D_{\text {cohen }}=0.59$. There was no difference in the number of admissions for age-groups 18-24 years and 45-64 years.

Case subject length of stay compared between and within age-groups, $p<.001$, $\eta^{2}=0.13, D_{\text {cohen }}=0.77$.

Differences between age-group ranked admissions for cases versus controls were statistically significant and confirmed by direct pairwise comparisons, $p<$ $.001, \eta^{2}=0.22, D_{\text {Cohen }}=1.05$. 
Control group differences in average length of stay by age-group, $p<.001, \eta^{2}=$ $0.30, D_{\text {Cohen }}=1.30$ and confirmed by pairwise comparison.

Case versus control subjects aged $18-24$ years number of admissions, $p<.001, \eta^{2}$ $=0.13, \mathrm{D}_{\text {Cohen }}=0.76$ and admission duration, $\mathrm{p}<.001, \eta^{2}=0.08, \mathrm{D}_{\text {Cohen }}=0.58$.

Age-group 75-84 control subjects versus controls, $p<.001, \eta^{2}=0.02, D_{\text {cohen }}=$ 0.29 . Average length of stay, $p<.001, \eta^{2}=0.002$, $D_{\text {Cohen }}=0.10$. 


\section{F.5 Example of outpatient unit costs for cost-analysis}

Table F.7: Example calculation for weighting unit costs using National Unit costs for Emergency Department attendance in 2015

\begin{tabular}{|c|c|c|c|c|c|c|c|c|c|}
\hline \multicolumn{10}{|c|}{ National Schedule of Reference Costs - Year 2014-15 - NHS trusts and NHS foundation trusts - Emergency Medicine } \\
\hline \begin{tabular}{|l|} 
Service \\
Code
\end{tabular} & Service Description & $\begin{array}{l}\text { Currency } \\
\text { Code }\end{array}$ & Currency Description & Attendances & $\begin{array}{l}\text { National } \\
\text { Average } \\
\text { Unit Cost } \\
\end{array}$ & $\begin{array}{l}\text { Lower } \\
\text { Quartile } \\
\text { Unit Cost } \\
\end{array}$ & $\begin{array}{l}\text { Upper } \\
\text { Quartile } \\
\text { Unit Cost } \\
\end{array}$ & $\begin{array}{l}\text { No. Data } \\
\text { Submissions }\end{array}$ & $\begin{array}{l}\text { Number of } \\
\text { attendances*National } \\
\text { average unit cost }\end{array}$ \\
\hline T01A & Type 01 Admitted & VB01Z & Emergency Medicine, Any Investigation with Category 5 Treatment & 29,523 & f417 & f253 & $f 560$ & 133 & $£ 12,314,719.19$ \\
\hline T01A & Type 01 Admitted & VB02Z & Emergency Medicine, Category 3 Investigation with Category 4 Treatment & 141,663 & f371 & $f 270$ & $£ 460$ & 136 & $f 52,537,385.33$ \\
\hline T01A & Type 01 Admitted & VB03Z & Emergency Medicine, Category 3 Investigation with Category 1-3 Treatment & 327,342 & $f 278$ & $f 221$ & $f 330$ & 139 & $£ 91,026,130.78$ \\
\hline T01A & Type 01 Admitted & VB04Z & Emergency Medicine, Category 2 Investigation with Category 4 Treatment & 787,182 & $£ 247$ & $f 198$ & f281 & 138 & $£ 194,285,369.36$ \\
\hline T01A & Type 01 Admitted & VB05Z & Emergency Medicine, Category 2 Investigation with Category 3 Treatment & 193,707 & $f 221$ & $f 174$ & f248 & 139 & $£ 42,800,957.00$ \\
\hline T01A & Type 01 Admitted & VB06Z & Emergency Medicine, Category 1 Investigation with Category 3-4 Treatment & 106,822 & f161 & $f 127$ & f192 & 139 & $£ 17,249,530.88$ \\
\hline T01A & Type 01 Admitted & VB07Z & Emergency Medicine, Category 2 Investigation with Category 2 Treatment & 525,452 & $£ 201$ & f158 & $£ 225$ & 139 & $f 105,547,785.39$ \\
\hline T01A & Type 01 Admitted & VB08Z & Emergency Medicine, Category 2 Investigation with Category 1 Treatment & $1,255,603$ & f184 & $£ 150$ & $£ 213$ & 140 & $£ 231,129,209.84$ \\
\hline T01A & Type 01 Admitted & VB09Z & Emergency Medicine, Category 1 Investigation with Category 1-2 Treatment & 483,365 & f133 & f101 & f146 & 140 & $£ 64,167,368.34$ \\
\hline T01A & Type 01 Admitted & VB10Z & Emergency Medicine, Dental Care & 9,132 & $f 72$ & f67 & $£ 67$ & 21 & $£ 657,584.86$ \\
\hline T01A & Type 01 Admitted & VB11Z & Emergency Medicine, No Investigation with No Significant Treatment & 190,837 & $f 109$ & $f 77$ & $£ 132$ & 138 & $£ 20,885,973.36$ \\
\hline T01A & Type 01 Admitted & VB99Z & Emergency Medicine, Patient Dead On Arrival & 73 & f155 & $f 75$ & $£ 251$ & 22 & $£ 11,320.66$ \\
\hline T01NA & Type 01 Non-Admitted & VB01Z & Emergency Medicine, Any Investigation with Category 5 Treatment & 13,581 & $£ 373$ & $£ 187$ & $£ 526$ & 133 & $£ 5,060,904.71$ \\
\hline T01NA & Type 01 Non-Admitted & VB02Z & Emergency Medicine, Category 3 Investigation with Category 4 Treatment & 42,274 & $£ 343$ & $£ 270$ & $£ 395$ & 134 & $£ 14,520,781.52$ \\
\hline T01NA & Type 01 Non-Admitted & VB03Z & Emergency Medicine, Category 3 Investigation with Category 1-3 Treatment & 273,027 & f249 & f205 & $£ 292$ & 137 & $f 68,103,569.13$ \\
\hline T01NA & Type 01 Non-Admitted & VB04Z & Emergency Medicine, Category 2 Investigation with Category 4 Treatment & 294,431 & $£ 224$ & $£ 186$ & $£ 240$ & 135 & $£ 65,878,121.46$ \\
\hline T01NA & Type 01 Non-Admitted & VB05Z & Emergency Medicine, Category 2 Investigation with Category 3 Treatment & 203,529 & f187 & f158 & $£ 210$ & 135 & $£ 38,001,656.59$ \\
\hline T01NA & Type 01 Non-Admitted & VB06Z & Emergency Medicine, Category 1 Investigation with Category 3-4 Treatment & 347,157 & $£ 132$ & $£ 108$ & $£ 147$ & 136 & $£ 45,958,183.51$ \\
\hline T01NA & Type 01 Non-Admitted & VB07Z & Emergency Medicine, Category 2 Investigation with Category 2 Treatment & $1,339,334$ & f162 & $£ 142$ & $£ 181$ & 136 & $£ 217,506,975.45$ \\
\hline T01NA & Type 01 Non-Admitted & VB08Z & Emergency Medicine, Category 2 Investigation with Category 1 Treatment & $2,815,693$ & f151 & $£ 134$ & $£ 165$ & 137 & $£ 426,326,017.19$ \\
\hline T01NA & Type 01 Non-Admitted & VB09Z & Emergency Medicine, Category 1 Investigation with Category 1-2 Treatment & $3,416,947$ & $£ 107$ & $f 88$ & $£ 122$ & 138 & $£ 366,452,455.00$ \\
\hline T01NA & Type 01 Non-Admitted & VB10Z & Emergency Medicine, Dental Care & 25,967 & $f 111$ & $£ 113$ & $£ 113$ & 65 & $£ 2,894,520.37$ \\
\hline T01NA & Type 01 Non-Admitted & VB11Z & Emergency Medicine, No Investigation with No Significant Treatment & $1,864,147$ & $£ 89$ & $f 67$ & $£ 106$ & 136 & $£ 165,450,349.47$ \\
\hline T01NA & Type 01 Non-Admitted & VB99Z & Emergency Medicine, Patient Dead On Arrival & 579 & $£ 279$ & f55 & $£ 251$ & 38 & $f 161,300.09$ \\
\hline
\end{tabular}




\begin{tabular}{|c|c|c|c|c|c|c|c|c|c|}
\hline T02A & Type 02 Admitted & VB01Z & Emergency Medicine, Any Investigation with Category 5 Treatment & 19 & f144 & f108 & f164 & 7 & $£ 2,728.05$ \\
\hline T02A & Type 02 Admitted & VB02Z & Emergency Medicine, Category 3 Investigation with Category 4 Treatment & 32 & f316 & f307 & f392 & 5 & $£ 10,099.62$ \\
\hline T02A & Type 02 Admitted & VB03Z & Emergency Medicine, Category 3 Investigation with Category 1-3 Treatment & 327 & f195 & f122 & f253 & 17 & $£ 63,799.36$ \\
\hline T02A & Type 02 Admitted & VB04Z & Emergency Medicine, Category 2 Investigation with Category 4 Treatment & 328 & $f 276$ & f299 & f299 & 9 & $£ 90,465.95$ \\
\hline T02A & Type 02 Admitted & VB05Z & Emergency Medicine, Category 2 Investigation with Category 3 Treatment & 270 & $f 242$ & $£ 230$ & $£ 293$ & 7 & $f 65,268.68$ \\
\hline T02A & Type 02 Admitted & VB06Z & Emergency Medicine, Category 1 Investigation with Category 3-4 Treatment & 553 & $f 130$ & f123 & f123 & 13 & $f 72,009.16$ \\
\hline T02A & Type 02 Admitted & VB07Z & Emergency Medicine, Category 2 Investigation with Category 2 Treatment & 3,359 & $f 152$ & f138 & $f 138$ & 9 & $£ 509,621.16$ \\
\hline T02A & Type 02 Admitted & VB08Z & Emergency Medicine, Category 2 Investigation with Category 1 Treatment & 7,933 & f146 & f143 & f143 & 16 & $£ 1,156,635.78$ \\
\hline T02A & Type 02 Admitted & VB09Z & Emergency Medicine, Category 1 Investigation with Category 1-2 Treatment & 32,885 & f89 & f89 & f89 & 19 & $f 2,933,940.38$ \\
\hline T02A & Type 02 Admitted & VB11Z & Emergency Medicine, No Investigation with No Significant Treatment & 4,182 & f96 & f96 & f96 & 17 & $£ 401,160.73$ \\
\hline T02A & Type 02 Admitted & VB992 & Emergency Medicine, Patient Dead On Arrival & 1 & $£ 8,724$ & $£ 8,724$ & $f 8,724$ & 1 & $£ 8,724.32$ \\
\hline T02NA & Type 02 Non-Admitted & VB01Z & Emergency Medicine, Any Investigation with Category 5 Treatment & 752 & $f 120$ & f86 & f142 & 13 & $£ 89,899.99$ \\
\hline T02NA & Type 02 Non-Admitted & VB02Z & Emergency Medicine, Category 3 Investigation with Category 4 Treatment & 49 & $£ 257$ & f152 & f339 & 9 & $£ 12,602.94$ \\
\hline T02NA & Type 02 Non-Admitted & VB03Z & Emergency Medicine, Category 3 Investigation with Category 1-3 Treatment & 9,511 & $f 182$ & f102 & $£ 253$ & 18 & $£ 1,733,939.90$ \\
\hline T02NA & Type 02 Non-Admitted & VB04Z & Emergency Medicine, Category 2 Investigation with Category 4 Treatment & 229 & f179 & £121 & f188 & 14 & $£ 40,989.48$ \\
\hline T02NA & Type 02 Non-Admitted & VB05Z & Emergency Medicine, Category 2 Investigation with Category 3 Treatment & 3,217 & $£ 93$ & $f 70$ & f85 & 17 & $£ 298,756.74$ \\
\hline T02NA & Type 02 Non-Admitted & VB06Z & Emergency Medicine, Category 1 Investigation with Category 3-4 Treatment & 12,891 & f82 & f46 & f98 & 18 & $f 1,054,086.73$ \\
\hline T02NA & Type 02 Non-Admitted & VB07Z & Emergency Medicine, Category 2 Investigation with Category 2 Treatment & 4,076 & $f 148$ & f146 & f146 & 15 & $£ 601,924.39$ \\
\hline T02NA & Type 02 Non-Admitted & VB08Z & Emergency Medicine, Category 2 Investigation with Category 1 Treatment & 62,374 & f108 & $f 42$ & f135 & 19 & $f 6,713,672.35$ \\
\hline T02NA & Type 02 Non-Admitted & VB09Z & Emergency Medicine, Category 1 Investigation with Category 1-2 Treatment & 228,094 & f83 & $f 51$ & f95 & 20 & $£ 19,018,553.63$ \\
\hline T02NA & Type 02 Non-Admitted & VB11Z & Emergency Medicine, No Investigation with No Significant Treatment & 148,397 & $f 85$ & $£ 51$ & $f 128$ & 21 & $£ 12,685,150.12$ \\
\hline T03A & Type 03 Admitted & VB012 & Emergency Medicine, Any Investigation with Category 5 Treatment & 20 & $f 182$ & f110 & $£ 140$ & 9 & $£ 3,632.81$ \\
\hline T03A & Type 03 Admitted & VB02Z & Emergency Medicine, Category 3 Investigation with Category 4 Treatment & 208 & $f 248$ & f229 & $f 229$ & 7 & $f 51,591.54$ \\
\hline T03A & Type 03 Admitted & VB03Z & Emergency Medicine, Category 3 Investigation with Category 1-3 Treatment & 689 & $£ 212$ & f177 & £177 & 16 & $£ 146,384.71$ \\
\hline T03A & Type 03 Admitted & VB04Z & Emergency Medicine, Category 2 Investigation with Category 4 Treatment & 2,333 & f141 & f110 & f119 & 18 & $£ 328,200.34$ \\
\hline T03A & Type 03 Admitted & VB05Z & Emergency Medicine, Category 2 Investigation with Category 3 Treatment & 1,203 & $f 118$ & f95 & f112 & 19 & $£ 142,473.11$ \\
\hline T03A & Type 03 Admitted & VB06Z & Emergency Medicine, Category 1 Investigation with Category 3-4 Treatment & 7,988 & f53 & £43 & f59 & 23 & $£ 424,476.94$ \\
\hline T03A & Type 03 Admitted & VB07Z & Emergency Medicine, Category 2 Investigation with Category 2 Treatment & 5,755 & f84 & f52 & f117 & 26 & $£ 483,199.76$ \\
\hline T03A & Type 03 Admitted & VB08Z & Emergency Medicine, Category 2 Investigation with Category 1 Treatment & 29,034 & $£ 58$ & $£ 36$ & f67 & 30 & $£ 1,685,513.68$ \\
\hline T03A & Type 03 Admitted & VB09Z & Emergency Medicine, Category 1 Investigation with Category 1-2 Treatment & 74,874 & f58 & $£ 43$ & f87 & 31 & $£ 4,329,419.72$ \\
\hline
\end{tabular}




\begin{tabular}{|c|c|c|c|c|c|c|c|c|c|}
\hline T03A & Type 03 Admitted & VB10Z & Emergency Medicine, Dental Care & 7 & $f 53$ & $f 38$ & $f 43$ & 3 & $£ 371.28$ \\
\hline T03A & Type 03 Admitted & VB11Z & Emergency Medicine, No Investigation with No Significant Treatment & 270,185 & f58 & f59 & $f 59$ & 33 & $£ 15,610,126.72$ \\
\hline T03NA & Type 03 Non-Admitted & VB01Z & Emergency Medicine, Any Investigation with Category 5 Treatment & 96 & f194 & f105 & $f 204$ & 20 & $£ 18,595.80$ \\
\hline T03NA & Type 03 Non-Admitted & VBO2Z & Emergency Medicine, Category 3 Investigation with Category 4 Treatment & 162 & $f 326$ & f204 & $f 398$ & 18 & f52,844.98 \\
\hline T03NA & Type 03 Non-Admitted & VB03Z & Emergency Medicine, Category 3 Investigation with Category 1-3 Treatment & 22,616 & $f 89$ & f78 & f78 & 28 & $£ 2,015,702.24$ \\
\hline T03NA & Type 03 Non-Admitted & VB04Z & Emergency Medicine, Category 2 Investigation with Category 4 Treatment & 37,396 & f84 & f70 & $f 70$ & 44 & $£ 3,149,254.26$ \\
\hline TO3NA & Type 03 Non-Admitted & VB05Z & Emergency Medicine, Category 2 Investigation with Category 3 Treatment & 85,397 & $f 70$ & f62 & $\mathrm{f62}$ & 44 & $£ 5,966,785.48$ \\
\hline T03NA & Type 03 Non-Admitted & VB06Z & Emergency Medicine, Category 1 Investigation with Category 3-4 Treatment & 69,579 & f79 & f39 & $f 105$ & 46 & $£ 5,513,453.59$ \\
\hline T03NA & Type 03 Non-Admitted & VB07Z & Emergency Medicine, Category 2 Investigation with Category 2 Treatment & 86,777 & $£ 120$ & $f 98$ & $£ 138$ & 44 & $£ 10,392,250.12$ \\
\hline T03NA & Type 03 Non-Admitted & VB08Z & Emergency Medicine, Category 2 Investigation with Category 1 Treatment & 203,695 & f96 & $f 73$ & f116 & 48 & $£ 19,482,590.16$ \\
\hline T03NA & Type 03 Non-Admitted & VB09Z & Emergency Medicine, Category 1 Investigation with Category 1-2 Treatment & 786,022 & $f 62$ & $f 50$ & $f 75$ & 54 & $£ 49,076,037.60$ \\
\hline T03NA & Type 03 Non-Admitted & VB10Z & Emergency Medicine, Dental Care & 15,418 & f94 & f94 & f94 & 8 & $£ 1,442,145.51$ \\
\hline T03NA & Type 03 Non-Admitted & VB11Z & Emergency Medicine, No Investigation with No Significant Treatment & 777,592 & f56 & f44 & f68 & 53 & $£ 43,679,369.98$ \\
\hline T03NA & Type 03 Non-Admitted & VB99Z & Emergency Medicine, Patient Dead On Arrival & 815 & $f 65$ & f64 & f64 & 4 & $£ 52,905.59$ \\
\hline T04A & Type 04 Admitted & VB04Z & Emergency Medicine, Category 2 Investigation with Category 4 Treatment & 1,731 & $£ 246$ & $f 246$ & $f 246$ & 1 & $£ 425,254.77$ \\
\hline T04A & Type 04 Admitted & VB06Z & Emergency Medicine, Category 1 Investigation with Category 3-4 Treatment & 46 & $f 55$ & f55 & f55 & 1 & $£ 2,507.00$ \\
\hline T04A & Type 04 Admitted & VB07Z & Emergency Medicine, Category 2 Investigation with Category 2 Treatment & 5 & f47 & f47 & f47 & 1 & $£ 233.60$ \\
\hline T04A & Type 04 Admitted & VB08Z & Emergency Medicine, Category 2 Investigation with Category 1 Treatment & 60 & $f 39$ & $f 39$ & $£ 39$ & 1 & $£ 2,335.80$ \\
\hline T04A & Type 04 Admitted & VB09Z & Emergency Medicine, Category 1 Investigation with Category 1-2 Treatment & 1,316 & $£ 31$ & £31 & f31 & 1 & $£ 40,980.24$ \\
\hline T04A & Type 04 Admitted & VB11Z & Emergency Medicine, No Investigation with No Significant Treatment & 1,436 & f16 & f16 & f16 & 1 & $f 22,358.52$ \\
\hline T04NA & Type 04 Non-Admitted & VB01Z & Emergency Medicine, Any Investigation with Category 5 Treatment & 67,532 & f45 & f44 & f45 & 4 & $£ 3,015,356.74$ \\
\hline T04NA & Type 04 Non-Admitted & VB03Z & Emergency Medicine, Category 3 Investigation with Category 1-3 Treatment & 146,929 & $£ 38$ & $£ 32$ & $f 43$ & 6 & $f 5,597,201.96$ \\
\hline T04NA & Type 04 Non-Admitted & VB04Z & Emergency Medicine, Category 2 Investigation with Category 4 Treatment & 2 & $f 70$ & $f 70$ & $f 70$ & 1 & $£ 140.14$ \\
\hline T04NA & Type 04 Non-Admitted & VB05Z & Emergency Medicine, Category 2 Investigation with Category 3 Treatment & 707 & $f 67$ & $f 53$ & $f 81$ & 4 & $£ 47,519.29$ \\
\hline TO4NA & Type 04 Non-Admitted & VB06Z & Emergency Medicine, Category 1 Investigation with Category 3-4 Treatment & 79,931 & f37 & f36 & $f 36$ & 6 & $f 2,946,388.46$ \\
\hline T04NA & Type 04 Non-Admitted & VB07Z & Emergency Medicine, Category 2 Investigation with Category 2 Treatment & 208,614 & $f 42$ & $f 38$ & $f 38$ & 6 & $f 8,861,370.44$ \\
\hline T04NA & Type 04 Non-Admitted & VB08Z & Emergency Medicine, Category 2 Investigation with Category 1 Treatment & 14,265 & f66 & $f 70$ & $f 70$ & 6 & $£ 934,834.48$ \\
\hline T04NA & Type 04 Non-Admitted & VB09Z & Emergency Medicine, Category 1 Investigation with Category 1-2 Treatment & 259,559 & $f 55$ & f38 & $f 70$ & 8 & $f 14,378,321.34$ \\
\hline T04NA & Type 04 Non-Admitted & VB10Z & Emergency Medicine, Dental Care & 6 & $£ 38$ & $£ 36$ & $£ 41$ & 2 & $£ 227.46$ \\
\hline T04NA & Type 04 Non-Admitted & VB11Z & Emergency Medicine, No Investigation with No Significant Treatment & 640,171 & £37 & f34 & f41 & 15 & $£ 23,769,564.90$ \\
\hline T04NA & Type 04 Non-Admitted & VB99Z & Emergency Medicine, Patient Dead On Arrival & 4 & $f 262$ & f262 & $f 262$ & 1 & $f 1,049.28$ \\
\hline
\end{tabular}




\begin{tabular}{|l|l|l|l|l|l|l|l|l|l|}
\hline & & & Summed total attendances & $\mathbf{1 9 , 1 0 7 , 0 2 1}$ & & & & Total & $\mathbf{f 2 , 5 2 0 , 5 8 5 , 1 6 9 . 2 8}$ \\
\hline & & & & & & & & & \\
\hline
\end{tabular}

\section{F.6 Threshold analysis}

Table F.8: Output of threshold analysis for healthcare services costs using changes in case subject costs. p-values attained from Mann-Whitney analysis case versus control subjects. Mean average costs ( $f$ ) (standard error of the mean)

\begin{tabular}{|c|c|c|c|c|}
\hline$\%$ of case subjects' costs & $\begin{array}{c}\text { Cases } \\
(n=657243)\end{array}$ & $\begin{array}{c}\text { Controls } \\
(n=2628972)\end{array}$ & Difference $(95 \% \mathrm{Cl})$ & p-value* (d) \\
\hline \multicolumn{5}{|l|}{ Total Primary care } \\
\hline 15 & $829.62(0.93)$ & $1121.34(1.21)$ & $-291.73(-294.72--288.74)$ & $<0.001(-0.26)$ \\
\hline 20 & $1106.15(1.24)$ & & $-15.19(-18.60--11.79)$ & $<0.001(-0.01)$ \\
\hline 20.2 & $1117.22(1.26)$ & & $-4.13(-7.54--0.71)$ & $<0.05(-0.003)$ \\
\hline 20.3 & $1122.75(1.26)$ & & $1.40(-2.02-4.83)$ & $0.422(0.001)$ \\
\hline 20.4 & $1128.28(1.27)$ & & $6.93(3.50-10.37)$ & $<0.001(0.01)$ \\
\hline 21 & $1161.46(1.31)$ & & $40.12(36.63-43.60)$ & $<0.001(0.03)$ \\
\hline 22 & $1216.77(1.37)$ & & $95.43(91.85-99.00)$ & $<0.001(0.07)$ \\
\hline 23 & $1272.08(1.43)$ & & $150.73(147.06-154.40)$ & $<0.001(0.11)$ \\
\hline 25 & $1382.69(1.56)$ & & $261.35(257.49-265.21)$ & $<0.001(0.18)$ \\
\hline \multicolumn{5}{|l|}{ Total Secondary Care } \\
\hline 80 & $7314.02(23.40)$ & $8009.06(16.27)$ & $-695.04(-750.89--639.18)$ & $<0.001(0.03)$ \\
\hline 86 & $7862.58(25.15)$ & & $-146.48(-205.20--87.77)$ & $<0.001(0.01)$ \\
\hline 87 & $7954.00(25.44)$ & & $-55.06(-114.25-4.14)$ & $0.068(-0.003)$ \\
\hline 87.5 & $7999.71(25.59)$ & & $-9.35(-68.78-50.09)$ & $0.758(0.00)$ \\
\hline 88 & $8045.43(25.74)$ & & $36.37(-23.31-96.05)$ & $0.232(0.002)$ \\
\hline 88.5 & $8091.14(25.88)$ & & $82.08(22.16-142.00)$ & $<0.05(0.004)$ \\
\hline 90 & $8228.28(26.32)$ & & $219.22(158.57-279.87)$ & $<0.001(0.01)$ \\
\hline 92 & $8411.13(26.91)$ & & $402.07(340.44-463.70)$ & $<0.001(0.02)$ \\
\hline 95 & $8685.40(27.78)$ & & $676.34(613.24-739.45)$ & $<0.001(0.03)$ \\
\hline
\end{tabular}




\begin{tabular}{|c|c|c|c|c|}
\hline \multicolumn{5}{|l|}{ Total costs } \\
\hline 50 & $8226.68(16.54)$ & $9757.27(16.77)$ & $-1530.60(-1576.76--1484.44)$ & $<0.001(-0.09)$ \\
\hline 55 & 9049.34 (18.19) & & $-707.93(-756.42--659.44)$ & $<0.001(-0.04)$ \\
\hline 58 & 9542.94 (19.19) & & $-214.33(-264.27--164.39)$ & $<0.001(-0.01)$ \\
\hline 58.5 & $9625.21(19.35)$ & & $-132.06(-182.25--81.88)$ & $<0.001(-0.01)$ \\
\hline 59 & $9707.48(19.52)$ & & $-49.79(-100.22--0.64)$ & $0.053(-0.003)$ \\
\hline 59.5 & $9789.74(19.68)$ & & $32.47(-18.20-83.15)$ & $0.209(0.002)$ \\
\hline 60 & $9872.01(19.85)$ & & $114.74(63.82-165-66)$ & $<0.001(0.01)$ \\
\hline 65 & $10694.68(21.50)$ & & $937.41(883.97-990.85)$ & $<0.001(0.05)$ \\
\hline 70 & $11517.35(23.16)$ & & $1760.08(1704.04-1816.11)$ & $<0.001(0.09)$ \\
\hline
\end{tabular}

Holm-Bonferroni confirmed it was correct to reject the null hypothesis 


\section{Appendix G Prescribing Persistence}

\section{G.1 Descriptive annual data for prescribing persistence}

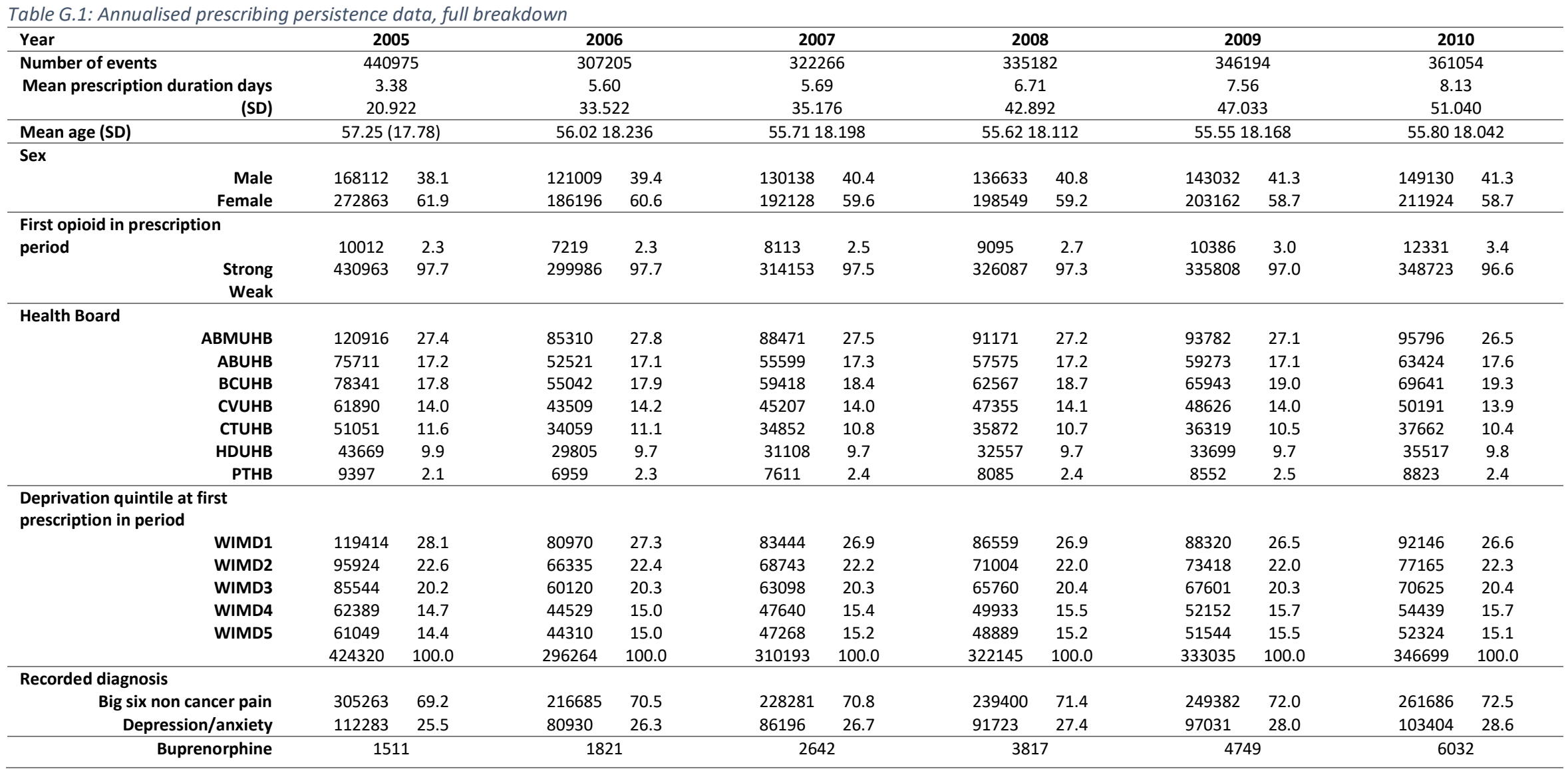




\begin{tabular}{|c|c|c|c|c|c|c|c|c|c|c|c|}
\hline Codeine & \multicolumn{2}{|c|}{248193} & \multicolumn{2}{|c|}{194023} & \multicolumn{2}{|c|}{208489} & \multicolumn{2}{|c|}{219476} & \multicolumn{2}{|c|}{226958} & 236636 \\
\hline Dextropropoxyphene & \multicolumn{2}{|c|}{40037} & \multicolumn{2}{|c|}{4272} & \multicolumn{2}{|c|}{1684} & \multicolumn{2}{|c|}{883} & \multicolumn{2}{|c|}{685} & 606 \\
\hline Dihydrocodeine & \multicolumn{2}{|c|}{95674} & \multicolumn{2}{|c|}{63929} & \multicolumn{2}{|c|}{62160} & \multicolumn{2}{|c|}{59877} & \multicolumn{2}{|c|}{57822} & 55898 \\
\hline Fentanyl & \multicolumn{2}{|c|}{1980} & \multicolumn{2}{|c|}{1723} & \multicolumn{2}{|c|}{1992} & \multicolumn{2}{|c|}{2354} & \multicolumn{2}{|c|}{2608} & 2866 \\
\hline Morphine & \multicolumn{2}{|c|}{4489} & \multicolumn{2}{|c|}{2994} & \multicolumn{2}{|c|}{3416} & \multicolumn{2}{|c|}{3924} & \multicolumn{2}{|c|}{4336} & 5398 \\
\hline Oxycodone & \multicolumn{2}{|c|}{1248} & 90 & & 116 & & 14 & & 202 & & 2556 \\
\hline Tapentadol & & & & & & & & & & & \\
\hline Tramadol & 464 & & 366 & & 399 & & 427 & & 463 & & 50443 \\
\hline Other & 135 & & 86 & & 78 & & 69 & & 65 & & 619 \\
\hline Year & 201 & & 201 & & 201 & & 20 & & 201 & & \\
\hline Number of events & 3771 & & 3971 & & 4120 & & 449 & & 5597 & & \\
\hline Mean prescription duration days & 8.5 & & 9.5 & & 9.7 & & 9.9 & & 15. & & \\
\hline (SD) & 53.4 & & 61.3 & & 61.6 & & 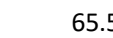 & & 118. & & \\
\hline Mean age (SD) & 55.761 & 3.001 & 55.881 & .928 & 56.101 & .848 & 56.361 & .708 & 56.781 & 7.388 & \\
\hline Sex & & & & & & & & & & & \\
\hline Male & 156156 & 41.4 & 164291 & 41.4 & 169455 & 41.1 & 184564 & 41.1 & 228299 & 40.8 & \\
\hline Female & 220956 & 58.6 & 232813 & 58.6 & 242555 & 58.9 & 264605 & 58.9 & 331463 & 59.2 & \\
\hline First opioid in prescription & & & & & & & & & & & \\
\hline period & 14163 & 3.8 & 17171 & 4.3 & 21558 & 5.2 & 27323 & 6.1 & 38340 & 6.8 & \\
\hline $\begin{array}{r}\text { Strong } \\
\text { Weak }\end{array}$ & 362949 & 96.2 & 379933 & 95.7 & 390452 & 94.8 & 421846 & 93.9 & 521422 & 93.2 & \\
\hline Health Board & & & & & & & & & & & \\
\hline ABMUHB & 99530 & 26.4 & 102392 & 25.8 & 104214 & 25.3 & 111294 & 24.8 & 141094 & 25.2 & \\
\hline АВUHB & 65956 & 17.5 & 69934 & 17.6 & 71937 & 17.5 & 77934 & 17.4 & 96513 & 17.2 & \\
\hline BCUHB & 73881 & 19.6 & 80452 & 20.3 & 88452 & 21.5 & 99911 & 22.2 & 123183 & 22.0 & \\
\hline CVUHB & 52052 & 13.8 & 55508 & 14.0 & 56909 & 13.8 & 61713 & 13.7 & 76817 & 13.7 & \\
\hline СTUHB & 39036 & 10.4 & 40734 & 10.3 & 41403 & 10.0 & 45034 & 10.0 & 55711 & 10.0 & \\
\hline HDUHB & 36779 & 9.8 & 37898 & 9.5 & 38006 & 9.2 & 41545 & 9.2 & 52499 & 9.4 & \\
\hline PTHB & 9878 & 2.6 & 10186 & 2.6 & 11089 & 2.7 & 11738 & 2.6 & 13945 & 2.5 & \\
\hline $\begin{array}{l}\text { Deprivation quintile at first } \\
\text { prescription in period }\end{array}$ & & & & & & & & & & & \\
\hline WIMD1 & 95575 & 26.4 & 101238 & 26.6 & 105037 & 26.5 & 114978 & 26.7 & 143403 & 26.8 & \\
\hline WIMD2 & 81013 & 22.4 & 85608 & 22.5 & 88023 & 22.2 & 95370 & 22.1 & 118945 & 22.2 & \\
\hline WIMD3 & 74003 & 20.4 & 76846 & 20.2 & 80709 & 20.4 & 88895 & 20.6 & 110162 & 20.6 & \\
\hline WIMD4 & 56965 & 15.7 & 59884 & 15.7 & 62349 & 15.8 & 67517 & 15.7 & 83403 & 15.6 & \\
\hline WIMD5 & 54539 & 15.1 & 57729 & 15.1 & 59594 & 15.1 & 64026 & 14.9 & 79883 & 14.9 & \\
\hline & 362095 & 100.0 & 381305 & 100.0 & 395712 & 100.0 & 430786 & 100.0 & 535796 & 100.0 & \\
\hline
\end{tabular}




\begin{tabular}{|c|c|c|c|c|c|c|c|c|c|c|}
\hline \multicolumn{11}{|l|}{ Recorded diagnosis } \\
\hline Big six non cancer pain & 275129 & 73.0 & 291646 & 73.4 & 303601 & 73.7 & 332683 & 74.1 & 418741 & 74.8 \\
\hline Depression/anxiety & 109881 & 29.1 & 118635 & 29.9 & 125358 & 30.4 & 138841 & 30.9 & 174963 & 31.3 \\
\hline Buprenorphine & \multicolumn{2}{|c|}{6870} & \multicolumn{2}{|c|}{7413} & \multicolumn{2}{|c|}{7966} & \multicolumn{2}{|c|}{8620} & \multicolumn{2}{|c|}{10135} \\
\hline Codeine & \multicolumn{2}{|c|}{247418} & \multicolumn{2}{|c|}{260215} & \multicolumn{2}{|c|}{268130} & \multicolumn{2}{|c|}{297982} & \multicolumn{2}{|c|}{379209} \\
\hline Dextropropoxyphene & \multicolumn{2}{|c|}{466} & \multicolumn{2}{|c|}{402} & \multicolumn{2}{|c|}{281} & \multicolumn{2}{|c|}{307} & \multicolumn{2}{|c|}{343} \\
\hline Dihydrocodeine & \multicolumn{2}{|c|}{54249} & \multicolumn{2}{|c|}{52528} & \multicolumn{2}{|c|}{52427} & \multicolumn{2}{|c|}{52869} & \multicolumn{2}{|c|}{62892} \\
\hline Fentanyl & \multicolumn{2}{|c|}{3025} & \multicolumn{2}{|c|}{3292} & \multicolumn{2}{|c|}{3720} & \multicolumn{2}{|c|}{3949} & \multicolumn{2}{|c|}{4454} \\
\hline Morphine & \multirow{2}{*}{\multicolumn{2}{|c|}{$\begin{array}{l}6720 \\
2925\end{array}$}} & \multicolumn{2}{|c|}{8909} & \multirow{2}{*}{\multicolumn{2}{|c|}{$\begin{array}{c}12822 \\
3363\end{array}$}} & \multicolumn{2}{|c|}{17788} & \multicolumn{2}{|c|}{26462} \\
\hline Oxycodone & & & & & & & \multirow{2}{*}{\multicolumn{2}{|c|}{$\begin{array}{l}3755 \\
373\end{array}$}} & \multicolumn{2}{|c|}{5008} \\
\hline Tapentadol & \multicolumn{2}{|c|}{31} & \multicolumn{2}{|c|}{$\begin{array}{l}3436 \\
114\end{array}$} & \multicolumn{2}{|c|}{195} & & & 71 & \\
\hline Tramadol & \multicolumn{2}{|c|}{54874} & 602 & & 626 & & 6305 & & 700 & \\
\hline Other & 53 & & 50 & & 47 & & 427 & & 51 & \\
\hline
\end{tabular}

\section{G.1.1 Annual descriptive data for prescribing persistence greater than $\mathbf{3 1}$ days}

Table G.2: Descriptive data with all single, not repeated within 31-day events removed

\begin{tabular}{|c|c|c|c|c|c|c|c|c|c|c|c|c|}
\hline Year & \multicolumn{2}{|l|}{2005} & \multicolumn{2}{|l|}{2006} & \multicolumn{2}{|l|}{2007} & \multicolumn{2}{|l|}{2008} & \multicolumn{2}{|l|}{2009} & \multicolumn{2}{|l|}{2010} \\
\hline Number of events & \multicolumn{2}{|l|}{22722} & \multicolumn{2}{|l|}{21331} & \multicolumn{2}{|l|}{23900} & \multicolumn{2}{|l|}{27490} & \multicolumn{2}{|l|}{31292} & \multicolumn{2}{|l|}{34415} \\
\hline Mean prescription duration days & \multicolumn{2}{|l|}{65.58} & \multicolumn{2}{|l|}{80.64} & \multicolumn{2}{|l|}{76.75} & \multicolumn{2}{|l|}{81.79} & \multicolumn{2}{|l|}{83.61} & \multicolumn{2}{|l|}{85.35} \\
\hline (SD) & \multicolumn{2}{|l|}{66.452} & \multirow{2}{*}{\multicolumn{2}{|c|}{$\begin{array}{l}100.665 \\
59.6316 .837\end{array}$}} & \multirow{2}{*}{\multicolumn{2}{|c|}{$\begin{array}{l}105.975 \\
58.6916 .883\end{array}$}} & \multirow{2}{*}{\multicolumn{2}{|c|}{$\begin{array}{l}127.634 \\
58.0216 .720\end{array}$}} & \multirow{2}{*}{\multicolumn{2}{|c|}{$\begin{array}{l}134.592 \\
57.7616 .720\end{array}$}} & \multirow{2}{*}{\multicolumn{2}{|c|}{$\begin{array}{l}144.018 \\
57.6816 .551\end{array}$}} \\
\hline Mean age (SD) & 61.53 & 565 & & & & & & & & & & \\
\hline \multicolumn{13}{|l|}{ Sex } \\
\hline Male & 8881 & 39.1 & 8420 & 39.5 & 9754 & 40.8 & 11545 & 42.0 & 13543 & 43.3 & 14955 & 43.5 \\
\hline Female & 13841 & 60.9 & 12911 & 60.5 & 14146 & 59.2 & 15945 & 58.0 & 17749 & 56.7 & 19460 & 56.5 \\
\hline \multicolumn{13}{|l|}{ First opioid in prescription } \\
\hline Strong & 21469 & 94.5 & 20725 & 97.2 & 23195 & 97.1 & 26636 & 96.9 & 30136 & 96.3 & 33010 & 95.9 \\
\hline \multicolumn{13}{|l|}{ Weak } \\
\hline \multicolumn{13}{|l|}{ Health Board } \\
\hline ABMUHB & 13797 & 60.7 & 13722 & 64.3 & 14365 & 60.1 & 15451 & 56.2 & 16816 & 53.7 & 17507 & 50.9 \\
\hline ABUHB & 2058 & 9.1 & 1764 & 8.3 & 2162 & 9.0 & 2614 & 9.5 & 3096 & 9.9 & 3658 & 10.6 \\
\hline BCUHB & 1690 & 7.4 & 1487 & 7.0 & 2040 & 8.5 & 2748 & 10.0 & 3548 & 11.3 & 4238 & 12.3 \\
\hline CVUHB & 1454 & 6.4 & 1159 & 5.4 & 1560 & 6.5 & 1949 & 7.1 & 2380 & 7.6 & 2740 & 8.0 \\
\hline CTUHB & 1443 & 6.4 & 1161 & 5.4 & 1265 & 5.3 & 1573 & 5.7 & 1857 & 5.9 & 2111 & 6.1 \\
\hline HDUHB & 1673 & 7.4 & 1348 & 6.3 & 1658 & 6.9 & 2081 & 7.6 & 2429 & 7.8 & 2827 & 8.2 \\
\hline PYHB & 607 & 2.7 & 690 & 3.2 & 850 & 3.6 & 1074 & 3.9 & 1166 & 3.7 & 1334 & 3.9 \\
\hline
\end{tabular}




\begin{tabular}{|c|c|c|c|c|c|c|c|c|c|c|c|c|}
\hline \multicolumn{13}{|l|}{$\begin{array}{l}\text { Deprivation quintile at first } \\
\text { prescription in period }\end{array}$} \\
\hline WIMD1 & 7003 & 32.1 & 6733 & 32.5 & 7411 & 32.0 & 8238 & 31.0 & 9086 & 30.0 & 9904 & 29.9 \\
\hline WIMD2 & 5143 & 23.5 & 4889 & 23.6 & 5290 & 22.9 & 6080 & 22.9 & 6916 & 22.9 & 7596 & 22.9 \\
\hline WIMD3 & 4333 & 19.8 & 4002 & 19.3 & 4597 & 19.9 & 5328 & 20.1 & 6039 & 20.0 & 6771 & 20.5 \\
\hline WIMD4 & 2508 & 11.5 & 2383 & 11.5 & 2815 & 12.2 & 3363 & 12.7 & 4061 & 13.4 & 4410 & 13.3 \\
\hline \multirow[t]{2}{*}{ WIMD5 } & 2863 & 13.1 & 2691 & 13.0 & 3021 & 13.1 & 3539 & 13.3 & 4150 & 13.7 & 4423 & 13.4 \\
\hline & 21850 & 100.0 & 20698 & 100.0 & 23134 & 100.0 & 26548 & 100.0 & 30252 & 100.0 & 33104 & 100.0 \\
\hline \multicolumn{13}{|l|}{ Recorded diagnosis } \\
\hline Big six non cancer pain & \multirow{2}{*}{\multicolumn{2}{|c|}{$\begin{array}{l}14165 \\
528223.2\end{array} \quad 62.3$}} & \multirow{2}{*}{\multicolumn{2}{|c|}{$\begin{array}{l}1512070.9 \\
568726.7\end{array}$}} & \multirow{2}{*}{\multicolumn{2}{|c|}{$\begin{array}{l}1742472.9 \\
6580 \quad 27.5\end{array}$}} & \multirow{2}{*}{\multicolumn{2}{|c|}{$\begin{array}{l}2062375.0 \\
784328.5\end{array}$}} & \multirow{2}{*}{\multicolumn{2}{|c|}{$\begin{array}{l}2361975.5 \\
925429.6\end{array}$}} & \multirow{2}{*}{\multicolumn{2}{|c|}{$\begin{array}{l}2617676.1 \\
1037630.1\end{array}$}} \\
\hline Depression/anxiety & & & & & & & & & & & & \\
\hline More than $\mathbf{3}$ months & 5122 & 22.5 & 5301 & 24.9 & 5396 & 22.6 & 6343 & 23.1 & 7395 & 23.6 & 8212 & 23.9 \\
\hline More than 6 months & 1736 & 7.6 & 2370 & 11.1 & 2359 & 9.9 & 2870 & 10.4 & 3277 & 10.5 & 3681 & 10.7 \\
\hline Year & \multicolumn{2}{|l|}{2011} & \multicolumn{2}{|l|}{2012} & \multicolumn{2}{|l|}{2013} & \multicolumn{2}{|l|}{2014} & \multicolumn{2}{|l|}{2015} & & \\
\hline Number of events & \multicolumn{2}{|c|}{37228} & \multicolumn{2}{|l|}{40898} & \multicolumn{2}{|l|}{43078} & \multicolumn{2}{|l|}{47201} & \multicolumn{2}{|l|}{61411} & & \\
\hline Mean prescription duration days & \multicolumn{2}{|l|}{86.11} & \multicolumn{2}{|l|}{92.34} & \multicolumn{2}{|l|}{92.86} & \multicolumn{2}{|l|}{94.74} & \multicolumn{2}{|c|}{142.24} & & \\
\hline (SD) & \multicolumn{2}{|c|}{149.226} & 169.99 & & 169.16 & & 181.190 & & 332.41 & & & \\
\hline Mean age (SD) & 57.50 & & 57.35 & 371 & 57.471 & & 57.561 & 189 & 57.76 & 040 & & \\
\hline Sex & & & & & & & & & & & & \\
\hline Male & 16303 & 43.8 & 18082 & 44.2 & 18980 & 44.1 & 20980 & 44.4 & 27370 & 44.6 & & \\
\hline Female & 20925 & 56.2 & 22816 & 55.8 & 24098 & 55.9 & 26221 & 55.6 & 34041 & 55.4 & & \\
\hline $\begin{array}{l}\text { First opioid in prescription } \\
\text { period }\end{array}$ & 1736 & 4.7 & 2305 & 5.6 & 3045 & 7.1 & 3865 & 8.2 & 6316 & 10.3 & & \\
\hline Strong & 35492 & 95.3 & 38593 & 94.4 & 40033 & 92.9 & 43336 & 91.8 & 55095 & 89.7 & & \\
\hline Weak & & & & & & & & & & & & \\
\hline Health Board & & & & & & & & & & & & \\
\hline АВMUHB & 17927 & 48.2 & 19022 & 46.5 & 19065 & 44.3 & 19907 & 42.2 & 25647 & 41.8 & & \\
\hline ABUHB & 4021 & 10.8 & 4629 & 11.3 & 4843 & 11.2 & 5369 & 11.4 & 7155 & 11.7 & & \\
\hline BCUHB & 5127 & 13.8 & 6068 & 14.8 & 7122 & 16.5 & 8624 & 18.3 & 11229 & 18.3 & & \\
\hline CVUHB & 3232 & 8.7 & 3598 & 8.8 & 3883 & 9.0 & 4417 & 9.4 & 5492 & 8.9 & & \\
\hline CTUHB & 2284 & 6.1 & 2468 & 6.0 & 2629 & 6.1 & 2855 & 6.0 & 3826 & 6.2 & & \\
\hline HDUHB & 3099 & 8.3 & 3478 & 8.5 & 3685 & 8.6 & 4035 & 8.5 & 5664 & 9.2 & & \\
\hline PYHB & 1538 & 4.1 & 1635 & 4.0 & 1851 & 4.3 & 1994 & 4.2 & 2398 & 3.9 & & \\
\hline $\begin{array}{l}\text { Deprivation quintile at first } \\
\text { prescription in period }\end{array}$ & & & & & & & & & & & & \\
\hline WIMD1 & 10379 & 29.0 & 11602 & 29.5 & 11941 & 28.8 & 13129 & 29.0 & 17264 & 29.5 & & \\
\hline WIMD2 & 8310 & 23.3 & 9068 & 23.0 & 9420 & 22.7 & 10024 & 22.1 & 13012 & 22.2 & & \\
\hline WIMD3 & 7390 & 20.7 & 7928 & 20.1 & 8530 & 20.6 & 9508 & 21.0 & 12132 & 20.7 & & \\
\hline WIMD4 & 4898 & 13.7 & 5413 & 13.7 & 5911 & 14.3 & 6574 & 14.5 & 8331 & 14.2 & & \\
\hline
\end{tabular}




\begin{tabular}{|c|c|c|c|c|c|c|c|c|c|c|}
\hline WIMD5 & $\begin{array}{l}4759 \\
35736\end{array}$ & $\begin{array}{l}13.3 \\
100.0\end{array}$ & $\begin{array}{l}5362 \\
39373\end{array}$ & $\begin{array}{l}13.6 \\
100.0\end{array}$ & $\begin{array}{l}5670 \\
41472\end{array}$ & $\begin{array}{l}13.7 \\
100.0\end{array}$ & $\begin{array}{l}6066 \\
45301\end{array}$ & $\begin{array}{l}13.4 \\
100.0\end{array}$ & $\begin{array}{l}7812 \\
58551\end{array}$ & $\begin{array}{l}13.3 \\
100.0\end{array}$ \\
\hline \multicolumn{11}{|l|}{ Recorded diagnosis } \\
\hline Big six non cancer pain & \multicolumn{2}{|c|}{2852476.6} & \multicolumn{2}{|c|}{3154377.1} & \multicolumn{2}{|c|}{3318677.0} & \multicolumn{2}{|c|}{3629276.9} & \multicolumn{2}{|c|}{4700676.5} \\
\hline Depression/anxiety & \multicolumn{2}{|c|}{1147630.8} & \multicolumn{2}{|c|}{1284931.4} & \multicolumn{2}{|c|}{1388432.2} & \multicolumn{2}{|c|}{1528232.4} & \multicolumn{2}{|c|}{2041733.2} \\
\hline More than 3 months & 8879 & 23.9 & 10380 & 25.4 & 10948 & 25.4 & 12037 & 25.5 & 19686 & 32.1 \\
\hline More than 6 months & 4038 & 10.8 & 4814 & 11.8 & 5080 & 11.8 & 5593 & 11.8 & 10560 & 17.2 \\
\hline
\end{tabular}




\section{G.2 Prescribing persistence by gender}

Women were recipients of $2,557,215$ periods of prescribing which equated to $59.4 \%$ of the total. The proportions of women compared to men remained similar for all the prescribing persistence categories examined (Table G.3). The difference in numbers of women compared to men was statistically significant (Mann-Whitney $\mathrm{p}<.001, \mathrm{~d}_{\text {Cohen }}=3.2, \eta^{2}=0.72$ ).

\section{G.2.1 Analysis by gender differences}

Examined by gender, men were noted to have an average prescribing persistence of $8.94 \pm 65.2$ days and women $8.36 \pm 61.9$ days (Figure G.1). There was no statistical difference in prescribing persistence between genders (MannWhitney $\mathrm{p}>0.05, \mathrm{~d}_{\text {cohen }}=0.1, \eta^{2}=0.002$ ). Prescription persistence increased 402.4\% (from 3.39 to 17.03 days) for men between 2005 and 2015 (Table G.3). In the same period, persistence increased $333.8 \%$ (from 3.37 to 14.62 days) for women.

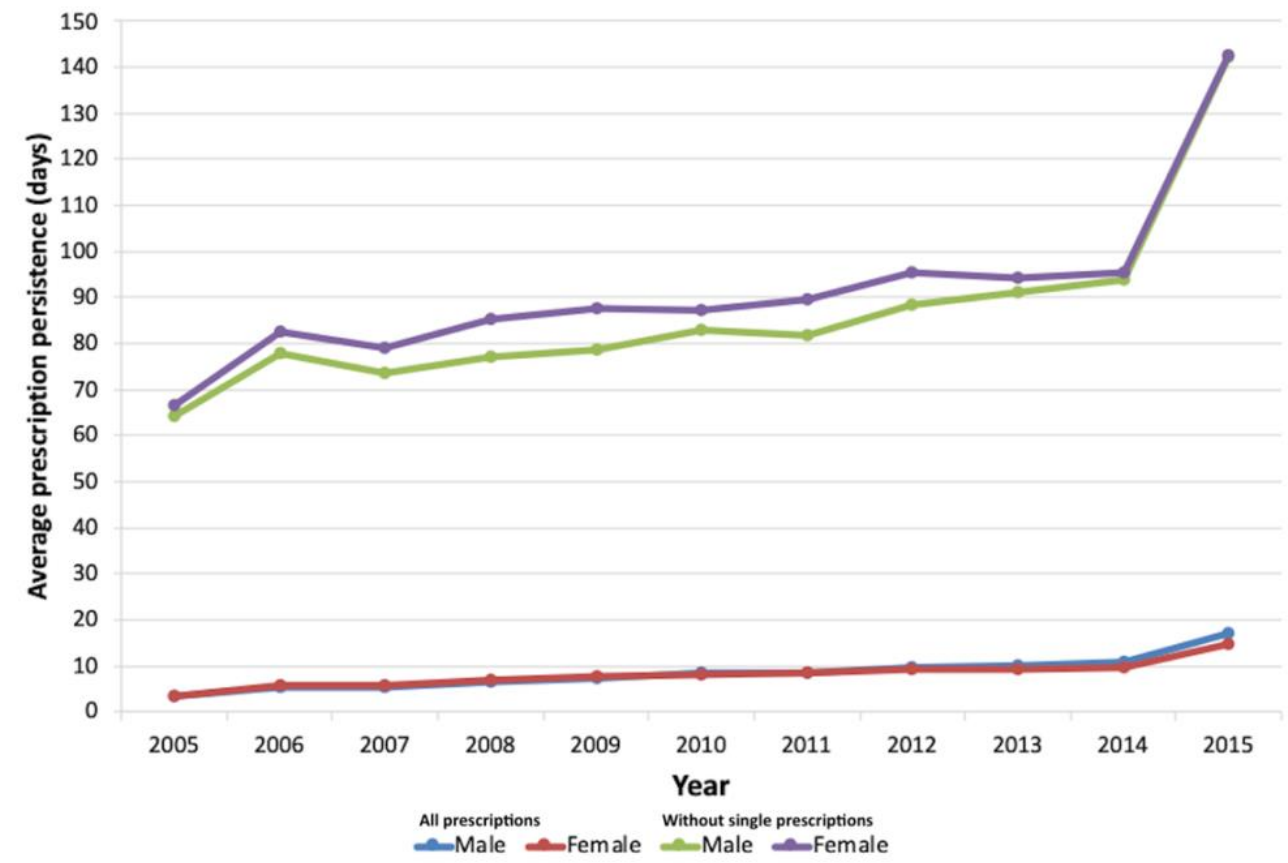

Figure G.1: Trends in prescribing persistence for all prescriptions and prescribing events with 2 or more consecutive prescriptions, stratified by gender

Although women received many more prescriptions than men during the study, there was very little difference between the average duration of prescribing persistence on an annual basis or when all data was examined (Figure G.1).

When one-off prescriptions were removed from analysis (where another prescription was not issued within 31 days from the preceding one), there was again, little difference between genders, in terms of days persistence (Table G.3). Men had a larger percentage increase in persistence but by the end of the 11 years examined, both genders were recorded as having the same prescribing persistence (Figure G.1). Unsurprisingly, a statistically significant difference in 
prescribing persistence between genders was not found (Mann-Whitney $p>0.05$, $\mathrm{d}_{\text {Cohen }}=0.63, \eta^{2}=.09$ ).

\section{G.2.2 Extended durations of prescribing persistence by gender}

Women had an average of $38 \%$ more prescribing events with a persistence of between 3 and 6 months although a greater percentage increase in the number of events was noted in men during the 11 years analysed (Table G.3). Despite the difference in the overall number of events, a statistically significant difference was not determined (Mann-Whitney $\mathrm{p} \geq .05$, $\mathrm{d}_{\text {Cohen }}=.94, \eta^{2}=.2$ ).

Table G.3: Trends in prescription persistence (in days), stratified by gender per 1000 gender-adjusted population.

\begin{tabular}{|c|c|c|}
\hline & Female & Male \\
\hline Mean duration (days) \pm SD & $8.36 \pm 62.0$ & $8.94 \pm 65.2$ \\
\hline 2005 & $3.4 \pm 21.1$ & $3.4 \pm 20.7$ \\
\hline 2015 & $14.6 \pm 114.4$ & $17.0 \pm 124.8$ \\
\hline Percentage increase (\%) & 333.8 & 402.4 \\
\hline Duration (days) without one-off doses \pm SD & $96.2 \pm 189.1$ & $92.8 \pm 190.7$ \\
\hline 2005 & $66.5 \pm 67.4$ & $64.2 \pm 65.0$ \\
\hline 2015 & $142.4 \pm 330.4$ & $142.1 \pm 335.0$ \\
\hline Percentage increase (\%) & 114.3 & 121.2 \\
\hline Persistence of $3-6$ months & $2813.6 \pm 979.0$ & $2033.8 \pm 866.6$ \\
\hline 2005 & 2084 & 1302 \\
\hline 2015 & 5117 & 4009 \\
\hline Percentage change (\%) & 145.5 & 207.9 \\
\hline \multicolumn{3}{|l|}{ Persistence of $3-6$ months per 1000} \\
\hline population & $2.32 \pm 0.80$ & $1.74 \pm 0.71$ \\
\hline 2005 & 1.76 & 1.15 \\
\hline 2015 & 4.18 & 3.37 \\
\hline Percentage change (\%) & 137.7 & 192.6 \\
\hline Persistence more than 6 months & $2478.8 \pm 1314.5$ & $1737.4 \pm 1120.6$ \\
\hline 2005 & 1115 & 621 \\
\hline 2015 & 5914 & 4646 \\
\hline Rate change (\%) & 430.4 & 648.1 \\
\hline Persistence more than 6 months per 1000 population & $2.04 \pm 1.06$ & $1.48 \pm 0.93$ \\
\hline 2005 & 0.94 & 0.55 \\
\hline 2015 & 4.83 & 3.91 \\
\hline Rate change (\%) & 413.5 & 610.9 \\
\hline
\end{tabular}

When adjusted to gender-population, women had 33\% more prescribing events with a persistence of between 3 and 6 months than men (2.32 versus 1.74 events per 1000 respectively) (Figure G.2). A larger percentage increase was noted in men (192.6\%, from 1.15 to 3.37 events per 1000 population) than women (137.7\%, from 1.76 to 4.18 events per 1000 population) (Table G.3). The average number of events per 1000 population, with persistence of between 3 to 6 months was not found to be statistically different (Mann-Whitney $p>.05, d_{\text {Cohen }}=$ $\left..91, \eta^{2}=.17\right)$. 


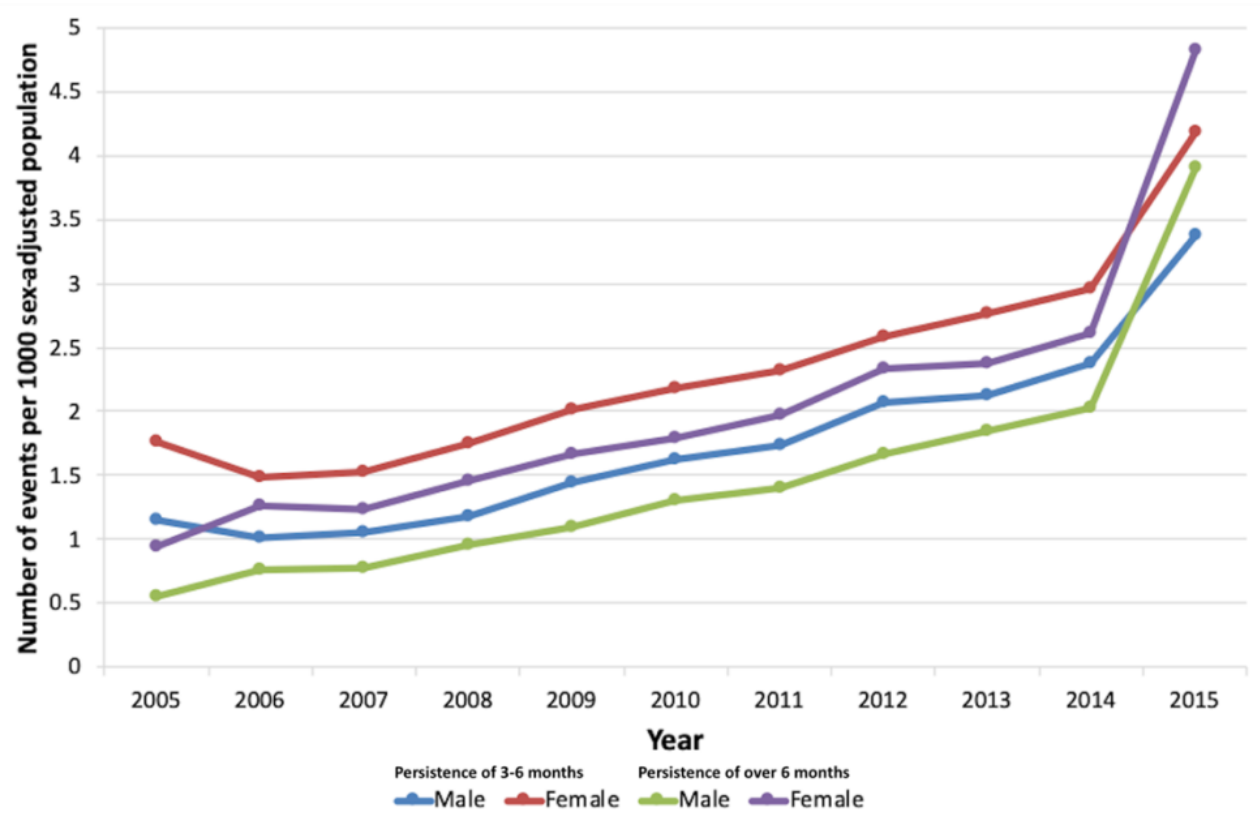

Figure G.2: Trends in the number of prescribing events with persistence of 3-6 months and over 6 months, stratified by gender

Both genders demonstrated a large increase in the number of prescribing events with a persistence of more than 6 months between 2005 and 2015 (Figure G.2). Men had the largest percentage increase (648.1\%, from 621 to 4646 events) although women had an average of $43 \%$ more events than men during the study ( 2478.8 versus 1737.4 events respectively). Despite the difference in average number of events, there was not a statistical difference between men and women, in respect of prescribing persistence of more than 6 months (MannWhitney $\mathrm{p}>0.05, \mathrm{~d}_{\text {Cohen }}=0.80, \eta^{2}=0.14$ ). Adjusting for population lead to little change in the differences noted between men and women (Table G.3) and no statistical difference was noted in number of events per 1000 (Mann-Whitney $p>0.5, d_{\text {cohen }}=0.73, \eta^{2}=0.12$ ) between 2005 and 2015 .

\section{G.3 Analysis by age-group}

Mean average prescribing persistence ranged from 1.9 days $(S D=0.65)$ for the 18-24 years age-group to 10.1 days $(S D=1.8)$ in the 85 years and over age-group (Table G.4). Average duration of prescription persistence, when all prescriptions were included, was significantly different between the 6 age-groups used in the study (Kruskal-Wallis $p<0.01, \mathrm{~d}_{\text {cohen }}=1.97, \eta^{2}=0.492$ ). Dunn's pairwise analysis confirmed statistically significant differences between the youngest (18-24 years) and all age groups aged 45 and over (Table G.5). Statistically significant differences did not exist between any of the other age groups, in relation to the average duration of prescribing persistence. 


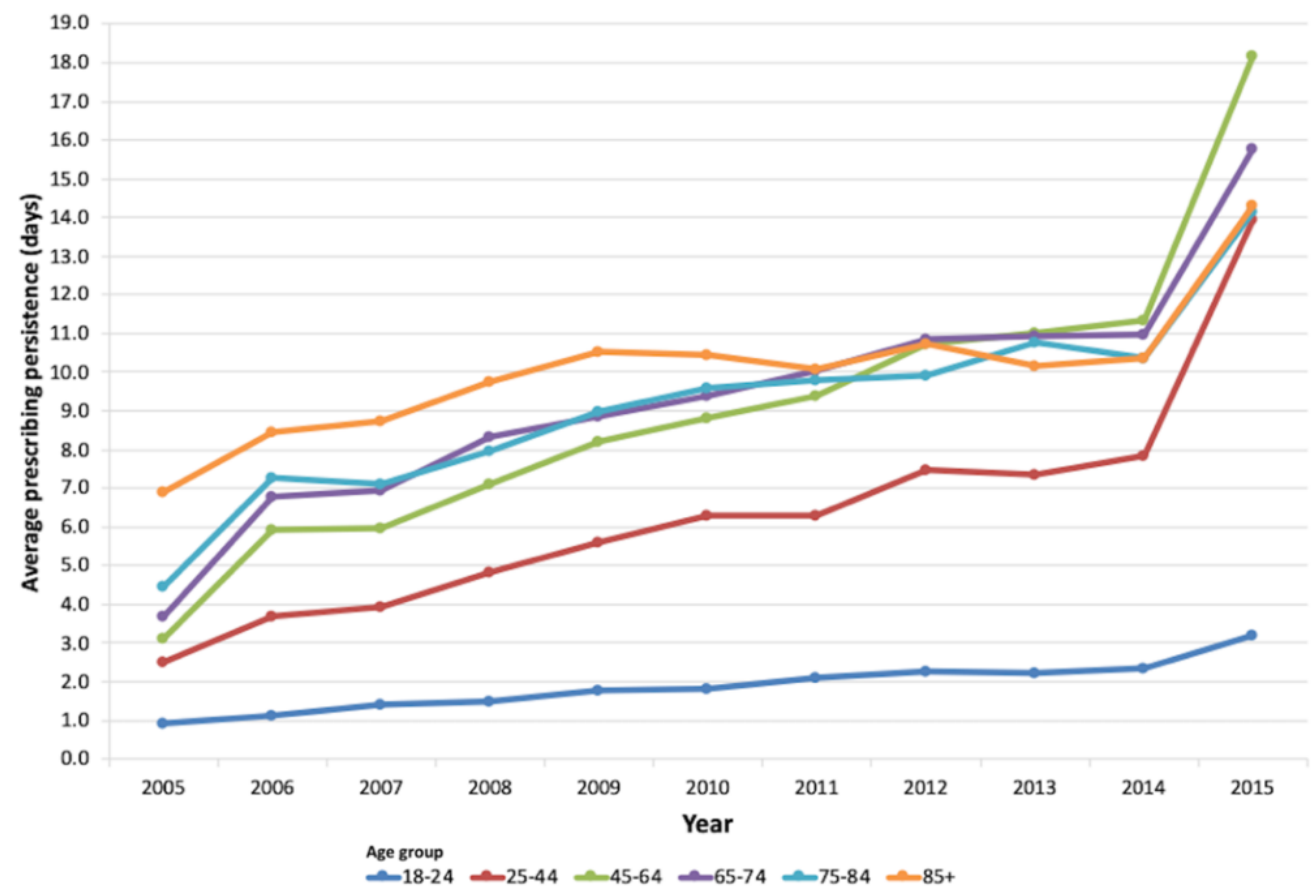

Figure G.3: Trends in average prescription persistence 2005 - 2015, stratified by age-group

Between 2005 and 2015, the largest increase in prescription persistence days was noted in the $45-64$ years group ( $481.5 \%$, from 3.1 to 18.2 days) (Figure G.3). That age-group had the longest average prescribing persistence (18.2 days) in 2015 , despite the oldest age-group ( $\geq 85$ years) having the highest average duration during the study (Table G.4).

Removing all single prescription events i.e. when a prescription was not followed by a subsequent prescription within 31 days, resulted in average durations lengthening (Table G.4) with a range from 43.7 days (18 -24 years) to 103.9 days ( $\geq 85$ years) - again, the older age groups had prescribing persistence of around double that of the youngest people receiving prescribed opioids (Figure G.4). 
Table G.4: Trends in prescribing persistence (in days), stratified by age group.

\begin{tabular}{|c|c|c|c|c|c|c|}
\hline \multirow[t]{2}{*}{ Mean annualised data $2005-2015$ (SD) } & \multicolumn{6}{|c|}{ Age group (years) } \\
\hline & $18-24$ & $25-44$ & $45-64$ & $65-74$ & $75-84$ & $\geq 85$ \\
\hline Duration in days & $1.9(0.65)$ & $6.4(3.1)$ & $9.1(3.9)$ & $9.3(3.1)$ & $9.2(2.5)$ & $10.1(1.8)$ \\
\hline 2005 & 0.9 & 2.5 & 3.1 & 3.7 & 4.5 & 6.9 \\
\hline 2015 & 3.2 & 14.0 & 18.2 & 15.8 & 14.2 & 14.3 \\
\hline Percentage increase (\%) & 245.2 & 456.2 & 481.5 & 325.9 & 217.0 & 107.5 \\
\hline Duration without one-off doses & $43.7(6.1)$ & $78.8(21.6)$ & $89.3(21.8)$ & $95.1(17.4)$ & $98.9(17.2)$ & $103.9(15.2)$ \\
\hline 2005 & 37.3 & 61.0 & 65.2 & 68.1 & 68.1 & 71.7 \\
\hline 2015 & 60.8 & 140.2 & 149.4 & 138.9 & 139.3 & 137.1 \\
\hline Percentage increase (\%) & 63.0 & 130.0 & 129.0 & 103.9 & 104.5 & 91.2 \\
\hline Number of annual events per 1000 & & & & & & \\
\hline population & $18988.1(1353.9)$ & 84505.7 (11169.9) & $142492.1(26426.9)$ & $67126.4(14737.1)$ & $44858.6(8747.4)$ & $17694.8(2615.0)$ \\
\hline Weak opioid & $65.8(3.5)$ & $111.8(16.4)$ & $178.2(31.4)$ & $220.8(38.5)$ & $236.8(41.6)$ & $243.5(36.6)$ \\
\hline per 1000 population & $185.5(122.0)$ & $2593.9(1562.1)$ & $6506.0(4351.8)$ & $2955.0(1861.0)$ & $2342.8(1170.4)$ & $1390.6(591.7)$ \\
\hline $\begin{array}{r}\text { Strong opioid } \\
\text { per } 1000 \text { population }\end{array}$ & $0.6(0.4)$ & $3.5(2.2)$ & $8.1(5.3)$ & $9.4(5.0)$ & $12.3(5.7)$ & $18.7(6.7)$ \\
\hline Number of events & & & & & & \\
\hline Male & $7274.4(1353.9)$ & 37882.7 (4701.1) & $65666.2(13277.6)$ & 28862.5 (7319.4) & 15326.9 (3629.8) & $4152.7(869.4)$ \\
\hline Per 1000 population & $51.5(2.3)$ & $102.2(14.1)$ & $167.4(31.3)$ & $192.9(35.7)$ & $164.7(34.0)$ & $115.9(19.5)$ \\
\hline Female & $11899.2(1243.0)$ & $49216.9(8068.4)$ & $83331.9(17270.9)$ & $41218.9(9137.7)$ & $31874.5(6168.6)$ & $14932.6(2259.2)$ \\
\hline Per 1000 population & $80.9(7.0)$ & $127.8(23.0)$ & $204.4(41.0)$ & $266.0(48.1)$ & $330.1(58.2)$ & $402.8(60.4)$ \\
\hline Persistence of 3-6 months & $51.7(19.1)$ & $754.2(290.9)$ & $2057.5(906.0)$ & $1011.7(395.4)$ & $668.8(183.1)$ & $303.5(77.3)$ \\
\hline Per 1000 population & $0.2(0.1)$ & $1.0(0.4)$ & $2.6(1.1)$ & $3.3(1.0)$ & $3.5(0.9)$ & $4.2(1.0)$ \\
\hline 2005 & 0.12 & 0.71 & 1.39 & 2.71 & 3.45 & 5.87 \\
\hline 2015 & 0.30 & 1.97 & 4.99 & 5.55 & 5.59 & 5.98 \\
\hline Rate increase (\%) & 144.2 & 178.0 & 258.2 & 104.9 & 62.0 & 1.7 \\
\hline Persistence more than 6 months & $32.9(18.1)$ & $673.9(439.3)$ & $1747.6(1132.0)$ & $888.2(490.5)$ & $592.1(267.9)$ & $281.5(90.1)$ \\
\hline Per 1000 population & $0.1(0.1)$ & $0.9(0.6)$ & $2.2(1.4)$ & $2.8(1.3)$ & $3.1(1.3)$ & $3.8(1.0)$ \\
\hline 2005 & 0.03 & 0.34 & 0.80 & 1.32 & 1.66 & 3.08 \\
\hline 2015 & 0.27 & 2.51 & 5.73 & 6.21 & 6.44 & 6.60 \\
\hline Rate increase (\%) & 703.8 & 641.0 & 619.8 & 370.8 & 287.6 & 114.0 \\
\hline Depression and/or anxiety & $6594.7(343.0)$ & 35005.5 (6516.5) & $46654.7(13720.1)$ & $14104.2(4380.4)$ & $7702.8(1722.6)$ & $2596.7(749.3)$ \\
\hline Per 1000 population & $22.9(1.7)$ & 46.4 (9.4) & $58.2(16.2)$ & $46.0(10.5)$ & $40.6(7.8)$ & $35.2(7.8)$ \\
\hline
\end{tabular}




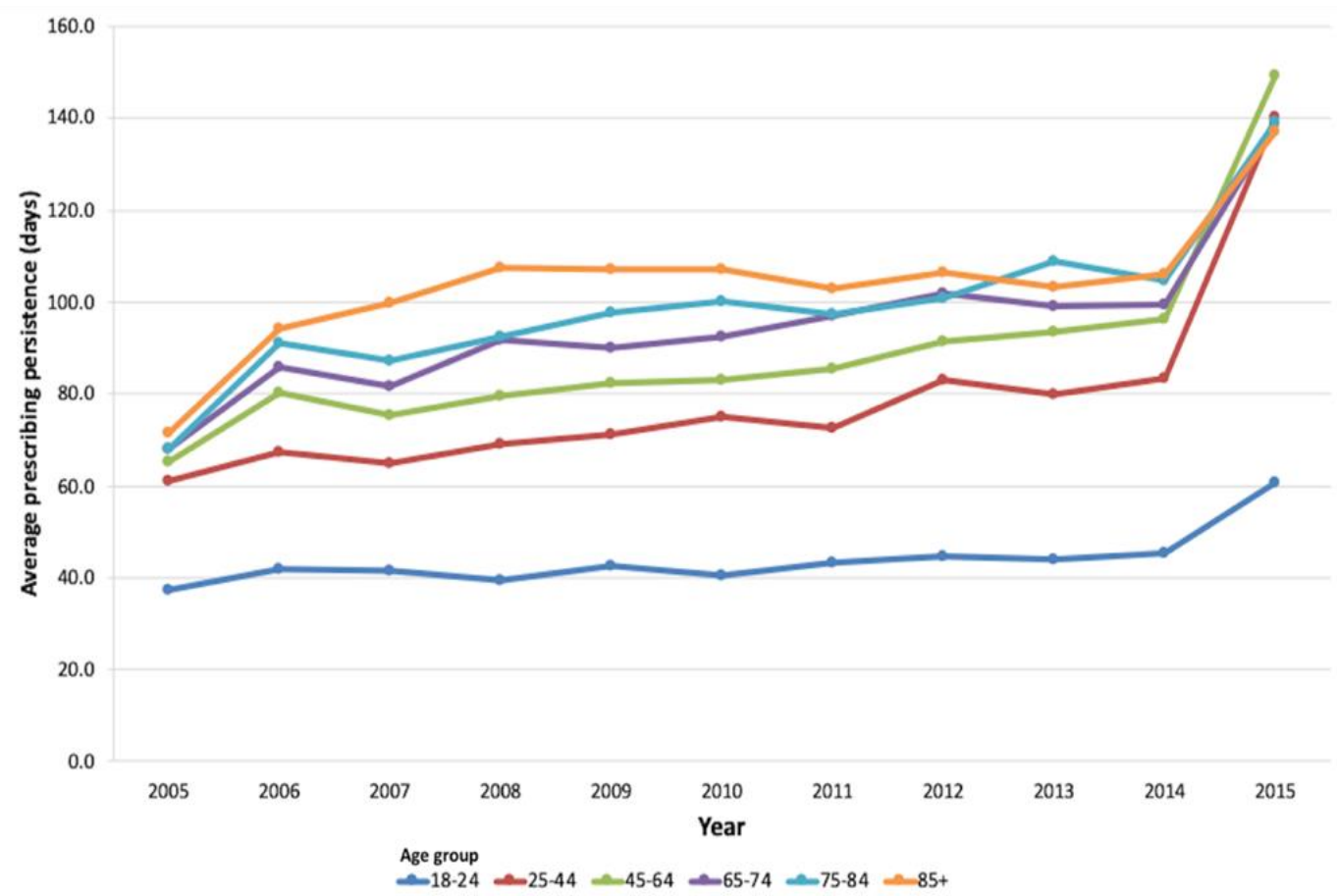

Figure G.4: Trends in prescription persistence 2005 - 2015 using data from people who received more than 1 prescription within a 31-day period, stratified by age group

The largest increase in prescription persistence (days) between 2005 and 2015, was noted in the $45-64$ years age-group, from 3.1 to 18.2 days (481.5\%). People aged 85 years and over had the smallest increase in prescribing persistence during the study period, even so, the duration doubled over the time examined. Statistically significant differences in the average duration of prescribing persistence were demonstrated between the age-groups (KruskalWallis $p<0.01, \mathrm{~d}_{\text {cohen }}=2.4, \eta^{2}=0.6$ ). Post-hoc analyses confirmed significant differences in average prescribing persistence between the youngest age group (18 - 24 years) and groups $\geq 65$ years and those aged $25-44$ and the oldest agegroup ( $\geq 85$ years). Statistical differences in prescribing persistence were not confirmed between any other age-groups (Table G.6).

All six age-groups were noted to have large increases in prescribing persistence (days) in 2015, this was due to the manner of data extraction and analysis resulting in people with the longest durations of prescribing, being recorded in 2015 rather than the year their prescriptions were initiated

Persistent periods of prescribing between 3 and 6 months, were most frequent in the $45-64$ years age-group (Figure G.5). When adjusted per 1000 age-group population however, the rate of prescribing persistence increased with increasing age-group. The largest percentage increase in prescribing persistence was in the $45-64$ years age-group (258.2\%, from 1.39 to 4.99 events per 1000 population). 


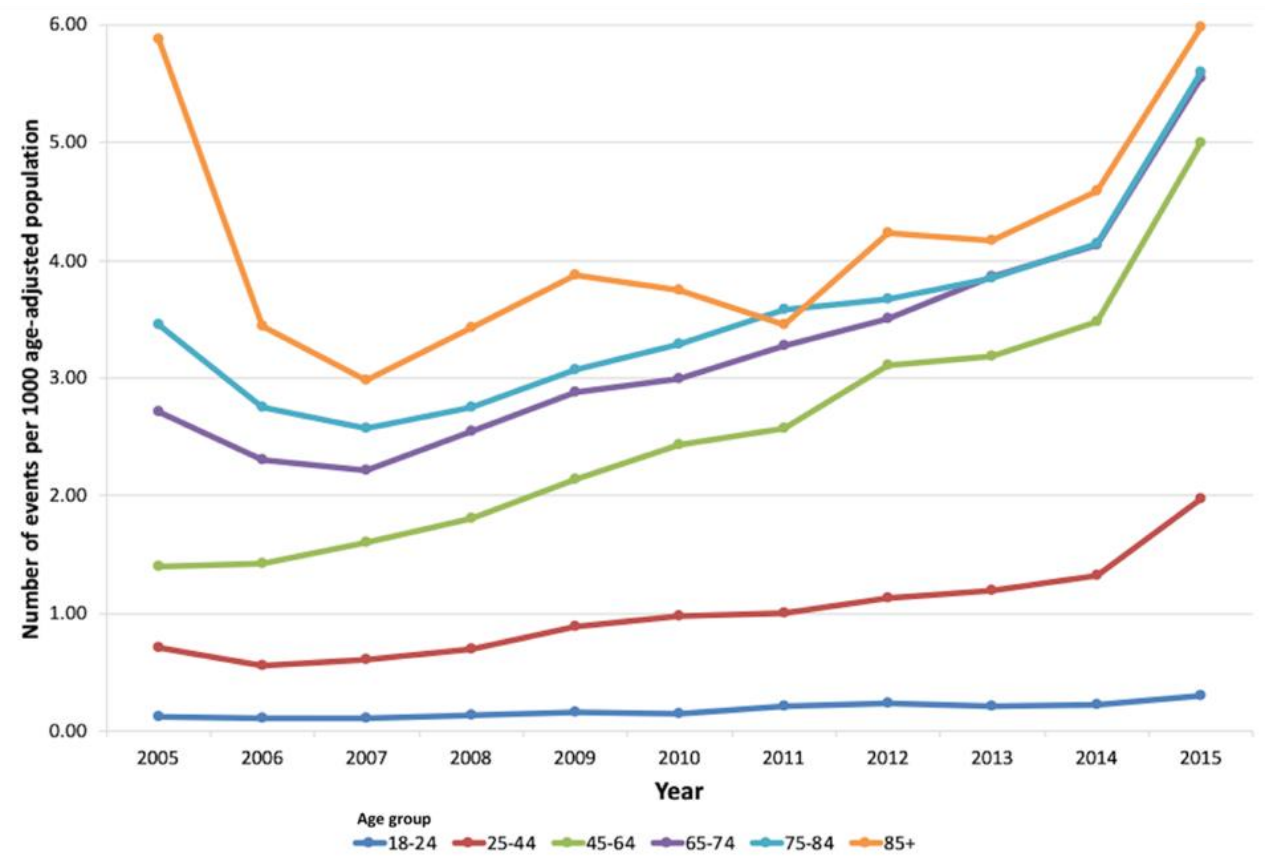

Figure G.5: Trends in the number of events with a prescribing persistence of between 3 and 6 months, between 2005 and 2015 and stratified by age-group

More than 40 times more events with a prescribing persistence of between 3 and 6 months were recorded in the $45-64$ years age-group compared to $18-24$ years. When adjusted to population, there was a 35 times difference between the average incidence in the youngest ( 0.2 per 1000 population) and oldest agegroup (4.2 per 1000 population) (Table G.8).

Average numbers of events where people received prescriptions for 3 and 6 months were determined to be significantly different $\left(p<.001, d_{\text {cohen }}=4.6, \eta^{2}\right.$ $=0.8$ ) between the six age-groups. Those differences were confirmed between the youngest age group ( $18-24$ years) and all age-groups between 25 and 84 years old. Statistically significant differences were not confirmed between the youngest and oldest age groups. Statistically significant differences were confirmed between the $65-74$ and $\geq 85$ years group and the $45-64$ years and $75-84$ years groups (Table G.7).

Table G.5: Dunn's pairwise comparisons and Bonferroni correction output for average prescribing duration of all prescribing events $2005-2015$, stratified by age-group

\begin{tabular}{rccccc}
\hline $\begin{array}{r}\text { Age group } \\
\text { (years) }\end{array}$ & $\mathbf{1 8 - 2 4}$ & $\mathbf{2 5 - 4 4}$ & $\mathbf{4 5 - 6 4}$ & $\mathbf{6 5 - 7 4}$ & $\mathbf{7 5 - 8 4}$ \\
\hline $\mathbf{2 5 - 4 4}$ & .291 & & & & \\
\hline $\mathbf{4 5 - 6 4}$ & .001 & $>.999$ & & & \\
\hline $65-74$ & $<.001^{*}$ & .567 & $>.999$ & & \\
\hline $75-84$ & $<.001^{*}$ & .852 & $>.999$ & $>.999$ & $>.999$ \\
\hline $85+$ & $<.001^{*}$ & .136 & $>.999$ & $>.999$ &
\end{tabular}

$* \mathrm{p}<0.05$ = statistically significant

Table G.6: Dunn's pairwise comparisons and Bonferroni correction output for average prescribing duration for events with at least two prescriptions issued per individual, $2005-2015$, stratified by age-group
Age group
18-24
25-44
45-64
65-74
75-84

(years) 


\begin{tabular}{rccccc}
\hline $\mathbf{2 5 - 4 4}$ & .347 & & & & \\
\hline $\mathbf{4 5 - 6 4}$ & $.014^{*}$ & $>.999$ & & & \\
\hline $\mathbf{6 5 - 7 4}$ & $<.001^{*}$ & .852 & $>.999$ & & \\
\hline $\mathbf{7 5 - 8 4}$ & $<.001^{*}$ & .170 & $>.999$ & $>.999$ & $>.999$
\end{tabular}

$* p<0.05$ = statistically significant

Table G.7: Dunn's pairwise comparisons and Bonferroni correction output for numbers of events with prescribing persistence between 3 and 6 months per 1000 population, 2005 - 2015, stratified by age-group

\begin{tabular}{rccccc}
\hline $\begin{array}{r}\text { Age group } \\
\text { (years) }\end{array}$ & $\mathbf{1 8 - 2 4}$ & $\mathbf{2 5 - 4 4}$ & $\mathbf{4 5 - 6 4}$ & $\mathbf{6 5 - 7 4}$ & $\mathbf{7 5 - 8 4}$ \\
\hline $\mathbf{2 5 - 4 4}$ & $>.999$ & & & & \\
\hline $\mathbf{4 5 - 6 4}$ & $.008^{*}$ & .567 & & & \\
\hline $\mathbf{6 5 - 7 4}$ & $<.001^{*}$ & $.034^{*}$ & $>.999$ & & \\
\hline $\mathbf{7 5 - 8 4}$ & $<.001^{*}$ & $.005^{*}$ & $>.999$ & $>.999$ & $>.999$
\end{tabular}

$* p<0.05=$ statistically significant 


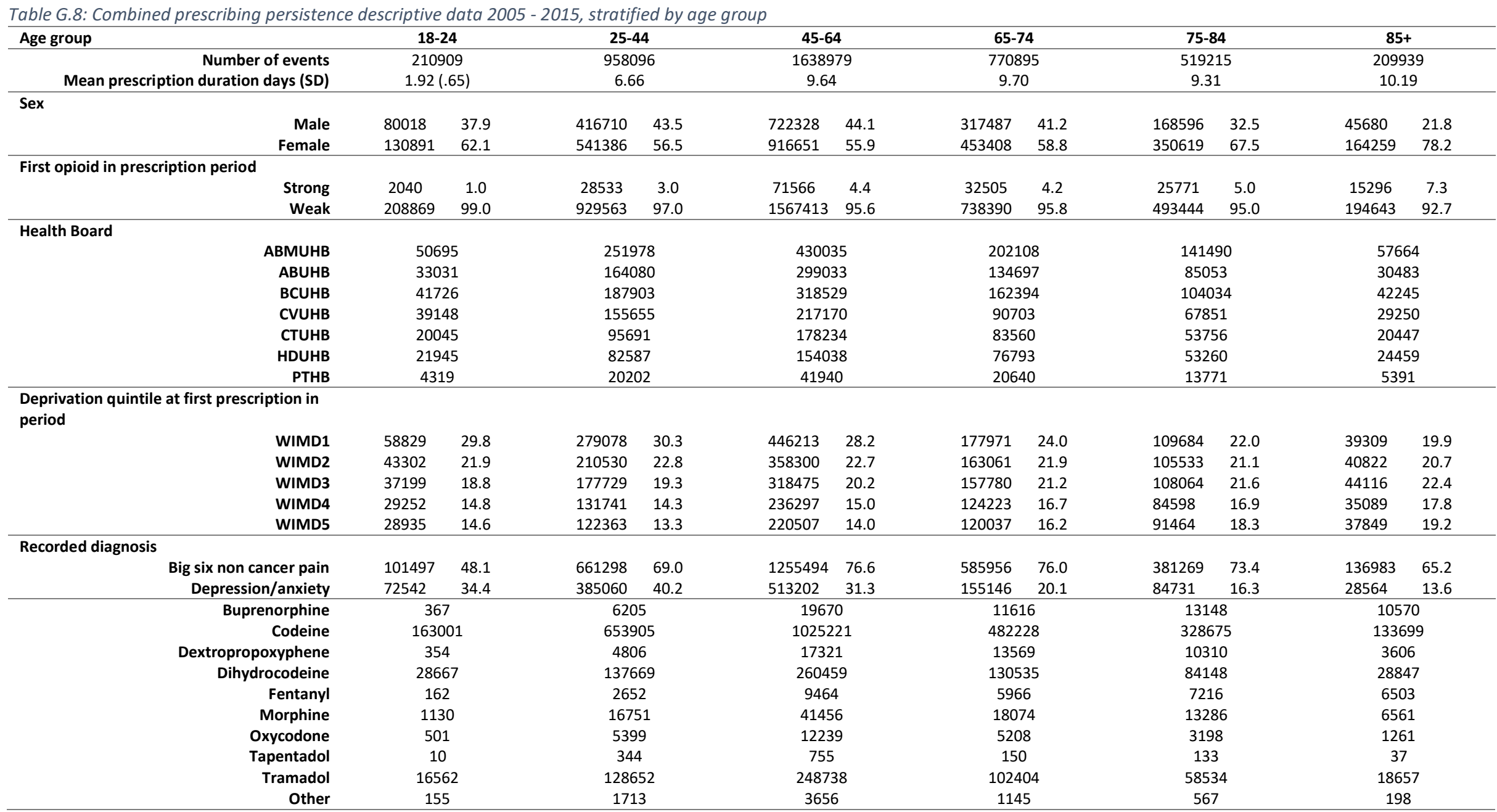




\section{G.3.1.1 Extended durations of prescribing persistence by age-group}

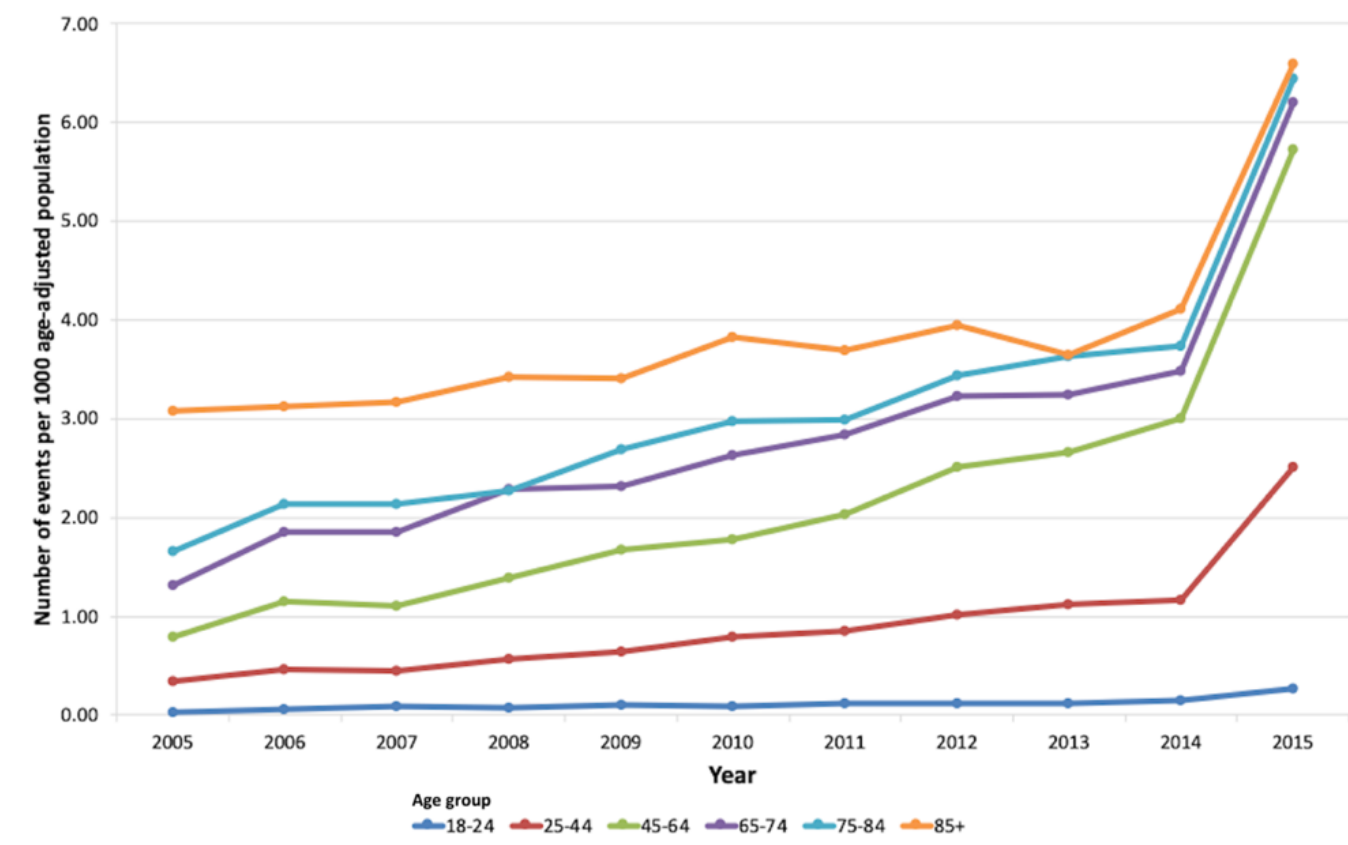

Figure G.6: Trends in the number of events with prescribing persistence of over 6 months, between 2005 and 2015 and stratified by age-group per 1000 age-adjusted population

Mean prescribing persistence of more than 6 months was highest by number of events in the $45-64$ years age-group (1747.6 events). Statistically significant differences were demonstrated between all the age groups $\left(p<.001 \mathrm{D}_{\text {cohen }}=3.6\right.$, $\eta^{2}=0.8$ ). Statistical significantly fewer events were confirmed in the youngest age group compared all those of 25 to 84 years. Significantly more events with persistence of more than six months were confirmed in those age 85 years and over compared to people aged between 45 and 74 years (Table G.9). Differences between all other age-groups receiving prescriptions for more than 6 months were not confirmed (Table G.9).

When adjusted to age-group population, the oldest age-group ( $\geq 85$ years) had the highest rate of prescribing persistence over 6 months (3.8 events per 1000 population) (Table G.4). The largest increase in the rate of persistence of over 6 months, was in the youngest age group $(703.8 \%$, from 0.03 to 0.27 events per 1000 population) between 2005 and 2015 . There were an average 38 times more prescribing events of over 6 months in the oldest age group in the same period although the percentage increase was much less, (114\% increase, from 3.08 to 6.6 events per 1000 population).

Statistical analysis confirmed significant differences between the rate of prescribing persistence for the different age-groups (Kruskal-Wallis $p<.001, \eta^{2}$ $=0.7, \mathrm{~d}_{\text {Cohen }}=3.4$ ). People aged $18-24$ years age-group were confirmed to have significantly lower prescribing rates compared to ages above 45 years but not with those aged $25-44$ years old (Table G.10). A significantly lower prescribing rate was also confirmed in the $25-44$ yeas age-group compared to the two oldest groups ( $\geq 75$ years old) (Table G.10). 
Table G.9: Dunn's pairwise comparisons and Bonferroni correction output for numbers of events with prescribing persistence greater than 6 months, 2005 -2015, stratified by age-group

\begin{tabular}{rccccc}
\hline $\begin{array}{r}\text { Age group } \\
\text { (years) }\end{array}$ & $\mathbf{1 8 - 2 4}$ & $\mathbf{2 5 - 4 4}$ & $\mathbf{4 5 - 6 4}$ & $\mathbf{6 5 - 7 4}$ & $\mathbf{7 5 - 8 4}$ \\
\hline $\mathbf{2 5 - 4 4}$ & $>.999$ & & & & \\
\hline $\mathbf{4 5 - 6 4}$ & $.013^{*}$ & $>.999$ & & & \\
\hline $\mathbf{6 5 - 7 4}$ & $<.001^{*}$ & .077 & $>.999$ & & \\
\hline $\mathbf{7 5 - 8 4}$ & $<.001^{*}$ & $.024^{*}$ & $>.999$ & $>.999$ & \\
\hline $85+$ & $<.001^{*}$ & $<.001^{*}$ & .091 & $>.999$ & $>.999$ \\
\hline
\end{tabular}

$*_{p}<0.05$ = statistically significant

Table G.10: Dunn's pairwise comparisons and Bonferroni correction output for numbers of events with prescribing persistence greater than 6 months per 1000 population, 2005 -2015, stratified by age-group

\begin{tabular}{llllll}
\hline $\begin{array}{l}\text { Age group } \\
\text { (years) }\end{array}$ & $\mathbf{1 8 - 2 4}$ & $\mathbf{2 5 - 4 4}$ & $\mathbf{4 5 - 6 4}$ & $\mathbf{6 5 - 7 4}$ & $\mathbf{7 5 - 8 4}$ \\
\hline $\mathbf{2 5 - 4 4}$ & $0.002^{*}$ & & & & \\
\hline $\mathbf{4 5 - 6 4}$ & $0.003^{*}$ & 0.212 & & & \\
\hline $\mathbf{6 5 - 7 4}$ & $<.001^{*}$ & $>.999$ & $>.999$ & & \\
\hline $\mathbf{7 5 - 8 4}$ & $0.003^{*}$ & $>.999$ & 0.150 & $>.999$ & \\
\hline $\mathbf{8 5 +}$ & $>.999$ & 0.352 & $<.001^{*}$ & $>.999$ & 0.481 \\
\hline
\end{tabular}

$* p<0.05$ = statistically significant

\section{G.4 Prescribing persistence by deprivation}

Mean duration (days) of prescription persistence was 54\% longer (10.3 days versus 6.7 days) in the most deprived areas (WIMD) of Wales, compared to the least deprived (WIMD5), between 2005 and 2015 respectively (Table G.15). Differences in mean persistence were statistically significant (Kruskal-Wallis $p<0.05, \eta^{2}=0.12$, $d_{\text {cohen }}=0.75$ ) when all quintiles were compared (Figure G.7). However, pairwise comparisons did not confirm any significant difference between any of the quintiles (Table G.11).

During the 11 years examined, persistence increased by more than 4 times in all quintiles (Figure G.7). The second least deprived areas (WIMD4) had the greatest increase (391.3\%, from 2.5 to 12.3 days). Most deprived areas (WIMD1), where persistence was longest throughout the study, had a similar level of increase (389.3, from 4.0 to 19.3 days) (Table G.15).

Prescription persistence, when single events were removed from the analysis, showed less variance in duration between the quintiles, with a 22\% (101.3 days versus 82.8 days) between WIMD1 and WIMD5 areas respectively (Table G.15). Prescription persistence was similar for all quintiles, when examining only multiple prescription issues (Figure G.8). This was the case until 2015 when there was a large increase in duration, due to the inclusion in that year's data of prescribed events which originated in the early years of the study.

Whilst a statistically significant difference between mean prescribing persistence was determined (Kruskall-Wallis $p<0.01, \eta^{2}=0.2$, $d_{\text {cohen }}=1.0$ ) pairwise comparison (Figure G.8) failed to confirm any significant differences between quintiles (Table G.12). 


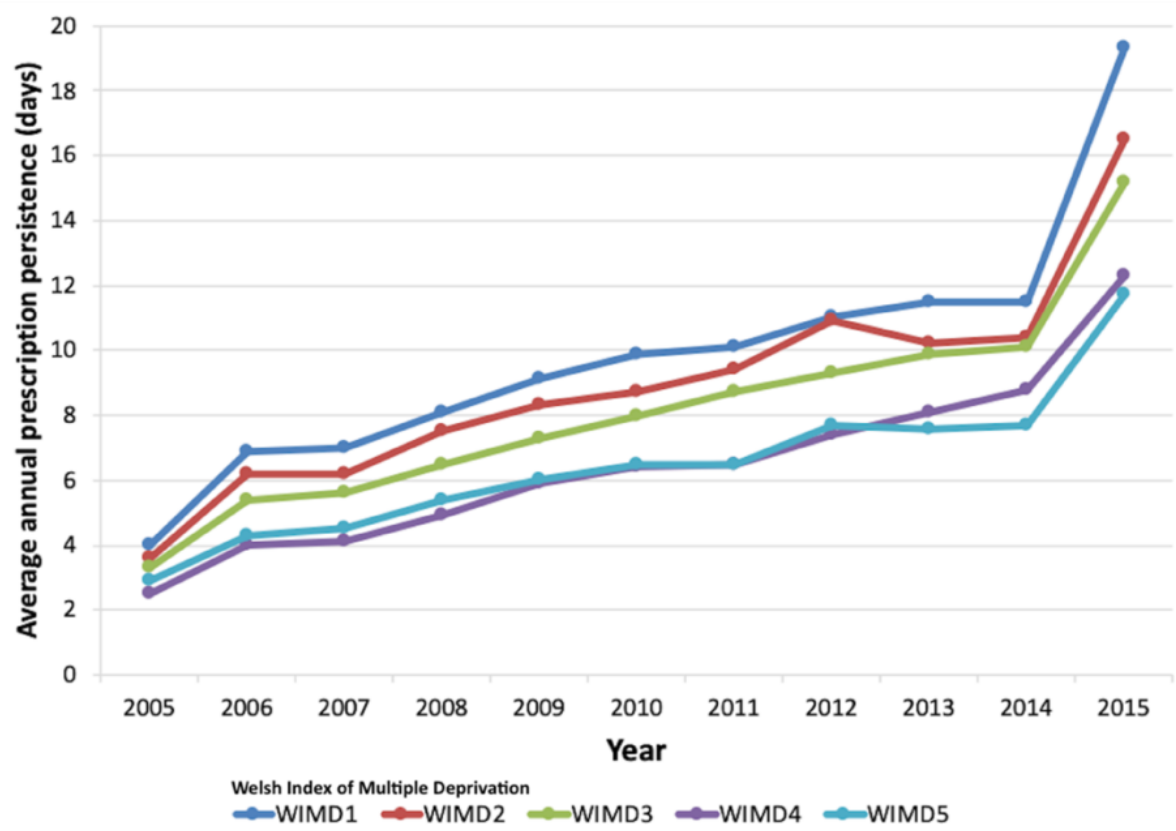

Figure G.7: Trends in average annual prescribing persistence (days) stratified by Welsh Index of Multiple Deprivation quintiles. WIMD1=most deprived, WIMD5 = least deprived

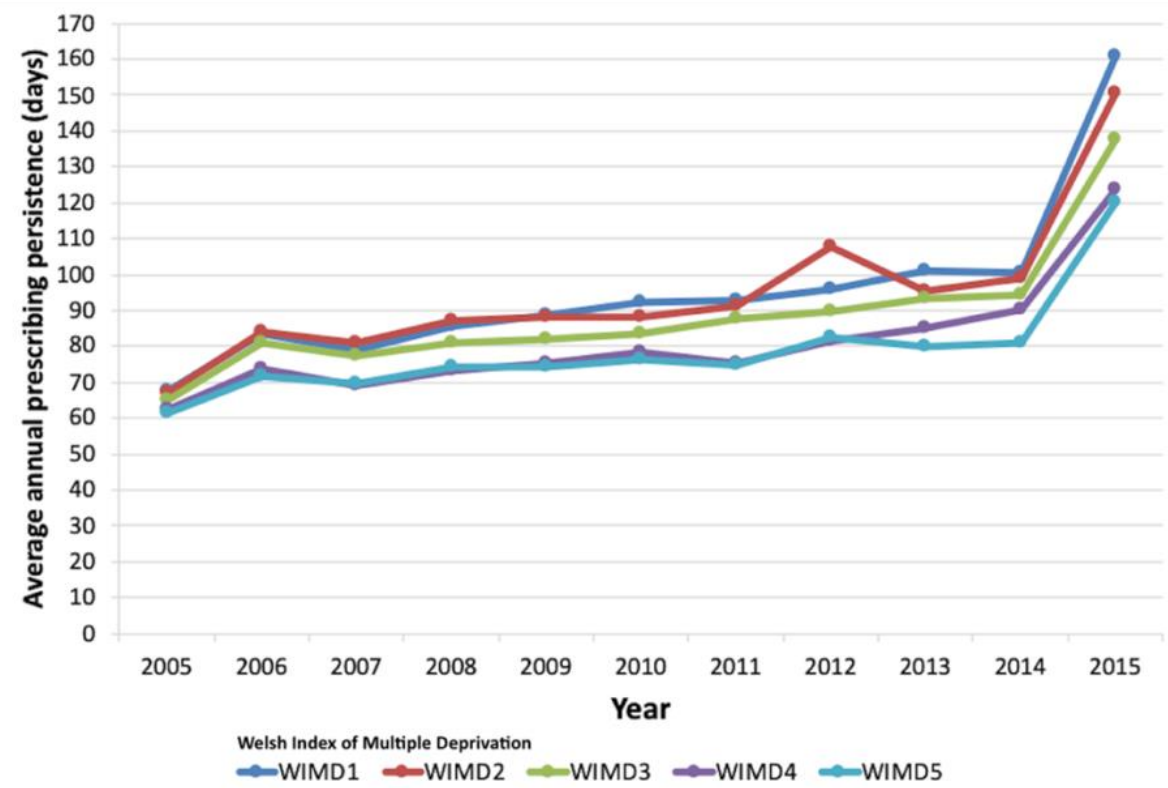

Figure G.8: Trends in average annual prescribing persistence (days) for events of two or more prescriptions issued within 32 days, stratified by Welsh Index of Multiple Deprivation quintiles. WIMD1=most deprived, WIMD5 = least deprived

\section{G.4.1.1 Extended durations of prescribing persistence by deprivation}

Prescribing events with a persistence of between 3 and 6 months, averaged more than double the number in the most deprived areas of Wales, compared to the least deprived (1467.3 in WIMD1 compared to 630.8 in WIMD5) (Table G.15). Mean event numbers showed a large variance between quintiles WIMD1-3, the most deprived areas, compared to WIMD4-5 areas, although there were an average $37 \%$ more prescribing events of between 3-6 months in WIMD1 areas compared to WIMD2 (1069.7) in the 11 years analysed. 
A statistically significant difference was demonstrated between the number of events with a persistence of between 3 and 6 months in each quintile (KruskalWallis $p<.001, \eta^{2}=0.4, d_{\text {cohen }}=1.8$ ). Pairwise comparison confirmed significant differences between the number of events occurring in WIMD4 areas compared to WIMD1 and WIMD2 areas respectively and WIMD5 and WIMD1 areas (Table G.13).

Table G.11: Dunn's pairwise comparison and Bonferroni post-hoc analysis of mean prescribing persistence (days), in the 5 different areas of deprivation as defined by the Welsh Index of Multiple Deprivation (WIMD)

\begin{tabular}{ccccc}
\hline \multicolumn{5}{c}{ Welsh Index of Multiple Deprivation } \\
\hline $\mathbf{2}$ & 1 & 2 & 3 & 4 \\
\hline $\mathbf{3}$ & $>.999$ & & & \\
\hline $\mathbf{4}$ & .117 & $>.999$ & & \\
\hline $\mathbf{5}$ & .123 & .570 & $>.999$ & $>.999$ \\
\hline
\end{tabular}

${ }^{*}$ p-value $<0.05$ = statistically significant WIMD1=most deprived, WIMD5-least deprived

Table G.12: Dunn's pairwise comparison and Bonferroni post-hoc analysis of mean prescribing persistence (days) when events not repeated within 31 days were removed from analysis, in the 5 different areas of deprivation as defined by the Welsh Index of Multiple Deprivation (WIMD)

\begin{tabular}{ccccc}
\hline \multicolumn{5}{c}{ Welsh Index of Multiple Deprivation } \\
\hline $\mathbf{2}$ & 1 & 2 & 3 & 4 \\
\hline $\mathbf{3}$ & $>.999$ & & & \\
\hline $\mathbf{4}$ & .1599 & $>.999$ & & \\
\hline $\mathbf{5}$ & $.046^{*}$ & .192 & $>.999$ & $>.999$ \\
\hline
\end{tabular}

$*_{\mathrm{p}}$-value $<0.05$ = statistically significant. WIMD1=most deprived, WIMD5-least deprived

Table G.13: Dunn's pairwise comparison and Bonferroni post-hoc analysis of average number of events with a prescribing persistence between 3 - 6 months, in the 5 different areas of deprivation as defined by the Welsh Index of Multiple Deprivation (WIMD)

\begin{tabular}{ccccc}
\hline \multicolumn{5}{c}{ Welsh Index of Multiple Deprivation } \\
\hline $\mathbf{2}$ & 1 & 2 & 3 & 4 \\
\hline $\mathbf{3}$ & .999 & & & \\
\hline $\mathbf{4}$ & $<.001^{*}$ & $>.999$ & & \\
\hline $\mathbf{5}$ & $<.001^{*}$ & $.040^{*}$ & .455 & $>.999$ \\
\hline
\end{tabular}

*p-value $<0.05$ = statistically significant WIMD1=most deprived, WIMD5-least deprived

Table G.14: Dunn's pairwise comparison and Bonferroni post-hoc analysis for number of events per 1000 population with prescribing persistence between $3-6$ months, in the 5 different areas of deprivation as defined by the Welsh Index of Multiple Deprivation (WIMD)

\begin{tabular}{|c|c|c|c|c|}
\hline \multicolumn{5}{|c|}{ Welsh Index of Multiple Deprivation } \\
\hline & 1 & 2 & 3 & 4 \\
\hline 2 & $>.999$ & & & \\
\hline 3 & .332 & $>.999$ & & \\
\hline 4 & $<.001^{*}$ & .093 & .431 & \\
\hline 5 & $<.001^{*}$ & $.027^{*}$ & .157 & $>.999$ \\
\hline
\end{tabular}

${ }^{*} \mathrm{p}$-value $<0.05$ = statistically significant WIMD1=most deprived, WIMD5-least deprived 
Table G.15: Trends in prescribing persistence between 2005 and 2015, stratified by Welsh Index of Multiple Deprivation (WIMD) quintile where WIMD1 = most deprived and WIMD5 = least deprived

\begin{tabular}{|c|c|c|c|c|c|}
\hline & \multicolumn{5}{|c|}{ Welsh Index of Multiple Deprivation quintile } \\
\hline & WIMD1 & WIMD2 & WIMD3 & WIMD4 & WIMD5 \\
\hline Mean duration (days) \pm SD & $10.3 \pm 72.0$ & $9.3 \pm 67.6$ & $8.5 \pm 61.4$ & $6.8 \pm 52.8$ & $6.7 \pm 51.5$ \\
\hline 2005 & $4.0 \pm 22.8$ & $3.6 \pm 21.6$ & $3.3 \pm 20.7$ & $2.5 \pm 18.2$ & $2.9 \pm 19.2$ \\
\hline 2015 & $19.3 \pm 140.1$ & $16.5 \pm 125.2$ & $15.2 \pm 112.5$ & $12.3 \pm 96.4$ & $11.7 \pm 96.4$ \\
\hline Percentage increase (\%) & 389.3 & 358.4 & 358.9 & 391.3 & 306.6 \\
\hline Duration without one-off doses (SD) & $101.3 \pm 204.8$ & $99.5 \pm 200.5$ & $93.7 \pm 183.2$ & $86.1 \pm 168.7$ & $82.8 \pm 162.7$ \\
\hline 2005 & $67.4 \pm 67.7$ & $67.0 \pm 66.5$ & $65.2 \pm 66.2$ & $62.5 \pm 65.8$ & $61.5 \pm 64.9$ \\
\hline 2015 & $160.6 \pm 374.6$ & $150.6 \pm 351.0$ & $137.7 \pm 313.0$ & $123.5 \pm 281.6$ & $120.0 \pm 286.6$ \\
\hline Percentage increase (\%) & 138.3 & 124.7 & 111.1 & 97.7 & 95.0 \\
\hline Persistence of $3-6$ months \pm SD & $1467.3 \pm 502.7$ & $1069.7 \pm 365.1$ & $934.6 \pm 366.2$ & $600.3 \pm 287.6$ & $630.8 \pm 274.4$ \\
\hline 2005 & 1091 & 793 & 630 & 353 & 370 \\
\hline 2015 & 2685 & 1866 & 1807 & 1232 & 1116 \\
\hline Percentage change (\%) & 146.1 & 135.3 & 186.8 & 249.0 & 201.6 \\
\hline Persistence of $3-6$ months per 1000 & & & & & \\
\hline population \pm SD & $3.0 \pm 1.0$ & $2.2 \pm 0.7$ & $2.0 \pm 0.8$ & $1.3 \pm 0.6$ & $1.2 \pm 0.5$ \\
\hline 2005 & 2.03 & 1.39 & 1.23 & 0.62 & 0.64 \\
\hline 2015 & 5.48 & 3.8 & 3.83 & 2.66 & 2.25 \\
\hline Percentage change & 138.1 & 126.4 & 173.4 & 231.4 & 190.4 \\
\hline Persistence more than 6 months \pm SD & $1308.6 \pm 738.0$ & $984.0 \pm 537.2$ & $824.6 \pm 479.6$ & $490.8 \pm 321.4$ & $508.5 \pm 308.2$ \\
\hline 2005 & 564 & 406 & 332 & 175 & 204 \\
\hline 2015 & 3262 & 2376 & 2051 & 1311 & 1114 \\
\hline Rate change (\%) & 478.4 & 485.2 & 517.8 & 649.1 & 446.1 \\
\hline Persistence more than 6 months \pm SD & $2.5 \pm 1.5$ & $2.0 \pm 1.1$ & $1.8 \pm 1.0$ & $1.1 \pm 0.7$ & $0.9 \pm 0.5$ \\
\hline 2005 & 1.19 & 0.86 & 0.74 & 0.40 & 0.43 \\
\hline 2015 & 6.65 & 4.84 & 4.35 & 2.84 & 2.24 \\
\hline Rate change & 459.5 & 463.0 & 488.9 & 611.4 & 425.7 \\
\hline
\end{tabular}


When adjusted to population, the average number of prescribing events with a persistence between 3 and 6 months was 2.5 times greater in the most deprived areas of Wales (WIMD1 $=3.0$ events per 1000 population) than the least deprived (WIMD5 = 1.2 events per 1000 population) (Figure G.9). Increases in the number of events per 1000 population were smallest in the two most deprived areas (Table G.15) although the overall numbers of events were higher in those areas compared to the lesser deprived quintiles. Significant differences were demonstrated between the number of events per 1000 population when all quintiles were compared (Kruskal-Wallis $p<.001, \eta^{2}=0.5, d_{\text {cohen }}=2.0$ ). WIMD1 quintile areas were confirmed to have significantly more events than the two least deprived quintile areas (WIMD4 and WIMD5 respectively). Confirmation of significantly more events in WIMD2 areas compared to WIMD5 areas was demonstrated from pairwise comparison as well. Significance was not confirmed when all other areas were compared pairwise

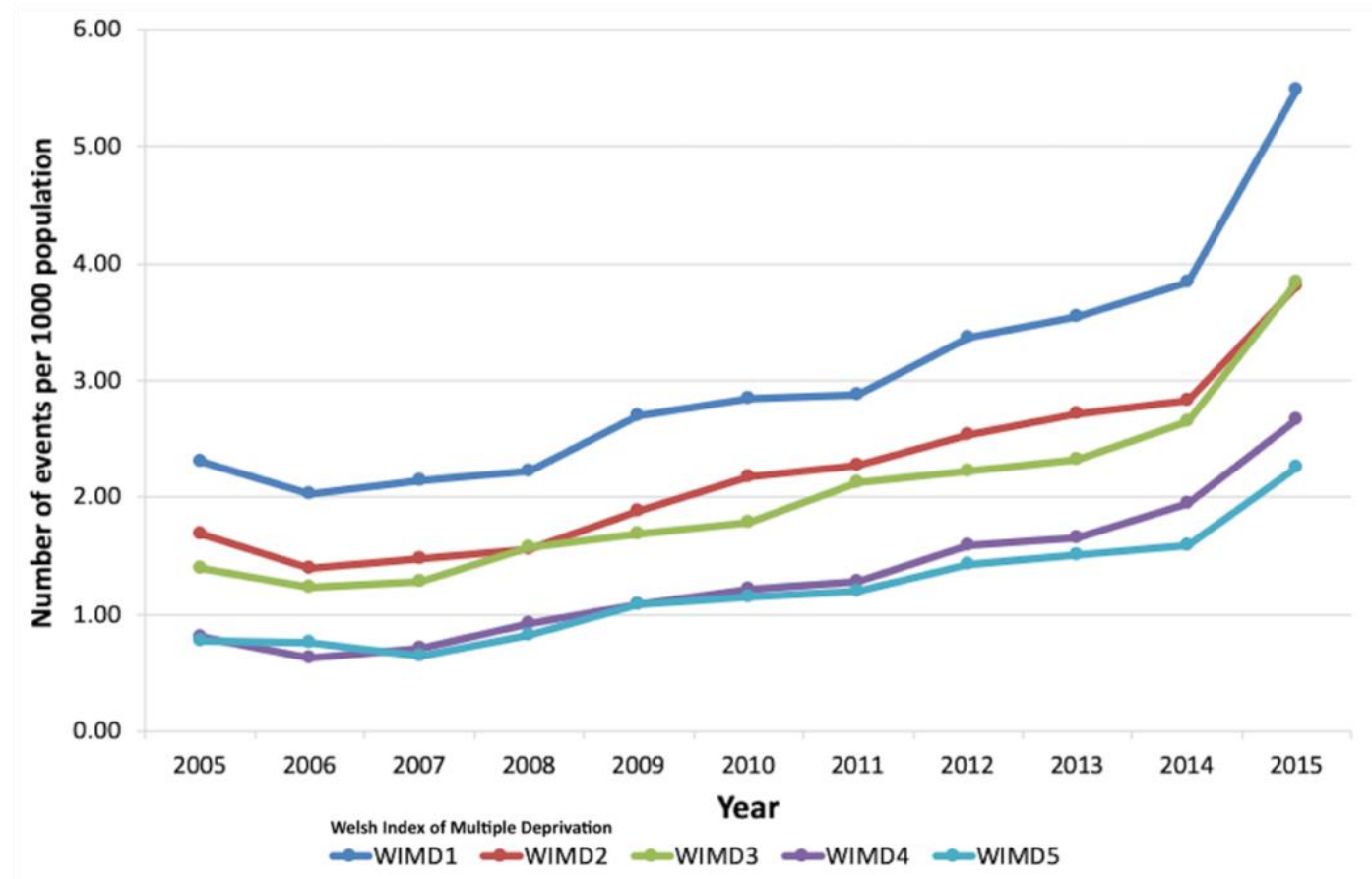

Figure G.9: Trends in prescription persistence of 3-6months in events per 1000 deprivation adjusted population. WIMD1 = most deprived, WIMD5 = least deprived

Data for prescribing persistence of over 6 months showed similar trends between 2005 and 2015, as shown for durations of 3 - 6 months (Table G.15). There were 2.5 times more events of over 6 months in the most deprived areas (WIMD1 $=1308.6$ events per 1000 population) compared to the least deprived (WIMD5 $=508.5$ events per 1000 population). In this instance, however, on average, there were fewer events with a persistence of over 6 months in WIMD4 areas (WIMD4 $=490.8$ events per 1000 population) (Figure-G.10). WIMD4 areas are classified as the second least deprived areas in Wales.

Initial analysis demonstrated a significant difference between quintiles in the number of events with more than 6 months persistence (Kruskal-Wallis $p<.001$, 
$\left.\eta^{2}=0.4, d_{\text {Cohen }}=1.5\right)$. Confirmation of those differences was only confirmed between the most deprived areas (WIMD1) and two least deprived quintiles (WIMD4 and WIMD5) when all groups were subjected to pairwise comparison).

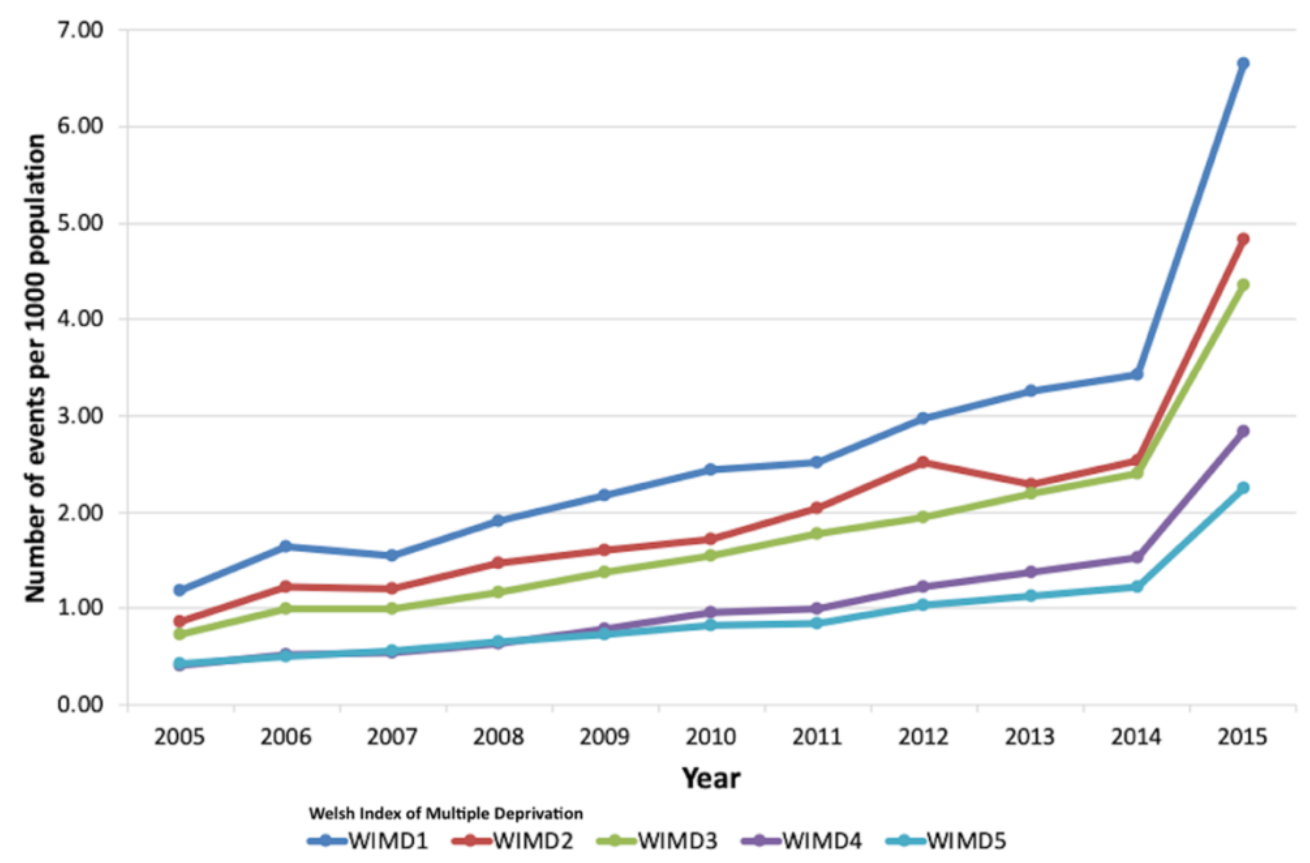

Figure-G.10: Trends in prescription persistence of over 6months in events per 1000 deprivation adjusted population. WIMD1 = most deprived, WIMD5 = least deprived

When adjusted to population, data regained a pattern of increasing number of events with increasing level of deprivation (Figure-G.10). Nearly three times as many events with a persistence of more than 6 months occurred on average in the most deprived areas (WIMD1 $=2.7$ events per 1000 population), compared with the least (WIMD5 $=0.93$ events per 1000 population). Large percentage increases were noted in the number of events per 1000 population with a persistence of over 6 months in all quintiles (Table G.15), the largest in WIMD4 areas (611.4\%, from 0.4 to 2.84 events per 1000 population). Again, a sudden increase in the number of events per 1000 population, were noted in all areas in 2015. This was due to the inclusion in that year's data of long-term prescribing the last date of which occurred in 2015. That there was a 3 times difference between the highest number of events and lowest suggests that long-term prescribing is more likely to occur in the most deprived areas of Wales (Figure-G.10).

Table G.16: Dunn's pairwise comparison and Bonferroni post-hoc analysis of average number of events with a prescribing persistence greater than 6 months, in the 5 different areas of deprivation as defined by the Welsh Index of Multiple Deprivation (WIMD)

\begin{tabular}{ccccc}
\hline \multicolumn{5}{c}{ Welsh Index of Multiple Deprivation } \\
\hline $\mathbf{2}$ & 1 & 2 & 3 & 4 \\
\hline $\mathbf{3}$ & $>.999$ & & & \\
\hline $\mathbf{4}$ & .653 & $.002^{*}$ & .999 & \\
\hline $\mathbf{5}$ & $.002^{*}$ & .068 & .580 & $>.999$ \\
\hline
\end{tabular}

*p-value $<0.05$ = statistically significant. WIMD1=most deprived, WIMD5-least deprived 
Table G.17: Dunn's pairwise comparison and Bonferroni post-hoc analysis for number of events per 1000 population with prescribing persistence greater than 6 months, in the 5 different areas of deprivation as defined by the Welsh Index of Multiple Deprivation (WIMD)

\begin{tabular}{ccccc}
\hline \multicolumn{5}{c}{ Welsh Index of Multiple Deprivation } \\
\hline $\mathbf{2}$ & 1 & 2 & 3 & 4 \\
\hline $\mathbf{3}$ & .999 & & & \\
\hline $\mathbf{4}$ & $.003^{*}$ & .078 & .536 & \\
\hline $\mathbf{5}$ & $.001^{*}$ & $.021^{*}$ & .192 & $>.999$ \\
\hline
\end{tabular}

$*_{p}$-value $<0.05$ = statistically significant. WIMD1=most deprived, WIMD5-least deprived

The difference in number of events per 1000 population were determined to be significant (Kruskal-Wallis $p<.001, \eta^{2}=0.4, d_{\text {cohen }}=1.6$ ). Significantly more events occurred in WIMD1 and WIMD2 areas when compared pairwise to the WIMD5 quintile. There were statistically significantly more events of $>6$ months persistence in WIMD1 than WIMD4 areas also (Table G.16).

\section{G.5 Prescribing persistence by opioid type}

Weak opioids, as defined for this study were prescribed at the end of $4,132,324$ (95.9\%, 158.4 events per 1000 population) of all the prescribing periods recorded between 2005 and 2015 (Table G.18). However, as persistence of consecutive prescriptions increased, so did the percentage of strong opioids. When prescriptions were issued for $\geq 181$ days, 4,279 (9.2\%, 0.2 events per 1000 population) of those periods ended with a strong opioid prescription.

Examination by individual drug showed codeine to be by far, the most frequently prescribed drug for all prescription persistence durations (Table G.18). For all durations of prescribing persistence, codeine was the final prescription for $64.7 \%$ $(2,786,730$ of $4,308,035$ events) of all events (Figure G.11). Codeine was also the drug of choice for nearly two thirds of short-term prescribing, where persistence was less than 32 days. As prescribing persistence increased, the percentage where codeine was given, reduced. When prescribing persisted for more than 6 months ( $\geq 181$ days), codeine reduced to $52.8 \%$ of prescriptions (Table G.18).

Table G.18: Prescribing persistence stratified by drug, totalled for 2005 - 2015

\begin{tabular}{rccccc}
\hline \multicolumn{5}{c}{ Number of prescribing events with duration prescription persistence (days) } \\
(\% of total for that duration)
\end{tabular}


Conversely, tramadol classified as a weak opioid for this study, increased its representation, in percentage terms as prescribing persistence increased (Table G.18). This was also the case with dihydrocodeine, the third most prescribed weak opioid, albeit the percentage change was small.

The drugs categorised as strong opioids; buprenorphine, fentanyl, morphine, oxycodone and tapentadol doubled in their percentage representation as prescribing persistence increased (Table G.18). Morphine accounted for $2.2 \%$ of periods with a persistence of less than 32 days, but $5.4 \%$ of periods with a duration of more than 6 months (Figure G.11).

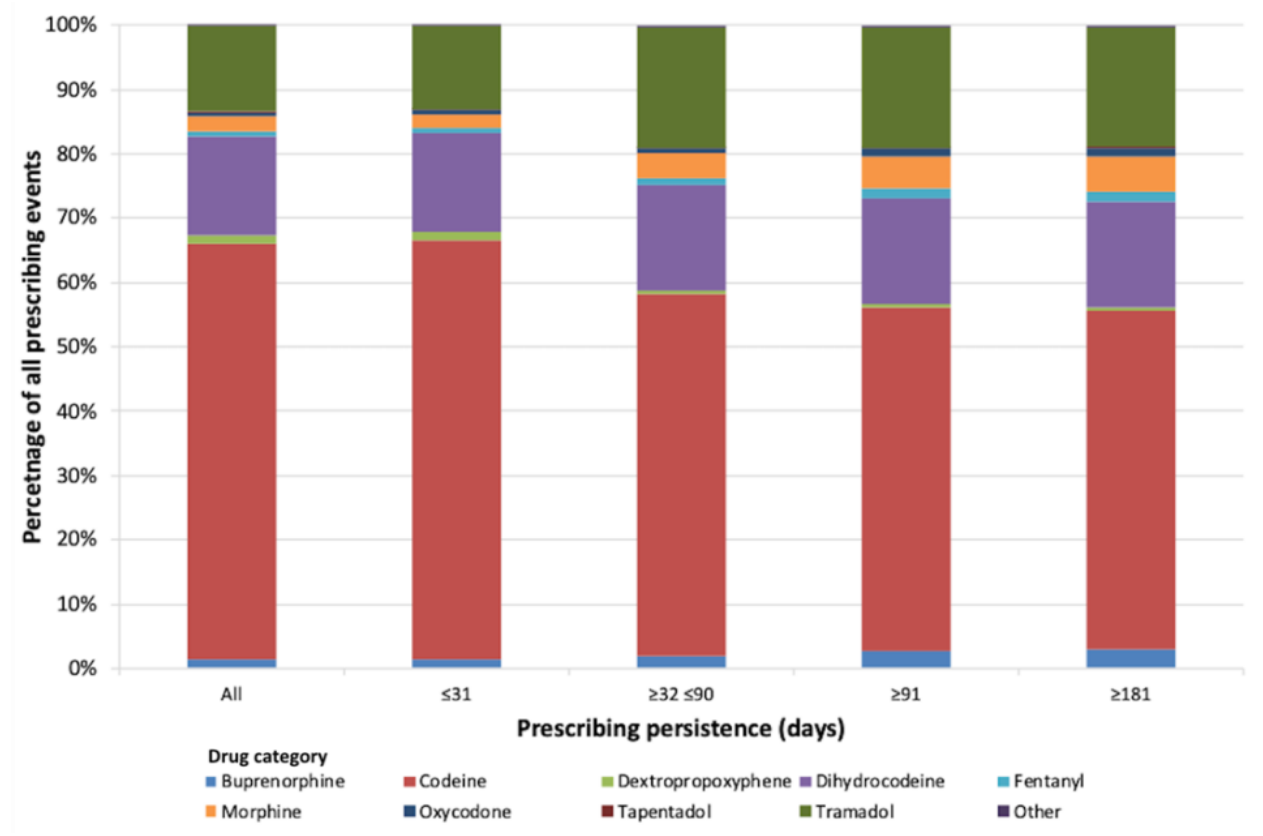

Figure G.11: Percentage contribution to prescribing persistence by drug, stratified by number of continuous days

Drugs classified as 'strong' opioids for this study, were demonstrated to have the longest average persistence in prescribing over the 11 years examined (Table G.21). Morphine, the most frequently prescribed strong opioid, had the longest average prescribing persistence when examined by all prescriptions $(97,258$ events in total, $19.6 \pm 117.1$ days) and when single prescriptions were excluded from analysis $(13,752$ events, $138.4 \pm 283.9$ days). Codeine, which was the most prescribed opioid in Wales between 2005 and 2015 had the second shortest average persistence $(2,786,729$ events, $7.1 \pm 55.0$ days and 223,077 events, 88.9 \pm 174.8 days for all data and 2 or more prescriptions in a period, respectively). The 'other' opioid group (Figure G.11) had the shortest prescribing persistence for all categories that were examined (Table G.21). 


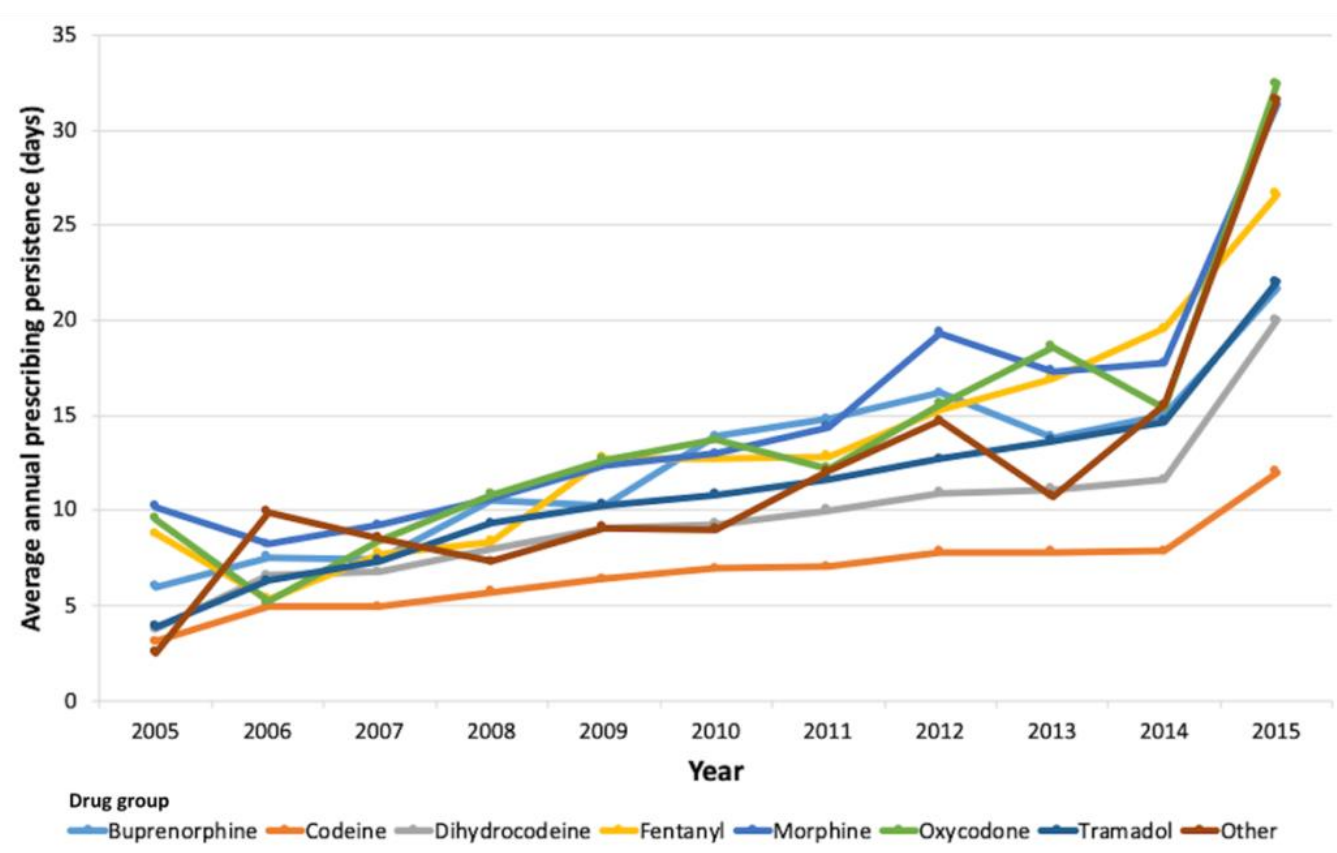

Figure G.12: Trends in average annual prescribing persistence between 2005 - 2015, stratified by drug group

The 'other' group of opioids had the largest percentage increase in average prescribing persistence (1177.2\%, from 2.5 to 31.6 days), which on examination was due to the inclusion of tapentadol in that group, causing a steep incline in persistence towards the end of the study. By 2015, the prescribing persistence of 'other' opioids was similar to that of morphine and oxycodone (Figure G.12 ).

No statistically significant difference was determined between the duration of prescribing persistence of each drug group (Kruskal-Wallis $p<0.005, D_{\text {Cohen }}=0.9, \eta^{2}$ $=0.2$ ). Pairwise comparison confirmed codeine had a significantly shorter duration of persistence compared to morphine, oxycodone, and fentanyl. No difference was confirmed between the persistence any of the other drug groups when directly compared (Table G.19).

Table G.19: Dunn's pairwise tests with Bonferroni corrections demonstrating relationships between average prescribing persistence (days) by drug for all prescriptions

\begin{tabular}{rccccccc}
\hline Drug & Buprenorphine & Codeine & Dihydrocodeine & Fentanyl & Morphine & Oxycodone & Tramadol \\
\hline Codeine & 0.052 & & & & & & \\
\hline Dihydrocodeine & $>.999$ & $>.999$ & & & & & \\
\hline Fentanyl & $>.999$ & $.032^{*}$ & $>.999$ & & & & \\
\hline Morphine & $>.999$ & $.003^{*}$ & .895 & $>.999$ & & & \\
\hline Oxycodone & $>.999$ & $.013^{*}$ & $>.999$ & $>.999$ & $>.999$ & & \\
\hline Tramadol & $>.999$ & .410 & $>.999$ & $>.999$ & $>.999$ & $>.999$ & \\
\hline Other & $>.999$ & .444 & $>.999$ & $>.999$ & $>.999$ & $>.999$ & $>.999$
\end{tabular}

${ }^{*} p<0.05$ is statistically significant

Table G.20: Dunn's pairwise tests with Bonferroni corrections demonstrating relationships between average prescribing persistence (days) by drug where events not repeated within 31 days were removed from analysis

\begin{tabular}{rccccccc}
\hline Drug & Buprenorphine & Codeine & Dihydrocodeine & Fentanyl & Morphine & Oxycodone & Tramadol \\
\hline Codeine & 0.405 & & & & & & \\
\hline Dihydrocodeine & $>.999$ & $>.999$ & & & & & \\
\hline
\end{tabular}




\begin{tabular}{rrrrrrrrl}
\hline Fentanyl & $>.999$ & $.017^{*}$ & .582 & & & & \\
\hline Morphine & $>.999$ & .173 & $>.999$ & $>.999$ & & & \\
\hline Oxycodone & $>.999$ & .061 & $>.999$ & $>.999$ & $>.999$ & & \\
\hline Tramadol & $>.999$ & $>.999$ & $>.999$ & .088 & .664 & .271 & \\
\hline Other & $>.999$ & $>.999$ & $>.999$ & .694 & $>.999$ & $>.999$ & $>.999$ \\
\hline
\end{tabular}

${ }^{*} p<0.05$ is statistically significant

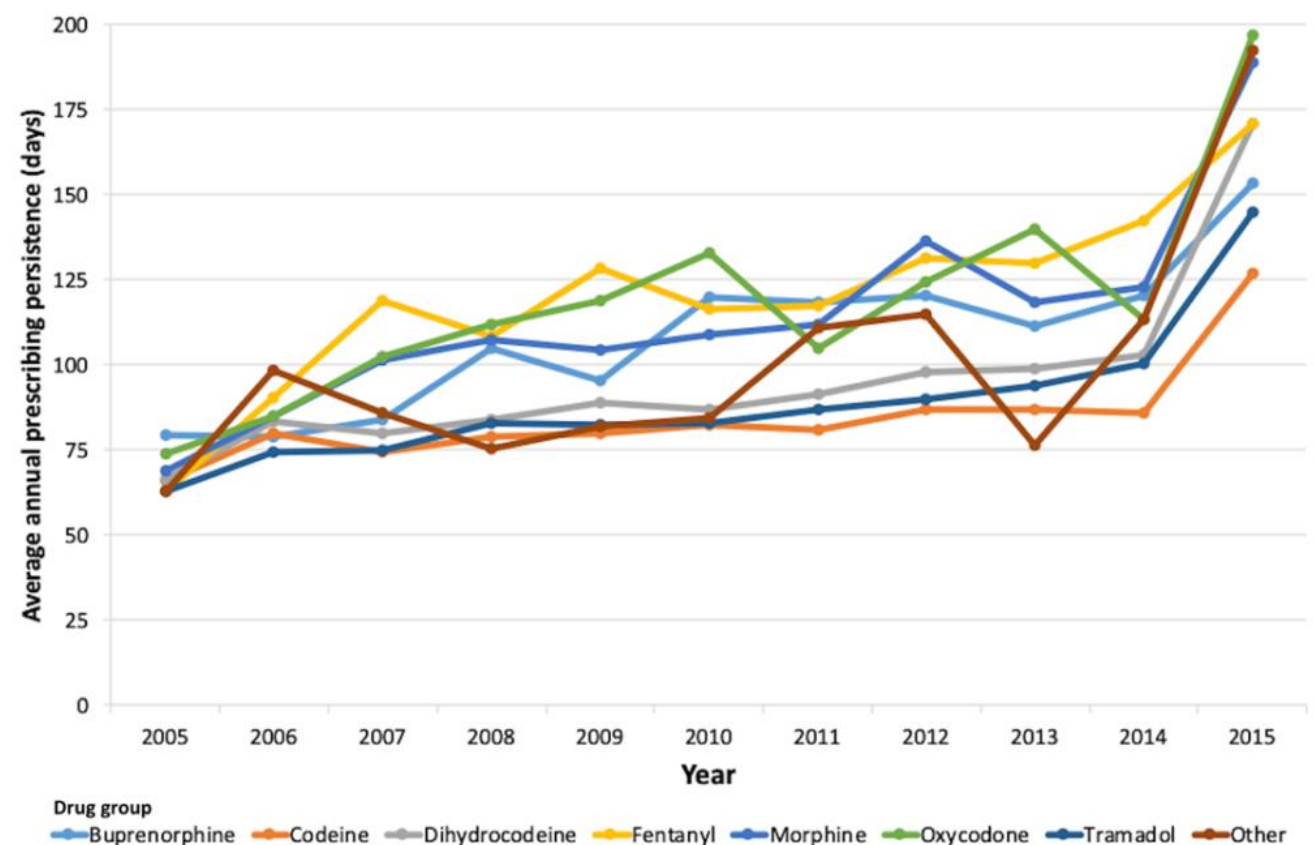

Figure G.13: Trends in average annual prescribing persistence, stratified by drug group and with >31-day repeats removed

When prescriptions which were not repeated within 31 days, were excluded from the analysis, 'other' opioids again demonstrated the largest percentage increase in average prescribing persistence between 2005 and 2015 (205.5\%, from 63.1 to 192.6 days) (Figure G.13). Average prescribing persistence for each drug group, excluding single prescriptions, were significantly different $(p<0.005$, $D_{\text {cohen }}=1.0, \eta^{2}=0.2$ ). Pairwise comparison analysis however, only confirmed that average codeine persistence was significantly less than that of fentanyl (Table $\mathrm{G}$.20) ( $\mathrm{p}<0.05)$. No difference was confirmed between the average durations of any other pairs of drug groups, when directly compared. 
Table G.21: Trends in prescription persistence between 2005 and 2015, stratified by drug group

\begin{tabular}{|c|c|c|c|c|c|c|c|c|}
\hline & \multicolumn{8}{|c|}{ Drug Group } \\
\hline & Buprenorphine & Codeine & Dihydrocodeine & Fentanyl & Morphine & Oxycodone & Tramadol & Other \\
\hline Mean duration (days) $\pm S D$ & $14.6 \pm 78.5$ & $7.1 \pm 55.0$ & $9.4 \pm 70.8$ & $15.0 \pm 90.2$ & $19.6 \pm 117.1$ & $17.1 \pm 101.0$ & $12.0 \pm 72.7$ & $5.33 \pm 49.6$ \\
\hline 2005 & 6.0 & 3.1 & 3.71 & 8.8 & 10.2 & 9.6 & 3.9 & 2.5 \\
\hline 2015 & 21.7 & 12.0 & 20.0 & 26.6 & 31.4 & 32.5 & 22.0 & 31.6 \\
\hline Percentage increase (\%) & 262.6 & 288.0 & 438.7 & 203.5 & 208.5 & 240.0 & 468.1 & 1177.2 \\
\hline Duration without one-off doses* (days) \pm SD & $120.2 \pm 195.5$ & $88.9 \pm 174.8$ & $98.0 \pm 209.2$ & $129.8 \pm$ & $138.4 \pm 283.9$ & $136.4 \pm 255.6$ & $95.4 \pm 185.1$ & $87.0 \pm 182.0$ \\
\hline 2005 & 79.4 & 65.9 & 66.3 & 235.8 & 68.9 & 74.0 & 62.7 & 63.1 \\
\hline 2015 & 153.7 & 127.2 & 171.0 & 63.0 & 189.1 & 197.1 & 144.9 & 192.6 \\
\hline Percentage increase (\%) & 93.6 & 93.0 & 158.0 & 171.2 & 170.7 & 166.3 & 131.0 & 205.5 \\
\hline & & & & 171.9 & & & & \\
\hline Number of events with persistence of $3-$ & & & & & & & & \\
\hline 6 months & 1401 & 28764 & 8810 & 702 & 2388 & 646 & 10107 & 503 \\
\hline Mean \pm SD & $127.4 \pm 90.4$ & $2,614.9 \pm 994.7$ & $800.9 \pm 98.3$ & $63.8 \pm 38.1$ & $217.1 \pm 217.6$ & $58.7 \pm 46.5$ & $918.8 \pm 423.7$ & $45.7 \pm 53.6$ \\
\hline 2005 & 23 & 1663 & 862 & 47 & 144 & 32 & 419 & 196 \\
\hline 2015 & 291 & 5009 & 1049 & 144 & 763 & 173 & 1656 & 41 \\
\hline Percentage change (\%) & 1165.2 & 201.2 & 21.7 & 206.4 & 429.9 & 440.6 & 295.2 & -79.1 \\
\hline Number of events with persistence more & & & & & & & & \\
\hline than 6 months & 1,355 & 24,480 & 7,638 & 702 & 2,483 & 655 & 8,673 & 392 \\
\hline Mean \pm SD & $123.2 \pm 95.3$ & $2,225.5 \pm$ & $694.4 \pm 240.9$ & $63.8 \pm 45.3$ & $225.7 \pm 305.4$ & $59.6 \pm 55.6$ & $788.5 \pm 493.5$ & $35.6 \pm 35.4$ \\
\hline 2005 & 13 & 1234.1 & 386 & 20 & 44 & 16 & 207 & 117 \\
\hline 2015 & 320 & 933 & 1351 & 157 & 1068 & 204 & 1940 & 56 \\
\hline Rate change (\%) & 2361.5 & 5464 & 250.0 & 685.0 & 2327.3 & 1175.0 & 837.2 & -52.1 \\
\hline & & 485.6 & & & & & & \\
\hline
\end{tabular}

*One-off doses $=$ where consecutive prescriptions had more than 31.5 days between issues

Table G.22: Dunn's pairwise tests with Bonferroni corrections demonstrating relationships between average prescribing persistence (days) by drug and where prescribing persistence was between 3 and 6 months

\begin{tabular}{|c|c|c|c|c|c|c|}
\hline Drug & Buprenorphine & Codeine & Dihydrocodeine & Fentanyl & Morphine & Oxycodone \\
\hline Codeine & $<.001^{*}$ & & & & & \\
\hline Dihydrocodeine & 0.143 & $>.999$ & & & & \\
\hline Fentanyl & $>.999$ & $<.001^{*}$ & $0.008^{*}$ & & & \\
\hline Morphine & $>.999$ & $0.004^{*}$ & 0.701 & $>.999$ & & \\
\hline
\end{tabular}




\begin{tabular}{llllllll}
\hline Oxycodone & $>.999$ & $<.001^{*}$ & $0.002^{*}$ & $>.999$ & $>.999$ & & \\
\hline Tramadol & 0.110 & $>.999$ & $>.999$ & $0.006^{*}$ & 0.563 & $0.002^{*}$ & \\
\hline Other & $>.999$ & $<.001^{*}$ & $<.001^{*}$ & $>.999$ & 0.563 & $>.999$ & $<.001^{*}$ \\
\hline
\end{tabular}

${ }^{*} p<0.05$ is statistically significant

Table G.23: Dunn's pairwise tests with Bonferroni corrections demonstrating relationships between average prescribing persistence (days) by drug and where prescribing persistence was over 6 months

\begin{tabular}{|c|c|c|c|c|c|c|c|}
\hline Drug & Buprenorphine & Codeine & Dihydrocodeine & Fentanyl & Morphine & Oxycodone & Tramadol \\
\hline Codeine & $<.001^{*}$ & & & & & & \\
\hline Dihydrocodeine & .145 & $>.999$ & & & & & \\
\hline Fentanyl & $>.999$ & $<.001 *$ & $.010^{*}$ & & & & \\
\hline Morphine & $>.999$ & $.005^{*}$ & .708 & $>.999$ & & & \\
\hline Oxycodone & $>.999$ & $<.001^{*}$ & $.004^{*}$ & $>.999$ & $>.999$ & & \\
\hline Tramadol & 0.116 & $>.999$ & $>.999$ & $.008^{*}$ & .588 & $.003^{*}$ & \\
\hline Other & $>.999$ & $<.001^{*}$ & $<.001^{*}$ & $>.999$ & .569 & $>.999$ & $<.001 *$ \\
\hline
\end{tabular}

${ }^{*} \mathrm{p}<0.05$ is statistically significant 


\section{G.5.1.1 Extended durations of prescribing persistence by drug}

Between 2005 and 2015, there were 53,321 prescribing events with a persistence of between 3-6 months. Occurrence of prescribing persistence between 3-6 months was highest for codeine, with a total 28,764 events. The other 7 groups of opioids had 24,557 events of $3-6$ months over the same period, around $17 \%$ fewer than for codeine alone (Table G.21). Codeine prescribing events increased by $201.2 \%$ over the study period (from 1,663 to 5,009 events per year). Tramadol was the second most prescribed opioid and had 3 times fewer events of 3-6 months than codeine, despite a 295.2\% (from 419 to 1,656 events per year) increase in the number of events during the study.

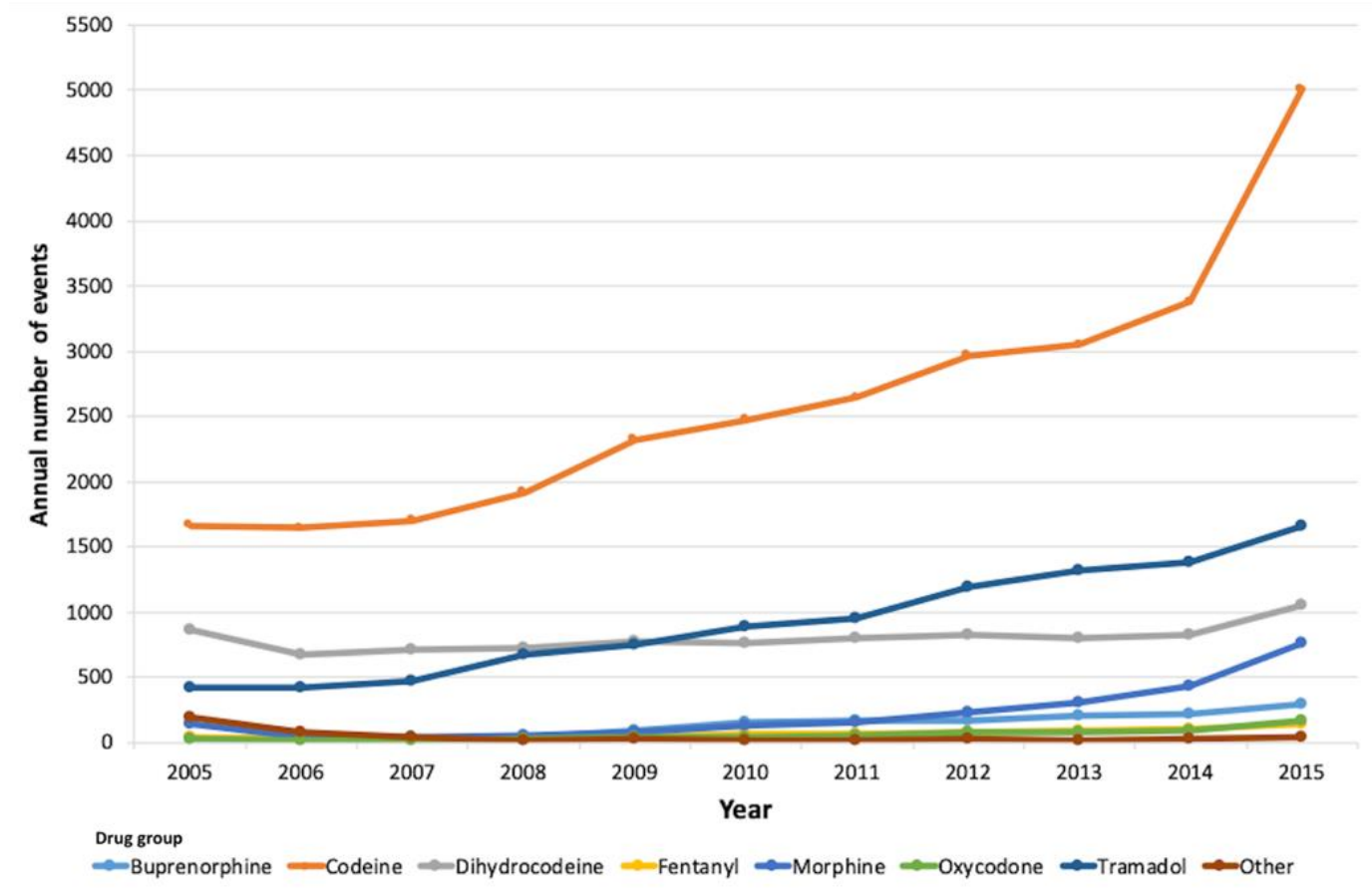

Figure G.14: Trends in prescribing persistence with duration of 3-6 months, between 2005 and 2015, stratified by drug group

Buprenorphine had the greatest percentage increase in the annual number of events of between 3-6 months (1165.2\%, from 23 to 291 events per year) (Figure G.14). This was likely due to the increasing use of low-dose buprenorphine patches which are used as an alternative to codeine. Whilst morphine and oxycodone had relatively small numbers of events each year, they both had a more than 5 times increase in the number of events per year between 2005 and 2015 (429.9\%, from 144 to 763 and 440.6\%, from 32 to 173 events per year respectively) (Figure G.14).

Statistical analysis demonstrated a significant difference in the annual number of events with a persistence of 3-6 months between then 8 groups of opioids examined $\left(p<.001, D_{\text {cohen }}=3.8, \eta^{2}=0.8\right)$. The number of events with codeine as the last prescribed medicine, were significantly greater than for morphine, oxycodone, fentanyl, buprenorphine, and the 'other' group of opioids. Whilst 
codeine events were greater in number than those for tramadol and dihydrocodeine, the difference was not significant.

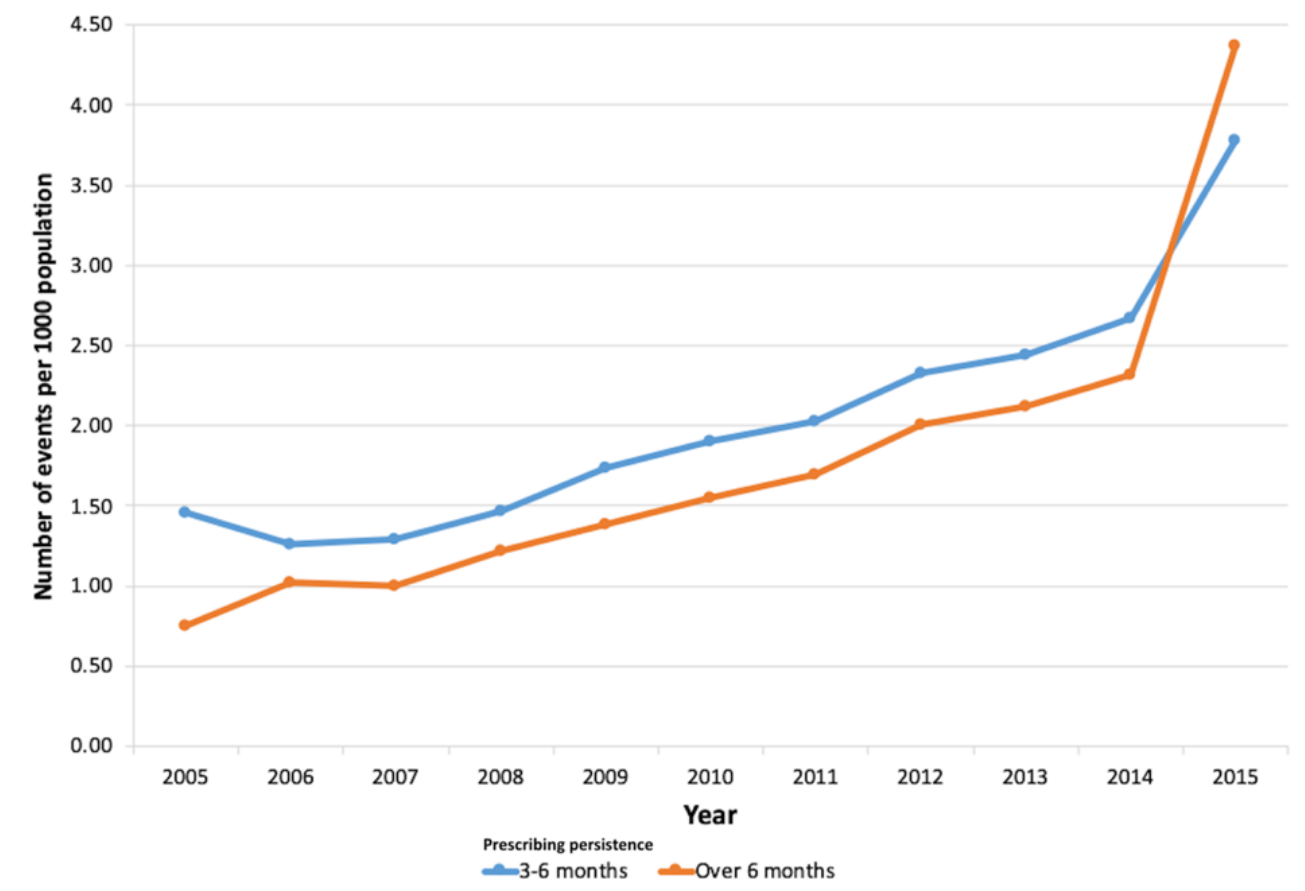

Figure-G.15: Trends in persistence of prescribing between 2005 and 2015, stratified by nominated duration

The average number of annual events with a persistence of 3-6 months for dihydrocodeine were significantly more than for oxycodone, fentanyl, and 'other' opioids, but not when compared to the other 4 groups compared. Tramadol showed the same pattern as dihydrocodeine, in terms of having significantly more events per year than oxycodone, fentanyl, and 'other' opioids, but not when compared pairwise to the other groups.

There were $15 \%$ fewer prescribing events which persisted for more than 6 months (46,378 events) than 3-6 months, during the study (Figure G.14). However, the number of events recorded in 2015 were higher for every drug group than those which persisted for 3-6 months (Table G.21). Between 2005 and 2015, the largest increases in the number of events were noted to be for strong opioids buprenorphine (2361.5\%, from 13 to 320 events per year), morphine (2327.3\%, from 44 to 1068 events per year) and oxycodone (1175\%, from 16 to 204 events per year). Tramadol, which was noted to have a reduction in the annual number of prescriptions issued over the study period had a large increase in prescribing persistence of more than 6 months (837.2\%, from 207 to 1,940 events per year).

Table G.24: Dunn's pairwise tests with Bonferroni corrections demonstrating relationships between average prescribing persistence (days) by drug and where prescribing persistence was between 3 and 6 months

\begin{tabular}{rccccccc}
\hline \multicolumn{1}{c}{ Drug } & Buprenorphine & Codeine & Dihydrocodeine & Fentanyl & Morphine & Oxycodone & Tramadol \\
\hline Codeine & $<.001^{*}$ & & & & & \\
\hline Dihydrocodeine & 0.143 & $>.999$ & & & & \\
\hline Fentanyl & $>.999$ & $<.001^{*}$ & $0.008^{*}$ & & \\
\hline Morphine & $>.999$ & $0.004^{*}$ & 0.701 & $>.999$ & & \\
\hline
\end{tabular}




\begin{tabular}{rccccccc}
\hline Oxycodone & $>.999$ & $<.001^{*}$ & $0.002^{*}$ & $>.999$ & $>.999$ & \\
\hline Tramadol & 0.110 & $>.999$ & $>.999$ & $0.006^{*}$ & 0.563 & $0.002^{*}$ & \\
\hline Other & $>.999$ & $<.001^{*}$ & $<.001^{*}$ & $>.999$ & 0.563 & $>.999$ & $<.001^{*}$ \\
\hline
\end{tabular}

$*_{p}<0.05$ is statistically significant

Table G.25: Dunn's pairwise tests with Bonferroni corrections demonstrating relationships between average prescribing persistence (days) by drug and where prescribing persistence was over 6 months

\begin{tabular}{|c|c|c|c|c|c|c|c|}
\hline Drug & Buprenorphine & Codeine & Dihydrocodeine & Fentanyl & Morphine & Oxycodone & Tramadol \\
\hline Codeine & $<.001^{*}$ & & & & & & \\
\hline Dihydrocodeine & .145 & $>.999$ & & & & & \\
\hline Fentanyl & $>.999$ & $<.001^{*}$ & $.010^{*}$ & & & & \\
\hline Morphine & $>.999$ & $.005^{*}$ & .708 & $>.999$ & & & \\
\hline Oxycodone & $>.999$ & $<.001^{*}$ & $.004^{*}$ & $>.999$ & $>.999$ & & \\
\hline Tramadol & 0.116 & $>.999$ & $>.999$ & $.008^{*}$ & .588 & $.003^{*}$ & \\
\hline Other & $>.999$ & $<.001^{*}$ & $<.001^{*}$ & $>.999$ & .569 & $>.999$ & $<.001^{*}$ \\
\hline
\end{tabular}

$* p<0.05$ is statistically significant

Comparison of the annual number of events with persistence of more than 6 months (Table G.21), demonstrated a significant difference between all 8 groups of opioids examined $\left(p<.001, \eta^{2}=0.8, D_{\text {cohen }}=3.5\right)$. Pairwise comparison confirmed that codeine had significantly more events of that duration than all groups, other than dihydrocodeine and tramadol. Dihydrocodeine had significantly more events with more than 6 months duration than fentanyl, oxycodone, and the 'other' group of opioids. Tramadol showed the same pattern and had significantly more events than fentanyl, oxycodone, and 'other' opioids. 


\section{Appendix H Prescribing trends stratified by recorded diagnosis of depression and anxiety}

\section{H.1 Read codes for depression and anxiety}

Table H.1: Read-codes for depression and anxiety

\begin{tabular}{|c|c|c|c|c|}
\hline COND_CAT & READ_VRS & READ_CD & TERM_ID & TERM_DETAIL \\
\hline DEPRESSION & 3 & 1285 & YaTgn & Family history of depression \\
\hline DEPRESSION & 3 & 1285 & Ya01E & FH: Depression \\
\hline DEPRESSION & 3 & 1465 & Ya04G & H/O: depression \\
\hline DEPRESSION & 3 & $62 T 1$. & Y01Fk & Postnatal depression \\
\hline DEPRESSION & 3 & $62 T 1$. & Y01FI & Postpartum depression \\
\hline DEPRESSION & 3 & $62 \mathrm{~T} 1$. & Y01Fm & Puerperal depression \\
\hline DEPRESSION & 3 & E1120 & Y01FR & Single major depressive episode, unspecified \\
\hline DEPRESSION & 3 & E1121 & Y01FS & Single major depressive episode, mild \\
\hline DEPRESSION & 3 & E1122 & Y01FT & Single major depressive episode, moderate \\
\hline DEPRESSION & 3 & E1123 & Y01FU & Single major depressive episode, severe, without mention of psychosis \\
\hline DEPRESSION & 3 & E1124 & Y01FV & Single major depressive episode, severe, with psychosis \\
\hline DEPRESSION & 3 & E1125 & Y01FW & Single major depressive episode, in partial or unspecified remission \\
\hline DEPRESSION & 3 & E1126 & Y01FX & Single major depressive episode, in full remission \\
\hline DEPRESSION & 3 & E112z & Y01FY & Single major depressive episode NOS \\
\hline DEPRESSION & 3 & E1130 & $\mathrm{Y01Fb}$ & Recurrent major depressive episodes, unspecified \\
\hline DEPRESSION & 3 & E1131 & Y01Fc & Recurrent major depressive episodes, mild \\
\hline DEPRESSION & 3 & E1132 & Y01Fd & Recurrent major depressive episodes, moderate \\
\hline DEPRESSION & 3 & E1133 & Y01Fe & Recurrent major depressive episodes, severe, without mention of psychosis \\
\hline DEPRESSION & 3 & E1134 & Y01Ff & Recurrent major depressive episodes, severe, with psychosis \\
\hline DEPRESSION & 3 & E1135 & Y01Fg & Recurrent major depressive episodes, in partial or unspecified remission \\
\hline
\end{tabular}




\begin{tabular}{|c|c|c|c|c|}
\hline DEPRESSION & 3 & E1136 & Y01Fh & Recurrent major depressive episodes, in full remission \\
\hline DEPRESSION & 3 & E1137 & YaYAt & Recurrent depression \\
\hline DEPRESSION & 3 & E113z & Y01Fi & Recurrent major depressive episode NOS \\
\hline DEPRESSION & 3 & E115. & Y01Ep & Bipolar affective disorder, current episode depression \\
\hline DEPRESSION & 3 & E1150 & Y01Er & Bipolar affective disorder, currently depressed, unspecified \\
\hline DEPRESSION & 3 & E1151 & Y01Es & Bipolar affective disorder, currently depressed, mild \\
\hline DEPRESSION & 3 & E1152 & Y01Et & Bipolar affective disorder, currently depressed, moderate \\
\hline DEPRESSION & 3 & E1153 & Y01Eu & Bipolar affective disorder, currently depressed, severe, without mention of psychosis \\
\hline DEPRESSION & 3 & E1154 & Y01Ev & Bipolar affective disorder, currently depressed, severe, with psychosis \\
\hline DEPRESSION & 3 & E1155 & Y01Ew & Bipolar affective disorder, currently depressed, in partial or unspecified remission \\
\hline DEPRESSION & 3 & E1156 & Y01Ex & Bipolar affective disorder, currently depressed, in full remission \\
\hline DEPRESSION & 3 & $\mathrm{E} 115 \mathrm{z}$ & Y01Ey & Bipolar affective disorder, currently depressed, NOS \\
\hline DEPRESSION & 3 & E130. & Y01FI & Psychotic reactive depression \\
\hline DEPRESSION & 3 & E2112 & YMBxo & Neurotic depression \\
\hline DEPRESSION & 3 & E2BO. & YaYB4 & Postviral depression \\
\hline DEPRESSION & 3 & E2B1. & YaYB5 & Chronic depression \\
\hline DEPRESSION & 3 & Eu313 & YMB3u & [X]Bipolar affective disorder, current episode mild or moderate depression \\
\hline DEPRESSION & 3 & Eu314 & YMB3v & [X]Bipolar affective disorder, current episode severe depression without psychotic symptoms \\
\hline DEPRESSION & 3 & R007z & YMOwl & [D]Postoperative depression \\
\hline DEPRESSION & 3 & xoos 8 & Y01Cd & Post-schizophrenic depression \\
\hline DEPRESSION & 3 & xoosb & Y01Gs & Anxiety depression \\
\hline DEPRESSION & 3 & Xooso & Y01FK & Agitated depression \\
\hline DEPRESSION & 3 & XOOSR & Y01FO & Endogenous depression \\
\hline DEPRESSION & 3 & xooss & Y01Fj & Endogenous depression first episode \\
\hline DEPRESSION & 3 & Xoosu & Y01Fo & Masked depression \\
\hline
\end{tabular}




\begin{tabular}{|c|c|c|c|c|}
\hline DEPRESSION & 3 & $\mathrm{X} 40 \mathrm{DI}$ & Y418V & Mild postnatal depression \\
\hline DEPRESSION & 3 & $\mathrm{X} 40 \mathrm{Dm}$ & Y418Y & Severe postnatal depression \\
\hline DEPRESSION & 3 & $\mathrm{X} 40 \mathrm{Dm}$ & Y418Y & Severe postnatal depression \\
\hline DEPRESSION & 3 & X7755 & Y7Flu & Single stimulus depression \\
\hline DEPRESSION & 3 & Xa110 & Y01Fn & Maternity blues \\
\hline DEPRESSION & 3 & ХаB9J & YMJar & Depression NOS \\
\hline DEPRESSION & 3 & $\mathrm{XaCHr}$ & $\mathrm{YMBxU}$ & [X] Single episode agitated depression without psychotic symptoms \\
\hline DEPRESSION & 3 & $\mathrm{XaCHs}$ & YMBXV & {$[\mathrm{X}]$ Single episode major depression without psychotic symptoms } \\
\hline DEPRESSION & 3 & $\mathrm{XaCls}$ & Yakyu & [X]Mild depression \\
\hline DEPRESSION & 3 & $\mathrm{XaCls}$ & Yabyn & Mild depression \\
\hline DEPRESSION & 3 & $\mathrm{XaClt}$ & Yabyo & Moderate depression \\
\hline DEPRESSION & 3 & XaClu & Yabyq & Severe depression \\
\hline DEPRESSION & 3 & XalmU & YalJv & Symptoms of depression \\
\hline DEPRESSION & 3 & XaK6d & YanJb & Depression annual review \\
\hline DEPRESSION & 3 & XaK6e & YanJc & Depression medication review \\
\hline DEPRESSION & 3 & XaLGO & YaoJo & Depression resolved \\
\hline DEPRESSION & 3 & XaNg1 & Yaqge & History of postnatal depression \\
\hline DEPRESSION & 3 & $\mathrm{XaXOC}$ & Yattn & Suspected depression \\
\hline DEPRESSION & 3 & XaX53 & Yatxk & Single major depressive episode, severe, with psychosis, psychosis in remission \\
\hline DEPRESSION & 3 & XaX54 & Yatxl & Recurrent major depressive episodes, severe, with psychosis, psychosis in remission \\
\hline DEPRESSION & 3 & $\mathrm{XaY} 2 \mathrm{C}$ & Yaudm & Antenatal depression \\
\hline DEPRESSION & 3 & XE1YO & Y01FQ & Single major depressive episode \\
\hline DEPRESSION & 3 & XE1YC & Y01FM & Neurotic depression reactive type \\
\hline DEPRESSION & 3 & XE1YC & Y01FL & Reactive depression \\
\hline DEPRESSION & 3 & XE1Zb & YMBxa & {$[\mathrm{X}]$ Depression NOS } \\
\hline
\end{tabular}




\begin{tabular}{|c|c|c|c|c|}
\hline DEPRESSION & 3 & $\mathrm{XM} 1 \mathrm{GC}$ & Y01FZ & Endogenous depression - recurrent \\
\hline DEPRESSION & 3 & XSEGJ & YSNUF & Major depression \\
\hline DEPRESSION & 3 & XSGok & YSRJY & Mild major depression \\
\hline DEPRESSION & 3 & XSGol & YSRJZ & Moderate major depression \\
\hline DEPRESSION & 3 & XSGom & YSRJa & Severe major depression without psychotic features \\
\hline DEPRESSION & 3 & XSGon & Yat7Y & {$[\mathrm{X}]$ Major depression, severe with psychotic symptoms } \\
\hline DEPRESSION & 3 & XSGon & YSRJC & Psychotic depression \\
\hline DEPRESSION & 3 & XSGon & YSRJb & Severe major depression with psychotic features \\
\hline DEPRESSION & 3 & $\mathrm{XSKr7}$ & YamzO & Cotard syndrome \\
\hline DEPRESSION & 3 & ZV790 & YaOqu & [V]Screening for depression \\
\hline DEPRESSION & 2 & 1285 & 0 & FH: Depression \\
\hline DEPRESSION & 2 & 1287 & 0 & FH: Manic depressive state \\
\hline DEPRESSION & 2 & 1465 & 0 & H/O: depression \\
\hline DEPRESSION & 2 & $1 \mathrm{~B} 1 \mathrm{U}$. & 0 & Symptoms of depression \\
\hline DEPRESSION & 2 & $1 \mathrm{JJ} .$. & 0 & Suspected depression \\
\hline DEPRESSION & 2 & $212 S$. & 0 & Depression resolved \\
\hline DEPRESSION & 2 & $9 \mathrm{H} 90$. & 0 & Depression annual review \\
\hline DEPRESSION & 2 & $9 \mathrm{H} 91$. & 0 & Depression medication review \\
\hline DEPRESSION & 2 & $9 \mathrm{H} 92$. & 0 & Depression interim review \\
\hline DEPRESSION & 2 & 9HAO. & 0 & On depression register \\
\hline DEPRESSION & 2 & E112. & 13 & Endogenous depression first episode \\
\hline DEPRESSION & 2 & E1120 & 0 & Single major depressive episode, unspecified \\
\hline DEPRESSION & 2 & $\mathrm{E} 1121$ & 0 & Single major depressive episode, mild \\
\hline DEPRESSION & 2 & E1122 & 0 & Single major depressive episode, moderate \\
\hline DEPRESSION & 2 & E1123 & 0 & Single major depressive episode, severe, without mention of psychosis \\
\hline
\end{tabular}




\begin{tabular}{|c|c|c|c|c|}
\hline DEPRESSION & 2 & E1124 & 0 & Single major depressive episode, severe, with psychosis \\
\hline DEPRESSION & 2 & E1125 & 0 & Single major depressive episode, in partial or unspecified remission \\
\hline DEPRESSION & 2 & E1126 & 0 & Single major depressive episode, in full remission \\
\hline DEPRESSION & 2 & E112z & 0 & Single major depressive episode NOS \\
\hline DEPRESSION & 2 & E113. & 11 & Endogenous depression - recurrent \\
\hline DEPRESSION & 2 & E1130 & 0 & Recurrent major depressive episodes, unspecified \\
\hline DEPRESSION & 2 & E1131 & 0 & Recurrent major depressive episodes, mild \\
\hline DEPRESSION & 2 & E1132 & 0 & Recurrent major depressive episodes, moderate \\
\hline DEPRESSION & 2 & E1133 & 0 & Recurrent major depressive episodes, severe, without mention of psychosis \\
\hline DEPRESSION & 2 & E1134 & 0 & Recurrent major depressive episodes, severe, with psychosis \\
\hline DEPRESSION & 2 & E1135 & 0 & Recurrent major depressive episodes, in partial or unspecified remission \\
\hline DEPRESSION & 2 & E1136 & 0 & Recurrent major depressive episodes, in full remission \\
\hline DEPRESSION & 2 & E1137 & 0 & Recurrent depression \\
\hline DEPRESSION & 2 & E113z & 0 & Recurrent major depressive episode NOS \\
\hline DEPRESSION & 2 & E115. & 11 & Manic-depressive - now depressed \\
\hline DEPRESSION & 2 & E1150 & 0 & Bipolar affective disorder, currently depressed, unspecified \\
\hline DEPRESSION & 2 & E1151 & 0 & Bipolar affective disorder, currently depressed, mild \\
\hline DEPRESSION & 2 & E1152 & 0 & Bipolar affective disorder, currently depressed, moderate \\
\hline DEPRESSION & 2 & E1153 & 0 & Bipolar affective disorder, currently depressed, severe, without mention of psychosis \\
\hline DEPRESSION & 2 & E1154 & 0 & Bipolar affective disorder, currently depressed, severe, with psychosis \\
\hline DEPRESSION & 2 & E1155 & 0 & Bipolar affective disorder, currently depressed, in partial or unspecified remission \\
\hline DEPRESSION & 2 & E1156 & 0 & Bipolar affective disorder, currently depressed, in full remission \\
\hline DEPRESSION & 2 & E115z & 0 & Bipolar affective disorder, currently depressed, NOS \\
\hline DEPRESSION & 2 & E11z2 & 0 & Masked depression \\
\hline DEPRESSION & 2 & E130. & 11 & Psychotic reactive depression \\
\hline
\end{tabular}




\begin{tabular}{|c|c|c|c|c|}
\hline DEPRESSION & 2 & E135. & 0 & Agitated depression \\
\hline DEPRESSION & 2 & E2003 & 0 & Anxiety with depression \\
\hline DEPRESSION & 2 & E204. & 11 & Postnatal depression \\
\hline DEPRESSION & 2 & E204. & 0 & Neurotic depression reactive type \\
\hline DEPRESSION & 2 & E2BO. & 0 & Postviral depression \\
\hline DEPRESSION & 2 & E2B1. & 0 & Chronic depression \\
\hline DEPRESSION & 2 & Eu204 & 0 & [X]Post-schizophrenic depression \\
\hline DEPRESSION & 2 & Eu32. & 13 & {$[\mathrm{X}]$ Single episode of reactive depression } \\
\hline DEPRESSION & 2 & Eu32. & 12 & [X]Single episode of psychogenic depression \\
\hline DEPRESSION & 2 & Eu320 & 0 & [X]Mild depressive episode \\
\hline DEPRESSION & 2 & Eu321 & 0 & [X]Moderate depressive episode \\
\hline DEPRESSION & 2 & Eu322 & 0 & [X]Severe depressive episode without psychotic symptoms \\
\hline DEPRESSION & 2 & Eu323 & 0 & [X]Severe depressive episode with psychotic symptoms \\
\hline DEPRESSION & 2 & Eu324 & 0 & [X]Mild depression \\
\hline DEPRESSION & 2 & Eu325 & 0 & {$[\mathrm{X}]$ Major depression, mild } \\
\hline DEPRESSION & 2 & Eu326 & 0 & [X]Major depression, moderately severe \\
\hline DEPRESSION & 2 & Eu327 & 0 & [X]Major depression, severe without psychotic symptoms \\
\hline DEPRESSION & 2 & Eu328 & 0 & [X]Major depression, severe with psychotic symptoms \\
\hline DEPRESSION & 2 & Eu329 & 0 & [X]Single major depressive episode, severe, with psychosis, psychosis in remission \\
\hline DEPRESSION & 2 & Eu32A & 0 & {$[\mathrm{X}]$ Recurrent major depressive episodes, severe, with psychosis, psychosis in remission } \\
\hline DEPRESSION & 2 & Eu32B & 0 & {$[\mathrm{X}]$ Antenatal depression } \\
\hline DEPRESSION & 2 & Eu32y & 0 & [X]Other depressive episodes \\
\hline DEPRESSION & 2 & Eu32z & 11 & {$[\mathrm{X}]$ Depression NOS } \\
\hline DEPRESSION & 2 & Eu32z & 14 & {$[\mathrm{X}]$ Reactive depression NOS } \\
\hline DEPRESSION & 2 & Eu32z & 0 & [X]Depressive episode, unspecified \\
\hline
\end{tabular}




\begin{tabular}{|c|c|c|c|c|}
\hline DEPRESSION & 2 & Eu32z & 0 & [X]Depressive episode, unspecified \\
\hline DEPRESSION & 2 & Eu33. & 13 & {$[\mathrm{X}]$ Recurrent episodes of reactive depression } \\
\hline DEPRESSION & 2 & Eu330 & 0 & {$[\mathrm{X}]$ Recurrent depressive disorder, current episode mild } \\
\hline DEPRESSION & 2 & Eu331 & 0 & {$[\mathrm{X}]$ Recurrent depressive disorder, current episode moderate } \\
\hline DEPRESSION & 2 & Eu332 & 0 & {$[\mathrm{X}]$ Recurrent depressive disorder, current episode severe without psychotic symptoms } \\
\hline DEPRESSION & 2 & Eu333 & 0 & {$[\mathrm{X}]$ Recurrent depressive disorder, current episode severe with psychotic symptoms } \\
\hline DEPRESSION & 2 & Eu334 & 0 & [X]Recurrent depressive disorder, currently in remission \\
\hline DEPRESSION & 2 & Eu33y & 0 & {$[\mathrm{X}] \mathrm{Other}$ recurrent depressive disorders } \\
\hline DEPRESSION & 2 & Eu33z & 0 & {$[\mathrm{X}]$ Recurrent depressive disorder, unspecified } \\
\hline DEPRESSION & 2 & Eu341 & 13 & {$[\mathrm{X}]$ Neurotic depression } \\
\hline DEPRESSION & 2 & Eu341 & 14 & [X]Persistant anxiety depression \\
\hline DEPRESSION & 2 & Eu412 & 11 & [X]Mild anxiety depression \\
\hline DEPRESSION & 2 & Eu530 & 11 & [X]Postnatal depression NOS \\
\hline DEPRESSION & 2 & Eu530 & 12 & [X]Postpartum depression NOS \\
\hline DEPRESSION & 2 & $\mathrm{R} 007 \mathrm{z}$ & 13 & [D]Postoperative depression \\
\hline DEPRESSION & 2 & ZV790 & 0 & [V]Screening for depression \\
\hline ANXIETY & 3 & $1 \mathrm{~B} 1 .$. & Y7D89 & General nervous symptoms \\
\hline ANXIETY & 3 & $1 \mathrm{~B} 1 \mathrm{Z}$. & Y7D86 & General nervous symptom NOS \\
\hline ANXIETY & 3 & E200. & Y01GY & Anxiety disorder \\
\hline ANXIETY & 3 & E200. & Y01Gq & Anxiety state \\
\hline ANXIETY & 3 & E2000 & Y01Gb & Anxiety state unspecified \\
\hline ANXIETY & 3 & E2002 & Y01Gp & Anxiety neurosis \\
\hline ANXIETY & 3 & E2002 & Y01Gm & GAD - Generalised anxiety disorder \\
\hline ANXIETY & 3 & E2002 & Y01GI & Generalised anxiety disorder \\
\hline ANXIETY & 3 & E2004 & YaYAu & Chronic anxiety \\
\hline
\end{tabular}




\begin{tabular}{|c|c|c|c|c|}
\hline ANXIETY & 3 & E2005 & YaYAv & Recurrent anxiety \\
\hline ANXIETY & 3 & $\mathrm{E} 200 \mathrm{z}$ & Y01Ga & Anxiety state NOS \\
\hline ANXIETY & 3 & E2020 & Y01GH & Phobia unspecified \\
\hline ANXIETY & 3 & E2021 & Y01GL & Agoraphobia with panic attacks \\
\hline ANXIETY & 3 & E2022 & Y01GK & Agoraphobia without mention of panic attacks \\
\hline ANXIETY & 3 & E2023 & Y01GQ & Social phobia, fear of eating in public \\
\hline ANXIETY & 3 & E2024 & Y01GP & Social phobia, fear of public speaking \\
\hline ANXIETY & 3 & E2025 & Y01GO & Social phobia, fear of public washing \\
\hline ANXIETY & 3 & E2026 & Y01GG & Acrophobia \\
\hline ANXIETY & 3 & E2027 & Y01GS & Animal phobia \\
\hline ANXIETY & 3 & E2028 & Y01GT & Claustrophobia \\
\hline ANXIETY & 3 & E2029 & Y01GF & Fear of crowds \\
\hline ANXIETY & 3 & E202C & YaYAy & Dental phobia \\
\hline ANXIETY & 3 & E202E & YaYBo & Fear of pregnancy \\
\hline ANXIETY & 3 & E2920 & YM1hy & Separation anxiety disorder \\
\hline ANXIETY & 3 & Eu40y & YMB4V & {$[\mathrm{X}]$ Other phobic anxiety disorders } \\
\hline ANXIETY & 3 & Eu410 & YMB4Y & [X]Panic disorder [episodic paroxysmal anxiety] \\
\hline ANXIETY & 3 & Ua1qa & YMGBQ & Fear of dentist \\
\hline ANXIETY & 3 & Ua1qc & YMGBS & Fear of not coping with treatment \\
\hline ANXIETY & 3 & Ua1qd & YMGBT & Fear of lifts \\
\hline ANXIETY & 3 & Ua1qe & YMGBU & Fear of thunderstorm \\
\hline ANXIETY & 3 & Ua1qf & YMGWS & Terrified \\
\hline ANXIETY & 3 & Ua1qo & Ya5FV & Anxiety about making mistakes \\
\hline ANXIETY & 3 & Ua1qp & Ya5FU & Anxiety about altered body image \\
\hline ANXIETY & 3 & Ua1qs & YMGBi & Fear of wetting self in public \\
\hline
\end{tabular}




\begin{tabular}{|c|c|c|c|c|}
\hline ANXIETY & 3 & Ua1qS & YMGBI & Panic attack \\
\hline ANXIETY & 3 & Ua1qt & YMGBj & Fear of losing control of bowels in public \\
\hline ANXIETY & 3 & Ua1qu & YMGBK & Fear of walking \\
\hline ANXIETY & 3 & Ua1qV & YMGBL & Fear of mobilising \\
\hline ANXIETY & 3 & Ua1qW & YMGBM & Fear of disconnection from ventilator \\
\hline ANXIETY & 3 & Ua1qX & YMGBN & Fear of being left alone during period of dependence \\
\hline ANXIETY & 3 & Ua1qY & YMGBO & Fear of being left alone \\
\hline ANXIETY & 3 & XOORP & Y017s & Organic anxiety disorder \\
\hline ANXIETY & 3 & Xoosa & Y01Gi & Non-situational panic attack \\
\hline ANXIETY & 3 & xoosb & Y01Gr & Mixed anxiety and depressive disorder \\
\hline ANXIETY & 3 & xoosc & Y01Gt & Anxiety hysteria \\
\hline ANXIETY & 3 & xoosd & Y01H8 & Stage fright \\
\hline ANXIETY & 3 & xoose & Y01H9 & Examination fear \\
\hline ANXIETY & 3 & xoose & Y01H9 & Examination fear \\
\hline ANXIETY & 3 & xoosv & Y01GI & Agoraphobia \\
\hline ANXIETY & 3 & xoosw & Y01GM & Social phobia \\
\hline ANXIETY & 3 & xoosx & YaOso & Specific phobia \\
\hline ANXIETY & 3 & XoosY & Y01GU & Needle phobia \\
\hline ANXIETY & 3 & xoosz & Y01Gh & Situational panic attack \\
\hline ANXIETY & 3 & X761a & Y7D8Q & Fear of swallowing \\
\hline ANXIETY & 3 & $\mathrm{X761b}$ & Y7D8R & Fear of collapsing \\
\hline ANXIETY & 3 & $\mathrm{X761c}$ & Y7D8S & Fear of fainting \\
\hline ANXIETY & 3 & X761d & Y7D8T & Fear of having a heart attack \\
\hline ANXIETY & 3 & X761e & Y7D8U & Fear of shaking \\
\hline ANXIETY & 3 & X761f & Y7D8V & Fear of sweating \\
\hline
\end{tabular}




\begin{tabular}{|c|c|c|c|c|}
\hline ANXIETY & 3 & X761g & Y7D8W & Fear of dying \\
\hline ANXIETY & 3 & X761h & Y7D8X & Fear of going crazy \\
\hline ANXIETY & 3 & $\mathrm{x} 761 \mathrm{i}$ & Y7D8a & Fear of losing emotional control \\
\hline ANXIETY & 3 & $\mathrm{x} 761 \mathrm{j}$ & Y7D8b & Fear of becoming fat \\
\hline ANXIETY & 3 & $\mathrm{X7611}$ & Y7D8f & Fear of appearing ridiculous \\
\hline ANXIETY & 3 & $\mathrm{X} 761 \mathrm{~m}$ & Y7D8g & Fear of saying the wrong thing \\
\hline ANXIETY & 3 & $\mathrm{X761N}$ & Y7D7s & Anxiety and fear \\
\hline ANXIETY & 3 & $x 761 n$ & Y7D8m & Fear of going out \\
\hline ANXIETY & 3 & X761p & Y7D8p & Fear of empty streets \\
\hline ANXIETY & 3 & X761P & Y7D8A & III-at-ease \\
\hline ANXIETY & 3 & $\times 761 q$ & Y7D8q & Fear of open spaces \\
\hline ANXIETY & 3 & $\mathrm{X} 761 \mathrm{r}$ & Y7D8r & Fear of crossing streets \\
\hline ANXIETY & 3 & X761T & Y7D8J & Anxiety about health \\
\hline ANXIETY & 3 & $\mathrm{x} 761 \mathrm{t}$ & Y7D8t & Fear of transport \\
\hline ANXIETY & 3 & $\mathrm{X761U}$ & Y7D8K & Fear of losing control of bowels \\
\hline ANXIETY & 3 & $\mathrm{x} 761 \mathrm{u}$ & Y7D8x & Social fear \\
\hline ANXIETY & 3 & $\mathrm{X} 761 \mathrm{v}$ & Y7D8y & Fear of activities in public \\
\hline ANXIETY & 3 & X761V & Y7D8L & Fear of wetting self \\
\hline ANXIETY & 3 & X761w & Y7D8z & Fear of eating in public \\
\hline ANXIETY & 3 & $\mathrm{X} 761 \mathrm{~W}$ & Y7D8M & Fear of vomiting in public \\
\hline ANXIETY & 3 & $\mathrm{x} 761 \mathrm{X}$ & Y7D8N & Fear of having a fit \\
\hline ANXIETY & 3 & $\mathrm{X} 761 \mathrm{x}$ & Y7D90 & Fear of public speaking \\
\hline ANXIETY & 3 & $\mathrm{X761Y}$ & Y7D80 & Fear of choking \\
\hline ANXIETY & 3 & $\mathrm{X761Y}$ & Y7D80 & Fear of choking \\
\hline ANXIETY & 3 & $\mathrm{x} 761 \mathrm{y}$ & Y7D91 & Fear of using public toilets \\
\hline
\end{tabular}




\begin{tabular}{|c|c|c|c|c|}
\hline ANXIETY & 3 & $\mathrm{X} 7612$ & Ya1tE & Anxiety about blushing \\
\hline ANXIETY & 3 & $\mathrm{X} 761 \mathrm{z}$ & Y7D92 & Fear of writing in public \\
\hline ANXIETY & 3 & $x 7620$ & Y7D93 & Fear of social group activities \\
\hline ANXIETY & 3 & X7621 & Y7D94 & Fear of being in a small group \\
\hline ANXIETY & 3 & $x 7622$ & Y7D95 & Fear of social gatherings \\
\hline ANXIETY & 3 & X7623 & Y7D96 & Fear of speaking on the phone \\
\hline ANXIETY & 3 & X7624 & Y7D97 & Fear of speaking to people in authority \\
\hline ANXIETY & 3 & X7625 & Y7D98 & Fear of being laughed at \\
\hline ANXIETY & 3 & X7626 & Y7D99 & Fear of being watched \\
\hline ANXIETY & 3 & $x 7627$ & YaOsq & Specific fear \\
\hline ANXIETY & 3 & X7628 & Y7D9B & Fear of natural phenomena \\
\hline ANXIETY & 3 & X7629 & Y7D9C & Fear of the dark \\
\hline ANXIETY & 3 & $\mathrm{X} 762 \mathrm{~A}$ & Y7D9D & Fear of animals \\
\hline ANXIETY & 3 & $\mathrm{X} 762 \mathrm{a}$ & Y7D9f & Fear of ghosts \\
\hline ANXIETY & 3 & $\mathrm{X} 762 \mathrm{~B}$ & Y7D9E & Fear of feathers \\
\hline ANXIETY & 3 & $x 762 b$ & Y7D9g & Fear of school \\
\hline ANXIETY & 3 & $\mathrm{X} 762 \mathrm{C}$ & Y7D9F & Fear of enclosed spaces \\
\hline ANXIETY & 3 & X762E & $\mathrm{Y7D9H}$ & Fear of tunnels \\
\hline ANXIETY & 3 & $\mathrm{X} 762 \mathrm{~F}$ & Y7D91 & Fear of phone boxes \\
\hline ANXIETY & 3 & $X 762 G$ & Y7D9J & Fear of flying \\
\hline ANXIETY & 3 & $\mathrm{X} 762 \mathrm{H}$ & Y7D9K & Flying phobia \\
\hline ANXIETY & 3 & $\mathrm{X} 7621$ & Y7D9L & Fear associated with illness and body function \\
\hline ANXIETY & 3 & X762J & Y7D9M & Fear of anaesthetic \\
\hline ANXIETY & 3 & $\mathrm{X} 762 \mathrm{~K}$ & Y7D9N & Fear of general anaesthetic \\
\hline ANXIETY & 3 & X762L & Y7D9P & Fear of awareness under general anaesthetic \\
\hline
\end{tabular}




\begin{tabular}{|c|c|c|c|c|}
\hline ANXIETY & 3 & $\mathrm{X762M}$ & Y7D9Q & Fear of not waking from general anaesthetic \\
\hline ANXIETY & 3 & $\mathrm{X} 762 \mathrm{~N}$ & Y7D9R & Fear of local anaesthetic \\
\hline ANXIETY & 3 & X7620 & Y7D9S & Fear of problem after anaesthetic \\
\hline ANXIETY & 3 & $\mathrm{X} 762 \mathrm{R}$ & Ya5sS & Fear of surgical masks \\
\hline ANXIETY & 3 & $\mathrm{X762S}$ & Y7D9X & Fear of hospitals \\
\hline ANXIETY & 3 & $\mathrm{X762T}$ & Y7D9Y & Fear of death \\
\hline ANXIETY & 3 & $\mathrm{X762U}$ & Y7D9Z & Fear of contracting disease \\
\hline ANXIETY & 3 & $\mathrm{X} 762 \mathrm{~V}$ & Y7D9a & Fear of infection \\
\hline ANXIETY & 3 & $\mathrm{X} 762 \mathrm{~W}$ & Y7D9b & Fear of contracting venereal disease \\
\hline ANXIETY & 3 & $\mathrm{x} 762 \mathrm{X}$ & Y7D9c & Fear of contracting HIV infection \\
\hline ANXIETY & 3 & $\mathrm{X} 762 \mathrm{Y}$ & Y7D9d & Fear of contracting radiation sickness \\
\hline ANXIETY & 3 & X762Z & Y7D9e & Fear of the bogey man \\
\hline ANXIETY & 3 & X78wp & Y7KRx & Apprehension \\
\hline ANXIETY & 3 & Xa00r & YaOss & Fear of heights \\
\hline ANXIETY & 3 & $\mathrm{XaO0s}$ & Ya0st & Fear of water \\
\hline ANXIETY & 3 & $\mathrm{XaOXB}$ & Ya1lw & Fear relating to body function \\
\hline ANXIETY & 3 & $\mathrm{XaOXG}$ & Ya1m3 & Anxiety about losing control of bowels \\
\hline ANXIETY & 3 & $\mathrm{XaOXH}$ & Ya1m4 & Anxiety about wetting self \\
\hline ANXIETY & 3 & $\mathrm{XaOXI}$ & Ya1m5 & Anxiety about vomiting in public \\
\hline ANXIETY & 3 & $\mathrm{XaOXJ}$ & Ya1m6 & Anxiety about having a fit \\
\hline ANXIETY & 3 & $\mathrm{XaOXK}$ & Ya1m7 & Anxiety about choking \\
\hline ANXIETY & 3 & $\mathrm{XaOXK}$ & Ya1m7 & Anxiety about choking \\
\hline ANXIETY & 3 & $\mathrm{XaOXM}$ & Ya1m9 & Anxiety about swallowing \\
\hline ANXIETY & 3 & $\mathrm{XaOXN}$ & Ya1mA & Anxiety about collapsing \\
\hline ANXIETY & 3 & $\mathrm{XaOXO}$ & Ya1mB & Anxiety about shaking \\
\hline
\end{tabular}




\begin{tabular}{|c|c|c|c|c|}
\hline ANXIETY & 3 & XaOXP & Ya1mc & Anxiety about sweating \\
\hline ANXIETY & 3 & $\mathrm{XaOXQ}$ & Ya1mD & Anxiety about dying \\
\hline ANXIETY & 3 & XaOXR & Ya1mF & Anxiety about going crazy \\
\hline ANXIETY & 3 & $\mathrm{XaOXU}$ & Ya1mk & Anxiety about becoming fat \\
\hline ANXIETY & 3 & $\mathrm{XaOXU}$ & Ya1mL & Anxiety about obesity \\
\hline ANXIETY & 3 & $\mathrm{XaOXX}$ & Ya1mo & Anxiety about fainting \\
\hline ANXIETY & 3 & $\mathrm{XaOXX}$ & Ya1mo & Anxiety about fainting \\
\hline ANXIETY & 3 & $\mathrm{XaOXY}$ & Ya1mP & Anxiety about having a heart attack \\
\hline ANXIETY & 3 & Ха19B & Ya2g2 & Performance anxiety \\
\hline ANXIETY & 3 & Xa1a8 & Ya3Bh & Examination phobia \\
\hline ANXIETY & 3 & Xa1Ev & Y7D9V & Fear of needles \\
\hline ANXIETY & 3 & $\mathrm{Xa3Vj}$ & Ya5rp & Panic \\
\hline ANXIETY & 3 & Xa3Vk & Ya5rq & Fear of insects \\
\hline ANXIETY & 3 & $\mathrm{Xa3VI}$ & Ya5rr & Fear of birds \\
\hline ANXIETY & 3 & ХaзWH & Ya5sR & Fear associated with healthcare \\
\hline ANXIETY & 3 & Xa3WI & Ya5sT & Fear of blood \\
\hline ANXIETY & 3 & Xa3WJ & $\mathrm{Ya} 5 \mathrm{su}$ & Fear of getting cancer \\
\hline ANXIETY & 3 & Xa3Xk & Ya5up & Level of anxiety \\
\hline ANXIETY & 3 & Xa7k9 & YaV9p & Parental anxiety \\
\hline ANXIETY & 3 & Xa7kB & YaV9s & Anxiety attack \\
\hline ANXIETY & 3 & Xa7kB & YaV9s & Anxiety attack \\
\hline ANXIETY & 3 & Xa7lj & YaVCF & Cancer phobia \\
\hline ANXIETY & 3 & Xa7lx & YaVCT & Pre-examination nerves \\
\hline ANXIETY & 3 & XaA5Z & YaYts & Beck anxiety inventory \\
\hline ANXIETY & 3 & Xaafv & YawVM & Anxiety about breathlessness \\
\hline
\end{tabular}




\begin{tabular}{|c|c|c|c|c|}
\hline ANXIETY & 3 & Хав96 & Ya6js & Other phobias \\
\hline ANXIETY & 3 & XaEGO & $\mathrm{Y} 50 \mathrm{~cm}$ & Dysmorphophobia \\
\hline ANXIETY & 3 & Xalvf & Yalgc & Fear of falling \\
\hline ANXIETY & 3 & XaMFU & YapP5 & Paruresis \\
\hline ANXIETY & 3 & XaP8d & Yarkm & Stranger anxiety \\
\hline ANXIETY & 3 & XaZJQ & YavR8 & Generalised anxiety disorder 2 scale \\
\hline ANXIETY & 3 & XEOra & Y7D7x & Nervousness \\
\hline ANXIETY & 3 & XEOrb & Ya5b6 & Anxiety \\
\hline ANXIETY & 3 & XEOri & Y7D8h & Fear \\
\hline ANXIETY & 3 & XE1Y7 & Y01Gd & Episodic paroxysmal anxiety disorder \\
\hline ANXIETY & 3 & XE1Y7 & Y01Gc & Panic disorder \\
\hline ANXIETY & 3 & XE1YA & Ya5am & Phobic anxiety disorder \\
\hline ANXIETY & 3 & XE1YB & Y01GE & Phobic disorder NOS \\
\hline ANXIETY & 3 & XE2Po & Y7DgD & Maternal concern \\
\hline ANXIETY & 3 & XMOOG & Y7D8C & Separation anxiety \\
\hline ANXIETY & 3 & XMOAk & Y01GV & School phobia \\
\hline ANXIETY & 3 & XMOeK & YM9X5 & Hospital anxiety and depression scale \\
\hline ANXIETY & 3 & XM1OD & Y71RO & Hydrophobia \\
\hline ANXIETY & 2 & ZV655 & 0 & [V]Person with feared complaint, no diagnosis made \\
\hline ANXIETY & 2 & 1288 & 0 & FH: Anxiety state \\
\hline ANXIETY & 2 & 1466 & 0 & $\mathrm{H} / \mathrm{O}$ : anxiety state \\
\hline ANXIETY & 2 & $173 f$. & 0 & Anxiety about breathlessness \\
\hline ANXIETY & 2 & $1 \mathrm{~B} 1 \mathrm{H}$. & 11 & Fear \\
\hline ANXIETY & 2 & $1 \mathrm{Bb} .$. & 0 & Specific fear \\
\hline ANXIETY & 2 & $1 \mathrm{Bb} 0$. & 0 & Fear of falling \\
\hline
\end{tabular}




\begin{tabular}{|c|c|c|c|c|}
\hline ANXIETY & 2 & $1 \mathrm{Bb} 1$. & 0 & Fear of getting cancer \\
\hline ANXIETY & 2 & $1 \mathrm{M} 61$. & 0 & Fearful with pain \\
\hline ANXIETY & 2 & $225 \mathrm{~K}$. & 0 & O/E - fearful mood \\
\hline ANXIETY & 2 & E200. & 0 & Anxiety states \\
\hline ANXIETY & 2 & E2000 & 0 & Anxiety state unspecified \\
\hline ANXIETY & 2 & E2001 & 0 & Panic disorder \\
\hline ANXIETY & 2 & E2002 & 0 & Generalised anxiety disorder \\
\hline ANXIETY & 2 & E2003 & 0 & Anxiety with depression \\
\hline ANXIETY & 2 & E2004 & 0 & Chronic anxiety \\
\hline ANXIETY & 2 & E2005 & 0 & Recurrent anxiety \\
\hline ANXIETY & 2 & E200z & 0 & Anxiety state NOS \\
\hline ANXIETY & 2 & E202. & 12 & Phobic anxiety \\
\hline ANXIETY & 2 & E2020 & 0 & Phobia unspecified \\
\hline ANXIETY & 2 & E2021 & 0 & Agoraphobia with panic attacks \\
\hline ANXIETY & 2 & E2022 & 0 & Agoraphobia without mention of panic attacks \\
\hline ANXIETY & 2 & E2023 & 0 & Social phobia, fear of eating in public \\
\hline ANXIETY & 2 & E2024 & 0 & Social phobia, fear of public speaking \\
\hline ANXIETY & 2 & E2025 & 0 & Social phobia, fear of public washing \\
\hline ANXIETY & 2 & E2026 & 0 & Acrophobia \\
\hline ANXIETY & 2 & E2027 & 0 & Animal phobia \\
\hline ANXIETY & 2 & E2028 & 0 & Claustrophobia \\
\hline ANXIETY & 2 & E2029 & 0 & Fear of crowds \\
\hline ANXIETY & 2 & E202A & 0 & Fear of flying \\
\hline ANXIETY & 2 & E202B & 0 & Cancer phobia \\
\hline ANXIETY & 2 & E202C & 0 & Dental phobia \\
\hline ANXIETY & 2 & E202D & 0 & Fear of death \\
\hline ANXIETY & 2 & E202E & 0 & Fear of pregnancy \\
\hline ANXIETY & 2 & E202z & 0 & Phobic disorder NOS \\
\hline
\end{tabular}




\begin{tabular}{|l|l|l|l|l|}
\hline ANXIETY & 2 & E227z & 11 & Fear of ejaculation \\
\hline ANXIETY & 2 & Eu054 & 0 & [X]Organic anxiety disorder \\
\hline ANXIETY & 2 & Eu341 & 14 & {$[$ X]Persistent anxiety depression } \\
\hline ANXIETY & 2 & Eu40. & 0 & {$[$ X]Phobic anxiety disorders } \\
\hline ANXIETY & 2 & Eu400 & 0 & {$[$ X]Agoraphobia } \\
\hline ANXIETY & 2 & Eu401 & 0 & {$[$ X]Social phobias } \\
\hline ANXIETY & 2 & Eu402 & 0 & {$[$ X]Specific (isolated) phobias } \\
\hline ANXIETY & 2 & Eu403 & 0 & {$[$ X]Needle phobia } \\
\hline ANXIETY & 2 & Eu40y & 0 & {$[$ X]Other phobic anxiety disorders } \\
\hline ANXIETY & 2 & Eu40z & 0 & {$[$ X]Phobic anxiety disorder, unspecified } \\
\hline ANXIETY & 2 & Eu41. & 0 & {$[$ X]Other anxiety disorders } \\
\hline ANXIETY & 2 & Eu410 & 0 & {$[$ X]Panic disorder [episodic paroxysmal anxiety] } \\
\hline ANXIETY & 2 & Eu411 & 0 & {$[$ X]Generalized anxiety disorder } \\
\hline ANXIETY & 2 & Eu411 & 0 & {$[$ X]Generalized anxiety disorder } \\
\hline ANXIETY & 2 & Eu412 & 0 & {$[$ X]Mixed anxiety and depressive disorder } \\
\hline ANXIETY & 2 & Eu413 & 0 & {$[$ X]Other mixed anxiety disorders } \\
\hline ANXIETY & 2 & Eu41y & 0 & {$[$ X]Other specified anxiety disorders } \\
\hline ANXIETY & 2 & Eu41z & 0 & {$[$ X]Anxiety disorder, unspecified } \\
\hline ANXIETY & 2 & Eu515 & 11 & {$[$ X]Dream anxiety disorder } \\
\hline ANXIETY & 2 & Eu931 & 0 & {$[$ X]Phobic anxiety disorder of childhood } \\
\hline ANXIETY & 2 & Eu932 & 0 & {$[$ X]Social anxiety disorder of childhood } \\
\hline & & & & \\
\hline
\end{tabular}




\section{H.2 Results}

In the eleven years of the study, the SAIL databank registered 475,085 individuals (aged $>18$ years) with a recorded diagnosis of depression or anxiety. When examined by more precise diagnosis, 275,232 (57.9\%) people had a recorded diagnosis of anxiety and 354,292 (74.6\%) a recorded diagnosis of depression, revealing a large cross-over in diagnosis.

\section{H.2.1 Trends in the number of people receiving prescriptions}

Over the 11 years of the study, opioid prescriptions were issued to a total of $277,988(n=1,099,026)$ people who also had a recorded diagnosis of depression and/or anxiety (RDDA) and no recorded diagnosis of cancer. Consequently, $58.5 \%$ of people with an RDDA recorded in the SAIL databank, received prescriptions for opioids between 2005 and 2015.

People with an RDDA equated to $25.3 \%$ of all people with a non-cancer diagnosis who received opioid prescriptions. A Mann-Whitney $U$ tests indicated there was a significant difference between the number of people receiving opioid prescriptions who had a recorded diagnosis of depression and/or anxiety and those without, $(\mathrm{U}=<.001, \mathrm{p}<.001, \mathrm{r}=0.8)$. The median number of people with an RDDA was 44.4 people per 1000 population and 104.3 people per 1000 population for those without.

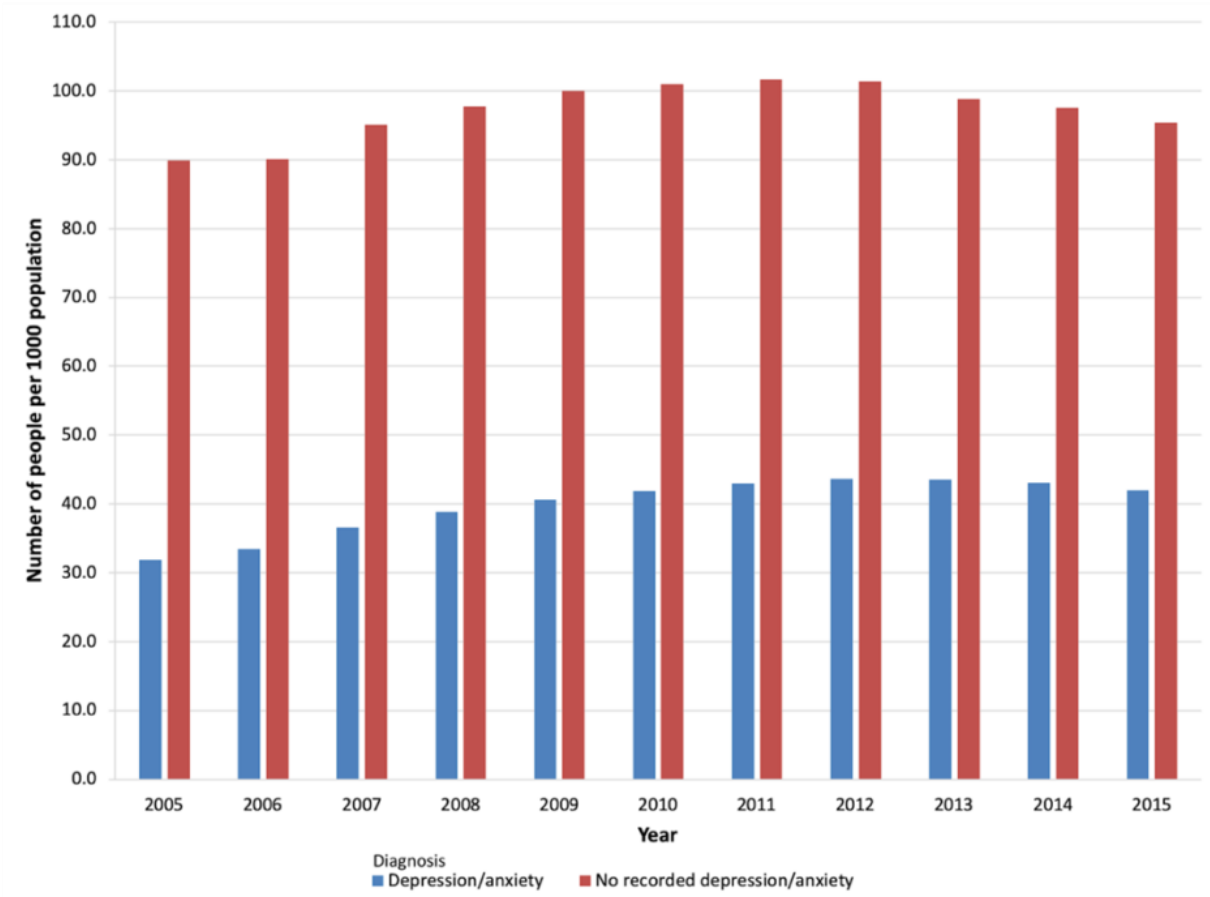

Figure H.1: Trends in the number of people per 1000 population, receiving prescriptions for opioids and with or without a recorded diagnosis of depression and/or anxiety

Annual trends demonstrated that percentage increases in the number of people receiving opioid prescriptions who also had an RDDA were much greater than the number of people without an RDDA (Table H.2). 
Table H.2: Annual trends in the number of people receiving opioid prescriptions with or without a recorded diagnosis of depression and/or anxiety. Data adjusted to number per 1000 population

People per 1000 population receiving opioid prescriptions

\begin{tabular}{rcccccc}
\hline & \multicolumn{3}{c}{$\begin{array}{c}\text { No recorded diagnosis of } \\
\text { depression/anxiety }\end{array}$} & \multicolumn{3}{c}{$\begin{array}{c}\text { Recorded diagnosis of } \\
\text { depression/anxiety }\end{array}$} \\
\hline & $\begin{array}{r}\text { Weak } \\
\text { opioid }\end{array}$ & $\begin{array}{c}\text { Strong } \\
\text { opioid }\end{array}$ & Total & $\begin{array}{c}\text { Weak } \\
\text { opioid }\end{array}$ & $\begin{array}{c}\text { Strong } \\
\text { opioid }\end{array}$ & Total \\
\hline $\mathbf{2 0 0 5}$ & 89.9 & 3.3 & 93.2 & 31.8 & 1.2 & 33.0 \\
$\mathbf{2 0 1 5}$ & 95.4 & 8.3 & 103.6 & 42.0 & 5.0 & 47.0 \\
\hline $\begin{array}{r}\text { Percentage } \\
\text { change (\%) } \\
\mathbf{2 0 0 5 - 2 0 1 5}\end{array}$ & 6.1 & 153.1 & 11.2 & 31.8 & 332.5 & 42.4 \\
\hline
\end{tabular}

Whilst there were 2.8 times more people without an RDDA receiving opioid prescriptions in 2005 compared to those with an RDDA; the annual number of people increased 3.8 times more in the latter group. By 2015, there were 2.2 times more people without an RDDA, receiving opioid prescriptions compared to those with that diagnosis.

In 2005, 3.5\% (3.3 people per 1000 population, $n=93.2$ people per 1000 population) of all people receiving opioid prescriptions and whom did not have an RDDA, received strong opioid prescriptions. There was a $153.1 \%$ increase (from 3.3 to 8.3 people per 1000 population) in the annual number of people receiving strong opioid prescriptions resulting in $8.0 \%$ (8.3 people per 1000 population, $n=103.6$ people per 1000 population) of all people in that category receiving strong opioids in 2015 (Table H.2).

There was a total of $27,358(30.4 \%, n=89,989)$ people who received strong opioid prescriptions over the study period and also had RDDA on their medical record. There was a significant difference $(U=13.0, p=.001, r=0.5)$ in the number of people receiving strong opioid prescriptions who had an RDDA (median=2.5 people per 1000 population) and those without (median=4.7 people per 1000 population). Whilst statistically significant, the effect size was moderate.

The percentage of people with an RDDA and receiving a prescription for a strong opioid as a total of all people with that diagnosis receiving opioid prescriptions, was $3.6 \%$ in 2005 . The annual number of people in that category increased $332.5 \%$ over the 11 years of the study, resulting in $10.6 \%$ (5.0 people per 1000 population, $n=47.0$ people per 1000 population) of the annual number of people with an RDDA receiving prescriptions for strong opioids in 2015 (Table H.2).

Over the 11 years examined, $29.1 \%$ of people receiving prescriptions for weak opioids had a recorded diagnosis of depression and/or anxiety. The annual number of people receiving weak opioid prescriptions increased by $31.8 \%$ (from 31.8 to 42.0 people per 1000 population) over the study period when there was also an RDDA (Table H.2: Annual trends in the number of people receiving opioid prescriptions with or without a recorded diagnosis of depression and/or anxiety. Data adjusted to number per 1000 population). This compared to $6.1 \%$ (from 89.9 to 95.4 people per 1000 population) where there was not an RDDA. 


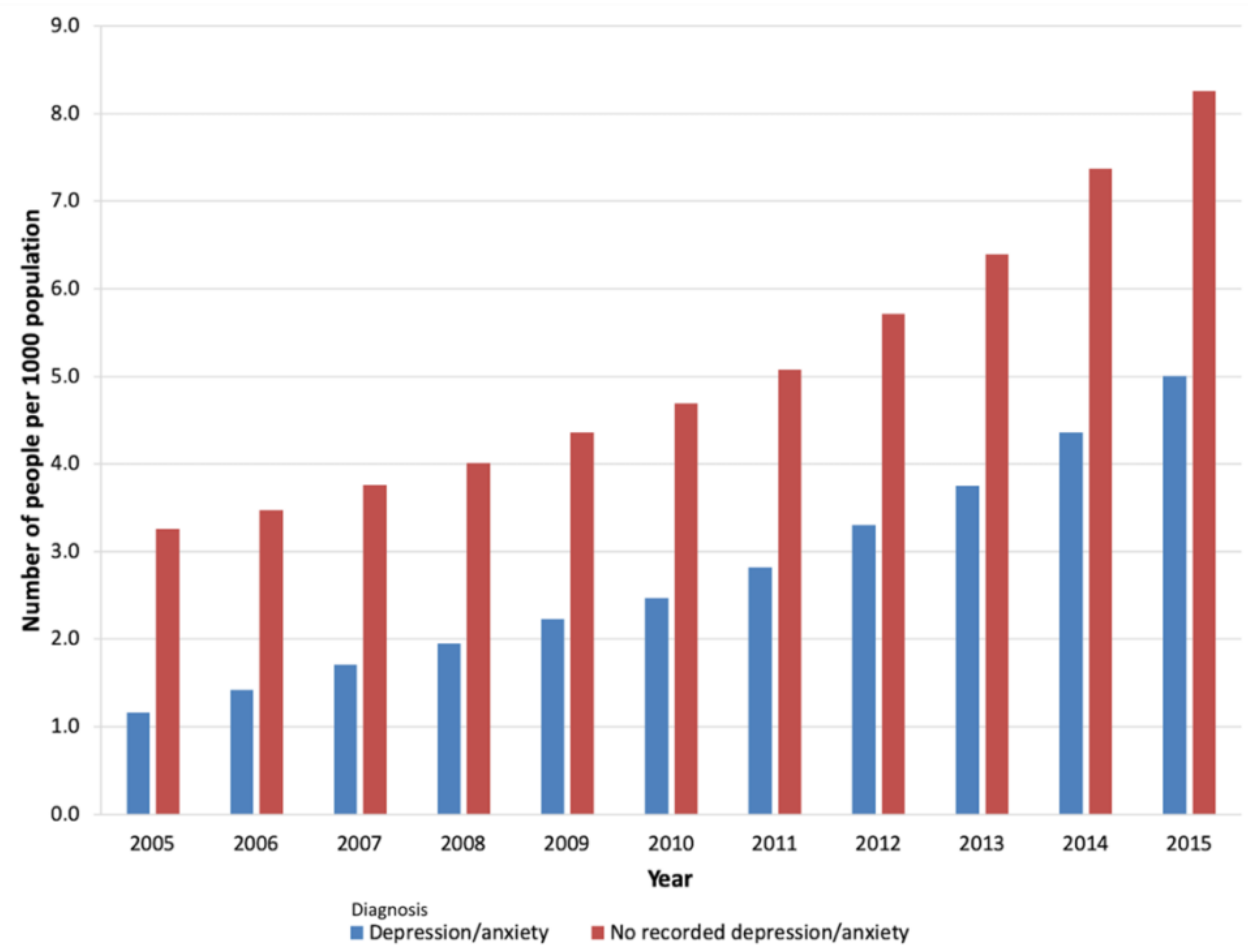

Figure H.2: Trends in the annual number of people per 1000 population receiving strong opioid prescriptions and whether they have a recorded diagnosis of depression and/or anxiety

Weak opioid prescriptions were issued to a total of 276,403 (25.4\%, $\mathrm{n}=1,089,618)$ people with RDDA. The median number of people receiving prescriptions for weak opioids without an RDDA was 97.8 people per 1000 population and 41.9 people per 1000 population for those with an RDDA. A Mann-Whitney $U$ test demonstrated the difference in the numbers of people receiving weak opioids, with or without an RDDA was significant, $\mathrm{U}=<.001$, $p<.001, r=0.8$.

The number of people without an RDDA and receiving weak opioids was 2.8 times higher than those with that diagnosis in 2005 (89.9 versus 31.8 people per 1000 population respectively) and changed to a 2.2 times difference by 2015 (95.4 versus 42.0 people per 1000 population). Weak opioid prescriptions and co-diagnoses of depression/anxiety accounted for 26.2\% (31.8 people per 1000 population, $n=121.8$ ) of all weak opioid prescriptions at the start of the study. By 2015 , the percentage of the annual number of people receiving weak opioid prescriptions who also had an RDDA had risen to 30.6\% (42.0 people per 1000 population, $\mathrm{n}=137.4$ ). 


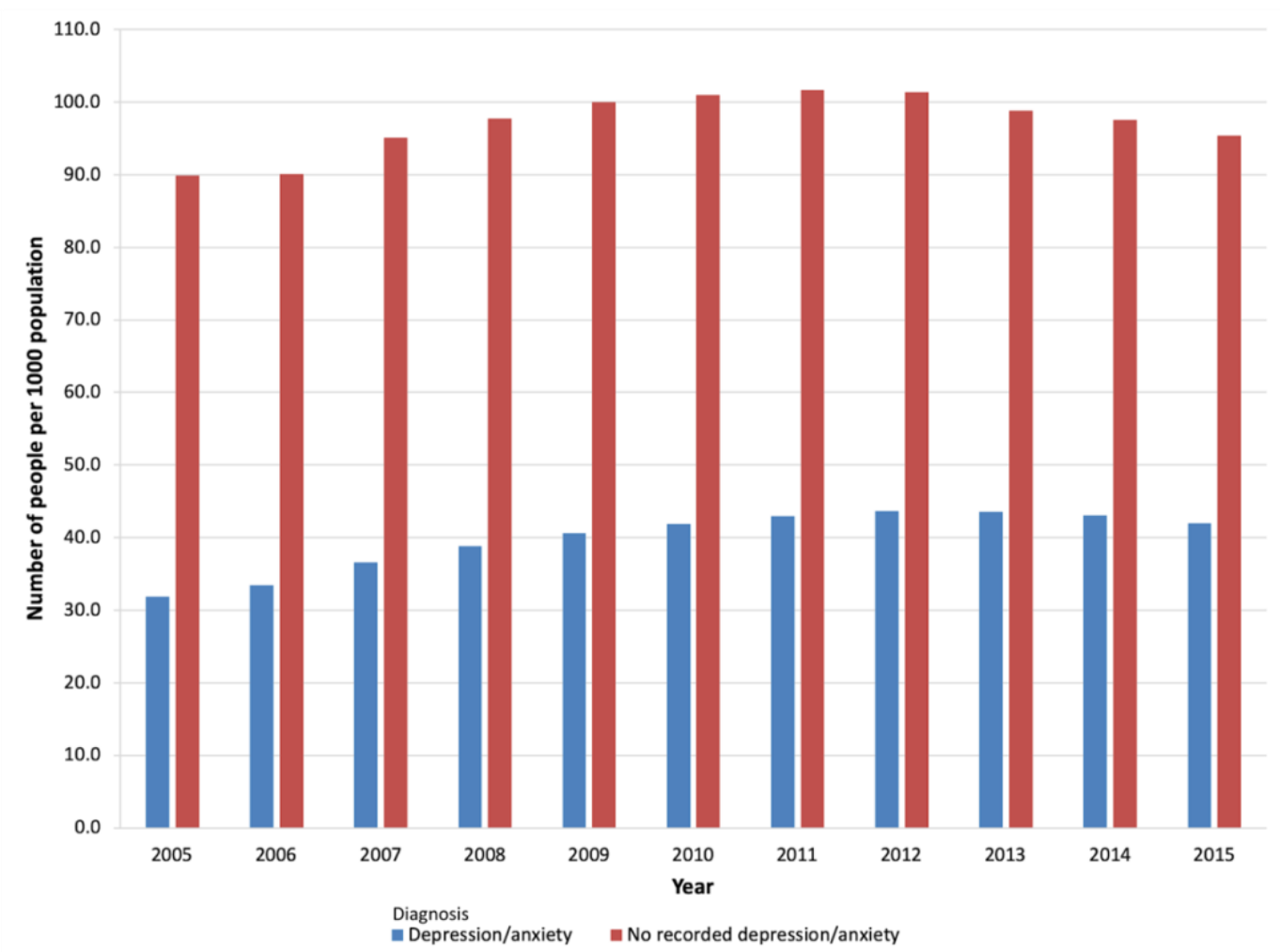

Figure H.3: Annual number of people per 1000 population receiving weak opioid prescriptions and whether they have a recorded diagnosis of depression and/or anxiety

\section{H.2.2 Trends in the number of prescriptions}

There was a total of $7,466,357$ ( $n=22,786.565)$ prescriptions for opioid medicines issued to people with a recorded diagnosis of depression and/or anxiety (RDDA) but no diagnosis of cancer (non-cancer). Prescriptions for opioid medicines for people with a recorded diagnosis of depression and/or anxiety comprised $32.7 \%$ (3140.0 of 9593.3 prescriptions per 1000 people) of all the opioid prescriptions issued across the 11 years of the study. The difference between the number of prescriptions for people with an RDDA (median=290.8 prescriptions per 1000 population) and those without (median $=597.4$ prescriptions per 1000 population), was determined significant by a MannWhitney $U$ test $(U=<.001, p<.001, r=0.8)$.

Strong opioid prescriptions for people with an RDDA comprised $4.4 \%(997,749)$ of the total of all opioid prescriptions issued and $41 \%(n=2,436,171)$ of the total of strong opioid prescriptions being prescribed. Prescriptions for weak opioids issued to people with an RDDA equated to $28.4 \%(6,468,608)$ of all opioids prescribed and $32 \%(n=20,350,394)$ of the total of weak opioids prescribed in the 11 years examined.

Table H.3: Annual trends in the number of opioid prescriptions with or without a recorded diagnosis of depression and/or anxiety. Data adjusted to number per 1000 sex-adjusted population

Prescriptions per 1000 population receiving opioid prescriptions

No recorded diagnosis of 


\begin{tabular}{rcccccc}
\hline & Weak opioid & $\begin{array}{c}\text { Strong } \\
\text { opioid }\end{array}$ & Total & $\begin{array}{l}\text { Weak } \\
\text { opioid }\end{array}$ & $\begin{array}{c}\text { Strong } \\
\text { opioid }\end{array}$ & Total \\
\hline $\mathbf{2 0 0 5}$ & 480.4 & 27.6 & 508.0 & 176.3 & 12.6 & 188.8 \\
$\mathbf{2 0 1 5}$ & 547.5 & 90.9 & 638.5 & 290.1 & 72.2 & 362.2 \\
\hline $\begin{array}{r}\text { Percentage } \\
\text { change (\%) } \\
\mathbf{2 0 0 5 - 2 0 1 5}\end{array}$ & 14.0 & 229.6 & 25.7 & 64.6 & 474.3 & 91.8 \\
\hline
\end{tabular}

The annual number of prescriptions for people with an RDDA increased $91.8 \%$ (from 188.8 to 362.2 prescriptions per 1000 population) between 2005 and 2015 . The number of prescriptions issued to people without an RDDA increased by $25.7 \%$ (from 508.0 to 638.5 prescriptions per 1000 population) although there were 2.1 times more prescriptions issued to people without depression or anxiety over the 11 years examined. In 2005, 2.7 times more prescriptions were issued to people without an RDDA compared to those with (508.0 versus 188.8 prescriptions per 1000 people respectively). This changed to a 1.8 times difference (638.5 versus 362.2 prescriptions per 1000 people respectively) in 2015.

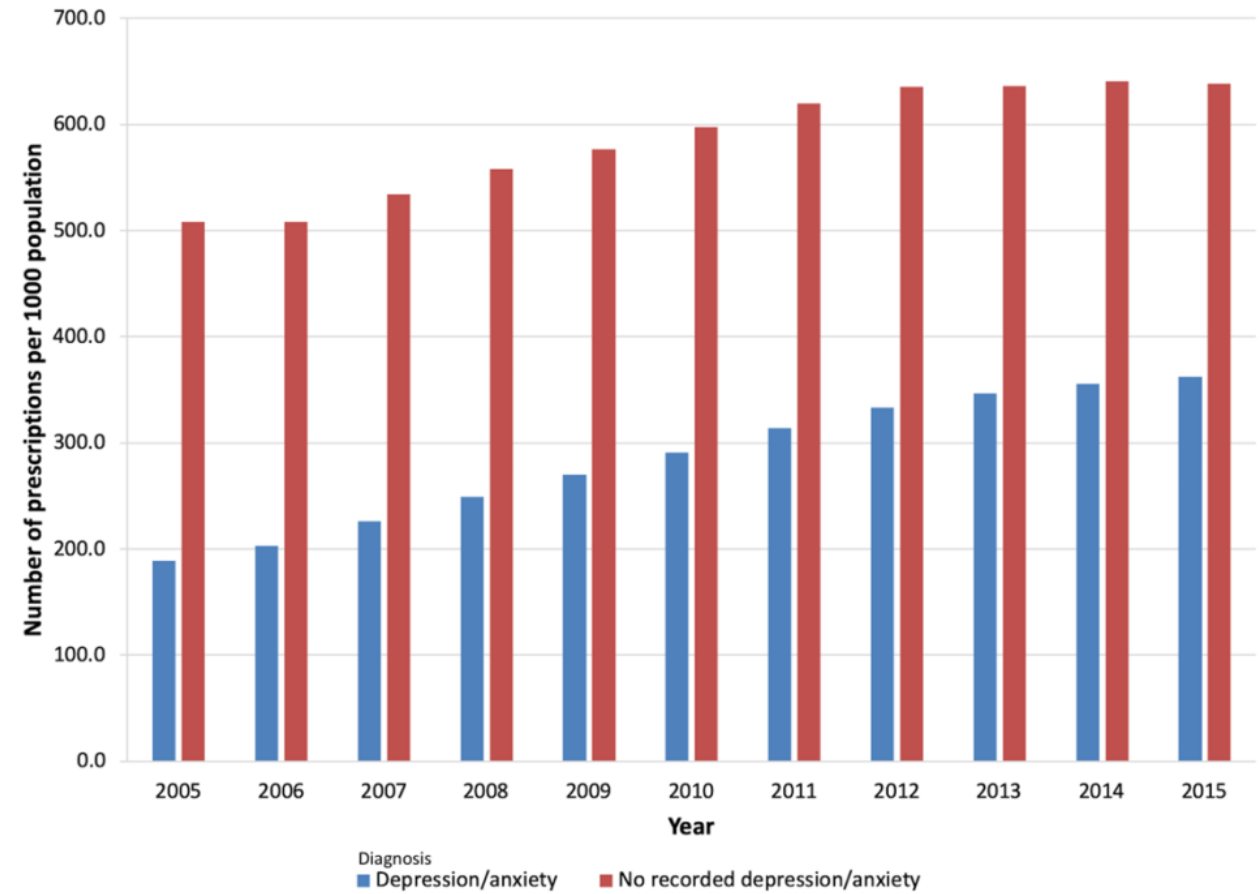

Figure H.4: Trends in the number of prescriptions per 1000 population with or without a recorded diagnosis of depression and/or anxiety

The annual number of prescriptions for strong opioids issued to people with an RDDA increased $474.3 \%$ (from 12.6 to 72.2 prescriptions per 1000 population) over the period examined, whereas the number for people without an RDDA increased $229.6 \%$ (from 27.6 to 90.9 prescriptions per 1000 population). There were 2.2 times fewer strong opioid prescriptions issued to people with a concurrent RDDA compared to those for people without such a diagnosis in 2005 (12.6 versus 27.6 prescriptions per 1000 population respectively). In 2015, the difference had reduced to 1.3 times (72.2 versus 90.9 prescriptions per 1000 population). The difference between the two groups; strong opioid prescriptions 
for people with an RDDA (median=34.4 prescriptions per 1000 population) and those without (median=51.1 prescriptions per 1000 population), was not significant $(U=37.0, p=.133, r=0.1)$.

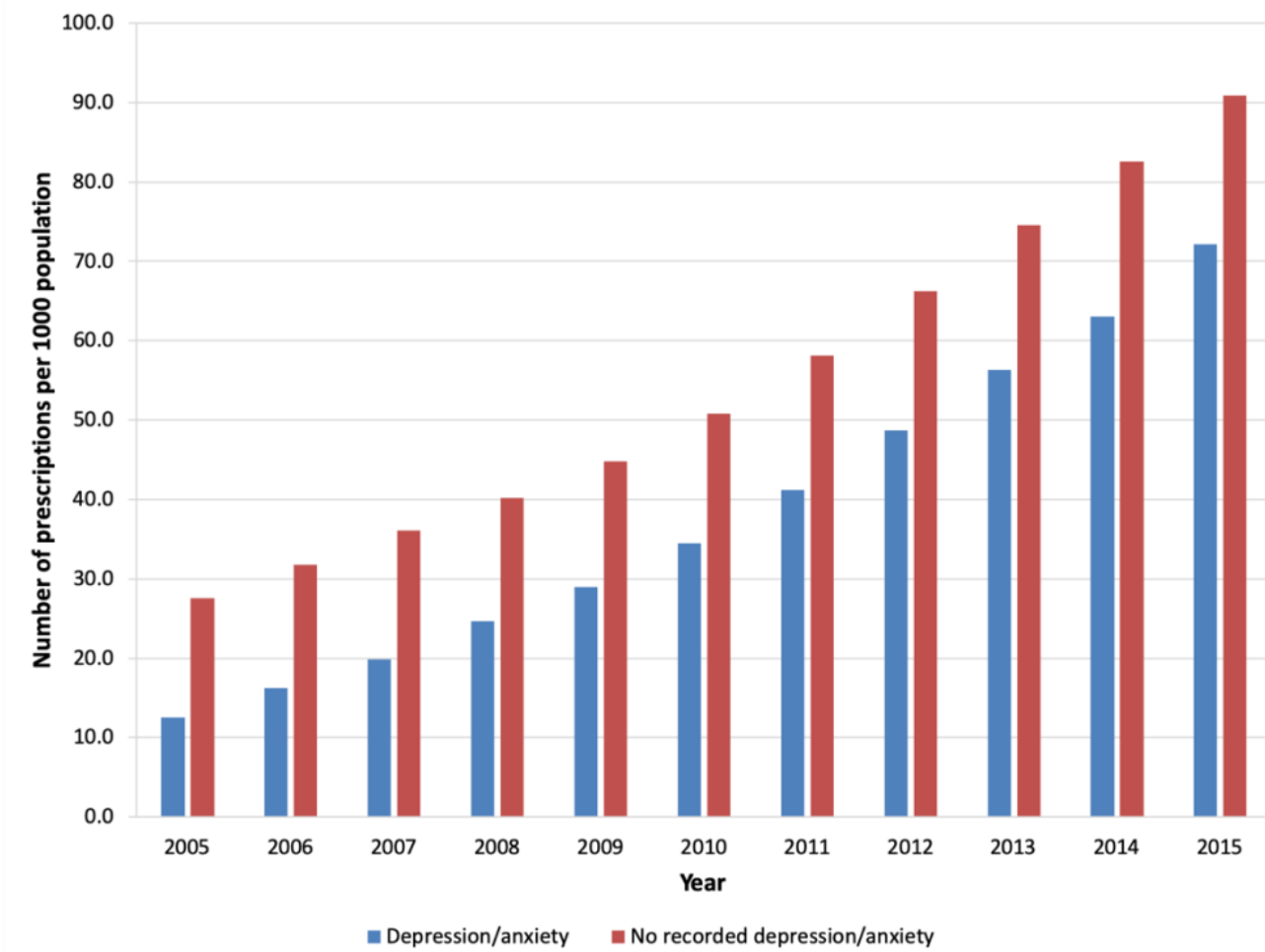

Figure H.5: Trends in the number of strong opioid prescriptions per 1000 population with or without a recorded diagnosis of depression and/or anxiety

Annual numbers of weak opioids being prescribed across the study period did not have such a high percentage increase as was seen with the strong opioid medicines. However, the annual number of weak opioid prescriptions for people with an RDDA increased 64.6\% (from 176.3 to 290.1 prescriptions per 1000 people) and those without by $14.0 \%$ (from 480.4 to 547.5 prescriptions per 1000 people) in the same period.

The difference in the number of weak opioid prescriptions over the 11-year period examined for those with an RDDA (median=256.4 prescriptions per 1000 population) and the number issued to those without that diagnosis (median $=546.6$ prescriptions per 1000 population) was significant as determined by Mann-Whitney $U$ test $(U=<.001, p<.001, r=0.8)$. 


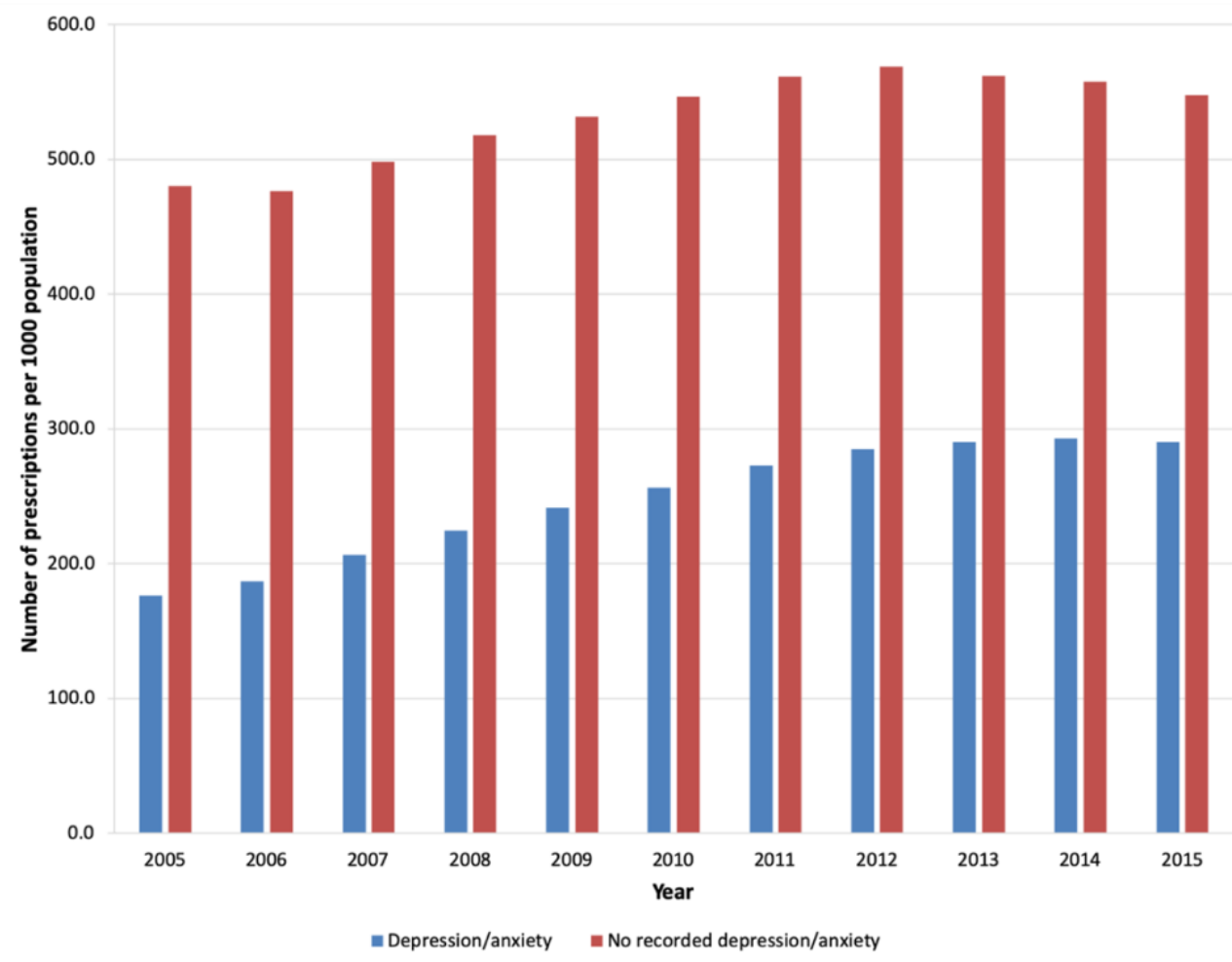

Figure H.6: Trends in the number of weak opioid prescriptions per 1000 population with or without a recorded diagnosis of depression and/or anxiety

Weak opioid prescriptions for people with an RDDA comprised $26.8 \%$ (176.3, $\mathrm{n}=656.6$ ) of the total of weak opioids examined in 2005 , rising to $34.6 \%$ (290.1, $n=547.5$ ) of the total in 2015. The difference between the number of prescriptions for people with an RDDA compared to those without changed from 2.7 times (176.3 versus 480.4 prescriptions per 1000 population) in 2005, to 1.8 times difference (290.1 versus 547.5 prescriptions per 1000 population) in 2015. There was a peak in the annual number of prescriptions for people without an RDDA in 2012. However, the peak in annual prescription numbers for people with an RDDA was seen in 2014.

\section{H.2.3 Trends in the number of people receiving prescriptions by gender}

Totalling annual data across the study period, twice as many women with a recorded diagnosis of depression and/or anxiety received prescriptions for opioids as men with the same recorded conditions (762,433 versus 350,391 respectively) in the 11 years examined. Over the 11 years examined, there was a median 28.7 men per 1000 male population who received prescriptions for opioid medicines and 59.4 women per 1000 female population who also had recorded diagnoses of depression and/or anxiety. Overall, $68 \%$ of the people with an RDDA and receiving opioid prescriptions were female. The difference between males and females with an RDDA and receiving opioid prescriptions was significant as determined by a Mann-Whitney $U$ test $(U=<.001, p<.001, r=0.8)$.

There were 3.3 times more men receiving opioid prescriptions who did not have an RDDA compared to those that did, averaged across the 11 years. Twice as 
many women without an RDDA received opioid prescriptions than those with either of those diagnoses.

Table H.4: Sex differences in prescribing of weak and strong opioids in people with and without recorded diagnoses of depression/anxiety. Data adjusted to people per 1000 sex-adjusted population

\begin{tabular}{rcccccc}
\hline Gender & \multicolumn{3}{c}{ People per $\mathbf{1 0 0 0}$ population receiving opioid prescriptions } \\
\cline { 2 - 7 } & \multicolumn{2}{c}{$\begin{array}{c}\text { No recorded diagnosis of } \\
\text { depression/anxiety }\end{array}$} & \multicolumn{3}{c}{$\begin{array}{c}\text { Recorded diagnosis of } \\
\text { depression/anxiety }\end{array}$} \\
\cline { 2 - 7 } & $\begin{array}{r}\text { Weak } \\
\text { opioid }\end{array}$ & $\begin{array}{c}\text { Strong } \\
\text { opioid }\end{array}$ & Total & Weak & Strong & Total \\
opioid & opioid & \\
\hline Male 2005 & 76.1 & 2.8 & 79.0 & 19.7 & 0.9 & 20.6 \\
Male 2015 & 86.5 & 6.7 & 93.3 & 26.8 & 3.4 & 30.1 \\
(\% change rate) & 13.7 & 136.5 & 18.1 & 36.1 & 277.1 & 46.6 \\
\hline Female 2005 & 103.1 & 3.7 & 106.7 & 43.4 & 1.4 & 44.9 \\
Female 2015 & 104.0 & 9.7 & 113.7 & 56.7 & 6.6 & 63.3 \\
(\% change rate) & 0.9 & 166.2 & 6.5 & 30.6 & 367.9 & 41.2 \\
\hline
\end{tabular}

Over the 11 years examined, there was a $41.2 \%$ (from 44.9 to 63.3 women per 1000 female population) increase in the number of women with an RDDA receiving prescriptions for opioid medicines. Over the same time, the number of women without those diagnoses increased 6.5\% (from 106.7 to 113.7 women per 1000 female population). There were peaks in the annual number of women receiving opioid prescriptions in 2012 for those without depression or anxiety and in $\mathbf{2 0 1 4}$ for those with. As a consequence, the percentage of the total annual number of women receiving opioid prescriptions; the number who had an RDDA changed from $29.6 \%$ (44.9, $n=151.6$ women per 1000 female population) in 2005 to $35.8 \%$ (63.3, $n=177$ women per 1000 female population).

The number of men with an RDDA receiving opioid prescriptions between 2005 and 2015 rose $46.6 \%$ (from 20.6 to 30.1 men per 1000 male population) (Figure $\mathrm{X}$ ). Men without an RDDA increased by $18.1 \%$ (from 79.0 to 93.3 men per 1000 male population) at the same time. Male data showed the same pattern of peak years as the female data already described although the percentage of the men receiving opioid prescriptions who had an RDDA changed from $20.7 \%(20.6, n=$ 99.6 ) in 2005 to $24.4 \%$ (30.1, $n=123.4$ men per 1000 male population). 


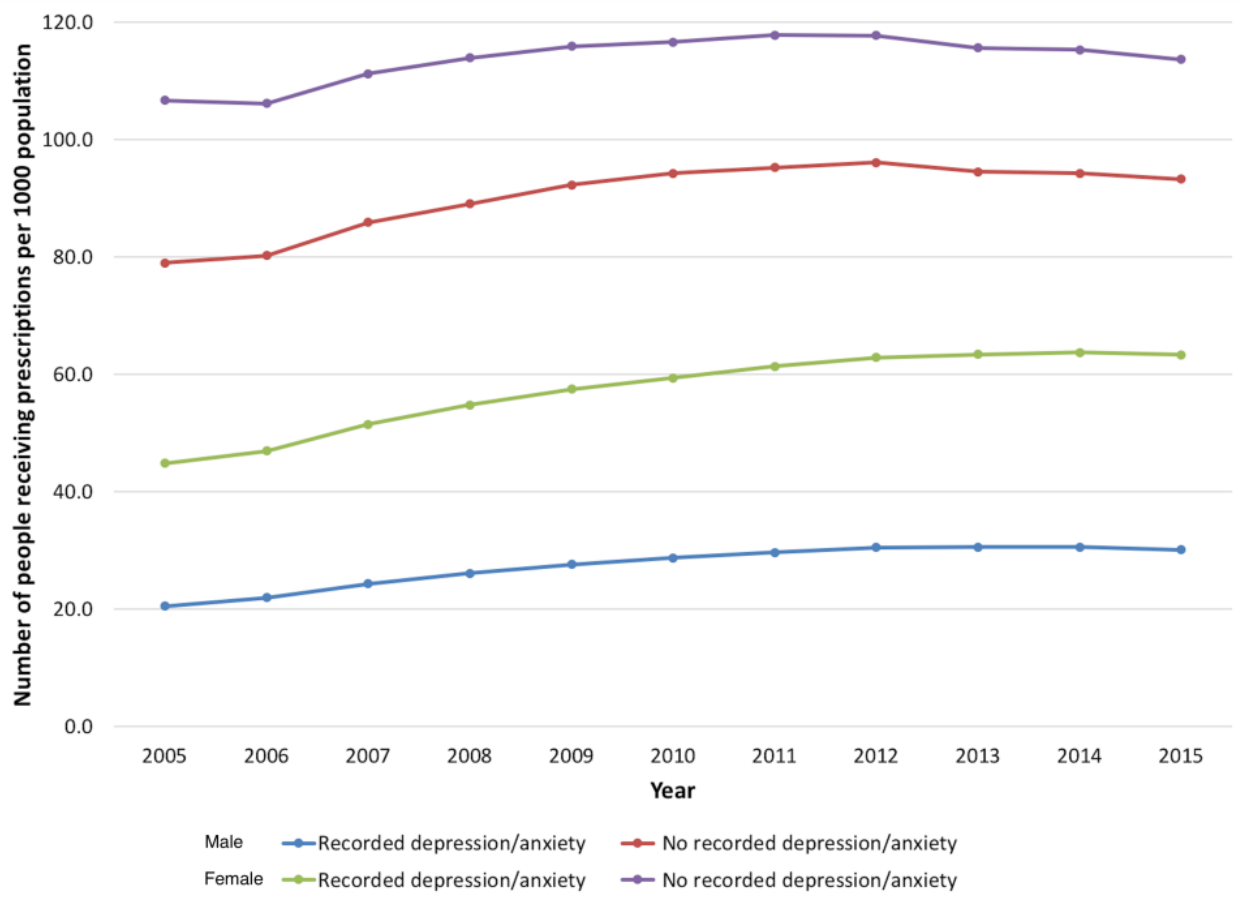

Figure H.7: Trends in opioid prescribing by sex and presence of a recorded diagnosis of depression/anxiety. Data presented by number of people per 1000 sex-adjusted population

The number of women with an RDDA receiving strong opioid prescriptions increased $367.9 \%$ (from 1.4 to 6.6 women per 1000 female population) in the 11 years analysed. Women without that recorded diagnosis increased in annual numbers by $166.2 \%$ (from 3.7 to .7 women per 1000 female population). There was a change in the difference between the two groups (RDDA versus none) of 2.6 times in 2005, reducing to 1.5 times difference in 2015. The difference between the two groups of women (median without an RDDA $=5.5$ women per 1000 female population, median with an RDDA $=3.2$ women per 1000 female population) receiving strong opioids was also determined significant although effect size was modest $(U=20.0, p=.007, r=0.3)$.

There was a $277.1 \%$ ( 0.9 to 3.4 men per 1000 male population) increase in the number of men receiving strong opioid prescriptions during the study period. Men without an RDDA increased in annual numbers by $136.5 \%$ (from 2.8 to 6.7 men per 1000 male population). There were 3 times more men receiving prescriptions for strong opioids and whom did not have an RDDA than those that did, in 2005 and this fell to a 2 times difference in 2015. Mann-Whitney $U$ tests demonstrated a significant difference $(U=5.0, p<.001, r=0.6)$ between the number of men receiving strong opioids who did not have an RDDA (median=3.8 men per 1000 male population) and those with those diagnoses (median=1.7 men per 1000 male population) between 2005 and 2015 .

Differences also existed between men and women receiving strong opioids with or without an RDDA. There were 1.6 times more women with an RDDA receiving strong opioid prescriptions than men in 2005 and this increased to 1.9 times difference in 2015. More women without an RDDA received strong opioid 
prescriptions than men and this also increased over the study period, from 1.3 to 1.5 times difference (2005 to 2015 respectively).

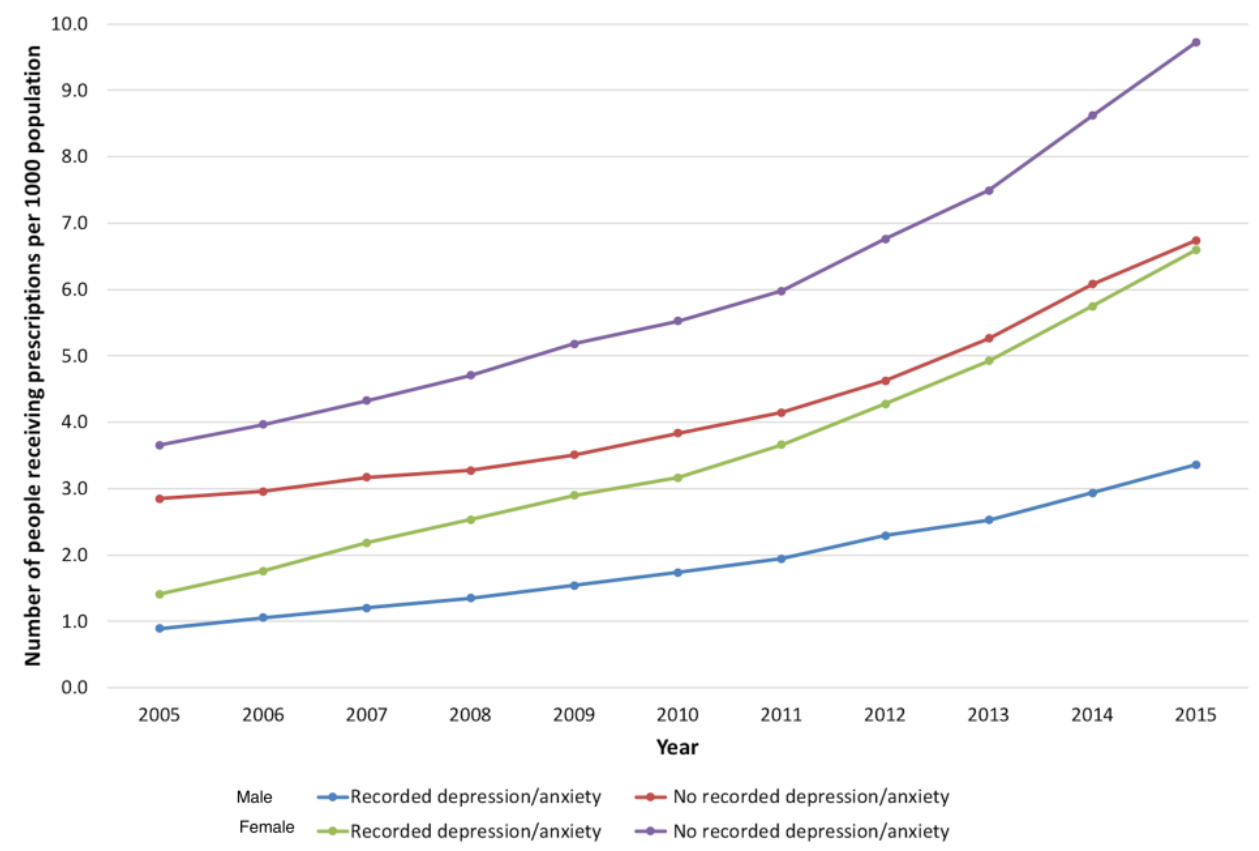

Figure H.8: Trends in strong opioid prescribing by sex and presence of a recorded diagnosis of depression/anxiety. Data presented by number of people per 1000 sex-adjusted population

As with strong opioids, the differences between women receiving weak opioids and who had an RDDA and those that did not, also reduced over the study period. The number of women with an RDDA receiving weak opioid prescriptions increased by $30.6 \%$ (from 43.4 to 56.7 women per 1000 female population). In contrast, women without an RDDA increased in number by $0.9 \%$ (from 103.1 to 104.0 women per 1000 female population) overall although there was a peak of 111.9 women per 1000 female population in 2011, which equated to an $8.6 \%$ increase from 2005. Although there was a greater percentage increase in the number of women with an RDDA receiving prescriptions, there were 1.8 times more women without an RDDA being issued with weak opioid prescriptions in 2015.

Using a Mann-Whitney $U$ test, a significant difference $(U=<.001, p<.001, r=0.8)$ between the number of women without an RDDA (median=108.1 women per 1000 female population) and women with that diagnosis (median=56.2 women per 1000 female population) was demonstrated. 


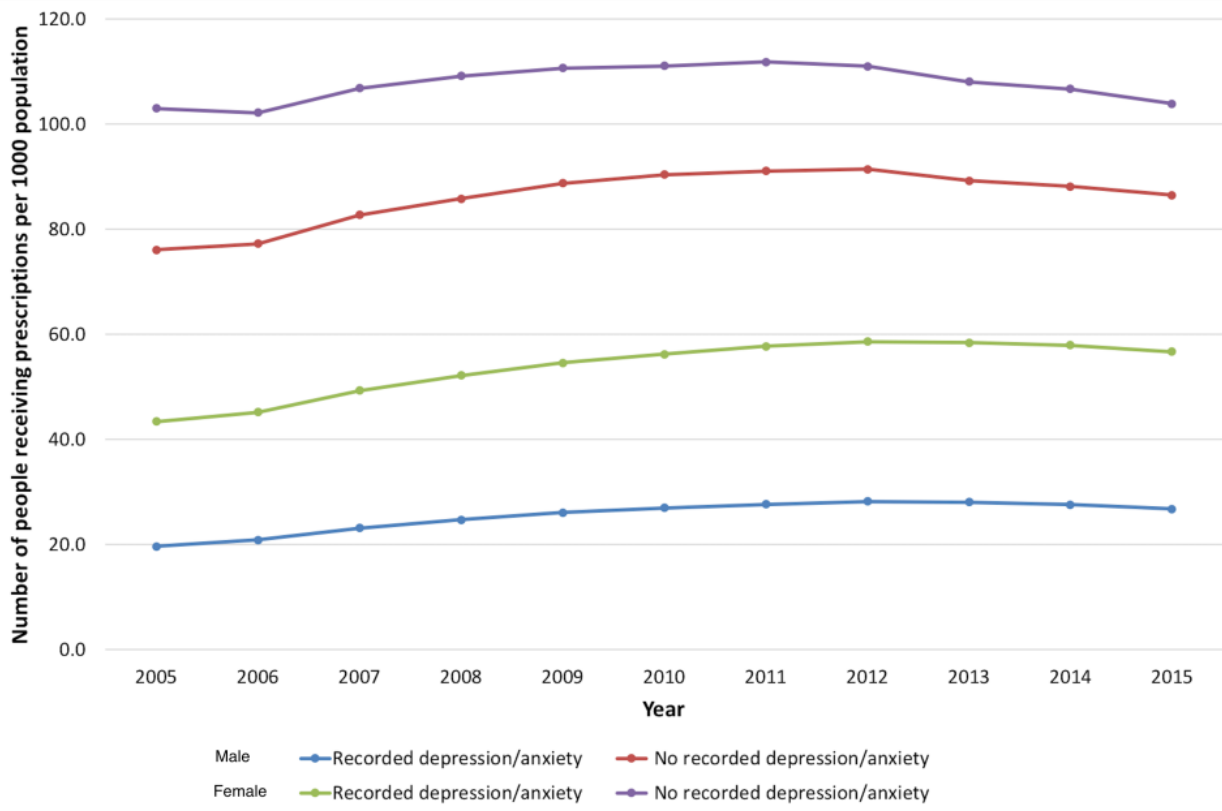

Figure H.9: Trends in weak opioid prescribing by sex and presence of a recorded diagnosis of depression/anxiety. Data presented by number of people per 1000 sex-adjusted population

Similarly, there was a greater percentage increase in the annual number of men with an RDDA receiving weak opioid prescriptions between 2005 and 2015, with a $36.1 \%$ (from 19.7 to 26.8 men per 1000 male population) in that time. Men without an RDDA being given weak opioid prescriptions however, rose in annual number by $13.7 \%$ (from 76.1 to 86.5 men per 1000 male population) but changed from being 3.9 times more men without an RDDA than those with in 2005, to being 3.2 times more in 2015.

The difference between the number of men without an RDDA (median=88.2 men per 1000 male population) and those with the diagnosis (median=26.8 men per 1000 male population), was statistically significant as derived by Mann-Whitney $U$ test $(U=<.001, p<.001, r=0.8)$.

\section{H.2.4 Trends in the number of prescriptions by gender}

As was seen with the number of people receiving prescriptions, there were twice as many opioid prescriptions issued to women with a recorded diagnosis of depression/anxiety as for men during the 11-year period examined $(4,975,365$ versus $2,490,992$ respectively). The difference between the number of prescriptions issued to men (median=198.4 prescriptions per 1000 male population) and to women (median=379.8 prescriptions per 1000 female population) was statistically significant as determined by a Mann-Whitney $U$ test $(U=<.001, p<.001, r=0.8)$.

There were however, 1.8 times more prescriptions issued for women without an $\operatorname{RDDA}(9,017,570$ actual prescriptions, median=689.1 prescriptions per 1000 female population). A Mann-Whitney $U$ test demonstrated the difference between the numbers of prescriptions issued to the two groups of women was 
significant $(\mathrm{U}=<.001, \mathrm{p}<.001, \mathrm{r}=0.8)$. Relatedly, 2.5 times more issued to men without an $\operatorname{RDDA}(6,302,638$ prescriptions, median $=502.2$ prescriptions per 1000 male population) than those with those recorded diagnoses respectively. This was also determined statistically significant $(U=<.001, p<.001, r=0.8)$.

Table H.5: Gender differences in prescription trends of weak and strong opioids with and without recorded diagnoses of depression/anxiety. Data adjusted to prescriptions per 1000 sex-adjusted population

\begin{tabular}{|c|c|c|c|c|c|c|}
\hline \multirow[b]{3}{*}{ Gender } & \multicolumn{6}{|c|}{ Prescriptions per 1000 population } \\
\hline & \multicolumn{3}{|c|}{$\begin{array}{l}\text { No recorded diagnosis of } \\
\text { depression/anxiety }\end{array}$} & \multicolumn{3}{|c|}{$\begin{array}{l}\text { Recorded diagnosis of } \\
\text { depression/anxiety }\end{array}$} \\
\hline & $\begin{array}{l}\text { Weak } \\
\text { opioid }\end{array}$ & $\begin{array}{l}\text { Strong } \\
\text { opioid }\end{array}$ & Total & $\begin{array}{l}\text { Weak } \\
\text { opioid }\end{array}$ & $\begin{array}{l}\text { Strong } \\
\text { opioid }\end{array}$ & Total \\
\hline Male 2005 & 383.3 & 25.1 & 408.4 & 113.1 & 11.6 & 124.6 \\
\hline Male 2015 & 474.0 & 77.3 & 551.3 & 191.7 & 52.7 & 244.4 \\
\hline (\% change rate) & 23.6 & 208.3 & 35.0 & 69.5 & 356.0 & 96.0 \\
\hline Female 2005 & 572.7 & 30.0 & 602.7 & 236.4 & 13.5 & 249.9 \\
\hline Female 2015 & 618.9 & 104.2 & 723.0 & 385.5 & 91.1 & 476.5 \\
\hline (\% change rate) & 8.1 & 247.5 & 20.0 & 63.1 & 573.0 & 90.7 \\
\hline
\end{tabular}

When adjusted to the relevant population, there was a $90.7 \%$ (from 249.9 to 476.5 prescriptions per 1000 population) increase in the number of prescriptions for women with an RDDA over the period examined. There were 2.4 times more prescriptions issued to women without an RDDA in 2005 and over the following 11 years, the annual number of prescriptions issued to that group increased by $20 \%$ (from 602.7 to 723.0 prescriptions per 1000 female population), reducing the difference to 1.5 times between the non-RDDA and RDDA groups respectively. These changes in the number of prescriptions issued resulted in prescriptions for women with an RDDA accounting for $29.3 \%$ of the total of prescriptions issued in 2005, increasing to $39.7 \%$ of the total in 2015 .

The annual number of prescriptions for men with an RDDA rose $96.0 \%$ (from 124.6 to 244.4 prescriptions per 1000 male population) whilst, for men without those diagnoses, annual prescription numbers grew $35.0 \%$ (from 408.4 to 551.3 prescriptions per 1000 male population). As a consequence of the changes in prescribing trends prescriptions for men without an RDDA changed from being 3.3 times greater in number in 2005 than for men with an RDDA, to 2.3 times greater in 2015. In terms of percentage of the total number of prescriptions being issued over those years, in 2005 opioid prescriptions for men with an RDDA comprised $23.4 \%$ and rose to $30.7 \%$ of the total in 2015 . Unlike with other trends already reported, there was no peak in the annual number of prescriptions issued to people of either sex who had a concurrent RDDA. The data demonstrated that the annual number of prescriptions for those groups of people increased every year between 2005 and 2015, although the rate of change appeared to slow in the last 3 years of the study period (Error! Reference source not found.). 


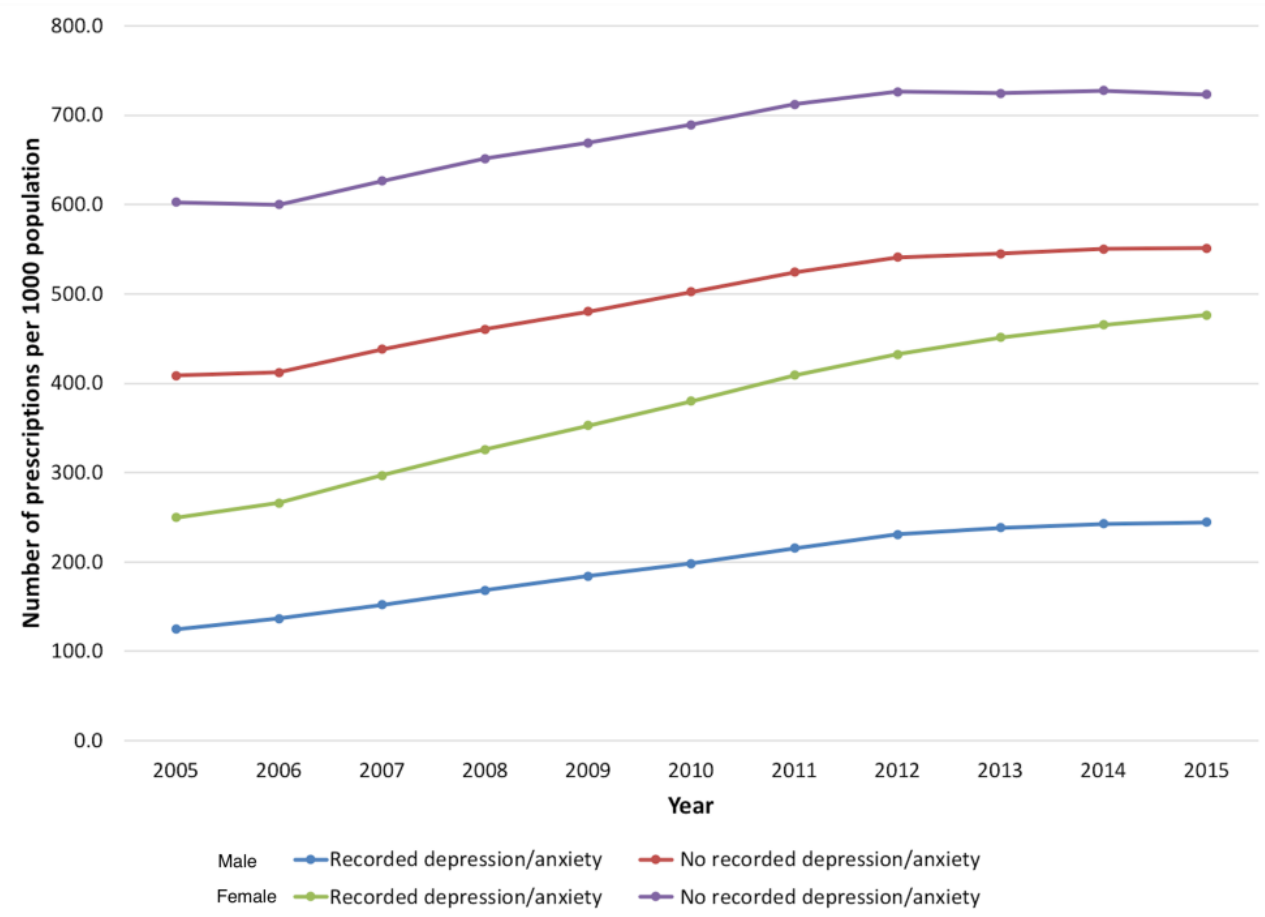

Figure H.10: Trends in the number opioid prescribing by gender and presence of a recorded diagnosis of depression/anxiety. Data presented by number of people per 1000 sex-adjusted population

Strong opioid prescriptions for women with an RDDA rose $573 \%$ (from 13.5 to 91.1 prescriptions per 1000 female population, median=42.0 prescriptions per 1000 female population) between 2005 and 2015. At the same time, the annual number of prescriptions for women without an RDDA increased $247.5 \%$ (from 30.0 to 104.2 prescriptions per 1000 female population, median $=59.0$ prescriptions per 1000 female population). Based on Mann-Whitney U tests, there was no significant difference between the number of strong opioid prescriptions being issued to women with or without an RDDA ( $U=37.0, p=.133$, $r=0.1)$.

Strong opioid prescriptions for women with an RDDA changed from accounting for $31.1 \%$ of the annual total being issued to women in 2005 , to $46.6 \%$ of the total in 2015. The faster rate of change in the annual number of prescriptions issued to women with an RDDA also resulted in a narrowing of the difference between that group and women without those diagnoses. Consequently, in 2005 there were 2.2 times more prescriptions issued to women without an RDDA compared to those with and this changed to a 1.14 times difference in 2015.

Men with an RDDA had a 356\% (from 11.6 to 52.7 prescriptions per 1000 population, median $=42.5$ prescriptions per 1000 male population) increase in the number of strong opioid prescriptions issued over the study period. Men without an RDDA had a $208.3 \%$ (from 25.1 to 77.3 prescriptions per 1000 population, median $=26.6$ prescriptions per 1000 population) increase in the annual number of prescriptions issued. Differently to women, there was a statistically significant difference between the number of strong opioid prescriptions being issued to men with and without an RDDA $(U=5.0, p<.001, r=0.6)$. 


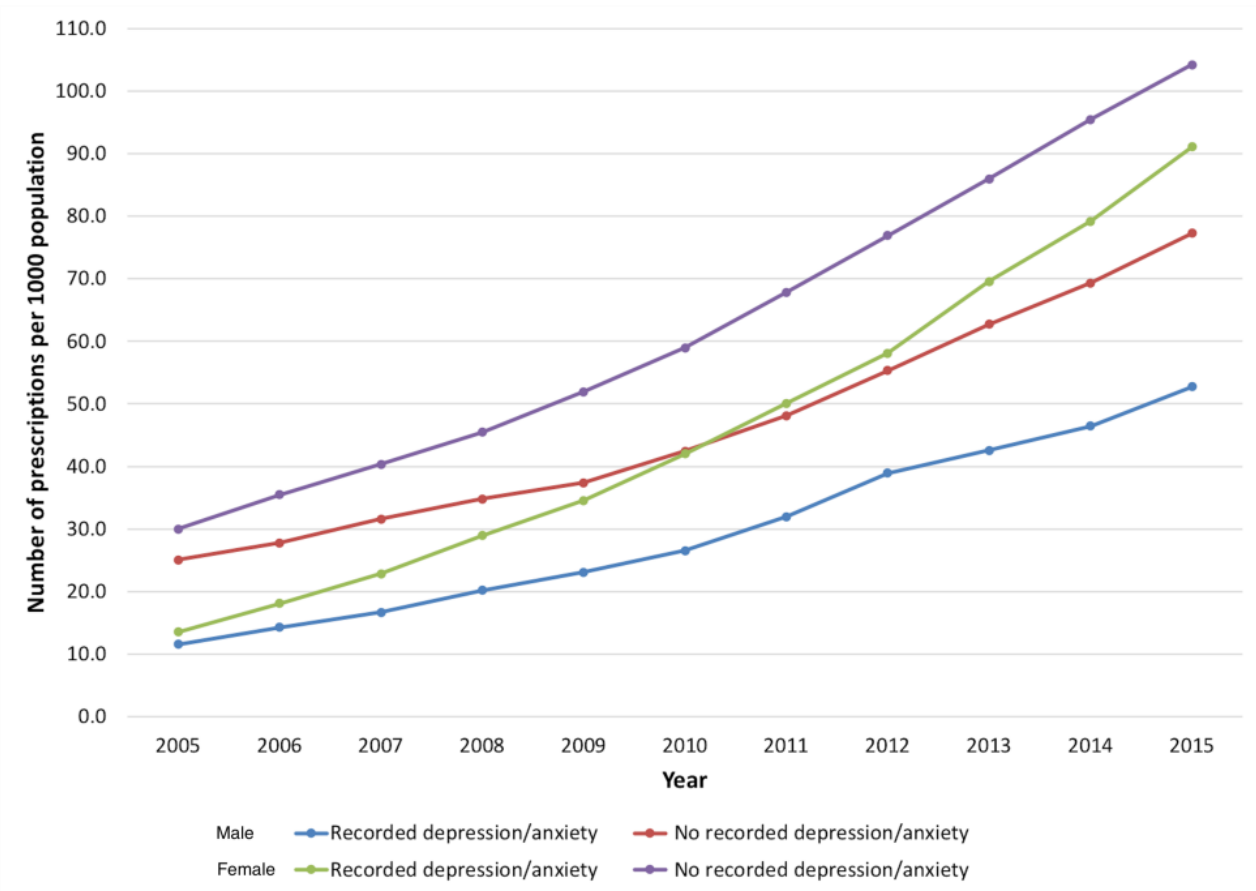

Figure H.11: Trends in the number of strong opioid prescriptions by sex and presence of a recorded diagnosis of depression/anxiety. Data presented by number of prescriptions per 1000 sex-adjusted population

Proportionally, the annual number of prescriptions for men with an RDDA was $31.6 \%(11.6, n=36.7)$ of the total number issued in 2005 and increased to $40.6 \%$ $(52.7, n=130)$ of the total number of prescriptions issued in 2015. As was seen in the female group, there was a narrowing of the difference between the two groups of men whose prescriptions were examined. There were 2.2 times more prescriptions issued to men without an RDDA in 2005 than men with the diagnoses. This changed to a 1.5 times difference in the number of prescriptions issued in 2015.

Annual number of weak opioid prescriptions for women with an RDDA increased 63.1\% (from 236.4 to 385.5 prescriptions per 1000 female population, median $=337.8$ prescriptions per 1000 female) compared to an $8.1 \%$ increase (from 572.7 to 618.9 prescriptions per 1000 female population, median=618.9 prescriptions per 1000 female population) for women without those diagnoses (Figure $X)$. Mann-Whitney $U$ tests determined the difference between the number of prescriptions issued to each group was significant $(U=<.001, p<.001$, $r=0.8)$.

The percentage of weak opioid prescriptions issued to women with an RDDA changed from $29.2 \%$ to $38.4 \%$ of the total of weak opioid prescriptions issued to women between 2005 and 2015. Further, in 2005 there were 2.4 times more weak opioid prescriptions issued to women without an RDDA than those with, which reduced to a 1.6 times difference between the groups in 2015 (Table X). 


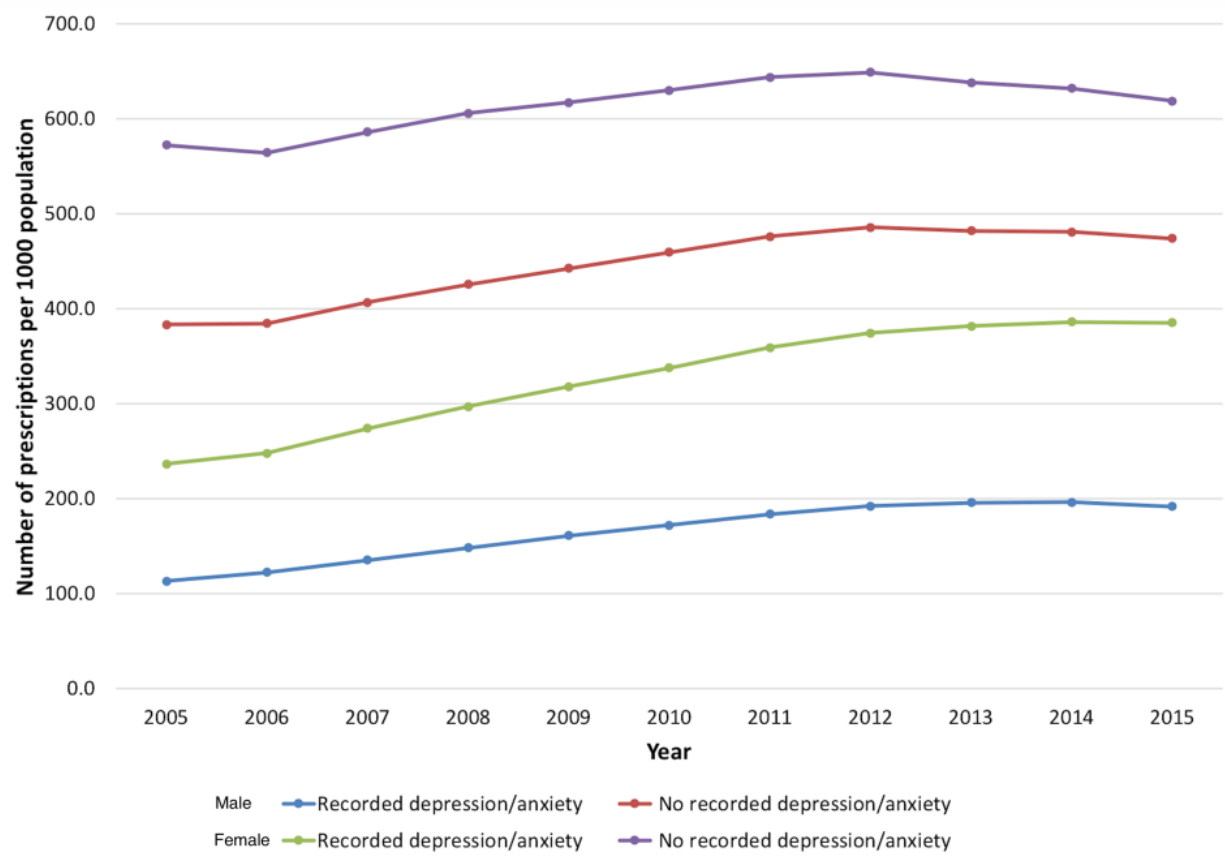

Figure H.12: Trends in the number of weak opioid prescribing by gender and presence of a recorded diagnosis of depression/anxiety. Data presented by number of prescriptions per 1000 gender-adjusted population

There were 3.4 times more weak opioid prescriptions issued to men without an RDDA in 2005 which changed to 2.5 times more in 2015. This corresponded with a $69.5 \%$ (from 113.1 to 191.7 prescriptions per 1000 male population, median=171.8 prescriptions per 1000 male population) increase in the annual number of weak opioid prescriptions issued to men with an RDDA compared to $23.6 \%$ (from 383.3 to 474.0 prescriptions per 1000 population, median $=459.7$ prescriptions per 1000 male population) in the men without those diagnoses. The difference in the number of prescriptions issued to each group was determined significant $(U=<.001, p<.001, r=0.8)$.

The percentage of the total weak opioid prescriptions, that were issued to men with an RDDA rose from $22.8 \%$ of the total in 2005 to $28.8 \%$ of the total issued in 2015. Unlike the annual number of prescriptions issued to women with an RDDA, which increased every year of the period examined, those for men with that diagnosis peaked in 2014. 


\section{Appendix I Results stratified by Health Board I.1 Health Board level data}

Since 1 October 2009, NHS health care has been delivered via 7 Integrated Local Health Boards in Wales (NHS Wales 2009). In April 2019, Abertawe Bro Morgannwg University Health Board and Cwm Taf University Board, had their borders and consequently, populations altered. The newly formed Swansea Bay University Health Board no longer contains the Bridgend locality which, transferred to newly named Cwm Taf Morgannwg University Health Board. Consequently, data since April 2019 reflects these changes and would need to be considered for future research.

Within each Health Board exist smaller geographic units known as Lower Super Output Areas (LSOA). The LSOA generally have around 1500 population and are used to assign their respective Welsh Index of Multiple Deprivation (WIMD) score. They were devised from areas used in the 2001 Census (StatsWales 2010). SAIL datasets were formulated using LSOA level data and linked to the appropriate Health Board. Whilst it is possible to examine data to an individual practice level; beyond deprivation and general geographic area (Health Board) it was decided not to explore the data to that level at this stage.

\section{I.2 Opioid prescribing by Health Board}

\section{I.2.1 Total opioid prescribing}

Population adjusted data identified differences in the numbers of people receiving opioid prescriptions between 2005 and 2015 in the seven different Health Boards providing primary care services in Wales (Figure I.1). 


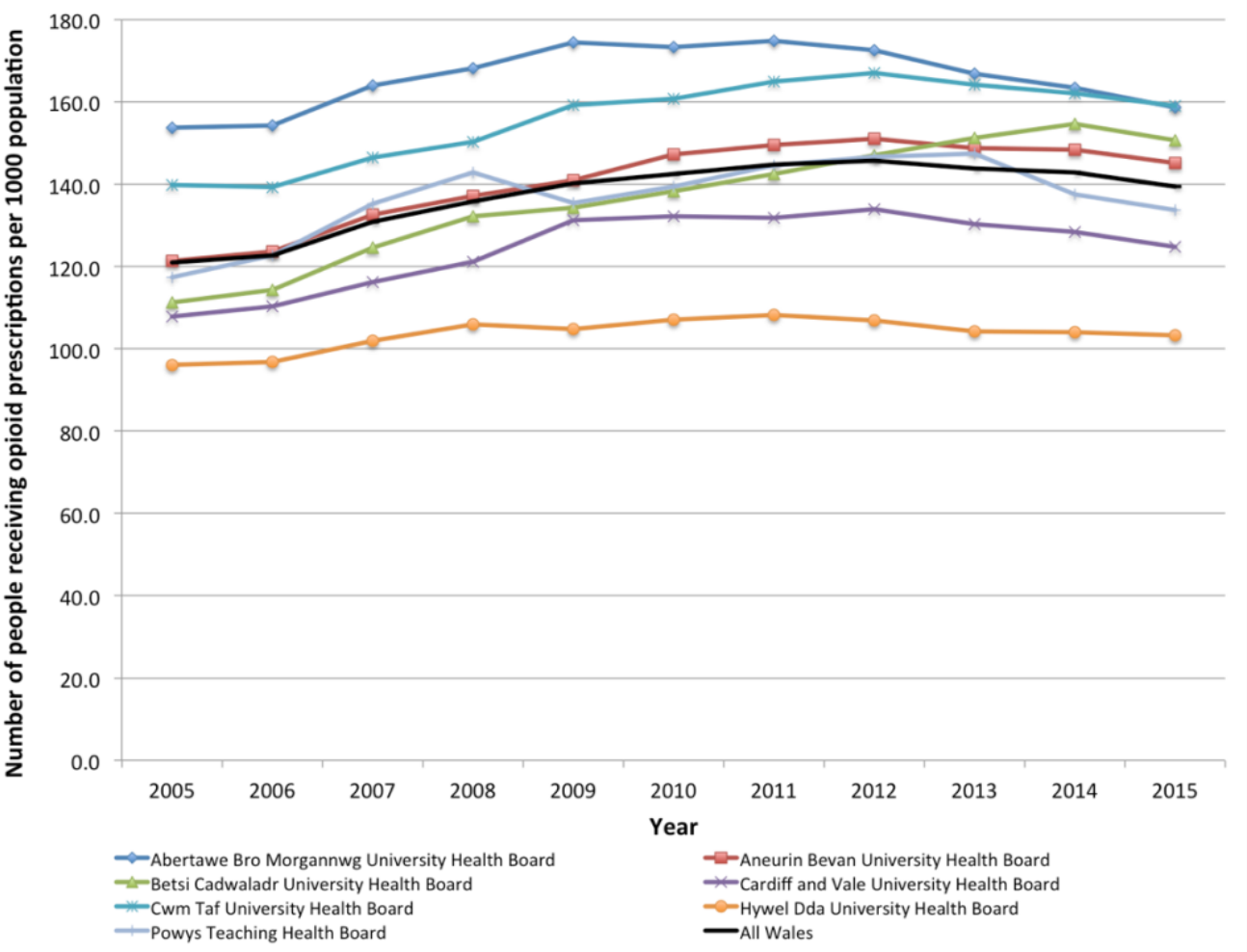

Figure I.1: Trend in the annual number of people per 1000 population receiving opioid prescriptions in each Health Board in Wales and compared to the National average. Data population adjusted to each Health Board

Abertawe Bro Morgannwg University Health Board (ABMUHB), Aneurin Bevan University Health Board (ABUHB) and Cwm Taf University Health Board (CTUHB) all had numbers of people receiving opioid prescriptions consistently above the National average for Wales (Table I.2) for the 11-year study period.

A Kruskal-Wallis test provided very strong evidence of a difference $(p<.001)$ between the mean ranks of the pairs of groups of the different Health Boards when examining the difference in the number of people receiving opioid prescriptions. Dunn's pairwise tests were carried out for the 21 pairs of groups and showed that 9 pairs of Health Board comparisons were significantly different for the number of people prescribed opioids. The remaining 12 pairs were not significantly different (Table I.1).

Table I.1: Dunn's pairwise comparison and Bonferroni post-hoc analysis of difference between the number of people receiving opioid prescriptions by Welsh Health Board between 2005 and 2015

\begin{tabular}{rcccccc}
\hline & \multicolumn{5}{c}{ Health Board** } \\
\cline { 2 - 6 } & ABMUHB & ABUHB & BCUHB & CVUHB & CTUHB & HDUHB \\
\hline ABUHB & .084 & & & & \\
\hline BCUHB & .021 & $>.999$ & & & \\
\hline CVUHB & $<.001^{*}$ & .830 & $>.999$ & & & \\
\hline CTUHB & $>.999$ & $>.999$ & .541 & $.002^{*}$ & & \\
\hline HDUHB & $<.001^{*}$ & $.004^{*}$ & $.018^{*}$ & $>.999$ & $<.001^{*}$ & \\
\hline PTHB & $.013^{*}$ & $>.999$ & $>.999$ & $>.999$ & .371 & $.030^{*}$ \\
\hline
\end{tabular}

${ }^{*} \mathrm{p}<0.05=$ statistically significant

**ABMUHB = Abertawe Bro Morgannwg University Health Board; $A B U H B=$ Aneurin Bevan University Health Board; $\mathrm{BCUHB}=$ Betsi Cadwaladr University Health Board; CVUHB = Cardiff and Vale University Health 
Board; $\mathrm{CTUHB}=$ Cwm Taf University Health Board; HDUHB = Hywel Dda University Health Board; PTHB = Powys Teaching Health Board

Population adjusted data demonstrated that whilst Powys has the smallest population in Wales, in terms of numbers; the number of people receiving opioid prescriptions per 1000 population were similar to some of the more densely populated Health Boards (Table I.2).

\section{I.2.2 Number of prescriptions issued}

Annual prescription numbers per 1000 population rose in every Health Board between 2005 and 2011 (Figure I.2). Betsi Cadwaladr University Health Board (BCUHB) had the largest increase in annual prescription numbers in Wales, with a $78.1 \%$ (from 527.9 to 940.5 prescriptions per 1000 population) rise over the 11 years of the study. 
Table I.2: Trends in the annual number of people receiving opioid prescriptions in each Health Board in Wales and compared to the National Average (All Wales). Percentage change over the 11 year study period included. Data population adjusted per Health Board (number of people per 1000 population)

\begin{tabular}{|c|c|c|c|c|c|c|c|c|c|c|c|c|c|c|c|c|}
\hline \multicolumn{17}{|c|}{ Health Board* } \\
\hline & \multicolumn{2}{|c|}{ ABMUHB } & \multicolumn{2}{|c|}{ ABUHB } & \multicolumn{2}{|c|}{ BCUHB } & \multicolumn{2}{|c|}{ CVUHB } & \multicolumn{2}{|c|}{ CTUHB } & \multicolumn{2}{|c|}{ HDUHB } & \multicolumn{2}{|c|}{ PTHB } & \multicolumn{2}{|c|}{ All Wales } \\
\hline \multicolumn{17}{|l|}{ Number of people - change rate(\%) } \\
\hline 2005 & 153.7 & & 121.3 & & 111.2 & & 107.8 & & 139.8 & & 96.1 & & 117.4 & & 120.9 & \\
\hline 2006 & 154.3 & 0.4 & 123.5 & 1.9 & 114.3 & 2.8 & 110.3 & 2.3 & 139.3 & -0.3 & 96.8 & 0.7 & 122.7 & 4.5 & 122.7 & 1.5 \\
\hline 2007 & 164.0 & 6.3 & 132.6 & 7.3 & 124.6 & 9.0 & 116.3 & 5.4 & 146.5 & 5.2 & 101.9 & 5.3 & 135.3 & 10. & 130.9 & 6.7 \\
\hline 2008 & 168.2 & 2.6 & 137.1 & 3.4 & 132.1 & 6.1 & 121.1 & 4.2 & 150.3 & 2.6 & 105.9 & 3.9 & 142.8 & 3 & 135.9 & 3.8 \\
\hline 2009 & 174.5 & 3.7 & 141.0 & 2.8 & 134.2 & 1.6 & 131.1 & 8.3 & 159.3 & 6.0 & 104.7 & -1.2 & 135.3 & 5.5 & 140.2 & 3.2 \\
\hline 2010 & 173.3 & -0.7 & 147.1 & 4.4 & 138.2 & 3.0 & 132.2 & 0.8 & 160.7 & 0.9 & 107.0 & 2.2 & 139.4 & -5.2 & 142.5 & 1.6 \\
\hline 2011 & 174.8 & 0.8 & 149.5 & 1.6 & 142.4 & 3.1 & 131.9 & -0.2 & 164.9 & 2.6 & 108.2 & 1.1 & 144.5 & 3.0 & 144.7 & 1.5 \\
\hline 2012 & 172.6 & -1.3 & 151.0 & 1.0 & 147.0 & 3.2 & 133.8 & 1.5 & 166.9 & 1.2 & 106.9 & -1.2 & 146.7 & 3.7 & 145.7 & 0.7 \\
\hline 2013 & 166.9 & -3.3 & 148.7 & -1.5 & 151.3 & 2.9 & 130.3 & -2.6 & 164.2 & -1.6 & 104.3 & -2.4 & 147.4 & 1.5 & 143.8 & -1.3 \\
\hline 2014 & 163.3 & -2.1 & 148.3 & -0.3 & 154.7 & 2.2 & 128.5 & -1.4 & 162.0 & -1.4 & 103.9 & -0.3 & 137.6 & 0.5 & 142.9 & -0.7 \\
\hline 2015 & 158.7 & -2.8 & 145.2 & -2.1 & 150.7 & -2.6 & 124.7 & -2.9 & 159.0 & -1.8 & 103.3 & -0.7 & 133.7 & -6.7 & 139.5 & -2.4 \\
\hline & & & & & & & & & & & & & & -2.8 & & \\
\hline Median & 166.9 & & 145.2 & & 138.2 & & 128.5 & & 159.3 & & 104.3 & & 137.6 & & 140.2 & \\
\hline Percentage change & 3.2 & & 19. & & 35.5 & & 15 & & 13. & & 7. & & 13. & & 15. & \\
\hline
\end{tabular}

*ABMUHB = Abertawe Bro Morgannwg University Health Board; ABUHB = Aneurin Bevan University Health Board; BCUHB = Betsi Cadwaladr University Health Board; CVUHB = Cardiff and Vale University Health Board; CTUHB = Cwm Taf University Health Board; HDUHB = Hywel Dda University Health Board; PTHB = Powys Teaching Health Board 


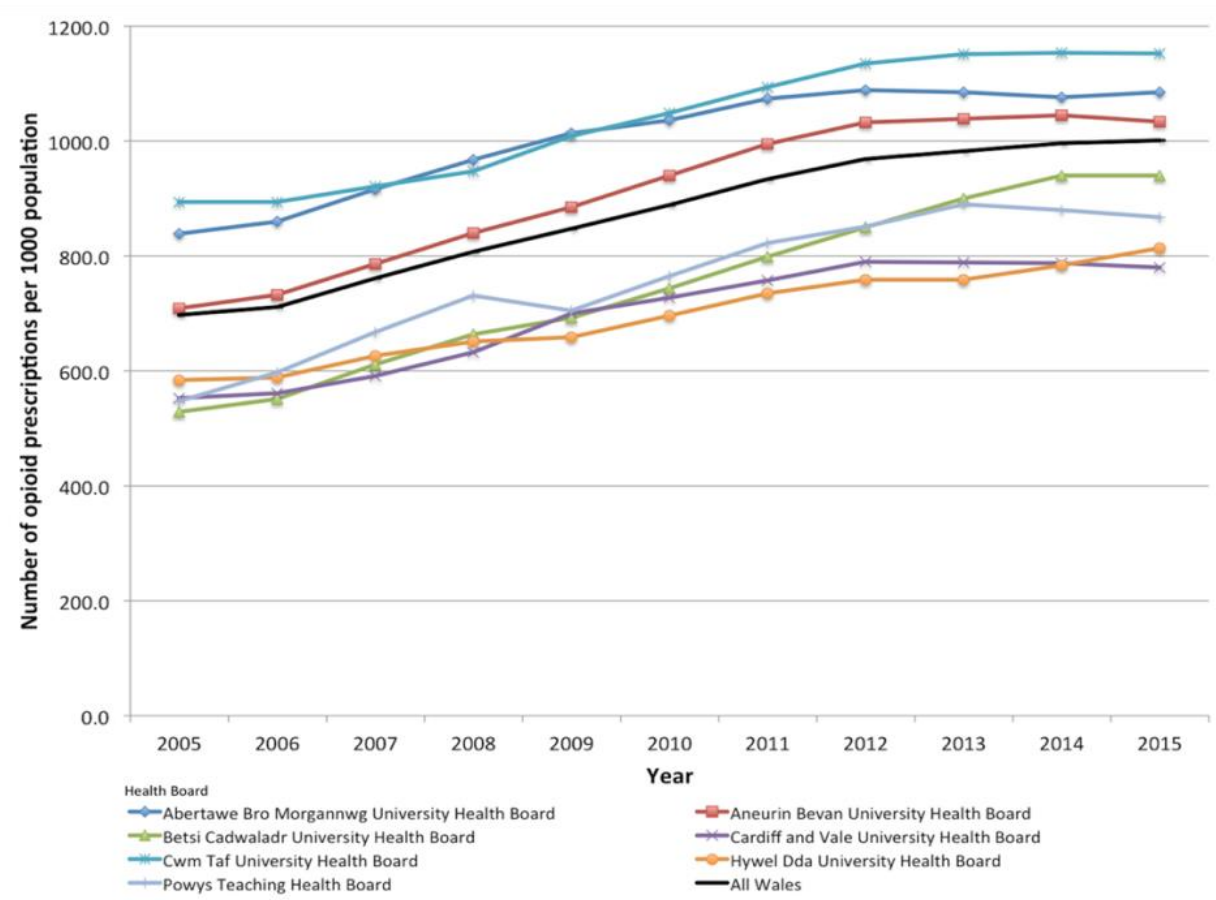

Figure 1.2: Trend in the annual number of prescriptions per 1000 population in each Health Board in Wales and compared to the National trend. Data population adjusted to each Health Board

Kruskal-Wallis test demonstrated a strong difference $(p<.001)$ between the number of opioid prescriptions issued between Health Boards across Wales over the 11 years examined. Dunn's pairwise comparisons and post-hoc Bonferroni tests highlighted significant difference between 8 pairs of comparisons of the number of prescriptions issued in the Health Boards.

Table 1.3: Dunn's pairwise comparison and Bonferroni post-hoc analysis of difference between the number of opioid prescriptions being issued by Welsh Health Board between 2005 and 2015

\begin{tabular}{rcccccc}
\hline \multicolumn{7}{c}{ Health Board** } \\
\hline ABUHB & $>.999$ & & & & \\
\hline BCUHB & $.014^{*}$ & .672 & & & & \\
\hline CVUHB & $.001^{*}$ & .080 & $>.999$ & & & \\
\hline CTUHB & $>.999$ & $>.999$ & $.003^{*}$ & $<.001^{*}$ & & \\
\hline HDUH & $<.001^{*}$ & .055 & $>.999$ & $>.999$ & $<.001^{*}$ & \\
B & & & & & & \\
\hline PTHB & $.019^{*}$ & .840 & $>.999$ & $>.999$ & $.004^{*}$ & $>.999$ \\
\hline
\end{tabular}

$* p<0.05=$ statistically significant

**ABMUHB = Abertawe Bro Morgannwg University Health Board; $A B U H B=$ Aneurin Bevan University Health Board; $\mathrm{BCUHB}=$ Betsi Cadwaladr University Health Board; $\mathrm{CVUHB}=$ Cardiff and Vale University Health Board; CTUHB $=$ Cwm Taf University Health Board; HDUHB = Hywel Dda University Health Board; PTHB = Powys Teaching Health Board

In 2005, CTUHB, the organisation with the highest number of annual prescriptions, had 1.7 times more prescriptions issued per 1000 population than $B C U H B$, which had the fewest number. However, due to the large increase in annual numbers of prescriptions in BCUHB, by 2015 it was the fourth highest prescriber. Cardiff and Vale University Health Board (CVUHB), despite having a $41.1 \%$ increase in annual prescription numbers over the study period was by 
2015, the Health Board with the fewest opioid prescriptions per 1000 population (Table I.4). 
Table I.4: Trends in the annual number of opioid prescriptions in each Health Board in Wales and compared to the National Average (All Wales). Rate change over the 11 year study period included. Data population adjusted per Health Board (number of people per 1000 population)

\begin{tabular}{|c|c|c|c|c|c|c|c|c|c|c|c|c|c|c|c|c|c|}
\hline \multirow{3}{*}{\multicolumn{2}{|c|}{ Number of people - change rate (\%) }} & \\
\hline & & \multicolumn{2}{|c|}{ ABMUHB } & \multicolumn{2}{|c|}{ ABUHB } & \multicolumn{2}{|c|}{ BCUHB } & \multicolumn{2}{|c|}{ CVUHB } & \multicolumn{2}{|c|}{ CTUHB } & \multicolumn{2}{|c|}{ HDUHB } & \multicolumn{2}{|c|}{ PTHB } & \multicolumn{2}{|c|}{ All Wales } \\
\hline & & & & & & & & & & & & & & & & & \\
\hline & 2005 & 838.2 & & 708.7 & & 527.9 & & 552.5 & & 893.9 & & 583.9 & & 547.2 & & 696.8 & \\
\hline & 2006 & 859.5 & 2.5 & 732.8 & 3.4 & 551.1 & 4.4 & 560.7 & 1.5 & 894.2 & 0.0 & 588.8 & 0.9 & 597.2 & 9.1 & 711.4 & 2.1 \\
\hline & 2007 & 916.2 & 6.6 & 786.6 & 7.3 & 610.6 & 10.8 & 590.6 & 5.3 & 921.6 & 3.1 & 625.8 & 6.3 & 667.4 & 11.8 & 760.6 & 6.9 \\
\hline & 2008 & 967.0 & 5.6 & 840.1 & 6.8 & 663.5 & 8.7 & 632.8 & 7.2 & 947.2 & 2.8 & 651.4 & 4.1 & 730.5 & 9.5 & 807.1 & 6.1 \\
\hline & 2009 & 1013.4 & 4.8 & 884.5 & 5.3 & 691.9 & 4.3 & 700.3 & 10.7 & 1009.1 & 6.5 & 658.6 & 1.1 & 705.4 & -3.4 & 846.8 & 4.9 \\
\hline & 2010 & 1035.9 & 2.2 & 940.5 & 6.3 & 743.3 & 7.4 & 727.5 & 3.9 & 1049.1 & 4.0 & 696.5 & 5.8 & 765.0 & 8.4 & 888.2 & 4.9 \\
\hline & 2011 & 1073.4 & 3.6 & 994.4 & 5.7 & 798.9 & 7.5 & 757.1 & 4.1 & 1093.9 & 4.3 & 734.2 & 5.4 & 821.7 & 7.4 & 933.8 & 5.1 \\
\hline & 2012 & 1089.0 & 1.5 & 1031.9 & 3.8 & 849.4 & 6.3 & 789.5 & 4.3 & 1134.4 & 3.7 & 759.0 & 3.4 & 851.5 & 3.6 & 968.7 & 3.7 \\
\hline & 2013 & 1084.7 & -0.4 & 1038.3 & 0.6 & 899.3 & 5.9 & 789.0 & -0.1 & 1150.6 & 1.4 & 758.8 & 0.0 & 890.0 & 4.5 & 982.8 & 1.5 \\
\hline & 2014 & 1076.5 & -0.8 & 1045.3 & 0.7 & 939.6 & 4.5 & 787.6 & -0.2 & 1153.5 & 0.3 & 784.1 & 3.3 & 879.6 & -1.2 & 996.2 & 1.4 \\
\hline & 2015 & 1085.3 & 0.8 & 1033.1 & -1.2 & 940.5 & 0.1 & 779.6 & -1.0 & 1152.4 & -0.1 & 813.9 & 3.8 & 867.6 & -1.4 & 1000.7 & 0.5 \\
\hline Median & & 1035.9 & & 940.5 & & 743.3 & & 727.5 & & 1049.1 & & 696.5 & & 765.0 & & 888.2 & \\
\hline \multicolumn{2}{|c|}{ Rate change 2005-2015 (\%) } & \multicolumn{2}{|c|}{29.5} & \multicolumn{2}{|c|}{45.8} & \multicolumn{2}{|c|}{78.1} & \multicolumn{2}{|c|}{41.1} & \multicolumn{2}{|c|}{28.9} & \multicolumn{2}{|c|}{39.4} & \multicolumn{2}{|c|}{58.6} & \multicolumn{2}{|c|}{43.6} \\
\hline
\end{tabular}

*ABMUHB = Abertawe Bro Morgannwg University Health Board; ABUHB = Aneurin Bevan University Health Board; BCUHB = Betsi Cadwaladr University Health Board; CVUHB = Cardiff and Vale University Health Board; CTUHB $=$ Cwm Taf University Health Board; HDUHB = Hywel Dda University Health Board; PTHB = Powys Teaching Health Board 


\section{I.2.3 Types of opioids being prescribed}

The trend in the numbers of prescriptions being issued in each Health Board across Wales varied depending on the type of opioid (weak or strong) being prescribed (Table I.5).

Table I.5: Trends in the number of opioid prescriptions per 1000 population, issued in each Health Board. Adjusted to annual Health Board population

\begin{tabular}{|c|c|c|c|c|c|c|c|}
\hline \multirow{2}{*}{$\begin{array}{l}\text { Number of } \\
\text { prescriptions }\end{array}$} & \multicolumn{7}{|c|}{ Health Board* } \\
\hline & ABMUHB & $A B \cup H B$ & BCUHB & CVUHB & CTUHB & HDUHB & PTHB \\
\hline \multicolumn{8}{|l|}{ Strong opioids } \\
\hline 2005 & 35.0 & 34.3 & 40.1 & 33.5 & 46.3 & 47.9 & 28.5 \\
\hline 2015 & 160.7 & 158.9 & 158.9 & 143.2 & 138.3 & 169.8 & 145.3 \\
\hline Median & 61.5 & 82.4 & 94.1 & 86.7 & 82.0 & 91.9 & 64.3 \\
\hline Change rate (\%) & 359.5 & 363.7 & 296.0 & 326.9 & 198.4 & 254.1 & 410.4 \\
\hline \multicolumn{8}{|l|}{ Weak opioids } \\
\hline 2005 & 803.3 & 674.5 & 487.8 & 519.0 & 847.5 & 535.9 & 518.7 \\
\hline 2015 & 924.6 & 874.2 & 781.5 & 636.4 & 1014.2 & 644.2 & 722.3 \\
\hline Median & 948.6 & 858.1 & 653.2 & 636.4 & 967.1 & 604.6 & 700.7 \\
\hline Change rate (\%) & 15.1 & 29.6 & 60.2 & 22.6 & 19.7 & 20.2 & 39.2 \\
\hline
\end{tabular}

*ABMUHB = Abertawe Bro Morgannwg University Health Board; ABUHB = Aneurin Bevan University Health Board; $B C U H B=$ Betsi Cadwaladr University Health Board; $C V U H B=$ Cardiff and Vale University Health Board; CTUHB = Cwm Taf University Health Board; HDUHB = Hywel Dda University Health Board; PTHB = Powys Teaching Health Board

Large increases in the number of strong opioid prescriptions being issued were observed in each Health Board (Figure I.3), the greatest seen in Powys Teaching Health Board (410.4\%, from 28.5 to 145.3 prescriptions per 1000 population). The lowest percentage increase in the annual number of strong opioid prescriptions was seen in Cwm Taff University Health Board although the annual number of prescriptions nearly tripled over the 11 years examined.

A Kruskal-Wallis test of the differences in the annual numbers of strong prescriptions between each Health Board did not demonstrate significance $(p=.695)$. As a result, Dunn's pairwise tests and post-hoc Bonferroni tests were not undertaken. 


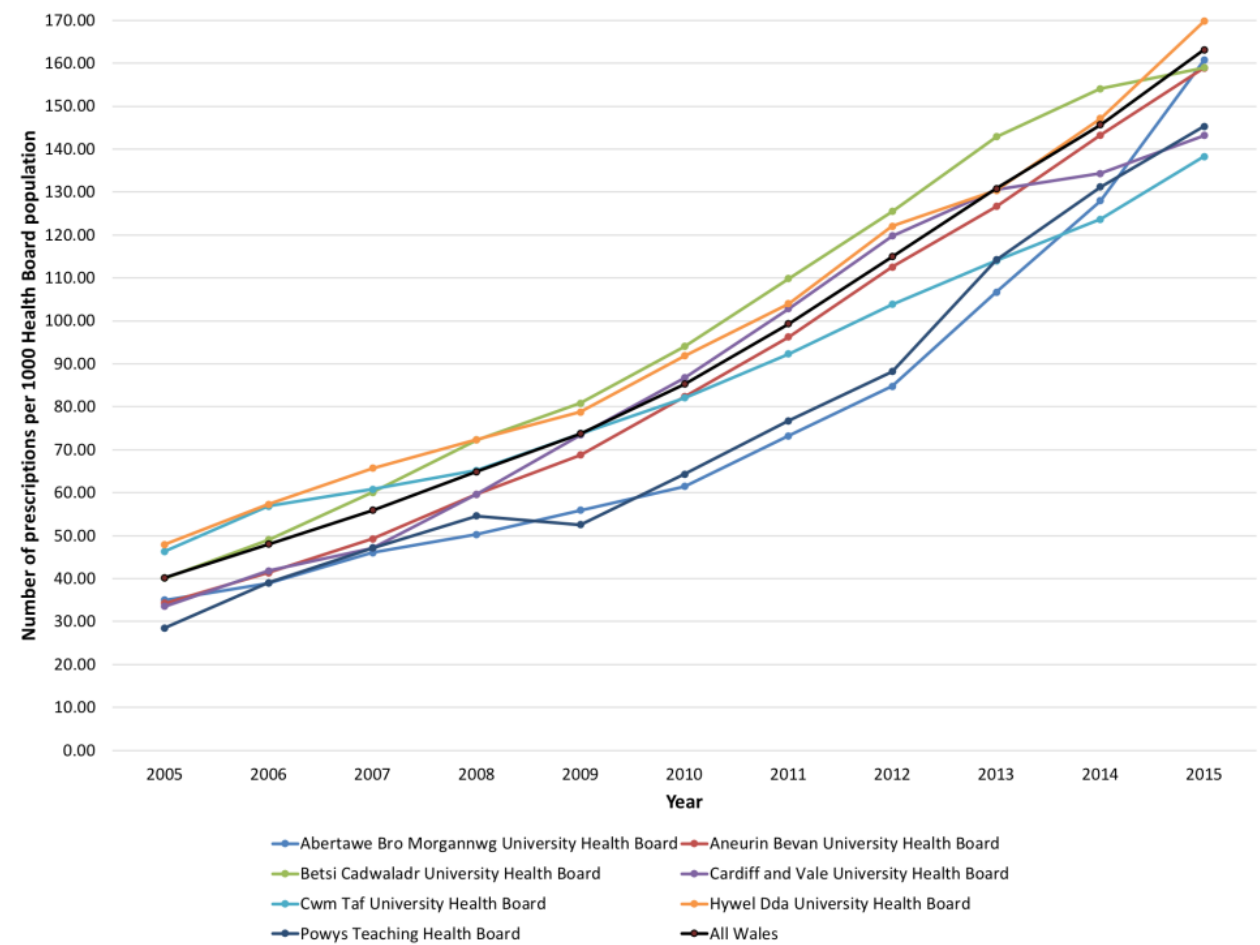

Figure 1.3: Trend in the annual number of strong opioid prescriptions in each Health Board and compared to the National trend. Data population adjusted to Health Board

Trends in the annual number of weak opioid prescriptions being issued within each of the seven Health Boards showed more variation than was seen with strong opioid prescriptions (Figure I.3).

Abertawe Bro Morgannwg University Health Board demonstrated the smallest increase in annual prescription numbers $(15.1 \%$, from 803.3 to 924.6 prescriptions per 1000 population) although had the second highest rate of prescribing in Wales. Again, as with the strong opioid prescriptions, Powys Teaching Health Board had the greatest percentage increase in annual prescription numbers from 2005 to 2015 (39.2\%, from 518.7 to 722.3 prescriptions per 1000 population). 


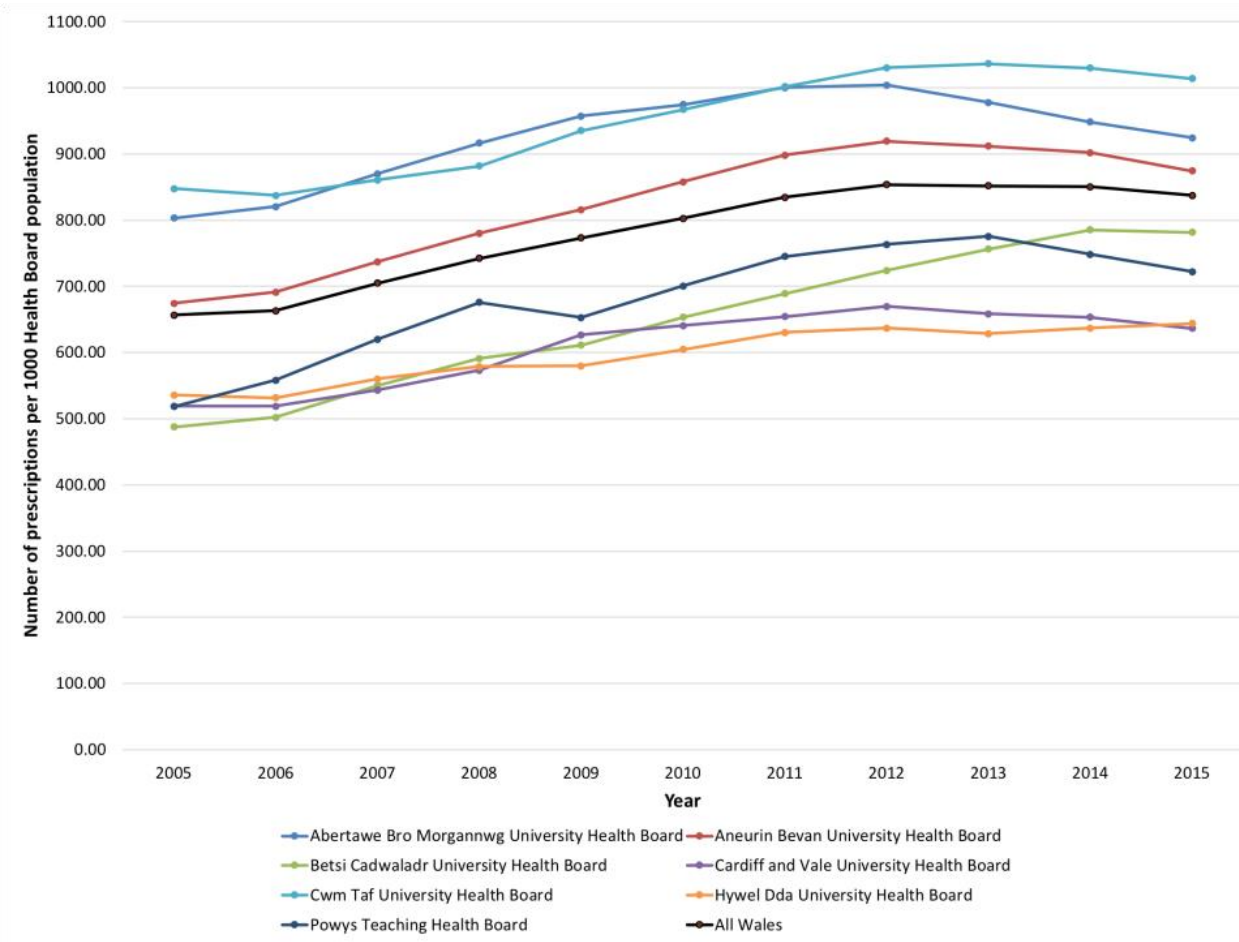

Figure 1.4: Trend in the annual number of weak opioid prescriptions in each Health Board and compared to the National trend. Data population adjusted to Health Board

A significant difference was demonstrated between the number of weak opioid prescriptions being issued in each Health Board, as determined by Kruskal-Wallis test $(p<.001)$. Dunn's pairwise and post-hoc Bonferroni tests showed significant differences between 10 pairs of Health Board data (Table I.6). The 11 other pairs were not statistically different.

Table I.6: Dunn's pairwise comparison and Bonferroni post-hoc analysis of difference between the number of weak opioid prescriptions being issued by Welsh Health Board between 2005 and 2015

\begin{tabular}{rcccccc}
\hline & \multicolumn{5}{c}{ Health Board** } \\
\cline { 2 - 7 } & ABMUHB & ABUHB & BCUHB & CVUHB & CTUHB & HDUHB \\
\hline ABUHB & $>.999$ & & & & \\
\hline BCUHB & $.002^{*}$ & .184 & & & \\
\hline CVUHB & $<.001^{*}$ & $.023^{*}$ & $>.999$ & & \\
\hline CTUHB & $>.999$ & $>.999$ & $.001^{*}$ & $<.001^{*}$ & \\
\hline HDUHB & $<.001^{*}$ & $.007^{*}$ & $>.999$ & $>.999$ & $<.001^{*}$ & \\
\hline PTHB & $.013^{*}$ & .757 & $>.999$ & $>.999$ & $.005^{*}$ & $>.999$
\end{tabular}

$*_{p}<0.05=$ statistically significant

**ABMUHB = Abertawe Bro Morgannwg University Health Board; $A B U H B=$ Aneurin Bevan University Health Board; $\mathrm{BCUHB}=$ Betsi Cadwaladr University Health Board; $\mathrm{CVUHB}=$ Cardiff and Vale University Health Board; $\mathrm{CTUHB}=\mathrm{Cwm}$ Taf University Health Board; HDUHB = Hywel Dda University Health Board; PTHB = Powys Teaching Health Board

\section{I.3 Opioid prescribing by deprivation per Health Board}

There was variance between the seven Health Boards in Wales, in respect of opioid prescribing in the different areas of deprivation (Table I.9). 


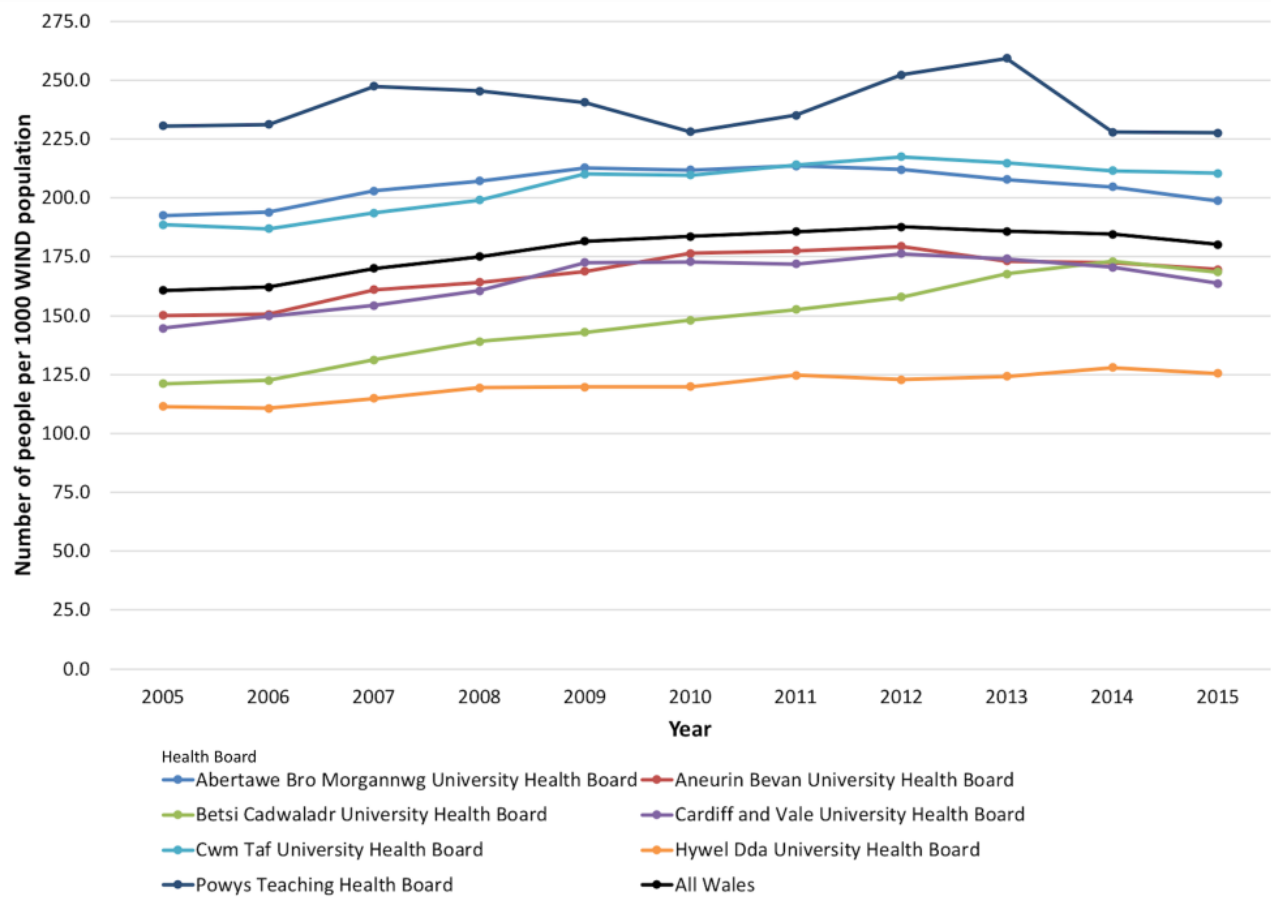

Figure 1.5: Trend in the number of people receiving prescriptions for opioid medicines in the most deprived areas (as per Welsh Index of Multiple Deprivation (WIMD)) in each Health Board in Wales. Data adjusted to population of WIMD1 areas in each Health Board

Powys Teaching Health Board (PTHB), whilst having the smallest population of all the Health Boards in Wales, had the greatest number of people in the most deprived areas, receiving opioid prescriptions over the whole 11 period examined, despite a small reduction in numbers within the Health Board itself (1.3\%, from 230.5 to 227.6 people per 1000 WIMD1 population) (Table I.4).

The greatest increase in number of people in WIMD1 areas $(39.2 \%$, from 121.1 to 168.5 people per 1000 WIMD1 population) receiving opioid prescriptions was in Betsi Cadwaladr University Health Board (BCUHB).

A Kruskal-Wallis test demonstrated a significant difference $(p<.001)$ between the number of people receiving opioid prescriptions in WIMD1 areas in the 7 Health Boards. Post-hoc analysis demonstrated significant difference $(p<.05)$ between 8 of the 21 pairs of comparators WIMD1 areas within the Health Board areas, the remaining pairs did not have a statistical difference between them.

Table I.7: Dunn's pairwise comparison and Bonferroni post-hoc analysis of difference between the number of people receiving opioid prescriptions being issued in WIMD1 areas (most deprived) by Welsh Health Board between 2005 and 2015

\begin{tabular}{rcccccc}
\hline & \multicolumn{5}{c}{ Health Board** } \\
\cline { 2 - 6 } & ABMUHB & ABUHB & BCUHB & CVUHB & CTUHB & HDUHB \\
\hline ABUHB & .411 & & & & & \\
\hline BCUHB & $.005^{*}$ & $>.999$ & & & & \\
\hline CVUHB & .189 & $>.999$ & $>.999$ & & & \\
\hline CTUHB & $>.999$ & .325 & $.004^{*}$ & .147 & & \\
\hline HDUHB & $<.001^{*}$ & .139 & $>.999$ & .309 & $<.001^{*}$ & \\
\hline PTHB & $>.999$ & $.001^{*}$ & $<.001^{*}$ & $>.999$ & $.005^{*}$ & $<.001^{*}$ \\
\hline
\end{tabular}


${ }^{*} p<0.05=$ statistically significant $* * A B M U H B=$ Abertawe Bro Morgannwg University Health Board; $\mathrm{ABUHB}=$ Aneurin Bevan University Health Board; $\mathrm{BCUHB}=$ Betsi Cadwaladr University Health Board; CVUHB = Cardiff and Vale University Health Board; CTUHB - Cwm Taf University Health Board; HDUHB = Hywel Dda University Health Board; PTHB = Powys Teaching Health Board

The highest numbers of people per 1000 population in WIMD2 areas receiving opioids, was again highest in PTHB (Table I.9), the area with the smallest population. Whilst there was variation over the 11 years examined (Figure I.6), there was overall a $17.1 \%$ (from 288.7 to 338.2 people per 1000 population) increase in the numbers between 2005 and 2015.

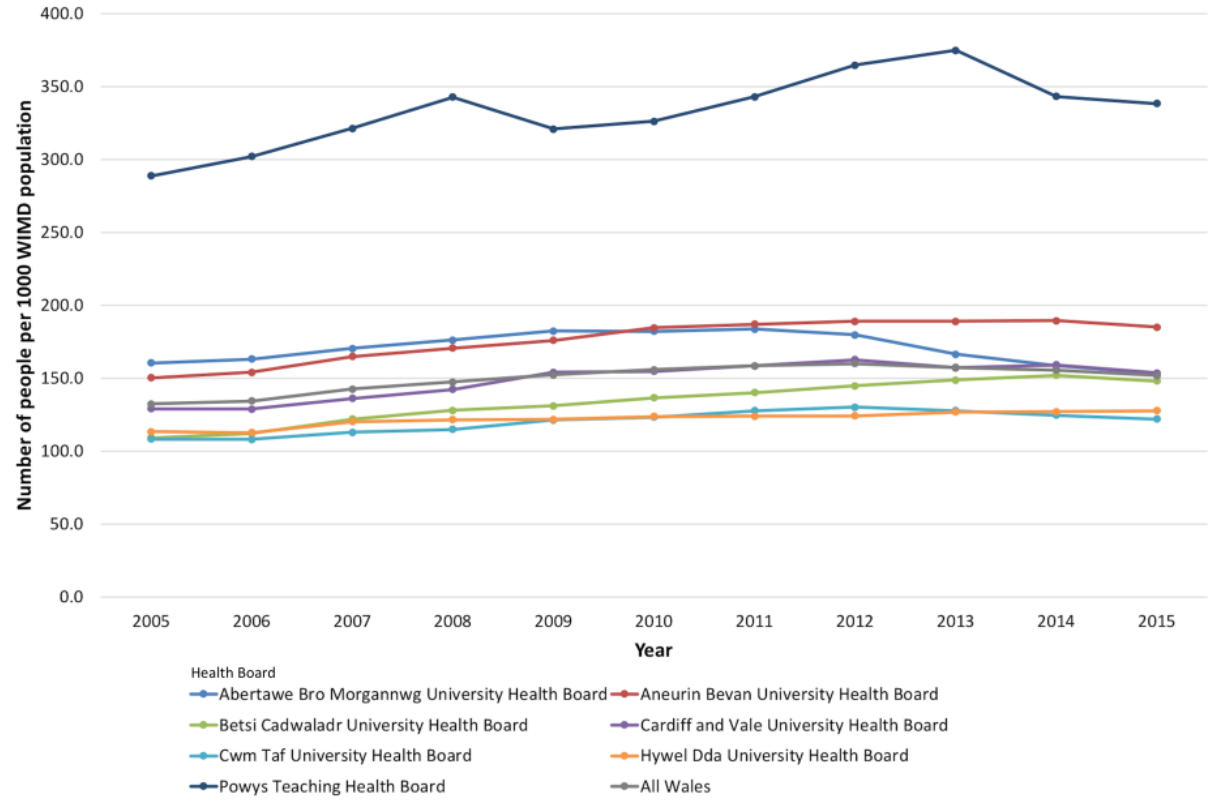

Figure 1.6: Trend in the number of people receiving prescriptions for opioid medicines in the 2nd most deprived areas (as per Welsh Index of Multiple Deprivation (WIMD)) in each Health Board in Wales. Data adjusted to population of WIMD2 areas in each Health Board

The greatest increase in the annual number of people receiving opioid prescriptions in WIMD2 areas was in BCUHB although 4 other Health Boards had larger levels of prescribing overall. The difference between the numbers of people living in WIMD2 areas and receiving opioid prescriptions was statistically significant as evaluated by Kruskal-Wallis tests $(p<.001)$. Dunn's pairwise tests and post-hoc Bonferroni tests showed that 9 out of 21 pairs of WIMD2 areas in different Health Boards had significant differences in the numbers of people receiving prescriptions (Table I.8).

Table I.8: Dunn's pairwise comparison and Bonferroni post-hoc analysis of difference between the number of people receiving opioid prescriptions being issued in WIMD2 areas by Welsh Health Board between 2005 and 2015

\begin{tabular}{|c|c|c|c|c|c|c|}
\hline & \multicolumn{6}{|c|}{ Health Board** } \\
\hline & ABMUHB & ABUHB & BCUHB & CVUHB & CTUHB & HDUHB \\
\hline ABUHB & $>.999$ & & & & & \\
\hline BCUHB & .099 & $.026 *$ & & & & \\
\hline CVUHB & $>.999$ & $>.999$ & $>.999$ & & & \\
\hline CTUHB & $.001^{*}$ & $<.001^{*}$ & $>.999$ & .165 & & \\
\hline HDUHB & $.002 *$ & $<.001^{*}$ & $>.999$ & .239 & $>.999$ & \\
\hline
\end{tabular}




$\begin{array}{llllll}\text { PTHB } & .850 & >.999 & <.001 * & .010 * & <.001 *\end{array}$

${ }^{*} p<0.05$ = statistically significant **ABMUHB = Abertawe Bro Morgannwg University Health Board; ABUHB $=$ Aneurin Bevan University Health Board; $B C U H B=$ Betsi Cadwaladr University Health Board; CVUHB = Cardiff and Vale University Health Board; CTUHB - Cwm Taf University Health Board; HDUHB = Hywel Dda University Health Board; PTHB = Powys Teaching Health Board

Cwm Taff University Health Board had overall the largest number of people per 1000 population in WIMD3 areas of any of the seven Health Boards and was subject to a $15.2 \%$ (from 157.3 to 181.2 people per 1000 population) between 2005 and 2015 (Table I.9).

Hywel Dda University Health Board had the least number of people receiving prescriptions in WIMD3 areas over the same period. Although the smallest increase in numbers appeared to be in PTHB (2.2\%, from 156.4 to 159.8 people per 1000 population), there was some fluctuation in the data from that Health Board over the study period.

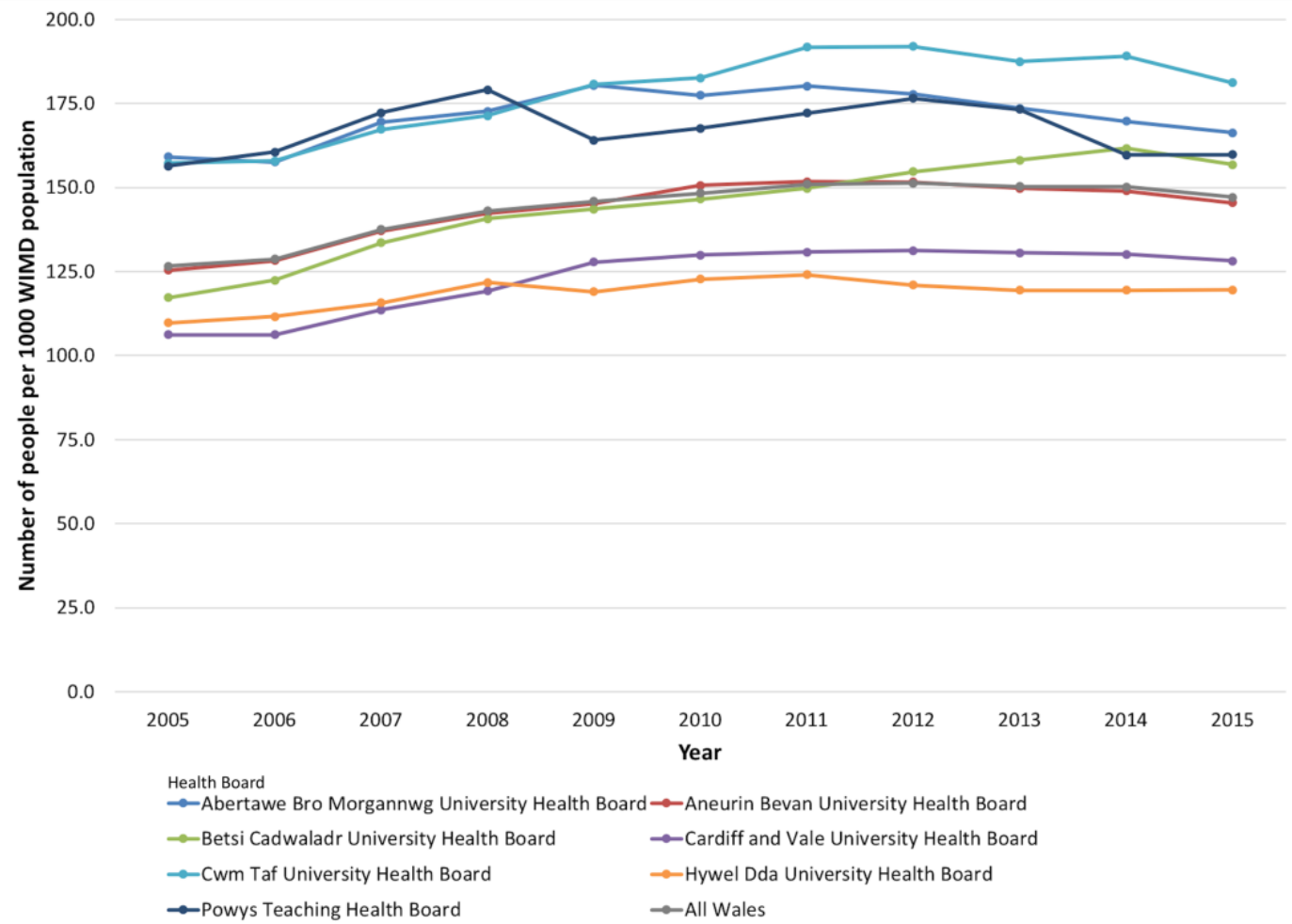

Figure 1.7: Trend in the number of people receiving prescriptions for opioid medicines in the WIMD3 areas (as per Welsh Index of Multiple Deprivation (WIMD)) in each Health Board in Wales. Data adjusted to population of WIMD3 areas in each Health Board 
Table I.9: Trends in the annual number of people receiving opioid prescriptions in each Health Board in Wales by Welsh Index of Multiple Deprivation (WIMD) area

\begin{tabular}{|c|c|c|c|c|c|c|c|}
\hline \multirow[b]{2}{*}{ Number of people per 1000} & \multicolumn{7}{|c|}{ Health Board } \\
\hline & ABMUHB & ABUHB & BCUHB & CVUHB & CTUHB & HDUHB & PTHB \\
\hline \multicolumn{8}{|l|}{ All opioids } \\
\hline WIMD1 & & & & & & & \\
\hline 2005 & 192.5 & 150.0 & 121.1 & 144.7 & 188.6 & 111.4 & 230.5 \\
\hline 2015 & 198.8 & 169.6 & 168.5 & 163.6 & 210.4 & 125.4 & 227.6 \\
\hline Median & 207.1 & 169.6 & 148.0 & 170.6 & 210.2 & 119.9 & 235.1 \\
\hline Rate change $2005-2015$ (\%) & 3.3 & 13.1 & 39.2 & 13.1 & 11.6 & 12.6 & -1.3 \\
\hline \multicolumn{8}{|l|}{ WIMD2 } \\
\hline 2005 & 160.5 & 150.2 & 109.1 & 129.0 & 108.2 & 113.2 & 288.7 \\
\hline 2015 & 153.0 & 185.0 & 147.9 & 153.6 & 121.8 & 127.8 & 338.2 \\
\hline Median & 170.4 & 184.4 & 136.4 & 154.0 & 121.8 & 123.7 & 338.2 \\
\hline Rate change 2005-2015 (\%) & -4.7 & 23.2 & 35.6 & 19.1 & 12.6 & 12.9 & 17.1 \\
\hline \multicolumn{8}{|l|}{ WIMD3 } \\
\hline 2005 & 159.1 & 125.4 & 117.3 & 106.3 & 157.3 & 109.7 & 156.4 \\
\hline 2015 & 166.3 & 145.5 & 156.8 & 128.2 & 181.2 & 119.6 & 159.8 \\
\hline Median & 172.7 & 145.5 & 146.6 & 128.2 & 181.2 & 119.5 & 167.6 \\
\hline Rate change 2005-2015 (\%) & 4.5 & 16.0 & 33.7 & 20.6 & 15.2 & 9.0 & 2.2 \\
\hline \multicolumn{8}{|l|}{ WIMD4 } \\
\hline 2005 & 154.0 & 89.1 & 76.7 & 95.3 & 116.4 & 99.0 & 69.1 \\
\hline 2015 & 167.1 & 115.5 & 104.6 & 114.0 & 130.6 & 101.3 & 83.3 \\
\hline Median & 172.1 & 115.5 & 96.8 & 115.7 & 134.2 & 106.3 & 88.1 \\
\hline Rate change 2005-2015 (\%) & 8.4 & 29.6 & 36.3 & 19.6 & 12.2 & 2.3 & 20.5 \\
\hline \multicolumn{8}{|l|}{ WIMD5 } \\
\hline 2005 & 101.9 & 71.7 & 112.2 & 84.9 & 103.3 & 31.5 & 75.8 \\
\hline 2015 & 111.2 & 92.1 & 147.0 & 95.9 & 130.1 & 28.2 & 106.7 \\
\hline Median & 114.0 & 87.8 & 140.3 & 98.5 & 131.7 & 33.0 & 109.8 \\
\hline Rate change 2005-2015 (\%) & 9.1 & 28.4 & 31.0 & 13.0 & 25.9 & -10.5 & 40.8 \\
\hline
\end{tabular}

*ABMUHB = Abertawe Bro Morgannwg University Health Board; ABUHB = Aneurin Bevan University Health Board; BCUHB = Betsi Cadwaladr University Health Board; CVUHB = Cardiff and Vale University Health Board; CTUHB $=$ Cwm Taf University Health Board; HDUHB = Hywel Dda University Health Board; PTHB = Powys Teaching Health Board. WIMD1=most deprived and

WIMD5=least deprived area. Rate change over the 11-year study period included. Data population adjusted per Health Board (number of people per 1000 population) 
A Kruskal-Wallis test demonstrated significance in the numbers of people receiving opioid prescriptions in the WIMD3 areas in each Health Board, although post-hoc testing demonstrated that this significance was between 8 of the 21 pairs of Health Board comparators (Table I.10).

Table I.10: Dunn's pairwise comparison and Bonferroni post-hoc analysis of difference between the number of people receiving opioid prescriptions being issued in WIMD3 areas by Welsh Health Board between 2005 and 2015

\begin{tabular}{rcccccc}
\hline & \multicolumn{5}{c}{ Health Board** } \\
\cline { 2 - 6 } & ABMUHB & ABUHB & BCUHB & CVUHB & CTUHB & HDUHB \\
\hline ABUHB & .067 & & & & \\
\hline BCUHB & .096 & $>.999$ & & & \\
\hline CVUHB & $<.001^{*}$ & $>.999$ & $>.999$ & & \\
\hline CTUHB & $>.999$ & $.008^{*}$ & $.013^{*}$ & $<.001^{*}$ & & \\
\hline HDUHB & $<.001^{*}$ & .508 & $>.999$ & $>.999$ & $<.001^{*}$ & \\
\hline PTHB & $>.999$ & .227 & .313 & $.001^{*}$ & $>.999$ & .375 \\
\hline
\end{tabular}

${ }^{*} p<0.05$ = statistically significant **ABMUHB = Abertawe Bro Morgannwg University Health Board; ABUHB $=$ Aneurin Bevan University Health Board; $B C U H B=$ Betsi Cadwaladr University Health Board; CVUHB = Cardiff and Vale University Health Board; CTUHB - Cwm Taf University Health Board; HDUHB = Hywel Dda University Health Board; PTHB = Powys Teaching Health Board

In the areas of Wales designated WIMD4, areas of lesser overall deprivation, Abertawe Bro Morgannwg University Health Board (ABMUHB) had the highest number of people receiving opioid prescriptions (Table I.9). Five of the remaining 6 Health Boards had significant differences in the number of people receiving prescriptions $(p<.05)$ based on Kruskal-Wallis analysis and post-hoc tests. Despite the higher rates of people receiving prescriptions in ABMUHB, that Health Board had an $8.4 \%$ (from 154.0 to 167.1 people per 1000 population) increase over the 11 years examined.

The smallest increase (2.3\%, from 99.0 to 101.3 people per 1000 population) was noted in HDUHB although the fewest people receiving prescriptions were in WIMD4 areas of PTHB. 


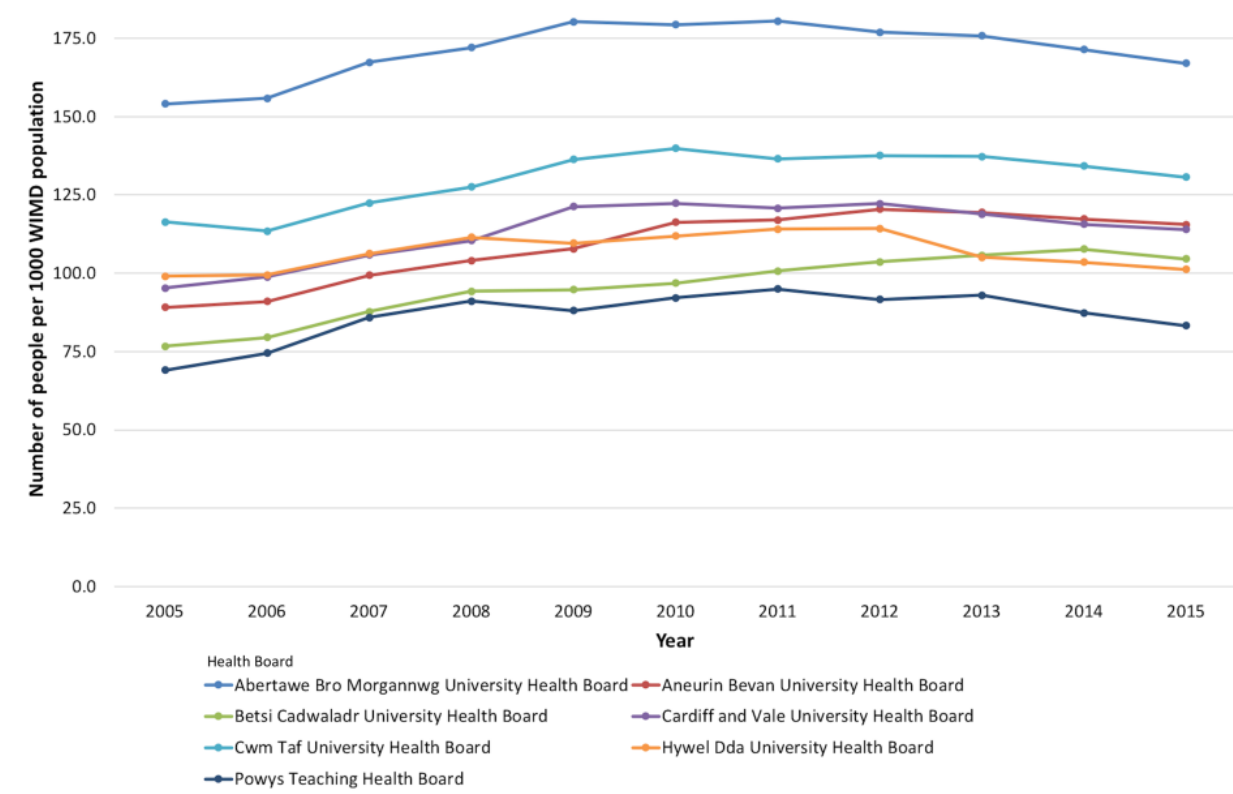

Figure I.8: Trend in the number of people receiving prescriptions for opioid medicines in the WIMD4 areas (as per Welsh Index of Multiple Deprivation (WIMD)) in each Health Board in Wales. Data adjusted to population of WIMD4 areas in each Health Board

Kruskal-Wallis analysis of the difference in the number of people in the WIMD4 areas in each Health Board demonstrated a significant difference (Table I.11). Post-hoc tests showed 8 of 21 pairs of Health Board comparators were significantly different. The remaining pairs of Health Boards which were compared did not demonstrate significance.

Table I.11: Dunn's pairwise comparison and Bonferroni post-hoc analysis of difference between the number of people receiving opioid prescriptions being issued in WIMD4 areas by Welsh Health Board between 2005 and 2015

\begin{tabular}{rcccccc}
\hline & \multicolumn{5}{c}{ Health Board** } \\
\cline { 2 - 7 } ABUHB & ABMUHB & ABUHB & BCUHB & CVUHB & CTUHB & HDUHB \\
\hline BCUHB & $<.001^{*}$ & $>.999$ & & & \\
\hline CVUHB & $.049^{*}$ & $>.999$ & .321 & & \\
\hline CTUHB & $>.999$ & .443 & $.001^{*}$ & $>.999$ & & \\
\hline HDUHB & $.001^{*}$ & $>.999$ & $>.999$ & $>.999$ & .158 & \\
\hline PTHB & $<.001^{*}$ & .082 & $>.999$ & $.009^{*}$ & $<.001^{*}$ & .246 \\
\hline
\end{tabular}

${ }^{*} p<0.05=$ statistically significant

**ABMUHB = Abertawe Bro Morgannwg University Health Board; $A B U H B=$ Aneurin Bevan University Health Board; $\mathrm{BCUHB}=$ Betsi Cadwaladr University Health Board; $\mathrm{CVUHB}=$ Cardiff and Vale University Health Board; CTUHB = Cwm Taf University Health Board; HDUHB = Hywel Dda University Health Board; PTHB = Powys Teaching Health Board

Betsi Cadwaladr University Health Board had the greatest number of people living in WIMD5 areas receiving opioid prescriptions in Wales between 2005 and 2015 (Table X). There was a significant difference $(p<.001)$ between BCUHB and HDUHB which had the lowest numbers of people receiving prescriptions and saw a decline in those numbers $(-10.5 \%$, from31.5 to 28.2 people per 1000 population) over the same period. 
The largest percentage increase in numbers (40.8\%, from 75.8 to 106.7 people per 1000 population) were seen in PTHB however.

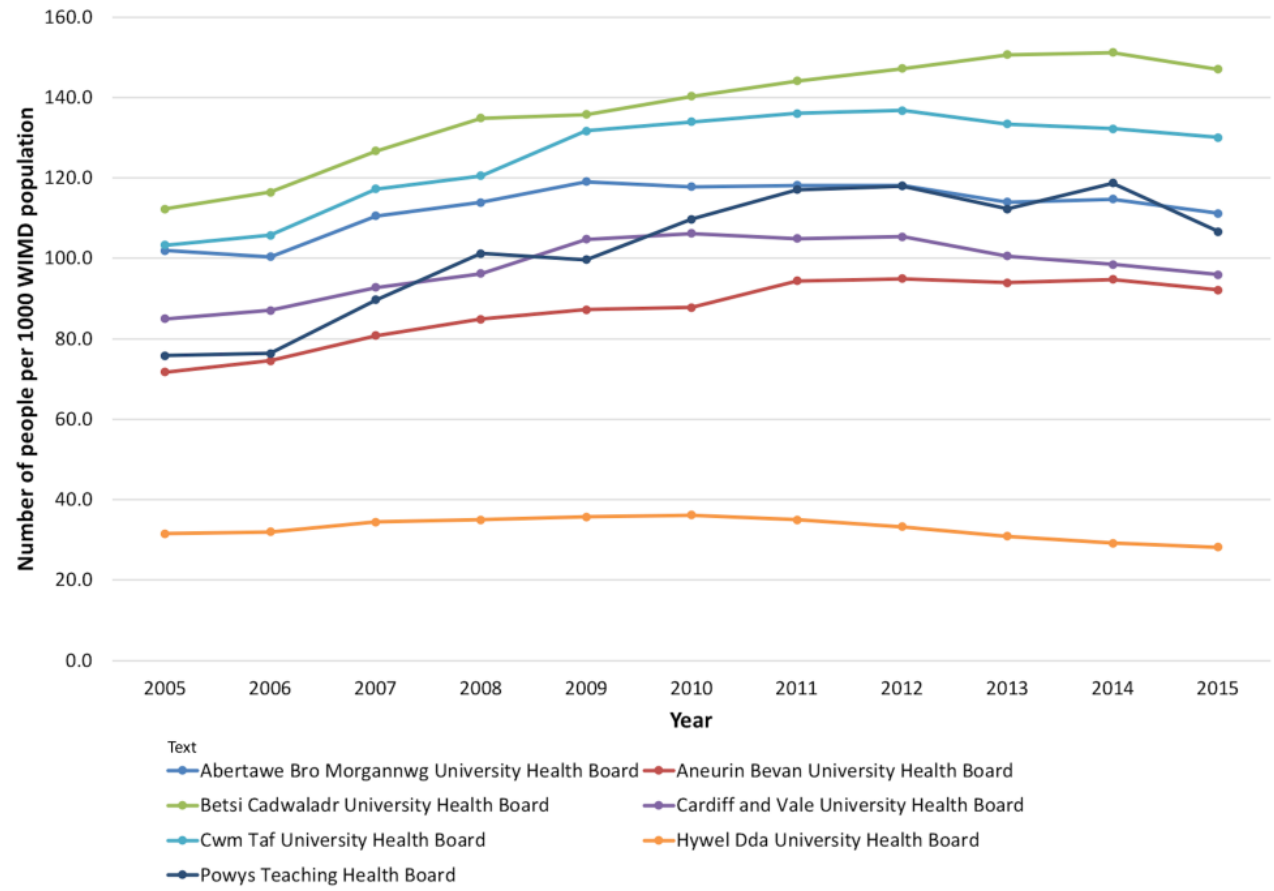

Figure 1.9: Trend in the number of people receiving prescriptions for opioid medicines in the least deprived areas (as per Welsh Index of Multiple Deprivation (WIMD)) in each Health Board in Wales. Data adjusted to population of WIMD5 areas in each Health Board

Comparison of the numbers of people receiving prescriptions in WIMD5 areas in each Health Board demonstrated significant difference $(p<.001)$. Post-hoc analysis revealed these differences predominated between HDUHB and 5 other Health Boards particularly (Table I.15).

Table I.12: Dunn's pairwise comparison and Bonferroni post-hoc analysis of difference between the number of people receiving opioid prescriptions being issued in WIMD4 areas by Welsh Health Board between 2005 and 2015

\begin{tabular}{rcccccc}
\hline & \multicolumn{5}{c}{ Health Board** } \\
\cline { 2 - 7 } & ABMUHB & ABUHB & BCUHB & CVUHB & CTUHB & HDUHB \\
\hline ABUHB & $.005^{*}$ & & & & & \\
\hline BCUHB & $<.001^{*}$ & $>.999$ & & & & \\
\hline CVUHB & $.049^{*}$ & $>.999$ & .321 & & & \\
\hline CTUHB & $>.999$ & .443 & $.001^{*}$ & $>.999$ & & \\
\hline HDUHB & $.001^{*}$ & $>.999$ & $>.999$ & $>.999$ & .158 & \\
\hline PTHB & $<.001^{*}$ & .082 & $>.999$ & $.009^{*}$ & $<.001^{*}$ & .246 \\
\hline
\end{tabular}

${ }^{*} \mathrm{p}<0.05=$ statistically significant

**ABMUHB = Abertawe Bro Morgannwg University Health Board; $A B U H B=$ Aneurin Bevan University Health Board; $\mathrm{BCUHB}=$ Betsi Cadwaladr University Health Board; $\mathrm{CVUHB}=$ Cardiff and Vale University Health Board; CTUHB - Cwm Taf University Health Board; HDUHB = Hywel Dda University Health Board; PTHB = Powys Teaching Health Board 


\section{I.3.1 Prescriptions by deprivation area}

The trends in the number of prescriptions issued within each designated area of deprivation were similar to those of the numbers of people receiving them, across Wales.

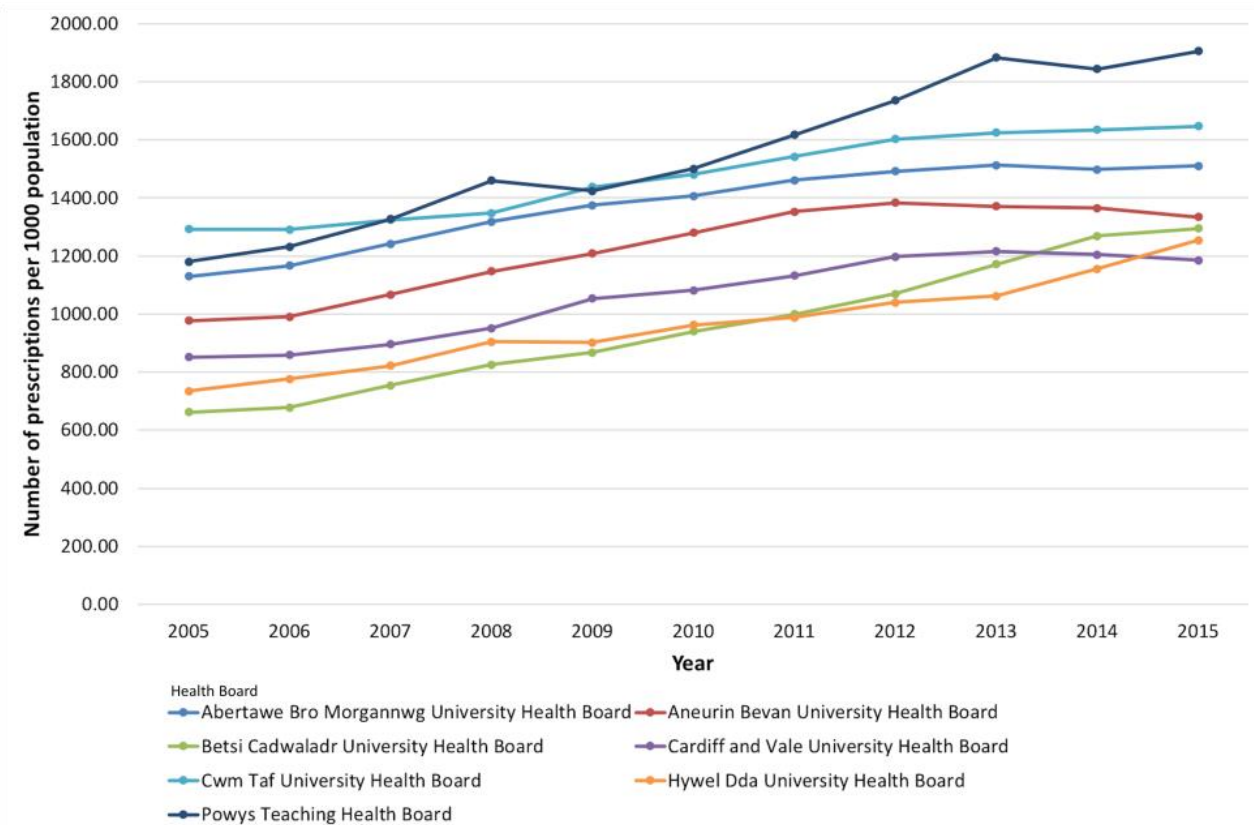

Figure 1.10: Trend in the number of opioid prescriptions in the most deprived areas (as per Welsh Index of Multiple Deprivation (WIMD)) in each Health Board in Wales. Data adjusted to population of WIMD1 areas in each Health Board

Powys Teaching Health Board had the highest number of prescriptions per 1000 population in WIMD1 areas, over the 11 years examined and experienced a $61.4 \%$ (from 1180.7 to 1905.7 prescriptions per 1000 population) increase in that time (Table I.15). The largest increase in the annual number of prescriptions issued in WIMD1 areas was in BCUHB (95.5\%, from 662.0 to 1294.2 prescriptions per 1000 population).

Statistical differences were examined by Kruskal-Wallis and were significant $(p<.001)$. Dunn's pairwise comparisons demonstrated significant differences between 8 of the 21 pairs of comparators (Table I.13).

Table I.13: Dunn's pairwise comparison and Bonferroni post-hoc analysis of difference between the number of opioid prescriptions being issued in WIMD1 areas by Welsh Health Board between 2005 and 2015

\begin{tabular}{rcccccc} 
& \multicolumn{5}{c}{ Health Board** } \\
\cline { 2 - 7 } & ABMUHB & ABUHB & BCUHB & CVUHB & CTUHB & HDUHB \\
\hline ABUHB & $>.999$ & & & & \\
\hline BCUHB & $.007^{*}$ & .582 & & & & \\
\hline CVUHB & .055 & $>.999$ & $>.999$ & & & \\
\hline CTUHB & $>.999$ & .626 & $<.001^{*}$ & $.003^{*}$ & & \\
\hline HDUHB & $.004^{*}$ & .400 & $>.999$ & $>.999$ & $<.001^{*}$ & \\
\hline PTHB & $>.999$ & .443 & $<.001^{*}$ & $.002^{*}$ & $>.999$ & $<.001^{*}$ \\
\hline
\end{tabular}

${ }^{*} \mathrm{p}<0.05$ = statistically significant

**ABMUHB = Abertawe Bro Morgannwg University Health Board; ABUHB = Aneurin Bevan University Health Board; $\mathrm{BCUHB}=$ Betsi Cadwaladr University Health Board; $\mathrm{CVUHB}=$ Cardiff and Vale University Health 
Board; CTUHB - Cwm Taf University Health Board; HDUHB = Hywel Dda University Health Board; PTHB = Powys Teaching Health Board

In WIMD2 areas, there were much greater number of prescriptions issued in PTHB (median=1616.9) compared to the other Health Boards in Wales (Figure I.11). Over the study period, there was a $61.4 \%$ (from 1180.7 to 1905.7 prescriptions per 1000 population) increase.

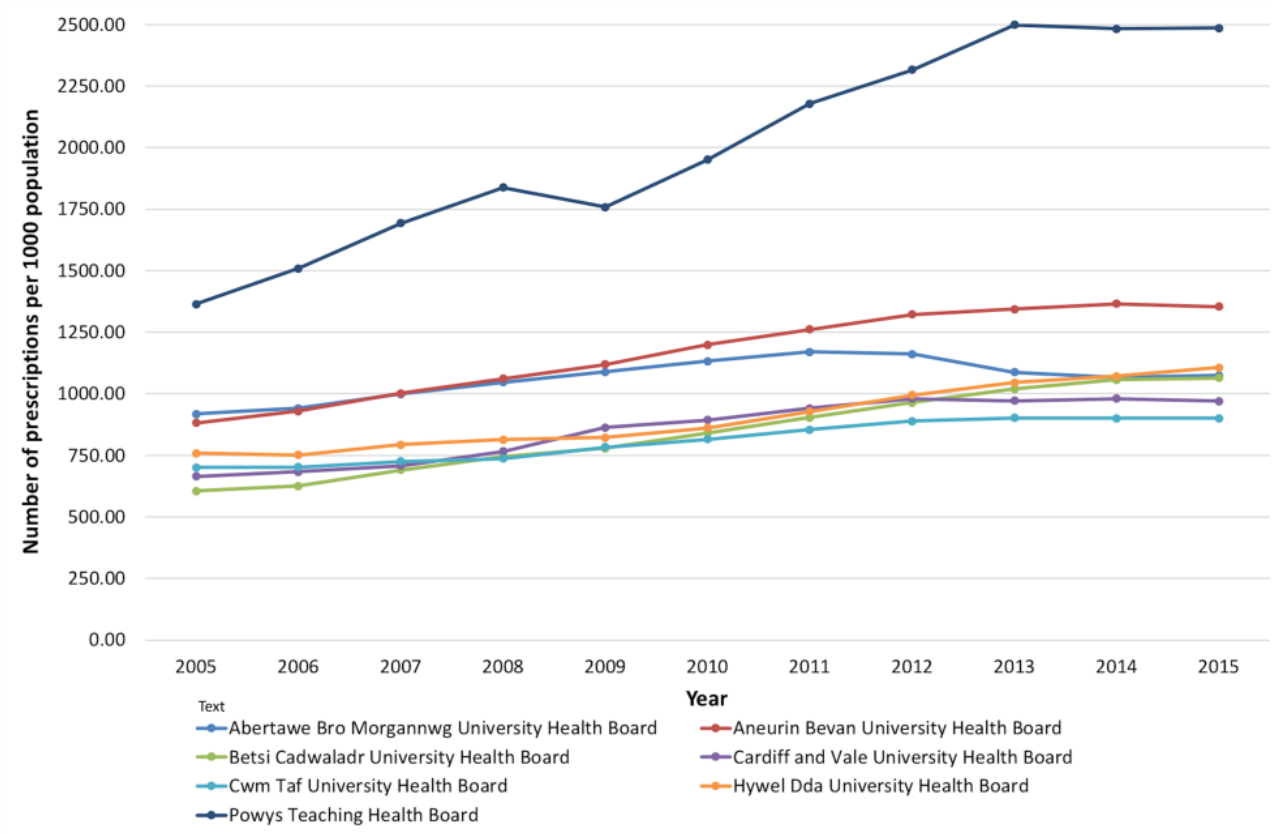

Figure 1.11: Trend in the number of prescriptions for opioid medicines in the 2nd deprived areas (as per Welsh Index of Multiple Deprivation (WIMD)) in each Health Board in Wales. Data adjusted to population of WIMD2 areas in each Health Board

When compared to WIMD2 areas in other Health Boards by Kruskal-Wallis there were significant differences ( $p<.001)$, which following Dunn's pairwise comparison were demonstrated between 7 of the 21 pairs of comparators (Table I.14).

Table I.14: Dunn's pairwise comparison and Bonferroni post-hoc analysis of difference between the number of opioid prescriptions being issued in WIMD2 areas by Welsh Health Board between 2005 and 2015

\begin{tabular}{rcccccc}
\hline & \multicolumn{5}{c}{ Health Board** } \\
\cline { 2 - 5 } & ABMUHB & ABUHB & BCUHB & CVUHB & CTUHB & HDUHB \\
\hline ABUHB & $>.999$ & & & & \\
\hline BCUHB & .156 & $.041^{*}$ & & & & \\
\hline CVUHB & .195 & .053 & $>.999$ & & & \\
\hline CTUHB & $.021^{*}$ & $.004^{*}$ & $>.999$ & $>.999$ & & \\
\hline HDUHB & .850 & .285 & $>.999$ & $>.999$ & $>.999$ & \\
\hline PTHB & .421 & $>.999$ & $<.001^{*}$ & $<.001^{*}$ & $<.001^{*}$ & $<.001^{*}$ \\
\hline
\end{tabular}

$* \mathrm{p}<0.05=$ statistically significant

**ABMUHB = Abertawe Bro Morgannwg University Health Board; $A B U H B=$ Aneurin Bevan University Health Board; $B C U H B=$ Betsi Cadwaladr University Health Board; $C V U H B=$ Cardiff and Vale University Health Board; CTUHB - Cwm Taf University Health Board; HDUHB = Hywel Dda University Health Board; PTHB = Powys Teaching Health Board 
Table I.15: Trends in the annual number of opioid prescriptions in each Health Board in Wales by Welsh Index of Multiple Deprivation (WIMD) area. WIMD1=most deprived and WIMD5=least deprived area. Rate change over the 11-year study period included. Data population adjusted per Health Board (number of prescriptions per 1000 population)

\begin{tabular}{|c|c|c|c|c|c|c|c|}
\hline \multirow[b]{2}{*}{ Number of prescriptions per 1000} & \multicolumn{7}{|c|}{ Health Board } \\
\hline & ABMUHB & $\mathrm{ABUHB}$ & BCUHB & CVUHB & CTUHB & HDUHB & PTHB \\
\hline \multicolumn{8}{|l|}{ All opioids } \\
\hline \multicolumn{8}{|l|}{ WIMD1 } \\
\hline 2005 & 1129.4 & 977.0 & 662.0 & 851.2 & 1292.4 & 734.3 & 1180.7 \\
\hline 2015 & 1510.3 & 1333.6 & 1294.2 & 1184.6 & 1646.1 & 1253.6 & 1905.7 \\
\hline Median & 1406.2 & 1280.0 & 939.8 & 1082.2 & 1481.0 & 962.7 & 1616.9 \\
\hline Rate change 2005-2015 (\%) & 36.5 & 36.5 & 95.5 & 39.2 & 27.4 & 70.7 & 61.4 \\
\hline \multicolumn{8}{|l|}{ WIMD2 } \\
\hline 2015 & 1073.9 & 1354.3 & 1064.3 & 970.7 & 901.2 & 1106.6 & 2486.0 \\
\hline Median & 1073.9 & 1199.0 & 840.8 & 893.7 & 814.9 & 861.1 & 2179.3 \\
\hline Rate change 2005-2015 (\%) & 53.5 & 53.5 & 75.9 & 46.0 & 28.5 & 45.7 & 82.2 \\
\hline \multicolumn{8}{|l|}{ WIMD3 } \\
\hline 2005 & 862.5 & 702.1 & 604.6 & 538.3 & 1014.1 & 655.7 & 774.6 \\
\hline 2015 & 1135.8 & 1004.1 & 1037.2 & 802.9 & 1244.7 & 887.0 & 1010.8 \\
\hline Median & 1039.1 & 905.4 & 840.0 & 715.1 & 1158.9 & 783.6 & 985.3 \\
\hline Rate change 2005-2015 (\%) & 31.7 & 43.0 & 71.5 & 49.2 & 22.7 & 35.3 & 30.5 \\
\hline 2005 & 770.5 & 466.6 & 362.1 & 462.9 & 628.0 & 560.9 & 300.3 \\
\hline 2015 & 1035.5 & 731.5 & 642.1 & 679.6 & 830.2 & 735.3 & 488.7 \\
\hline Median & 967.7 & 645.3 & 521.6 & 649.0 & 769.7 & 678.2 & 488.7 \\
\hline Rate change 2005-2015 (\%) & 34.4 & 56.8 & 77.3 & 46.8 & 32.2 & 31.1 & 62.8 \\
\hline \multicolumn{8}{|l|}{ WIMD5 } \\
\hline 2005 & 470.6 & 343.7 & 494.9 & 373.4 & 486.5 & 166.8 & 309.4 \\
\hline 2015 & 630.9 & 529.0 & 867.7 & 501.4 & 750.6 & 203.7 & 565.3 \\
\hline Median & 581.3 & 452.8 & 698.2 & 492.8 & 698.4 & 180.2 & 558.8 \\
\hline Rate change 2005-2015 (\%) & 34.1 & 53.9 & 75.3 & 34.3 & 54.3 & 22.1 & 82.7 \\
\hline
\end{tabular}

*ABMUHB = Abertawe Bro Morgannwg University Health Board; ABUHB $=$ Aneurin Bevan University Health Board; BCUHB $=$ Betsi Cadwaladr University Health Board; CVUHB $=$ Cardiff and Vale

University Health Board; CTUHB = Cwm Taf University Health Board; HDUHB = Hywel Dda University Health Board; PTHB = Powys Teaching Health Board 
A different trend in prescribing was seen in WIMD3 areas (Table I.15) with the largest increase in the number of prescriptions issued occurring in BCUHB (75.9\%, from 604.6 to 1037.2 prescriptions per 1000 population).

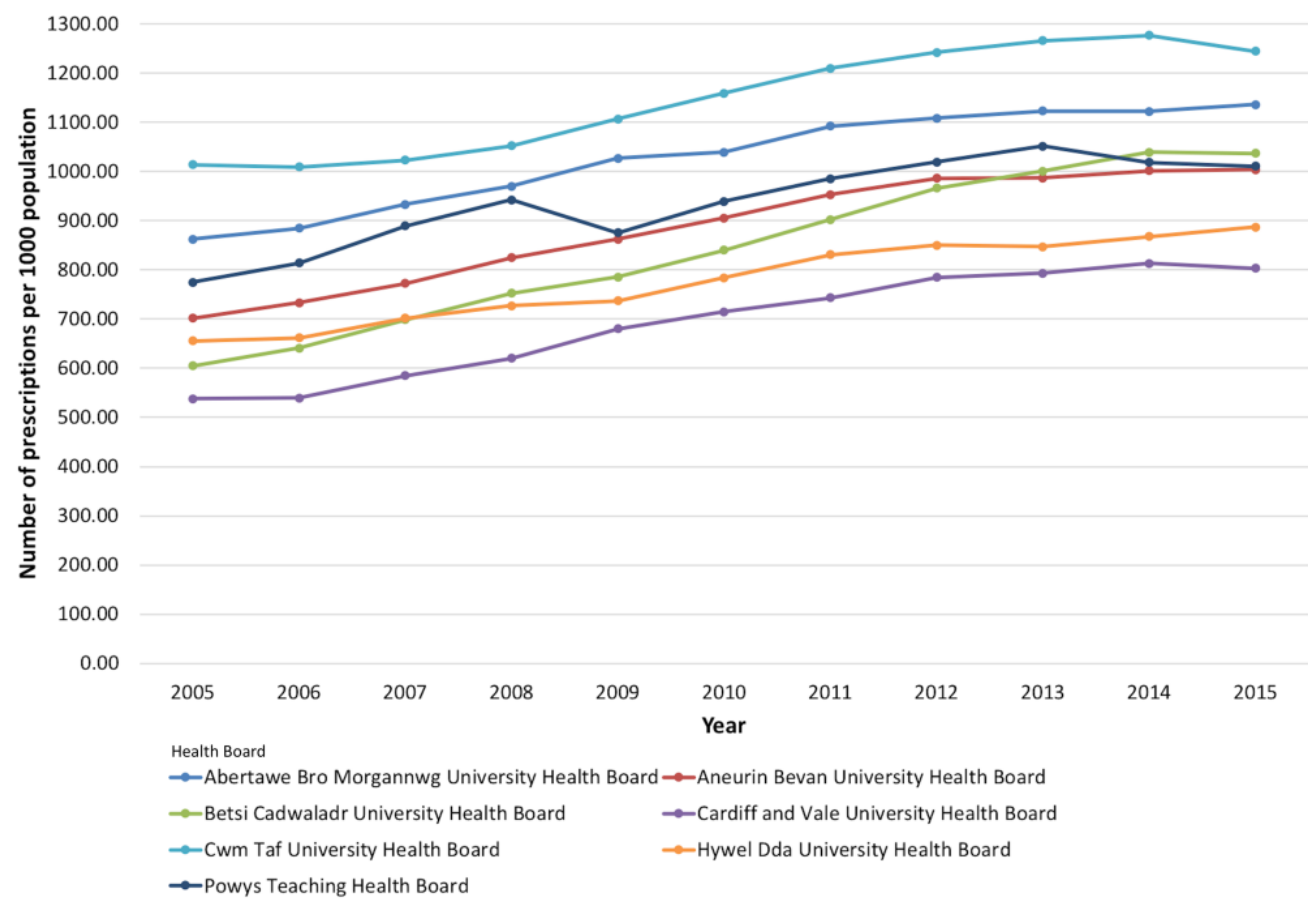

Figure 1.12: Trend in the number of prescriptions for opioid medicines in the 3rd most deprived areas (as per Welsh Index of Multiple Deprivation (WIMD)) in each Health Board in Wales. Data adjusted to population of WIMD3 areas in each Health Board

The largest number of prescriptions issued in WIMD3 areas was in CTUHB (median=1158.9) (Figure I.12) and that was also the Health Board which had the smallest increase $(22.7 \%$, from 1014.1 to 1244.7 prescriptions per 1000 population) over the 11 years examined. Cardiff and Vale University Health Board (median=715.1 prescriptions per 1000 population) had the lowest number of prescriptions issued in WIMD3 areas of any Health Board in Wales.

Table I.16: Dunn's pairwise comparison and Bonferroni post-hoc analysis of difference between the number of opioid prescriptions being issued in WIMD3 areas by Welsh Health Board between 2005 and 2015

\begin{tabular}{rcccccc}
\hline & \multicolumn{5}{c}{ Health Board** } \\
\cline { 2 - 5 } & ABMUHB & ABUHB & BCUHB & CVUHB & CTUHB & HDUHB \\
\hline ABUHB & $>.999$ & & & & \\
\hline BCUHB & .156 & $.041^{*}$ & & & & \\
\hline CVUHB & .195 & .361 & $>.999$ & & & \\
\hline CTUHB & $.021^{*}$ & $.004^{*}$ & $>.999$ & $>.999$ & & \\
\hline HDUHB & .850 & .285 & $>.999$ & $>.999$ & $>.999$ & \\
\hline PTHB & .421 & $>.999$ & $<.001^{*}$ & $.022^{*}$ & $<.001^{*}$ & $<.001^{*}$ \\
\hline
\end{tabular}

${ }^{*} \mathrm{p}<0.05=$ statistically significant

**ABMUHB = Abertawe Bro Morgannwg University Health Board; ABUHB = Aneurin Bevan University Health Board; $\mathrm{BCUHB}=$ Betsi Cadwaladr University Health Board; $\mathrm{CVUHB}=$ Cardiff and Vale University Health Board; CTUHB - Cwm Taf University Health Board; HDUHB = Hywel Dda University Health Board; PTHB = Powys Teaching Health Board

Kruskal-Wallis test revealed a significant difference $(p<.001)$ in the number of prescriptions issued in WIMD3 areas in the 7 Health Board. Dunn's pairwise 
analysis highlighted 7 pairs of comparators where significant difference existed in the number of prescriptions (Table I.16).

Abertawe Bro Morgannwg University Health Board had the highest number of prescriptions issued in WIMD4 areas over the study and a $34.4 \%$ (from 770.5 to 1035.5 prescriptions per 1000 population) increase between 2005 and 2015 (Figure I.13).

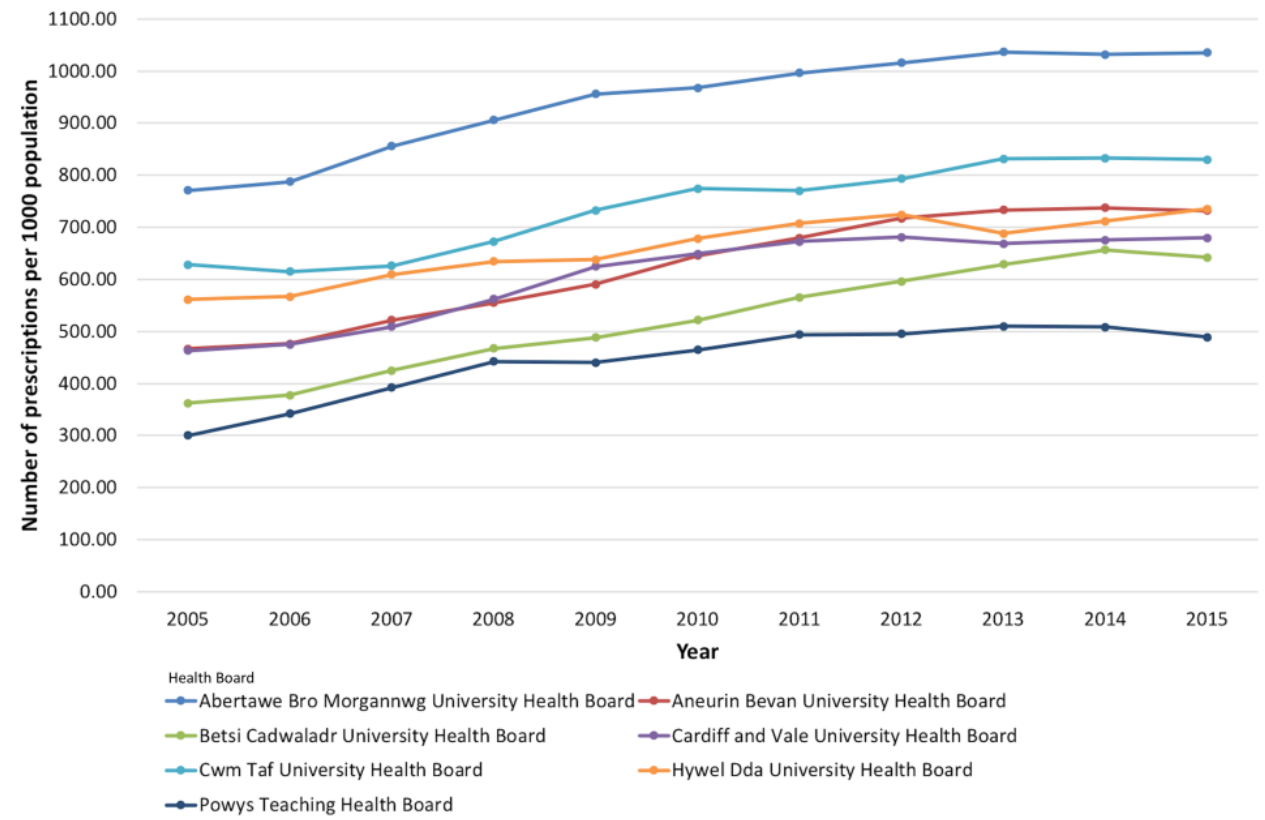

Figure I.13: Trend in the number of prescriptions for opioid medicines in the less deprived areas (WIMD4 as per Welsh Index of Multiple Deprivation (WIMD)) in each Health Board in Wales. Data adjusted to population of WIMD4 areas in each Health Board

The largest increase in annual prescription numbers was seen in BCUHB (77.3\% increase, from 362.1 to 642.1 prescriptions per 1000 population) although that Health Board had around 49\% fewer prescriptions issued in WIMD4 areas than ABMUHB.

As with previous analyses, there was an overall significance in the differences seen in the number of prescriptions issued in WIMD4 areas of each Health Board $(p<.001)$ when determined by Kruskal-Wallis test. Post-hoc analysis confirmed significant differences existed between 7 of 21 pairs of comparators (Table I.17). The remaining pairs were not considered to have significant differences between them.

Table I.17: Dunn's pairwise comparison and Bonferroni post-hoc analysis of difference between the number of opioid prescriptions being issued in WIMD4 areas by Welsh Health Board between 2005 and 2015

\begin{tabular}{ccccccc}
\hline & \multicolumn{5}{c}{ Health Board** } \\
\cline { 2 - 5 } & ABMUHB & ABUHB & BCUHB & CVUHB & CTUHB & HDUHB \\
\hline ABUHB & $.011^{*}$ & & & & \\
\hline BCUHB & $<.001^{*}$ & $.041^{*}$ & & & \\
\hline CVUHB & $.002^{*}$ & $>.999$ & $>.999$ & & \\
\hline CTUHB & $>.999$ & $>.999$ & $.014^{*}$ & .757 & \\
\hline HDUHB & .072 & $>.999$ & .466 & $>.999$ & $>.999$ \\
\hline
\end{tabular}




PTHB $<.001 *$
*p $<0.05=$ statistically significant
**ABMUHB = Abertawe Bro Morgannwg University Health Board; ABUHB = Aneurin Bevan University Health
Board; BCUHB = Betsi Cadwaladr University Health Board; CVUHB = Cardiff and Vale University Health
Board; CTUHB - Cwm Taf University Health Board; HDUHB = Hywel Dda University Health Board; PTHB =
Powys Teaching Health Board

Two Health Boards, BCUHB (median=698.2 prescriptions per 1000 population) and CTUHB (median=698.4 prescriptions per 1000 population) had similar patterns of prescribing (Figure $\mathrm{X}$ ) in the least deprived areas over the study period and with very similar median values, although a larger percentage increase was seen in BCUHB (Table X).

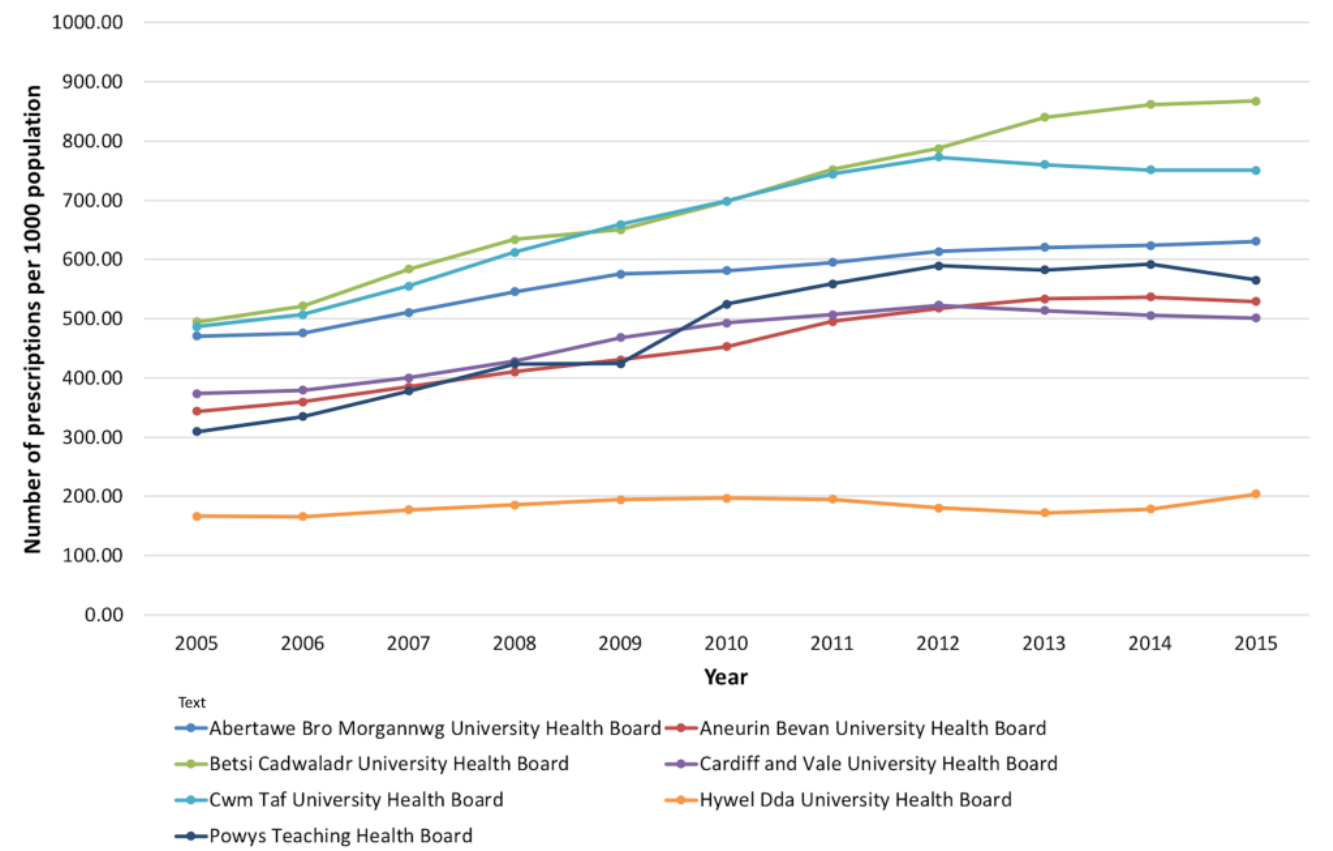

Figure 1.14: Trend in the number of prescriptions for opioid medicines in the least deprived areas (as per Welsh Index of Multiple Deprivation (WIMD)) in each Health Board in Wales. Data adjusted to population of WIMD5 areas in each Health Board

The largest percentage increase in the annual number of prescriptions was in PTHB (82.7\%, from 309.4 to 565.3 prescriptions per 1000 population) which had overall, the $4^{\text {th }}$ highest level of prescribing during the study in the least deprived areas. Hywel Dda University Health Board had a lot fewer prescriptions in the least deprived areas than other Health Boards. Post-hoc statistical testing resulted in adjusted statistical significance between 4 of the 6 other Health Boards (Table I.18).

Table I.18: Dunn's pairwise comparison and Bonferroni post-hoc analysis of difference between the number of opioid prescriptions being issued in WIMD5 areas by Welsh Health Board between 2005 and 2015

\begin{tabular}{ccccccc} 
& \multicolumn{5}{c}{ Health Board** } \\
\cline { 2 - 6 } & ABMUHB & ABUHB & BCUHB & CVUHB & CTUHB & HDUHB \\
\hline ABUHB & .830 & & & & \\
\hline BCUHB & $>.999$ & $.011^{*}$ & & & \\
\hline CVUHB & .739 & $>.999$ & $.009^{*}$ & & \\
\hline CTUHB & $>.999$ & $.037^{*}$ & $>.999$ & $.032^{*}$ & \\
\hline HDUHB & $<.001^{*}$ & .263 & $<.001^{*}$ & .301 & $<.001^{*}$ \\
\hline
\end{tabular}




\begin{tabular}{lllllll}
\hline PTHB & $>.999$ & $>.999$ & .089 & $>.999$ & .256 & $.038^{*}$ \\
\hline
\end{tabular}

${ }^{*} \mathrm{p}<0.05=$ statistically significant

**ABMUHB = Abertawe Bro Morgannwg University Health Board; $A B U H B=$ Aneurin Bevan University Health Board; $\mathrm{BCUHB}=$ Betsi Cadwaladr University Health Board; $\mathrm{CVUHB}=$ Cardiff and Vale University Health Board; CTUHB - Cwm Taf University Health Board; HDUHB = Hywel Dda University Health Board; PTHB = Powys Teaching Health Board

Kruskal-Wallis test demonstrated the differences between the number of opioid prescriptions issued in WIMD5 areas across the 7 Health Boards were significant $(p<.001)$. Further post-hoc analysis confirmed statistical differences between 8 of the 21 pairs of comparators (Table I.18).

\section{I.3.2 Weak opioid prescribing by deprivation}

There were similar trends in the number of weak opioid prescriptions that were issued across Wales, depending on the level of deprivation of the area in which the prescription was issued (Table I.19). In 2005, there were $77 \%$ more prescriptions issued in the most deprived areas of the Health Board with the highest level of prescribing (CTUHB, 1223.6 prescriptions per 1000 population) compared to the one with the least (BCUHB, 620.1 prescriptions per 1000 population). By 2015, the difference had reduced to 52\% (PTHB, 1468.5 prescriptions per 1000 population versus CVUHB, 952.3 prescriptions per 1000 population).

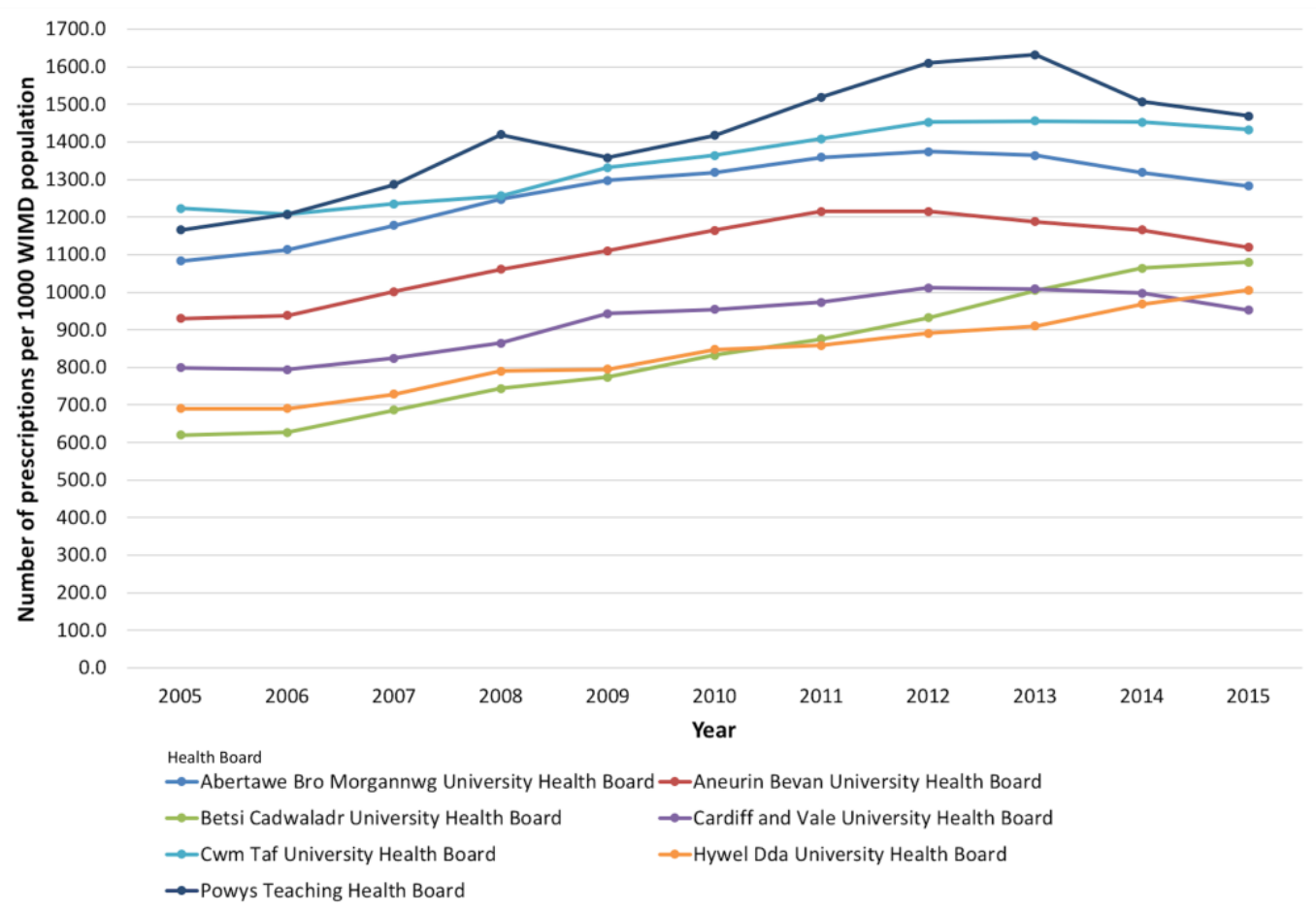

Figure 1.15: Trend in the number of people receiving prescriptions for opioid medicines in the most deprived areas (as per Welsh Index of Multiple Deprivation (WIMD)) in each Health Board in Wales. Data adjusted to population of WIMD1 areas in each Health Board 


\begin{tabular}{|c|c|c|c|c|c|c|c|}
\hline \multirow[t]{2}{*}{ Number of prescriptions per 1000} & \multicolumn{7}{|c|}{ Health Board } \\
\hline & ABMUHB & $A B \cup H B$ & BCUHB & CVUHB & CTUHB & HDUHB & PTHB \\
\hline \multicolumn{8}{|l|}{ All opioids } \\
\hline \multicolumn{8}{|l|}{ WIMD1 } \\
\hline 2005 & 1082.9 & 930.4 & 620.1 & 798.7 & 1223.6 & 690.5 & 1165.3 \\
\hline 2015 & 1282.6 & 1119.7 & 1079.9 & 952.3 & 1432.3 & 1005.9 & 1468.5 \\
\hline Median & 1297.5 & 1119.7 & 831.9 & 952.3 & 1363.7 & 847.8 & 1419.7 \\
\hline Rate change 2005-2015 (\%) & 18.4 & 20.4 & 74.2 & 19.3 & 17.1 & 45.7 & 26.0 \\
\hline \multicolumn{8}{|l|}{ WIMD2 } \\
\hline 2005 & 877.2 & 842.8 & 555.5 & 630.4 & 666.3 & 688.6 & 1325.9 \\
\hline 2015 & 906.3 & 1135.5 & 896.2 & 791.2 & 801.8 & 882.4 & 2136.1 \\
\hline Median & 984.2 & 1093.9 & 737.2 & 791.2 & 751.2 & 761.4 & 1826.4 \\
\hline Rate change 2005-2015 (\%) & 3.3 & 34.7 & 61.3 & 25.5 & 20.3 & 28.1 & 61.1 \\
\hline \multicolumn{8}{|l|}{ WIMD3 } \\
\hline 2005 & 832.9 & 666.1 & 554.7 & 509.6 & 965.8 & 600.6 & 721.1 \\
\hline 2015 & 964.7 & 866.4 & 853.7 & 651.8 & 1096.3 & 692.0 & 841.3 \\
\hline Median & 975.6 & 836.4 & 631.2 & 631.2 & 1072.8 & 677.3 & 856.4 \\
\hline Rate change 2005-2015 (\%) & 15.8 & 30.1 & 53.9 & 27.9 & 13.5 & 15.2 & 16.7 \\
\hline \multicolumn{8}{|l|}{ WIMD4 } \\
\hline 2005 & 735.8 & 441.0 & 334.1 & 431.8 & 590.7 & 516.9 & 280.7 \\
\hline 2015 & 892.7 & 614.7 & 533.1 & 557.3 & 734.6 & 583.8 & 401.5 \\
\hline Median & 895.1 & 578.1 & 450.4 & 556.1 & 708.9 & 569.6 & 401.5 \\
\hline Rate change 2005-2015 (\%) & 21.3 & 39.4 & 59.6 & 29.0 & 24.4 & 12.9 & 43.0 \\
\hline \multicolumn{8}{|l|}{ WIMD5 } \\
\hline 2005 & 450.5 & 327.0 & 461.4 & 349.2 & 459.2 & 155.7 & 292.8 \\
\hline 2015 & 545.8 & 458.8 & 716.2 & 419.7 & 672.1 & 165.9 & 481.1 \\
\hline Median & 545.8 & 415.1 & 609.3 & 421.5 & 641.5 & 158.7 & 481.1 \\
\hline Rate change 2005-2015 (\%) & 21.2 & 40.3 & 55.2 & 20.2 & 46.4 & 6.5 & 64.3 \\
\hline
\end{tabular}

*ABMUHB = Abertawe Bro Morgannwg University Health Board; ABUHB = Aneurin Bevan University Health Board; BCUHB = Betsi Cadwaladr University Health Board; CVUHB = Cardiff and Vale University Health Board; CTUHB $=$ Cwm Taf University Health Board; HDUHB $=$ Hywel Dda University Health Board; PTHB = Powys Teaching Health Board WIMD1=most deprived and WIMD5=least deprived area. Rate change over the 11-year study period included. Data population adjusted per Health Board (number of prescriptions per 1000 population) 
The greatest percentage increase in the annual number of weak opioid prescriptions issued within WIMD1 areas was in BCUHB (74.2\%, from 620.1 to 1079.9 prescriptions per 1000 population) and the lowest was seen in CTUHB (17.1\%, from 1223.6 to 1432.3 prescriptions per 1000 population). Powys Teaching Health Board saw an increase in the number of prescriptions of $26 \%$ (from 1165.3 to 1468.5 prescriptions per 1000 population) but had the largest overall numbers of prescriptions issued during the study period.

Statistical analysis demonstrated a significant difference in the number of weak opioid prescriptions issued in the WIMD1 areas of each Health Board $(p<.001)$. Dunn's pairwise comparisons confirmed statistically significant differences between 9 of 21 pairs of comparators, the remaining 11 not being considered as significantly different (Table I.20).

Table 1.20: Dunn's pairwise comparison post-hoc analysis of difference between the number of weak opioid prescriptions being issued in WIMD1 areas by Welsh Health Board between 2005 and 2015

\begin{tabular}{rcccccc}
\hline & \multicolumn{5}{c}{ Health Board** } \\
\cline { 2 - 7 } & ABMUHB & ABUHB & BCUHB & CVUHB & CTUHB & HDUHB \\
\hline ABUHB & $>.999$ & & & & \\
\hline BCUHB & $.001^{*}$ & .454 & & & & \\
\hline CVUHB & $021^{*}$ & $>.999$ & $>.999$ & & & \\
\hline CTUHB & $>.999$ & .309 & $<.001^{*}$ & $.001^{*}$ & & \\
\hline HDUHB & $.001^{*}$ & .309 & $>.999$ & $>.999$ & $<.001^{*}$ & \\
\hline PTHB & $>.999$ & .098 & $<.001^{*}$ & $<.001^{*}$ & $>.999$ & $<.001^{*}$ \\
\hline
\end{tabular}

${ }^{*} \mathrm{p}<0.05=$ statistically significant

**ABMUHB = Abertawe Bro Morgannwg University Health Board; $A B U H B=$ Aneurin Bevan University Health Board; $\mathrm{BCUHB}=$ Betsi Cadwaladr University Health Board; $\mathrm{CVUHB}=$ Cardiff and Vale University Health Board; CTUHB - Cwm Taf University Health Board; HDUHB = Hywel Dda University Health Board; PTHB = Powys Teaching Health Board

In the second most deprived areas of Wales, PTHB had 51\% more weak opioid prescriptions per 1000 population than the next highest prescribing Health Board in 2005 (Table I.19). The difference increased to 88\% difference in 2015, although the trends in each Health Board were either slowing or declining from around 2012 onwards (Figure I.16). There was a $61.1 \%$ increase in the annual number of prescriptions in PTHB over the study period. The smallest percentage increase in WIMD2 weak opioid prescription numbers was in ABMUHB (3.3\%, from 877.2 to 906.3 prescriptions per 1000 population). 


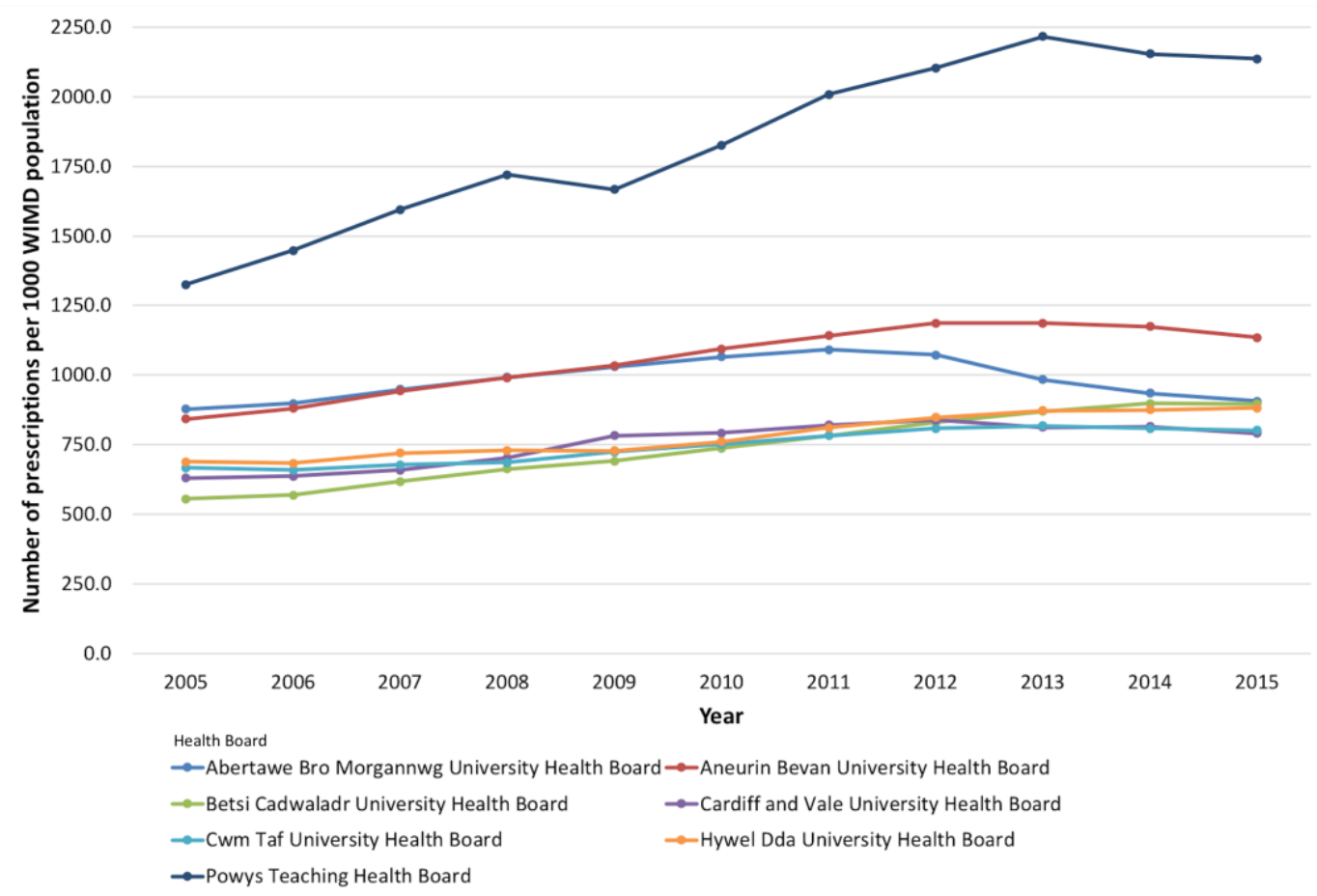

Figure l.16: Trend in the number of people receiving prescriptions for weak opioid medicines in the 2nd most deprived areas (as per Welsh Index of Multiple Deprivation (WIMD)) in each Health Board in Wales Data adjusted to population of WIMD2 areas in each Health Board

A Kruskal-Wallis test determined a statistically significant difference between the number of prescriptions issued in the WIMD2 areas of each Health Board across Wales. Post-hoc analysis confirmed significant differences between 11 of 21 pairs of comparators (Table I.21).

Table I.21: Dunn's pairwise comparison post-hoc analysis of difference between the number of weak opioid prescriptions being issued in WIMD2 areas by Welsh Health Board between 2005 and 2015

\begin{tabular}{rcccccc}
\hline \multicolumn{7}{c}{ Health Board** } \\
\hline ABUHB & $>.999$ & ABUHB & BCUHB & CVUHB & CTUHB & HDUHB \\
\hline BCUHB & $.025^{*}$ & $.006^{*}$ & & & & \\
\hline CVUHB & $.028^{*}$ & $.006^{*}$ & $>.999$ & & & \\
\hline CTUHB & $.012^{*}$ & $.002^{*}$ & $<.001^{*}$ & $>.999$ & & \\
\hline HDUHB & .139 & $.037^{*}$ & $>.999$ & $>.999$ & $<.001^{*}$ & \\
\hline PTHB & .953 & $>.999$ & $<.001^{*}$ & $<.001^{*}$ & $<.001^{*}$ & $<.001^{*}$ \\
\hline
\end{tabular}

${ }^{*} \mathrm{p}<0.05=$ statistically significant

${ }^{* *} \mathrm{ABMUHB}=$ Abertawe Bro Morgannwg University Health Board; $\mathrm{ABUHB}=$ Aneurin Bevan University Health Board; $\mathrm{BCUHB}=$ Betsi Cadwaladr University Health Board; $\mathrm{CVUHB}=$ Cardiff and Vale University Health Board; CTUHB - Cwm Taf University Health Board; HDUHB = Hywel Dda University Health Board; PTHB = Powys Teaching Health Board

Trends in the number of weak opioid prescriptions in WIMD3 areas across Wales were similar in most Health Boards, although there were approximately $36 \%$ fewer prescriptions per 1000 population issued overall, compared to the most deprived areas (WIMD1) over the study period (Table I.19). 


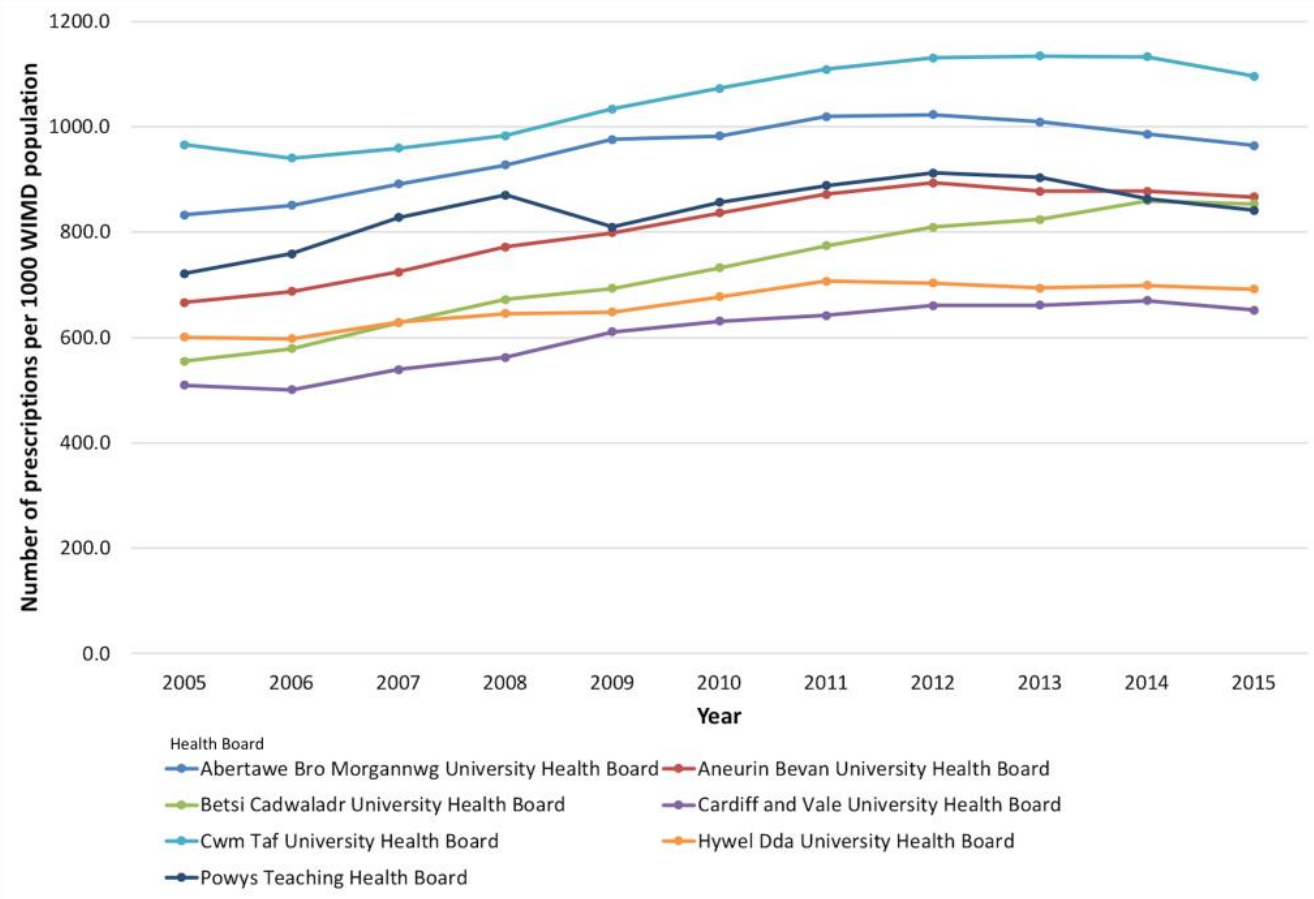

Figure 1.17: Trend in the number of people receiving prescriptions for weak opioid medicines in the 3rd most deprived areas (as per Welsh Index of Multiple Deprivation (WIMD)) in each Health Board in Wales Data adjusted to population of WIMD3 areas in each Health Board

Cwm Taf University Health Board had the highest number of weak opioid prescriptions issued in WIMD3 areas between 2005 and 2015 but also had the smallest percentage increase (13.5\%, from 965.8 to 1096.3 prescriptions per 1000 population) over that period. The largest percentage increase was seen in BCUHB (53.9\%, from 554.7 to 853.7 prescriptions per 1000 population), which was consistent with the increases seen in the other areas of deprivation (Table I.19). Cardiff and Vale University Health Board had the lowest number of weak opioid prescriptions issued over the study period in WIMD3 areas.

Statistical analysis by Kruskal-Wallis test demonstrated a significant difference $(p<.001)$ in the number of weak opioid prescriptions issued in WIMD3 areas in the 7 Health Boards across Wales. Dunn's pairwise comparison highlighted statistically significant differences between 9 of the 21 paired comparators.

Table I.22: Dunn's pairwise comparison post-hoc analysis of difference between the number of weak opioid prescriptions being issued in WIMD3 areas by Welsh Health Board between 2005 and 2015

\begin{tabular}{rcccccc}
\hline & \multicolumn{5}{c}{ Health Board** } \\
\cline { 2 - 6 } & ABMUHB & ABUHB & BCUHB & CVUHB & CTUHB & HDUHB \\
\hline ABUHB & .793 & & & & & \\
\hline BCUHB & $.011^{*}$ & $>.999$ & & & & \\
\hline CVUHB & $<.001^{*}$ & $.035^{*}$ & $>.999$ & & & \\
\hline CTUHB & $>.999$ & $.045^{*}$ & $<.001^{*}$ & $<.001^{*}$ & & \\
\hline HDUHB & $<.001^{*}$ & .528 & $>.999$ & $>.999$ & $<1^{*}$ & \\
\hline PTHB & $>.999$ & $>.999$ & $>.999$ & $.006^{*}$ & .206 & .135 \\
\hline
\end{tabular}

$* \mathrm{p}<0.05$ = statistically significant

**ABMUHB = Abertawe Bro Morgannwg University Health Board; $A B U H B=$ Aneurin Bevan University Health Board; $\mathrm{BCUHB}=$ Betsi Cadwaladr University Health Board; $\mathrm{CVUHB}=$ Cardiff and Vale University Health Board; CTUHB - Cwm Taf University Health Board; HDUHB = Hywel Dda University Health Board; PTHB = Powys Teaching Health Board 
In the areas of lesser deprivation (WIMD4) across Wales, there were more than double the numbers of weak opioid prescriptions issued in ABMUHB $(21.3 \%$ increase, from 735.8 to 892.7 prescriptions per 1000 population) (Figure I.17) which had the highest prescribing and PTHB (43.0\% increase, from 280.7 to 401.5 prescriptions per 1000 population), which had the least.

As with the analysis of previous areas of deprivation, there was a significant difference between the number of weak opioid prescriptions issued in the 7 Health Boards $(p<.001)$. Post-hoc analysis highlighted significant differences between ABMUHB and 5 of the 6 other Health Boards (Table I.23). There were significant differences between PTHB and three other Health Boards also (Table I.23).

Table I.23: Dunn's pairwise comparison post-hoc analysis of difference between the number of weak opioid prescriptions being issued in WIMD4 areas by Welsh Health Board between 2005 and 2015

\begin{tabular}{rcccccc}
\hline & \multicolumn{5}{c}{ Health Board** } \\
\cline { 2 - 6 } ABUHB & ABMUHB & ABUHB & BCUHB & CVUHB & CTUHB & HDUHB \\
\hline BCUHB & $<.999$ & & & & & \\
\hline CVUHB & $.001^{*}$ & .421 & $>.999$ & $>.999$ & & \\
\hline CTUHB & $>.999$ & $>.999$ & .092 & $>.999$ & & \\
\hline HDUHB & $.035^{*}$ & $>.999$ & .301 & $>.999$ & $>.999$ & \\
\hline PTHB & $<.001^{*}$ & $.025^{*}$ & $<.001^{*}$ & $>.999$ & $<.001^{*}$ & $.016^{*}$ \\
\hline
\end{tabular}

${ }^{*} p<0.05=$ statistically significant

${ }^{* *} \mathrm{ABMUHB}=$ Abertawe Bro Morgannwg University Health Board; $\mathrm{ABUHB}=$ Aneurin Bevan University Health Board; $B C U H B=$ Betsi Cadwaladr University Health Board; $C V U H B=$ Cardiff and Vale University Health Board; CTUHB - Cwm Taf University Health Board; HDUHB = Hywel Dda University Health Board; PTHB = Powys Teaching Health Board

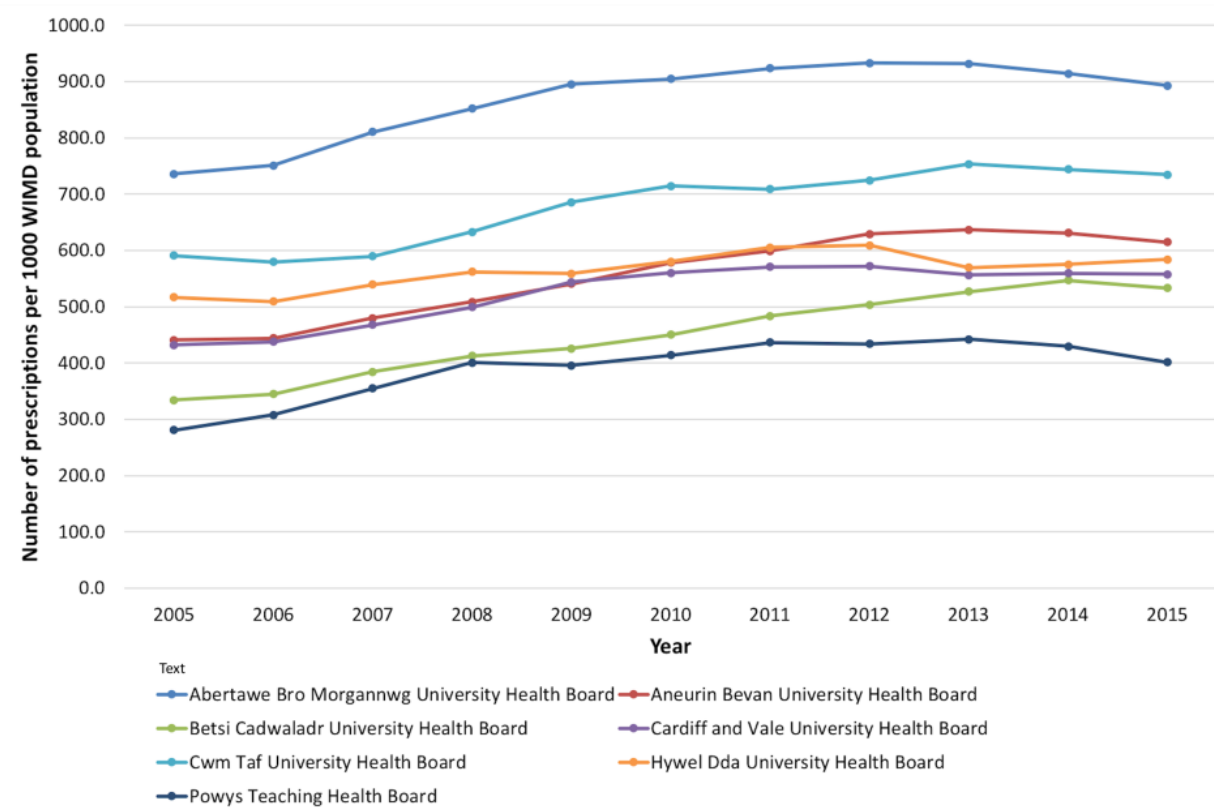

Figure 1.18: Trend in the number of people receiving prescriptions for weak opioid medicines in the lesser deprived areas (as per Welsh Index of Multiple Deprivation (WIMD)) in each Health Board in Wales Data adjusted to population of WIMD4 areas in each Health Board 
In 2005, there were nearly three times more weak opioid prescriptions issued in BCUHB (461.4 prescriptions per 1000 population), the Health Board with the highest rate of prescribing in the least deprived areas (WIMD5), compared to HDUHB (155.7 prescriptions per 1000 population) which had the least (Figure X). This rose to a 4.3 times difference in 2015 (716.2 versus 165.9 prescriptions per 1000 population respectively).

Hywel Dda University Health Board had the smallest percentage increase (6.5\%, from 155.7 to 165.9 prescriptions per 1000 population) in prescribing of weak opioids in the least deprived areas. The highest percentage increase in the number of prescriptions issued was in PTHB (64.3\%, from 292.8 to 481.1 prescriptions per 1000 population).

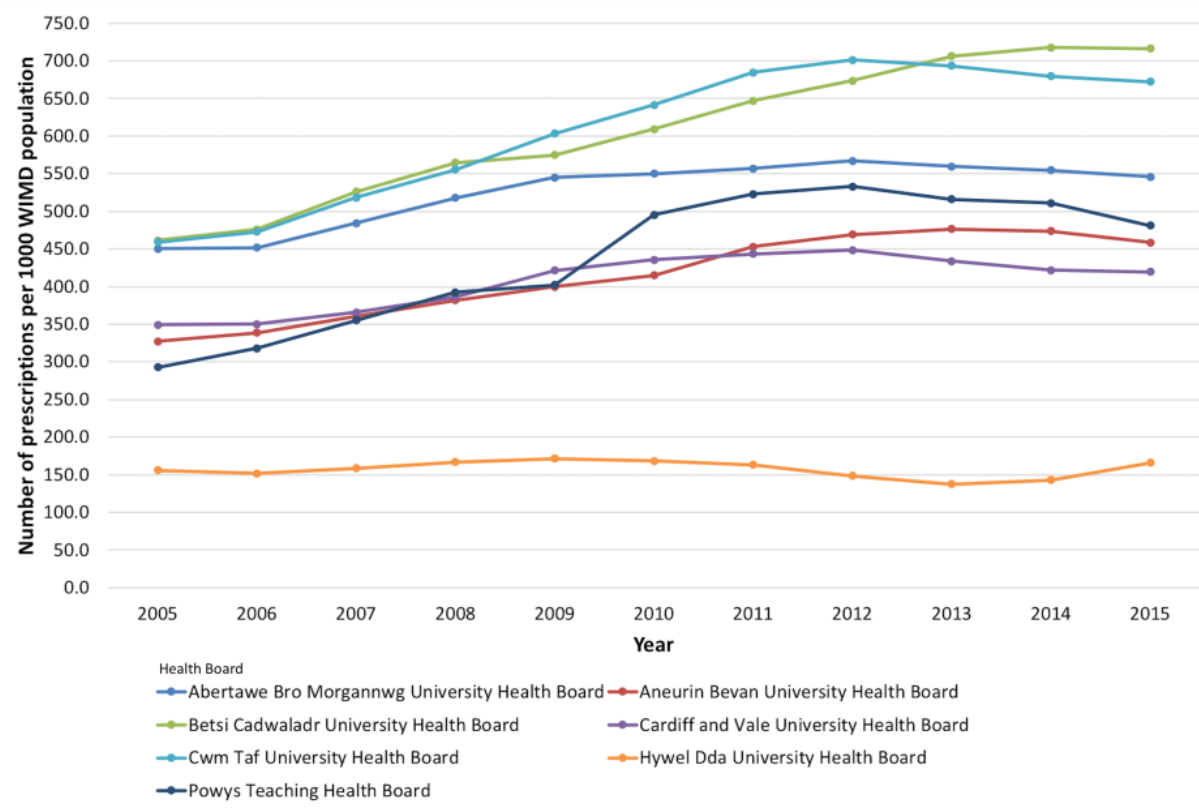

Figure I.19: Trend in the number of people receiving prescriptions for weak opioid medicines in the least deprived areas (as per Welsh Index of Multiple Deprivation (WIMD)) in each Health Board in Wales Data adjusted to population of WIMD5 areas in each Health Board

As with the other areas examined, there was a significant difference $(p<.001)$ in the number of weak opioid prescriptions issued in WIMD5 areas of the 7 Health Boards. Dunn's pairwise comparison of 21 comparators confirmed significant differences between 8 pairs of Health Board areas.

Table I.24: Dunn's pairwise comparison post-hoc analysis of difference between the number of weak opioid prescriptions being issued in WIMD5 areas by Welsh Health Board between 2005 and 2015

\begin{tabular}{ccccccc}
\hline & \multicolumn{5}{c}{ Health Board** } \\
\cline { 2 - 6 } & ABMUHB & ABUHB & BCUHB & CVUHB & CTUHB & HDUHB \\
\hline ABUHB & .301 & & & & \\
\hline BCUHB & $>.999$ & $.007^{*}$ & & & \\
\hline CVUHB & .120 & $>.999$ & $.002^{*}$ & & \\
\hline CTUHB & $>.999$ & $.012^{*}$ & $>.999$ & $.004^{*}$ & & \\
\hline HDUHB & $<.001^{*}$ & .343 & $<.001^{*}$ & .775 & $<.001^{*}$ & \\
\hline PTHB & $>.999$ & $>.999$ & .072 & $>.999$ & .107 & $.048^{*}$ \\
\hline$*_{p}^{*}<0.05=$ statistically significant & & & &
\end{tabular}


**ABMUHB = Abertawe Bro Morgannwg University Health Board; $A B U H B=$ Aneurin Bevan University Health Board; $B C U H B=$ Betsi Cadwaladr University Health Board; $C V U H B=$ Cardiff and Vale University Health Board; CTUHB - Cwm Taf University Health Board; HDUHB = Hywel Dda University Health Board; PTHB = Powys Teaching Health Board

\section{I.3.3 Strong opioid prescribing by deprivation}

There were large percentage increases in the number of strong opioid prescriptions issued in all 5 deprivation areas across Wales. In the most deprived areas (WIMD1) PTHB (2739.2\%, from 15.4 to 437.2 prescriptions per 1000 population) had the largest increase by some margin (Table I.25).

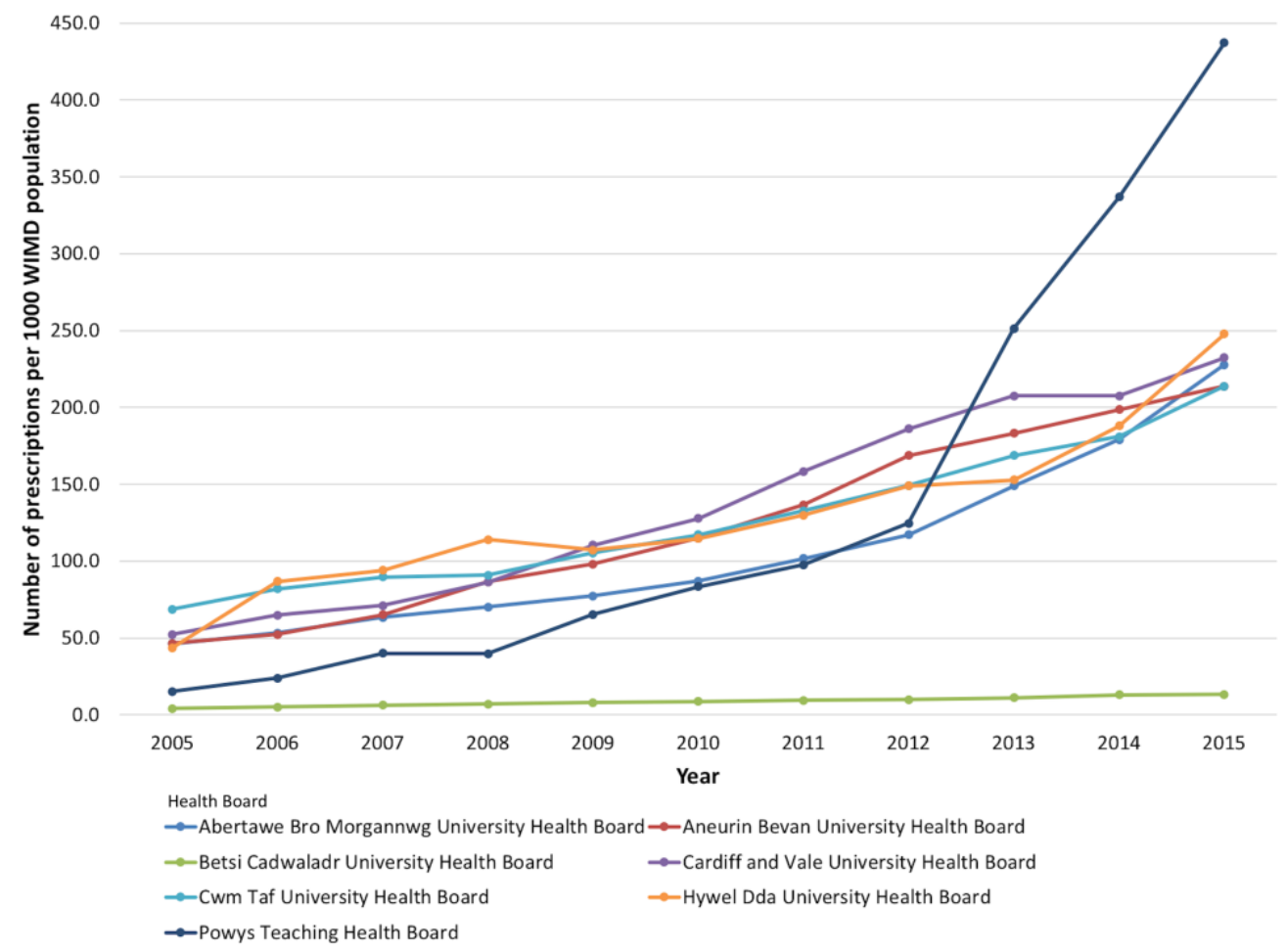

Figure 1.20: Trend in the number of people receiving prescriptions for strong opioid medicines in the most deprived areas (as per Welsh Index of Multiple Deprivation (WIMD)) in each Health Board in Wales Data adjusted to population of WIMD1 areas in each Health Board

There was a comparatively low level of strong opioid prescribing in the WIMD1 areas of BCUHB, despite a tripling of the annual number of prescriptions in the 11 years examined (212.2\% increase, from 4.3 to 13.4 prescriptions per 1000 population). In 2015, there were 32 times more strong opioid prescriptions per WIMD1 1000 population in PTHB compared to BCUHB (Figure I.20).

The remaining 5 Health Boards had similar levels of strong opioid prescribing over the study period (Table I.25). 
Table I.25: Trends in the annual number of weak opioid prescriptions in each Health Board in Wales by Welsh Index of Multiple Deprivation (WIMD) area. WIMD1=most deprived and WIMD5=least deprived area. Rate change over the 11-year study period included

\begin{tabular}{|c|c|c|c|c|c|c|c|}
\hline \multirow[t]{2}{*}{ Number of prescriptions per 1000} & \multicolumn{7}{|c|}{ Health Board } \\
\hline & ABMUHB & ABUHB & $\mathrm{BCUHB}$ & CVUHB & CTUHB & HDUHB & PTHB \\
\hline \multicolumn{8}{|l|}{ All opioids } \\
\hline \multicolumn{8}{|l|}{ WIMD1 } \\
\hline 2005 & 46.5 & 46.7 & 4.3 & 52.5 & 68.9 & 43.8 & 15.4 \\
\hline 2015 & 227.7 & 213.9 & 13.4 & 232.3 & 213.8 & 247.7 & 437.2 \\
\hline Median & 87.0 & 115.1 & 8.7 & 127.9 & 117.3 & 114.9 & 83.5 \\
\hline Rate change 2005-2015 (\%) & 390.1 & 358.3 & 212.2 & 342.5 & 210.5 & 466.2 & 2739.2 \\
\hline \multicolumn{8}{|l|}{ WIMD2 } \\
\hline 2005 & 41.4 & 39.6 & 8.3 & 34.4 & 34.8 & 70.8 & 38.6 \\
\hline 2015 & 167.6 & 218.8 & 18.6 & 179.5 & 99.4 & 224.1 & 349.9 \\
\hline Median & 66.5 & 105.1 & 13.7 & 101.2 & 117.3 & 114.9 & 83.5 \\
\hline Rate change $2005-2015(\%)$ & 304.5 & 452.9 & 123.1 & 421.7 & 185.6 & 216.5 & 806.5 \\
\hline \multicolumn{8}{|l|}{ WIMD3 } \\
\hline 2005 & 29.6 & 36.0 & 10.1 & 28.7 & 48.3 & 55.1 & 53.5 \\
\hline 2015 & 171.1 & 137.6 & 25.7 & 151.2 & 148.4 & 195.0 & 169.5 \\
\hline Median & 57.0 & 69.0 & 17.4 & 83.9 & 86.1 & 106.3 & 82.9 \\
\hline Rate change 2005-2015 (\%) & 477.0 & 282.2 & 153.7 & 426.6 & 207.4 & 253.7 & 216.9 \\
\hline \multicolumn{8}{|l|}{ WIMD4 } \\
\hline 2005 & 34.7 & 25.6 & 12.3 & 31.0 & 37.2 & 44.1 & 19.5 \\
\hline 2015 & 142.9 & 116.8 & 31.9 & 122.3 & 95.6 & 151.5 & 87.2 \\
\hline Median & 63.1 & 67.2 & 24.4 & 88.6 & 59.9 & 97.7 & 50.7 \\
\hline Rate change 2005-2015 (\%) & 311.9 & 356.2 & 159.1 & 294.0 & 156.7 & 243.8 & 346.1 \\
\hline \multicolumn{8}{|l|}{ WIMD5 } \\
\hline 2005 & 20.1 & 16.7 & 5.7 & 24.2 & 27.3 & 11.1 & 16.6 \\
\hline 2015 & 85.1 & 70.2 & 17.6 & 81.7 & 78.5 & 37.8 & 84.2 \\
\hline Median & 31.1 & 37.8 & 12.0 & 57.0 & 56.9 & 28.6 & 31.4 \\
\hline Rate change 2005-2015 (\%) & 322.7 & 321.3 & 209.8 & 237.7 & 187.5 & 241.1 & 406.4 \\
\hline
\end{tabular}

*ABMUHB = Abertawe Bro Morgannwg University Health Board; ABUHB = Aneurin Bevan University Health Board; BCUHB = Betsi Cadwaladr University Health Board; CVUHB = Cardiff and Vale University Health Board; CTUHB $=$ Cwm Taf University Health Board; HDUHB $=$ Hywel Dda University Health Board; PTHB = Powys Teaching Health Board. Data population adjusted per Health Board (number of prescriptions per 1000 population) 
A Kruskal-Wallis test demonstrated a significant difference in the number of strong opioid prescriptions issued in the WIMD1 areas of each of the Health Boards. Dunn's pairwise comparison confirmed that the only statistically significant differences existed between BCUHB and the other 6 Health Boards.

Table I.26: Dunn's pairwise comparison post-hoc analysis of difference between the number of strong opioid prescriptions being issued in WIMD1 areas by Welsh Health Board between 2005 and 2015

\begin{tabular}{rcccccc} 
& \multicolumn{5}{c}{ Health Board** } \\
\cline { 2 - 6 } & ABMUHB & ABUHB & BCUHB & CVUHB & CTUHB & HDUHB \\
\hline ABUHB & $>.999$ & & & & \\
\hline BCUHB & $.011^{*}$ & $.001^{*}$ & & & \\
\hline CVUHB & $>.999$ & $>.999$ & $<.001^{*}$ & & \\
\hline CTUHB & $>.999$ & $>.999$ & $<.001^{*}$ & $>.999$ & \\
\hline HDUHB & $>.999$ & $>.999$ & $<.001^{*}$ & $>.999$ & $>.999$ & \\
\hline PTHB & $>.999$ & $>.999$ & $.012^{*}$ & $>.999$ & $>.999$ & $>.999$ \\
\hline
\end{tabular}

${ }^{*} \mathrm{p}<0.05$ = statistically significant

**ABMUHB = Abertawe Bro Morgannwg University Health Board; $A B U H B=$ Aneurin Bevan University Health Board; $\mathrm{BCUHB}=$ Betsi Cadwaladr University Health Board; $\mathrm{CVUHB}=$ Cardiff and Vale University Health Board; CTUHB - Cwm Taf University Health Board; HDUHB = Hywel Dda University Health Board; PTHB = Powys Teaching Health Board

A similar pattern in the number of strong opioid prescriptions issued in the second most deprived areas (WIMD2) in each Health Board to the most deprived areas was noted. As with the most deprived areas (WIMD1), BCUHB had the lowest rate of prescribing and the smallest percentage increase $(123.1 \%$ increase, from 8.3 to 18.6 prescriptions per 1000 population) seen in any of the Health Boards.

There was a large increase in prescribing in PTHB with a particularly rapid rise from 2010 onwards. Overall, there was an $806.5 \%$ (from 38.6 to 349.9 prescriptions per 1000 population) increase in the annual number of strong opioid prescriptions issued in WIMD1 areas of PTHB

A statistically significant difference $(p<.001)$ was noted in the number of strong opioid prescriptions issued in the WIMD2 areas across Wales. Post-hoc analysis however, confirmed statistically significant differences existed between 6 Health Boards and BCUHB, the remaining 15 comparisons were not significant (Table I.25). 


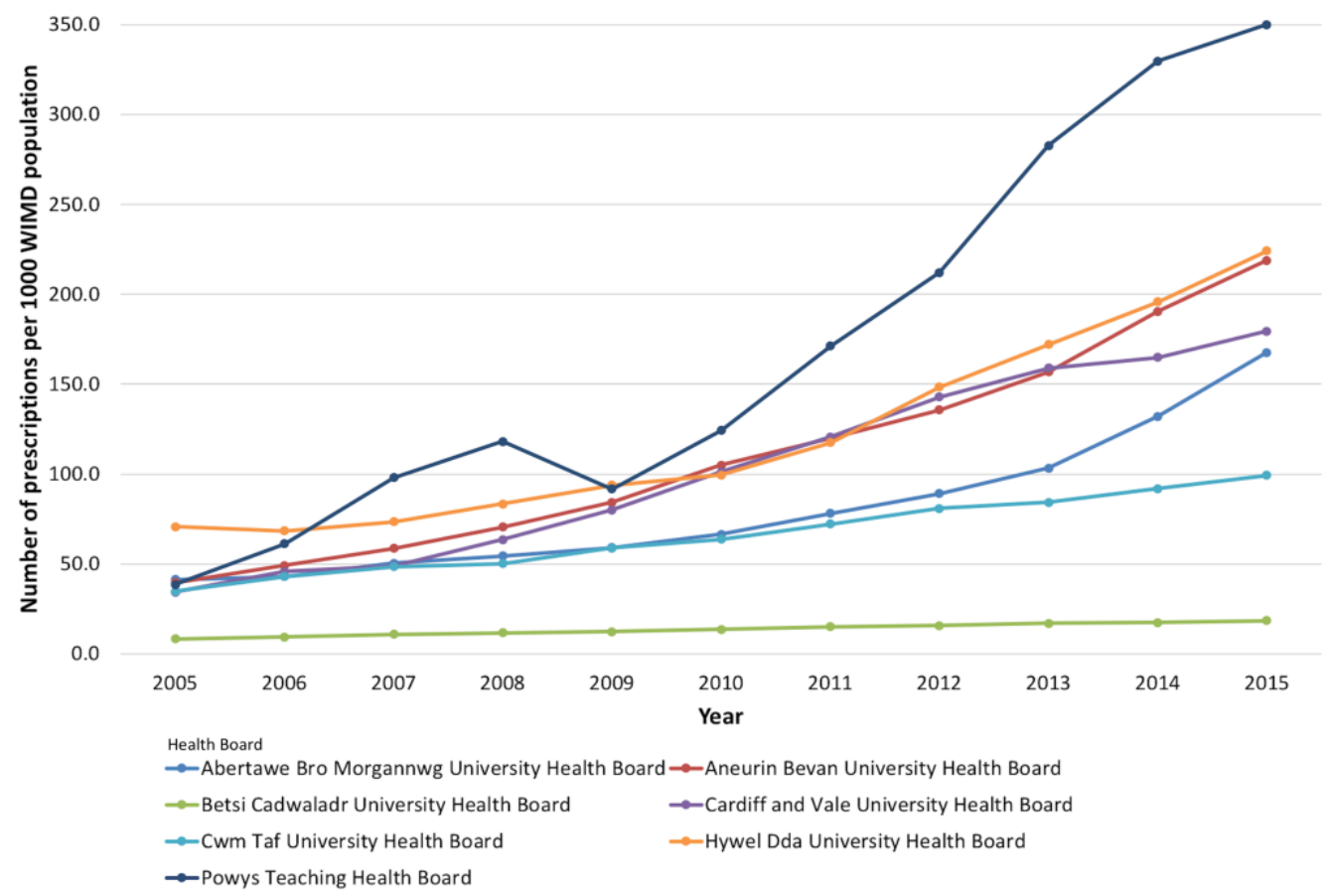

Figure 1.21: Trend in the number of people receiving prescriptions for strong opioid medicines in the 2nd most deprived areas (as per Welsh Index of Multiple Deprivation (WIMD)) in each Health Board in Wales Data adjusted to population of WIMD2 areas in each Health Board

Table 1.27: Dunn's pairwise comparison post-hoc analysis of difference between the number of strong opioid prescriptions being issued in WIMD2 areas by Welsh Health Board between 2005 and 2015

\begin{tabular}{rcccccc} 
& \multicolumn{5}{c}{ Health Board** } \\
\cline { 2 - 5 } & ABMUHB & ABUHB & BCUHB & CVUHB & CTUHB & HDUHB \\
\hline ABUHB & $>.999$ & & & & \\
\hline BCUHB & $.030^{*}$ & $<.001^{*}$ & & & \\
\hline CVUHB & $>.999$ & $>.999$ & $.001^{*}$ & & \\
\hline CTUHB & $>.999$ & $>.999$ & .160 & $>.999$ & \\
\hline HDUHB & $>.999$ & $>.999$ & $<.001^{*}$ & $>.999$ & .656 & \\
\hline PTHB & .850 & $>.999$ & $<.001^{*}$ & $>.999$ & .212 & $>.999$
\end{tabular}

${ }^{*} p<0.05$ = statistically significant **ABMUHB = Abertawe Bro Morgannwg University Health Board; ABUHB $=$ Aneurin Bevan University Health Board; BCUHB = Betsi Cadwaladr University Health Board; CVUHB = Cardiff and Vale University Health Board; CTUHB - Cwm Taf University Health Board; HDUHB = Hywel Dda University Health Board; PTHB = Powys Teaching Health Board

In the areas of moderate deprivation (WIMD3), the highest rate of prescribing was in HDUHB (Figure I.21). The largest increase in the annual number of prescriptions was seen in ABMUHB however (477.0\% increase, from 29.6 to 171.1 prescriptions per 1000 population).

The lowest rate of prescribing was again seen in BCUHB, which also had the lowest percentage increase in the annual number of prescriptions $(153.7 \%$ increase, from 10.1 to 25.7 prescriptions per 1000 population) (Table I.25). 


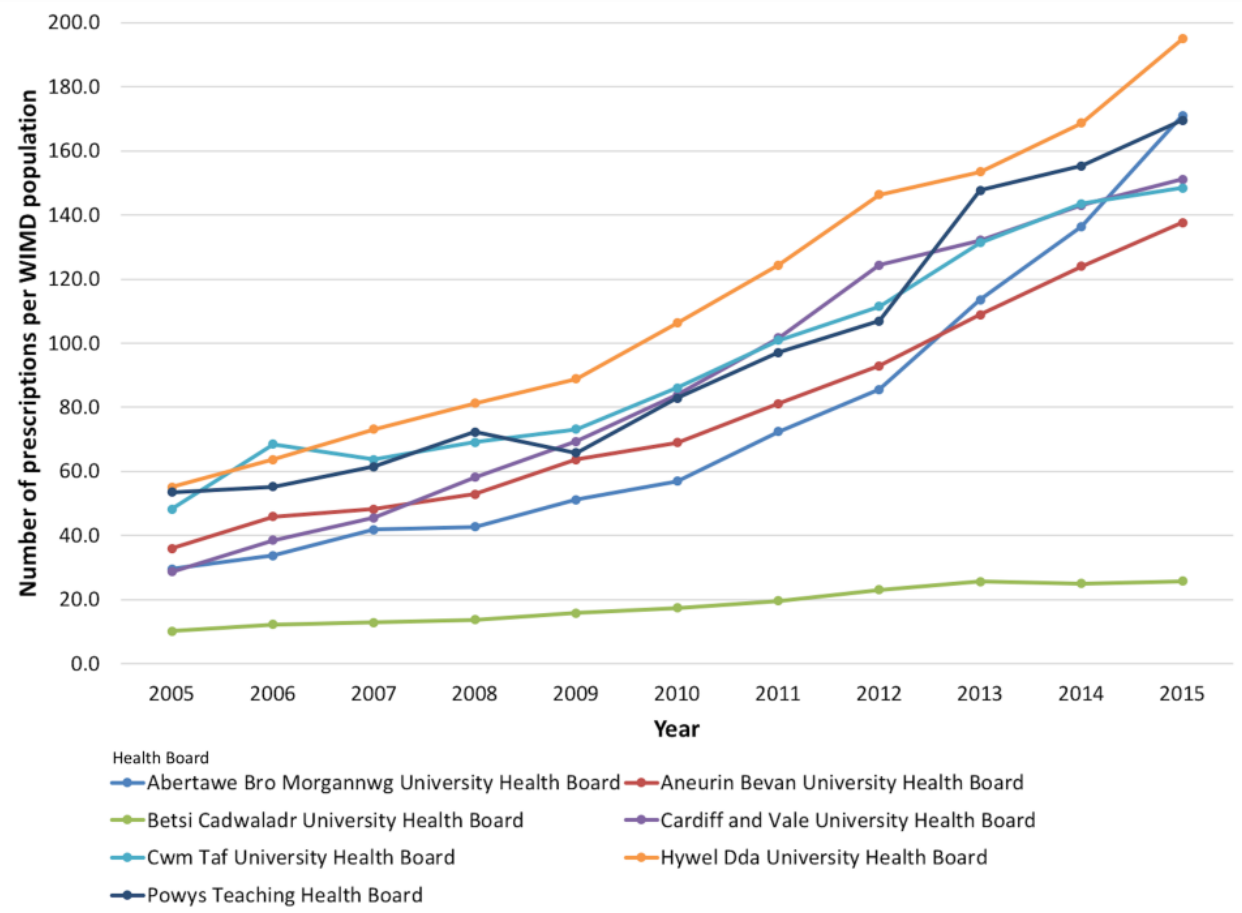

Figure 1.22: Trend in the number of people receiving prescriptions for strong opioid medicines in the 3rd most deprived areas (as per Welsh Index of Multiple Deprivation (WIMD)) in each Health Board in Wales Data adjusted to population of WIMD3 areas in each Health Board

The remaining 6 Health Boards had similar levels of prescribing in WIMD3 areas and this was reflected in the statistical analysis where Kruskal-Wallis demonstrated statistical significance $(p<.001)$ but post-hoc analysis clarified significant differences existed between BCUHB and the 6 other Health Boards (Table I.28).

Table I.28: Dunn's pairwise comparison post-hoc analysis of difference between the number of strong opioid prescriptions being issued in WIMD3 areas by Welsh Health Board between 2005 and 2015

\begin{tabular}{rcccccc}
\hline & \multicolumn{5}{c}{ Health Board** } \\
\cline { 2 - 7 } & ABMUHB & ABUHB & BCUHB & CVUHB & CTUHB & HDUHB \\
\hline ABUHB & $>.999$ & & & & \\
\hline BCUHB & $.033^{*}$ & $.015^{*}$ & & & \\
\hline CVUHB & $>.999$ & $>.999$ & $.002^{*}$ & & \\
\hline CTUHB & $>.999$ & $>.999$ & $<.001^{*}$ & $>.999$ & \\
\hline HDUHB & $>.999$ & $>.999$ & $<.001^{*}$ & $>.999$ & $>999$ & \\
\hline PTHB & 1.00 & $>.999$ & $<.001^{*}$ & $>.999$ & $>.999$ & $>.999$
\end{tabular}

${ }^{*} \mathrm{p}<0.05=$ statistically significant ${ }^{* *} \mathrm{ABMUHB}=$ Abertawe Bro Morgannwg University Health Board; ABUHB

$=$ Aneurin Bevan University Health Board; $\mathrm{BCUHB}=$ Betsi Cadwaladr University Health Board; CVUHB =

Cardiff and Vale University Health Board; CTUHB - Cwm Taf University Health Board; HDUHB = Hywel Dda

University Health Board; PTHB = Powys Teaching Health Board

Hywel Dda University Health Board also had the highest rate of strong opioid prescribing in WIMD4 areas, which have lower overall levels of deprivation (Figure I.23). The highest percentage increase in the annual number of strong opioid prescriptions was in ABUHB (356.2\%, from 25.6 to 116.8 prescriptions per 1000 population).

As with the more deprived areas, BCUHB had the lowest rates of strong opioid prescribing (Figure I.23) and a lower percentage increase in annual numbers of 
prescriptions issued (159.1\% increase, from 12.3 to 31.9 prescriptions per 1000 population) (Table I.25).

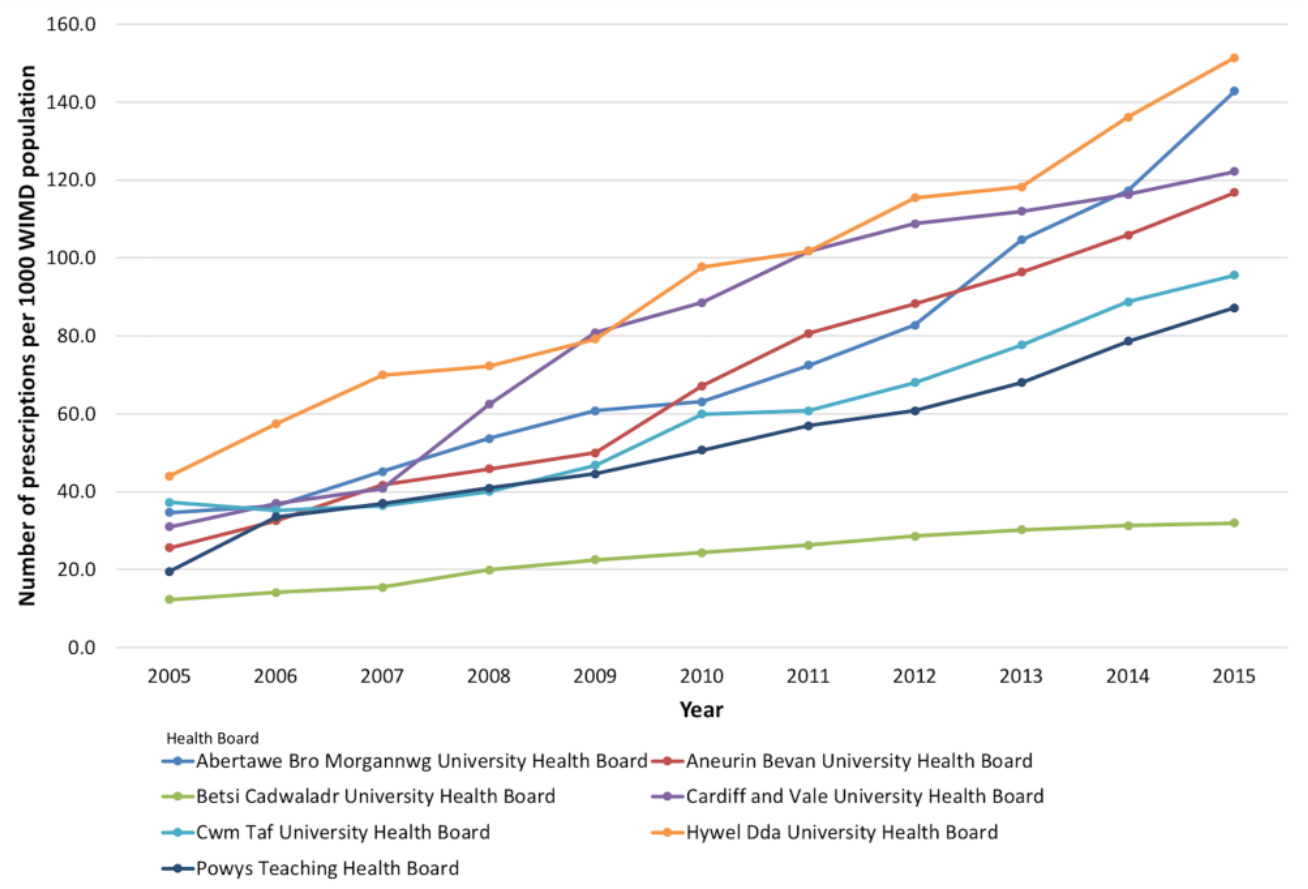

Figure 1.23: Trend in the number of people receiving prescriptions for strong opioid medicines in the lesser deprived areas (as per Welsh Index of Multiple Deprivation (WIMD)) in each Health Board in Wales Data adjusted to population of WIMD4 areas in each Health Board

A Kruskal-Wallis test determined statistically significant differences $(p<.001)$ between the number of strong opioid prescriptions issued in the WIMD4 areas in the 7 Health Boards. Dunn's pairwise comparison highlighted significant differences between BCUHB and 5 of the 6 other Health Boards. There was not a statistically significant difference between PTHB and BCUHB ( $p=.167)$ (Table I.29).

Table I.29: Dunn's pairwise comparison post-hoc analysis of difference between the number of strong opioid prescriptions being issued in WIMD4 areas by Welsh Health Board between 2005 and 2015

\begin{tabular}{ccccccc} 
& \multicolumn{5}{c}{ Health Board** } \\
\cline { 2 - 6 } & ABMUHB & ABUHB & BCUHB & CVUHB & CTUHB & HDUHB \\
\hline ABUHB & $>.999$ & & & & \\
\hline BCUHB & $.001^{*}$ & $.005^{*}$ & & & & \\
\hline CVUHB & $>.999$ & $>.999$ & $<.001^{*}$ & & \\
\hline CTUHB & $>.999$ & $>.999$ & $.036^{*}$ & $>.999$ & & \\
\hline HDUHB & $>.999$ & $>.999$ & $<.001^{*}$ & $>.999$ & .722 & \\
\hline PTHB & $>.999$ & $>.999$ & .167 & $>.999$ & $>.999$ & .198 \\
\hline
\end{tabular}

${ }^{*} p<0.05$ = statistically significant **ABMUHB = Abertawe Bro Morgannwg University Health Board; ABUHB $=$ Aneurin Bevan University Health Board; $B C U H B=$ Betsi Cadwaladr University Health Board; CVUHB = Cardiff and Vale University Health Board; CTUHB - Cwm Taf University Health Board; HDUHB = Hywel Dda University Health Board; PTHB = Powys Teaching Health Board

The patterns of strong opioid prescribing in the least deprived areas of the Health Boards, were most different to those seen in the other areas of deprivation. However, the overall rates of prescribing in the least deprived areas were lower overall than in the other areas of deprivation (Table I.25). 


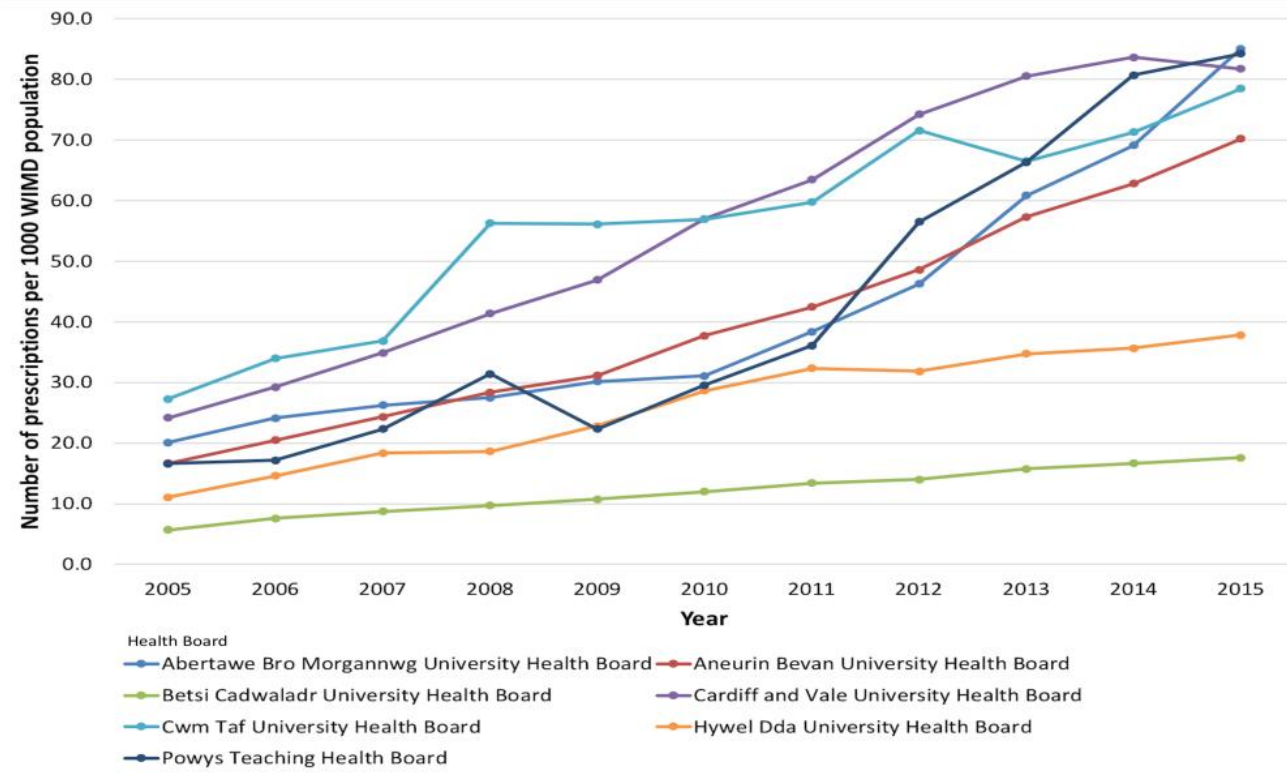

Figure 1.24: Trend in the number of people receiving prescriptions for strong opioid medicines in the least deprived areas (as per Welsh Index of Multiple Deprivation (WIMD)) in each Health Board in Wales Data adjusted to population of WIMD5 areas in each Health Board

Whilst BCUHB remained the Health Board with the lowest rates of prescribing in all 5 areas of deprivation, PTHB was the Health Board with the highest percentage increase in the number of prescriptions issued in the 11 years examined (406.4\%, from 16.6 to 84.2 prescriptions per 1000 population). Similar rates of strong opioid prescribing to those seen in PTHB, were seen in ABMUHB (Table $X$ ) where a large percentage increase in annual prescription numbers was seen (322.7\%, from 20.1 to 85.1 prescriptions per 1000 population), particularly from 2012 (Figure I.24).

Table I.30: Dunn's pairwise comparison post-hoc analysis of difference between the number of strong opioid prescriptions being issued in WIMD5 areas by Welsh Health Board between 2005 and 2015

\begin{tabular}{rcccccc} 
& \multicolumn{5}{c}{ Health Board** } \\
\cline { 2 - 7 } & ABMUHB & ABUHB & BCUHB & CVUHB & CTUHB & HDUHB \\
\hline ABUHB & $>.999$ & & & & \\
\hline BCUHB & $.004^{*}$ & $.006^{*}$ & & & & \\
\hline CVUHB & $>.999$ & $>.999$ & $<.001^{*}$ & & & \\
\hline CTUHB & $>.999$ & $>.999$ & $<.001^{*}$ & $>.999$ & & \\
\hline HDUH & $>.999$ & $>.999$ & .554 & .092 & .092 & \\
B & & & & & & \\
\hline PTHB & $>.999$ & $>.999$ & $.008^{*}$ & $>.999$ & $>999$ &
\end{tabular}

${ }^{*} \mathrm{p}<0.05$ = statistically significant ${ }^{*} \mathrm{ABMUHB}=$ Abertawe Bro Morgannwg University Health Board; $A B U H B$ $=$ Aneurin Bevan University Health Board; $\mathrm{BCUHB}=$ Betsi Cadwaladr University Health Board; CVUHB = Cardiff and Vale University Health Board; CTUHB - Cwm Taf University Health Board; HDUHB = Hywel Dda University Health Board; PTHB = Powys Teaching Health Board

Statistical analysis using a Kruskal-Wallis test showed there was a significant difference $(p<.001)$ in the number of strong opioid prescriptions issued in each Health Board. Post-hoc analysis however, confirmed that significant statistical difference was present between BCUHB and 5 other Health Boards (Table I.30). Differences between the other pairs of Health Boards that were compared, were not significant. 


\section{I.3.4 Log-linear regression}

Split by LHB

$\mathrm{LHB}=1$

Rsquare is worse, 0.009 , classification percentage is $86.7 \%$ which is high. Most influential factor is WIMD1, least influential factor is depression

Table 1.31: Logistic regression for strong opioid prescribing in ABMUHB

Variables in the Equation

\begin{tabular}{lll|l|l|l|l|l} 
& & B & S.E. & Wald & df & Sig. & Exp(B) \\
\hline Step 1 & WIMD1 & .049 & .005 & 103.329 & 1 & $<.001$ & 1.050 \\
\cline { 2 - 8 } & WIMD3 & -.115 & .004 & 747.369 & 1 & $<.001$ & .891 \\
\cline { 2 - 8 } & WIMD4 & -.103 & .004 & 537.645 & 1 & $<.001$ & .902 \\
\cline { 2 - 8 } WIMD5 & -.062 & .005 & 160.378 & 1 & $<.001$ & .940 \\
\cline { 2 - 8 } & Male & -.063 & .003 & 443.156 & 1 & $<.001$ & .939 \\
\cline { 2 - 8 } DEPRESSION & -.410 & .003 & 19220.547 & 1 & $<.001$ & .664 \\
\hline & Constant & 2.102 & .004 & 338243.005 & 1 & $<.001$ & 8.185
\end{tabular}

a. Variable(s) entered on step 1: WIMD1, WIMD3, WIMD4, WIMD5, Male, DEPRESSION.

$\mathrm{LHB} 2=1$

$85.7 \%$ classification correct

R square 0.015

Table 1.32: Logistic regression for strong opioid prescribing in $A B \cup H B$

Variables in the Equation

\begin{tabular}{lll|l|l|l|l|l} 
& & B & S.E. & Wald & df & Sig. & Exp(B) \\
\hline Step 1 & aIMD1 & .022 & .007 & 9.261 & 1 & .002 & 1.022 \\
\cline { 2 - 8 } & WIMD3 & -.113 & .005 & 532.942 & 1 & $<.001$ & .893 \\
\cline { 2 - 8 } & WIMD4 & -.093 & .005 & 290.979 & 1 & $<.001$ & .911 \\
\cline { 2 - 8 } & WIMD5 & -.059 & .010 & 33.584 & 1 & $<.001$ & .942 \\
\cline { 2 - 8 } & Male & -.277 & .004 & 5300.321 & 1 & $<.001$ & .758 \\
\hline & DEPRESSION & -.487 & .004 & 15211.620 & 1 & $<.001$ & .614 \\
\hline & Constant & 2.128 & .004 & 223658.892 & 1 & $<.001$ & 8.395
\end{tabular}

a. Variable(s) entered on step 1: WIMD1, WIMD3, WIMD4, WIMD5, Male, DEPRESSION.

Same conclusions as LHB1

$\mathrm{LHB}=3$

R-square 0.006

Classification $92.3 \%$

WIMD3 most influential, depression least WIMD5 should be omitted

Table l.33: Logistic regression for strong opioid prescribing in BCUHB

Variables in the Equation

\begin{tabular}{lll|l|l|l|l|l} 
& & B & S.E. & Wald & df & Sig. & Exp(B) \\
\hline \multirow{3}{*}{ Step 1 $1^{\text {a }}$} & WIMD1 & -.058 & .006 & 109.783 & 1 & $<.001$ & .944 \\
\cline { 2 - 9 } & WIMD3 & -.075 & .007 & 119.045 & 1 & $<.001$ & .927 \\
\cline { 2 - 9 } & WIMD4 & -.145 & .007 & 476.134 & 1 & $<.001$ & .865 \\
\hline
\end{tabular}




\begin{tabular}{ll|l|l|l|l|l}
\hline WIMD5 & -.046 & .006 & 60.189 & 1 & $<.001$ & .955 \\
\hline Male & -.069 & .004 & 354.612 & 1 & $<.001$ & .933 \\
\hline DEPRESSION & -.426 & .004 & 14256.663 & 1 & $<.001$ & .653 \\
\hline Constant & 2.194 & .005 & 176920.239 & 1 & $<.001$ & 8.971 \\
\hline
\end{tabular}

a. Variable(s) entered on step 1: WIMD1, WIMD3, WIMD4, WIMD5, Male, DEPRESSION.

$\mathrm{LHB}=4$

R square $=0.01$

Classification $87.3 \%$

Table I.34: Logistic regression for strong opioid prescribing in CVUHB

Variables in the Equation

\begin{tabular}{lll|l|l|l|l|l} 
& & B & S.E. & Wald & df & Sig. & Exp(B) \\
\hline Step 1 & WIMD1 & -.034 & .006 & 38.019 & 1 & $<.001$ & .967 \\
\cline { 2 - 8 } & WIMD3 & -.015 & .007 & 4.840 & 1 & .028 & .985 \\
\cline { 2 - 8 } & WIMD4 & .013 & .011 & 1.433 & 1 & .231 & 1.013 \\
\cline { 2 - 8 } & WIMD5 & -.044 & .009 & 22.650 & 1 & $<.001$ & .957 \\
\cline { 2 - 8 } & Male & -.223 & .005 & 2399.196 & 1 & $<.001$ & .800 \\
\hline & DEPRESSION & -.472 & .005 & 10576.403 & 1 & $<.001$ & .624 \\
\hline
\end{tabular}

a. Variable(s) entered on step 1: WIMD1, WIMD3, WIMD4, WIMD5, Male, DEPRESSION.

WIMD 4 out

WIMD 3 highest, Depression lowest

$\mathrm{LHB}=5$

R square $=0.011$

Classification $91.6 \%$

Table 1.35: Logisitic regression for strong opioid prescribing in CTUHB

Variables in the Equation

\begin{tabular}{lll|l|l|l|l|l} 
& & B & S.E. & Wald & df & Sig. & Exp(B) \\
\hline Step 1 & WIMD1 & -.050 & .004 & 138.388 & 1 & $<.001$ & .951 \\
\cline { 2 - 9 } & WIMD3 & .074 & .005 & 201.619 & 1 & $<.001$ & 1.076 \\
\cline { 2 - 8 } & WIMD4 & -.169 & .006 & 780.658 & 1 & $<.001$ & .844 \\
\cline { 2 - 8 } WIMD5 & .064 & .007 & 92.654 & 1 & $<.001$ & 1.066 \\
\cline { 2 - 8 } & Male & -.049 & .003 & 205.111 & 1 & $<.001$ & .952 \\
\hline & DEPRESSION & -.321 & .003 & 8458.097 & 1 & $<.001$ & .726 \\
\hline & Constant & 2.378 & .004 & 397735.485 & 1 & $<.001$ & 10.785 \\
\hline
\end{tabular}

a. Variable(s) entered on step 1: WIMD1, WIMD3, WIMD4, WIMD5, Male, DEPRESSION.

WIMD3 high influence, Depression lowest influence

$\mathrm{LHB}=6$

R square $=0.05$

Classification $=90.3 \%$

$\mathrm{LHB}=7$

$\mathrm{R}$ square $=0.005$ 
Classification $=89.8$

Most influential variable Male

Least Depression

Table 1.36: Logistic regression for strong opioid prescribing in PTHB

Variables in the Equation

\begin{tabular}{|c|c|c|c|c|c|c|c|}
\hline & & B & S.E. & Wald & df & Sig. & $\operatorname{Exp}(B)$ \\
\hline \multirow[t]{7}{*}{ Step $1^{a}$} & WIMD1 & -.065 & .021 & 9.600 & 1 & .002 & .937 \\
\hline & WIMD3 & -.233 & .014 & 272.209 & 1 & $<.001$ & .792 \\
\hline & WIMD4 & -.384 & .013 & 827.583 & 1 & $<.001$ & .681 \\
\hline & WIMD5 & -.062 & .024 & 6.869 & 1 & .009 & .940 \\
\hline & Male & .117 & .011 & 123.383 & 1 & $<.001$ & 1.124 \\
\hline & DEPRESSION & -.397 & .010 & 1449.612 & 1 & $<.001$ & .673 \\
\hline & Constant & 2.465 & .012 & 42092.408 & 1 & $<.001$ & 11.769 \\
\hline
\end{tabular}

a. Variable(s) entered on step 1: WIMD1, WIMD3, WIMD4, WIMD5, Male, DEPRESSION.

\section{I.4 Abertawe Bro Morgannwg University Health Board}

Over the course of 11 years, there was a $7.5 \%$ increase (from 74,488 to 80,050 ) in the annual number of people receiving prescriptions within all categorised deprivation areas - the smallest increase seen in Wales. When adjusted to the annual Health Board population over the same time, a 3.2\% (from 153.7 to 159.7 people per 1000 population) increase was noted.

Table I.37: Annual change in the number of people per 1000 population receiving opioid prescriptions and annual change rate (percentage change) in Abertawe Bro Morgannwg University Health Board (ABMUHB)

\begin{tabular}{|c|c|c|c|c|c|c|c|c|c|c|}
\hline & \multicolumn{10}{|c|}{ Welsh Index of Multiple Deprivation (WIMD) } \\
\hline & \multicolumn{2}{|c|}{1} & \multicolumn{2}{|c|}{2} & \multicolumn{2}{|c|}{3} & \multicolumn{2}{|c|}{4} & \multicolumn{2}{|c|}{5} \\
\hline & $\begin{array}{c}\text { People } \\
\text { per } \\
1000\end{array}$ & $\begin{array}{c}\text { Change } \\
\text { rate } \\
(\%)\end{array}$ & $\begin{array}{c}\text { People } \\
\text { per } \\
1000\end{array}$ & $\begin{array}{c}\text { Change } \\
\text { rate } \\
(\%)\end{array}$ & $\begin{array}{c}\text { People } \\
\text { per } \\
1000\end{array}$ & $\begin{array}{c}\text { Change } \\
\text { rate } \\
(\%)\end{array}$ & $\begin{array}{c}\text { People } \\
\text { per } \\
1000\end{array}$ & $\begin{array}{c}\text { Change } \\
\text { rate } \\
(\%)\end{array}$ & $\begin{array}{c}\text { People } \\
\text { per } \\
1000\end{array}$ & $\begin{array}{c}\text { Change } \\
\text { rate } \\
(\%)\end{array}$ \\
\hline 2005 & 192.5 & & 160.5 & & 159.1 & & 154.0 & & 101.9 & \\
\hline 2006 & 193.9 & 0.7 & 163.0 & 1.6 & 157.6 & -0.9 & 155.8 & 1.2 & 100.4 & -1.5 \\
\hline 2007 & 203.0 & 4.7 & 170.4 & 4.5 & 169.5 & 7.5 & 167.4 & 7.4 & 110.6 & 10.1 \\
\hline 2008 & 207.1 & 2.0 & 176.1 & 3.4 & 172.7 & 1.9 & 172.1 & 2.8 & 113.9 & 3.0 \\
\hline 2009 & 212.8 & 2.7 & 182.3 & 3.5 & 180.4 & 4.5 & 180.3 & 4.8 & 119.1 & 4.5 \\
\hline 2010 & 211.8 & -0.5 & 182.2 & -0.1 & 177.4 & -1.7 & 179.4 & -0.5 & 117.8 & -1.1 \\
\hline 2011 & 213.6 & 0.8 & 183.6 & 0.8 & 180.3 & 1.6 & 180.5 & 0.6 & 118.2 & 0.4 \\
\hline 2012 & 211.9 & -0.8 & 179.5 & -2.2 & 177.7 & -1.4 & 177.0 & -1.9 & 118.1 & -0.1 \\
\hline 2013 & 207.8 & -2.0 & 166.4 & -7.3 & 173.6 & -2.4 & 175.8 & -0.7 & 114.0 & -3.5 \\
\hline 2014 & 204.6 & -1.5 & 158.3 & -4.9 & 169.7 & -2.2 & 171.4 & -2.5 & 114.7 & 0.6 \\
\hline 2015 & 198.8 & -2.9 & 153.0 & -3.4 & 166.3 & -2.0 & 167.1 & -2.5 & 111.2 & -3.1 \\
\hline $\begin{array}{l}\text { erall rate } \\
\text { ange (\%) }\end{array}$ & 3.3 & & -4.7 & & 4.5 & & 8.4 & & 9.1 & \\
\hline
\end{tabular}

Welsh Index of Multiple Deprivation (WIMD) where WIMD1 = most deprived and WIMD5 = least deprived

Statistically significant difference between areas of deprivation was noted by one-way ANOVA $\left(F(4,50)=168.6, p<.001, \eta^{2}=0.93\right)$. Bonferroni post-hoc testing however, demonstrated that there was not a statistically significant difference between WIMD2 (mean $=170.5, \mathrm{SD}=10.9)$, WIMD3 $($ mean $=171.3$, $S D=7.9)$ and WIMD4 areas (mean $=171.0, S D=9.3, p=>.999)$. 
The areas of least deprivation (WIMD-4 and WIMD-5) had the largest increases in numbers of people receiving prescriptions $8.4 \%$ (from 154.0 to 167.1 per 1000 population) and $9.1 \%$ (from 101.9 to 111.2 per 1000 population) respectively.

ABMUHB had 5,470,667 opioid prescriptions issued over 11 years, which accounted for $24.9 \%$ of recorded opioid prescriptions issued in categorised areas of Wales $(22,001,509$ total). This was population adjusted to $16.2 \%$ of opioid prescriptions issued in Wales.

The most deprived areas of ABMUHB had $35.6 \%(1,947,126)$ of all opioid prescriptions issued in that Health Board over the study period. This was $38 \%$ more prescriptions than were issued in the two least deprived categories of area $(667,473$ in WIMD-4 and 742,625 in WIMD-5 areas).

There were increases in opioid prescribing in all deprivation areas over the 11 years that the study examined although the 2.4 times difference between the number of prescriptions being issued in the most deprived areas versus the least, remained across the whole period (Table I.38).

There was the same difference in the number of weak opioid prescriptions per 1000 population seen between the most and least deprived areas in ABMUHB (Table I.38). The difference between the number of strong opioid prescriptions per 1000 population between the most deprived and least deprived areas of the Health Board increased from 2.3 times more in 2005 to 2.7 times larger in 2015 (Table I.38).

Table l.38: Changes in the number of opioid prescriptions issued by deprivation area within Abertawe Bro Morgannwg University Health Board between 2005 and 2015

\begin{tabular}{rccccc}
\hline & \multicolumn{5}{c}{ Number of prescriptions per 1000 } \\
\cline { 2 - 6 } & WIMD1 & WIMD2 & WIMD3 & WIMD4 & WIMD5 \\
\hline All Opioids & & & & & \\
$\mathbf{2 0 0 5}$ & 1129.4 & 918.6 & 862.5 & 770.5 & 470.6 \\
$\mathbf{2 0 1 5}$ & 1510.3 & 1073.9 & 1135.8 & 1035.5 & 630.9 \\
Change rate (\%) & 33.7 & 16.9 & 31.7 & 34.4 & 34.1 \\
\hline Weak opioids & & & & & \\
$\mathbf{2 0 0 5}$ & 1082.9 & 877.2 & 832.9 & 735.8 & 450.5 \\
$\mathbf{2 0 1 5}$ & 1282.6 & 906.3 & 964.7 & 892.7 & 545.8 \\
Change rate (\%) & 18.4 & 3.3 & 15.8 & 21.3 & 21.2 \\
\hline Strong opioids & & & & & \\
$\mathbf{2 0 0 5}$ & 46.5 & 41.4 & 39.6 & 34.7 & 20.1 \\
$\mathbf{2 0 1 5}$ & 227.7 & 167.6 & 171.1 & 142.9 & 85.1 \\
Change rate (\%) & 390.1 & 304.5 & 477.0 & 311.9 & 322.7 \\
\hline
\end{tabular}

Population adjusted data presented and change rate calculated between 2005 and 2015

Changes in the number of prescriptions issued for weak opioids were relatively modest with the greater increases seen in the least deprived areas. Strong opioid prescribing however appreciably increased in all areas although WIMD3 areas had the biggest growth over the study period. 

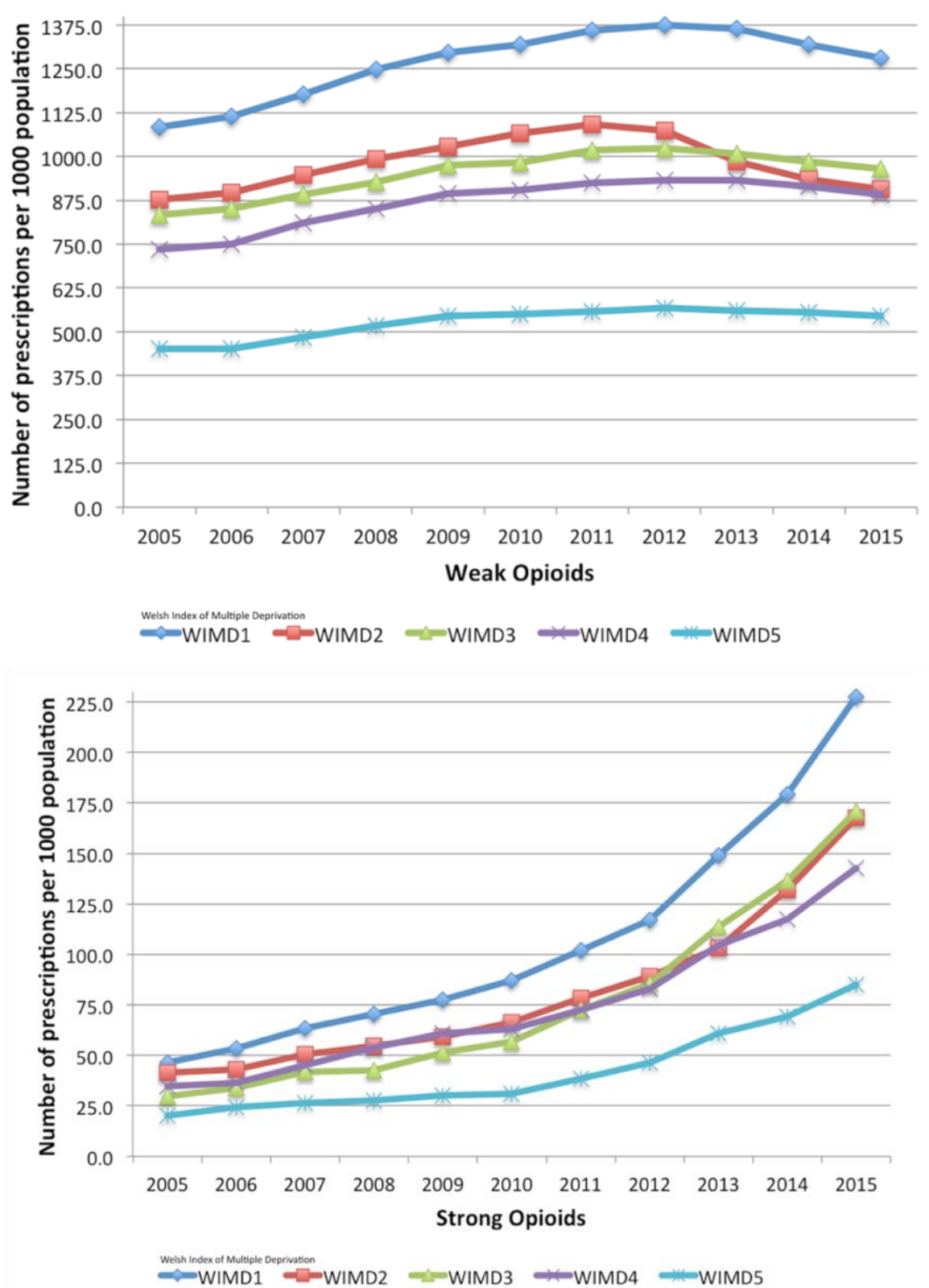

Figure 1.25: Trends in the number of weak or strong opioid prescriptions issued per 1000 population in Abertawe Bro Morgannwg University Health Board by deprivation area (WIMD)

Welsh Index of Multiple Deprivation (WIMD) where WIMD1 = most deprived and WIMD5 = least deprived

\section{I.5 Aneurin Bevan Local Health Board}

There was a $23.5 \%$ (from 47,888 to 59,132 ) increase in the number of people receiving opioid prescriptions in the Health Board area of Aneurin Bevan. When adjusted to Health Board population, a 19.7\% (from 121.3 to 145.2 people per 1000 population) increase was observed (Table I.39).

Table I.39: Annual change in the number of people per 1000 population receiving opioid prescriptions and annual change rate (percentage change) in Aneurin Bevan University Health Board (ABUHB)

\begin{tabular}{cccccccccc}
\multicolumn{2}{c}{ WIMD1 } & \multicolumn{2}{c}{ WIMD2 } & \multicolumn{2}{c}{ WIMD3 } & \multicolumn{2}{c}{ WIMD4 } & \multicolumn{2}{c}{ WIMD5 } \\
\hline People & Change & People & Change & People & Change & People & Change & People & Change \\
per & rate & per & rate & per & rate & per & rate & per & rate \\
1000 & $(\%)$ & 1000 & $(\%)$ & 1000 & $(\%)$ & 1000 & $(\%)$ & 1000 & $(\%)$ \\
\hline
\end{tabular}




\begin{tabular}{rrrrrrrrrrr}
\hline $\mathbf{2 0 0 5}$ & 150.0 & & 150.2 & & 125.4 & & 89.1 & & 71.7 \\
$\mathbf{2 0 0 6}$ & 150.5 & 0.3 & 154.1 & 2.6 & 128.3 & 2.3 & 90.9 & 2.0 & 74.5 & 3.9 \\
$\mathbf{2 0 0 7}$ & 161.0 & 7.0 & 164.8 & 7.0 & 137.1 & 6.8 & 99.3 & 9.2 & 80.8 & 8.5 \\
$\mathbf{2 0 0 8}$ & 164.2 & 2.0 & 170.6 & 3.5 & 142.4 & 3.9 & 104.1 & 4.7 & 84.8 & 5.0 \\
$\mathbf{2 0 0 9}$ & 168.9 & 2.9 & 175.9 & 3.1 & 145.2 & 2.0 & 107.8 & 3.6 & 87.3 & 2.9 \\
$\mathbf{2 0 1 0}$ & 176.4 & 4.5 & 184.4 & 4.8 & 150.7 & 3.8 & 116.2 & 7.8 & 87.8 & 0.6 \\
$\mathbf{2 0 1 1}$ & 177.5 & 0.6 & 186.9 & 1.3 & 151.7 & 0.7 & 117.0 & 0.7 & 94.4 & 7.5 \\
$\mathbf{2 0 1 2}$ & 179.4 & 1.1 & 189.0 & 1.1 & 151.6 & -0.1 & 120.5 & 3.0 & 94.9 & 0.6 \\
$\mathbf{2 0 1 3}$ & 173.2 & -3.4 & 188.9 & 0.0 & 149.8 & -1.2 & 119.4 & -0.9 & 93.9 & -1.0 \\
$\mathbf{2 0 1 4}$ & 172.4 & -0.5 & 189.4 & 0.3 & 149.0 & -0.5 & 117.3 & -1.7 & 94.7 & 0.8 \\
$\mathbf{2 0 1 5}$ & 169.6 & -1.6 & 185.0 & -2.4 & 145.5 & -2.4 & 115.5 & -1.5 & 92.1 & -2.7 \\
\hline Change & 13.1 & & 23.2 & & 16.0 & & 29.6 & & 28.4 &
\end{tabular}

Welsh Index of Multiple Deprivation (WIMD) where WIMD1 = most deprived

There were twice as many people receiving opioid prescriptions in the most deprived areas when compared to the least in 2005 (150.0 versus 71.7 people per 1000 population respectively). By 2015 the difference had reduced to result in 2.7 times more people in the most deprived areas receiving opioid prescriptions compared to the least deprived $(17,963$ versus 6,754$)$.

Aneurin Bevan Local Health Board (ABLHB) issued 4,041,844 opioid prescriptions over the 11 year period examined, which equated to $18.4 \%$ of the total for Wales. This was population adjusted to $16.2 \%$ of prescriptions issued. Within the Health Board area, $34.9 \%(1,410,654)$ of all prescriptions issued were within the areas of highest deprivation compared to $9 \%$ in the areas of least deprivation (362,148 prescriptions).

Categories 3, 4 and 5 of the WIMD2011 scale accounted for 1,493,301 prescriptions between 2005 and 2015, which was only 6\% more than the total prescribed within the most deprived areas of the Health Board.

Overall, there was a $50.3 \%$ (from 279,896 to 420,731 ) increase in the annual number of all opioid prescriptions in ABUHB between 2005 and 2015. Increases in the annual number of prescriptions were noted in all deprivation areas across $A B U H B$ in that 11-year period.

In 2005, the number of weak opioid prescriptions issued in the areas of greatest deprivation in ABUHB was 2.9 times the number in the least deprived (Table I.40). There was a reduction in the difference over the 11 years examined to 2.4 times in 2015 , although weak opioid prescribing continued to predominate in the areas of greater deprivation.

Table I.40: Changes in the number of opioid prescriptions issued by deprivation area within Aneurin Bevan University Health Board between 2005 and 2015

\begin{tabular}{|c|c|c|c|c|c|}
\hline & \multicolumn{5}{|c|}{ Number of prescriptions per 1000} \\
\hline & WIMD1 & WIMD2 & WIMD3 & WIMD4 & WIMD5 \\
\hline \multicolumn{6}{|l|}{ All Opioids } \\
\hline 2005 & 977.0 & 882.4 & 702.1 & 466.6 & 343.7 \\
\hline 2015 & 1333.6 & 1354.3 & 1004.1 & 731.5 & 529.0 \\
\hline Change rate (\%) & 36.5 & 53.5 & 43.0 & 56.8 & 53.9 \\
\hline
\end{tabular}




\begin{tabular}{rrrccc}
\hline Weak opioids & & & & \\
$\mathbf{2 0 0 5}$ & 930.4 & 842.8 & 666.1 & 441.0 & 327.0 \\
$\mathbf{2 0 1 5}$ & 1119.7 & 1135.5 & 866.4 & 614.7 & 458.8 \\
Change rate (\%) & 20.4 & 34.7 & 30.1 & 39.4 & 40.3 \\
\hline Strong opioids & & & & \\
$\mathbf{2 0 0 5}$ & 46.7 & 39.6 & 36.0 & 25.6 & 16.7 \\
$\mathbf{2 0 1 5}$ & 213.9 & 218.8 & 137.6 & 116.8 & 70.2 \\
Change rate (\%) & 358.3 & 452.9 & 282.2 & 356.2 & 321.3 \\
\hline \multicolumn{5}{l}{ Population adjusted data presented and change rate calculated between 2005 and 2015 }
\end{tabular}

Strong opioid prescribing was higher in the more deprived areas of ABUHB also. The most deprived areas had 2.8 times more prescriptions issued in 2005 than the least deprived. There were significant increases in all areas, between 2005 and 2015 but the difference between most and least deprived also increased so that by 2015 , there were three times more strong opioid prescriptions being issued in WIMD1 areas compared to WIMD5. The greatest increase, however, was seen in WIMD2 areas (Table I.40).

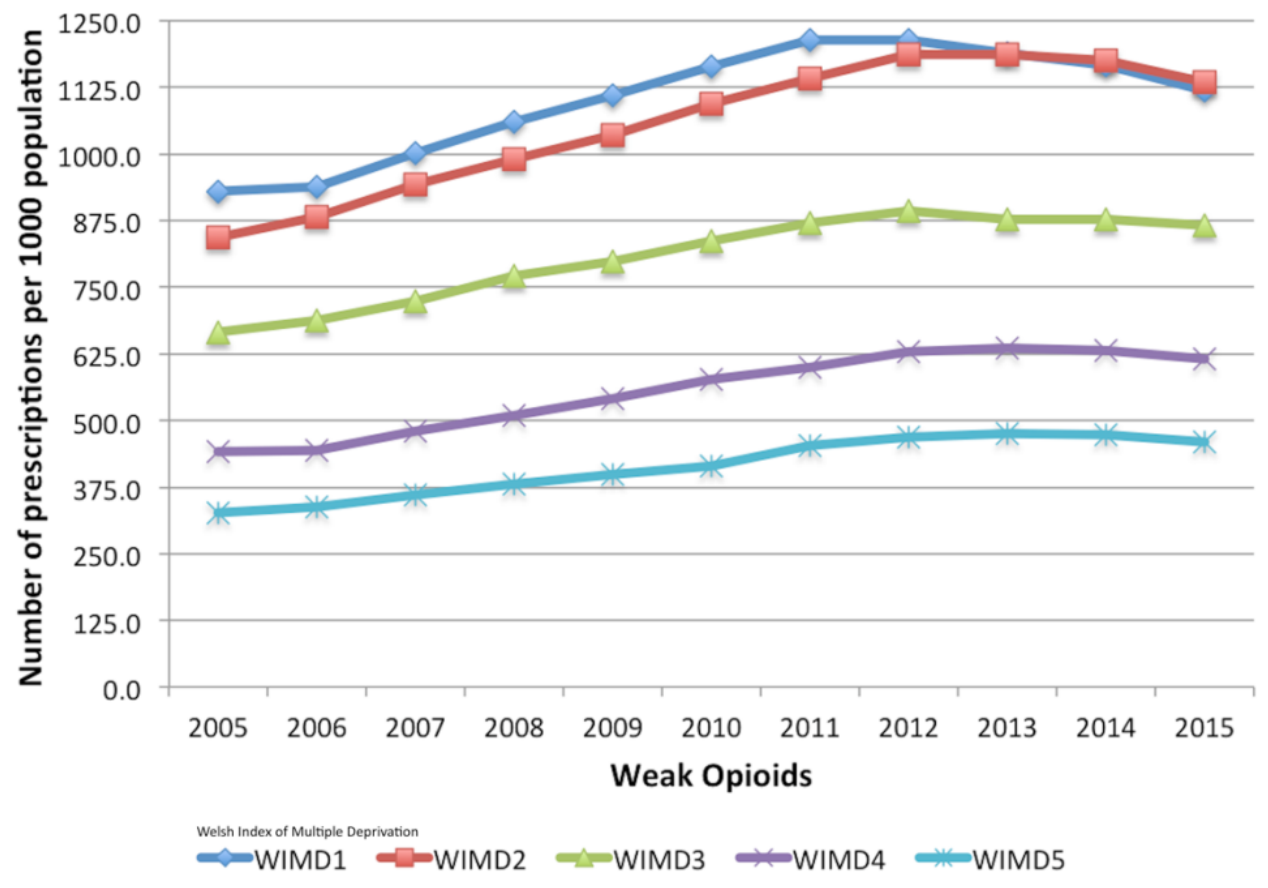




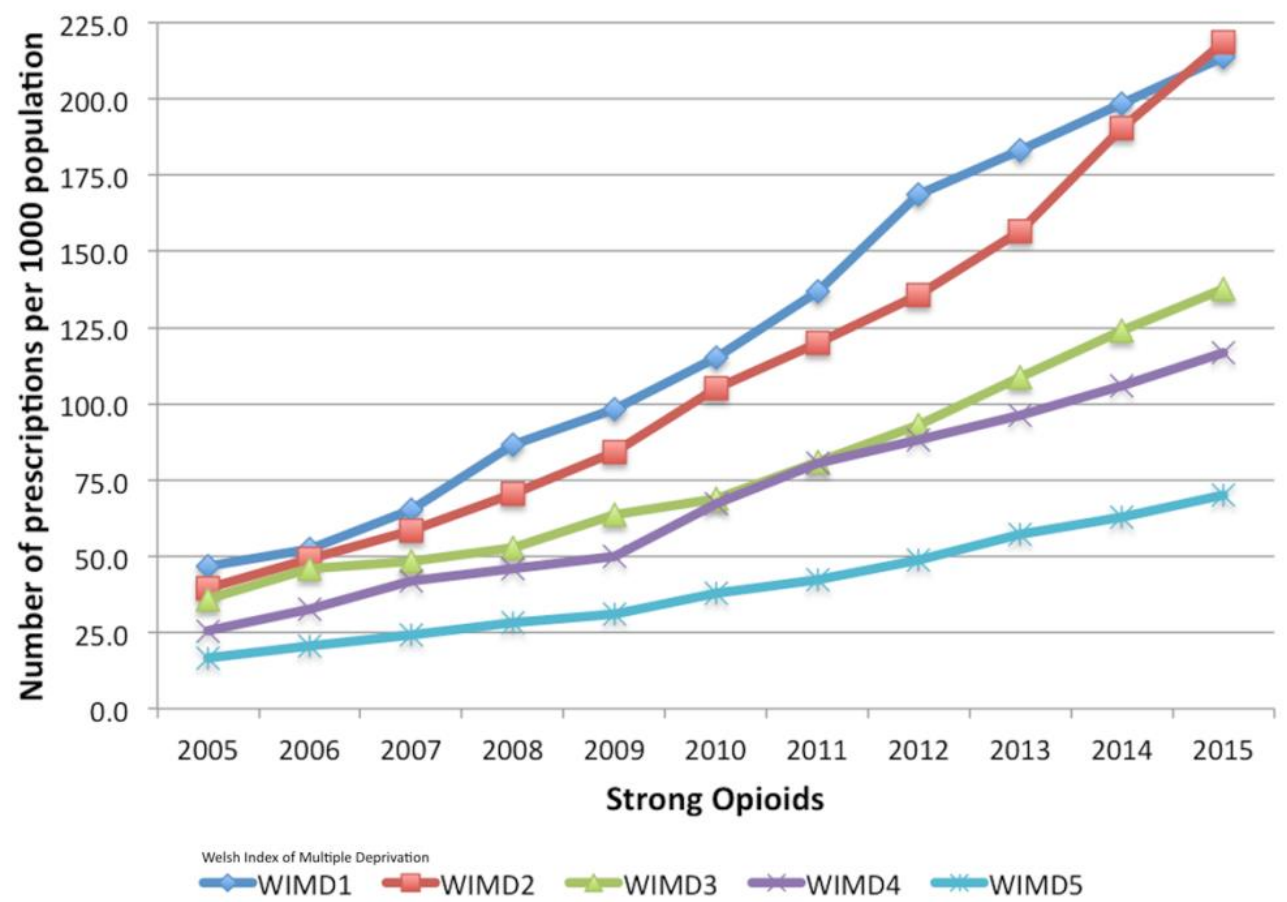

Figure I.26: Trends in the number of weak or strong opioid prescriptions issued per 1000 population in Aneurin Bevan University Health Board by deprivation area (WIMD)

Welsh Index of Multiple Deprivation (WIMD) where WIMD1 = most deprived and WIMD5 = least deprived

\section{I.6 Betsi Cadwaladr University Health Board}

In the eleven years studied, there was a $44.1 \%$ (from 54,464 to 78,483 ) increase in the annual number of people receiving any opioid prescription in all areas of Betsi Cadwaladr University Health Board (population adjusted to 35.5\% increase). This was the highest increase seen in any Health Board in Wales over the 11 years of the study (Table I.41).

Table I.41: Annual change in the number of people per 1000 population receiving opioid prescriptions and annual change rate (percentage change) in Betsi Cadwaladr University Health Board (BCUHB)

\begin{tabular}{|c|c|c|c|c|c|c|c|c|c|c|}
\hline & \multicolumn{2}{|c|}{ WIMD1 } & \multicolumn{2}{|c|}{ WIMD2 } & \multicolumn{2}{|c|}{ WIMD3 } & \multicolumn{2}{|c|}{ WIMD4 } & \multicolumn{2}{|c|}{ WIMD5 } \\
\hline & $\begin{array}{c}\text { People } \\
\text { per } \\
1000\end{array}$ & $\begin{array}{c}\text { Change } \\
\text { rate } \\
(\%)\end{array}$ & $\begin{array}{c}\text { People } \\
\text { per } \\
1000\end{array}$ & $\begin{array}{c}\text { Change } \\
\text { rate } \\
(\%)\end{array}$ & $\begin{array}{c}\text { People } \\
\text { per } \\
1000\end{array}$ & $\begin{array}{c}\text { Change } \\
\text { rate } \\
(\%)\end{array}$ & $\begin{array}{c}\text { People } \\
\text { per } \\
1000\end{array}$ & $\begin{array}{c}\text { Change } \\
\text { rate } \\
(\%)\end{array}$ & $\begin{array}{c}\text { People } \\
\text { per } \\
1000\end{array}$ & $\begin{array}{c}\text { Change } \\
\text { rate } \\
(\%)\end{array}$ \\
\hline 2005 & 121.1 & & 109.1 & & 117.3 & & 76.7 & & 112.2 & \\
\hline 2006 & 122.4 & 1.2 & 112.0 & 2.7 & 122.4 & 4.4 & 79.5 & 3.6 & 116.5 & 3.8 \\
\hline 2007 & 131.3 & 7.2 & 121.9 & 8.8 & 133.6 & 9.1 & 87.7 & 10.4 & 126.7 & 8.8 \\
\hline 2008 & 139.0 & 5.9 & 127.8 & 4.9 & 140.7 & 5.4 & 94.2 & 7.4 & 134.9 & 6.5 \\
\hline 2009 & 142.9 & 2.8 & 130.9 & 2.4 & 143.6 & 2.0 & 94.7 & 0.6 & 135.8 & 0.7 \\
\hline 2010 & 148.0 & 3.6 & 136.4 & 4.2 & 146.6 & 2.1 & 96.8 & 2.2 & 140.3 & 3.3 \\
\hline 2011 & 152.7 & 3.1 & 140.1 & 2.7 & 149.8 & 2.2 & 100.7 & 4.0 & 144.1 & 2.7 \\
\hline 2012 & 157.8 & 3.4 & 144.7 & 3.3 & 154.8 & 3.3 & 103.6 & 2.8 & 147.2 & 2.1 \\
\hline 2013 & 167.7 & 6.2 & 148.6 & 2.7 & 158.2 & 2.2 & 105.7 & 2.0 & 150.7 & 2.4 \\
\hline 2014 & 173.0 & 3.2 & 152.1 & 2.3 & 161.7 & 2.3 & 107.7 & 1.8 & 151.2 & 0.4 \\
\hline 2015 & 168.5 & -2.6 & 147.9 & -2.7 & 156.8 & -3.0 & 104.6 & -2.8 & 147.0 & -2.8 \\
\hline $\begin{array}{r}\text { Change } \\
\text { (\%) }\end{array}$ & 39.2 & & 35.6 & & 33.7 & & 36.3 & & 31.0 & \\
\hline
\end{tabular}

Welsh Index of Multiple Deprivation (WIMD) where WIMD1 = most deprived 
There was less difference in the annual number of people being issued opioid prescriptions between deprivation areas in BCUHB, other than WIMD4 area which maintained a 1.6 times difference in numbers compared to the most deprived area (WIMD1).

Nineteen percent of all opioid prescriptions in Wales $(4,199,945$ of $22,001,509)$ were issued within BCUHB between 2005 and 2015 (12.6\% when adjusted to population). BCUHB demonstrated the largest increases in opioid prescribing of the seven Health Boards in Wales between 2005 and 2015. However, the least deprived areas (WIMD5) were not where the least prescriptions were issued. Instead WIMD4 areas were where the fewest prescriptions were issued per 1000 population (Table I.42).

The increases in weak opioids were also greater than those seen in other Health Boards (Table I.42). More weak opioid prescriptions were issued in the most deprived areas of BCUHB than other areas. There were 1.8 times more weak opioid prescriptions per 1000 population issued in the most deprived areas of BCUHB compared to WIMD4 areas which had the least in 2005. Despite significant increases in all areas, the difference between WIMD1 and WIMD4 rose to 2 times by 2015 (Table I.42).

Table I.42: Changes in the number of opioid prescriptions issued by deprivation area within Betsi Cadwaladr University Health Board between 2005 and 2015

\begin{tabular}{|c|c|c|c|c|c|}
\hline & \multicolumn{5}{|c|}{ Number of prescriptions per 1000} \\
\hline & WIMD1 & WIMD2 & WIMD3 & WIMD4 & WIMD5 \\
\hline \multicolumn{6}{|l|}{ All Opioids } \\
\hline 2005 & 662.0 & 605.2 & 604.6 & 362.1 & 494.9 \\
\hline 2015 & 1294.2 & 1064.3 & 1037.2 & 642.1 & 867.7 \\
\hline $\begin{array}{l}\text { Change rate } \\
\text { (\%) }\end{array}$ & 95.5 & 75.9 & 71.5 & 77.3 & 75.3 \\
\hline \multicolumn{6}{|l|}{ Weak opioids } \\
\hline 2005 & 620.1 & 555.5 & 554.7 & 334.1 & 461.4 \\
\hline 2015 & 1079.9 & 896.2 & 853.7 & 533.1 & 716.2 \\
\hline $\begin{array}{l}\text { Change rate } \\
\text { (\%) }\end{array}$ & 74.2 & 61.3 & 53.9 & 59.6 & 55.2 \\
\hline Strong opioids & & & & 12.3 & 5.7 \\
\hline 2005 & 4.3 & 8.3 & 10.1 & 31.9 & 17.6 \\
\hline 2015 & 13.4 & 18.6 & 25.7 & 159.1 & 209.8 \\
\hline $\begin{array}{l}\text { Change rate } \\
\text { (\%) }\end{array}$ & 212.2 & 123.1 & 153.7 & & \\
\hline
\end{tabular}

Strong opioid prescribing was less prevalent in the most deprived areas of BCUHB than in WIMD4 areas - the opposite of weak opioid prescribing (Table I.42). However, there was a $212 \%$ increase (from 4.3 to 13.4 prescriptions per 1000 population) in WIMD1 areas between 2005 and 2015 (Figure I.27). There was a reduction in the difference between the number of strong opioid prescriptions in WIMD4 versus WIMD1areas over the study period, from 2.9 to 2.4 times more respectively. 

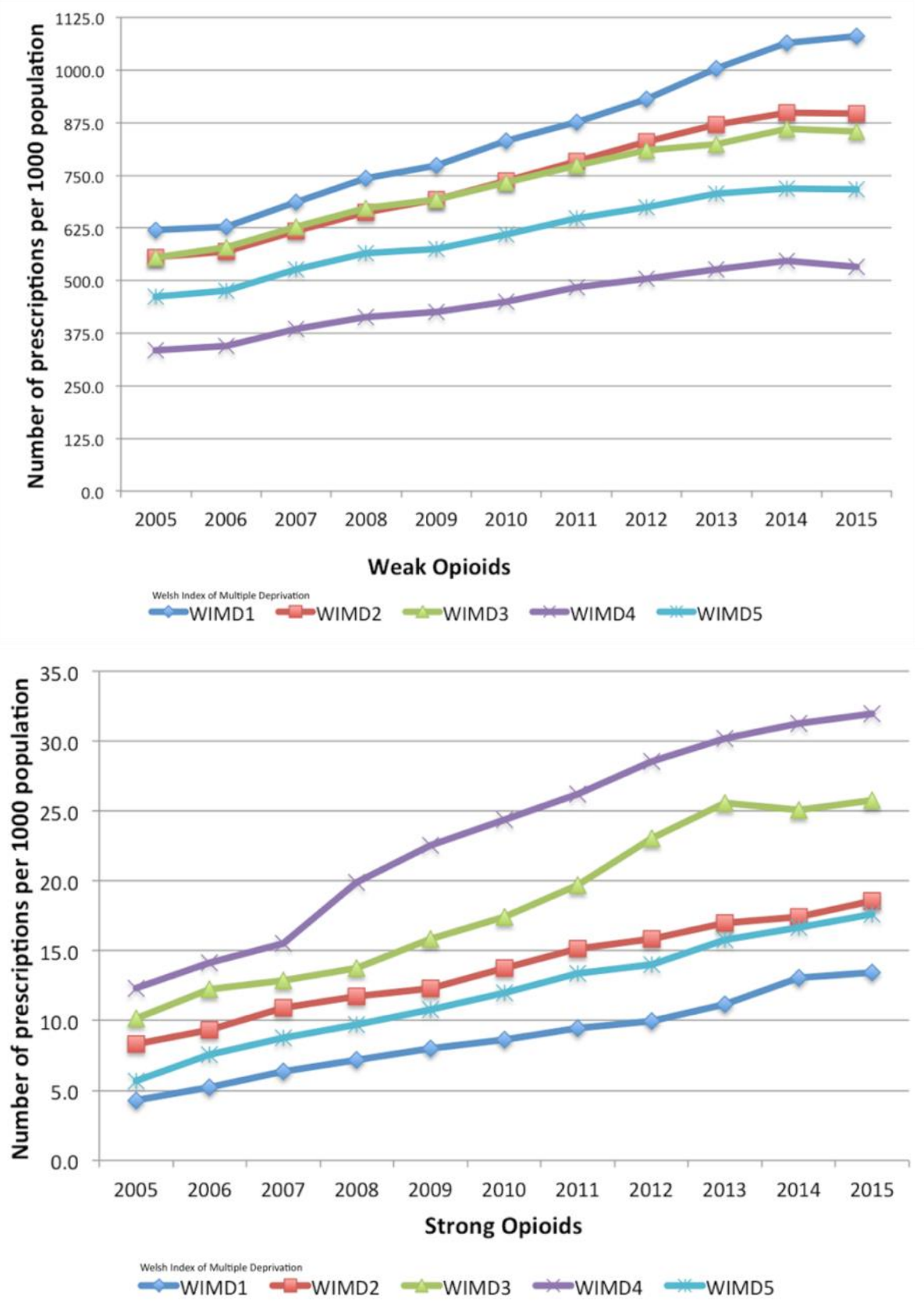

Figure 1.27: Trends in the number of weak or strong opioid prescriptions issued per 1000 population in Betsi Cadwaladr University Health Board by deprivation area (WIMD)

Welsh Index of Multiple Deprivation (WIMD) where WIMD1 = most deprived and WIMD5 = least deprived

\section{I.7 Cardiff and Vale University Health Board}

The number of people receiving opioid prescriptions in the designated areas of CVUHB rose by $18 \%$ between 2005 and 2015 (from 40,891 to 48,367) or 15.7\% (from 107.8 to 124.7 people per 1000 population) when adjusted to the population.

There was maintained throughout the 11 years examined, a 1.7 times difference between the numbers of people (per thousand) receiving opioid prescriptions in the most deprived areas compared to the least (Table I.43). 
Table I.43: Annual change in the number of people per 1000 population receiving opioid prescriptions and annual change rate (percentage change) in Cardiff and Vale University Health Board (CVUHB)

\begin{tabular}{rcccccccccc}
\hline & \multicolumn{2}{c}{ WIMD1 } & \multicolumn{2}{c}{ WIMD2 } & \multicolumn{2}{c}{ WIMD3 } & \multicolumn{2}{c}{ WIMD4 } & \multicolumn{2}{c}{ WIMD5 } \\
\cline { 2 - 11 } & $\begin{array}{c}\text { People } \\
\text { per } \\
1000\end{array}$ & $\begin{array}{c}\text { Change } \\
\text { rate } \\
(\%)\end{array}$ & $\begin{array}{c}\text { People } \\
\text { per } \\
1000\end{array}$ & $\begin{array}{c}\text { Change } \\
\text { rate } \\
(\%)\end{array}$ & $\begin{array}{c}\text { People } \\
\text { per } \\
1000\end{array}$ & $\begin{array}{c}\text { Change } \\
\text { rate } \\
(\%)\end{array}$ & $\begin{array}{c}\text { People } \\
\text { per } \\
1000\end{array}$ & $\begin{array}{c}\text { Change } \\
\text { rate } \\
(\%)\end{array}$ & $\begin{array}{c}\text { People } \\
\text { per } \\
1000\end{array}$ & $\begin{array}{c}\text { Change } \\
\text { rate } \\
(\%)\end{array}$ \\
\hline $\mathbf{2 0 0 5}$ & 144.7 & & 129.0 & & 106.3 & & 95.3 & & 84.9 & \\
$\mathbf{2 0 0 6}$ & 149.9 & 3.6 & 128.7 & -0.2 & 106.2 & -0.1 & 98.8 & 3.7 & 87.1 & 2.5 \\
$\mathbf{2 0 0 7}$ & 154.4 & 3.0 & 136.1 & 5.7 & 113.6 & 7.0 & 105.8 & 7.0 & 92.8 & 6.5 \\
$\mathbf{2 0 0 8}$ & 160.6 & 4.0 & 142.3 & 4.6 & 119.3 & 5.0 & 110.5 & 4.4 & 96.2 & 3.7 \\
$\mathbf{2 0 0 9}$ & 172.6 & 7.5 & 154.0 & 8.2 & 127.9 & 7.2 & 121.3 & 9.8 & 104.7 & 8.9 \\
$\mathbf{2 0 1 0}$ & 172.9 & 0.2 & 154.6 & 0.3 & 129.9 & 1.6 & 122.4 & 0.9 & 106.2 & 1.4 \\
$\mathbf{2 0 1 1}$ & 172.0 & -0.5 & 158.4 & 2.5 & 130.8 & 0.7 & 120.7 & -1.3 & 104.9 & -1.2 \\
$\mathbf{2 0 1 2}$ & 176.3 & 2.5 & 162.5 & 2.6 & 131.2 & 0.3 & 122.3 & 1.3 & 105.3 & 0.4 \\
$\mathbf{2 0 1 3}$ & 174.1 & -1.3 & 157.2 & -3.3 & 130.6 & -0.5 & 118.9 & -2.7 & 100.6 & -4.5 \\
$\mathbf{2 0 1 4}$ & 170.6 & -2.0 & 159.2 & 1.3 & 130.1 & -0.3 & 115.7 & -2.7 & 98.5 & -2.0 \\
$\mathbf{2 0 1 5}$ & 163.6 & -4.1 & 153.6 & -3.5 & 128.2 & -1.5 & 114.0 & -1.5 & 95.9 & -2.6 \\
\hline Change & 13.1 & & 19.1 & & 20.6 & & 19.6 & & 13.0 & \\
$(\%)$ & & & & & & & & & &
\end{tabular}
(\%)

Welsh Index of Multiple Deprivation (WIMD) where WIMD1 = most deprived

Whilst the most deprived areas in CVUHB had the highest number of people receiving opioid prescriptions, it was observed that those same areas had the lowest rate of increase over the study period. Deprivation areas WIMD2, 3 and 4 had similar rates of increase in the number of people receiving opioid prescriptions. However, there was around $26 \%$ difference in numbers between WIMD2 and WIMD4 areas (Table I.43).

Cardiff and Vale University Health Board (CVUHB) had 2,923,204 opioid prescriptions issued between 2005 and 2015, representing 13.3\% of prescriptions in Wales over that time ( $12.9 \%$ when adjusted to population).

Prescriptions issued within the most deprived areas of CVUHB accounted for $36.4 \%(1,064,009)$ of all prescriptions issued within that Health Board in the study period. The least deprived areas of CVUHB had the second highest number of prescriptions issued, 698,999 (23.9\% of total). However, adjusted to the populations for those particular areas changed these proportions to $29 \%$ (WIMD1) and $12.6 \%$ (WIMD5) respectively.

Table I.44: Changes in the number of opioid prescriptions issued by deprivation area within Cardiff and Vale University Health Board between 2005 and 2015

\begin{tabular}{rccccc}
\hline & \multicolumn{5}{c}{ Number of prescriptions per 1000 } \\
\cline { 2 - 6 } & WIMD1 & WIMD2 & WIMD3 & WIMD4 & WIMD5 \\
\hline All Opioids & & & & & \\
$\mathbf{2 0 0 5}$ & 851.2 & 664.8 & 538.3 & 462.9 & 373.4 \\
Change rate (\%) & 1184.6 & 970.7 & 802.9 & 679.6 & 501.4 \\
\hline Weak opioids & 39.2 & 46.0 & 49.2 & 46.8 & 34.3 \\
$\mathbf{2 0 0 5}$ & 798.7 & 630.4 & 509.6 & 431.8 & 349.2 \\
$\mathbf{2 0 1 5}$ & 952.3 & 791.2 & 651.8 & 557.3 & 419.7 \\
Change rate (\%) & 19.2 & 25.5 & 27.9 & 29.0 & 20.2 \\
\hline Strong opioids & & & & & \\
$\mathbf{2 0 0 5}$ & 52.5 & 34.4 & 28.7 & 31.0 & 24.2 \\
$\mathbf{2 0 1 5}$ & 232.3 & 179.5 & 151.2 & 122.3 & 81.7 \\
\hline
\end{tabular}




$\begin{array}{llllll}\text { Change rate (\%) } & 342.5 & 421.7 & 426.6 & 294.0 & 237.7\end{array}$

All deprivation areas had increases in the number of opioid prescriptions issued although there were marked differences in the number of prescriptions of weak and strong opioids in the most deprived areas compared the areas of least deprivation (Table I.44).

Weak opioid prescriptions were issued 2.3 times more often in the most deprived areas of CVUHB compared to the least deprived areas. The larger percentage increases in annual prescriptions numbers were however, seen in the WIMD areas 2 - 4 i.e. neither the most nor least deprived areas.

Strong opioid prescribing predominated in the areas of greater deprivation with more than double the number of prescriptions issues in the most deprived areas compared to the least deprived (Table I.44). This difference increased from 2.2 to 2.9 times more prescriptions per 1000 population between 2005 and 2015 (Figure I.28).

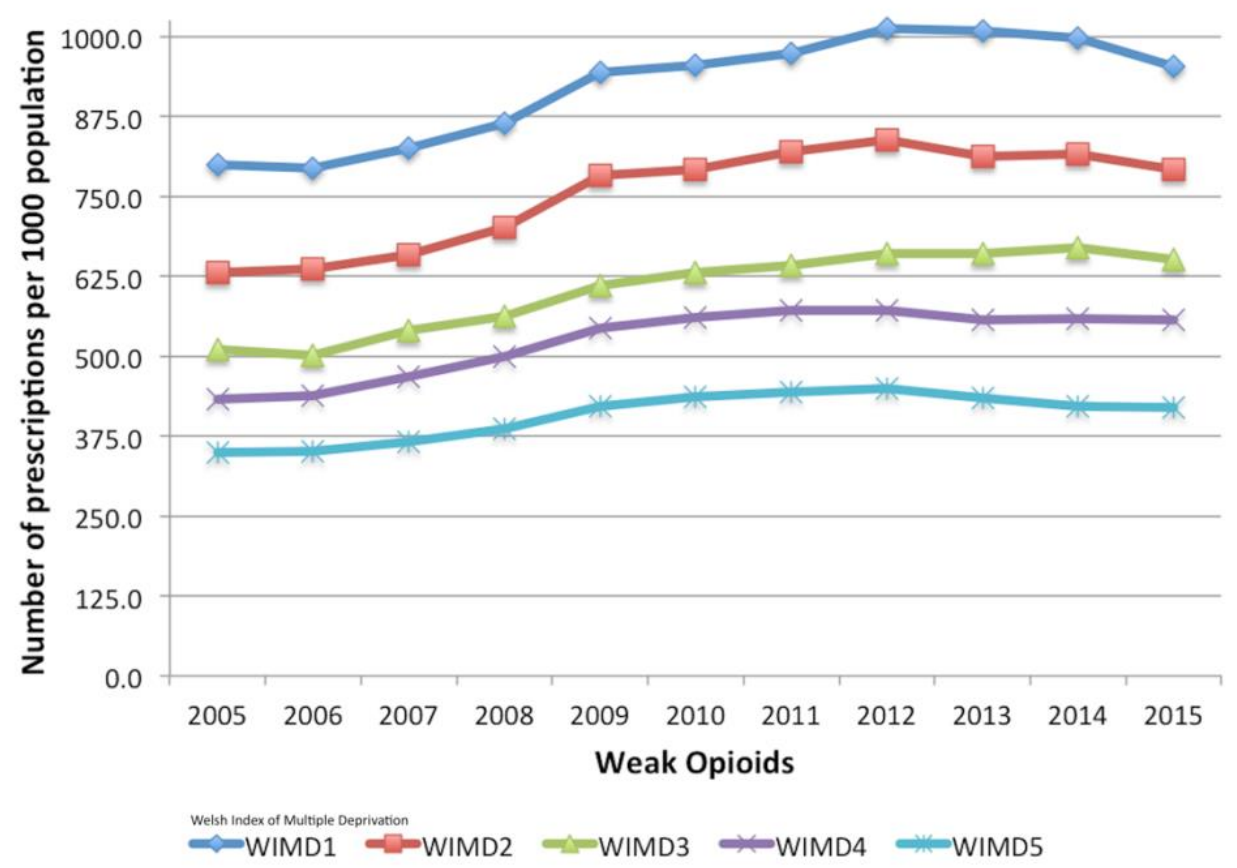




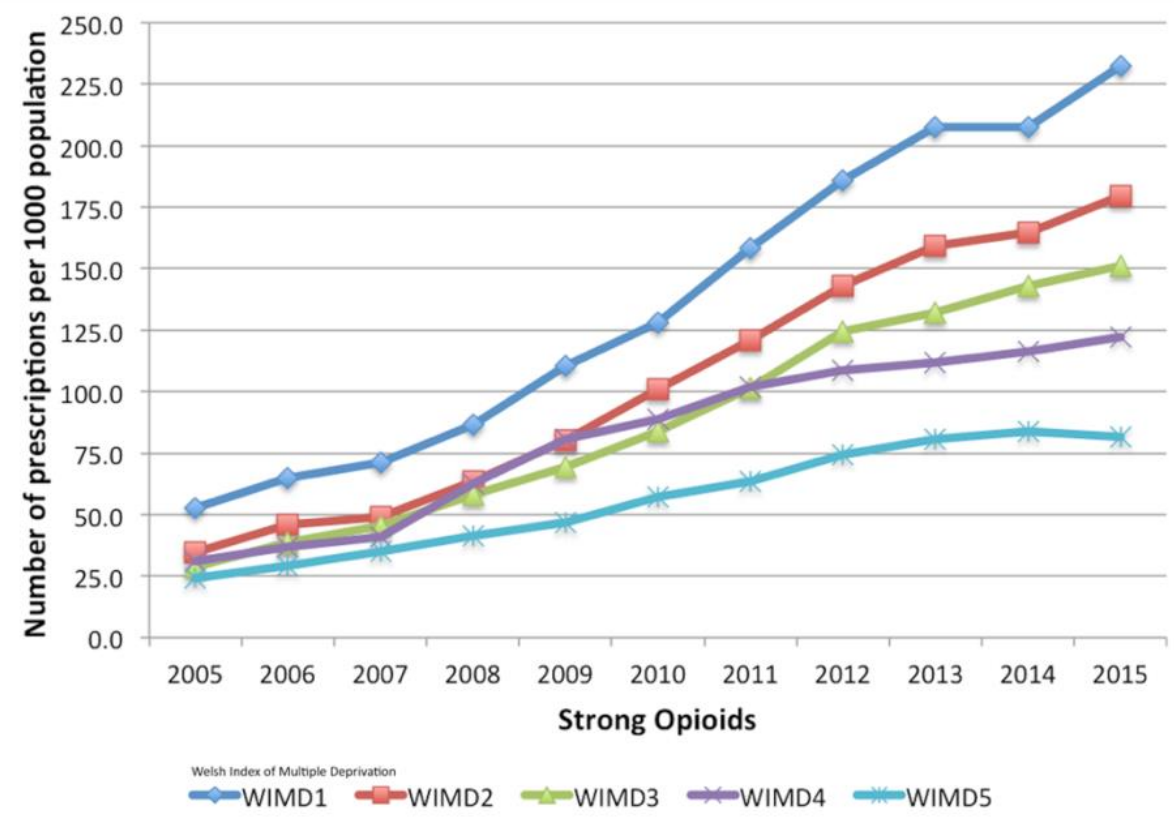

Figure 1.28: Trends in the number of weak or strong opioid prescriptions issued per 1000 population in Cardiff and Vale University Health Board by deprivation area (WIMD)

Welsh Index of Multiple Deprivation (WIMD) where WIMD1 = most deprived and WIMD5 = least deprived

\section{I.8 Cwm Taf University Health Board}

There was a $13.7 \%$ increase in the number of people receiving opioid prescriptions each year in designated deprivation areas, across the whole of Cwm Taf University Health Board (CTUHB) between 2005 and 2015 (13.8\% population adjusted increase).

Table 1.45: Annual change in the number of people per 1000 population receiving opioid prescriptions and annual change rate (percentage change) in Cwm Taf University Health Board (CTUHB)

\begin{tabular}{|c|c|c|c|c|c|c|c|c|c|c|}
\hline & \multicolumn{10}{|c|}{ Welsh Index of Multiple Deprivation (WIMD) } \\
\hline & \multicolumn{2}{|c|}{1} & \multicolumn{2}{|c|}{2} & \multicolumn{2}{|c|}{3} & \multicolumn{2}{|c|}{4} & \multicolumn{2}{|c|}{5} \\
\hline & $\begin{array}{c}\text { People } \\
\text { per } \\
1000\end{array}$ & $\begin{array}{c}\text { Change } \\
\text { rate } \\
(\%)\end{array}$ & $\begin{array}{c}\text { People } \\
\text { per } \\
1000\end{array}$ & $\begin{array}{c}\text { Change } \\
\text { rate } \\
(\%)\end{array}$ & $\begin{array}{c}\text { People } \\
\text { per } \\
1000\end{array}$ & $\begin{array}{c}\text { Change } \\
\text { rate } \\
(\%)\end{array}$ & $\begin{array}{c}\text { People } \\
\text { per } \\
1000\end{array}$ & $\begin{array}{c}\text { Change } \\
\text { rate } \\
(\%)\end{array}$ & $\begin{array}{c}\text { People } \\
\text { per } \\
1000\end{array}$ & $\begin{array}{c}\text { Change } \\
\text { rate } \\
(\%)\end{array}$ \\
\hline 2005 & 188.6 & & 108.2 & & 157.3 & & 116.4 & & 103.3 & \\
\hline 2006 & 186.8 & -0.9 & 107.9 & -0.3 & 157.9 & 0.4 & 113.4 & -2.6 & 105.8 & 2.4 \\
\hline 2007 & 193.6 & 3.6 & 112.8 & 4.5 & 167.3 & 5.9 & 122.5 & 8.0 & 117.3 & 10.9 \\
\hline 2008 & 199.1 & 2.9 & 114.8 & 1.8 & 171.4 & 2.5 & 127.6 & 4.2 & 120.6 & 2.8 \\
\hline 2009 & 210.2 & 5.6 & 121.2 & 5.5 & 180.7 & 5.4 & 136.3 & 6.9 & 131.7 & 9.2 \\
\hline 2010 & 209.6 & -0.3 & 123.2 & 1.6 & 182.6 & 1.0 & 139.9 & 2.6 & 134.0 & 1.7 \\
\hline 2011 & 214.1 & 2.1 & 127.6 & 3.6 & 191.8 & 5.0 & 136.6 & -2.4 & 136.0 & 1.5 \\
\hline 2012 & 217.4 & 1.6 & 130.0 & 1.9 & 192.1 & 0.1 & 137.5 & 0.7 & 136.8 & 0.6 \\
\hline 2013 & 214.9 & -1.2 & 127.5 & -1.9 & 187.5 & -2.4 & 137.3 & -0.2 & 133.4 & -2.5 \\
\hline 2014 & 211.5 & -1.6 & 124.4 & -2.4 & 189.2 & 0.9 & 134.2 & -2.2 & 132.2 & -0.9 \\
\hline 2015 & 210.4 & -0.5 & 121.8 & -2.1 & 181.2 & -4.2 & 130.6 & -2.7 & 130.1 & -1.6 \\
\hline Change & 11.6 & & 12.6 & & 15.2 & & 12.2 & & 25.9 & \\
\hline
\end{tabular}

Welsh Index of Multiple Deprivation (WIMD) where WIMD1 = most deprived and WIMD5 = least deprived

The areas of CTUHB of least deprivation had the largest increase $(25.9 \%$, from 103.3 to 130.1 people per 1000 ) in the annual number of people receiving opioid prescriptions. However, there were 1.8 times more people receiving opioid 
prescriptions in the most deprived areas compared to the least, in 2005, which changed to 1.6 times more in 2015 (Table I.45).

There was a peak in the number of people receiving opioid prescriptions in 2012, in all areas of CTUHB.

Cwm Taf Local Health Board had a total of 2,600,096 opioid prescriptions issued between 2005 and 2015; equivalent to $11.8 \%$ of the total issued in Wales during that time. This figure was $15.8 \%$ when adjusted to population. Prescribing increased by $28.8 \%$ (from 204,369 to 263,317 ) over the period examined. This was adjusted by population to $28.9 \%$ (from 893.9 to 1152.4 prescriptions per 1000 population) which, was the lowest percentage increase of any Health Board in Wales.

As with other Health Boards, the highest number of prescriptions was issued in the most deprived areas of CTUHB (Table I.46). The second highest area of prescribing was WIMD3 although these areas has around $25 \%$ fewer prescriptions issued than the most deprived areas during the study period.

Table 1.46: Changes in the number of opioid prescriptions issued by deprivation area within Cwm Taf University Health Board between 2005 and 2015

\begin{tabular}{rccccc}
\hline & \multicolumn{5}{c}{ Number of prescriptions per 1000 } \\
\cline { 2 - 6 } & WIMD1 & WIMD2 & WIMD3 & WIMD4 & WIMD5 \\
\hline All Opioids & & & & & \\
$\mathbf{2 0 0 5}$ & 1292.4 & 701.1 & 1014.1 & 628.0 & 486.5 \\
$\mathbf{2 0 1 5}$ & 1646.1 & 901.2 & 1244.7 & 830.2 & 750.6 \\
Change rate (\%) & 27.4 & 28.5 & 22.7 & 32.2 & 54.3 \\
\hline Weak opioids & & & & & \\
$\mathbf{2 0 0 5}$ & 1223.6 & 666.3 & 965.8 & 590.7 & 459.2 \\
$\mathbf{2 0 1 5}$ & 1432.3 & 801.8 & 1096.3 & 734.6 & 672.1 \\
Change rate (\%) & 17.1 & 20.3 & 13.5 & 24.4 & 46.4 \\
\hline Strong opioids & & & & & \\
$\mathbf{2 0 0 5}$ & 43.8 & 70.8 & 55.1 & 44.1 & 11.1 \\
$\mathbf{2 0 1 5}$ & 247.7 & 224.1 & 195.0 & 151.5 & 37.8 \\
Change rate (\%) & 466.2 & 216.5 & 253.7 & 243.8 & 241.1 \\
\hline
\end{tabular}

Population adjusted data presented and change rate calculated between 2005 and 2015

Weak opioid prescribing increased in all areas of CTUHB. The least deprived areas of the Health Board had the greatest increase in the number of weak opioid prescriptions issued although there were 2.6 times more prescriptions issued in the most deprived areas in 2005 which, changed to 2.2 times more in 2015 (Table I.46).

Strong opioid prescribing was highest in WIMD2 areas of CTUHB in 2005 (Table I.46). However, due to a significant increase (466.2\%, from 43.8 to 247.7 prescriptions per 1000 population) in the most deprived areas, by 2015 the highest number of strong opioid prescriptions were being issued there. Although there was a substantial increase in the number of strong opioid prescriptions issued in the least deprived areas of CTUHB, there 6.6 times more prescriptions issued in the areas of highest prescribing in 2015 (Figure I.29). 

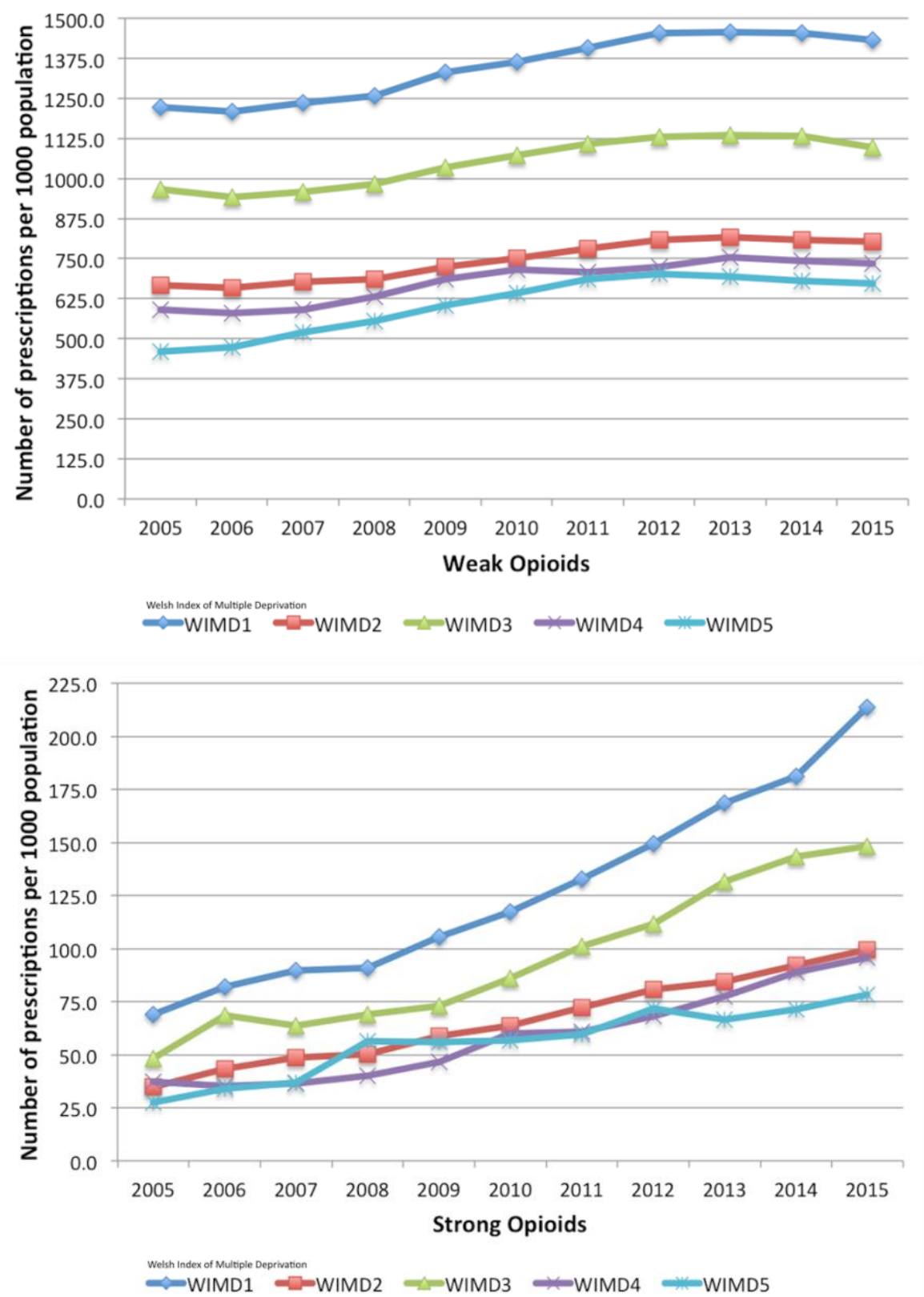

Figure I.29: Trends in the number of weak or strong opioid prescriptions issued per 1000 population in Cwm Taf University Health Board by deprivation area (WIMD)

Welsh Index of Multiple Deprivation (WIMD) where WIMD1 = most deprived and WIMD5 = least deprived

\section{I.9 Hywel Dda Local Health Board}

Hywel Dda LHB had a $15.6 \%$ increase in the annual number of people receiving opioid prescriptions between 2005 and 2015 (from 27,732 to 32,051). When adjusted to the annual Health Board population, this was $7.5 \%$ (from 96.1 to 103.3 people per 1000 population).

Table I.47: Annual change in the number of people per 1000 population receiving opioid prescriptions and annual change rate (percentage change) in Hywel Dda University Health Board (HDUHB) 


\begin{tabular}{rcccccccccc}
\hline & $\begin{array}{c}\text { People } \\
\text { per } \\
1000\end{array}$ & $\begin{array}{c}\text { Change } \\
\text { rate } \\
(\%)\end{array}$ & $\begin{array}{c}\text { People } \\
\text { per } \\
1000\end{array}$ & $\begin{array}{c}\text { Change } \\
\text { rate } \\
(\%)\end{array}$ & $\begin{array}{c}\text { People } \\
\text { per } \\
1000\end{array}$ & $\begin{array}{c}\text { Change } \\
\text { rate } \\
(\%)\end{array}$ & $\begin{array}{c}\text { People } \\
\text { per } \\
1000\end{array}$ & $\begin{array}{c}\text { Change } \\
\text { rate } \\
(\%)\end{array}$ & $\begin{array}{c}\text { People } \\
\text { per } \\
1000\end{array}$ & $\begin{array}{c}\text { Change } \\
\text { rate } \\
(\%)\end{array}$ \\
\hline $\mathbf{2 0 0 5}$ & 111.4 & & 113.2 & & 109.7 & & 99.0 & & 31.5 & \\
$\mathbf{2 0 0 6}$ & 110.7 & -0.6 & 112.6 & -0.5 & 111.6 & 1.7 & 99.5 & 0.5 & 32.0 & 1.6 \\
$\mathbf{2 0 0 7}$ & 114.8 & 3.7 & 120.1 & 6.7 & 115.7 & 3.6 & 106.3 & 6.8 & 34.5 & 7.7 \\
$\mathbf{2 0 0 8}$ & 119.4 & 4.1 & 121.5 & 1.1 & 121.7 & 5.2 & 111.5 & 4.9 & 35.0 & 1.6 \\
$\mathbf{2 0 0 9}$ & 119.6 & 0.2 & 121.7 & 0.2 & 119.1 & -2.2 & 109.6 & -1.7 & 35.7 & 1.9 \\
$\mathbf{2 0 1 0}$ & 119.9 & 0.2 & 123.7 & 1.6 & 122.8 & 3.2 & 111.8 & 2.1 & 36.2 & 1.3 \\
$\mathbf{2 0 1 1}$ & 124.6 & 3.9 & 124.0 & 0.2 & 124.0 & 1.0 & 114.0 & 2.0 & 35.0 & -3.1 \\
$\mathbf{2 0 1 2}$ & 122.7 & -1.5 & 124.1 & 0.1 & 121.0 & -2.4 & 114.3 & 0.2 & 33.3 & -5.0 \\
$\mathbf{2 0 1 3}$ & 124.1 & 1.1 & 126.7 & 2.1 & 119.5 & -1.3 & 105.1 & -8.1 & 30.9 & -7.2 \\
$\mathbf{2 0 1 4}$ & 128.0 & 3.1 & 126.9 & 0.2 & 119.5 & 0.0 & 103.5 & -1.5 & 29.2 & -5.6 \\
$\mathbf{2 0 1 5}$ & 125.4 & -2.0 & 127.8 & 0.7 & 119.6 & 0.1 & 101.3 & -2.1 & 28.2 & -3.3 \\
\hline Change & 12.6 & & 12.9 & & 9.0 & & 2.3 & & -10.5 & \\
(\%) & & & & & & & & & \\
\hline
\end{tabular}

Welsh Index of Multiple Deprivation (WIMD) where WIMD1 = most deprived and WIMD5 = least deprived

The least deprived areas of Hywel Dda University Health Board saw a reduction in the numbers of people receiving opioid prescriptions over the study period. By 2015, there were 4.5 times more people receiving prescriptions in the areas of highest numbers (WIMD2) compared to the areas with the least population receiving prescriptions (Table 1.47).

The areas which had the highest number of people to whom opioid prescriptions were issued (WIMD2), also had the higher increase in numbers between 2005 and $2015,12.9 \%$ (from 113.2 to 127.8 people per 1000 population).

A total of 2,325,837 opioid prescriptions were issued within the Hywel Dda Local Health Board (HDLHB) area in the eleven years examined by this study. That figure corresponds to $10.6 \%$ of all opioid prescriptions in Wales or $11.4 \%$ when adjusted to the population.

The annual number of prescriptions issued in HDUHB rose $49.9 \%$ over the period (from 168,515 to 252,654 ). This was adjusted to $45.5 \%$ by population (from 2877.1 to 4186 prescriptions per 1000 population).

Table I.48: Changes in the number of opioid prescriptions issued by deprivation area within Cwm Taf University Health Board between 2005 and 2015

\begin{tabular}{|c|c|c|c|c|c|}
\hline & \multicolumn{5}{|c|}{ Number of prescriptions per 1000} \\
\hline & WIMD1 & WIMD2 & WIMD3 & WIMD4 & WIMD5 \\
\hline \multicolumn{6}{|l|}{ All Opioids } \\
\hline 2005 & 734.3 & 759.4 & 655.7 & 560.9 & 166.8 \\
\hline 2015 & 1253.6 & 1106.4 & 887.0 & 735.3 & 203.7 \\
\hline Change rate $(\%)$ & 70.7 & 45.7 & 35.3 & 31.1 & 22.1 \\
\hline \multicolumn{6}{|l|}{ Weak opioids } \\
\hline 2005 & 690.5 & 688.6 & 600.6 & 516.9 & 155.7 \\
\hline 2015 & 1005.9 & 882.4 & 692.0 & 583.8 & 165.9 \\
\hline Change rate (\%) & 45.7 & 28.1 & 15.2 & 12.9 & 6.5 \\
\hline \multicolumn{6}{|l|}{ Strong opioids } \\
\hline 2005 & 43.8 & 70.8 & 55.1 & 44.1 & 11.1 \\
\hline 2015 & 247.7 & 224.1 & 195.0 & 151.5 & 37.8 \\
\hline Change rate (\%) & 466.2 & 216.5 & 253.7 & 243.8 & 241.1 \\
\hline
\end{tabular}

Population adjusted data presented and change rate calculated between 2005 and 2015 
There were increases in weak opioid prescribing in all areas within HDUHB. The largest increase in prescribing was in the area of greatest deprivation (Table I.48) which also had 4 times the number of prescriptions issued in 2005 compared to the area with least number of prescriptions (WIMD5).

The annual number of weak opioid prescription rose least in the area of least deprivation. Subsequently, this led to a 6 times difference in the number of weak opioid prescriptions issued in the most deprived areas of HBUHB compared to the least deprived (Table I.48).

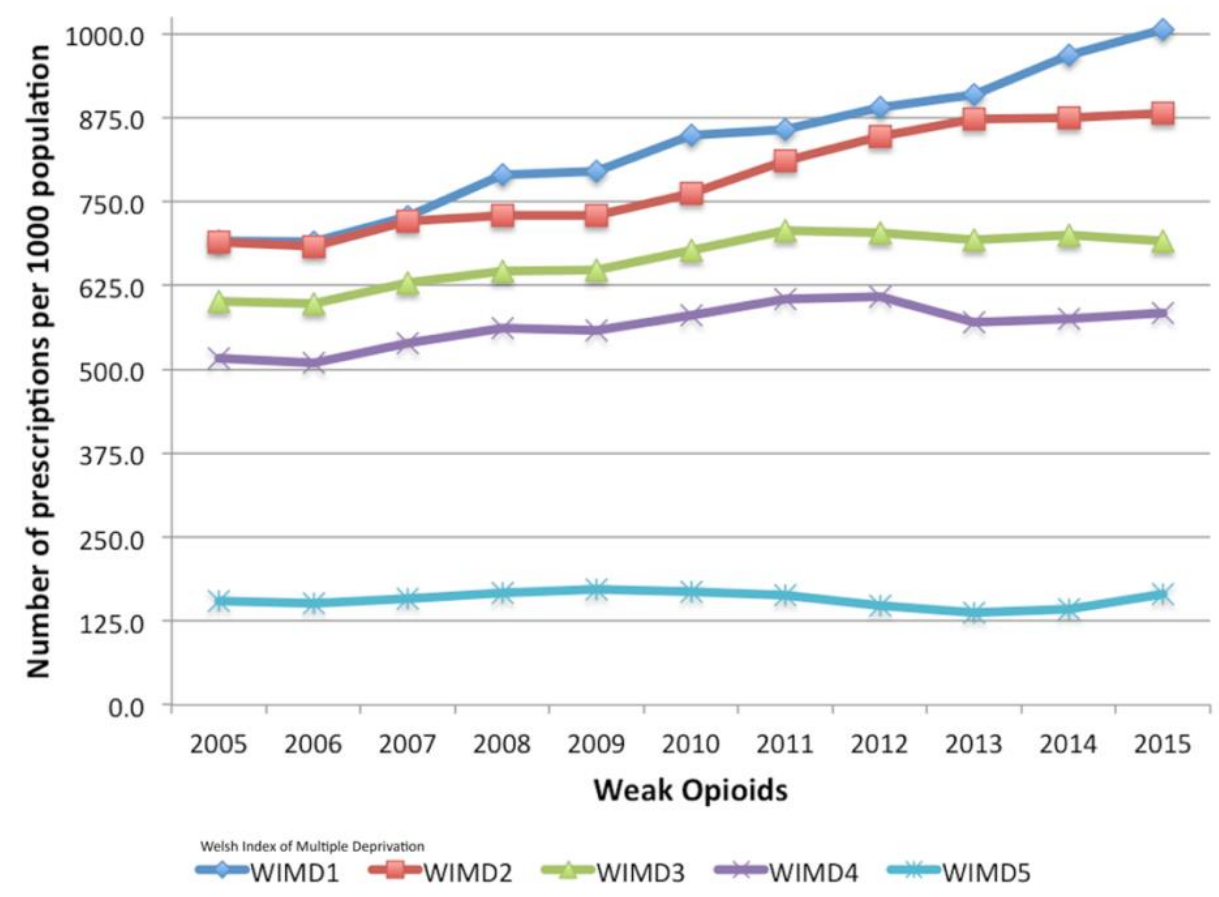




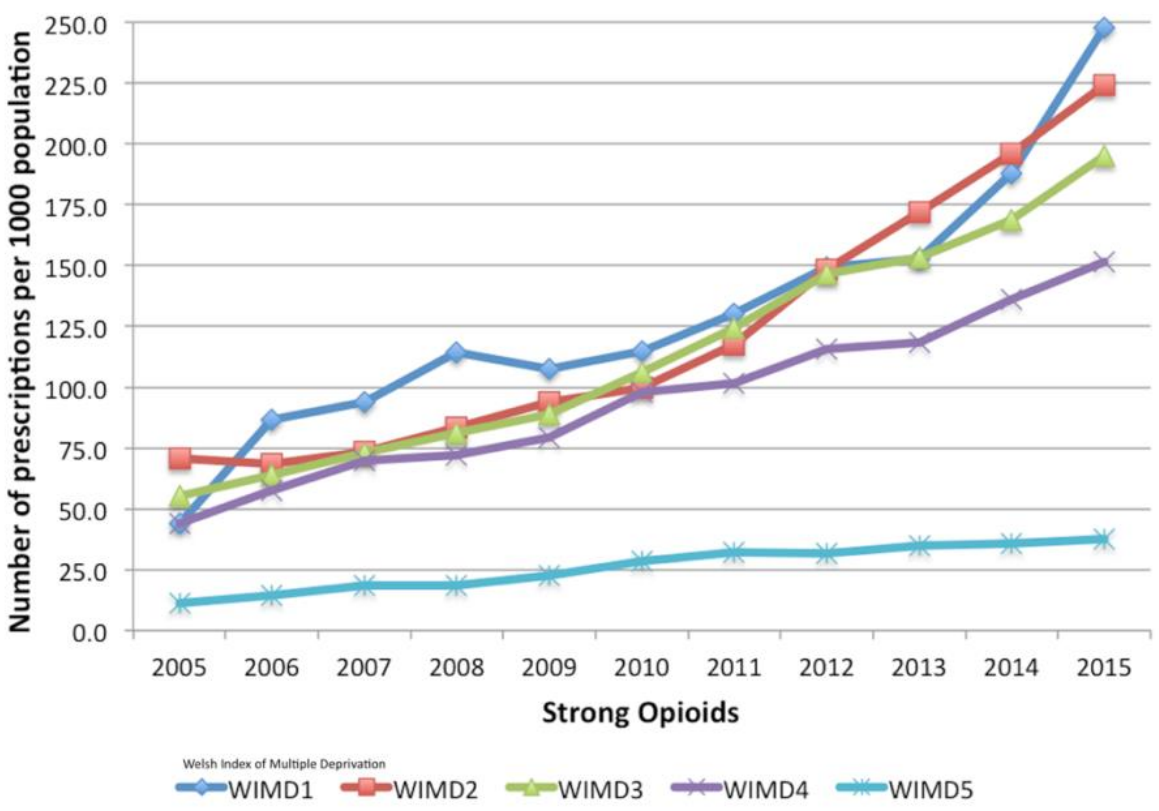

Figure I.30: Trends in the number of weak or strong opioid prescriptions issued per 1000 population in Hywel Dda University Health Board by deprivation area (WIMD)

Welsh Index of Multiple Deprivation (WIMD) where WIMD1 = most deprived and WIMD5 = least deprived

Strong opioid prescribing increased significantly in all areas of HDUHB but predominated in areas of greater deprivation levels. Prescribing in the areas of greatest deprivation were lower in 2005 than in three other categorised areas (WIMD2, 3 and 4). However, due to an increase in the number of annual prescriptions of $466.2 \%$ over the study period; by 2015 the number of prescriptions issued in the most deprived areas was the highest in the Health Board and 6.6 times more prescriptions than in the least deprived area where fewest prescriptions were issued (Figure I.30).

\section{I.10 Powys Teaching Health Board}

The predominantly rural Powys Teaching Health Board (PTHB) appeared, based on actual numbers of people, to have the fewest people in Wales receiving opioid prescriptions. There were 439,916 opioid prescriptions issued in PTHB during the period surveyed; $2 \%$ of the total in Wales. However, when adjusted to population this rose to $14.2 \%$ of people per 1000 population, which placed it above more populated Health Boards such as Betsi Cadwaladr and Cardiff and Vale.

Annual numbers of people rose $27.2 \%$ (from 5,712 to 7,272 ) over the 11 years or 13.9\% (from 117.4 to 133.7 people per 1000 ) when adjusted to population.

Table I.49: Annual change in the number of people per 1000 population receiving opioid prescriptions and annual change rate (percentage change) in Powys Teaching Health Board (PTHB)

\begin{tabular}{ccccccccccc}
\multicolumn{10}{c}{ Welsh Index of Multiple Deprivation (WIMD) } \\
\hline \multicolumn{10}{c}{2} & \multicolumn{2}{c}{3} & \multicolumn{4}{c}{4} & \multicolumn{2}{c}{5} \\
\hline People & Change & People & Change & People & Change & People & Change & People & Change \\
per & rate & per & rate & per & rate & per & rate & per & rate \\
1000 & $(\%)$ & 1000 & $(\%)$ & 1000 & $(\%)$ & 1000 & $(\%)$ & 1000 & $(\%)$ \\
\hline
\end{tabular}




\begin{tabular}{rcccccccccc}
$\mathbf{2 0 0 5}$ & 230.5 & & 288.7 & & 156.4 & & 69.1 & & 75.8 & \\
$\mathbf{2 0 0 6}$ & 231.2 & 0.3 & 302.0 & 4.6 & 160.6 & 2.7 & 74.5 & 7.9 & 76.4 & 0.8 \\
$\mathbf{2 0 0 7}$ & 247.5 & 7.0 & 321.3 & 6.4 & 172.3 & 7.3 & 85.9 & 15.2 & 89.7 & 17.4 \\
$\mathbf{2 0 0 8}$ & 245.3 & -0.9 & 342.6 & 6.6 & 179.1 & 3.9 & 91.1 & 6.0 & 101.2 & 12.8 \\
$\mathbf{2 0 0 9}$ & 240.5 & -2.0 & 320.9 & -6.3 & 164.1 & -8.4 & 88.1 & -3.3 & 99.7 & -1.5 \\
$\mathbf{2 0 1 0}$ & 228.1 & -5.2 & 326.2 & 1.7 & 167.6 & 2.1 & 92.2 & 4.7 & 109.8 & 10.1 \\
$\mathbf{2 0 1 1}$ & 235.1 & 3.1 & 342.9 & 5.1 & 172.1 & 2.7 & 94.9 & 3.0 & 117.1 & 6.7 \\
$\mathbf{2 0 1 2}$ & 252.3 & 7.3 & 364.5 & 6.3 & 176.5 & 2.6 & 91.7 & -3.5 & 118.0 & 0.7 \\
$\mathbf{2 0 1 3}$ & 259.2 & 2.7 & 374.7 & 2.8 & 173.3 & -1.9 & 93.0 & 1.4 & 112.3 & -4.8 \\
$\mathbf{2 0 1 4}$ & 227.9 & -12.1 & 343.2 & -8.4 & 159.7 & -7.8 & 87.3 & -6.1 & 118.8 & 5.7 \\
$\mathbf{2 0 1 5}$ & 227.6 & -0.2 & 338.2 & -1.5 & 159. & 0.1 & 83.3 & -4.6 & 106.7 & -10.2 \\
\hline Change & -1.3 & & 17.1 & & 2.2 & & 20.5 & & 40.8 & \\
$(\%)$ & & & & & & & & &
\end{tabular}

Welsh Index of Multiple Deprivation (WIMD) where WIMD1 = most deprived and WIMD5 = least deprived

More people residing in WIMD2 areas received prescriptions for opioids than those in the most deprived areas or those living WIMD4 areas where fewest people were issued prescriptions. Four times more people received prescriptions in the WIMD2 area compared to WIMD4 areas (Table I.49) across the study period. There was a small reduction in the annual number of people in the most deprived areas of PTHB receiving opioid prescriptions between 2005 and 2015 (Table I.49).

Powys Teaching Health Board had an increase of $77.1 \%$ (from 26,646 to 47,183 ) in annual opioid prescription numbers between 2005 and 2015. When adjusted to population, this was $58.6 \%$ (from 547.2 to 867.6 prescriptions per 1000 ), which was the second highest increase in Wales over the time period studied.

Although the most deprived areas of PTHB were not the areas with the highest numbers of prescriptions, there were more than 3 times the number of prescriptions issued in WIMD1 areas compared to WIMD5 (Table I.50).

Table 1.50: Changes in the number of opioid prescriptions issued by deprivation area within Powys Teaching Board between 2005 and 2015

\begin{tabular}{rccccc}
\hline & \multicolumn{5}{c}{ Number of prescriptions per 1000 } \\
\cline { 2 - 6 } & WIMD1 & WIMD2 & WIMD3 & WIMD4 & WIMD5 \\
\hline All Opioids & & & & \\
$\mathbf{2 0 0 5}$ & 1180.7 & 1364.5 & 774.6 & 300.3 & 309.4 \\
Change rate (\%) & 1905.7 & 2486.0 & 1010.8 & 488.7 & 565.3 \\
\hline Weak opioids & 61.4 & 82.2 & 30.5 & 62.8 & 82.7 \\
$\mathbf{2 0 0 5}$ & 1165.3 & 1325.9 & 721.1 & 280.7 & 292.8 \\
$\mathbf{2 0 1 5}$ & 1468.5 & 2136.1 & 841.3 & 401.5 & 481.1 \\
Change rate (\%) & 26.0 & 61.1 & 16.7 & 43.0 & 64.3 \\
\hline Strong opioids & & & & & \\
$\mathbf{2 0 0 5}$ & 15.4 & 38.6 & 53.5 & 19.5 & 16.6 \\
$\mathbf{2 0 1 5}$ & 437.2 & 349.9 & 169.5 & 87.2 & 84.2 \\
Change rate (\%) & 2739.2 & 806.5 & 216.9 & 346.1 & 406.4 \\
\hline
\end{tabular}

The largest increase in the annual number of weak opioid prescriptions issued in Powys occurred in the least deprived areas (Table 1.50). A similar increase, however, was seen in the annual number of weak opioid prescriptions issued in WIMD2 areas, the areas of highest overall prescription numbers. The areas with 
the highest number of prescriptions issued (WIMD2) had 1.5 times more prescriptions than the next nearest area (WIMD1) of higher prescribing in 2015.
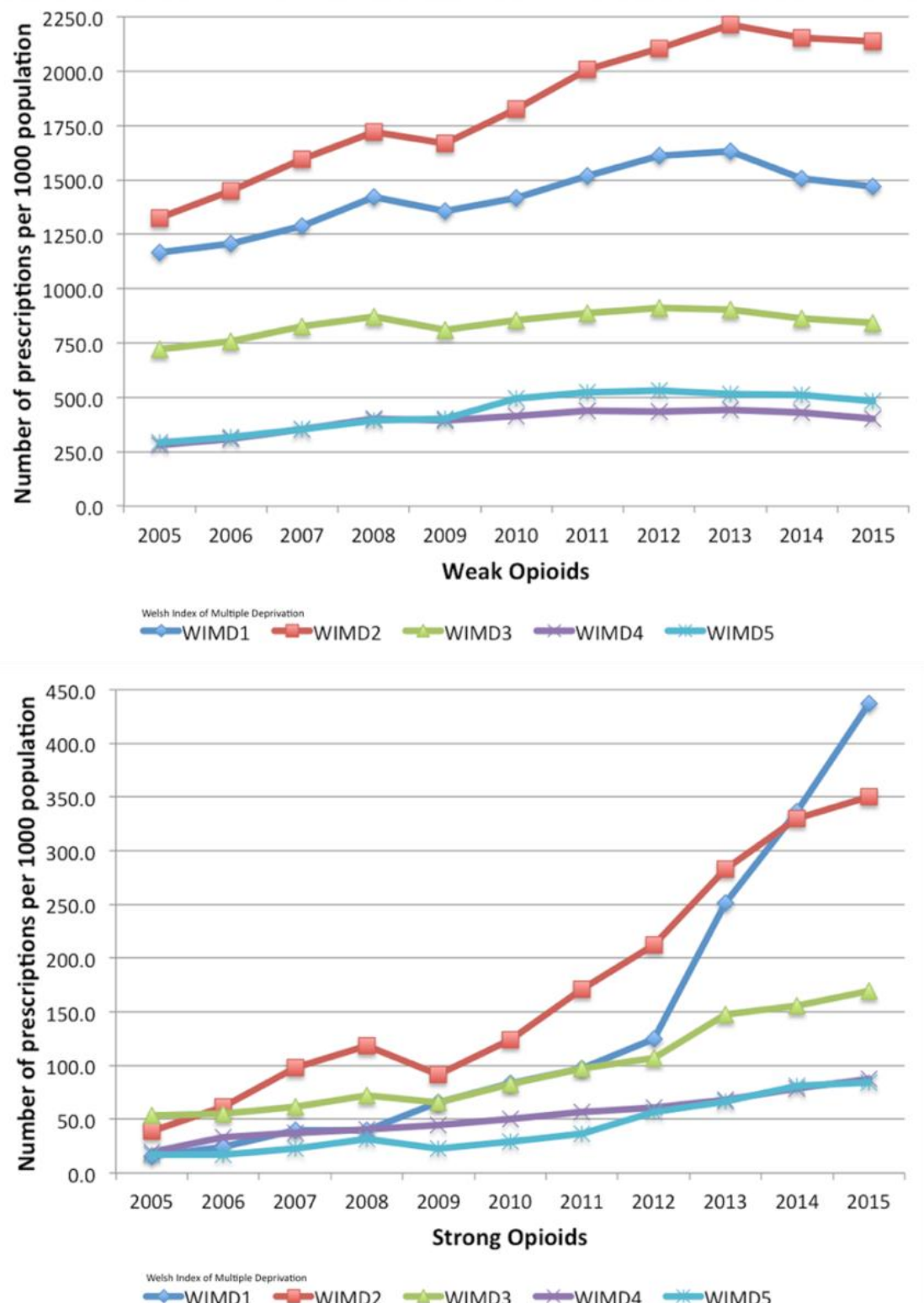

Figure 1.31: Trends in the number of weak or strong opioid prescriptions issued per 1000 population in Powys Teaching Health Board by deprivation area (WIMD)

Welsh Index of Multiple Deprivation (WIMD) where WIMD1 = most deprived and WIMD5 = least deprived

Strong opioid prescribing increased 685\% (from 143.7 to 1128.1 prescriptions per 1000 population) in Powys between 2005 and 2015. The most deprived areas of the Health Board had the least number of prescriptions issued in 2005 but rose significantly (2739.2\%, from 15.4 to 437.2 prescriptions per 1000 population) over the study period. This was the largest increase in strong opioid prescription numbers seen anywhere in Wales in the time period examined. By 2015, there were 5 times more prescriptions being issued in the most deprived areas compared to the least deprived (Table I.50). 


\title{
Appendix J Published papers, abstracts and presentations
}

\author{
2nd Prize, Royal Pharmaceutical Society Winter Conference, 2017
}
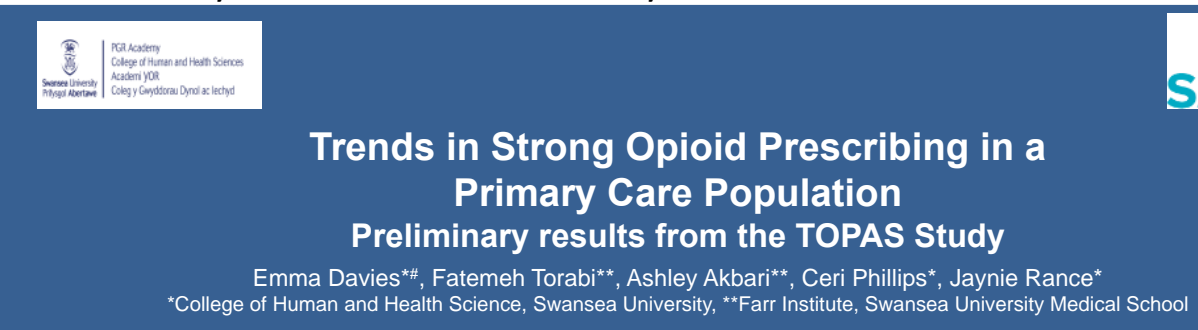

\section{Introduction}

Previous studies have demonstrated dramatic increases in United Kingdom opioid prescribing over the last 20 years ${ }^{1,2}$. Given that up to $44 \%$ of the United Kingdom (UK) population have been estimated to live with pain ${ }^{3}$ and there is little evidence to support the use of opioids in chronic non-cancer pain ${ }^{4}$; it is important to understand more about the people receiving these medicines. This is the first, large-scale observational study of opioid prescribing in Wales.

\section{Method}

Data on all prescriptions for opioid containing medications issued between 2005 and 2015, were taken from the Secure Anonymised Information Linkage databank (SAIL). People with a diagnosis for cancer during the study period were excluded from the detailed analysis. Annual number of prescriptions, numbers of patients, age, gender, duration of prescribing, social deprivation and co-diagnosis of depression were measured in repeated crosssections and analysed using descriptive statistics.

\section{Results}

Between 2005 and 2015 there were $26,180,200$ prescriptions for opioids issued to 1,223,503 people (55.9\% female, $89.9 \%$ non-cancer) in the participating practices in Wales (78\% of all practices). Strong opioid prescriptions for non-cancer pain increased by $323.4 \%$ (from 92,976 to 393,697 ) over the study period and comprised $11.5 \%$ of all opioid prescriptions.
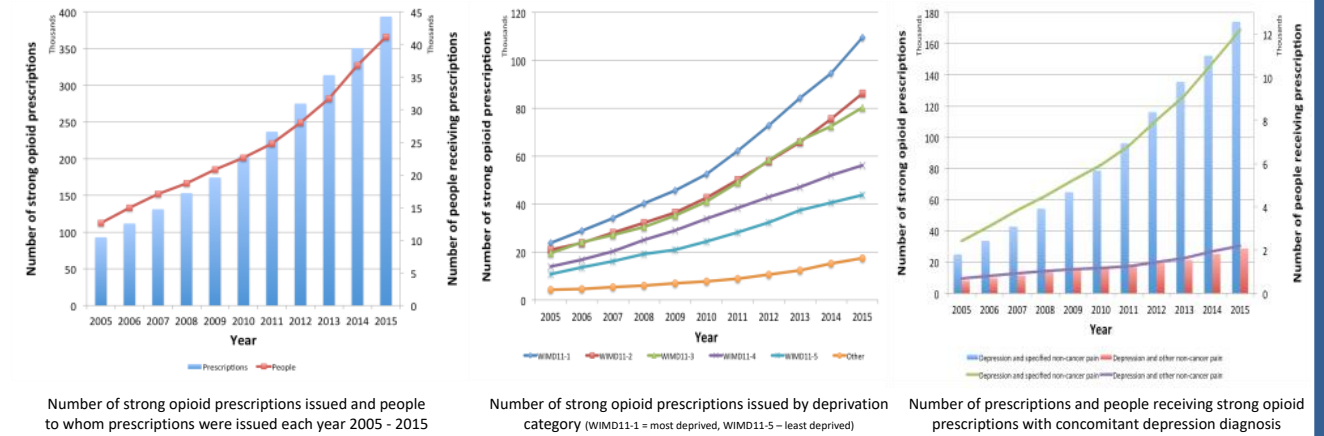

There was a greater increase in opioid prescribing for females than males (233\% compared to $184 \%$ ). The areas with the highest level of deprivation (WIMD11-1) saw the greatest rise in strong opioid prescribing for non-cancer pain (380\% over the study period). There was a 598.4\% increase (from 24,933 to 174,134) in strong opioid prescriptions for people with co-diagnoses of either osteo- or rheumatoid arthritis, back or neck pain, fibromyalgia or neuropathic pain and depression.

\section{Conclusion}

There was an enormous rise in strong opioid prescribing in Wales between 2005 and 2015, in line with findings from other UK-based studies ${ }^{1,2}$. This is the first study of its type in the Principality and highlights some areas of concern, such as the link between strong opioid prescribing and depression. Whilst data quality is determined by the accuracy of the input at source; further analysis is planned to determine the significance of the results from a public health perspective and their clinical impact.

References

Zin, C. S., Chen, L. C. \& Knaggs, R. D. Eur J Pain 18, 1343-1351 (2014)

Ruscitto, A., Smith, B. H. \& Guthrie, B. Eur J Pain 19, 59-66 (2014)

Fayaz, A, P Croft, R M. Langford, L J. Donaldson, and G T. Jones. 2016. BMJ open 6(6):e010364

Chou, Roger, Jane C. Ballantyne, Gilbert J. Fanciullo, Perry G. Fine, and Christine Miaskowski. 2009. J Pain 10(2):147-59.
Acknowledgements

Research costs have been provided via a Galen Personal Pharmacy Research UK Award from Pharmacy Research UK

\#PhD fellowship partly funded by Welsh Government through Health and Care Research Wales and the Research Capacity
Building Collaboration programme

Davies, E. et al. 2017. Abstract: Trends in strong opioid prescribing in a primary care population: preliminary results from the Trends in Opioid Prescribing and Associated Health Care Utilisation in Wales Study (TOPAS). International Journal of Pharmacy Practice 25(Suppl 2), p. 14. doi: 10.1111/ijpp.12417. 
$1^{\text {st }}$ Prize College of Human and Health Sciences Post-Graduate Conference, September 2017
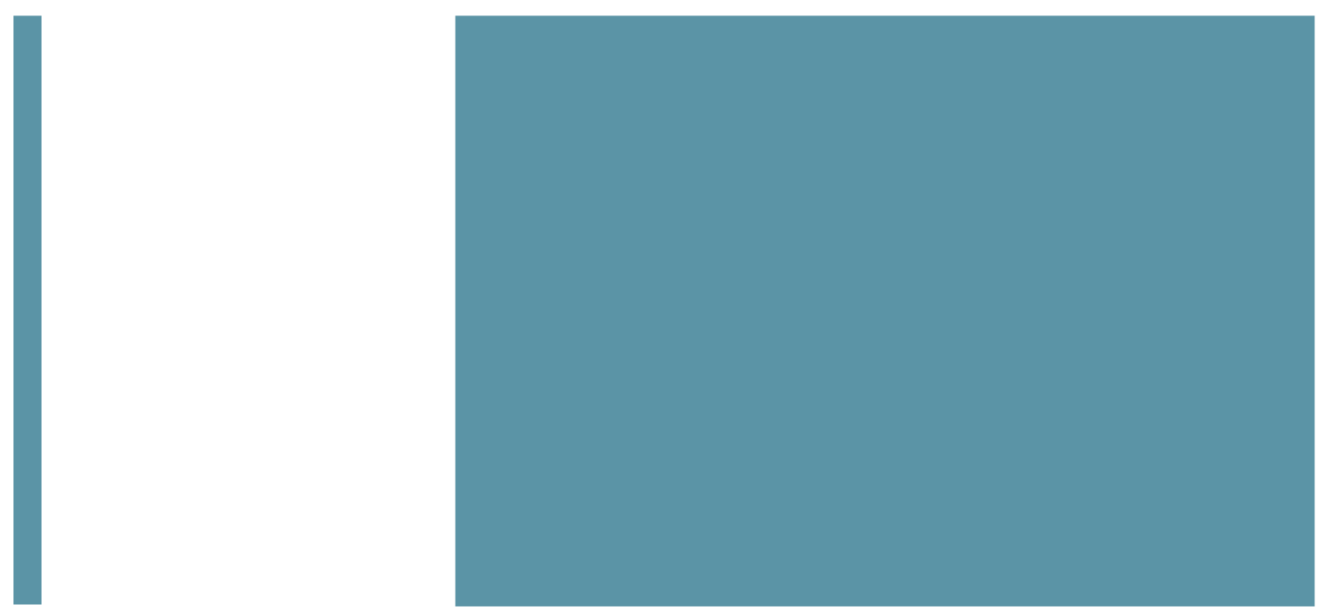

Trends in Opioid Prescribing and Associated Healthcare Utilisation in Wales (TOPAS)

Emma Davies

Pharmacy Research UK

"rebcunales 
Trends in Opioid Prescribing by Deprivation in a Primary Care Population: Results from the TOPAS* Study

"Trends in Opioid Prescrbing and Associaled Resource Utlisation in Wales

Emma Davies

College of Human and Health Science, Swarese University

\section{Introduction}

It has been estimated that up to $48 \%$ of the population in the United Kingdom (UK) live with pain ${ }^{1}$. Pain prevalence has been shown to be higher in areas of greatest socio-economic deprivation ${ }^{2}$. This section of the TOPAS study examines trends in opioid prescribing by deprivation across Wales, between 2005 -2015.

\section{Method}

Data on all prescriptions for opioid containing medications issued between 2005 and 2015, were taken from the Secure Anonymised Information Linkage databank (SAIL). People with a diagnosis for cancer during the study period were excluded from the detalled analysis. Annual number of prescriptions, numbers of patients and social deprivation were measured in repeated cross-sections, adjusted for population and analysed using descriptive statistics.

\section{Results}

There were significantly more people recelving opioid prescriptions in the areas of Wales with highest levels of deprivation (WIMD1) (One-way ANOVA: WIMD1 mean 183.6, $S D=11.1$, WIMD5 mean 105.1, $\left.S D=8.2, F(4,50)=109.6, p=.000, n^{2}=0.90\right)$. There were $75 \%$ more people recelving weak opioids and $80 \%$ more people receiving strong opioids in the most deprived areas compared to the least deprived areas of the country.

Nearly a third of all opioid prescriptions in Wales between 2005 and 2015, were issued in the most deprived areas. Those areas had a $347 \%$ increase in the annual number of strong opioid prescriptions and $140 \%$ more prescriptions issued than the least deprived areas of Wales. Weak opioid prescribing increased more in WIMD4 areas (lesser deprivation) although there were $140 \%$ more prescriptions issued in the areas of highest|deprivation compared to the areas with least.

\section{Conclusion}

The substantial increase in opioid prescribing and the concentration of it, within areas of high deprivation have been noted in other UK-based studies. The reasons for greater pain prevalence and consequently, higher rates of opioid prescribing in areas of socio-economic deprivation are not well understood. Some authors have postulated that prescribing may be a substitute for timely access to good quality pain services. 
Royal Pharmaceutical Society Wales Conference, 2018
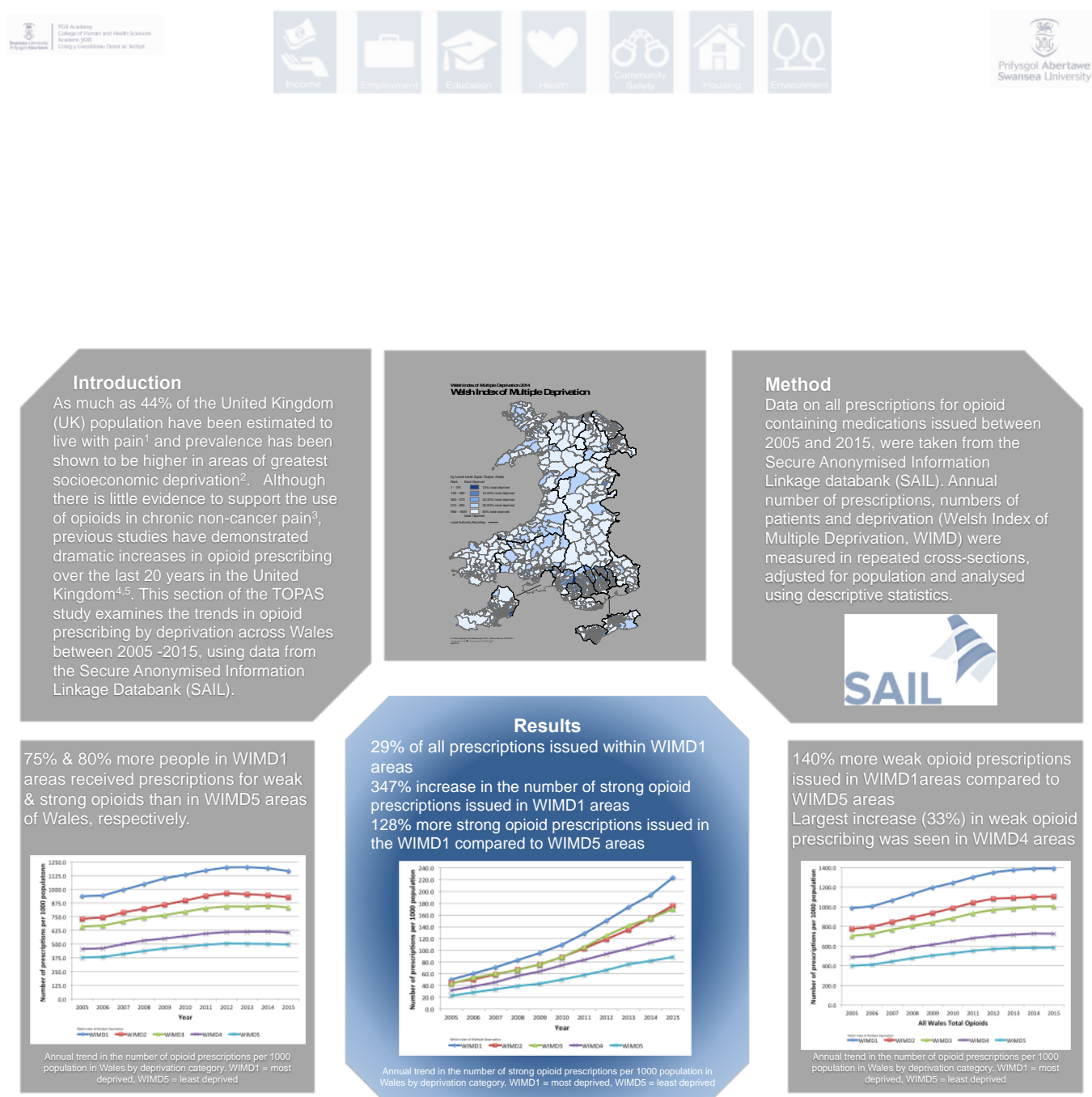

Results

Method

Data on all prescriptions for opioid

containing medications issued betwee

2005 and 2015, were taken from the

Secure Anonymised information

Linkage databank (SAIL). Annual

number of prescriptions, numbers of

patients and deprivation (Welsh Index o

Multiple Deprivation, WIMD) were

measured in repeated cross-sections,

using descriptive statistics.

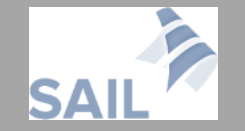

$29 \%$ of all prescriptions issued within WIMDi

$347 \%$ increase in the number of strong opioid prescriptions issued in WIMD1 areas prescriptions issued in the WIMD1 compared to WIMD5 areas
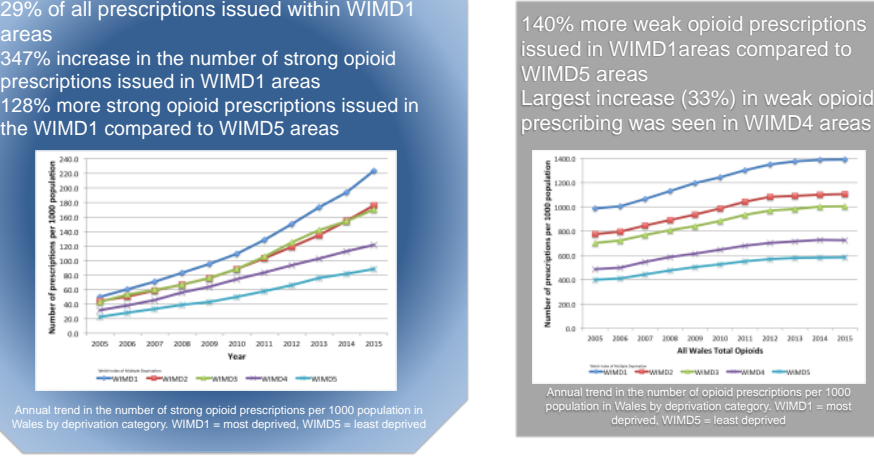
issued in WIMD1areas compared to WIMD5 areas

Largest increase $(33 \%)$ in weak opioid prescribing was seen in WIMD4 areas
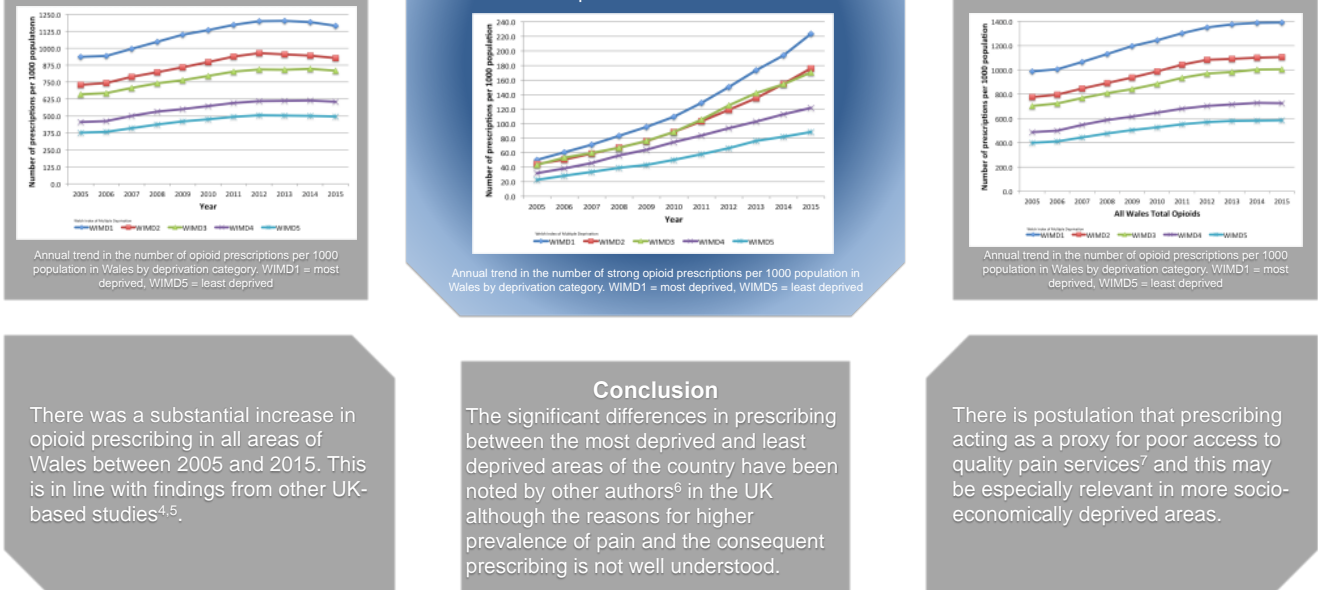

Conclusion The significant differences in prescribin between the most deprived and least deprived areas of the country have been noted by other authors ${ }^{6}$ in the UK although the reasons for higher prevalence of pain and the consequent prescribing is not well understood.

then
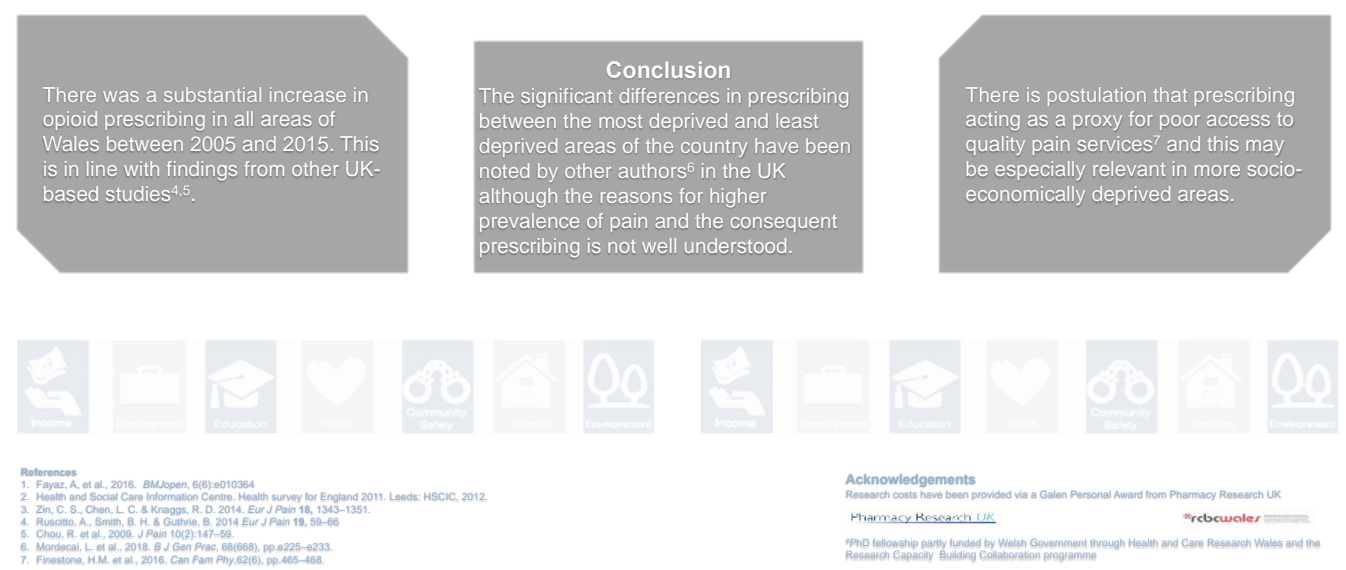


\title{
Examining patterns in opioid prescribing for non-cancer-related pain in Wales: preliminary data from a retrospective cross-sectional study using large datasets
}

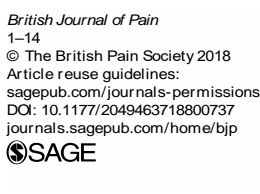

Emma Davies $^{1}$ (D), Ceri Phillips ${ }^{1,2}$, Jaynie Rance ${ }^{1}$ and Berni Sewell ${ }^{2}$

\begin{abstract}
Objectives: To examine trends in strong opioid prescribing in a primary care population in Wales and identify if factors such as age, deprivation and recorded diagnosis of depression or anxiety may have influenced any changes noted.

Design: Trend, cross-sectional and longitudinal analyses of routine data from the Primary Care General Practice database and accessed via the Secure Anonymised Information Linkage (SAIL) databank. Setting: A total of 345 Primary Care practices in Wales.

Participants: Anonymised records of $1,223,503$ people aged 18 or over, receiving at least one opioid prescription between 1 January 2005 and 31 December 2015 were analysed. People with a cancer diagnosis $(10.1 \%)$ were excluded from the detailed analysis.

Results: During the study period, $26,180,200$ opioid prescriptions were issued to 1,223,503 individuals (55.9\% female, $89.9 \%$ non-cancer diagnoses). The greatest increase in annual prescribing was in the $18-24$ age group $(10,470 \%)$, from 0.08 to 8.3 prescriptions $/ 1000$ population, although the $85+$ age group $18-24$ age group $(10,470 \%)$, from 0.08 to 8.3 prescriptions/ 1000 population, although the $85+$ age group
had the highest prescribing rates across the study period (from 149.9 to 288.5 prescriptions/1000 population). The number of people with recorded diagnoses of depression or anxiety and prescribed strong opioids increased from 1.2 to 5.1 people/ 1000 population (328\%). The increase was $366.9 \%$ in areas of highest deprivation compared to 310.3 in the least. Areas of greatest deprivation had more than twice the rate of strong opioid prescribing than the least deprived areas of Wales.

Conclusion: The study highlights a large increase in strong opioid prescribing for non-cancer pain, in Wales between 2005 and 2015. Population groups of interest include the youngest and oldest adult age groups and people with depression or anxiety particularly if living in the most deprived communities. Based on this evidence, development of a Welsh national guidance on safe and rational prescribing of opioids in chronic pain would be advisable to prevent further escalation of these medicines.

Summary points

This is the fir st large-scale, observational study of opioid prescribing in Wales.

Over 1 million individual, anonymised medical records have been searched in order to develop the study cohort, thus reducing recall bias.

Diagnosis and intervention coding in the Primary Care General Practice database is limited at input and may lead to under-reporting of diagnoses.
\end{abstract}

'College of Human and Health Scienc es, Swansea Univer sity, Swansea, UK

(t)

Thalth Sciences, Swansea University, Swansea, UK
Corresponding author:

Emma Davies, College of Human and Health Sciences, Swansea University, Singleton Park, Swansea SA2 8PP, UK.

Davies, E. et al. 2018. Examining patterns in opioid prescribing for non-cancer related pain in wales: preliminary data from a retrospective cross-sectional study using large datasets. British Journal of Pain 13(3), pp. 145-158. doi: $10.1177 / 2049463718800737$. 
$1^{\text {st }}$ Prize HSRRP Conference 2019, Birmingham University

GENDER-BASED TRENDS IN OPIOID PRESCRIBING IN A PRIMARY CARE

SAIL POPULATION WITH RECORDED DIAGNOSES OF DEPRESSION OR ANXIETY: Using a retrospective, cross-sectional analysis of a large dataset

Emma Davies

Background

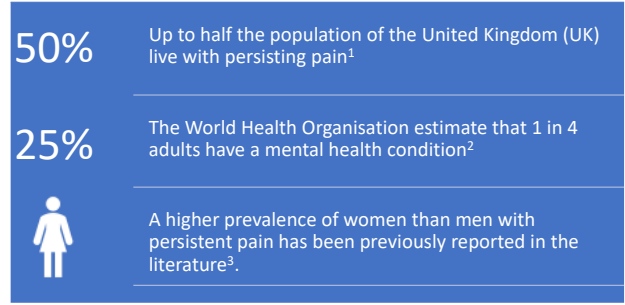

Method

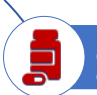

国
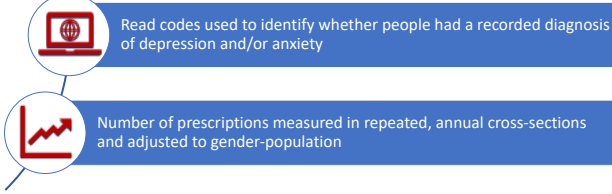

Number of prescriptions measured in repeated, annual cross-sections

Resulfis
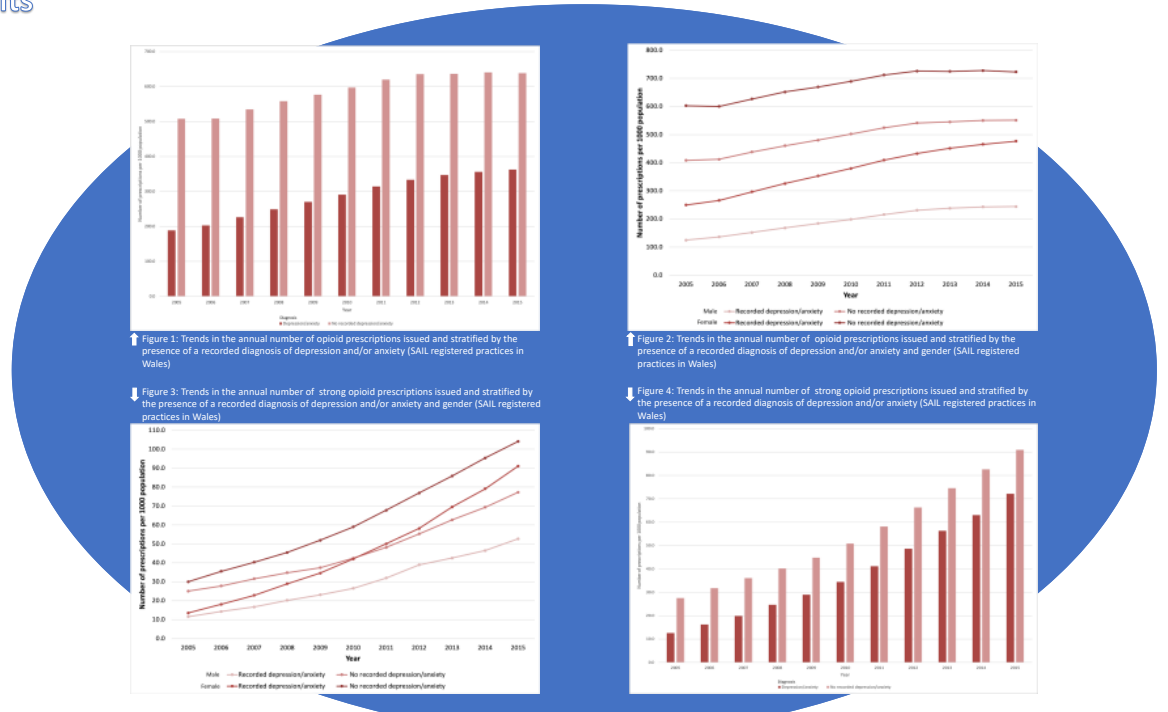

Resulits
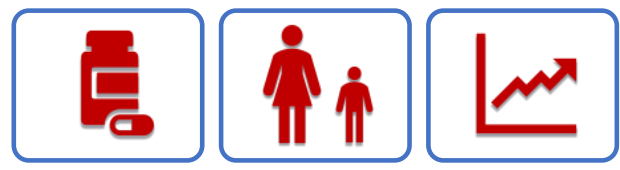

Discussion
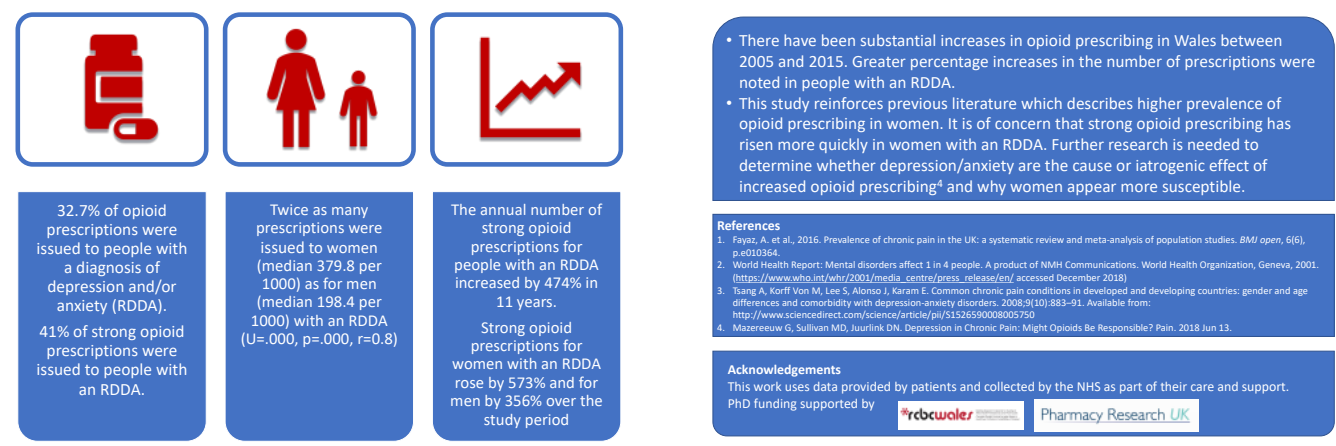

Davies, E. 2019. Gender-based trends in opioid prescribing in a primary care population with recorded diagnoses of depression or anxiety; using a retrospective, cross-sectional analysis of a large dataset - Poster Abstracts. International Journal of Pharmacy Practice 27(Supplement_2), pp. 27-60. Available at: https://academic.oup.com/ijpp/articlepdf/27/Supplement_2/27/36130946/ijpp12533.pdf. 
RCBC Conference May 2019

$1^{\text {st }}$ Prize College of Human and Health Sciences Post-Graduate Conference, June 2019

\section{$r$ \\ 政 \\ GENDER-BASED TRENDS IN OPIOID PRESCRIBING IN A PRIMARY CARE POPULATION WITH RECORDED DIAGNOSES OF DEPRESSION OR ANXIETY: \\ Using a retrospective, cross-sectional analysis of a large dataset \\ Emma Davies*, Berni Sewell**, Mari Jones**, Ceri Phillips*, Jaynie Rance* \\ * College of Human and Health Sciences, ** Swansea Centre for Health Economics}
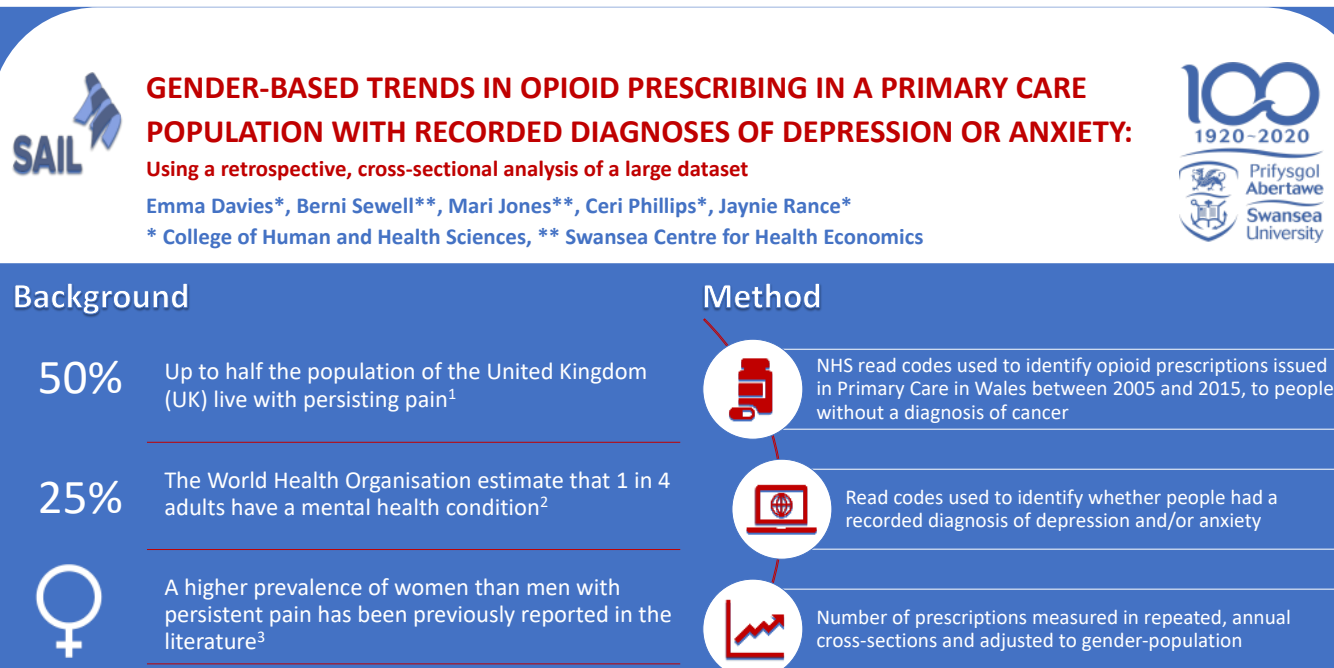

Method

Results

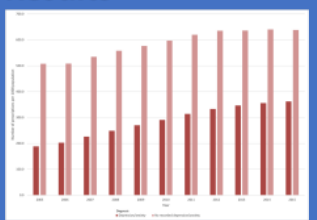

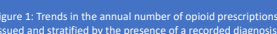

Results
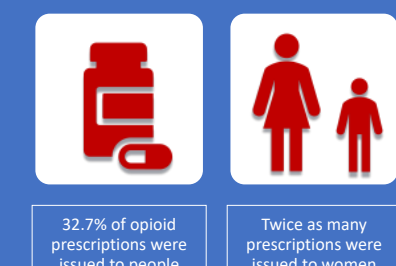
prescriptions were
issued to women (median 379.8 per
1000 ) as for men 1000) as for men
(median 198.4 per (median 198.4 per
1000) with an $\operatorname{RDDA}(U=.000$
$p=.000, r=0.8$
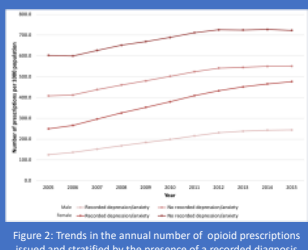

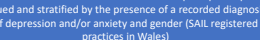

$32.7 \%$ of opioid
prescriptions were issued to people
with a diagnosis of with a diagnosis of
depression and/or anxiety (RoD

$41 \%$ of strong

opioid
prescriptions were

issued to people

with an RDDA

$\underbrace{\text { with an nDDA }}$
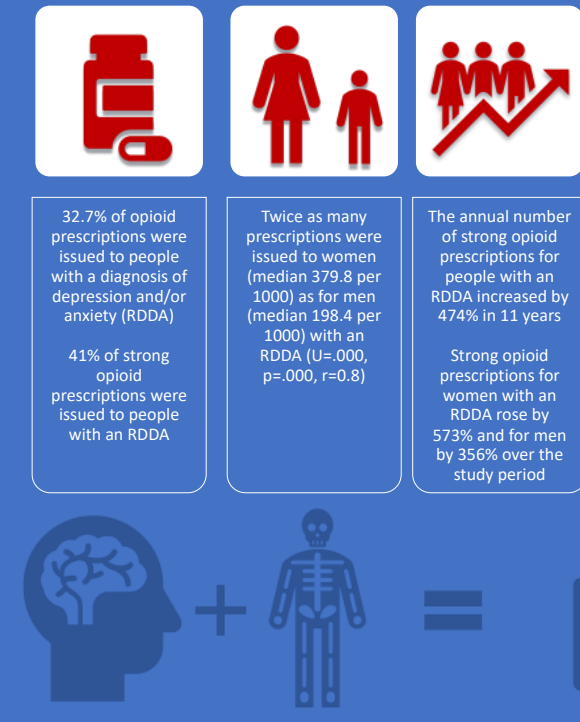

of strong opioid

of strong opioid
prescriptions for

people with an
RDDA increased by

$474 \%$ in 11 years

Strong opioid

Strong opioid
prescriptions for

women with an

RDDA rose by
$573 \%$ and for $m e$

$573 \%$ and for men
by $356 \%$ over the by $356 \%$ over the
study period

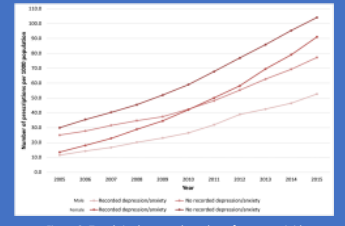

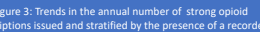

Discussion

There have been substantial increases in opioid prescribing in Wales between 2005 and 2015. Greater percentage increases in the number of prescriptions were noted in people with an RDDA

This study reinforces previous literature which describes higher prevalence of opioid prescribing in women. It is of concern that strong opioid prescribing has risen more quickly in women with an RDDA. Further research is needed to determine whether depression/anxiety are the cause or iatrogenic effect of increased opioid prescribing ${ }^{4}$ and why women appear more susceptible

Acknowledgements

This work uses data provided by patients and collected by the NHS as part of their care and support

PhD funding supported by Frclocwoles

References

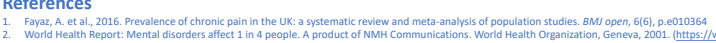


Oral Presentation, HSRPP conference 2020

consultation, using chi-squared tests. Any questionnaire free text comments were qualitatively explored for insight.

Resulfs: In the first six months of the service, 741 patients were referred to one of the community pharmacies enrolled in the service $(n-24)$ to provide advice $(15 \%)$ treatment with an over-the-counter product $(47 \%)$ of same day referral back to the GP $(14 \%)$. Six patients were
Introduction: Opioid prescribing has risen considerably in the UK over the last 20 years ${ }^{1}$ and with it, comes increased risks to population health ${ }^{2}$ Opioid dose is an indicator of potential harm, but understanding the bur den of different opioid medicines can be hampered by the numerous doses, strengths and products available. Aims: The study aimed to describe trends in prescribing of opioid analgesics for non-canoer pain acrose Wales

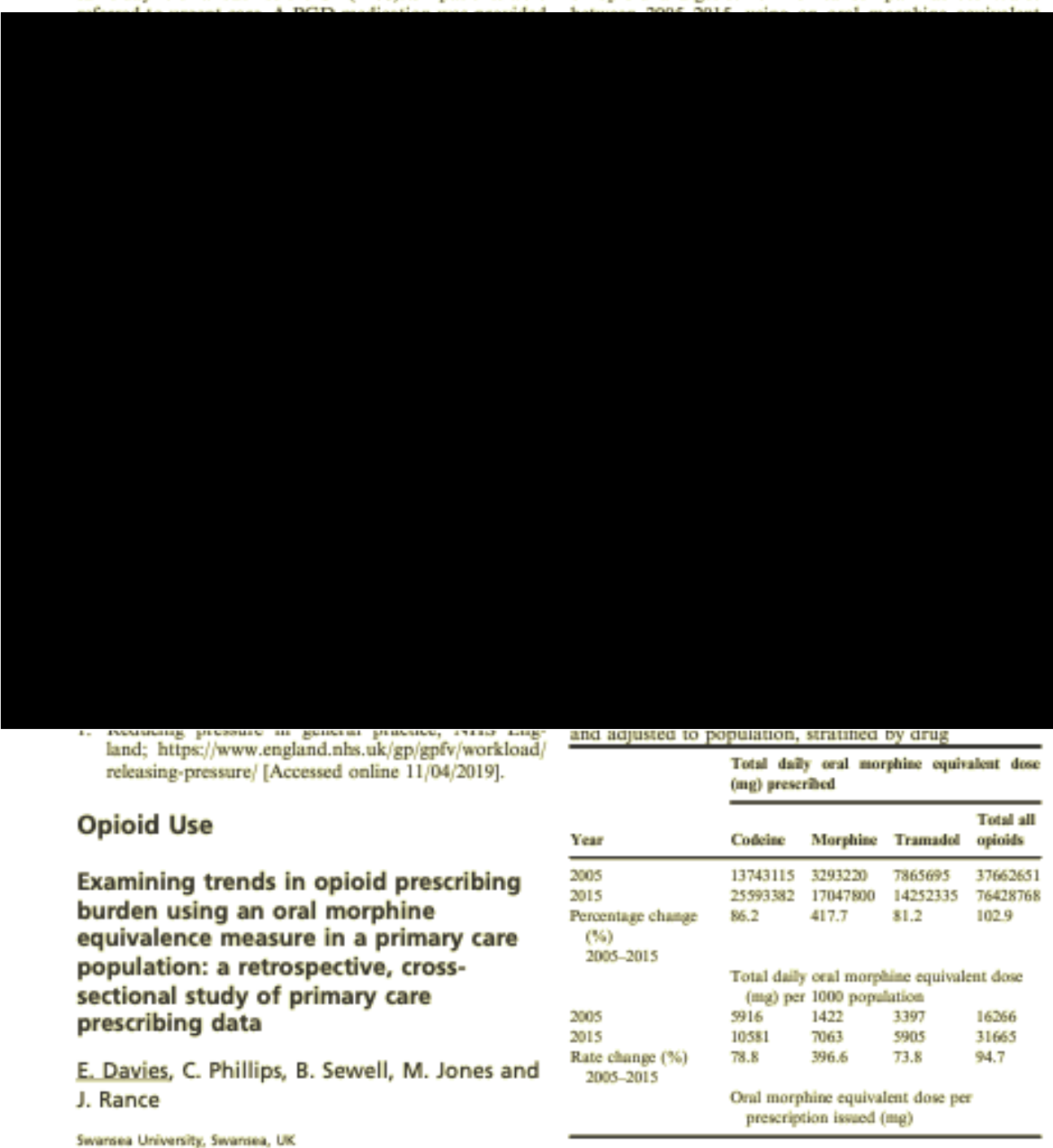

Swarsea Uriversity, Swariea, UK 
Table L. (Continued)

\begin{tabular}{|c|c|c|c|c|}
\hline \multirow[b]{2}{*}{ Year } & \multicolumn{4}{|c|}{$\begin{array}{l}\text { Total daily oral motphine equisuleat duse } \\
\text { (mig) prescribed }\end{array}$} \\
\hline & Cedeine & Merpaine & Tramadol & $\begin{array}{l}\text { Total all } \\
\text { apsiaids }\end{array}$ \\
\hline 2005 & 17 & 86 & 36 & 23 \\
\hline 2015 & 19 & 68 & 38 & 32 \\
\hline $\begin{array}{l}\text { Percentage change } \\
\text { (\%) } \\
2005-2015\end{array}$ & 16.2 & -20.6 & 5.7 & 35.5 \\
\hline
\end{tabular}

Conchusion: Large increases in OMED burden were
Many of these deaths include those who inject illici drugs, so may access NSP and people currently or recently in treatment for their addiction. Evidences sug. gest OST reduces both overdose and overall mortality but more needs to be done to prevent these deaths. UK guidance describes pharmacy practice to reduce overdose bat the extent to which such guidance is imple. mented is questionable ${ }^{1}$.

Ain: To describe CPs' self-reported adherence to guide: lines for preventing overdose deaths.

Methods: A cross-sectional quantitative telephone sur. vey was undertaken with CPs in England. A random stratified sampling technique was adapted to get repre. sentative sample from $6 \%$ of registered pharmacy pre

Davies, E. et al. 2020. Examining trends in opioid prescribing burden using an oral morphine equivalence measure in a primary care population: a retrospective, cross- sectional study of primary care prescribing data. International Journal of Pharmacy Practice 28(S1), pp. 9-10. Available at: https://onlinelibrary.wiley.com/doi/abs/10.1111/ijpp.12606. 


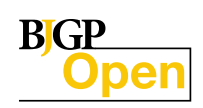

\section{Examining opioid prescribing trends for non-cancer pain using an estimated oral morphine equivalence measure: a retrospective cohort study between 2005 and 2015}

Emma Davies ${ }^{1,2 \star}$, Bernadette Sewell ${ }^{3}$, Mari Jones $^{4}$, Ceri J Phillips ${ }^{5}$, Jaynie Y Rance ${ }^{6}$

${ }^{1} \mathrm{PhD}$ Research Fellow, College of Human and Health Sciences, Swansea University, Swansea, UK; ${ }^{2}$ Advanced Pharmacist Practitioner in Pain Management, Pharmacy and Medicines Management, Cwm Taf Morgannwg University Health Board, Abercynon, UK; ${ }^{3}$ Senior Lecturer in Health Economics, Swansea Centre for Health Economics, Swansea University, Swansea, UK; ${ }^{4}$ Research Officer, Swansea Centre for Health Economics, Swansea University, Swansea, UK; ${ }^{5}$ Professor of Health Economics, College of Human and Health Sciences, Swansea University, Swansea, UK; ${ }^{6}$ Professor of Public Health, Policy and Social Sciences, College of Human and Health Sciences, Swansea University, Swansea, UK

*For correspondence: 878970@ swansea.ac.uk

Competing interest: The author declare that no competing

interests exist.

Received: 19 May 2020 Accepted: 22 June 2020 Published: 02 December 2020

(๑) This article is Open Access: $C C$ BY license (https://creativecommons.org/licenses/by/4.0/)

Author Keywords: primary health care, social deprivation, cohort studies, opioid prescribing, analgestics, opioid

Copyright $\odot$ 2020, The Authors;

DOI:10.3399/

bjgpopen20X101122

\section{Abstract}

Background: Over the past 20 years prescription of opioid medicines has markedly increased in the UK, despite a lack of supporting evidence for use in commonly occurring, painful conditions. Prescribing is often monitored by counting numbers of prescriptions dispensed, but this may not provide an accurate picture of clinical practice.

Aim: To use an estimated oral morphine equivalent $\left(\mathrm{OMEQ}_{\mathrm{e}}\right)$ dose to describe trends in opioid prescribing in non-cancer pain, and explore if opioid burden differed by deprivation status.

Design \& setting: A retrospective cohort study using cross-sectional and longitudinal trend analyses of opioid prescribing data from Welsh Primary Care General Practices (PCGP) took place. Data were used from the Secure Anonymised Information Linkage (SAIL) databank.

Method: An $\mathrm{OMEQ}_{\mathrm{e}}$ measure was developed and used to describe trends in opioid burden over the study period. $\mathrm{OMEQ}_{\mathrm{e}}$ burden was stratified by eight drug groups, which was based on usage and deprivation.

Results: An estimated 643436843 milligrams $(\mathrm{mg}) \mathrm{OMEQ}_{\mathrm{e}}$ was issued during the study. Annual number of prescriptions increased 44\% between 2005 and 2015, while total daily $\mathrm{OMEQ}_{\mathrm{e}}$ per 1000 population increased by $95 \%$. The most deprived areas of Wales had $100711696 \mathrm{mg}$ more OMEQ. prescribed than the least deprived over the study period.

Conclusion: Over the study period, $\mathrm{OMEQ}_{\mathrm{e}}$ burden nearly doubled, with disproportionate OMEQ prescribed in the most deprived communities. Using $\mathrm{OMEQ}_{\mathrm{e}}$ provides an alternative measure of prescribing and allows easier comparison of the contribution different drugs make to the overall opioid burden.

Davies, E. et al. 2020. Examining opioid prescribing trends for non-cancer pain using an estimated oral morphine equivalence measure: a retrospective cohort study between 2005 and 2015. BJGP Open 17(8), p. bjgpopen20X101122-13. Available at: http://bjgpopen.org/lookup/doi/10.3399/bjgpopen20X101122. 
Emma Davies $^{1} \square$, Ceri J Phillips ${ }^{1}$, Mari Jones ${ }^{2}$ and Bernadette Sewell ${ }^{1}$

\begin{abstract}
Objective: To examine differences in healthcare utilisation and costs associated with opioid prescriptions for non-cancer pain issued in primary care.

Method: A longitudinal, case-control study retrospectively examined Welsh healthcare data for the period 1 January 2005-31 December 2015. Data wer e extracted from the Secure Anonymised Information Linkage (SAIL) databank. Subjects, aged 18 years and over, were included if their primary care record contained at least one of six overarching pain diagnoses during the study period. Subjects were excluded if their record also contained a cancer diagnosis in that time or the year prior to the study period. Case subjects also received at least one prescription for an opioid analgesic. Controls were matched by gender, age, pain-diagnosis and socioeconomic deprivation. Healthcare use included primary care visits, emergency department (ED) and outpatient (OPD) attendances, inpatient (IP) admissions and length of stay. Cost analysis for healthcare utilisation used nationally derived unit costs for 2015. Differences between case and control subjects for resource use and costs were analysed and further stratified by gender, prescribing persistence (PP) and deprivation. Results: Data from 3,286,215 individuals were examined with 657,243 receiving opioids. Case subjects averaged 5 times more primary care visits, 2.8 times more OPD attendances, 3 times more ED visits and twice as many IN admissions as controls. Prescription persistence over 6 months and greater deprivation were associated with significantly greater utilisation of healthcare resources. Opioid prescribing was associated with $69 \%$ greater average healthcare costs than in control subjects. National Health Service (NHS) healthcare service costs for people with common, pain-associated diagnoses, receiving opioid analgesics were estimated to be £0.9billion per year between 2005 and 2015 .

Conclusion: Receipt of opioid prescriptions was associated with significantly greater healthcare utilisation and accompanying costs in all sectors. Extended prescribing durations are particularly important to address and should be considered at the point of initiation.

Keywords

Opioid analgesics, non-cancer pain, resource utilisation, healthcare costs, cost analysis
\end{abstract}

How this fits in

It is known that opioid analgesics can have long-term, harmful effects other than misuse and dependence. Previous studies examined the association between healthcare utilisation and the presence of opioid-induced adverse effects or misuse. This study examined the relationship between opioid prescribing for a range of pain-

\section{${ }^{1}$ College of Human and Health Sciences, Swansea University,} Swansea, UK

2Swane, UK for Health Economics, Swansea University College of Human and Health Sciences, Swansea, UK

Corresponding author:

Emma Davies, College of Human and Health Sciences, Swansea University, Singleton Park, Sketty Lane, Swansea, Wal UK.

Email: esdavies500@ymail.com

Davies E. et al. 2021 Healthcare resource utilisation and cost analysis associated with opioid analgesic use for non-cancer pain: A case-control, retrospective study between 2005 and 2015. Br J Pain 204946372110458 (2021) doi:10.1177/20494637211045898. 